\title{
Al1100 988046
}

NATL INST OF STANDARDS \& TECH R.IC.

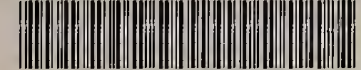

$$
\text { A11100988046 }
$$

Materials Research 

R.I.C. LOAN SLIP
INTERLIBRARY LEA 3061
PHONE $975 \quad$

DATE DUE: $\frac{6-25-91}{1804104}$

$\because$ Please return this publication by the date due, to the address below

DO NOT REMOVE THIS SLIP

National Institute of Standards and Tectroblogy

Research Information Center - IIL

india. Bldg; Room A-47

Gäthersuris, 10 id 20899 
<smiles>[CH]=C</smiles> 


\section{0th MATERIALS. \\ RESEARGH SYMPOSIUM}

\section{ON GHARAGTERIZATION OF HIGH TEMPERATURE VAPORS AND GASES}

\section{NBS SPECIAL PUBLICATION 561 Volume 2}

$Q C$

100

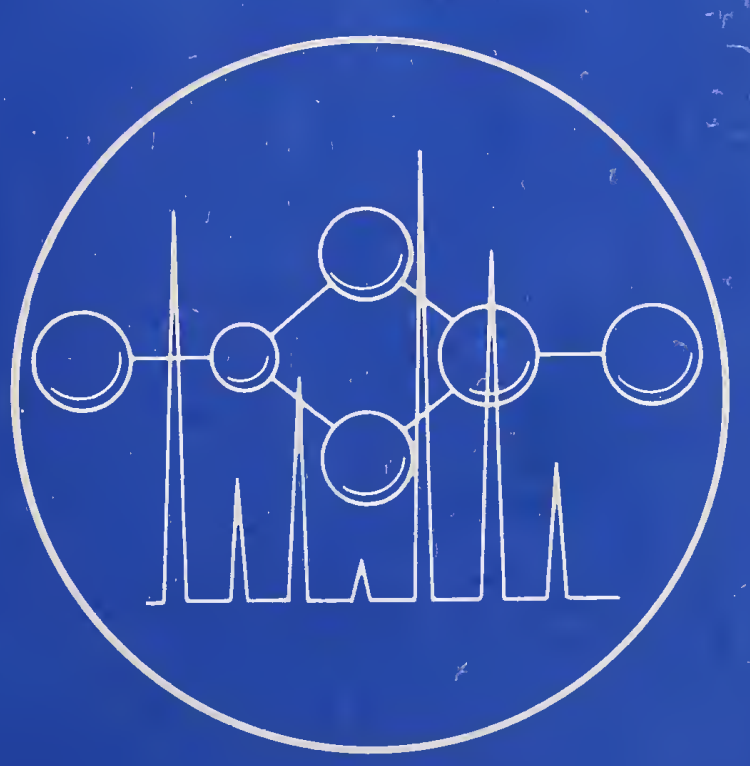

.457

N0561

V.2 


\section{NATIONAL BUREAU OF STANDARDS}

The National Bureau of Standards' was established by an act of Congress on March 3, 1901. The Bureau's overall goal is to strengthen and advance the Nation's science and technology and facilitate their effective application for public benctit. To this end, the Bureau conderts research and provides: (1) a basis for the Nation's physical measurement system, (2) scientilic and technological services for industry and government, (3) at technical basis for equity in trade, and (4) technical services to promote public safety. The Bureau's technical work is performed by the National Measurement Laboratory, the National Engincermg Laboratory, and the Institute for Computer Sciences and Technology.

THE NATIONAL MEASUREMENT LABORATORY provides the nation.l system of physical and chemical and materials measurement; coordinates the system with measurement systems of other nations and furnishes essential services leading to accurate and unitorm physical and chemical measurement throughout the Nation's scientific community, industry, and commerce; conducts materials research leading to improved methods of measurement, standards, and data on the properties of materials needed by industry, commerce, educational institutions, and Government; provides advisory and research services to other Government agencies: develops, produces, and distributes Standard Reterence Materials; and provides calibration services. The Laboratory consists of the following centers:

Absolute Physical Quantities² - Radiation Research - Thermodynamics and

Molecular Science - Analytical Chemistry - Materials Science.

THE NATIONAL ENGINEERING LABORATORY provides technology and technical services to the public and private sectors to address nattonal needs and to solve nattonal problems: conducts research in engineering and applied science in support of these cflorts; builds and maintains competence in the necessary disciplines required to carry out this research and technical service; develops enginecring data and meastrement capabilities; provides engineering measurement traceability vervices; develops test methods and proposes engincering standards and code changes; develops and proposes new engineering practices: and develops and improves mechanisms to transler results of its rescarch to the ultimate user. The Laboratory consists of the following centers:

Applied Mathematics - Electronics and Electrical Enginecring ${ }^{2}$ - Mechanical

Engineering and Process Technology $y^{2}$ - Building Technology - Fire Research -

Consumer Product Technology - Field Methods.

THE INSTITUTE FOR (OMPLTER SCIENCES AND TECHNOIOCYY conducts research and provides scientific and technical services to aid $\mathrm{t}$ ederal atgencies in the sclection, acquisition, application, and use of computer technology to improve cllectiveness and economy in Government operations in accordance with Public Law 89-306 (40 U.S.C. 759), relevant Fxecutive Orders, and other directives; carries out this mission by managing the Federal Information Processing Standards Program, developing Federal ADP standards guidelines, and managing Federal participation in ADP voluntary standardization activities; provides scientific and technological advisory services and assistance to Federal agencies; and provides the technical foundation for computer-related policies of the Federal Government. The Institute consists of the following centers:

Programning Science and Technology - Computer Systems Engmeering.

'Headquarters and Laboratories at Gitithersburg, MD, unless otherwise noted: mailing address Washington, DC 20234.

Some divisions within the center are located at Boulder, CO 80303. 


\section{CHARACTERIZATION OF HIGH TEMPERATURE VAPORS AND GASES \\ Volume 2}

Proceedings of the 10th

Materials Research Symposium

held at the National Bureau of Standards

Gaithersburg, Maryland

September 18-22, 1979

Edited by:

John W. Hastie

Center for Materials Science

National Measurement Laboratory

National Bureau of Standards

Washington, DC 20234

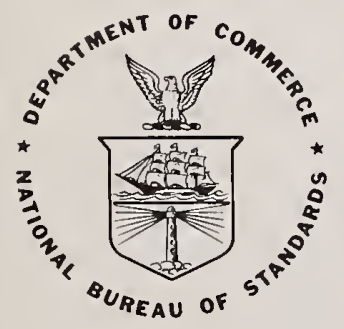

U.S. DEPARTMENT OF COMMERCE, Juanita M. Kreps, Secretary

Luther H. Hodges, Jr., Under Secretary

Jordan J. Baruch, Assistant Secretary for Science and Technology

NATIONAL BUREAU OF STANDARDS, Ernest Ambler, Director

Issued September 1979 


\section{Library of Congress Catalog Card Number: $79-600152$}

\section{National Bureau of Standards Special Publication 561/2}

Nat. Bur. Stand. (U.S.) Spec. Publ. 561/2, 895 pages (Sept. 1979)

CODEN: XNBSAV

\section{U.S. GOVERNMENT PRINTING OFFICE}

WASHINGTON: 1979

For sale by the Superintendent of Documents, U.S. Government Printing Office, Washington, D.C. 20402 Stock No. 003-003-02124-5 Price $\$ 20$. (Sold in sets only) 
During the week of September 18-22, 1978, the 10th Materials Research Symposium was held at NBS, Gaithersburg, on the subject of "Characterization of High Temperature Vapors and Gases". The Symposium aim was to assess the state-of-the-art and future directions and characterization methods for high temperature vapors including, where appropriate, gases and, to a lesser extent, plasmas. Modern technology increasingly requires materials and processes to function at high temperatures--a condition where the vapor, gaseous or plasma phase becomes significant. For example, the future success of new energy technologies such as coal-fired magnetohydrodynamic generators, coal gasification, and nuclear fusion depends largely on materials performance in hot chemically reactive gaseous atmospheres. Characterization of such atmospheres, or components thereof, is a challenging problem requiring adaptation of existing and development of new experimental and theoretical techniques. An assessment of these techniques for application in modern science and technology had not been previously attempted. The Symposium addressed this question by assembling internationally recognized experts in the measurement science and technology of high temperature vapors, gases, flames, and, to a lesser extent, plasmas. Key foreign laboratories, including those of the USSR, France, England, Japan, West Germany, Canada and Mexico are represented in the formal proceedings. In the United States, academic, industrial and Government institutions each made a substantial contribution to the Symposium.

From this Symposium, a group of invited and pertinent contributed papers were selected for publication. Also, in order to prevent, as far as possible, serious omissions of important new or improved techniques a number of papers not delivered at the Symposium were solicited for inclusion in this publication. Each paper has been subjected to a critical review process. Further, those papers delivered verbally at the Symposium were discussed by the attendant body and the edited discussion included at the end of each paper. I believe that this interactive process between authors, reviewers, symposium attendees, and the editor has resulted in a valuable published account of the current status of "Characterization of High Temperature Vapors and Gases."

The discussion following most of the papers in these volumes was prepared as follows. During the Symposium both written (question and answer forms) and taped discussion material.was generated. Editing of this material was designed mainly to eliminate remarks that were adequately covered in the main text. Additional constraints were imposed by the usual problems of incoherence and human error in taping. A light handed approach was used in the editing process in order to retain something of the conference atmosphere and the personalities involved. Those scientists involved in the discussion process may be located by reference to the list of Symposium Participants. 
These volumes deal with both classical and recently developed experimental techniques, ranging from the historically significant and still active area of Knudsen effusion to sophisticated spectroscopic methods such as Coherent AntiStokes Raman Spectroscopy ${ }^{1,2}$. What results from such developments is the generation of new basic data and diagnostic techniques for monitoring complex laboratory or real-1ife systems ${ }^{3}$. Such data and measurement tools provide the necessary ingredients to develop and validate computerbased models of complex technologically important systems such as combustion, corrosion, materials preparation and so on. Though the main emphasis of these volumes is on characterization methods, a useful number of examples of application to practical problems through modeling have been included.

Researchers concerned both with the basic properties of hot vapors and gases and the applied problems resulting from the extremes of temperature, pressure and chemical activity should find these volumes of particular interest. Throughout, the authors have made an effort to cite useful follow-up references and I have listed here additional major reference sources representing the past decade of research in this or related areas ${ }^{4}$.

It is a particular pleasure to acknowledge the following members of the Symposium Review Committee who willingly and enthusiastically assisted with the important review process; in alphabetical order they are: Dr. Dave Bonne11, Dr. Ray Di1s, Dr. Mike Drake, Dr. Dick Gann, Dr. Bob Hauge, Dr. Bill Horton, Dr. Marilyn Jacox, Mr. Ralph Krause, Dr. Ken McCulloh, Dr. Ernie Plante, Dr. Gerd Rosenblatt, and Dr. Pete Schenck. The mammoth task of formatting, typing and proofing the manuscripts was managed primarily by the NML Text Editing Facility. other conference management activities were expertly arranged by the offices of Mr. Ron Johnson and Joanne Lordon.

\footnotetext{
${ }^{1}$ In the interests of accuracy and clarity in describing various items of equipment or apparatus, mention has been made of commercial sources or brand names. This in no way implies endorsement of such products by the U.S. Government.
} 
2Units: Several systems of units are used in this publication. Conversion factors to the International System of Units (SI) may be found in the representative publications listed below. The more common units and their conversion factors, as used in this publication are as follows.

Physical Quantity

Unit Equivalent SI Unit

\begin{tabular}{lc}
\hline length & inch \\
length & angstrom \\
mass & pound \\
mass & atomic mass unit \\
force & kilogram-force \\
pressure & atmosphere \\
pressure & torr \\
energy & calorie(thermochem.) \\
energy & electron volt \\
temperature & Celsius
\end{tabular}

$2.54 \times 10^{-2} \mathrm{~m}$ $10^{-10} \mathrm{~m}$

$0.45359237 \mathrm{~kg}$

$21.66041 \times 10^{-27} \mathrm{~kg}$

$9.80665 \mathrm{~N}$

$101325 \mathrm{~N} \mathrm{~m}^{-2}$

$(101325 / 760) \mathrm{N} \mathrm{m}^{-2}$

$4.184 \mathrm{~J}$

$\sim 1.6021 \times 10^{-19} \mathrm{~J}$

kelvin - 273.15

References: Manual of Symbols and Terminology for Physiochemical Quantities and Units, Pure and Applied Chemistry 21, 1-44 (1970). Policy for NBS Usage of SI Units, J. Chem. Ed. 48, 569-572 (1971). SI Units in Physico-Chemical Calculations, A. C. Norris, J. Chem. Ed. $\underline{48}, 797-800$ (1971).

${ }^{3} \mathrm{~A}$ recurrent theme throughout these proceedings is the very limited bank of basic high temperature data, and the widespread usage of the JANAF Thermochemical Tables.

${ }^{4}$ High Temperature Vapors and Gases--Major References:

1975

1967

1967-1971

Current

1976
High Temperature Vapors (J. W. Hastie; Academic Press, New York) Characterization of High Temperature Vapors (J. Margrave, ed., Wiley, New York). Advances in High Temperature Chemistry (series, Academic Press, New York) Symposium (Int.) Combustion (Combustion Inst., Pittsburgh, Pennsylvania). Combustion Measurements (R. Goulard, ed., Academic Press, New York). 



\section{WELCOME}

Good morning. I am pleased to have this opportunity to welcome the participants in this Symposium on "Characterization of High Temperature Vapors and Gases." This is the first conference of its kind devoted to the important measurement problems associated with the high temperature gaseous phase. Our charter as the National Measurement Laboratory makes it quite appropriate that we host this unique conference.

Some of you may be visiting NBS for the first time and may not be familiar with the range of activities at the Bureau. I would like to take a few minutes to describe some of our activities.

The Bureau was established in 1901 to develop and disseminate the national standards of measurement, to determine physical constants and properties of important materials, to develop test methods, to aid in the establishment of standard practices, and to provide technical services to other government agencies. Recent legislation has given NBS new and specific responsibilities in the areas of materials conservation, energy conservation, computer technology, standard reference data, and fire research. A new organizational structure was implemented on April 9, 1978 at NBS. Presently, the work of the Bureau is carried out in three major organizational units: The National Measurement Laboratory, the National Engineering Laboratory, and the Institute for Computer Science and Technology. Measurements, standard practices, and accurate data are common themes in all of our work, and we are perhaps best described as the nation's measurement laboratory.

NBS also has a long history of involvement in characterizing hot vapors and gases. Examples of notable past activities include the early work of Broida on flame spectroscopy, the application of matrix isolation methods to high temperature species as carried out by Milligan and Jacox, the development of specialized microwave spectroscopic techniques for structural determinations of high temperature species by Lide and coworkers, and the deve1opment of metal vapor pressure standards by Paule and associates. Current NBS interests in this area range from understanding flame inhibition to the thermodynamic characteristics of slag vapors important in the development of new magnetohydrodynamic and coal gasification technologies.

In recent years this field has seen rapid development, as evidenced by the variety and number of papers to be delivered this week. In order that the reports of these new activities receive rapid and widespread attention the conference committee and support staff has made every effort to ensure a rapid publication of the proceedings. I believe that this conference will enhance greatly the understanding of high temperature gases and vapors and their importance in developing new technologies. Once again, welcome to NBS, Gaithersburg and have a good meeting.

John D. Hoffman, Director

National Measurement Laboratory

National Bureau of Standards, 
This book is based on the proceedings of the 10th Materials Research Symposium, held at the National Bureau of Standards, Gaithersburg, Maryland, September 18-22, 1978. The symposium was sponsored by the National Measurement Laboratory (formerly Institute for Materials Research). Modern technology increasingly requires materials and processes to function at high temperatures--a condition where the vapor, gaseous or plasma phase becomes significant. Characterization of such atmospheres, or components thereof, is a challenging problem requiring adaptation of existing and development of new experimental and theoretical techniques. The aim of the symposium was to assess the state-of-the-art and future directions and characterization methods for high temperature vapors including, where appropriate, gases and, to a lesser extent, plasmas. In order to prevent, as far as possible, serious omissions of important new or improved techniques, a number of papers not delivered at the symposium were solicited for inclusion in this publication. A total of eighty-six papers (invited and contributed) or chapters representing nine countries are contained in these volumes. The discussion, which followed most of the delivered papers, is also included.

Key words: Combustion; equilibrium; high temperature; isolation; lasers; mass spectrometry; matrix; modeling; vapors. 
Preface

Welcome

J. D. Hoffman . . . . . . . . . . . . . . . . . . . . . vi

Abstract ............................................. vii

VOLUME I

I. CLASSICAL VAPORIZATION METHODS

THE EFFUSION METHOD AT AGE 69: CURRENT STATE OF THE ART . . . . . . . . . . .

E. David Cater

GENERAL THEORETICAL ANGULAR NUMBER DISTRIBUTION FUNCTIONS OF KNUDSEN FLOW THROUGH THE FOLLOWING SINGLE AND DOUBLE-SLIT ORIFICE GEOMETRIES: CIRCULAR, RECTANGULAR, AND CYLINDRICAL . . . . . . . . . . . . . . . . . . .

F. Miller

AUTOMATIC DATA ACQUISITION BY THE TORSION-EFFUSION METHOD . . . . . . . . . . .

J. G. Edwards

APPLICATION OF LASER PULSE HEATING FOR THE STUDY OF HIGH TEMPERATURE

VAPORS, PHASE TRANSITIONS AND EQUATION OF STATE . . . . . . . . . . . . . . . .

R. W. Ohse, J. F. Babelot, L. Cercignani, P. R. Kinsman, K. A. Long,

J. Magill, and A. Scotti

A HIGH TEMPERATURE TRANSPIRATION APPARATUS FOR THE STUDY OF THE ATMOSPHERE ABOVE VISCOUS INCONGRUENTLY VAPORIZING MELTS . . . . . . . . . . . . . . . . .

D. M. Sanders and W. K. Haller

VAPORIZATION THERMODYNAMICS AND KINETICS OF HEXAGONAL SILICON CARBIDE

R. G. Behrens and G. H. Rinehart

A VERY ACCURATE PYROMETER FOR GENERAL LABORATORY USE . . . . . . . . . . . . .

E. K. Storms and B. A. Mueller

OPERATION OF NEAR IDEAL HEAT PIPES . . . . . . . . . . . . . . . . . . . . .

L. A. Melton

II. MASS SPECTROMETRIC TECHNIQUES--KNUDSEN EFFUSION

ATTAINMENT OF CHEMICAL EQUILIBRIUM IN EFFUSIVE BEAM SOURCES OF THE HETEROGENEOUS REACTION TYPE

D. L. Hildenbrand

THERMODYNAMIC AND PHYSICO-CHEMICAL BEHAVIOR OF THE INTERACTIONS BETWEEN KNUDSEN-EFFUSION-CELLS AND THE SYSTEMS UNDER INVESTIGATION. ANALYSIS BY HIGH TEMPERATURE MASS SPECTROMETRY . . . . . . . . . . . . . . . . .

C. Chatillon, M. Allibert, and A. Pattoret

\footnotetext{
${ }^{1}$ The grouping of papers is based primarily on the method rather than the system under study. Papers having either a fundamental or review emphasis are usually grouped at the beginning of a section.
} 
CHARACTERIZATION OF HIGH TEMPERATURE VAPORS BY ANGULAR DISTRIBUTION MASS SPECTROMETRY

R. T. Grimley and J. A. Forsman

THE HIGH TEMPERATURE VAPORIZATION AND THERMODYNAMICS OF THE TITANIUM OXIDES. XIV. RELATIVE IONIZATION CROSS-SECTIONS OF Ti, TiO, AND $\mathrm{TiO}_{2}$

R. I. Sheldon and P. W. Gilles

CONGRUENT VAPORIZATION AND THERMODYNAMICS IN THE PRASEODYMIUM-SULFUR SYSTEM

E. D. Cater, B. H. Mueller, and J. A. Fries

VAPOR PRESSURE MEASUREMENTS OF POTASSIUM OVER $\mathrm{K}_{2} \mathrm{O}-\mathrm{SiO}_{2}$ SOLUTIONS BY A KNUDSEN EFFUSION MASS SPECTROMETRIC METHOD .

E. R. Plante

MASS SPECTROMETRIC INVESTIGATION OF VAPOR SPECIES OVER LIQUID GERMANIUM DIOXIDE

T. Sasamoto, M. Kobayashi, and T. Sata

MASS SPECTROMETRIC DETERMINATION OF ATOMIZATION ENERGIES OF INORGANIC MOLECULES AND THEIR CORRELATION BY EMPIRICAL MODELS OF BONDING

K. A. Gingerich

III. MASS SPECTROMETRIC TECHNIQUES--HIGH PRESSURE SAMPLING

HIGH PRESSURE MOLECULAR BEAM MASS SPECTROMETRIC SAMPLING OF HIGH TEMPERATURE MOLECULES

C. A. Stearns, F. J. Kohl, G. C. Fryburg, and R. A. Miller

TRANSPIRATION MASS SPECTROMETRY OF HIGH TEMPERATURE VAPORS

D. W. Bonnell and J. W. Hastie

IN SITU ANALYSIS OF MINOR GASEOUS SPECIES IN CRYSTAL GROWTH AMBIENTS USING MOLECULAR BEAM MASS SFECTROMETRY . . . . . . . . . . . . . . . . . .

H. Dun, B. L. Mattes, and D. A. Stevenson

MOLECULAR BEAM MASS SPECTROMETRIC SAMPLING OF MINOR SPECIES FROM COAL DUST-AIR FLAMES

F. T. Greene, J. E. Beachey, and T. A. Milne

THE USE OF FLAMES AS MEDIA FOR THE STUDY OF ION-MOLECULE THERMOCHEMISTRY . . .

W. J. Miller

MASS SPECTROMETRIC DETERMINATION OF NEGATIVE AND POSITIVE ION CONCENTRATIONS IN COAL-FIRED MHD PLASMAS ........................

J. Wormhoudt and C. E. Kolb

MASS SPECTROMETRIC SAMPLING OF CONDENSED SYSTEM FLAMES ${ }^{2}$

O. P. Korobeinichev and A. G. Tereshenko

\footnotetext{
${ }^{2}$ Not presented at Symposium.
} 
IV. MOLECULAR STRUCTURE DETERMINATION--MATRIX ISOLATED SPECIES

MATRIX ISOLATION STUDIES OF HIGH TEMPERATURE SPECIES

R. H. Hauge and J. L. Margrave

THE CHARACTERIZATION OF HIGH TEMPERATURE MOLECULES USING MATRIX ISOLATION

AND ISOTOPE FINE STRUCTURE . . . . . . . . . . . . . . . . . . . .

J. S. Ogden

MATRIX ISOLATION OF HIGH TEMPERATURE SPECIES AND MATRIX PERTURBATION EFFECTS .

F. W. Froben

MATRIX ISOLATION STUDY OF THE VIBRATIONAL AND ELECTRONIC SPECTRA OF PO AND HPO

M. Larzilliere and M. E. Jacox

ESR MATRIX ISOLATION OF ATH ${ }^{+}$FORMED DURING THE HIGH TEMPERATURE VAPORIZATION OF $\mathrm{Al}_{2} \mathrm{O}_{3}$

L. B. Knight, Jr., R. L. Martin and E. R. Davidson

MATRIX ISOLATION STUDIES OF MAGNESIUM AND IRON ATOM REACTIONS WITH WATER

R. H. Hauge, S. E. Gransden, J. W. Kauffman, and J. L. Margrave

PHOTOSELECTIVE BIMETALLIC AGGREGATION: A NEW ROUTE TO BIMETALLIC CLUSTERS . . .

W. E. Klotzbucher and G. A. Ozin

TRANSITION-METAL MOLECULES AND WALSH'S RULES--RATIONALIZATION OF OPTICAL

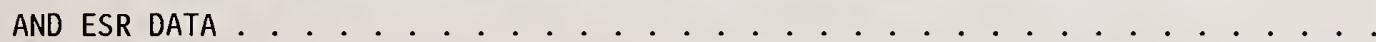

W. Weltner, Jr.

V. MOLECULAR STRUCTURE DETERMINATION--VAPOR SPECIES

THE DETERMINATION OF THE MOLECULAR SHAPES OF 'HIGH TEMPERATURE' SPECIES . . .

I. Beattie and D. A. Greenhalgh

RAMAN SPECTROSCOPY IN HIGH TEMPERATURE CHEMISTRY . . . . . . . . . . . . .

609

M. C. Drake and G. M. Rosenblatt

RESONANCE RAMAN SPECTRA OF METAL HALIDE VAPOR COMPLEXES

G. N. Papatheodorou

INFRARED SPECTRAL MEASUREMENTS OF SPECIES IN A HIGH TEMPERATURE FLOW

TUBE

M. E. Gersh and C. E. Kolb

ROTATIONAL SPECTROSCOPY OF HIGH TEMPERATURE MOLECULES ${ }^{3}$

T. Torring and E. Tiemann

MODERN HIGH-TEMPERATURE ELECTRON DIFFRACTION ${ }^{3}$

V. P. Spiridonov and E. Z. Zasorin

PHOTOIONIZATION MASS SPECTROMETRY AND PHOTOELECTRON SPECTROSCOPY OF HIGH TEMPERATURE VAPORS

J. Berkowitz

${ }^{3}$ Not presented at Symposium. 
THE PHOTOELECTRON SPECTROSCOPIC CHARACTERIZATION OF VAPORS OVER HEATED INORGANIC SOLIDS . . . . . . . . . . . . . . . . . . . . .

R. A. Bowling, T. W. Lassiter, D. 0. Vick, and G. K. Schweitzer $\underline{A B}$ INITIO MOLECULAR ORBITAL STUDIES OF SOME HIGH TEMPERATURE METAL HALIDE COMPLEXES

L. A. Curtiss SYMPOSIUM PARTICIPANTS . . . . . . . . . . . . . . . . . . . 803 AUTHOR INDEX

VOLUME II

VI. SPECTROSCOPIC DIAGNOSTIC TECHNIQUES--SPECIES CONCENTRATION

NEW OPTICAL DIAGNOSTIC TECHNIQUES FOR TEMPERATURE AND SPECIES CONCENTRATION MEASUREMENTS IN FLAMES

J. R. McDonald, A. P. Baronavski, L. Pasternack, S. Lamont, and A. B.

Harvey

LASER-ABSORPTION METHODS FOR CHARACTERIZING HIGH TEMPERATURE GASES . . . . . .

W. C. Gardiner, Jr.

NEAR-RESONANCE RAYLEIGH SCATTERING AND ATOMIC FLAME FLUORESCENCE SPECTROSCOPY

C. Chan and J. W. Daily

LASER INDUCED REACTIONS OF LITHIUM IN FLAMES

C. H. Muller, III, K. Schofield, and M. Steinberg OPTO-GALVANIC SPECTROSCOPY: A NEW LOOK AT ATOMS AND MOLECULES

K. C. Smyth, P. K. Schenck, W. G. Mallard, and J. C. Travis $\mathrm{SiCl}_{2}$ FLUORESCENCE AS A CHEMICAL VAPOR DEPOSITION REACTOR PROBE

T. O. Sedgwick and G. U. Arbach

RAMAN SCATTERING OF THE GASEOUS PHASE AT HIGH TEMPERATURE

R. Hillel, J. Bouix, and A. Machaelides

VII. SPECTROSCOPIC DIAGNOSTIC TECHNIQUES AND TEMPERATURE MEASUREMENT

DIAGNOSTIC TECHNIQUES IN COMBUSTION MHD FLOWS

S. A. Self

CARS DIAGNOSTIC INVESTIGATIONS OF FLAMES

A. C. Eckbreth and R. J. Hall

COMPARISON OF THERMOCOUPLE, GAS SAMPLING AND RAMAN MEASURED TEMPERATURES IN AN AFTERBURNING TURBOJET ENGINE PLUME

W. M. Roquemore and P. O. Yaney

USE OF THERMOCOUPLES FOR GAS TEMPERATURE MEASUREMENTS IN GAS TURBINE COMBUSTORS

R. R. Dils and P. S. Follansbee 
MOVING THERMOCOUPLE MEASUREMENTS OF HEAT TRANSFER IN HOT GASES . . . . . . . .

L. W. Hunter, C. H. Hoshall, C. Grunfelder, and R. M. Fristrom APPLICATION OF THE TWO LINE ATOMIC FLUORESCENCE TECHNIQUE TO THE TEMPORAL MEASUREMENT OF SMALL VOLUME FLAME TEMPERATURES . . . . . . . . . . . .

J. Bradshaw, J. Bower, S. Weeks, K. Fujiwara, N. Omenetto, H. Haraguchi, and J. D. Winefordner

A COMPARISON OF SPECTROSCOPIC FLAME TEMPERATURE MEASUREMENTS: Na-D LINE REVERSAL, ROTATIONAL AND VIBRATIONAL RAMAN, AND OH ABSORPTION SPECTROSCOPY . .

M. C. Drake, L. H. Grabner, and J. W. Hastie OPTICAL DIAGNOSTICS IN CVD REACTORS

G. H. Miller, A. J. Mulac, and P. J. Hargis, Jr. LASER-GENERATED OPTO-ACOUSTIC PULSES IN AN ATMOSPHERIC PRESSURE FLAME

W. R. Anderson, J. E. Allen, Jr., T. D. Fansler, and D. R. Crosley MEASUREMENT OF ROTATIONAL DISTRIBUTIONS WITHIN OH $\left(A^{2} \Sigma^{+}, v^{\prime}=0\right)$ IN FLAMES

M. A. DeWilde and D. R. Crosley ROTATIONAL DEPENDENCE OF TRANSITION PROBABILITIES IN THE OH A-X BAND SYSTEM

I. L. Chidsey and D. R. Crosley

VIII. RATE PROCESSES IN GASES--HOMOGENEOUS SYSTEMS

CHEMICAL PROCESSES IN HIGH REYNOLDS NUMBER TURBULENT DIFFUSION FLAMES

D. E. Jensen

MODELING STUDY OF FLAME STRUCTURE IN LOW-PRESSURE, LAMINAR, PRE-MIXED

METHANE FLAMES

J. R. Creighton and C. M. Lund

PREDICTIVE MODELING OF DIBORANE OXIDATION

W. M. Shaub and M. C. Lin

NEW COMPUTATIONAL ALGORITHMS FOR CHEMICAL KINETICS ${ }^{4}$

D. T. Pratt

MEASURES OF EFFECTIVENESS AND MECHANISMS FOR CHEMICAL AND PHYSICAL INHIBITION OF QUENCHED AND ADIABATIC PREMIXED FLAMES

J. J. Reuther

INFLUENCE OF WATER VAPOR ON HYDROGEN COMBUSTION ${ }^{4}$

A. Feugier

INHIBITION ON THE PROPAGATION OF FLAME ${ }^{4}$

T. Inomata, S. Okazaki, T. Moriwaki, and M. Suzuki

MEASUREMENTS OF SOME ELEMENTARY HYDROCARBON REACTIONS AT HIGH TEMPERATURES . . .

P. Roth and T. H. Just

THE WALL-LESS REACTOR. A TECHNIQUE FOR THE STUDY OF GAS PHASE OXIDATIONS AND PYROLYSES OF HYDROCARBONS 4

J. E. Taylor

\footnotetext{
${ }^{4}$ Not presented at Symposium.
} 
STUDIES OF THE ELEMENTARY REACTION KINETICS OF HIGH-TEMPERATURE SPECIES

USING OPTICAL MEASUREMENT TECHNIQUES .

1359

A. Fontijn

DIFFUSION OF CARBON DIOXIDE AT HIGH TEMPERATURES

J. R. Ferron, D. L. Kerr, and A. U. Chatwani

IX. RATE PROCESSES IN GASES--HETEROGENEOUS SYSTEMS

THE COMBUSTION OF PUVERIZED COALS--AN ASSESSMENT OF RESEARCH NEEDS

1407

D. R. Hardesty and J. H. Pohl

TRANSPORT, THERMODYNAMIC AND KINETIC ASPECTS OF SALT/ASH DEPOSITION

RATES FROM COMBUSTION GASES .......................

1451

D. E. Rosner, K. Seshadri, J. Fernandez de la Mora, G. Fryburg, F. Kohl,

C. Stearns, and G. Santoro

THE MODELING OF MODERN HIGH PRESSURE ARC LAMPS

R. J. Zollweg

THE FUTURE OF GAS TRANSPORT REACTIONS 5 .

V. P. Orlovskii

NEW MOLECULES INVOLVED IN CHEMICAL TRANSPORT REACTIONS: GASEOUS HALIDE COMPLEXES 5 .

H. Schafer

METAL HALIDE--GROUP III HALIDE VAPOR COMPLEXES WITH EMPHASIS ON ALUMINUM CHLORIDE $^{5}$

H. A. Øye and D. M. Gruen

FORMATION OF CHROMATES FROM THE REACTION OF ALKALI CHLORIDES WITH $\mathrm{Cr}_{2} \mathrm{O}_{3}$

AND OXYGEN

C. Hirayama and C. Y. Lin

CATALYTIC REACTIONS STUDIES BY A MATRIX ISOLATION TECHNIQUE: DECOMPOSITION OF $\mathrm{HCOOH}$ ON Pt.

D. Tevault and M. C. Lin

$X$. THERMODYNAMIC DATA AND APPLICATIONS

REFERENCE DATA FOR HIGH TEMPERATURE SYSTEMS . . . . . . . . . . . . 1571

L. N. Gevantman, H. J. White, Jr., and D. R. Lide, Jr.

EVALUATION AND COMPILATION OF THE THERMODYNAMIC PROPERTIES OF HIGH

TEMPERATURE SPECIES

M. W. Chase, J. R. Downey and A. N. Syverud

DIMENSIONAL ANALYSIS OF PARTITION FUNCTIONS FOR IONIC MOLECULES:

THERMODYNAMIC PROPERTIES OF VAPORS

D. J. Frurip and M. Blander

${ }^{5}$ Not presented at Symposium. 
THERMODYNAMICS OF NON-RIGID MOLECULES AT HIGH TEMPERATURE ${ }^{6}$. . . . . . . . . .

P. Ip and H. L. Strauss

GRAPH THEORY PREDICTION OF THE PRESSURE SECOND VIRIAL COEFFICIENTS OF

SOME HYDROCARBONS, FLUOROCARBONS, AND THEIR MIXTURES ${ }^{6} \ldots \ldots \ldots \ldots$ 1619

W. Brostow

MECHANISMS OF OXIDATION AND HOT CORROSION OF METALS AND ALLOYS AT TEMPERATURES

OF 1150 TO $1450 \mathrm{~K}$ UNDER FLOW . . . . . . . . . . . . . . . . . . . . .

E. A. Gulbransen and G. H. Meier

POTASSIUM SEED ACTIVITIES IN OPEN CYCLE MHD SYSTEMS: PREDICTIONS USING A MULTIPHASE MULTICOMPONENT SOLUTION MODEL . . . . . . . . . . . . . . . . . . . 1683

C. C. Hsu and C. E. Johnson

COMPUTER MODELING OF GAS-CONDENSED PHASE EQUILIBRIA: PRESENT STATUS AND

SOME FUTURE NEEDS IN SCIENCE AND TECHNOLOGY . . . . . . . . . . . . . . . . . . 1707

M. Blander

SYMPOSIUM PARTICIPANTS . . . . . . . . . . . . . . . . . . . 1717

AUTHOR INDEX . . . . . . . . . . . . . . . . . . . . . . . . . 1725

$\overline{{ }^{6} \text { Not presented at Symposium }}$ 



\section{SPECTROSCOPIC DIAGNOSTIC TECHNIQUES-- SPECIES CONCENTRATION}



National Bureau of Standards Special Publication 561, Proceedings of the 10th Materials Research Symposium on Characterization of High Temperature Vapors and Gases held at NBS, Gaithersburg, Maryland, September 18-22, 1978. Issued October 1979.

\title{
NEW OPTICAL DIAGNOSTIC TECHNIQUES FOR TEMPERATURE AND SPECIE CONCENTRATION MEASUREMENTS IN FLAMES
}

\author{
J. R. McDonald, A. P. Baronavski, L. Pasternack, \\ S. Lemont, and A. B. Harvey \\ Naval Research Laboratory, Chemistry Division \\ Washington, DC 20375
}

\section{Introduction}

In combustion systems and in plasmas or discharges, typical sampling and physical probing techniques for final product analysis such as gas chromatography and mass spectrometry are often impractical because the species which one wishes to monitor are transient or highly reactive in nature. Classical optical spectroscopic techniques, such as absorption and chemiluminescence, are also often impractical because instantaneous measurements must be made, long path lengths are not available for measurement, and the systems are fraught with chemical and spectroscopic interferences. With the advent of laser light sources, light scattering spectroscopic diagnostic techniques are assuming an ever increasing role in a broad range of physical diagnostic applications in hostile environments.

In the combustion diagnostics program at NRL we are developing two such techniques which show considerable promise for species and temperature quantification in combustors. The two techniques, CARS and Laser Induced Saturation Fluorescence Spectroscopy, are complimentary because the former has greatest application for relatively high concentration species $\left(\mathrm{CH}_{4}, \mathrm{~N}_{2}, \mathrm{O}_{2}, \mathrm{CO}_{2}\right.$, etc. $)$, while the latter is most sensitive for low concentration radicals, transients, and metastables $\left(\mathrm{OH}, \mathrm{CH}, \mathrm{C}_{2}, \mathrm{NH}, \mathrm{CN}\right.$, etc.). Each technique is amenable for in-situ temperature measurements and allows noninterfering, essentially point source measurements, in real time within hot combustion zones. The methods are relatively new and are developing rapidly. By using examples from NRL we will show, how the measurement techniques are experimentally designed, the fundamental theoretical basis for the phenomena, typical experimental measurements in combustion systems, and their applications. The literature review should enable the interested reader to follow up recent developments and applications in the field.

\section{Coherent Anti-Stokes Raman Spectroscopy (CARS)}

Coherent Anti-Stokes Raman Spectroscopy (CARS) is a relatively new and powerful technique which has been successfully used as a diagnostic for probing gaseous systems $[1]^{1}$.

${ }^{1}$ Figures in brackets indicate the literature references at the end of this paper. 
For example, at the NRL, we have applied CARS in gas phase studies of spectroscopy [1], photochemistry [1], electric discharges [2,3], and combustion [4,5-7].

The detailed theory of CARS is presented elsewhere $[6,7]$. Briefly, CARS is a thirdorder, nonlinear optical mixing process which relies on the use of two high power lasers, as shown in figure 1 below. One of these is a frequency doubled, Q-switched Nd:YAG laser at fixed frequency $w_{1}$, while the other is a YAG-pumped dye laser, tunable at $w_{2}$. The two frequencies are mixed by the third-order electric susceptibility when $w_{2}$ is adjusted such that $w_{1}-w_{2}$ matches the frequency of some Raman-active resonance of the molecule of interest. Consequently, a coherent, laser-like, anti-Stokes beam is produced at a frequency $w_{3}=2 w_{1}-w_{2}$. In gas phase CARS experiments, the two laser beams are focused colinearly in the sample to achieve maximum phase matching and power conversion to $w_{3}$. After exciting the sample, the beams are recollimated. The CARS emission at $w_{3}$ is then spectrally separated from $w_{1}$ and $w_{2}$, whereupon it is detected by a photomultiplier and averaged using a boxcar integrator. The CARS spectrum is recorded by simply observing the CARS power generated versus frequency shift, $w_{1}-w_{2}$, as the dye laser is tuned.

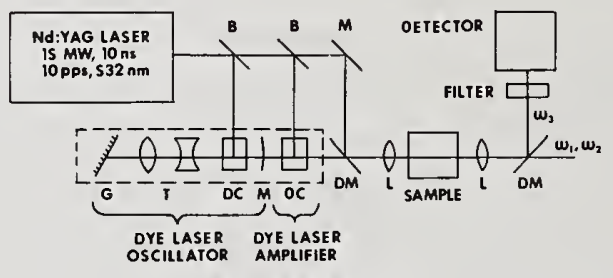

CARS APPARATUS

Figure 1. Schematic diagram of the CARS apparatus $(B=$ beam-splitter, $M=$ mirror, $G=$ grating, $T=$ beam expanding telescope, $D C=$ dye ce $11, D M=$ dichroic mirror, and $\mathrm{L}=$ lens).

As a tool for general spectroscopic studies, CARS is several orders of magnitude more efficient than normal Raman spectroscopy. In addition, the possibility for high (and ultra-high) resolution spectroscopy exists using narrow bandwidth lasers. Collection efficiencies are excellent, and a monochromator is usually not required for observing Raman shifts $\left(>500 \mathrm{~cm}^{-1}\right)$ because CARS is generated in a laser-like beam, well separated in frequency from the other two beams. Furthermore, since CARS is a process involving four waves, there may be more information potentially available for measuring polarization ratios. Therefore, it may be possible to make better assignments of normal modes to their correct irreducible representations. The coherent and anti-Stokes character of the CARS emission makes CARS an important analytical tool for probing gas molecules in electric discharges, plasmas, combustion systems, etc., since interference from laser-induced fluorescence and chemiluminescence is minimized at the anti-Stokes frequency. 


\subsection{Photochemistry}

The sensitivity of the CARS technique and the short 10 ns duration of the probing pulses make CARS potentially useful for studying photofragments, excited states, and the products of chemical reactions. The $10 \mathrm{~ns}$ laser pulse width enhances the study of processes occurring at moderate pressures under collision-free conditions. Our interest in CARS probes of photochemical reactions began when we obtained the spectrum of photolyzed benzene vapor represented in figure 2. The $532 \mathrm{~nm} \mathrm{Nd}$ :YAG laser beam was doubled to produce coherent light at $266 \mathrm{~nm}$. The ultraviolet beam and the CARS diagnostic beams were then focused and overlapped spatially and temporally in the sample. It was generally thought that a number of complex photochemical products were produced as a result of $266 \mathrm{~nm}$ photolysis [8]. In the absence of the UV beam, only a single strong CARS line is observed in the $\mathrm{CH}$ stretching region of benzene $\left(\nu_{1}\right)$. Upon UV irradiation, the benzene $v_{1}$ signal intensity decreases significantly, and evidence for acetylenic, olefinic and saturated organic groups was suggested from the resulting spectrum. Benzene photochemistry is uncharacterized at $266 \mathrm{~nm}$, but it is known that vacuum ultraviolet photolysis by single photon processes (corresponding to two $266 \mathrm{~nm}$ photons in energy) produces classes of compounds like the ones suggested in this case. It was therefore postulated that photolysis of benzene occurs via two photon absorption of the high intensity, focused laser beam at $266 \mathrm{~nm}$. However, subsequent (and as yet preliminary) experiments at Oregon State University [9] suggest that the spectrum shown in figure 2 may be that of the $c_{2}$ radical which is resonantly enhanced. The conclusion that the spectrum is not that of hydrocarbon radicals lies in the fact that essentially the same CARS spectrum is obtained with photolysis of other hydrocarbon molecules, including benzene- $d_{6}$. If it is found that the spectrum appearing in figure 2 is indeed that of $C_{2}$, it means that CARS may also be a useful diagnostic tool for detecting certain radicals, as well as bulk species.

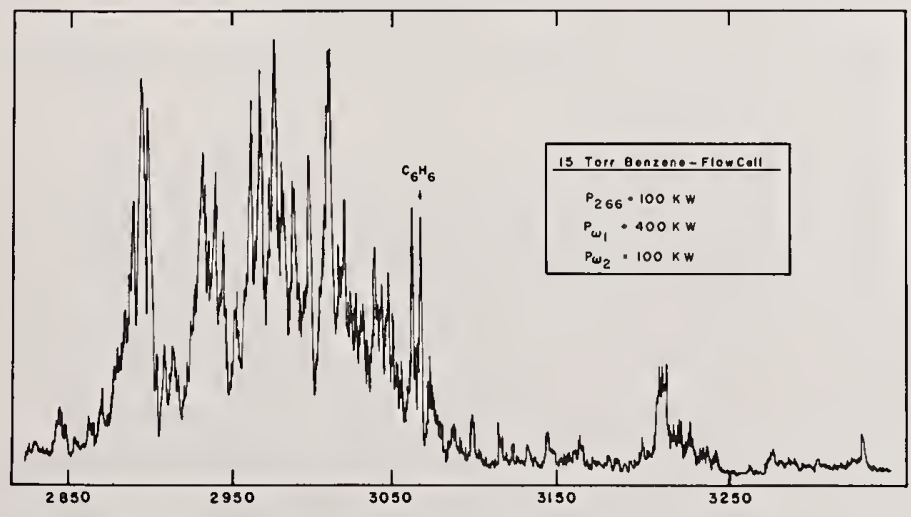

Figure 2. A spectrum observed when benzene vapor is subjected to a $266 \mathrm{~nm}$ laser beam overlapped temporally and spatially with the two CARS beams. 


\subsection{Electric discharge diagnostics}

We have successfully used the CARS technique to probe electric discharges of $D_{2}$ [2] and $\mathrm{N}_{2}$ [3] and to determine rotational and vibrational temperatures and vibrational populations distributions in these systems. Measurements were made at the center of the discharge where luminescence is a maximum. Yet, the CARS signal is unhindered by the strongly luminescent background.

Figure 3 shows a spectrum obtained for $D_{2}$ gas in an electric discharge at $10 \mathrm{kV}$ and a current of $4 \mathrm{~mA}$. We11-resolved Q-branch lines were observed for both the ground and first excited state. Vibrational temperatures, $T_{v}$, can be extracted from CARS spectra of this type by measuring $v=1: v=0$ peak height ratios for given Q-branch lines. Similarly, the rotational temperature, $T_{r}$, (assumed to be equal to the translational temperature under most circumstances) may be found by measuring relative intensities of the Q-branch lines for a particular vibrational state. Applying this treatment to the spectra in figure 3 , it was determined that $T_{r} \approx 400 \mathrm{~K}$ and $T_{v} \approx 1050 \mathrm{~K}[2]$.

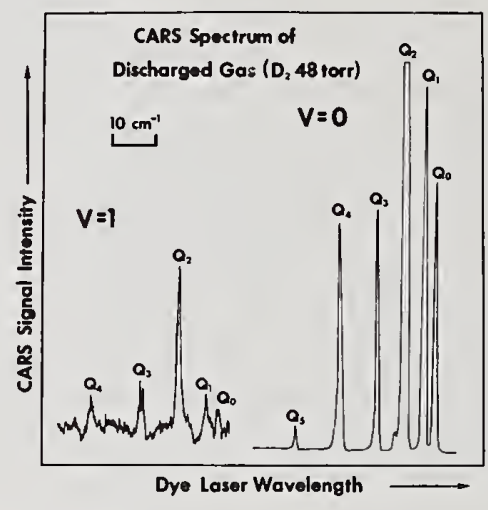

Figure 3. CARS spectrum of $\mathrm{D}_{2}$ in an electric discharge.

The cross-section for excitation of $\mathrm{N}_{2}$ by electron impact in an electric discharge is much greater than for $\mathrm{D}_{2}$. For an $\mathrm{N}_{2}$ discharge with conditions roughly similar to those for $D_{2}$, vibrationally excited $N_{2}$ is observed up to $v=7$ [3]. However, the vibrational population distribution reflected by the Na spectrum is clearly non-Boltzman. On the assumption that a Boltzman equilibrium does exist among only the lowest vibrational levels, a method has been developed to permit the direct determination of the vibrational populations of all other levels [3]. A computer program has been developed which generates intensity ratios for the lowest vibrational levels as a function of $T_{v}[10]$. The program also computes the square of the total third-order susceptibility, convoluted over a finite laser linewidth, as a function of frequency shift, to give an adequate representation of the CARS spectral profile. Experimental intensity ratios are subsequently compared with theoretical ratios to obtain a value for $T_{v}$. This may then be used to compute $A_{0}$, the 
fractional population difference between the $v=0$ and $v=1$ levels, from which other vibrational populations may be calculated. The detailed theoretical analysis and experimental results are available in [3].

Future experiments are planned for further electric discharge diagnostics in known and potential laser systems. Vibrational temperatures and populations may be monitored as a function of applied voltage, current, gas pressure and flow rate.

\subsection{Combustion diagnostics}

The CARS technique is useful for the measurement of temperatures in flames and other combustion systems, which, of course, may also be measured using thermocouples, or other physical or optical temperature-measuring devices. However, CARS allows one to spatially profile temperature within small volume elements without disturbing the system. Furthermore, Eckbreth has demonstrated that CARS spectra may be obtained under adverse conditions such as are present in sooty flames [11]. One can also use CARS to measure number densities of species in combustors, but, in this report, we will concentrate on the measurement of temperature.

We have used our apparatus to probe combustion processes in a flat flame burner. Burners of this type are used in research because of their ability to stabilize combustion over a wide range of flame speed and pressure conditions, and because they produce a flat, disk-shaped, steady flame. It is generally assumed that the composition and temperature profiles are also uniform across the flame. In a collaborative study, similar measurements are being conducted at Wright-Patterson AFB and Battelle where identical burners exist. In our burner, we have been operating with a stoichiometric $\mathrm{CH}_{4}$-air mixture at pressures in the range 80-760 Torr. The flame is 2.5 inches in diameter and 0.5-2 $\mathrm{mm}$ thick. A typical spectrum of $\mathrm{N}_{2}$ in the flame is shown in figure 4 . This spectrum was measured at $1 \mathrm{~atm}$ total pressure at a height of $2 \mathrm{~mm}$ above the flame holder. The dye laser linewidth is $0.4 \mathrm{~cm}^{-1}$. The partially resolved $\mathrm{N}_{2} \mathrm{Q}$-branch is depicted, showing rotational lines out to $\mathrm{J}=32$.

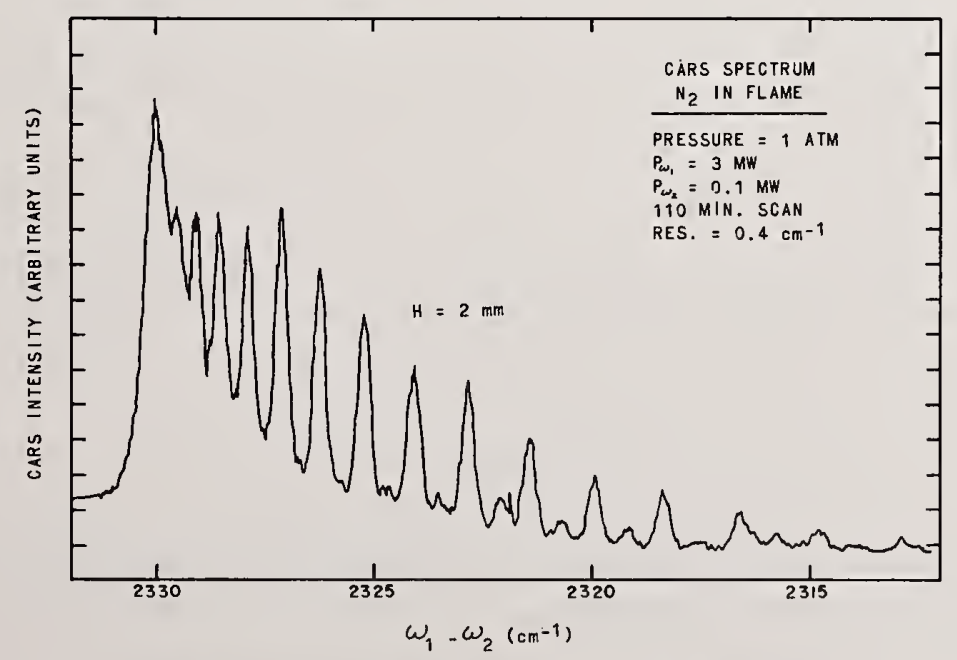

Figure 4. CARS spectrum of $\mathrm{N}_{2}$ in a flame. 
A simple method of data analysis for the purpose of temperature measurement has been suggested by Taran and coworkers [12]. In this treatment, one assumes a Boltzman rotational population distribution and the slope of the logarithm of the Q-branch envelope of the $\mathrm{N}_{2}$ spectrum is related to the temperature by a simple expression. Although easy to apply, this method is only approximate and temperature measurement errors result from its general application. Comparison of the results using this technique to computer generated CARS spectra have shown this to be the case, and the results are found to be especially sensitive to changes in system parameters such as dye laser design and spectral resolution [13]. We conclude that the determination of temperature from $\mathrm{N}_{2}$ CARS spectra are best accomplished by comparison with computer generated spectra.

One extremely important parameter to consider in performing combustion diagnostics is the length of the region being probed by the laser beams. By translating a thin piece of glass through the laser beam focal region, and measuring the nonresonant CARS signal [1,6, 7] generated in the glass as a function of its position, we find that 95 percent of the collected CARS signal is generated over a length shorter than $9 \mathrm{~mm}$ for the 25-cm focal length lens being used to focus the laser beams. Tighter focusing decreases this length. However, we have found that the CARS probe region can be greatly decreased by purposely crossing the two laser beams. Of course, when the beams are crossed in gases, some signal is lost since the beams are no longer perfectly phase matched $[6,7]$. Intensity losses resulting from the use of this technique are no more than a factor of ten for crossing angles up to $2^{\circ}$. Since the CARS signals are usually large, this loss is tolerable in many cases. When the two input beams are crossed, the CARS output beam at $w_{3}$ emerges between the other two beams. Excellent spatial resolution is achieved as the CARS probe region is kept shorter than $2 \mathrm{~mm}$, and the emerging beams are almost totally separated in space. This technique is suited for probing practical combustors in which the CARS focal region must be kept short, but lenses of short focal length cannot be introduced in close proximity to the system when probed. The application of the beam crossing technique would also be valuable for observing small Raman shifts where the frequencies $w_{1}, w_{2}$, and $w_{3}$ are spectrally close and difficult to separate by filtering. Hence, the crossed beam method could be used for observing low frequency vibrations and pure rotational Raman spectra. We are currently in the process of working on a detailed theoretical analysis of crossed CARS beams in gases.

Eckbreth has developed an elegant crossed beam technique, known as BOXCARS, in which the $w_{1}$ beam is separated into two separate beams. These two, along with the third beam at $w_{2}$, are crossed and phase matched in the sample to be probed [14]. This technique produces excellent results.

\subsection{Broad band CARS}

Usually, CARS spectra are recorded using two narrow band lasers, one being tunable to probe for vibrational resonances in the sample. In contrast to this, it is also possible to record spectra using one narrow band laser and one broad band laser. We have performed. 
broad band CARS experiments using the narrow band Nd:YAG laser described previously in conjunction with a broad band dye laser. A monochromator is used to sort out CARS resonances in this case. Since the CARS signal intensities are usually quite large, the monochromator slits may be narrowed to achieve high resolution. The major advantages of this technique over the conventional method are simplicity of dye laser design, increased system stability, and the ability to record "single shot" spectra by using an optical multichannel analyzer (OMA) or photographic plate as detector. This last feature is valuable in experiments designed to probe turbulent systems, ultra-fast chemical reactions, and events at modest pressures in collisionless environments.

Figure 5 shows a spectrum of a mixture of gases obtained in a single laser shot and demonstrates that excellent signal-to-noise can be obtained on a single pulse basis for samples at low pressures. Unfortunately, we have found that unless a high dispersion monochromator is used with an OMA free from channel cross talk, the spectra obtained via single shot CARS are unresolved and distorted [1].

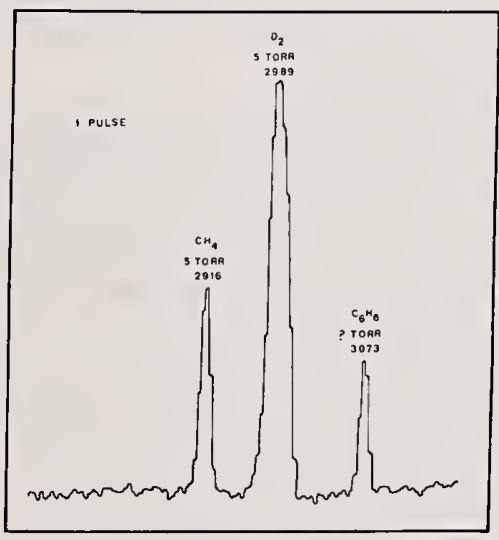

Figure 5. CARS spectrum of a mixture of gases $\left(\mathrm{CH}_{4}, \mathrm{D}_{2}\right.$ and $\left.\mathrm{C}_{6} \mathrm{H}_{6}\right)$ obtained in a single laser shot using a broad band dye laser probe source and an optical multichannel analyzer for detection.

Alternatively, we11-resolved broad band CARS spectra may be obtained by narrowing the monochromator slits and scanning through the region of interest while using a photomultiplier detector. Using this technique, we have obtained spectra of $D_{2}$ in an electric discharge and $\mathrm{N}_{2}$ in a flame, comparable to those obtained using the conventional CARS technique described earlier. However, we find that the conventional setup with a gratingtuned dye laser is much more sensitive, since much signal intensity is lost in multiplex CARS by spreading the dye laser output over a broad spectral range. 
In summary, it is clear that CARS is a powerful analytical tool for the chemist, physicist, and engineer. There are numerous high temperature applications. Combustion analysis, photochemistry and electrical discharge interrogation are notable examples illustrated in this report.

\section{Saturated Fluorescence Excitation Spectroscopy}

The technique of saturated fluorescence spectroscopy is based upon the special properties of the pulsed laser which make it possible to use a high power, narrow bandwidth laser pulse tuned to within the Doppler bandwidth of a resonant absorption feature. The laser beam, because of its low order mode structure, is capable of being focused to an extremely small cross-section. Under these conditions it is possible to saturate, or nearly saturate, optical transitions in molecules. When these conditions are met, the fluorescence behavior of the excited molecules follows rules which are very different from those which govern lower intensity excitation. For instance, when the transition is completely saturated, the observed fluorescence intensity is independent of such variables as laser intensity, fluorescence lifetime, quenching rates, and quantum yield. The evaluation of populations requires only evaluation of detection geometry and efficiency and known numerical and molecular constants.

The technique of saturation fluorescence spectroscopy has recently been both theoretically [15,16] and experimentally [17-21] evaluated for determination of the absolute concentrations of radical species in atmospheric pressure combustion sources. This is possible because in the saturation regime one is unhampered by the inability to measure insitu excited state lifetimes and quenching rates.

The laser induced fluorescence signal, S, can be written as [18]:

$$
S=h v \frac{A_{21}}{4 \Pi} \Omega \int N_{2} d V
$$

for a two-level system measured under the condition that the laser line width is greater than the absorption line width. Here $A_{21}$ is the Einstein coefficient for spontaneous emission, $\Omega$ is the solid angle of the collection optics, $N_{2}$ is the upper level population, and $V$ is the focal volume sampled by the detection optics. For a laser pulse which is long compared to the inverse of the quenching rate for the upper state, steady state conditions prevail, and it has been previously $[15,16]$ shown that the upper state concentration is given by

$$
N_{2}=\frac{N_{1}^{\circ} B_{12} I_{v}}{\left[Q+A_{21}+\left(B_{12}+B_{21}\right) I_{v}\right]}
$$


where $N_{1}^{\circ}$ is the initial concentration of the lower state prior to the -excitation pulse, $B_{12}$ and $B_{21}$ are the Einstein coefficients for induced absorption and emission, $Q$ is the total quenching rate of molecules in level 2 by all mechanisms, and $I_{\nu}$ is the laser intensity at frequency $\nu$. Inherent in eq. (2) are the assumptions that $N_{1}^{\circ} \gg N_{2}^{0}$ and that no intermediate levels serve as metastable sinks for excited molecules.

Baronavski and McDonald [17], assuming a rectangular laser beam profile in the region of near saturation, showed that the fluorescence signal takes the form

$$
S=h \nu \frac{A_{21}}{4 \Pi} \Omega \Pi r^{2} L \frac{B_{12}}{B_{12}+B_{21}}\left[N_{1}^{0}-\frac{N_{1}^{o}\left(Q+A_{21}\right)}{\left(B_{12}+B_{21}\right) I_{\nu}}\right] .
$$

Hence, a plot of fluorescence intensity vs the inverse of the laser power is expected to exhibit straight line behavior with the intercept proportional to the lower state number density and slope to intercept ratio proportional to the quenching rate. Equation (3) has been shown to represent the experimental behavior very well for several atomic and molecular systems in various combustors. A typical experimental arrangement for fluorescence saturation measurements is shown in figure 6 .

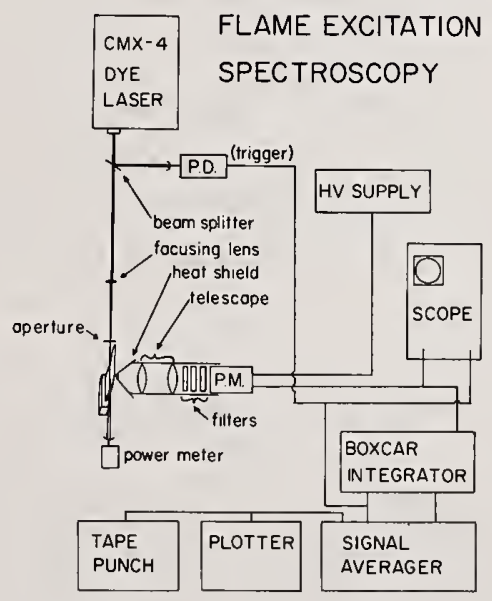

Figure 6. Schematic of the experimental apparatus used for making fluorescence saturation measurements and measuring fluorescence excitation spectra. 


\section{1 Combustors}

The combustor used in these experiments [18] is a 10- $\mathrm{cm}$ slot burner using premixed acetylene and air. The burner (from a Perkin-Elmer Model 503 Atomic Absorption Instrument) is fitted for aspirating solutions homogeneously into the premixed gases prior to burning. The gas flow system is precisely controlled with micrometering valves and a careful calibration of gas delivery rates has been made. The aspiration system has been calibrated and the delivery rate of aspirated solutions into the combustion region determined. This combustor system, thus modified, operates in a highly reproducible manner over long periods of time. Other combustors have been used for experimental measurements including a variety of hand torches, Bunsen burners and flat flame burners.

\subsection{Laser excitation}

Fluorescence, when using slot burners, is excited along the long dimension of the slot burner using a pulsed flashlamp pumped tunable dye laser (Chromatix CMX-4) with a pulse width of $1 \mu \mathrm{s}$. The laser has a bandwidth of $0.25 \mathrm{~cm}^{-1}$ when using an intracavity etalon and $3 \mathrm{~cm}^{-1}$ without the etalon. The laser beam shape is determined by scanning a pinhole backed by a photodiode across the attenuated laser beam. The beam is approximately gaussian over a $2.5-\mathrm{mm}$ cross-section with a 1/e intensity diameter of $1.44 \mathrm{~mm}$. Beyond this central region the laser intensity drops to zero because of a beam limiting aperture which is used, as shown in figure 6 . These beam profile measurements do not include refractive index gradient effects of the flame or particle scattering effects. However, in the far field the flame is observed to have a vanishingly small effect on the beam profile. Other laser systems such as $\mathrm{Nd}$ :YAG pumped dye lasers have also been used to make these saturation measurements.

\subsection{Fluorescence signal detection}

The laser induced fluorescence emission passing a $2.4-\mathrm{mm}$ beam stop is collimated and refocused using a $1 X$ telescope onto the detector, an RCA 31000B photomultiplier. Combinations of glass and dielectric filters are used to isolate the fluorescence wavelength region to be observed. These filters discriminate against background chemiluminescence and, when monitoring wavelengths different from the excitation laser line for Mg0, they also discriminate against scattered laser light. Although particle scattering, particularly forward scattering, may be significant in some flame systems due to the relatively high concentration of particulates, the laser interaction with particles does not contribute to our measured fluorescence signals. The fluorescence signals from the photomultiplier are measured using transient digitizers or a boxcar integrator. 


\subsection{Fluorescence excitation spectra}

Prior to measurement of concentrations we often measure the laser induced fluorescence excitation spectra. For example, exciting $M g 0, X^{1} \Sigma^{+}$to $B^{1} \Sigma^{+}$, and observing the $B^{1} \Sigma^{+}-A^{7} \Pi$ fluorescence emission [18] (fig. 7); and by exciting the metastable $A^{1} \Pi$ to the $B^{1} \Sigma^{+}$state and observing fluorescence emission on the $B^{7} \Sigma^{+}-X^{7} \Sigma^{+}$transition (fig. 8) one can monitor the rovibrational populations of species in flames. Based on these excitation spectra, the rotational distribution within the electronic levels for MgO is thermal with a Boltzman temperature of $\sim 2500 \mathrm{~K}$. The fluorescence excitation spectra for Mg0 very closely track the electronic transitions of the molecular species being probed. When the laser frequency is off resonance, we see no measurable laser induced fluorescence. These observations strongly indicate that laser induced fluorescence does not result from laser interaction with particulates in the flame. Such measurements of fluorescence excitation spectra clearly demonstrate that it is possible to measure flame temperatures by rotational population distributions of the systems being probed.

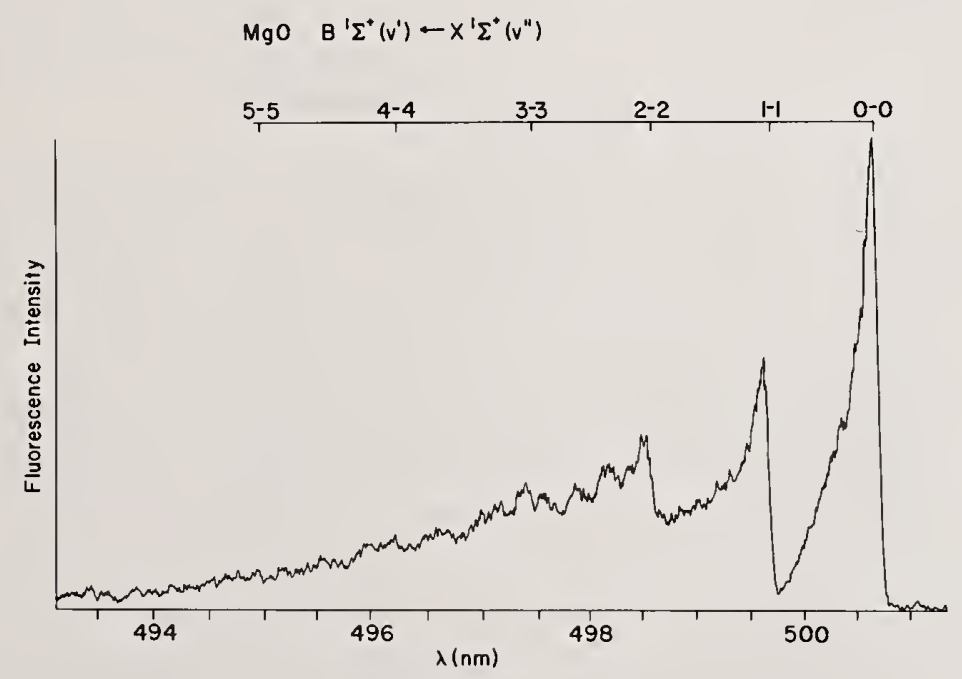

Figure 7. Laser induced fluorescence excitation spectrum for the $x^{1} \Sigma^{+}-B^{1} \Sigma^{+}$ transition of MgO. 


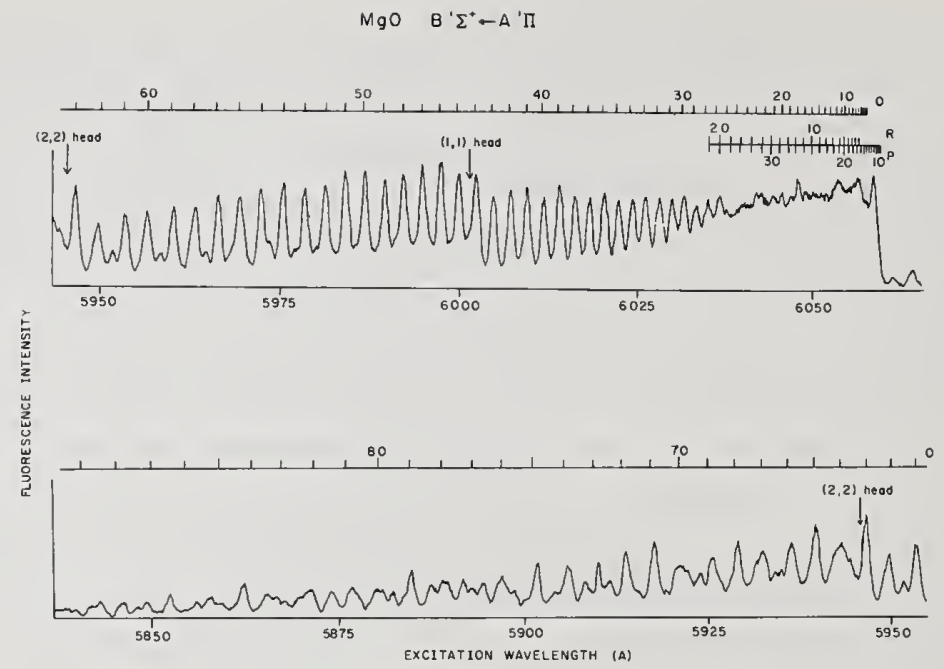

Figure 8. Laser induced fluorescence excitation spectrum for the $A^{\top} \Pi-B^{1} \Sigma^{+}$ transition of $\mathrm{MgO}$.

\subsection{Concentration measurements}

We have found it most convenient to measure absolute concentrations by working in the near saturation region for excitation as described in eq. (3). A typical measurement [17] for a common flame radical, $C_{2}$, is shown in figure 9 . Here, a plot of fluorescence signal vs the inverse of laser power shows the predicted linear behavior. The slope of the plot gives the total quenching rate for the $c_{2}{ }^{3} \Pi_{g}$ state while the intercept is used to evaluate the ${ }^{3} \Pi_{u}$ state number density. A similar plot [18] is shown in figure 10 for $\mathrm{MgO}$ in an acetylene flame. In the latter case $\mathrm{MgO}$ is created by aspirating $\mathrm{MgCl}_{2}$ into the flame of a slot burner.

The concentration measurements arrived at by saturation fluorescence measurements have been carefully checked by making classical absorption measurements in the slot burner flames for $\mathrm{Na}$ and $\mathrm{MgO}$. These two independent measurements give satisfactory agreement (within the uncertainties of the experiments) for concentrations for species which have highly different spectroscopic properties.

The fluorescence signal for sodium was measured under saturating laser power conditions at a variety of concentrations ranging over three orders of magnitude [18]. Figure 11 demonstrates that the saturation spectrosccpy measurements give a linear fluorescence signal dependence over a concentration range of from 100 parts per trillion to 100 parts per billion in the flames. Higher concentration measurements were not made because of burner corrosion problems. The practical limits of sensitivity for concentration measurements with our present experimental setup are on the order of ten parts per trillion for atomics and about a factor of ten higher for most molecular species. 


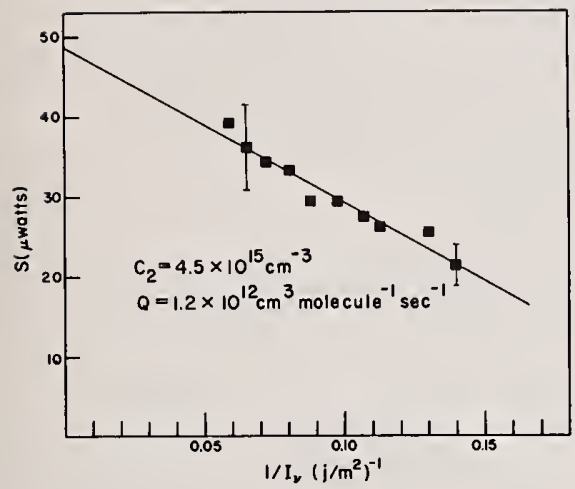

Figure 9. A plot of $C_{2}$ fluorescence emission signal vs $1 / I_{\nu}$. The fluorescence signal is corrected for efficiency factors. The slope of the plot gives the total quenching rate of the excited state while the intercept is used to evaluate the lower state population.

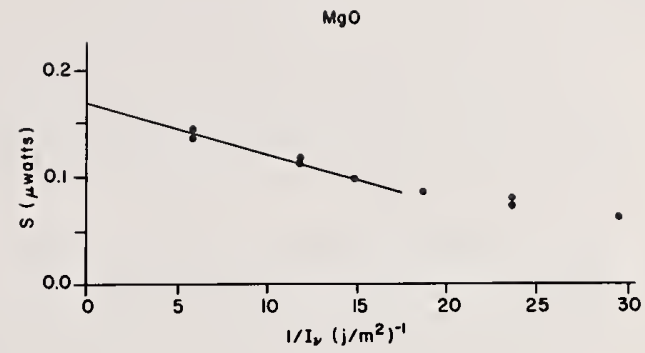

Figure 10. Plot of the Mg0 $X$ state emission signal vs inverse laser intensity. The solid line represents the best fit to the data for a rectangular beam profile.

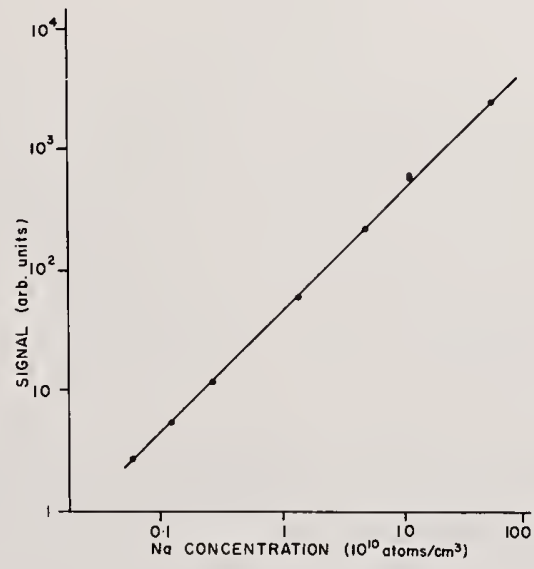

Figure 11. Plot of fluorescence signal vs $\mathrm{Na}$ concentration in the flame. 
We have shown that the values for the ground state concentrations and electronic excited state quenching rates of species in turbulent atmospheric pressure combustion systems can be measured by employing the techniques of saturation spectroscopy.

The technique employed here has the following advantages:

(1) Only modest laser powers and bandwidths are required.

(2) Real time measurements can be made upon turbulent flames.

(3) Measurements are tolerent of high background luminescence and particulate scattering.

(4) Point measurements $\left(10^{-5} \mathrm{~cm}^{3}\right)$ can be made.

(5) One can simultaneously obtain measurements of the effective steady state rotational and vibrational temperatures of the species in the flame by monitoring unsaturated fluorescence excitation spectra.

(6) The optical technique can be used for remote sensing of stationary flame systems.

The authors would like to thank S. Lemont, J. W. Nibler, W. M. Shaub, R. W. Gammon, W. M. Tolles, R. N. DeWitt, and W. Sanders for their contribution to the CARS projects at NRL.

\section{References}

[1] Harvey, A. B. and Nibler, J. W., Coherent Anti-Stokes Raman Spectroscopy of Gases. To be published. Additional references are given in this work.

[2] Nibler, J. W., McDonald, J. R., and Harvey, A. B., Opt. Commun. 18, 371 (1976).

[3] Shaub, W. M., Nibler, J. W., and Harvey, A. B., J. Chem. Phys. 67, 1883 (1977).

[4] Lemont, S. and Harvey, A. B., Applications of coherent anti-Stokes Raman spectroscopy at NRL, Advances in Laser Chemistry, A. H. Zewail, ed. (Springer-Verlag, Berlin, 1978).

[5] Harvey, A. B., Proceedings of the 6th International Conference on Raman Spectroscopy, Bangalore, India, Sept. 4-9, 1978.

[6] Tolles, W. M., Nibler, J. W. , McDonald, J. R., and Harvey, A. B., Appl. Spectrosc. 31, 253 (1977).

[7] DeWitt, R. N., Harvey, A. B., and Tolles, W. M., Theoretical development of thirdorder susceptibility as related to coherent anti-Stokes Raman spectroscopy (CARS), NRL Memorandum Report 3260, Naval Research Laboratory, Washington, D.C. (Apri1 1976).

[8] Nibler, J. W., McDonald, J. R., and Harvey, A. B., Coherent anti-Stokes Raman spectroscopy of gases, in Proceedings of the Fifth International Conference on Raman Spectroscopy, Sept. 2-8, 1976, E. D. Schmidt et al., eds. (H.F.S. Verlag, Fresburg, Germany, 1976). 
[9] Nibler, J. W. , private communication.

[10] Shaub, W. M., Lemont, S., and Harvey, A. B., CARS Spectral Profiles for Homonuclear Diatomic Molecules, submitted for publication.

[11] Eckbreth, A. C., 10th Materials Research Symposium on The Characterization of High . Temperature Vapors and Gases, National Bureau of Standards, Gaithersburg, Md., September 1978.

[12] Moya, F., Druet, S., Pealat, M. and Taran, J. P., Flame investigation by coherent anti-Stokes Raman scattering, AIAA 14th Aerospace Sciences Meeting, Washington, D.C., Jan. 26-28, 1976, AIAA Paper No. 76-29.

[13] Shaub, W. M., Lemont, S., and Harvey, A. B., Interpretation of CARS Spectral Profiles, submitted for publication.

[14] Eckbreth, A. C., Appl. Phys. Lett. 32, 421 (1978).

[15] Daily, J. W., Appl. Opt. 15, 955 (1976); 16, 568 (1977).

[16] Daily, J. W., Appl. Opt. 17, 225 (1978).

[17] Baronavski, A. P. and McDonald, J. R., Appl. Opt. 16, 1897 (1977); J. Chem. Phys. $\underline{66}$, 3300 (1977).

[18] Pasternack, L., Baronavski, A. P., and McDonald, J. R., J. Chem. Phys., in press.

[19] Daily, J. W. and Chan, C., submitted to Combustion and Flame.

[20] Lucht, R. P. and Laurendeau, N. M., submitted to Combustion and Flame.

[21] Eckbreth, A. C., Bonczyk, P. A. and Shirley, J. A., private communication. These authors have recently completed saturated fluorescence measurements on the radicals $\mathrm{CN}$ and $\mathrm{CH}$ in flame systems.

\section{Discussion}

Comment (Steinberg): The inapplicapability of the two level models is induced chemistry. I think it is correct --- I have recently had occasion to do an analysis including the chemistry in the alkali metal stuff that I will be discussing later (see chapter by Mueller, et al.). But, what it does to your analysis is, you still get the straight line when you plot your fluorescent intensity under saturated conditions against one over your intensity. But the slope doesn't give you the quenching cross section in any simple way because there is chemistry involved, some of which I am sure you and I don't understand. And the intercept gives you a low value relative to your absorption measurement, which is precisely what this model should do. And, your $\mathrm{C}_{2}$ data, and MgO data suggest that the same thing is operative. It may be that you are inducing chemistry of your excited Mgo.

Response (McDonald): We anticipate that, that likely is the case. However, I think it should also be pointed out that we are still able to make semi-quantitative measurements at concentrations levels, probably $10^{4}$ times lower than you can measure by any other technique in these hostile environments, and they are probably right within a factor of two, even given the excited state chemistry. 
Comment (Daily): This is neat. This is fun stuff. I have got to say that. One of the things that we discovered with sodium, although we haven't really observed it with $\mathrm{OH}$ yet, which is what we are working with now, is that if you look at the fluorescent signal, you can actually see chemical decay.

What we did, we just sort of ran across it by chance. We11, it was suggested by Alkemade originally, and then we went back and looked. If you look at a scope trace of the fluorescent signal and change the air to fuel ratio, and you are lucky and the chemical decay times are about the length of the laser pulse, which happens to us, then you will see that laser pulse will fall off faster and faster as you go to leaner, and leaner flames. So, what we have done is gone to clipping the signal; that is, we gate only our detector on for the leading edge of the pulse, so we miss the chemistry that takes place later on.

We seem, at least for sodium, to have been able to get rid of the chemistry effects by doing that. We don't know what the scoop is with $\mathrm{OH}$.

Response (McDonald): We observed that also with sodium. Under appropriate concentration temperature conditions, you see a spike on the leading edge of the long CMX-four pulse, which is indicative of the chemistry that is going on there.

We agreed with you. We do see it. We don't see it for any other molecule, or atom that we have probed though.

Question (Crosley): We don't see the same kind of spike, and apparently in our experiments I think the chemistry is not determining the concentration over the same kind of time regime that John Daily is.

I was going to ask you though, you had mentioned experimental uncertainties. You listed focal volume and your measurements always come out lower on the fluorescence for the concentration that you get from absorption. So do ours. In fact, I think everything does measure lower than with the absorption.

Did you take into account the focal volume in looking at the Gaussian profile of the beam the way that John Daily had worked out. That has affected our measurements to some degree.

Response (McDona1d): We have a fairly long paper coming out which tries to treat that in some detail with the experimental measurements which we have done here.

We do not use Gaussian beams. We use a fairly broad Gaussian beam which we aperture and clip the wings on completely.

We have done the treatment of the extraction of the data from this using the appropriate real beam situation and it does not make a very large difference, a few percent.

\section{Clarification (Crosley): Even in a numerical simulation sense?}

Response (McDonald): No. We have rederived the equations for using a Gaussian beam, and cutting off the Gaussian beam where we really cut it off only makes a few percent difference in our situation. 
Question (Gardiner): The CARS technique you describe uses a pulsed dye Taser for generating signals. Can you please comment on the practical applicability (i.e. detection sensitivity and time resolution) of CARS to real time observations of species profiles? The first demonstrations of CARS scattering used scattering from the gas to be probed $\left(\mathrm{H}_{2}\right)$ for . generating $w_{s}$; would this simplification be possible for a CW source?

Response (McDonald): I believe you are asking whether CARS can be used in real time for $\mathrm{CW}$ measurements of specific concentrations. Several groups, including some excel lent work at Stanford University, have been using $\mathrm{CW}$ lasers to measure very high resolution spectra of simple gases. Because of the high order of dependence of CARS signals on laser power, the sensitivity of the $\mathrm{CW}$ techniques is much less than for pulsed systems. Much of this lack of sensitivity can be made up in signal averaging using the very stable CW sources. I can't give you numbers off the top of my head relating to detection limits. The answers are readily available from the literature.

With respect to the second part of your question about generating the probe beam by scattering with the pump source in a static gas, this is a stimulated Raman process which has a high threshold. Stimulated Raman stokes shifting cannot be done with Taboratory $\mathrm{CW}$ Tasers. 
National Bureau of Standards Special Publication 561, Proceedings of the 10th Materials Research Symposium on Characterization of High Temperature Vapors and Gases held at NBS, Gaithersburg, Maryland, September 18-22, 1978. Issued October 1979.

\title{
LASER ABSORPTION METHODS FOR CHARACTERIZING HIGH TEMPERATURE GASES
}

\author{
W. C. Gardiner, Jr. \\ Chemistry Department \\ University of Texas \\ Austin, TX 78712
}

The special problems which arise in using laser absorption spectroscopy to characterize high temperature gases are reviewed. Examples are given of successful applications of the method for the study of reactive flow in shock tubes, and possible future developments are suggested.

\section{Introduction}

Absorption spectroscopy has been used as a standard method for measuring the concentrations of chemical species in flowing as well as stationary high temperature gases for many years. The instrumentation required for the method is in essence no different from that required for the more widespread applications of absorption spectroscopy in liquid solutions. There is really only one special subclassification of the instrumentation, namely when time resolution is required, in which case the term "kinetic absorption spectroscopy" applies. With or without time resolution, absorption spectroscopy of high temperature gases does pose a number of problems that are of little importance in dealing with liquid solutions. When laser sources are contemplated for absorption spectroscopy of gases, these problems often take on special forms.

Basic to all applications is the Beer-Lambert Law

$$
I=I_{0} 10^{-a l c}
$$

where the symbols have their usual meanings: $I$ = transmitted intensity, $I_{0}=$ source intensity, $\mathrm{a}=$ decadic absorption coefficient or absorptivity $\left./ \mathrm{cm}^{2} \mathrm{~mol}\right]^{-1}, \ell=$ path length/cm, and $c=$ concentration of absorbing species $/ \mathrm{mol} \mathrm{cm}{ }^{-3}$. The absorptivity in gases always depends not only upon wavelength, but also upon the total gas pressure (or density), the nature of other gases that may be present, and as discussed in more detail later, upon temperature. In most applications, one is dealing with a line absorption spectrum rather than a continuous one. Equation (1) then is valid only for limited ranges of concentration also, whatever the nature of the source lamp may be.

of conventional sources, the type which has traditionally been used to achieve the greatest specificity and sensitivity is the resonance line source, in which the lamp excites emission of the same transition(s) that are absorbed in the sample gas. There 
is then no or little contribution to $I_{0}$ from background continuum or from unwanted lines, and the absorptivity is determined by the relative shapes of the source and absorber lines. For convenience in operation, at a sacrifice in sensitivity and specificity, continuum sources have also been used for a long time in diverse applications for the characterization of species concentrations in gases. Using laser sources, especially tunable ones, there is now a prospect that one can combine the best aspects of both source types for virtually every application, including kinetic absorption spectroscopy.

The first special problem presented by temperature in absorption spectroscopy of high-temperature gases is that of stimulated emission. At high temperatures, it is readily possible to achieve significant thermal populations of the upper states of transitions that one wishes to use for absorption spectroscopic analysis. Even if one is able by careful optical arrangements to exclude virtually all thermal emission from the detection system, there is still the inevitable consequence that the probability per unit time of stimulated downward transitions is equal to that of stimulated upward transitions. For the absorption of a given spectral transition, one then can modify eq. (1) to take stimulated emission into account by isolating the temperature dependence of stimulated emission through

$$
a=a^{\circ}\left(1-e^{-c}{ }^{\omega / T}\right)
$$

where $w$ is the wavenumber of the transition observed, $c_{2}=1.439 \mathrm{Kcm}$ is the second radiation constant, and $a^{\circ}$ is the value that the absorptivity would have if stimulated emission did not exist.

If $c$ in eq. (1) is to refer to the total concentration of some species in the usual chemical sense, then temperature also affects a through the thermal population of the absorbing state. The fractional population $f_{i}(T)$ of absorbing state $i$ depends upon temperature according to

$$
f_{i}(T)=\frac{g_{j} e^{-c_{2} E_{i} / T}}{Q(T)}
$$

where $g_{i}$ and $E_{j}$ are the degeneracy and energy of state $i$ and $Q(T)$ is the internal partition function of the species. Since $Q(T)$ increases with $T$ approximately as $T^{n}$, where $n$ is a small number, the dominant temperature dependence is from the Boltzmann factor in the energy of the absorbing state for large $E_{i}$ and from $Q(T)$ for small $E_{i}$. In any event, the absorbing state population may generally be expected to decrease fairly rapidly with temperature due to redistribution of the overall population into other states of the species. This would appear in $a^{\circ}$ measured at two temperatures as

$$
\frac{a^{\circ}\left(T_{2}\right)}{a^{0}\left(T_{1}\right)}=\frac{e^{-C_{2} E_{i} / T_{2} Q\left(T_{1}\right)}}{e^{-C_{2} E_{i} / T_{1} Q\left(T_{2}\right)}}
$$


which may prove to be a fairly small ratio if $T_{1}$ refers to room temperature and $T_{2}$ is a few kK. For the special case that the absorbing state is the ground state of the species then

$$
\frac{a^{0}\left(T_{2}\right)}{a^{0}\left(T_{1}\right)}=\frac{Q\left(T_{1}\right)}{Q\left(T_{2}\right)} .
$$

Since both the Boltzmann factor and the partition function increase with temperature, the net effect upon $a^{0}$ is determined by their ratio and thus by the magnitudes of $E_{j}$ and $n$. For atoms and diatomic molecules, the depopulation effect may be relatively unimportant compared to line shape effects discussed below, while for larger molecules $n$ may be so large that depopulation can have major effects upon $a^{\circ}$ even at only slightly elevated temperatures.

The third effect of temperature is upon the absorption line shape, and here the use of a laser source presents a special situation when compared with either continuum or conventional line sources. This is due, of course, to the need to know the laser lineshape itself, which one is usually not in a position to measure experimentally and must therefore obtain from theoretical knowledge of the laser operation. As an example, we take the case of absorption of the neon $2948 \mathrm{~cm}^{-1}$ line by its coincidence absorption with methane $[1]^{1}$. For a gas laser in a cavity of length $L$, one assumes a Gaussian line profile whose half width at half maximum $\Delta w_{1 / 2}$ is given by [2]

$$
\Delta w_{1 / 2}=(1-R) / 4 \pi L
$$

where $R$ is the reflectivity of the output mirror of the laser. This profile is compared to profiles of the methane absorbing line in figure 1.

One notes several features of the comparison. First, while the laser line is, as expected, quite narrow compared with the absorber lines at both high and low temperatures, it is by no means infinitely narrow, and the absorptivity may be expected to be affected by the finite amount of convolution over the absorber profile that will be due to the finite source line width. Second, one notes that there is a substantial loss of absorptivity due to the spreading of the line at increased temperature, i.e. that Doppler broadening is having its expected effect for laser absorption just as it would for a conventional line source. Finally, one notes that the assumptions one makes about the form of the absorber line profile, as well as about the parameters which are adopted for whichever profile is assumed, will have an influence upon the computed temperature dependence of the absorptivity.

${ }^{1}$ Figures in brackets indicate the literature references at the end of this paper. 


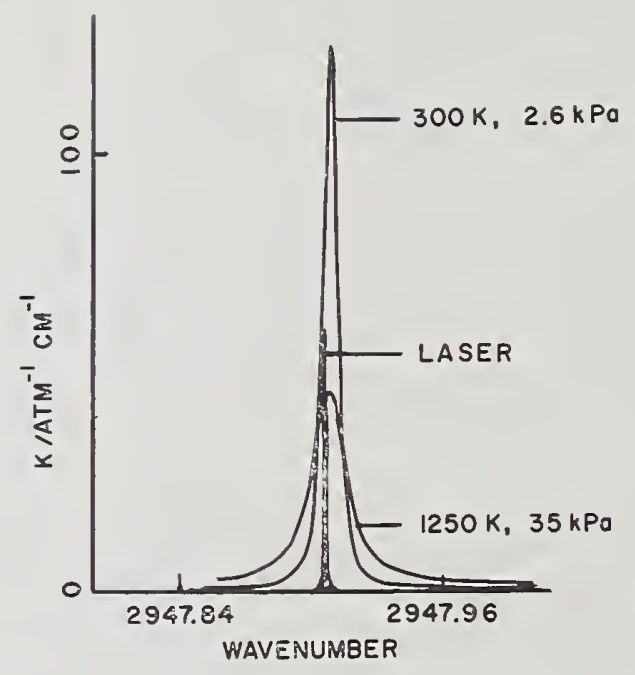

Figure 1. Comparison of laser profile with $\mathrm{CH}_{4} \mathrm{~V}_{3}(\mathrm{P} 7)$ line profiles at low and high temperatures, using a Galatry profile [1].

Of these temperature effects, only the effect of stimulated emission can be readily accounted for in all cases. Thermal depopulation of the absorbing state can be taken into account to the accuracy with which one can compute the partition function of the species, which for high temperatures may be a limitation. The spectral lineshape at high temperatures, even when pressure broadening is not the major source of broadening, is a matter of controversy [1]. A theoretical analysis of the absorptivity for a laser line as a function of temperature may thus be considered to be on shaky enough grounds that an experimental determination of it is to be preferred in virtualiy every case.

\section{Accuracy Considerations for Kinetic Absorption Spectroscopy}

The basic intention of using a laser source for absorption spectroscopy is to achieve better results than with a conventional source, either as to specificity of the analysis or as to accuracy of the concentration measurement. With a tunable laser, the situation with respect to specificity amounts to a question of the wavelength accuracy of the laser spectrometer, which, of course, depends on the type of laser used and the care with which it is operated. Especially in terms of convenient operation, it is hard to see how laser absorption spectroscopy could offer greater specificity than a hollow cathode source lamp, although a very good laser can be tuned from one transition to another more rapidly than hollow cathode lamps can be changed. Even with a tunable laser, there are more coincidence absorptions than one would think at first, and so the question of specificity of laser absorption sources is one that is debatable. 
More definitive statements can be made about the accuracy of a concentration measurement. We assume for simplicity that eq. (1) is valid and that errors in c will arise only through random uncorrelated measurement errors in I, I, and a. Solving eq. (1) for $c$ and applying the propagation of errors formula gives after some algebra

$$
\begin{aligned}
(\Delta \mathrm{c} / \mathrm{c})^{2}= & (\mathrm{allc})^{-2}\left[\left(\Delta \mathrm{I}_{0} / \mathrm{I}_{0}\right)^{2}+(\Delta \mathrm{I} / \mathrm{I})^{2}\right] \\
& +(\Delta \mathrm{a} / \mathrm{a})^{2}+(\Delta \ell / \ell)^{2}
\end{aligned}
$$

where the $\Delta^{\prime} s$ on the right-hand side refer to estimates of the measurement errors in each quantity. We are interested in the case that the amount of absorption is small, i.e., that $I_{0} / I \simeq 1$. Then, only the first two terms on the right-hand side of eq. (7) will be important in determining $\Delta \mathrm{c} / \mathrm{c}$. For small absorptions, the denominators of these terms will be nearly the same. The measurement error in I will be, for these purposes, the same

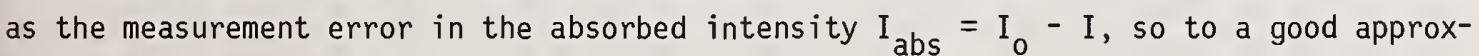
imation

$$
(\Delta \mathrm{c} / \mathrm{c})^{2}=\left(\mathrm{alcl} \mathrm{I}_{0}\right)^{-2}\left(\Delta \mathrm{I}_{0}^{2}+\Delta \mathrm{I}_{\mathrm{abs}}^{2}\right)
$$

It is quite possible that the measurement errors in $I_{0}$ and $I_{\text {abs }}$ could be quite different; $I_{0}$ could be measured in a $\mathrm{CW}$ arrangement that would permit much better accuracy than in measuring $I_{\text {abs }}$ in a kinetic absorption spectroscopy experiment, or the converse could be the case, when a fluctuating $I_{0}$ during an experiment could not be recorded as accurately as the absorbed intensity change $I_{\text {abs. }}$. If we provisionally assume that these two are comparable to one another in magnitude, then the relative measurement error in concentration scales with the relative measurement error in intensity (approximately) as

$$
\Delta c / c=\sqrt{2} \Delta \mathrm{I}_{0} / \mathrm{alc} \mathrm{I}_{0}
$$

Equation (9) shows three possibilities for enhancing measurement accuracy for smal concentrations through use of laser sources rather than conventional ones. First, by tuning to the center of an absorption line, one can increase a. Second, the better collimation afforded by lasers permits one to use longer pathlengths $\ell$, perhaps through multiple-pass cells, more conveniently than with conventional sources. Third, one can anticipate that a carefully operated laser may permit smaller values of $\Delta I_{0}$ to be attained than with conventional sources.

This third point bears further examination. For kinetic spectroscopy applications, one may use a laser source either in a continuous-wave or a pulse mode. A continuous wave application that would strain the ability of the laser source to deliver intensity would be one in which time resolution of $1 \mu \mathrm{s}$ is sought. The least intense continuous wave laser that one might consider using would still produce $10 \mu \mathrm{W}$ of power. This would correspond to the fewest quanta per second for a visible or UV laser. At $500 \mathrm{~nm}$, for example, one 
would have from this laser about $2 \times 10^{7}$ quanta per microsecond. The shot noise in the laser beam itself would be then at the $2 \times 10^{-4}$ level. Even allowing for conversion efficiency into photoelectrons, this is a much better noise level than one is accustomed to expect for an absorption spectroscopic source. For this extreme example of a weak laser and a fast experiment, it is clear that the real sources of noise are not to be found in the finite number of photons. They are instead to be found either in the detector electronics or in the plasma (or other) instabilities of the laser itself. In the following section, we will see what measurement error level is achieved in practice with present lasers. While there have not yet been any reports of using pulsed lasers for absorption spectroscopy of high temperature gases, a similar argument to the above will show that even very weak laser pulses will provide plenty of quanta for use as absorption sources, and that laser stability and detector noise will also be the limiting factors for pulsed laser applications in this area.

One may also be concerned that a high intensity laser source might introduce an error in concentration measurements due to a disturbance of the thermal equilibrium populations of the lower and upper absorbing states by the absorbed photons. If the absorbed intensity is high enough and the relaxation rates removing molecules from the two states are low enough, then the concentration difference between the two states, and hence the absorptivity, will go to zero. This phenomenon, known as the "optical pumping effect" or "hole burning" would probably become of critical importance for possible applications of pulsed lasers in absorption studies at high temperatures. For continuous wave lasers, however, a detailed analysis of the magnitude of the decreases in absorptivity for realistic relaxation rates and a fairly high power $\mathrm{CO}_{2}$ laser source showed that the effect was in fact, negligibly small [3].

\section{Survey of Applications}

In this section, an up-to-date survey is made of the applications of laser absorption spectroscopy to characterization of high temperature gases.

The first laser considered for absorption spectroscopy of gases was the $3.39 \mu \mathrm{m} \mathrm{He}-$ Ne laser, whose coincidence absorption by a methane rotational line led to a number of early investigations [4]. As far as high temperature applications are concerned, the first one appears to have been that of Emrich and Soloukhin [5], who investigated the temperature dependence of the absorptivity up to $1000 \mathrm{~K}$ using a shock tube. Further investigations of this temperature dependence for the purpose of discovering the physical processes underlying the absorption led to a fairly complete picture [1]. In the temperature range 500-2000 $\mathrm{K}$, the absorptivity decreases by about two orders of magnitude due to the combined effects of thermal depopulation of the absorbing state and broadening of the absorber line shape. Above $2000 \mathrm{~K}$, there is no longer a decrease in absorptivity with temperature, implying that an additional absorbing state (or states) begins to contribute to the absorption. A more-or-less satisfactory description of the temperature dependence below $2000 \mathrm{~K}$ is possible in conventional line-broadening theory, while only speculative interpretation could be given to the observed lack of temperature dependence above $2000 \mathrm{~K}$. 
Chemical kinetics studies in shock waves based upon $\mathrm{CH}_{4}$ absorption of the $3.39 \mu \mathrm{m}$ $\mathrm{He}-\mathrm{Ne}$ laser 1 ine have been carried out [6-11]. These include measurements of the thermal decomposition rate $[6,7,10]$, the rate of disappearance of $\mathrm{CH}_{4}$ in combustion [11], and the isotope exchange rate with $D_{2}[9]$. The results supplement and are in good accord with results obtained by other methods. An illustration of the quality of data obtainable is given in figure 2 .

Since the $3.39 \mu \mathrm{m}$ laser line is within the $\mathrm{C}-\mathrm{H}$ stretch region of the ir spectrum, it was to be expected that other hydrocarboris should also show absorption. Zeeman-tuned lasers were used to search for these absorptions [12-14], and many were indeed found in room temperature experiments. The essence of the results is that small hydrocarbons proved to have a number of lines within the $\pm 1 \mathrm{~cm}^{-1}$ tuning range of the spectrometers, while large ones showed continuous absorption. At high temperature, however, it proves to be the case that hydrocarbons, in general, show absorption of the $3.39 \mu \mathrm{m}$ line, the amount of absorption increasing with molecular size and decreasing slowly with increasing temperature [15] (fig. 3). This general absorption indicates that even with narrow source lines it will not be possible to discriminate among hydrocarbons at high temperature using laser absorption spectroscopy. In order to find useful applications, one must instead use chemical sense to develop experiments in which changes in absorption can be correlated with progress of chemical reaction despite the lack of spectroscopic discrimination. This is, in fact, facilitated by the fact that hydrocarbon thermal decomposition and oxidation reactions lead to products that absorb the $3.39 \mu \mathrm{m}$ line only very weakly or not at a11 $[16,17]$.

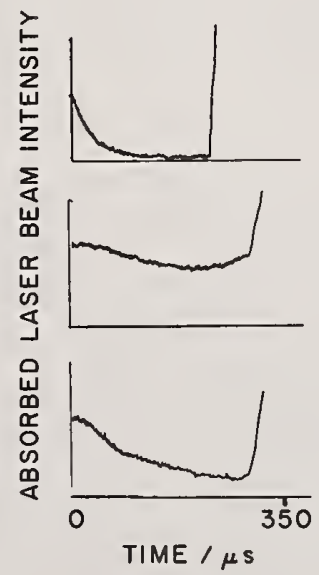

Figure 2. Kinetic absorption spectroscopy applied to concentration changes of $\mathrm{CH}_{4}$. Shown are oscilloscope trace photographs of absorption changes with time after incident shock heating from a starting pressure of $1.3 \mathrm{kPa}$. The baseline corresponds to 100 percent transmission. The large change in transmission signals the arrival of the contact surface. Upper trace: $\mathrm{CH}_{4} / \mathrm{Ar}=10 / 90, \mathrm{~T}=2500 \mathrm{~K}$; Middle trace: $\mathrm{CH}_{4} / \mathrm{Ar}=10 / 90, \mathrm{~T}=2070 \mathrm{~K}$; Lower trace: $\mathrm{CH}_{4} / \mathrm{D}_{2} / \mathrm{Ar}=10 / 10 / 80, \mathrm{~T}=2070 \mathrm{~K}$. 


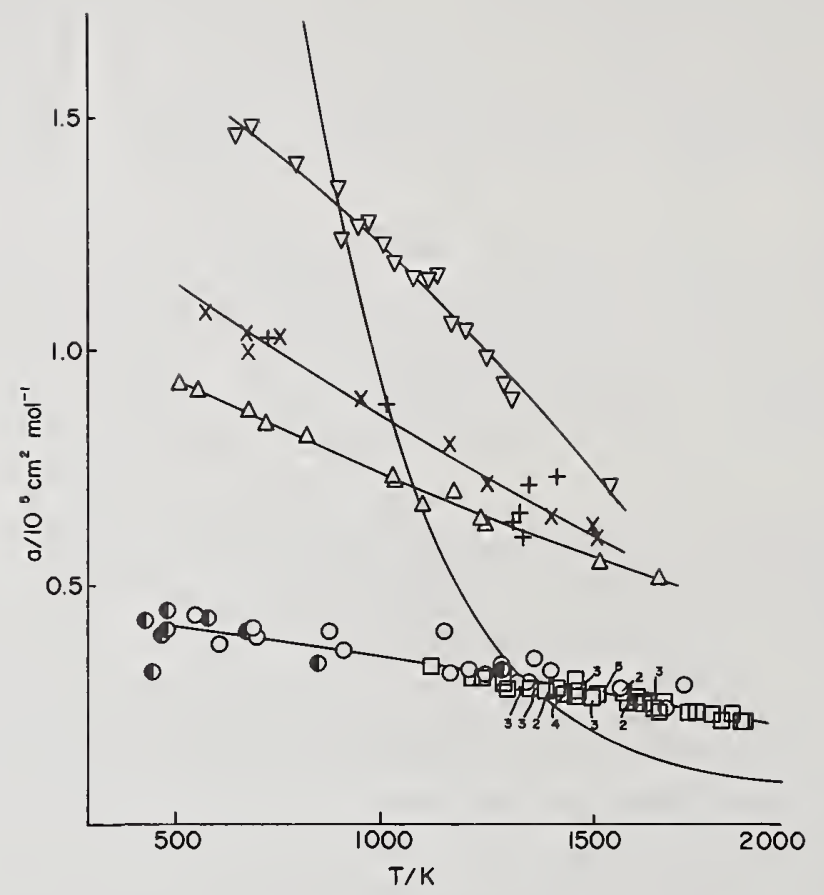

Figure 3. Dependence of absorptivity upon temperature for the $3.39 \mu \mathrm{m}$ line of the He-Ne laser. $\quad \nabla n-C_{5} H_{12} \quad x,+n_{-}-C_{4} H_{10} \quad \Delta C_{3} H_{8}$. Other symbols are for various $\mathrm{C}_{2} \mathrm{H}_{6}$ mixtures [15]. In contrast to the negative temperature dependence of absorptivity shown by these molecules, $\mathrm{C}_{2} \mathrm{H}_{4}$ shows a weak absorptivity which increases with temperature [17]. The steeply falling line shows the absorptivity of $\mathrm{CH}_{4}[1]$.

We now turn to a high temperature experiment in which a laser was used to measure the concentration of the lasing molecule itself, that of Eckstrom and Bershader on $\mathrm{CO}_{2}[3,18]$. The object of their experiments was to measure the vibrational relaxation time for $\mathrm{CO}_{2}$ over the temperature range 500-2000 $\mathrm{K}$ by monitoring the time-dependent concentration of specific rotational levels of the 100 and 200 vibrational levels in shock-heated $\mathrm{CO}_{2}$ test gas. It was found that the time constants for the two levels were the same and in agreement with thermal measurements of the overall vibrational relaxation time made earlier by interferometry under the assumption of series excitation of the higher-energy vibrational states. In this study, the final absorptions after attainment of vibrational equilibrium ranged from 3 to 10 percent, and could be measured with time resolution better than $1 \mu s$ at signal-to-noise ratios better than 50. It was suggested that a dependence of vibrational relaxation time upon rotational state may have been observed in the results $[19,20]$. 
Another experiment in which the lasing molecule itself was monitored was one by Dibble and Bowen on $\mathrm{CO}$ [21]. Their goal was to probe the vibrational states of $c 0$ produced in the highly exothermic elementary reaction $\mathrm{CS}+0=\mathrm{CO}{ }^{*}+\mathrm{S}$. The experiment consisted of shock-heating 5 percent $\mathrm{CS}_{2}+95$ percent $\mathrm{O}_{2}$ test gas to $860 \mathrm{~K}, 80 \mathrm{kPa}$, and monitoring the gain or absorption of a $C 0$ laser beam passing through the reacting test gas. The lasing transitions had lower vibrational levels from $v=8$ to $v=12$. While it could be confirmed that the vibrational levels probed were changing at the time of fastest reaction in the explosion, difficulties in reproducing the gain data prevented the authors from comparing their results quantitatively to those obtained in similar room-temperature experiments.

A CO laser can also be used to monitor NO concentration, due to the existence of coincidences among the vibration-rotation lines of the two molecules [22]. This has been exploited to measure the thermal decomposition rate of NO and the rate of the important air chemistry reaction $0+\mathrm{N}_{2}=\mathrm{NO}+\mathrm{N}[23,24]$. Shock tube methods were used to heat NOcontaining or NO-forming test gas mixtures to temperatures as high as $3850 \mathrm{~K}$. In these applications, the accuracy of the laser absorption measurements of NO concentration was checked directly by making parallel experiments using infrared emission to monitor the No concentration. It was found throughout that the technique yielded results that were in good accord with earlier work. This laser technique could then be applied to measure No concentrations where spectral interferences preclude measurements by the more traditional techniques of ultraviolet absorption spectroscopy or chemiluminescence.

The great advances made in recent years in the technology of tunable lasers lead one to assume that tunable lasers have greater potential than fixed-frequency lasers for absorption spectroscopy of high temperature gases. The considerable expense of these instruments, however, has led to delay in their application. Moreover, other laser spectroscopic methods such as conventional [25] or coherent versions [26] of Raman spectroscopy [27], laser-stimulated fluorescence [28], and now photo-acoustic [29] and optogalvanic [30] spectroscopy have drawn more attention. While in specific situations these techniques may have greater sensitivity or other special advantages, one can readily show that laser absorption also offers unique potential, if not the novelty of the alternatives.

Laser absorption spectroscopy of high temperature gases in the ultraviolet or visible spectral regions has received scant attention, presumably for lack of a perceived need. The necessary tunable (dye) lasers themselves are readily available commercially. The only applications to absorption spectroscopy of gases of which we are aware, however, is a study of $\mathrm{OH}$ absorption in a water vapor discharge [31] and in a methane-air flame [32,33], and detection of $633 \mathrm{~nm}$ absorption by unidentified polyacetylene radicals [34]. Visible ( $\mathrm{Ar}$ and $\mathrm{He}-\mathrm{Ne}$ ) lasers have also been used to follow the concentration of soot aerosol formed in pyrolytic or oxidative processes $[34,35]$ and the oxidation of carbon particles suspended in high temperature gases [36]. The use of two laser wavelengths permits one to find the average size of the aerosol droplets as well as the volume fraction of carbon present [35]. 
After a long gestation period, tunable diode lasers for the infrared spectral region are now available commercially also [37,38]. Typical diodes have a tuning range of about $100 \mathrm{~cm}^{-1}$, and beams that can be focused to very small diameters. For high temperature applications, what remains to be demonstrated in full is the sensitivity for important species, absence of spectral interference, and, for kinetic spectroscopy applications, adequate signal-to-noise ratio. The first application was reported by Hanson [39], who scanned single $\mathrm{CO}$ absorption lines in a shock-heated $\mathrm{CO}-\mathrm{H}_{2}-\mathrm{Ar}$ mixture at $3340 \mathrm{~K}$. The line profiles recorded were found to be in agreement with theoretical profiles calculated from the shock temperature and reasonable values for the collisional broadening parameter. An application to Co concentration measurements in a flame was reported by Hanson, Kuntz, and Kruger [40]. A propane-air flame burning at atmospheric pressure and having a flame temperature of $2000 \mathrm{~K}$ gives a $\mathrm{CO}$ partial pressure of about $0.06 \mathrm{~atm}$. For these conditions, it was found that only one out of the approximately 20 lines studied had a spectral interference. In a later report by Hanson and Falcone [41], the technique was extended to vibrational temperature measurements in flat flames.

It should be noted that diode lasers are by no means the only possible sources for tunable laser absorption spectroscopy in the infrared. Color-center lasers, spin-flip lasers, optical parametric oscillators, and Zeeman-tuned gas lasers have all been shown to provide useful tunability and stability, and there is already a considerable literature on spectroscopic applications of them. It would appear, however, that diode lasers will prove to offer a sufficient advantage in convenience over their competitors to make them the source of choice, in the foreseeable future, for high temperature applications.

\section{Conclusions}

Laser absorption spectroscopy has found useful applications for the characterization of high temperature gases. The advent of tunable lasers with high spectral resolution, wavelength and power stability promises that further applications will be forthcoming. Of particular interest are line-of-sight concentration measurements of species concentrations in flames and shock tubes. Other applications to temperature measurements, lineshape studies, and general spectroscopy are possible.

The laser absorption spectroscopy research cited from our laboratory has been carried out in connection with shock tube investigations of high temperature decomposition and combustion reactions. Acknowledgment is made to the donors of the Petroleum Research Fund, administered by the American Chemical Society, for partial support of this research. This research was also supported by the Robert A. Welch Foundation and the U.S. Army Research Office. 
[1] Mallard, W. G. and Gardiner, Jr., W. C., J. Quant. Spectrosc. Radiat. Transfer (1978), in press.

[2] Bennett, Jr., W. R., Appl. Opt. Supp. 2, 3 (1965).

[3] Eckstrom, D. J. and Bershader, D., J. Chem. Phys. 57, 632 (1972).

[4] Edwards, B. N. and Burch, D. E., J. Opt. Sci. Am. 55, 174 (1965).

[5] Emrich, R. J. and Soloukhin, R. I., Astronautica Acta, 17, 639 (1972).

[6] Owen, J. H. and Gardiner, Jr., W. C., Seventeenth International Symposium on Combustion, Leeds, 1978 (The Combustion Institute, 1979), p. 720, in press.

[7] Gardiner, Jr., W. C., Owen, J. H., Clark, T. C., Dove, J. E., Bauer, S. H., Miller, J. A., and McLean, W. J., in Fifteenth International Symposium on Combustion, Tokyo, 1974 (The Combustion Institute, 1975), p. 857.

[8] Heffington, W. M., Parks, F. E., Sulzmann, K. G. P., and Penner, S. S., J. Quant. Spectrosc. Radiat. Transfer, 16, 839 (1976).

[9] Gardiner, Jr., W. C., Herrmann, J. M., Mallard, W. G., and Owen, J. H., Int. J. Chem. Kin. 8,111 (1976).

[10] Heffington, W. M., Parks, F. E., Sulzmann, K. G. P., and Penner, S. S., in Sixteenth International Symposium on Combustion, MIT, 1976 (The Combustion Institute, 1977), p. xxx. [11] 01son, D. B. and Gardiner, Jr., W. C., Combust. Flame, 32, 151 (1978).

[12] Gerritsen, H., in Proceedings of the Third International Congress on Quantum Electronics, Paris, 1963, P. Grivet and N. Bloembergen, eds. (Columbia University Press, 1964), p. 581 .

[13] Gerritsen, H. J. and Heller, M. E., App1. Opt. Supp1. 2, 73 (1965).

[14] Goldring, H., Szöke, A., Zamir, E., and Ben-Reuven, A., J. Chem. Phys. 49, 4253 (1968).

[15] 01 son, D. B., Mal1ard. W. G., and Gardiner, Jr., W. C., App1. Spectrosc. 32, 489 (1978).

[16] 01 son, D. B., Tanzawa, T., and Gardiner, Jr., W. C., Int. J. Chem. Kin. (1978), in press.

[17] Koike, T. and Gardiner, Jr., W. C., unpublished research.

[18] Eckstrom, D. J. and Bershader, D., J. Chem. Phys. 53, 2978 (1970).

[19] Simpson, C. J. S. M. and Simmie, J. M., J. Chem. Phys. 55, 5842 (1971).

[20] Eckstrom, D. J. and Bershader, D., J. Chem. Phys. $\underline{55}, 5842$ (1971).

[21] Dibble, R. W. and Bowen, J. R., J. Chem. Phys. 65, 2028 (1976).

[22] Hanson, R. K., Monat, J. P., and Kruger, C. H., J. Quant. Spectrosc. Radiat. Transfer 16, 705 (1976).

[23] Hanson, R. K., Monat, J. P., Flower, W. L., and Kruger, C. H., in Tenth International Shock Tube Symposium, Kyoto, 1975, G. Kamimoto, ed. (Shock Tube Research Society, Japan, 1975), p. 536.

[24] Monat, J. P., Hanson, R. K., and Kruger, C. H., in Seventeenth International Symposium on Combustion, Leeds, 1978 (The Combustion Institute, 1979), in press.

[25] Rosenblatt, G. M. and Drake, M. C., this volume. 
[26] Eckbreth, A. C., this volume.

[27] Lapp, M. and Penney, C. M. , in Laser Raman Gas Diagnostics (Plenum Press, NY, 1974).

[28] McDonald, J. R., Barenauski, A. P., Pasternack, L., Lemont, S., and Harvey, A. B., this volume.

[29] Anderson, W. R., Allen, Jr., J. E., Fansler, T. D., and Crosley, D. R., this volume.

[30] Smith, K. C., Schenck, P. K., Mallard, W. G., and Travis, J. C., this volume.

[31] Wang, C. C. and Ki11inger, D. K., Phys. Rev. Lett. 39, 929 (1977).

[32] Lück, K. C. and Müller, F. J., J. Quant. Spectrosc. Radiat. Transfer, 17, 403 (1977).

[33] Lück, K. C. and Thielen, W., J. Quant. Spectrosc. Radiat. Transfer, 20, 71 (1978).

[34] Tanzawa, T. and Gardiner, Jr., W. C., in Seventeenth International Symposium on Combustion, Leeds, 1978 (The Combustion Institute, 1979), in press.

[35] Graham, S. C., Homer, J. B., and Rosenfeld, J. L. J., Proc. Roy. Soc. London, A344, 259 (1975).

[36] Park, C. and Appleton, J. P., Recent developments in shock tube research, Proceedings of the Ninth International Shock Tube Symposium, D. Bershader and W. Griffith, eds. (Stanford University Press, 1973), p. 793.

[37] Ni11, K. W., in Laser Focus (February 1977), p. 32.

[38] Hinkley, E. D., Nill, K. W., and Blum, F. A., in Laser Spectroscopy of Atoms and Molecules, H. Walther, ed. (Springer Verlag, Heidelberg, 1976), chap. 2.

[39] Hanson, R. K., App 1. 0pt. 16, 1479 (1977).

[40] Hanson, R. K., Kuntz, P. A., and Kruger, C. H., Appl. 0pt. 16, 2045 (1977).

[41] Hanson, R. K. and Falcone, P. K., Appl. 0pt. 17, 2477 (1978). 
National Bureau of Standards Special Publication 561, Proceedings of the 10th Materials Research Symposium on Characterization of High Temperature Vapors and Gases held at NBS, Gaithersburg, Maryland, September 18-22, 1978. Issued October 1979.

\title{
NEAR-RESONANT RAYLEIGH SCATTERING AND ATOMIC FLAME FLUORESCENCE SPECTROSCOPY 1
}

\author{
Calvin Chan and John W. Daily \\ Department of Mechanical Engineering \\ University of California \\ Berkeley, CA 94720
}

\begin{abstract}
Near-resonant Rayleigh scattering and collision induced resonance fluorescence of sodium in a combustion environment at atmospheric pressure has been observed. Sodium atoms in the equilibrium region of a flat flame burner were excited by a pulsed dye laser. The behavior of the two signals as the laser was detuned from the resonance line was studied and the behavior agrees with theoretical predictions. The results have important implications for atomic flame fluorescence spectroscopy in that trapping is avoided at large atom number densities.
\end{abstract}

\section{Introduction}

Laser induced fluorescence spectroscopy has proven to be a most useful technique for the analytic chemist in recent years. The high degree of resolution afforded by narrow, tunable laser sources combined with their high power results in a very selective and sensitive spectroscopic probe.

The major problems associated with laser fluorescence continue to be quenching at all concentrations of the analytic species, and radiative trapping at large number densities. We and others have attempted to overcome the quenching problem through the use of saturated fluorescence method.

Recently, we have been studying near resonant scattering from atomic species in flames and have found that by utilizing the resonantly enhanced Rayleigh signal, radiative trapping can be reduced significantly.

In the following, we discuss the nature of near resonant scattering and its implications for atomic spectroscopy. In Section 3, we present experimental results for sodium.

${ }^{1}$ This work was performed under Air Force Grant AFOSR-77-3357. 


\section{Theoretical Considerations}

Spectral analysis of near-resonant scattering of laser light by atomic vapor has been studied by several workers $[1,2,3,4]^{2}$ in recent years and can be demonstrated using the energy level diagram of figure 1 . When a monochromatic light wave impinges on an atom near one of its resonance lines, part of the light is scattered. The spectrum of the scattered light is centered on the incident wavelength, and is known as Rayleigh scattering. If the atom that scatters light collides with other atoms, the scattered light has a second component emitted at the resonance frequency, known as fluorescence. This process is known as collisional redistribution. The practical importance of this for atomic fluorescence flame spectroscopy lies in the fact that the Rayleigh component is not on one of the absorption lines, and hence, it is not trapped even at quite high atom concentrations.

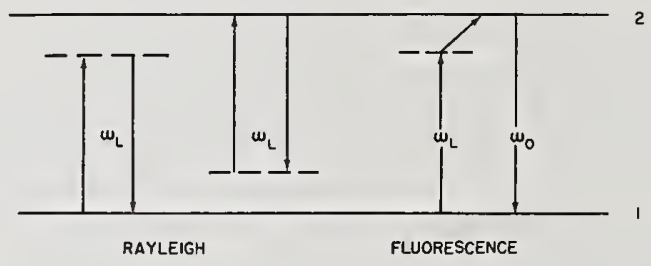

Figure 1. Energy level diagram.

The theory of collisional redistribution at high laser intensity has been developed by Mollow [2]. The integrated Rayleigh scattering per unit volume, $I_{R}$, can be shown to be

$$
I_{R}=N \frac{\left(\gamma_{N} / 2\right) \Omega^{2}}{\Delta^{2}+\Omega^{2}}
$$

where $N$ is the atomic number density. $\gamma_{N}$ is the natural line width; $\Delta$ is the amount of detuning from the resonant frequency, and $\Omega=\mu E / \hbar$ is the Rabi frequency associated with the incident laser field $\vec{E}(t)=\hat{\varepsilon} E(t) \cos w t$. $\hat{\varepsilon}$ is the laser's polarization vector and $\mu=e(2|\hat{\varepsilon} \cdot r| 1)$ is the electric dipole matrix element. Thus, $\Omega^{2}$ is proportional to the laser intensity. In the low intensity limit, i.e., $\Omega \ll \Delta, I_{R}$ should be proportional to $1 / \Delta^{2}$. Moreover, it is directly proportional to the atomic number density. Hence, if all other quantities are known, $I_{R}$ can be used as a direct measurement of the concentration of that atomic species.

The behavior of the collision induced fluorescence signal is given by

$$
I_{F}=\frac{N\left(\gamma_{N} / 4\right) \Omega^{2}\left(\Omega^{\prime}+\Delta\right)\left[\eta\left(\Omega^{\prime}+\Delta\right)-\Delta\right]}{\left(\Delta^{2}+\Omega^{2}\right)\left(\Delta^{2}+\eta \Omega^{2}\right)},
$$

${ }^{2}$ Figures in brackets indicate the literature references at the end of this paper. 
where $\eta=\left(\gamma_{N}+\gamma_{I}+\gamma_{E}\right) /\left(2 \gamma_{N}+2 \gamma_{I}\right)$ and $\gamma_{I}$ and $\gamma_{E}$ are the half widths of broadening due to inelastic and elastic collisions, respectively. $\Omega^{\prime}=\Delta+\delta$ where $\delta$ is the stark shift given by $\delta=\Delta\left\{\left[1+(\Omega / \Delta)^{2}\right]^{\frac{1}{2}}-1\right\}$. The dependence of $I_{F}$ is not as easily seen, since $\gamma_{E}$ is usually a function of $\Delta$ also [3].

of importance for analytic purposes is the ratio of the integrated Rayleigh signal to that of the resonant fluorescence signal so that signal to noise considerations can be assessed.

For multilevel systems, the resonant fluorescence scattering per unit volume may be written as [5]

$$
I_{F}=N \gamma_{N} \beta\left(\frac{E_{\nu}}{E_{v}^{S}+E_{v}}\right)
$$

where $E_{\nu}$ is the spectral irradiance of the laser and $E_{\nu}{ }^{S}$, the saturation irradiance, which implicitly accounts for collisional and radiative decay processes.

$$
\beta=\left[1+\frac{g_{j}}{g_{k}}+\sum_{j \neq k}\left(\frac{N_{j}}{N_{k}}\right)^{*}\right]^{-1}
$$

is a parameter which reflects syphoning of population to other levels. $\left(\frac{N_{j}}{N_{k}}\right)^{*}$ is the quasiequilibrium population ratio. $E_{\nu}$ is directly related to $\Omega^{2}$ by the relation (for plane monochromatic waves)

$$
\Omega^{2}=\frac{\mu^{2}}{\varepsilon_{0} c \hbar^{2}} E_{\nu} \delta \nu_{L}
$$

where $\varepsilon_{0}$ is the dielectric constant of free space, $\mu$ is the dipole moment for the transition being considered; $c$ is the speed of light, $\hbar$ is the Plank's constant, and $\delta v_{L}$ is the laser band width.

Combining eqs. (1), (3), and (5), one obtains

$$
\frac{I_{R}}{I_{F_{R}}}=\frac{\Omega_{S}^{2}+\Omega^{2}}{\Delta^{2}+\Omega^{2}},
$$

where $\Omega_{s}$ corresponds to the saturation irradiance in units of Rabi frequency. $\beta$ drops out because the $\mathrm{N}$ in eq. (1) is the number density of the ground and the excited state only. Here, a two level system with $g_{1}=g_{2}$ has been assumed.

In the fully saturated limit, $I_{R} / I_{F_{R}}$ goes to unity and there is no distinction between the Rayleigh and fluorescence scattering. The more usual situation is for either the weak limit to hold, in which case $\Omega \ll \Omega_{\mathrm{s}}, \Delta$ and 


$$
I_{R} / I_{F_{R}} \rightarrow \Omega_{S}{ }^{2} / \Delta^{2}
$$

or for the laser power to be large enough to saturate the resonance fluorescence, so that $\Omega \gg \Omega_{\mathrm{s}}$ and

$$
I_{R} / I_{F_{R}} \rightarrow \frac{\Omega^{2}}{\Delta^{2}+\Omega^{2}}
$$

For most atomic species, $\Omega_{\mathrm{s}}$ is of the order of $10^{9} \mathrm{~s}^{-1}$ or $0.02 \AA$. Thus, for $\Delta$ equal to about $1 \AA$, the Rayleigh signal will be about 0.04 percent of the resonant fluorescence signal or larger. Our laser has an intensity of about $10^{10} \mathrm{~W} / \mathrm{m}^{2}$ over a bandpass of $0.1 \AA$, which corresponds to $\Omega \simeq 10^{11} \mathrm{~s}^{-1}$. Thus, at a detuning of $1 \AA \mathrm{I}_{\mathrm{R}} / \mathrm{I}_{\mathrm{F}_{\mathrm{R}}}$ is about one third.

Figure 2 shows how $I_{R} / I_{F}$ varies (eq. (6)) with detuning for various values of $\Omega$ assuming a typical atomic species with $\Omega_{\mathrm{s}}=10^{9} \mathrm{~s}^{-1}(0.02 \AA)$.

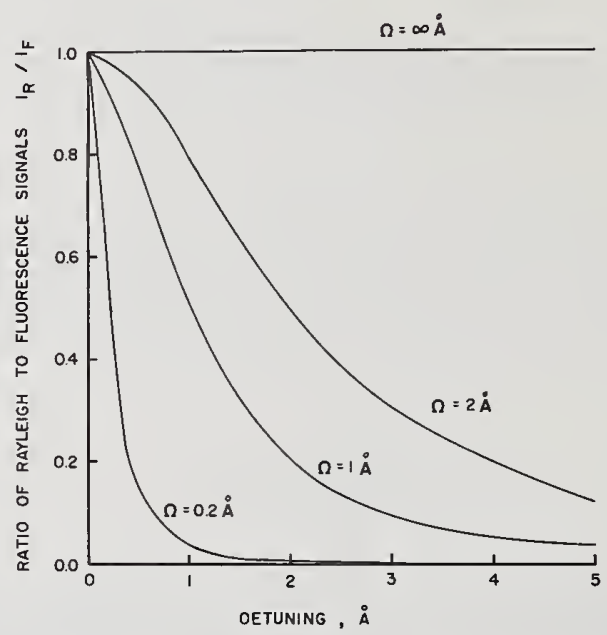

Figure 2. Ratio of Rayleigh to fluorescence signal as a function of detuning.

Atomic line widths are typically quite small. For many atomic lines, the pressure and Doppler widths are about the same, on the order of $0.01 \mathrm{~A}$. The amount of detuning actually used in an experiment is determined by practical considerations, and typically the smaller monochromators seen in analytic work can be used effectively to detunings of about $1 \AA$. Thus, trapping of the Rayleigh component should be completely negligible. This is likely to always be the case because in order to separate the Rayleigh and fluorescent components, the laser detuning must be outside the fluorescent, and thus absorption, line width. So one would always operate in the thin wings. 


\section{Experimental Apparatus and Results}

We have used a CMX-4 flash lamp pumped tunable dye laser to study the scattered spectrum for near-resonant excitation. The laser was tuned to near the $5890 \mathrm{~A}$ sodium 1 ine and focused into the equilibrium region of a methane air flat flame seeded with sodium. The diameter of the burner is $1.25 \mathrm{~cm}$. The dye laser has a pulse width of $1.5 \mu \mathrm{s}$, a spectral width of 0.1 $\AA$, and an energy of $1-2 \mathrm{~mJ}$. The focal volume has a diameter of about $0.4 \mathrm{~mm}$, thus a Rabi frequency of about $10^{11} \mathrm{~s}^{-1}$, meaning that we would saturate the resonant fluorescence signal. The emission was measured at $90^{\circ}$ to the laser beam with $\mathrm{f} / 3.5$ collection optics. The spectrum was resolved using a $0.5 \mathrm{~m}$ monochromator with a spectral resolution of $0.1 \mathrm{~A}$. The laser intensity was monitored using a photo diode. The output of the photomultiplier, placed at the exit slit of the monochromator, was fed into a dual channel gated ( $2 \mu \mathrm{s})$ analog integrator. It was then normalized by the laser pulse. Signal averaging was used to improve the signal-to-noise ratio.

A spectral scan, taken under the above conditions, is shown in figure 3 . The laser was set $2 \AA$ on the red side of the resonance 1 ine. As shown in the figure, we observed the two peaks. Figure 4 shows the behavior of the Rayleigh scattering signal and the induced fluorescence signal as a function of detuning. Only detuning to the red of $5896 \AA 1$ ine and to the blue of $5890 \AA$ are shown. Since the two sodium lines are so close together, the enhanced Rayleigh scattering for excitation between the two lines will have contribution from both lines which can make the phenomenon more complex. As shown in the figure, for detuning up to $5 \AA$, the Rayleigh component shows the expected $1 / \Delta^{2}$. As mentioned before, the dependence of the fluorescence component can be more complicated. The error bars indicated on the graphs represent the standard deviation of the fluctuation of the signal. The single sample uncertainties are relatively small compared to this fluctuation.

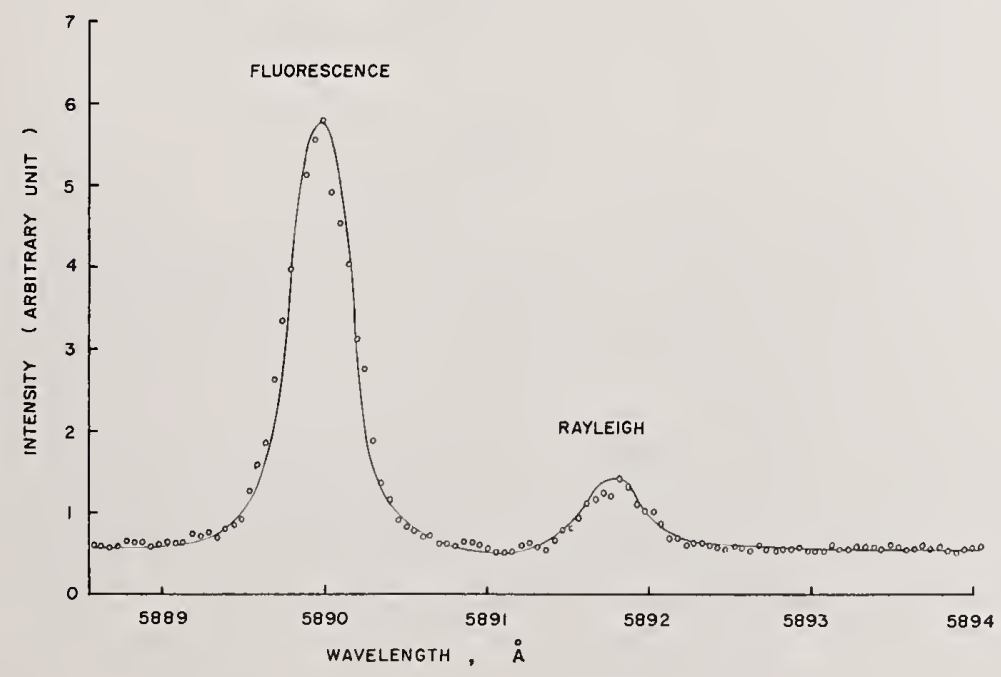

Figure 3. Scattered spectrum from near-resonant excitation. 


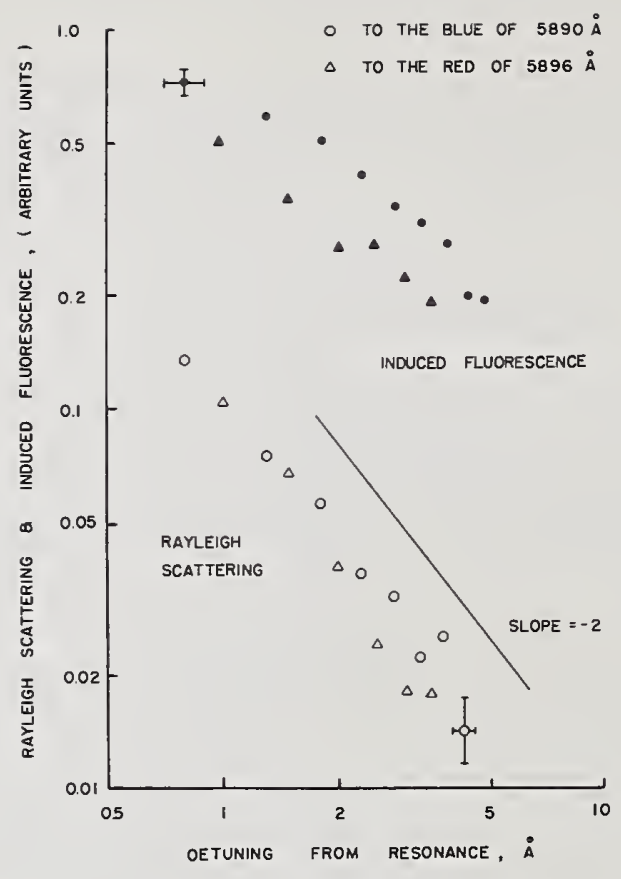

Figure 4. Rayleigh scattering and induced fluorescence signal as a function of detuning.

The effect of trapping is demonstrated in figures 5 and 6 which show the behavior of the collision induced fluorescence and Rayleigh signals as the trapping distance increases. Figure 5 is for the optically thick limit and figure 6 for the optically thin limit. As can be seen, the Rayleigh signal is not trapped. $A_{T}$ is the total absorption factor determined using a tungsten lamp in a conventional absorption measurement.

\section{Summary}

In summary, we have observed resonantly enhanced Rayleigh scattering and fluorescence caused by collisional redistribution for sodium in a flame and the results agree with theoretical predictions. It has also been shown that the Rayleigh scattering signal is not trapped in optically thick situations. Moreover, the enhanced Rayleigh scattering signal can be quite large compared to the resonant fluorescence signal for detunings large enough to avoid the trapping effect. The results have important implications for atomic flame fluorescence spectroscopy in that the Rayleigh scattering signal can be used to obtain a linear curve of growth when the atom number density is high. 


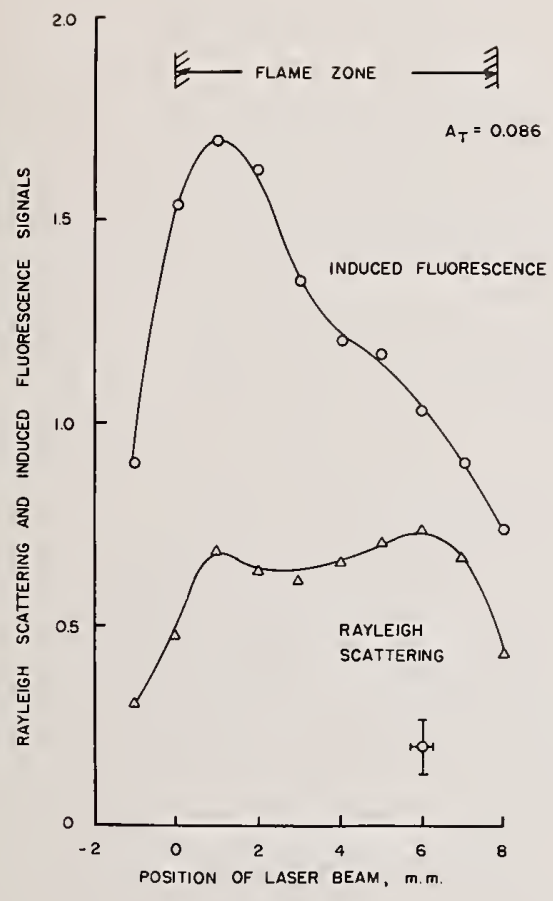

Figure 5. Comparison of Rayleigh and fluorescence trapping for optically thick flame.

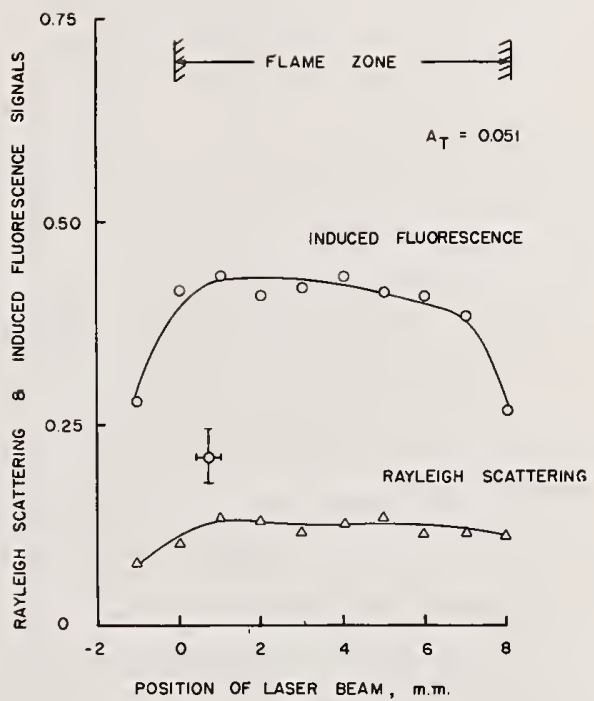

Figure 6. Comparison of Rayleigh and fluorescence trapping for optically thin flame.

\section{References}

[1] Omont, A., Smith, E., and Cooper, J., The Astrophysical Journal, 175, 185 (1972).

[2] Mollow, B. R., Phys. Rev. A 15, 1023 (1977).

[3] Carlsten, J. L., Szöke, A., and Raymer, M. G., Phys. Rev. A 15, 1029 (1977).

[4] Vriens, L., J. App1. Phys. 48, 653 (1977).

[5] Daily, J. W., Applied Optics, 17, 2 (1978). 

National Bureau of Standards Special Publication 561, Proceedings of the 10th Materials Research Symposium on Characterization of High Temperature Vapors and Gases held at NBS, Gaithersburg, Maryland, September 18-22, 1978. Issued October 1979.

\title{
LASER INDUCED REACTIONS OF LITHIUM IN FLAMES
}

\author{
C. H. Muller III, K. Schofield, and M. Steinberg \\ Quantum Institute \\ University of California \\ Santa Barbara, CA 93106
}

\begin{abstract}
Laser induced chemical reactions of electronically excited $\operatorname{Li}\left(2^{2} \mathrm{P}_{3 / 2,1 / 2}\right)$ have been observed from fluorescence measurements in rich $\mathrm{H}_{2} / \mathrm{O}_{2} / \mathrm{N}_{2}$ flames under laser saturated conditions. The enhanced removal rate of excited 1 ithium by $\mathrm{H}_{2} \mathrm{O}$ and $\mathrm{H}_{2}$ is shown to be sufficiently fast relative to the laser pulse duration to significantly modify the free lithium concentration. A mathematical model consistent with previously measured quenching rates is proposed to explain the laser induced chemical reactions. Differences between the model and experiment are less than 15 percent.
\end{abstract}

\section{Introduction}

Laser fluorescence techniques provide a high level of sensitivity for monitoring trace constituents, particularly atomic species. Also, with the advent of sufficiently powerful pulsed tunable lasers, it has become possible to operate such systems quite routinely in a saturated absorption mode. This has the additional advantages of eliminating the need for any information or assumptions concerning collisional quenching of the excited state or the stability and magnitude of the laser power $[1,2]^{1}$. It has been suggested as an alternate analytical mode $[3,4]$.

Although such systems have been studied in detail for atomic sodium, both theoretically $[1,2,5-10]$ and experimentally $[3-7,11-13]$, the possibility of laser induced chemistry was consistently overlooked. Recently, however, laser induced chemical reactions of electronically excited sodium $\left(3^{2} \mathrm{P}_{3 / 2,1 / 2}\right)$ were reported [14]. We now report the first measurements of laser induced chemical reactions of $\mathrm{Li}\left(2^{2} \mathrm{P}_{3 / 2,1 / 2}\right)$ in rich hydrogen-oxygen-nitrogen flames doped with trace additions of $\mathrm{LiCl}$. These measurements have significant implications not only for the interpretation of fluorescence data obtained in the saturated absorption mode, but also provide a means for obtaining high temperature data for electronically excited states which, for most species, are not generally available.

${ }^{1}$ Figures in brackets indicate the literature references at the end of this paper. 


\section{Experimental}

A scaled drawing of the optical system is shown in figure 1. Emission from the flame is collected by mirror, $M_{1}(15.2 \mathrm{~cm}$ diameter and $81 \mathrm{~cm}$ radius of curvature) and directed via $M_{2}$ into an image rotator, $R$, which images a horizontal slice of the central flame into the vertical entrance slit of the monochromator. The image rotator consists of 2 mirrors at $45^{\circ}$ to the horizontal, one face above the other, with their planes of reflection offset by $90^{\circ}$ about a vertical axis. The emission collection optics and monochromator have $\mathrm{f} / 5.3 \mathrm{aper}-$ tures. The detector is an EMI 9558QBM photomultiplier. Physical constraints forced the use of reflective optics $\left(M_{3}\right)$ in the output from the monochromator to the photomultiplier. A quartz (Suprasi1) lens, $L_{\eta}$, is positioned so that the cathode subtends the exit beam from the monochromator over the spectral range from 180 to $700 \mathrm{~nm}$.

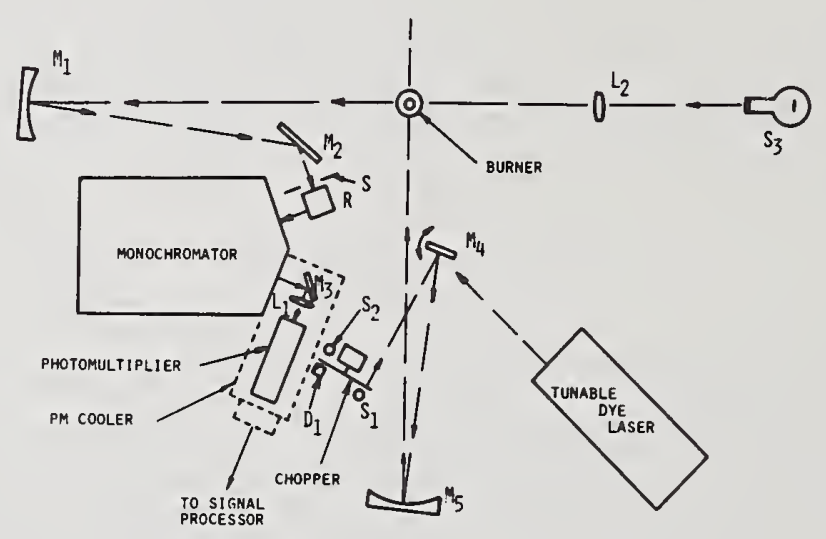

Figure 1. Optical system schematic. Fluctuations in the laser power are monitored by gathering a small fraction of the laser signal with an oblique placed between the laser and mirror $M_{4}$.

The entrance slit of the monochromator is $1 \mathrm{~cm}$ high and sees a horizontal slice of the central flame $1 \mathrm{~cm}$ wide. The spatial resolution above the flame is determined by the monochromator slit width with depth of field corrections for the $f / 5.3$ optics. For fluorescence measurements the photomultiplier output is a.c. coupled to a current/voltage and voltage/voltage amplifying system and then fed into a boxcar integrator synchronized to the laser output pulse. 
The exciting light source for the Li measurements is a tunable, flashlamp pumped dye laser equipped with intracavity etalons for line narrowing and doubling crystals (used primarily in our concurrent sulfur flame chemistry experiments).

The third arm of the optical layout in figure 1 is for sodium 1 ine reversal flame temperature measurements using the tungsten filament lamp $S_{3}$ and injecting sodium chloride solution into the flame with an ultrasonic nebulizer [15]. A stop, $S$, is positioned at the conjugate focus of lens $L_{2}$ in mirror $M_{1}$ to guarantee that the solid angle of emitted radiation into the detector is equal to the solid angle sampled from the image of the tungsten filament at the flame [16].

A series of premixed $\mathrm{H}_{2} / \mathrm{O}_{2} / \mathrm{N}_{2}$ flames of varying composition were stabilized on the $2 \mathrm{~cm}$ diameter flat-flame Padley-Sugden burner [17] with the burnt gases exhibiting nearly isothermal, one-dimensional, vertical flows. Typical flame temperatures varied from 1700 to $2200 \mathrm{~K}$ and burnt gas flow velocities from $(0.6-1.3) \times 10^{3} \mathrm{~cm} \mathrm{~s}^{-1}$. An aerosol of a solution of $\mathrm{LiCl}$ was added to the premixed flow to the central core $(1.1 \mathrm{~cm}$ diam) of the flame with the ultrasonic nebulizer. Atomic lithium is generated by the very rapid dissociation of $\mathrm{LiCl}$ in the flame reaction zone. Complications arising from optical trapping were avoided by maintaining the atomic lithium concentrations at $10^{9}$ to $10^{10} \mathrm{~cm}^{-3}$ with a $1 \mathrm{~cm}$ optical path.

The lithium doublet $(670.776$ and $670.791 \mathrm{~nm})$ was excited to near saturation conditions by the dye laser while resonance fluorescence was monitored at $670.8 \mathrm{~nm}$ with a $0.15 \mathrm{~nm}$ bandpass. The laser line was narrowed to approximately $0.5 \mathrm{~cm}^{-1}(0.02 \mathrm{~nm})$ with an intracavity etalon and had a beam diameter of $0.1 \mathrm{~cm}$. The laser was operated at $15 \mathrm{pulses} / \mathrm{s}$ with a $2 \mu$ sulse duration. Small variations in pulse to pulse laser power have been shown to be unimportant in the near saturated mode of operation [1,2], and these variations were not considered when analyzing this fluorescence data. However, the nonsaturated fluorescence data were normalized to the laser power. Temporal variations of the emission and fluorescence intensities (saturated and unsaturated conditions) in the burnt gases of the various flames were recorded by raising or lowering the burner relative to the optical measurement axes. H-atom axial concentration profiles were measured for the flame series using the $\mathrm{Na} / \mathrm{Li}$ method [18].

\section{Results and Discussion}

Unlike ground state atomic sodium, atomic lithium is not inert in fuel rich flames, but exists as a mixture of atomic lithium and LiOH according to the equilibrium

$$
\left.\mathrm{Li}^{2}{ }^{\mathrm{S}} \mathrm{S}_{1 / 2}\right)+\mathrm{H}_{2} \mathrm{O} \stackrel{\mathrm{LiOH}}{\leftarrow} \mathrm{H} . \quad \Delta \mathrm{H}_{2000 \mathrm{~K}}^{0}=+85.1 \mathrm{~kJ} \mathrm{~mol}{ }^{-1} .
$$

Consequently, thermal emission profiles of $\mathrm{Li}$ in isothermal flames exhibit a significant decay with increasing distance above the burner, reflecting the approach of the $\mathrm{H}$-atom concentrations towards their equilibrium values. This process serves as the basis for the $\mathrm{Na} / \mathrm{Li}$ technique for monitoring $\mathrm{H}$-atom concentrations in flames. 
Fluorescence profiles for Li obtained with low laser power (nonsaturated conditions) exhibit decay profiles in agreement with such emission data. This behavior is illustrated in figure 2 for an $\mathrm{H}_{2} / \mathrm{O}_{2} / \mathrm{N}_{2}=3 / 1 / 4$ flame. However, as also shown in figure 2 under near saturated laser excitation conditions, the fluorescence decay is strikingly increased. There is a markedly enhanced removal of Li during the $2 \mu \mathrm{s} 1$ laser pulse. The laser induced chemistry found responsible for the removal of sodium [14] applies equally well to the removal of lithium in near saturated conditions and is described by the following reaction scheme,

$$
\mathrm{Li}\left({ }^{2} \mathrm{P}_{3 / 2,1 / 2}\right)+\mathrm{H}_{2} \mathrm{O} \underset{\leftarrow}{\mathrm{LiOH}}+\mathrm{H} \quad \Delta \mathrm{H}_{2000 \mathrm{~K}}^{0}=-93.2 \mathrm{~kJ} \mathrm{~mol}{ }^{-1}
$$

followed by

$$
\left.\mathrm{LiOH}+\mathrm{H} \underset{\leftarrow}{\mathrm{LiO}}{ }^{2} \mathrm{~S}_{1 / 2}\right)+\mathrm{H}_{2} 0 . \quad \Delta \mathrm{H}_{2000 \mathrm{~K}}^{0}=-85.1 \mathrm{~kJ} \mathrm{~mol}{ }^{-1} .
$$

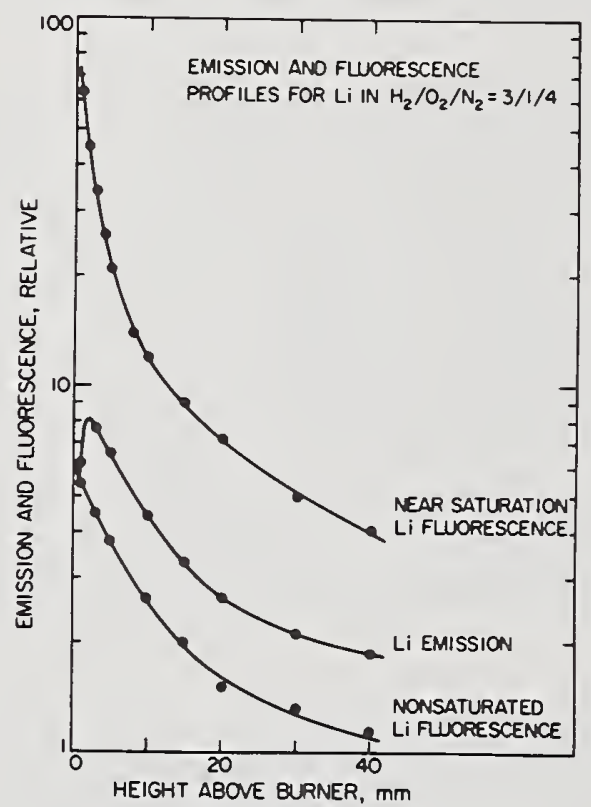

Figure 2. Emission and fluorescence profiles for $\mathrm{Li}$ in an $\mathrm{H}_{2} / \mathrm{O}_{2} / \mathrm{N}_{2}=$ $3 / 1 / 4$ flame.

The reaction of $\mathrm{H}_{2} \mathrm{O}$ with $\mathrm{Li}\left({ }^{2} \mathrm{P}\right)$ should be at least $10^{2}$ times faster than with $\mathrm{Li}\left({ }^{2} \mathrm{~S}\right)$. Some contribution from an additional laser induced process

$$
\mathrm{Li}\left({ }^{2} \mathrm{P}_{3 / 2,1 / 2}\right)+\mathrm{H}_{2} \vec{\leftarrow} \mathrm{LiH}+\mathrm{H} \quad \Delta \mathrm{H}_{2000 \mathrm{~K}}^{0}=+27.6 \mathrm{~kJ} \mathrm{~mol}{ }^{-1}
$$


followed by

$$
\mathrm{LiH}+\mathrm{H} \rightarrow \mathrm{Li}\left({ }^{2} \mathrm{~S}_{1 / 2}\right)+\mathrm{H}_{2} \Delta \mathrm{H}_{2000 \mathrm{~K}}^{0}=-205.8 \mathrm{~kJ} \mathrm{~mol}^{-1}
$$

is also evident. These reaction schemes are summarized in figure 3 . The reactions are expected to proceed at rates sufficient to permit the rapid establishment of steady state $\mathrm{LiOH}$ and $\mathrm{LiH}$ concentrations during the initial rise of the $2 \mu \mathrm{s}$ laser pulse.

Li LASER INDUCED CHEMISTRY MODEL

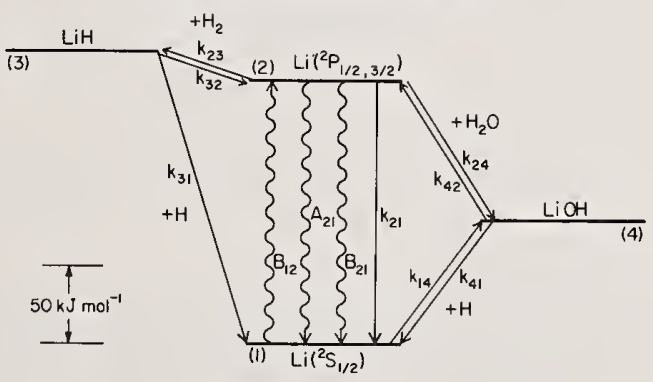

Figure 3. Li laser induced chemistry model.

The high non-equilibrium concentration of $\mathrm{H}$-atom early in these fuel rich flames tends to suppress the $\mathrm{LiOH}$ and $\mathrm{LiH}$ buildups. However, as $\mathrm{H}$-atom decays toward equilibrium in the flow above the burner, increased $\mathrm{LiOH}$ and $\mathrm{LiH}$ concentrations will exist during the laser pulse resulting in reduced $L i\left({ }^{2} P_{1 / 2,3 / 2}\right)$ fluorescence intensities.

Since complete mixing of the $\operatorname{Li}\left({ }^{2} \mathrm{P}_{1 / 2}\right)$ and $\mathrm{Li}\left({ }^{2} \mathrm{P}_{3 / 2}\right)$ states can be assumed in these atmospheric pressure $f 1$ ames $[12,13]$, the rate relationships for the processes shown in figure 3 are

$$
\begin{aligned}
\frac{d n_{2}}{d t}=n_{1} B_{12} I_{v}-n_{2}\left[A_{21}+B_{21} I_{v}+k_{21}(M)\right. & \left.+k_{24}\left(H_{2} O\right)+k_{23}\left(H_{2}\right)\right] \\
& +\left[n_{4} k_{42}+n_{3} k_{32}\right](H) \\
\frac{d n_{4}}{d t}=\left(k_{14} n_{1}+k_{24} n_{2}\right)\left(H_{2} 0\right)-\left(k_{41}+k_{42}\right) n_{4}(H) & \\
\frac{d n_{3}}{d t}= & k_{23} n_{2}\left(H_{2}\right)-\left(k_{31}+k_{32}\right) n_{3}(H)
\end{aligned}
$$


where $n_{1}$ through $n_{4}$ are the number densities of the species as labeled. Note that $n_{2}$ is the total $L i\left({ }^{2} P_{J}\right)$ concentration. $A_{21}, B_{12}$, and $B_{21}$ are the Einstein transition probabilities for emission, absorption, and induced emission. $I_{v}$ is the intensity of the laser beam.

For saturation conditions of $\operatorname{Li}\left({ }^{2} P_{3 / 2,1 / 2}\right)$, steady state conditions for $n_{2}, n_{3}$, and $n_{4}$ during the laser pulse, and noting that the fluorescence signal

$$
I_{f}=A_{21} n_{2}
$$

this kinetic model predicts the relationship

where

$$
\frac{\mathrm{n}_{0}}{\mathrm{I}_{\mathrm{f}}}=\frac{4}{3 \mathrm{~A}_{21}}+\frac{1}{\mathrm{~A}_{21}}\left[\frac{\mathrm{k}_{14} / 3+\mathrm{k}_{24}}{\mathrm{k}_{41}+\mathrm{k}_{42}}\left(\mathrm{H}_{2} 0\right)+\frac{\mathrm{k}_{23}}{\mathrm{k}_{31}+\mathrm{k}_{32}}\left(\mathrm{H}_{2}\right)\right] \frac{1}{(\mathrm{H})}
$$

$$
n_{0}=n_{1}+n_{2}+n_{3}+n_{4}
$$

is the total lithium density in the flame.

For any one specific flame, since the concentrations of the major species $\mathrm{H}_{2} \mathrm{O}$ and $\mathrm{H}_{2}$ are essentially constant in the burnt gases and close to their calculated equilibrium values, eq. (9) predicts a direct relationship between $n_{0} / I_{f}$ and $1 /[\mathrm{H}]$. As indicated in figure 4 , this is found to be the case for all the flames studied with a maximum variation of 15 percent.

Previous experiments in flames have indicated that $\mathrm{H}_{2} \mathrm{O}$ and $\mathrm{H}_{2}$ quench 1 ithium resonance radiation with cross sections of about $6 x$ and $16 \times 10^{-16} \mathrm{~cm}^{2}$, respectively [12]. The quenching cross section with $\mathrm{H}_{2} 0$, about 10 percent of the gas kinetic value, is surprisingly small and unexplained. The exact nature of these interactions, namely the relative importance of physical to reactive quenching remains unknown. Nevertheless, such cross sections are compatible with our data. If the $6 \times 10^{-16} \mathrm{~cm}^{2}$ cross section with $\mathrm{H}_{2} 0$ refers to the reactive process, characterized by the rate constant $k_{24}$, then a significant steady state concentration of $\mathrm{LiOH}$ relative to the free lithium atoms will occur during the initial rise-time of the $2 \mu \mathrm{s}$ laser pulse if $\mathrm{k}_{41}$ for the $\mathrm{LiOH}+\mathrm{H}$ reaction is somewhat less than its gas kinetic value. Formation of $\mathrm{LiH}$ from $\left.\mathrm{Li}^{2}{ }^{2} \mathrm{P}\right)$ and $\mathrm{H}_{2}$ is $27.6 \mathrm{~kJ} \mathrm{~mol}^{-1}$ endothermic. Coupled with a gas kinetic cross section of about $28 \times 10^{-16^{2}} \mathrm{~cm}^{2}$ for this process, we obtain an optimum effective reactive quench cross section of about $5.3 \times 10^{-16} \mathrm{~cm}^{2},\left[28 \times 10^{-16} \exp \left(-\Delta \mathrm{H}_{23} / \mathrm{RT}\right)\right]$, at $2000 \mathrm{~K}$. Such a cross section is compatible with LiH also being a significant lithium sink. The relative importance of these channels leading from $\operatorname{Li}\left({ }^{2} \mathrm{P}_{3 / 2}, 1 / 2\right)$ to the hydroxide and hydride is verified in figure 4 . Here, the best fit to our near saturation fluorescence data is obtained by assuming approximately equal reactive cross sections for the formation of $\mathrm{LiOH}$ and $\mathrm{LiH}$ from $\mathrm{Li}\left({ }^{2} \mathrm{P}_{3 / 2,1 / 2}\right)$. It would therefore appear that whereas the $6 \times 10^{-16} \mathrm{~cm}^{2}$ 
quenching cross sections for $\mathrm{Li}\left({ }^{2} \mathrm{P}\right)$ by $\mathrm{H}_{2} \mathrm{O}$ refers predominantly to a reactive quench mechanism, only about 30 percent of the quenching cross section for $L i\left({ }^{2} P\right)$ by $\mathrm{H}_{2}$ is reactive in nature.

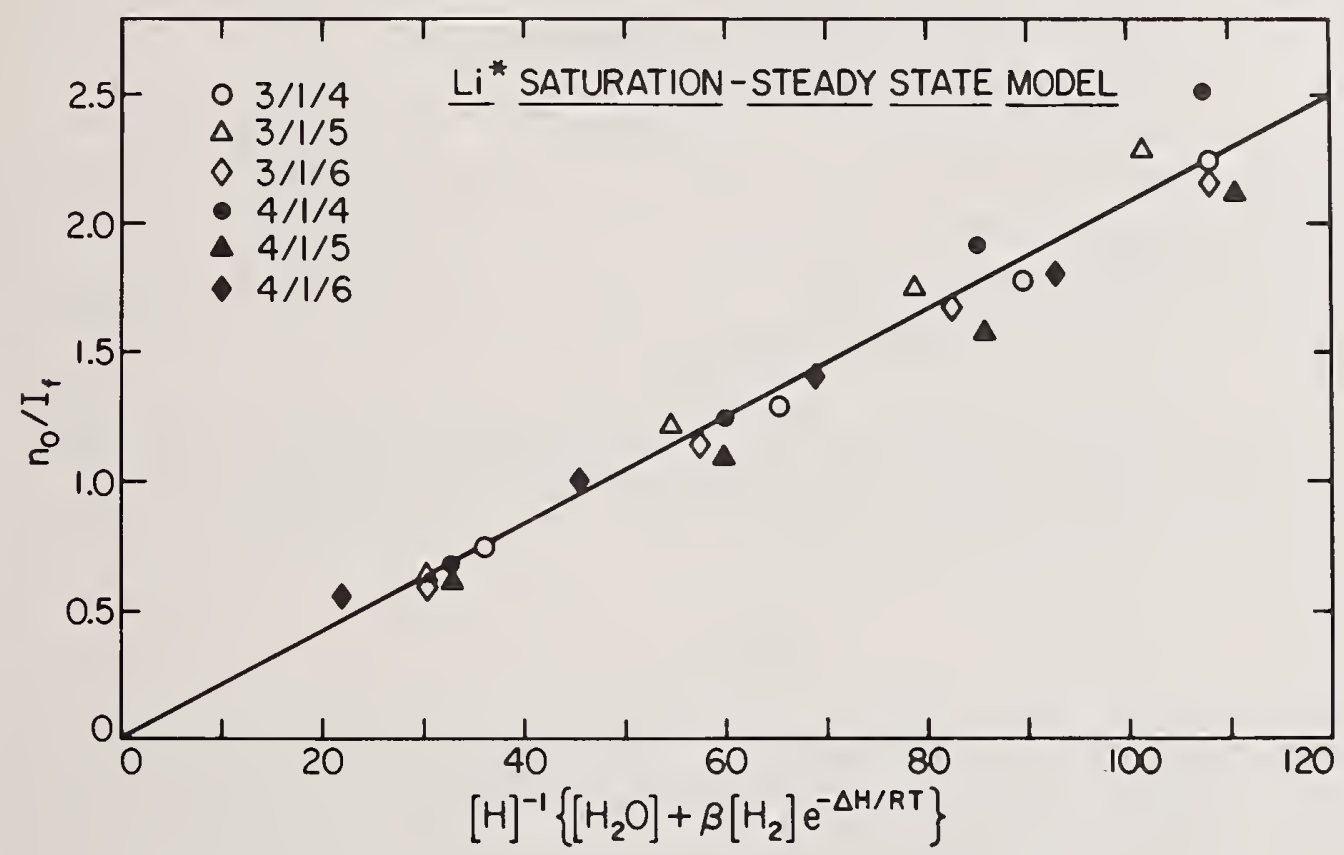

Figure 4. Comparison of experiment and theory for the $L i^{*}$ saturation steady state model. Best fit to experimental data is obtained with $\beta=2.80$.

The enhanced thermal rate of ionization of $\mathrm{Li}\left({ }^{2} \mathrm{P}_{3 / 2,1 / 2}\right)$ or reaction with $\mathrm{OH}$ to yield $\mathrm{Li}^{+}$and $\mathrm{OH}^{-}$are also plausible laser induced reactions in these flames via

$$
\mathrm{Li}\left({ }^{2} \mathrm{P}_{3 / 2,1 / 2}\right)+\mathrm{M} \rightarrow \mathrm{Li}^{+}+\mathrm{e}^{-}+\mathrm{M} \quad \Delta \mathrm{H}_{2000 \mathrm{~K}}^{0}=+383.5 \mathrm{~kJ} \mathrm{~mol}{ }^{-1}
$$

or

$$
\mathrm{Li}\left({ }^{2} \mathrm{P}_{3 / 2,1 / 2}\right)+\mathrm{OH} \rightarrow \mathrm{Li}^{+}+\mathrm{OH}^{-} . \quad \Delta \mathrm{H}_{2000 \mathrm{~K}}^{0}=+165.2 \mathrm{~kJ} \mathrm{~mol}^{-1} .
$$

Even though these adverse enthalpies are much reduced below those corresponding to ground state atoms, nevertheless, calculations of the times for a 1/e approach to equilibrium ionization for flames temperatures of $\leq 2300 \mathrm{~K}$ yields values too long to make significant contributions within the life of the laser pulse $(2 \mu \mathrm{s})$. 


\section{Conclusions}

A similar laser induced flame chemistry has been noted for lithium as was previously found with near saturated laser excited sodium [14]. However, in that case ground state sodium is essentially inert in the flame and does not react with $\mathrm{H}_{2} \mathrm{O}$ within the few millisecond time scale available in the flame flows. Nevertheless, the results in both cases are explained by essentially the same kinetic model.

Such laser induced chemistry presents a new approach for obtaining high temperature data for electronically excited states. The relative importance of laser induced ionization processes and chemical interactions with flame species such as $\mathrm{H}_{2} \mathrm{O}, \mathrm{H}_{2}, \mathrm{O}_{2}$, $\mathrm{CO}$, and $\mathrm{CO}_{2} \mathrm{Can}$ be established for a wide range of elements. The nature of the various sub-states and their mixing cross sections may be investigated at high temperatures and compared to that of the ground state.

Also, the occurrence of such laser induced chemistry has a potentially considerable impact on the development of quantitative saturation fluorescence techniques which still make no allowance for its possible occurrence [19].

\section{Appendix}

The laser induced chemistry models used to explain the near saturation sodium [14] and lithium data are identical with respect to the excited state chemical reactions considered. It has been shown by our group recently, however, that additional reactions to those shown in figure 3 are important in stoichiometric flames. These additional reactions (probably the formation of sodium oxides) are not presently well understood. Consequently, the $\mathrm{Na}^{*}$ near saturation data in reference [14] has been reanalyzed without the stoichiometric flames $(2 / 1 / 4-2 / 1 / 6)$. The comparison of experiment and theory for the $\mathrm{Na}^{*}$ data (fue 1 rich flames only) is shown in figure 5 . Here, the best fit to theory is obtained with a $\beta$ of 28.75 which yields a maximum difference between experiment and theory of about 5 percent. This $\beta$ implies that the channel leading to $\mathrm{NaH}$ from $\mathrm{Na}\left({ }^{2} \mathrm{P}\right)$ and $\mathrm{H}_{2}\left(\Delta \mathrm{H}_{23}=+40.6 \mathrm{~kJ} \mathrm{~mol}{ }^{-1}\right)$ is approximately 2.5 times $\left[28.75 \exp \left(-\Delta \mathrm{H}_{23} / \mathrm{RT}\right)\right]$ more important than that leading to $\mathrm{NaOH}$ via $\mathrm{Na}\left({ }^{2} \mathrm{P}\right)$ and $\mathrm{H}_{2} \mathrm{O}$ at $2000 \mathrm{~K}$. 


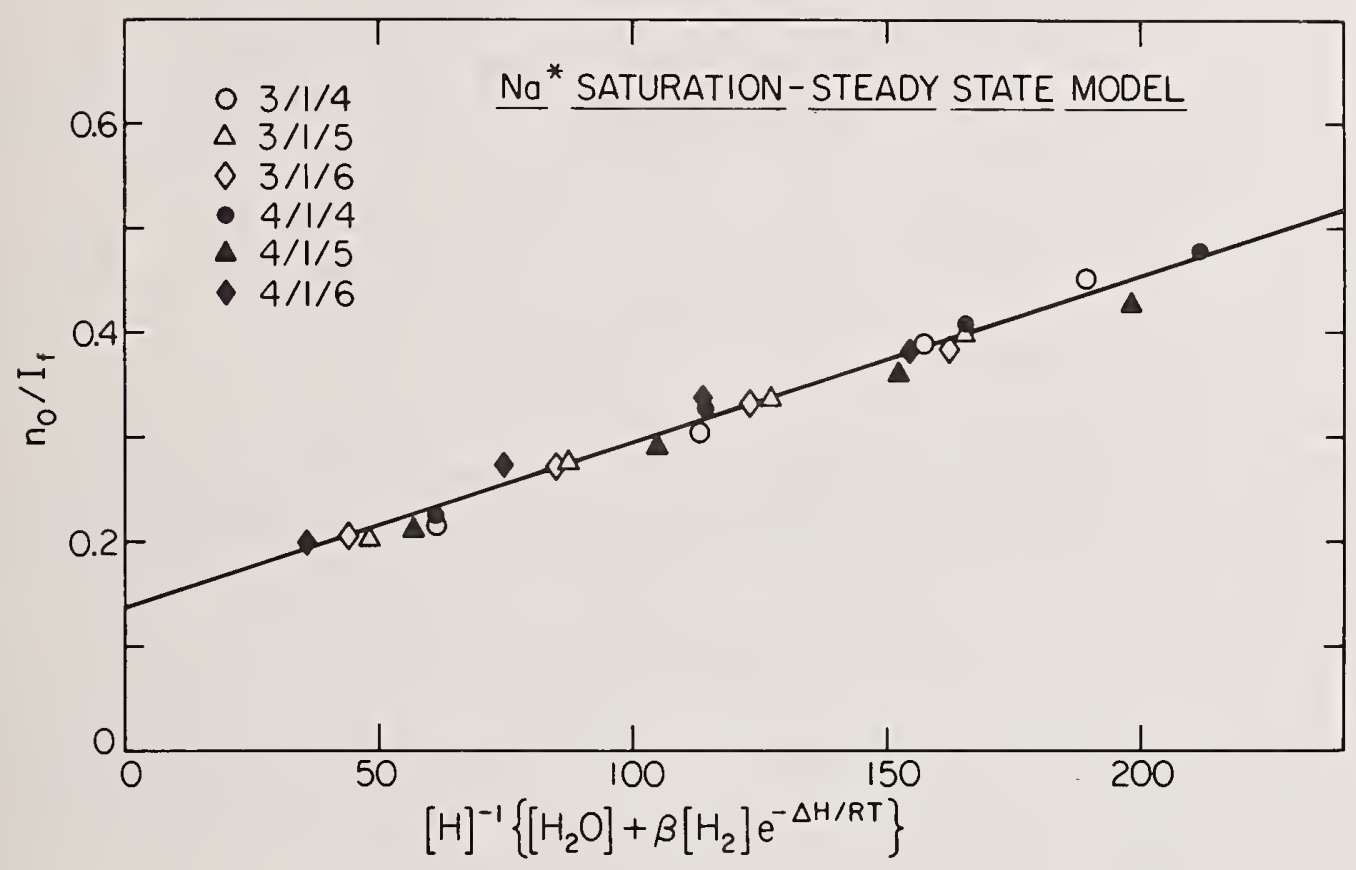

Figure 5. Comparison of experiment and theory for the $\mathrm{Na}^{*}$ saturation steady state mode1. Best fit to experimental data is obtained with $\beta=28.75$.

\section{References}

[1] Piepmeier, E. H., Spectrochim. Acta, 27B, 431 (1971).

[2] Daily, J. W. , App1. Optics, 16, 568 (1977).

[3] Gelbwachs, J. A., Klein, C. F., and Wesse1, J. E., Appl. Phys. Letters, 30, 489 (1977).

[4] Smith, B., Winefordner, J. D., and Omenetto, N., J. Appl. Phys. 48, 2676 (1977).

[5] Kuh1, J., Neumann, S., and Kriese, M., Z. Naturforsch. 28a, 273 (1973).

[6] Sharp, B. L. and Goldwasser, A., Spectrochim. Acta, 31B, 431 (1976).

[7] Eckbreth, A. C., Bonczyk, P. A., and Verkieck, J. F., Review of laser Raman and fluorescence techniques for practical combustion diagnostics, United Technologies Research Center Report EPA-600/7-77-066, PB269653, (June 1977).

[8] Omenetto, N., Winefordner, J. D., and Alkemade, C. Th. J., Spectrochim. Acta, 30B, 335 (1975). 
[9] Daily, J. W., App1. Optics, 15, 955 (1976).

[10] 01 ivares, D. R. and Hieftje, G. M., Saturation of energy levels in analytical atomic fluorescence spectrometry. I. theory, Dept. of Chemistry, Indiana University Report No. 3, (December 1976): AD/A 033543 (to be published in Spectrochim. Acta, 33B (1978)).

[11] Jenkins, D. R., Spectrochim. Acta, 25B, 47 (1970).

[12] Linjnse, P. L., Review of literature on quenching, excitation and mixing collision cross sections for the first resonance doublets of the alkalis, Fysisch Laboratorium Report 398, Rijksuniversiteit Utrecht, The Netherlands (February 1972).

[13] Krause, L., Advan. Chem. Phys., 28, 267 (1975).

[14] Muller III, C. H., Schofield, K., and Steinberg, M., Chem. Phys. Letters, 59, 364 (1978).

[15] Denton, M. B. and Swartz, D. B., Rev. Sci. Instr. $\underline{45}, 81$ (1974).

[16] Thomas, D. L., Combustion Flame, 12, 569 (1968).

[17] Padley, P. J. and Sugden, T. M., Proc. Roy. Soc. A248, 248 (1958).

[18] Bulewicz, E. M., James, C. G., and Sugden, T. M., Proc. Roy. Soc. A235, 89 (1956). [19] Mailander, M. , J. Appl. Phys. 49, 1256 (1978).

\section{Discussion}

Question (Daily): One of the things that I am a little curious about is that your beta is a parameter that puts all of the curves together. Does that beta give a fixed ratio of hydride to the hydroxide? Does it give a fixed ratio of the rates?

Response (Steinberg): It gives a fixed ratio of the rates, the fluxes that go into the hydride and the fluxes that go into the hydroxide.

Question (Daily): And you expect as you went down in temperature you would start seeing more hydroxide?

Response (Steinberg): Yes. The endothermicity for the hydride formation is rather small, so it is not a strong temperature dependence, and our temperature ranges from 1700 to 2300 Kelvin. It is a relatively narrow temperature range.

Comment (Hauge): The work that we do in matrices with metal atoms and water, where we are apparently exciting the metal atom in order to get it to react with the water, seems similar to what you are suggesting in your studies, and it rather looks like most metal atoms would undergo these kinds of reactions rather rapidly from what we have seen.

Response (Steinberg): It is not surprising, a lot of laser induced chemistry is now operative by organic chemists, inorganic chemists, physical chemists, mechanical engineers, and--I am looking for civil engineers to get into the game. 
National Bureau of Standards Special Publication 561, Proceedings of the 10th Materials Research Symposium on Characterization of High Temperature Vapors and Gases held at NBS, Gaithersburg, Maryland, September 18-22, 1978. Issued October 1979.

\title{
OPTO-GALVANIC SPECTROSCOPY: A NEW LOOK AT ATOMS AND MOLECULES
}

\author{
Kermit C. Smyth, Peter K. Schenck, W. Gary Mallard,
} and

John C. Travis

\author{
National Bureau of Standards \\ Washington, DC 20234
}

Opto-galvanic spectroscopy is a "new" method to obtain absorption spectra of atoms and molecules by measuring voltage and current changes upon laser irradiation of discharges and flames. This paper describes a variety of experiments which demonstrate the sensitivity and versatility of this technique. In addition, many of the important mechanisms which produce the observed voltage and current signals have been identified.

\section{Introduction}

Opto-galvanic spectroscopy measures current and voltage changes which are produced upon visible-uv irradiation of flames and discharges. These electrical signals are due to absorption of photons whose energy corresponds to electronic transitions. Spectroscopic and analytical applications of this effect may be studied without monitoring either the absorption from the incident light beam or the re-emitted fluorescence. Conventional absorption techniques suffer the inherent disadvantage of comparing two large signals, i.e., attempting to observe a small decrease in a large photon flux. In contrast, opto-galvanic spectroscopy monitors signals against a much reduced background noise, determined by the electrical stability of the flame or discharge. Thus, common optical interference sources, such as background emission, scattered excitation light, and ambient light, are completely avoided.

Workers at the National Bureau of Standards were the first to apply opto-galvanic spectroscopy to the study of flames $[1]^{1}$. On the other hand, in discharges such observations were made fifty years ago by Penning [2], who irradiated one neon tube with light from a second neon discharge. Despite this early start, however, very few similar experiments have been carried out [3-7]. More recently, opto-galvanic effects (i.e, voltage and current changes) have been observed in the discharges of gas lasers, in particular for helium-neon $[8,9]$, xenon [10], and $\mathrm{CO}_{2}[11]$. The substantial observed voltage changes have been employed

${ }^{1}$ Figures in brackets indicate the literature references at the end of this paper. 
to frequency stabilize a $\mathrm{CO}_{2}$ laser [12], and hollow cathode lamps can be used to lock tunable dye lasers to specific atomic transitions [13]. In addition, the convenience of opto-galvanic spectroscopy in discharges for spectral calibration and bandwidth determination has been demonstrated [14].

Other photoelectric effects in gases have been used to study excitation mechanisms and photoionization cross sections. Of special relevance are experiments which utilize thermionic diodes to detect ion formation [15-18]. These diodes are operated under spacecharge-limited conditions, and deliberate care is taken to avoid a self-sustained discharge. Nevertheless, the effects of collisional ionization of highly excited species are directly pertinent to our interpretation of recent discharge experiments $[19,20]$.

In this paper, we focus attention upon opto-galvanic spectroscopy performed in both discharges and flames during the last two years. In discharges, we have made detailed measurements on neon in order to elucidate the important mechanisms involved in producing the observed voltage changes [20]. Most of the data can be readily explained in terms of photon-induced processes which either enhance or decrease ionization rates in the discharge. This ionization model has been substantiated by two mass spectrometer experiments [21,22]. Also, absorption by atomic ions has been detected in hollow cathode discharges [23].

In the flame studies, analytical applications have been explored successfully for trace element detection. Sensitivity comparable to or better than conventional optical methods has been demonstrated for a large number of elements [24]. Most recently, we have reported the first results on molecular absorption using opto-galvanic spectroscopy [25], in which extensive data have been obtained in premixed flames on LaO, YO, and ScO.

\section{Discharge Experiments}

Most of our discharge experiments have been carried out in commercial hollow cathode lamps. A schematic diagram of the apparatus and detection circuitry is shown in figure 1. Typically, the visible output beam from a $\mathrm{cw}$ tunable dye laser is directed axially down the bore of the discharge. When the wavelength of this light corresponds to an electronic transition for a species in the discharge, a change in the anode-cathode voltage is produced. These voltage changes have been measured by chopping the laser beam and using phase sensitive detection to observe the voltage modulation. In the circuit shown in figure $1, a$ ballast resistor is placed in series with the discharge tube and a constant dc voltage source. Thus, an increase in the current upon irradiation is accompanied by a corresponding decrease in the discharge voltage, and vice-versa. 


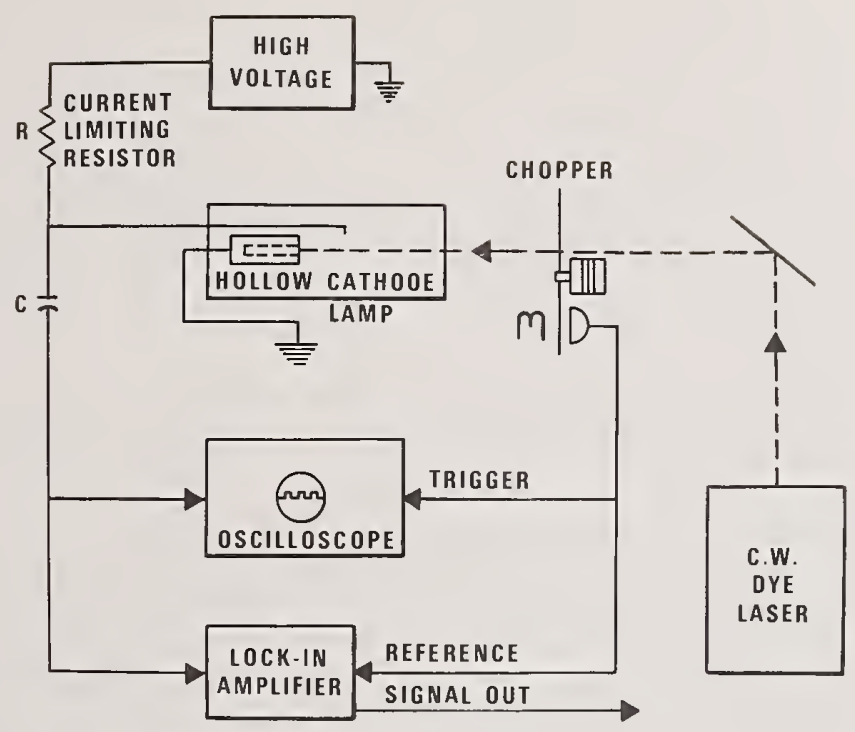

Figure 1. Schematic diagram of the discharge apparatus. Typical conditions employ $\mathrm{CW}$ laser operation (20-65 mW), 80-4000 Hz modulation frequency, 190-250 VDC anodecathode voltage, 2-25 mA discharge current, $22.5 \mathrm{k} \Omega$ current limiting resistor, and a $1.0 \mu \mathrm{F}$ coupling capacitor.

\section{1 Neon experiments}

The opto-galvanic spectroscopy of neon discharges has helped to identify many of the processes important in producing the observed voltage signals. In the 572-654 nm wavelength region, a total of 75 absorption transitions have been detected [20], a 11 of which originate in high-lying electronic states. Figure 2 shows a $10 \mathrm{~nm}$ portion of the neon spectrum and serves to illustrate several important features of this technique. First, we observe a11 but the very weakest known neon transitions in this wavelength range. Second, both positive and negative voltage signals are detected, and finally, this spectrum exhibits signal-tonoise ratios which exceed 1000 on the strong lines. This sensitivity, obtained in a few minutes without long signal averaging, is perhaps the most attractive feature of optogalvanic spectroscopy. 


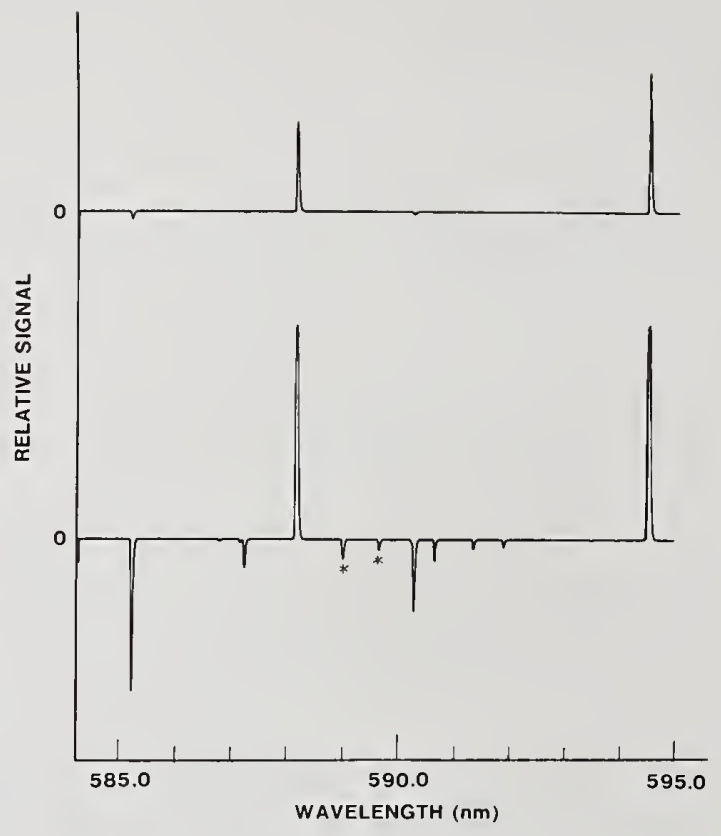

Figure 2. Opto-galvanic spectrum from a commercial sodium-neon hollow cathode lamp operating at $10 \mathrm{~mA}$, using an $0.02-\mathrm{nm}$ bandwidth, 10-mW laser beam. The two starred lines in the lower trace are sodium transitions; all other signals are due to neon. The lower trace has been amplified by a factor of 25 , to show the negative voltage signals (the two positive lines are off scale).

The absorption transitions observed in neon can be broadly classified into two groups, $1 s \rightarrow 2 p$ and $2 p \rightarrow 3 s, 4 d, \ldots$ in Paschen notation. The interpretation of our results is based on photon-induced ionization changes in the neon discharge. Three processes will make important contributions to ionization:

$$
\begin{array}{ll}
\mathrm{Ne}+\mathrm{e}^{-} \rightarrow \mathrm{Ne}^{+}+2 \mathrm{e}^{-} & \text {direct ionization } \\
\mathrm{Ne}^{*}+\mathrm{e}^{-} \rightarrow \mathrm{Ne}^{+}+2 \mathrm{e}^{-} & \begin{array}{l}
\text { collisional ionization of } \\
\text { metastables by electrons }
\end{array} \\
\mathrm{Ne}^{*}+\mathrm{Ne}^{*} \rightarrow \mathrm{Ne}^{+}+\mathrm{Ne}+\mathrm{e}^{-} & \begin{array}{l}
\text { metastable-metastable } \\
\text { collisions. }
\end{array}
\end{array}
$$


The majority of transitions detected in the 572-654 $\mathrm{nm}$ region are $2 \mathrm{p} \rightarrow 3 \mathrm{~s}, 4 \mathrm{~d}, \ldots$, and all of these lines exhibited negative voltage signals, corresponding to increased currents upon laser irradiation [20]. There are two ways in which ionization can be enhanced and lead to the observed voltage changes. First, collisional ionization by electrons will increase as the excitation energy of neon increases, since there are more electrons with sufficient energy to cause ionization. Second, the associative ionization channel becomes energetically allowed for neon atoms excited to the $3 \mathrm{~s}, 4 \mathrm{~d}$, and higher-lying electronic states:

$$
\mathrm{Ne} e^{* *}+\mathrm{Ne} \rightarrow \mathrm{Ne}_{2}^{+}+\mathrm{e}^{-} \quad \text { associative ionization }
$$

The $1 \mathrm{~s} \rightarrow 2 \mathrm{p}$ transitions form the more interesting group, since many of these 1 ines showed voltage signals which changed sign as a function of discharge current [20]. These transitions also exhibited the largest voltage signals, which is not surprising in view of the fact that the $1 \mathrm{~s}$ states are either truly metastable $\left(1 s_{5}\right.$ and $\left.1 s_{3}\right)$ or quasi-metastable due to radiative trapping $\left(1 s_{4}\right.$ and $\left.1 s_{2}\right)$. Therefore, these states have large populations and play an important role in discharge ionization due to processes (2) and (3) above.

Our results on the $1 \mathrm{~s} \rightarrow 2 \mathrm{p}$ transitions can be understood in terms of photon-induced changes in ionization rates, but the interpretation is complex [20]. At low discharge currents $(\sim 2 \mathrm{~mA})$ radiative decay of the $2 p$ states to the $1 \mathrm{~s}$ states is most important, particularly with regard to its effect on the populations of the true metastable states, $1 s_{5}$ and $1 s_{3}$. At currents of $15-25 \mathrm{~mA}$ collisional ionization of the excited neon $2 \mathrm{p}$ states becomes more important and eventually dominates (i.e., all voltage signals are negative at high currents). In addition, coupling of nearby electronic states by collisions with both electrons and ground state neon atoms is competitive with radiative decay and collisional ionization, thus complicating considerably the energy transfer in a neon discharge. For example, if one monitors the fluorescence following excitation of a specific $1 s \rightarrow 2 p$ transition, one observes emission from nearby $2 p$ states lying both higher and lower in energy [20]. Similar fluorescence results have been reported in a He-Ne discharge [26].

The competition between radiative and collision processes is also illustrated by the temporal behavior of the observed voltage changes [20]. Most of the $1 \mathrm{~s} \rightarrow 2 \mathrm{p}$ transitions showed signals with two components of opposite polarity, with different characteristic time constants; the relative amplitudes of the two components varied with the discharge current. This temporal information is potentially an important probe of discharge processes. However, the comparison of several different neon discharges reveals that the temporal behavior is extremely sensitive to the discharge parameters. The observed time dependence is influenced by the neon pressure, the modulation frequency of the laser beam, the beam diameter (which affects what portion of the discharge is being irradiated), the anode and cathode geometries, as well as the discharge current [21]. It is clear that systematic studies are required in order to extract meaningful information from a temporal analysis of the voltage signals. 
Recently, Bridges [27] and Johnston [28] have observed opto-galvanic signals in discharges. Bridges studied a number of different discharge gases and configurations; the polarity of his voltage signals in a neon tube was similar to our results [20,21]. Johnston was able to obtain Doppler-free spectra in a He-Ne discharge using saturation techniques.

\subsection{Mass spectrometer experiments}

The ionization model used to interpret our neon results is essentially an extension of ideas first proposed by Penning [2] and followed by subsequent workers [3-6]. A second plausible mechanism for producing the voltage changes is that photon excitation alters the energy partitioning in inelastic $\mathrm{e}^{-}-\mathrm{Ne}^{*}$ collisions and thereby changes the electron energy distribution (or temperature) in the discharge $[6,27]$. Clearly, it is of interest to directly test this ionization model, and for this purpose a mass spectrometer experiment was performed at Los Alamos Scientific Laboratory [21]. A hollow cathode discharge was coupled to a time-of-flight mass spectrometer, which was used to directly monitor the $\mathrm{Ne}^{+}$signal sampled from the discharge upon laser irradiation. The laser was tuned to the same $1 s \rightarrow 2 p$ and $2 p \rightarrow 3 s, 4 d, \ldots$ transitions studied earlier [20], and changes in the $\mathrm{Ne}^{+}$concentration and in the discharge voltage were detected simultaneously.

Figure 3 shows the most dramatic result of these measurements. Irradiation of the neon discharge at $614.31 \mathrm{~nm}$, corresponding to excitation of the $1 \mathrm{~s}_{5}$ (10west metastable state) $\rightarrow$ $2 \mathrm{p}_{6}$ transition in neutral neon, produced a substantial positive voltage change across the discharge and a corresponding decrease in the $\mathrm{Ne}^{+}$signal of 38 percent! In fact, all of the $1 s \rightarrow 2 p$ transitions showed ion concentration changes of at least 1 percent (the observed changes were at least a factor of 20 smaller for the $2 p \rightarrow 3 s, 4 d, \ldots$ transitions). However, these $\mathrm{Ne}^{+}$signal changes probably do not quantitatively reflect the overall photon-induced changes in the $\mathrm{Ne}^{+}$density, since the neon ions were sampled from a very small volume of the discharge [21].

Table 1 summarizes the results of the mass spectrometer work; for each transition the sign of the observed $\mathrm{Ne}^{+}$change is that expected on the basis of the detected voltage change. For example, a positive voltage change upon laser irradiation corresponds to a decrease in discharge current, and a decrease in $\mathrm{Ne}^{+}$concentration was measured in each such case. For all of the $1 \mathrm{~s} \rightarrow 2 \mathrm{p}$ transitions, the sign of the voltage changes was consistent with photon-induced changes in the metastable atom population due to radiative $2 \mathrm{p} \rightarrow 1 \mathrm{~s}$ decay [20]. The large $\mathrm{Ne}^{+}$changes observed for the $1 \mathrm{~s} \rightarrow 2 \mathrm{p}$ lines, relative to the $2 p \rightarrow 3 s, 4 s, \ldots$ transitions, indicate that the metastable neon states do play the dominant role in the ionization processes in a neon discharge under our experimental conditions. In addition, for the first time we have direct corroboration that ionization changes accompany the observed voltage signals. The large neon ion changes strongly suggest that the photoninduced voltage changes arise directly from enhanced and decreased ionization. 


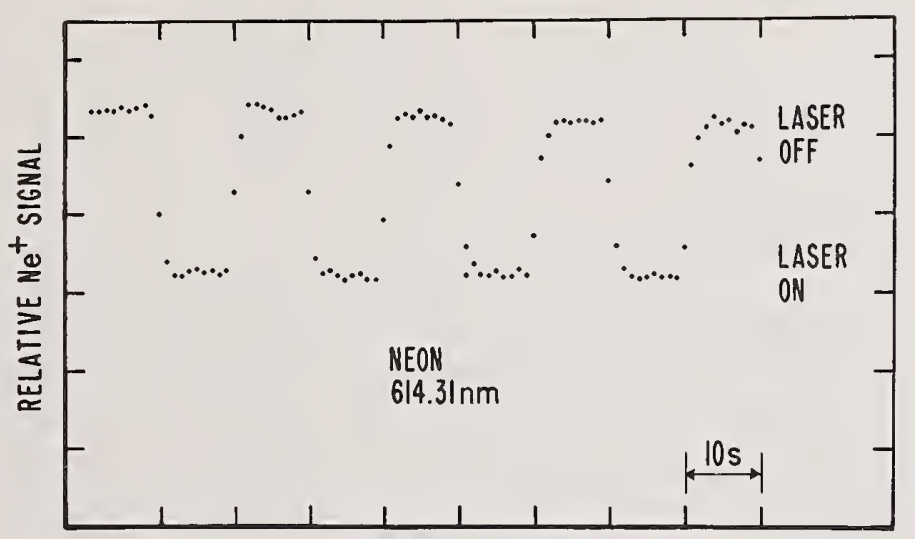

TIME (1S/CHANNEL)

Figure 3. ${ }^{20} \mathrm{Ne}^{+}$signal for irradiation of the neutral neon transition at $614.31 \mathrm{~nm}$ $\left(1 s_{5} \rightarrow 2 p_{6}\right)$. The laser beam is chopped at $0.1 \mathrm{~Hz}$, and the mass spectrometer output is accumulated in a multi-channel analyzer with a dwell time of $1 \mathrm{~s}$ per channel. Experimental conditions: 0.41 Torr (55 Pa) neon pressure, $27 \mathrm{~mA}$ discharge current, and $230 \mathrm{~mW}$ laser power. The photon-induced signal change is -38 percent.

Table 1. Voltage and $\mathrm{Ne}^{+}$changes observed upon irradiation of a neon discharge.

$\begin{array}{lcc}\text { Neon Transitions } & \text { Voltage Change } & \mathrm{Ne}^{+} \text {Change } \\ 1 s_{5}, 1 s_{3} \rightarrow 2 \mathrm{p} & + & - \\ 1 s_{4}, 1 s_{2} \rightarrow 2 \mathrm{p} & - & + \\ 2 \mathrm{p} \rightarrow 3 s, 4 \mathrm{~d} & - & +\end{array}$

A second mass spectrometer experiment was performed, at the University of Virginia, for the purpose of investigating the processes important in ionizing the minor species in a neon discharge [22]. In this work, a quadrupole mass spectrometer was coupled to a hollow cathode discharge, and the concentrations of several ion species were monitored upon laser irradiation at wavelengths corresponding to neutral neon transitions. Again, substantial changes (20-30 percent) in the ion signals were found when exciting $1 s \rightarrow 2 p$ neon transitions and thereby altering the metastable neon atom population. The observed voltage and ion signal changes for the neon species were consistent with the earlier experiments [20,21].

In a neon discharge there are four processes by which a minor species $M$ may be ionized: 


$$
\begin{array}{ll}
\mathrm{Ne}^{*}+M \rightarrow \mathrm{M}^{+}+\mathrm{Ne}+\mathrm{e}^{-} & \text {Penning ionization } \\
M+\mathrm{h} v \rightarrow \mathrm{M}^{+}+\mathrm{e}^{-} & \text {photoionization } \\
\mathrm{M}+\mathrm{e}^{-} \rightarrow \mathrm{M}^{+}+2 \mathrm{e}^{-} & \text {electron impact } \\
\mathrm{Ne}^{+}+\mathrm{M} \rightarrow \mathrm{M}^{+}+\mathrm{Ne} & \text { charge transfer. }
\end{array}
$$

Photon excitation of $1 s_{5} \rightarrow 2 p$ transitions decreases the metastable neon population and produces positive voltage changes. Thus, one expects the rates of (1) and (4) to decrease, (2) to increase, and (3) to remain unchanged [22]. Experimentally, the ion signals for the minor species always decreased upon irradiation at wavelengths corresponding to $1 s_{5} \rightarrow 2 p$ neon transitions. Since the cross sections for asymmetric charge transfer are very small $\left(<10^{-18} \mathrm{~cm}^{2}\right.$ ), Penning ionization is most likely the dominant mode of ionization for the minor species. These results were obtained at low discharge currents. At high currents ( $40 \mathrm{~mA}$ ) Penning ionization was found to play a minor role at best, and electron impact ionization (3) is probably the most important ionization process.

\subsection{Detection of atomic ions}

In our experiments on neon discharges, no absorption by $\mathrm{Ne}^{+}$was detected in the 570-660 $\mathrm{nm}$ wavelength range. This observation is perhaps not surprising for two reasons: (1) The visible absorption spectrum of $\mathrm{Ne}^{+}$originates in high-lying states $(\geq 26.9 \mathrm{eV}$ above the ground state of $\left.\mathrm{Ne}^{+}[29,30]\right)$; and (2) the second ionization potential of neon is also very high $(41.07 \mathrm{eV})$. In a neon discharge, there are few electrons whose energy exceeds $25 \mathrm{eV}$ [6]. Thus, it will be difficult to perturb the rate of

$$
\mathrm{Ne}^{+}+\mathrm{e}^{-} \rightarrow \mathrm{Ne}^{++}+2 \mathrm{e}^{-}
$$

and the overall ionization in a neon discharge by the absorption of $2 \mathrm{eV}$ photons. Therefore, species with low second ionization potentials were chosen for investigation, with the hope that a visible photon would alter the rate of

$$
M^{+}+e^{-} \rightarrow M^{++}+2 e^{-}
$$

and thereby result in a detectable voltage change. 
Figure 4 shows a spectrum obtained in a barium/argon hollow cathode lamp. For Ba transitions, the observed voltage changes were negative, corresponding to enhanced ionization in the discharge, but for $\mathrm{Ba}^{+}$lines at 585.37 and $614.17 \mathrm{~nm}$, positive voltage signals were . detected corresponding to decreased ionization [23]. Positive voltage changes were also observed for three $\mathrm{Eu}^{+}$lines. These results are unexpected and show that the mechanism for producing the voltage signals is not as simple as outlined above. Laser-induced fluorescence experiments have not thus far revealed strong collisional coupling between excited $\mathrm{Ba}^{+}$and neutral argon. Nevertheless, the most plausible explanation for the positive voltage changes is that collisions between the excited ions and neutral argon act to decrease the argon metastable atom population and thus decrease ionization.

All of the transitions observed for $\mathrm{Ba}^{+}$and $\mathrm{Eu}^{+}$originate in low-lying electronic states, and all have large oscillator strengths [31]. Thus, opto-galvanic spectroscopy has not as yet proven to be a particularly sensitive or versatile (or well understood) tool for detecting ionic species. However, in specific favorable cases this technique can be most useful.

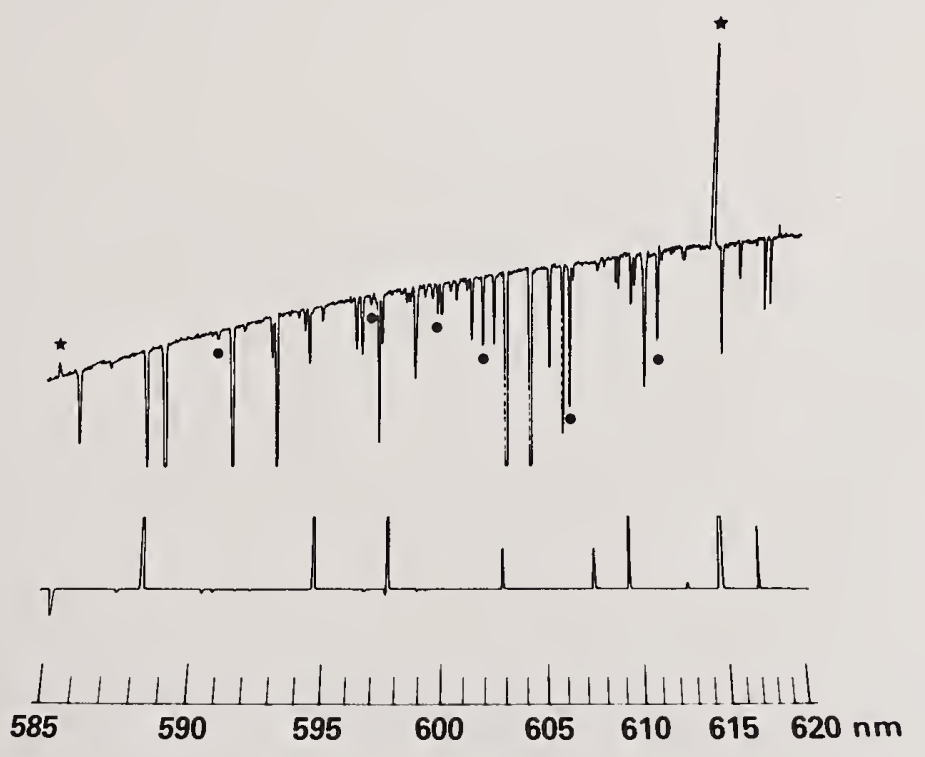

Figure 4. Opto-galvanic spectrum of a barium/argon hollow-cathode lamp. The upper spectrum is the signal obtained by scanning a cw dye laser from $585 \mathrm{~nm}$ to $620 \mathrm{~nm}$ while irradiating a commercial hollow-cathode lamp operating at $15 \mathrm{~mA}$. The laser power was stabilized at $150 \mathrm{~mW}$ throughout the scan. The starred peaks are due to barium ion transitions and the dotted peaks are neutral barium transitions. All other lines are due to neutral argon. The lower spectrum is simultaneously generated from a hollow-cathode lamp containing neon. This spectrum is used to calibrate the non-linear wavelength scan of the dye laser. 


\section{Flame Experiments}

As mentioned previously, only in the last two years has opto-galvanic spectroscopy been applied in a flame environment. Figure 5 illustrates a typical experimental arrangement, in which the opto-galvanic spectra are obtained by monitoring photon-induced current changes as a function of laser wavelength. The apparatus has three parts: (1) the burner-electrode assembly, (2) the tunable dye laser, and (3) the data acquisition electronics. Aqueous samples are aspirated into premixed air-acetylene and air-hydrogen flames, which are maintained at atmospheric pressure on a $5 \mathrm{~cm}$ long slot burner. The signal is detected with a pair of $1 \mathrm{~mm}$ diameter tungsten welding rods, placed parallel to the slot and in close proximity to opposite sides of the flame. These rods are used as the cathode (at $\sim-1000 \mathrm{~V}$ ), while the anode is the burner head. Flashlamp-pumped [24], nitrogen-pumped [25], and continuous-wave [1] tunable dye lasers have been employed as excitation sources. For the pulsed laser work, the photon-induced current signals are separated from the dc background current with a high-pass filter, amplified, and then processed by a boxcar averager. When using $\mathrm{CW}$ lasers, the current signals are observed with phase-sensitive detection.

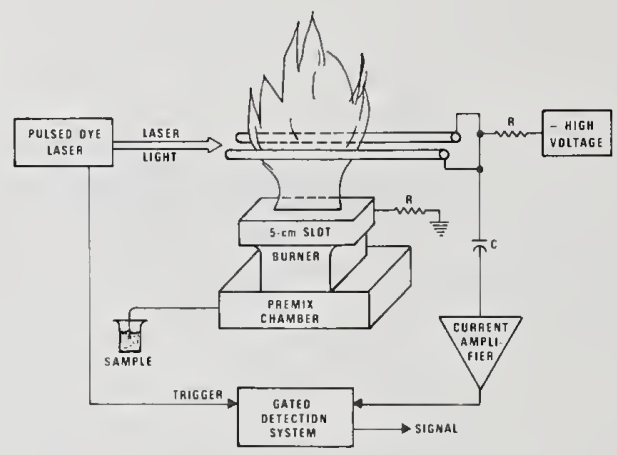

Figure 5. Schematic diagram of the flame apparatus. For detecting the opto-galvanic signal, the two tungsten rods are held at $-1000 \mathrm{~V}$. The second electrode is the burner head, and the ground return is through a $10 \mathrm{k} \Omega$ resistor. Using a nitrogen pumped dye laser, typical powers are 10-100 kW with a bandwidth of $0.03 \mathrm{~nm}$. In a data run (see fig. 6), 1500 points are acquired in approximately ten minutes.

In the flame studies, the laser-induced current changes are found to be always positive, and thus the mechanism for producing opto-galvanic signals is considerably simpler than in the discharge experiments. Our observations can be interpreted in terms of a two-step process, initial photon absorption followed by collisional ionization: 


$$
\begin{array}{ll}
A+h \nu \rightarrow A^{*} & \text { absorption } \\
A^{*}+M \rightarrow A^{+}+M+e^{-} & \text {collisional ionization. }
\end{array}
$$

The enhanced current signals are due to the fact that ionization of species in flames is more probable from an excited electronic state than from the ground state. This mechanism suggests that opto-galvanic spectroscopy will be especially sensitive for optical transitions which excite an atom or molecule to a state very near its ionization limit--a prediction which has been verified by experiment.

\section{1 Trace element detection}

Using the two-step mechanism outlined above, the observed signal intensity depends upon the lower state population, the transition probability for photon absorption, and the collisional ionization probability of the excited state. An approximate figure of merit can be calculated based on these considerations and is useful for predicting the relative sensitivity of various atomic transitions:

$$
\text { Figure of Merit }=\beta I_{\nu} B_{\ell u} \text { exp }\left[-\Delta E_{i} / k T\right] \exp \left[-E_{\ell} / k T\right] g_{\ell} / g_{0} \text {. }
$$

Here, $\beta$ is the atomization efficiency of the element in the flame; $I_{\nu}$ the laser spectral irradiance; $B_{\ell u}$ the Einstein transition probability of absorption from state $\ell$ to $u ; \Delta E_{i}$ the ionization potential less the energy of the upper state of the transition; $E_{\ell}$ the energy of the lower state of the transition; $g_{\ell}$ and $g_{0}$ the lower state and ground state statistical weights; $T$ the flame temperature; and $k$ the Boltzmann constant. The first exponential term expresses the relative probability that a collision in the flame will provide the thermal energy required $\left(\Delta \mathrm{E}_{i}\right)$ to complete the ionization process. The second exponential term, multiplied by the statistical weight ratio, is simply the Boltzmann population of the lower level of the transition, relative to the ground state.

Thus, many transitions which originate in an excited electronic state can be used for analytical investigations, since the loss in sensitivity resulting from the small fractional population of the lower state is counterbalanced by the decrease in $\Delta E_{j}$. Table 2 lists atomic detection limits obtained using opto-galvanic spectroscopy. When these limits are plotted against the corresponding figures of merit on a log-log scale, the data fall roughly on a line of slope -1 [32], which is expected due to the nominal reciprocal relationship between detection limit and sensitivity. This result supports the mechanism involving photon absorption followed by collisional ionization. 
Table 2. Comparative detection limits (in $\mathrm{ng} / \mathrm{m} 1$ ) by opto-galvanic spectroscopy (OGS), flame atomic absorption (FAA), flame atomic emission (FAE), flame atomic fluorescence (FAF) and laser induced flame atomic fluorescence (laser FAF).

\begin{tabular}{|c|c|c|c|c|c|}
\hline Element & $\underline{O G S}$ & $F A A^{a}$ & $\mathrm{FAE}^{\mathrm{a}}$ & $\mathrm{FAF}^{\mathrm{a}}$ & Laser FAF \\
\hline $\mathrm{Cr}$ & 2 & 2 & 2 & 5 & 1 \\
\hline $\mathrm{Cu}$ & 100 & 4 & 10 & 0.5 & 1 \\
\hline $\mathrm{Fe}$ & 2 & 4 & 5 & 8 & 30 \\
\hline $\mathrm{Ga}$ & 0.07 & 50 & 60 & 10 & 0.9 \\
\hline In & 0.008 & 30 & 0.4 & 100 & 0.2 \\
\hline K & 1 & 3 & 0.05 & -- & -- \\
\hline $\mathrm{Mg}$ & 0.1 & 3 & 70 & 0.1 & 0.2 \\
\hline Mn & 0.3 & 0.8 & 1 & 1 & 0.4 \\
\hline $\mathrm{Na}$ & 0.05 & 0.8 & 0.5 & -- & 0.1 \\
\hline $\mathrm{Ni}$ & 8 & 5 & 20 & 3 & 2 \\
\hline $\mathrm{Pb}$ & 0.6 & 10 & 100 & 10 & 13 \\
\hline Sn & 6 & 50 & 100 & 50 & -- \\
\hline $\mathrm{T} 1$ & 0.09 & 20 & 20 & 8 & 4 \\
\hline
\end{tabular}

\footnotetext{
aTaken from Winefordner, J. D., Fitzgerald, J. J., and Omenetto, N., Appl. Spectrosc. 29 , 369 (1975).

beeks, S. J., Haraguchi, H., and Winefordner, J. D., Anal. Chem. 50, 360 (1978).
}

Table 2 also compares our detection limits with those reported for other flame spectrometric techniques. With the one exception of $\mathrm{Cu}$, the limits determined using opto-galvanic spectroscopy are comparable to or better than those obtained with the other methods. For $\mathrm{Cu}$ : $\Delta \mathrm{E}_{i}$ is larger than any of the other transitions studied, and so this technique is insensitiv for the wavelength range easily accessible with tunable dye lasers.

Given the favorable detection limits of table 2 and the spectral selectivity available with tunable lasers, are there any interferences peculiar to analysis by opto-galvanic spectroscopy? Chemical interferences will generally be the same as those experienced by other flame spectroscopic methods. For spectral interferences, some of the documented interfering transitions will be inconsequential due to their low figure of merit, and conversely, some undocumented coincidences of weak lines may become important due to a high figure of merit. In general, however, such spectral interferences can be avoided because of the large number of electronic transitions which can be utilized. Opto-galvanic spectroscopy is impervious 
to such optical background interferences as flame emission, ambient light, and scattered light from the excitation source, but it is subject to unique electrical interferences. Two types may be identified: (1) the effect of electron density in the flame on ionization and recombination rates, and (2) the effect of electron and positive ion densities on the signal collection process.

\subsection{Detection of molecular species}

Recently, we reported the first results on absorption by molecular species using optogalvanic spectroscopy [25]. Extensive measurements have been made in premixed flames on La0 in the 360-750 $\mathrm{nm}$ wavelength region, and somewhat less data were obtained for Sc0 and Y0 to complete the homologous series. Only fragmentary laboratory results on absorption and excitation spectra for these molecules have been published previously.

Figure 6 shows several band systems of LaO. Our spectra exhibit excellent signal-tonoise with a resolution limited by the laser bandwidth. Numerous emission studies have been carried out previously on LaO, YO, and ScO (particularly for LaO), with the result that the large majority of band systems observed in our work can be readily identified. For example, transitions are detected in LaO which involve four excited electronic states and a total of eighteen sequences. For the $B^{2} \Sigma^{+}-x^{2} \Sigma^{+}, c^{2} \Pi_{1 / 2}-x^{2} \Sigma^{+}$, and $c^{2} \Pi_{3 / 2}-x^{2} \Sigma^{+}$transitions, five new sequences have been assigned on the basis of known molecular constants and their characteristic structure. Table 3 summarizes our observations on these molecules.
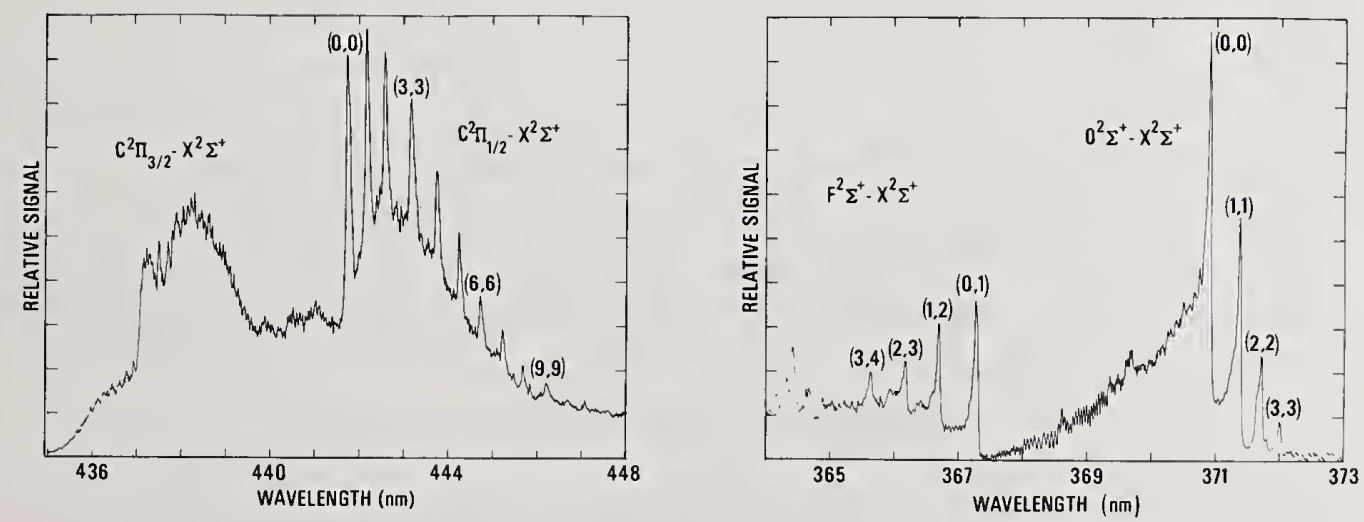

Figure 6. Opto-galvanic absorption signal plotted as a function of laser wavelength for known band systems of $\mathrm{LaO}$ in a premixed $\mathrm{H}_{2}$ /air flame. The electronic states and $\left(v^{\prime}, v^{\prime \prime}\right)$ sequence members are indicated. The individual members of the $c^{2} \Pi_{3 / 2}-$ $\mathrm{X}^{2} \Sigma^{+}$sequence could be resolved using a $\mathrm{C}_{2} \mathrm{H}_{4}-\mathrm{N}_{2} \mathrm{O}$ flame. The data are normalized for laser power, and the laser bandwidth is $0.03 \mathrm{~nm}$. 
Table 3. Electronic states and sequences of metal oxides observed using opto-galvanic spectroscopy.

Molecule

La0

YO

Sc0

\section{Transition}

$B^{2} \Sigma^{+}-x^{2} \Sigma^{+}$

$c^{2} \Pi 1 / 2-x^{2} \Sigma^{+}$

$c^{2} \Pi_{3 / 2}-x^{2} \Sigma^{+}$

$D^{2} \Sigma^{+}-x^{2} \Sigma^{+}$

$F^{2} \Sigma^{+}-x^{2} \Sigma^{+}$

$A^{2} \Pi 1 / 2^{-x^{2} \Sigma^{+}}$

$A^{2} \Pi_{3 / 2}-x^{2} \Sigma^{+}$

$\mathrm{B}^{2} \Sigma^{+}-\mathrm{x}^{2} \Sigma^{+}$

$A^{2} \Pi_{1 / 2}, 3 / 2^{-x^{2} \Sigma^{+}}$

$B^{2} \Sigma^{+}-x^{2} \Sigma^{+}$
Number of Sequences

7

4

4

2

1

2

1

5

2

5

Again, our results indicate that the mechanism for producing the opto-galvanic signals involves initial photon absorption, followed by collisional ionization. However, these data were obtained using a pulsed laser with high peak powers (typically 600-800 $\mu \mathrm{J}$ in $7 \mathrm{~ns}$ or $\sim 100 \mathrm{~kW})$, and it is possible that two- and three-photon ionization may also contribute to our observed signals, particularly at higher photon energies. A number of observations point to photon-assisted collisional ionization as the dominant mechanism:

(1) Many of the transitions detected for $\mathrm{Sc} 0, \mathrm{YO}$, and LaO require three photons in a direct photoionization process, yet the data closely match previously reported emission results and are usually free of unidentified structure (which might correspond to two-photon resonances).

(2) For several strong features in the La0 spectrum the signal strength is linear with laser power.

If we assume that ionization of the electronically excited molecules is due to collisions, then the detection sensitivity will increase as the energy separation between the upper state and the ionization continuum decreases. Thus, an opto-galvanic spectrum is uniquely sensitive to absorption transitions which reach highly excited electronic states, and therefore, it is complementary to conventional emission methods, in which detection of high-lying states is limited by low Boltzmann populations. Our results on LaO illustrate this behavior and provide an interesting comparison to the extensive emission studies which have been reported. In our work, the $A^{2} \Pi-x^{2} \Sigma^{+}$systems have not been observed definitively, the $B^{2} \Sigma^{+}-x^{2} \Sigma^{+}$bands are detected with moderate intensity, and transitions to all higherlying states of LaO are very strong. 
The premixed flame constitutes an environment where electronic excitation is low compared to conventional arc sources employed for emission studies. Thus, in these flames one can be reasonably assured that the opto-galvanic molecular spectra will involve transitions. which originate in the ground electronic state. Of course, this technique is also ideally suited to probe interesting flame chemistry which produces excited electronic states (however, we see no evidence for the $c^{2} \Pi-A^{\prime 2} \Delta$ absorption in LaO under our experimental conditions). In addition to the results of table 3 , transitions known from emission studies have been observed in $\mathrm{SrOH}$ (centered around $606 \mathrm{~nm}$ ), BaOH/BaO (centered around $512 \mathrm{~nm}$ ), and $\mathrm{BaCl}$ (bands in the $c^{2} \Pi-x^{2} \Sigma^{+}$transition). Also, complex and congested spectra have been detected when solutions containing salts of $\mathrm{Dy}, \mathrm{Er}$, Eu, and $U$ were aspirated into the flame. From these results, we are most encouraged about the prospects for detecting species with low ionization potentials not conveniently accessible using other optical methods.

\subsection{Two-photon spectroscopy}

The sensitivity of opto-galvanic spectroscopy for detecting highly-excited electronic states suggests the possibility of studying multiple-photon excitation processes. Beginning with the well known two-photon transitions in sodium [33-35], we have detected over thirty two-photon lines in $\mathrm{Na}, \mathrm{K}, \mathrm{Ca}, \mathrm{Li}$, and In using the same experimental configuration as shown in figure 5 [36]. The intensity of a two-photon transition is greatly enhanced by the existence of a dipole-allowed one-photon transition at an energy close to half of the two-photon energy. For many of the observed transitions, this intermediate state lies $>1000 \mathrm{~cm}^{-1}$ away from the one photon energy of the laser beam. Yet the two-photon signals can still be detected, due to efficient collisional ionization of the high-lying electronic states.

For two-photon spectroscopy, the flame is a more convenient and versatile reservoir than an oven, or heat pipe, or molecular beam apparatus. In addition, opto-galvanic spectroscopy obviates the need for optical detection of fluorescence. One important disadvantage is the broadening of the energy levels due to collisions at atmospheric pressure.

\section{Conclusions and Prognosis}

The main conclusions of our studies are the following:

(1) Opto-galvanic spectroscopy is a most sensitive way by which to detect the absorption spectra of atoms [1,24] and molecules [25] in a flame environment. For trace element detection of many species, this technique is certainly competitive with, and often superior to, other flame spectrometric methods. In the case of molecular absorption, opto-galvanic spectroscopy may be restricted to molecules with low ionization potentials ( $\leq$ approximately $7 \mathrm{eV}$ ). However, at least for some of these molecules, spectra of truly high quality can be obtained. This has been demonstrated 
for La0, where new sequences having very low Franck-Condon factors $\left(\sim 10^{-5}\right)$ have been identified.

(2) The mechanism for producing the observed voltage and current signals can be understood in terms of photon-induced changes in the ionization rates of a number of processes in the discharges and flames. However, a full understanding of the important mechanisms is not available, and further work on this subject is underway.

(3) In discharges, opto-galvanic spectroscopy provides interesting, and sometimes unique, insights into the important radiative and collision processes which affect ionization. In this environment, essentially all atomic species can be detected in a large number of electronic states $[19,20]$.

(4) For spectroscopy of atoms and molecules in a flame, opto-galvanic spectroscopy is a fundamentally new tool, which in many ways complements emission and fluorescence techniques. Of particular interest is its sensitivity for observing high-lying electronic states, which can be efficiently ionized by collisions.

What does the future hold for opto-galvanic spectroscopy? Already, this technique is widely used in laser laboratories as a convenient means of calibrating wavelength, determining bandwidth (of broadband lasers), and stabilizing frequency. of much greater importance, however, is its utility for probing radiative and collision processes in ionized media of all kinds. Opto-galvanic spectroscopy in discharges is most sensitive to those atomic levels which are strongly coupled to the discharge. These should be the same states which are important in lasing action, and thus, this technique should be helpful in the search for new, high efficiency laser lines [28]. Similarly, opto-galvanic spectroscopy is likely to become a valuable tool for studying plasmas. Bridges has pointed out that it may be possible to create interesting new properties, such as "low-ionization-potential" atoms and cold-electron plasmas [27]. He also plans to use the technique in conjunction with a laser isotope enrichment process which involves discharge cataphoresis separation [27]. In addition to the study of atoms in discharges, experiments on molecules are being actively pursued [37].

Thus far, opto-galvanic spectroscopy in flames has utilized the flame environment, but not probed the flame properties themselves. It is most likely that this situation will change in the near future. For example, a variety of temperature measurements are now possible, such as electronic temperature (using two states in In [38]) and vibrationalrotational temperatures (using the molecular $\mathrm{LaO}$ and $\mathrm{YO}$ absorption spectra). This technique is also well suited to probe interesting flame chemistry, such as oxidation processes, reactions producing excited electronic states, and chemi-ionization processes $(\mathrm{CH}+0 \rightarrow$ $\left.\mathrm{CHO}^{+}+\mathrm{e}^{-}\right)$. Also, there is considerable evidence that the initial nucleation stages in soot formation involve ionic species [39]. Opto-galvanic spectroscopy perturbs the ion production rate in the flame and thus may well influence the production of soot. 0ptogalvanic spectroscopy may also provide a breakthrough in the detection of hydrogen atoms, 
which are of key importance in flame propagation. Recently, a three-photon ionization technique has been demonstrated with both high sensitivity for $H$ and $D$ atoms and high spatial resolution [40].

\section{References}

[1] Green, R. B., Keller, R. A., Schenck, P. K., Travis, J. C., and Luther, G. G., J. Am. Chem. Soc. $\underline{98}, 8517$ (1976).

[2] Penning, F. M., Physica $\underline{8}, 137$ (1928); 10, 47 (1930); 11, 183 (1931); 12, 65 (1932); Z. Physik 46 , 335 (1928); 57, 723 (1929); Phil. Mag. 11, 961 (1931); Druyvesteyn, M. J. and Penning, F. M., Rev. Mod. Phys. 12, 87 (1940); 13, 72 (1941).

[3] Pike, E. W., Phys. Rev. 49. Two articles starting on p. 513 and 515 (1936).

[4] Kenty, C., Phys. Rev. 80, 95 (1950).

[5] Meissner, K. W. and Miller, W. F., Phys. Rev. 92, 896 (1953).

[6] Drouet, M. G. and Novak, J. P., Phys. Letters 34A, 199 (1971).

[7] Kopeika, N. S., Rosenbaum, J., and Kastner, R., Appl. 0pt. 15, 1610 (1976), and references therein.

[8] Garscadden, A., Bletzinger, P., and Friar, E. M., J. Appl. Phys. 35, 3432 (1964); Garscadden, A. and Adams, S. L., Proc. IEEE, 54, 427 (1966).

[9] Schiffner, G. and Seifert, F., Proc. IEEE, 53, 1657 (1965).

[10] Freiberg, R. J. and Weaver, L. A., J. Appl. Phys. 38, 250 (1967).

[11] Carswe11, A. I. and Wood, J. I., J. App 1. Phys. 38, 3028 (1967).

[12] Skolnick, M. L., IEEE J. Quantum Electron., QE-6, 139 (1970).

[13] Green, R. B., Keller, R. A., Luther, G. G., Schenck, P. K., and Travis, J. C., IEEE J. Quantum Electron., QE-13, 63 (1977).

[14] King, D. S., Schenck, P. K., Smyth, K. C., and Travis, J. C., Appl. 0pt. 16, 2617 (1977).

[15] Foote, P. D. and Mohler, F. L., Phys. Rev. 26, 195 (1925); Mohler, F. L., Foote, P. D., and Chenault, R. L., Phys. Rev. 27, 37 (1926).

[16] Collins, C. B., et al., Phys. Rev. A 14, 1662 (1976), and earlier references therein.

[17] Marr, G. V. and Wherrett, S. R., J. Phys. B $\underline{5}, 1735$ (1972).

[18] Harvey, K. C., and Stoicheff, B. P., Phys. Rev. Letters, 38, 537 (1977).

[19] Green, R. B., Keller, R. A., Luther, G. G., Schenck, P. K., and Travis, J. C., Appl. Phys. Letters, 29, 727 (1976).

[20] Smyth, K. C. and Schenck, P. K., Chem. Phys. Letters, 55, 466 (1978).

[21] Smyth, K. C., Keller, R. A., and Crim, F. F., Chem. Phys. Letters, 55, 473 (1978).

[22] Smyth, K. C., Bentz, B. L., Bruhn, C. G., and Harrison, W. W. (submitted for publication).

[23] Schenck, P. K. and Smyth, K. C., J. 0pt. Soc. Am. 68, 626 (1978).

[24] Turk, G. C., Travis, J. C., DeVoe, J. R., and 0'Haver, T. C., Anal. Chem. 50, 817 (1978). 
[25] Schenck, P. K., Mallard, W. G., Travis, J. C., and Smyth, K. C., J. Chem. Phys. (in press).

[26] Weaver, L. A. and Freiberg, R. J., J. App1. Phys. 37, 1528 (1966).

[27] Bridges, W. B., J. 0pt. Soc. Am. 68, 352 (1978).

[28] Johnston, Jr., T. F., Laser Focus, 14, 58 (1978).

[29] Moore, C. E., NSRDS-NBS 35, 76 (1971).

[.30] Persson, W., Phys. Scripta, 3 , 133 (1971).

[31] Meggers, W. F., Corliss, C. H., and Scribner, B. F., Nat. Bur. Stand. (U.S.), Monogr. 145, Part I, p. 7 and 76 (1975).

[32] Travis, J. C., Schenck, P. K., and Turk, G. C. (submitted for publication).

[33] Hansch, T. W., Harvey, K. C., Meisel, G., and Schawlow, A. L., Opt. Comm. 11, 50 (1974).

[34] Levenson, M. D. and Salour, M. M., Phys. Lett. 48A, 331 (1974).

[35] Bjorkholm, J. E. and Liao, P. F., Phys. Rev. Lett. $\underline{33}, 128$ (1974).

[36] Schenck, P. K., Mallard, W. G., Travis, J. C., and Smyth, K. C. (unpublished).

[37] Keller, R. A. (Los Alamos Scientific Laboratory), Robin, M. B. and Heath, B. (Bell Laboratories, Murray Hill, N.J.) private communications.

[38] Browner, R. F. and Winefordner, J. D., Anal. Chem. 44, 247 (1972); Haraguchi, H., Smith, B., Weeks, S., Johnson, D. J., and Winefordner, J. D., App 1. Spec. 31, 156 (1977).

[39] Place, E. R. and Weinberg, F. J., 11 th Symp. Comb. (International), p. 245 (1967).

[40] Bjorklur.d, G. C., Ausschnitt, C. P., Freeman, R. R., and Storz, R. H., J. Opt. Soc. Am. 68, 675 (1978).

\section{Discussion}

Comment (Crosley): Something that this effect might be useful for is to excite out of two different ground state levels to a single excited state, an optogalvanic version of Winefordner's two line fluorescence technique.

If you excite to a single excited state then any uncertainties in the collision cross section for ionization are not going to bother you, and you might be able to very sensitively get some good temperature information.

Response (Mallard): We11, we tried that with indium. The problem that we have had so far is simply that we have got severe saturation problems. We are saturating both of those transitions.

That was part of the origin of the two-photon work. We would like to do exactly that sort of an experiment, only going to the states with two photons each. So yes, I think that is a very good idea.

Question (Dewing): What is the order of magnitude of the currents you observed? 
Response (Mallard): That is very much a function of the laser that we are talking about. Most of the experiments that I discussed, all of the spectroscopy, was done with a nitrogen pump dye laser. We are dealing there with about 10 micro-amps, peak currents, but only for about three nanoseconds. The currents are much larger in the case of something like sodium with a continuous wave laser, and you can often observe currents in the micro-amp region. You can get photo induced currents in about the micro-amp region, but that is the very high extreme. Typicaliy, we don't operate anywhere near that high. 

National Bureau of Standards Special Publication 561, Proceedings of the 10th Materials Research Symposium on Characterization of High Temperature Vapors and Gases held at NBS, Gaithersburg, Maryland, September 18-22, 1978. Issued October 1979.

\title{
$\mathrm{SiCl}_{2}$ FLUORESCENCE AS A CHEMICAL VAPOR DEPOSITION REACTOR PROBE
}

\author{
T. Sedgwick and G. V. Arbach
}

International Business Machines Corp.

T. J. Watson Research Center

Yorktown Heights, NY 10598

$\mathrm{SiCl}_{2}$ is the dominant silicon containing species in the gas phase at high temperatures in $\mathrm{Si}$ deposition and etching reactions in the $\mathrm{Si}-\mathrm{Cl}-\mathrm{H}$ system. $\mathrm{SiCl}_{2}$ fluoresces in $4880 \AA$ light, and it has been possible using a fast (equilibrium) reaction between $\mathrm{HCl}$ and $S i$ to calibrate a fluorescence measuring technique to make quantitative local concentration measurements of $\mathrm{SiCl}_{2}$ near the growing (etching) Si surface. The role of $\mathrm{SiCl}_{2}$ as the initial product in the etching-reaction and as an intermediate in some deposition reactions will be discussed. These results are discussed in reference to recent studies of the $\mathrm{Si}-\mathrm{Cl}-\mathrm{H}$ system using mass spectrometry and vapor phase chromatography.

\section{Introduction}

The growth of Si epitaxial layers by chemical vapor deposition (CVD) on Si substrates has been studied extensively over the last two decades. One of the most frequently used processes is the reduction of $\mathrm{SiCl}_{4}$ (alternatively $\mathrm{SiCl}_{3} \mathrm{H}$ or $\mathrm{SiCl}_{2} \mathrm{H}_{2}$ ) by hydrogen at 1050$1200{ }^{\circ} \mathrm{C}$ in an open tube flow reactor at one atmosphere pressure. Most previous studies have had the pragmatic goal of developing a reliable process to deposit uniform, smooth, perfect crystalline layers with a known growth rate and a desired dopant level. However, there have been a continuing number of investigations attempting to probe the fundamental chemical processes involved. Initially, investigations focused on thermodynamic studies of the vapor phase and hydrodynamic flow modeling to describe the growth rate, the only experimentally accessible measure of system operation.

Recently, mass spectrometry $[1-3]^{1}$, vapor phase chromatography (VPC) [4], and Raman and fluorescence spectroscopy [5-9], and infrared absorption spectroscopy [10-11] have been used to identify the actual species and measure their concentration profiles in reactors. Reported results indicate that $\mathrm{SiCl}_{2}$ is the major gas phase species containing Si produced at high temperature under conditions closely duplicating actual growth conditions when either $\mathrm{SiCl}_{4}$ or $\mathrm{SiCl}_{2} \mathrm{H}_{2}$ and $\mathrm{H}_{2}$ are used as reactants.

\footnotetext{
${ }^{1}$ Figures in brackets indicate the literature references at the end of this paper.
} 
However, the data from all these sources have been found wanting, i.e., from the mass spectrometric studies there is only a single measured $\mathrm{SiCl}_{2}$ gradient curve for one set of experimental conditions, from the VPC studies the $\mathrm{SiCl}_{2}$ concentration must be inferred since the high temperature species cannot be detected directly, and finally, the data from the fluorescence studies has yielded only relative $\mathrm{SiCl}_{2}$ concentrations to date.

In the present paper, we first describe a calibration technique which enables quantitative $\mathrm{SiCl}_{2}$ measurements to be made by the fluorescence technique. Secondly, we briefly review the results and conclusions from previous studies of the system in light of the present quantitative measurements.

\section{Experimental Apparatus and Procedures}

2.1 Optical equipment and measuring techniques

The optical equipment and the measuring techniques for concentration gradient measurements above a surface have been previously described [7] and are shown schematically in figure 1. Briefly, the light source was a Spectra Physics Mode1 170 Argon Ion Laser ( $8 W$, $4880 \AA$, or $5145 \AA$ ), alined so the beam passed down the axis of the reactor. For a short length along the laser beam (called the sampling volume), the radiation scattered by the gas molecules at right angles to the laser beam is collected by a f/1.5, $135 \mathrm{~mm}$ focal length lens which images the light into the entrance slit (usually $500 \mu$ wide) of a Spex $1301 \mathrm{f} / 7$ double monochromator. After passing through the monochromator, the light is detected by an RCA C31034 photomultiplier tube, and photon counting is accomplished by an SSR model 1110 digital synchronous computer.

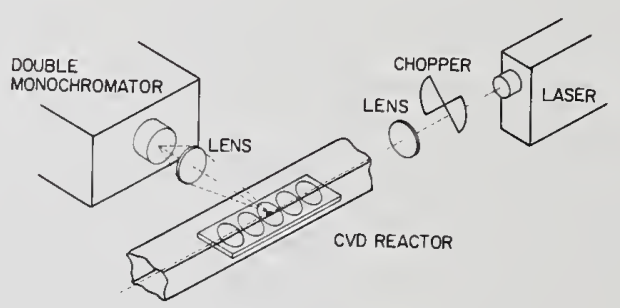

Figure 1. Schematic of Raman scattering and chemical deposition apparatus.

The sampling volume is small. It is a cylinder about $0.1 \mathrm{~mm}$ in diameter by $0.5-1.0$ $\mathrm{cm}$ long. The entire reaction chamber is movable along the $x, y$, and $z$ axes, so that the sampling volume can be located at any point above the susceptor. In the $\mathrm{Si}-\mathrm{Cl}-\mathrm{H}$ system, the spectrum is dominated by the fluorescence of $\mathrm{SiCl}_{2}$. Figure 2 shows the fluorescence spectrum for $\mathrm{SiCl}_{2}$ obtained in the CVD reactor (described below) at about $870{ }^{\circ} \mathrm{C}$ susceptor temperature using $\mathrm{SiCl}_{4}$ and $\mathrm{H}_{2}$ as input reactants. Following our previous procedure [5-9], 
we have measured the intensity, i.e., photon counts in (10 s x 50 percent duty cycle equals $5 \mathrm{~s}$ ) of the scattered light downshifted $565 \mathrm{~cm}^{-1}$ from the laser line at $4880 \AA$ and assumed it is proportional to the $\mathrm{SiCl}_{2}$ concentration, subject only to the following . correction. The system optical transmission efficiency is measured at room temperature with only $\mathrm{H}_{2}$ present to correct for variations in window transmission and variations in geometrical light collection efficiency as a function of position of sampling volume in the reaction zone. The procedure for converting these relative measurements to actual $\mathrm{SiCl}_{2}$ partial pressure values is described below.

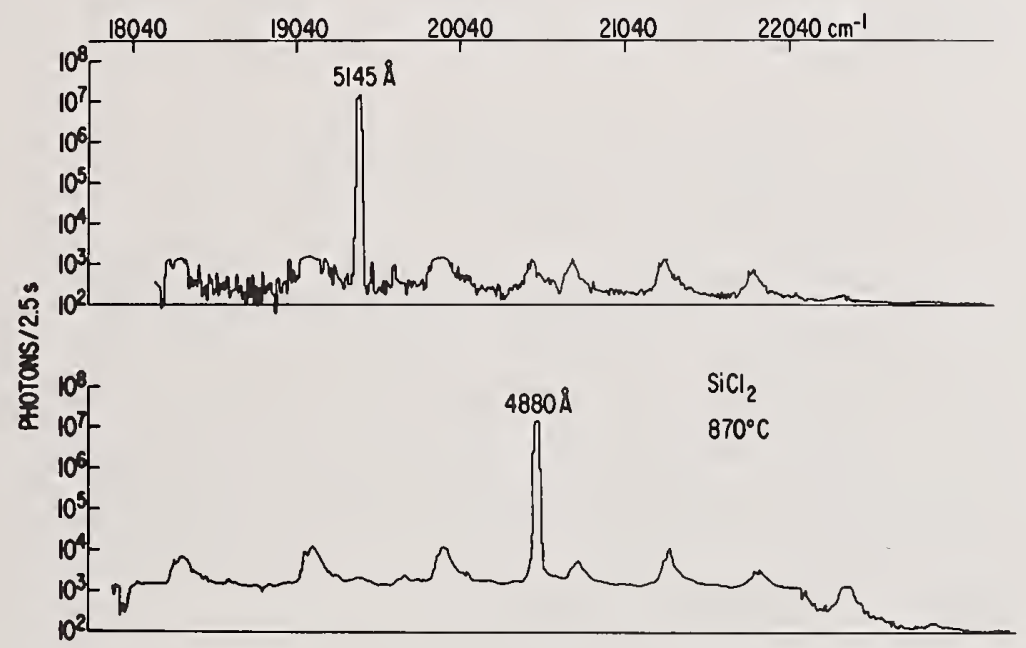

Figure 2. Fluorescence spectrum of $\mathrm{SiCl}_{2}$ using $4880 \AA$ and $5145 \AA$ incident light produced from an input mixture of $\mathrm{SiCl}_{4}$ and $\mathrm{H}_{2}$ and a susceptor temperature of $870{ }^{\circ} \mathrm{C}$.

\subsection{CVD reactor and gas flow conditions}

A detail of the reactor chamber is shown in figure 3. The reactor was a horizontal quartz chamber with square cross section. A rectangular silicon carbide coated susceptor was placed on the bottom of the tube leaving a free space $1.1 \mathrm{~cm}$ high, $3.55 \mathrm{~cm}$ wide, and $16 \mathrm{~cm}$ long above it. The quartz walls float thermally during the process. The measuring positions designated 1,3 , and 6 were respectively $1.1,5.5$, and $12.1 \mathrm{~cm}$ from the leading edge of the susceptor. 


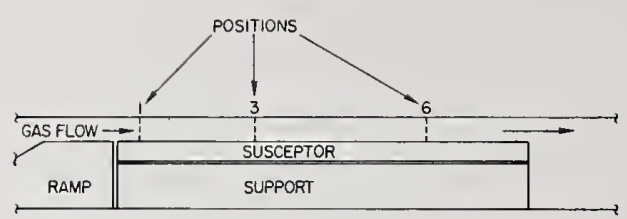

Figure 3. Horizontal quartz chamber showing silicon carbide coated susceptor and location of measuring positions 1,3 , and 6 .

Small silicon chips placed on the rf-heated susceptor were brought to temperatures of 1050 or $1200^{\circ} \mathrm{C}$ (optical). Palladium purified hydrogen was used as a carrier gas. The $\mathrm{SiCl}_{2} \mathrm{H}_{2}$ was provided in a gas cylinder by Matheson and mixed with hydrogen to make up the total flow. The gas mixture flowed through the $3.9 \mathrm{~cm}^{2}$ cross-sectional area above the susceptor at an average velocity (room temperature) of $41.7 \mathrm{~cm} / \mathrm{s}$.

Growth rate measurements were made using heavily doped $n$ type $\langle 100\rangle$ Si chips in each position (1,3, and 6). Prior to deposition, the chips were Huang cleaned and then hydrogen etched for 10 minutes in the reactor at $1150^{\circ} \mathrm{C}$. After deposition they were scored in half, etched in HF, and a photomicrograph was taken of the cross section to measure the thickness of the layer deposited.

\section{Calibration Cel1}

The apparatus used for calibration of the measuring system for $\mathrm{SiCl}_{2}$ concentration is shown in figure 4. Unlike the reactor in figure 1 , this calibration cell is placed in a furnace which is designed to provide a constant temperature over the whole of the cell. A helical resistance winding over the length of the furnace including the viewing window (not shown in the figure) was used. During measurements, the window always remained clean. Careful thermocouple probing did not show a significant temperature drop in the viewing window region. The space between the perforated disks was $6.2 \mathrm{~cm}$ long by $3.1 \mathrm{~cm}$ diameter and was filled with crushed Si particles $0.3-0.6 \mathrm{~mm}$ in diameter. A dilute mixture of $\mathrm{HCl}$ in $\mathrm{H}_{2}$ was passed from left to right through the packed bed at several velocities and the light scattered from the sampling volume was viewed through the viewing window (through the furnace windings). The laser light was incident from the right and passed through the sampling volume before it passed down the center tube and through the Brewster angle window gas barrier before exiting the cell. 


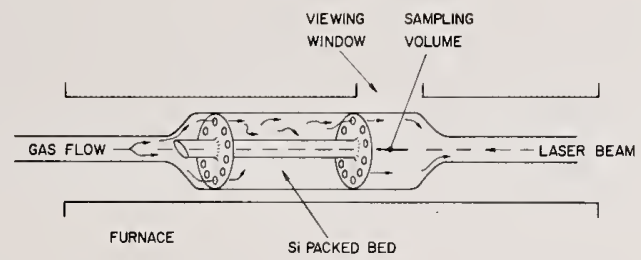

Figure 4. Calibration cell for $\mathrm{SiCl}_{2}$ partial pressure measurement.

4. Results and Discussion

$4.1 \mathrm{SiCl}_{2}$ concentration calibration

The basic idea involved in the calibration procedure is to produce a known concentration of $\mathrm{SiCl}_{2}$ in the calibration cell. We used a transpiration technique where a reaction which reaches equilibrium rapidly and whose thermodynamic properties were known was chosen. The fluorescence intensities due to $\mathrm{SiCl}_{2}$ measured in the $\mathrm{CVD}$ reactor were then compared to the intensity measured in this cell to obtain quantitative values. In order to compensate for differences in light collection efficiency between the calibration cell and the CVD reactor, the intensity values were compared to a Raman signal from $\mathrm{N}_{2}$ in air at room temperature, but at otherwise identical conditions in both the reactor and the cel1. The reaction chosen with its corresponding $k_{p}$ was

$$
\begin{gathered}
2 \mathrm{HCl}+\mathrm{Si}(\mathrm{s}) \underset{\leftarrow}{\rightarrow} \mathrm{SiCl}_{2}+\mathrm{H}_{2} \\
K_{p}=\frac{\mathrm{P}_{\mathrm{SiCl}}{ }_{2} \mathrm{P}_{2}}{\mathrm{P}_{\mathrm{HCl}}^{2}}=2.2 \text { at } 1100{ }^{\circ} \mathrm{C} \text { [12] }
\end{gathered}
$$

where from thermodynamic calculations [13], it was clear that for a $\mathrm{Cl} / \mathrm{H}$ ratio of about 0.01 and at $1100{ }^{\circ} \mathrm{C}$ or higher, only the species indicated in (1) are present in significant concentration. The etching of $\mathrm{Si}$ by $\mathrm{HCl}$ is found to be fast $[4,14]$ and equilibrium is readily obtained. We used a 1.2 percent $\mathrm{HCl}$ in $\mathrm{H}_{2}$ input mixture where the $\mathrm{HCl}$ concentration in the gas was measured at room temperature by comparing the intensity of the Raman scattering due to $\mathrm{HCl}$ in the mixture to that of a 100 percent $\mathrm{HCl}$ reference.

Under conditions of sufficiently low flow to achieve transpiration, $P_{H_{2}}=1, P_{H C l}=0.012$ and $\mathrm{P}_{\mathrm{SiCl}}=3.4 \times 10^{-4} \mathrm{~atm}$ from eq. (1). The fluorescence intensity was measured as a function of flow in two separate experiments at $1100{ }^{\circ} \mathrm{C}$ and the results are shown in 
figure 5. The difference in slope of the data taken on two different days is not understood, but may possibly be due to differences in surface area or condition of the Si in the packed bed. The equilibrium partial pressure of $\mathrm{SiCl}_{2}$ was assumed to be closely approximated at the lowest flow, ca $1 \mathrm{~cm} / \mathrm{s}$. Extrapolation of the curves to zero flow gives an average fluorescent intensity of $1.45 \times 10^{16}$ for the standard count interval.

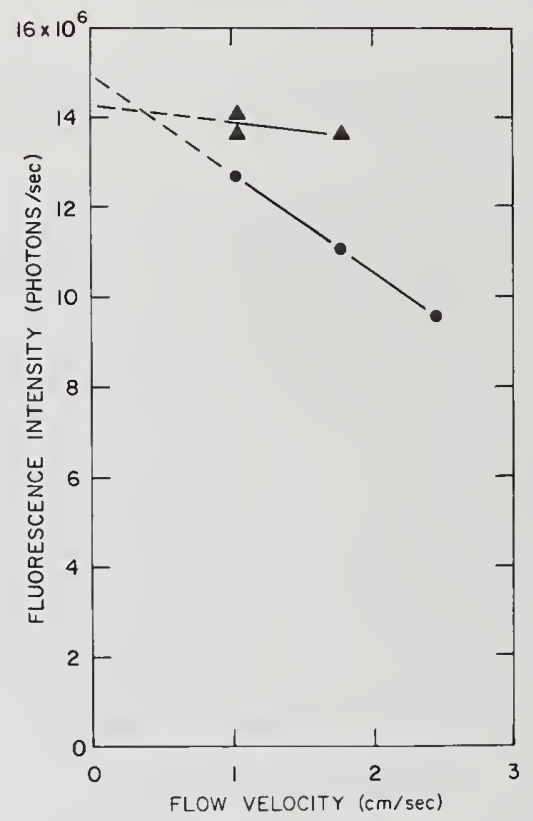

Figure 5. Fluorescence intensity of $\mathrm{SiCl}_{2}$ vs flow rate of 1.2 percent $\mathrm{HCl}$ in $\mathrm{H}_{2}$ in calibration cell sampling chamber at $1100^{\circ} \mathrm{C}$. $\Delta$ Run 1, Run 2.

The Raman scattering intensity of $\mathrm{N}_{2}$ in air (vibrational Stokes line at $18,161 \mathrm{~cm}^{-1}$ ) at room temperature measured under otherwise identical conditions was $8.9 \times 10^{4}$ counts. By taking into consideration the concentration of $\mathrm{N}_{2}$ in air, the partial pressure of $\mathrm{SiCl}_{2}$ as calculated above and the gas density difference at $1100{ }^{\circ} \mathrm{C}$ as compared to that at room temperature for $\mathrm{N}_{2}$, it is easy to show that the apparent fluorescent cross section, $\sigma_{\mathrm{SiCl}_{2}}$ at $4880 \AA$ and under these specific conditions for $\mathrm{SiCl}_{2}$ relative to the Raman scattering cross section of $\mathrm{N}_{2}, \sigma_{\mathrm{N}_{2}}$ is

$$
\frac{{ }^{\sigma_{\mathrm{SiCl}}}{ }_{2}}{{ }_{\mathrm{N}_{2}}}=1.69 \times 10^{5} .
$$


This large value points out the overwhelming sensitivity advantage of fluorescence scattering as compared to Raman scattering for detection of low species concentrations in reactive gas systems.

By comparing the Raman scattering of $\mathrm{N}_{2}$ in air at room temperature in both the calibration cell and the CVD reactor, the CVD reactor was found to allow 1.27 times more scattered light to be detected under otherwise identical conditions.

Finally, the fluorescence intensity per atmosphere of $\mathrm{SiCl}_{2}$ in the CVD reactor is given by

$$
\frac{1.45 \times 10^{6} \text { counts } \times 1.27}{3.4 \times 10^{-4} \text { atm } \mathrm{SiCl}_{2}}=5.37 \times 10^{9} \text { counts/atm of } \mathrm{SiCl}_{2} \text { in } \mathrm{CVD} \text { reactor } 2 .
$$

This calibration may be applied to the data in references [5] and [9], but it is clearly only applicable to the data measured with the apparatus described and the specific geometry used. Since count levels as low as 100 are possible (see fig. 5, [7]), this demonstrates the ability to measure concentrations down to the $10-100 \mathrm{ppb}$ range.

We do not know to what extent, if any, the fluorescence is quenched by the gaseous environment of the $\mathrm{SiCl}_{2}$ molecules. However, the above calibration procedure which is made at the same total pressure, $1 \mathrm{~atm}$, in the carrier, $\mathrm{H}_{2}$ and in the same temperature and concentration ranges would minimize any errors due to possible quenching.

\section{$4.2 \mathrm{SiCl}_{2}$ in $\mathrm{Si}$ etching by $\mathrm{SiCl}_{4}$}

Figure 6 shows the $\mathrm{SiCl}_{2}$ partial pressure as a function of distance above the susceptor when $\mathrm{SiCl}_{4}$ in $\mathrm{He}$ was passed over a $\mathrm{Si}$ chip. The reaction which takes place at the surface is

$$
\mathrm{SiCl}_{4}+\mathrm{Si}(\mathrm{s}) \rightarrow 2 \mathrm{SiCl}_{2} .
$$

The monotonically decreasing $\mathrm{SiCl}_{2}$ concentration as a function of distance above the susceptor is expected since the $\mathrm{SiCl}_{2}$ must be formed at the Si surface before it diffuses away into the bulk of the gas stream. However, even at $1200^{\circ} \mathrm{C}$, the $\mathrm{SiCl}_{2}$ partial pressure at the surface (extrapolated) is less than $5 \times 10^{-5}$ atm. Although equilibrium considerations would indicate the $\mathrm{SiCl}_{4}$ to be essentially converted to $\mathrm{SiCl}_{2}$, the small concentration of $\mathrm{SiCl}_{2}$ observed shows that this reaction is slow and surface rate limited.

\section{$4.3 \mathrm{SiCl}_{2}$ in $\mathrm{Si}$ deposition from $\mathrm{SiCl}_{4}$ and $\mathrm{SiCl}_{2} \mathrm{H}_{2}$}

Figures 7 and 8 show $\mathrm{SiCl}_{2}$ partial pressures as a function of height above the susceptor at a flow velocity of $41.7 \mathrm{~cm} / \mathrm{s}$ (room temperature) when $\mathrm{SiCl}_{4} \mathrm{plus}_{2}$ or 


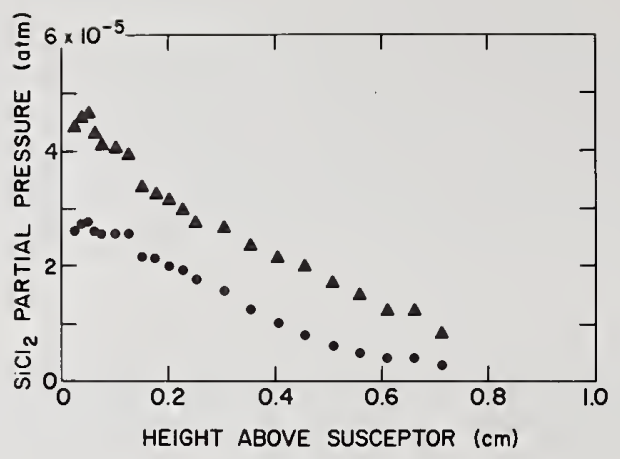

Figure 6. Partial pressure profiles of $\mathrm{SiCl}_{2}$ produced from the etching reaction of 0.276 percent $\mathrm{SiCl}_{4}$ in $\mathrm{He}$ and with previously deposited silicon at susceptor temperatures of $1050{ }^{\circ} \mathrm{C}(\Theta)$ and $1200{ }^{\circ} \mathrm{C}(\Delta)$. The flow velocity is $41.7 \mathrm{~cm} / \mathrm{s}$.

$\mathrm{SiCl}_{2} \mathrm{H}_{2}$ plus $\mathrm{H}_{2}$, respectively, is passed over Si. The Si chip temperature was held at 1050 and $1200{ }^{\circ} \mathrm{C}$, and profiles were measured at positions 1,3 , and 6 . In this case, $\mathrm{Si}$ is deposited and these figures also show the $\mathrm{Si}$ growth rate for these same conditions.
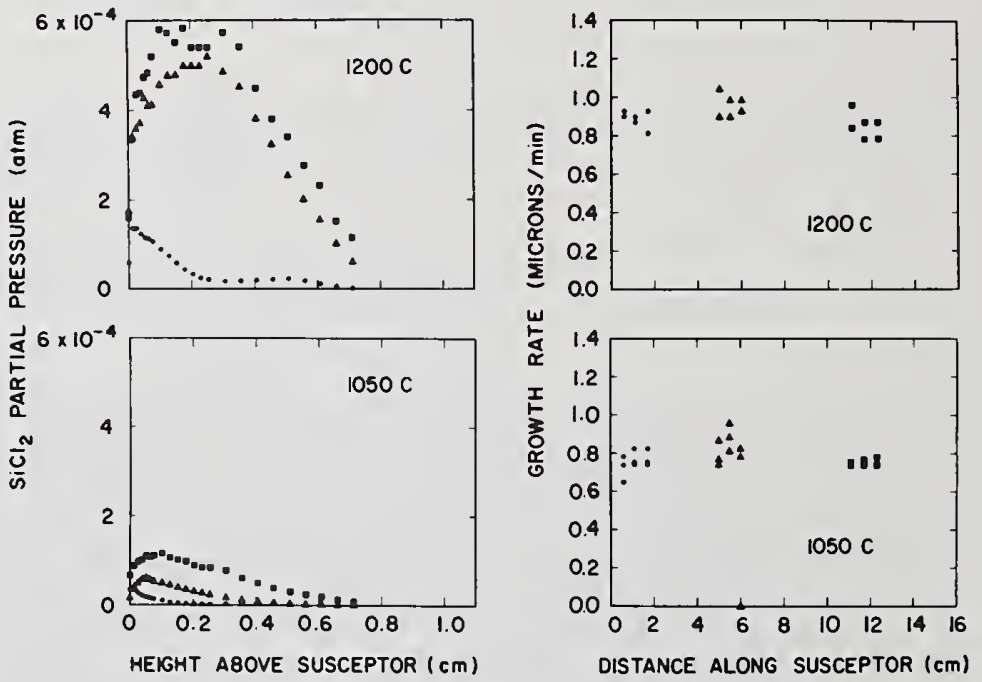

Figure 7. Partial pressure profiles of $\mathrm{SiCl}_{2}$ and epitaxial rates of deposited silicon at positions $1(0), 3(\Delta)$, and $6(\mathbb{D})$, produced from 0.276 percent $\mathrm{SiCl}_{4}$ in $\mathrm{H}_{2}$ flowing at an average velocity of $41.7 \mathrm{~cm} / \mathrm{s}$. 

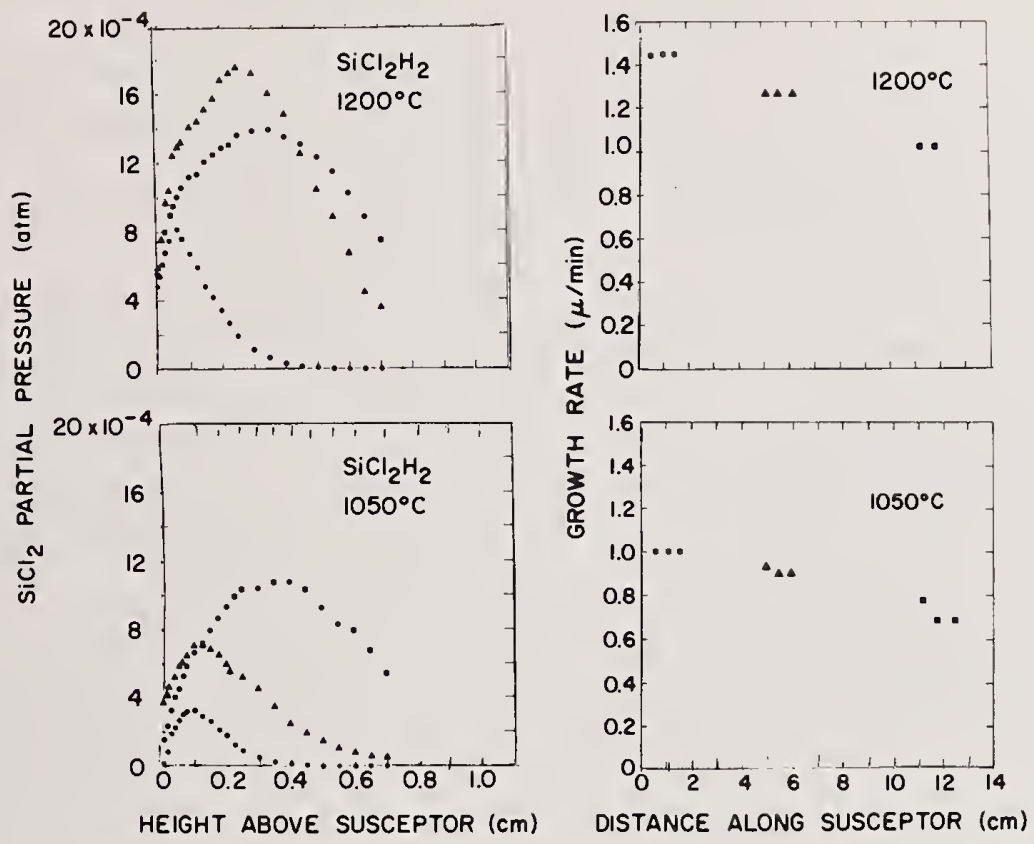

Figure 8. Partial pressure profiles of $\mathrm{SiCl}_{2}$ and epitaxial growth rates of deposited silicon at positions $1(\mathbf{O}), 3(\boldsymbol{\Delta})$, and $6(\square)$, produced from 0.276 percent $\mathrm{SiCl}_{2} \mathrm{H}_{2}$ in $\mathrm{H}_{2}$ flowing at an average velocity of $41.7 \mathrm{~cm} / \mathrm{s}$.

Some of the data curves in figures 7 and 8 show well-defined peaks at $0.05-0.4 \mathrm{~cm}$ above the susceptor. These peaks were first observed in the $\mathrm{H}_{2}$ reduction of $\mathrm{SiCl}_{4}$ with the fluorescence technique which has the ability of obtaining data close to the surface [7]. One of the important advantages of this technique is the ability to obtain data down to about $50 \mu$ above the surface. The peak is attributed to the homogeneous formation $[5,6,15]$ of $\mathrm{SiCl}_{2}$ in the gas phase represented by reactions (3) and (4)

$$
\begin{aligned}
& \mathrm{SiCl}_{4}+\mathrm{H}_{2} \rightarrow \mathrm{SiCl}_{2}+2 \mathrm{HCl} \\
& \mathrm{SiCl}_{2} \mathrm{H}_{2} \rightarrow \mathrm{SiCl}_{2}+\mathrm{H}_{2} .
\end{aligned}
$$

Mass spectrometric measurements confirmed the existence of the peak and showed that under typical growth conditions, $\mathrm{SiCl}_{2}$ was the only major product species present other than $\mathrm{HCl}[2,3]$. In the case involving $\mathrm{SiCl}_{2} \mathrm{H}_{2}$, additional unpublished fluorescence data at lower temperatures shows that this homogeneous formation occurs in the gas phase when the surface temperature is $900{ }^{\circ} \mathrm{C}$ or higher. 
The decreasing $\mathrm{SiCl}_{2}$ partial pressure as the surface is approached implies that the $\mathrm{SiCl}_{2}$, in fact, diffuses to the surface and is reduced to $\mathrm{Si}$ there by reaction (5):

$$
\mathrm{SiCl}_{2}+\mathrm{H}_{2} \rightarrow \mathrm{Si}(\mathrm{s})+2 \mathrm{HCl} \text { (surface). }
$$

However, the relative importance of this reaction in the overall reduction process is a question of some concern. If the input of $\mathrm{SiCl}_{4}$ molecules were completely reduced to $\mathrm{SiCl}_{2}$ homogeneously in the gas phase sufficiently far from the surface, then a maximum partial pressure of $2.76 \times 10^{-3}$ atm of $\mathrm{SiCl}_{2}$ would be observed. In the case of $\mathrm{SiCl}_{4}$ (see fig. 7), the maximum partial pressure of $\mathrm{SiCl}_{2}$ observed at $1050{ }^{\circ} \mathrm{C}$ is only $1 \times 10^{-4}$ atm, which leads us to believe that homogeneous $\mathrm{SiCl}_{2}$ formation and subsequent diffusion and reduction at the $S i$ surface is only a secondary parallel process for Si growth. At $1200{ }^{\circ} \mathrm{C}$ where the maximum observed partial pressure is $6 \times 10^{-4} \mathrm{~atm}$ it appears that homogeneous formation and subsequent diffusion is becoming an important process.

In the case of $\mathrm{SiCl}_{2} \mathrm{H}_{2}$ (fig. 8), however, as much as 40 percent at $1050{ }^{\circ} \mathrm{C}$ or 65 percent at $1200{ }^{\circ} \mathrm{C}$ of the input $\mathrm{SiCl}_{2} \mathrm{H}_{2}$ appears as $\mathrm{SiCl}_{2}$ in the gas phase. Thus, $\mathrm{SiCl}_{2}$ is a primary reaction intermediate produced in the gas phase and growth at the surface proceeds directly as a result of $\mathrm{SiCl}_{2}$ diffusion. In addition, it has been found that the $\mathrm{SiCl}_{2}$ gradient at the surface is proportional to growth rate [9]. These resuits are consistent with the fact that $\mathrm{Si}$ growth from $\mathrm{SiCl}_{2} \mathrm{H}_{2}$ is largely temperature independent above $1050{ }^{\circ} \mathrm{C}$ and is thought to be diffusion controlled. These results are in contradiction to previous speculation which has suggested that the $\mathrm{SiCl}_{2} \mathrm{H}_{2}$ diffuses all the way to the surface before reaction.

In addition, concentration profiles of the reactant $\mathrm{SiCl}_{2} \mathrm{H}_{2}$ above the growing surface obtained by vapor phase chromatography [4] show a characteristic S shape, shown schematically in figure 9. This data shows that even at $970{ }^{\circ} \mathrm{C}$, the $\mathrm{SiCl}_{2} \mathrm{H}_{2}$ begins to decompose in the gas phase. At $1100{ }^{\circ} \mathrm{C}$ this decomposition is sufficiently complete that only small amounts of $\mathrm{SiCl}_{2} \mathrm{H}_{2}$ exist near the $\mathrm{Si}$ surface at high temperature. Figure 9 shows schematically the species concentration profiles obtained from the fluorescence and VPC results.

It is important to point out that the three ana?ytical techniques being brought to bear on the $\mathrm{Si}-\mathrm{Cl}-\mathrm{H}$ system are producing very complementary types of data. For example, in mass spectrometry, the concentrations of all the species can be measured but the sampling probe tends to clog in a deposition reaction especially close to the surface. To date, fluorescence spectroscopy has been most successful in measuring $\mathrm{SiCl}_{2}$ concentrations close to the surface but the strong fluorescence signal masks the weaker Raman scattering from the other molecules of interest so that only $\mathrm{SiCl}_{2}$ is detectable. Vapor phase chromatography has a larger sampling probe which does not clog, but the gases are cooled before detection, thus making direct measurement of $\mathrm{SiCl}_{2}$ or any other species which only exists at high temperatures impossible. It is also interesting to note that recent 


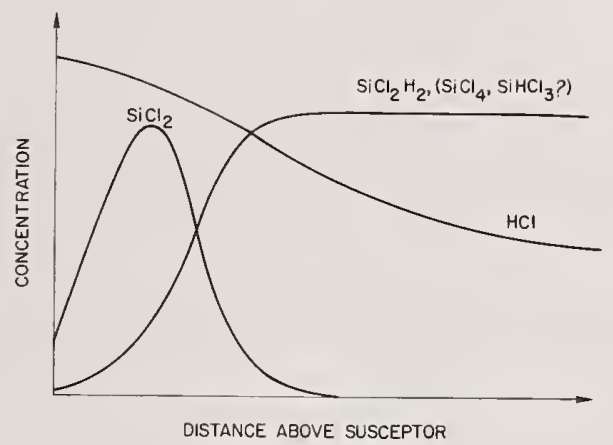

Figure 9. Schematic of gaseous reactant, intermediate and product curves in $\mathrm{Si}$ growth from $\mathrm{SiCl}_{2} \mathrm{H}_{2}$.

thermodynamic calculations of the surface coverage of $\mathrm{Si}$ at high temperature in the $\mathrm{Si}-\mathrm{Cl}-\mathrm{H}$ system indicate that $\mathrm{SiCl}_{2}$ is the only $\mathrm{Si}-\mathrm{Cl}_{\text {species with appreciable equilibrium }}$ surface coverage $[16,17]$.

\section{Conclusions}

By using a packed $\mathrm{Si}$ bed and making transpiration experiments involving the fast reaction between $\mathrm{HCl}$ and $\mathrm{Si}$ to produce $\mathrm{SiCl}_{2}$, it has been possible to calibrate the fluorescent technique to make quantitative measurements of the $\mathrm{SiCl}_{2}$ species partial pressure which is dominant at high temperatures in the $\mathrm{Si}-\mathrm{C} \mathrm{l}-\mathrm{H}$ system. In this case the observed fluorescence cross section of $\mathrm{SiCl}_{2}$ is $1.69 \times 10^{5}$ greater than the Raman scattering of $\mathrm{N}_{2}$ indicating the great sensitivity advantage in fluorescence measurements in reactive gas systems.

Using a laminar flow cold wall Si epitaxial CVD reactor, concentration gradient measurements of $\mathrm{SiCl}_{2}$ have been made over a range of about $1 \mathrm{~cm}$ and to within $50 \mu$ of the r.f. heated $\mathrm{Si}$ deposition surface. Results show that in the case of $\mathrm{SiCl}_{2} \mathrm{H}_{2}$ as reactant, $\mathrm{SiCl}_{2}$ is the major intermediate product formed homogeneously in the gas phase. The $\mathrm{SiCl}_{2}$ then diffuses to the surface where subsequent surface reduction takes place.

The authors are indebted to Richard Ghez for many helpful discussions and to Ray Brengs for construction of the calibration cell. 
[1] Ban, V. S. and Gilbert, S. L., J. Electrochem. Soc. 122, 1382 (1975); Ban, V. S., J. Electrochem. Soc. 122, 1380 (1975).

[2] Ban, V. S., Abs. 229, Oct. 1976 Meeting of the Electrochem. Soc., Las Vegas, Nevada.

[3] Ban, V. S., Proc. of the Sixth Intl. Conf. on Chem. Vapor Deposition, L. F. Donaghey, et al., ed., p. 66 (The Electrochem. Soc., Princeton, N.J. 1977).

[4] Duchemin, J., Revue Technique, Thomson-CSF 9 , No. 1, 33 (March 1977).

[5] Sedgwick, T. 0., Arbach, G. V., and Ghez, R., Proc. of the Sixth Int1. Conf. on Chem. Vapor Deposition, L. F. Donaghey, et al., ed., p. 79 (The Electrochem. Soc., Princeton, N.J., 1977).

[6] Sedgwick, T. 0., Smith, J. E., Jr., Ghez, R., and Arbach, G. V., Recent Newspaper 366, October 1976 Meeting of the Electrochem. Soc., Las Vegas, Nevada.

[7] Sedgwick, T. 0., Smith, J. E., Jr., Ghez, R., and Cowher, M. E., J. Crystal Growth, 31, 264-273 (1975).

[8] Smith, J. E., Jr. and Sedgwick, T. 0., Thin Solid Films, 40, 1-11 (1977).

[9] Sedgwick, T. 0. and Arbach, G. V., J. Japan Assoc. Crystal Growth, 5, 93-102 (1978).

[10] Nishizawa, J. and Nihira, H., Proc. ICVGE-4, J. Crystal Growth, 45 (1978).

[11] Nishizawa, J., Murphy, T.U.M.S., Yoneda, M., and Shimbo, M., Proc. ICVGE-4, J. Crystal Growth, 45 (1978).

[12] JANAF Thermochemical Tables (Pub1. U.S. Department of Commerce, 1971).

[13] Sirt1, E., Hunt, L. P., and Sawyer, D. H., J. Electrochem. Soc. 121, 919 (1974).

[14] van der Putte, P., Giling, L. J., and Bloem, J., J. Crystal Growth, 41, 133 (1977).

[15] Ghez, R., Abstract 228, October 1976 Meeting of the Electrochem. Soc., Las Vegas, Nevada.

[16] Chernov, A. A., J. Assoc. Crystal Growth, $\underline{5}$ (1978).

[17] Chernov, A. A. and Rusaikin, M. P., Proc. ICVGE-4, J. Crystal Growth, 45 (1978).

\section{Discussion}

Question (Hastie): I wasn't sure what temperature you were using there, and did that temperature correspond with the temperature that you showed in the equilibrium plot where $\mathrm{SiCl}_{2}$ is the predominant product?

Response (Sedgwick): Yes, it does. And, it is right in between the two experimentally measured temperatures where I have profiles, 1050 , and 1200. It was $1100{ }^{\circ} \mathrm{C}$; okay?

I make the following comment: we have heard a lot about quenching, and of course quenching may occur here, but the approach I have taken simply is to make my calibration check under conditions that are very close to the experimentally determined profiles, that is the same total pressure--one atmosphere, the same majority carrier gas which is hydrogen, and the same minor species, which are $\mathrm{HCl}$ and so on, and at the same basic concentration level. 
Comment (Hastie): So, I would deduce from what you are saying, that the thermodynamic data for silicon dichloride is in pretty good shape, because the temperature matches (i.e., where predicted and observed $\mathrm{SiCl}_{2}$ maximum concentration occurs).

Response (Sedgwick): I think it is. It has been determined by at least weight loss measurements and Bourdon gage measurements from reaction of silicon tetrachloride, and silicon. So, the thermodynamic information, I think, is in fairly good shape.

Question (Rosner): The silicon deposition/etching systems you described appear to be characterized by the simultaneous presence of large temperature gradients and vapor species of widely disparate molecular weight (i.e. $\mathrm{H}_{2}$ and $\mathrm{SiCl}_{4}$ ). I suspect that in such cases thermal (Soret) diffusion will play an important role and wonder whether you or your CVD colleagues have had occasion to investigate this in such work. We have recently found [ 1 . Rosner, D. E., "Thermal (Soret) Diffusion Effects on Interfacial Mass Transport Rates," Proc. 1978 International (Levich) Conference on Physicochemical Hydrodynamics, (in Press (1979)] and [2. Srivastava, R. and Rosner, D. E., "A New Approach to the Correlation of Boundary Layer Mass Transfer Rates with Thermal Diffusion and/or Variable Properties," Int. J. Heat Mass Transfer (in Press (1979)o that Soret transport and vapor species profiles are those of blowing (suction) and homogeneous chemical sources (sinks)--indeed this can form the basis of a useful correlation/prediction scheme.

Thus, as in some premixed flames, quantitative inferences on homogeneous (and heterogeneous) chemical kinetics from the analysis of steady-state concentration profiles require a transport model which includes multicomponent Fick and Soret diffusion.

We have found that Soret transport causes noticeable shifts in the predicted "dew point" in partial condensation situations, e.g. deposition of salts from combustion gases [3. See chapter of Rosner et al; elsewhere in this volume on "Transport, Thermodynamic and Kinetic Aspects of Salt/Ash Deposition Rates from Combustion Gases," and the references cited therein.] By analogy, I would expect Soret transport to influence important "boundaries" (e.g. etching/deposition, "active"/"passive" transition temperatures, etc.).

Response (Sedgwick): We have not done so, but Jan Bloom from Phillips Einhoven in the Netherlands, has concerned himself about thermal diffusion in this kind of system, and he feels it does play a significant role.

I have so far avoided drawing very quantitative conclusions, and I think one would have to factor that in. 

National Bureau of Standards Special Publication 561, Proceedings of the 10th Materials Research Symposium on Characterization of High Temperature Vapors and Gases held at NBS, Gaithersburg, Maryland, September 18-22, 1978. Issued October 1979.

\title{
RAMAN SCATTERING OF THE GASEOUS PHASE AT HIGH TEMPERATURE
}

\author{
R. Hillel, J. Bouix, and A. Michaelides \\ Laboratoire de Physicochimie Minérale I \\ (associé au C.N.R.S. N ${ }^{\circ} 116$ ) \\ Université Claude Bernard Lyon I \\ 43 Boulevard du 11 Novembre 1918 \\ 69621 Villeurbanne, France
}

\section{Introduction: Choice of Raman Scattering as Analytical Method}

During these last five years, we have been interested in the analysis of gaseous mixtures in equilibrium at high temperature. Our purpose was the study of solid-vapour reactions, chiefly in the case of crystal growth of inorganic solids by chemical transport.

This study presents two different problems. On one hand, the mixture is in equilibrium and the analytical method should disturb as little as possible this equilibrium. On the other hand, this mixture is at high temperatures which causes experimental problems concerning the nature of usable cell materials, especially since the vapour is in many cases highly corrosive.

Because of the first requirement, we preferred to use a method other than mass spectroscopy, which is, however, the most common in gas analysis $[1-8]^{1,2}$. Mass spectrometry has been used by different authors to study chemical vapour deposition of solids [9-14]. A small silica capillary introduced into the reactor and connected with the spectrometer, al lows a pressure of about $10^{-5}$ Torr to be maintained in the mass spectrometer. As far as we are concerned, this method suffers two important disadvantages. First, the sample is expanded to a very low pressure, quite different from that in the reactor. If, pressure sensitive equilibria takes place in the gaseous mixture, for example, decomposition of unstable species may occur and the sample will be highly disturbed and not representative of the mixture ${ }^{3}$. Second, the sample beam is bombarded with a high-power electron beam many molecules. In the mass spectrum, it is not always easy to distinguish between parent and fragment.

${ }^{1}$ Figures in brackets indicate the literature references at the end of this paper. ${ }^{2}$ Editor's note: These particular citations are only representative examples of the many mass spectrometric developments in the literature.

${ }^{3}$ Editor's note: This problem has been addressed in detail in the chapter by Bonne 17 and Hastie elsewhere in this volume. 
A second possible method is uv-visible spectroscopy [15-18]. By testing this method, we immediately found that, with our current apparatus (Cary-17), it was not possible to record a spectrum over $700^{\circ} \mathrm{C}$. The monochromator detector receives all the energy radiated by the furnace and reactor and becomes saturated or even damaged. For this reason, we used this method only below $700{ }^{\circ} \mathrm{C}$, and generally with good results with very simple molecules, like diatomics, but with much less success in the case of more complex species. For example, the system $\mathrm{In} / \mathrm{I}_{2}$ that we studied within the scope of the chemical transport of $\mathrm{In}_{2} \mathrm{Se}_{3}$ by iodine, exhibits two gaseous iodides of indium, InI and $\mathrm{InI}_{3}$. Below $600{ }^{\circ} \mathrm{C}$, we obtained by uv-visible absorption a spectrum with a wide maximum at $3100 \AA$. For the same conditions, as will be seen later, the Raman spectrum shows plainly the three vibrations of the planar molecule $\mathrm{InI}_{3}$, which is impossible to identify by uv-visible spectroscopy. On the other hand, above $600{ }^{\circ} \mathrm{C}$ a high resolution band system corresponding to InI ( $f i g$. 1) shows up clearly in the uv-visible spectra. We determined that the limit of sensitivity by this method was about 0.1 Torr for this compound, whereas it is 100 times higher by Raman spectroscopy. Thus, in the case of $\mathrm{InI}_{3}-\mathrm{InI}$, these two techniques are complementary.

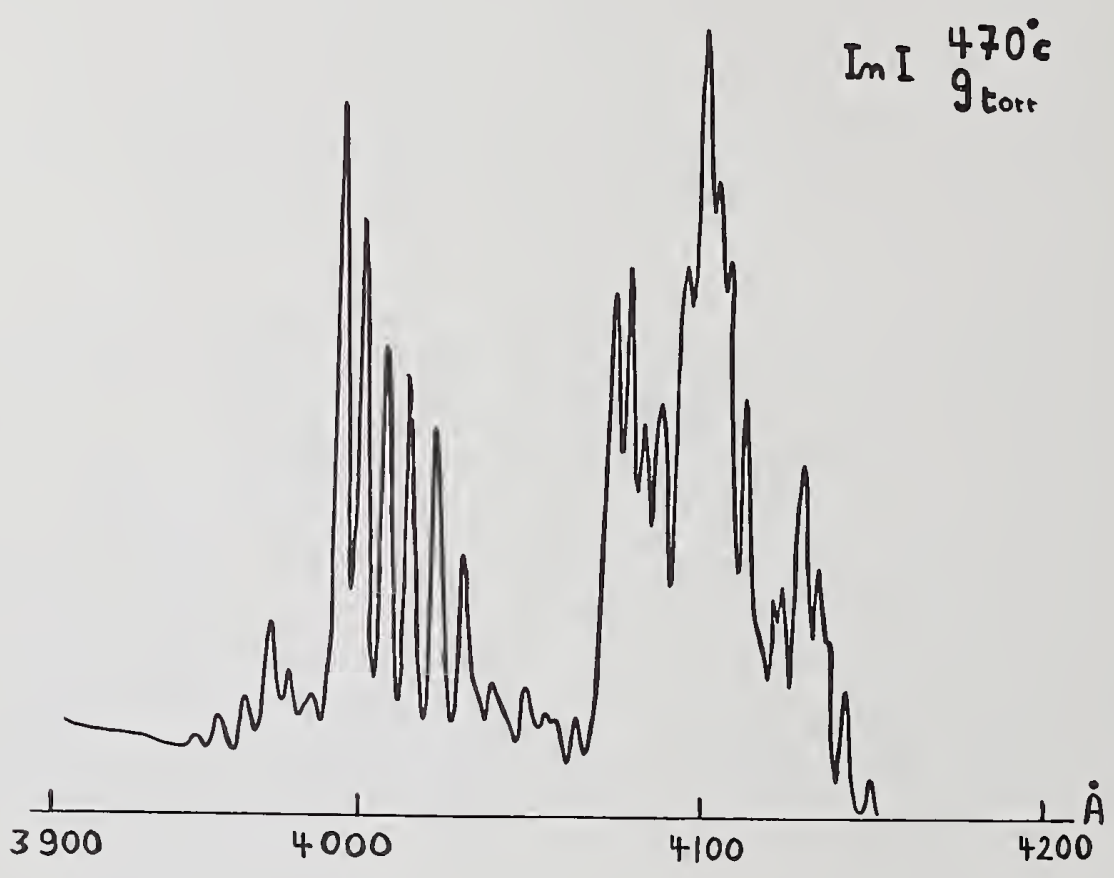

Figure 1. uv-visible spectrum of InI.

Because of the temperature limit for uv-visible absorption, we decided to try the vibrational spectroscopic methods of infrared absorption and Raman scattering. With infrared absorption, the main advantage is its high sensitivity, but window materials are a limitation. They must satisfy three conditions: (1) to be transparent in the interesting spectral domain (in our case between about 30 and $600 \mathrm{~cm}^{-1}$; (2) to have a good thermal 
stability at high temperatures (about $1000{ }^{\circ} \mathrm{C}$ ); and ( 3 ) to resist chemical attack by the gaseous mixtures containing, in general, very corrosive species at high temperature, like halogens, hydracids, oxygen or water. We have never found such windows.

For our conditions, the only convenient method is Raman spectroscopy. The source is a visible laser beam, allowing an in situ analysis without sensible perturbation of the gaseous sample. Moreover, the scattered Raman radiation is in the visible, and it is possible to use optical materials like silica which withstand high temperatures. Interpretation of spectra and identification of unknown molecules are generally possible using group-theory and force-field calculations.

High temperature Raman spectroscopy of gases has been extensively used by Beattie [19] and recently applied by Sedgwick $[20,21]$ to chemical vapour deposition. The vibrational Raman spectra of most of the molecules studied here have been obtained previously which aids in our assignments. During this study, we have often observed the Raman resonance effect which means a considerable enhancement of one or more modes of vibration of the resonant molecule. For example, the complex resonance of tellurium was for us a difficult problem in studying the chemical transport of vanadium oxide with tellurium tetrachloride because it concealed the spectrum of the other components. In contrast, the transport of SiAs by halogens forms the dihalide $\mathrm{SiX}_{2}$, whose resonance gives only one peak plus its overtones. This resonance provides very high detection sensitivity without perturbing the rest of the spectrum.

\section{Experimental}

We have developed a simple apparatus for recording Raman spectra of gaseous mixtures up to $1000{ }^{\circ} \mathrm{C}[22,23]$. This apparatus is shown in figure 2. The reactants are introduced in a cylindrical silica cel1-1- which is sealed under vacuum at the temperature of liquid air. This cell is prolonged at one end by a silica rod-2- and centered in the axis of the furnace by adjusting screws -3-. When the studied gaseous mixture is strongly colored, the cell, at its second end -4-, which is also cylindrical but with a smaller diameter is used to reduce the absorption of the laser beam.

The furnace consists of three independent heating elements -8-, separately regulated by three Pt-Pt.Rh thermocouples -5 - to obtain the desired temperature profile along the axis. The outside of the furnace is water-cooled. Two diametrically opposite holes are made in the whole structure for the direct and, if required, reflected laser beams. When working at very high temperature, the spectrometer is protected by a water-cooled pyrex cell with parallel sides -7-. The spectrometer is a Coderg PHO model with a photomultiplier cooled by the Peltier effect. The laser source is a Spectra Physics 165.

The Bourdon manometer [24] shown in figure 3 is a silica apparatus whose essential part is a thin membrane with a long curved shape - $a$ - connected to by a silica needle - $b^{-}$.

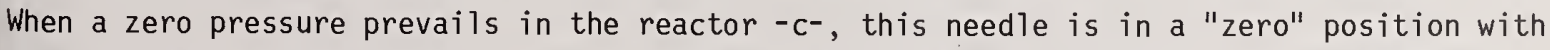
regard to the silica tip $-d-$. When the pressure increases in the reactor, the membrane deforms and the needle deviates from its initial position. It is reset to this position 


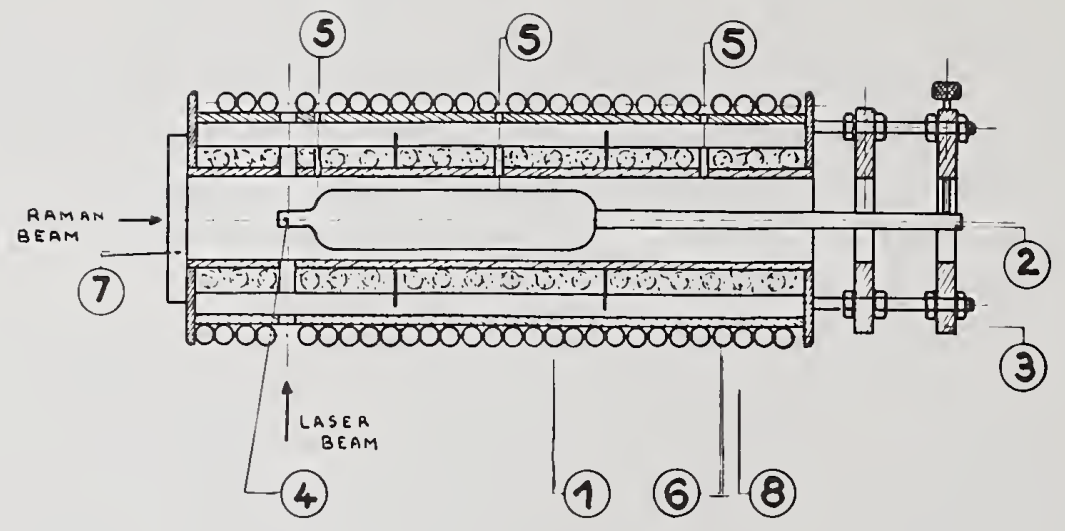

Figure 2. Apparatus for Raman scattering at high temperatures.

by introducing in the upper part of the manometer an argon pressure equal to the one prevailing in the reactor. Pressures up to one atmosphere are measured in this manner to the nearest one-half Torr.

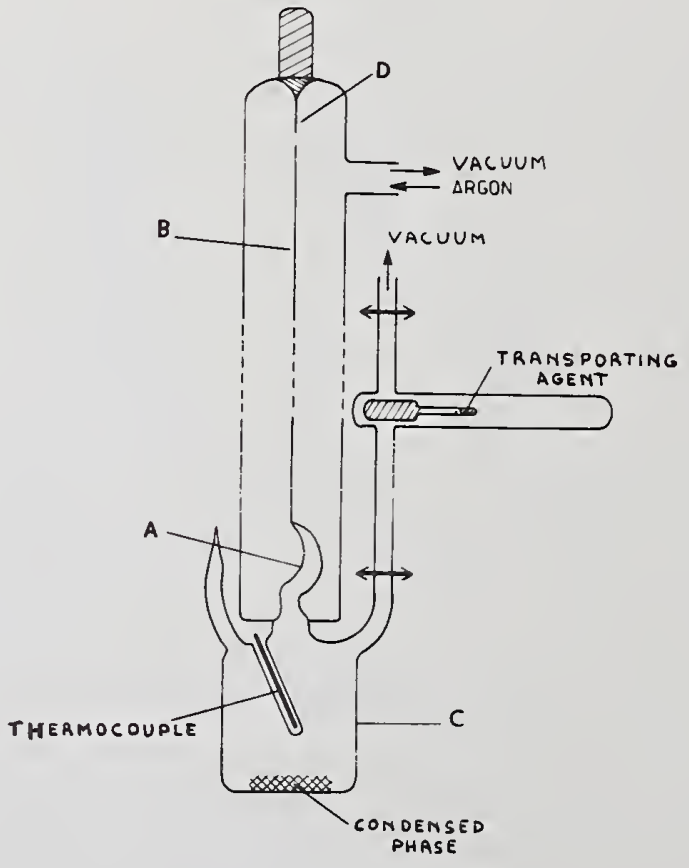

Figure 3. Bourdon manometer. 


\section{Results}

Among the numerous systems that we have studied, we summarize here some results obtained for the P-S system and for chemical transport of solids.

\subsection{P-S system [25]}

Only sparse information exists in the literature concerning the behaviour of $\mathrm{P}-\mathrm{S}$ derivatives $\left(\mathrm{P}_{4} \mathrm{~S}_{3}, \mathrm{P}_{4} \mathrm{~S}_{7}, \mathrm{P}_{4} \mathrm{~S}_{10}\right)$ in the gaseous state at high temperature. Besides the vapor density study of gaseous $\mathrm{P}_{4} \mathrm{~S}_{3}$ and $\mathrm{P}_{4} \mathrm{~S}_{7}$ by Stock in 1908 [26], the pressure of saturated [27] and unsaturated [28] vapours of $\mathrm{P}_{4} \mathrm{~S}_{3}$ and $\mathrm{P}_{4} \mathrm{~S}_{10}$ have been determined and the Raman spectra recorded in the solid, liquid, and gaseous states [29]. For our part, we observe by Raman scattering that $\mathrm{P}_{4} \mathrm{~S}_{3}$ is quite stable up to $700{ }^{\circ} \mathrm{C}$ and begins to decompose reversibly above this temperature according to the gaseous reaction:

$$
P_{4} S_{3} \underset{\leftarrow}{m} P_{m}+\frac{3}{n} S_{n}
$$

Gaseous $\mathrm{P}_{4} \mathrm{~S}_{7}$ decomposes reversibly over $400{ }^{\circ} \mathrm{C}$ with formation of $\mathrm{P}_{4} \mathrm{~S}_{3}$ and sulfur:

$$
\mathrm{P}_{4} \mathrm{~S}_{7} \text { (vap.) } \underset{\leftarrow}{\rightarrow} \mathrm{P}_{4} \mathrm{~S}_{3} \text { (vap.) }+\frac{4}{n} \mathrm{~S}_{n} \text { (vap.) }
$$

Lastly, $\mathrm{P}_{4} \mathrm{~S}_{10}$ vaporizes with irreversible decomposition giving $\mathrm{P}_{4} \mathrm{~S}_{7}$ and sulfur:

$$
\mathrm{P}_{4} \mathrm{~S}_{10} \rightarrow \mathrm{P}_{4} \mathrm{~S}_{7}+\frac{3}{\mathrm{n}} \mathrm{S}_{n}
$$

Knowing the nature of the equilibrated decompostion of $\mathrm{P}_{4} \mathrm{~S}_{3}$ and $\mathrm{P}_{4} \mathrm{~S}_{7}$, Bourdon manometry allowed us to determine the constants of these equilibria and some of the thermodynamic properties of these compounds.

\section{$3.2 \mathrm{In}_{2} \mathrm{Se}_{3}-\mathrm{I}_{2}$ system [30]}

Our objective was to perfect the single-crystal growing of $\mathrm{In}_{2} \mathrm{Se}_{3}$ under its different allotropic forms by chemical transport with iodine. Using together uv-visible and Raman spectroscopies, we established that the gaseous mixture contains chiefly $\mathrm{InI}_{3}, \mathrm{I}_{2}$, and $\mathrm{Se}_{\mathrm{n}}$ below $650{ }^{\circ} \mathrm{C}$ and $\mathrm{InI}, \mathrm{I}_{2}$, and $\mathrm{Se}_{\mathrm{n}}$ above $650{ }^{\circ} \mathrm{C}$. The system is perfectly reversible on cooling. Figure 4 shows the Raman spectrum of $\mathrm{InI}_{3}$ at $450{ }^{\circ} \mathrm{C}$ under a pressure of 100 Torr. Figures 1 and 4 show the excellent limits of detection of $\mathrm{InI}_{\text {and }} \mathrm{InI}_{3}$ owing to the simultaneous utilization of these two spectroscopies. 


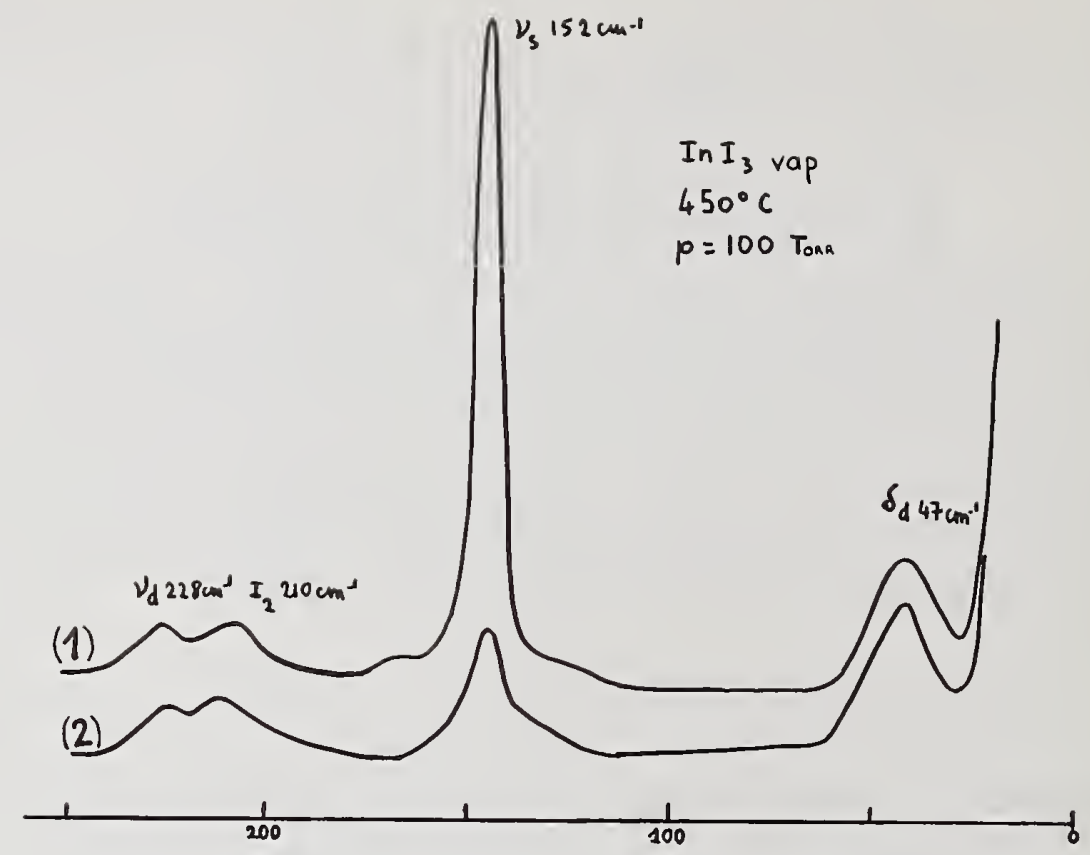

Figure 4. Raman spectrum of $\operatorname{InI}_{3},(1): I_{\|}+I_{\perp},(2): 2 I_{\perp}$

It is clear that two different equilibria take part in this transport:

$$
\begin{aligned}
& \mathrm{In}_{2} \mathrm{Se}_{3} \text { (sol.) }+3 \mathrm{I}_{2} \text { (vap.) } \underset{\leftarrow}{\rightarrow} \mathrm{InI}_{3} \text { (vap.) }+\frac{3}{n} \mathrm{Se}_{n} \text { (vap.) } \\
& \text { (below } 650{ }^{\circ} \mathrm{C} \text { ) }
\end{aligned}
$$

and

$$
\begin{aligned}
& \mathrm{In}_{2} \mathrm{Se}_{3} \text { (sol.) }+\mathrm{I}_{2} \text { (vap.) } \underset{\leftarrow}{(} \mathrm{InI} \text { (vap.) }+\frac{3}{\mathrm{n}} \mathrm{Se}_{\mathrm{n}} \text { (vap.) } \\
& \text { (above } 650{ }^{\circ} \mathrm{C} \text { ) }
\end{aligned}
$$

The pressure measurement as a function of temperature for different amounts of iodine (fig. 5) allowed us to determine the thermodynamic properties of these equilibria: the first reaction is exothermic and the second endothermic. Details are discussed elsewhere [30]. Therefore, for the crystal growth of the low temperature form of $\mathrm{In}_{2} \mathrm{Se}_{3}$, it is necessary to perform the transport with a positive temperature gradient around $500{ }^{\circ} \mathrm{C}$ [31]. For such a temperature, at which this form is stable, the exothermic equilibrium is indeed responsible for the transport. In contrast, to obtain the high temperature form, the transport should be carried out around $700{ }^{\circ} \mathrm{C}$ with a negative gradient. 


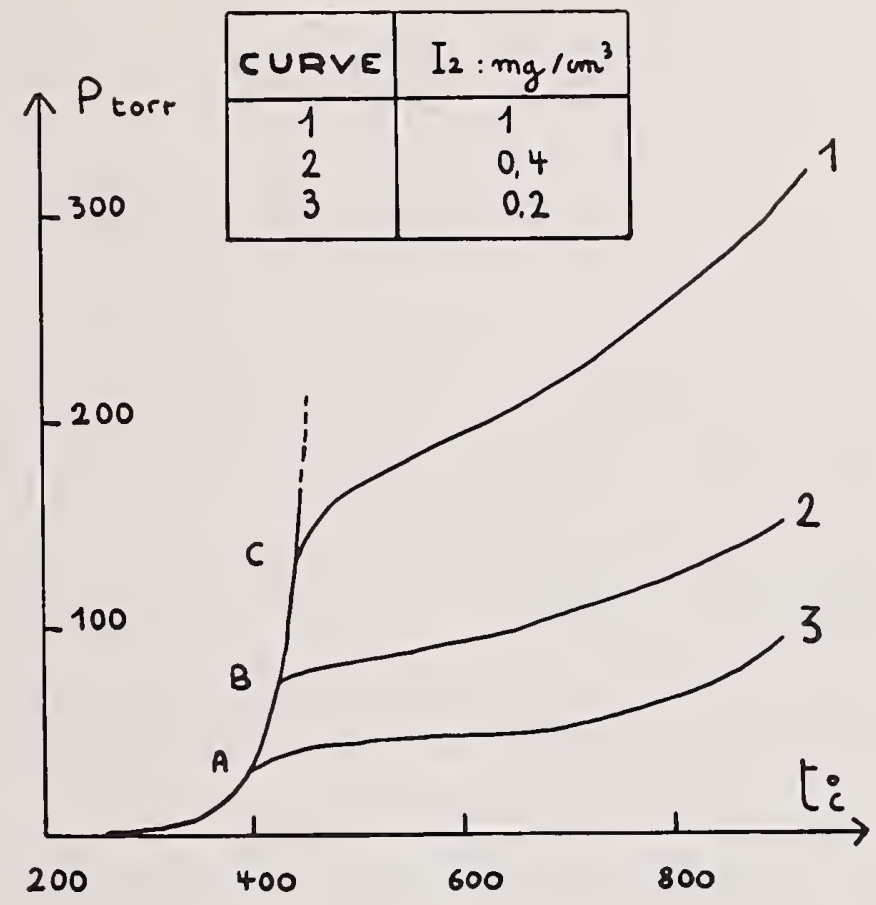

Figure 5. The total pressure in the $\mathrm{In}_{2} \mathrm{Se}_{3}-\mathrm{I}_{2}$ system.

\section{$3.3 \mathrm{Ge}-\mathrm{I}_{2}$ system [24]}

In this case, it was necessary to know the composition of the vapour phase during the transport of germanium by iodine to determine the transition between diffusion and convection conditions. Starting from a mixture containing $0.45 \cdot 10^{-5} \mathrm{~mol} \cdot \mathrm{cm}^{-3}$ of iodine and an excess of germanium, we noticed at $200{ }^{\circ} \mathrm{C}$ an almost total reaction giving the tetrahedral molecule $\mathrm{GeI}_{4}$ (fig. 6). The peak at $213 \mathrm{~cm}^{-1}$ corresponds to remaining iodine and that at $228 \mathrm{~cm}^{-1}$ to the symmetric stretching of $\mathrm{GeI}_{2}$ formed in small amount.

Above $400{ }^{\circ} \mathrm{C}$, the intensity of the $\mathrm{GeI}_{4}$ spectrum decreases, while there appears a system of three peaks at 75,228 (polarized) and $242 \mathrm{~cm}^{-1}$ (depolarized) as shown in figure 7. This result is consistent with the triatomic bent molecule $\mathrm{GeI}_{2}$ of $\mathrm{C}_{2 v}$ symmetry. The existence of overtones at $150\left(2 v_{2}\right), 300\left(v_{1}+v_{2}\right), 375\left(2 v_{1}-v_{2}\right), 445\left(2 v_{1}\right)$ and 520 $\left(2 v_{1}+v_{2}\right)$ and combinations of the symmetric fundamental vibrations indicates a pre-resonance effect. 


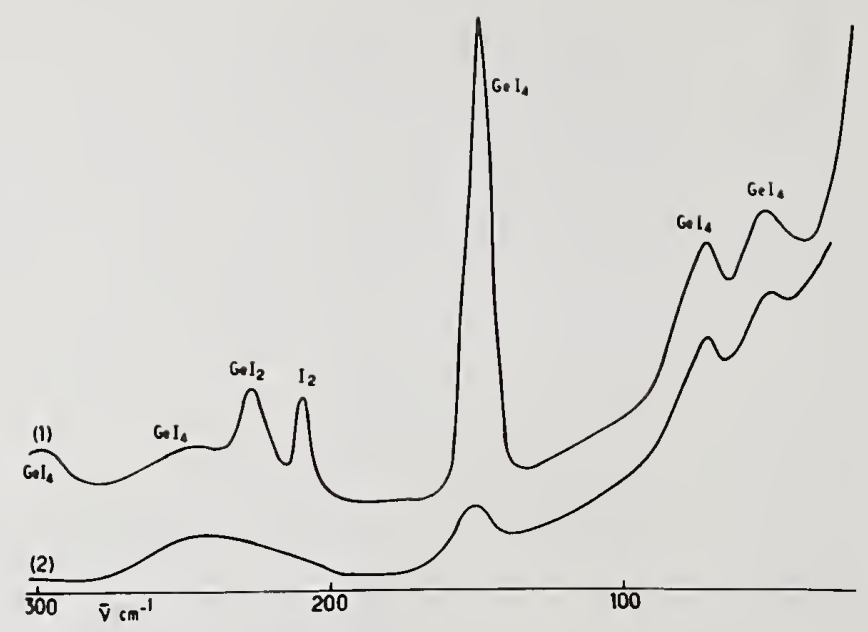

Figure 6. Raman spectrum of the Ge $-\mathrm{I}_{2}$ system at $200^{\circ} \mathrm{C}$.

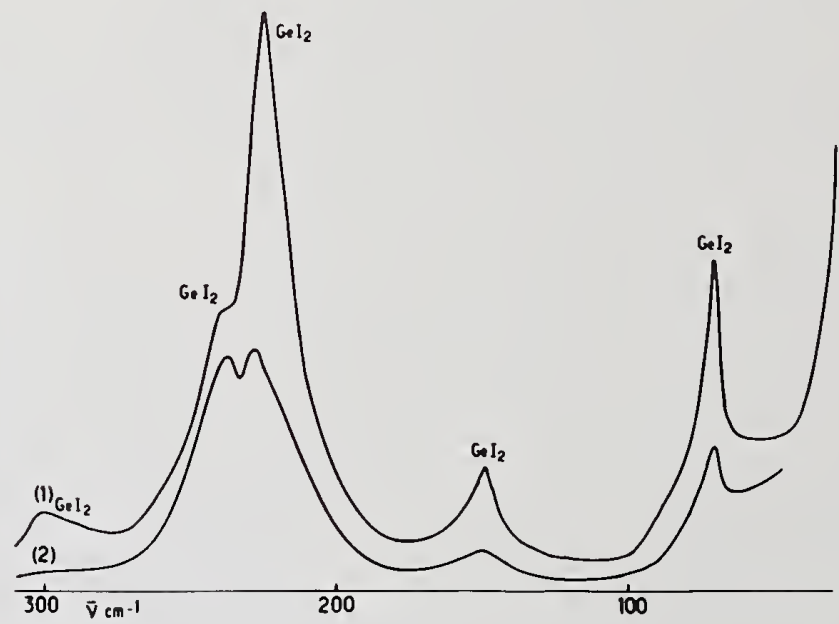

Figure 7. Raman spectrum of the Ge $-\mathrm{I}_{2}$ system above $400{ }^{\circ} \mathrm{C}$.

Above $800{ }^{\circ} \mathrm{C}$, there appears a new peak at $243 \mathrm{~cm}^{-1}$, indicating a reversible formation of GeI (fig. 8). From known electronic spectra, the GeI frequency is $245 \mathrm{~cm}^{-1}$. 


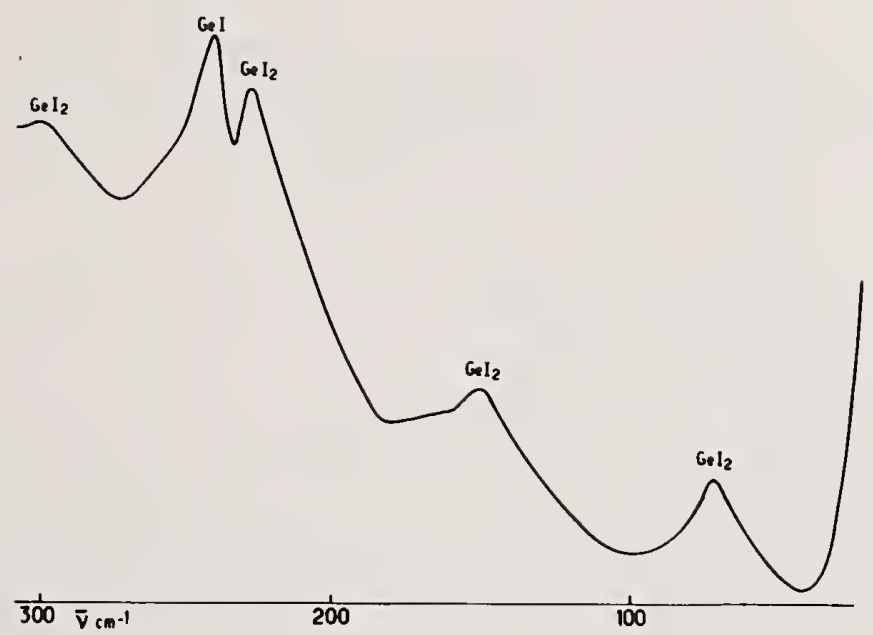

Figure 8. Raman spectrum of the $\mathrm{GeI}_{2}-\mathrm{I}_{2}$ system above $800{ }^{\circ} \mathrm{C}$.

In figure 8 , the upslope of the background with increasing Raman frequency results from the thermal emission of the cell and furnace.

The transport mechanism of germanium with iodine is then the following:

$$
\begin{aligned}
& \mathrm{Ge}(\text { sol. })+2 \mathrm{I}_{2} \text { (vap.) } \rightarrow \mathrm{GeI}_{4} \text { (vap.) above } 200{ }^{\circ} \mathrm{C} \\
& \mathrm{Ge}(\text { sol. })+\mathrm{GeI}_{4} \text { (vap.) } \underset{\leftarrow}{ } 2 \mathrm{GeI}_{2} \text { (vap.) above } 400{ }^{\circ} \mathrm{C} \\
& \mathrm{Ge}\left(\text { sol.) }+\mathrm{GeI}_{2} \text { (vap.) } \underset{\leftarrow}{ } 2 \mathrm{GeI} \text { (vap.) above } 800^{\circ} \mathrm{C} .\right.
\end{aligned}
$$

In this case, these equilibria are endothermic and the transport occurs at any temperature from hot to cold. In figure 9 the calculated partial pressures of the gaseous reactants are plotted as a function of temperature, and the calculated and experimental total pressures for different initial amounts of iodine are shown in figure 10 . They show the good agreement between manometric, thermodynamic and spectroscopic results. 


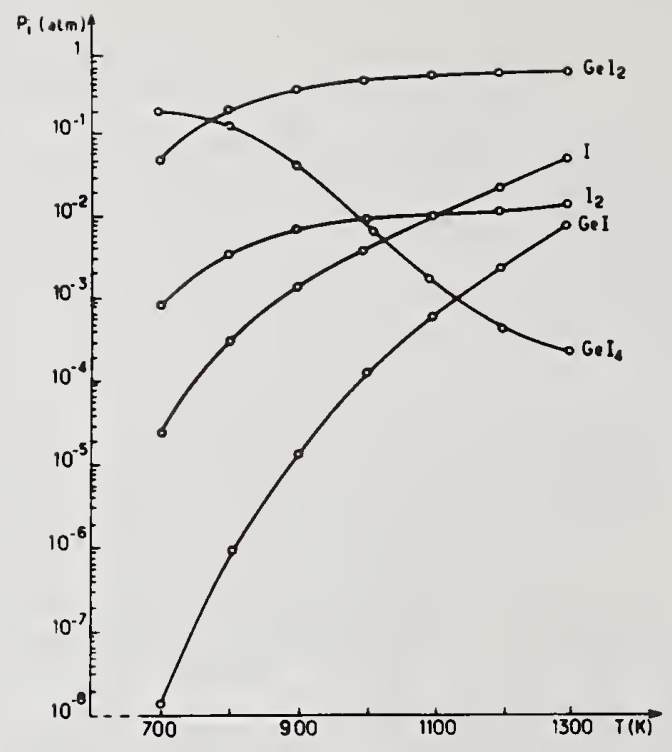

Figure 9. The calculated partial pressures of the gaseous reactants in the Ge $-\mathrm{I}_{2}$ system for $\mathrm{CI}_{2}=2 \mathrm{mg} / \mathrm{cm}^{3}$.

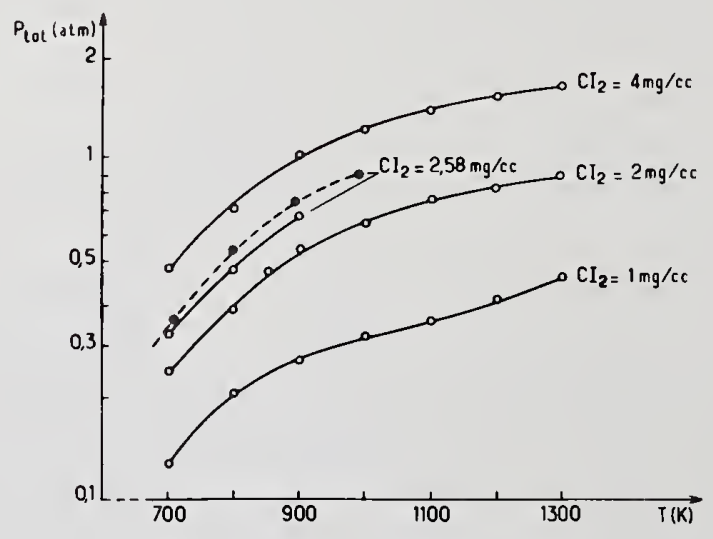

Figure 10. Calculated (O) and experimental (O) total pressures in the Ge $-I_{2}$ system for different initial amounts of iodine. 


\subsection{SiAs and GeAs - halogens systems $[32,33]$}

Chemical transport is the best way to obtain single-crystals of these important semiconductors because of their instability at high temperature, which prevents the utilization of liquid-solid methods, especially in the case of GeAs.

Raman spectroscopy shows that the first step of the transport consists of a reaction giving as an intermediate state a mixture of arsenic trihalide and $\mathrm{Si}$ or Ge tetrahalide, followed by the tetrahalide and arsenic reacting according to the irreversible global reaction:

$$
\operatorname{MAs}(\text { sol. })+2 X_{2} \text { (vap.) } \rightarrow M X_{4}(\text { vap. })+\frac{1}{4} A s_{4}(\text { vap. })
$$

This reaction begins between $150{ }^{\circ} \mathrm{C}\left(\mathrm{GeAs}-\mathrm{I}_{2}\right.$ ) and $400^{\circ} \mathrm{C}\left(\mathrm{SiAs}-\mathrm{Cl}_{2}\right)$. At higher temperatures, the Raman peaks from the tetrahalide decrease. At the same time, there appears a new set of bands. In the case of GeAs, this set contains the three fundamental vibrations of the $\mathrm{GeX}_{2}$ molecule ( $s$ and $\delta$ polarized, a depolarized). With SiAs, the spectra are complicated by the Raman resonance effect from the deformation of the $\mathrm{SiCl}_{2}$ and $\mathrm{SiI}_{2}$ molecule and the stretching of $\mathrm{SiBr}_{2}$. Figure 11 shows the spectrum of the SiAs$\mathrm{I}_{2}$ system at $350(\mathrm{~A}), 400(\mathrm{~B})$ and $780^{\circ} \mathrm{C}(\mathrm{C})$. The latter shows the resonance of the $\mathrm{SiI}_{2}$ deformation at $88 \mathrm{~cm}^{-1}$ (fundamenta1), and 175, 262, 350, $437 \mathrm{~cm}^{-1}$ (overtones).

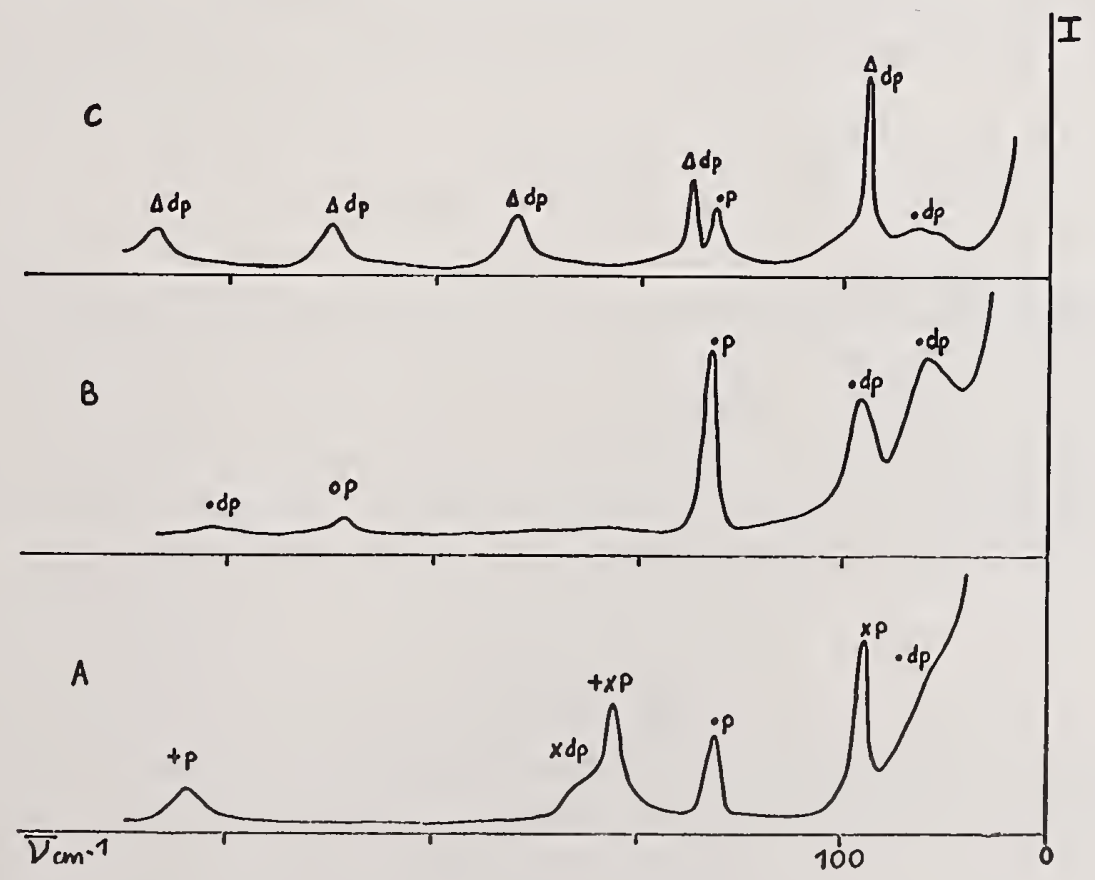

Figure 11. Raman spectra of the SiAs $\mathrm{I}_{2}$ system, A: $350{ }^{\circ} \mathrm{C}, \mathrm{B}: 400^{\circ} \mathrm{C} ; \mathrm{C}: 780^{\circ} \mathrm{C}$; p: polarized; dp: depolarized; +: $\mathrm{I}_{2}, \mathrm{x}: \mathrm{AsI}_{3}, 0: \mathrm{SiI}_{4}, 0: \mathrm{As}_{4}, \Delta: \mathrm{SiI}_{2}$. 
We have never observed in this transport the formation of a monohalide of silicon or germanium. The transport equilibrium is then:

$$
\operatorname{MAs}(\text { sol. })+M X_{4} \text { (vap.) } \underset{\leftarrow}{\rightarrow} 2 \mathrm{MX}_{2} \text { (vap.) }+\frac{1}{4} \mathrm{As}_{4} \text { (vap.) }
$$

This equilibrium is endothermic and the transport is carried out from hot to cold according to our experiments.

\section{Future Prospects}

The main drawback of the Raman technique is the existence of a maximal temperature of about $1000{ }^{\circ} \mathrm{C}$. This limit results from the energy radiated by the cell and the furnace which increases with the temperature and becomes stronger than the Raman energy scattered by the gaseous sample. We are now planning to adopt the same solution as Sedgwick [20], consisting of laser beam modulation and using a synchronous detector to filter out all non-modulated signals including the thermal radiation. It may be possible then to study chemical transport where the mechanisms are unknown, like those of the transition metals borides. Another future prospect concerns the study of the mechanism of chemical vapour deposition of solids, especially fibers for composite materials, by determining the composition of the gaseous mixture as a function of distance from the deposition surface [34]. This improvement requires some modifications for our apparatus that we are now carrying out.

\section{Conclusion}

Raman scattering is a demonstrated method for in situ gaseous phase analysis of high temperature equilibrium. For a majority of molecules, the limit of detectability is probably a few Torr, sometimes much less in the case of Raman resonance. Used together with uv-visible absorption, Bourdon manometry, and thermodynamic calculation, this method allowed us to determine mechanisms of chemical transport, generally with the object of establishing the best experimental conditions of crystal growth from the vapour phase.

We thank Dr. Y. Monteil and Dr. H. Vincent for their useful help in the study of the $\mathrm{P}-\mathrm{S}$ system and Mr. Favre for his technological help.

References

[1] Goldfinger, P. and Drowart, J., J. Chem. Phys. 55, 721 (1958).

[2] Drowart, J., Am. Rev. Phys. Chem. 13, 459 (1962). 
[3] Shchukarev, S. A., Semenov, G. A., and Rat'kouskii, I. A., Russ. J. Inorg. Chem. 14, 1 (1969).

[4] Goldfinger, P. and Jeunehomme, M., Trans. Faraday. Soc. 59, 2857 (1963).

[5] Drowart, J. et al., Trans. Faraday. Soc., 64, 2611 (1968); 64, 3006 (1968); 66, 809 (1970).

[6] Drowart, J., et al., Z. Naturforsh, 26a, 327 (1971); 26a, 1934 (1971).

[7] Farber, M., Can. Met. Quart. 13, 373 (1974).

[8] Semenov, G. A., in Symposium, Silicates and Oxides in High Temperature Chemistry, p. 228 (Moscow, 1963).

[9] Tietjen, J. J. and Amick, J. A., J. Electrochem. Soc. 113, 724 (1966).

[10] Tietjen, J. J., Maruska, H. P., and Clough, R. B., J. Electrochem. Soc. 116, 492 (1969).

[11] Ban, V. S., J. Electrochem. Soc. 118, 1473 (1971), 119, 761 (1972).

[12] Ban, V. S., J. Crystal Growth, 17, 19 (1972).

[13] Ban, V. S. and White, E. A. D., J. Electrochem. Soc. 122, 1382 (1975).

[14] Ban, V. S. and White, E. A. D., J. Crystal Growth, 33, 365 (1976).

[15] Nitsche, R. and Richman, D., Z. Elektrochem 66, 709 (1962).

[16] Richman, D., R. C. A. Rev. 24, 596 (1963).

[17] Kuniya, Y., Tanizawa, Y., and 0saka, M. H., Denki Kagaku 41, 108 (1973).

[18] Salki, M., J. Crystal Growth 36, 77 (1976).

[19] Vibrational Spectroscopy - Moderns Trends, A. J. Barns and W. F. Orville-Thomas, eds. p. 89 (Elsevier, 1977).

[20] Sedgwick, T. 0. and Smith, J. E., J. Electrochem. Soc. 123, 254 (1976).

[21] Sedgwick, T. 0., Smith, J. E., Ghez, R., and Cowher, M. E., J. Crystal Growth, 31, 264 (1976).

[22] Hille1, R., Bouix, J., and Favre, R., Bu11. Soc. Chim. (France), 11/12, 2458 (1975).

[23] Bouix, J. and Hillel, R., J. Less Common Met. 47, 67 (1976).

[24] Choukroun, S., Launay, J. C., Pouchard, M., Hagenmuller, P., Bouix, J., and Hillel, R., J. Crystal Growth, 43, 597 (1978).

[25] Bouix, J., Hillel, R., Vincent, H., and Montei1, Y., J. Thermal Analysis, 12, 371 (1977).

[26] Stock, A. and Von Bezold, H., Chem. Ber. 41, 657 (1908).

[27] Forthmann, R. and Schneider, A., Z. Physik. Chem. 49, 22 (1966).

[28] Kukushkina, E. A. et al., Zh. Fiz. Khim 48, 1885 (1974).

[29] Gardner, M., J. Chem. Soc. Dalton, 691 (1973).

[30] Grinberg, J. K., Boriakova, V. A., Chevel'kov, V. F., Hillel, R., and Bouix, J., Inorg. Nuclear Chemistry, 38, 383 (1976).

[31] Likformand, A., Carre, D., and Hi1le1, R., Acta Cryst. B14, 1 (1978).

[32] Bouix, J., Hillel, R., and Michaelides, A., J. Raman Spectroscopy, in press.

[33] Hillel, R., Bouix, J., and Michaelides, A., to be published.

[34] Smith, Jr., J. E. and Sedgwick, T. 0., Thin Solid Films, 40, 1 (1977). 


\section{Discussion}

Question (Berkowitz): In your indium selenide plus iodine studies, you had some $\mathrm{Se}_{\mathrm{n}}$ vapor species. And, there is some information about the distribution of the different kinds of vapor species at certain temperatures and pressures. I don't know how distinct they would be, that is whether one species would be dominant, but did you look for absorptions characteristic of these species?

And, can you provide perhaps some information about the vibrational frequency of one or another of these species?

Response (Bouix): No, we didn't obtain observations of this kind. But, for the thermodynamic calculation, of course, we introduced all of the different species of selenium, and in general the equilibrium constants are known and for us it was no problem for thermodynamic calculations.

But, about the structure of the gaseous selenium, I have no special information.

Question (Rosenblatt): Did you do your Raman spectroscopy with selenium in the vapor phase?

Response (Bouix): The vapor selenium, yes, of course.

Question (Rosenblatt): Did you have any trouble with fluorescence from the selenium?

Response (Bouix): The fluorescence of selenium is not a difficult problem--it is possible to record the spectra, but I tried with tellurium, and in that case the fluorescence is too intense and it's impossible.

But, with selenium we had some problems but not too much. It was possible to measure the spectra without disappearance of all the components. 


\section{SPECTROSCOPIC DIAGNOSTIC TECHNIQUES AND TEMPERATURE MEASUREMENT}



National Bureau of Standards Special Publication 561, Proceedings of the 10th Materials Research Symposium on Characterization of High Temperature Vapors and Gases held at NBS, Gaithersburg, Maryland, September 18-22, 1978. Issued October 1979.

\title{
DIAGNOSTIC TECHNIQUES IN COMBUSTION MHD FLOWS
}

\author{
Sidney A. Self \\ High Temperature Gasdynamics Laboratory \\ Standord University, CA 94305
}

\section{Introduction}

The U.S. national program in MHD electric power generation is predicated on the direct firing of pulverized coal, using MHD as a topping cycle for a regular steam plant. Extensive system studies [1] $]^{1}$ predict the thermal efficiency of a large combined MHD/Steam plant will be in the range 50-55 percent compared with 35-40 percent for a conventional steam plant.

The status of this technology is reported in a recent US-USSR joint report [2] and in the proceedings of the annual Symposia on Engineering Aspects of MHD Power Generation [3], and the International Conferences on MHD Electric Power Generation [4].

In the next section the major features of an MHD power generation system are briefly outlined, and the important problem areas identified. This is followed, in Section 3 , by a discussion of those aspects related primarily to the characterization of the combustion products plasma as they affect generator performance. In Section 4, the sub-scale MHD facility at Stanford is briefly described, and the principal areas of the past and ongoing research program outlined. A major focus of this work is understanding the detailed magnetoplasma mechanics of the generator channel flow. A vital component of the program is the development and use of advanced diagnostic techniques [5] for determining the plasma and flow properties inside an operating generator channel. These techniques are described in Section 5. It goes without saying that the extreme conditions and hostile environment pose a considerable challenge to most measurement methods.

\section{Combustion MHD Power Generation}

In combustion, or open-cycle, MHD electric power generation, the basic idea is to burn a fossil fuel at a very high temperature ( $3000 \mathrm{~K}$ ), seed it with an easily ionizable material (K, Cs) and expand the resulting combustion products plasma through a nozzle into a generator channel at high (usually subsonic) velocity $u \lesssim c_{s} \sim 1000 \mathrm{~m} / \mathrm{s}$. The generator channel is immersed in a strong transverse magnetic field $B$, and is fitted with electrodes in the walls for electric power take-off. The induced electric field in the plasma is

${ }^{1}$ Figures in brackets indicate the literature references at the end of this paper. 


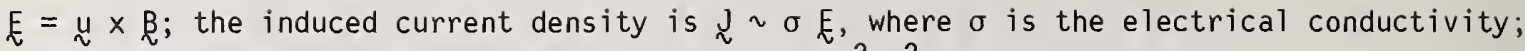
and the generated power density is $P \sim J \cdot E \sim \sigma u^{2} B^{2}$.

To be economically competitive, the power density should be of the order of $100 \mathrm{MW} / \mathrm{m}^{3}$. Since $u$ is fixed at $\lesssim 1000 \mathrm{~m} / \mathrm{s}$, and the practical limit of large scale superconducting magnets is $B \sim 5 T$, this leads to a requirement for a conductivity of $\sim 10 \mathrm{mho} / \mathrm{m}$, and corresponding fields and currents of $5 \mathrm{kV} / \mathrm{m}$ and $5 \times 10^{4} \mathrm{~A} / \mathrm{m}^{2}$.

The electrical conductivity $\sigma=e n_{e} \mu_{e}$ is determined by the product of the concentration $n_{e}$ of electrons and their mobility $\mu_{e}$ calculated as a weighted mean for the various neutral and ionized species. Since combustion plasmas, whether fired by gas, oil or coal, are essentially in thermal equilibrium (except perhaps in current concentrations near the electrodes), the electron concentration can be calculated from Saha equilibrium in terms of the concentration of easily ionizable seed [6]. Moreover, for typical combustion MHD conditions at a pressure of $\mathrm{p} \sim 1 \mathrm{Atm}$, the effective electron mobility is $\mu_{\mathrm{e}} \sim 0.5 \mathrm{~m}^{2} / \mathrm{V} \cdot \mathrm{s}$ and is a slow function of temperature. This leads to a requirement of electron concentration $\mathrm{n}_{\mathrm{e}} \sim 10^{20} \mathrm{~m}^{-3}$. At practical levels of seed concentration, e.g. $\sim 1$ percent by mass of $\mathrm{K}$, Saha's equation then leads to a requirement for temperatures in the range $2800-3000 \mathrm{~K}$ at the generator input.

To achieve fossil fuel combustion temperatures of this order requires a high degree of air preheat ( $\sim 1500-1800 \mathrm{~K}$ ) (oxygen enrichment is uneconomic on a large scale). This is provided by regenerative, pebble-bed type preheaters, heated by the exhaust from the MHD generator. The exhaust temperature, 2000-2200 $\mathrm{K}$, is determined by the fact that at this temperature the electrical conductivity has fallen to such a low value that further MHD power extraction is uneconomic.

The high combustion temperatures lead not only to formidable materials' problems for the walls of the flow train, but also to questions of $\mathrm{NO}_{x}$ formation, though the latter appear to be soluble [7]. Another important factor is that at the required potassium seed concentrations, it is economically necessary to recover the seed for re-use. In the case of sulfur bearing fuels, the potassium combines with the sulfur and must be recovered as potassium sulfate, which incidentally fulfills the necessary sulfur emission clean-up process. In the case of coal-firing, the potassium sulfate must be separated from the mineral ash particulates in the exhaust.

While a considerable body of experience has been accumulated with clean-fuel MHD, the additional problems associated with coal-firing have received less attention until recently, except for the abandoned British program [8]. The principal new problems relate to the presence of mineral ash in the pulverized coal--typically 10 percent by mass with principal constituents in order $\mathrm{SiO}_{2}, \mathrm{Al}_{2} \mathrm{O}_{3}, \mathrm{Fe}_{2} \mathrm{O}_{3}, \mathrm{CaO}, \mathrm{MgO}$, as well as $\mathrm{SO}_{3}$.

Since the combustion temperature is much higher than in conventional coal-fired plants, a higher fraction of the ash is vaporized, and the particulate ash droplets appear to be distributed over a smaller size range (perhaps $\sim 1 \mu \mathrm{m}$ instead of $10 \mu \mathrm{m}$ mass mean diameter). It is anticipated that a multi-stage cyclone combustor will be used to remove a large fraction of the ash, but still, a significant fraction ( 10 percent) of the mineral matter will be carried over into the generator in the form of vapor and particulates. This has two 
important implications: first, as regards the effect on the plasma conductivity in the volume of the channel; and second, with regard to the operation of the generator channel with slagging walls.

The characterization of the combustion products plasma, as it affects generator performance is discussed in the next section. Some of the problems related to the operation of generator channels with slagging walls may be briefly mentioned here.

The first question concerns the achievement of a thin, stable slag layer coating the channel surfaces. Studies [9] of the equilibrium mechanics of the slag layer show that it is maintained by a balance of condensation/evaporation of mineral vapor, by ash droplet deposition through turbulent diffusion across the boundary layer, and by surface run-off. The strong temperature dependence of the slag viscosity and thermal conductivity, results in the buildup of a solid layer with a front surface, in contact with the gas, which is liquid at $T \sim 1600 \mathrm{~K}$, and exhibits rippled flow. The slag layer thickness depends primarily on the substrate temperature, and the layer can be regarded as a thermal barrier which protects the underlying electrode and insulator substrate from corrosive effects of the high temperature combustion gas.

The mechanism of current transfer between electrodes and plasma through the slag layer is extremely complex and the subject of active research [10]. It appears that, depending on conditions such as slag thickness and average current density, current transfer may be diffuse or in concentrated arc spots. Depending on ash composition, current transfer through the slag layer is more or less ionic in character, and is accompanied by plating out of negative oxygen ions at the anode and potassium ions at the cathode. Research on electrode and insulator materials which can resist the resulting electrochemical attack for adequate operational lifetimes is a critical problem area.

Another critical problem related to the channel surface concerns the control of electrical breakdown across the insulators separating adjacent electrodes, due to the induced Hall field [11].

\section{Characterization of Combustion Products Plasma Flow}

In this section we focus attention on the properties of the combustion products plasma flow as they affect performance in the MHD generator channel.

Given the input flow rates and temperatures of fuel, oxidant and seed material into the combustor, and knowing the combustor heat losses, the enthalpy, temperature and composition of the resulting plasma can be calculated from an equilibrium properties computer code [7, 12] if all the relevant thermochemical data are known [13]. For clean hydrocarbon fuels this is relatively straightforward. For example, the composition of the combustion products resulting from the stoichiometric combustion of ethanol in oxygen-enriched air (which we commonly use) seeded to $\sim 1$ percent $\mathrm{K}$ (as $\mathrm{KOH}$ ) at $\mathrm{T}=2800 \mathrm{~K}$ and atmospheric pressure is shown in table 1 . 


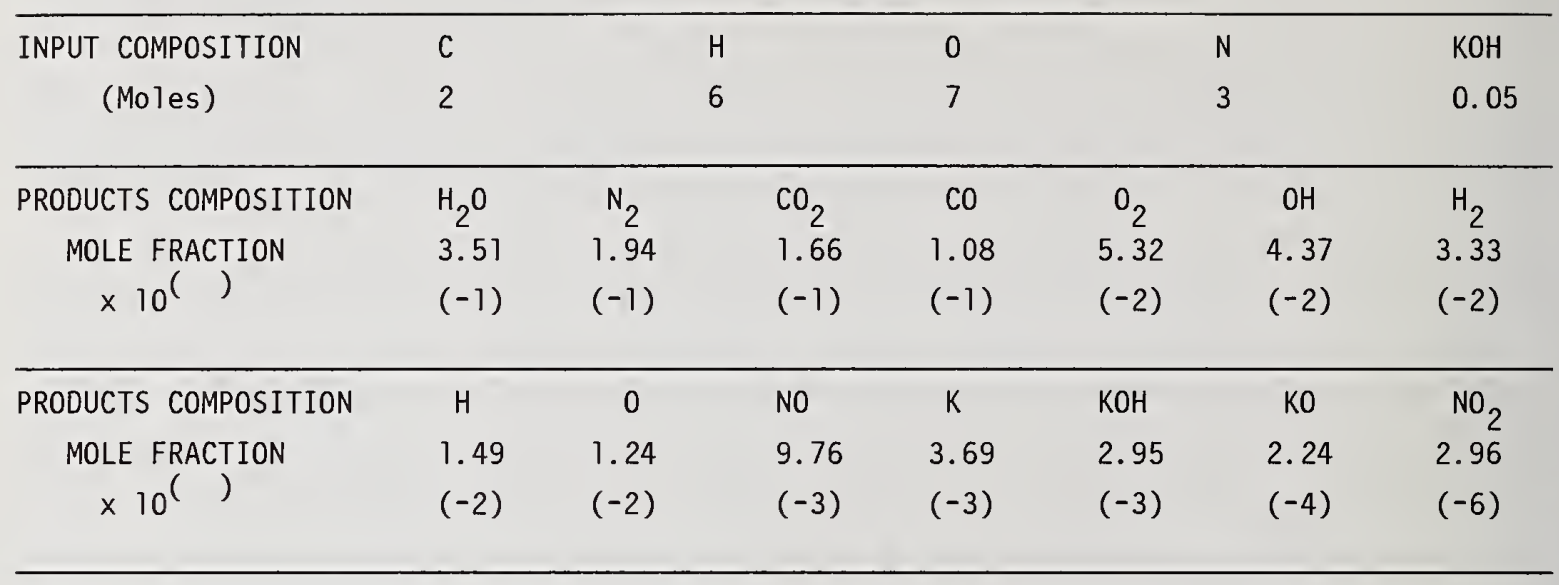

Several facts are apparent from the table. Most of the $\mathrm{H}$ is oxidized to $\mathrm{H}_{2} \mathrm{O}$, but small percentages remain as $\mathrm{OH}, \mathrm{H}_{2}$, and $\mathrm{H}$. Of the carbon, some 60 percent is oxidized to $\mathrm{CO}_{2}$, while 40 percent remains as $\mathrm{CO}$. A majority of the $\mathrm{KOH}$ is dissociated to $\mathrm{K}$, with a significant fraction occurring as $\mathrm{K} 0$. Also, the plasma contains approximately 1 percent mole fraction of $\mathrm{NO}$, and a few ppm of $\mathrm{NO}_{2}$.

The concentrations of free electrons $n_{e}$, and of singly ionized $K^{+}$atoms $\left(n_{K}+\approx n_{e}\right)$ are also calculated. They are essentially given in terms of the $K$ concentration, as a balance between thermal ionization and three-body ( $\left.e^{-} e^{-i}\right)$ recombination, by the Saha relation [6].

$$
\frac{n_{e}^{2}}{n_{k}} \approx \frac{n_{e} n_{K^{+}}}{n_{k}}=2 \frac{z_{K^{+}}}{z_{K}}\left(\frac{2 \pi m_{e} k T}{n^{2}}\right)^{3 / 2} \exp -\left(\varepsilon_{K}+/ k T\right) .
$$

Thus, $n_{e}$ is proportional to $n_{K}^{1 / 2}$ and is a strong function of temperature $\left(T / n_{e}\right)\left(d n_{e} / d T\right) \sim 10$. With attaching species present, the $\mathrm{K}^{+}$positive space charge is balanced by a combination of electrons and negative ions, with the result that $n_{e}$ (and $\sigma$ ) is reduced. To calculate this reduction requires accurate data for the electron affinities of the relevant negative ions, including $\mathrm{OH}^{-}, \mathrm{O}^{-}, \mathrm{NO}^{-}$and possibly $\mathrm{K}^{-}, \mathrm{KO}^{-}$and $\mathrm{H}_{2} \mathrm{O}^{-}$(for which no estimates are available).

The net electron mobility is determined by the weighted sum of the electron mobilities for all the heavy species, combined according to Blanc's law. These mobilities, or equivalently the average cross-sections for momentum transfer are generally available [14] from quantum mechanical calculations or from electron beam or swarm experiments. Two species that contribute significantly to the mobility are the polar molecules $\mathrm{KOH}$ and $\mathrm{H}_{2} \mathrm{O}$ for which no very reliable data are available.

Table 2 gives the electron concentration, mobility and scalar electrical conductivity for the same plasma considered in table 1, but as a function of temperature over the range 
2800-2000 K. This might correspond to the changing conditions along the length of the MHD generator channel. The $n_{e}$ values include corrections for attachment, which results in a reduction of $\sim 12$ percent. The strong dependence of $n_{e}$ and $\sigma$ on temperature are apparent in table 2.

Table 2.

\begin{tabular}{llcc}
\hline$T(K)$ & $n_{\mathrm{e}}\left(\mathrm{m}^{-3}\right)$ & $\mu_{\mathrm{e}}\left(\mathrm{m}^{2} / \mathrm{V} \cdot \mathrm{s}\right)$ & $\sigma(\mathrm{mho} / \mathrm{m})$ \\
\hline 2800 & $2.09\left(\times 10^{20}\right)$ & 0.449 & 15.06 \\
2600 & $0.901\left(\times 10^{20}\right)$ & 0.402 & 5.81 \\
2400 & $0.324\left(\times 10^{20}\right)$ & 0.355 & 1.84 \\
2200 & $0.093\left(\times 10^{20}\right)$ & 0.311 & 0.462 \\
2000 & $0.020\left(\times 10^{20}\right)$ & 0.272 & 0.087 \\
\hline
\end{tabular}

For "clean" fuels, for which most MHD experience has hitherto been accumulated, it is generally believed that the necessary molecular data are probably well enough known that $\sigma$ can be calculated with a confidence of perhaps \pm 20 percent. It should be added, however, that few reliable measurements of $\sigma, n_{e}$ or $\mu_{e}$ for precisely documented conditions have been reported to substantiate this belief, owing to the difficulty of making such measurements in combustion MHD flows.

For coal-fired plasmas the situation is much less satisfactory. The mineral ash content leads to the presence of various negative ions whose effect in depressing the electron concentration cannot be calculated with confidence due to lack of reliable attachment data. Calculations accounting for $\mathrm{ArO}_{2}{ }^{-}, \mathrm{BO}_{2}{ }^{-}, \mathrm{PO}_{2}{ }^{-}, \mathrm{SO}_{2}{ }^{-}$, and $\mathrm{Cl}^{-}$on the basis of the "best" estimates give reductions of $\sim 20$ percent [15]. The contributions of ash vapor species to the mobility are likewise difficult to estimate because the relevant collision cross-sections are unknown, but are thought to be small because of the low concentrations.

In addition, the electron concentration (and hence the conductivity) may be reduced by recombination on ash droplets in the flow, which depends primarily on the surface area available. At the exit of the combustor, measurements [16] indicate droplet diameters in the range of $1 \mu \mathrm{m}$, and at ash loadings of interest (e.g. with 10 percent ash carry-over from the combustor), the specific surface area is probably too small to have a significant effect. However, calculations [17] indicate that as the temperature falls through the generator, the ash vapors can become supersaturated and undergo homogeneous nucleation, which could cause a significant reduction in $n_{e}$ through electron-ion recombination on the sub-micron aerosol surface. The possible significance of this process has yet to be firmly established.

The performance of an MHD generator depends not onty on the characterization of the plasma as regards to species concentrations and electrical properties, as described above, but also on the fluid mechanics and heat transfer associated with the turbulent channel 
flow. The latter determine the spatial distribution of temperature in the channel, and hence, the distribution of composition and electrical properties.

Turbulent channel flow computer codes have been developed $[18,19]$ which calculate the flow and temperature field by matching a two-dimensional boundary layer model to the core flow, and include the electromagnetic interaction terms. The latter comprise the $\underset{\sim}{J} \times \underset{\sim}{B}$ force term in the momentum conservation equation and the $\underset{\sim}{J} \cdot \underset{\sim}{E}$ joule dissipation term in the energy conservation equation. The inclusion of these terms can result in considerable modification of the ordinary gasdynamic flow excluding electromagnetic interaction, and produce significant changes in the wall friction and heat transfer coefficients.

The turbulent boundary layers can have a profound effect on MHD generator performance because the wall temperature is necessarily appreciably lower than the core plasma temperature. The resulting thermal boundary layer has a lower electrical conductivity and can give rise to significant internal resistance (or voltage drop) in the generator. Consequently, detailed understanding of the boundary layers, on both the electrode wall [18] and insulating sidewall [19] is very important in calculating generator performance.

Finally, in this section, mention should be made of the significance of temporal fluctuations of conductivity on the performance of MHD generators. Calculations indicate that such fluctuations result in an effective conductivity which is lower than the timeaverage value and results in a degradation of generator performance. The effect is stronger the higher the fluctuation amplitude and increases with magnetic field strength. Such fluctuations may be induced by combustion instabilities or possibly from inherent magnetoplasma instabilities of the magneto-acoustic type. Thus, the study of fluctuations of pressure, temperature and electrical conductivity in MHD generator channels [20] is of vital concern to the evaluation of MHD power generation.

\section{Stanford MHD Research Facility}

The High Temperature Gasdynamics Laboratory's MHD facility is a sub-scale system designed for flexibility in research into the basic physics of MHD phenomena rather than the demonstration or development of efficient power generation systems. The latter can only be effectively carried out in facilities of pilot plant scale, say $100 \mathrm{Mw}$ (thermal), because of the inevitable effects of unfavorable surface to volume ratio in sub-scale systems.

The present hardware centers around either of two combustors, the M-2 system rated at $2 \mathrm{Mw}$ (th) and the $M-8$ system rated at $8 \mathrm{Mw}$ (th). These are liquid fuel spray combustors, stabilized by counter swirling injection of oxygen and nitrogen (used as a diluent to control the temperature). Simulation of coal-firing is achieved by admixture of a pulverized coal slurry to the fuel--either ethanol or JP4. Potassium seed, up to $\sim 2$ percent by mass of $\mathrm{K}$, is conveniently added by dissolving $\mathrm{KOH}$ in ethanol fuel.

The combustor normally feeds into a MgO brick-lined plenum chamber to provide adequate residence time for mixing and to reduce fluctuations of plasma properties. The exit of the plenum is fitted with a nozzle to accelerate the flow into various experimental channel sections which are constructed to suit the purpose of particular experimental studies. 
Sometimes, especially for preliminary development of diagnostic techniques, use is made of the free jet issuing from the nozzle.

The channels are usually constructed in a water-cooled metal body lined with insulators and electrodes, depending on the configuration required. The insulators are normally of MgO firebrick (occasionally dense $\mathrm{Al}_{2} \mathrm{O}_{3}$ ) and the electrodes are stainless steel and watercooled to run at a surface temperature $\sim 1200 \mathrm{~K}$. Below this temperature seed material $\left(\mathrm{K}_{2} \mathrm{CO}_{3}\right)$ condenses on the surface, while much higher temperatures result in corrosion. With coal-firing, the walls are usually of water-cooled metal, grooved and filled with castable ceramic to ensure attachment of the slag layer, which does not adequately wet plain metal surfaces.

The channels are normally sized to fit the available electromagnet. This is a conventional iron yoke, water-cooled copper magnet producing fields up to 2.7 Tesla (27 k Gauss) in a working volume between pole-pieces which allow channel internal dimensions of $5 \mathrm{~cm}$ wide (B field direction) $\times 10 \mathrm{~cm}$ high $\times 70 \mathrm{~cm}$ long.

The channel exit attaches to a diffuser and exhaust section which includes a water spray quench and scrubber system for removing particulates.

Storage capacity of fuel, oxygen and nitrogen are sufficient to allow tests of several hours duration. Most tests are run under subsonic conditions ( $<1000 \mathrm{~m} / \mathrm{s}$ ); but occasionally supersonic tests have been run. Total mass flow rates may range up to $1 \mathrm{lb} / \mathrm{s}$.

In addition, a small superconducting magnet capable of $6 \mathrm{~T}$ is available, and for the future a large superconducting magnet with a field of $7 \mathrm{~T}$, a bore of $55 \mathrm{~cm}$, and a working length of $1.5 \mathrm{~m}$ will be available.

Metering of fuel, oxygen, nitrogen, and potassium seed is routinely measured, as are wall temperatures and pressures. The outputs from the various transducers are coupled into a minicomputer for on-line display. A patch panel allows the electrodes to be connected to appropriate loads in a variety of configurations, and currents and voltages are recorded via the minicomputer. To enhance the currents and MHD interaction to simulate full scale generator operation, the electrodes are often powered from a battery pack.

A great variety of diagnostic techniques have been developed and used to measure the properties of the plasma channel flow and to study slag layer dynamics and electrode current transfer and breakdown effects. These are described in the next section.

A major problem with any diagnostic technique is set by the difficulty of access and the hostility of the environment. For optical diagnostics, ports and windows are normally shuttered and purged with dry $\mathrm{N}_{2}$ to prevent condensation of water and potassium carbonate (dew point $\sim 1000^{\circ} \mathrm{C}$ ). The bulk of the surrounding magnet particularly makes for difficult access problems. In addition, the stray field precludes the use of photomultipliers, oscilloscopes, etc. in close proximity to the rig.

\section{Diagnostic Techniques}

As noted above, a major component of the Stanford program in MHD research is the development and use of diagnostic techniques for characterizing the plasma flow inside an 
operating generator, with the purpose of studying the basic physical processes, and providing engineering data useful in the design of large scale systems.

Both the plasma properties, especially the electrical conductivity, and the flow properties are important in determining generator behavior, and the boundary layers, in particular, are of interest since they play an important role in determining generator performance. This places a premium on measurement techniques capable of high spatial resolution. Moreover, as noted earlier, fluctuations also have an important effect on generator performance, so that temporal resolution, (to $\sim 1-10 \mathrm{kHz}$ ) is also highly desirable.

A considerable variety of techniques are available for measuring the various quantities of interest, which include velocity (including turbulence characterization), temperature, electrical conductivity, electron concentration, electron mobility, and seed $(K)$ concentration. In addition, for coal-fired flows, the ash loading and particulate size distribution is important, as is the thickness and surface temperature of the surface slag layers. The majority of the techniques are non-intrusive optical ones, though some material probe methods are valuable, in spite of the fact that they must be either heavily cooled or restricted to very short insertion times.

An extensive review of the status of diagnostic techniques for combustion MHD flows, as of two years ago, was given in reference [5]. A more recent review, including Soviet contributions in this area, appears in Chapter 14 of reference [2]. In the following, the status of the various techniques will be summarized according to the quantity measured; for further details the cited references should be consulted.

\section{1 Velocity}

Boundary layer profiles of average streamwise velocity and turbulence intensity have been measured using laser anemometry $[21,22]$ in two distinct configurations, the configurations being determined primarily by the access possibilities. In laser anemometry, the velocity is determined from the Doppler frequency shift of light scattered by small particles entrained in the flow, as they traverse the measurement volume, defined by the intersection of a laser beam focused into the flow and the axis of the collection optics.

In the first configuration used to measure the electrode wa11 boundary layer [18], the only possibility for access was from a port in the downstream channel sidewall looking upstream into the magnet, as shown in figure 1. This single-beam backscatter configuration, aligned at a small angle to the flow axis, combined with the high velocities, results in a Doppler frequency shift (several $\mathrm{GHz}$ ) which is too high to measure by heterodyne techniques. Consequently, it was measured as a wavelength shift using a scanning Fabry-Perot interferometer. In this system, and in that described below, micron-sized $\mathrm{ZrO}_{2}$ powder was added to the flow to act as scattering centers. 


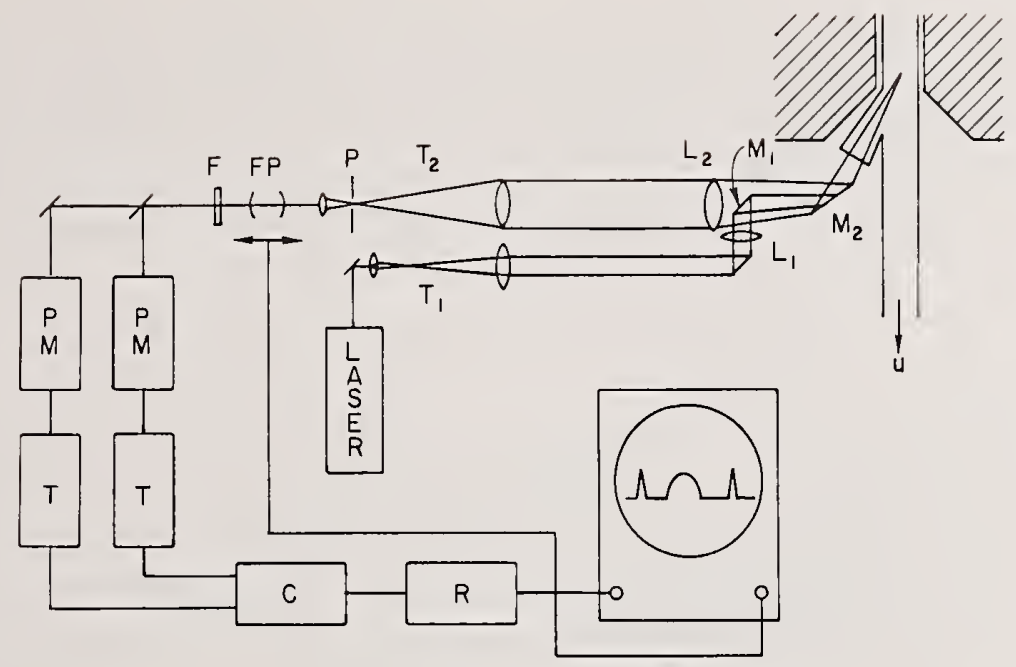

Figure 1. Single beam backscatter anemometer for electrode wall boundary layer.

Boundary layer profiles with a spatial resolution of $\sim 0.1 \mathrm{~mm}$ were made to within $\sim 0.3 \mathrm{~mm}$ of the electrode surface, at both subsonic and supersonic core velocities. An example of a measured profile at a free stream velocity of $1600 \mathrm{~m} / \mathrm{s}$ is shown in figure 2 .

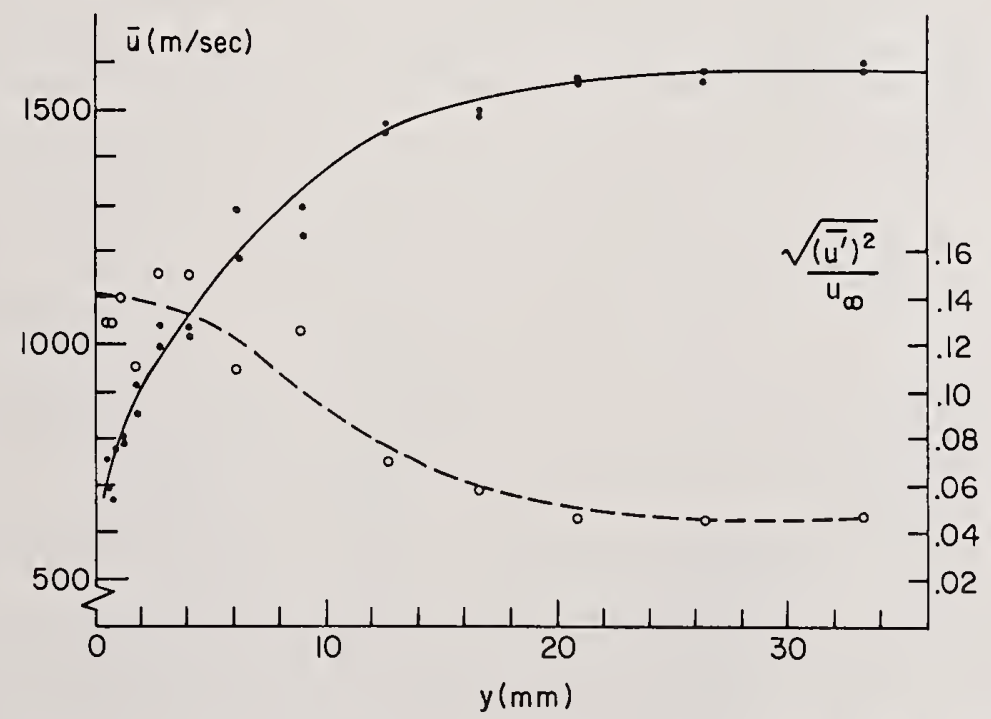

Figure 2. Electrode wall boundary-layer velocity profiles from single beam anemometer. Mean velocity--solid line and dots; turbulence intensity-broken line and circles.

In the second configuration, used to measure the insulating sidewall boundary layer [19], access was possible between the magnet pole pieces through the top and bottom electrode walls. This allowed the use of the more conventional dual beam, real fringe technique 
in (near) forward scatter, as shown in figure 3, which shows how access and scanning of the measurement point was achieved using a series of mirrors. Figure 4 compares the measured and computed profiles of average velocity with and without electromagnetic interaction. The marked change in profile is due to the fact that the $\mathrm{J} \times \boldsymbol{\beta}$ braking force is strongest in the core flow, because the current is concentrated there due to the lower conductivity in the sidewall thermal boundary layer. This results in significant changes in the friction and heat transfer coefficients. A reduction of turbulence intensity was observed in the presence of magnetic field due to magnetic damping of turbulence.

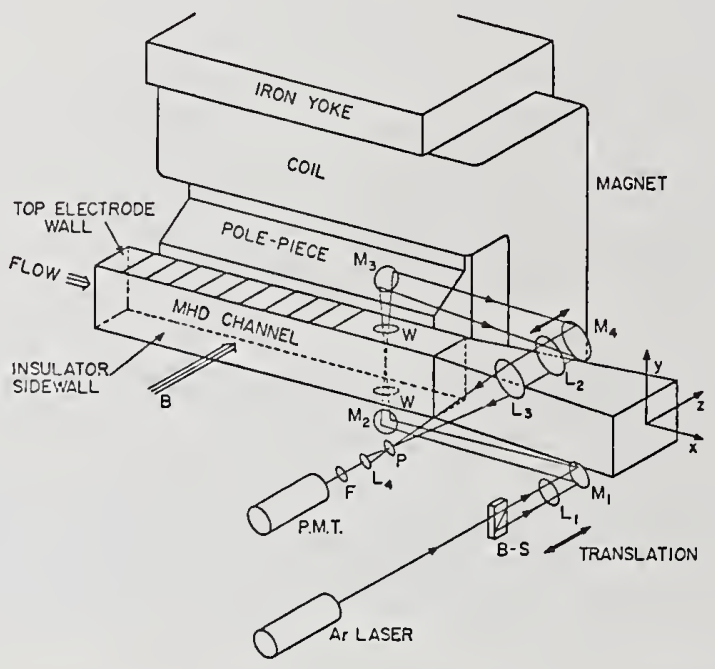

Figure 3. Dual beam forward scatter anemometer for sidewall boundary layer.

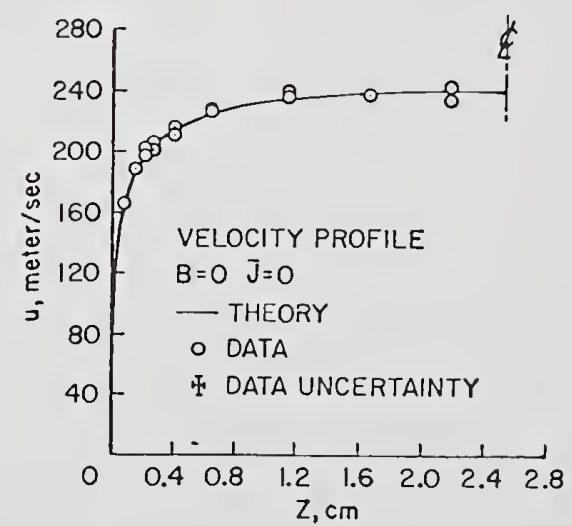

(a)

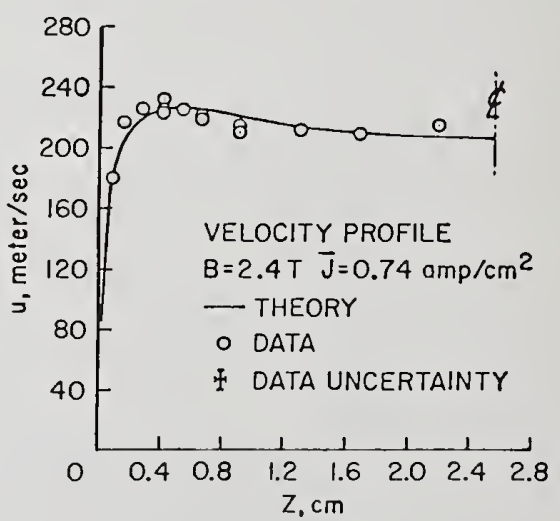

(b)

Figure 4. Sidewall boundary layer profiles of average streamwise velocity:

(a) without MHD interaction, (b) with MHD interaction.

Further details of both laser anemometer systems is given in reference [23]. 


\subsection{Temperature}

The gas temperature is among the most critical parameters for operation of MHD generators. Since, for subsonic operation, thermochemical equilibrium prevails, except in near electrode arcs, the temperature is a measure of the enthalpy and composition of the gas and, to the extent that the seed atom concentration is known, of the electron concentration and electrical conductivity.

\subsubsection{Resonance line reversal method}

The old-established resonance line reversal method, which yields a line-of-sight average temperature, has been adapted to measure boundary layer temperature profiles in MHD channels $[18,24]$. Modification of the standard reversal procedure is necessary for two reasons. First, because the plasma temperature is higher than the temperature available from standard tungsten ribbon lamps, the true reversal condition cannot be attained, and some sort of extrapolation technique is necessary. Second, under typical conditions using small concentrations of sodium seed, the temperature and seed concentration gradients in the boundary layers along the line of sight, result in the center of the emission line being self-reversed. To overcome this problem, measurements are made in the line wing where the optical depth is $\lesssim 0.5$, and the radiative transfer equation [25] is solved, using an assumed boundary layer temperature profile to obtain the core temperature [26]. The effects of fluctuations and non-uniformities have also been taken into account [27]. Figure 5 shows a schematic of the optical system used to make electrode wall boundary layer profile measurements with a spatial resolution of $0.3 \mathrm{~mm}$ to within $0.4 \mathrm{~mm}$ of the electrode. Figure 6 compares experimental and theoretical profiles with and without current drawn to the electrode. The temperature rise due to Joule heating is clearly evident.

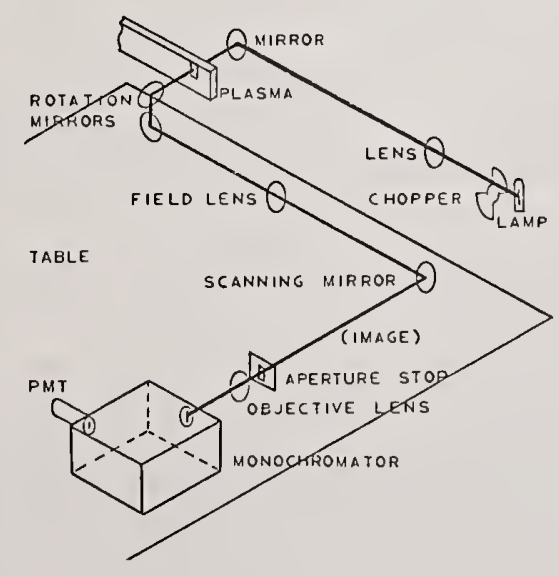

Figure 5. Optical system for spectroscopic measurements of temperature and electron concentration.

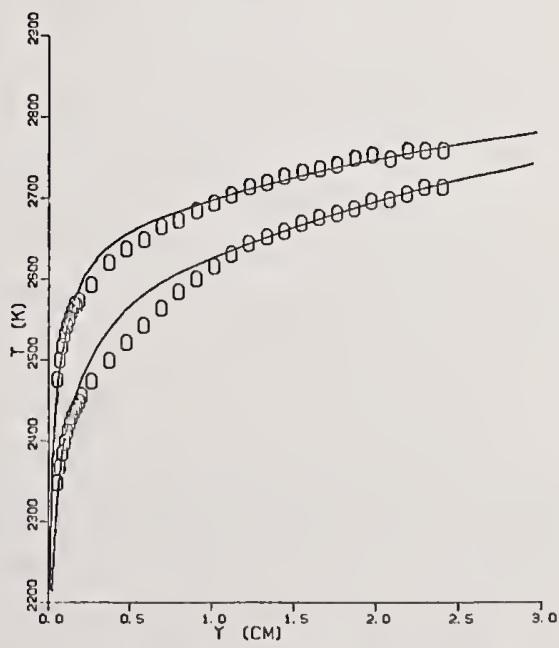

Figure 6. Comparison of measured and computed electrode wall boundary layer temperature profiles: lower curve $\mathrm{J}=0$; upper curve $\mathrm{J}=1.54 \mathrm{~A} / \mathrm{cm}^{2}$. $\quad(u \sim 350 \mathrm{~m} / \mathrm{s})$. 
Soviet workers have developed an automatic line reversal temperature measuring system [28] for use on the pilot scale U-25 MHD system at the High Temperature Institute in Moscow, which has a channel width of $\sim 1 \mathrm{~m}$. By chopping techniques, signals from the lamp alone, the plasma alone, and the lamp plus plasma are continuously recorded, and the temperature is automatically calculated with a response time of $\sim 30 \mathrm{~ms}$.

\subsubsection{Laser fluorescence technique}

As an alternative to line-of-sight emission/absorption techniques, a "point" measurement technique has recently been developed at Stanford $[29,30]$ using laser fluorescence. A tunable dye laser is used to pump transitions from an excited electronic level of $\mathrm{Na}$ or $\mathrm{K}$ to a higher level. Since the population of the absorbing level is exponentially dependent on temperature, the resulting fluorescence re-radiation is a sensitive function of relative temperature and temperature fluctuations. Observations of this signal along a path orthogonal to the exciting beam provides a spatial resolution of $\sim 1 \mathrm{~mm}^{3}$. Spatially resolved measurements, relative temperature profiles and temperature fluctuations, as well as two point fluctuation correlations, should be feasible in MHD plasma using this technique.

\subsection{Electrical conductivity}

There is no known non-intrusive technique for directly measuring the electrical conductivity. However, four-wire conductive probes have been used at Stanford. While reliable measurements can be made in seeded Argon flows from an arc-jet [31], their use in combustion MHD flows poses serious problems due to seed condensation, probe heating and electrode corrosion [32].

Soviet workers have developed rf inductive probes [33] which avoid such problems by enclosing the coils in a protective dielectric housing of airfoil shape. The change in $Q$ of the coil due to eddy currents induced in the surrounding plasma as the probe is rapidly inserted and withdrawn from the plasma, is used to measure the plasma conductivity. The probe is calibrated by immersion in conducting salt solutions. Corrections must be applied for the conductivity profile in the boundary layer surrounding the probe, and the absolute accuracy is probably no better than \pm 50 percent.

Vendell has used a two-coil inductive probe [34] in combustion MHD flows at Stanford.

\subsection{Electron concentration}

Methods for the measurement of electron concentration that have found use in combustion MHD flows include electric (Langmuir) probes, a spectroscopic line intensity method, and submillimeter laser interferometry. 


\subsubsection{Electric (Langmuir) probes}

The use of Langmuir probes in $\mathrm{fl}$ ames and MHD-type plasmas has been reviewed in reference [35]. At Stanford, the utility of such probes for electron concentration measurements in combustion MHD flows was demonstrated by Clements [36]. The appropriate theory [37] predicts the ion current collected by a negatively biased spherical probe, under conditions that the sheath thickness is larger than the gasdynamic boundary layer, but smaller than the probe radius, as (MKS units):

$$
I \approx 12\left(\mu_{i} \varepsilon_{0}\right)^{1 / 4}\left(n_{e} \text { e } u\right)^{3 / 4} v^{1 / 2} r_{p}^{5 / 4}
$$

Here $\mu_{i}$ is the ionic mobility, $\varepsilon_{0}$ the vacuum permittivity, e the electron charge, $u$ the gas velocity, $V$ the probe potential and $r_{p}$ the probe radius. The probe actually measures the ion flux $\left(n_{e} u\right)$ and separate knowledge of $u$ is required to determine $n_{e}$.

The practical problems caused by probe heating due to the high temperatures and velocities is overcome by using very short residence times (< $100 \mathrm{~ms}$ ). An incidental problem resulting from the use of a cold probe is the deposition of potassium carbonate on the surface. However, this is very soluble and can be washed off between insertions. The typical probe construction is shown in figure 7 , where stainless steel balls in the range 3-6 mm diameter have been used. Recently probe measurements have been made [38] inside a combustion MHD channel using a fast reciprocating probe mechanism driven by an air cylinder as shown in figure 8 . While the absolute accuracy of the probe is not high ( \pm 50 percent), it is simple and inexpensive, and gives good spatial and temporal resolution for relative measurements.

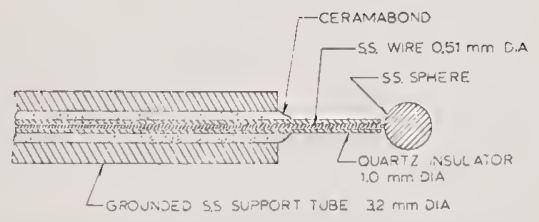

Figure 7. Spherical Langmuir probe: construction details.

\subsubsection{Spectroscopic method}

A method for measuring the electron concentration has been developed $[18,24,30]$ using the absolute emission intensity of high lying electronic levels of potassium seed atoms. The measurement yields line of sight averages which must be unfolded to yield core values, and while the absolute accuracy is not high the technique offers high spatial resolution ( $(\sim 0.3 \mathrm{~mm})$ for boundary layer measurements. The apparatus is essentially the same as that used for line-reversal temperature measurements (fig. 5). A feature of the technique is that the high lying electronic levels should be in thermal equilibrium with the electron temperature, even though the lower levels may not be. This allows it to be used to explore 
non-equilibrium effects in MHD boundary layers. Figure 9 compares measured $n_{e}$ profiles with those calculated and with those deduced from Saha equilibrium using the measured temperature. Within $2 \mathrm{~mm}$ of the surface a small electron concentration non-equilibrium is suggested, but the increase of $n_{e}$ relative to equilibrium is considerably less than that predicted by kinetic considerations using conventional three-body electron recombination coefficients.

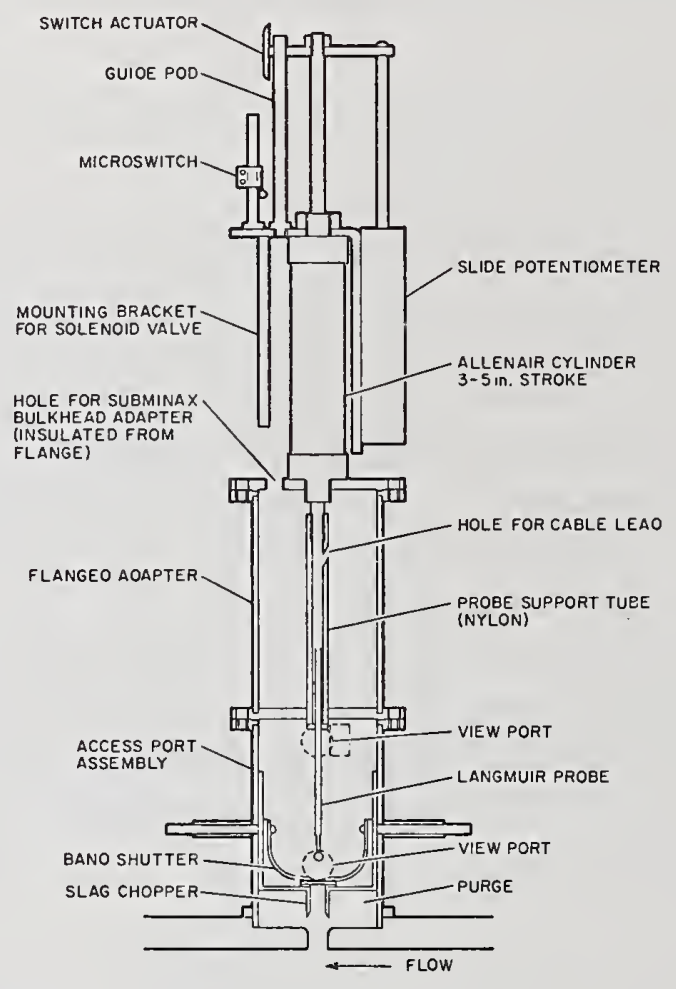

Figure 8. Probe access port and sweep mechanism.

\subsubsection{Submillimeter laser interferometry}

The utility of submillimeter (far infrared) laser wave propagation as a non-intrusive technique for accurate measurements of electron concentration, $n_{e}$, and mobility, $\mu_{e}$, in combustion MHD flows has been established by preliminary measurements $[39,40]$. A laser operating at $394 \mu \mathrm{m}$ and $496 \mu \mathrm{m}$, optically pumped by a $\mathrm{CO}_{2}$ laser, was used with a Michelson interferometer (figure 10) to measure the contribution to the refractive index by free electrons [41] from the phase shift observed with the addition of potassium seed to the free jet from the M-8 combustor. The mobiiity was obtained from a measurement of the absorption of the submillimeter radiation by the plasma. From the separate measurements the electrical conductivity $\sigma=e n_{e} \mu_{e}$ is given directly. 


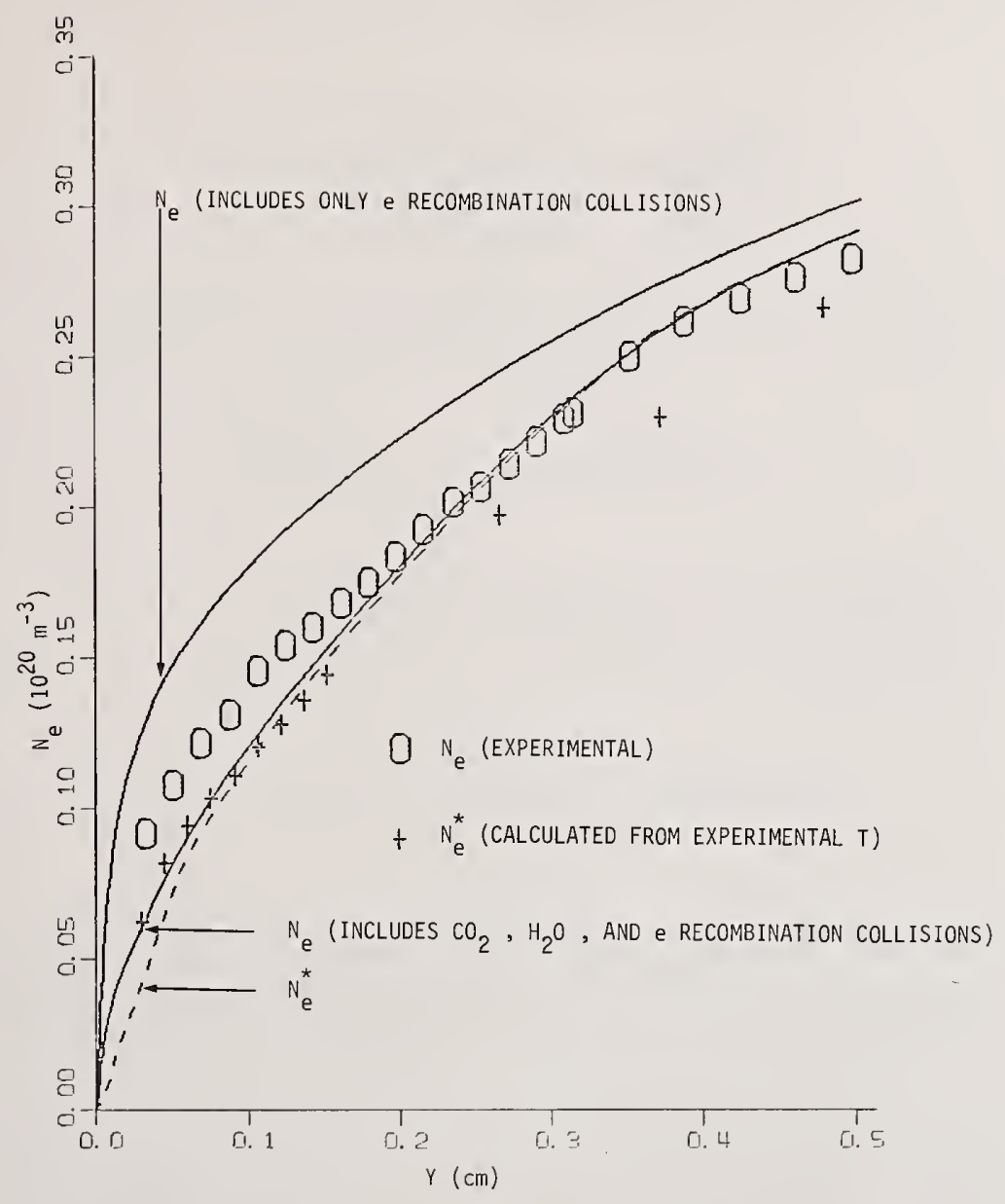

Figure 9. Comparison of experimental and calculated profiles of $\mathrm{n}_{e}$ in the electrode wall boundary layer.

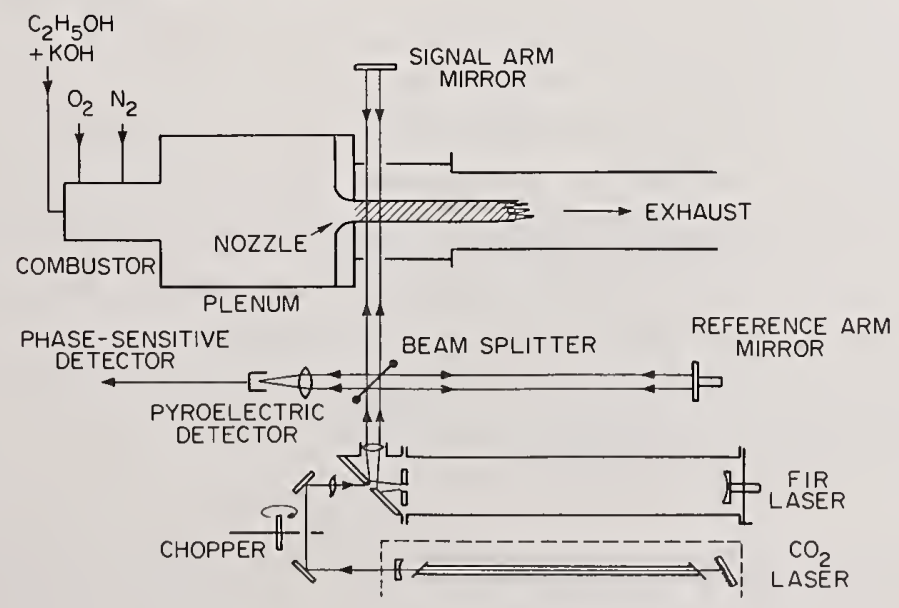

Figure 10. Schematic of submillimeter laser interferometer system. 
For a Michelson interferometer the number of fringe shifts $\mathrm{N}$ is given by

$$
N=\frac{1}{n_{c} \lambda} \int_{0}^{L} n_{e} d x
$$

where $L$ is the plasma width, $\lambda$ is the free-space wavelength and (MKS units)

$$
n_{c}=\frac{\varepsilon_{0} m_{e} c^{2}}{4 \pi^{2} e^{2} \lambda^{2}} \approx \frac{1.12 \times 10^{15}}{\lambda^{2}}
$$

is the critical density above which free propagation ceases.

The double pass transmission is given by

$$
\operatorname{Tr}=\exp -\frac{2}{n_{c} c} \int_{0}^{L} n_{e} v d x
$$

where $\nu$ is the effective electron collision frequency for momentum transfer $\left(\mu_{e}=e / m_{e} \nu\right)$.

The choice of working frequency is set by the requirement that the number of fringe shifts should be neither too large nor too small, and that the attenuation be not too large. For typical combustion MHD plasma conditions $\mathrm{n}_{\mathrm{e}} \sim 10^{20} \mathrm{~m}^{-3}, \nu \sim 3 \times 10^{11} \mathrm{~s}^{-1}$, and for small installations this leads to a requirement for submillimeter waves. For instance for $L=$ $50 \mathrm{~mm}$ and $\lambda=0.5 \mathrm{~mm}$, we find $\mathrm{N} \approx 2$ and $T r \approx 0.14$. In practice the shift can be determined to $\sim 1 / 50$ fringe, leading to an accuracy of $\sim 1$ percent in the determination on $\bar{n}_{e}$. For larger systems the use of shorter wavelengths is indicated to maintain reasonable transmission.

Figure 11 shows measured $n_{e}$ values plotted against $\sqrt{K}$, where $K$ is the input potassium seed concentration to the combustor. The broken lines show the equilibrium $n_{e}$ values calculated from the equilibrium properties program for three temperatures at intervals of $20 \mathrm{~K}$. Simultaneous measurements were made of the temperature by line reversal and the temperature was also calculated from the metered combustor inputs and measured heat losses. The measured $n_{e}$ values agreed with those calculated from equilibrium for both the measured and calculated temperatures all to within their respective estimated uncertainties. Because the electron concentration is such a strong function of temperature it is preferable to deduce $T$ from a measurement of $n_{e}$ than vice versa. 


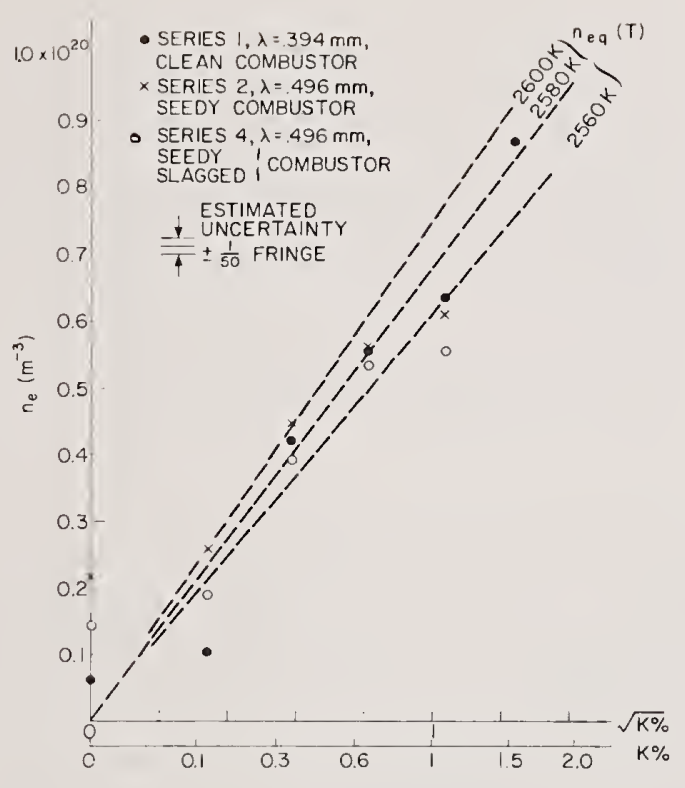

Figure 11. Measurements of electron concentration in free jet by submillimeter laser interferometer versus square root of potassium seed concentration: broken lines show values computed from equilibrium for temperatures shown.

Measured values of the electron mobility versus $\sqrt{K}$ are shown in figure 12 , where they are compared with those calculated from the best estimates of collision cross-sections for the species present. It is anticipated that more accurate measurements will be possible with refinement of the technique.

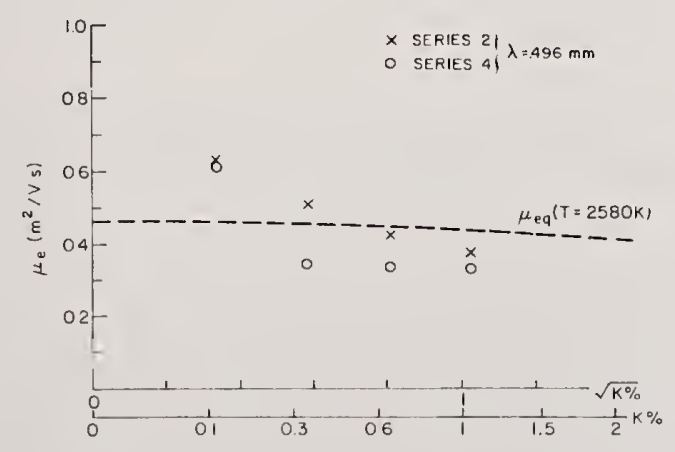

Figure 12. Mobility measured by submillimeter wave attenuation compared with theoretical estimate. 
A considerable advantage of this technique is that it should be unaffected by the presence of ash droplets, since the Rayleigh scattering should be negligible at these long wavelengths. This should be particularly valuable on large coal-fired systems which are optically thick due to scattering and thermal radiation at visible wavelengths.

The technique also appears to be useful for evaluating the effects of electron attachment and the measurement of collision frequencies for ash vapor species under MHD conditions. By introducing species of interest one at a time into a seeded argon arc jet flow, the relevant molecular data may be obtained directly under MHD conditions.

\subsection{Potassium seed concentration}

Although, in principle, the concentration of potassium (or other) seed atoms can be calculated from thermochemical equilibrium if the temperature and seed input rate to the combustor is known, in practice it is useful to be able to measure the concentration directly because of difficulties of controlling and metering the input (especially in dry power form) and the effects of condensation, absorption and desorption on the flow train surfaces, especially ceramic ones. The concentration of alkali seed atoms has been measured in MHD flows by Soviet workers [42] from the radiative broadening of resonance emission lines. Spectral line absorption techniques on suitable higher energy transitions from the ground state might also offer a useful technique for this measurement.

\subsection{Particulates}

In coal-fired MHD flows it is important to know the ash droplet loading and size distribution. Assuming a cyclone type combustor is used to reject $\sim 90$ percent of the ash, the mass fractional loading at the input to the generator channel may be of the order of 1 percent or less. In view of the higher combustion temperature and rejection of large particles in the cyclone, the mean droplet size entering the channel may be expected to be much smaller (say $\sim 1 \mu \mathrm{m}$ ) than in conventional pulverized coal-fired boilers which typically produce a mass mean diameter of $10 \mu \mathrm{m}$.

Because the problems of using sampling probes to capture high velocity, high temperature liquid droplets are formidable, our attention has focused on non-intrusive optical techniques [43], using lasers to facilitate the rejection of background radiation by spectral filtering.

The simplest of these is the transmissometer which measures the extinction of a collimated light beam resulting from scattering and absorption by the particles. This is a well-established technique for cold flows, which effectively yields a measure of the total projected area of all the particles in the beam. To deduce a mean particle diameter, a separate measure is required of the fractional loading of ash. 


\subsubsection{Laser transmissometer}

Figure 13 shows a two-pass transmissometer, employing a He-Ne laser $(\lambda=632.8 \mathrm{~nm})$ which has been used [16] to measure the average particle size in the plasma issuing from a channel $(5 \times 10 \mathrm{~cm})$ attached to the $M-8$ combustor fired with a slurry of pulverized coal in ethanol, at input ash mass fractions of $\sim 0.50$ percent. The ash mass fraction at the measurement station was estimated as $\sim 0.23$ percent, taking account of los sy evaporation, and particle deposition in the combustor. Under these conditions the extinction is small $\sim 1$ percent, and considerable care must be taken to obtain reliable measurements. The Sauter mean diameter obtained from these measurements was $1.5 \mu \mathrm{m}$ with an estimated uncertainty of \pm 50 percent.

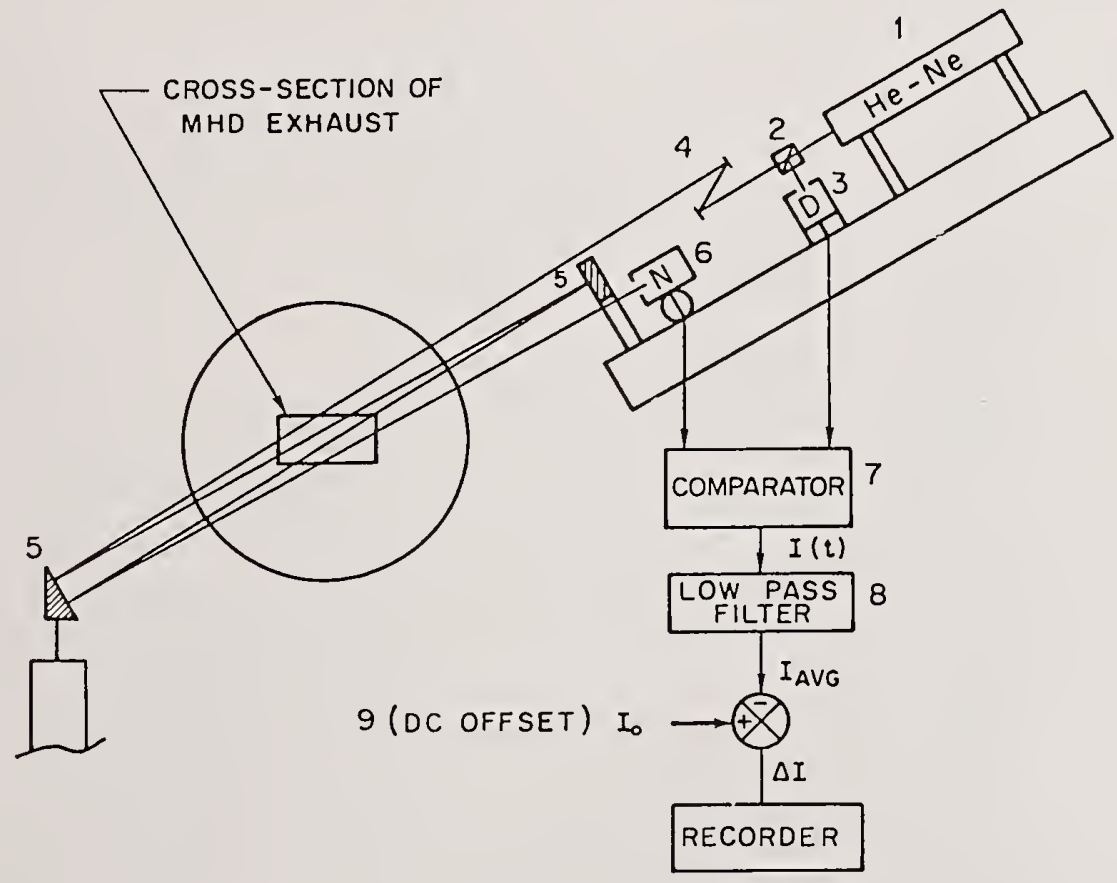

Figure 13. Schematic of He-Ne transmissometer for ash droplet measurements.

\subsubsection{Multiple wavelength laser transmissometer}

To avoid the necessity of estimating the ash mass fraction, a new two-wavelength laser transmissometer system is being constructed, which should allow the measurement of smaller extinction values. By using two widely separated wavelengths, it is possible to determine the mean particle size and ash loading.

Figure 14 shows the calculated ratios of the extinction coefficients as a function of the Sauter mean diameter, $D_{32}$, for a dispersion of droplets of refractive index $m=1.6+$ $i 0$, for various pairs of readily available laser wavelengths. It can be seen that the 
combination of $\lambda_{1}=0.325 \mu \mathrm{m}$ (He-Cd laser) and $\lambda_{2}=3.39 \mu \mathrm{m}$ (He-Ne laser) should allow $\mathrm{D}_{32}$ values in the range $0.1-3.0 \mu \mathrm{m}$ to be determined, while the combination of $\lambda_{1}=$ $0.442 \mu \mathrm{m}$ ( $\mathrm{He}-\mathrm{Cd}$ laser) and $\lambda_{2}=10.6 \mu \mathrm{m}$ ( $\mathrm{CO}_{2}$ laser) should cover the range $0.2-10 \mu \mathrm{m}$. However, the lower limit in either case may be excluded by the fact that the extinction for the longer wavelength may be too small on subscale systems at ash loadings of interest. On larger scale systems the extinction would be correspondingly greater and should facilitate the measurement.

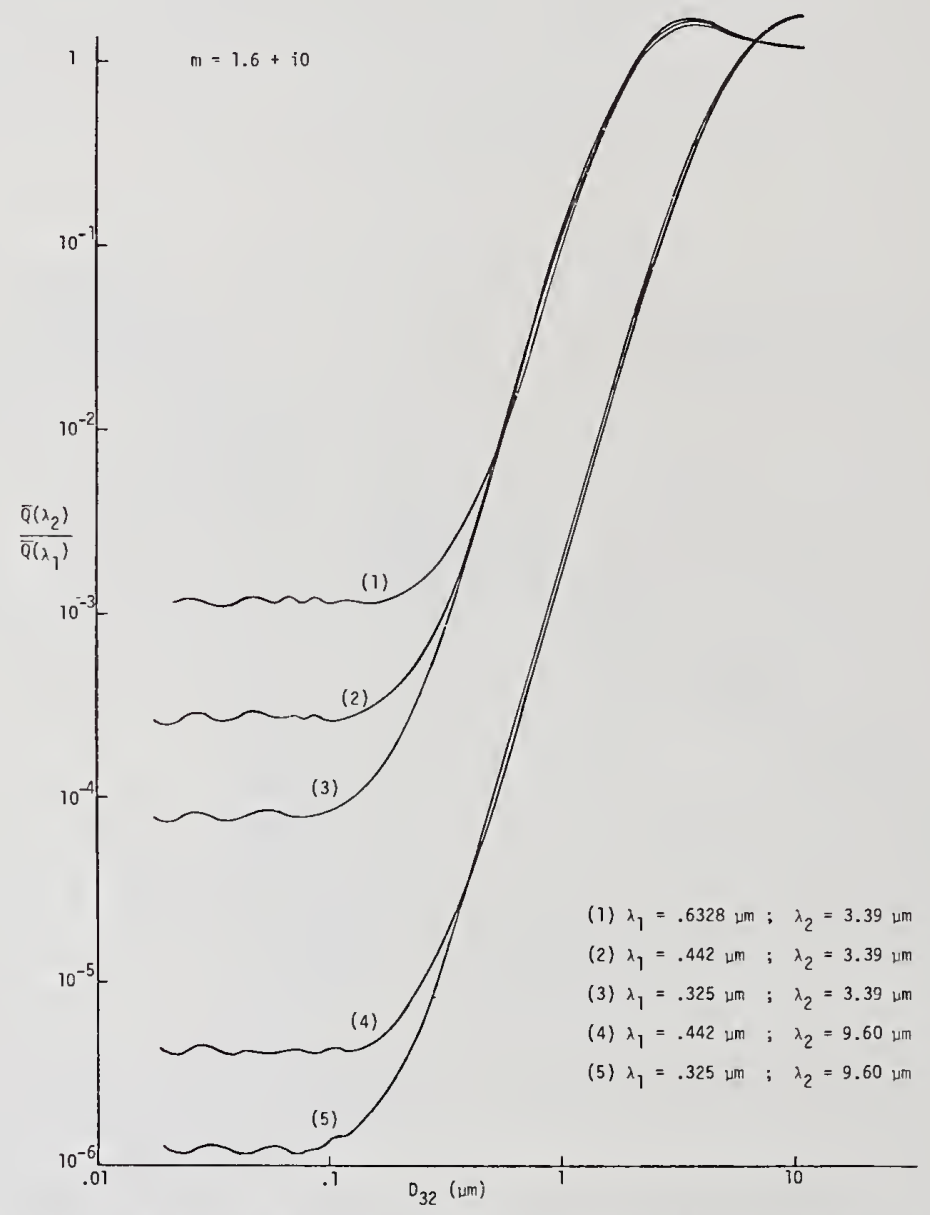

Figure 14. Ratio of average extinction efficiencies for selected pairs of laser wavelengths versus Sauter mean diameter.

\subsubsection{In-situ particle-sizing counter}

In separate work, a laser scattering device has been developed which determines the particle size distribution from the amplitude distribution of scattered light pulses as individual particles pass through the focus of a laser beam. It is essentially an extension of the commercially available sampling type of optical sizing counter to allow in-situ measurements, and is reported in more detail in references $[44,45]$. 
The experimental configuration is shown in figure 15. The beam from a $2 \mathrm{~mW} \mathrm{He}-\mathrm{Ne}$ laser is focused by cylindrical lenses to a measurement volume with Gaussian waist dimensions of $\sim 100 \mu \mathrm{m} \times 300 \mu \mathrm{m}$ (to $1 / \mathrm{e}^{2}$ of the one-axis intensity). Light scattered by particles passing through the measurement volume in the forward direction is collected by the lens pair ( $f=25 \mathrm{~cm}, F / 2.8$ ), and imaged onto the pinhole (100 $\mu \mathrm{m}$ diameter) preceding the spectral filter and photomultiplier detector. The pulses due to the transit of individual particles are amplified and sorted in a pulse height analyzer.

The use of forward scatter is advantageous in that for particles with $D \gtrsim \lambda$, the forward diffraction lobe is strong and well characterized. Unlike side or back scatter, the scattering amplitude is insensitive to refractive index and particle shape. However, compared with side scatter, forward scatter does imply greater difficulty in creating a small measurement volume, which is determined as the intersection of the illuminated and viewed volumes.

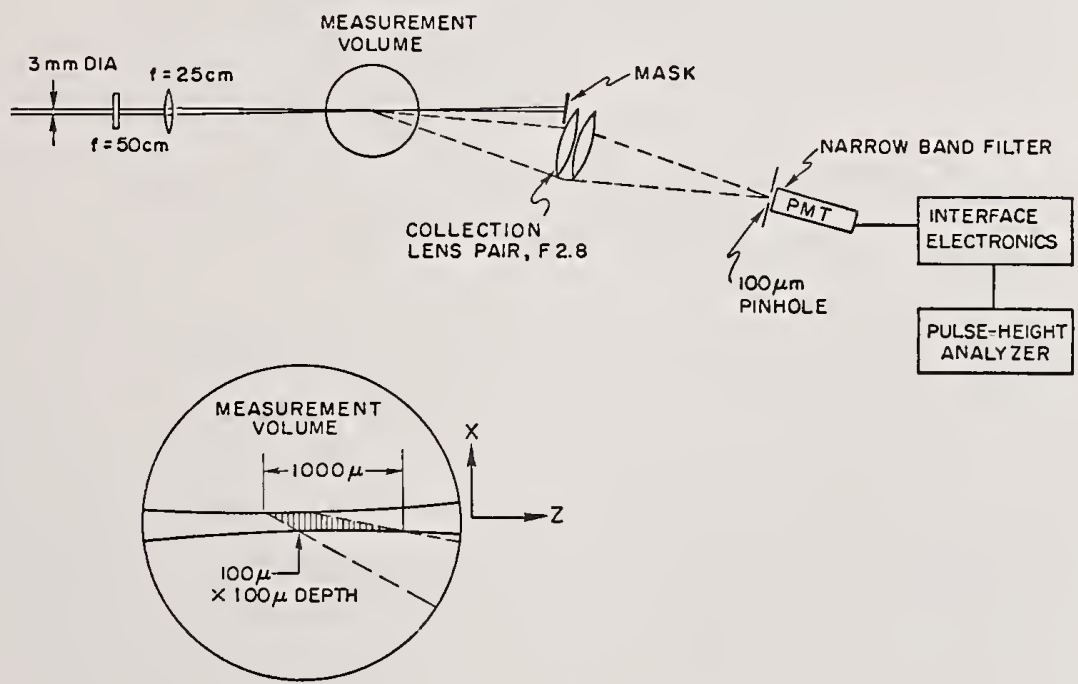

Figure 15. Schematic of optical particle-sizing counter.

As presently configured, the collection axis is at $12^{\circ}$ to the forward direction, and allows measurements of particles in the size range 2-30 $\mu \mathrm{m}$. The measurement volume is then $\delta V \sim 10^{-5} \mathrm{~cm}^{3}$, which allows concentrations as high as $10^{5} \mathrm{~cm}^{-3}$ to be measured without coincidence problems.

A desideratum of an optical sizing counter is that the response should be monotonic with particle size, but this is difficult to achieve exactly. Nevertheless, in practice it can be satisfied sufficiently well to allow adequate size resolution for most applications. Figure 16 shows the calculated and measured response for our instrument as a function of the size parameter $\alpha \equiv \pi \mathrm{D} / \lambda$. Monodisperse droplets of oleic acid of known size were generated by a Berglund-Liu generator. It will be noted that the response has a resonance at $\alpha \sim 5$ and weaker ones at larger values of $\alpha$. Although this resonance eliminates size discrimination between $1-2 \mu \mathrm{m}$ for $\lambda=0.633 \mu \mathrm{m}$, overall resolution is satisfactory for practical aerosol sizing requirements. 


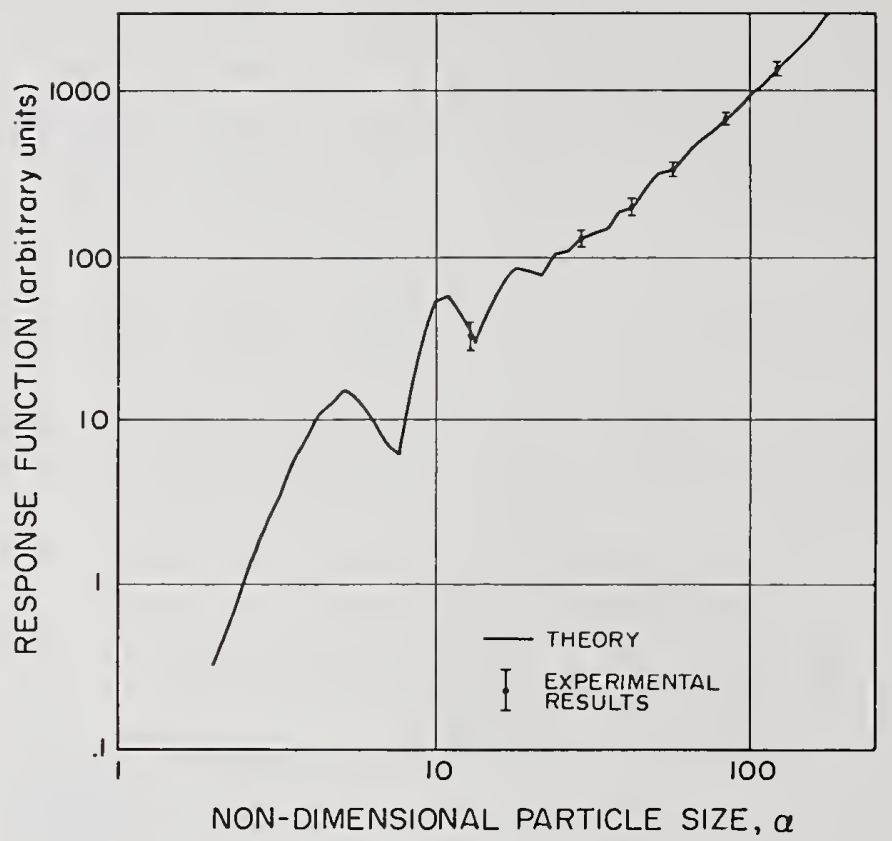

Figure 16. Experimental results for 0leic acid particles compared with theoretical response function.

Compared with a sampling type optical counter, where the sampled and diluted particles are passed through a uniformly illuminated jet, a major problem with the in-situ device is that the scattered signal amplitudes depend not only on particle size, but also on their trajectory through the measurement volume. Thus, a monodispersion will yield a signal pulse height distribution with a sharp upper cut-off, corresponding to particles passing through the center of the measurement volume, and extending down to small values, corresponding to particles passing off the center.

A novel and vital feature of the present instrument is the use of an on-line computerbased inversion technique, which unfolds the measured count distribution of pulse heights to yield the true particle size distribution. The ability of the inversion technique to unfold the fine structure of a polydispersion artificially created by the addition of four separate monodispersions was demonstrated [44].

Measurements have also been made of the size distribution of glass beads introduced into a bench scale flat flame burner, to test the instrument's immunity to background radiation and refractive index fluctuations. Figure 17 compares the measured size distribution for a nominal 1-30 $\mathrm{m}$ log normal distribution, with the burner both 1 it and unlit, with the manufacturer's curve. The deficit in the concentration of large particles is attributed to particle deposition in the feed tube between the particle feeder and the burner tube. 


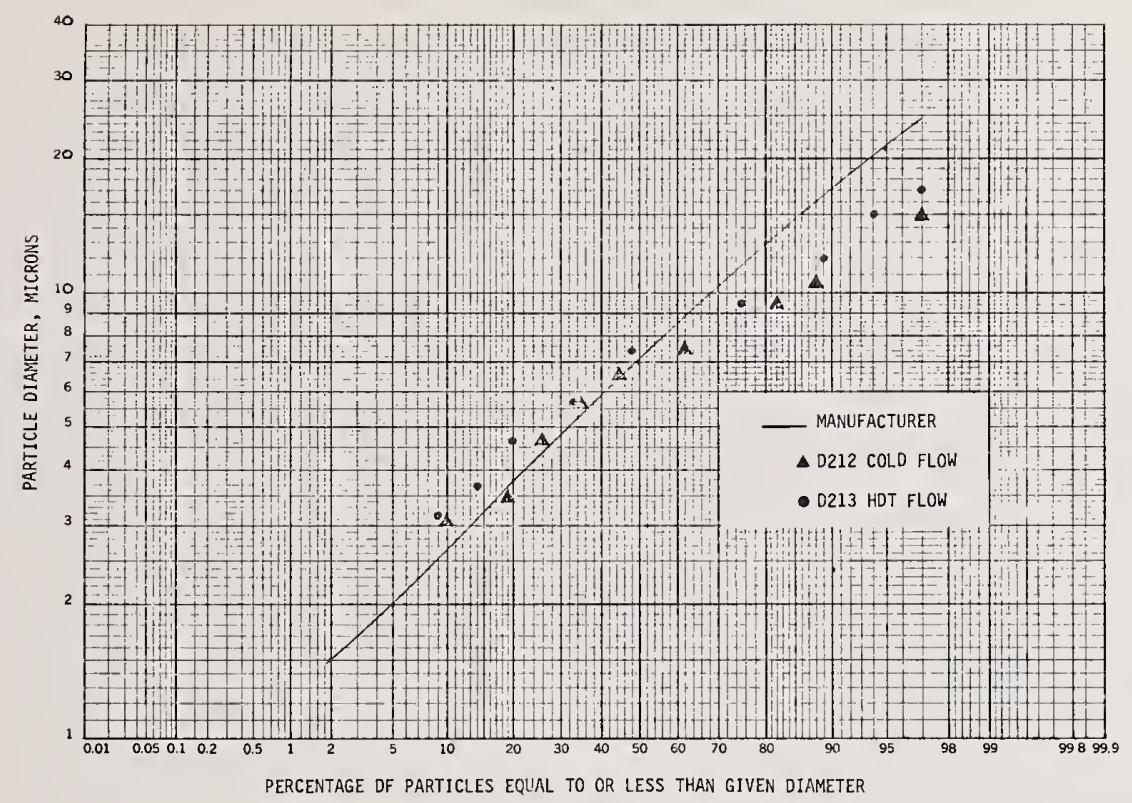

Figure 17. Comparison of results for cold and hot flows for nominal 1-30 $\mu \mathrm{m}$ particle distribution. Solid line is distribution supplied by particle manufacturer.

At present, the instrument has been demonstrated to be capable of measuring particle size distributions in the range 2-30 $\mu \mathrm{m}$ at concentrations up to $10^{5} \mathrm{~cm}^{-3}$ in smal1 1 aboratory flames. By modifying the configuration the range can probably be extended from 0.5 to $50 \mu \mathrm{m}$ or more. Its extension to the high velocity, high temperature conditions of coal-fired MHD plasma flows poses a number of problems, primarily the need for very much faster pulse-processing electronics to handle the very short pulses resulting from the high velocity.

\section{Conclusion}

The particular diagnostic measurement needs for understanding and monitoring the performance of combustion MHD generator systems have been described. Despite the hostile environment and access difficulties, a considerable variety of techniques have been developed for measuring velocity, gas temperature, electron concentration and mobility, electrical conductivity, alkali seed atom concentration and ash droplet loading and size distribution. Many of these techniques have been used as research tools in sub-scale laboratory systems. Their extension to use for monitoring important performance-determining parameters in pilot scale systems is, in principle, straightforward, but will present some challenging technical problems. 
It is a pleasure to record the vital contributions and active support of many members of the High Temperature Gasdynamics Laboratory to the work described herein. Particular mention should be made of Professor.R. H. Eustis, Laboratory Director, for constant encouragement, of Professor C. H. Kruger who has supervised the spectroscopic work, of Dr. D. Holve who was instrumental in the particulate measurements, and of Prof. R. M. Clements who, as a visiting Professor, taught us the mysteries of Langmuir probe measurements in hostile environments and assisted materially with the submillimeter laser technique. Last, but by no means least, the contributions of numerous graduate students and of the technical staff, especially Mr. F. 0 . Reigel, to the diagnostic measurement efforts are gratefully acknowledged.

This work has been supported by the Department of Energy under Contract EX 76-C-01-2341, by the National Science Foundation under Grant ENG-76-04116-A01, and by the Office of Naval Research through Project SQUID.

\section{References}

[1] Seike1, G. R., Sovie, R. J., Burns, R. K., Barna, G. J., Burkhark, J. A., Nainiger, J. J., and Smith, J. M., A summary of energy conversion alternatives study, Performance and costs results for MHD systems, 15th Symposium Engineering Aspects of MHD, Philadelphia (May 1976), paper III.4.

[2] Joint US-USSR Report on the Status of Open Cycle MHD Power Generation, Argonne National Laboratory (1978).

[3] Annual Symposia on Engineering Aspects of MHD, 15th, Philadelphia, 1976; 16th, Pittsburgh, 1977; 17th, Stanford, 1978.

[4] International Conferences on MHD Electric Power Generation: 6th, Washington, D.C. (June 1975).

[5] Self, S. A. and Kruger, C. H., Diagnostic methods in combustion MHD flows, AIAA J. Energy, 1, 25-43 (1977); also Self, S. A., Vasil'eva, I., and Nefedov, A., Diagnostics, in Joint US-USSR Report on The Status of Open Cycle MHD Power Generation. Argonne National Laboratory (1978), Chap. 14, to be published.

[6] Mitchner, M. and Kruger, C. H., Partially Ionized Gases (Wiley Interscience, 1973).

[7] Pepper, J. W., Eustis, R. H., and Kruger, C. H., NO concentration in MHD steam power plant systems, 12th Symposium on Engineering Aspects of MHD, Argonne National Laboratory (March 1972).

[8] Heywood, J. and Womack, G., Open Cycle MHD Power Generation (Pergamon Press, 1969).

[9] Rodgers, M. E. and Kruger, C. H. , Fluid mechanics and thermal behavior of MHD channel slag 1ayers, 17th Symposium on Engineering Aspects of MHD, Stanford (March 1978).

[10] Koester, J. K. and Nelson, R. M., Discharge characteristics of slagging metal electrodes, ibid. 
[11] Unkel, W. and Kruger, C. H., Axial field limitiations in MHD generators, 16th Symposium on Engineering Aspects of MHD, Pittsburgh (May 1977).

[12] Gordon, Zeleznik and Huff, A general method for automatic computation of equilibrium composition and theoretical performance of propellants, NASA TND-132 (0ct. 1959).

[13] JANAF Thermochemical Tables, D. R. Stull and H. Prophet, eds., National Bureau of Standards, U.S. Department of Commerce, 2nd Edition, 1971.

[14] Spencer, F. E. and Phelps, A. V., Momentum transfer cross sections and conductivity integrals for gases of MHD interest, 15th Symposium on Engineering Aspects of MHD (1976). Also revisions in Tech. Progress Rep. to ERDA, PERC, July 1976.

[15] Spencer, Jr., F. E. Hendrie, Jr., J. C., and Bienstock, D., Equilibrium electron density of coal-combustion mixtures--computer study and comparison with experiment, 13th Symposium on Engineering Aspects of MHD, 1973.

[16] Holve, D. and Self, S. A., Optical measurements of mean particle size in the exhaust of a coal-fired MHD generator, Western States Meeting of the Combustion Institute, San Diego (October 1976).

[17] Kolb, C. E., Yousefian, V., Wormhoudt, J., Martinez-Sanchez, M., and Kerrebrock, J. L., Characterization of open-cycle coal-fired MHD generators, Quarterly Report for 10-1-76 to 12-31-76 on ERDA Contract No. EX-76-C-01-2478, Aerodyne Research Inc.

[18] Daily, J. W., Kruger, C. H., Self, S. A., and Eustis, R. H., Boundary layer profile measurements in a combustion-driven MHD generator, J. AIAA, 14, 997-1005 (1976).

[19] Rankin, R. R., Self, S. A., and Eustis, R. H., A study of the MHD insulating wall boundary 1ayer, 16th Symposium on Engineering Aspects of MHD, Pittsburgh, May 1977.

[20] Barton, J. P., Koester, J. K., and Mitchner, M., An experimental and analytical investigation of fluctuation phenomena within a subsonic combustion MHD generator, 17 th Symposium on Engineering Aspects of MHD, Stanford (March 1978).

[21] Durst, F., Melling, A., and Whitelaw, J. H., Principles and Practice of Laser-Doppler Anemometry (Academic Press, NY, 1976).

[22] Self, S. A. and Whitelaw, J. D., Laser anemometry for combustion research, International J. Combustion Science \& Technology, Special Issue on Turbulent Reactive Flows, Vo1. 13, Nos. 1-6, 171-198 (1976).

[23] Self, S. A., Boundary layer measurements in combustion MHD channels, 3rd International Workshop on Laser Velocemetry, W. Stevenson and D. Thompson, eds., Purdue University, July 1978.

[24] James, R. K. and Kruger, C. H., Boundary layer profile measurements in the electrode wall of a combustion driven MHD channel, 17th Symposium on Engineering Aspects of MHD, Stanford, March 1978.

[25] Vincenti, W. G. and Kruger, C. H., Introduction to Physical Gas Dynamics, Sec. 8, Chap. XI (Wiley, NY, 1965).

[26] Daily, J. W. and Kruger, C. H., Effect of cold boundary layers on spectroscopic temperature measurements in combustion gas flows, J. Quant. Spectrosc. Radiat. Transfer, 17, 327-338 (1977). 
[27] Kowalik, R. M. and Kruger, C. H., The effects of fluctuation and non-uniformities on line-reversal temperature measurements, ibid, 18, 627-636 (1977).

[28] Vasil'eva, I. A., Kirillov, V. V., Maksimov, I. A., Malyuzhonok, G. P., and Novosadov, V. B., Measurements of plasma temperature by a spectroscopic method with continuous automatic detection, Teplofizika Vysokikh Temperature, 2, 838 (1973).

[29] Kowalik, R. M. and Kruger, C. H., Laser fluorescence temperature measurements, Combustion and Flame (to be published).

[30] Kruger, C. H., Kowalik, R. M., and James, R. K., Spectroscopic measurements of temperature electron density and temperature, fluctuation, 4th US/USSR Colloquium on MHD, Department of Energy, Washington, D.C., October 1978.

[31] Hower, N., Measurements of electrical conductivity of MHD plasmas with four-pin probes, HTGL Report 108, Stanford University (February 1978).

[32] Nelson, R., High Temperature Gasdynamics Laboratory, Stanford University, private communication.

[33] Gapanov, I. M., Poberezhshky, L. P., and Chernov, Yu G., Study of the electrical conductivity of plasma of combustion products with seeding in the U-02 MHD generator channel and on a laboratory installation, Combustion and Flame, 23, 29 (1974).

[34] Vende11, E. W., Free jet electrical conductivity profiles of a seeded MHD combustion plasma, 15th Symposium on Engineering Aspects of MHD, Philadelphia, May 1976.

[35] Clements, R. M. and Smy, P. R., Collection of ions by electric probes in combustion MHD plasmas: an overview, AIAA J. Energy, 2, 53-58 (1978).

[36] Clements, R. M., Some measurements of the ion current to a spherical probe in an atmospheric pressure combustion MHD plasma, Combustion and Flame, 25, 393 (1975).

[37] Clements, R. M. and Smy, P. M., Ion current to a spherical probe in a flowing high pressure plasma under thin-sheath conditions, Proc. IEE, 117, 1721 (1970).

[38] Reigel, F. 0. and Self, S. A. (1978), unpublished work.

[39] Self, S. A., Reigel, F. 0., Clements, R. M., and James, R. K., Electron concentration measurements in combustion MHD flows by submillimeter laser interferometry, AIAA J. Energy, 1, 206-211 (1977).

[40] Kuzmenko, P., Reigel, F. 0., and Self, S. A., Measurement of electron concentration and mobility in slag-laden MHD flows by submillimeter interferometry, 4th US/USSR Colloquium on MHD, Department of Energy, Washington, D.C. , October 1978.

[41] Heald, M. A. and Wharton, C. B., Plasma Diagnostics with Microwaves (Wiley, NY, 1965).

[42] Vasil'eva, I. A., Deputatova, L. V., and Nefedov, A. P., Investigation of the far wings of resonance 1 ines of alkaline metals in combustion products plasma, 2nd Joint US/USSR Colloquium on MHD, Washington D.C., June 1975.

[43] Ariessohn, P., Holve, D., and Self. S. A., Techniques for measurement of ash droplet size in coal-fired MHD flows, 4th US/USSR Colloquium on MHD, Department of Energy, Washington, D.C., October 1978.

[44] Holve, D. and Self, S. A., An optical particle-sizing counter for in-situ measurements, Technical Report SU-2-PU, Project SQUID, Purdue University, January 1978. 
[45] Holve, D. and Self, S. A., An optical particle-sizing counter for in-situ measurements, International Workshop on Laser Velocimetry, W. Stevenson and D. Thompson, eds., Purdue University, July 1978.

\section{Discussion}

Question (McDonald): Could you comment on the technique by which you use laser fluorescence of sodium to measure temperature?

Response (Self): Yes, although this has actually been done by a colleague of mine, Charles Kruger, and a student of his, they have used visible dyelaser pumping in the yellow up from an excited electronic level whose population depends on the temperature.

So, the absorption on that level is a measure of the temperature. They measure the fluorescent radiation from the level, the high level they have pumped at right angles, and the system can be calibrated, or from the absolute intensity you can calculate the population of the level you were pumping from and hence get the temperature.

Moderator (McDonald): Is that work published?

Response (Self): I think it is accepted, but I don't think it has actually come out. 

National Bureau of Standards Special Publication 561, Proceedings of the 10th Materials Research Symposium on Characterization of High Temperature Vapors and Gases held at NBS, Gaithersburg, Maryland, September 18-22, 1978. Issued October 1979.

\title{
CARS DIAGNOSTIC INVESTIGATIONS OF FLAMES ${ }^{1}$
}

\author{
Alan C. Eckbreth and Robert J. Hall \\ United Technologies Research Center \\ East Hartford, CT 06108
}

Coherent anti-Stokes Raman spectroscopy (CARS) investigations in a variety of $f l a m e s$ are presented. Thermometry has received the primary emphasis in the research to be reported, but some species sensitivity studies will be described as well. CARS is generated by mixing a $10 \mathrm{pps}$, frequency-doubled neodymium "pump" laser with a spectrally broadband, laser-pumped, "Stokes" dye laser. This approach obviates the requirement to frequency scan the dye laser and generates the entire CARS spectrum with each pulse permitting "instantaneous" measurements of medium properties. A crossed-beam, phase-matching technique termed BOXCARS is described which leads to greatly enhanced and unambiguous spatial resolution in contrast to the conventionally employed collinear phasematching approaches. Using this technique, moderate resolution ( $\sim \mathrm{cm}^{-1}$ ) CARS spectra from hot $\mathrm{N}_{2}$, obtained by scanning the spectrum in premixed laminar flames, show excellent agreement with computer generated model predictions. Temperatures determined by computer fitting the experimental CARS spectra agree with those determined by sodium line reversal to within $50 \mathrm{~K}$. Lower resolution $\left(2.7 \mathrm{~cm}^{-1}\right) \mathrm{col}^{-}$ linear phase-matched CARS spectra of flame $\mathrm{N}_{2}$ have been obtained in a single 10 nanosecond pulse using an optical multichannel analyzer. These single pulse spectra also display good agreement with predicted spectra and demonstrate the feasibility of single pulse thermometry. Measurements in a highly sooting, laminar propane diffusion flame revealed the existence of a coherent spectral interference arising from electronic-resonance CARS generation from $C_{2}$. The $C_{2}$ is produced by the laser vaporization of the soot. Reduction of the Stokes laser bandwidth and use of polarization filters permitted low distortion $\mathrm{N}_{2}$ CARS spectra to be obtained. These spectra, when computer fitted, allowed determination of the temperature in the highly sooting flame marking the first measurement in such flames by a remote, spatially precise diagnostic technique. CARS species sensitivity was examined

${ }^{1}$ Research supported in part under EPA Contract 68-02-2176 and Project SQUID (ONR) Contract (8960-28). 
in a study of flame co detectability levels. Very good agreement between CARS CO spectra and the computer model was obtained at the 4 percent co level. With the fluctuations in the experimental apparatus, it was difficult to detect co below the 1-2 percent level. The computer calculations indicate that co would be barely detectable to about 0.5 percent using conventional CARS approaches.

\section{Introduction}

Coherent anti-Stokes Raman spectroscopy (CARS) has recently come to prominence for combustion diagnostics based upon the pioneering investigations of Taran and his coworkers $[1-5]^{2}$ at ONERA in France. The effect was originally discovered in the early sixties by Maker and Terhune [6] and remained essentially in the province of nonlinear optics until Taran's application of it for gas phase diagnostics. In the United States, Byer and Harvey and their coworkers [7-11] have conducted numerous investigations into the technique. Barrett has demonstrated both $\mathrm{CW}$ CARS generation [12] and pure rotational CARS [13]. Broadband CARS generation in a single pulse important for instantaneous measurements has also been obtained [14]. Crossed-beam phase-matched CARS generation in gases, required for good spatial resolution, has been demonstrated by Eckbreth [15]. A11 of the experimental work to be reported herein has been broadband or multiplex CARS for reasons to be elaborated upon later. Publications describing investigations into CARS are appearing at an ever increasing rate and the technique apparently will have a major impact in molecular structure and biological studies. Several very good reviews of CARS have appeared recently $[9,11,16]$.

CARS is probably best understood by reference to figure 1 . Incident laser beams at frequencies $w_{1}$ and $w_{2}$ (often termed the pump and Stokes beams respectively) interact through the third order nonlinear susceptibility $x^{(3)}\left(-w_{3}, w_{1}, w_{7},-w_{2}\right)$ to generate a polarization field which produces coherent radiation at frequency $w_{3}=2 w_{1}-w_{2}$. When the frequency difference $w_{1}-w_{2}$ is close to the frequency of a Raman active resonance, the magnitude of the radiation at $w_{3}$, then at the anti-Stokes frequency relative to $w_{1}$, can become very large. Large enough, for example, that with the experimental arrangement described herein, the CARS signals from room air $\mathrm{N}_{2}$ are readily visible. The incident beams, however, must be so alined that the four wave mixing process is properly phased. For gases which are nearly dispersionless, phase matching occurs when the beams are mixed collinearly, i.e., alined. Although easy to implement, collinear phase matching possesses a drawback from a diagnostic standpoint, namely, potentially poor and ambiguous spatial resolution. A technique to circumvent this difficulty, which employs a crossed-triple-beam phase-matching scheme, will be described shortly. Assuming a fixed narrowband pump frequency, $w_{7}$, the CARS spectrum at $w_{3}$ can be mapped out piecewise by scanning a variable frequency, narrow-band laser source at $w_{2}$. Or, as depicted, if a broadband source at $w_{2}$ is employed, the entire CARS spectrum can be generated simultaneously permitting fast, time-resolved measurements of fluctuating phenomena [14].

${ }^{2}$ Figures in brackets indicate the literature references at the end of this paper. 


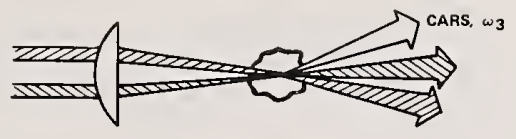

- energy level Diagram

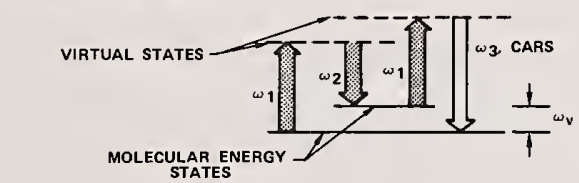

- SPECTRUM

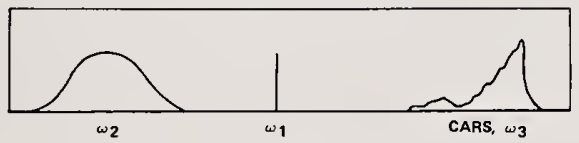

Figure 1. Coherent anti-Stokes Raman spectroscopy (CARS).

CARS offers very promising potential for the diagnostic probing of high interference environments such as those typical of combustion processes for two reasons. First, in contrast to spontaneous Raman phenomena, CARS is a fairly strong process leading to signal levels typically many orders of magnitude larger than those from Raman scattering. Second, the CARS signals are coherent. Consequently, all of the CARS radiation can be collected. Contrast this with the situation pertaining in the normal Raman process where photons are scattered over $4 \pi \mathrm{sr}$ and are collected only over a limited solid angle, $\Omega$. For f/3 optics, only 1 percent of the signal is collected in an isotropic, incoherent scattering process. Furthermore, since the CARS radiation can be collected in an extremely small solid angle, discrimination against background luminosity and laser induced particulate interferences, e.g., incandescences [17], fluorescences, is greatly facilitated. Thus, CARS is expected to offer signal to interference ratio (S/I) improvements of many orders of magnitude over spontaneous Raman scattering. Based upon the calculations presented in [18], CARS appears capable of probing practical combustion environments successfully over a broad range of operating conditions.

Before CARS can be practically implemented, however, a number of questions needed to be addressed such as spatial resolution, computer synthesis of CARS flame spectra, feasibility and practicality of single pulse thermometry, the effects of soot particulates, and species sensitivity limitations. The investigations reported herein sought to answer these questions and to provide an experimental foundation on which to base future avenues of relevant development. In the section which follows, BOXCARS, a nomen for a crossed-beam, phase-matching technique will be described. BOXCARS provides fine and unambiguous spatial resolution for CARS diagnostics of spatially inhomogeneous media. Then, the experimental apparatus employed 
in these investigations will be described. Thermometry investigations, scanned and single pulse, in premixed flat flames are described. Then, thermometry investigations in a highly sooting diffusion flame are described. The paper concludes with an initial examination of CARS $\mathrm{CO}$ detection sensitivity in flames.

\section{BOXCARS: Crossed-Beam Phase Matching}

As mentioned previously, the incident pump and Stokes laser beams must be alined in a precise manner so that the CARS generation process is properly phased. The general phasematching diagram for four wave mixing is shown in figure $2 a$ and requires that $2 \vec{k}_{1}=\vec{k}_{2}+\vec{k}_{3}$. $\vec{k}_{i}$ is the wave vector at frequency $w_{i}$ with absolute magnitude equal to $w_{i} n_{j} / c$, where $c$ is the speed of light and $n_{i}$, the refractive index at frequency $w_{i}$. Since gases are virtually dispersionless, i.e., the refractive index is nearly invariant with frequency, the energy conservation condition $w_{3}=2 w_{1}-w_{2}$ indicates that phase matching occurs when the input laser beams are alined parallel to each other, figure $2 \mathrm{~b}$. Collinearity, however, possesses a problem in regard to spatial resolution. Since the CARS signal is coherent and undergoes an integrative growth process, the spatial resolution cannot be well defined by imaging techniques such as those successfully employed in spontaneous Raman scattering approaches to yield fine resolution. Since the CARS signal generation scales as the intensity product $\mathrm{I}_{1}{ }^{2} \mathrm{I}_{2}$, the incident laser beams are generally tightly focused for diagnostic purposes when collinear phase matching is employed. For diffraction-limited beams, the interaction is found to occur primarily within a cylindrical volume of diameter $\phi$ and length $6 \ell$ given by [4].

$$
\phi=\frac{4 \lambda f}{\pi D} \quad \ell=\frac{\pi \phi^{2}}{2 \lambda}
$$

where $f$ is the focusing lens focal length; $D$, the beam aperture incident on the lens; and $\lambda$, the wavelength. In table 1 , the probe volume focal diameter, cross-sectional area, and sample length are tabulated for various focal length lenses for a $1 \mathrm{~cm}$ diameter beam at $5320 \mathrm{~A}$. Depending on the specific diagnostic circumstance, i.e., the focusing lens to measurement point separation, the spatial resolution may be less than that which is desired. Although the resolution is very good for short focal length lenses, gas breakdown may 1 imit the input beam intensities leading to diminished CARS signal levels.

Many laser beams are not diffraction-limited, resulting in much poorer spatial resolution than that tabulated here. For example, for a "three times diffraction-limited" beam divergence angle, the linear resolution would be about an order of magnitude poorer. Specifically, in figure 3 , CARS radiation curves of growth are displayed for beam divergence angles of 1 and 2 milliradians and various practical focal length lenses. The calculations were made using a plane wave analysis applicable to the parameter range selected [19] corresponding to so-called "loose focusing." As is readily apparent in figure 3, significant CARS generation occurs well before the focal region in the prefocal regions. If the coherence 
(a)

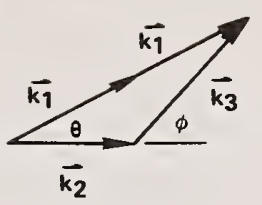

(b)

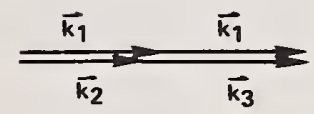

(c)

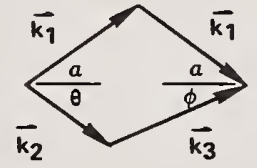

Figure 2. CARS phase-matching approaches: (a) general two beam scheme, (b) collinear, and (c) crossed-beam or BOXCARS.

Table 1. Collinear phase-matched CARS probe volume

\begin{tabular}{cccc} 
Focal length $(\mathrm{cm})$ & Diameter $(\mathrm{cm})$ & Area $\left(\mathrm{cm}^{2}\right)$ & $\begin{array}{c}\text { Sample } \\
\text { length }(\mathrm{cm})\end{array}$ \\
10 & $6.11(-4)$ & $2.93(-7)$ & $7.32(-2)$ \\
20 & $1.22(-3)$ & $1.17(-6)$ & $2.93(-1)$ \\
50 & $3.06(-3)$ & $7.35(-6)$ & 1.83 \\
100 & $6.11(-3)$ & $2.93(-5)$ & 7.32 \\
\hline
\end{tabular}

length is sufficiently large, significant growth could occur beyond the focal region as well. To make the situation worse, in the presence of density gradients, the resolution further degrades since CARS scales as the square of the gas density. For probing of a hot flame operating at atmospheric pressure, significant contributions to the CARS signal may originate from the cool, high density regions adjacent to the flame. For example, in the experimental setup used here, initial attempts to record flame spectra with $40 \mathrm{~cm}$ focal length lenses on a 7.5-cm diameter burner were unsuccessful due to a dominance of room air $\mathrm{N}_{2}$ contributions. With a piece of Schott glass GG495, which transmits the laser and Stokes beams but absorbs the CARS beam, it was determined that about 10-15 percent of the total room air CARS signal 
was generated in the first $32 \mathrm{~cm}$ from the lens. With the flame ignited, the CARS signal from the hot, low density flame gases was substantially less than the cold, room air contribution.

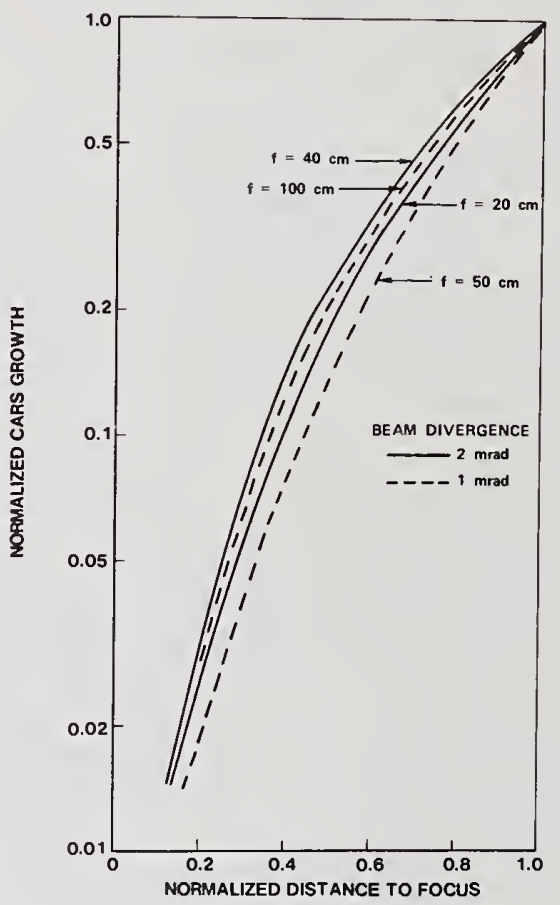

Figure 3. Collinear phase-matched CARS radiation growth curves for various focal length lenses and laser beam angular divergences.

CARS signal contributions may derive also from the various elements in the optical train, e.g., lenses, when collinear phase matching is used. These could be significant when low gas densities or weak resonances are being probed. Clearly, it would be desirable to avoid beam overlap and potential four wave mixing in all regions except the desired measurement location. In an effort to avoid collinearity, one could attempt to introduce the pump and Stokes beams at a slight angle to one another. As phase mismatch is deliberately introduced in this manner, the CARS signal generating efficiency will diminish. At $\Delta k \ell \approx 3$, where $\Delta k$ is the magnitude of the phase mismatch, i.e., $\left|2 \vec{k}_{1}-\vec{k}_{2}-\vec{k}_{3}\right|$, the CARS efficiency will have decreased by an order of magnitude [9]. Accepting this loss of power for the moment and if a $0.1 \mathrm{~cm}$ spatial resolution is desired, then a $\Delta \mathrm{k}$ of $30 \mathrm{~cm}^{-1}$ would be tolerable. At wavelengths of $5320 \AA\left(w_{1}\right)$ and $6073 \AA$ (the $N_{2}$ ground vibrational state Stokes wavelength), a $30 \mathrm{~cm}^{-1}$ phase mismatch would be produced at an angular separation of only $1^{\circ}$ assuming no dispersion. Although one could operate in this manner, it is clearly inefficient and the actual spatial resolution will depend very critically on the precise angular 
separation. CARS has been generated using crossed $w_{1}$ and $w_{2}$ beams as will be described later in the $C O$ studies, but no systematic study of the scaling of the signal generation efficiency or spatial resolution was attempted.

A method which permits large angular separation of the input frequencies, while still satisfying the phase matching requirement is depicted in figure 2c [15]. In this approach the $w_{1}$ pump beam is split into two components which are crossed at a half angle of $\alpha$. The $\omega_{2}$ Stokes beam is introduced at angle $\theta$ producing phase-matched CARS at angle $\phi$. Based upon the shape of the phase-matching diagram, figure $2 \mathrm{c}$, this technique has been termed BOXCARS. The appropriate phase-matching angles are readily related from simple geometric considerations as follows:

$$
\begin{gathered}
\mathrm{n}_{2} \omega_{2} \sin \theta=\mathrm{n}_{3} \omega_{3} \sin \phi \\
\mathrm{n}_{2} \omega_{2} \cos \theta+\mathrm{n}_{3} \omega_{3} \cos \phi=2 \mathrm{n}_{1} \omega_{1} \cos \alpha .
\end{gathered}
$$

Although the interaction zone has not been analyzed in detail, BOXCARS generation can clearly occur only where all three beams overlap leading to unambiguous spatial resolution. By proper design, this interaction zone can be made acceptably small for most diagnostic applications, e.g., less than $1 \mathrm{~mm}$.

At larger angles and for the longer focal lengths required in practical applications, a single conventional focusing lens generally would be quite heavy and expensive. One can then first cross the beams and use individual lenses on each leg. An alternative would be to investigate the use of large, corrected, acrylic Fresnel lenses.

An interesting aspect of BOXCARS that has not yet been explored concerns the employment of crossed polarizations [20]. In isotropic media such as gases, the third order nonlinear resonant susceptibility can be expressed as [21]

$$
x_{i j k 1}\left(-w_{3}, w_{1}, w_{1},-w_{2}\right)=x_{x x x x}\left[(1-2 p) \delta_{i j k} \delta_{k l}+\rho\left(\delta_{i k} \delta_{j 1}+\delta_{i 1} \delta_{j k}\right)\right]
$$

where the indices $i, j, k, 1$ refer to the polarization orientation of the frequencies $w_{3}$, $w_{1}, w_{1}$ and $w_{2} ; \rho$ is the depolarization of the Raman mode and $\delta_{i j}$, the Kroneker delta $(=1$, $i=j ;=0, i \neq j$ ). In $N_{2}, \rho$ is extremely small and equal to 0.022 [22]. Hence,

$$
x_{i j k 1} \quad x_{x x x x} \delta_{i j} \delta_{k 1}
$$

With conventional CARS, where only one $w_{1}$ input pump beam is employed, $j \equiv k$, and hence, for CARS to be observed, the Stokes polarization must be parallel to the pump polarization, $k=1$. The CARS radiation will then exhibit the same polarization, $i=j$, as the 


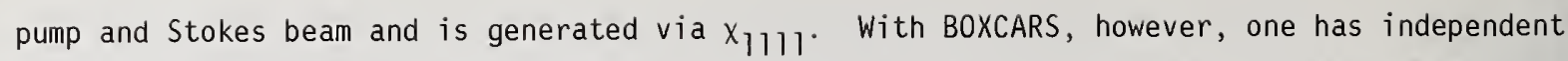
control of the $j$ and $k$ polarizations. If $j$ and $k$ are parallel, then the Stokes beam must have the same polarization for CARS to be generated, as in conventional CARS. However, if $j$ and $k$ are orthogonally polarized, and the Stokes beam is alined along one of the pump components, then the CARS radiation will emerge orthogonally polarized to the Stokes beam.

This gives rise to an interesting instrumental approach to thermometry. One way to measure temperature is to ratio the CARS from a hot band, e.g., $v=1 \rightarrow 2$ to CARS from the ground state band, $v=0 \rightarrow 1$. If the Stokes beam contains two orthogonally polarized components corresponding respectively to wavelengths necessary to generate CARS from the hot and ground state band, then the CARS so generated from each band would be orthogonally polarized. The two CARS components could be separated with a polarization splitter and temperature ascertained from the ratio of the two. In contrast to the broadband techniques, such an approach offers back end (detection) simplicity. It obviously would be more complicated on the front end (laser) side than the broadband CARS approach. Hence, in addition to obvious spatial resolution advantages, BOXCARS may offer other instrumental benefits as we 11 .

\section{Experimental Approach}

Although CARS has no threshold per se and can be generated with $\mathrm{Cw}$ laser sources [12], high intensity pulsed laser sources are required for the probing of high interference environments to generate CARS signals well in excess of the various sources of interference. Because CARS is highly nonlinear in its temperature and density dependences, signal averaging in temporally fluctuating media will obscure and distort the CARS spectrum rendering it of little utility or leading to measurement errors. Thus, measurements in fluctuating environments require the CARS spectrum to be generated and captured with each laser pulse. Hence, the individual laser pulses must be energetic enough to provide a statistically large number of CARS signal photons in each spectral detection interval. So restricted, laser selection narrows to a choice between ruby and frequency-doubled neodymium $(2 \times N d)$ with the latter probably preferable.

$2 x N d$ lasers can be operated at a repetition rate generally an order of magnitude higher than ruby, at least $10 \mathrm{pps}$ versus $1 \mathrm{pps}$. In an instrumental application, this obviously expedites data collection. In the laboratory, it permits the use of boxcar averagers and spectral scanning techniques (when applicable). At $10 \mathrm{pps}$ or better, the experiment behaves much like a cw experiment permitting "tweaking" of adjustments, while the experiment is running, an important feature for the critical alinement requirements of CARS experiments. If a portion of the pump laser is split off to pump a Stokes beam dye laser, $2 \times N d$ lasers at $5320 \AA$ can pump very efficient dyes in the 5500-7000 $\AA$ region of the spectrum, while ruby lasers must pump lower efficiency near ir dyes. Furthermore, due to the wavelength scaling of the Raman cross-sections and, hence, the third order nonlinear susceptibility, the CARS signals generated from $2 \times N d$ are larger than those from ruby for equivalent laser power levels. In the case of $\mathrm{N}_{2}$, for example, this factor is 6.5. Also, the CARS radiation from 
$2 \times N d$ resides in a region of higher photomultiplier tube quantum efficiency than does the CARS from $6943 \AA$, a small advantage. The ruby laser wavelength disadvantages are mitigated by frequency doubling to $3471 \AA$. Whether one would encounter desirable or undesirable (from background constituents) two-photon resonant-enhancement effects for combustion diagnosis is not known at this time. This is also true of using the third harmonic of neodymium at $3533 \AA$. As an aside, one interesting feature of operating in this region is that the CARS signals would be about 144 times stronger from $2 x$ ruby relative to ruby, and 19.5 times stronger for $3 \times \mathrm{Nd}$ than $2 \times \mathrm{Nd}$ on the basis of wavelength scaling alone. Considerations of harmonic generation efficiencies would diminish these gains however. For the investigations reported herein, a frequency-doubled neodymium laser was selected based on the foregoing rationale. In some early investigations of CARS [1], the Stokes beam was generated via stimulated Raman scattering of the pump beam. Such an approach is limited to probing only stable species from which stimulated Raman is readily produced, is not suitable for broadband CARS generation, and thus, is not very versatile. A more flexible scheme is to employ a tunable dye laser, generally pumped by splitting off a portion of the pump laser. CARS configurations employing $2 \times N d$ lasers and dye lasers pumped by a fraction of the $2 \times N d$ output are also being used at, among other places, the Naval Research Laboratory, Stanford University, and Oregon State University.

In figure 4 is shown a schematic of the UTRC CARS setup. In figure 5, a photograph of the experimental setup is shown. Referring to figure 4, the output of a Quanta-Ray neodymium laser (Model DCR-1A) is frequency doubled to generate a horizontally polarized, "primary" green beam at $5320 \mathrm{~A}$. The primary green and residual $1.06 \mu$ are separated in a splitter section and the residual $1.06 \mu$ doubled to generate a "secondary" green beam. Depending on the condition of the flashlamps in the laser and the frequency doublers, the primary green is typically between 1.5 to $2.5 \mathrm{~W}$ (150 to $250 \mathrm{~mJ}$ pulses, $10 \mathrm{pps}, 10^{-8} \mathrm{~s}$ pulse duration). As seen in the figure, part of the primary is split off by the beamsplitter, BS, reflected by the mirror $M$, and focused to pump (off axis) a flowing dye cell oscillator. The output from the dye laser is amplified in a flowing dye cell off-axis pumped by the secondary green. The secondary green passes through a piece of KG3 air-cooled, Schott glass placed at the Brewster angle to absorb any remaining $1.06 \mu$. The secondary green is then turned by the mirrors, $M$, and focused by lens, $L$, to pump the dye in the amplifier cell. The oscillator and amplifier cells are in flow series. A small magnetically coupled, stainless steel certrifugal pump circulates the dye from a 1.51 iter reservoir through a $0.6 \mu$ filter to remove any large air bubbles present. The dye cells are oriented at Brewster's angle and, produce a horizontally polarized output; actual oscillator efficiencies vary between 20-30 percent, while the amplifier operates between 40 and 50 percent efficiency.

Figure 4 shows the disposition of three laser beams at frequencies $w_{1}, w_{1}$, and $w_{2}$ as utilized for crossed-beam phase matching. The primary pump beam after passing through the first beamsplitter is split at a second beamsplitter, BS', and sent to an additional mirror. The remaining $w_{7}$ beam reflects from the beam combining dichroic, $D$, but appropriately displaced from $w_{2}$. Optical delay can be inserted into the pump beam leg to ensure that the pump and Stokes laser pulses are temporally overlapped. A11 three beams, $w_{1}, w_{1}$ and $w_{2}$ are 


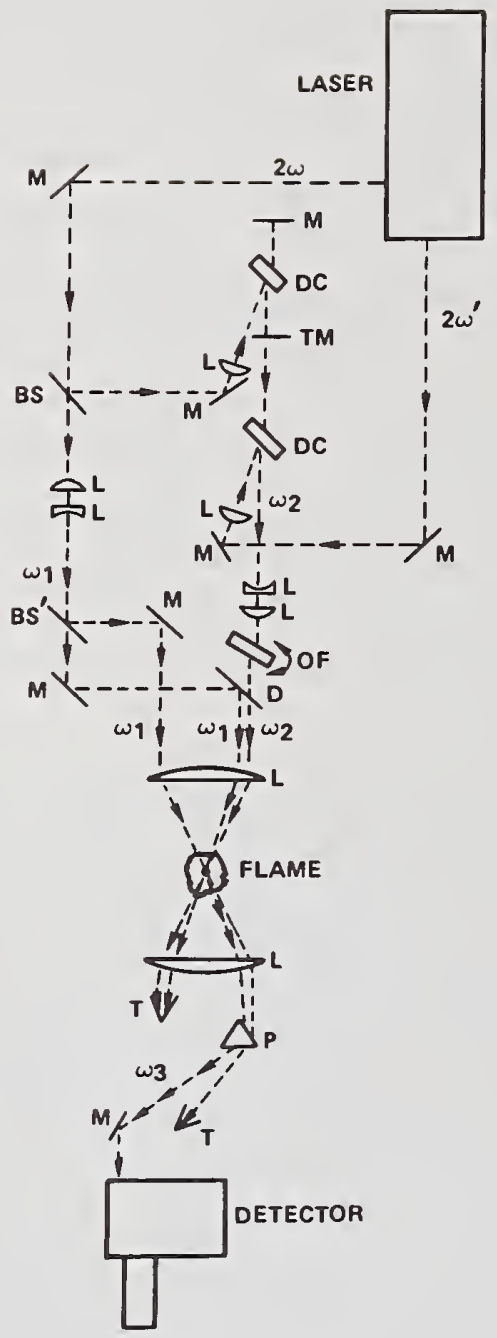

Figure 4. Schematic illustration of the CARS experimental arrangement. Code: BS, beamsplitter; L, lens; D, dichroic; OF, optical flat; $P$, prism; F, filter; DC, dye cel1; T, trap; TM, partially transmitting mirror; $M$, mirror.

alined parallel to each other in a single plane and sent to the focusing lens. If the beams are alined properly, they will cross at the focal point of the lens, by definition. However, due to wavefront sphericity, they may not necessarily waist at the crossing point. To ensure that the minimum beam waist occurs at the crossing point, adjustable telescopes are added in each leg. At high laser. intensities, the beams are readily visualized near the focal region via the room air Rayleigh scattering, the telescopes are then adjusted to visually produce minimum waisting at the crossing point. Also inserted in the $w_{2}$ leg is a rotatable optical flat. Rotation of the flat permits displacement of the $w_{2}$ beam on the focusing/crossing lens permitting the phase-matching angle $\theta$ to be varied. After passing through the crossing point, the four beams, i.e., CARS at $w_{3}, w_{1}, w_{1}, w_{2}$ are recollimated by 


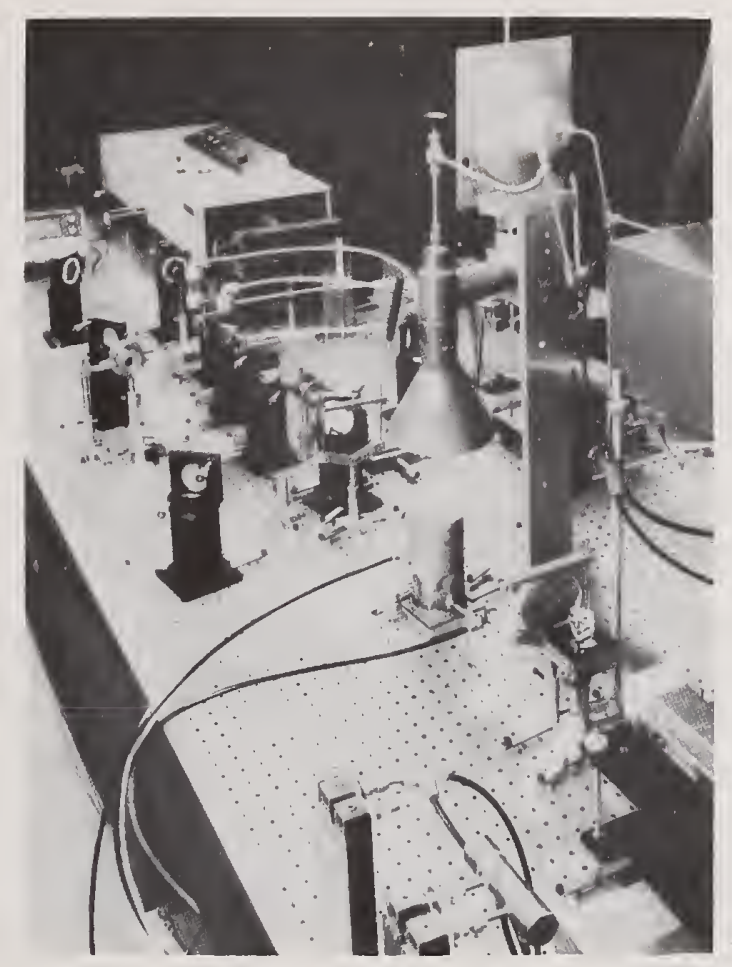

Figure 5. Photograph of UTRC CARS facility.

a second lens, generally the same focal length as the focusing lens. Two of the components, $w_{1}$ and $w_{2}$, are trapped although they could be sent to a reference leg if desired to generate a normalizing signal. At the small crossing angles usually employed for BOXCARS, the CARS and one pump beam, although angularly separated, are not spatially separated. Additional angular separation is achieved with a double extra dense flint prism. After a suitable distance, the one pump beam is trapped and the CARS passes through cutoff filters prior to analysis by a $1-\mathrm{m}$ double monochromator (Ramanor HG2S, Jobin Yvon) or to an optical multichannel analyzer, OMA (Princeton Applied Research) fitted to a 1/4-m spectrograph equipped with either a 1200 or $2400 \mathrm{gr} / \mathrm{mm}$ holographic grating. Recently, the $1-m$ double monochromator has been modified to accept the OMA as well. For operation in the collinear mode, the beamsplitter, BS', is removed. The pump and Stokes beams are combined at the dichroic and collinearly focused into the region under study to generate CARS. In this mode, the experimental configuration is capable of very intense CARS generation. It is strong enough, that the CARS generation from $\mathrm{N}_{2}$ in room air is readily visible and easily photographed.

To produce the broadband Stokes laser output desired for single pulse CARS work, a variety of dye oscillator cavity configurations were tested. Bandwidths on the order of $150 \mathrm{~cm}^{-1}$ are desired since the vibrational band separation in $\mathrm{N}_{2}$ is about $30 \mathrm{~cm}^{-1}$. A flat Fabry-Perot oscillator arrangement was adopted and the bandwidth centered by selection 
of appropriate dyes and dye concentration [14]. Small adjustments in the Stokes laser center frequency are also possible by varying the cavity mirror separation. Bandwidths vary from $100 \mathrm{~cm}^{-1}$ to $200 \mathrm{~cm}^{-1}$ depending on oscillator pump energy and whether the dye amplifier is used. By using mixtures of two different dyes, very broad bandwidths are possible, i.e., 250 to $300 \mathrm{~cm}^{-1}$. For $\mathrm{N}_{2}$ thermometry, which requires the Stokes laser to be centered at $6073 \AA$, Rhodamine 640 (Exciton Chemical Co.) at a concentration of about $10^{-4} \mathrm{M}$ in ethanol is employed.

In broadband CARS, either the linewidth of the pump laser or the resolution of the monochromator determine the ultimate resolution of the spectrum. The 1-m double monochromator has a limiting resolution of about $0.5 \mathrm{~cm}^{-1}$. The pump laser with no intracavity etalon has a linewidth of $\sim 1.2 \mathrm{~cm}^{-1}$ and thus limited the spectral resolution to around this value. The large pump laser linewidth may be detrimental, however, in regard to the strength of the CARS radiation. In this regard, it would be desirable to have the pump linewidth comparable to the Raman linewidths of the hot $f 1$ ame gases, $20.1 \mathrm{~cm}^{-1}$. Narrower 1 inewidths than used for the studies reported here are obtainable with intracavity etalons and active line narrowing accessories.

For scanning the CARS spectrum from laminar flames, the output of the monochromator photomultiplier is fed to one channel of a two channel boxcar averager (PAR Model 162) fitted with gated integrators (PAR Mode1 164). For broadband Stokes wave CARS generation, the monochromator signal can be normalized to the total spectrally integrated CARS signal. The latter is obtained by splitting off a small fraction of the CARS signal which is monitored by a second photomultiplier fitted with a narrowband interference filter. The latter signal is sent to the second or normalizing channel of the boxcar averager. In this manner, shot to shot laser power fluctuations are accounted for.

\section{Flat Flame Thermometry}

Unlike spontaneous Raman spectra which depend linearly on and, hence, mirror quantum state population distributions, CARS spectra are much more complex representatives of the nonlinear nature of the four wave mixing process. Although some simple schemes were first proposed to derive temperature information from CARS spectra, computer modeling will probably be required for accurate temperature measurements. This is particularly true for CARS thermometry from molecules with small rotational line separation. In such instances, interference effects between neighboring lines are significant and difficult to approximate analytically. In an attempt to assess the accuracy of CARS thermometry, CARS spectra were recorded from flat flames at various temperatures. The flame temperatures were obtained over a limited range from radiation corrected thermocouple readings; the radiation corrections were obtained via sodium line reversal temperature measurements on the flames near the actual conditions used for the CARS experiments. The temperature from the CARS spectra was ascertained from the best computer synthesized spectral fit and compared with the corrected thermocouple reading. 


\section{1 Flat flames}

Premixed flames of $\mathrm{CH}_{4}$ /air were studied over burner surfaces consisting either of a $2.5 \mathrm{~cm}$ diam hexagonal or $7.5 \mathrm{~cm}$ diam circular array of $0.30 \mathrm{~cm}$ diam stainless steel hypo tubes. For approximately the same total mass flow rates, the two burners ran at considerably different temperatures due to the difference in the volumetric heat release. Airflow rates were in the range from 200 to $320 \mathrm{~cm}^{3} / \mathrm{s}, \mathrm{CH}_{4}$ in the range from 19 to $28 \mathrm{~cm} / \mathrm{s}$. Temperatures were measured with fine wire $\mathrm{Pt} / \mathrm{Pt}-10 \% \mathrm{Rh}$ thermocouples coated with a 90 percent $Y_{2} \mathrm{O}_{3}-10$ percent Be0 coating. Two thermocouples were used: one $0.0762 \mathrm{~cm}$ in diameter, the other $0.127 \mathrm{~cm}$; each with appropriate radiation corrections agreed in regard to temperatures. The radiation corrections were obtained from sodium line reversal measurements of the flame temperature. The sodium was injected into the flame through a center tube by bubbling either the fuel/air mixture or nitrogen through a saturated salt water solution. The choice of which gas to bubble through the solution was based upon minimizing the flame temperature perturbation of the sodium injection. The temperature change was typically less than $25 \mathrm{~K}$. Line reversal measurements were performed using a tungsten filament source and a 1/2-m Jarrell Ash monochromator and observing the normal cautionary procedures $[23,24]$. The discrepancy between the thermocouple indication and the line reversal temperature was taken as the radiation correction and used over a limited temperature range, $\simeq 50 \mathrm{~K}$, about the calibration point. Corrections were determined at several different temperatures.

\subsection{CARS computer code}

A computer model to synthesize CARS spectra as a function of density, temperature, and background susceptibility has been formulated by one of the authors (RJH) [Appendix I, 25]. The model is currently capable of generating $\mathrm{N}_{2}, \mathrm{CO}, \mathrm{O}_{2}, \mathrm{H}_{2}$, and $\mathrm{H}_{2} \mathrm{O}$ spectra. Upon inputting selection of the molecular species of interest together with density and temperature, the model calculates the state populations $n_{v, J}$ and energies, $h w_{v, J}$, where $h$ is Planck's constant and $v, J$ the vibrational and rotational quantum numbers for the state of interest. Next, the program computes the complex third order nonlinear susceptibility. The CARS radiation is proportional to the square of the absolute value of the susceptibility. The third order nonlinear susceptibility may be written as

$$
x^{(3)}=\sum_{j}\left(x^{\prime}+i x^{\prime \prime}\right)_{j}+x^{n r}
$$

where $\left(x^{\prime}+i x^{\prime \prime}\right)_{j}$ is the resonant susceptibility associated with transition $j$ and $x^{n r}$ is the nonresonant susceptibility contribution of the electrons and remote resonances. The resonant contribution may be expressed as 


$$
\left(x^{\prime}+i x^{\prime \prime}\right)_{j}=k_{j} \frac{\Gamma_{j}}{2 \Delta w_{j}-i \Gamma_{j}}
$$

where the detuning frequency $\Delta w_{j}=w_{j}-\left(w_{1}-w_{2}\right)$ has been introduced. $w_{j}$ is the frequency of a particular possible transition from $v, J \rightarrow v^{\prime}, J^{\prime}$ and equal to $\left|w_{v, J}-w_{v^{\prime}, J^{\prime}}\right|$. Selection rules govern which transitions are allowed. $\Gamma_{j}$ is the Raman linewidth for the transition denoted by $j . K_{j}$ is the modulus of the susceptibility and equal to

$$
k_{j}=\frac{2 c^{4}}{\pi w_{2}^{4}} n \Delta_{j} g_{j}\left(\frac{\partial \sigma}{\partial \Omega}\right)_{j} \frac{1}{\Gamma_{j}}
$$

where $\hbar$ is Planck's constant divided by $2 \pi ; n$, the total species number density; $\Delta_{j}$, the normalized population difference between the levels involved in the transition; $g_{j}$, linestrength factor equal to $\left(v_{j}+1\right)$; and $(\partial \sigma / \partial \Omega)_{j}$, the Raman cross-section for the transition. The susceptibility is calculated by summing over all appropriately allowed transitions and selecting a value for the nonresonant susceptibility. The susceptibility emerges from this subroutine as a function of $w_{1}-w_{2}$. Next, the square of the absolute value of the susceptibility is calculated together with a convolution over the function $g\left(w_{1}\right)^{2} g\left(w_{2}\right)$, where $g\left(w_{j}\right)$ is the laser lineshape at frequency $w_{1}$. This accounts for the scaling of the CARS radiation as $\mathrm{I}_{1}{ }^{2} \mathrm{I}_{2}$ where $\mathrm{I}_{i}$ is the laser intensity at frequency $w_{i}$. In the program, Gaussian fits to the laser lineshapes are used. The $w_{1}$ halfwidth is $1.2 \mathrm{~cm}^{-1}$ and $w_{2}$ linewidth appropriate to the experimental value, typically 150 to $300 \mathrm{~cm}^{-1}$. Finally the CARS spectrum is convoluted over a monochromator slit function, generally taken to be triangular. At the display terminal, plots are available of the susceptibility, and the CARS spectrum before and after the monochromator slit convolution.

\section{3 CARS temperature measurements}

CARS spectra from $\mathrm{N}_{2}$ in atmospheric pressure, flat flames sustained on the previously described burners were obtained by scanning the broadband CARS spectrum with the $1-m$ double monochromator. Measurements were typically performed about $1.3 \mathrm{~cm}$ above the burner surface. BOXCARS was employed with angles of $\alpha=2.14^{\circ}, \theta=2.5^{\circ}$ and $\phi=1.95^{\circ}$. The spatial resolution was determined by generating CARS from within a translatable $0.015-\mathrm{cm}$ thick microscope slide cover. Signal generation occurred entirely within a $0.5-\mathrm{cm}$ extent with over 90 percent of the signal originating from within $3 \mathrm{~mm}$.

In figure 6 is displayed the CARS spectrum (solid line) obtained at a nominal spectral resolution of $1 \mathrm{~cm}^{-1}$ above the $2.5 \mathrm{~cm}$ diameter hexagonal burner operating at $2110 \mathrm{~K}$ as determined by the radiation corrected thermocouple. The dotted curve is the computer synthesized spectrum which gave the best agreement with the experimental trace. Best agreement was obtained at $2150 \mathrm{~K}$ using constant $0.1 \mathrm{~cm}^{-1}$ linewidths throughout the program. 
Variable (i.e., with rotational quantum number) linewidths have not been studied as yet. In figure 6 , the computed CARS spectrum is displayed for a $0.75 \mathrm{~cm}^{-1}$ monochromator resolution. The monochromator typically possesses better spectral resolution than the nominal resolution indicated by the slit width. As seen, very good agreement with the experimental trace has been obtained. In the $0 \rightarrow 1$ band, the $10 \mathrm{w} \mathrm{J} Q$ branches are unresolved. At higher rotational quantum numbers, the splitting of the band due to the vibration-rotation interaction, $a_{e} J(J+1)$, is sufficiently large to permit the resolution of individual even $J Q$ branches, ranging from $Q(28)$ to $Q(40)$. The odd $Q$ branches, which have a nuclear spin weighting equal to half of the even numbered branches, are reduced in intensity by about a factor of four and do not stand out. For Q branches beyond Q (40), overlap with the $1 \rightarrow 2$ band transitions occurs, giving rise to two prominent peaks in the "hot" band. Reproducing these features in the computer spectrum places stringent requirements on the vib-rotational energy level positions used in the calculation. Because the homogeneous linewidths are expected to be $\sim 0.1 \mathrm{~cm}^{-1}$ or less, the energy level positions must be calculated to an accuracy of much less than $0.1 \mathrm{~cm}^{-1}$ if these spectral coincidences are to be predicted accurately. The predicted hot band features have been found to be very sensitive to the values selected for the vibration-rotation interaction parameter, $\alpha_{e}$, and the line positions $v=0,1$, and 2 . It has been found that the use of the more recent $\alpha_{e}$ values [26,27] in conjunction with the various sets of vibrational anharmonic constants $[28,29]$ or measured line positions [30] all fail to reproduce the observed spectral overlaps. The agreement shown in figure 6 was obtained by employing the $\alpha_{e}$ and $w_{01}$ values of Bendtsen [26] together with an adjusted $w_{02}$ value of $4631.34 \mathrm{~cm}^{-1}$. This value is within $0.05 \mathrm{~cm}^{-1}$ of that inferred from examination of the Lyman-Birge-Hopfield system [31].

Broadening of the Raman Q-branches is mainly due to rotationally inelastic collisions and, therefore, the cross-section may be a complicated function of temperature and concentration. In the foregoing computer calculation, a J-independent value of $0.1 \mathrm{~cm}^{-1}$ was employed as mentioned. An indication of the sensitivity of these predictions to assumed linewidth is shown in figure 7 where the calculation of figure 6 is compared to one based on a linewidth value of $0.0375 \mathrm{~cm}^{-1}$. This is the value of the linewidth that results from a $T^{-1 / 2}$ scaling of the room temperature linewidth. As seen, the predicted spectrum is moderately sensitive to this variation in linewidth. As the linewidth is decreased, the slope of the fundamental band hot side and the relative intensity of the hot band decrease. The discrepancy in the spectra in figure 6 would be worse for 1 inewidths less than $0.1 \mathrm{~cm}^{-1}$. As the linewidth is reduced, higher temperatures would be required to match the computed and experimental spectra, increasing the discrepancy between the CARS and thermocouple measurement of temperature. Furthermore, temperatures above the adiabatic flame temperature of $\mathrm{CH}_{4} /$ air, $2236 \mathrm{~K}$ [32], would be required to force a fit at reduced 1inewidths. At larger linewidths, e.g., $0.125 \mathrm{~cm}^{-1}$, a sharp peak is predicted on the $\mathrm{N}_{2} \mathrm{v}=0 \rightarrow 1$ bandhead which is not seen experimentally. For the flames studied, the $\mathrm{N}_{2}$ Raman Q-branch 1 inewidths appear to be $\sim 0.1 \mathrm{~cm}^{-1}$. While the sensitivity of $\mathrm{N}_{2}$ CARS thermometry to the unknown homogeneous widths is not extreme, more information about the temperature and concentration dependences of these widths would obviously be desirable for very accurate work. 


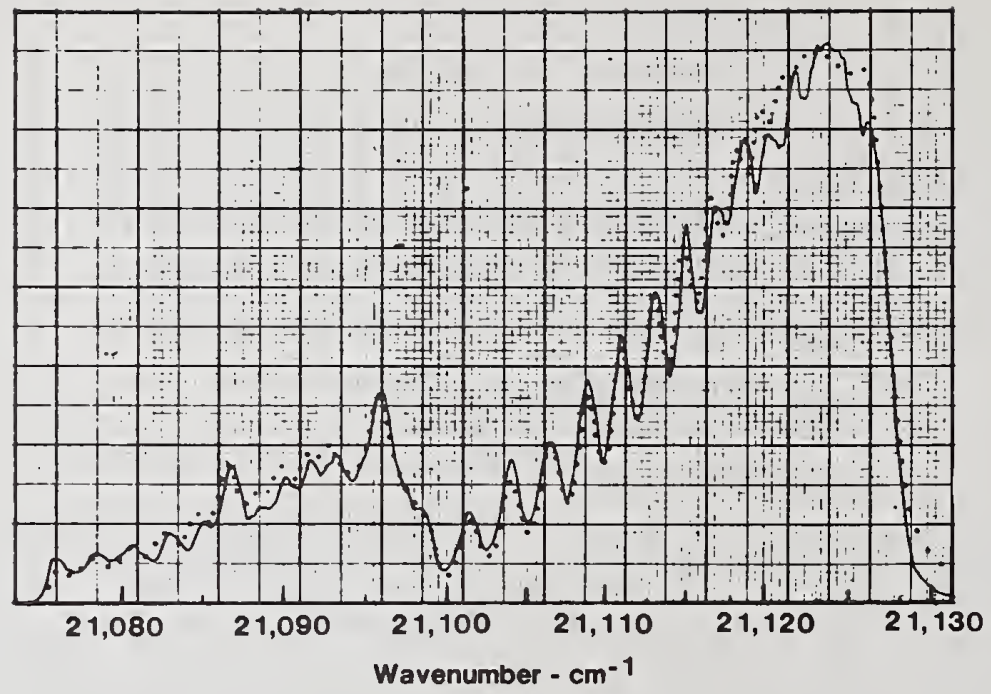

Figure 6. BOXCARS spectrum of $\mathrm{N}_{2}$ over $2.5-\mathrm{cm}$ diameter hexagonal flat flame burner operating on $\mathrm{CH}_{4}$ /air at $2110 \mathrm{~K}$ and nominal $1 \mathrm{~cm}^{-1}$ spectral resolution. Dotted curve is the best computer fit at $2150 \mathrm{~K}, 0.8 \mathrm{~cm}^{-1} \mathrm{slit}$, and $0.1 \mathrm{~cm}^{-1}$ Raman linewidths.

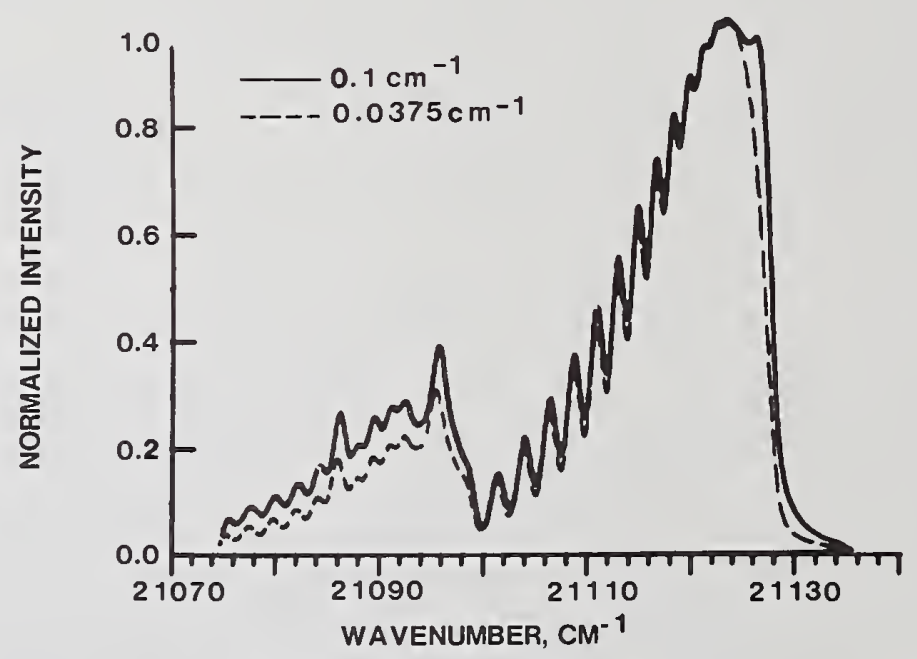

Figure 7. Sensitivity of computed $\mathrm{N}_{2}$ CARS spectrum at $2150 \mathrm{~K}$ to assumed homogeneous linewidth for $0.8 \mathrm{~cm}^{-1} \mathrm{slit}$. 
At lower spectral resolution $\left(2.7 \mathrm{~cm}^{-1}\right.$ ) the individual Q-branches can no longer be resolved as seen in figure 8 . The theoretical prediction for $T=2150 \mathrm{~K}$ is again in good agreement with the experimental trace. For these low resolution experiments, the energy level accuracy becomes much less important in fitting the theoretical to the experimental spectrum. In figure 9 is shown the BOXCARS spectrum taken over the $7.5 \mathrm{~cm}$ diameter burner. The computed spectrum producing the best fit occurred at a temperature of $1650 \mathrm{~K}$, in good agreement with the corrected thermocouple measurements of $1610 \mathrm{~K}$. As seen in figure 10 these lower resolution spectra are a fairly sensitive function of temperature. The width and slope of the fundamental band and the relative intensity of the hot band provide a basis for accurate thermometry at low resolution. From the computed sensitivity shown and on the basis of the above computed fitted temperature determinations, measurements appear possible at this time to within $50 \mathrm{k}$.

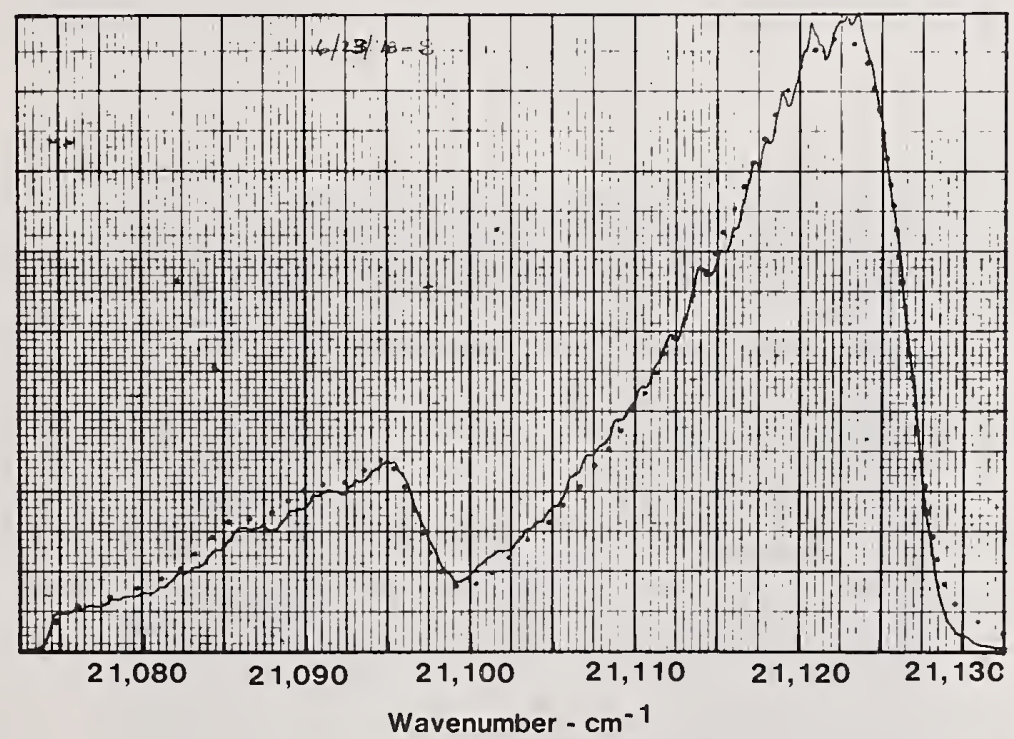

Figure 8. BOXCARS spectrum of $\mathrm{N}_{2}$ for conditions of figure 6 and nominal $2.7 \mathrm{~cm}^{-1}$ spectral resolution and dotted computer fit at $2150 \mathrm{~K}$.

\subsection{Single pulse thermometry}

For turbulent flame work, the CARS spectrum must be captured in a single pulse due to the highly nonlinear dependence of CARS on temperature and density. Scanning the spectrum over a period of time would result in an accumulation of terms involving self-and crosscorrelations between the density and temperature fluctuations. This obviously would render the spectrum ambiguous from a data reduction standpoint. Furthermore, single shot thermometry leads to a determination of the temperature probability distribution function from which the magnitude of turbulent temperature fluctuations can be obtained. 


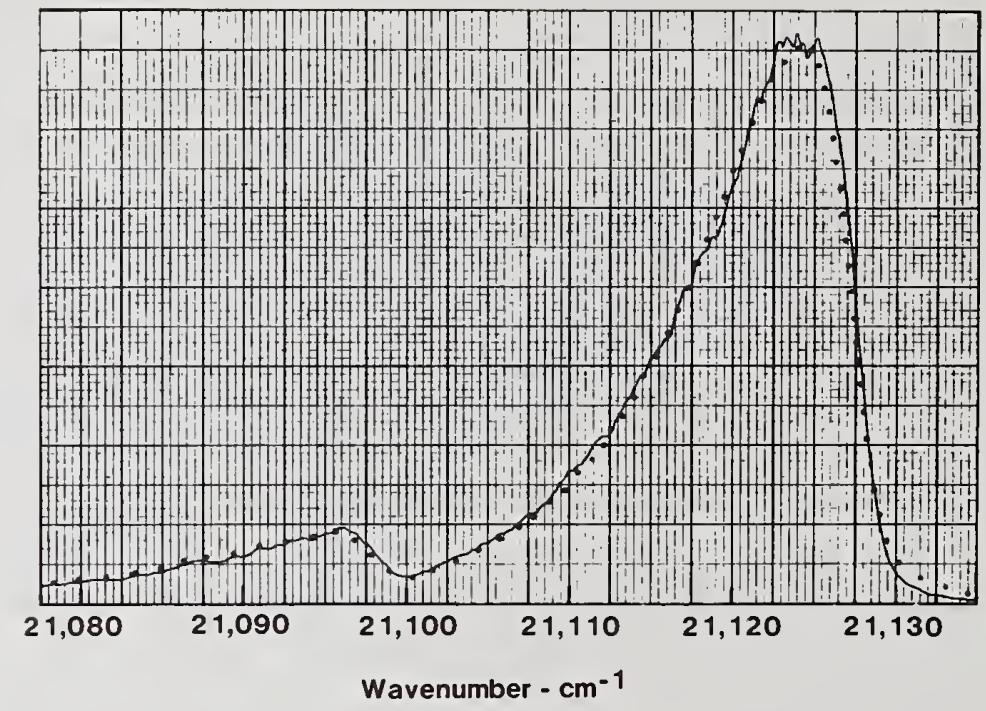

Figure 9. BOXCARS spectrum of $\mathrm{N}_{2}$ over $7.5-\mathrm{cm}$ diameter circular flat flame burner operating on $\mathrm{CH}_{4} /$ air at $1610 \mathrm{~K}^{2}$ and nominal $2.7 \mathrm{~cm}^{-1}$ spectral resolution. Dotted curve is the best computer fit at $1650 \mathrm{~K}, 0.1 \mathrm{~cm}^{-1}$ 1inewidths and $2.7 \mathrm{~cm}^{-1}$ slit.

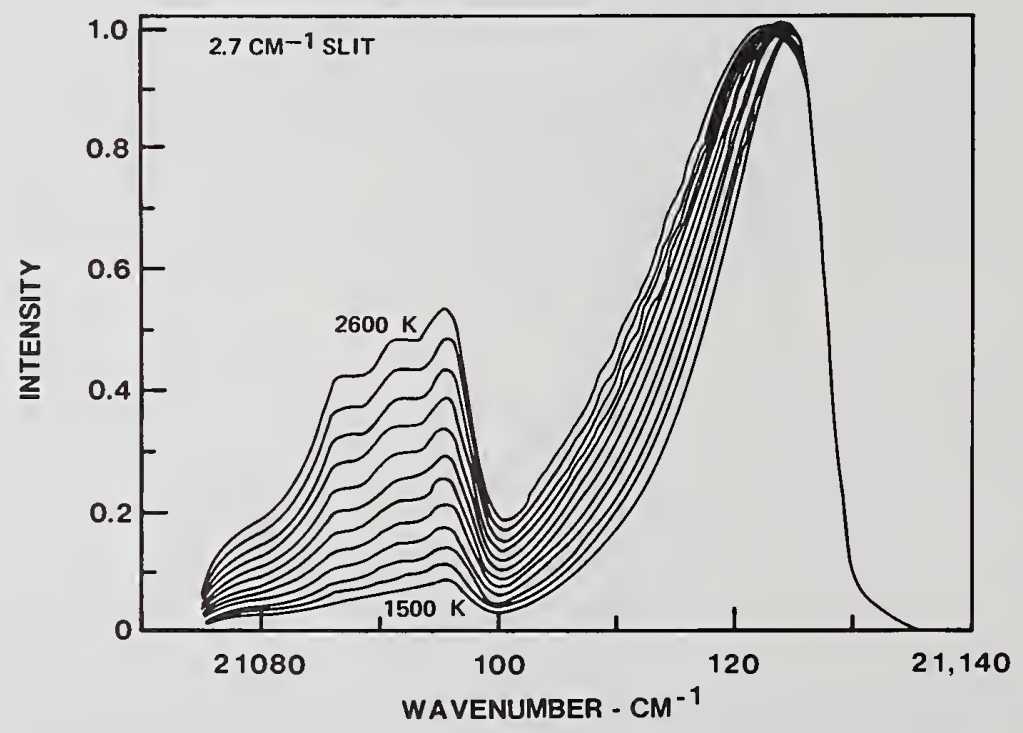

Figure 10. Computed temperature variation of $\mathrm{N}_{2}$ CARS spectra in $100 \mathrm{~K}$ steps from $1500 \mathrm{~K}$ to $2600 \mathrm{~K}$ for $2.7 \mathrm{~cm}^{-1}$ slit width.

Single pulse, CARS spectra of flame $\mathrm{N}_{2}$ were obtained using an optical multichannel analyzer (OMA, PAR) fitted to a $1 / 4-\mathrm{m}$ monochromator containing a $2400-\mathrm{gr} / \mathrm{mm}$ holographic 
grating. A single CARS pulse was captured using a photographic shutter. Since the laser operated at $10 \mathrm{pps}$, setting the shutter at 90 millisecond duration guarantees capturing only one pulse and with high probability. In figure 11 is shown the single pulse CARS spectrum of flame $\mathrm{N}_{2}$ from a specially built $2.5 \mathrm{~cm} \times 15 \mathrm{~cm}$ rectangular flat flame burner. Collinear phase matching was employed since these particular tests preceded the demonstration of crossed-beam phase matching. The long flame path eliminated potential room air contributions to the CARS signal. Each point on the spectrum corresponds to $0.39 \AA$ $\left(1.74 \mathrm{~cm}^{-1}\right)$. Also shown as the thin solid line is the computed CARS spectrum at $1700 \mathrm{~K}$ but now convolved over a $2.7 \mathrm{~cm}^{-1}$ slit function corresponding to the combined monochromatorOMA resolution. This was determined by examining the spectrum of a thin atomic line. As seen, the agreement between the OMA trace and the model prediction is quite good. No independent radiation corrected thermocouple measurement was made in this instance. The measured temperature is reasonable based on the geometry and mass flow rates employed. The data presented in figure 11 thus demonstrate the feasibility of single pulse CARS thermometry.

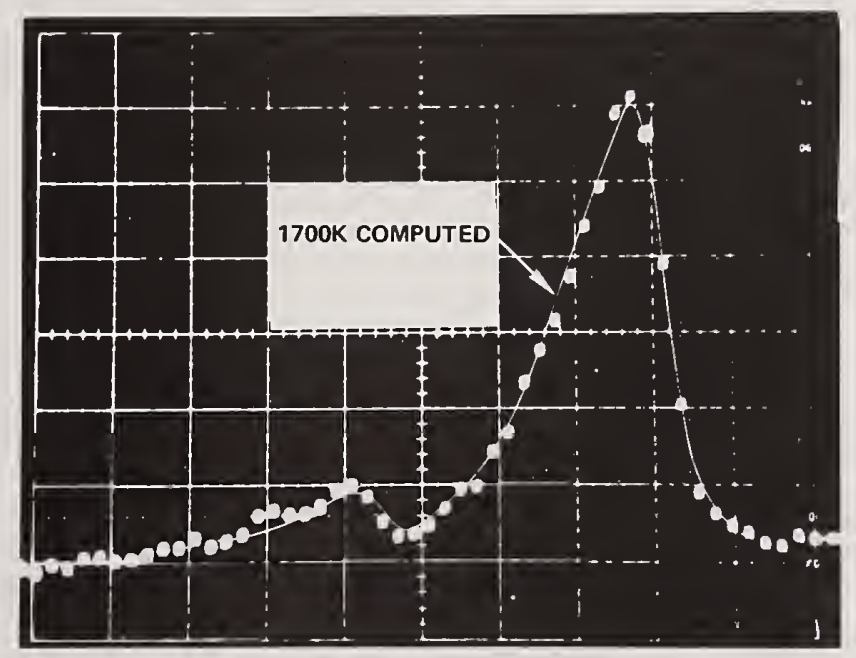

Figure 11. Single pulse CARS spectrum of flame $\mathrm{N}_{2}$ captured with an optical multichannel analyzer. Each dot represents $0.4 \mathrm{~A}$. The solid curve is the computer prediction for $\mathrm{N}_{2}$ at $1700 \mathrm{~K}$ and $2.7 \mathrm{~cm}^{-1}$ resolution.

\section{Sooting Flame Temperature Measurements}

CARS is of great interest because of its potential for operation in the highly particle laden environments typical of practical devices. To investigate this potential, CARS generation was studied in a laminar, sooting, propane diffusion flame. Such a flame was studied 
extensively in conjunction with studies of laser modulated particulate incandescence interferences in Raman scattering diagnostics [17]. The flame is sustained on a stainless steel tube, 0.6 to $1.27 \mathrm{~cm}$ i.d., and established by merely flowing propane through the tube. The flame resembles that from a candle and is highly sooting. Mie scattering diagnostics indicated a soot number density on the order of $10^{10} \mathrm{~cm}^{-3}$ with an average particle diameter of $400 \AA$. This is a very high soot level producing an attenuation in transmitted light of tenths of percent per $\mathrm{mm}$ pathlength. The requisite spatial resolution was achieved with BOXCARS using an $89 \mathrm{~mm}$ diameter, $305 \mathrm{~mm}$ focal length lens. The phase-matching angles of $\alpha=5.8^{\circ}$ and $\theta=6.5^{\circ}$ gave a resolution of approximately $0.4 \mathrm{~mm}$ in diameter by $1 \mathrm{~mm}$ long experimentally determined by generating CARS in a translatable glass slide. With a two dye component mixture in the dye laser, which produces very broad bandwidths $\left(\sim 270 \mathrm{~cm}^{-1}\right)$, the BOXCARS signals were weak, necessitating an increase in the monochromator slit width to $400 \mathrm{~m}$, or a resolution of $4 \mathrm{~cm}^{-1}$. With $4 \mathrm{~cm}^{-1}$ resolution, the broadened ground vibrational state and first hot band are still readily apparent with sufficient resolution for temperature determinations.

In figure 12 is shown one of the first BOXCARS spectra from a highly sooting, laminar propane diffusion flame sustained on a $0.95-\mathrm{cm}$ diameter tube. As is apparent, this spectrum bears no resemblance to the previously displayed hot $\mathrm{N}_{2}$ spectra. The strong spectral feature seen arises from $C_{2}$ which has a major Swan band transition at $4737 \mathrm{~A}$ midway between the $\mathrm{N}_{2}$ bands at $4733 \AA(v=0)$ and $4740 \AA(v=1)$. Swan bands are known to be degraded to the violet (higher wave numbers) in contrast to the $N_{2}$ CARS spectrum. The signal in figure 12 consists of both incoherent and, unfortunately, coherent features. The incoherent contribution is ascertained by blocking one of the $w_{1}$ or $w_{2}$ beams, or rotating the optical flat in the $w_{2}$ Stokes beam to phase mismatch the four wave mixing process. Incoherent interferences are generally suppressible in coherent spectroscopy. Coherent features are obviously more problematical. Here, the coherent feature arises from four wave mixing in laser produced $\mathrm{C}_{2}$. At the high laser intensities used to produce CARS, significant soot vaporization will occur even on a nanosecond time scale [17]. A major product of vaporizing soot is $C_{2}$. CARS can be electronically resonantly enhanced when either the CARS radiation itself or the pump component resides near an electronic transition [33]. The CARS generation in the laser produced $C_{2}$ vapor is resonantly enhanced due to the coincidence of the CARS and $\mathrm{C}_{2}$ electronic transition frequencies. In figure 12, the coherent contribution was approximately 25 percent of the total interference and roughly comparable to the CARS $\mathrm{N}_{2}$ ground state peak. The shoulder in the slope of the Swan spectrum arises from CARS from the $\mathrm{N}_{2}$ ground state. In the absence of soot particles, $C_{2}$ may actually be measurable via electronically enhanced CARS.

An optical collection system was set up at right angles to the central BOXCARS axis and the laser induced soot emissions were examined through blocking and narrowband interference filters. Emissions were examined at 4733, 5050, and $6864 \AA$ and exhibited risetimes comparable to the laser pulse ( $\sim 10 \mathrm{~ns} F W H H$ ). This is due to the fact that there is no effective soot heat transfer process other than vaporization [17] resulting in flashed 


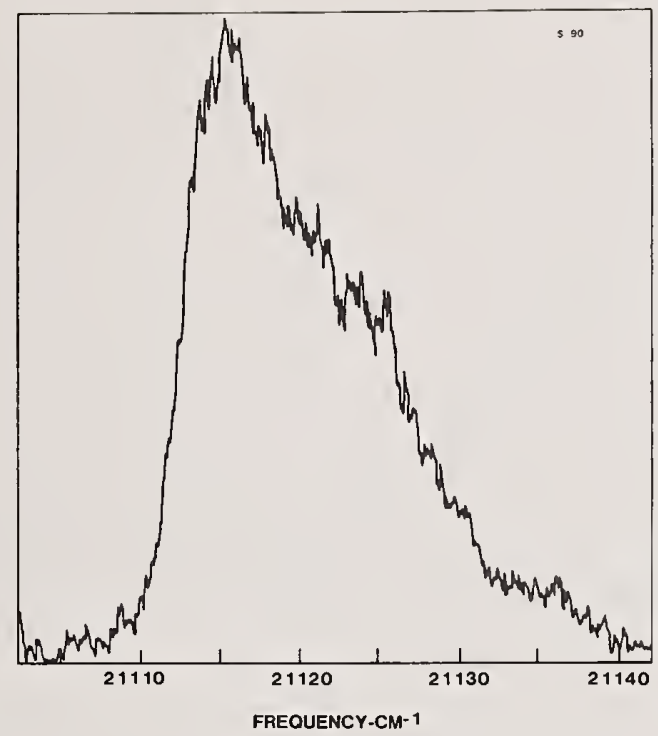

Figure 12. BOXCARS spectrum from a laminar propane diffusion flame displaying the laser-produced $\mathrm{C}_{2}$ spectral interference.

vaporization of the soot surface. The emissions at 5050 and $6864 \AA$ are laser modulated soot incandescences, while that at $4733 \mathrm{~A}$ is incandescence and primarily Swan emission at $4737 \AA$ within the filter bandpass.

Quantitatively, the emissions are quite interesting. By comparing those at $4733 \AA$ and $5050 \AA$, the Swan emissions are found to be about an order of magnitude larger than the incandescence contribution. Furthermore, the two emissions display substantially different response to the exciting laser wavelength. For example, the two high intensity $w_{1}(5320 \AA)$ beams produce slightly more incandescence than does the lower intensity $w_{2}$ beam $(6073 \AA$ ). This is expected due to the saturation behavior of the incandenscence. The Swan emissions at $4737 \mathrm{~A}$ are much more pronounced with the $6073 \mathrm{~A}$ excitation, however, than with the 5320 $\AA$, probably due to absorption of the broadband $6073 \AA$ by three Swan bands residing within the dye laser profile. The Stokes dye laser width of $270 \mathrm{~cm}^{-1}$ centered at $16466 \mathrm{~cm}^{-1}$ encompassed three strong Swan transitions at 16,333, 16,502, and 16,653 $\mathrm{cm}^{-1}$. Similar results were found for the incoherent interference component when recording the BOXCARS spectrum. Whether the enhanced Swan emission at $4733 \AA$ by the Stokes beam (6073 $\AA$ ) is via a direct vapor excitation process or vapor phase absorption leading to increased heating of the laser irradiated soot particle has not been determined.

The result did suggest decreasing the dye laser spectral bandwidth to decrease the $c_{2}$ Swan absorptions and to increase the CARS signal strength. Returning to a single dye component mixture decreased the Stokes laser bandwidth by about a factor of two to $150 \mathrm{~cm}^{-1}$ (still quite sufficient for single pulse multiplex CARS) and quite significantly resulted in about a factor of two increase in dye laser energy as wel1. Furthermore, since the 
CARS signal from $\mathrm{N}_{2}$ using horizontally polarized laser sources is horizontally polarized, an additional factor of two discrimination against incoherent interferences is obtained by insertion of a polarization filter in the CARS detection system. In so doing, the spectrum shown in figure 13 was obtained. Also shown for comparison is the computed CARS spectrum at $2300 \mathrm{~K}$ for a $4 \mathrm{~cm}^{-1}$ slit width resolution and $0.1 \mathrm{~cm}^{-1}$ assumed Raman linewidths. As seen, the computer model prediction fits the experimental spectrum fairly wel1. The adiabatic flame temperature for propane-air is $2250 \mathrm{~K}$ [32]. The rough character of the experimental spectral trace reflects the compromise made in the number of pulses averaged over, the desire to record the spectrum in a reasonable period of time $\left(10 \mathrm{~cm}^{-1} / \mathrm{min}\right)$ and use of a very short output time constant on the averager. Also shown is the location of the beginning of the Swan band peak at $21,110 \mathrm{~cm}^{-1}$ which peaks at $21,115 \mathrm{~cm}^{-1}$ and degrades to higher wave numbers. A small amount of electronically resonantly-enhanced CARS from laser produced $\mathrm{C}_{2}$ is probably present, but its contribution is quite small.

With a quieter, higher sensitivity photomultiplier tube fitted to the monochromator, improved resolution spectra have been obtained as seen in figure 14 . There, a BOXCARS spectrum is displayed at a nominal resolution of $1 \mathrm{~cm}^{-1}$ from a laminar sooting propane diffusion flame sustained on a 1.27-cIn diameter tube. The CARS measurement was made on the centerline approximately midway between the inner and outer flame cones. The best computer fit occurred at a temperature of $2000 \mathrm{~K}$. The slightly lower temperature relative to figure 13 probably results from more distributed heat release from the flame sustained on the larger tube. The foregoing results are very encouraging since the soot densities probed are quite high which bodes well for the practical utility of CARS diagnostics.

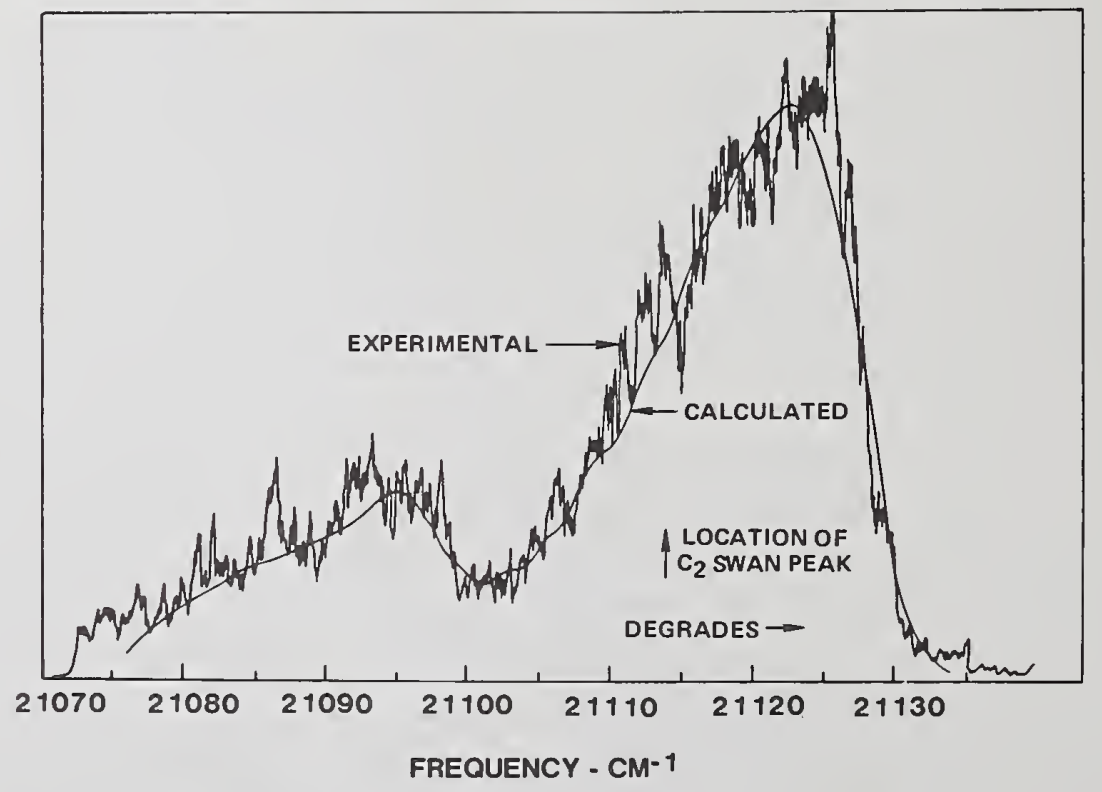

Figure 13. BOXCARS spectrum of $\mathrm{N}_{2}$ in laminar propane diffusion flame $(0.095-\mathrm{cm}$ diameter tube) with $4 \mathrm{~cm}^{-1}$ spectral resolution. The solid curve is the computed CARS spectrum at $2300 \mathrm{~K}, 4 \mathrm{~cm}^{-1}$ resolution, and $0.1 \mathrm{~cm}^{-1}$ Raman linewidths. 


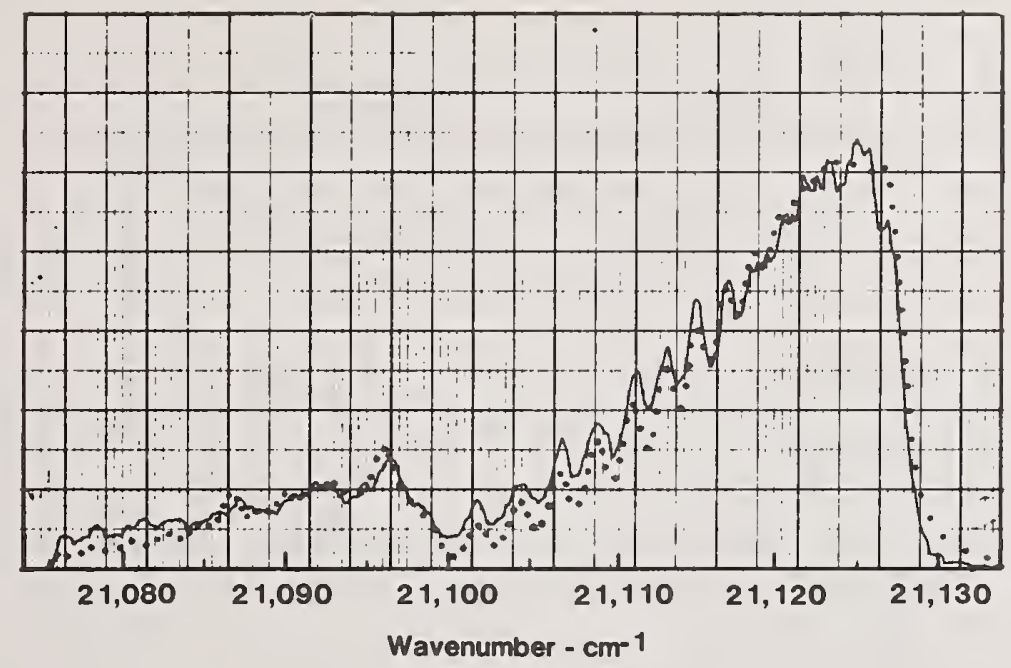

Figure 14. BOXCARS spectrum of $\mathrm{N}_{2}$ in laminar propane diffusion flame (1.27-cm diameter tube) with $1 \mathrm{~cm}^{-1}$ spectral resolution. The dotted curve is the computed CARS spectrum at $2000 \mathrm{~K}, 1 \mathrm{~cm}^{-1}$ resolution, and $0.1 \mathrm{~cm}^{-1}$ Raman linewidths.

\section{Species Concentration Measurements}

Species detection sensitivity is limited for conventional CARS approaches not due necessarily to an inadequacy of signal as is often the case in spontaneous Raman scattering, but due to the presence of the background nonresonant susceptibility. The nonresonant susceptibility is essentially an electronic contribution from all of the molecules present. Species sensitivity limitation is best illustrated by considering the case of a single resonance in the presence of background. The susceptibility may be expressed as before

$$
x=x^{\prime}+i x^{\prime \prime}+x^{n r}
$$

where $x^{\prime}, x^{\prime \prime}$, and $x^{n r}$ are, respectively, the resonant and nonresonant components of the susceptibility. The square of the absolute value of the susceptibility is

$$
|x|^{2}=x^{\prime 2}+2 x^{\prime} x^{n r}+x^{n r^{2}}+x^{\prime \prime}
$$

or in the case of a very weak resonance

$$
|x|^{2} \approx x^{n r^{2}}+2 x^{\prime} x^{n r}
$$


Since $\chi^{\prime}$ varies from negative to positive about line center, the presence of a species in low concentrations is apparent by a similar modulation in the CARS background susceptibility profile. In broadband CARS, the background nonresonant susceptibility profile merely mirrors the Stokes laser profile. In narrowband, scanned CARS, it reflects the tuning power variation of the Stokes laser. When the species concentration becomes very low, i.e., $x^{\prime} \ll x^{n r}$, the "signal" is lost in the background susceptibility profile.

\subsection{Model calculations}

The foregoing situation is quite evident in the detailed computer calculations of flame Co, some of which are summarized in figure 15. There, computer calculations are displayed as a function of CO concentration in the postflame region of an atmospheric pressure $\mathrm{CH}_{4}$-air flame at $1700 \mathrm{~K}$. The background susceptibility was calculated from the values [34] at $6943 \AA$ assuming a postflame composition of 70 percent $\mathrm{N}_{2}, 20$ percent $\mathrm{H}_{2} \mathrm{O}$, and 10 percent $\mathrm{CO}_{2}$. Any variation in the nonresonant susceptibility with wavelength was not accounted for. In the absence of data on the nonresonant susceptibility of $\mathrm{H}_{2} \mathrm{O}$ vapor, it was assumed to be that of methane. Since $\mathrm{H}_{2} \mathrm{O}$ is a major constituent resulting from hydrocarbon combustion, knowledge of its nonresonant susceptibility would be quite valuable. Changes in the composition of flame products which result when varying amounts of $\mathrm{CO}$ are produced are not accounted for in the model. The spectra in figure 15 have not been convoluted with a spectrometer slit function, but do reflect the limited resolution attainable with a $1.2 \mathrm{~cm}^{-1}$ pump laser line. Also, note that the ordinate scale is not the same from plot to plot. In all cases shown, the destructive interference dip caused by the resonant portion of the susceptibility going negative is apparent, but clearly less so as the concentration decreases. At the larger concentrations shown, the co spectrum clearly rises out of the nonresonant background and displays a pronounced hot band. The computer calculations display vividly the species sensitivity limitations of CARS. At the 0.5 percent CO level, the destructive interference causes the spectrum to dip by only about 12 percent and at 0.1 percent Co only by 2 percent. For scanned, laminar flame studies, this places very stringent requirements on the pulse-to-pulse stability of the CARS signal [5]. For single pulse work, it places high resolution requirements on the detection system. For the nonresonant susceptibility selected for these calculations, it would be difficult to measure co levels below a few tenths of a percent. If the nonresonant susceptibility were larger, the detection sensitivity would be less. Not surprising, the calculated spectra are quite sensitive to the nonresonant susceptibility value selected.

\subsection{Experimental approach}

To produce large levels of Co, a water-cooled porous plug burner $7.5 \mathrm{~cm}$ in diameter was operated fuel rich, either with excess $\mathrm{CH}_{4}$ or $\mathrm{CO}$. At these high equivalence ratios, the flat flame exhibited some diffusion flame behavior, i.e., a blue conical outer secondary 
$0.1 \%$

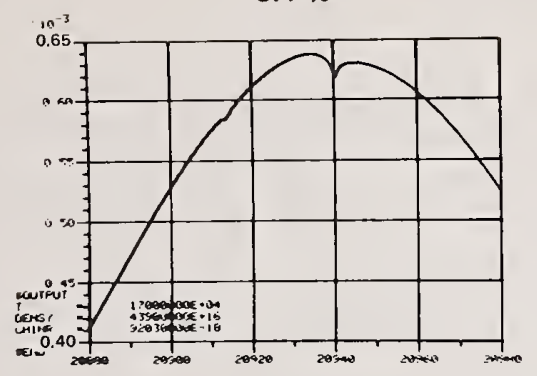

$1 \%$

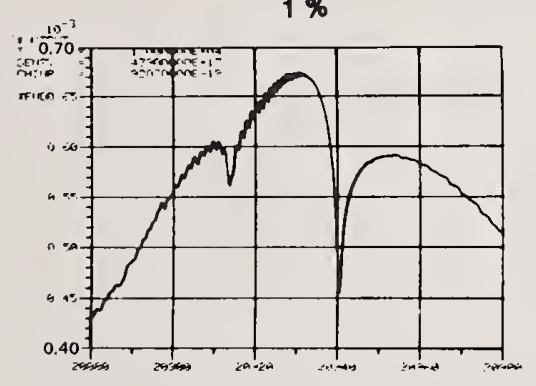

$0.5 \%$

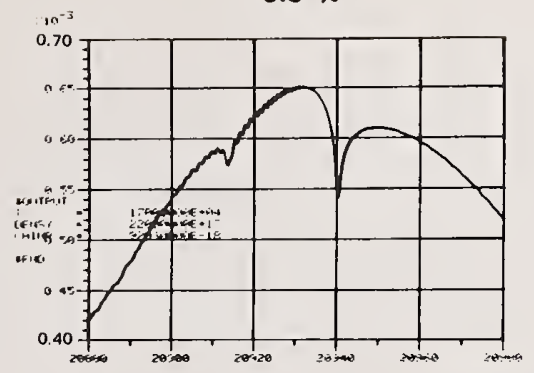

$4 \%$

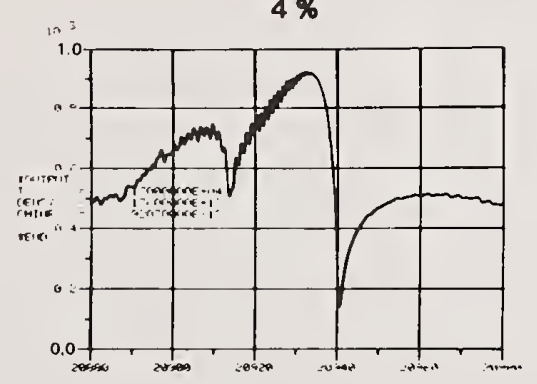

Figure 15. Computed CO CARS spectra in a $\mathrm{CH}_{4} /$ air flame at $1700 \mathrm{~K}$ for various $\mathrm{CO}$ concentration levels.

flame zone was clearly evident. Lack of agreement between collinear phase-matched c0 spectra and the computer code predictions suggested that the experimental results were spatially averaged. With BOXCARS, the signals were too weak even for small crossing angles. Apparently the spatial resolution was too fine; recall that CARS scales as the square of the interaction length. The CARS signal was found to be adequately large, however, when the $w_{1}$ and $w_{2}$ beams were merely crossed. Although the resultant phase mismatch decreases the signal conversion efficiency, this is apparently more than compensated for by the increased interaction length. A $305-\mathrm{mm}$ focal length lens was used and the pump and Stokes lasers were crossed at an angle of $1.8^{\circ}$. At this angular separation, the beams intersected only over the burner and near its centerline. Hence, no contributions from the cool regions outside the burner were possible. The spatial resolution was checked in the usual way by monitoring the CARS signal generated from a translatable microscope slide. All of the signal was generated within a $2.5-\mathrm{cm}$ extent, but the CARS originated predominantly within $\pm 0.5 \mathrm{~cm}$ of the crossing point. To measure the amount of $\mathrm{CO}$ produced in the flames, an uncooled quartz microprobe was used to sample the centerline co concentration, the level of which was indicated by a Beckman Model 864 ndir analyzer. Sampling with uncooled probes in high pressure flames is subject to some uncertainty. However, water-cooling of the probe resulted in little change in the Beckman readings. 


\section{3 Measurements}

In figure 16 is shown the CARS spectrum recorded for an ndir reading of 3.9 percent $C 0$ in a $\mathrm{CO}_{-} \mathrm{CH}_{4}$-air "flat" flame. The spectrum was obtained $7 \mathrm{~mm}$ above the burner surface. As seen due to various fluctuations in the laser, flame, etc., even the averaged CARS signal is subject to fluctuations on the order of about \pm 10 percent. In the spectrum shown, $100 \mu$ slits corresponding to a nominal $1-\mathrm{cm}^{-1}$ resolution were employed. Also shown, as points, is the 4 percent $C O$ model calculation in figure 15 convolved over a $1-\mathrm{cm}^{-1}$ slit function. As seen, the agreement between the experimental spectrum and the model calculation is quite good.

The quality of the spectra degraded rapidly, however, at lower co concentrations indicated by the quartz microprobe sampling system. For given $\mathrm{CH}_{4}$-air settings, the $\mathrm{CO}$ spectra obtained at lower $\mathrm{CO} f \mathrm{flame}$ levels as the CO doping level was reduced did not behave as those shown in figure 15. Modulation of the CARS spectrum was apparent at the 2 percent level and to the trained eye at 1 percent in the presence of the random spectral fluctuations. The spectra had the qualitative appearance of CO levels within a higher nonresonant susceptibility. CARS spectral differences were also noted at the nominal 4 percent level depending upon how the $\mathrm{CO}$ was produced, i.e., with $\mathrm{CO}$ addition or high $\mathrm{CH}_{4}$ fractions. This may be due to a change in the nonresonant susceptibility. $\mathrm{CH}_{4}$ has a nonresonant susceptibility over twice that of $\mathrm{N}_{2}$ and to produce the 4 percent co level, over twice the $\mathrm{CH}_{4}$ flow relative to that required for good flame behavior.

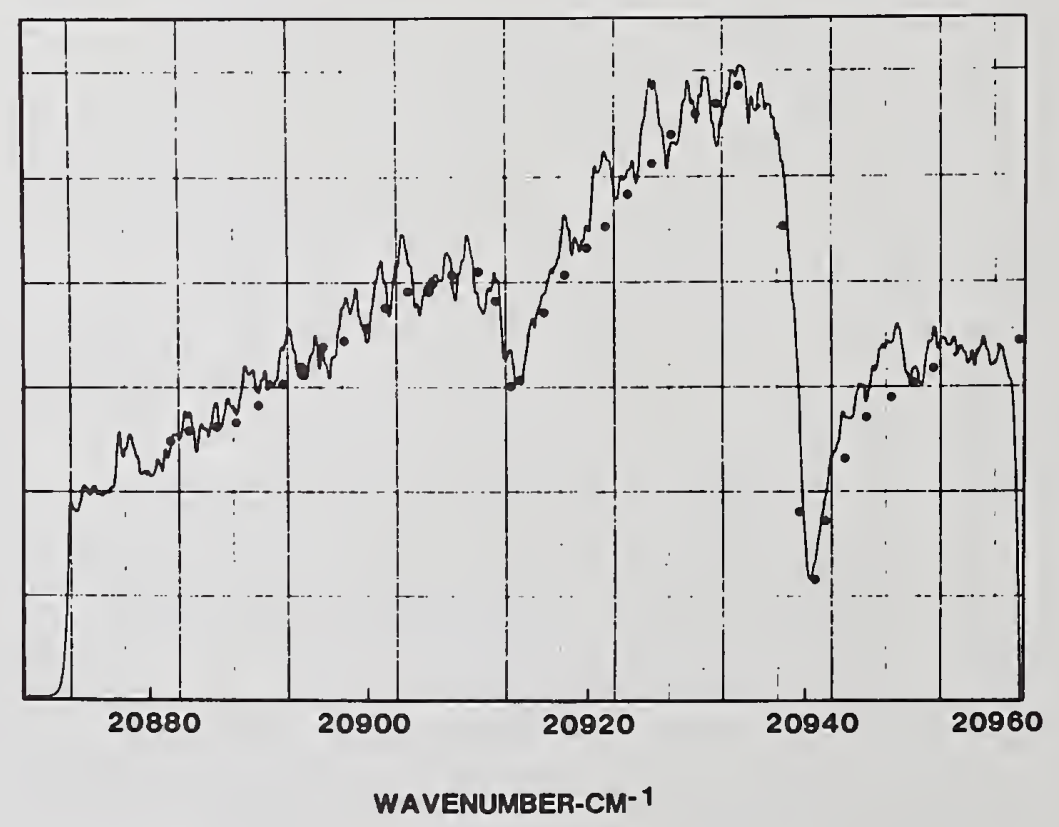

Figure 16. Crossed-beam, phase-mismatched CARS spectrum of 3.9 percent $C 0$ at a nominal spectral resolution of $1 \mathrm{~cm}^{-1}$. The points are the computer prediction for 4 percent $\mathrm{CO}$ at $1700 \mathrm{~K}$ and a nonresonant susceptibility of $9.2\left(10^{-19}\right) \mathrm{cm}^{3} / \mathrm{erg}$. 
CARS measurements of $\mathrm{CO}$ species concentration in the presence of an uncertain nonresonant susceptibility require a three-parameter model fit involving the concentration, temperature, and nonresonant susceptibility. Parametric variations with $N$ and $x_{N R}$ show, however, that these solutions are not unique, and that the inferred $\mathrm{CO}$ mole fraction is subject to an uncertainty determined by the uncertainty in $x_{N R}$. In the limit of small $N$, eq. (11) gives

$$
|x|^{2} \sim x_{N R}\left(x_{N R}+2 x_{R}^{\prime}\right)=x_{N R}^{2}\left(1+\frac{2 N}{x_{N R}} \bar{x}_{R}^{\prime}(w)\right)
$$

where $\bar{x}_{R}^{\prime}$ is the molecular resonant susceptibility. Thus, there will be a family of solutions determined by $N / x_{N R}=$ const. that have the same dispersive profile. A calculation with the computer model confirms that the experimental spectrum of figure 16 can be fit with a $\mathrm{CO}$ mole fraction of 0.03 if $x_{N R}$ is taken to be 0.75 times its nominal value. This result demonstrates the need for a measurement of the $\mathrm{H}_{2} \mathrm{O}$ nonresonant susceptibility, and suggests that CARS minority species measurements would be most accurately performed in conjunction with computer modeling of the majority species concentration profiles. If the nonresonant susceptibility of $\mathrm{H}_{2} \mathrm{O}$ is comparable to that of $\mathrm{O}_{2}$ and $\mathrm{N}_{2}$ which are nearly equal [34], concentration measurements can be performed without the requirement to know the gas composition very accurately. In such an instance, changes in composition would have little effect on the value of the nonresonant susceptibility. Note that in the presence of a known background susceptibility, concentration measurements over a limited range can be performed from the shape of the CARS spectrum (fig. 15); absolute intensity determinations are not necessary.

In the present case, measurements were possible to only the 1-2 percent level at best. Analytically, species concentration measurements appear to be practically possible to about the 0.5 percent level or so (fig. 15) for the nonresonant susceptibility assumed. Clearly, CARS approaches which lead to a cancellation of the background nonresonant susceptibility, some of which are reviewed in [18], would seem desirable for species concentration measurements.

\section{Summary}

Coherent anti-Stokes Raman spectroscopy (CARS) is capable of both temperature and species concentration measurements in flames with good spatial resolution. A crossed-beam, phase-matching approach, termed BOXCARS, has been described which can provide very high spatial precision and, quite importantly, eliminates the spatial ambiguities associated with conventional collinear phase matching. Using this technique, CARS temperature measurements have been performed in premixed, flat flames at various temperatures. The experimenta] 
CARS spectra show very good agreement with computer synthesized spectra permitting temperature to be ascertained. These measurements agreed to within $50 \mathrm{~K}$ of those determined by radiation corrected thermocouples. Single shot CARS spectra from flame $\mathrm{N}_{2}$ obtained with an optical multichannel analyzer show good agreement with computer code predictions and demonstrate the feasibility of single pulse thermometry. CARS temperature measurements have been performed in highly sooting flames. Although interferences were encountered, they were not serious and were suppressible. CARS thus appears capable of measurements in practical combustion environments. CARS spectra of $\mathrm{CO}$ also show very good agreement with code predictions. These spectra were obtained using a purposely phase-mismatched crossbeam technique and demonstrated the limited species detection sensitivity of CARS with aligned field polarizations.

\section{References}

[1] Regnier, P. R. and Taran, J. P. E., On the possibility of measuring gas concentrations by stimulated anti-Stokes scattering, Appl. Phys. Lett. 23, 240-242 (September 1973).

[2] Regnier, P. R., Moya, F., and Taran, J. P. E., Gas concentration measurement by coherent Raman anti-Stokes scattering, AIAA Paper 73-702, AIAA 6th Fluid and Plasma Dynamics Conference, Palm Springs, CA (July 1973).

[3] Moya, F. S., Druet, S. A. J., and Taran, J. P. E., Gas spectroscopy and temperature measurement by coherent Raman anti-Stokes scattering, Opt. Comm. 13, 169-174 (February 1975).

[4] Regnier, P. R., Moya, F., and Taran, J. P. E., Gas concentration measurement by coherent Raman anti-Stokes scattering, AIAA J. 12, 826-831 (June 1974).

[5] Moya, F., Druet, S., Pealat, M., and Taran, J. P., Flame investigation by coherent antiStokes Raman scattering, in Experimental Diagnostics in Gas Phase Combustion Systems, B. T. Zinn, ed. (AIAA, New York, NY, 1977), pp. 549-575.

[6] Maker, P. D. and Terhune, R. W., Study of optical effects due to an induced polarization third order in electric field strength, Phys. Rev. 137, A801-818 (February 1965).

[7] Begley, R. F., Harvey, A. B., and Byer, R. L., Coherent anti-Stokes Raman spectroscopy, App. Phys. Letts. $\underline{25}$, 387-390 (October 1974).

[8] Harvey, A. B., McDonald, J. R., and Tolles, W. M., Analytical applications of a new spectroscopic tool: coherent anti-Stokes Raman spectroscopy (CARS), in Progress in Analytical Chemistry (Plenum Press, 1977).

[9] Tolles, W. M., Nibler, J. W., McDonald, J. R., and Harvey, A. B., A review of the theory and application of coherent anti-Stokes Raman spectroscopy (CARS), Appl. Spect. 31, 253-272 (1977).

[10] Nibler, J. W., McDonald, J. R., and Harvey, A. B., CARS measurement of vibrational temperature in electric discharges, 0pt. Comm. 18, 371-373 (August 1976).

[11] Nibler, J. W., Shaub, W. M., McDonald, J. R., and Harvey, A. B., Coherent anti-Stokes Raman spectroscopy, in Vibrational Spectra and Structure, Vol. 6, J. R. Durig, ed. (Elsevier, Amsterdam, 1977), pp. 173-225. 
[12] Barrett, J. J. and Begley, R. F., Low-power CW generation of coherent anti-Stokes Raman radiation in $\mathrm{CH}_{4}$ gas, Appl. Phys. Letts. 27, 129-131 (1975).

[13] Barrett, J. J., Generation of coherent anti-Stokes rotational Raman radiation in hydrogen gas, App1, Phys. Letts. 29, 722-724 (December 1976).

[14] Roh, W. B., Schreiber, P. W., and Taran, J. P. E., Single-pulse coherent anti-Stokes Raman scattering, Appl. Phys. Letts. 29, 174-176 (August 1976).

[15] Eckbreth, A. C., BOXCARS: crossed-beam phase-matched CARS generation in gases, App 1 . Phys. Lett. 32, 429-423 (Apri1 1978).

[16] Nibler, J. W. and Knighten, G. V., Coherent anti-Stokes Raman spectroscopy, in Topics in Current Physics, Chapter 7, A. Weber, ed. (Springer Verlag, Stuttgart, 1977).

[17] Eckbreth, A. C., Effect of laser-modulated particulate incandescence on Raman scattering diagnostics, J. App 1. Phys. 48, 4473-4479 (November 1977).

[18] Eckbreth, A. C., Bonczyk, P. A., and Verdieck, J. F., Laser Raman and fluorescence techniques for practical combustion diagnostics, Appl. Spect. Rev. 13, 15-164 (January 1978).

[19] Shaub, W. M., Harvey, A. B., and Bjorklund, G. C., Power generation in coherent antiStokes Raman spectroscopy with focused laser beams, J. Chem. Phys. 67, 2547-2550 (September 1977).

[20] Rahn, L., Sandia Laboratories, Livermore, CA. Private communication.

[21] Hellwarth, R. W., Third-Order Optical Susceptibilities of Liquids and Solids (Pergammon Press, 0xford, 1977).

[22] Penney, C. M., Goldman, L. M., and Lapp, M., Raman scattering cross sections, Nat. Phys. Sci. 235, 110-112 (February 1972).

[23] Sasaki, Y., Some remarks on the temperature measurement of nonisothermal flames by the line reversal technique, Jap. J. Appl. Phys. 5, 439-446 (May 1966).

[24] Snelleman, W., Errors in the method of 1 ine reversal, Combustion and Flame, 11, 453463 (December 1967).

[25] Eckbreth, A. C., Bonczyk, P. A., and Shirley, J. A., Investigation of saturated laser flourescence and CARS spectroscopic techniques for combustion diagnostics, Report EPA-600/7-78-104 (June 1978).

[26] Bendtsen, J., The rotational and rotational-vibrational Raman spectra of ${ }^{14} \mathrm{~N}_{2},{ }^{14} \mathrm{~N}$ ${ }^{15} \mathrm{~N}$, and ${ }^{15} \mathrm{~N}_{2}$, J. Raman Spec. 2, 133-145 (1974).

[27] Barrett, J. J. and Harvey, A. B., Vibrational and rotational-translational temperatures in $\mathrm{N}_{2}$ by interferometric measurement of the pure rotational Raman effect, J. Opt. Soc. Am. $65,392-398$ (1975).

[28] Benesch, W., Tilford, S. G., Vanderslice, J. T., and Wilkinson, P. G., Potential curves for the observed states of $\mathrm{N}_{2}$ below $11 \mathrm{eV}$, Astrophys. J. 142, 1227-1240 (1965).

[29] Rosetti, F., Phys. Rev. 34, 367 (1929); Z Physik, 6], 598 (1930).

[30] Lofthus, A., Spectroscopic Report No. 2, Dept. of Physics, University of 0s1o (1960).

[31] Lofthus, A. and Krupenie, P. H., The spectrum of molecular nitrogen, J. Phys. Chem. Ref. Data, $\underline{6}, 113-307$ (1977). 
[32] Kanury, A. M., Introduction to Combustion Phenomena (Gordon and Breach, New York, 1975), p 131.

[33] Hudson, B., Hetherington III, W. , Cramer, S., Chabay, I., and Klauminzer, G. K., Resonance enhanced coherent anti-Stokes Raman scattering, Proc. Nat1. Acad. Sci. U.S.A. 73, 3798-3802 (November 1976).

[34] Rado, W. G., The nonlinear third order dielectric susceptibility coefficients of gases and optical third harmonic generation, App1. Phys. Letts. 11, 123-125 (August 1967).

\section{Discussion}

Comment (Shaub): In discussing advantages of BOXCARS over CARS for spatial resolution, the speaker mentions that CARS signal generation occurs over a considerable length of the probe gas. This argument is valid when considering solution of the wave equation assuming plane fields. However, if one assumes Gaussian electric fields it can be shown that the CARS power generation is confined to a very small volume of the probed gas sample, particularly when using tight focusing optics.

Also, regarding power generation, in CARS the signal scales with laser power as $\alpha \mathrm{I}_{1}{ }^{2} \mathrm{I}_{2}$. In BOXCARS, the proportionality is $\alpha\left(1 / 2 \mathrm{I}_{1}\right)\left(1 / 2 \mathrm{I}_{1}\right) \mathrm{I}_{2}$ or $1 / 4 \mathrm{I}_{1}{ }^{2} \mathrm{I}_{2}$, a factor of four reduction in signal strength, if one assumes the $I_{1}$ beam is split in half. 
National Bureau of Standards Special Publication 561, Proceedings of the 10th Materials Research Symposium on Characterization of High Temperature Vapors and Gases held at NBS, Gaithersburg, Maryland, September 18-22, 1978. Issued October 1979.

\author{
COMPARISON OF THERMOCOUPLE, GAS SAMPLING, AND RAMAN MEASURED
} TEMPERATURES IN AN AFTERBURNING TURBOJET ENGINE PLUME

\author{
W. M. Roquemore \\ Air Force Aero Propulsion Laboratory \\ Wright-Patterson AFB, OH 45433 \\ and \\ Perry Pappas Yaney ${ }^{1}$ \\ Department of Physics \\ University of Dayton \\ Dayton, $\mathrm{OH} 45469$
}

This paper describes the results of plume temperature measurements made with three different probes: a Pt,13\% Rh-Pt thermocouple probe, a gas sampling probe, and a spontaneous-Raman-scattering optical probe. Measurements were made on the centerline of the chemically reactive plume of an afterburning 3855 turbojet engine, about one meter from the nozzle exit. The engine was operated at 12 different fuel-air ratios ranging from 0.0094 to 0.0517 . Thermocouple measurements were corrected for radiation and recovery. A combination gas-sampling-andpressure probe was used to measure carbon dioxide, carbon monoxide and total hydrocarbon concentrations, and total gas stream pressures. Temperatures were calculated from the concentration measurements. Raman scattering measurements were made in a near-backscattering geometry using a pulsed nitrogen gas laser ( $3371 \AA$ ), a computer-controlled SPEX double spectrometer, and an 8850 RCA photomultiplier tube with EGG/ ORTEC time-gated current-integration detection apparatus. Raman temperatures were determined by fitting calculated spectra to the experimental spectra of the nitrogen gas in the plume. Simultaneous Raman and gas sampling measurements made at nearly the same axial location in the plume were compared. An observed correlation between engine fuel-air ratio and temperature, was also used to compare the

${ }^{1}$ Work reported here was performed through the auspices of the Research Institute of the University of Dayton. 
three techniques. The temperatures measured by the Raman, gas-sampling, and thermocouple techniques were in agreement within the experimental error of about 5 percent except at maximum engine power where the thermocouple deviated by about 10 percent.

\section{Introduction}

Thermocouple and gas sampling probes are state-of-the-art techniques for measuring temperatures in practical combustion devices. Because they are simple to make, easy to use, rugged and inexpensive, probes will remain the standard methods of measuring temperatures in combustion devices for the forseeable future. However, there are concerns that probes can seriously perturb certain combustion environments to the extent that the measured temperatures are not representative of the environment when the probes are removed. This type of effect is not new. The indicated temperature of a thermocouple in the plume of an afterburning jet engine is not the true temperature of the plume. Radiation and recovery effects can result in more than a 5 percent bias error. These effects are understood and the true temperature can be estimated using correction factors. However, there are other effects that are not well understood which could lead to significant errors. Such effects include: catalytic or other surface reactions that could occur on the tip of noble metal thermocouples, (2) gas samples which are altered because of continued reactions in the probe or sampling line, and (3) the change in the combustion process due to the presence of a watercooled probe in a turbulent reaction zone. Most of the potentially biasing effects occur because a foreign object has been placed in the combustion zone.

Laser Raman scattering offers the potential of making non-intrusive, point temperature measurements in combusting gases. However, there are uncertainties associated with Raman measurements. The largest uncertainty is whether one can obtain sufficiently defined Raman spectra that will permit temperature determinations. This concern arises because spontaneous Raman scattering is an extremely weak effect. For example, with a laser pulse containing about $10^{12}$ photons, one can normally observe one Raman photon from a typical Raman band of a molecular gas. Because of this, Raman scattering is susceptible to laser-induced and nonlaser-induced background radiation which tends to mask the spectra. Normally, one cannot determine a priori whether Raman will or will not work in a specific practical combustion environment.

This paper reports the results of using Raman scattering to measure temperatures in the plume of an afterburning J85-5 engine. The remainder of this paper is divided into five sections. The next section (Section 2) presents background information about thermocouple, gas sampling and Raman techniques as combustion diagnostic tools. The instruments used in this study and their theory of operation are described in Section 3. The experiment is described in Section 4 with the results given in Section 5 . Our summary and conclusions are presented in Section 6 . 


\section{Background}

The development of thermocouple technology over the last 30 years has firmly established the general principles of operation for these devices. Many different materials and designs have been evaluated for a wide variety of aircraft engine applications. As a result, thermocouple capabilities and limitations are reasonably well understood. The cause of errors have been identified and methods for correcting some of the bias errors have been developed. The literature is too voluminous to review in this paper. A comprehensive source of information on thermocouples is given in [1] ${ }^{2}$. Specific information that was extracted from the literature in performing this study, is referenced in Section 3. 1 .

Gas sampling probes are routinely used to measure gaseous emissions from gas turbine engines. Emissions measurements are used to determine local fuel-air ratios, combustion efficiencies, pollutant concentrations, and local temperatures. Because all the probe elements can be water-cooled, they can be used in very high temperature environments where thermocouple elements would normally melt. Thus, gas sampling probes are indispensable in combustor development programs.

Gas sampling technology has been developing over the last 20 years with most of the work occurring within the last 10 years. A stimulus for recent developments has been the need to standardize measurement procedures for accessing the impact of aircraft pollutants on the environment. In 1968, a special committee, E-31, under the auspicies of the Society of Automotive Engineers, was established for the purpose of developing standard procedures for measuring emissions in aircraft engine exhausts. This resulted in the Aerospace Recommended Practices for measuring gaseous emissions given in [2]. Other studies have resulted in recommended procedures for measuring emissions in afterburning engines [3].

The measurement errors associated with using gas-sampling probes in different environments are not as well understood as they are for thermocouples. Studies are in progress to determine probe and sampling line effects on certain emissions such as CO and NO species [4]. It is likely that such studies will result in modification of the standard procedures. Procedures for calculating temperatures from emissions measurements have not been standardized. The principles behind the calculations are well understood; however, the temperature calculations are involved and there are numerous details that probably vary among different laboratories. Section 3.2 gives the procedures we used in calculating temperatures from emissions measurements.

Raman spectroscopy is a relatively new technique for studying combustion gases. Raman technology has been expanding over the past 10 years with most of the combustion-centered research occurring within the last five years. A large volume of literature has resulted in this short time. A collection of reports on most of the early Raman gas diagnostic studies is given by Lapp and Penney [5].

Numerous Raman scattering experiments have been performed in flames as noted in a review by Lapp and Penney in [6]. The theory is we11 developed for making temperature and species

${ }^{2}$ Figures in brackets indicate the literature references at the end of this paper. 
concentration measurements in high temperature gases. Experiments in flames have demonstrated the validity of the theory. Lederman [7] has given an in-depth review of Raman theory, experimental configurations, and experimental results of Raman measurements in gases. A Raman technique has been used with laser Doppler velocimetry in a common experiment [7] to obtain nearly instantaneous measurements of temperatures, major species concentrations, and gas velocities in turbulent laboratory flames. From this body of information, one can conclude that Raman scattering can be effectively used in studying simple laboratory flames.

The use of Raman scattering in practical combustion environments has met with some successes and some failures. Leonard [8] has described Raman measurements made in a jet engine exhaust. At idle engine power condition, a broad-band, laser-induced background was observed which masked all Raman spectra. The background decreased rapidly with increasing engine power, permitting Raman measurements of $\mathrm{CO}_{2}$ concentrations and $\mathrm{O}_{2} / \mathrm{N}_{2}$ ratios over a wide range of engine conditions. The intensity of the background varied directly with total unburned hydrocarbons. This suggested that the background was due to fluorescence from some of the gaseous hydrocarbons. Bailly, et al. [9] also observed a broad-band background in the exhaust of a small supersonic rocket burning kerosene fuel. This background was also believed to be due to fluorescence. When the exhaust was above $1000 \mathrm{~K}$, the background disappeared and Raman measurements of $\mathrm{N}_{2}, \mathrm{O}_{2}, \mathrm{CO}_{2}$, and $\mathrm{H}_{2} \mathrm{O}$ concentrations were made. Setche11, et a1. [10] encountered laser-induced fluorescence in Raman studies of the exhaust gases from a single-cylinder research engine. The fluorescence was strongly dependent on fuel type and engine fuel-air ratios. Fuel-rich combustion, with equivalence ratios of 1.7 , resulted in the most intense fluorescence. The fluorescence disappeared when the exhaust gases were passed through a $0.3 \mu \mathrm{m}$ filter. It was concluded that the fluorescence was due to aerosols of hydrocarbons or other organic compounds. Another form of laser-induced background has been observed by Eckbreth [11] in particulate-1aden flames and in a model research combustor. The background prevented successful Raman measurements. A detailed study showed that incandescence from particulates heated by the highpower laser beam was the probable cause of the background. Williams, et al. [12] describe rotational Raman measurements of temperatures and gas density in the plume of a rocket burning $M M H / \mathrm{N}_{2} \mathrm{O}_{4}$ liquid fuel. Neither laser-induced fluorescence or particulate incandescence was observed during these experiments. This was contributed, in part, to the clean burning of the rocket engine.

In addition to the properties of the combustion medium, the characteristics of the Raman systems used in the various studies are an important consideration because the Raman system could influence the outcome of the experiment. For example, fluorescence has been observed primarily with nitrogen or argon lasers operated in the near-ultraviolet wavelength regions. Questions pertaining to whether a "red" or a "green" laser will change the experimental outcome or to which electronic detection scheme is best suited to a given measurement must be answered by future research. Goulard [13] has assembled a comprehensive survey of these research needs including state-of-the-art developments in both probe and optical combustion diagnostic techniques. 
It is clear that Raman scattering is in the early stages of development as a tool for studying practical combustion environments. For a given combustion device and a given Raman system, there appear to be conditions where Raman measurements can be made while there are . other conditions where the measurements cannot be made. It is not clear that a priori determinations can accurately predict the outcome of a given experiment. We believe that additional studies are needed in various combustion environments using a variety of different Raman systems to clearly establish the capabilities and limitations of Raman scattering as a combustion diagnostic tool. This paper provides additional information about the application of Raman scattering as a tool for measuring temperature in an environment where Raman measurements have not been previously attempted.

\section{Measurement Techniques}

This section is divided into three parts. In Section 3.1, the thermocouple probe used in this study is described and the theory is outlined for correcting thermocouple measurements for recovery and radiation errors. The procedure used in estimating the static temperatures is included. The gas sampling probe and associated instruments used in this study are described in Section 3.2. Also, the calculations performed to obtain the gas temperatures from the gas sampling emissions are outlined. The Raman system used in this work, and the theory and calculations used to obtain the Raman temperatures are described in Section 3.3.

\subsection{Thermocouple probe description}

The combination thermocouple-and-total-pressure probe used in this study was designed and built at NASA Lewis Research Center [14]. The probe consists of a thermocouple element, a total-pressure tap, and a cooling jacket. The thermocouple was enclosed in a separate tube which could be removed from the cooling jacket. A photograph of the probe and a thermocouple insert are shown in figure 1. North, et a1. [14] used the probe with a different thermocouple element to measure temperature and velocity profiles in an after-burning engine.

The total-pressure tap was made of a $6.35 \mathrm{~mm}$ (1/4 in) copper tube with a chamfered inlet leading to a $1.52 \mathrm{~mm}(0.06 \mathrm{in})$ passage. This passage was connected to a $2.03 \mathrm{~mm}(0.08 \mathrm{in})$ inconel tube which extended the length of the probe. The pressure tap extended $12.7 \mathrm{~mm}(1 / 2$ in) from the leading edge of the probe. Water-cooling flow rate of $19 \mathrm{l} / \mathrm{min}$ was adequate for the internal elements of the probe and the copper pressure tap. The water jacket shell was an elliptically shaped inconel tube with an overall length of $1.1 \mathrm{~m}$.

The thermocouple was a crossflow, bare-wire $0.813 \mathrm{~mm}(0.032 \mathrm{in})$ platinum 13 -percent rhodium-platinum (Pt $13 \% \mathrm{Rh}-\mathrm{Pt}$ ) element with a length-to-diameter (L/d) ratio of 31 . Before the test, the tip was $U$-shaped as shown by the close-up in figure 1 . The junction was $10^{-}$ cated about $10 \mathrm{~mm}$ above the tip of the probe. During test, the high velocity exhaust distorted the shape of the thermocouple and bent it towards the tip of the probe. A $1.02 \mathrm{~mm}$ ( $0.040 \mathrm{in}$ ) diameter Pt $13 \% \mathrm{Rh}-\mathrm{Pt}$ thermocouple was also used in the afterburner plume. However, the thermocouple wire broke near the tip of the probe before statistically meaningful data could be collected. 


\subsubsection{Theory}

A thermocouple probe immersed in an afterburning plume of a turbojet engine attains a thermal equilibrium at a temperature different from the temperature of the gas. This is a result of a heat balance between the probe and its surroundings. In general, there are four modes of heat transfer: (1) the rate of convective heat transfer between the gas stream and the thermocouple junction, (2) the rate of net radiant energy exchange between the gas stream and the thermocouple junction, (3) the rate at which heat is conducted out of the junction through the thermocouple wire, and (4) the rate of conversion of kinetic energy to thermal energy at and around the thermocouple junction. From detailed theoretical treatments of

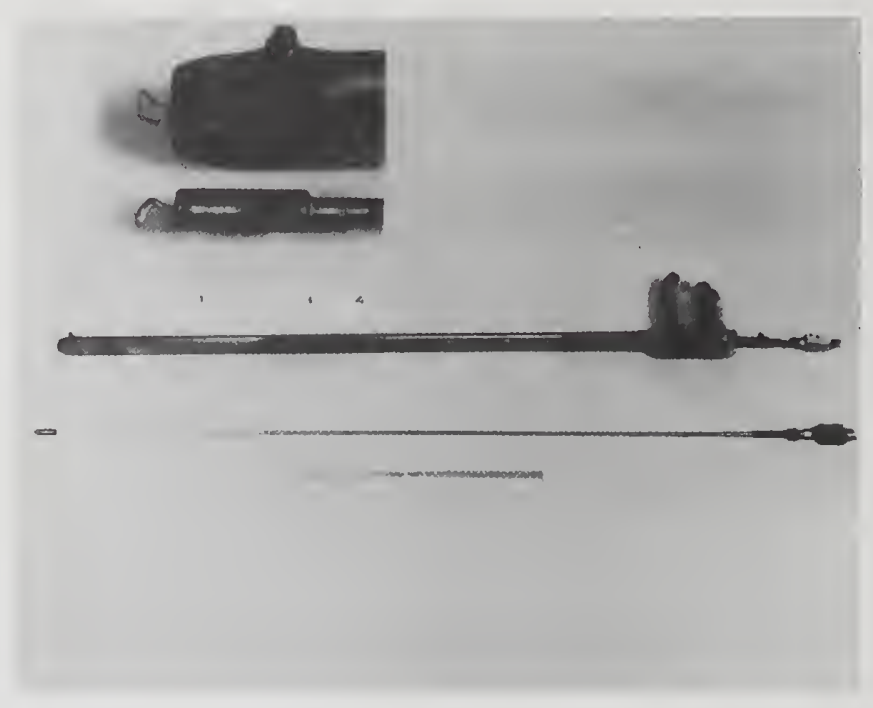

Figure 1. The combination thermocouple-and-total-pressure probe used in this study. The assembled probe with a thermocouple insert are shown. The inset gives two closeup views of the thermocouple tip. The upper view shows the total pressure orifice in the assembled probe.

these processes it may be concluded that a steady-state gas temperature can be estimated by adding recovery, radiation, and conduction corrections to the indicated thermocouple temperature [15]. Furthermore, theoretical and empirical mathematical expressions have been developed for the different correction factors [16]. We neglected conduction errors because the large $\mathrm{L} / \mathrm{d}$ ratio of the thermocouple reduced these errors to less than $2 \mathrm{~K}$ for a11 test conditions. However, recovery and radiation corrections were made to our data. These corrections are discussed in the next two sections. 


\subsubsection{Recovery - correction factor}

There are two temperatures associated with a moving gas stream: (1) the static temperature, and (2) the stagnation or total temperature. The static temperature is the temperature measured by a sensing element moving at the same velocity as the gas stream. It is a measure of the mean kinetic energy of the random motion of the gas molecules. The total temperature is measured if the gas stream is brought to rest adiabatically. It is the sum of the static temperature $T \sigma$ and a factor called the impact temperature which is a measure of the temperature corresponding to the mean translational kinetic energy of the gas stream. Both static and total temperatures have practical applications. The total temperature can be used to determine the combustion efficiency of an engine. Whereas, the chemical kinetics of the reacting species are a function of the static temperature. The total temperature $T_{T}$ of a moving gas stream is defined as

$$
T_{T}=T_{s}+\frac{u^{2}}{2 J C_{p}},
$$

$$
\text { where } \begin{aligned}
T_{T}= & \text { total or stagnation temperature }(\mathrm{K}), \\
T_{\mathrm{S}}= & \text { static temperature }(\mathrm{K}), \\
U= & \text { stream velocity }(\mathrm{m} / \mathrm{s}), \\
\mathrm{J}= & \text { mechanical equivalent of heat } \\
& (4186 \text { joules } / \mathrm{kcal}), \\
\mathrm{C}_{\mathrm{p}}= & \text { specific heat of gas at constant } \\
& \text { pressure }(\mathrm{kcal} / \mathrm{kg} / \mathrm{K}) .
\end{aligned}
$$

Only part of the mean translational kinetic energy of a high velocity gas stream is recovered by a bare-wire thermocouple. It is convenient to correct for this aerodynamic recovery error by using,

$$
T_{T}=T_{I}(1-\Delta)^{-1} \cong T_{I}+T_{I} \Delta \text { for } \Delta<<1
$$

where $\Delta$ is the recovery correction factor and $T_{I}$ is the indicated thermocouple temperature [17]. The recovery temperature correction is $T_{I} \Delta$. Glawe, et al. [17] determined the dependence of $\Delta$ on Mach number and static pressure for several different thermocouple designs. We used the data in [17] for a $1.01 \mathrm{~mm}$ diameter, bare-wire, crossflow chromel-alumel thermocouple to obtain values of $\Delta$ from measured values of Mach number ${ }^{3}$. A small correction, $\Delta^{*}\left(d / d_{0}\right)^{1 / 5}$ was also made to account for the difference in wire diameter of the thermocouple used in collecting the data in figure $2\left(d_{0}\right)$ and our thermocouple diameter $(d)$. The corrected $\Delta$ was used in eq. (2) to determine the corrected gas temperature. However, this is

${ }^{3}$ The procedure for obtaining the Mach number is given in Section 3.1.1.3. 
still not the true total temperature because the radiation correction has not yet been made.

\subsubsection{Radiation correction}

Detailed theoretical treatments of thermocouple radiation corrections are given by Scadron and Warshawsky [15] and by Williamson and Stanforth [18]. The important equations have been summarized by Scadron, et al. [16] and by Glawe, et al. [17]. In this section, we shall briefly outline how our data were corrected for radiation losses.

A thermocouple in the plume of an afterburning engine can exchange energy with the surrounding air by radiation transfer. At equilibrium, the thermocouple obtains some temperature between that of the gas plume and the surrounding air. The equilibrium state is determined when the radiation heat loss by the thermocouple is balanced by the convective heat gain of the plume gas. The mathematical development of these ideas were used by Scadron and Warshawsky [15] to derive the radiation correction factor given by,

$$
\text { Rad. Corr. }=2.976 \varepsilon_{W}\left(d / M P{ }_{S}\right)^{0.5}\left(T_{I} / 555.5\right)^{3.82} \text {, }
$$

where $P_{S}$ is the static pressure in atmospheres, $M$ is the stream Mach number, and $\varepsilon_{W}$ is the total hemispherical emittance of the thermocouple wire. A radiation term for the air surrounding the afterburning plume was assumed to be small and was neglected when eq. (3) was derived. Equation (3) applies for a bare-wire, crossflow, Pt $13 \%$ Rh-Pt thermocouple used at a reference temperature of $555.5 \mathrm{~K}$ and was used to make radiation corrections. Glawe, et a1. [19] measured $\varepsilon_{W}$ for $\mathrm{Pt} 13 \% \mathrm{Rh}-\mathrm{Pt}$ bare-wire crossflow thermocouples with different surface conditions obtained by exposure to different combustion environments. Their results for a thermocouple with a dull grey appearance were used to obtain values of $\varepsilon_{W}$.

In summary, the thermocouple measures a temperature resulting from an energy balance of various heat transfer processes. We estimated the total gas temperature by adding the recovery temperature, $T_{I} \Delta$ given in eq. (2) and the radiation correction given by eq. (3) to the indicated thermocouple temperature $T_{I}$. For this study, we wished to compare the temperatures measured by the thermocouple and Raman scattering techniques; however, the Raman technique measures a static temperature. The next section describes how we estimated the static temperature of the gas using the total temperature measured by the thermocouple.

\subsubsection{Static temperature calculations}

The static temperature was estimated using

$$
T_{S}=T_{T}\left[1+0.5(\gamma-1) M^{2}\right]
$$




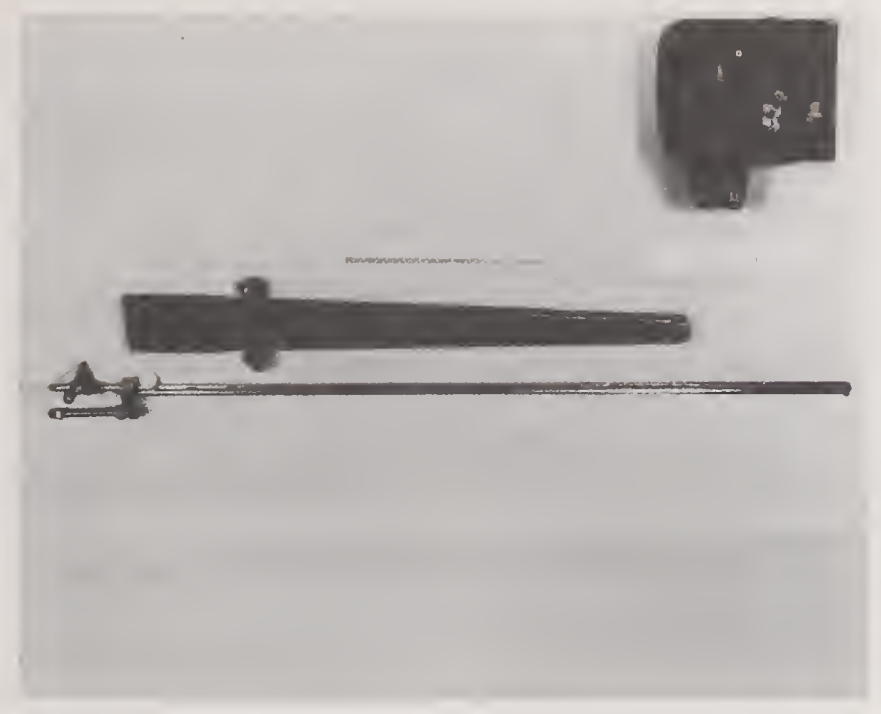

Figure 2. The combination gas-sampling-and-total-pressure probe used in this study. The probe is shown disassembled from the cooling jacket. The inset is a closeup of the probe tip showing the two orifices.

This equation can be derived from eq. (1) by using Bernoulti's equation for a compressible gas. The Mach number, M, was calculated using [19]

$$
M=\left[2\left[\left(P_{T} / P_{S}\right)(\gamma-1) / \gamma_{-1}\right] /(\gamma-1)\right]^{0.5}
$$

The total pressure, $P_{T}$, was measured from the pressure tap on the thermocouple probe. The static pressure, $P_{s}$, was assumed to be the same as the ambient pressure. The remaining problem of calculating $M$ and $T_{s}$ becomes one of estimating gamma, $\gamma$. In general, $\gamma$ is a function of static temperature and gas composition. In our case, combustion of JP-4 fue 1 was taking place in the afterburner plume; thus, the temperature and composition of the combustion products determined $\gamma$.

Gordon and McBride [21] developed a computer code which calculates $\gamma$ and $T_{s}$, as we11 as other parameters, for the combustion of different fuels. This code was used to generate values of $\gamma$ and $T_{s}$ for the combustion of JP-4 fuel. A curve fit to these data resulted in an equation given by

$$
\gamma=1.316-1.096 \times 10^{-11} \mathrm{~T}_{\mathrm{S}}^{3}+1.861 \times 10^{4} \mathrm{~T}_{\mathrm{S}}{ }^{-4}
$$

The fitting error of this equation was less than 0.5 percent for temperatures between 600 and $2000 \mathrm{~K}$. To use eq. (6), $\mathrm{T}_{\mathrm{s}}$ was needed; however, this is the parameter that was being sought. We obtained an approximate value of $\gamma$ by using the indicated junction temperature, $T_{I}$, in place of $T_{s}$ in eq. (6). This value of $\gamma$ along with the measured values of total and static 
pressures were used in eq. (5) to calculate M. The static temperature was then calculated with eq. (4), using these values of $\gamma$ and $M$ and the total temperature obtained as described in the previous section.

The use of $T_{I}$ for $T_{S}$ in eq. (6) resulted in a bias error in the calculated static temperature of about 0.5 percent. A larger error resulted in the calculation of the gas sampling static temperatures. As will be discussed in the next section, our gas sampling analysis resulted in the estimate of a total temperature. We used the same procedure for calculating the gas sampling static temperature as was applied to the thermocouple calculations. The only difference was that $T_{T}$ was used in eq. (6) to get an estimate of $\gamma$. This resulted in bias errors between 0.5 percent and 1.3 percent, depending on the test conditions. The larger errors occurred at the maximum afterburning power condition. An iterative procedure could have eliminated these errors. The magnitude of these errors does not have significant impact on the results presented in this paper; therefore, a complete recalculation was deemed unwarranted.

\subsection{Gas sampling}

The gas sampling probe and the gas sampling instruments used to measure $\mathrm{CO}_{2}$, $\mathrm{CO}$, and total hydrocarbon concentrations are described in this section. Also, the theory is outlined for calculating total temperatures from emissions concentrations.

\subsubsection{Description of gas sampling probe and emissions instruments}

The gas sampling probe used in this study was designed and built on an Air Force contract with General Electric ${ }^{4}$. It was designed specifically for measuring emissions in plumes of afterburning engines. Lyon, et a1. [3] used the probe to measure emissions profiles at different axial stations in the afterburning plumes of a J85-5 and J79-15 engines. Probe design information and a description of their test program are given in [3].

The probe is a combination gas-sampling-and-total-pressure probe. The probe assembly has three parts: the probe, the probe support body, and the probe support arm. Figure 2 shows a photograph of the disassembled probe and the support body. The mounted probe was 1.5 $\mathrm{m}$ long. The probe shel1 was made of two $12.7 \mathrm{~mm} \mathrm{0.D.} \mathrm{(0.5} \mathrm{in)} \mathrm{stainless} \mathrm{steel} \mathrm{tubes.} \mathrm{Each}$ tube contained a smaller $6.35 \mathrm{~mm} 0.0$. (0.25 in) tube which connected to the total pressure and gas sampling orifices at the tip of the probe. These small tubes were for transporting gas samples and pressure to the external transport line connections at the end of the probe. The total pressure and gas sampling orifices in the tip of the probe were separated by 4.78 $\mathrm{mm}$. Water circulating through the shell cooled the transport tubes and the copper tip of the probe. The cooling water was maintained at a temperature above $330 \mathrm{~K}$ to prevent water and hydrocarbons from condensing in the probe.

${ }^{4}$ Air Force Contract Number F33616-73-C-2047. 
When the probe was in the afterburning plume of the engine, the gases entering the probe tip underwent chemical reactions. It was desirable to freeze or quench these reactions so the sample transported to the emissions instruments were representative of those in the plume. The gas sampling orifice consisted of a diverging passage leading away from the sample entrance. A pressure ratio greater than 5:1 was provided across the probe tip. The combination of the diverging passage and high pressure drop, created a short, supersonic expansion which reduced the temperature of the reacting gas. While in the supersonic flow, the gas transferred additional energy to the water-cooled walls of the copper tip. Supposedly, this cooling was sufficient to maintain the gas below the reaction temperature even after the supersonic-to-subsonic transition which occurred downstream of the probe tip. Because of this quenching action, probes of this type are sometimes referred to as quick-quench probes.

The recommended procedures for measuring aircraft emissions are given in references [2] and [22]. Our gas sampling system was designed in accordance with these procedures. A Meta1-Bellows-Corporation Model MB601HT pump was used to pull the sample through the probe and sampling lines. A heated, $6.35 \mathrm{~mm}$ (1/4 in) $0 . D$. stainless steel tube was used to transport the sample to various analytical instruments. Carbon dioxide and carbon monoxide concentrations were measured with two Beckman Model 864 nondispersive infrared instruments. Total hydrocarbon concentrations (THC) were measured with a Beckman Model 402 flame ionization detector. The water in the sample was removed by a refrigerated dryer before going into the $\mathrm{CO}_{2}$ and $\mathrm{CO}$ instruments. The concentrations of these two species are reported on a dry basis.

\subsubsection{Calculation of total temperature}

This section is divided into five parts. The first four parts give background information needed to understand the calculation procedure. An outline of the steps used to calculate total and static temperature from measured emissions is given in the final part.

\subsubsection{Conservation of total enthalpy}

The fundamental principles of gas-turbine engine combustion are given by $\mathrm{Blazowski}$ and Henderson [23]. Some of these principles will be reviewed before outlining the procedures used to calculate total and static temperature from emissions measurements. Combustion in the plume of an afterburning engine is a constant pressure process; hence, total enthalpy is conserved. This means that the total enthalpy of the air and fuel must be equal to the total enthalpy of the products. Assuming that the total enthalpy of the air just before it enters the engine is all sensible enthalpy (thermal), then the conservation of total enthalpy gives 


$$
\int_{T_{R}}^{T} c_{p A}(T) d T+f_{L} n Q_{L}=\left(1+f_{L}\right) \int_{T_{R}}^{T} C_{p P}(T) d T .
$$

The left-hand side of eq. (7) represents the sum of the sensible enthalpy of the air and the actual heat energy released by the fuel $\left(f, \eta Q_{L}\right)$. The right-hand side of eq. (7) is the total enthalpy of the products after they are brought to rest adiabatically.

To calculate the total flame temperature, $T_{T}$, using eq. ( 7 ), the following parameters must be known: the specific heat of air as a function of temperature, $C_{p A}(T)$, the specific heat of the combustion products as a function of temperature, $C_{p P}(T)$, the local fuel-air ratio, $f_{L}$, the local combustion efficiency, $\eta$, the lower heating value of the fuel, $Q_{L}$, the reference temperature, $T_{R}$, and the air inlet temperature, $T_{j}$. The average value of $Q_{L}$ for JP-4 fuel $(10,392 \mathrm{kcal} / \mathrm{kg})$ was used. The value of $T_{i}$ was measured during the experiments. The following paragraphs discuss how the other parameters were determined.

\subsubsection{Specific heats $\left(C_{p A}, C_{p P}\right)$}

The equation for the specific heat of air $C_{p A}$ is given by VanWylen and Sommstay [24]. The specific heat of the products was not as easily obtained. The NASA computer code for ideal flame calculations was used to generate $C_{p P}$ versus $T$ data for the combustion of $\mathrm{JP}-4$ fuel over a fuel-air ratio range of 0.0 to $0.06[21]^{5}$. These $C_{p P}-v s-T$ data were curve fit to a polynominal with both positive and negative exponents. This polynomial was used for calculating $C_{p P}$. This equation is complicated and is not included in this paper.

\subsubsection{Local fuet-air ratio $\left(f_{L}\right)$}

The fuel-air ratio can be obtained by balancing the chemical equation describing the combustion of a mixture of JP-4 fuel and air. This equation is solved in [2]. The result is given by

$$
f_{L}=\left(\mathrm{CO} / 10^{4}+\mathrm{CO}_{2}+\mathrm{THC} / 10^{4}\right)\left(207+\mathrm{CO}_{2}\right)^{-1}
$$

which represents dry samples of $\mathrm{CO}_{2}$ and $\mathrm{CO}$, since the water in the samples was removed before going into the $\mathrm{CO}_{2}$ and $\mathrm{CO}$ instruments. The $\mathrm{CO}_{2}$ concentration is expressed in percent by volume and the concentrations of $\mathrm{CO}$ and THC are given in parts per million (ppm) by volume. These concentrations are measured with the gas sampling probe. The molecular weight of the products $\left(M_{p}\right)$ can also be obtained from the chemical balance of the reaction equation. This was used in the determination of $\eta$.

\footnotetext{
${ }^{5}$ The NASA computer code could be used to calculate ideal flame temperatures. However, it is too large for routine data analysis.
} 


\subsubsection{Combustion efficiency $(\eta)$}

The combustion efficiency is the ratio of the actual energy released by the fuel, $Q$ (actual), to the total energy available for combustion, $Q$ (ideal). The latter quantity can vary with the environment. For example, non-afterburning engines have relatively low exhaust temperatures, typically $950 \mathrm{~K}$. Under these conditions, all chemical reactions have ceased. The ideal energy is determined by assuming that all the fuel is converted to $\mathrm{CO}_{2}$ and $\mathrm{H}_{2} \mathrm{O}$. All other products, such as $\mathrm{CO}, \mathrm{THC}$, and $\mathrm{H}_{2}$ contain energy which was not used during combustion. Thus, they represent combustion inefficiencies. In an afterburning plume, the temperatures are normally high enough so that the chemical equilibrium effects due to the dissociation of $\mathrm{CO}_{2}$ and $\mathrm{H}_{2} \mathrm{O}$ are important. The water-gas reaction is a simple expression of this equilibrium and is given by

$$
\mathrm{CO}+\mathrm{H}_{2} \mathrm{O} \stackrel{\rightarrow}{\leftarrow} \mathrm{H}_{2}+\mathrm{CO}_{2}
$$

The chemical energy associated with the equilibrium concentration of $\mathrm{CO}$ and $\mathrm{H}_{2}$ is not available for combustion. However, this energy can never be expended and does not represent a combustion inefficiency. Thus, dissociation reduces Q (ideal). Blazowski, et al. [23] describes how to calculate equilibrium concentrations of $\mathrm{CO}$ and $\mathrm{H}_{2}$ if the static temperature is known. Rosfjord [25] considered dissociation in deriving the expression for $\eta$ given by

$$
\begin{aligned}
& 1-\eta=a\left(E I_{C O}-E I_{C O} \text {, eq. }\right)+b\left(E I_{H_{2}}-E_{H_{2}, \text { eq. }}\right)+c E I_{T H C}, \\
& a=10^{-3} Q_{L, C 0} / Q_{L, \text { Fuel }}\left(2.32 \times 10^{-4} \text { for } J P-4 \text { fuel }\right), \\
& b=10^{-3} Q_{L, H_{2}} / Q_{L, \text { Fuel }}\left(2.75 \times 10^{-3} \text { for } J P-4 \text { fuel }\right), \\
& c=10^{-3} \text { for JP-4 fuel. }
\end{aligned}
$$

Equation (10) is the same as that used by Lyon, et al. [3] for non-afterburning engines except for the equilibrium index of $\mathrm{CO}$ and $\mathrm{H}_{2}$. The emission indices for $\mathrm{CO}$ and $\mathrm{H}_{2}$ are defined by

$$
E I_{Y} \equiv 10^{3} Y\left(1+f_{L}\right) M_{Y} / M_{P}
$$

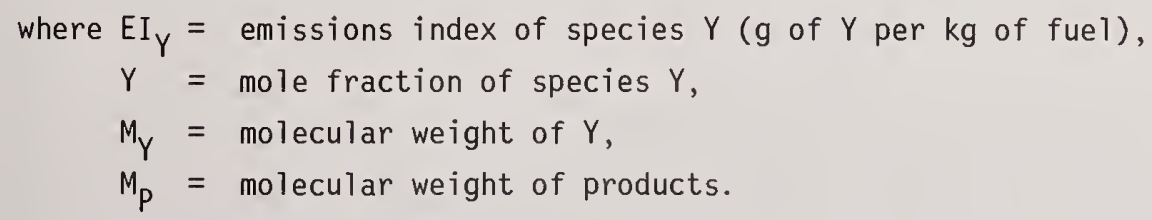

Note that mole fractions of $\mathrm{CO}, \mathrm{THC}$, and $\mathrm{H}_{2}$ are involved in calculating $\eta$. We did not have 
the capability of measuring $\mathrm{H}_{2}$ concentrations during our experiments. We assumed that the only $\mathrm{H}_{2}$ present in the plume was due to chemical equilibrium.

\subsubsection{Outline of the steps used to calculate $T_{T}$ and $T_{S}$}

With this background, we can outline the procedure used to calculate total and static temperatures.

Step 1: The local fuel-air ratio corresponding to the sampling volume at the tip of the probe was calculated with eq. ( 8 ) by using the measured concentrations of $\mathrm{CO}_{2}, \mathrm{CO}$, and THC. Also, the molecular weight of the products was calculated by using an equation given in [2].

Step 2: An iterative procedure was performed on eq. (7) to determine the total ideal temperature $T_{T I}$. In this case $\eta=1.0$ and $T_{j}$ was measured in the experiment. The left-hand side (LHS) of eq. (7) was computed using the known equations for $C_{p A}(T)$ and $C_{p P}(T)$. The right-hand side of eq. (7) was made equal to the LHS by varying $T_{T I}$. Thus, $T_{T I}$ (ideal) was obtained. However, this was not the true flame temperature because the true combustion efficiency was not used. The remaining steps were required to obtain the value of $\eta$.

Step 3: TTI was used to calculate the ideal static temperature, $T_{S I}$, following the procedures described in Section 3.1.1.3.

Step 4: $T_{S I}$ was used to calculate the equilibrium concentration and emission index of $\mathrm{CO}$ assuming the water-gas reaction in eq. (9). The emission indices of $\mathrm{CO}_{2}$ and THC were also calculated.

Step 5: The emissions indices determined in Step 4 were used in eq. (10) to calculate the local combustion efficiency $\eta$.

Step 6: Step 2 was repeated using $\eta$ in order to determine the local total temperature observed at the tip of the gas sampling probe.

Step 7: Step 3 was repeated to determine the local static temperature, $T_{s}$, so that it could be compared with Raman scattering measurements.

\section{3 Raman scattering}

Light scattering is the analysis of the intensity, polarization, and spectral content of the light collected from a well-defined region of a medium which is illuminated by an intense, monochromatic light source of known characteristics. Raman scattering is concerned specifically with that portion of the scattered light which is spectrally shifted (i.e., in wavelength) from the wavelength of the incident light due to the molecular oscillations of the medium. The use of Raman scattering as an optical diagnostic probe for temperature measurement spans three areas of overlapping endeavor. These are (1) the theoretical analysis of the basis for the measurement, (2) the design and operation of the experimental apparatus, and (3) the analysis of the data. The approaches taken in this work in these three areas are the subjects of this section. In Section 3.3.1, the apparatus used to make the measurements and the features important to its operation are described. A brief outline of spontaneous Raman scattering theory is given in Section 3.3.2 with the application of this theory to the calculation of temperature covered in Section 3.3.3. 


\subsubsection{Description of the Raman system}

The Raman system used in this work was a modified version of the system described by Leonard [8]. It consisted of two separated subsystems. One subsystem was the optical transceiver unit which was located in the test cell with the engine. The other subsystem consisted of the signal-handling electronics, a Data General Nova 1220 minicomputer, and various power supplies. Two of the important features of these subsystems are: (1) the size and shape of the scattering volume referred to as the scattering "geometry," and (2) the electronic signal detection scheme. These features as well as the subsystems are described in the following sections.

\subsubsection{The transceiver}

The transceiver and its position relative to the engine is illustrated in figure 3 . The transceiver components were mounted on a custom-made, aluminum pallet with dimensions of $2.9 \mathrm{~m}$ by $1.4 \mathrm{~m}$. The laser was an AVCO model $C 5000$ pulsed, nitrogen-gas laser with emission at $3371 \mathrm{~A}$. It was operated at $250 \mathrm{pps}$ with about $100 \mathrm{~mW}$ average power. Its beam pattern consisted of a complex array of parallel "lines" of light in the far field having an overall measured half-angle divergence of approximately $1.2 \mathrm{mr}$ by $10.4 \mathrm{mr}$. The beam at the laser (near field) consisted primarily of two very narrow, bright lines of light separated by $3.2 \mathrm{~mm}$ and $50 \mathrm{~mm}$ in length. The beam was directed and focused by means of a lens, a 135-cm-focal length, 45-cm-diameter spherical concave mirror, and five large, flat, beamfolding mirrors. The mirrors were adjusted to focus the two lines of light into a single line. Since the pulse length was about $10 \mathrm{~ns}$, the pulse energy was $400 \mu \mathrm{J}$, and with a peak power of about $40 \mathrm{~kW}$, the peak intensity at focus was about $0.6 \mathrm{MW} / \mathrm{cm}^{2}$. The laser beam was monitored by a ITT type F4000 high-current, biplanar, vacuum photodiode with 55 cathode response. The signal from this photodiode was used to monitor the laser power and to provide trigger signals for the electronics.

The receiver portion of the transceiver used a mode $111740-\mathrm{cm}$-diameter Cassegrain telescope supplied by Group 128, Inc. as the collecting optic. This telescope had a primary focal ratio of $f / 3$ with an overall ratio of $f / 11$. It was set up to give near unity magnification with the laser-illuminated volume at about three meters from the telescope. The light from the telescope was focused on the entrance slit of a SPEX model 1402 double spectrometer. The detector was an RCA 8850 photomultiplier tube (PMT) connected for fast-pulse detection.

\subsubsection{Scattering geometry}

The transceiver was positioned so that the axis of the collecting telescope was approximately horizontal and intersected the axis of the engine. The laser beam was directed from below the telescope up through the engine axis so that the long dimension of the beam was coincident with the vertical plane containing the telescope axis. This 


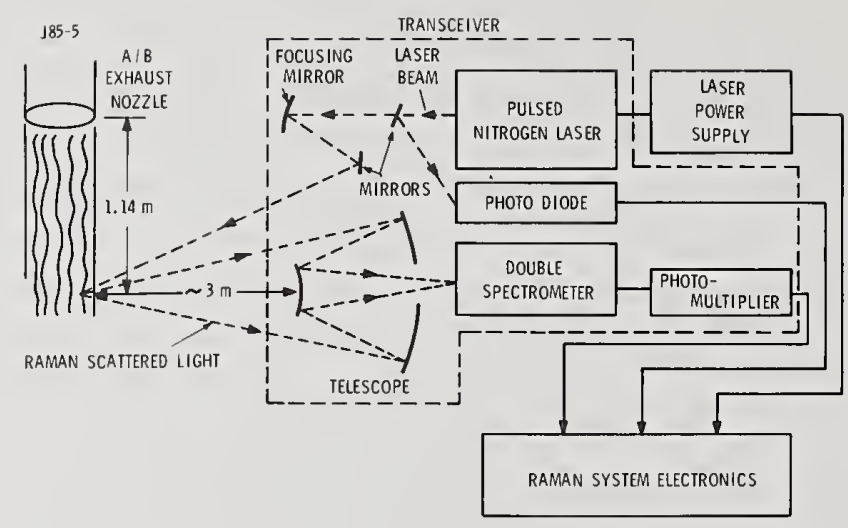

Figure 3. Raman transceiver subsystem with a schematic of the optical arrangement.

arrangement not only placed the long dimension of the laser beam parallel to the spectrometer slit, but it also allowed the transceiver to be placed at a safe distance from the combustion plume; however, it created a rather large, odd-shaped scattering volume from which the Raman signal was gathered. Because of the large divergence of the long dimension of the laser beam, the focused beam was originally almost $50 \mathrm{~mm}$ long. By replacing the final, 30-cm-long, beam-directing, flat mirror with a segmented mirror consisting of three 10-cm-long, flat mirrors each adjusted to project its beam segment onto a common line; the focused beam was reduced to a length of about $23 \mathrm{~mm}$. The scattering volume as viewed from the engine nozzle is illustrated in figure 4. The "thickness" of this volume was determined by the width of the laser beam which was about $0.3 \mathrm{~mm}$ at the engine axis.

The scattering geometry was nearly "backscattering" with an angle between the beam direction the direction of the collected light along the telescope axis of $155^{\circ}$. A disadvantage of this near-backscattering geometry was that signals from across the whole diameter of the combustion plume could be detected as indicated in figure 4 . The effective "depth-of-focus" of this geometry was determined by translating an ordinary microscope slide along the axis of the telescope and measuring the fluorescence signal from the slide due to the laser beam. These measurements are plotted in figure 5 along with the total temperature profile reported by General Electric for this same type engine [3] at the 1.14 $m$ position from the nozzle of the afterburner. These graphs indicate that about 90 percent of the observed Raman signals came from within the constant temperature portion of the plume. This fact was the deciding factor in the choice of this axial station for the measurements. The remaining 10 percent of the observed signal which may have included the cooler portions of the plume could have biased our temperature measurements low by as much as 1 percent. However, since it was not possible to account for this error in any detailed manner, it had to be neglected. 


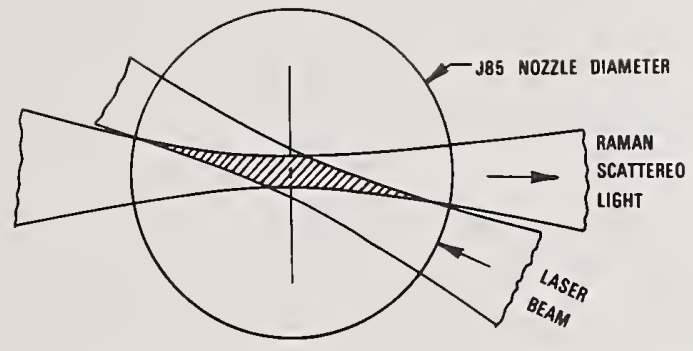

Figure 4. Scattering geometry as viewed along the axis and from the exit nozzle of the engine.

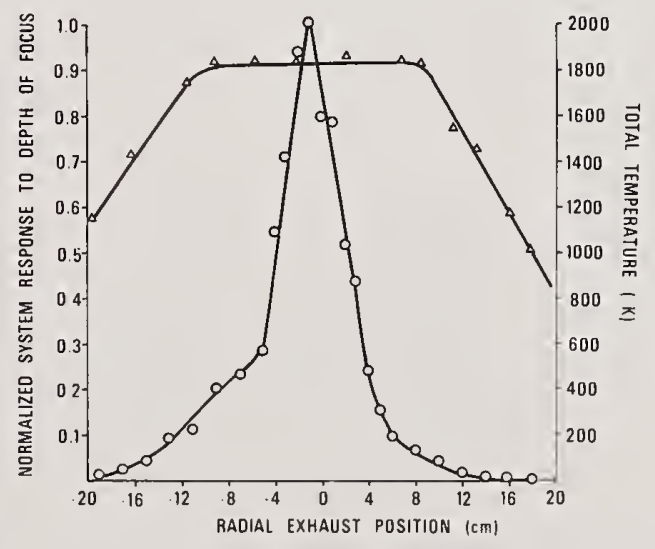

Figure 5. Measured radial response of the Raman transceiver (circles, left axis) compared to the radial temperature profile (triangles, right axis) reported by Lyon, et al. [3] $1.14 \mathrm{~m}$ from the plane of the exit nozzle. 


\subsubsection{The electronics}

A block diagram of the electronics subsystem is given in figure 6 . The subsystem was separated from the transceiver by about $15 \mathrm{~m}$ of cable and was located in the control room next to the test cell. As shown in figure 6 , the system was completely controlled by the Nova computer. Three different signals were recorded for each laser pulse. The data signal from the PMT, which was synchronous with the laser pulse and carried the Raman signal, was recorded first followed by a recording of the laser power signal which came from the photodiode. After $600 \mu \mathrm{s}$ from each laser pulse, the signal from the PMT was again sampled to determine the non-laser-induced background signal. This sequence was repeated for the number of laser pulses programmed. The three signals were accumulated in the computer during this signal-acquisition time. At the end of this period, the accumulated background signal was substracted from the accumulated data signal and the result was divided by the normalized value of the integrated laser power during the period. This net signal as well as the three raw signals were recorded on punched paper tape for subsequent analysis. After this process was completed, the spectrometer was stepped to the next programmed wavelength and the sequence was repeated.

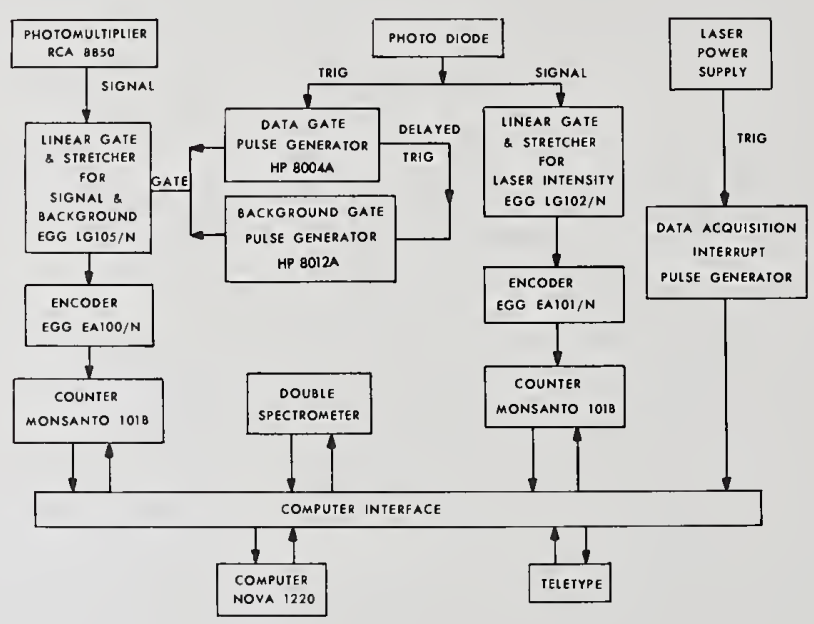

Figure 6. Raman electronics subsystem.

\subsubsection{Signal detection}

Signal detection using a linear-gate-and-stretcher circuit is an analog method (versus pulse counting) in that the pulses from the detector are integrated in real time. The output of this circuit is a relatively long pulse $(\sim 3 \mu \mathrm{s})$ whose amplitude is linearly related to the total electric charge received at the input. Since the anode current in a PMT or a photodiode is proportional to the number of photons incident on the photocathode 
per second, integrating the anode pulse gives a signal proportional to the number of photons detected. The pulses from the PMT were about 20 ns in width across their base. A 20 ns gate signal was applied to the linear gate module attached to the PMT, and its time delay was adjusted to be synchronous with the room-air nitrogen $\left(\mathrm{N}_{2}\right)$ Raman signal. In a similar manner, a gate signal delayed by $600 \mu$ s was applied to the module in order to sample the non-laser-induced background signal.

This gated current-integrating detection scheme had the advantage of a low duty cycle, namely $5 \times 10^{-6}$ for our case, which suppressed signals that arose from ambient sources such as room lights, combustion plume radiation, etc. A second advantage came from the discrimination against fluorescence processes which had lifetimes equal to or greater than about 10 ns. Although many of the processes occurring in combustion zones appear to have lifetimes shorter than $10 \mathrm{~ns}$, we have found that there is almost always some fluorescence emission present beyond 10 ns from the laser-pulse peak.

The main disadvantage of this detection technique is that the limit on the dynamic range of signal measurement is set by the linear-gate-and-stretcher module. The module used with the PMT was specified to have a linear range of at least 3 to 100 pico-Coulombs $(\mathrm{pC})$. This required that the voltage applied to the PMT be adjusted so that the maximum anticipated signal did not exceed the $100 \mathrm{pC}$ value. As shown in figure 6 , the output of the module was sent to an encoder which produced a burst of $40 \mathrm{MHz}$ pulses. The number of pulses in the burst were proportional to the input pulse amplitude. The counter recorded the number of pulses and sent the value to the computer. Our concern was that the entire signal detection and recording scheme was linear over the largest possible range. Calibration measurements were made with the linear gate, encoder, and counter combination using attenuated test pulses into the linear gate as well as with the complete detection system using attenuating optical (neutral density) filters taken while sitting on a peak of the room-air $\mathrm{N}_{2}$ Raman 1 ine. These measurements showed that the response was linear to within \pm 1 percent over a range of at least 50 to one, with signals down by a factor of 100 being usefully detected. This was precisely the range needed in our studies to record the Raman spectrum of $\mathrm{N}_{2}$ with the engine at "Military" power. At this power, the signal dynamic range was the largest of all the observed spectra due to the very low laserinduced background.

\subsubsection{Spontaneous Raman scattering}

In the simplest terms, Raman scattering arises from the modulation of an incident electromagnetic wave by the temporal fluctuations of the dielectric constant of the medium being probed. This modulation produces the "side bands" which are electromagnetic emissions that are shifted in frequency in a symmetric manner above and below the frequency of the incident wave as illustrated in figure $7(a)$. In the case of a simple diatomic gas, the dielectric constant depends on the electric polarizability of its molecules. As a molecule vibrates and rotates, its polarizability oscillates at frequencies which are characteristic of the vibrational and rotational states that can be occupied by that molecule. The 
relative intensities of the side bands are determined by the populations of these states which in turn are governed by the rules of quantum mechanics and the temperature of the medium. The scattered light intensity is linearly dependent on the intensity of the incident light and, in general, occurs in all directions from the scattering medium. These features are characteristic of spontaneous scattering. In the two following sections, the Raman scattering from diatomic molecules and the manner in which it depends on temperature are briefly reviewed.

\subsubsection{The Raman spectrum of a diatomic molecule}

The theory of Raman spectra in general as well as of diatomic molecules in particular has been wel1 documented $[26,27,28]$. Furthermore, the theory has been reviewed a number of times in publications concerned with Raman thermometry $[5,6,7,8,11]$. Therefore, we will only outline those features of the theory which clarify the rationale and methods used to obtain the Raman temperatures.

As shown in figure $7(a)$, the Raman emissions shifted to frequencies higher than the incident laser frequency form the anti-Stokes spectrum, while those shifted lower are called the Stokes spectrum. In addition to the Raman spectra, a medium will scatter the incident light without any shift of frequency. This is called Rayleigh scattering; however, in many measurement circumstances, additional unshifted light is scattered by various sizes and types of particles (e.g., soot) in the medium and, as such come under the topic of Mie or Tyndall scattering [29]. To characterize the strength of spontaneous Raman emissions, it is noted that Rayleigh scattering from a pure gas is generally not visible (i.e., a bright beam of light viewed from the side on a dark, clear night cannot be seen). The Raman emissions from $\mathrm{N}_{2}$ gas are on the order of a thousand times weaker than its Rayleigh scattering. Thus, the need for high-power lasers and sophisticated detection equipment is clearly dictated.

In figure $7(b)$, a portion of the energy level scheme of a diatomic molecule is schematically represented. The vibrational energy levels labeled with the quantum number $v$ show a decreasing spacing with increasing energy. The quantum mechanical selection rules require that if the vibrational state of the molecule changes, it can only do so if the change in $v$ is by one unit, that is, $\Delta v= \pm 1$. In addition, in any vibrational state a molecule can have rotational energy specified by the quantum number $\mathrm{J}$. The rule governing changes in rotational energy which appear in the Raman spectrum is $\Delta J=0, \pm 2$. In figure 7 (b), only six of the many possible transitions are shown all of which have $\Delta J=0$. For example, in the transition labeled " $0, "$ the molecule is excited into the first vibrational level $(v=1)$ by the incident laser light of frequency $\cup L$ with the Stokes Raman light appearing at " 0 " in figure $7(a)$. Thus, with the inclusion of all the permitted lines, it can be seen that the position, density, and spread of the lines in the Raman spectrum is determined by the energy level scheme of the molecule. 

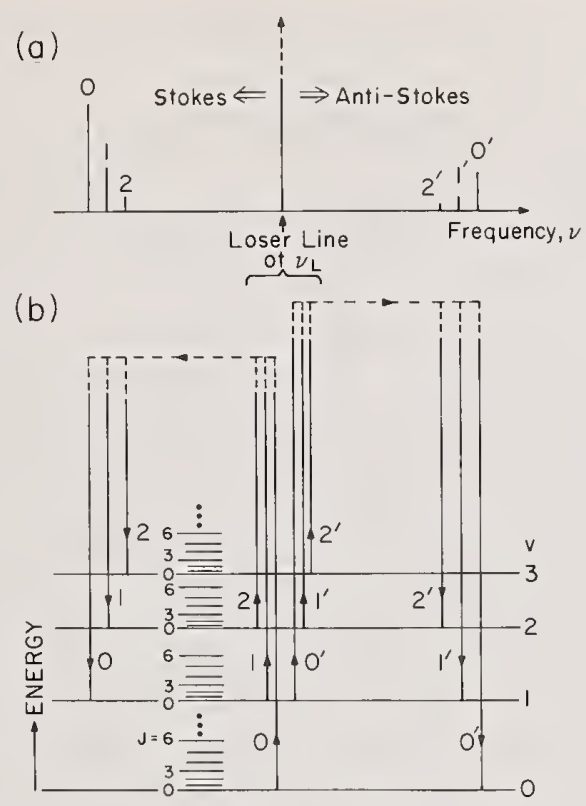

Figure 7. (a) Simplified Raman spectrum. (b) Schematic representation of the energy levels and selected $Q$ branch transitions of a diatomic molecule.

The vibrational-rotational Raman spectrum of a diatomic molecule is divided into three branches of spectra. The group of 1 ines for which $\Delta \mathrm{J}=0$, a small portion of which is shown in figure 7 , is called the $Q$ branch. On either side of the $Q$ branch are broad (20 to $40 \AA$ wide) groups of weak lines which result from a change in the rotational energy given by $\Delta J= \pm 2$. The band on the high-frequency side of the $Q$ branch is called the 0 branch $(\Delta J=-2)$ with the remaining band being the $S$ branch $(\Delta J=+2)$. Generally, the intensities of these two branches are about two orders of magnitude weaker than the peak of the $Q$ branch [8]. Because of the relatively low intensity of these branches, they are often neglected for high temperature measurement purposes [30,31]; however, since our studies included measurements of temperatures below $\sim 900 \mathrm{~K}$, we calculated the contributions of these branches to the Raman spectra.

\subsubsection{Temperature dependence}

Raman scattered light is directly proportional to the concentration of molecules in the observed volume. Because of the unequal spacing of the vibrational energy levels, as illustrated in figure $7(\mathrm{~b})$, the $Q$ branch spectrum is made up of one, two, or more bands or peaks corresponding to the initial energy levels of the transitions responsible for the bands. The relative intensity of a band depends on the fraction of the observed molecules present in the initial level of the band (i.e., the population). This fraction depends on the temperature. As the temperature increases, the population of the higher levels $(v>0$ 
in figure $7(b)$ ) increases at the expense of the lowest level $(v=0)$. Thus, the corresponding bands of lines of which two are represented by the single lines labeled " 1 " and " 2 " in figure $7(a)$, will become more intense relative to "band 0." For this reason, these bands are commonly called "hot bands." As a convenience, we shall refer to band " 0 " as the "main peak" since it is commonly the strongest feature in the Raman spectra of diatomic molecules for temperatures less than $3000 \mathrm{~K}$.

We performed temperature measurements by making use of the fact that the intensity envelope of the Stokes $Q$ branch provides a unique signature for every temperature. Typical Stokes Raman spectra (as a function of wavelength, not frequency) calculated for $\mathrm{N}_{2}$ gas are given in figure 8 . The laser wavelength used for these spectra was $3371 \AA$. Clearly, as the temperature increases, the intensity of the hot band labeled $v=1$ relative to the main peak labeled $v=0$ also increases. A finite spectral width of the laser line plus a wide slit setting of the spectrometer were assumed in order to obtain the trapezoidal slit function shown in the figure. This slit function integrates or smooths out the contributions from the individual Q branch lines described above. It should be noted that these spectra have their peak intensities normalized to unity to provide for a convenient format. In practice there is a decrease in the intensity of the main peak with increasing temperature, not only due to shifts of energy-level populations, but also due to a loss of gas density as given by the gas law.

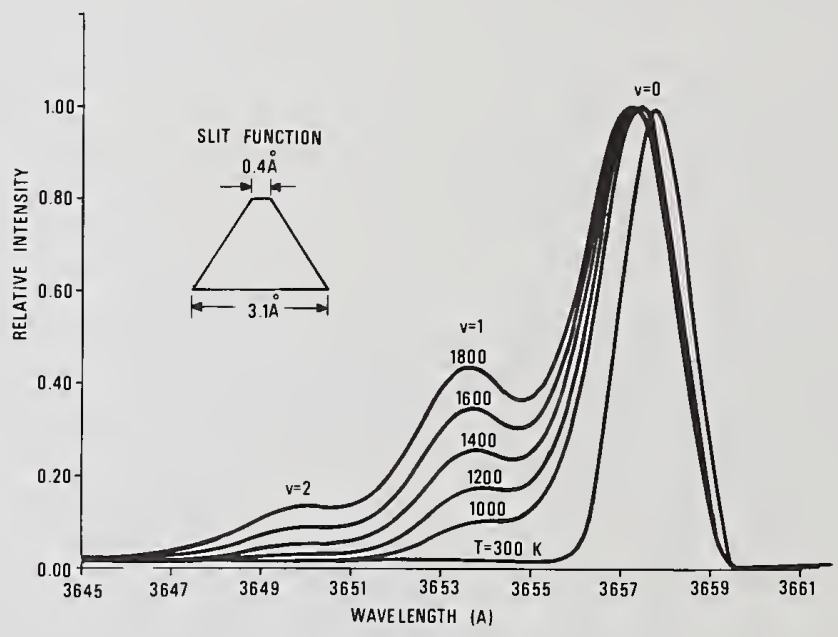

Figure 8. Calculated Stokes $Q$ branch spectra of $N_{2}$ on a normalized intensity scale for $3371 \mathrm{~A}$ excitation and a trapezoidal slit function. 
A second approach to temperature measurement uses the result that as the higher vibrational levels become occupied with increasing temperature, the anti-Stokes spectrum $(\Delta v$ $=-1$ ) becomes more intense. Thus, the temperature can be calculated from the ratio of the total anti-Stokes intensity to the total Stokes intensity. However, in the studies reported here, the engine-induced vibration of the spectrometer prevented the use of the first-order gratings needed to record the anti-Stokes spectrum.

In addition to Raman spectra involving both vibrational and rotational transitions, pure rotational Raman can be observed wherein only $\Delta \mathrm{J}= \pm 2$ and $\Delta \mathrm{v}=0$. In this case, a very closely spaced array of lines appear on both sides and in the immediate vicinity of the laser line. Although, the pattern and intensity envelope of these groups of lines are temperature dependent, this region is occupied by pure rotational Raman spectra of all molecular gases. It is possible to pick out specific rotational lines for temperature measurement [12] providing that the spectral line width of the laser is sufficiently narrow. In these studies, the line width of the nitrogen laser was about $1.0 \mathrm{~A}$ which is larger than the spacing of the Raman lines in the pure rotational spectrum of $\mathrm{N}_{2}$ gas. Thus, it was not possible to resolve this spectrum in this work.

\subsubsection{Calculation of Raman temperature}

The primary fact used in attempting to make remote temperature measurements in a gaseous atmosphere using any particular Raman technique is that the strength, shape, and extent of the Raman spectrum of a gas in equilibrium is unique for any given temperature. This is the case for pure rotational Raman scattering as well as for vibrational-rotational Raman scattering regardless of whether one of the complicated coherent Raman techniques is used or the simple spontaneous Raman technique used in these studies is chosen. Thus, if the spectral shape can be sufficiently resolved or if it can be sampled in such a way as to detect the features which are most temperature sensitive, then a temperature can be deduced.

Regardless of the approach chosen, it is absolutely essential that some means be provided to distinguish between the portion of the observed signal that is due to the desired Raman scattering and that which arises from other effects such as fluorescence, radiation from hot particles (i.e., soot), and other spurious sources. This is probably the single most significant impediment to making accurate measurements using a Raman technique. This is true for both the spontaneous Raman techniques as well as for the coherent Raman techniques such as coherent anti-Stokes Raman scattering (CARS) spectroscopy wherein the onset of additional Raman and other interferring phenomena produce "background" signal levels for which an accounting must be made [32].

The merits of one approach compared to another is not of concern here except that it is suggested that such comparisons deal in "trade-offs." That is, to gain an advantage in one area, a loss of something invariably arises in another area.

The following three sections outline the method of calculating the theoretical Raman spectra, the procedure used to fit the theoretical spectra to the experimental spectra to obtain temperatures, and the method of determining certain important parameters. 


\subsubsection{Calculation of Raman spectra}

In the studies presented here, the Stokes vibrational-rotational Raman spectrum of the $\mathrm{N}_{2}$ gas present in the combustion plume of the engine was scanned and recorded, point by point. The strength of this approach is in the complete theoretical description of the spontaneous Raman scattering of $\mathrm{N}_{2}$ that is available (see Section 3.3.2.1). That is, we can calculate the spectrum quite accurately for any temperature. The weakness of this approach is that the observed zone must remain in a steady-state for a time period long enough to permit the spectrum to be scanned. In these studies, this period was usually five to eight minutes. Experience with this engine indicated that this time period was sufficiently short to permit the steady-state assumption to be reasonably valid. Thus, the approach used here was to find the temperature for which the calculated spectrum gave the "best fit" to the observed spectrum.

The observed wavelength of a Stokes Raman line, $\lambda_{r}$, due to an incident laser beam of wavelength $\lambda_{L}$ can be written as

$$
\begin{aligned}
\lambda_{r} & =\left(\lambda_{L}^{-1}-\bar{\nu}_{r}\right)^{-1} \\
\text { with } \quad \bar{v}_{r} & =\left[E\left(v^{\prime}, J^{\prime}\right)-E(v, J)\right] / h c,
\end{aligned}
$$

where $E\left(v^{\prime}, J^{\prime}\right)$ and $E(v, J)$ are, respectively, the energies of the final and initial states of the transition (see fig. 7), $h$ is Planck's constant, $c$ is the speed of light, and $\bar{v}_{r}$ is in units of $\mathrm{cm}^{-1}$. The energies are made up of a sum of the vibrational and rotational contributions $[26,27,28]$. The first and second order terms of the vibrational part plus the first order term of the rotational energy were found to give sufficient accuracy $(\sim 0.02 \mathrm{~A})$ for these calculations.

The Raman scattering efficiency, $\varepsilon$, can be defined as the observed photoelectron count rate, $N_{R}$, divided by the incident laser power, $P$, (photons/s). For a given scattering geometry and a scattering molecule in a state given by $\mathrm{J}$ and $\mathrm{v}$, the efficiency becomes

$$
\varepsilon \equiv N_{R} / P \propto n \eta^{\prime}\left(\lambda_{r}\right) S_{J J^{\prime}}(v+1) \lambda_{r}^{-4},
$$

where $J^{\prime}$ is the final rotational state quantum number, $\mathbf{n}$ is the population of molecules in the state given by $v$ and $J, \eta^{\prime}\left(\lambda_{r}\right)$ is the quantum efficiency of the detection system at $\lambda_{r}$, and $S_{J J^{\prime}}$ is the rotational strength factor [26]. The strength factor is a function of $J$ and it derives from three expressions, one each for the 0 branch, $(\Delta J=-2)$, the $Q$ branch, $(\Delta J=0)$, and the $S$ branch, $(\Delta J=+2)$. For a simple homonuclear, diatomic molecule, these expressions depend only on the polarizations of the incident and scattered light and on the nuclear spin. These expressions and their use are given in references $[26,27,28]$.

Assuming that the observed medium is in thermal equilibrium, the molecular population $n$ in the initial level of a transition is related to the concentration of the molecular species, $n_{0}$, being probed through a Boltzmann factor. We have 


$$
n \propto n_{0}(2 J+1) \exp [-1.43879 E(v, J) / T]
$$

where $E(v, J)$ is the energy of the level in $\mathrm{cm}^{-1}$ measured relative to the $v=0, \mathrm{~J}=0$ level; and $T$ is the absolute temperature in $K$. By accounting for various characteristics of the experimental setup, the intensity profile of a Raman spectrum was calculated for a specific temperature using eqs. (14) and (15) and compared to the observed spectrum. An iterative procedure was used to find the best match-up between the observed and calculated spectra thereby providing the temperature.

\subsubsection{Computer fitting procedure}

The technique used to determine the "best fit" is the method of non-linear least squares and comes under the general name of multiple regression analysis. The measure of goodness of fit used is the quantity chi-square given by

$$
x^{2} \equiv \sum_{i=1}^{N}\left[\left[y_{i}-y\left(\lambda_{i}\right)\right]^{2} / \sigma_{i}^{2}\right]
$$

where $\sigma_{i}^{2}$ are the variances in the observed data values $y_{i}, y\left(\lambda_{i}\right)$ are the calculated values of the Raman spectrum at the wavelengths $\lambda_{j}$, and $N$ is the number of spectral positions recorded. In general, the observed spectrum depends on a set of parameters any subset of which can become free variables in any particular sequence of measurements. For the measurements reported here, the free parameters were temperature, the background signal level and slope, and the wavelength correction. A computer program was developed which minimized $x^{2}$ with respect to the chosen set of parameters by an iterative search process. All that was required was that a set of reasonable starting values be provided. The program determined the best fit and generated standard errors for each fitted parameter. These errors were derived from calculations that effectively characterized the size and shape of the minimum in the hypersurface that was defined by the functional dependence of $x^{2}$ on the free parameters. Hence, the errors in effect specify how well-defined the minimum was with respect to the corresponding parameter.

One of the more interesting problems that arose in the search for an efficient leastsquares fitting computer routine [34] was that certain parameters when made free showed a high degree of correlation. For example, the correlation coefficient between the laserinduced background and the temperature was typically between 0.4 and 0.7 . Some fitting routines were found to be unable to find the best fit with such correlations. The program used in this work was derived from the program CURFIT [33]. Its speed and accuracy in handling correlated parameters when used with a CDC 6600 computer has been satisfactory to date.

The digital data values recorded during a measurement were on an arbitrary scale set by the apparatus as described in Section 3.3.1.4. In order, to make the calculations of $x^{2}$ and 
the parameter errors numerically correct, it was necessary to determine the average number of observed counts per photoelectron. Weak processes such as light scattering follow Poisson statistics [33], wherein the variance of the count value is equal to the count value. It was found for the PMT voltages used, about 250 counts corresponded to one photoelectron. Since the dynamic range of our detection apparatus was about 1250 observed counts per laser pulse, we observed up to five photoelectrons from each laser pulse. If the combined efficiency of the optics, spectrometer, and PMT was 10 percent, then this amounts to a maximum of 50 scattered photons per laser pulse.

The spectrum calculations and the computer fits were performed on the photoelectron values of the data. This insured that the variances in eq. (16) were statistically related to the data values. Since the variances weight the individual observed calculated differences in eq. (16), their values determine the magnitude of $\chi^{2}$ which, in turn, fixes the magnitudes of the parameter errors. The value of $\chi^{2}$ reflects both the degree of agreement between the functional forms of the calculated and observed spectra and the amount of fluctuation or noise in the data. A useful quantity is the reduced chi-square, $x_{r}^{2}$, which is $x^{2}$ divided by the number of degrees of freedom ( $\mathrm{N}$ minus the number of free parameters). The ideal value for $x_{r}^{2}$ is 1.0 ; however, in practice, values $<1.5$ are acceptable [33]. Except for (1) the low temperature ( $<1000 \mathrm{~K})$ spectra where the approximations in the slit function were visible in the fit, and (2) the spectra having high, and therefore noisy, backgrounds, the $x_{r}^{2}$ values obtained satisfied this criterion. With one exception, the $x_{r}^{2}$ values for all the Raman fits reported here were $<2.0$.

\subsubsection{Determination of fixed parameters}

In order to perform the computer fit described in the previous section, it was necessary to computationally duplicate the integrating effect of the wide spectrometer slits used plus the $\sim 1 \AA$ width of the incident laser light on the dense line spectrum described in Section 3.3.2. This was done by determining the effective overall slit function. This function had to be known in order that the theoretical spectrum determined for a minimum $x_{r}^{2}$ was calculated under conditions that had a one-to-one correspondence to the conditions under which the experimental spectrum was obtained.

There are several approaches to determining the slit function; however, because of its large width relative to the spacing of the individual lines in the $\mathrm{N}_{2}$ Raman spectrum, it was possible to greatly simplify the required calculations by approximating the effective slit function as a trapezoid such as shown in figure 8 where the top and bottom dimensions were determined operationally. This was accomplished by fitting the parameters of the trapezoidal slit function to the Raman spectrum of $\mathrm{N}_{2}$ in room-temperature air using the same slit settings that were used with the engine measurements. The $\mathrm{N}_{2}$ Raman transitions at room temperature ranges up to $\mathrm{J}=15$ to 20 in the $Q$ branch which spans a range of less than $0.1 \AA$ with $3371 \AA$ excitation. Thus, the observed shape of the room-temperature Raman $Q$ branch "line" is, in effect, the effective slit function. 
The spectrometer was operated in third order with entrance/middle/exit slit dimensions of $1.0 \mathrm{~mm} / 1.5 \mathrm{~mm} / 1.0 \mathrm{~mm}$. The trapezoidal slit function fitted to the room temperature data had a slit-bottom half width of $1.481 \pm 0.026 \mathrm{~A}$ and a slit top-to-bottom width ratio (ST/BWR) of $0.121 \pm 0.038$. This gave a full width at half-maximum (FWHM) of $1.660 \pm$ $0.062 \mathrm{~A}$.

The importance of the careful determination of a valid effective slit function can be seen in a study [35] of the dependence of the fitted temperature on the two slit parameters with all other parameters held fixed. It was found that the temperature shifted up about $2.5 \mathrm{~K}$ for a 10 percent increase in ST/BWR while a downward shift of about $31 \mathrm{~K}$ occurred for a 10 percent increase in the FWHM. Clearly, the opportunities for accumulation of systematic errors are significant in a circumstance such as this one where a number of parameters are correlated. The point, of course, is that there are many "good" fits possible to a given set of data depending on which parameters are permitted to be free. Therefore, to obtain the "correct" fit, it is necessary to minimize the number of free parameters while choosing values for the fixed parameters that can be justified on physical grounds (i.e., on measurements).

As the result of the sensitivity to the slit parameters, an additional complication was brought on by the intense vibrations induced in the spectrometer by the engine as discussed in Section 5. The observed value of FWHM increased by $\sim 5$ percent while ST/BWR decreased by $\sim 20$ percent when the engine power was increased from the minimum afterburning $(A / B)$ condition to the maximum $A / B$ condition. From the above-mentioned study [35], both of these changes would shift the fitted temperature upwards if constant slit parameters equal to the room-temperature values were used. The shift was found to be $\sim 20 \mathrm{~K}$. The slit parameters were varied in a linear way with engine $A / B$ power between the minimum and maximum $A / B$ power values in order to correct for this bias.

For the most part, the scattering by a diatomic molecule is isotropic (viz., the socalled "trace" scattering). That is, the scattering follows the electric dipole induced by the oscillating electric field (i.e., the polarization) of the incident wave. The intensity due to trace scattering $\mathrm{I}_{\mathrm{T}}$ is the primary contribution to the $Q$ branch. Due to the molecule's non-spherical shape, there is also an anisotropic part to the polarizability which not only contributes a small amount to the Q branch intensity but also determines the strength of the 0 and $S$ branches. This anisotropic part produces a scattering intensity $I_{A}$ that contains a portion which is polarized perpendicular to the incident polarization. Thus, the total intensity of the $Q$ branch can be operationally defined as $I_{T}$ $+\mathrm{bI}_{A}$ where $b$ is a small fraction which accounts for the polarization properties of the optical system (viz., the laser beam and the spectrometer).

Ordinarily, it is sufficient to calculate only the $Q$ branch contributions [30,31]; however, in our case it was found necessary to deal with an additional feature. Scans of the scattered laser light at $3371 \AA$ (Rayleigh scattering) revealed that the laser emission included a weak "hot-band-type" 1 ine at about $3367 \AA$. The strength of this "spurious" emission line was about 2 percent of the "Rayleigh" peak. Since the Raman scattering from this peak nearly coincides with the first hot band in the $\mathrm{N}_{2}$ Raman spectrum of the $3371 \AA$ 
laser line, it was necessary to measure it accurately and to include it in the calculations. This was accomplished by recording the $\mathrm{N}_{2}$ Raman spectrum of room air over large portions of the 0 and $S$ branches as well as the Q branch. A signal range of over 1000:1 was recorded by operating the PMT at two voltages and recording the three branches using overlapping spectra. The calculated spectrum, including the spurious line, was fitted to this observed spectrum. The fractional contribution of $I_{A}$ specified by $b$, the spurious line height, and the line's position were made free parameters along with the slit parameters. By means of a series of fittings, the best fit of these parameters was obtained. The value of $b$ was found to be $0.174 \pm 0.016$ with the spurious line shifted down by 4.713 $\pm 0.036 \AA$ with a strength ratio relative to the main line of $0.0203 \pm 0.0012$. The value of $b$ is acceptably close to the value of 0.156 predicted for unpolarized laser light and polarization insensitive detection $[27,36]$.

A linear background was added to the calculated spectrum described above. The parameters for this background were the intercept at $3657 \mathrm{~A}$ and the slope. These two parameters, the temperature, and a wavelength correction parameter were made free parameters in the fitting procedure. All the calculations included the 0 and $S$ branch contributions, the strengths of which relative to the $Q$ branch were fixed by the constant $b$. However, several of these calculations were repeated with $b=0$, and it was found that the fitted background increased slightly while the fitted temperature decreased. The temperature decrease ranged from about 0.8 percent at $\sim 800 \mathrm{~K}$ to 0.2 percent at $\sim 1600 \mathrm{~K}$. These results suggest that in many cases the anisotropic part of scattering can be neglected.

\section{The Experiment}

This section is divided into four parts. The objective of the experiment is described in Section 4.1. A description of the experimental configuration is given in Section 4.2. Section 4.3 gives the test schedule, and Section 4.4 gives the test conditions and test procedures.

\section{1 Objective}

The objective of the experiment was to determine if spontaneous Raman scattering could be used to measure temperatures in the plume of an afterburning J85-5 turbojet engine. To accomplish this objective, we believe that two claims must be demonstrated: (1) that Raman spectra of sufficient quality to permit temperature determinations can be measured, and (2) that these tempe:atures will be accurate within reasonable limits. The demonstration of (1) was straightforward. We recorded quality Raman spectra at different engine power conditions and then determined if temperatures could be obtained from these spectra. Our approach to (2) was to make Raman, thermocouple, and gas sampling temperature measurements at nearly the same spatial location in the plume and at the same engine power conditions and compare the results. 
The time required per Raman measurement was a secondary consideration in this study. Since this was the first time Raman measurements had been attempted in an afterburning engine plume, we directed our attention towards obtaining meaningful Raman spectra. Typical measurement times were five to eight minutes. No attempts were made to reduce this time, although this could probably have been accomplished in one of several ways. However, the impact of reducing the measurement time on the accuracy of the temperature measurements would have required careful examination. Such an examination was not included as part of this study.

\subsection{Experimental configuration}

The J85-5 engine is used in the F-5 fighter and the T-38 trainer aircraft. It is a high thrust-to-weight engine. At the maximum afterburning ( $A / B)$ power condition, it develops about $17 \mathrm{kN}$ (3800 lbf) of thrust and weighs about $265 \mathrm{~kg}$ (584 lbm). Afterburning is used primarily in military aircraft to augment thrust during takeoff or combat operations. This is accomplished by injecting and burning fuel downstream of the turbine. In our experiment, a bright blue flame extended about $3 \mathrm{~m}$ beyond the exit plane demonstrating that combustion occurred over this distance.

The experiments were performed in a sea level test stand at the Air Force Aero Propulsion Laboratory. This facility is described in [37]. Figure 3 illustrates the experimental arrangement. A photograph of the experimental set-up is shown in figure 9. The Raman transceiver unit, which is located in the photograph to the left of the engine, was about $3 \mathrm{~m}$ from the engine centerline. At this location, it was estimated that the Raman system experienced an overall sound power level of about $172 \mathrm{~dB}$, referenced to $10^{-12}$ $W$. The gas sampling probe attached to an $X-Y$ positioning device is also shown in figure 9. The probe was positioned so the probe tip was on the engine centerline at an axial distance of $116 \mathrm{~cm}$ from the nozzle exit. The thermocouple probe junction was located at this same position when it was being tested.

The Raman measurement volume was located $114 \mathrm{~cm}$ from the nozzle exit and was centered about the engine centerline. This location was chosen primarily so that a uniform temperature profile occurred within the Raman measurement volume (see Section 3.3.1.2). However, other benefits were gained from making the measurements at the $114 \mathrm{~cm}$ axial station. First, our results could be compared with those reported by Lyon, et al. [3]. Second, the emissions profiles were uniform at this station and extended almost the entire diameter of the exhaust. This meant that the local fuel-air ratio measured with a gas sampling probe at the engine centerline was about the same as the overall engine fuel-air ratio ( $F / A)$. Thus, by comparing the local F/A with the engine F/A, we could check the validity of the emissions measurements. 


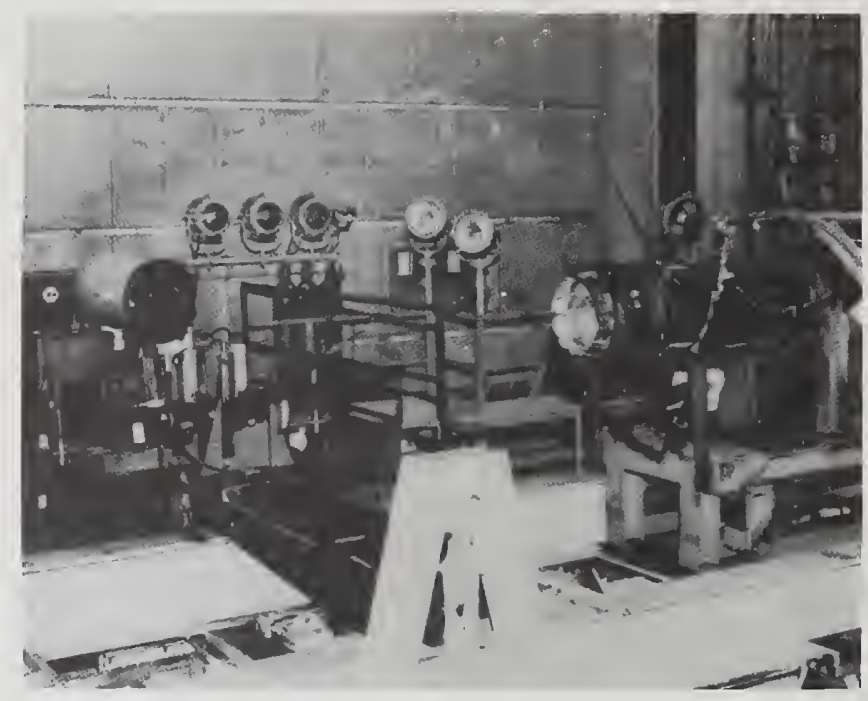

Figure 9. The J85-5 test stand showing the afterburner portion of the engine (right side of photo) and the Raman transceiver and gas sampling probe installations (facing out and at left side of photo).

\subsection{Test schedule}

Two sequences of Raman measurements were accomplished wherein spectra were obtained as a function of engine power. The first series was made in March 1977 [38]. Simultaneous Raman and gas-sampling measurements were planned in these tests. Unfortunately, the gas sampling probe developed an internal leak and could not be used at that time. The Raman data obtained during this sequence was the most self-consistent of the two data sets in that repeated runs at the same engine condition did not show any extraordinary deviations. of the 12 runs recorded, 10 were obtained within a two-day period. A11 of the 12 spectra gave viable measurements of temperature.

The second series of measurements occurred in July 1977. The afterburning section of the engine had been replaced in June because of a burn-through that occurred during another test program. The replaced afterburner was the same type as that used in the March tests. During the July tests, gas-sampling probe measurements were made simultaneously with the Raman measurements. Thirty-five Raman runs were recorded in a four-day period. However, many of these runs were of poor quality due to loss of laser power or due to the occasional occurrence of high, noisy background signals of unknown origin. The high ambient temperatures in the cell often caused the laser to go off during a run due to overheating. Also, the high humidity conditions caused condensation to slowly fog the laser windows reducing the available laser power. As the result, only 15 of the 35 Raman runs could be accepted as valid measurements. 
After the July tests, cracks developed in the afterburner liner and it had to be replaced. Additional gas-sampling data were collected over a two-day period in 0ctober 1977. The gas-sampling temperatures measured in 0ctober were about 3 percent lower than those measured in July. This may have been due to the different liner. The thermocouple measurements were made over a two-day period in November 1977.

\subsection{Test conditions and procedures}

Twelve different engine power conditions were established for our experiments. As noted in table I, conditions ranged from Idle $(45 \% \mathrm{rpm})$ to Max A/B. The Idle, Mid Idle, and Military power conditions were defined by the engine rpm. The rpm was used by the engine operator for repeating test conditions. The $A / B$ power conditions were repeated by controlling the afterburner fuel flow rates. For example, the $3000 \mathrm{lbm} \mathrm{A} / \mathrm{B}$ condition corresponds to a flow rate of $3000 \mathrm{lbm} / \mathrm{hr}$ of fuel to the afterburner. Since most of the Raman measurements were made at $A / B$ conditions, the data at Idle and Mid Idle conditions were not considered when comparing the different techniques.

Slightly different procedures were followed for the simultaneous Raman and gassampling measurements than were followed for the independent measurements made with the thermocouple and the gas-sampling probes. For the simultaneous tests, the engine was operated at an A/B condition for a sufficient time to collect one or two consecutive Raman spectra. This required about 20 minutes. During the test period, the PMT voltage was adjusted so that the signal levels obtained from the main $\mathrm{N}_{2}$ Raman peak was just within the upper limit of the dynamic range of the linear-gate-and-stretcher module. Each Raman scan was generally chosen to cover 51 to 61 steps at $0.3 \AA$ per step centered about the first hot band at $3653.8 \AA$. This insured that the laser-induced background signal beyond the second hot band was recorded as well as the background on the main-peak side of the spectrum. Normally, gas-sampling measurements were made near the start and completion of the test period. Each gas sampling measurement took about three minutes. The engine would be throttled back to a lower power non-A/B condition for about three minutes before going to the next $A / B$ condition. With just the gas-sampling probe or thermocouple probe, measurements were made at all $A / B$ conditions before returning to the low power condition.

\section{Results}

The results of the gas sampling, thermocouple, and Raman measurements made in the J85-5 afterburning plume are summarized in this section. The results are presented in five subsections. Our gas sampling probe measurements and those made by Lyon, et a1. [3] are compared in Section 5.1. The Raman data are presented and discussed in Section 5.2. Section 5.3 contains the results of the simultaneous gas sampling and Raman measurements. The thermocouple data are presented in Section 5.4 and a comparison between all the avai1able data is given in Section 5.5. 


\subsection{Comparison of APL and GE gas sampling measurements}

Figure 10 illustrates the total and static engine temperature rise measured during our tests at the Aero Propulsion Laboratory (APL) along with the results of measurements made by Lyon, et al. [3] at a General Electric (GE) test facility. The engine temperature rise is the measured temperature minus the engine inlet temperature. The same model engine was used in both tests. The APL and GE measurements were made with the same gas sampling probe at nearly the same axial location in the plume. The maximum fuel-air (F/A) ratio for the GE data was about 0.062 . This is close to the stoichiometric value of 0.067 where the peak flame temperature occurs [25]. Because of this, second order polynomial curves (dashed lines) were required to fit the GE data. The maximum F/A ratio for the APL tests was about 0.052 . This was sufficiently removed from the stoichiometric value that linear curves (solid lines) gave good fits to our data.

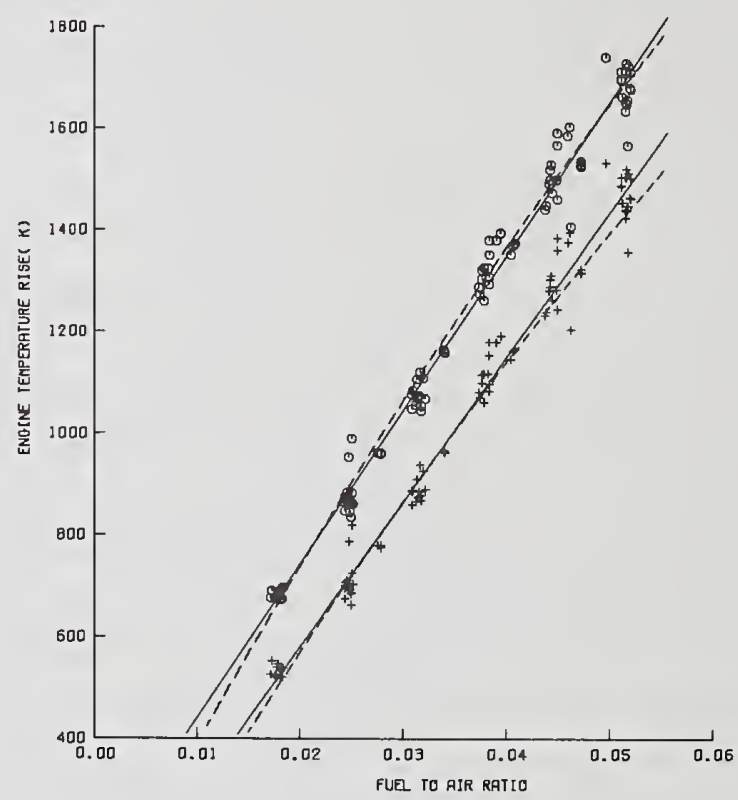

Figure 10. Gas sampling data for the J85-5 engine. The upper curves are the total temperature rise (circles) and the lower curves are the static temperature rise (crosses). The solid curves are linear fits to the data reported in this study $(A P L)$. The dashed curves are second order polynomial curve fits to the data reported by Lyon, et a1. [3] of General Electric for the same type engine. To simplify presentation, only the data points reported in the APL study are shown. 
Lyon, et al. [3] made measurements at different radial positions for four different engine test conditions. For each of the test conditions, all data points within a $10 \mathrm{~cm}$ radius of the plume centerline, corresponding to the flat portion of the plume as shown by figure 5 , were used in establishing the dashed curves in figure 10 . The engine $F / A$ ratios were used in plotting the APL data. Because Lyon, et al. did not measure the engine $F / A$ ratios, local values were used in plotting the GE data.

The curves in figure 10 representing the GE and APL gas sampling measurements are in better agreement than one might expect. The difference between the fitted APL and GE curves of static temperature rise was less than 3.5 percent for all F/A ratios between 0.017 to 0.055 and was less than 1 percent for the total temperature rise for the same F/A ratio range. About 1.3 percent of the deviation in static temperature rise is a result of a bias error in our calculations of gamma (see Section 3.1.1.3). The standard deviations about the linear regression lines gave fitting errors of about the same magnitude for the $\mathrm{APL}$ and GE measurements of static and total temperature rise and varied between 2 percent and 8 percent depending on F/A ratio. The average precision was about 5 percent which easily accounts for the observed difference in APL and GE measurements. This excellent agreement reinforced our confidence in using gas sampling as a standard method to compare with the Raman technique and it demonstrated the good repeatability of the J85-5 engine as a combustion source.

Two problems occurred while we were making gas sampling measurements. Early in the test program, a large discrepancy was observed between the APL and GE measurements. The problem was traced to the non-linear $\mathrm{CO}_{2}$ calibration curve. The instrument had been "calibrated," as suggested by the manufacturer, by introducing a known calibration gas and adjusting the meter to read the appropriate value on the manufacturer's calibration curve. Suspecting that the calibration curve had changed, we established our own curve by using six different calibration gases which covered the range of the instrument. The new calibration curve, which is the one used in the final data analysis, was significantly different from the manufacturer's curve. Periodic checks on the new calibration curve showed that it did not change during the course of the experiments. This example demonstrates the importance of making comparative measurements to check out measurement procedures.

The second problem occurred during an afterburner test. An internal leak developed within the probe. Coolant was forced through the static pressure line and then ejected through the static pressure orifice at the tip of the probe. The coolant mixture was sucked into the gas sampling orifice and through the sampling lines. Fortunately, filters prevented the coolant from reaching the analytical instruments. However, the sampling lines had to be cleaned and reconditioned before they could be used again. This type of problem is typical of those that can occur with gas sampling probes in hostile combustion environments. 


\subsection{Raman data}

Examples of the recorded Raman spectra are presented in figures 11 and 12 . The data in figure 11 are plotted on logarithmic scales in order to reveal the character of the low data values on either side of the main peak. Note the beginning of the 0 and $S$ branches in the "Military" scan and the absence of these features in the low afterburner (A/B) spectrum. In figure 12 , the data are plotted on linear scales. The $A / B$ data shown in figure 11 were included to aid comparisons between the three power conditions. The onset of the second hot band is seen in the $4000 \mathrm{lb} / \mathrm{hr} A / B$ spectrum. The noise due to the high background in the $1775 \mathrm{lb} / \mathrm{hr}$ data (nearly three times the Raman hot band) compared to the $4000 \mathrm{lb} / \mathrm{hr}$ data is clearly evident. The fit of the calculated curves shown in the two figures are typical of all the Raman data reported here.

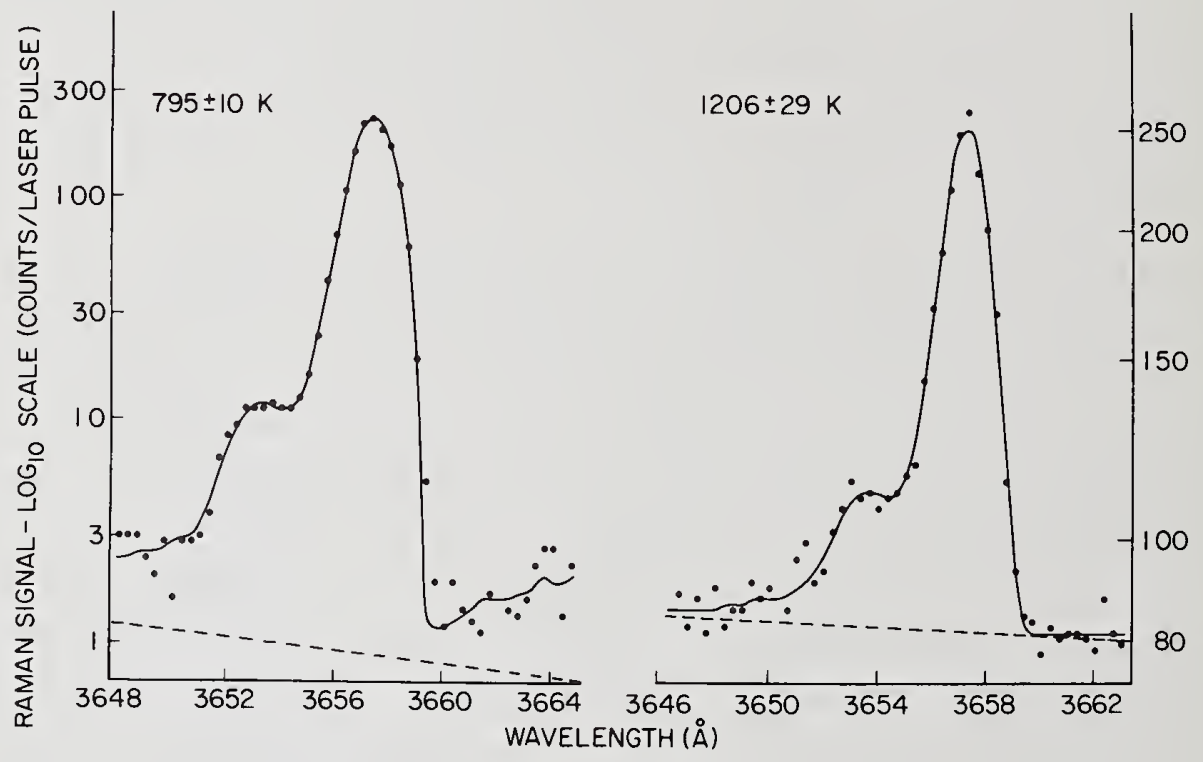

Figure 11. Observed (points) and fitted theoretical (solid lines) spectra of the $\mathrm{N}_{2}$ Q branch Raman transitions on logarithmic scales for the Military (left) and 1775 1bs/hr afterburner power (right) conditions of the J85-5 engine obtained in March 1977.

The July data are presented in table II along with the temperatures measured simultaneously with the gas sampling probe and reported in table I. The March data are given in table III. The engine $F / A$ ratios given in table III permit a comparison of the Raman temperature with the gas-sampling-probe temperatures obtained from the July tests. 


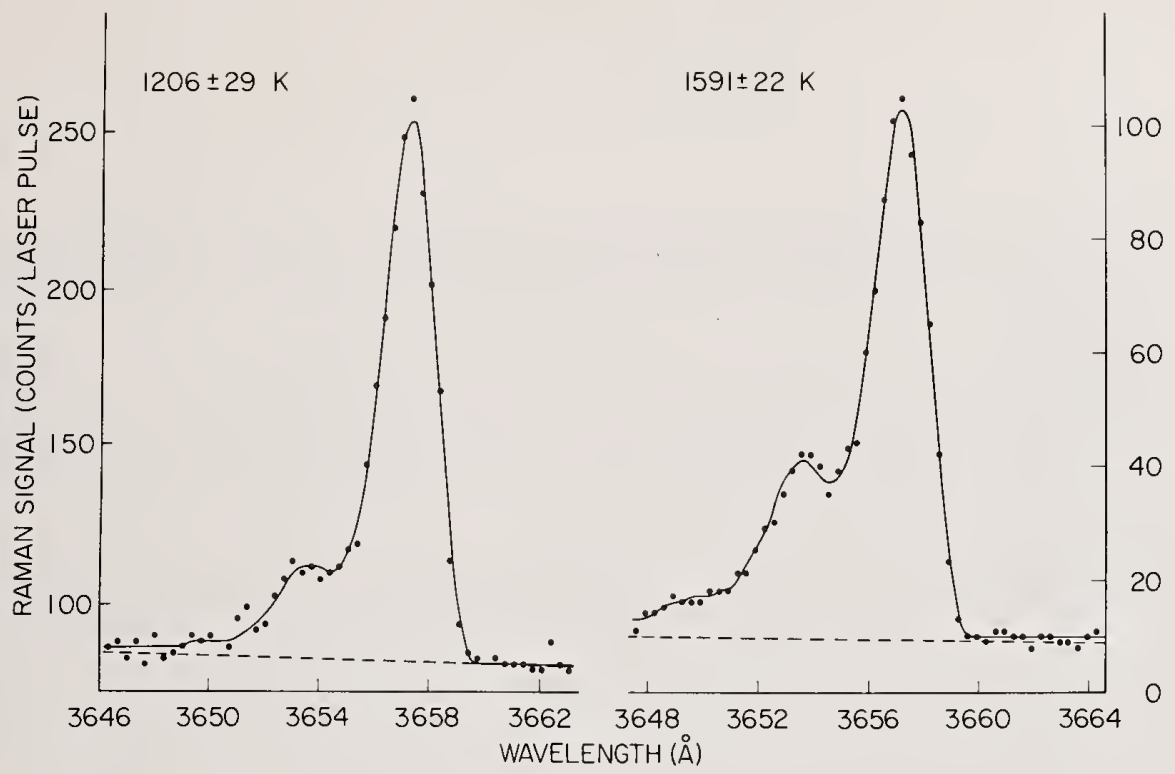

Figure 12. Observed (points) and fitted theoretical (solid lines) spectra of the $\mathrm{N}_{2} \mathrm{Q}$ branch Raman transitions on linear scales for the $1775 \mathrm{lbs} / \mathrm{hr}$ (left) and $4000 \mathrm{lbs} / \mathrm{hr}$ (right) afterburner power conditions of the J85-5 engine obtained in March 1977.

\subsubsection{Background signals}

The Raman temperature values and the fitting errors obtained from the March and July tests were in good accord with each other and with the gas sampling probe data. However, the similarity ends there. As table III shows, the March data has a well-defined trend in the laser-induced background signals and slopes. This is consistent with the uninterrupted sequence in which ten out of the twelve runs were carried out. In the July tests, a large variability in the background signals and slopes was recorded. Some of these features are apparent in table II. For example, the background signal drops sharply and the slope reverses sign at the $3000 \mathrm{lb} / \mathrm{hr} A / B$ condition. Also, run $T 6 B / 07 / 14 / 77$ shows an extraordinarily high background relative to the Raman hot band signal, especially for the Max $A / B$ condition. High backgrounds occurred at other engine conditions too, but the data were too noisy to give viable fits. There is no clear basis for understanding why the two tests had such a different character. The gas sampling probe data did not have any characteristics which could account for these differences. Although speculations can be made to explain the data, it is suffice here to suggest that the Taser-induced background signal carries information concerning the engine operation which is not commonly accessible.

The laser-induced background signal was the most intense at low afterburning ( $A / B)$ conditions and decreased rapidly as the $A / B$ power was increased. For example, as the fuel flow to the afterburner was increased from $2000 \mathrm{lb} / \mathrm{hr}$ to $3000 \mathrm{lb} / \mathrm{hr}$, table II shows that 


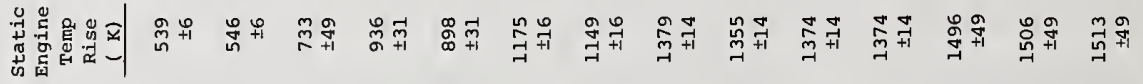

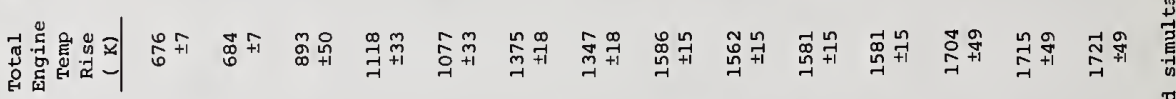

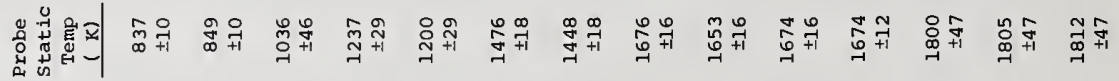

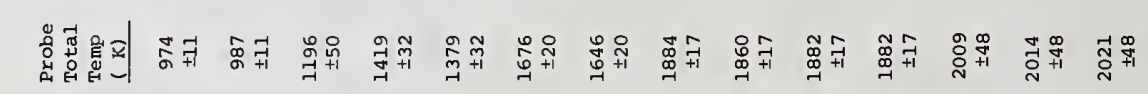

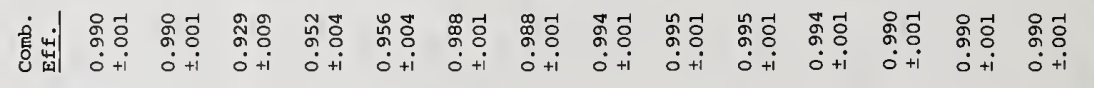

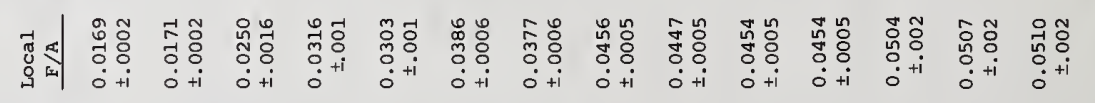

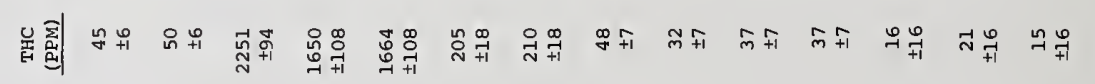

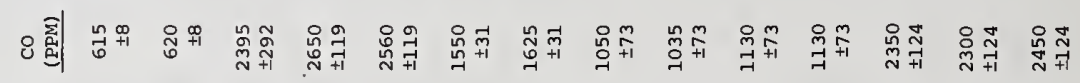

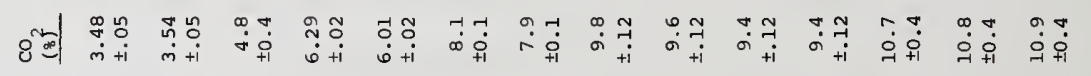

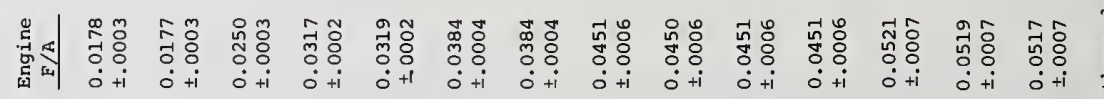

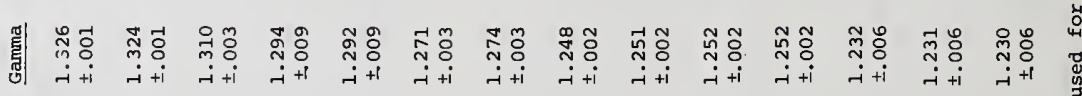

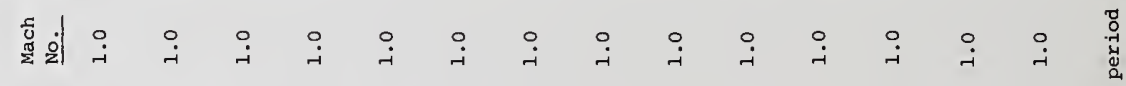

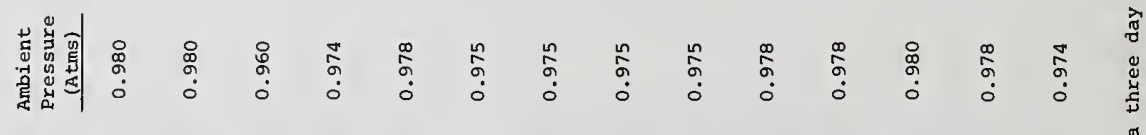

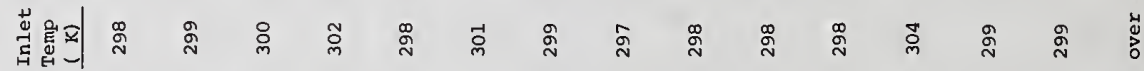

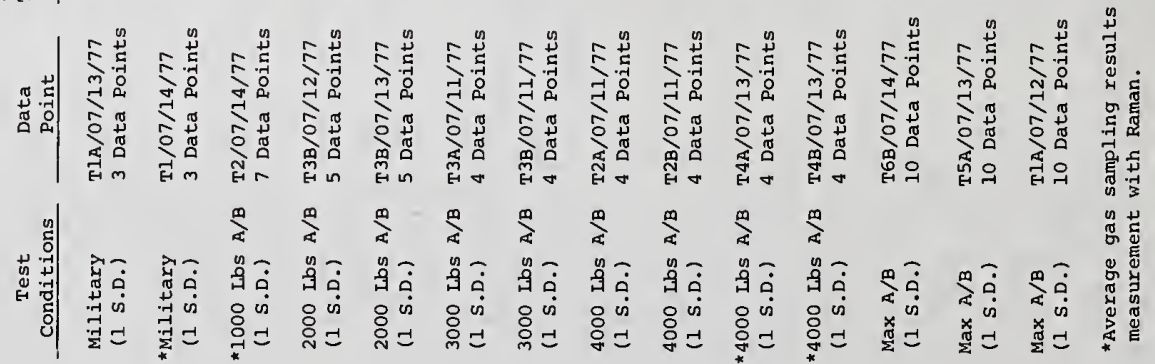




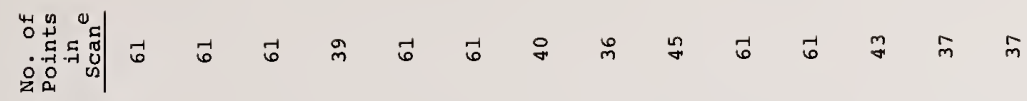

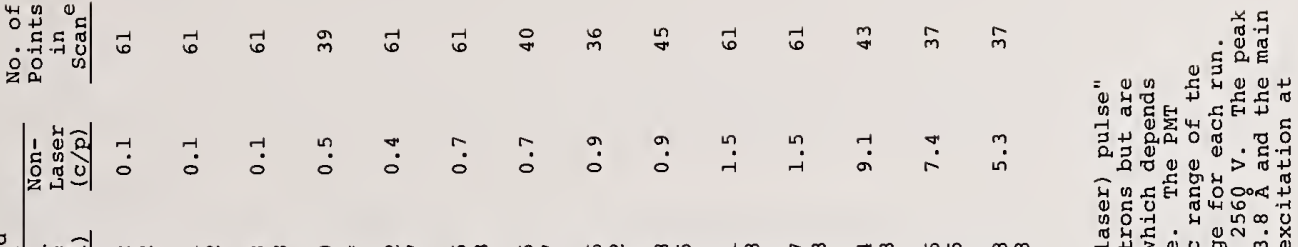

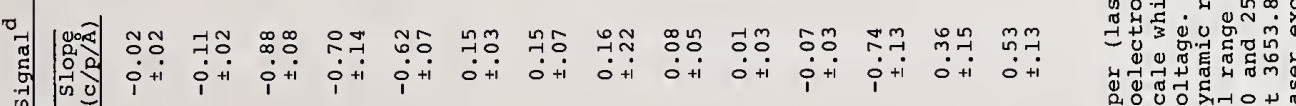

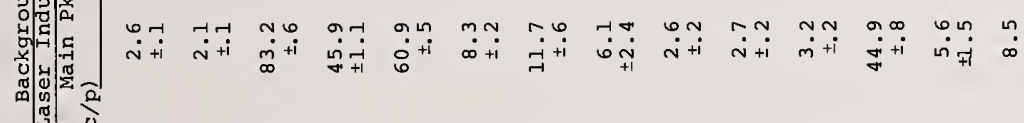

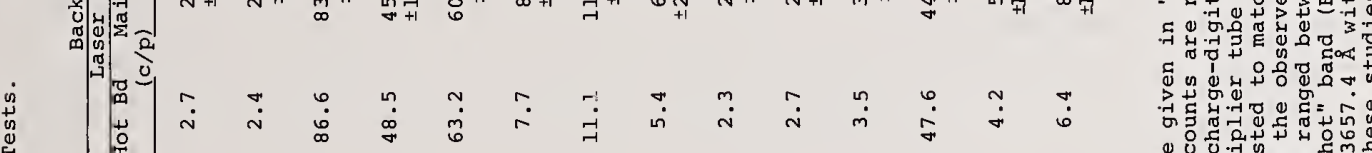

s.

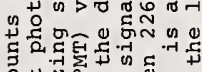

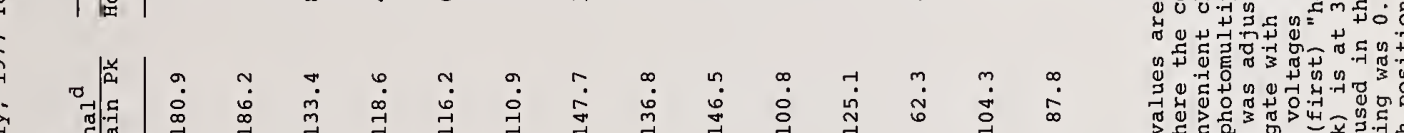

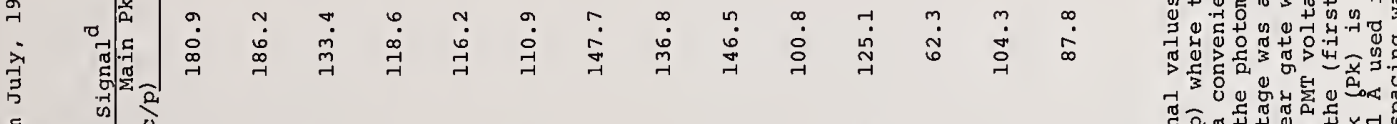

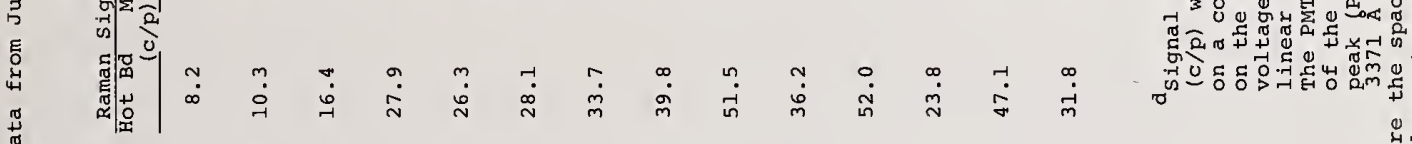

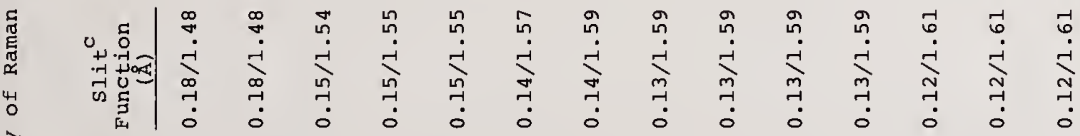

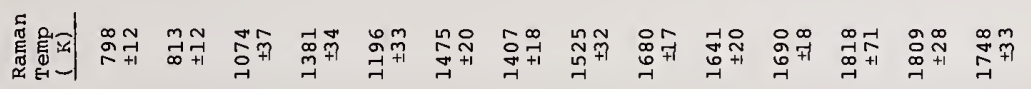

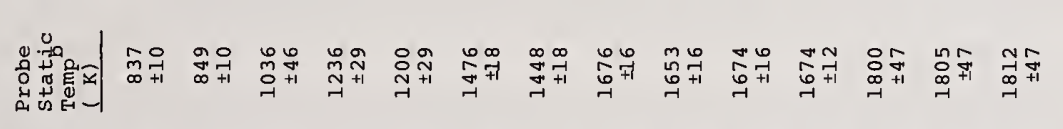

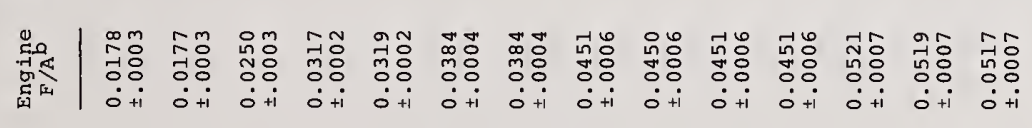

HIIIIIIM

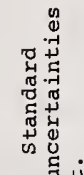

.5 $\quad 0^{0}$

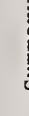


TABLE III. Summary of Raman Data from March, 1977 Tests.

\begin{tabular}{|c|c|c|c|c|c|c|c|c|c|c|c|}
\hline \multirow{4}{*}{$\frac{\begin{array}{c}\text { Test } \\
\text { Condition }\end{array}}{\begin{array}{c}\text { Military } \\
\text { (1 S.D.) }\end{array}}$} & \multirow{4}{*}{$\begin{array}{c}\text { Run/Date } \\
\text { F1A/03/30/77 }\end{array}$} & \multirow{4}{*}{$\begin{array}{c}\begin{array}{c}\text { Engine } \\
\text { F/A b }\end{array} \\
0.0179\end{array}$} & \multirow{4}{*}{$\begin{array}{l}\begin{array}{l}\text { Raman } \\
\text { Termp } \\
(\mathrm{K})\end{array} \\
816 \\
\pm 12\end{array}$} & \multirow{4}{*}{$\begin{array}{c}\begin{array}{c}\text { Slit }{ }^{c} \\
\text { Function } \\
(\AA)\end{array} \\
0.18 / 1.48\end{array}$} & \multirow{3}{*}{\multicolumn{2}{|c|}{$\begin{array}{l}\text { Raman Signal }{ }^{\mathrm{d}} \\
\text { Hot Bd Ma in } \overline{P k} \\
\text { (c/p) }\end{array}$}} & \multicolumn{4}{|c|}{ Background signal $^{d}$} & \multirow{4}{*}{$\begin{array}{c}\begin{array}{c}\text { No. of } \\
\text { Points } \\
\text { in } \mathrm{e} \\
\text { Scan }\end{array} \\
46\end{array}$} \\
\hline & & & & & & & \multirow{2}{*}{\multicolumn{2}{|c|}{$\begin{array}{l}\text { Laser Indu } \\
\text { Hot Bd Main pk } \\
(c / p)\end{array}$}} & \multirow{3}{*}{$\begin{array}{c}\begin{array}{c}\text { slope } \\
(\mathrm{c} / \mathrm{p} / \AA)\end{array} \\
-0.03 \\
\pm .03\end{array}$} & \multirow{3}{*}{$\begin{array}{c}\begin{array}{l}\text { Non- } \\
\text { Laser } \\
(c / p)\end{array} \\
0.2\end{array}$} & \\
\hline & & & & & & & & & & & \\
\hline & & & & & 9.9 & 216.4 & 1.0 & $\begin{array}{l}0.9 \\
\pm .1\end{array}$ & & & \\
\hline $\begin{array}{l}\text { Military } \\
\text { (I S.D.) }\end{array}$ & $\mathrm{F} 1 \mathrm{~B} / 03 / 30 / 77$ & 0.0179 & $\begin{array}{l}795 \\
\pm 10\end{array}$ & $0.18 / 1.48$ & 10.3 & 222.7 & 1.0 & $\begin{array}{l}0.9 \\
\pm .1\end{array}$ & $\begin{array}{r}-0.03 \\
\pm .02\end{array}$ & 0.2 & 51 \\
\hline $\begin{array}{l}1775 \text { Lbs A/B } \\
\text { (1 S.D.) }\end{array}$ & $\mathrm{F} 1 \mathrm{~A} / 03 / 29 / 77$ & 0.0300 & $\begin{array}{r}1130 \\
\pm 33\end{array}$ & $0.15 / 1.55$ & 29.4 & 184.1 & 84.6 & $\begin{array}{r}83.3 \\
\pm .9\end{array}$ & $\begin{array}{r}-0.38 \\
\pm .12\end{array}$ & 0.6 & 51 \\
\hline $\begin{array}{l}1775 \text { Lbs A/B } \\
(1 \text { S.D.) }\end{array}$ & $F 1 B / 03 / 29 / 77$ & 0.0303 & $\begin{array}{r}1181 \\
\pm 27\end{array}$ & $0.15 / 1.55$ & 30.6 & 172.3 & 81.3 & $\begin{array}{r}80.2 \\
\pm .7\end{array}$ & $\begin{array}{r}-0.32 \\
\pm .10\end{array}$ & 0.7 & 51 \\
\hline $\begin{array}{l}1775 \text { Lbs } A / B \\
(1 \text { S.D.) }\end{array}$ & $\mathrm{FlC} / 03 / 29 / 77$ & 0.0301 & $\begin{array}{r}1206 \\
\pm 29\end{array}$ & $0.15 / 1.55$ & 29.2 & 179.0 & 82.4 & $\begin{array}{r}81.5 \\
\pm .8\end{array}$ & $\begin{array}{r}-0.25 \\
\pm .11\end{array}$ & 0.6 & 51 \\
\hline $\begin{array}{c}2600 \text { Lbs } A / B \\
(1 \text { S.D. })\end{array}$ & $F 2 A / 03 / 29 / 77$ & 0.0358 & $\begin{array}{r}1299 \\
\pm 36\end{array}$ & $0.14 / 1.57$ & 31.8 & 150.5 & 47.1 & $\begin{array}{r}46.7 \\
\pm .8\end{array}$ & $\begin{array}{r}-0.12 \\
\pm .12\end{array}$ & 1.5 & 50 \\
\hline $\begin{array}{l}2600 \text { Lbs } A / B \\
(1 \text { S.D. })\end{array}$ & $F 2 B / 03 / 29 / 77$ & 0.0357 & $\begin{array}{r}1333 \\
\pm 24\end{array}$ & $0.14 / 1.57$ & 38.6 & 150.0 & 46.1 & $\begin{array}{r}45.3 \\
\pm .5\end{array}$ & $\begin{array}{r}-0.23 \\
\pm .06\end{array}$ & 1.4 & 61 \\
\hline $\begin{array}{c}2600 \text { L.bs } A / B \\
(1 \text { S.D. })\end{array}$ & $\mathrm{F} 2 \mathrm{C} / 03 / 29 / 77$ & 0.0355 & $\begin{array}{r}1350 \\
\pm 29\end{array}$ & $0.14 / 1.57$ & 35.8 & 151.1 & 44.4 & $\begin{array}{r}43.8 \\
\pm .6\end{array}$ & $\begin{array}{r}-0.15 \\
\pm .08\end{array}$ & 1.4 & 61 \\
\hline $\begin{array}{c}3450 \text { Lbs } A / B \\
(1 \text { S.D.) }\end{array}$ & $\mathrm{F} 2 \mathrm{~A} / 03 / 30 / 77$ & 0.0417 & $\begin{array}{r}1481 \\
\pm 25\end{array}$ & $0.13 / 1.58$ & 35.1 & 114.3 & 16.6 & $\begin{array}{r}16.2 \\
\pm .4\end{array}$ & $\begin{array}{r}-0.09 \\
\pm .05\end{array}$ & 2.2 & 57 \\
\hline $\begin{array}{c}3450 \text { Lbs A/B } \\
(1 \text { S.D.) }\end{array}$ & $\mathrm{F} 2 \mathrm{~B} / 03 / 30 / 77$ & 0.0415 & $\begin{array}{r}1438 \\
\pm 28\end{array}$ & $0.13 / 1.58$ & 29.4 & 114.8 & 16.9 & $\begin{array}{r}16.5 \\
\pm .4\end{array}$ & $\begin{array}{r}-0.10 \\
\pm .06\end{array}$ & 2.2 & 56 \\
\hline $\begin{array}{l}4000 \text { Lbs } A / B \\
(1 \text { S.D. }\end{array}$ & $\mathrm{F} 2 / 03 / 21 / 77$ & 0.0446 & $\begin{array}{r}1591 \\
\pm 22\end{array}$ & $0.13 / 1.59$ & 32.8 & 95.6 & 9.4 & $\begin{array}{l}9.2 \\
\pm .3\end{array}$ & $\begin{array}{r}-0.04 \\
\pm .04\end{array}$ & 2.7 & 51 \\
\hline $\begin{array}{l}4000 \text { Lbs } A / B \\
(1 \text { S.D. })\end{array}$ & $\mathrm{F} 2 / 03 / 24 / 77$ & 0.0450 & $\begin{array}{r}1521 \\
\pm 21\end{array}$ & $0.13 / 1.59$ & 22.1 & 68.3 & 11.3 & $\begin{array}{r}11.1 \\
\pm .2\end{array}$ & $\begin{array}{r}-0.05 \\
\pm .03\end{array}$ & 3.0 & 61 \\
\hline
\end{tabular}

a After burner (A/B) fuel flow in lbs per hour. Standard deviations (S.D.) for the Raman data are the uncertainties obtained from the fitting procedure. See text.

bingine data can be derived from Table I.

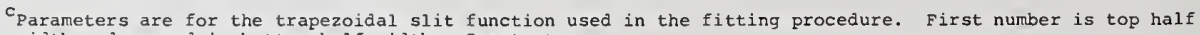
width and second is bottom half width. See text.

a Signal values are given in "counts per (laser) pulse" (c/p), where the counts are not photoelectrons but are on a convenient charge-digitizing scale which depends on the photomultiplier tube (PMT) voltage. The PMT voltage was $2400 \mathrm{~V}$ for all runs except "Military" which used $2350 \mathrm{~V}$ giving a gain reduction to about 798 of the $2400 \mathrm{~V}$ value. The peak of the (first) "hot" band (Bd) is at $3653.8 \AA$ and the main peak (Pk)

$e_{\text {Number }}$ of spectral positions recorded where the spacing was $0.3 \AA$. The signal was recorded for 20001 aser pulses at each position except for Max A/B which was recorded for 4000 pulses and at 250 pps. 
the ratio of the laser-induced-background signal to the Raman-hot-band signal decreased from about two to about 0.3. From table I, the total hydrocarbon concentration also decreased from about $1600 \mathrm{ppm}$ to $200 \mathrm{ppm}$ with the static temperature increased from about 1200 to $1400 \mathrm{~K}$. We believe this behavior signifies that the laser-induced background signals observed in this study were due to fluorescence from unburned hydrocarbons. Laserinduced soot incandescence was unlikely because this engine generated very little soot [3]. Also, the laser intensity in the focal volume was quite low which from the work of Eckbreth, et al. [11] suggests that soot incandescence did not make strong contributions to the backgrounds in our runs.

The non-laser-induced background signal was essentially constant during each scan. The values given in tables II and III resulted from averaging over each scan. Presumably, this signal comes from the luminosity of the plume since it follows the expected trend. Namely, the higher the temperature the greater is the emission at short wavelengths, and hence, the greater the non-laser-induced signal in the 3646-3664 $\AA$ region scanned in these studies. Again, the March values were larger, by about a factor of two for equivalent conditions, than the July values for no apparent reason.

\subsubsection{Inaccurate background measurements}

A precise determination of the laser-induced background levels on both sides of the spectrum was important for all A/B conditions. Without adequate pinning of the background on both sides of the spectrum, the apparent strength of the hot band can vary widely, easily \pm 30 percent. This occurs when the computer search fits a background that is shifted either too high or too low. When this happens, there is a negatively correlated shift in the fitted temperature. The result is that the fitted temperature is in error by as much as 10 percent. An example of this effect is the T2A/07/11/77 run in table II. The fitted temperature is clearly 10 percent low while the laser-induced background is about a factor of two higher than the other values at the $4000 \mathrm{lb} / \mathrm{hr} \mathrm{A} / \mathrm{B}$ condition. In addition, the standard deviation (S.D.) values for the background signal and slope are very high. This scan was incomplete in that it was short (36 points), and it was cut off before the high wavelength background was scanned. Thus, by comparisons with the other data, it is apparent that the low temperature and its high standard deviation (S.D.) as well as the unusual background parameter and S.D. values resulted from a lack of well defined background data on both sides of the Raman spectrum. This run was retained in the data set to provide an illustration of this problem area.

Another likely, but somewhat more subtle example of the problem of pinning the background is run $\mathrm{T} 3 \mathrm{~B} / 07 / 12 / 77$ in table II. None of the measured or fitted values for this run by themselves suggest a problem. However, only 39 points were used in the scan. This would not be a problem if the background signal was small, as in the last two Max A/B runs in the table. It is evident from the other data that the fitted temperature is 10 percent too high. As it turned out, only two background data points were obtained on the high wavelength side of the Raman spectrum with four points on the low side. With so few 
points, the background signal was inadequately determined. Although a good fit was obtained, the fitted temperature was incorrect because the statistical fluctuations in the background signal were not averaged out over a sufficient number of points.

\subsubsection{Error analysis}

The errors in the Raman temperatures were mainly comprised of contributions from the measuring apparatus and from the analysis of the data. The errors in the measured variables, signal strength and wavelength, were quite small. The wavelength errors were generally within $\pm 0.05 \AA$, and therefore, were negligible because of the wide slits used in these studies. The performance and use of the detection apparatus is discussed in Section 3.3.1.4. Clearly, a nonlinearity in the response of the apparatus would introduce a bias error in the fitted temperature. The response became slightly nonlinear below about 25 counts/pulse $(c / p)$ such that the measured value was high. This increased the recorded weak background levels obtained with the Military engine condition thereby lowering the recorded strength of the weak hot band. This shifted the fitted temperatures low for the Military condition. Comparison of these temperatures with the probe values in table II shows a shift of about 4 percent. For the remaining runs, this nonlinearity introduced onty slight errors in the fitted background signals and did not affect significantly the temperatures because of the high background or hot band signals, or both.

There were two kinds of errors in the analysis of the data. There were bias errors that arose in the fitting procedure which resulted from various approximations that were made to simplify the analysis of the data and bias errors that could occur due to the large Raman scattering volume. Also, there were the errors due to the statistical fluctuations in the data. The bias errors in the fitting procedure and the scattering volume are described in Sections 3.3.3 and 3.3.1.2. If these bias errors were adequately considered in our computations, and if the theoretical description of the $\mathrm{N}_{2}$ Raman spectrum used in this work was sufficiently accurate, then the fitting errors in the temperature given in tables II and III for the A/B power conditions characterize the accuracy of this approach to Raman thermometry.

One half or more of the temperature fitting error for an $A / B$ condition was due to the noise associated with the background. A dramatic example of this is the first Max $A / B$ run in table II (TGB/07/14/77). This run had a laser-induced background that was about 10 times higher than the remaining Max A/B runs which more than doubled the S.D. in the temperature fit.

This dependence of the fitting error on the background was revealed in a more general way by using the fact that the hot-band-to-main-peak ratio was a linear function of temperature [37] for our choice of slit function. Thus, if $V$ is this ratio, then

$$
V=m\left(T-T_{0}\right) \text {, }
$$


where $m$ is the slope and $T_{0}$ is the temperature intercept. From eq. (17), the temperature error is given by

$$
\sigma_{T}=\left(\sigma_{V} / V\right)\left(T-T_{0}\right)
$$

By applying eq. (18) to the March data in table III and adjusting the calculation to make $\sigma_{T}$ on the average equal to the fitting errors in table III, the contributions of the background to the temperature errors were revealed. The results of these calculations are shown in figure 13. These curves are presented only to illustrate this characteristic dependence of the temperature error on the background signals. Since the signal-to-noise ratio is proportional to the square root of the time interval over which the signal is recorded, then a longer scan time will give a smaller error. However, the probed medium must remain steady during this longer scan. In our case, this meant that the engine performance had to be repeatable throughout the test sequence. We found it difficult to achieve this in blocks of time greater than four days. Thus, our scan times were indirectly influenced by the constraints set by the number of runs planned and the number of days available for the runs.

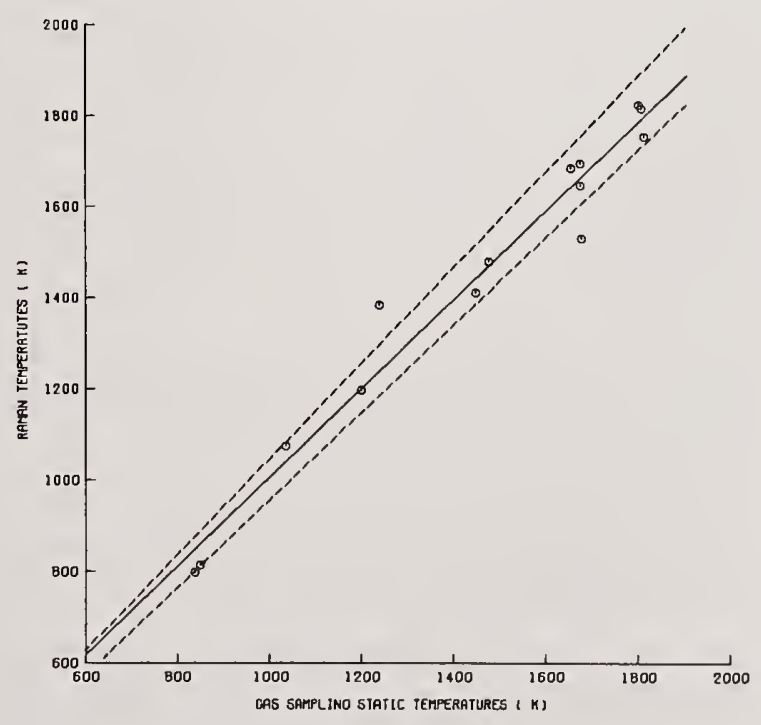

Figure 13. Temperature error analysis of the March 1977 Raman data calculated from the linear dependence of the first-hot-band-to-main-peak ratio on the temperature for the ten afterburner conditions. The curves are best fits to the data points shown. 


\subsubsection{Vibration problem}

Initially, the plans were to record both the Stokes and anti-Stokes spectra and to obtain the temperature from these measurements. The spectrometer was equipped with firstorder gratings blazed at $3000 \AA$ and calibrated. The Raman spectra of $N_{2}$ occur at 3658 and $3125 \AA$ for an excitation wavelength of $3371 \AA$. However, as the engine power was increased, the Stokes and anti-Stokes spectra of $\mathrm{N}_{2}$ dropped in intensity and broadened in wavelength. To identify the problem, the spectrum of a mercury lamp placed on the table supporting the spectrometer was recorded for different engine operating conditions. As the engine power was increased, vibration of the linkage between the two gratings of the spectrometer also increased causing the gratings to vibrate about their rotational axes. This caused the image on the third (output) slit to sweep back and forth creating the observed effect. Every effort was made to reduce the vibration of the spectrometer but it was to no avail.

The recorded spectra of the $3125 \AA$ ine of mercury as affected by this vibration are shown in figure 14 (a), (b), and (c). Because the spacing between the main peak of the $\mathrm{N}_{2}$ Stokes spectrum and the first hot band is $3.6 \mathrm{~A}$, it was desirable to use slit widths that give a spectral resolution of $2.0 \AA$ or less. With the first order gratings, this corresponded to $333 \mu \mathrm{m}$ wide slits. However, judging from the amount of engine-induced broadening shown in figure 14 (c), the effective amplitude of the vibration at the exit slit was between 100 and $200 \mu \mathrm{m}$. This loss of spectral resolution made it untenable for us to proceed with the first-order gratings.

The solution to the vibration problem was to change to third order gratings. This permitted the slits to be increased by a factor of three to $1000 \mu \mathrm{m}$ thereby greatly reducing the influence of the vibration. However, because of the $3333 \AA$ blaze of these gratings, only the Stokes spectrum could be efficiently recorded which meant that the temperature had to be extracted from the profile of this spectrum. The spectra of the $3125 \AA$ line of mercury using the third-order gratings are shown in figure 14 (d), (e), and ( $f$ ) as a function of engine power. Clearly, the vibration-induced broadening is quite smal1 compared to figure 14 (c). The effect of this residual broadening and the procedure followed to correct for it are described in Section 3.3.3.3.

\subsection{Comparison of simultaneous Raman and gas sampling measurements}

The static temperature values from the two measurement techniques are summarized in table II along with the Raman data. The detailed gas sampling probe data are given in table I. The standard deviation (S.D.) values in table I were calculated from the number of data points given for each engine condition. The S.D. values in table II were obtained from the computer curve-fitting procedure. Except for two runs, namely $T 3 B / 07 / 12 / 77$ and $\mathrm{T} 2 \mathrm{~A} / 07 / 11 / 77$ discussed in Section 5.2.2, the two sets of temperatures for the $A / B$ conditions are in good agreement. The disparity in the temperatures for the Military condition is covered in Section 5.2.3. 

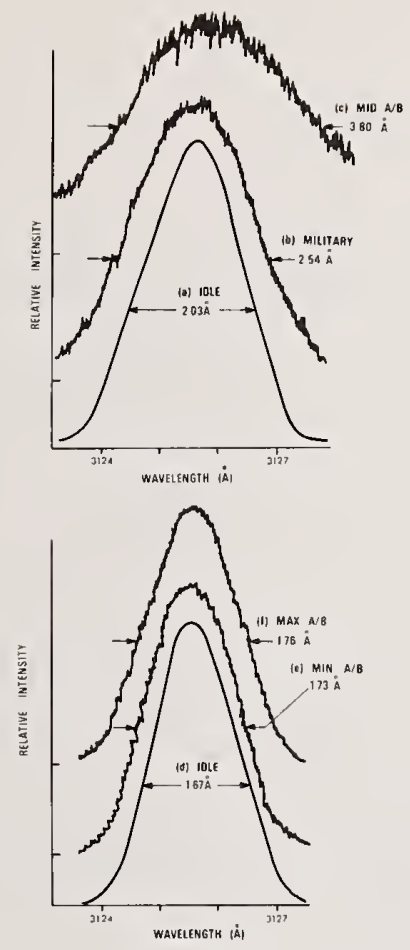

Figure 14. Spectral line shapes of the $3125 \AA$ line from a mercury lamp resulting from engine-induced vibrations. The upper curves, (a), (b), and (c), were obtained with the first order gratings and $333 \mu \mathrm{m} / 500 \mu \mathrm{m} / 333 \mu \mathrm{m}$ slits. The lower curves, (d), (e), and ( $f$ ), were obtained with the third order gratings and $1000 \mu \mathrm{m} / 1500$ $\mu \mathrm{m} / 1000 \mu \mathrm{m}$ slits. The measured full widths at half maximum are given for each curve.

The simultaneously measured temperatures are plotted in figure 15. Ideally, the data should fall on the line of unity slope. The line shown is a least squares fit to the data. It falls well within the \pm 4.5 percent error range shown in the figure. The two points which are outside this range are the two aforementioned runs. Neglecting these two results and the Military runs, the average difference between the two temperature measurements given in table II is 1.6 percent. Including the Military data increases this difference to 2.1 percent. The average S.D. values taken over all 14 runs in the table are 1.8 percent for the gas sampling temperatures and 1.9 percent for the Raman temperatures. The bias error in the gas sampling measurements discussed in Section 3.1.1.3 can increase the overall average gas sampling error to as high as 3.1 percent. Thus, since the differences between the two measurements are well within the uncertainties, we can consider the gas sampling and Raman measured temperatures to be in very good agreement. 


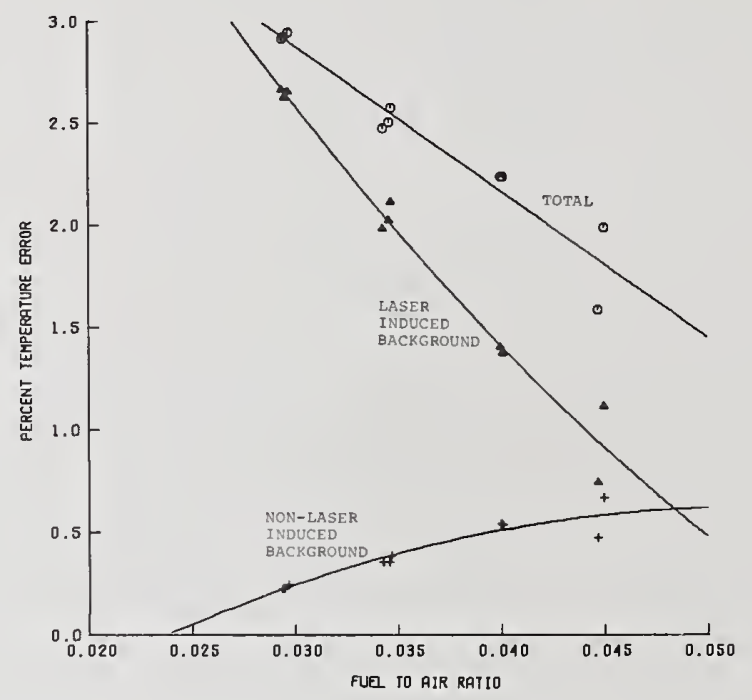

Figure 15. Comparison of static temperatures obtained from simultaneous Raman and gas sampling measurements carried out during the July 1977 tests. The solid line is the linear curve fit to the points. The dashed lines identify the \pm 4.5 percent deviation from the unity slope 1 ine and encompass 86 percent of the data.

\subsection{Thermocouple measurements}

The results of the thermocouple measurements are given in table IV. The table includes the average values of the various engine and thermocouple parameters as we 11 as the standard deviations and the number of data points used in establishing the average values. Note that both the radiation and recovery temperature corrections increase with increasing engine power. If these corrections were not made, a bias error of about 6.8 percent would result at the Max A/B condition.

\subsubsection{Errors}

The estimated accuracy of our thermocouple measurements was based on five sources of error. These errors are summarized as follows: 


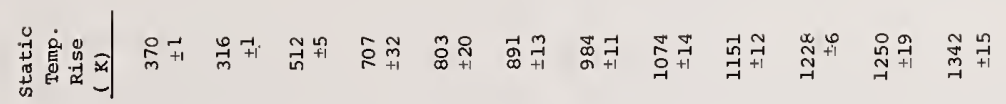

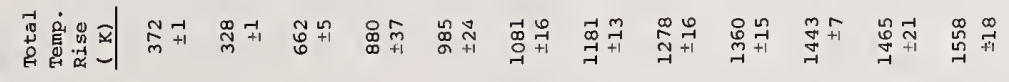

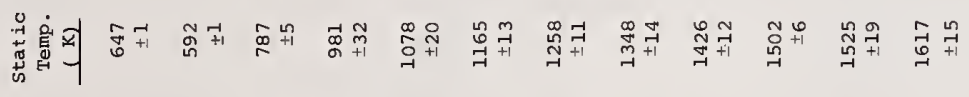

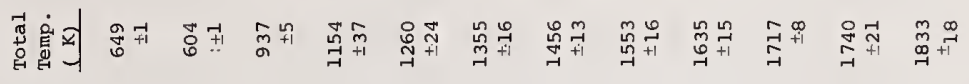

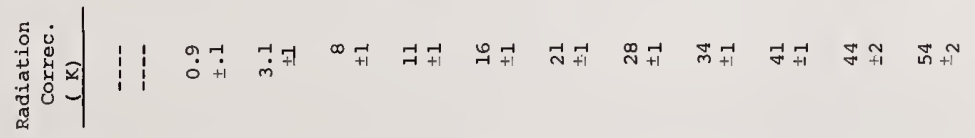

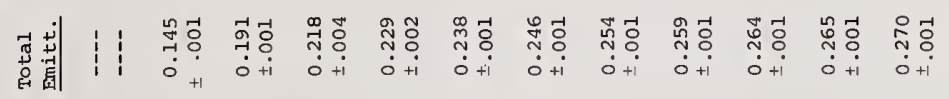

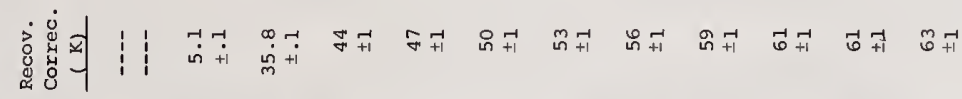

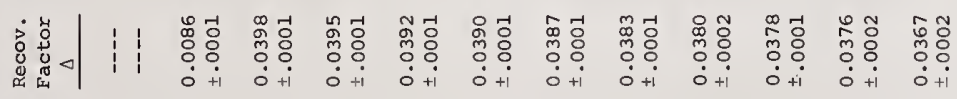

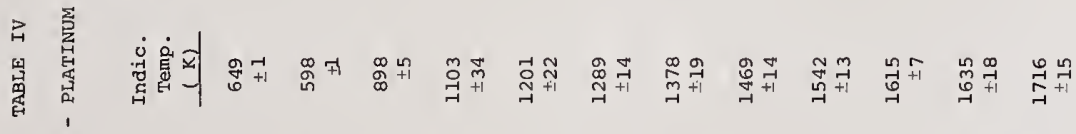

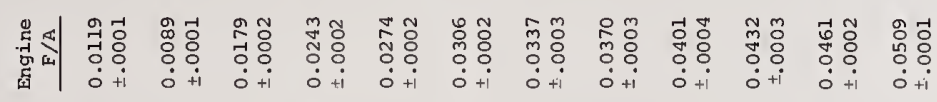

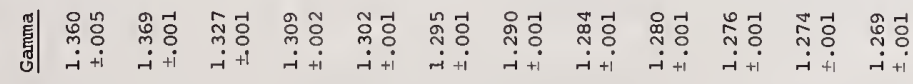

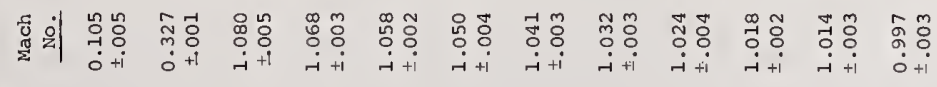

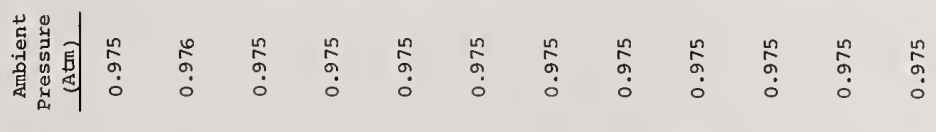

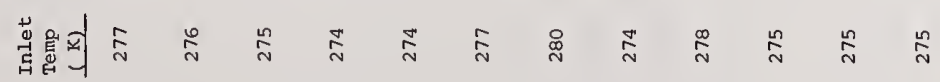

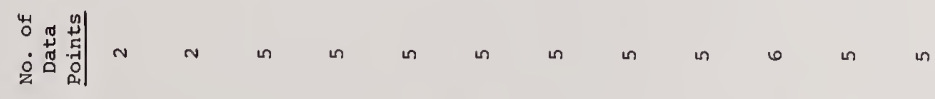

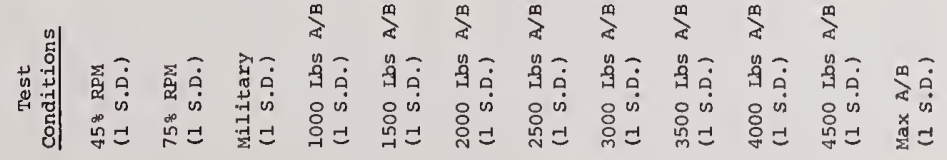


(1) thermocouple calibration tolerance [17] (0.025\%),

(2) error in recovery factor [19] (10\% of correction),

(3) error in radiation correction [19] (20\% of correction),

(4) error due to the calculation of gamma (Sec. 3.1.2.3) (0.5\%), and

(5) precision error in the measurements (1.5\%).

The first four are bias errors. The total bias error, estimated by taking the square root of the sum of the squares of the individual errors, was 1.8 percent for the static temperature rise and a 0.8 percent for the total temperature rise. The total errors, respectively, for the static and total temperature rise were 3.3 percent and 2.3 percent. They were calculated by adding the 1.5 percent precision error associated with the measurements in the afterburning plume.

\subsubsection{Experimental problems}

Three problems were observed while making the thermocouple measurements. First, an attempt to use a $1 \mathrm{~mm}$ diameter thermocouple failed because the thermocouple broke under the strain of the high gas velocities and high temperatures. The second problem occurred while making measurements with the smaller $0.81 \mathrm{~mm}$ diameter thermocouple. The high gas velocities distorted the shape of the thermocouple and bent the junction to within about 5 $\mathrm{mm}$ of the probe tip. The impact of the distorted shape of the thermocouple on the temperature measurements is unknown. However, it is likely that the junction could have been cooled as a result of being close to the cooled tip of the probe. The third problem definitely resulted in cooling of the thermocouple junction. A leak in the probe coolant section caused the coolant to be sprayed over the thermocouple junction, significantly reducing its temperature. This problem was identified early in the test program while inspecting the probe tip after a test. All data from these tests were rejected.

\subsection{Comparison of thermocouple, gas-sampling, and Raman measurements}

As discussed in Section 4.3, most of the measurements with the three techniques were not made simultaneously. However, the engine F/A ratios were measured for all the tests. Figure 10 demonstrates that engine $F / A$ ratios can be used as a parameter for comparing temperature measurements made in the $185-5$ afterburning plume.

Regression analyses were performed on the engine static-temperature rise data obtained with the three techniques. The results are presented in figure 16. Linear curves gave the best fit to the APL gas sampling and Raman data; whereas, quadratic curves were needed to fit the GE gas-sampling and thermocouple data. The error bars in figure 16 correspond to a nominal uncertainty of $\pm 50 \mathrm{~K}$ in all the fits. This suggests that the quality of the four measurements was essentially equivalent. The data used in these fits did not include the bias errors discussed in Sections 3.1.1.3, 5.2.2, 5.2.3, and 5.4.1. It is of interest to note that the thermocouple fitting error was about the same magnitude as the estimated total error given in Section 5.4.1 for a $1300 \mathrm{~K}$ temperature. 


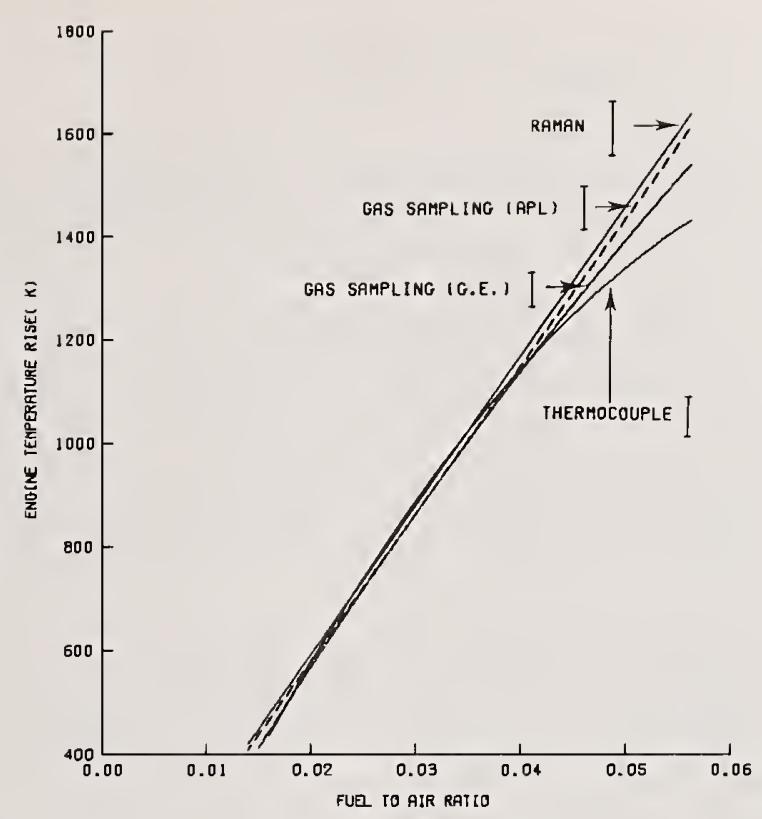

Figure 16. Regression analyses of the Raman, gas sampling, and thermocouple data. All valid data were used, viz., 26 points of Raman data, 117 points of gas sampling data reported here (APL), 25 points of General Electric (GE) gas sampling data, and 55 points of thermocouple data. The error bar given with each curve is the standard deviation of the data from the fitted curve.

Figure 16 shows that the static temperature rise measured with the Raman and gas sampling techniques, agree within their fitting errors for the measured $F / A$ ratios between 0.017 to 0.052 . The thermocouple measurements agree for $F / A$ ratios up to 0.045 , where a significant bending in the curve takes place. We believe that this bending was a result of the thermocouple junction being pushed towards the cooled probe tip as discussed in Section 5.4.2.

\section{Summary and Conclusions}

The objective of this study was to assess the feasibility of using spontaneous Raman scattering to measure temperatures in the plume of an afterburning 385-5 turbojet engine. This assessment required Raman spectral measurements of sufficiently good quality to permit temperature determinations and confirmation that the temperatures were correct within reasonable error limits. Our basic approach was to use gas sampling as a standard technique to be compared with the Raman technique. Simultaneous gas sampling and Raman measurements were made at about the same time and axial location in the plume (114 cm from nozzle exit plane) and for different engine afterburning conditions. Additional gas sampling measurements were made later in the test program and thermocouple measurements were also made. The simultaneous measurements were directly compared. A11 the measurements 
were then compared by plotting temperature rise versus engine fuel-air ratio. The conclusions from these measurements are summarized below:

(1) Our gas sampling measurements and those made by Lyon, et al. [3], for nearly the same test conditions, agreed within an experimental error of less than 5 percent. This established our confidence for using gas sampling as a standard technique.

(2) Raman $N_{2} Q$ branch spectra were obtained with sufficient resolution to permit temperature measurements over the entire operating range of the afterburner. The temperatures were determined by fitting theoretical spectral profiles to experimental data. This technique for obtaining temperatures provided all the information essential for estimating the probable errors associated with the Raman temperature measurements.

(3) The error in the Raman temperatures estimated from the spectral-profile fitting procedure varied between about 1 percent and 4 percent depending on test conditions. These errors may be a little low but appear reasonable based on the comparisons with other techniques.

(4) Simultaneous Raman and gas sampling temperatures agreed within experimental error of about 3 percent.

(5) Engine static temperature rise was measured by Raman, gas sampling, and thermocouple techniques over an engine F/A ratio range of 0.017 to 0.052 . A plot of these data versus engine $F / A$ ratio showed that Raman and gas sampling measurements agreed within an experimental error of less than 5 percent. The thermocouple measurements were in agreement up to a F/A ratio of 0.045 . At higher $F / A$ ratios, the thermocouple measurements were significantly below the temperatures measured by the other techniques. We believe that this occurred because the thermocouple junction was cooled when it was bent towards the cooled tip of the probe by the high gas velocities.

(6) The non-laser-induced background was not a serious problem for these experiments because of the low duty cycle $\left(5 \times 10^{-6}\right)$ provided by the 20 ns time-gated detection electronics.

(7) The laser-induced background was the most serious Raman-related problem encountered during the test program. It was the primary factor that determined the accuracy of the temperature measurements. We believe that the background was due to fluorescence from unburned hydrocarbons similar to that observed by Leonard [8] and could have been influenced by the plume temperature as suggested by Bailly, et al. [9].

The uncertain nature of the laser-induced background raises questions about whether our Raman system could be used to measure temperatures at other locations within the afterburner plume. According to the gas sampling measurements made by Lyon, et al. [3], the total hydrocarbon concentrations can be as high as $6000 \mathrm{ppm}$ at the engine exit plane and the static temperatures are just under $1300 \mathrm{~K}$. Under these conditions, we would expect a strong laser-induced background signal. Additional experiments are needed to determine whether Raman temperature measurements can be made in this environment. 
The authors gratefully acknowledge the effort and dedication of Mr. P. R. Hemmer who assembled and developed the Raman computer fitting routine. Special appreciation is extended to Mr. T. H. Hemmer for his assistance in the July, 1977 tests and for performing the computer analyses of the Raman data presented in this paper. The authors thank Dr. J. Ay for his valued assistance in setting up and performing the early measurements in this program and for preparing several figures presented in this paper.

The authors would like to express their appreciation to Mr. L. Angello, Mr. R. Whitlock, and Mr. J. Carnes for operating and maintaining the engine during the experiments and Mr. R. Allen, Mr. T. Campbell, and Mr. J. Malone for calibrating and operating the various instruments associated with the test cell. Special acknowledgments are given to Dr. T. Rosfjord for developing the theory used to calculate temperatures from emissions measurements and Mr. S. Farenbach and Mr. B. Eresman for writing the computer codes for making the calculations. The authors would also like to thank Mr. F. Voll and Mr. R. Hornbuckle for assisting in the data reduction and Mr. R. Bradley for aiding in plotting various data. Special appreciation is extended to Mr. G. Glawe at NASA Lewis for providing the thermocouple probe used in this study, to $\mathrm{Mr}$. T. Lyon for his discussions about their probe measurements, and to Dr. Marshall Lapp for sharing the results of his $\mathrm{N}_{2}$ Raman calculations with us.

\section{References}

[1] Temperature, Its Measurement and Control in Science and Industry, Vo1. III, Part 2 (Reinhold, New York, 1962).

[2] Procedure for the Continuous Sampling and Measurement of Gaseous Emissions from Aircraft Turbine Engines, Society of Automotive Engineers, Aerospace Recommended Practices No. 1256 (Oct. 1971).

[3] Lyon, T. F., Calley, W. C., Kenworth, J. J., and Bahr, D. W., Development of Emissions Measurement Techniques for Afterburning Turbine Engines, AFAPL-TR-75-52 (1975).

[4] Samulson, G. S. and Harmen, J. N., Chemical transformation of nitrogen oxides while sampling combustion products, Air Pollution Control Association, 27, 648-655 (July 7 , 1977).

[5] Lapp, M. and Penney, C. M., eds., Laser Raman Gas Diagnostics (Plenum Press, New York, 1974).

[6] Lapp, M. and Penney, C.M., Raman measurements on flames, in Advances in Infrared and Raman Spectroscopy, J. H. Clark and R. E. Hester, eds. (Heyden and Son Ltd., London, 1977).

[7] Lederman, S., The use of laser Raman diagnostics in flow fields and combustion, Prog. Energy Combust. Sci. 3 , 1-34 (1977).

[8] Leonard, D. A. , Field Tests of a Laser Raman Measurement System for Aircraft Engine Exhaust Emissions, AFAPL-TR-74-100 (0ct. 1974). 
[9] Bailly, R., Pealat, M., and Taran, J. P. E., Opt. Comm. 17, [1], 6873 (Apri1 1976).

[10] Setche11, R. E. and Aeschliman, D. P., Fluorescence Interferences in Raman Scattering from Combustion Products, Sandia Laboratories Energy Report SAND76-8705 (Feb. 1978).

[11] Eckbreth, A. C., Applicability of Laser Raman Scattering Diagnostic Techniques to Practical Combustion Systems, Project SQUID Technical Report UTRC4PU (0ct. 1976); also Eckbreth, A. C., Bonczyk, P. A., and Verdieck, J. F., Practical considerations for laser light scattering diagnostics, The Winter Annual Meeting of the American Society of Mechanical Engineers, Atlanta, Georgia, November 27-December 2, 1977 , Session 4A, in Gas Turbine and Combustion Fuels Technology, E. K. Bastress, ed.

[12] Williams, W. D., Powe11, H. M., McCay, T. D., Jones, J. H. and McGuire, R. L., Exhaust plume gas dynamic and radiation measurements on a 500 LBF thrust liquid rocket engine at simulated flight conditions, Part II: Laser Raman/Mass Spectrometer Diagnostics, AEDC-TR-78-17 (May 1978).

[13] Goulard, R., ed., Combustion Measurements: Modern Techniques and Instrumentation (Hemisphere Publishing and Co., Washington, D.C., 1976)

[14] North, J., Callaghan, E., and Lanzo, D., Investigation of Field and Velocity Profiles of an Afterburning Engine, NACA-RM-E54G07 (1954).

[15] Scadron, D., and Warshawsky, I., Experimental Determination of Time Constants and Nusselt Numbers for Bare-Wire Thermocouples in High-Velocity Air Streams and Analytical Approximation of Conduction and Radiation Errors, NACA-TN-2599 (1952).

[16] Scadron, D., Warshawsky, I., and Gettelman, C. D., Thermocouples for jet-engine gas temperature measurement, Prac. Instr. Soc. Am., Paper No. 52123, 7, 142-148 (1952).

[17] Glawe, G. E., Simmons, F. S., and Stickney, F. M., Radiation and Recovery Corrections and Time Constants of Several Chromel-Alumel Thermocouple Probes in High-Temperature, High-Velocity Gas Streams, NACA-TN-3766 (1956).

[18] Williamson, R. C. and Stanforth, C. M., Measurements of Jet Engine Combustion Temperature by the Use of Thermocouples and Gas Analysis, SAE Paper 690433 (1969).

[19] Glawe, G. E. and Shepard, C. E., Some Effects of Exposure to Exhaust-Gas Streams on Emittance and Thermoelectric Power of Bare-Wire Platinum Rhodium-Platinum Thermocouples, NACA-TN-3253 (1954).

[20] Baumeister, T. and Marks, L. S., eds., Standard Handbook for Mechanical Engineers, Seventh Edition (McGraw-Hi11, New York, 1967).

[21] Gordon, S. and McBride, B. J., Computer Program for Calculation of Complex Chemical Equilibrium Compositions, Rocket Performance, Incident and Referenced Shocks, and Chapman Jouquet Detonations, NASA Special Publication, 273 (1971).

[22] U.S. Environmental Protection Agency, Control of air pollution from aircraft and aircraft engines, Federal Register, 38, [136] (July 17, 1973).

[23] Blazowski, W. S., and Henderson, R. E., A Review of Turbopropulsion Combustion, AFAPL-TR-77-41 (June 1977).

[24] VanWylen, G. J., and Sonntag, R. E., in Fundamentals of Statistical Thermodynamics (Wiley, New York, 1965). 
[25] Rosfjord, T. J., Efficiency Determined from Exhaust Measurements, Internal Air Force Aero Propulsion Laboratory Paper.

[26] Herzberg, G., Molecular Spectra and Molecular Structure, I. Spectra of Diatomic Molecules (D. Van Nostrand Co., New York, 1950).

[27] Widhopf, G. F. and Lederman, S., Species Concentrations Measurements Utilizing Raman Scattering of a Laser Beam, Polytechnic Institute of Brooklyn, PIBAL Report No. 69-46 (November 1969).

[28] Koningstein, J. A., in Introduction to the Theory of the Raman Effect (Reide] Publishing Co., Dordrecht, Holland, 1972).

[29] Van De Hulst, H. C., in Light Scattering by Smal] Particles (Wjley, New York, 1957).

[30] Lapp, M. , Penney, C. M., and St. Peters, R. L., Laser Raman Pr be for Flame Temperature, Project Squid Technical Report GE-1-PU (April 1973).

[31] Lapp, M., Goldman, L. M., and Penney, C. M., Raman scattering from flames, Science, $175,1112-1115$ (1972).

[32] Roh, W. B., Coherent Anti-Stokes Raman Scattering of Molecular Gases, AFAPL-TR-7747, (August 1977).

[33] Bevington, P. R., in Data Reduction and Error Analysis for the Physical Sciences (McGraw-Hil1, New York, 1969).

[34] Hemmer, P. R., Yaney, P. P., and Roquemore, W. M., Computer fits of calculated Raman spectra of $\mathrm{N}_{2}$ to observed spectra, Bull. Am. Phys. Soc. 22, 1039 (1977).

[35] Hemmer, T. H., Yaney, P. P., and Hemmer, P. R., Effect of variations in instrument function on the temperature calculated from the Raman spectrum of $N_{2}, B u 11$. Am. Phys. Soc. 23,162 (1978).

[36] Gilson, T. R., Hendra, P. J., Laser Raman Spectroscopy (Wiley-Interscience, New York, 1970).

[37] Yaney, P. P., Hemmer, P. R., Hemmer, T. H., and Roquemore, W. M., Determination of temperatures in the combustion zone of a J85-5 jet engine using the laser-excited vibrational-rotational Raman spectrum of nitrogen, in Thirty-Third Annual Molecular Spectroscopy Symposium (Ohio State University, June, 1976); also Ay, J., Roquemore, W. M., Yaney, P. P., and Hemmer, P. R., Raman measurements in the J85-5 afterburning turbojet engine plume, The Winter Annual Meeting of the American Society of Mechanical Engineers, Atlanta, Georgia, November 27-December 2, 1977, postdead1ine paper, Session $4 A$.

[38] Vol1, F. and Eresman, B., C-Stand Stationary Turbojet Engine Test Faci]ity and Data Acquisition and Data Reduction, AFAPL-SFF-TM-77-24 (1977).

[39] Lyon, T. F., Colley, W. C., Kenworth, J. J. and Baker, D. W., Development of Emissions Measurement Techniques for Afterburning Turbine Engines, Supplement 1-Engine Emissions Test Data, AFAPL-TR-75-52-Supplement 1 (Oct. 1975). 


\section{Discussion}

Question (Rosenblatt): You showed what are apparently the most precise vibrational Raman temperatures which I am directly aware of, temperatures of 1500 Kelvin, and uncertainties of plus or minus 22 degrees, I think was the number. Could you define what kind of uncertainty that was, that 22 degrees? Is that a standard deviation, or was that an overall accuracy?

Response (Roquemore): We11, it is not an accuracy because we don't know how to get an accuracy. We could get a precision. Professor Yaney will probably be the best to comment on that, he was doing the Raman and I was doing the thermocoupling gas sampling. Perry Yaney, would you like to explain?

Response (Yaney): You would have asked that. That is a good question. Basically what we had is a fitting program, a Chi-squared minimization program. It is a standard kind of routine where you get the curvature matrix for the minimum and you can invert that and get an error. So, this is a fitting error.

Question (Rosenblatt): So, that is one standard deviation?

Response (Yaney): Right. One standard deviation. It is as simple as that. It is still pretty good, considering all of that.

Question (Rosner): You stressed the role of unburned hydrocarbons in influencing the laser method, but I wonder if you would comment on the role of unburned hydrocarbons in interpreting the thermocouple probe data. It does seem that in using the heat capacity equations mentioned in connection with the difference between the static and the total temperature, that you are basically assuming that at the position of the measurement the fuel has in fact combusted and you are dealing with the conversion of ordered energy into thermal energy, but nowhere have you mentioned the fact that there is a certain amount of chemical energy that may not have actually been released at the point of the measurement.

Response (Roquemore): Yes, that is true. The unburned hydrocarbons could really show up in several different ways. One way is that you could get a blackening of the probe,--of the element itself, and this could cause errors in the radiation correction factor. So, it could show up that way as well as chemical effects that would show up on the element. There is a technique whereby you can look at the thermocouple probe and make an estimate as to how much sooting took place on the probe, and how it would change your radiation correction factors, and this is described in the paper. It was based on some data that NASA-Lewis had collected on bare wire thermocouples back in the ' 50 's. 
As far as the chemical effects are concerned, we really did not know how to handle that problem, and there was nothing we could do, so we assumed that they were not present. If you have got some suggestions on how we could handle it, we would be very interested in knowing.

Question (Rosner): I was wondering if you see different sorts of results at different distances from the exit of the engine in that regard, and whether such things as surface catalytic combustion on the thermocouple surface played some role?

Response (Roquemore): It could, but it is in the same nature. We can't get a handle on it. 
National Bureau of Standards Special Publication 561, Proceedings of the 10th Materials Research Symposium on Characterization of High Temperature Vapors and Gases held at NBS, Gaithersburg, Maryland, September 18-22, 1978. Issued October 1979.

USE OF THERMOCOUPLES FOR

GAS TEMPERATURE MEASUREMENTS IN GAS TURBINE COMBUSTORS

R. R. Dils

National Bureau of Standards

Washington, DC 20234

and

P. S. Follansbee

Carnegie-Mellon University

Pittsburgh, PA 15213

Widebandwidth gas temperature measurements within a model gas turbine combustor show that large scale gas temperature gradients and fluctuations occur throughout the combustor. The local average temperatures vary from the inlet temperature to the stoichiometric flame temperature; the local fluctuating temperatures are contained within a $0-1000 \mathrm{~Hz}$ bandwidth and exhibit RMS values from 0.05 to 0.25 of the temperature rise across the combustor. The average gas temperatures are used to describe the location and size of recirculation zones and the trajectories of the combustion air jets; the fluctuating temperatures provide new information on the combustion dynamics. The performance of fine wire noble metal thermocouples used to measure these temperatures is reviewed and a demonstration of the related measurement techniques and data analyses is presented. It is shown that the oscillatory state in the combustor affects the uniformity of the time-average temperatures in the combustor exhaust. Regenerative amplification of the longitudinal resonant acoustic pressure modes of the burner is accompanied by significant spacial correlation of the heat release rate within the combustor. The correlated heat release rate fluctuations cause extensive motion of the large primary and secondary air jets and an attendant improvement of the uniformity of the time average gas temperatures in the combustor exhaust. 


\section{Introduction}

The gases within gas turbine combustors display a number of special features which obtain from the combustor design and operation. Primarily, the gases are only partially mixed and this unmixedness results in large gas temperature and velocity variations throughout the combustor. It is generally recognized that the mixing processes in the combustor influence the combustion efficiency, high altitude relight characteristics, pollutant formation, combustor durability, and the uniformity of the exhaust gas temperatures. The significant role of the oscillatory state in the combustor in affecting many of these same engineering parameters has not been fully appreciated. The combustors nonlinearly amplify acoustic and flow disturbances within a 0-1000 $\mathrm{Hz}$ bandwidth and, as a result, large scale, correlated, temporal temperature and flow fluctuations are observed within this bandwidth. These fluctuating properties of the combustor gases are related to both the performance of the combustor and the durability of downstream components. In preceding papers, it has been shown that the temporal temperature fluctuations in the combustor exhaust affect the rate of oxidation of downstream turbine components $[1]^{1}$ and can be used to measure local heat transfer coefficients on the turbine components [2].

The importance of the temporal fluctuations and the presence of frequent and large spacial temperature gradients within the combustor require that a technique to measure the local gas temperatures must have a uniform frequency response over the $0-1000 \mathrm{~Hz}$ bandwidth and high spacial resolution. As a practical matter, it is equally important that the technique can be adapted to the harsh environment generated by a large gas turbine. Over the past decade, it has been found that fine wire noble metal thermocouples can be used in the combustor exhaust gases on a regular basis in full scale engine tests and in laboratory combustors $[3,4]$. The intent of this paper is to show how these thermocouples can be used to obtain accurate, high resolution measurements of the local gas temperatures within the combustors and to demonstrate how these data can be used to obtain a new understanding of several features of the combustion and flow within the combustor. Of specific interest is the role of the oscillatory state in determining the time average temperature uniformity of the exhaust gas temperatures. The design of the thermocouples and the operation of the combustion chamber are reviewed. Application of the technology in a laboratory combustor provides the demonstration of the nature of the experiments and analysis. A brief discussion of the effects of temporal and spacial gas temperature fluctuations on the measurement of combustor gas temperatures with nonlinear devices is included in an appendix.

\section{1 Heat transfer to fine wire thermocouples in gas turbine combustion chambers}

Extensive discussions of the design and operation of a wide variety of anemometric devices in simple gas streams are available in the gas dynamics literature $[5,6]$. Applications of the technology to simple flames using the fine wire thermocouples are frequent

${ }^{1}$ Figures in brackets indicate the literature references at the end of this paper. 
[7-10] and in an earlier paper, the authors have described the performance of fine wire thermocouples in gas turbine combustors [4]. Here, it is sufficient to summarize those elements critical to the present experiments. Through proper design and the special features of the combustor gases, the response of the thermocouples is straightforward. Specifically, for the thermocouple design shown in figure 1, where a 0.003 in. diameter, Pt-6 percent Rh/Pt-30 percent Rh laser butt welded thermocouple is suspended between 0.010 in. diameter support posts of similar material and the length to diameter ratios of the thermocouple and support posts are $30 / 1$ and $15 / 1$, respectively, heat transfer to the thermocouple junction is primarily by convection from the gas.

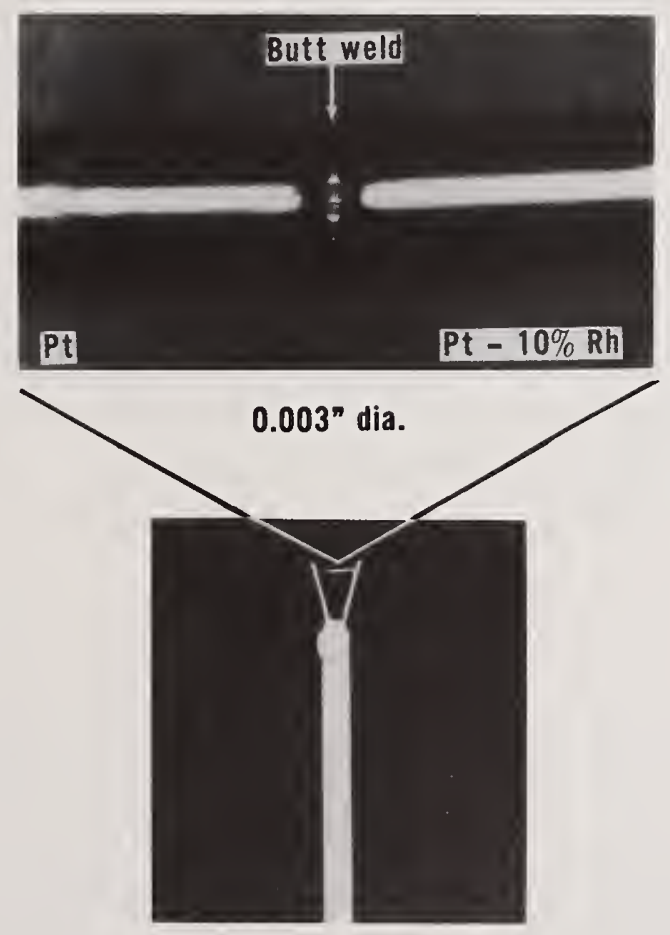

Figure 1. Fine wire thermocouple.

For wires of this size, the differential equation describing the response of the wire to the convective transfer is

$$
\frac{\rho_{w}{ }^{c} w_{w}{ }^{1.5}}{1.92 K \sqrt{\frac{\rho}{\mu} \sqrt{u}}} \quad \frac{d T_{w}}{d t}=T-T_{w}
$$


where

$$
\begin{aligned}
\rho_{\mathrm{W}} & =\text { wire density, } 1 \mathrm{bm} / \mathrm{ft}^{3}, \\
c_{\mathrm{w}} & =\text { wire specific heat, } \mathrm{Btu} / 1 \mathrm{bm}{ }^{\circ} \mathrm{R}, \\
d_{\mathrm{w}} & =\text { diameter of the wire, } \mathrm{ft}, \\
\mathrm{K} & =\text { gas thermal conductivity, } \mathrm{Btu} / \mathrm{ft} \mathrm{s}^{\circ} \mathrm{F}, \\
\rho & =\text { gas density, } 1 \mathrm{bm} / \mathrm{ft}^{3}, \\
\mathrm{u} & =\text { gas velocity, } \mathrm{ft} / \mathrm{s}, \\
\mu & =\text { gas viscosity, } 1 \mathrm{bm} / \mathrm{s} \mathrm{ft}, \\
T_{\mathrm{W}} & =\text { wire temperature, }{ }^{\circ} \mathrm{F}, \\
T & =\text { gas temperature, }{ }^{\circ} \mathrm{F},
\end{aligned}
$$

and $K, \rho, \mu$, and $u$ vary with time.

Initially, it would be suspected that for the complex flows and large temperature fluctuations encountered in the gas turbine combustor, it would be difficult to obtain $T$ from a measurement of $T_{w}$. Fortuitously, the group $K \sqrt{\frac{\rho}{\mu}}$ is not a strong function of gas temperature. For example, the value of $K \sqrt{\frac{\rho}{\mu}}$ at $2500^{\circ} \mathrm{F}$ is only 1.014 times the value of $K$ $\sqrt{\frac{\rho}{\mu}}$ at $1500^{\circ} \mathrm{F}$. Therefore, the gas properties can be evaluated at the average gas temperature with neglible error. In addition, it has been shown that the turbulent intensity within the 0-1000 Hz bandwidth is small and only partially correlated with the gas temperature fluctuations. Solving eq. (1) using the actual time varying values of $u$ and $T$ results in a rate of heat accumulation in the wire which is less than 1.5 percent higher than the rate obtained assuming a constant velocity equal to the average gas velocity [4]. Therefore, eq. (1) reduces to the simple first order differential equation

$$
\tau \frac{d T_{w}}{d t}=T-T_{W}
$$

where $\tau=$ the thermocouple time constant and is equal to the time average values of the constants in eq. (1).

Solutions to eq. (2) are we11-known [11]. The wire element acts as a simple low pass filter of gas temperature fluctuations with a transfer function, $Y(w)$, and cutoff frequency, $f_{c}$, equal to

$$
Y(\omega)=\frac{1}{1+j \omega \tau}
$$

and

$$
f_{c}=\frac{1}{2 \pi \tau}, H z \text {, }
$$

respectively. 
For the gas turbine combustor exhaust gases, the effects of conduction, radiation, and catalysis on the measurement of average and dynamic temperatures is small. The conduction to the support posts is limited by maintaining a large length to diameter ratio of the thermocouple and support posts. Radiation does not affect the transfer function of the thermocouple above $f_{c}$ and through the use of small wires, average gas temperatures are significantly affected by radiation only in low velocity, low pressure, high temperature stream tubes. Catalytic heat transfer to the thermocouples is effectively eliminated by coating the thermocouples with a few hundred angstroms of quartz or alumina. The compilation in table 1 indicates the magnitude of the conduction and radiation errors introduced by considering only the convective heat transfer to fine wire thermocouples in a gas turbine combustor. The accuracy of the average temperature measurements can be improved by computing the radiative and conductive heat transfer to the wire [4], but in most cases the extra effort is unnecessary. Therefore, the error in most measurements of the average gas temperature can be maintained between 0 and -5 percent.

Table 1. Fine wire thermocouple errors assuming simple convective heat transfer. Reference [4].

\begin{tabular}{|l|c|c|c|c|}
\hline \multirow{2}{*}{$\mathrm{T},{ }^{\circ} \mathrm{F}$} & \multicolumn{2}{|c|}{$1 \mathrm{ATM}$} & \multicolumn{2}{c|}{$20 \mathrm{ATM}$} \\
\cline { 2 - 5 } & $100 \mathrm{ft} / \mathrm{sec}$ & $1000 \mathrm{ft} / \mathrm{sec}$ & $100 \mathrm{ft} / \mathrm{sec}$ & $1000 \mathrm{ft} / \mathrm{sec}$ \\
\hline & & & & \\
& $-58^{\circ} \mathrm{F}$ & $-4^{\circ} \mathrm{F}$ & $-1^{\circ} \mathrm{F}$ & $0.1^{\circ} \mathrm{F}$ \\
& & & & \\
Radiation & & & & \\
1000 & 6.9 & 2.1 & 1.5 & 0.5 \\
1500 & 1.7 & 0.5 & 0.4 & 0.1 \\
2000 & -8.1 & -3.2 & -2.2 & -0.7 \\
2500 & -13.7 & -4.4 & -3.1 & -1.0 \\
3000 & -58.5 & -19.1 & -13.5 & -4.2 \\
\hline
\end{tabular}

The transfer function of the thermocouples above $f_{C}$ is only a function of the convective heat transfer and can be described by eqs. (1-3). Error in the transfer function in a known gas stream is due to variations in the wire diameter at the butt welded junction. Experience over an extended period of time has shown that with proper inspection of the junctions, the transfer functions can be computed with an accuracy of at least \pm 5 percent. This accuracy is sufficient to describe the critical features of the combustor gases and applies to the present experiments. 
In the unknown gas streams within the combustor, the same error bounds apply to the average temperature measurements, but the transfer function can vary. Since the local gas flows are generally not known, it is necessary to experimentally determine the cutoff frequencies of the thermocouples. One technique which has been found useful is based on a comparison of the uncompensated signals of two wires of different diameter at a given location within the burner. The ratio of the transfer functions is

$$
\frac{Y_{1}}{Y_{2}}=\sqrt{\frac{1+\left(2 \pi f \tau_{2}\right)^{2}}{1+\left(2 \pi f \tau_{1}\right)^{2}}}=\sqrt{\frac{1+\left[2 \pi f \tau_{1}\left(\frac{d_{2}}{d_{1}}\right)^{3 / 2}\right]^{2}}{1+\left[2 \pi f \tau_{1}\right]^{2}}}
$$

where $f=w / 2 \pi, H z, d_{1}, \tau_{1}$, and $Y_{1}$, are the diameter, time constant and transfer function of the larger wire and $d_{2}, \tau_{2}$, and $Y_{2}$ are the equivalent parameters for the small wire. For high frequencies,

$$
\frac{Y_{1}}{Y_{2}}=\frac{\tau_{2}}{\tau_{1}}=\left(\frac{d_{2}}{d_{1}}\right)^{3 / 2}
$$

This limit and the ratio of the transfer functions can be used to determine the time constants of each wire in an unknown gas stream with an accuracy of \pm 5 percent. An example of the ratio of transfer functions of 0.0031 in. diameter and 0.0053 in. diameter thermocouples in a known gas stream is shown in figure 2. In practice it has been found that the time constants vary on the order of \pm 10 percent over most of the combustor volume and therefore, as a first approximation, the widebandwidth gas temperatures can be continuously measured within the combustor using an average time constant. This approximation was employed in the present experiments.

The preceding description applies to $\mathrm{Pt}-6$ percent $\mathrm{Rh} / \mathrm{Pt}-30$ percent $\mathrm{Rh}$ fine wire thermocouples in cross flow in combustion gases with average gas temperatures from $1500^{\circ} \mathrm{F}$ to $3300{ }^{\circ} \mathrm{F}$, average gas velocities from $100 \mathrm{ft} / \mathrm{s}$ to $1000 \mathrm{ft} / \mathrm{s}$ and average pressures from 1 atm to $20 \mathrm{~atm}$. In these gas streams, it has been found that the thermocouple lifetime is approximately $20 \mathrm{hrs}$, which is much longer than is normally required to complete a test. The durability and the simple response of the thermocouples allows practical widebandwidth gas temperature measurements to be conducted throughout a gas turbine combustor operating with a low inlet temperature, $\left(\mathrm{T}_{\text {inlet }} \approx 100^{\circ} \mathrm{F}\right.$ ), and in many areas of a combustor operating with a moderate inlet temperature, $\left(\mathrm{T}_{\text {inlet }} \approx 800-1000^{\circ} \mathrm{F}\right)$. Recently, Hayhurst and Kittelson [10] have shown that fine wire Indium-Rhodium thermocouples can operate to approximately $4000^{\circ} \mathrm{F}$ in simple flames and thermocouple devices using these alloys may extend the useful temperature range of fine wire thermocouple measurements in gas turbine combustors. 


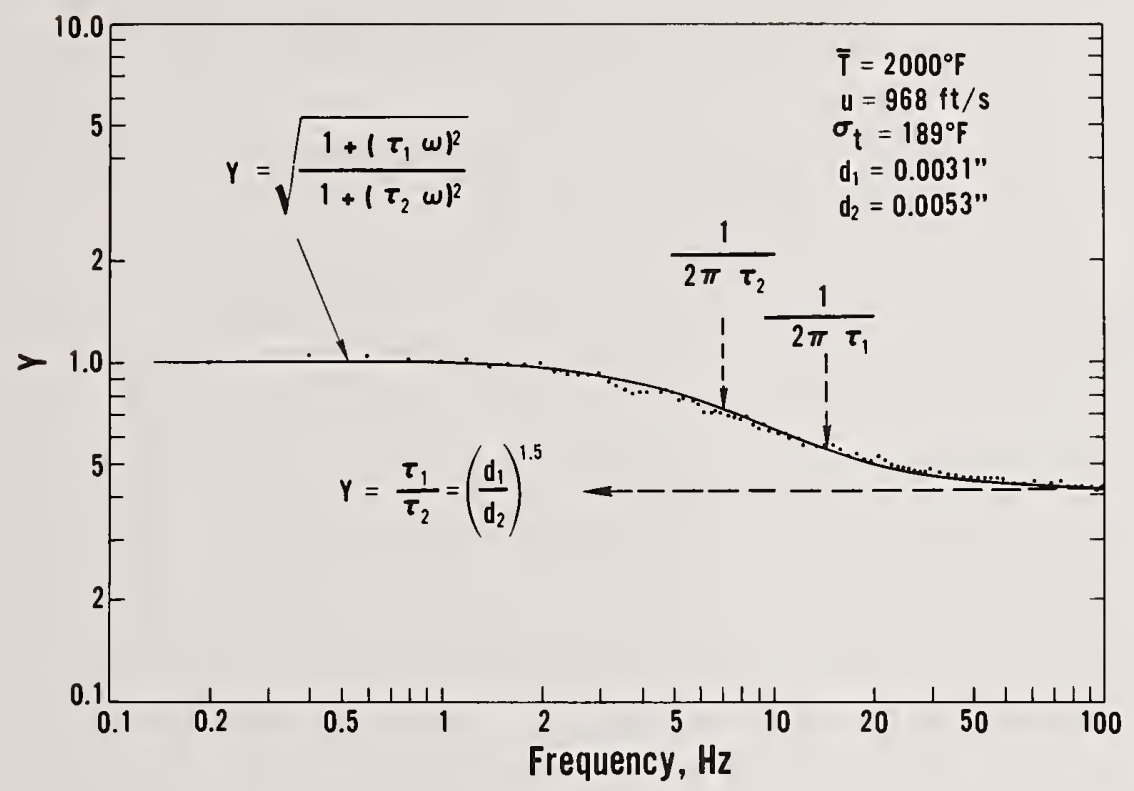

Figure 2. Two-wire method of determining fine wire thermocouple time constant. Reference [4].

\subsection{Combustor operation}

In a conventional gas turbine main burner, liquid fuel is injected directly into the combustor where it is dispersed, vaporized, mixed with air and burned. In order to obtain rapid and complete combustion, the equivalence ratio in the forward section is maintained near one (stoichiometric fuel/air ratio) and the hot combustion products are mixed with secondary air in the back of the combustor in order to cool the gases to a temperature level acceptable to the downstream turbine components. The rate of combustion (heat release) is determined by the liquid fuel vaporization rate, the combustion rate and the rate of mixing of the fuel and air and the combustion products; there are characteristic times associated with each event, i.e., $\tau_{v}, \tau_{c}$ and $\tau_{m}$, respectively. Vranos [12] has provided an order of magnitude estimate of these times for typical operating conditions and a range of mixtures; as shown in table 2 , in most cases, $\tau_{m} \gg \tau_{v}, \tau_{c}$. Experimentally, it is observed that the overall reaction times in gas turbine combustors are approximately two orders of magnitude larger than would be predicted by "stirred reactor" kinetics. The kinetic energy feed rate of the combustion jets furnishes the power for stirring the combustion zone and the large value of $\tau_{\mathrm{m}}$ is due to the engine design constraints which limit the injection pressure of the jets to 2-4 percent. Approximately 50 percent of the 
air introduced in these combustors is introduced as cooling air along the side of the combustion chamber and the actual values of $\tau_{m}$ for such a nonuniform initial distribution of the air in the combustor are greater than the estimated $10 \mathrm{~ms}$. In fact, the mixing remains incomplete as the gases leave the combustor exhaust nozzle $[3,4]$.

Tabie 2. Some characteristic combustion and mixing times in a gas turbine combustor $\left(P=15 \mathrm{~atm}, T_{\text {inlet }}=800^{\circ} \mathrm{F}, \mathrm{JP}-5\right.$ fuel $)$. Reference [11].

\begin{tabular}{|c|c|}
\hline $\begin{array}{c}\text { Equivalence } \\
\text { Ratio }\end{array}$ & $\tau_{c}$ \\
\hline 0.7 & $0.20 \mathrm{~ms}$ \\
0.8 & 0.17 \\
0.9 & 0.15 \\
1.0 & 0.13 \\
1.1 & 0.16 \\
1.2 & 0.2 \\
1.4 & 0.4 \\
\hline
\end{tabular}

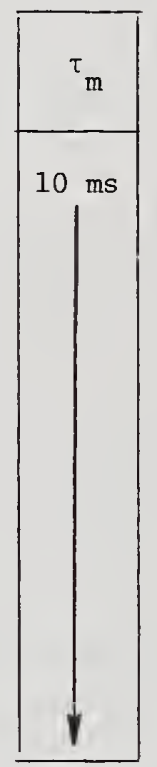

\begin{tabular}{|c|c|}
\hline $\begin{array}{c}\text { Drop } \\
\text { Diameter }\end{array}$ & $\tau_{\mathrm{v}}$ \\
\hline $150 \mu$ & $9.8 \mathrm{~ms}$ \\
$100 \mu$ & 3.6 \\
$50 \mu$ & 1.2 \\
$25 \mu$ & 0.4 \\
$10 \mu$ & 0.2 \\
& \\
\hline
\end{tabular}

The disparity in the time scales affects the combustion processes in a significant manner. As described by Vranos, the large value of $\tau_{m}$ precludes the formation of a wide range of burning mixtures. Initially, near the vaporizing fuel droplet, the mixtures are ab the rich flammability limit and $\tau_{m} \ll t_{c}$. Dilution proceeds without combustion until $\tau_{\mathrm{m}} \approx t_{\mathrm{c}}$, and then dilution and combustion proceed simultaneously. Further dilution causes $\tau_{c}$ to decrease rapidly; combustion is limited by the rate of arrival of air; unburned mixtures below the rich flammability limits are not formed; and the equivalence ratio at the flame front remains near one. This strong dependence of the combustion rate on the rate of mixing introduces the possibility of combustion system oscillations $[3,13]$. For $\tau_{m} \gg \tau_{c}, \tau_{v}$, if the rate of mixing is a function of the rate of combustion, the combustion in the burner will be unsteady. When the linear component of the feedback between the mixing and combustion processes is large enough and has the correct phase, regenerative amplification of the combustion disturbances will occur. Both pressure and flow perturbations can affect the heat release rate. In the recirculation zones in the forward sections of the burner, the recirculatory flow can provide a feedback loop for some of the fourier components of a pressure, flow or combustion disturbance. Characteristic times around the recirculation zones are $2-5 \mathrm{~ms}$, and with $\tau_{\mathrm{m}} \approx 10 \mathrm{~ms}$ and $\tau_{\mathrm{v}}, \tau_{\mathrm{c}} \approx 1 \mathrm{~ms}$, the 
mixing and combustion can be expected to respond to disturbances within the 10-1000 $\mathrm{Hz}$ bandwidth; the gain within the bandwidth will be both nonuniform and nonlinear $[3,13]$.

In this paper, the steady and widebandwidth gas temperatures within a laboratory com- . bustion chamber are cataloged in an effort to provide a general description of the mixing and combustion throughout the combustor and to identify the potential feedback loops which can lead to regeneration. Of specific interest is the regenerative nonlinear amplification of longitudinal acoustic pressure modes within the combustor, since evidence of these oscillations has been obtained in previous widebandwidth gas temperature measurements in gas turbine combustor exhaust gases [3]. In the present experiments, the combustor was operated at a much lower pressure and inlet temperature than is characteristic of normal engine operation ( $1 \mathrm{~atm}$ vs $15 \mathrm{~atm} ; 100^{\circ} \mathrm{F}$ vs $800^{\circ} \mathrm{F}$ ). The test conditions were chosen to permit easy access to the interior of the combustor and to reduce the stoichiometric flame temperature to $3620^{\circ} \mathrm{F}$, thereby enabling the majority of the combustor volume to be traversed with the simple type $B$ fine wire thermocouples. Even with the changes in inlet temperature and pressure $\tau_{m}$ is still greater than $\tau_{v}$ and $\tau_{c}$ and, therefore, the model combustor conditions are adequate to investigate the macroscopic features of the combustion and flow within the combustor. Experimentally, it is observed that the features of the combustor exhaust gases generated by the model combustor are identical with those measured earlier in full scale gas turbine combustors $[3,4]$.

\section{Experimental Procedures}

The experiments were conducted in a laboratory combustor in which the mixing and combustion closely approximate those of many conventional gas turbine main burners [1, 14]. The combustor was composed of five modules: a fuel nozzle plate, primary and secondary diffusers and burner liners, an instrumentation liner and a 3 in. diameter conical exhaust nozzle (fig. 3). The primary and secondary sections were supplied by independent air supplies. Several configurations were employed in the test matrix shown in table 3 . The combustion and cooling hole patterns were sized to reproduce the major features of the axial air distribution in a conventional aircraft gas turbine $[3,4]$; the axial air distributions are shown in figure 4 and the hole patterns for the convectively cooled and louver cooled laboratory combustor liners are described in table 4. A perforated plate with uniformly spaced 0.375 in. holes was used to simulate the 76 percent exhaust nozzle blockage introduced by the first stage vanes in a gas turbine. As indicated in table 3, the type of liner cooling, fuel nozzle and exhaust nozzle blockage were sequentially varied to examine the influence of each combustor design element on the combustion and flow within the burner. The majority of the tests were conducted with a 3 percent pressure drop across the burner liner and an inlet flow of 0.364 $\mathrm{bbm} / \mathrm{s}$ at $100^{\circ} \mathrm{F}$; the average exhaust gas temperature was maintained near $2000^{\circ} \mathrm{F}$, which corresponds to an overall air to fuel ratio of approximately $36 / 1$. 


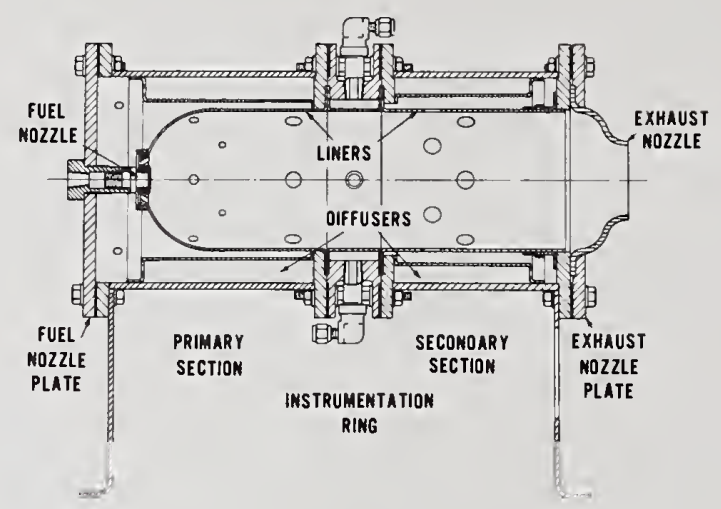

Figure 3. Laboratory combustor.

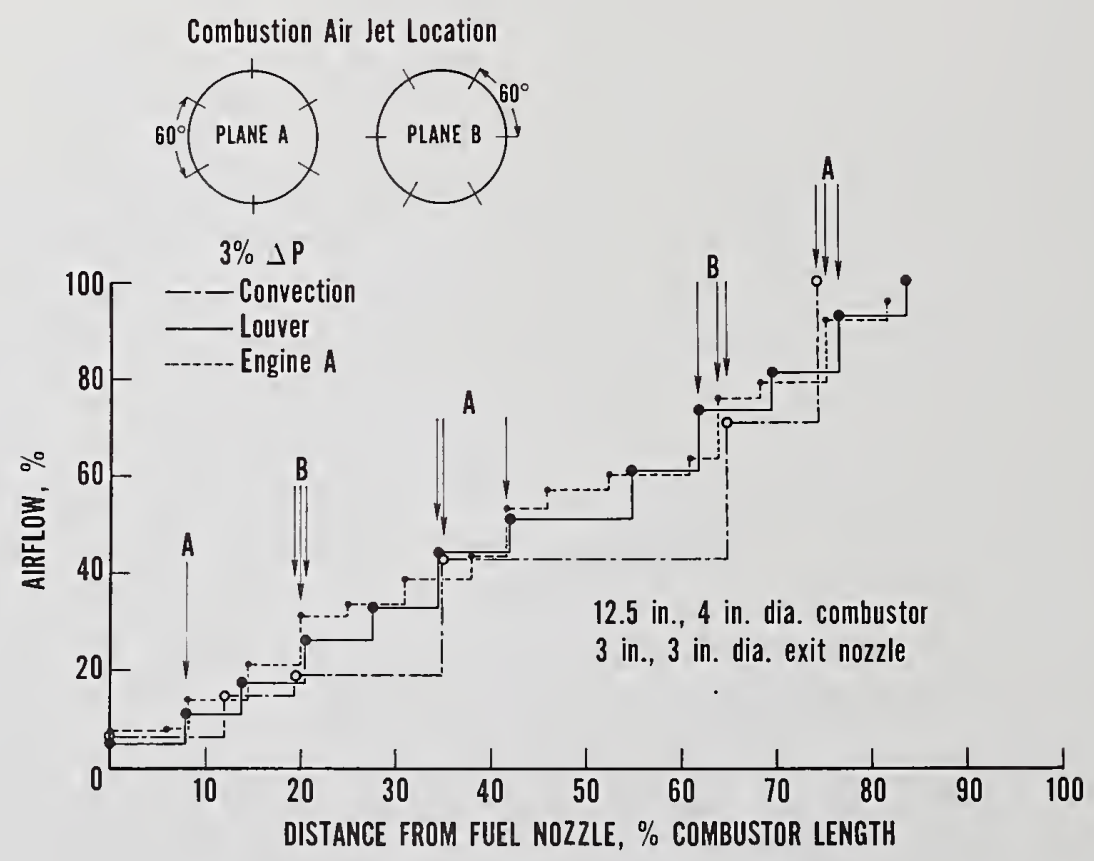

Figure 4. Comparison of axial air distributions in the laboratory combustor and a gas turbine main burner. 


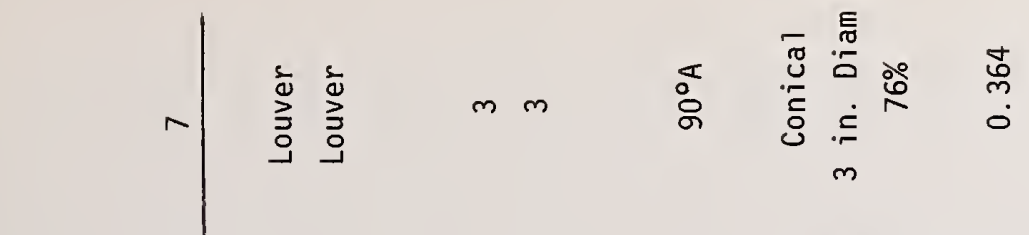

$$
\begin{aligned}
& \text { 引ँ }
\end{aligned}
$$

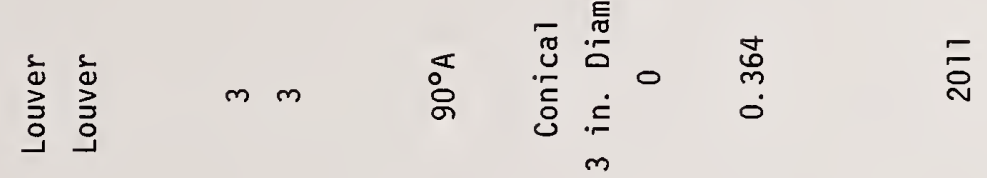

$$
\begin{aligned}
& \text { 它 }
\end{aligned}
$$

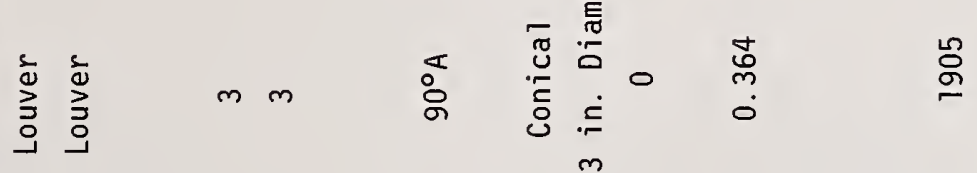

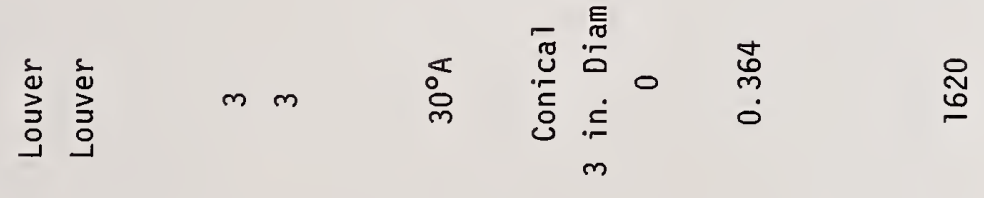

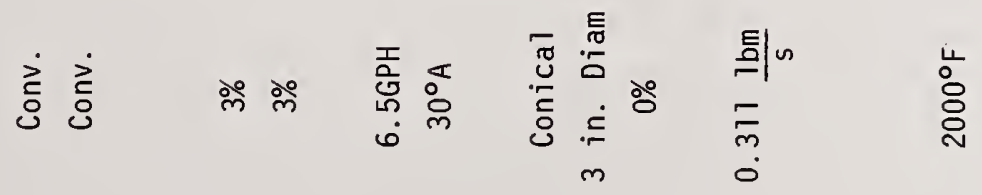

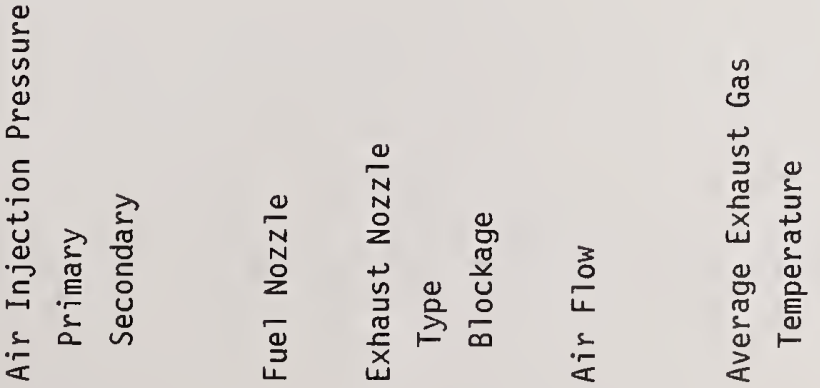


Table 4. Axial airflow distributions in the laboratory combustor.

\begin{tabular}{|c|c|c|c|c|c|c|c|}
\hline \multirow{2}{*}{$\begin{array}{l}\text { Axial Distance } \\
\text { From } \\
\text { Fue1 Nozzle }\end{array}$} & \multicolumn{4}{|c|}{ COMBUSTION AIR } & \multicolumn{3}{|c|}{ COOLING AIR } \\
\hline & Plane & No & Dia & $\begin{array}{c}\text { W/o } \\
\text { Airflow }\end{array}$ & No & Dia & $\begin{array}{c}\text { w/o } \\
\text { Airflow }\end{array}$ \\
\hline $\begin{array}{l}\text { Convectively } \\
\text { Cooled Liner }\end{array}$ & & & & & & & \\
\hline swirler & & 16 & $0.125 \mathrm{in}$ & $6.35 \%$ & & & \\
\hline $1.55 \mathrm{in}$ & A & 6 & 0.265 & 8.09 & & & \\
\hline 2.5 & B & 6 & 0.200 & 4.61 & & & \\
\hline 4.36 & A & 6 & 0.450 & 23.3 & & & \\
\hline 8.15 & B & 6 & 0.500 & 28.8 & & & \\
\hline 9.3 & A & 6 & 0.500 & 28.8 & & & \\
\hline $\begin{array}{l}\text { Louver Cooled } \\
\text { Liner }\end{array}$ & & & & & & & \\
\hline swirler & & 16 & $0.125 \mathrm{in}$ & $4.90 \%$ & & & \\
\hline fuel nozzle & & & & 0.50 & & & \\
\hline $1.375 \mathrm{in}$ & & & & & 72 & $0.059 \mathrm{in}$ & $5.13 \%$ \\
\hline 2.185 & & & & & 72 & 0.059 & 5.13 \\
\hline 2.935 & B & 6 & 0.312 & 8.39 & & & \\
\hline 3.855 & & & & & 72 & 0.069 & 7.00 \\
\hline 4.680 & A & 6 & 0.344 & 10.82 & & & \\
\hline 5.625 & & & & & 72 & 0.069 & 7.00 \\
\hline 7.250 & & & & & 72 & 0.082 & 10.00 \\
\hline 8.140 & B & 6 & 0.375 & 12.79 & & & \\
\hline 9.140 & & & & & 72 & 0.069 & 7.00 \\
\hline 9.978 & A & 6 & 0.375 & 12.79 & & & \\
\hline 10.980 & & & & & 72 & 0.069 & 7.00 \\
\hline
\end{tabular}


The combustor gas temperatures were measured with the fine wire thermocouples described in the preceding section. The $0.010 \mathrm{in}$. diameter support posts were cemented in an 0.062 in. diameter aluminum oxide insulator which was cemented to an 0.125 in. diameter platinum tube; the thermocouple extended $1.5 \mathrm{in}$. forward of the platinum tube and $3 \mathrm{in}$. forward of an 0.375 in. diameter stainless steel water-cooling jacket. The platinum tube and cooling jacket provided mechanical support for the thermocouple [4]. The combustor was traversed along the centerline and at the $0.5,1.0,1.5$, and $1.75 \mathrm{in}$. radii in two planes of symmetry. Signals from the thermocouples were amplified, differentiated, band passed from 0 to $1000 \mathrm{~Hz}$, and recorded on a Hewlett Packard 3960 magnetic tape recorder with FM record and playback

modules. The equivalent input noise of the system was $0.6 \mu \mathrm{V}\left(0.09^{\circ} \mathrm{F}\right)$ within the $0-1000 \mathrm{~Hz}$ bandwidth; signal to noise ratios were typically $2500 / 1$.

Several data reduction procedures were employed. The signals were filtered with a $1 \mathrm{~Hz}$ cutoff frequency low pass filter to obtain the average gas temperature; the RMS values of the gas temperature waves were measured with a Hewlett Packard 3400A true RMS meter and averaging circuit. The gas temperature power spectral density functions were measured with Federal Scientific UA-500 five hundred channel and 444a four hundred channel spectrum analyzers; for most measurements, the bandwidth per channel was $2 \mathrm{~Hz}$. Probability density functions and cross correlation functions of the gas temperature waves were measured with a Saicor SAI-43A Correlation and Probability Analyzer.

\section{Results and Discussion}

Since widebandwidth measurements within gas turbine type combustors are not common, it is necessary to explain the elementary features of the data before attempting to use the data to examine the relationships between the combustor design elements and the combustion and mixing within the combustor. In these experiments, the gas temperature is used as a marker, in the same manner that particles are used to follow the gas flows in conventional water tunnel simulations of combustors. In combination with previous descriptions of the fluid mechanics within the combustors, the average gas temperatures can be used to infer time average values of the local mixing and combustion within the combustor and many features of the combustion can be identified just from such steady state data. A simple example of these data is obtained using a convectively cooled liner and the test \#1 conditions described in table 3. The combustor traverses are shown in figures 5 and 6 where it is apparent that the local average gas temperatures do vary markedly within the combustor. The first feature of the combustor operation that can be determined from this type of data is the location of the combustion in the combustor; the areas are indicated by high temperatures. It appears that combustion primarily occurs near the liner walls and extends significantly downstream of the primary section. The primary and secondary air jets are also easily identified in these traverses as discrete areas of low temperature. Even a cursory examination of the data shows that the penetration of the aft row of primary air jets is much larger than the larger diameter secondary air jets. 

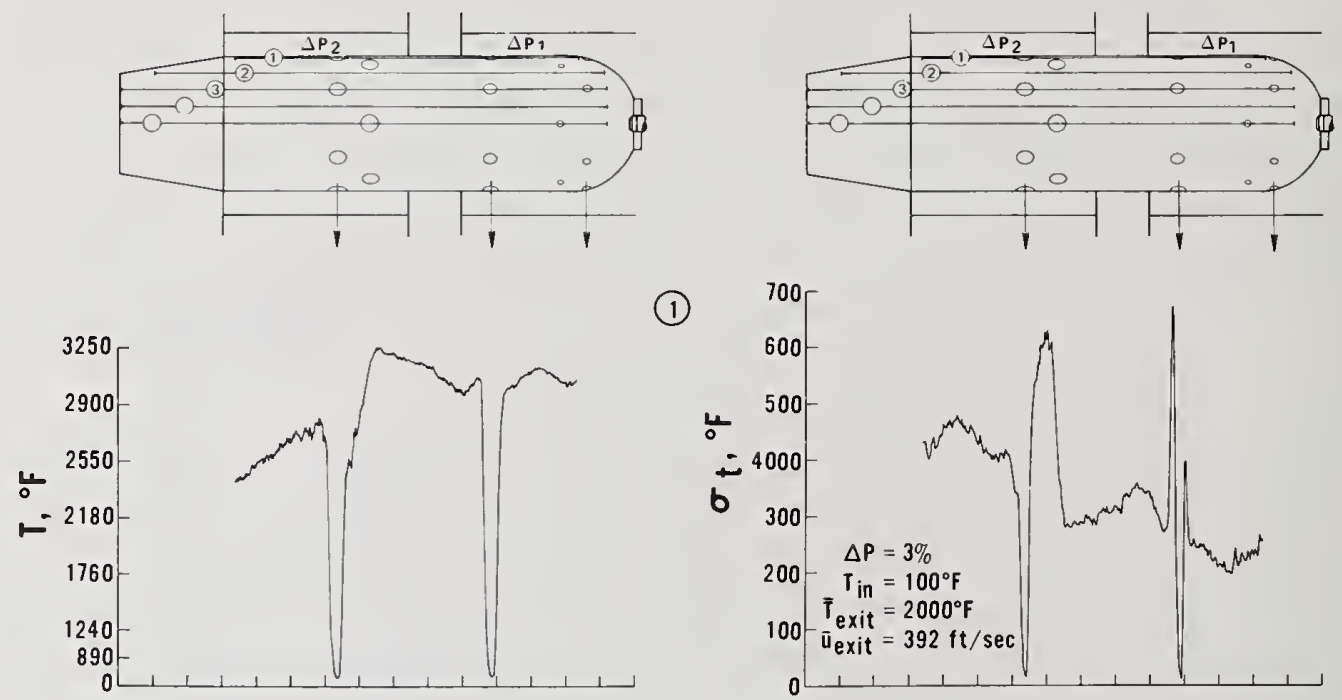

(1)
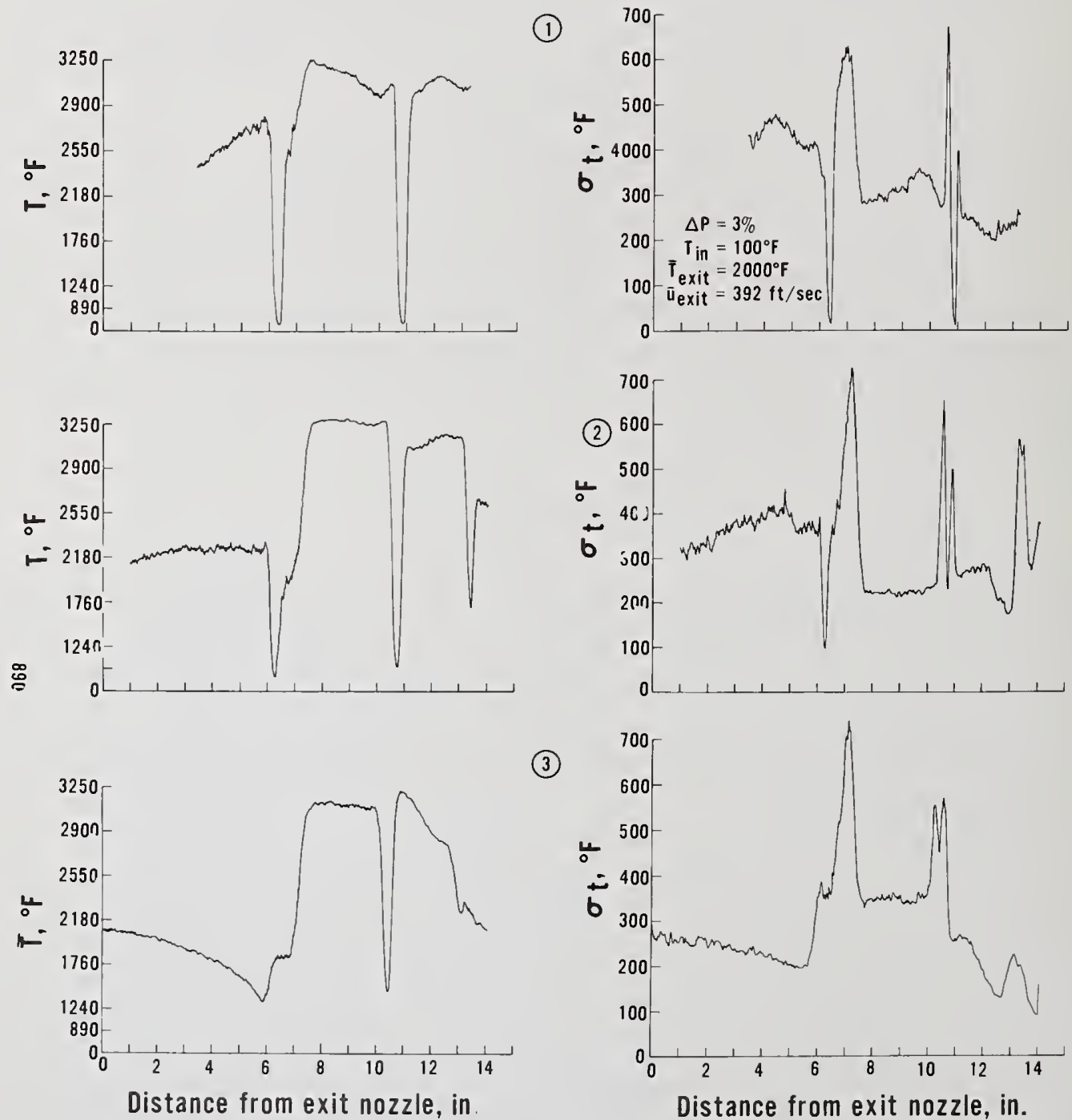

(3)

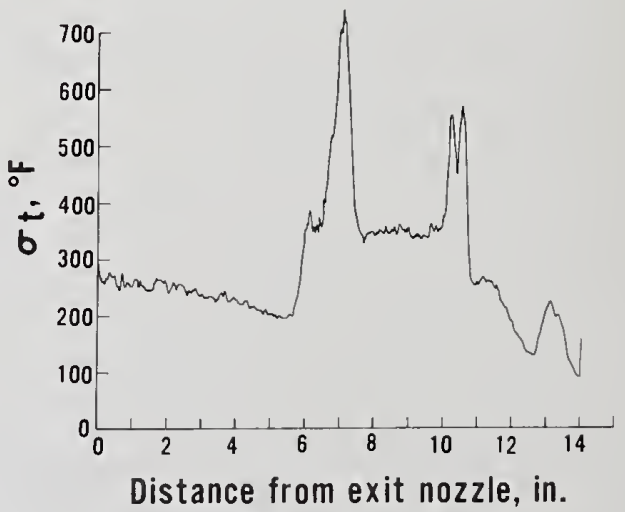

Figure 5. Average and widebandwidth gas temperatures along axial combustor traverses near combustor wall. 

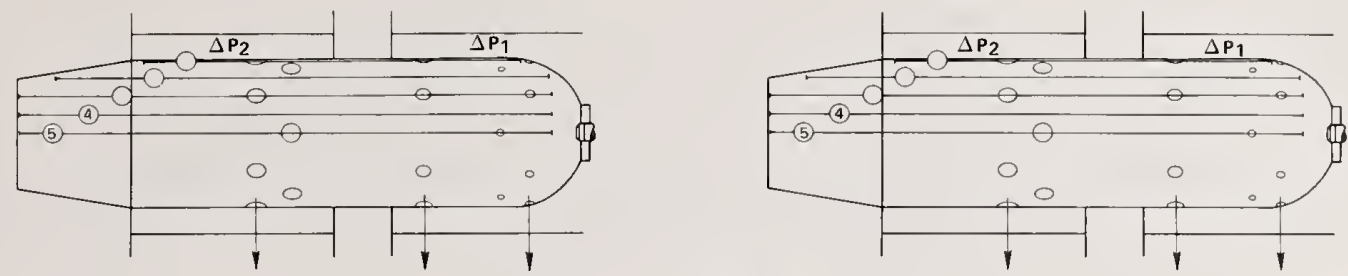

(4)
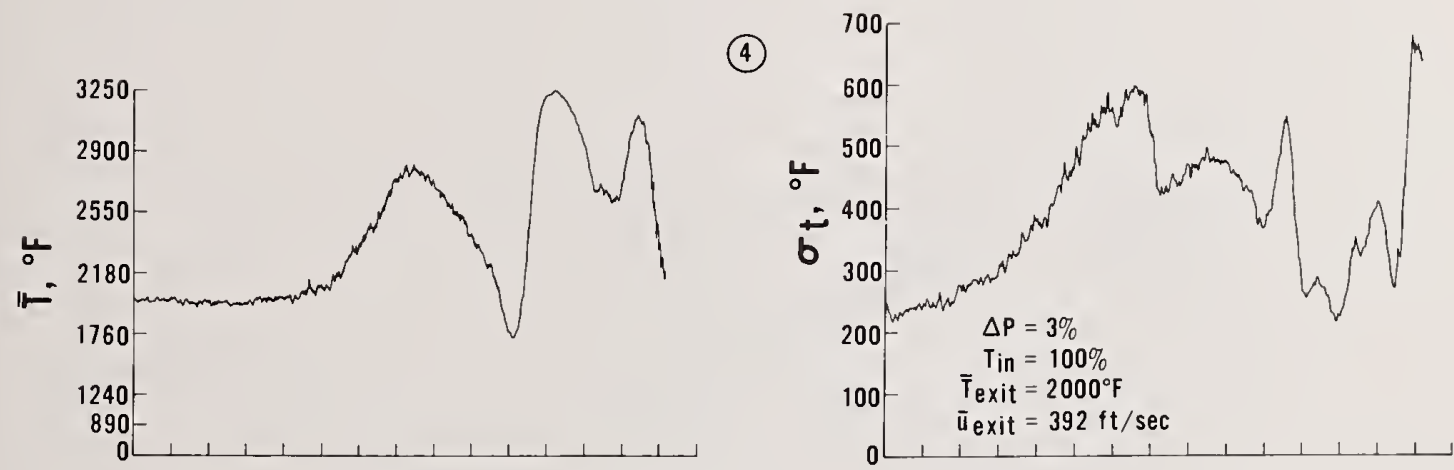

(5)
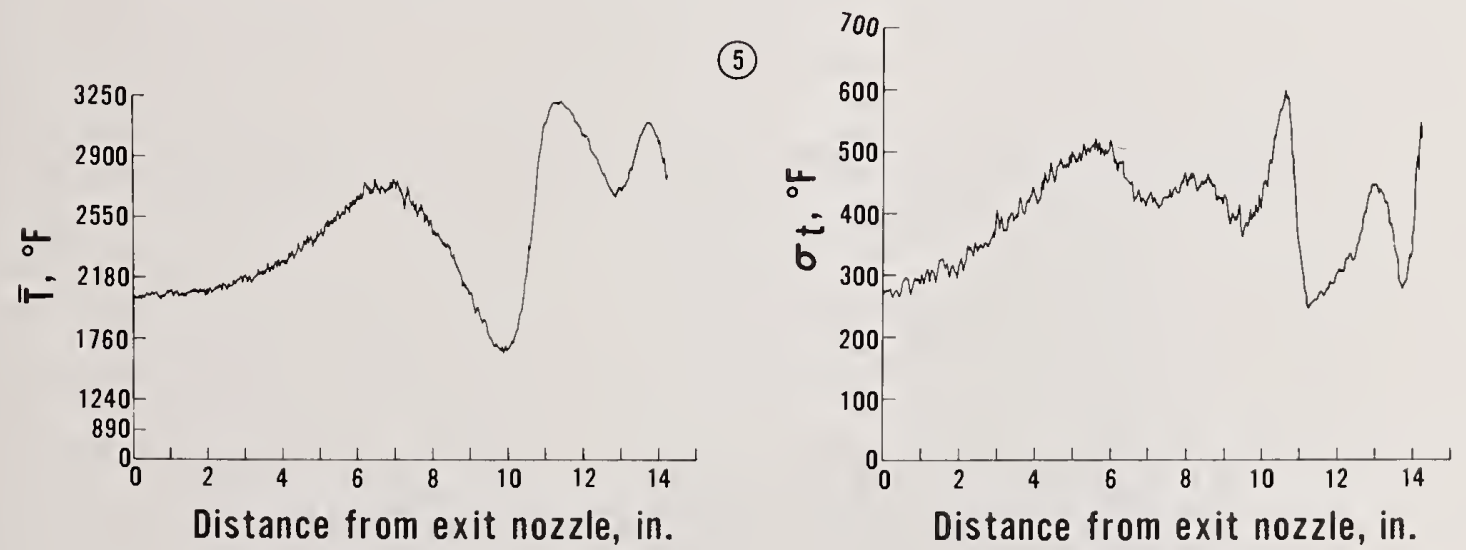

Figure 6. Average and widebandwidth gas temperatures along axial combustor traverses near combustor centerline.

A clearer picture of the jet behavior and the combustion is obtained by transforming these data to isotherm data in the form shown in figures 7 and 8 . The data are presented as one millivolt increment isotherms due to the large amount of data on a single isotherm map of the burner; the corresponding temperatures are listed in table 5 [15]. These data are strikingly similar in appearance to the flow field data obtained by conventional water tunnel simulations of this type of combustor [16], thereby simplifying the task of identifying the origin of some of the features of the isotherms. The location of the jets should be apparent at B, D, and G; the locations coincide with the location of the combustion holes in the liner. Again, it is seen that the aft row of primary jets penetrates much farther than the secondary air jets; this behavior is in agreement with previous 
engine data, where it is observed that the temperature at the centerline of the burner exhaust is sensitive to changes in the aft row of primary jets. In the future, by using the average gas temperature isotherms to monitor the behavior of the jets in the combustor, it should be possible to adjust the large scale gas flows within the combustor much more systematically and rapidly than has been possible with the conventional technique of changing combustor hole patterns and observing the effects only in the combustor exit.

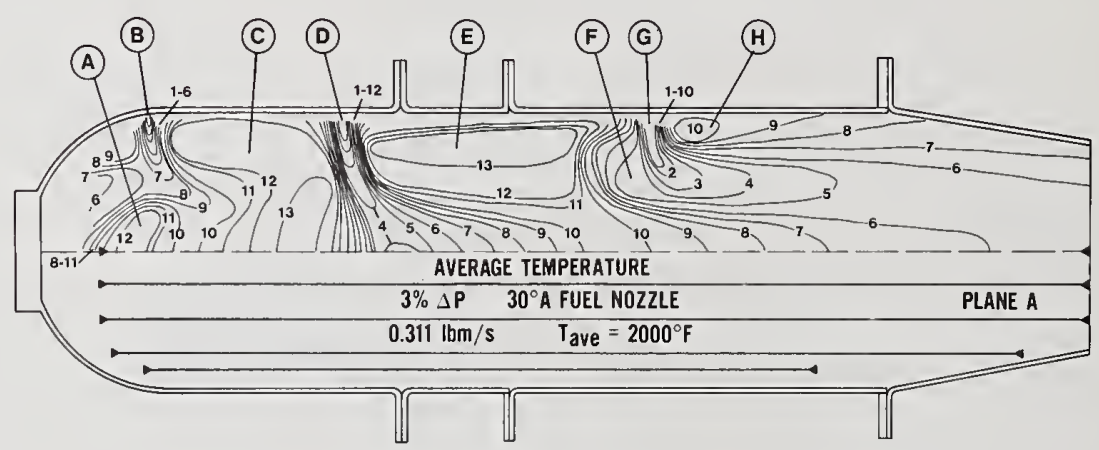

Figure 7. Average gas temperature isotherms; test \#1, plane A.

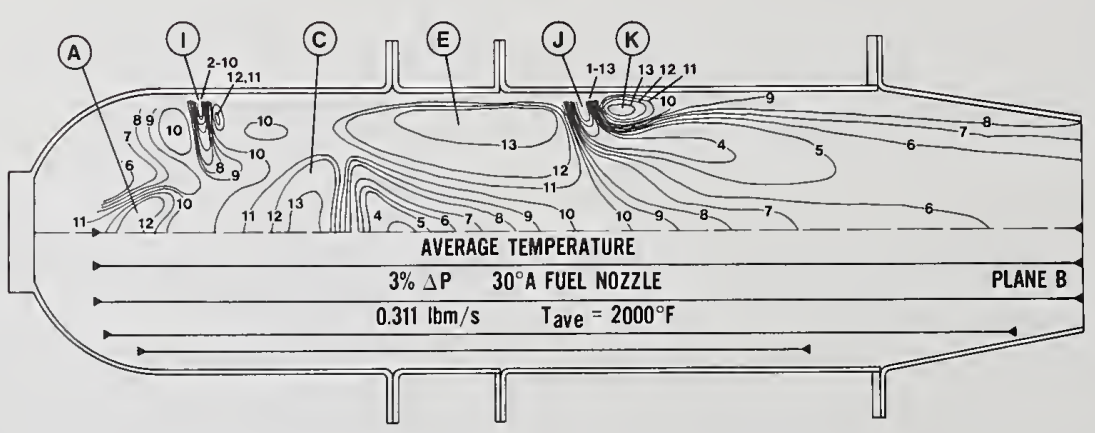

Figure 8. Average gas temperature isotherms; test \#1, plane B. 
Table 5. Selected type B thermocouple EMFs. Reference [15].

Average Temperature Isotherm Maps

\begin{tabular}{|l|l|l|l|}
\hline $\mathrm{mv}$ & $\overline{\mathrm{T}}$ & $\mathrm{mv}$ & $\overline{\mathrm{T}}$ \\
\hline $1 \mathrm{mv}$ & $841^{\circ} \mathrm{F}$ & $8 \mathrm{mv}$ & $2398^{\circ} \mathrm{F}$ \\
2 & 1173 & 9 & 2560 \\
3 & 1435 & 10 & 2717 \\
4 & 1661 & 11 & 2872 \\
5 & 1865 & 12 & 3026 \\
6 & 2053 & 13 & 3181 \\
7 & 2230 & & \\
\hline
\end{tabular}

0-1000hz RMS Temperature Isotherm Maps

\begin{tabular}{|c|c|c|c|}
\hline Leve1 & $\mathrm{mv}$ & $\overline{\mathrm{T}}=2000^{\circ} \mathrm{F}$ & $\overline{\mathrm{T}}=3000^{\circ} \mathrm{F}$ \\
\hline 1 & $0.5 \mathrm{mv}$ & $78^{\circ} \mathrm{F}$ & $90^{\circ} \mathrm{F}$ \\
2 & 1.0 & 155 & 180 \\
3 & 1.5 & 228 & 270 \\
4 & 2.0 & 310 & 360 \\
5 & 2.5 & 398 & 459 \\
6 & 3.0 & 465 & 540 \\
7 & 3.5 & 548 & 630 \\
8 & 4.0 & 620 & 720 \\
9 & 4.5 & 698 & 810 \\
\hline
\end{tabular}

The shapes of the isotherms at $A, C, E$ and $H$ suggest that recirculation zones occur at these locations; it would be expected that such zones would occur at these locations. Recirculation of the air from jet $B$ forms the zone at $A$; the high temperatures in the center of the zone indicate that the fuel is entrained and burned in this area. The apparent recirculation zone generated by jet $D$ is much more irregular, an influence of jet $B$ and jet $I$ in plane B (fig. 8). Again, intense combustion is sustained at the center of this area. Another major combustion zone $E$ is developed between jets $D$ and $G$; this zone is commonly observed during normal combustor operation. Smaller recirculation zones are observed just forward of jets B and I and aft of jets $G$ (zone $H$ ), I, and $J$ (zone $K$ ). Notice the amount of combustion occurring well past the midpoint of the combustor at zone K. 
As mentioned earlier, the actual temperature at a given location is fluctuating markedly with time. In figures 5 and 6 , RMS values of the waves along the traverse vary from 200 to $700^{\circ} \mathrm{F}$. The gas temperature density functions are generally asymmetrical, with the average value of the wave below the mean value of the wave in fuel lean areas (fig. 9) and above the mean value in fuel rich areas (fig. 10). In figures 9 and 10 , $\sigma_{t}$ is the RMS value of the wave and $\bar{T}$ is the time average gas temperature. The asymmetries are identical to those measured previously in large gas turbines [3]. Such asymmetries indicate that the PSD functions of the waves are not constant over the indicated bandwidth and that there are discrete, fixed phase relationships between some of the harmonic components of the waves. The fixed phase relationships can result from active nonlinear combustion processes [3] or even passive mixing and sampling processes when the size of initial populations of the hot and cold gases are significantly different [4]. The peak to peak values of the oscillations are approximately 50 but are skewed about the average value of the wave. It is interesting to note that in some areas of the combustor, the temperature oscillates approximately $3500^{\circ} \mathrm{F}$, which is quite close to the difference between the $100{ }^{\circ} \mathrm{F}$ inlet temperature and the $3620^{\circ} \mathrm{F}$ inlet temperature and the $3630^{\circ} \mathrm{F}$ stoichiometric flame temperature. Apparently, in some locations a stationary sensor can sample burned stoichiometric fuel/air mixtures and inlet gas.

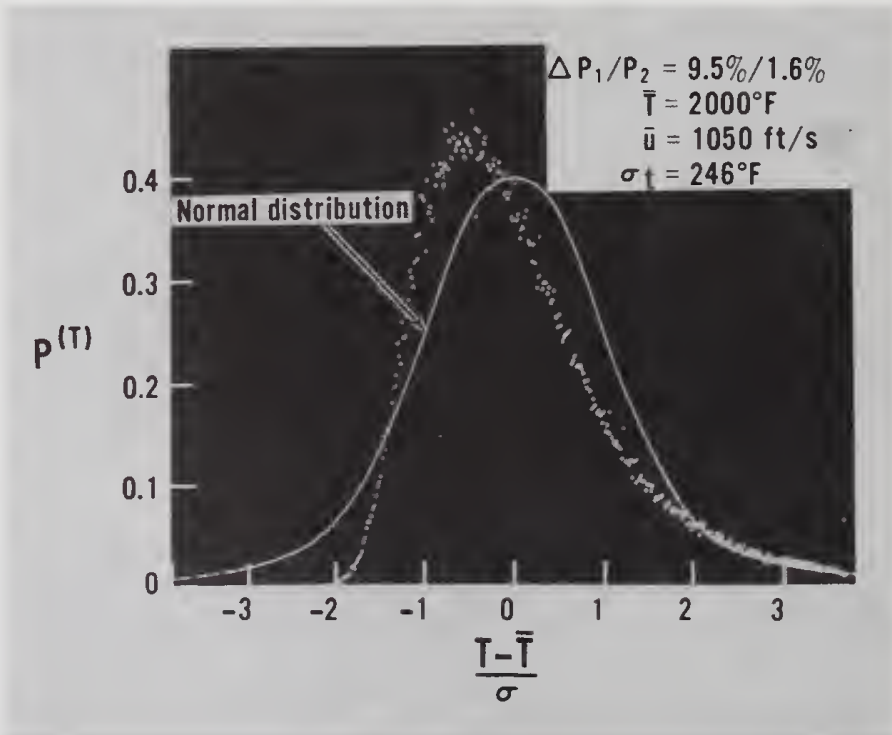

Figure 9. Gas temperature probability density function at combustor exhaust nozzle. 


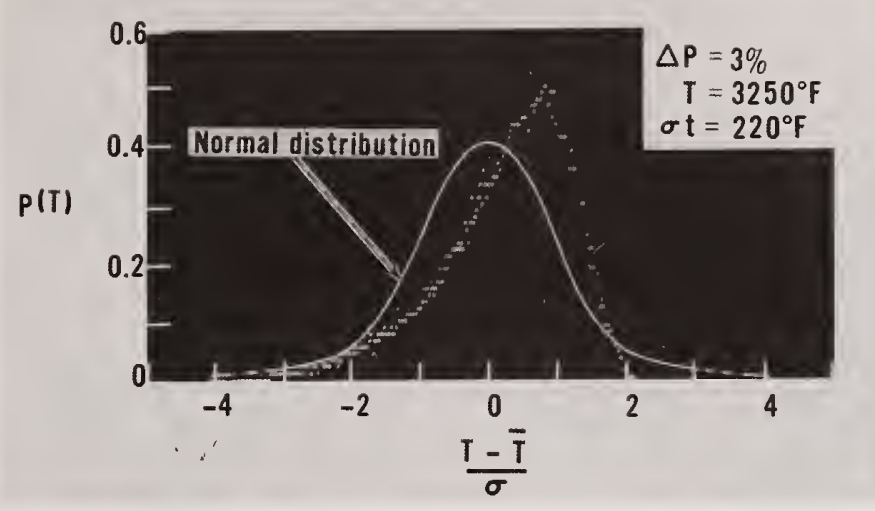

Figure 10. Gas temperature probability density function in foward recirculation zone.

The gas temperature waves are composed of $0-1000 \mathrm{~Hz}$ components and, depending on the operation of the combustor, the fourier components can be distributed uniformly over the bandwidth or limited to a few discrete frequencies [4]. Power spectral density, PSD, functions measured along a traverse can vary significantly or exhibit only small and gradual changes; again, the PSD functions are dependent on the operation of the combustor. As will be shown in a subsequent section of this paper, analysis of the distribution of power in the PSD functions within the combustor provides added insight to the large scale combustion dynamics and mixing within the combustor. Example exhaust gas PSD functions are presented in figure 11.

Remembering that the $0-1000 \mathrm{~Hz}$ components do represent gas stream tubes over $1 \mathrm{ft}$ long in a $1000 \mathrm{ft} / \mathrm{s}$ gas stream, and over $0.1 \mathrm{ft}$ in a $100 \mathrm{ft} / \mathrm{s}$ gas stream, the widebandwidth data provide the first direct information on the fluctuations of the large scale flows in the combustor; even without supporting velocity measurements, the data can be used to better understand the combustor aerodynamics. First, the importance of local unmixedness in the burner can be appreciated by comparing the amplitudes of the waves with those measured in simple flames [17], and premixed/prevaporized flames stabilized on a perforated plate flameholder $[18,19]$. In the simpler flames, gas temperature oscillations due to the axial motion of the flame front are 2-6 times smaller than those observed in figures 5 and 6 . Second, widebandwidth data provide additional information on the behavior of the primary and secondary air jets. In figures 5 and 6 it is observed that near the combustor wall, $\sigma_{t}$ in the vicinity of the jets is the derivative of $\bar{T}$, and large oscillations are observed only over a narrow distance at the edge of the jets. These data show that the jet motion is less than a few tenths of an inch and that very little of the entraining hot gas penetrates to the center of the jet. In contrast, near the burner centerline $\sigma_{t}$ is highest at the center of the jets, indicating that the jets have become unstable and are disintegrating. By monitoring the widebandwidth behavior of the jets it is possible to better understand how the jets are being turned and accelerated by the entraining hot gas stream. 


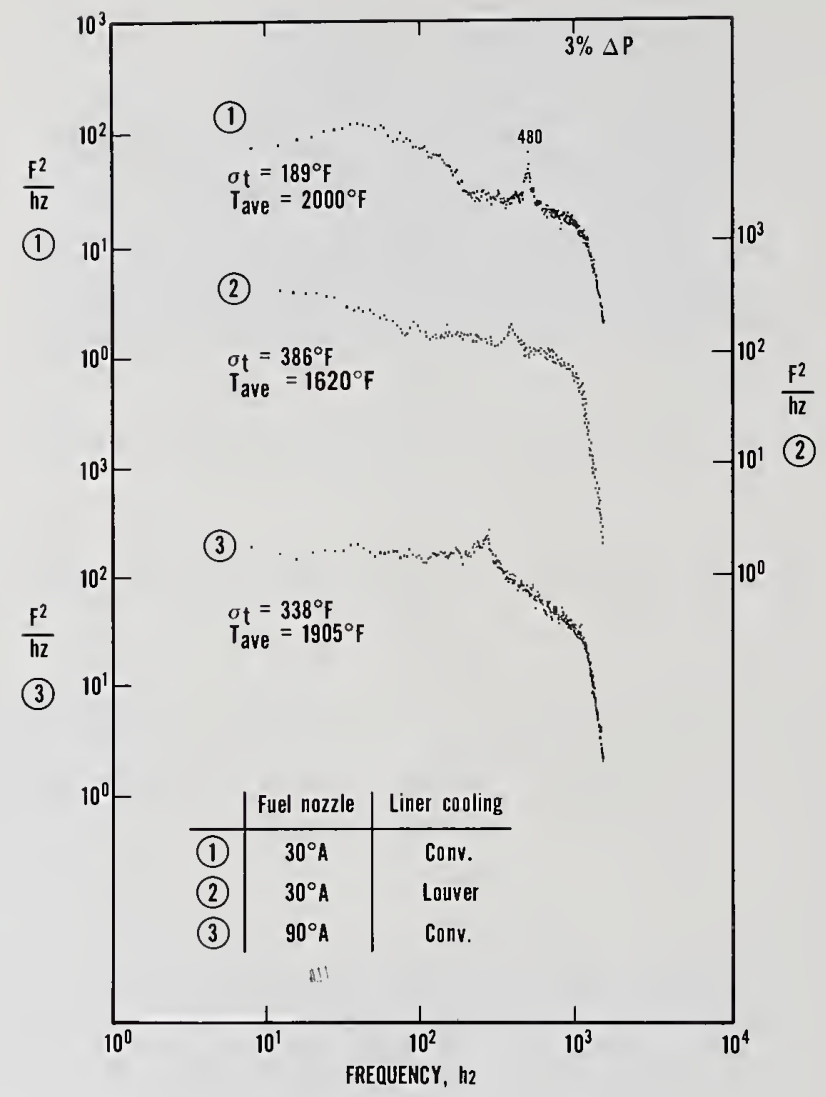

Figure 11. Exhaust gas temperature power spectral density functions; tests \#1, 2, and 4.

The widebandwidth data can be transformed to RMS isotherms as shown in figure 12 where a 0.5 millivolt increment isotherm map of plane $A$ is presented; the corresponding temperatures are listed in table 5 . Evidence of small jet motion and large temperature gradients is apparent near jet $D$ and the presence of jet $G$ is obvious. Notice that the small jet $B$ appears to oscillate and break up quite near the burner wall; this is in agreement with the average temperature data in figure 7 . In fact, the relationship between the average and dynamic gas temperatures is best appreciated by superimposing the two maps. It then becomes apparent that the largest values of $\sigma_{t}$ occur near the largest gradients in $\bar{T}$; examples are areas $L$ in figure 12 and $A$ in figure 7 , the area below 


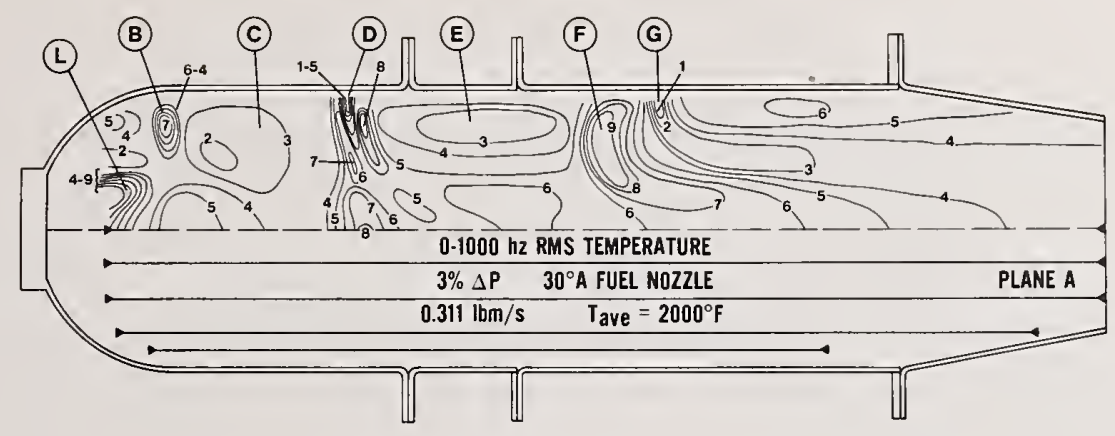

Figure 12. Widebandwidth gas temperature RMS isotherms; test \#1, plane A.

jet $D$ in figures 7 and 12, and area $F$ in figures 7 and 12. The intense mixing in area $F$ is due to both the presence of jet $J$ in plane $B$ and the oscillation of jet $G$. Small values of $\sigma_{t}$ occur where the average temperature is relatively uniform over the oscillation distances of the gas stream tubes, i.e., areas $C, E$ and the combustor exit in figures 7 and 12.

Let us now combine the $T$ and $\sigma_{t}$ isotherm maps and the PSD functions in a demonstration study of an evaluation of some of the simpler relationships between the combustor design elements listed in table 3 and the combustion and mixing in the combustor.

\subsection{Effects of the liner cooling}

Use of one-half of the combustor airflow to cool the liner wall has a profound effect on the combustion and mixing within the combustor. To demonstrate the effects, test 2 was conducted with the same fuel nozzle and air injection pressure used in test 1; the combustor liner was changed to a louvered configuration, small adjustments to the combustion hole configuration were made in order to maintain the axial air distribution, and the mass flow rate was increased to $0.365 \mathrm{lbm} / \mathrm{s}$ in order to maintain the injection pressure at 3 percent. The results are presented in figure 13 and 14 ; the parallel convectively cooled liner data are shown in figures 7 and 8 . Comparing these two sets of figures, it is apparent that the extensive use of airflow for liner cooling modifies both the character and location of the combustion and the behavior of the combustion air jets. The penetration of jets $C$ in figure 13 is much smaller than the corresponding jet $D$ in figure 7 . While similar recirculation zones occur ( $A$ in figures 7 and $13, C$ in figure 7 , and $B$ in figure 13), the intensity of combustion in the associated recirculation zones is much smaller and peak temperatures in the forward sections of the combustor are $500^{\circ} \mathrm{F}$ lower than in the convectively cooled liner. There is a well-developed recirculation zone at $F$ in figure 14 , 
but most of the combustion is occurring near the burner centerline with the peak temperature in the aft third of the combustor (zone $E$ ). The large combustion zone at $E$ in figure 7 has disappeared. It would appear that the strong primary jets in the convectively cooled liner help to displace the fuel to the outside of the combustor. The penetration of the jets $D$ and $I$ in figures 13 and 14, respectively, is remarkably weak; in general it has been found that the penetration of these jets in louver cooled liners is small for the range of sizes and injection pressures which can be used in a gas turbine combustor.

Normally, a combustor would not be operated in this manner since the fuel is only partially burned and the exhaust gas exit temperature is quite nonuniform. In this series of experiments, the tests were conducted only to demonstrate the significant changes in the combustion and mixing in the combustor due to the extensive use of liner cooling air.

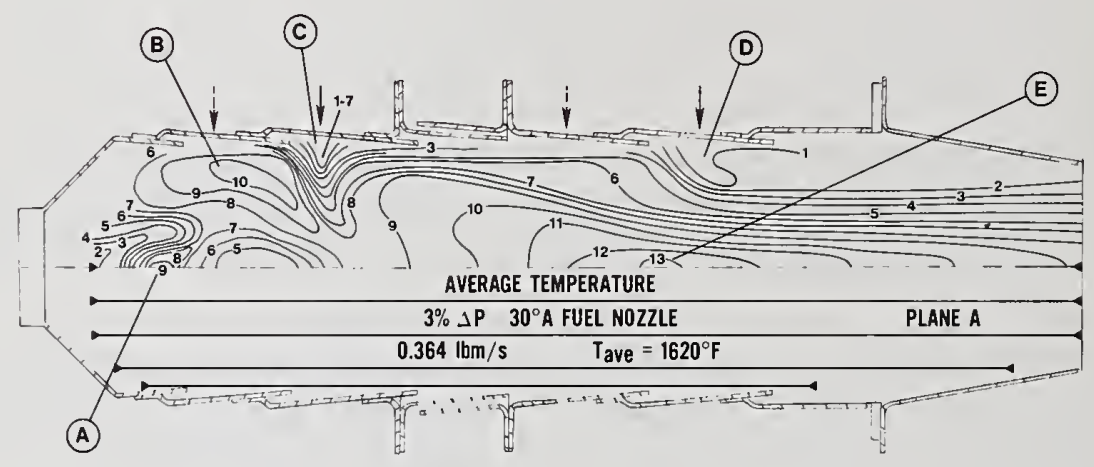

Figure 13. Average gas temperature isotherms; test \#2, plane A.

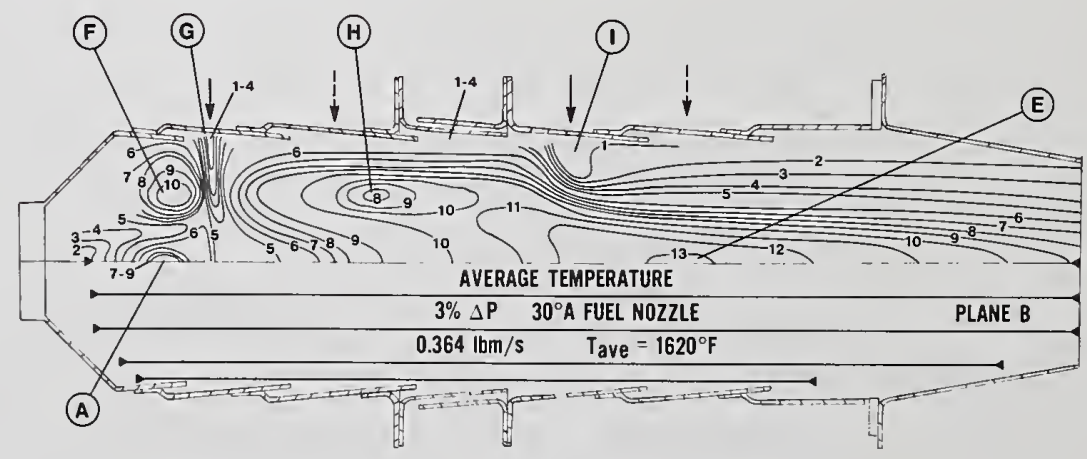

Figure 14. Average gas temperature isotherms; test \#2, plane B. 


\subsection{Effects of fuel nozzle spray angle}

Changes in the fuel nozzle spray angle also affect the events within the combustor. In test 3 , the conditions of test 2 were maintained with only a change in the fuel nozzle spray angle from $30^{\circ}$ to $90^{\circ}$. The results are presented in figures 15 and 16 ; figure 15 is comparable to figure 13. Comparing the isotherm maps of figures 13 and 15 , it is observed that the $90^{\circ}$ spray angle re-establishes intense combustion in the front end recirculation zone and the major combustion zone at $C$ in figure 15. While there is no change in the penetration of the secondary air jets, the uniformity of the exhaust gas temperatures is improved by simply generating hotter gases near the walls of the combustor upstream of the jets; these gases mix and provide a hotter average gas temperature near the edge of the exit nozzle. Coincidentally, reduction of the combustion at the centerline reduces the average gas temperature at that location, thereby further improving the uniformity of the combustor exhaust gases. However, the improvement is not large and further redistribution of the combustion near the liner walls will introduce significant burner liner durability problems with marginal gain in the uniformity of the combustor exhaust gases. In a much more extensive study, it has been shown that it is difficult to achieve significantly better burner exhaust profiles through simple adjustment of the average fuel and airflows within the combustor [20].

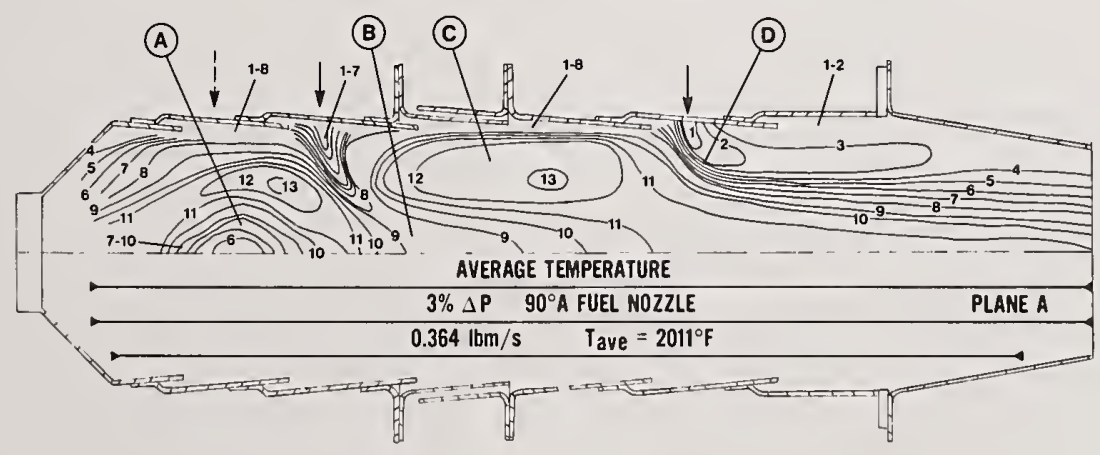

Figure 15. Average gas temperature isotherms; test \#3, plane A. 


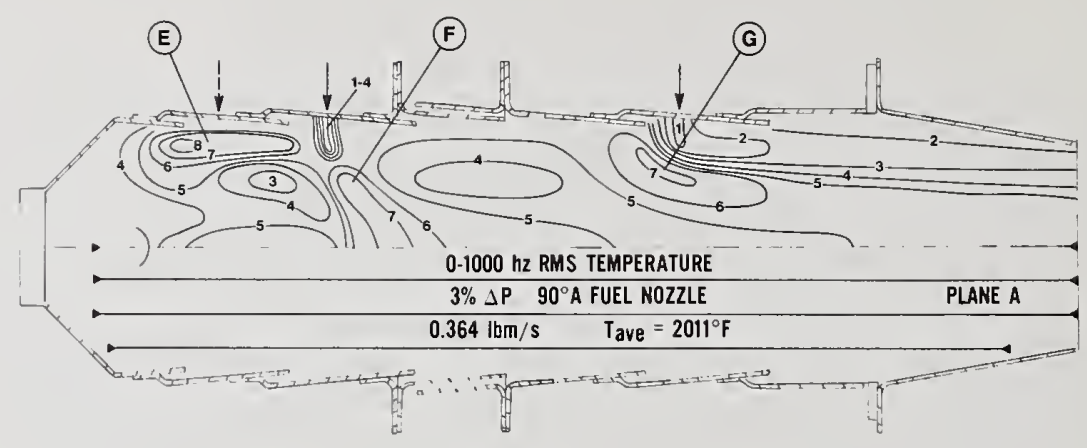

Figure 16. Widebandwidth gas temperature RMS isotherms; test \#3, plane A.

The RMS isotherm map in figure 16 indicates a straightforward behavior of the combustion air jets and the recirculation zones. The RMS levels in the uniform average temperature areas are similar to those presented in figure 9. Evidence of some recirculation zone oscillation is present in area $E$ of figure 16. The aft row of primary air jets breaks up near the liner at $F$. Notice that the motion of the secondary jets is limited to a narrow region just forward of the jets and there is only a small influence of the secondary air jets located in plane B.

Finally, to complete the test matrix, let us now combine the effects of primary air jet peneration and fuel nozzle spray angle to generate an extremely uniform exhaust temperature profile. In this test, test \#4 in table 3 , the mass flow and fuel nozzle are similar to test \#3, and the convectively cooled liner is operated with a 7.8 percent primary injection pressure and a 3.5 percent secondary air pressure. The results are shown in figure 17, where it is seen that the strong penetration of the primary air jets at $B, D$, and $E$ in figure 17 combine with the wide spray angle fuel nozzle to form intense, high temperature recirculation zones at $C$ and $F$. Therefore, even though the penetration of the jets $G$ in figure 7 and 17 are similar, the exhaust temperature profile is more uniform in figure 17 due to the redistribution of the combustion forward near the liner wall. It is interesting to note that while the results are of no direct interest to the gas turbine combustor, the configuration has been found useful in application of the combustor to materials testing where uniform average and dynamic gas temperatures must be sustained $[1,14,21]$. The superior performance of the materials test combustor was obtained by measuring and adjusting the combustion and flow within the combustor using the techniques and analyses described in this paper. 


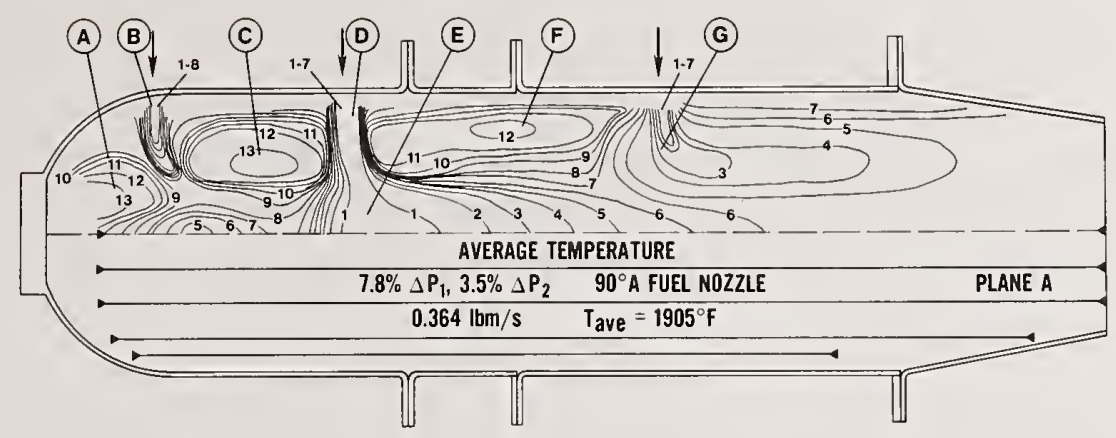

Figure 17. Average gas temperature isotherms; test \#4, plane A.

\subsection{Some details of the combustion oscillations}

In the preceding sections, the gas temperature fluctuations have been introduced in only a qualitative manner to identify jet motion and the scale of the gas stream tube movements within the combustor. At this point it is necessary to extend the discussion to examine some relationships between the details of the oscillatory state and the combustion and flow within the combustor. As an example of the importance of a single design element of the combustor on the oscillatory state, a louvered liner was operated with and without exhaust nozzle blockage (table 3 , tests 3,5,6, and 7). A perforated flat plate was used to provide 76 percent exhaust nozzle blockage and to provide the proper boundary conditions to sustain closed tube longitudinal acoustic pressure resonant modes in the combustor. From the outset, it is important to recognize that several elements of the combustor design must be properly tuned to sustain a strong oscillatory state. Elements which are known to significantly affect the oscillatory state are the mixing and flow within the recirculation zones and the physical shapes of the combustor and exhaust nozzle [20]. To obtain regenerative amplification of the longitudinal acoustic resonances by the combustion processes, it is necessary to increase the gain of each element until the gain around the feedback loop exceeds the nonlinear losses due to combustion, flow or improper acoustic boundary conditions within the combustor. When the gain around the loop is greater than one, regeneration occurs (Barkhausen Criteria). In the present experiments, the combustor design was such that incorporation of a flat exhaust nozzle blockage plate was sufficient to cause regeneration.

\subsubsection{Effects on exhaust gases}

\subsubsection{Power spectral density functions}

Several changes in the exhaust gases are caused by the blockage plate. There is a striking change in the PSD functions measured 1 inch forward of the exhaust nozzle exit at the burner centerline (fig. 18). Incorporation of the blockage plate increases the $\sigma_{t}$ 
value two and one-half times with the new power in the wave almost entirely contained within a few discrete fourier components. The peaks in the PSD function correspond to the first, second, third and fifth order of the longitudinal closed tube acoustic pressure resonance; the $Q$ values ( $w / \Delta w$, where $\Delta w$ is the width of a Lorentzian peak at one-half maximum intensity) are greater than 100 . Since the $Q$ values of this type of combustor without combustion are less than 10 [3], it can be concluded that the combustion processes have regeneratively amplified the acoustic resonances.

\subsubsection{Cross correlation functions}

There is also a marked change in the degree of correlation of the gas temperatures in the exhaust nozzle. The cross correlation functions of signals from a thermocouple located at the burner centerline and a thermocouple located at a radial position, $r$, away

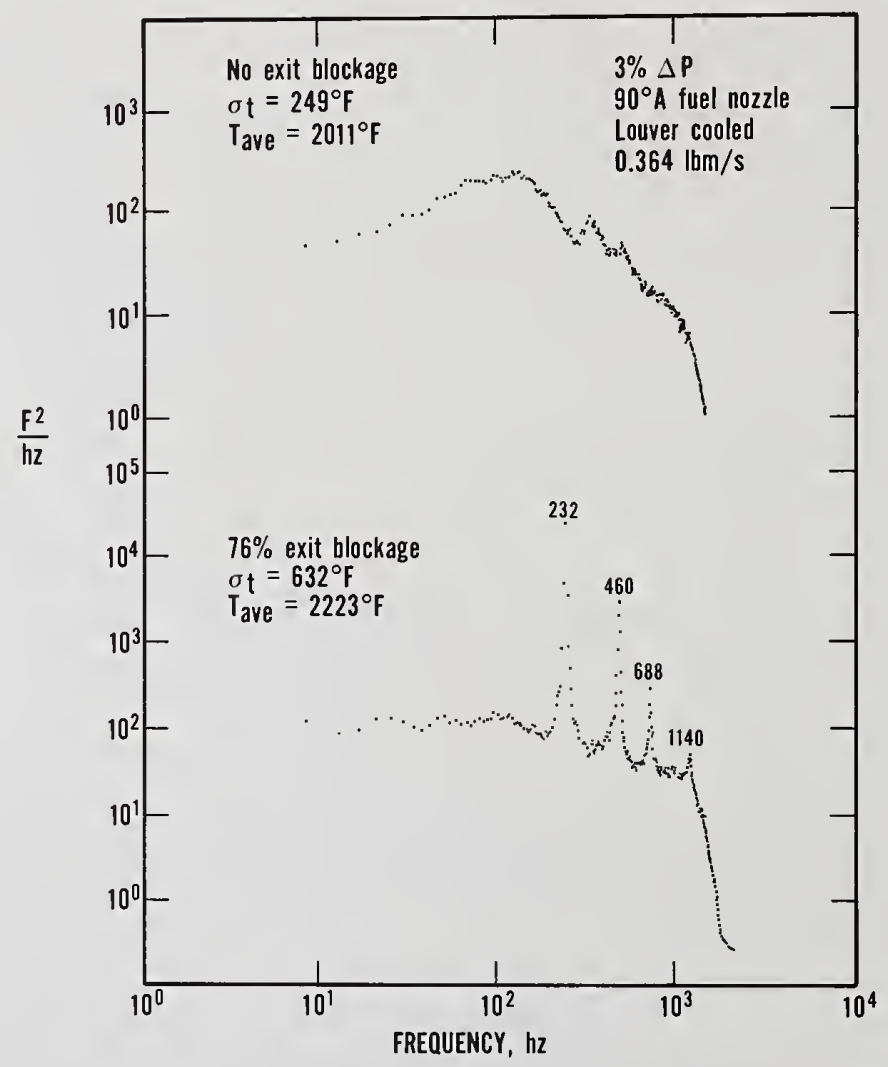

Figure 18. Exhaust gas temperature power spectral density functions; tests 6 and 7. 
from the centerline, $c_{0, r}(\tau)$, where $\tau$ is the time delay between the signals and is set equal to 0 , are shown in figure 19. In the absence of regeneration, the values of $C_{0, r}$ $(\tau=0)$ gradually decrease, reaching a value of approximately 0.15 for all the fourier components. In contrast, the resonant modes are positively correlated and the $C_{0, r}(\tau=0)$ values remain above 0.4 even at the edge of the duct.
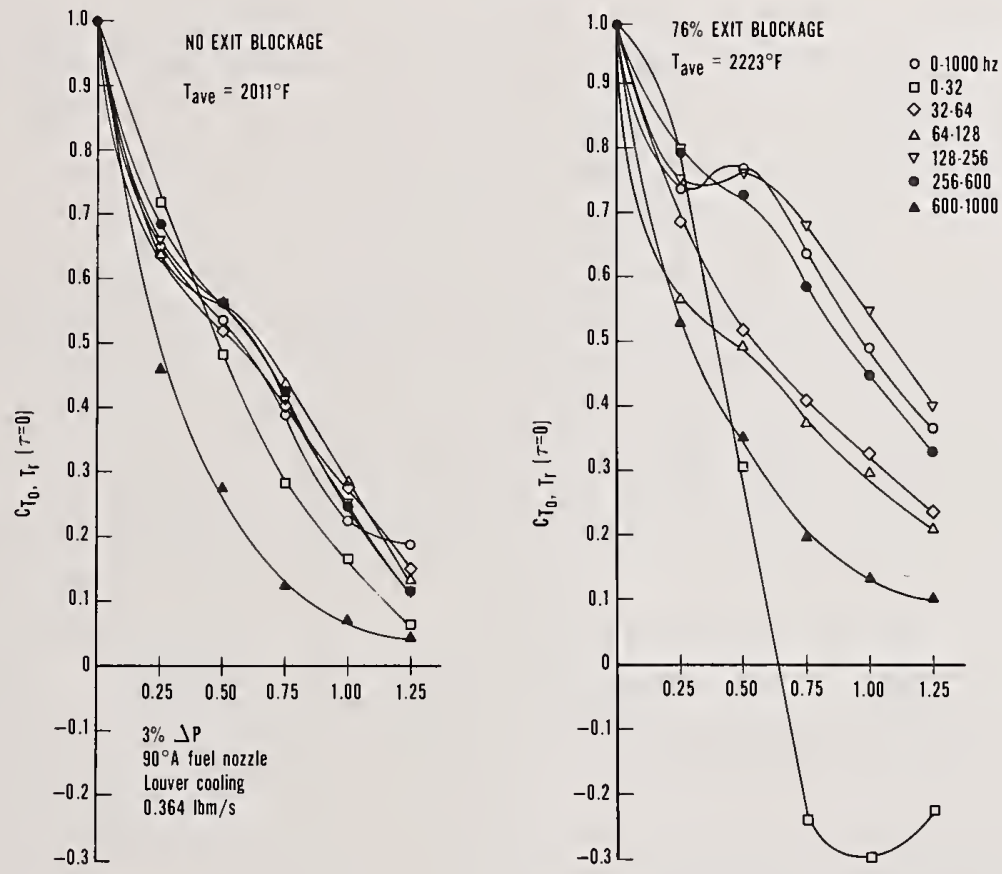

Figure 19. Cross correlation functions $(\tau=0)$ between exhaust gas temperatures at the centerline and at several radial positions; tests 6 and 7 .

An estimate of the coherent duct wide average fluctuations of the rate of heat release can be obtained by comparing the narrow and broadband $C_{0, r}(\tau=0)$ values with and without regeneration.

$$
c_{0, r}(\tau=0)=\frac{\sum_{i=1}^{i=\infty} T_{0, t_{j}} \cdot T_{r, t_{i}}}{\sigma_{0}, \sigma_{r}} \text {, }
$$


where,

$$
\begin{aligned}
& T_{0, t_{i}}=\text { the temperature at the burner centerline at time } t_{i},{ }^{\circ} \mathrm{F}, \\
& \begin{aligned}
\mathrm{T}_{r, t_{i}} & =\text { the temperature at the radius } r \text { at time } t_{i},{ }^{\circ} \mathrm{F}, \\
\sigma_{0} & =\text { the RMS value of the wave at the burner centerline, }{ }^{\circ} \mathrm{F}, \\
\text { and } \sigma_{r} & =\text { the RMS value of the wave at radius } r,{ }^{\circ} \mathrm{F} .
\end{aligned}
\end{aligned}
$$

In the absence of regeneration, both broadband and narrowband $C_{0, r}(\tau=0)$ values reflect the micro and macroscale of the turbulent structure and contain some information due to partially correlated heat release rates which occur even without regeneration. The turbulence is quite nonhomogeneous and contains extensive structure due to both the structure of the primary combustion gases and the proximity of the origin of the secondary air jets. No attempt is made to separate these effects here, but only to point out that, for those bandwidths which do not contain the resonant modes, $\sigma_{r}$ and $\sigma_{0}$ and $c_{0, r}(\tau=0)$ values are similar with or without regeneration. The onset of regeneration does not appear to affect these components and a conservative estimation of the effects of the macroscale of the nonresonant components of the temperature oscillation, $\lambda^{\prime}$, can be obtained from these values of $C_{0, r}(\tau=0)$. The problem can be thought of as similar to an extension in time problem, where a wave is composed of white noise of bandwidth, $B$, and a steady state signal; for times much greater than $1 / B$, the cross correlation function will become a constant and equal to the power in the steady state component. The extension in space problem is complicated by the presence of microturbulence near the origin, but for $r$ values greater than $0.5 \mathrm{in}$, the difference between the $c_{0, r}(\tau=0)$ functions of the nonresonant frequencies near the resonant modes and the $C_{0, r}(\tau=0)$ functions of the resonant modes is relatively constant. For $r>\lambda^{\prime}$,

$$
c_{0, r>\lambda^{\prime}}(\tau=0)=\frac{\sum_{i}^{i=n} T_{o c, t_{i}}-T_{r c, t_{i}}}{\sigma_{0} \sigma_{r}} \text {, }
$$

where $T_{o c}, t_{i}$ and $T_{r c, t_{i}}$ are the temperature components at 0 and $r$ which are due to the coherent resonant modes. The majority of the resonant power is contained in the 232 and $460 \mathrm{~Hz}$ components. Examination of the $128-256 \mathrm{~Hz}$ and $256-600 \mathrm{~Hz}$ bandwidth $\mathrm{C}_{0, r}(\tau)$ functions for the regenerative case shows they are harmonic and of constant amplitude for large values of $r$ and $\tau$. This indicates that the correlated components of each of the narrowband waves are single harmonic components which are in phase. For this case,

$$
c_{0, r}>\lambda^{\prime}(\tau=0)=\frac{\sigma_{o c} \sigma_{r c}}{\sigma_{0} \sigma_{r}} \text {, }
$$


where $\sigma_{o c}$ and $\sigma_{r c}$ are the RMS values of the coherent resonant frequencies at 0 and $r$. The 0-1000 Hz bandwidth $\sigma_{r c}$ values are simply the RMS sum of the two large fourier components.

The constant difference between the $c_{0, r}(\tau=0)$ functions of the correlated components and the uncorrelated components suggests that $\sigma_{r c} / \sigma_{r}$ is relatively constant over the duct. For this case,

$$
\frac{\sigma_{r c}}{\sigma r} \approx \sqrt{c_{0, r>\lambda^{\prime}}(\tau=0)}
$$

and for the $0-1000 \mathrm{~Hz}$ bandwidth,

$$
c_{0, r>\lambda^{\prime}}(\tau=0) \approx 0.2 \text { and } \frac{\sigma_{r c}}{\sigma r} \approx 0.45
$$

Note that approximately 60 percent of the power in the gas temperature wave with regeneration is contained in the resonant modes, and it appears that approximately 75 percent of the power in these components is correlated across the entire duct.

The amount of duct wide correlation can also be estimated from the narrowband data for the 128-256 Hz and 256-600 Hz bands which contain the large first and second order resonant modes. Again for large values of $r$,

$$
\begin{aligned}
& \left.\frac{\sigma_{r c}}{\sigma_{r}}\right|_{128-256}=\sqrt{0.3} \text { and }\left.\frac{\sigma_{r c}}{\sigma_{r}}\right|_{256-600}=\sqrt{0.2}, \\
& \sigma_{r c} \approx 0.42 \sigma_{r 28-256} \text { and } \sigma_{r c}=0.44 \sigma_{r} .
\end{aligned}
$$

The total coherent power in the two bandwidths is $0.43 \sigma_{t}$, which is very close to the value derived from the widebandwidth cross correlation functions. Assuming a constant value of $\sigma_{r c} / \sigma_{r}$ across the duct, $\sigma_{r c}$ is obtained from the local values of $\sigma_{r}$. The average correlated variation in temperature across the entire duct, $\bar{\sigma}_{c}$, is

$$
\bar{\sigma}_{c}=\frac{2}{R^{2}} \int_{0}^{R} r \sigma_{r c} d r
$$

where $R$ is the duct radius. Evaluating eq. (11) leads to a value of $\bar{\sigma}_{c}$ equal to 10 percent of the average temperature. This corresponds to an RMS value of correlated heat release rate gscillations equal to 10 percent, which indicates an unusual degree of coupling between the resonant acoustic modes and the rate of heat release. 


\subsubsection{Uniformity of exhaust gases}

The uniformity of the time average exhaust gas temperatures is also improved with regeneration. In figure 20 , reduced radial profiles show that the peak temperature is significantly smaller with regeneration. The data are normalized with respect to $T_{r}$, the temperature rise across the combustor; $T_{r}=T_{\text {ave }}-T_{\text {inlet }}$, where $T_{\text {ave }}$ is the average of the time average temperatures in the exhaust nozzle. The parameter is commonly used in descriptions of the uniformity of combustor exhaust gases. For the present single combustor, the maximum value of $\left(T-T_{a v e}\right) / T r$ is not only equal to the radial profile, but is also approximately equal to the burner pattern factor. The burner pattern factor is related to the variance, $\sigma_{s}$, of the spacial distribution of the time average temperatures across the exhaust duct by the approximation (4) $\rfloor$

$$
B P F \approx 2 \sigma_{s} / T_{r}
$$

Regeneration causes a 50 percent reduction in the maximum value of $\left(T-T_{\text {ave }}\right) / T_{r}$.

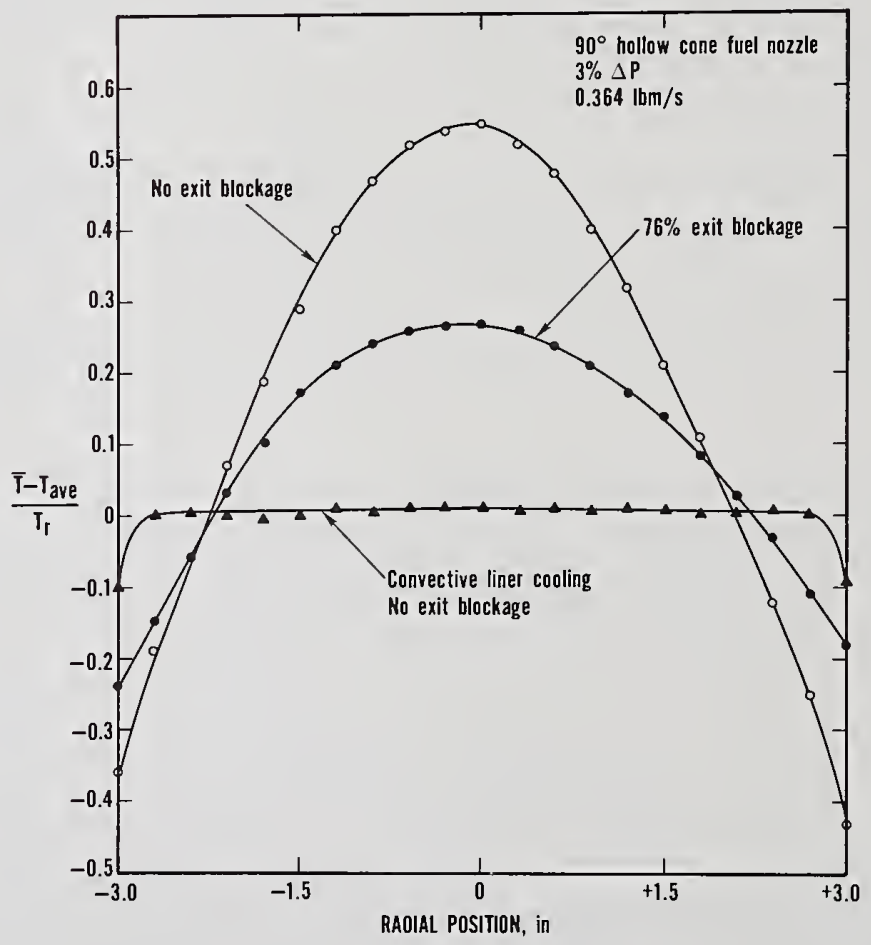

Figure 20. Radial profiles at combustor exhaust nozzle; tests 6 and 7 , plane A. 
In an earlier paper, the authors introduced the parameter $\sigma_{m}$, the RMS value of the unmixedness of the exhaust gases. $\sigma_{m}$ can be obtained by measuring $\sigma_{t}$ and $\sigma_{s}$; the total unmixedness and the temperature distributions in time and space are related by the approximation [4]

$$
\sigma_{\mathrm{m}} \approx \sqrt{\sigma_{\mathrm{s}}^{2}+\bar{\sigma}_{\mathrm{t}}^{2}}
$$

where $\bar{\sigma}_{t}$ is the duct average value of $\sigma_{t}$. Values of these parameters for the blocked and unblocked exhaust nozzle experiments are presented in table 6 where it is seen that an increase in $\sigma_{t}$ is accompanied by a decrease in $\sigma_{s}$, while $\sigma_{m}$ remains unchanged. The $\sigma_{m}$ values in the laboratory combustor exhaust gases are also equal to those measured in the full scale gas turbine combustor exhaust gases [4]. In general, the features of the exhaust gases observed in these tests are identical with those observed during the past decade in a series of full scale engine tests $[3,4,20]$.

Table 6. Unmixedness of the combustor exhaust gases.

\begin{tabular}{|c|c|c|c|}
\hline COMBUSTOR & $\bar{\sigma}_{t} / \mathrm{T}$ & $\sigma_{s} / T_{r}$ & $\sigma_{m} / T_{r}$ \\
\hline $\begin{array}{l}\text { Test } 6 \text {. No Exhaust } \\
\text { Nozzle Blockage }\end{array}$ & 0.09 & 0.28 & 0.294 \\
\hline Engine $C$, Ref 4 & 0.07 & 0.26 & 0.269 \\
\hline $\begin{array}{l}\text { Test } 7.76 \% \text { Exhaust } \\
\text { Nozzle Blockage }\end{array}$ & 0.24 & 0.15 & 0.283 \\
\hline Engine A, Ref 4 & 0.245 & 0.13 & 0.277 \\
\hline $\begin{array}{l}\text { Test } 4 \text { Convective } \\
\text { Liner Cooling }\end{array}$ & 0.19 & 0.05 & 0.19 \\
\hline
\end{tabular}


The results of the convectively cooled liner test (test 4 , table 2 ) have also been included in figure 20 and table 6 to demonstrate the importance of the louver cooling air in establishing the radial profile and to show that there is no necessary relationship between $\sigma_{t}$ and $\sigma_{s}$ away from the gas turbine main burner design point. In the absence of louver cooling, the combustion and flow in the forward sections of the burner can be adjusted to reduce $\sigma_{s}$ with no necessary increase in $\sigma_{t}$; comparable adjustments are not feasible in the full scale gas turbine combustor. In fact, the gas turbine main burner must satisfy so many performance requirements (e.g., efficiency, stability, turn down ratio, emissions, high altitude relight, burner durability, BPF and vane profile) that there is only a narrow permissible design range and it is for this reason that $\sigma_{m}$ is reasonably constant for most practical gas turbine combustors.

\subsubsection{0scillations within the combustor}

In order to understand the origin of the regenerative combustion oscillations, it is necessary to look inside the combustor and trace the changes in the local gas temperatures that accompany the onset of regeneration (figs. 21-24). Starting at the front of the combustor in figure 21 , it is seen that subtle changes occur in the average temperature and $\sigma_{t}$ values in the forward recirculation zones. The changes indicate that the combustion occurs closer to the front of the combustor and is more uniformly distributed due to larger flow oscillations within the zone. The increased motion is observed through the appearance of the combustion jet from plane $B$, the uniformity of the $T$ values within the zone, and the induced motion of the jets in the aft row of holes in the primary section. The flow disturbances travel downstream and induce extensive oscillations of the secondary air jets (area $D$ in fig. 21). The lack of change in area $C$ in figure 21 does not imply that the flow disturbances are not transmitted through this zone, but only that the resultant motion is not sufficient to significantly change $\bar{T}$. (In a uniform temperature zone, large scale flow fluctuations can occur and not be detected by the fine wire thermocouples.) The increased motion of the secondary air jets appears as an abrupt change in $\overline{\mathrm{T}}$ values and an increase in $\sigma_{t}$ in area $D$. Notice that in figure $21, \sigma_{t}$ increases before the second row of secondary air jets. With increased jet motion, the secondary air jets located in plane $B$ can be sampled in plane $A$ at the one inch radius.

The changes in the centerline profiles shown in figure 22 are more subtle and show gradual changes in the $\overline{\mathrm{T}}$ and $\sigma_{t}$ values in figures 21 and 22 are not markedly increased with regeneration and there are only quantitative changes in the time average size and structure of the recirculation zones and the flow within the combustor. Therefore, it would appear that the increased jet motion and resultant increased exhaust gas temperature uniformity are due to an increase in the coherence of the fluctuations in heat release rate within the combustor, rather than an increase in the magnitude or location of the fluctuations in heat release rate. 

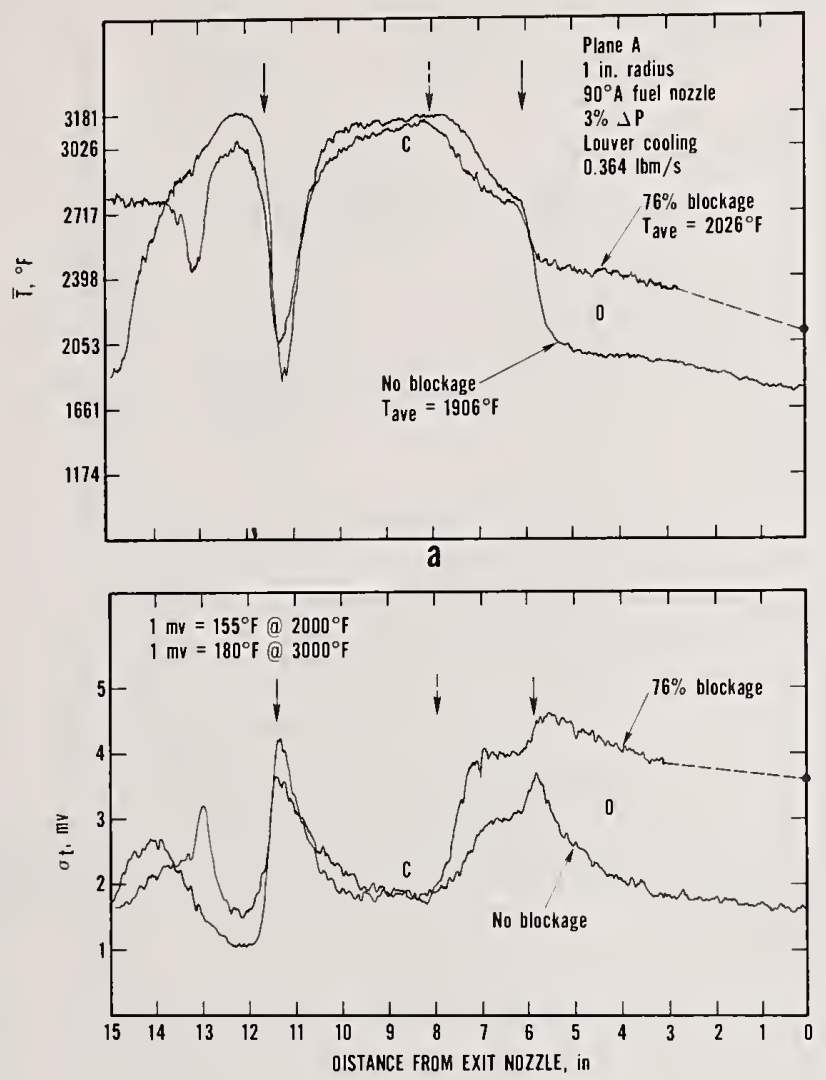

b

Figure 21. Effects of regeneration on the average (a) and widebandwidth (b) gas temperatures along the 1 in. radius traverse; tests 3 and 5 , plane $A$.

The importance of the coherent wave components can be shown by following the individual fourier components along the burner traverses. Carpet displays of the PSD functions along the two burner traverses are presented in figure 23 , where it is seen that the distribution of power within the gas temperature waves is a strong function of position within the combustor. The first, second and third order multiples of the closed tube longitudinal acoustic resonance appear within the burner. In figure 24, the large first and second order terms are followed along the traverses in a quantitative manner; with regeneration there is a significant increase in $\sigma_{t}(B)$ at these frequencies. 

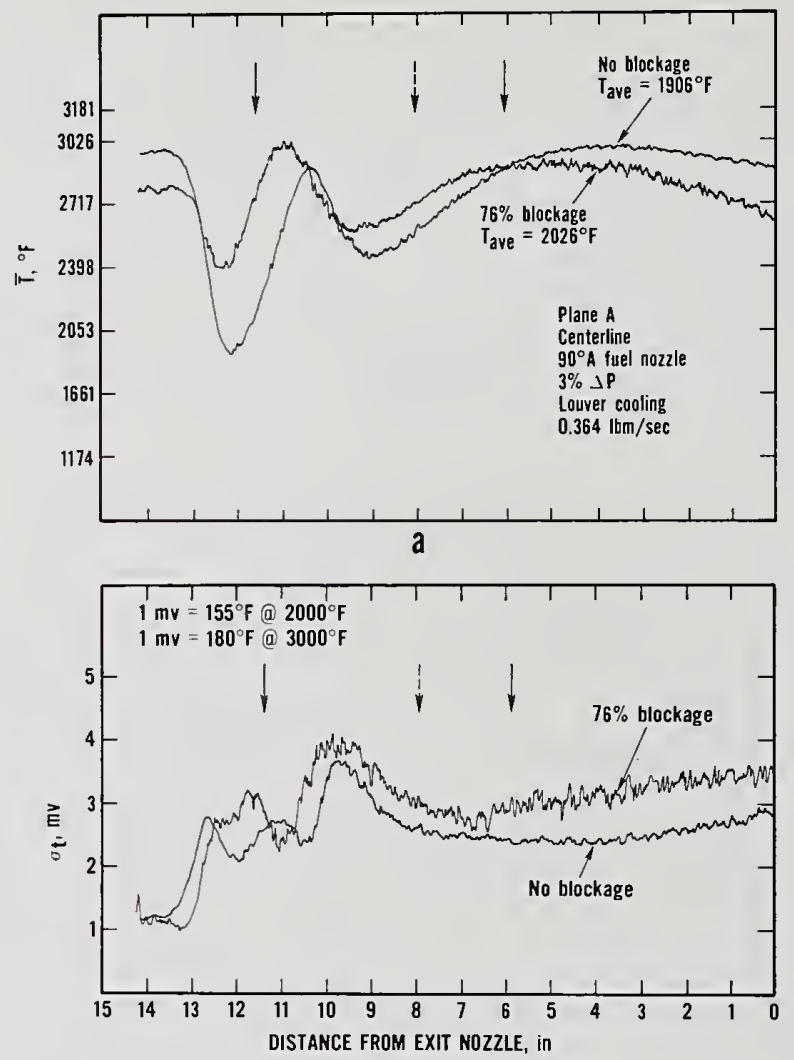

b

Figure 22. Effects of regeneration on the average (a) and widebandwidth (b) gas temperatures along the combustor centerline traverse; tests 3 and 5, plane A.

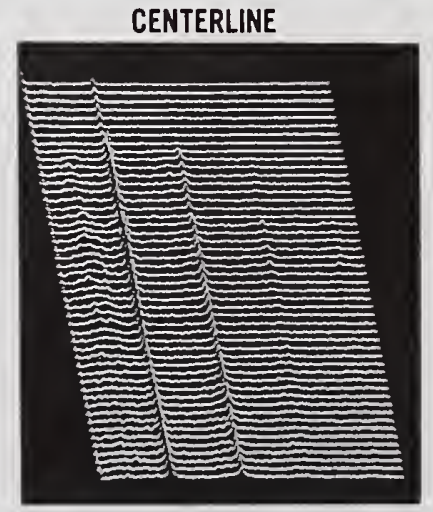

a

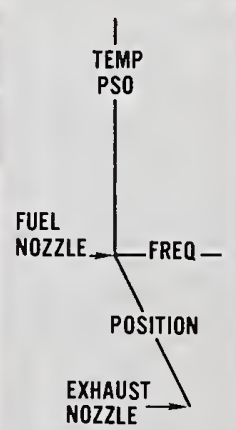

NOZZLE

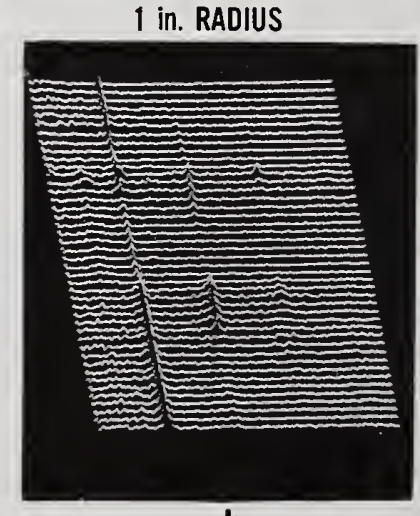

b

Figure 23. Carpet displays of $0-1000 \mathrm{~Hz}$ PSD functions; test 5, plane A. 


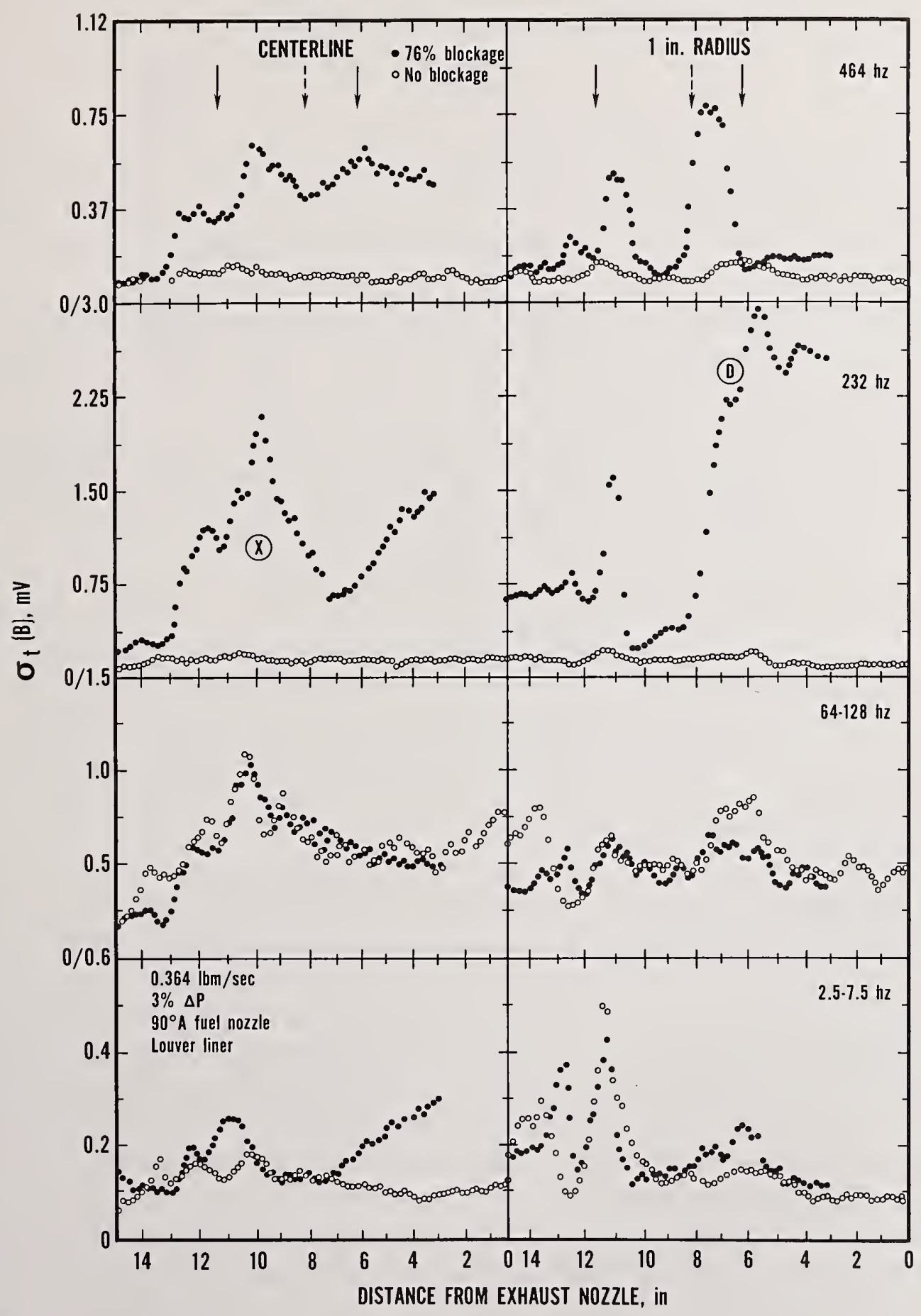

Figure 24. Effects of regeneration on selected gas temperature fourier components within combustor; tests 3 and 5 ; plane A. 
At the 1 in. radius, the motion of the large jets produces both first and second order terms. The second order term appears to result from sampling parts of two jets. For example, at point $D$ the large second order term occurs between the jets located in plane $A$ and $B$ and forward of the large first order term associated with motion of the jets in plane A.

As the jets penetrate to the burner centerline they become less distinct and the axial variations of the resonant modes become less abrupt. The peak in the first order term at $x$ corresponds to the axial location of the aft row of the primary air jets seen in figure 15; the gradual increase in the first order term at the back of the combustor is due to the secondary air jets.

Also shown in figure 24 is the RMS value of the wave contained within the $64-128 \mathrm{~Hz}$ bandwidth. 0scillations within this bandwidth are commonly observed within this type of combustor and appear to be associated with the large forward recirculation zone [20]. The flow around this zone does not appear to be strongly correlated in space and only a low $Q(\leq 3)$ oscillator is produced. The flow oscillations appear to be rather independent of the presence of regenerative amplification of the acoustic resonances, which is characteristic of the fourier components away from the resonant frequencies.

Finally, the power contained within the $2.5-7.5 \mathrm{~Hz}$ bandwidth is also shown in figure 24. These fourier components exhibit negative $C_{0, r}(\tau=0)$ values in figure 19; similar negative $C_{0, r}$ ( $(\tau=0)$ values have been observed in earlier engine measurements $[3,4]$. The $2.5-7.5 \mathrm{~Hz}$ components first appear in the forward recirculation zone. The components could be actual linear components of the wave. However, the oscillator is a marginal oscillator with significant amplitude modulation and the modulation frequency will appear as a fourier component of the PSD function if the wave is nonlinear (figs. 9 and 10) or the average local temperature is a function of $\sigma_{t}$ (fig. 20)

In the combustor exhaust, the dependence of $\overline{\mathrm{T}}$ on $\sigma_{t}$ appears to be the actual cause of the low frequency PSD components. The uniformity of the exhaust gases is a function of $\sigma_{t}$, and an increase in $\sigma_{t}$ is accompanied by a decrease in the average gas temperatures near the centerline and an increase in the average gas temperatures near the edge of the duct (fig. 24). This effect will result in the negative values of the cross correlation functions shown in figure 19. Note that this is a different interpretation of these low frequency oscillations than was originally proposed $[3,13]$ and derives principally from the examination of the behavior of the jets within the combustor where it is seen that the jets do not remain distinct enough nor is there enough jet motion to generate negative values of $c_{0, r}(\tau=0)$ within this bandwidth.

To summarize, the data show that the regenerative amplification of the first order quarter wavelength longitudinal acoustic resonances by the combustion processes causes a significant fraction of the heat release rate variations to be spacially correlated across the combustor. The correlated fluctuations in heat release rate cause extensive motion of the aft row of jets in the primary zone and the secondary air jets. 


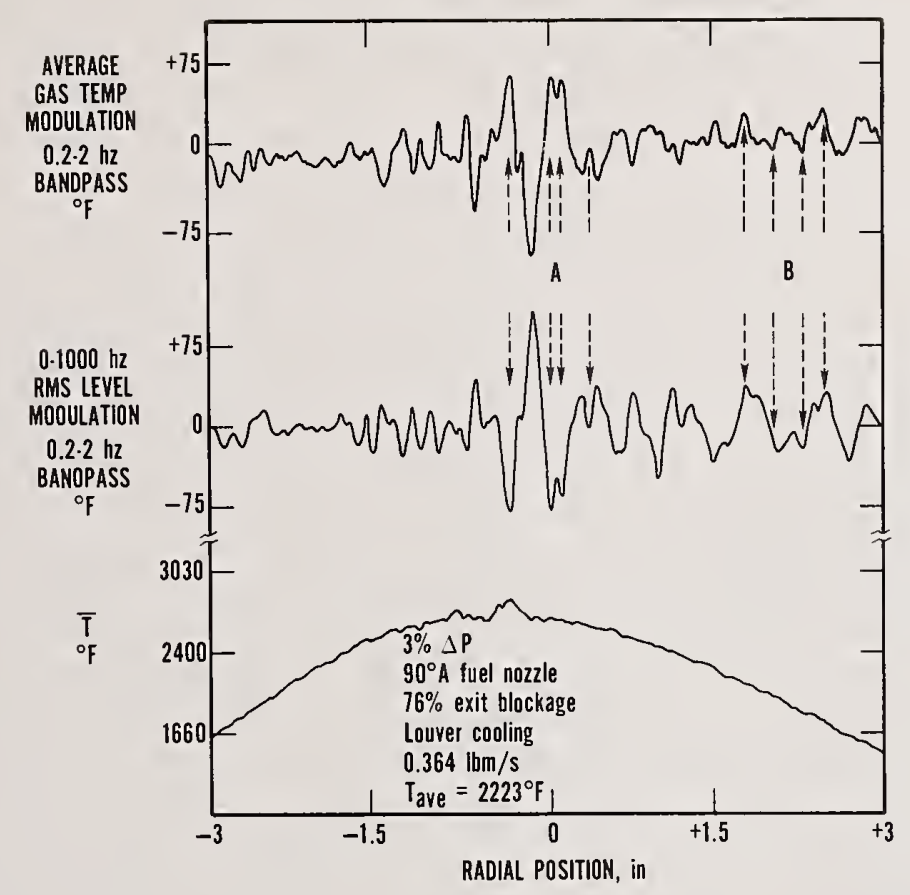

Figure 25. Effects of low frequency amplitude modulation on average gas temperatures at the combustor exhaust nozzle; test \#7, plane A.

The enhanced jet motion primarily occurs due to increases in the spacial coordination of the heat release rate fluctuations in the forward sections of the burner rather than a general increase in the amplitude of the heat release fluctuations throughout the burner. The oscillator developed in the forward sections of the combustor is a marginal oscillator with substantial amplitude modulation. The jet motion improves the uniformity of the time average exhaust gas temperatures.

The results are in agreement with earlier engine data, which showed comparable interrelationships between the radial profiles, BPF, PSD functions and cross correlation coefficients of the exhaust gases $[3,4,20]$. However, the engine data was obtained with a wide range of available engine designs and combustor configurations and, therefore, it was not possible to unambiguously isolate the effects of regeneration. Historically, the combustion and mixing in gas turbine main burners has been described by partial modeling techniques in air or water tunnels [16] and from investigations of full scale combustors in rigs and engines [22]. In the past few years, a number of computer codes have been developed to model some part of the combustion processes [23]. Descriptions of the correlated large scale fluctuations are notably absent from the past and present efforts and the present gas turbine designs reflect this lack of understanding of the importance of the correlated large scale flows. The recent use of annual liners with lean front ends 
has resulted in poor oscillators and straightforward increases in the nonuniformity of the combustor exhaust gases. From the data presented in the preceding sections of this paper and in earlier papers $[3,4]$ it would appear prudent to acknowledge, understand, and learn to use the correlated large scale flow structure.

\subsubsection{Feedback mechanisms}

Unfortunately, the picture is still incomplete with regard to the details of the correlation of the flows within the combustor and the nature of the feedback mechanism. The large scale and amplitude of the heat release rate fluctuations combined with the degree of correlation of the heat release rate fluctuations do simplify identification of the feedback mechanism to some extent. For example, the amplitudes of the pressure fluctuations measured in main burners are less than 0.3 percent of the chamber pressure [3] and, therefore, it can be concluded that they are incapable of independently causing significant changes in the mass flow within the combustor or the observed secondary jet motions. By elimination, it would appear that large fluctuations in the heat release rate are required in the forward sections of the burner to drive the large jets. This suggestion is in agreement with the experimental data. Furthermore, the origin of the large scale fluctuations in the heat release rate can be traced to the recirculation zones in the forward section of the burner. Prior to such a zone, feedback to the forward sections of the burner is only through transmission of pressure waves and their amplitude is also insufficient to directly cause large variations in the heat release rate in unidirectional flow. In the recirculation zones, the recirculatory flow can provide a high gain for some of the fourier components of a pressure, combustion or flow disturbance. Again, this suggestion is in agreement with the experimental data which shows evidence of extensive fluctuations within the primary recirculation zone through the large scale motion induced in the downstream jets.

The large scale and amplitude of the correlated flows and the period of the fourier components also point in the direction of a feedback mechanism that depends on a several millisecond time delay around the feedback loop, rather than the few hundredths of a millisecond characteristic time associated with the microscale of turbulence. One possible feedback mechanism that relates the pressure oscillations to the rate of heat release through primary air jet instabilities can be suggested [13]. In this hypothesis, the acoustic pressure resonances produce small changes in the pressure drop across the burner liner and these pressure drop perturbations affect the primary jet trajectories and flow in the recirculation zone. Since the rate of heat release is limited by the air supplied to the forward section of the burner and the rate of mixing, the fluctuations in the airflow to the recirculation zone generate a fluctuation in the heat release rate, which reinforces the pressure fluctuations to cause enhanced instability of the primary air jets. For example, introducing a small increase in the pressure drop across the liner causes an increase in the jet penetration and a positive perturbation in the mass flow into the recirculation zone. After a time delay, $\tau_{r}$, where $\tau_{r}$ is primarily a function of 
$\tau_{m}$ and the time around the recirculation zone, the fuel and air mix and burn forward of the jet, increasing the temperature and velocity of the gases and causing a small deflection of the jet downstream. A decrease in the pressure drop across the liner will produce the same downstream deflection. As the jet is deflected downstream, less air enters the recirculatory flow and, after a time delay approximately equal to $\tau_{r}$, burning occurs and produces a smaller than average temperature and velocity forward of the jet. Such a decrease in heat release rate would cause an upstream perturbation of the primary jet in the same manner as the small increase in pressure drop across the liner. All that is required for regenerative amplification of the pressure wave is for $\tau_{r}$ to be near one-half the period of the resonant mode and for the linear terms of the rate of heat release to be sufficiently high that the gain around the loop exceeds the dissipative losses.

It turns out this general description is in agreement with the experimental data. As an exercise, the reader can add and subtract the coherent component of the local $\sigma_{t}$ values to each isotherm in figure 15 to obtain a rough indication of the magnitude of the variations in the recirculation zones and the variations in the trajectories of the large jets; the resultant isotherms show significant variations in the size and temperatures of the recirculation zones. However, much more detailed information on the large scale flows in the recirculation zones is required to understand the actual feedback mechanism. Additional cross correlation measurements within the recirculation zones are required to determine the actual changes in shape and the relative motion of the recirculating flows and the large air jets. Both narrow bandwidth and widebandwidth data are required. The techniques and analysis developed in this paper can help. In combination with other methods, the fine wire thermocouple measurements can lead to further understanding of the feedback mechanisms and in a more general sense, provide additonal information on the combustion and flow throughout the aircraft gas turbine combustor.

\section{Conclusions}

The results of widebandwidth gas temperature measurements within a model gas turbine combustor have been presented in this paper. The performance of 0.003 in. diameter Pt- 6 percent Rh/Pt-30 percent Rh thermocouples in the combustor gases are reviewed and a demonstration of the related measurement techniques and data analyses is presented. It has been shown that the simple fine wire thermocouples can provide useful information on the combustion and mixing within the combustor. The local average gas temperature provides information on the location and size of the recirculation zones and the trajectories of the combustion air jets; average gas temperatures from the inlet temperature to the stoichiometric flame temperature are measured within the combustor. Widebandwidth gas temperatures provide new information on the combustion dynamics; the gas temperature fluctuations are composed of fourier components within the $0-1000 \mathrm{~Hz}$ bandwidth and local RMS values of the waves vary from 0.05 to 0.25 of the temperature rise across the combustor.

The application of the technique focused on the role of the oscillatory state in determining the uniformity of the time average combustor exhaust gas temperatures. It was 
shown that the combustion processes can regeneratively amplify longitudinal acoustic resonant modes. Regeneration is accompanied by significant spacial correlation of the exhaust gas temperatures; analyses of the data indicate the presence of heat release rate fluctuations which are correlated across the entire duct. The correlated heat release rate fluctuations within the combustor cause extensive motion of the large primary and secondary air jets and an attendant improvement of the uniformity of the time average gas temperatures in the combustor exhaust.

\section{References}

[1] Dils, R. R. and Follansbee, P. S., Dynamic oxidation and corrosion in power generating units, Corrosion, 33 , No. 11, p. 385 (1977).

[2] Dils, R. R. and Follansbee, P. S., Heat transfer coefficients around cylinders in crossflow in combustor exhaust gases, Journal of Engineering for Power, Trans. ASME, 99 , No. 4, p. 497 (1977).

[3] Dils, R. R., Dynamic gas temperature measurements in a gas turbine transition duct exit, Journal of Engineering for Power, Trans. ASME, Series A, 95, No. 3, p. 265 (1973).

[4] Dils, R. R. and Follansbee, P. S., Widebandwidth gas temperature measurements in combustor exhaust gases, Instrumentation in the Aerospace in Industry, 22, Proceedings of the 22nd International Instrumentation Symposium, ISA, San Diego, CA, p. 307 (1976).

[5] Hinze, J. 0., Turbulence, Second Edition (McGraw-Hi1l, New York, 1975), chap. 2.

[6] Corrsin, S., Turbulence: experimental methods, Encyclopedia of Physics, Fluid Dynamics II, S. Flugge, ed., Vol. VIII/2, Springer Verlag, p. 555 (1963).

[7] Toong, Tan-Yi, Salant, R. F., Stopford, J. M., and Anderson, C. Y., Mechanisms of combustion instability, Tenth Symposium (International) on Combustion, The Combustion Institute, p. 1301 (1965).

[8] Gupta, A. K., Syred, N., and Beer, J. M., Fluctuating temperature and pressure effects on the noise output of swirl burners, Fifteenth Symposium (International) on Combustion, The Combustion Institute, p. 1367 (1974).

[9] Ho, C. M., Jakus, K., and Parker, J. H., Temperature fluctuations in a turbulent flame, Combustion and Flame, 27, p. 113 (1976).

[10] Hayhurst, A. N. and Kittelson, D. B., Heat and mass transfer considerations in the use of electrically heated thermocouples of iridium versus an iridium/rhodium alloy in atmospheric pressure flames, Combustion and Flame, 28, No. 3, p. 301 (1977).

[11] Eckert, E.R.G. and Drake, R. M., Heat and Mass Transfer, P. 76 (McGraw-Hi11, New York, 1959).

[12] Vranos, A., Turbulent mixing and $\mathrm{NO}_{\mathrm{x}}$ formation in gas turbine combustors, Combustion and Flame, 22, p. 253 (1974).

[13] Dils, R. R., Measurement of engine temperatures at the front plane of a JT8D-1 first stage nozzle guide vane; Part II: a nonlinear, active electronic analog of the combustion area, Pratt and Whitney Aircraft Report No. 68-038, p. 106 (November 1969). 
[14] Dils, R. R., A New Materials Test Combustor, presented at 5 th Annual Spring Meeting, Philadelphia, PA (May 1973).

[15] Powe11, R. L., Ha11, W. J., Hyink, C. H., Sparks, L. L., Burns, G. W., Scroger, M. G. , and Plumb, H. H., Thermocouple reference tables based on the IPTS-68, NBS Monograph 125, p. 47 (1974).

[16] Spalding, D. B., The art of partial modeling, Ninth Symposium (International) on Combustion, The Combustion Institute, p. 833 (1965).

[17] Lockwood, F. C. and 0didi, A. 0. 0., Measurement of mean and fluctuating temperature and ion concentration in round free-jet turbulent diffusion and premixed flames Fifteenth Symposium (International) on Combustion, p. 561, The Combustion Institute, (1974).

[18] Semerjian, H. and Vranos, A., $\mathrm{NO}_{x}$ formation in premixed turbulent flames, Sixteenth Symposium (International) on Combustion, The Combustion Institute, p. 169 (1976).

[19] Dils, R. R. and Follansbee, P. S., unpublished data.

[20] Dils, R. R., unpublished data.

[21] Materials Test Combustor, Data Sheet 2, BECON, Inc., 46 Schweir Road, South Windsor, CT 06074 .

[22] Lefebvre, A. H., Progress and problems in gas-turbine combustion, Tenth Symposium (International) on Combustion, p. 1129, The Combustion Institute (1965).

[23] Mellor, A. M., Turbulence-combustion interaction models for practical high intensity combustors, Seventeenth Symposium (International) on Combustion, The Combustion Institute, Paper No. 40, (1978). 
Appendix

Measurement of Combustor Gas Temperatures with Nonlinear Devices

The effect of the temporal and spacial gas temperature fluctuations on measurements of gas turbine combustor gas temperatures with nonlinear devices is described below. Various optical and speed of sound techniques are proposed from time to time to avoid the problems associated with inserting a probe in the harsh gas turbine combustor environment. In many cases the signal may be quite a nonlinear function of temperature. Let us consider representative techniques in which the output current is proportional to $\sqrt{\mathrm{T}}$ (speed of sound devices), $T$ (thermocouples and several Ramans/CARS techniques), $\sqrt{T^{4}}$ (black and grey body radiators), and $\exp (-2.1 \mathrm{eV} / \mathrm{kT})$ (sodium $\mathrm{D}-1$ ine reversal method). For a point analysis at $x_{j}$ within the combustor, considering only the effect of the unmixedness on signal generation at that point,

$$
I_{x_{i}}=C_{1} \int_{0}^{\infty} \rho_{x_{i}}(T) f(T) d t
$$

where

$I_{x_{i}}=$ time average signal intensity at point $x_{i}$, arbitrary units,

$C_{i}=$ normalization constant,

$\rho_{x_{i}}(T)=$ gas temperature density function at point $x_{i}$, and

$f(T)=$ functional dependence of the signal on temperature.

Again, considering only signal generation, the signal intensity measured along a line within the combustor is

$$
I=C_{2} \int_{0}^{L} I_{x_{i}}(x) d x
$$

where

$I=$ measured signal along the line $x=0$ to $x=L$, arbitrary units, and $\mathrm{C}_{2}=$ normalization constant.

In gas turbine combustors, the gas temperature waves are nonlinear, the distributions in space may be quite irregular, and, as a result, the actual values of $\rho_{x_{i}}$ (T) must be used (see figs. 9 and 10). However, a gaussian or normal density function can be used to demonstrate the effect of the unmixedness. Typical RMS values at a point and across a radius in a gas turbine combustor are $250{ }^{\circ} \mathrm{F}$ and $500^{\circ} \mathrm{F}$, respectively. $\mathrm{I}_{x_{i}}$ and $\mathrm{I}$ have been computed using these values, an average temperature of $2500^{\circ} \mathrm{F}$, a normal 
density function, and signals which are proportional to $\sqrt{T}, T, T^{4}$ and exp $(-2.1 \mathrm{ev} / \mathrm{kT})$. An example of the relative signal density functions is shown in figure $A 1$. In table $A 1$, the results are compared with a signal that would be obtained in a uniform gas stream with the same average temperature. It is observed that in most cases the errors due to the unmixedness are surprisingly small. The most sensitive measurement is the sodium D-line measurement, and even in this case, the error remains below 15 percent. Therefore, it can be concluded that in most cases the nonlinearities of signal generation will probably not limit the use of a technique for many control and diagnostic purposes. In fact, the calculations assume the investigator is unaware of the existence of the gas temperature oscillations. With prior knowledge of the oscillations, it is possible to use a compensating circuit with a sufficiently fast response time to compensate the signal for the detector nonlinearities before linearly integrating the signal, thereby avoiding the problem entirely. In general, it can be anticipated that refractive bending and nonlinear absorption of light beams by the large scale fluctuating stream tubes, in combination with the limited access and extreme mechanical vibrational fields characteristic of full scale engines, will 1 imit the optical transmission and detection in the various nonintrusive optical techniques far more than the effects of nonlinear signal generation. Optical gas temperature measuring techniques are not in use in gas turbines main burners at the present time.

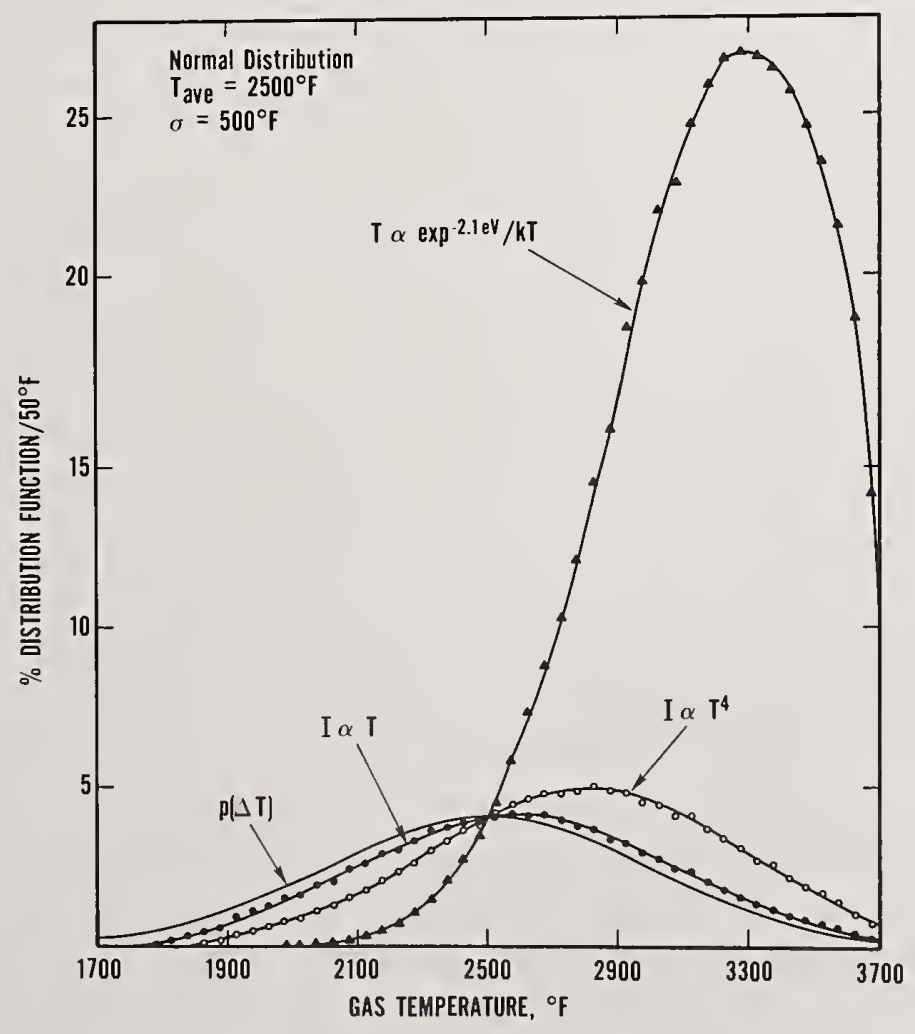

Figure A1. Example signal density functions. 
Table 1A. Comparison of signal generation errors.

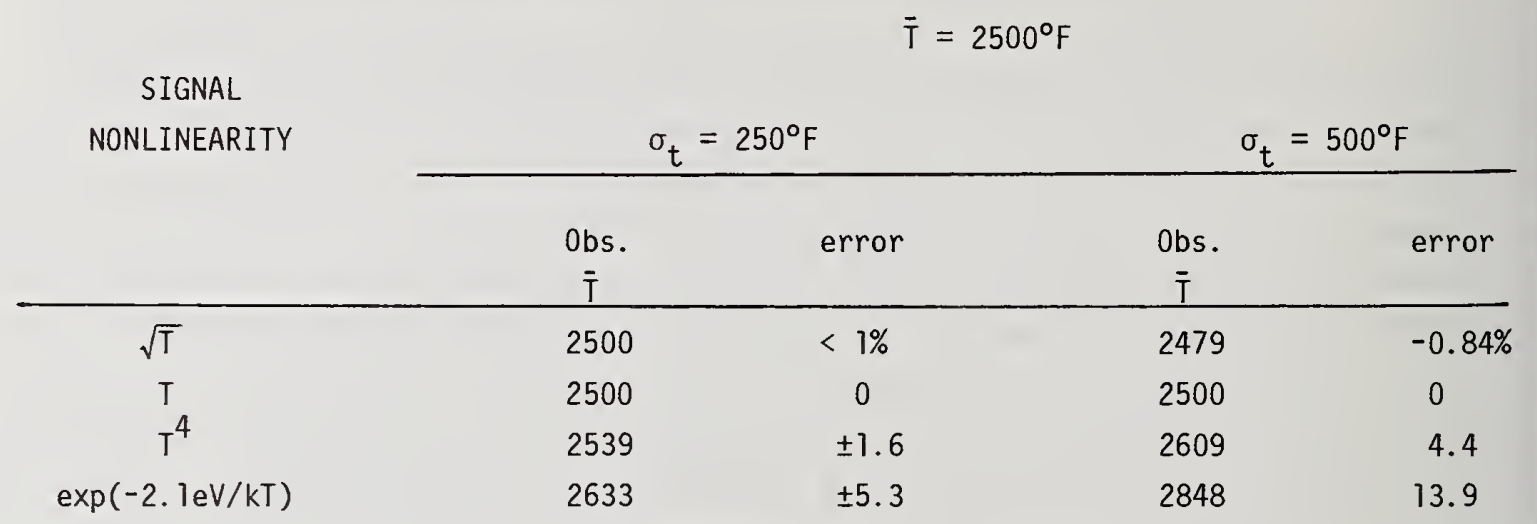

The authors wish to thank R. Beaulieu for his enthusiastic and competent assistance in performing the experiments. The experimental work was conducted at Pratt and Whitney Aircraft, Middletown, Connecticut. 
National Bureau of Standards Special Publication 561, Proceedings of the 10th Materials Research Symposium on Characterization of High Temperature Vapors and Gases held at NBS, Gaithersburg, Maryland, September 18-22, 1978. Issued October 1979.

\section{MOVING THERMOCOUPLE MEASUREMENTS OF HEAT TRANSFER IN HOT GASES ${ }^{1}$}

L. W. Hunter, C. H. Hoshal1, C. Grunfelder, and R. M. Fristrom The Johns Hopkins University

Applied Physics Laboratory Laure 1, MD 20810

A new technique was developed for measuring the convective heat transfer coefficient from a hot gas to a small diameter circular cylinder in cross flow. A thermocouple is stretched taut and drawn in the direction of its axis through the gas. The measurement can be made in the presence of radiant heating.

\section{Introduction}

An important property of a flowing high temperature gas is its convective heat transfer coefficient to a solid surface. The heating depends on the shape and size of the solid. This paper focuses on the coefficient for a cylinder. In an older technique for measuring this quantity [1-3] ${ }^{2}$, Hg flows through a stainless steel tube immersed in the hot gas. This technique has not been applied at diameters of $1 \mathrm{~mm}$ or less. However, the principle of the method can be applied at such small diameters. In a technique introduced by Hunter and Grunfelder [4], the flowing $\mathrm{Hg}$ is replaced by a moving wire thermocouple, stretched out and drawn in the direction of its axis (fig. 1). The junction is butt-welded to give it the same diameter as the lead wires. Reference [4] validates the technique and gives typical results at normal pressure. The present report describes the experimental method and apparatus in greater detail, and demonstrates results obtained at reduced pressure.

The moving thermocouple junction responds like a slug calorimeter, its temperature rising at a rate proportional to the net influx of heat. On the other hand, when radiation accompanies the convection, the net flux entering the thermocouple contains a larger convective fraction (and a smaller radiative fraction) than does the net flux entering the (typically larger) slug calorimeter.

One application of the measurements made with the moving thermocouple is to the moving wire technique (MWT) for studying polymer combustion $[5,6]$. In the MWT, a polymer of interest is coated on a supporting metal wire (or fiberglass yarn). The wire is then drawn at a controlled speed in the direction of its axis across the wake of a premixed gas burner

\footnotetext{
${ }^{1}$ Supported by the National Bureau of Standards, U.S. Department of Commerce, Grant G7-9016.
}

${ }^{2}$ Figures in brackets indicate the literature references at the end of this paper. 
flame. The technique produces a steady polymer flame which is convenient for chemical studies. A knowledge of the heat transfer coefficient facilitates calculation of the polymer surface temperature at ignition $[6,7]$.

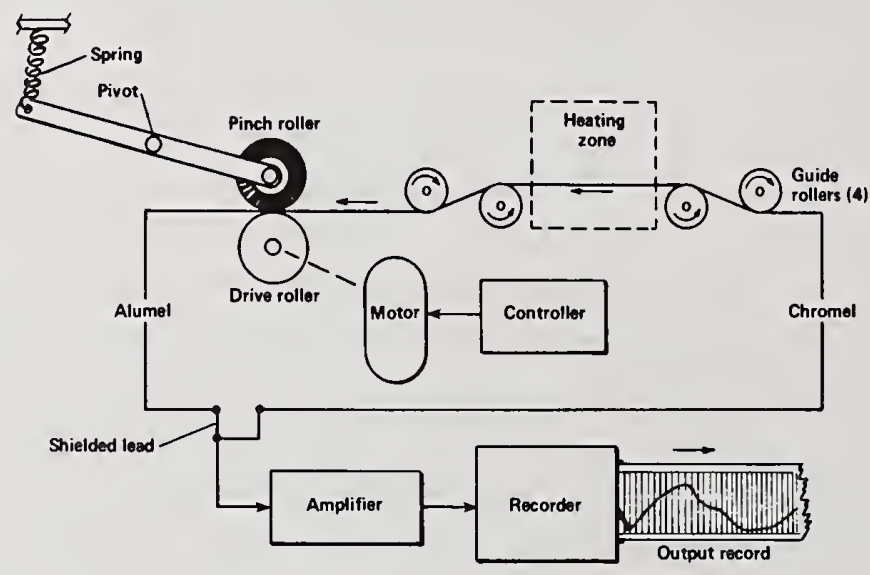

Figure 1. The basic moving thermocouple apparatus. The path of the thermocouple is oriented to follow a line of constant gas temperature as far as possible.

\section{Data Analysis}

This section summarizes the data analysis procedure and its justification developed in reference [4].

The rate of rise of the thermocouple junction temperature, $T$, determines the net influx of heat, $F$ :

$$
F=\frac{a C}{2} \frac{\partial T}{\partial t}
$$

where $a$ is the thermocouple radius, $C$ is its heat capacity, and $t$ is time.

The net influx is written as the sum of radiative and convective terms:

$$
F=H\left(T_{\text {gas }}-T\right)+\varepsilon F_{R}-\varepsilon \sigma T^{4},
$$

where $H$ is the heat transfer coefficient to be measured, $T_{\text {gas }}$ is the gas temperature, $\varepsilon$ is the emissivity, $F_{R}$ is an applied radiant influx, and $\sigma$ is the Stefan-Boltzmann constant. Equations (1) and (2) make a number of assumptions, readily met in practice, but whose validity should be checked during any measurement of $H$. Radial temperature gradients have an effect less than 1 percent when 


$$
N \equiv \frac{\mathrm{aH}}{\mathrm{k}}<0.01,
$$

where $k$ is the thermal conductivity of the wire. Longitudinal heat conduction is negligible ( $\leq 1$ percent) when the wire speed $v$ is fast enough:

$$
\mathrm{v}^{*} \equiv \frac{\mathrm{aCv}}{2 \mathrm{kN}^{1 / 2}}>5
$$

and the gas temperature is locally uniform along the path of the junction. Finally, differences in thermal properties of the two lead wires have only a small effect (usually less than 5 percent) which can be assessed by reversing the direction of the thermocouple motion.

The convective influx to a 0.010 inch diameter thermocouple is commonly from 5 to $40 \mathrm{~W}$ $\mathrm{cm}^{-2}$ (50 to $400 \mathrm{kJm}^{-2} \mathrm{~s}^{-1}$ ). By keeping $\mathrm{T}<700 \mathrm{~K}$, the radiative loss is less than $0.2 \mathrm{~W} \mathrm{\textrm {cm } ^ { 2 }}$ and can be neglected. Now consider the slope of a plot of $F$ VS $T$ :

$$
\frac{\partial F}{\partial T}=-H+\left(\frac{\partial \varepsilon}{\partial T}\right) F .
$$

A typical value of $\mathrm{H}$ is $0.3 \mathrm{kJm}^{-2} \mathrm{~s}^{-1} \mathrm{~K}^{-1}$ while $\partial \varepsilon / \partial \mathrm{T}$ is typically $1.5 \times 10^{-4} \mathrm{~K}^{-1}$. It follows that even for $F=10 \mathrm{~W} \mathrm{~cm}^{-2}\left(100 \mathrm{kJm}^{-2} \mathrm{~s}^{-1} \mathrm{~K}^{-1}\right)$, the second term is only 5 percent of the first. Hence, the second term can be neglected.

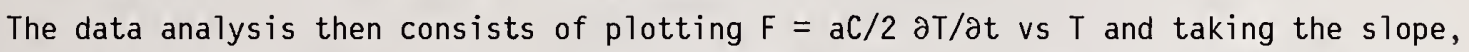
which is $-H$. The measurement does not require $T_{\text {gas }}, F_{R}$, or $\varepsilon$. The wire speed need only be estimated to check eq. (4). The only remaining question is whether the wire motion affects H. The effect of wire motion is small because gas flow speeds are usually more than an order of magnitude larger than wire speeds, and it is the relative velocity which is important.

\section{Thermocouple Junction}

The thermocouple is butt-welded to ensure uniformity of radius with the lead wires. The junction can be made with a spot welder.

The butt-welding technique for 0.010 inch diameter chromel and alumel wires is as follows. Each wire is held in a jeweler's pin vise (fig. 2). The wire is then cut with a wire cutter and withdrawn into the jaws so that less than $0.5 \mathrm{~mm}$ protrudes. The vise is tightened, but not enough to notch the wire. To remove the burr left by the cutter, the cut face is rubbed several times across abrasive paper ( 320 grit).

The spot welder ${ }^{3}$ is set to 2 joule energy. With a pin vise held in each hand, the two wires are pressed together, and the welder is energized by foot pedal. The weld should withstand reasonable tension and be well alined. Any metal flash at the weld is removed

${ }^{3}$ Weldmatic United Model 1-016-04. 
with pattern maker's files, and the junction is smoothed with fine abrasive paper (600 grit) The junction is then heated to a dull red color in the flame of a propane torch.

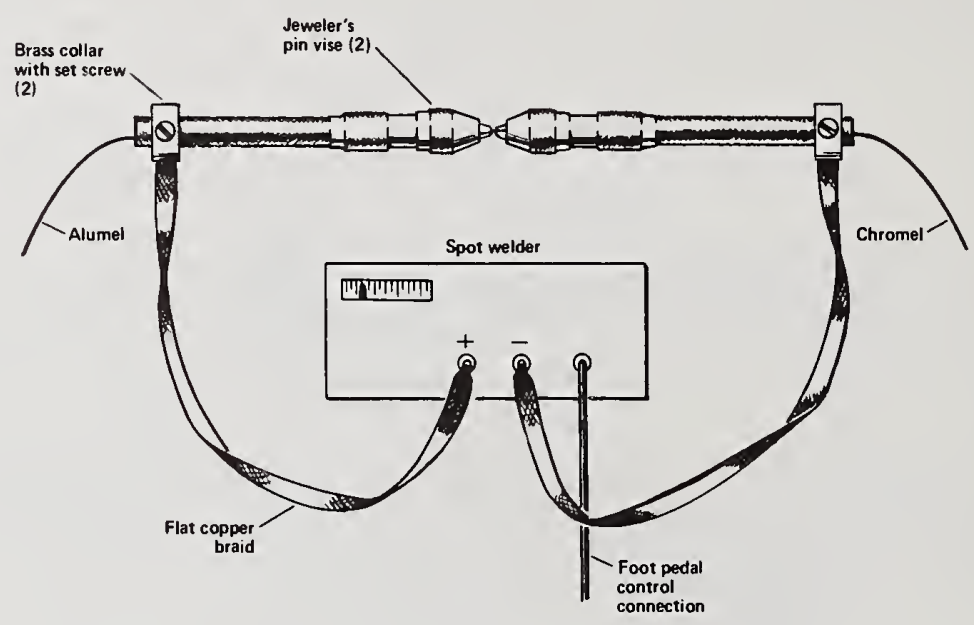

Figure 2. Butt-welding technique.

\section{Experimental Method}

One pass of the thermocouple through the hot gas is, in principle, sufficient for measuring $H$. The thermocouple temperature $T$ is recorded as it rises with time $t$. The slopes OT/at are plotted against $T$. The minimum apparatus necessary is a wire-pulling mechanism and a recording system fast enough to accurately follow the temperature rise. The minimum apparatus is described first and then a number of optional features, which are added conveniences, are described. For example, to study heat transfer in a low pressure flame, the thermocouple is best spooled in low pressure chambers connected to the combustion chamber. Also, a reversing mechanism makes it possible to keep the thermocouple in motion while adjustments to the flame are being made; the thermocouple should be in motion before the flame is ignited in case the thermocouple might melt.

The basic apparatus is shown in figure 1. The thermocouple is pulled by a drive roller turned by a controlled speed motor ${ }^{4}$. The thermocouple is guided through the heating zone by 4 grooved teflon rollers having ball bearings. The output of the thermocouple is amplified 5 and fed to a high speed (16 inch $\mathrm{s}^{-1}$ ) chart recorder ${ }^{6}$ which marks time every $0.1 \mathrm{~s}$.

Three designs of reels are convenient, depending on the application (fig. 3). The feed and take-up reels shown are unidirectional. The reversible reel uses "Negator" springs ${ }^{7}$

${ }^{4}$ G. K. Heller Motor and Controller Model T2.

${ }^{5}$ Accudata 117 DC.

${ }^{6}$ Consolidated Electrodynamics.

${ }^{7}$ Ametek/Hunter Spring Division. 
which maintain a constant tension. The end of each thermocouple wire can emerge from the side of its reel through a hole near the axis. At this point, the thermocouple wire is soldered to a stranded wire connected to the recorder. The stranded wire twists as the reel turns. This avoids a more elaborate rubbing contact.
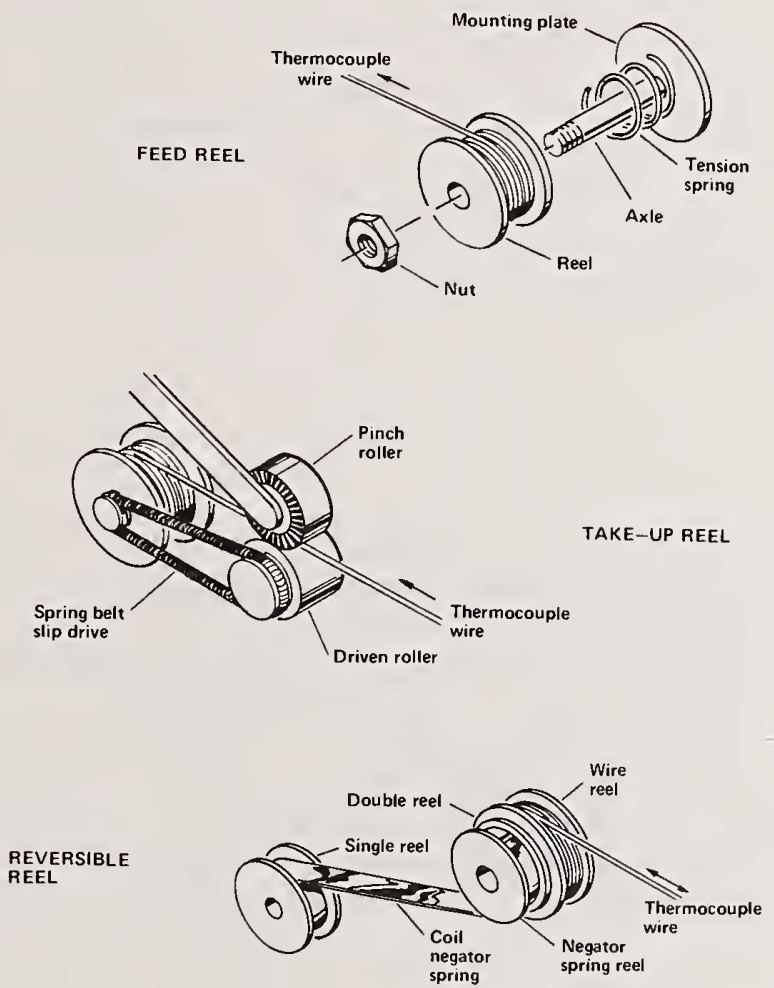

Figure 3. Reels for spooling the thermocouple in various applications.

With appropriate spools, the motor can be reversed manually. However, a good junction is invisible to the eye. Once located with a hot soldering iron, its location may be followed by means of a mechanical counter ${ }^{8}$ geared to the motor drive shaft. Alternatively, an electronic position indicator (fig. 4) can be attached to a reel. Then motor reversal can be achieved automatically, using comparator circuits which sense the potentiometer voltage. We used another method in which a square wave generator ${ }^{9}$ with offset control reversed the motor at regular time intervals. The motor controller was modified to permit remote reversal.

\section{Results}

Reference [4] presents measurements (at normal pressure) of heat transfer in hot flowing $\mathrm{N}_{2}$, in a premixed flat $\mathrm{CH}_{4}-\mathrm{O}_{2}-\mathrm{N}_{2}$ flame, and in a black body oven where convection and

${ }^{8}$ Veeder-Root.

${ }^{9}$ Krohn-Hite, Model 5700. 
radiation occurred together. Here, the flame studies are extended to reduced pressure. The present results made it possible [6] to calculate the surface temperature at ignition of a polymer whose flame structure was measured at reduced pressure.

The flame data are given in table 1 and figure 5. In figure 5, the radiant flux lost from the thermocouple was less than 2 percent of the influx at $T<900 \mathrm{~K}$. Up to this temperature, the data was grouped around straight lines which intersected the $T$-axis at the flame gas temperature. The variation in $H$, given in the first three lines of table 1 , is roughly correlated in reference [4] with the change in thermocouple radius a.

The last four lines in table 1 compare heat transfer under conditions where the Reynolds number remains constant, being on the order of $R e=1$. The observed decrease in $H$ as pressure is lowered, reflects a change in the degree of completeness of the flame reactions at the location of the thermocouple.
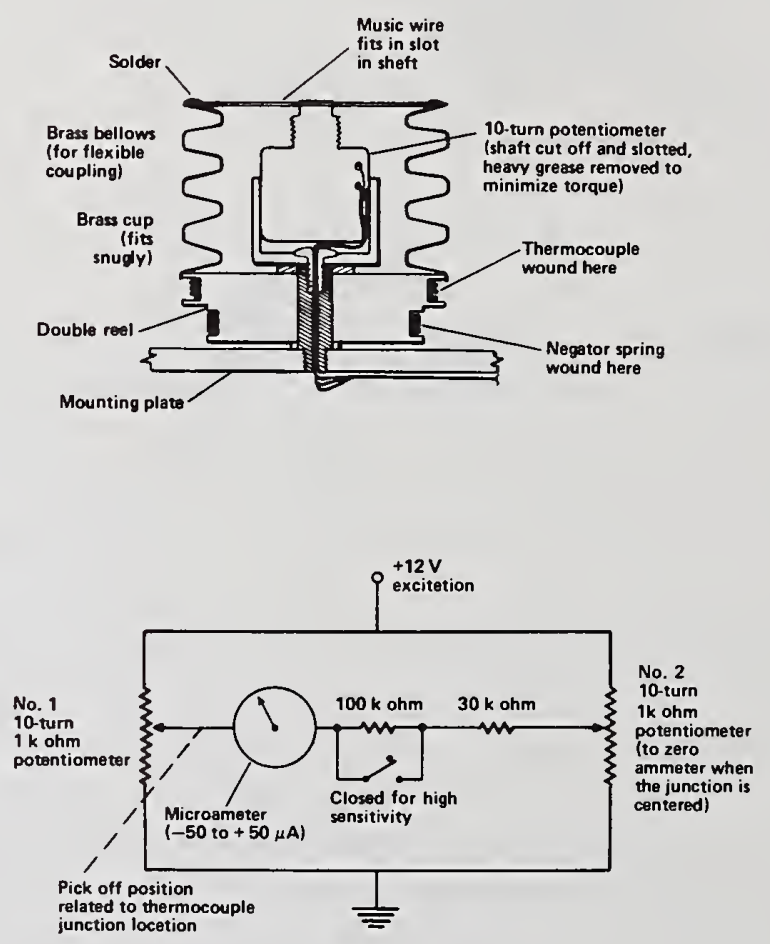

Figure 4. Electronic junction position indicator. The microammeter reads negative on one side of the heating zone, positive on the other side and zero in the middle of the zone. 
Table 1. Heat transfer in the wake of flat premixed $\mathrm{CH}_{4}-\mathrm{O}_{2}-\mathrm{N}_{2}$ flames.

\begin{tabular}{lllll} 
Pressure & $\begin{array}{c}\text { Gas } \\
\text { temperature }\end{array}$ & $\begin{array}{l}\text { Hot flow } \\
\text { velocity }\end{array}$ & $\begin{array}{l}\text { Thermocouple } \\
\text { diameter }\end{array}$ & $\begin{array}{l}\text { Heat transfer } \\
\text { coefficient }\end{array}$ \\
\hline $\begin{array}{ll}\mathrm{T}_{\text {gas }} \\
\mathrm{k}\end{array}$ & - & $2 \mathrm{a}$ & $\mathrm{H}$ \\
1.0 & 1870 & $\mathrm{~ms}^{-1}$ & $\mathrm{~mm}$ & $\mathrm{kWm}^{-2} \mathrm{~K}^{-1}$ \\
1.0 & 1870 & 0.94 & 0.13 & 0.46 \\
1.0 & 1870 & 0.94 & 0.25 & 0.27 \\
1.0 & 1870 & 0.94 & 0.38 & 0.19 \\
0.25 & $1390^{\mathrm{a}}$ & 0.95 & 0.25 & 0.27 \\
0.20 & $1350^{\mathrm{a}}$ & 0.93 & 0.25 & 0.26 \\
0.10 & $1410^{\mathrm{a}}$ & 0.97 & 0.25 & 0.26 \\
\hline
\end{tabular}

${ }^{a}$ Different $\mathrm{H}_{2} \mathrm{O}$-cooled burners were used for the normal and reduced pressure studies. The reduced pressure burner extracted more heat from the flame.

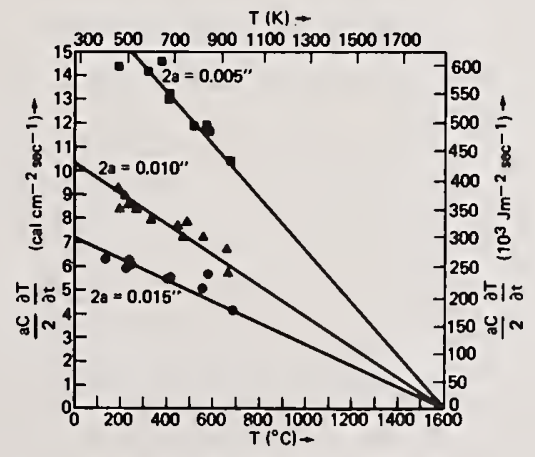

Figure 5. Flux intake by chromel-alumel thermocouples in the wake of a premixed flat $\mathrm{CH}_{4}-\mathrm{O}_{2}-\mathrm{N}_{2}$ flame at normal pressure. The diameter of the thermocouple wires is $2 \mathrm{a}$.

\section{References}

[1] Rosner, D. E., Convective Heat Transfer with Chemical Reaction, Report ARL 99 of Aeronautical Research Laboratory, Office of Aerospace Research, USAF, Wright-Patterson Air Force Base (Aug. 1961).

[2] Nettleton, H. R., On a new method of determining thermal conductivity, Proc. Physical Society of London, 22, 278 (1910).

[3] Nettleton, H. R., On the thermal conductivity of mercury by the impressed velocity method, Proc. Physical Society of London, 26, 28 (1913). 
[4] Hunter, L. W. and Grunfelder, C., Heat transfer measurements in the moving wire technique for studying polymer flammability, Comb. Flame (1978), in press.

[5] Schacke, H., Hunter, L. W., Fristrom, R. M., and Grunfelder, C., Combustion of poly (vinyl chloride) studied by the moving wire technique, in Sixteenth Symposium (International) on Combustion (The Combustion Institute, 1977); and other papers cited therein.

[6] Hunter, L. W., Grunfelder, C., Hoshal1, C. H., and Fristrom, R. M., Combustion of polyvinyl chloride studied by a low pressure moving wire technique, submitted to Comb. Flame for publication.

[7] Hunter, L. W. and Favin, S., Steady state temperature distribution in a solid cylinder moving in the direction of its axis through a cross flow of hot gas, J. Heat Transfer, 99, 668 (1977).

[8] Eckert, E. R. G. and Soehngen, E., Distribution of heat transfer coefficients around circular cylinders in cross flow at Reynolds numbers from 20 to 500 , Trans. ASME, 74, 343 (1952).

[9] Schlichting, H., Boundary Layer Theory (McGraw-Hi11, 1960). 
National Bureau of Standards Special Publication 561, Proceedings of the 10th Materials Research Symposium on Characterization of High Temperature Vapors and Gases held at NBS, Gaithersburg, Maryland, September 18-22, 1978. Issued October 1979.

\author{
APPLICATION OF THE TWO LINE ATOMIC \\ FLUORESCENCE TECHNIQUE TO THE TEMPORAL MEASUREMENT \\ OF SMALL VOLUME FLAME TEMPERATURES ${ }^{1}$
}

J. Bradshaw, J. Bower, S. Weeks ${ }^{2}$, K. Fujiwara ${ }^{3}$, N. Omenetto ${ }^{4}$, H. Haraguchi ${ }^{5}$, and J. D. Winefordner

\author{
Department of Chemistry \\ University of Florida \\ Gainesville, FL 32611
}

Temperatures of flames can be measured by a variety of techniques including line reversal, two-line emission, two-line absorption, Raman scatter, and two-line fluorescence. Of these, only the Raman scatter and the two-line fluorescence techniques give temperature information for small flame volumes without the need to deconvolute the line of sight measurements taken at various flame positions. The two-line fluorescence technique has the advantage of increased signal level and signal-to-noise ratio over the Raman scatter methods, while also maintaining immunity from scatter (observed wavelengths being either Stokes or anti-Stokes to the pump wavelength). A discussion of both continuum (xenon arc/interference filter) source and pseudo-continuum (dye-laser) source excitation methods will be presented pointing out the advantages and limitations of both approaches. A further discussion of the theoretical merits of several approaches to obtain temporal as well as spatial temperature distributions will also be presented. The information content, expected precision, and accuracy of each of the above methods will also be discussed, thereby showing where each method may be best applied.

${ }^{1}$ Work supported by Wright Patterson Air Force Base, Contract No. F33615-78-C-2038.

2Present Address: National Bureau of Standards, Analytical Chemistry Section, Gaithersburg, MD 20760.

${ }^{3}$ On Leave: Department of Chemistry, The University of Tokyo, Bunkyo-Ku, Tokyo, Japan. ${ }^{4} 0$ n Leave: Institute of Inorganic and General Chemistry, University of Pavia, Viale Taramelli 12, 27100 Pavia, Italy. 5Present Address: Department of Chemistry, The University of Tokyo, Bunkyo-Ku, Tokyo 113 , Japan. 


\section{Introduction}

A flame is a free flow of principally gaseous matter of rather high temperature. The temperature is attained through conversion of chemical energy of the initial components into mostly thermal energy of the products. In order to recognize the limitation of the concept of temperature in a flame, it is necessary to compare the actual state of the gases with the state of thermodynamic equilibrium. A volume of gas is said to be in thermodynamic equilibrium at a temperature, $T$, when this temperature, $T$, describes:

(i) the radiation density in the volume according to the Planck's radiation formula;

(ii) the distribution of energy in the internal and external degrees of freedom of the gas according to the Maxwe11-Boltzmann formula;

(iii) the degrees of ionization and dissociation according to the Saha formula and mass action law, respectively.

Only when all of these requirements have been fulfilled, has the temperature, $T$, a unique meaning. To avoid confusion, "temperatures" are named after the processes to which they apply, e.g., translational temperature, $\mathrm{T}_{\text {trans }}$, excitation temperature, $\mathrm{T}_{\text {exc }}$ (excitation can involve electronic states, $\mathrm{T}_{\text {exc,el }}$, vibrational states, $\mathrm{T}_{\text {exc, }}$ ib, and rotational states, $\mathrm{T}_{\text {exc, rot }}$ ), ionization temperature, $\mathrm{T}_{\text {ion }}$, dissociation temperature, $\mathrm{T}_{\mathrm{dis}}$, and the radiational temperature, $T_{\text {rad }}$. The translational temperature, deduced from Maxwell's formula for the velocity distribution of particles is considered the most true temperature from which others deviate, because kinetic energy is established after the fewest number of collisions, say 10 collisions (at standard pressure, this requires $\sim 10 \mathrm{~ns}$ ). On the other hand, excitation temperatures (electronic, vibrational, or rotational) may be slightly to significantly different for every type of particle [1-24]6.

It is impossible here to review thoroughly all measurement methods of flame temperature. However, the essential features of the major flame temperature measurement methods are given in table 1. The general aspects of the instrumental setup are shown in figure 1. Based upon the figures of merit given in table 1 , namely, the spatial and temporal characteristics, the excellent sensitivity and selectivity, the relatively simple instrumental setup, analytical procedure, and data interpretation, and the convenient configuration (see fig. 1), the two-line atomic fluorescence method $[23,24]$, especially with the pulsed laser $\left(\mathrm{N}_{2}\right.$-dye or $\mathrm{Nd}-\mathrm{YAG}$-dye), seems to us to be the optimum means of accurate, precise, rapid measurement of spatially, temporally resolved temperatures in laboratory flames and in combustors.

${ }^{6}$ Figures in brackets indicate the literature references at the end of this paper. 


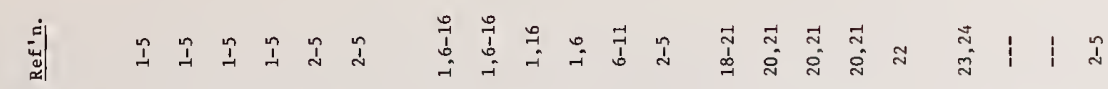

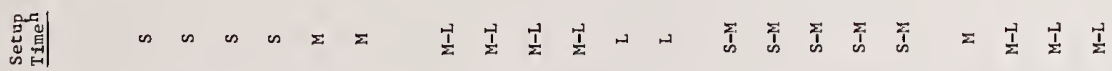

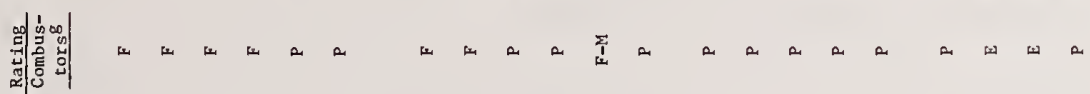

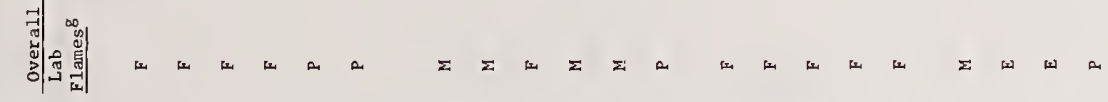

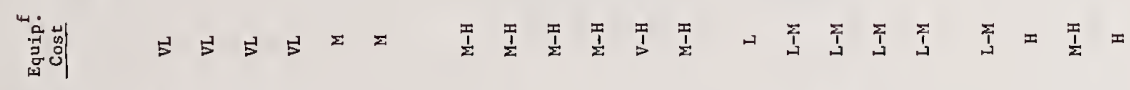

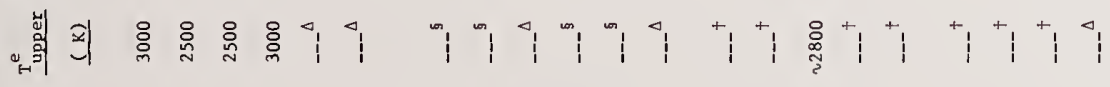

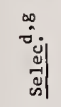

要

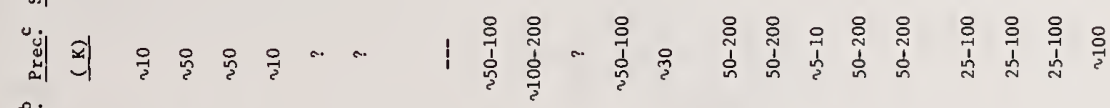
c.

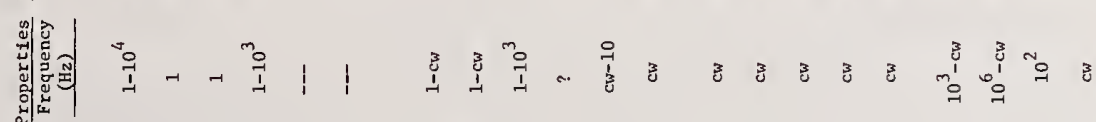

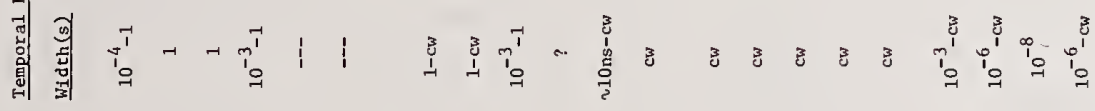

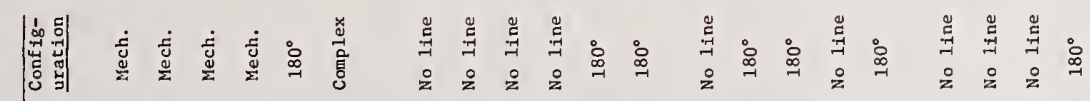

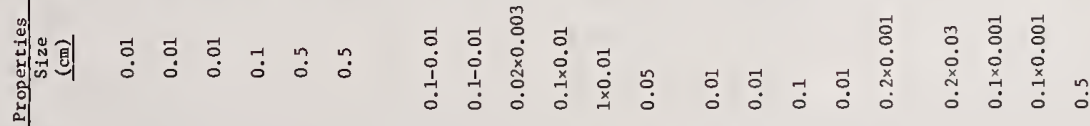

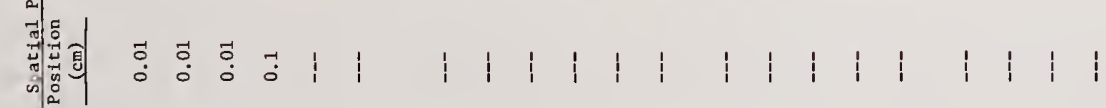

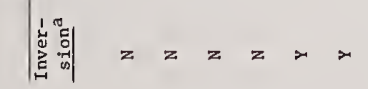

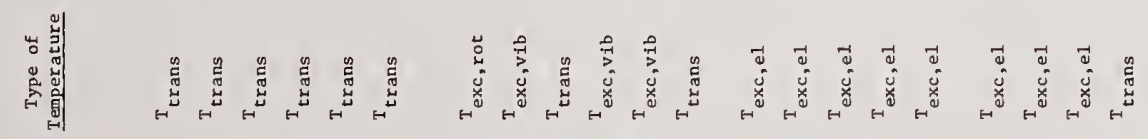

$\frac{0}{\frac{\pi}{\circ}}$
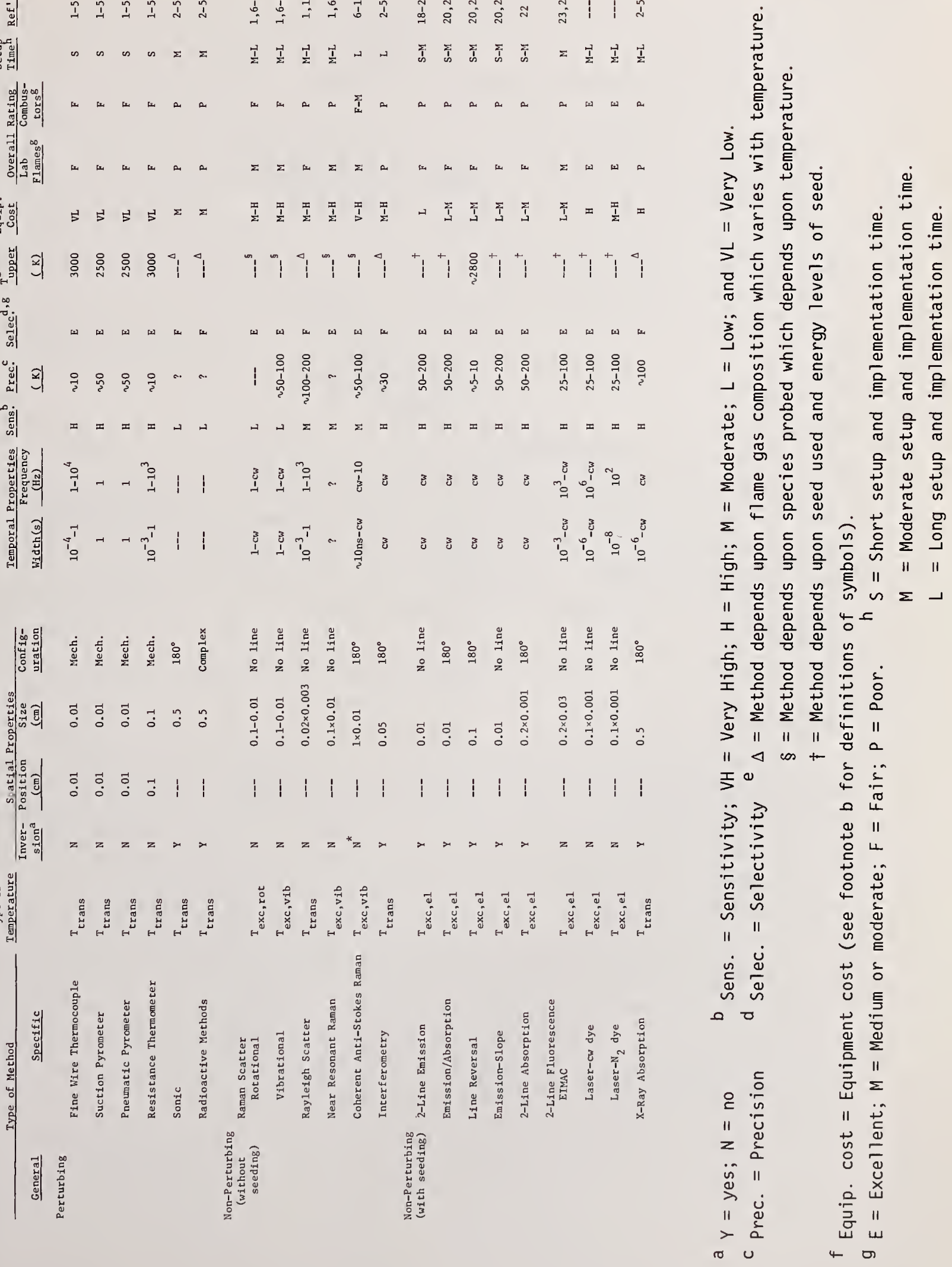

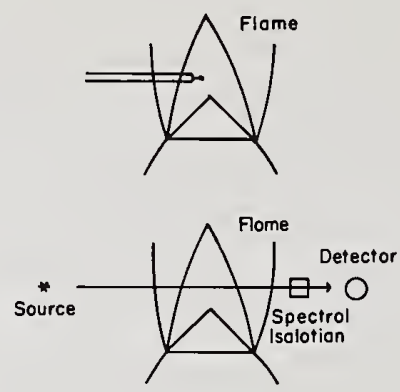

c

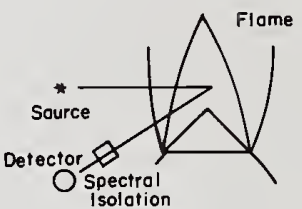

Figure 1. Schematic representation of methods: (a) Thermocouple/pyrometer devices (thermocouple, suction pyrometer, pneumatic pyrometer, resistance thermometer). (b) $180^{\circ}$ methods (sonic, CARS, absorption, two-line absorption, $x$-ray absorption, interferometry). (c) Methods with no limitations (Raman, Rayleigh, emission, two-line fluorescence).

\section{Two-Line Atomic Fluorescence Method}

\section{1 General}

The two-line atomic fluorescence method (2LF) has the advantages of increased signal level and signal-to-noise ratio over the Raman scatter methods. Therefore, the increased sensitivity and reduced noise should allow both temporal and spatial temperature information in flames to be achieved especially if laser sources are used. If the excitation source is

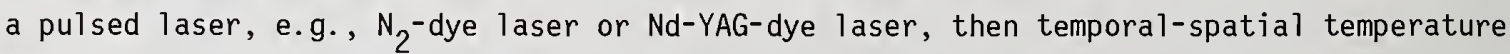
measurements should be possible on a pulse-to-pulse basis at a fairly low repetition rate. If the excitation source is a modulated $\mathrm{CW} \mathrm{Kr}$-dye laser, then temporal-spatial temperature measurements should be possible at a much higher repetition rate, but on a longer time scale, assuming adequate computer facilities are available. A comparison of the temporal-spatial characteristics of the 2LF method for several different sources are given in table 2. In our laboratory, we are in the process of investigating the $\mathrm{N}_{2}$-dual dye lasers-filter system for temporal-spatial flame temperature measurements. 


\subsection{Basis of $2 \mathrm{LF}$ method}

The 2LF method involves excitation of an atomic probe, e.g., $\mathrm{Pb}$, In, $\mathrm{Tl}, \mathrm{Ga}$, etc., which have low metastable electronic levels (three-level systems), in the flame (see table 3 for their atomic characteristics). Excitation is performed at two wavelengths (see fig. 2). First excitation is at $\lambda_{02}$ (spectral irradiance, $\mathrm{J} \mathrm{s}^{-1} \mathrm{~m}^{-2} \mathrm{~nm}^{-1}$, of excitation $=E_{\lambda_{02}}$ ) corresponding to excitation of the upper most state (2) via the ground state (0) and measurement of the fluorescence occurring at $\lambda_{21}$ (irradiance, $\mathrm{J} \mathrm{s}^{-1} \mathrm{~m}^{-2}$, of fluorescence $=$ $E_{F}\left(\lambda_{21}\right)$ ). Also, excitation is at $\lambda_{12}$ (spectral irradiance of excitation $=E_{\lambda_{12}}$ ) corresponding to excitation of the upper most, 2 , state via the metastable state, 1 , state, and

Table 2. Comparison of several excitation sources for two-line atomic fluorescence temperature measurements.

\begin{tabular}{|c|c|c|c|c|}
\hline \multirow[t]{2}{*}{ Source-Optics } & \multirow{2}{*}{$\begin{array}{l}\text { Spatial } \\
(\operatorname{mm} \times m m \times m m)\end{array}$} & \multicolumn{2}{|c|}{ Temporal } & \multirow{2}{*}{$\operatorname{Cos} t^{a, d}$} \\
\hline & & Pulse Width(s) & Frequency (Hz) & \\
\hline \multirow{3}{*}{$\begin{array}{l}\text { EIMAC Xenon (150 W) } \\
\text { Arc Lamp-Filters }\end{array}$} & & & & \\
\hline & $0.3 \times 2 \times 5$ & $\mathrm{cw}$ & $\mathrm{Cw}$ & $\sim \$ 6,000$ \\
\hline & $0.3 \times 2 \times 5$ & $0.001^{b}$ & 500 & 10,000 \\
\hline $\begin{array}{l}\mathrm{N}_{2} \text {-dual dye lasers- } \\
\text { filters } \\
(5 \mathrm{~ns}, 50 \mathrm{~Hz}) \\
(1 \mu \mathrm{s} \text { delay between } \\
\text { pulses) }\end{array}$ & $<0.3 \times 0.3 \times 0.3$ & $<10^{-7}$ & 50 & $\sim \$ 25,000$ \\
\hline $\begin{array}{l}\text { Nd-YAG-dual dye } \\
\text { lasers-Filters } \\
(10 \mathrm{~ns}, 510 \mathrm{kHz})\end{array}$ & $<0.3 \times 0.3 \times 0.3$ & $<10^{-6}$ & $>10^{4}$ & $\sim \$ 60,000^{c}$ \\
\hline $\begin{array}{l}\text { Kr-dual modulated } \\
\text { dye lasers-Filters }\end{array}$ & $<0.3 \times 0.3 \times 0.3$ & $10^{-3}$ & $10^{3}$ & $v \$ 50,000^{c}$ \\
\hline
\end{tabular}

a Cost does not include computer facilities.

b Mechanical amplitude modulation.

C Computer cost due to high data rate will be much greater than for $\mathrm{N}_{2}$-dual dye laser system.

d A typical CARS system costs $\$ 100,000$ according to Eckbreth [6].

measurement of the fluorescence occurring at $\lambda_{20}$ (irradiance of fluorescence $=E_{F}\left(\lambda_{20}\right)$ ). The former case [25] is an example of Stokes type direct line fluorescence, SDLF, and the latter case [25] is an example of Anti-Stokes type direct line fluorescence, ASDLF. By measuring Stokes and anti-Stokes direct line fluorescence, scatter is minimized even for sooty flames. 
Table 3. Characteristics of two-line fluorescence seeds $[23,24]$

$\begin{array}{cccc}\text { ATOM } & \begin{array}{c}\text { METASTABLE LEVEL } \\ \text { ENERGY, } \mathrm{V}_{1}, \mathrm{eV}\end{array} & \lambda_{02}(\mathrm{~nm}) & \lambda_{12}(\mathrm{~nm}) \\ \mathrm{Ga} & 0.109 & 417.2 & 403.3 \\ \mathrm{In} & 0.274 & 451.1 & 410.2 \\ \mathrm{~T} 1 & 0.966 & 535.0 & 377.6 \\ \mathrm{~Pb} & 1.32 & 405.8 & 283.3\end{array}$

a. SDLF

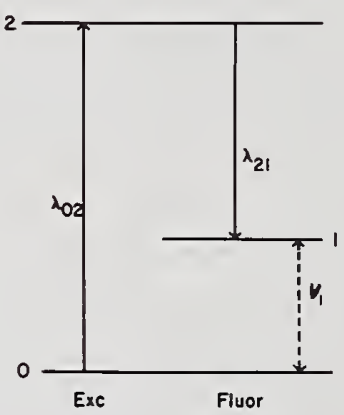

b. ASDLF

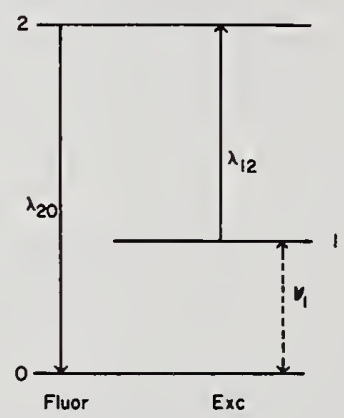

Figure 2. Schematic representation of two-line atomic fluorescence method for flame temperature measurement: (a) Stokes type direct line fluorescence (SDLF), (b) Anti-Stokes type direct line fluorescence (ASDLF).

Assuming steady state excitation-fluorescence is achieved (as long as the excitation pulse is $\widetilde{>}_{5} \mathrm{~ns}$, steady state conditions should be achieved for atoms in combustion flames [26], and assuming low intensity excitation conditions, i.e., not saturation [27], then the expression for the flame temperature, $T$, is given by 


$$
T=\frac{5040 v_{1}}{\log \left(\frac{E_{\lambda_{12}}}{E_{\lambda_{02}}}\right)+6 \log \left(\frac{\lambda_{12}}{\lambda_{02}}\right)+\log \left(\frac{E_{F}\left(\lambda_{21}\right)}{E_{F}\left(\lambda_{20}\right)}\right)}
$$

where $V_{1}$ is the excitation energy, in eV, of the metastable state (see fig. 2) and all other terms have been defined. Because $V_{1}, \lambda_{12}$, and $\lambda_{02}$ are known precisely and accurately and because $E_{\lambda_{12}} / E_{\lambda_{02}}$ can be measured from calibration of the optical system by means of a standard reference source, then $T$ can be measured (corresponding to the temporal and spatial coordinates) by measuring the fluorescence ratio $E_{F}\left(\lambda_{21}\right) / E_{F}\left(\lambda_{20}\right)$, using the optical calibration of the optical measurement system. The temperature ranges possible via the atomic probes, $\mathrm{Pb}, \mathrm{In}, \mathrm{Ga}$, and $\mathrm{Tl}$ are shown in figure 3 for excitation with an Eimac xenon arc lamp.

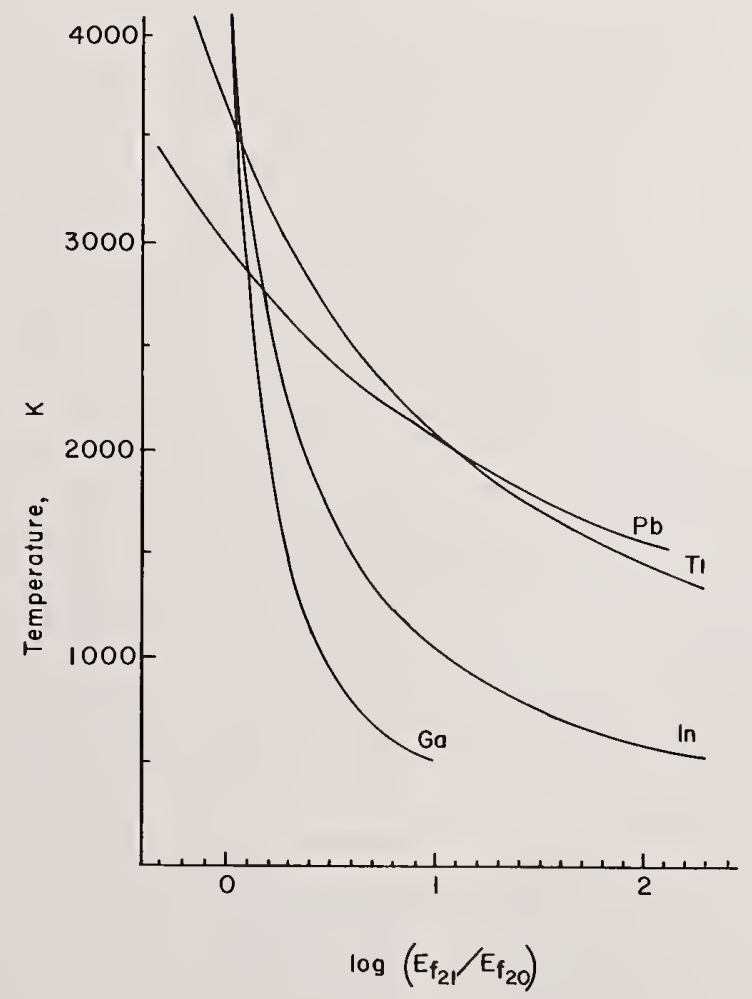

Figure 3. Variation of $E_{F}\left(\lambda_{21}\right) / E_{F}\left(\lambda_{20}\right)$ with flame temperature for $P b, I n, G a$, and T1. $\left[V_{1}(T 1)=0.966 \mathrm{eV} ; V_{1}(P b)=1.32 \mathrm{eV} ; V_{1}(I n)=0.274 \mathrm{eV}\right.$; and $V_{1}(G a)=0.109$ eV] $E_{\lambda_{12}} / E_{\lambda_{02}}$ taken for an Eimac xenon arc light source (this ratio will vary the slope of $T_{u s} \log E_{F_{21}} / E_{F_{20}}$ ). 
The error in the measured flame temperature can be estimated from the $T$ expression above, assuming $a 11$ errors are random and reside in the estimates of the "intensity" ratios, i.e.,

$$
s_{T}^{2}=\left[\frac{\partial T}{\partial E_{S_{\lambda}}}\right]^{2} s^{2} E_{S_{\lambda}}+\left[\frac{\partial T}{\partial E_{F}}\right]^{2} s^{2} E_{F}
$$

where $\mathrm{s}_{\mathrm{T}}$ corresponds to the standard deviation in the flame temperature, ${ }^{\mathrm{S}_{\mathrm{S}}}$ corresponds to the standard deviation in the source spectral irradiance ratio, $E_{S \lambda}=E_{\lambda_{12}} / E_{\lambda_{02}}$, ${ }^{S} E_{F}$ corresponds to the standard deviation in the fluorescence irradiance ratio, $E_{F}=E_{F}\left(\lambda_{21}\right) /$ $E_{F}\left(\lambda_{20}\right)$, and the partial derivatives correspond to the sensitivities of flame temperature witr respect to source spectral irradiance and fluorescence irradiance changes. Solving for $s^{2} T$ and assuming the major error is contributed by the random error in measuring the fluorescence irradiance ratio, then

$$
s_{T}^{2}=T^{2}\left[\frac{\partial T}{T}\right]^{2}=T^{2}\left\{\left(\frac{0.434}{5040 V_{1}}\right)^{2} T^{2}\left[\frac{\partial E_{F}}{E_{F}}\right]^{2}\right\}
$$

The absolute and percentage errors in temperatures produced by random errors in $E_{F}$ for In, $\mathrm{Ga}, \mathrm{Tl}$ and $\mathrm{Pb}$ are given in table 4 . As can be noted, the temperature relative errors witl Ga are too large for diagnostic purposes, e.g., a 2-percent error in $E_{F}$ results in a 146 , 82 , and $36 \mathrm{~K}$ errors for flames of temperatures 2000 , 1500 , and $1000 \mathrm{~K}$, respectively. On the other hand, the relative temperature errors for $\mathrm{Pb}$ and $\mathrm{Tl}$ are low and similar in size.

Several other limitations must also be kept in mind when using the $2 \mathrm{LF}$ method for flame temperature measurements: (i) $\mathrm{Tl}$ and $\mathrm{Pb}$ cannot be used for the determination of temperatures below $\sim 1400 \mathrm{~K}$ because the relative error in $\mathrm{T}$ asymptotically approaches large values (see fig. 3); and (ii) the accurate-precise measurement of $E_{F}$ is difficult when studying flames with a wide temperature range, i.e., for best results the ratio $E_{F}\left(\lambda_{21}\right) / E_{F}\left(\lambda_{20}\right)$ should vary between about 5 and about 50 . Therefore, the optimum seed for flames are as follows:

$$
\begin{array}{rr}
\text { (i) Ga: } & \sim 500-1000 \mathrm{~K} \\
\text { (ii) In: } & \sim 800-1700 \mathrm{~K} \\
\text { (iii) Tl: } & \sim 1400-3500 \mathrm{~K} \\
\text { (iv) Pb: } & \sim 1400-3000 \mathrm{~K}
\end{array}
$$

It should be pointed out that random errors increase for measurements of flame temperatures near the flame fringes (approaches room temperature) and near the flame center in some cases (e.g., use of In with hot flames as $\mathrm{C}_{2} \mathrm{H}_{2} / \mathrm{N}_{2} \mathrm{O}$ ). Near the flame fringes where temperatures are lower, the $\mathrm{S} / \mathrm{N}$ for the ASDLF component is poor when using $\mathrm{Pb}$ or $\mathrm{Tl}$ as a seed, but is 
Table 4. Errors in flame temperature produced by errors in measured fluorescence intensity ratios for indium, [24] gallium, [24] thallium, and lead.

$00 \Delta E_{F} / E_{F}, \%$

$\Delta \mathrm{T}, \mathrm{K}(100 \Delta \mathrm{T} / \mathrm{T}, \%)$

$$
\text { I. Ga }\left(V_{1}=0.109 \mathrm{eV}\right)
$$

$2000 \mathrm{~K}$

$1500 \mathrm{~K}$

$1000 \mathrm{~K}$

$500 \mathrm{~K}$

\begin{tabular}{rrrrrrrr}
\hline 316 & $(15.8)$ & 177 & $(11.8)$ & 79 & $(7.9)$ & 20 & $(4.0)$ \\
158 & $(7.9)$ & 87 & $(6.4)$ & 39 & $(4.0)$ & 10 & $(2.0)$ \\
126 & $(6.4)$ & 71 & $(4.7)$ & 32 & $(3.2)$ & 9 & $(1.7)$ \\
95 & $(4.7)$ & 53 & $(3.6)$ & 24 & $(2.4)$ & 6 & $(1.2)$ \\
63 & $(3.2)$ & 36 & $(2.4)$ & 16 & $(1.6)$ & 4 & $(0.9)$ \\
32 & $(1.6)$ & 18 & $(1.2)$ & 8 & $(0.8)$ & 2 & $(0.5)$
\end{tabular}

II. In $\left(V_{1}=0.274 \mathrm{eV}\right)$

$2500 \mathrm{~K}$

$2000 \mathrm{~K}$

$1500 \mathrm{~K}$

$1000 \mathrm{~K}$

\begin{tabular}{rrrrrrrr}
\hline 197 & $(8.0)$ & 127 & $(6.4)$ & 71 & $(4.7)$ & 32 & $(3.3)$ \\
98 & $(4.0)$ & 63 & $(3.2)$ & 35 & $(2.4)$ & 16 & $(1.6)$ \\
79 & $(3.3)$ & 50 & $(2.5)$ & 28 & $(1.8)$ & 13 & $(1.3)$ \\
59 & $(2.5)$ & 38 & $(1.9)$ & 21 & $(1.4)$ & 10 & $(1.0)$ \\
39 & $(1.6)$ & 25 & $(1.3)$ & 14 & $(1.0)$ & 6 & $(0.6)$ \\
19 & $(0.8)$ & 13 & $(0.7)$ & 7 & $(0.5)$ & 3 & $(0.3)$
\end{tabular}

III. TI $\left(V_{1}=0.966 \mathrm{eV}\right)$

$3000 \mathrm{~K}$

$2500 \mathrm{~K}$

$2000 \mathrm{~K}$

$1500 \mathrm{~K}$

\begin{tabular}{rrrrrrrr}
\hline 80 & $(2.7)$ & 56 & $(2.2)$ & 36 & $(1.8)$ & 20 & $(1.4)$ \\
40 & $(1.4)$ & 28 & $(1.1)$ & 18 & $(0.9)$ & 10 & $(0.7)$ \\
32 & $(1.0)$ & 22 & $(0.9)$ & 14 & $(0.7)$ & 8 & $(0.6)$ \\
24 & $(0.8)$ & 17 & $(0.7)$ & 11 & $(0.6)$ & 6 & $(0.4)$ \\
16 & $(0.5)$ & 11 & $(0.4)$ & 7 & $(0.4)$ & 4 & $(0.3)$ \\
8 & $(0.3)$ & 5 & $(0.2)$ & 4 & $(0.2)$ & 2 & $(0.2)$
\end{tabular}

IV. $\mathrm{Pb}\left(\mathrm{V}_{1}=1.32 \mathrm{eV}\right)$

$3500 \mathrm{~K}$

$3000 \mathrm{~K}$

$2500 \mathrm{~K}$

$2000 k$

\begin{tabular}{rrrrrrrr}
\hline 80 & $(2.3)$ & 59 & $(2.0)$ & 41 & $(1.6)$ & 26 & $(1.4)$ \\
40 & $(1.1)$ & 30 & $(1.0)$ & 21 & $(0.8)$ & 13 & $(0.7)$ \\
32 & $(0.9)$ & 24 & $(0.8)$ & 16 & $(0.7)$ & 11 & $(0.6)$ \\
24 & $(0.7)$ & 19 & $(0.6)$ & 13 & $(0.5)$ & 8 & $(0.4)$ \\
16 & $(0.4)$ & 12 & $(0.4)$ & 8 & $(0.4)$ & 6 & $(0.3)$ \\
8 & $(0.2)$ & 6 & $(0.2)$ & 4 & $(0.2)$ & 3 & $(0.2)$
\end{tabular}


considerably increased when using In or especially Ga. On the other hand, in the center of a hot flame $(T \sim 2500 \mathrm{~K})$, the $\mathrm{S} / \mathrm{N}$ for the ASDLF component is good, but the relative errors due to variation in the fluorescence ratio are large for In and especially Ga but much lower for $\mathrm{Pb}$ or $\mathrm{Tl}$.

\subsection{Experimental systems}

The experimental system used by Haraguchi, et a]. $[24,28,30]$ is shown in figure 4 . Because of the low intensity output of the EIMAC-xenon arc lamp over the excitation lines used in the $2 \mathrm{LF}$ method, the local measured volume was limited to $0.3 \mathrm{~mm} \times 2 \mathrm{~mm} \times 5 \mathrm{~mm}$ (refer to figs. 5 and 6 ). Even so, poor $S / N$ ratios (refer to fig. 6) resulted in marginally reliable results near the flame fringes, especially when using $T 1$ as the seed in the lower temperature $\mathrm{H}_{2}$-based flames and in the flame center of hot flames, e.g., $\mathrm{C}_{2} \mathrm{H}_{2}-\mathrm{N}_{2} \mathrm{O}$, when using $\mathrm{T} 1$ or In as the seed (refer to figs. 5 and 6 ). In the Appendix and in table 5, information is given as to the experimental conditions related to the seed and the expected signal levels. It should be stressed that the signal levels (see table 5 ) in the two-line fluorescence method are many orders of magnitude greater than with the Raman methods which simplify the instrumental requirements and improve the precision and accuracy of the spatialtemporal measurements of flame temperatures.

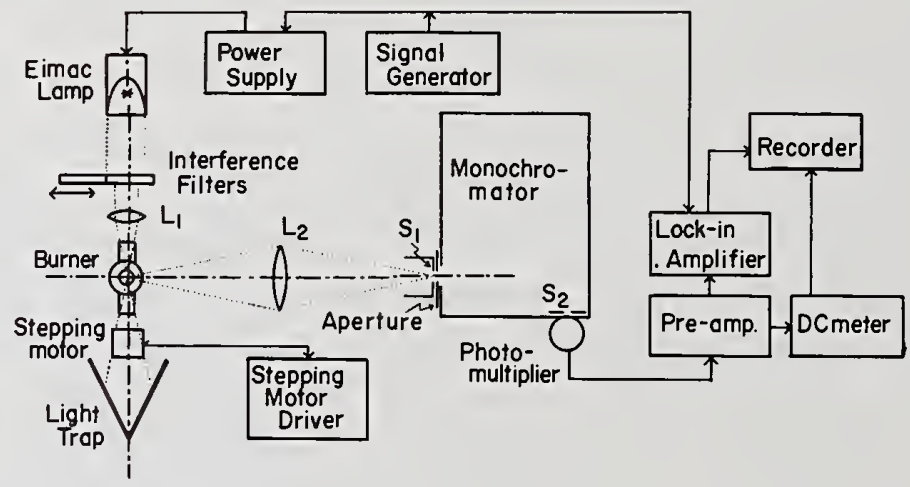

Figure 4. Experimental system for local two-line atomic fluorescence temperature measurements of flames as described by $H$. Haraguchi, et al. [24]. 


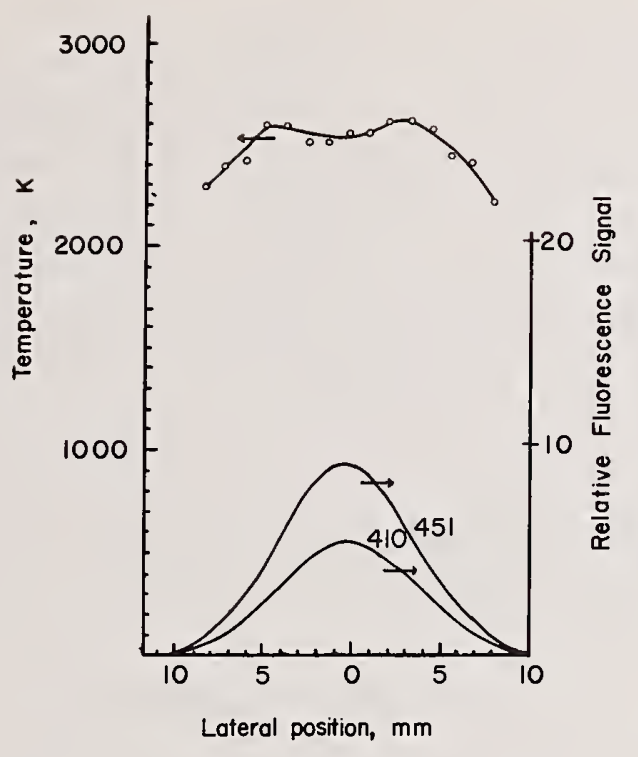

Figure 5. Temperature profile of air-acetylene flame and atomic fluorescence intensity profiles of In at 410.18 and $451.13 \mathrm{~nm}$. Experimental conditions: flame height above burner head: $32 \mathrm{~mm}$; Airflow rate: $8.25 \mathrm{Lmin}^{-1}$; Acetylene flow rate: $1.15 \mathrm{Lmin}^{-1}$. Arrows point to proper axes.

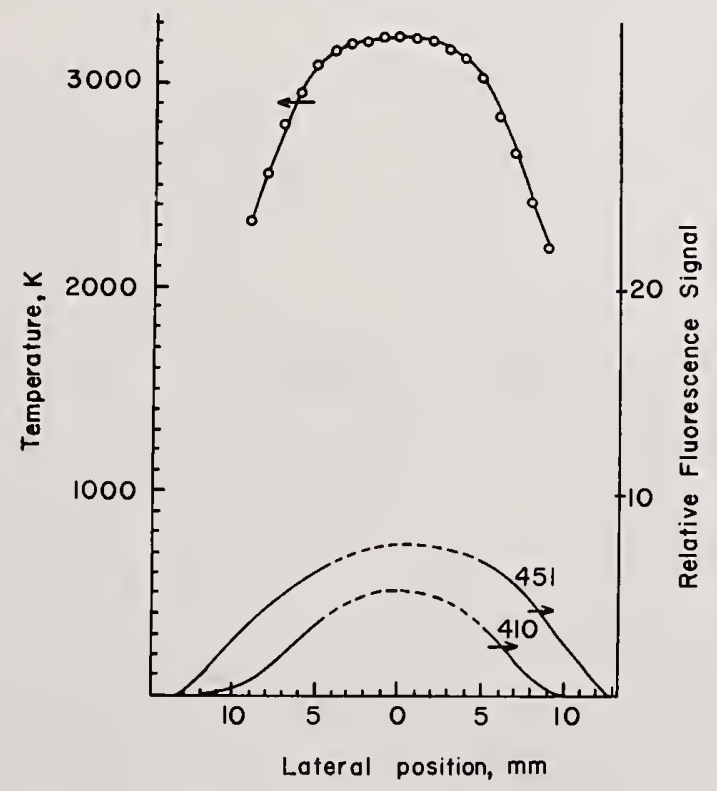

Figure 6. Temperature profile of argon-separated nitrous oxide-acetylene flame and atomic fluorescence intensity profiles of In at 410.18 and $451.13 \mathrm{~nm}$. Experimental conditions: flame height: $24 \mathrm{~mm}$; Nitrous oxide flow rate: ca. $8.0 \mathrm{~L} \mathrm{~min}^{-1}$; Acetylene flow rate: $2.5 \mathrm{Lmin}^{-1}$; Argon flow rate: ca. $10 \mathrm{Lmin}^{-1}$. Arrows point to proper axes. 
The experimental systems proposed for accurate, precise, rapid spatial-temporal measurements are shown in figures 7 and 8 . In figure 7 , a single $\mathrm{N}_{2}-1$ aser pump ( $\left.>400 \mathrm{~kW}\right)$ will be used to pump two-dye lasers (one at $\lambda_{02}$ and one at $\lambda_{12}$ ) which are temporally delayed by $\sim 10$ ns with respect to their pulses (the pulsing order will be to pump the $\lambda_{12}$ line first and then $10 \mathrm{~ns}$ later to pump the $\lambda_{02}$ line in order to avoid any changes in the steady state ground state atom population ( $I n$ and $T 1$ will be used as seeds)). By means of two photomultiplier detectors with interference filters centered at $\lambda_{20}$ and $\lambda_{21}$, respectively, and a ratio boxcar (gated detector) integrator to obtain the fluorescence ratio for each set of successive pulses and with a computer interface to a minicomputer system, flame temperatures for each submicrosecond time interval (each pulse will be $\sim 5$ ns FWHM but will be separated by $\sim 10 \mathrm{~ns}$ ) will be obtained at a repetition rate of $\sim 50 \mathrm{~Hz}$. With photomultiplier detection, temperature measurements will be made on a point-by-point (temporal and spatial points) basis.

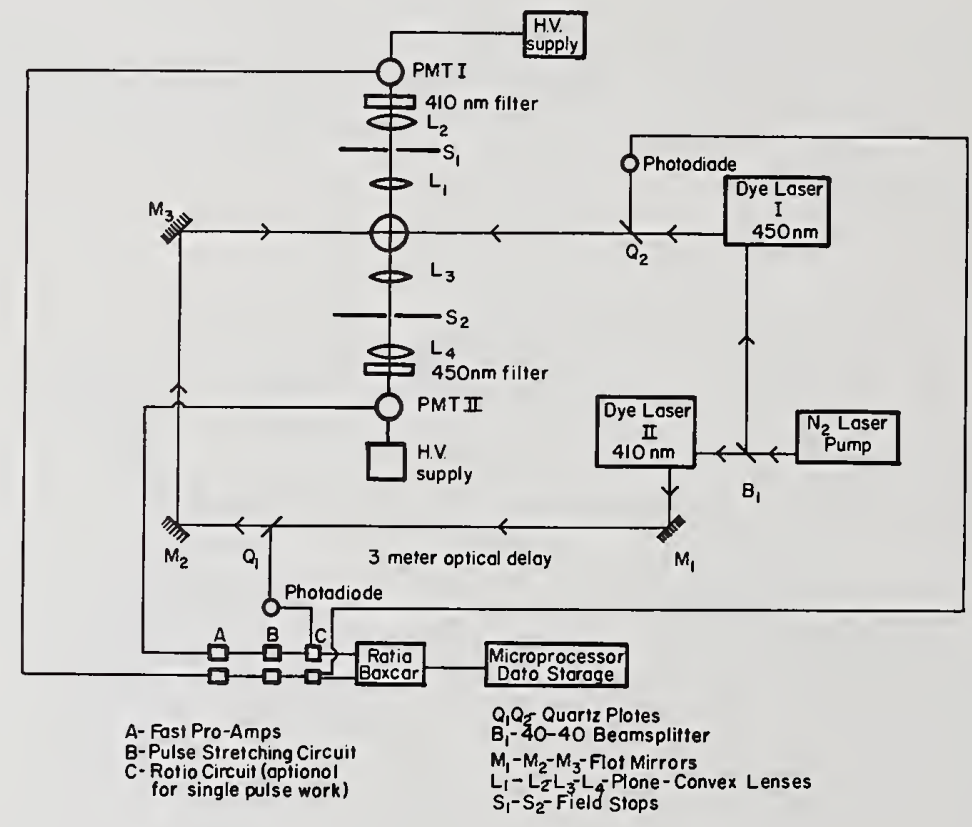

Figure 7. Proposed experimental system for spatial-temporal temperature measurements using the $\mathrm{N}_{2}$-pumped dual dye laser system and two photo multiplier detectors for the atomic fluorescence two-line method. 


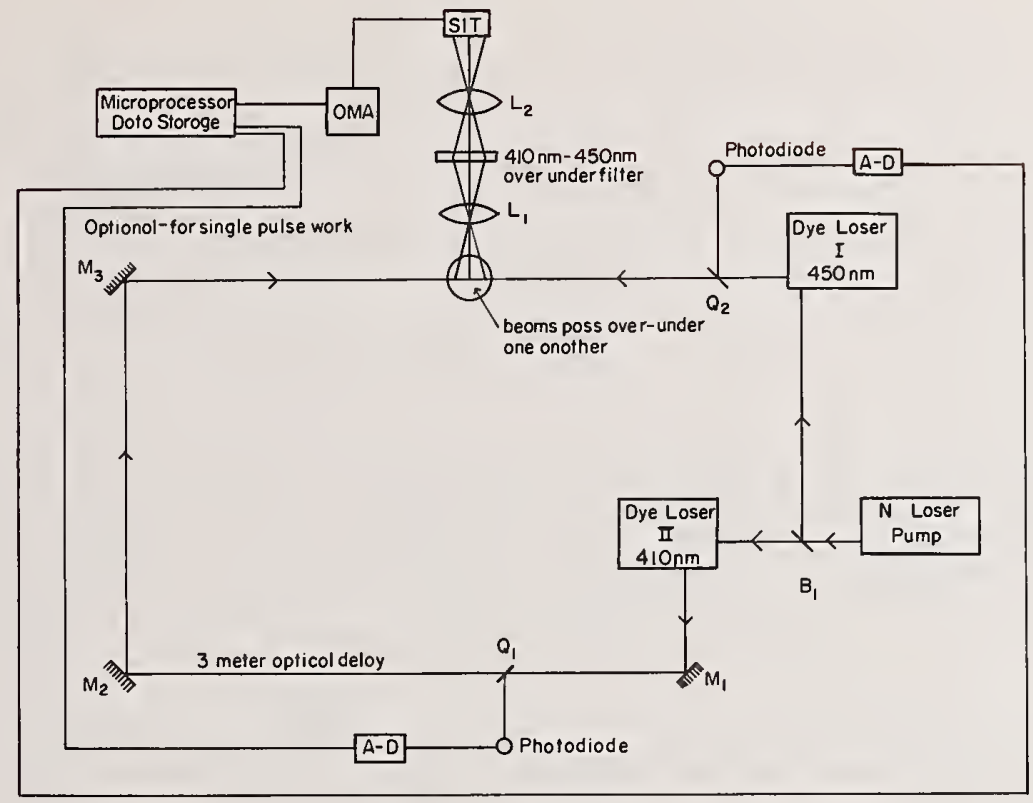

Figure 8. Proposed experimental system for spatial-temporal temperature measurements using the $\mathrm{N}_{2}$-pumped dual dye laser system and an SIT-Vidicon detector for the atomic fluorescence two-line method.

In figure 8, a more sophisticated 2LF system with a SIT detector and an under-over filter system should allow both the SDLF and the ASDLF lines to be measured simultaneously. With a SIT detector (an array of 512 diodes, $12.5 \mathrm{~mm}$ in length) and two interference filters, an array of flame temperature measurements across any flame region can be made as long as the flame region to be studied is parallel to the spectrometer entrance slit and is imaged upon it. In the case of the SIT (an under-over filter system will be used so that the SDLF line will be on one-half of the SIT face and the ASDLF line will be on the other half--thus each channel of the 512 diodes--12.5 mm long--will represent a different vertical (or horizontal, if a dove prism is used) region of the flame. However, since the scan time of the OMA (optical multichannel analyzer) is $32.8 \mathrm{~ms}$, the data rate must be reduced to $\widetilde{<} 30 \mathrm{~Hz}$. Such a system will require great amounts of computer core memory to handle the high data rate. Nevertheless, spatial temperatures corresponding to $\sim 500$ various vertical and/or horizontal segments of the flame could be measured at a rate of $\sim 30 \mathrm{~s}^{-1}$ (with temporal resolution of $\ll 1 \mu s$ ). 


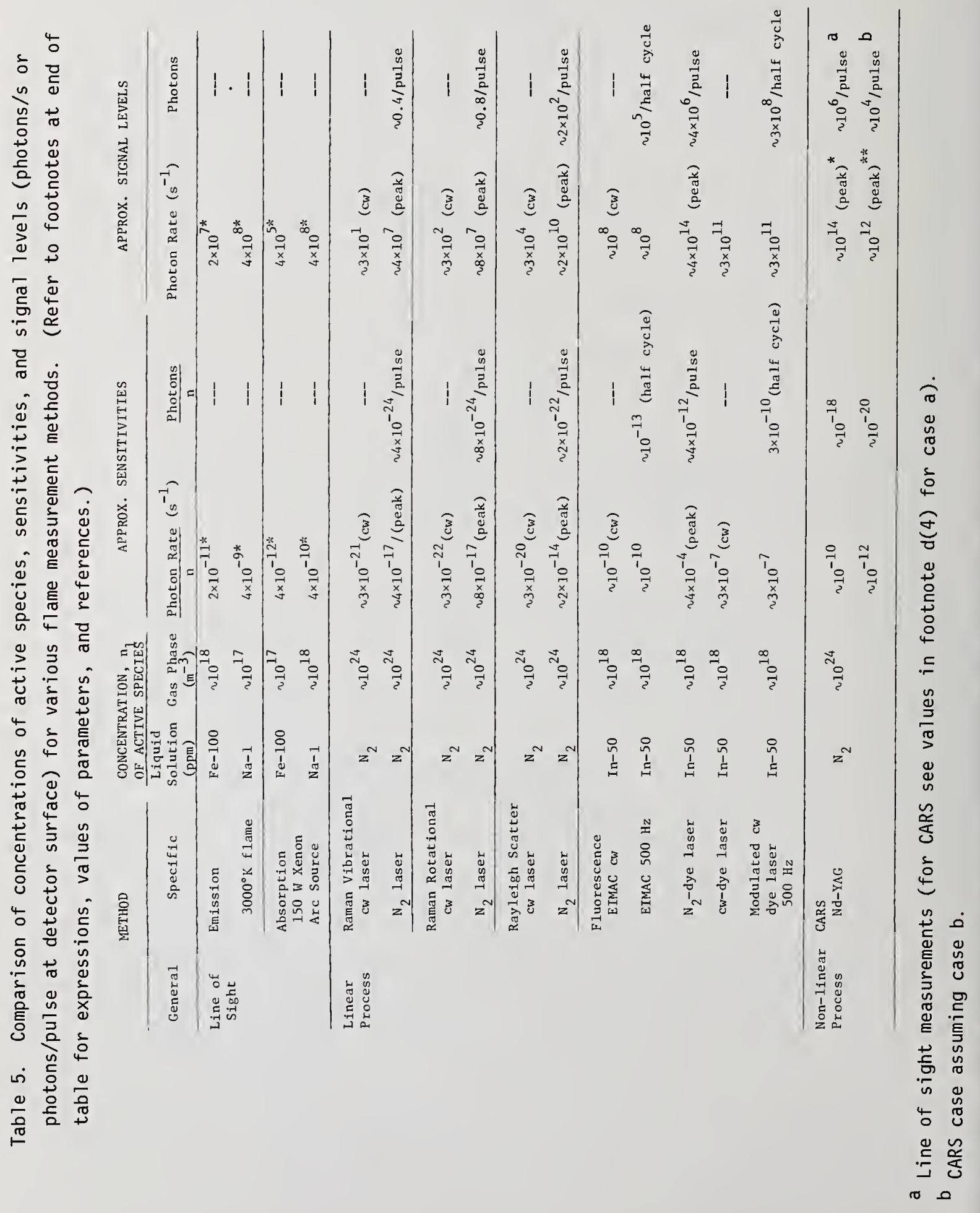


1. Estimation of Concentrations and Signals for Spectroscopic Methods

a. Estimation of $n^{\prime} s$ in $\mathrm{Flames}$

(1) Natural Molecular Species in Flame [A1]

The concentration, $n_{x}$, of a species $X\left(m^{-3}\right)$ is given by

$$
n_{x}=f_{x} \frac{P_{T}}{k T}
$$

where $p_{T}$ is the total pressure (atm) of the flame gases, $T$ is the flame temperature $(K), k$ is the Boltzmann constant $\left(k=1.37 \times 10^{-16} \mathrm{~atm} \mathrm{~m}^{-3} \mathrm{~K}^{-1}\right)$, and $f_{x}$ is the molecule fraction of species $X$ (partial pressure $\approx f_{X}$ ). For example, a $C_{2} H_{2} /$ air flame, assuming $f_{x}=0.75$, has $n_{N} \cong 2.5 \times 10^{24} \mathrm{~m}^{-3}$.

(2) Seeds Added to Flame

The concentration, $n_{y}$, of a seed added to the flame is given by

$$
n_{y}=6 \times 10^{29}\left(\frac{F \varepsilon \beta c y}{e_{f} Q_{t}}\right)\left(\frac{g_{0}}{Z_{T}}\right)
$$

where $F$ is the transport rate of seed solution into the nebulizer $\left(\mathrm{cm}^{3} \mathrm{~s}^{-1}\right), \varepsilon$ is the efficiency of nebulization and vaporization, i.e., the fraction of seed material which actually enters the flame gases as submicroscopic species (atoms and molecules) (no units), $\beta$ is the fraction of the submicroscopic species which are in the desired form (generally atomic) (no units), $c_{y}$ is the concentration of seed solution being aspirated (moles $\mathrm{cm}^{-3}$ ), $e_{f}$ is the flame gas expansion factor (no units), $Q_{t}$ is the flow rate of unburnt gases into the $\mathrm{flame}\left(\mathrm{cm}^{3} / \mathrm{s}\right)$ and $\mathrm{g}_{0} / \mathrm{Z}_{\mathrm{T}}$ accounts for losses of ground state atoms via excitation $\left(\mathrm{g}_{0}=\right.$ ground state electronic statistical weight and $\mathrm{Z}_{\mathrm{T}}=$ electronic partition function). For example, assuming the use of $\mathrm{Na}, \mathrm{Fe}, \mathrm{In}, \mathrm{Ga}, \mathrm{Tl}$, or $\mathrm{Pb}$ probes (introduction of these species into the flame is generally as chlorides or nitrates) and assuming a nebulizerflame system as described by Haraguchi, et al. [24,28-30], then $\varepsilon \beta \sim 0.05$. For an $\mathrm{C}_{2} \mathrm{H}_{2}$-air flame $(\mathrm{T} \sim 2200 \mathrm{~K}$ ) with room temperature flow rates of $7.6 \mathrm{l} / \mathrm{min}$ air and $1.0 \mathrm{l} / \mathrm{min} \mathrm{CH}, \mathrm{e}_{\mathrm{f}} \mathrm{Q}_{\mathrm{t}} \cong 10^{3} \mathrm{~cm}^{3} \mathrm{~s}^{-1}$. The rate of introduction of seed solution into the nebulizer-chamber prior to transfer of the small droplets to the flame gases in $\mathrm{F} \approx 3 \mathrm{~cm}^{3} / \mathrm{min}$ or $0.05 \mathrm{~cm}^{3} \mathrm{~s}^{-1}$. Assuming $\mathrm{g}_{0} / \mathrm{Z}_{\mathrm{T}} \sim \mathrm{L}$, then the gas phase concentration, $n_{y}$, of atoms, $Y$, in the flame gases $\left(m^{-3}\right)$ is related to the liquid phase concentration of seed nebulized, $c_{y}$ by 


$$
n_{y}=1.5 \times 10^{18} c_{y}
$$

In the case of the line reversal method, a typical $\mathrm{Na}$ (chloride) concentration, $\mathrm{c}_{\mathrm{Na}}$, is $1 \mathrm{ppm}$ ( $1 \mathrm{\mu g} \mathrm{Na} / \mathrm{ml}$ solution) or $4.3 \times 10^{-5} \mathrm{~mol} / \mathrm{e}$ or $4.3 \times 10^{-8} \mathrm{~mol} / \mathrm{cm}^{3}$. The value of $n_{\mathrm{Na}}$ for the above typical experimental conditions is then $6.5 \times 10^{10}$ atoms/ $\mathrm{cm}^{3}$. In the case of the atomic emission/atomic absorption methods, a typical valuk of $\mathrm{C}_{\mathrm{Fe}}$ is $100 \mathrm{ppm}\left(100 \mu \mathrm{g} \mathrm{Fe} / \mathrm{ml}\right.$ solution) or $1.8 \times 10^{-3} \mathrm{~mol} / 2$ or $1.8 \times 10^{-6} \mathrm{~mol} / \mathrm{cm}^{3}$. The value of $n_{\mathrm{Fe}}$ for the above typical experimental conditions is $2.7 \times 10^{12}$ atoms/ $\mathrm{cm}^{-3}$. In the case of the two-line atomic fluorescence method with the EIMAC excit: tion source, a typical indium (chloride) concentration, $C_{\text {In }}$ is $50 \mathrm{ppm}(50 \mu \mathrm{g} \mathrm{In} / \mathrm{m} 1$ solution) or $4.3 \times 10^{-4} \mathrm{~mol} / 2$ or $4.3 \times 10^{-7} \mathrm{~mol} / \mathrm{cm}^{3}$. The value of $\mathrm{n}_{3}$ fn for the above typical experimental conditions is then $n_{\text {In }}=6.5 \times 10^{11}$ atoms $/ \mathrm{cm}^{3}$. In may be interesting to point out that the detection limit of In via laser excited atomic fluorescence flame spectrometry is $0.2 \mathrm{ng} / \mathrm{ml}$ or $1.7 \times 10^{-9} \mathrm{~mol} / \mathrm{e}$ or $1.7 \times 10^{-12} \mathrm{~mol} /$ $\mathrm{cm}^{3}$. Converted to a gas phase concentration in the flame, $\mathrm{n}_{\text {In }}=2.5 \times 10^{6}$ In atoms per $\mathrm{cm}^{3}$ of flame gases. Therefore, the high sensitivity of atomic fluorescence spectrometry (especially with laser excitation) is evident. Also, it is evident that the concentration of $50 \mathrm{ppm}$ In (as chloride) will produce an extremely large signal and signal-to-noise ratio.

b. Rate equations (photons/s) for each method are given below (low optical density of the analyte assumed in all emission, absorption, and fluorescence cases).

(1) Line of Sight Methods--Aborption of Emission $[\mathrm{Al}]^{1}$

The photon flux, $\phi_{p}$, photons $s^{-1} \mathrm{~m}^{-2}$ of any species for absorption or emission techniques is given by

$$
\phi_{p}=B_{p s_{v}}\left({ }_{0}\right) \Omega l \int_{0}^{\infty} k_{v} d v
$$

where: $B_{p s}\left(\nu_{0}\right)=$ spectral radiance (photons $s^{-1} \mathrm{~m}^{-2} \mathrm{sr}^{-1}$ ) for a black body radiator in the case of flame (thermal) emission or the spectral radiance (photons $\mathrm{s}^{-1} \mathrm{~m}^{-2} \mathrm{sr}^{-1}$ ) for an excitation spectral continum source in the case of atomic absorption spectrometry;

$\Omega=$ solid angle $(s r)$ of radiation collected from the flame in emission measurements or from the source in atomic absorption measurements;

$\ell=$ absorption or emission path length (m); and

$$
\int_{0}^{\infty} k_{v} d v=\text { integrated absorption coefficient for spectral } 1 \text { ine of atom of }
$$

The integrated absorption coefficient is given [1] by

\footnotetext{
${ }^{1}$ Figures in brackets refer to the Appendix references.
} 


$$
\int_{0}^{\infty} k_{\nu} d v=\left(\frac{e^{2}}{4 \varepsilon_{0} m c}\right) n_{\ell} f=\left(2.7 \times 10^{-6} \underline{m}^{2} \underline{H z}\right) n_{\ell} f
$$

where: $e$ and $m=\operatorname{charge}\left(1.610^{-19} \mathrm{C}\right)$ and mass $\left(9.1 \times 10^{-31} \mathrm{~kg}\right)$ of the electron;

$\varepsilon_{0}=$ permittivity of vacuum $\left(8.85 \times 10^{-12} \mathrm{C}^{2} /(\mathrm{Nm})^{2}\right)$;

$\mathrm{n}_{\ell}=$ concentration $\left(\mathrm{m}^{-3}\right)$ of analyte in lower state of transition; and

$f=$ oscillator strength for the absorption or emission process (dimensionless).

The photon flux (in $s^{-1}$ ) reaching the detector surface after transferral through an optical train is

$$
R_{p}=\phi_{p} \cdot A \tau
$$

where: $\quad A=$ limiting area $\left(\mathrm{m}^{2}\right)$ of radiation measured $=$ monochromator slit area, wh, in most studies;

$w=$ slit width $(\mathrm{m})$;

$\mathrm{h}=$ slit height $(\mathrm{m})$; and

$\tau=$ transmission of entire optical system (dimensionless).

(2) Linear Processes, Spontaneous Raman Scatter, Rayleigh Scatter, and Fluorescence.

For the linear processes (signal depending upon first power of source power and analyte density of Raman and Rayleigh Scatter), the photon rate at the detector surface is given [A2] by

$$
R_{p}=R_{S} n_{\ell} \frac{d \sigma}{d \Omega} \Omega \ell \cdot \frac{A \tau}{S}
$$

where: $\quad \mathrm{R}_{\mathrm{S}}=$ photon rate from source (photons $/ \mathrm{s}$ );

$\mathrm{n}_{\ell}=$ concentration or density $\left(\mathrm{m}^{-3}\right)$ of active species in level, $\ell$;

$\mathrm{do} / \mathrm{d} \Omega=$ differential cross-section for process of concern $\left(\mathrm{m}^{2} \mathrm{sr}\right)$;

$\Omega=$ solid angle of observation ( $s r)$;

$\ell=$ path length over which observed process occurs in the direction of detection $(\mathrm{m})$;

$\tau=$ transmittance of optical system (dimensionless);

$S=i 11$ uminated area of flame $\left(\mathrm{m}^{2}\right)$; and

$A=1$ imiting aperture $\left(\mathrm{m}^{2}\right)$. 
The above expression is also valid for atomic fluorescence assuming the use of a narrow line source (source FWHM « absorption line analyte in the flame); however, if the condition in the parenthesis is not valid, then the expression for $R_{S}$ is unduly complicated with the convolution of the source and absorption line profiles By using a pseudo-continuum or real continuum source (source FWHM $\gg$ absorption 1i! width), then $[\mathrm{Al}]$

$$
R_{P}=\phi_{A b s} \cdot Y \cdot \frac{A \tau \Omega_{F}}{4 \pi}
$$

where: $\quad \phi_{A b s}=$ absorption photon flux (photons $\mathrm{s}^{-1} \mathrm{~m}^{-2}$ ) as given by eq. (A4);

$$
\begin{aligned}
Y= & \text { quantum (power) efficiency of fluorescence process (dimension- } \\
& \text { less); and } \\
\Omega_{\mathrm{F}} / 4 \pi= & \text { fractional solid angle of fluorescence transferred by optical } \\
& \text { train. }
\end{aligned}
$$

If a pulsed source is used, then the number of photons per pulse reaching the detector surface is given [A1] by

$$
N_{p}=R_{p} t_{p}
$$

where: $\quad t_{p}=$ pulse width (FWHM in $s$ ).

(3) Non-Linear Process, CARS

of the many non-linear processes, only CARS has been used to any appreciable extent for diagnostic studies, and so only CARS will be of concern here. The photon, rate, $R_{p}$, for CARS is given [A2] by

$$
R_{p} \cong\left(\frac{4 \pi^{2} w_{A S}}{c^{2}}\right) \phi_{L}^{2} \phi_{S} K_{j}^{2} z^{2} \tau
$$

where: $\quad w_{A S}=$ frequency $(\mathrm{Hz})$ of anti-Stokes component;

$\mathrm{c}=$ speed of 1ight $\left(\mathrm{m} \mathrm{s}^{-1}\right)$;

$\phi_{L}=$ energy flux of laser pump beam $\left(\mathrm{J} \mathrm{s}^{-1} \mathrm{~m}^{-2}\right)$;

$\phi_{S}=$ photon flux of laser Stokes beam (photons $\mathrm{s}^{-1} \mathrm{~m}^{-2}$ );

$z$ = distance $(m)$ over which phase-matched interaction occurs; and 


$$
\begin{aligned}
k_{j}= & \frac{2 c^{4}}{2 \pi h \omega_{s}^{4}} n_{j} \Delta_{j} g_{j} \quad \frac{\partial \sigma}{\partial \Omega} \frac{l}{\Gamma_{j}}\left(m^{3} j^{-1}\right) ; \\
\omega_{s}= & \text { frequency of Stokes component }(\mathrm{Hz}) ; \\
n_{j}= & \text { concentration of active species }\left(\mathrm{m}^{-3}\right) ; \\
\Delta_{j}= & \text { relative population difference between levels involved in the } \\
\left(\frac{\partial \sigma}{\partial \Omega}\right)_{j}= & \text { dransition (dimensionless); } \\
\Gamma_{j}= & \text { Raman line width }(\mathrm{Hz}) ; \text { and } \\
\frac{A \tau}{S}= & \text { overall transmission and collection efficiency of optical } \\
& \text { system. }
\end{aligned}
$$

\section{c. Detector Count Rates or Counts}

To convert any of the photon rates, $R_{p}$, to count rates, it is only necessary to multiply $R_{p}$ by $\eta \varepsilon$ where $\eta$ is the photocathode efficiency (electrons produced for photons reaching the surface) and $\varepsilon$ is the first dynode efficiency. To convert any of the photons/pulse to counts/pulse, it is only necessary to multiply again by $\eta \varepsilon$.

d. Estimation of Sensitivities, Photon Rates, and Photons/Pulse

(1) Parameters Used to Estimate Line of Sight Values are:

Black Body [A3] Radiator ( $T=$ flame temperature) $(\Omega=0.1 \mathrm{sr})$

$B_{p v}\left(\begin{array}{l}\lambda=306 \mathrm{~nm} \\ T=3000 \mathrm{~K}\end{array}\right) \Omega=4 \times 10^{5}$ photons $\mathrm{s}^{-1} \mathrm{~m}^{-2} \mathrm{~Hz}^{-1}$

$B_{p \nu}\left(\begin{array}{l}\lambda=589 \mathrm{~nm} \\ T=3000 \mathrm{~K}\end{array}\right) \Omega=3 \times 10^{6}$ photons $\mathrm{s}^{-1} \mathrm{~m}^{-2} \mathrm{~Hz}^{-1}$

Xenon Arc Source [A1,A3] ( $\varepsilon=$ emissivity; $T=$ plasma temperature) $(\Omega=0.1 \mathrm{sr})$

$$
\begin{aligned}
\mathrm{B}_{\mathrm{p} \nu}\left(\begin{array}{l}
\lambda=306 \mathrm{~nm} \\
\mathrm{~T}=6000 \mathrm{~K} \\
\varepsilon=0.07
\end{array}\right) \Omega & =1 \times 10^{5} \text { photons } \mathrm{s}^{-1} \mathrm{~m}^{-2} \mathrm{~Hz}^{-1} \text { (image at flame) }{ }^{2} \\
\mathrm{~B}_{\mathrm{p} \nu}\left(\begin{array}{l}
\lambda=589 \mathrm{~nm} \\
\mathrm{~T}=6000 \mathrm{~K} \\
\varepsilon=0.07
\end{array}\right) \Omega & =5 \times 10^{5} \text { photons } \mathrm{s}^{-1} \mathrm{~m}^{-2} \mathrm{~Hz}^{-1} \text { (image at flame) }{ }^{2} \\
\ell & =1.0 \mathrm{~cm} \times 1 \times 10^{-2} \mathrm{~m} \text { (reference A1) } \\
& =0.03 \text { (reference A4) } \\
\mathrm{f}_{\mathrm{Fe}-306 \mathrm{~nm}} & =0.65 \text { (reference A4) } \\
\mathrm{f}_{\mathrm{Na}-589 \mathrm{~nm}} & =0.01 \mathrm{~cm} \times 0.1 \mathrm{~cm}=10^{-3} \mathrm{~cm}^{2}=10^{-7} \mathrm{~m}^{2} \text { (typical value) } \\
\mathrm{A}=\mathrm{Wh} & =0.5 \text { (typical value) }
\end{aligned}
$$

"Spectral radiances are corrected for magnification of source image at flame "surface" ( $1000 \times$ magnification). 
(2) Parameters Used to Estimate Fluorescence Values are:

CW EIMAC or Modulated EIMAC $[A 1, A 3, A 5](\lambda=410$ or $451 \mathrm{~nm})(T=6000 \mathrm{~K}, \varepsilon=0.07$, $\Omega=2 \pi)$

$B_{p \nu}(410$ or $451 \mathrm{~nm}) \Omega=2 \times 10^{8} \frac{\text { photons }^{2}}{\mathrm{~s} \mathrm{~m}^{2} \mathrm{~Hz}}$ (image at flame)

Modulated EIMAC $(500 \mathrm{~Hz})$ : half cycle period = $1 \mathrm{~ms}$;

$\mathrm{B}_{\mathrm{p} v}(410$ or $451 \mathrm{~nm}) \Omega=2 \times 10^{11} \frac{\text { photons }}{\mathrm{s} \mathrm{m}^{2} \mathrm{~Hz}}$

$\mathrm{N}_{2}$-Dye Laser $[\mathrm{Al}, \mathrm{A6}](\lambda=410$ or $451 \mathrm{~nm})\left(50 \mathrm{pps}, 30 \mathrm{~W} / \mathrm{pulse}^{3}, 10 \mathrm{~ns}\right.$ pulses; $0.3 \mathrm{~mm}$ diameter; $0.1 \AA$ bandwidth)

$$
E_{p v}(410 \text { or } 451 \mathrm{~nm}) \sim 3 \times 10^{16} \frac{\text { photons }}{\mathrm{s} \mathrm{m}^{2} \mathrm{~Hz}} \text { (image at flame) }
$$

CW Dye Laser [A1,A7] $(\lambda=410$ or $451 \mathrm{~nm})$ or modulated CW Dye Laser $(0.1 \mathrm{~W} ; 0.5 \mathrm{~mm}$ diameter; $10^{11} \mathrm{~Hz}$ bandwidth)

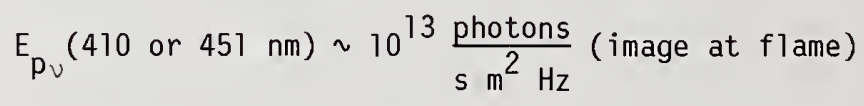

Modulated Dye Laser $(500 \mathrm{~Hz})$ : half cycle period = $1 \mathrm{~ms}$;

$$
\begin{aligned}
& B_{p_{\nu}}(410 \text { or } 451 \mathrm{~nm}) \Omega \cong 10^{13} \frac{\text { photons }}{\mathrm{s} \mathrm{m}^{2} \mathrm{~Hz}} \\
& \ell=1.0 \mathrm{~cm}=1 \times 10^{-2} \mathrm{~m} \text { for EIMAC }(\text { reference } \mathrm{Al}) ;=0.03 \mathrm{~cm} \text { for } \mathrm{N}_{2} \text { Dye laser; } \\
& =0.05 \mathrm{~cm} \text { for } \mathrm{cw} \text { dye laser } \\
& f_{\text {In }} 410 \text { or } 451 \cong 0.3 \text { (reference } \mathrm{A} 4 \text { ) } \\
& Y=0.1 \text { (reference } A 1) \\
& A \tau=5 \times 10^{-8} \mathrm{~m}^{2} \text { (same as line of sight methods) (typical value) } \\
& \Omega_{\mathrm{F}} / 4 \pi=0.01 \text { (typical value) }
\end{aligned}
$$

\footnotetext{
${ }^{3}$ This power is about an order of magnitude below that needed to produce saturation and is many orders of magnitude below typical peak power from pulsed lasers.
} 
(3) Parameters Used to Estimate Spontaneous Raman Scatter and Rayleigh Scatter Values are:

CW Laser $\left(\mathrm{Ar}^{+}\right)[\mathrm{Al}, \mathrm{A} 7](\lambda=488 \mathrm{~nm})(1 \mathrm{~W} ; 1.5 \mathrm{~mm}$ diameter $)$

$$
R_{S}=\frac{1 \mathrm{Js}^{-1}}{h \nu}=2.5 \times 10^{18} \text { photons } \mathrm{s}^{-1}(\mathrm{cW})
$$

$\mathrm{N}_{2}$-Laser $[\mathrm{A} 1, \mathrm{~A} 6](\lambda=337 . \mathrm{nm})\left(400 \mathrm{~kW} ; 0.63 \mathrm{~cm}^{2} ; 10 \mathrm{~ns}\right.$ pulse width $)$

$$
\begin{aligned}
& R_{S}=\frac{400,000 \mathrm{~J} \mathrm{~s}^{-1}}{h \nu}=1 \times 10^{24} \text { photons } \mathrm{s}^{-1} \text { (at peak) } \\
& \mathrm{N}_{S}=R_{S} t_{p}=1 \times 10^{16} \text { photons } / \text { pulse }
\end{aligned}
$$

$$
\begin{aligned}
& \left(\frac{d \sigma}{d \Omega}\right)_{\mathrm{N}_{2}-Q_{\text {vib }}}=\begin{array}{l}
3.5 \times 10^{-34} \mathrm{~m}^{2} \mathrm{sr}^{-1}(337 \mathrm{~nm}) \\
7 \times 10^{-35} \mathrm{~m}^{2} \mathrm{sr}^{-1}(488 \mathrm{~nm})
\end{array} \quad \begin{array}{l}
(\text { reference A2) } \\
(\text { reference A2) }
\end{array} \\
& \left(\frac{\mathrm{d} \sigma}{\mathrm{d} \Omega}\right)_{\mathrm{N}_{2}-\operatorname{rot}}=5.4 \times 10^{-34} \mathrm{~m}^{2} \mathrm{sr}^{-1} \quad(\text { reference } \mathrm{A} 2)
\end{aligned}
$$

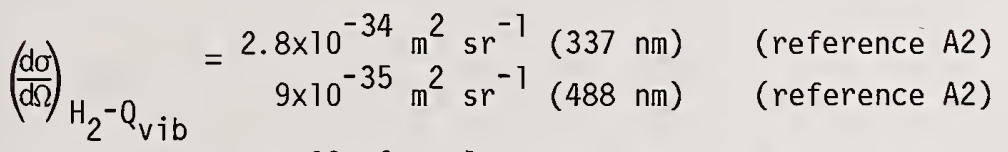

$$
\begin{aligned}
& \left(\frac{d \sigma}{d \Omega}\right)_{\text {Rayleigh }}=\begin{array}{lll}
10^{-30} \mathrm{~m}^{2} \mathrm{sr}^{-1} & (337 \mathrm{~nm}) & \text { (reference A2) } \\
10^{-31} \mathrm{~m}^{2} \mathrm{sr}^{-1}(488 \mathrm{~nm}) & \text { (reference A2) }
\end{array} \\
& \ell=0.03 \mathrm{~cm}=3 \times 10^{-4} \mathrm{~m} \quad(\text { reference } \mathrm{Al} \text { ) } \\
& \Omega=0.1 \mathrm{sr} \quad \text { (typical value) } \\
& \frac{A \tau}{S}=5 \times 10^{-3} \quad \text { (typical value) }
\end{aligned}
$$

(4) Parameters Used to Estimate CARS Values [A2].

$$
\left.\begin{array}{rl}
K_{j} & =2.5 \times 10^{-16} \mathrm{~m}^{3} \mathrm{~J}^{-1} \text { for } \mathrm{N}_{2} \\
\mathrm{z} & =1 \mathrm{~cm}=10^{-2} \mathrm{~m} \\
\Phi_{\mathrm{L}} & =5 \times 10^{13} \mathrm{~J} \mathrm{~s}^{-1} \mathrm{~m}^{-2}(532 \mathrm{~nm}) \\
\phi_{S} & =1 \times 10^{24} \text { photons } \mathrm{s}^{-1}(607 \mathrm{~nm})
\end{array}\right\} \begin{aligned}
& \text { Nd-YAG Laser (Doub } \\
& (10 \mathrm{~ns} \text { pul ses) }
\end{aligned}
$$




$$
\begin{aligned}
\frac{A \tau}{S}= & 10^{-1} \text { considering line of sight measurements with interaction length } \\
& \text { length of } 1 \mathrm{~cm} \text { limiting the resolution (Case a) } \\
\frac{A \tau}{S}= & 10^{-3} \text { (assuming the same spatial resolution as for the other techniques } \\
& \text { techniques in table } 5 \text { ) (Case } b \text { ) }
\end{aligned}
$$

2. Discussion of table 5 and Significance of Results

a. Signal-to-Noise of Techniques

No definitive estimations of or expressions for signal-to-noise ratios, SNR, will be given here because they have been discussed elsewhere [A2,A8]. However, it should be pointed out that shot and flicker noises from various sources are present in all techniques. It is especially significant that in those cases where spatial-temporal measurements are made and where the number of photons striking the detector surface are small, e.g., any of the pulsed Raman ${ }^{4}$ techniques ( $<100$ photons/pulse or $<25$ counts/ pulse), signal shot noise (the best possible condition) will seriously impair the SNR (SNR $\sim 5$ in the case designated here). Of course, background shot noises as well as signal related and background related flicker noises will lead to a further degradation of the SNR. The fluorescence (and emission or absorption) technique will result in considerably greater SNRs primarily as a result of their larger signals.

b. Flame Background Interference of Techniques

In general, any of the $\mathrm{CW}$ measurement systems will be prone to a high degree of background interference (i.e., soot emission, and flame gas molecular emission) since the duty factor of the measurement system is unity. The modulated systems will discriminate against non-source induced flicker noise in the interference signal as long as the frequency of modulation is high enough. However, shot noise on the interference signal (with a white frequency spectrum) may contribute significantly to the total noise of the system in high background regions will low analyte signal levels (i.e., modulated Raman or Rayleigh scatter techniques). The pulsed source gated detector techniques will have the highest immunity from flame background emission. With a duty factor of $210^{-6}$ ( $10 \mathrm{~ns}$ detector gate, $100 \mathrm{~Hz}$ repetition rate) background levels may be reduced to $<10$ photons per detector gate cycle reaching the detector. Only the Rayleigh and Raman techniques will be affected by the shot noise on this small count rate, while CARS and fluorescence signals will be many orders of magnitude greater. It should be pointed out that when using longer gate widths (i.e., for longer laser pulses or for signal pulse stretching), the duty factor on background emission is also increased and may cause the interference signal to present a serious problem even for CARS and fluorescence.

c. Scatter Effects of Techniques

0nly the absorption and Rayleigh scatter techniques will be greatly affected by Mie type scatter since all of the other techniques measure radiation Stokes and/or anti-Stokes to the pump wavelength.

${ }^{4}$ Not including CARS. 


\section{d. Conclusions}

Based upon the results in table 5 and the above discussion, the two line fluorescence and CARS methods are similar in terms of signal levels and probably in terms of signal-to-noise ratios. However, the two-line fluorescence method is superior to CARS in terms of lower cost, simplicity of implementation and operation, and spatial resolution.

\section{e. References to Table 5}

[1] Winefordner, J. D., Schulman, P. G., and O'Haver, T. C., Luminescence in Analytical Chemistry (John Wiley, New York, 1972).

[2] Eckbreth, A. C. G., Bonczyk, P. A., and Verdieck, J. F., Review of Laser Ramon and Fluorescence Techniques for Practical Combustion Diagnostics, R77-952665-6, Technical Report, United Technolgoies Research Center (Feb. 1977).

[3] Pivonosky, M. and Nagel, M. R., Tables of Blackbody Radiation Functions (The MacMillan Company, New York, 1961).

[4] Corliss, C. H. and Bozman, W. R., Experimental Transition Probabilities for Spectral Lines of Seventy Elements, NBS Monograph 53, U.S. Government Printing Office, Washington, D.C. (1961).

[5] Varian Eimac Literature, Varian EIMAC, San Carlos, California.

[6] Molectron Literature, Molectron Corp., Sunnyvale, California.

[7] Spectra Physics Literature, Spectra Physics, Mountain View, California.

[8] Alkemade, C. Th. J., Snelleman, A., Boutilier, G. D., Pollard, B. D., Winefordner, J. D., Chester, T. L., and Omenetto, N., Spectrochim. Acta, B, in press.

[9] Boutilier, G. D., Pollard, B. D., Winefordner, J. D., Chester, T. L., and Omenetto, N., Spectrochim. Acta, $\underline{B}$, in press.

\section{References}

[1] Lapp, M. and Penney, C. M., Laser Raman Gas Diagnostics (P1enum Press, New York, 1974).

[2] Gaydon, A. G., The Spectroscopy of Flames (Chapman and Ha11, Ltd., London, 1974).

[3] Fristrom, R. M. and Westenberg, A. A., Flame Structure (McGraw-Hi11, New York, 1965).

[4] Beer, J. M. and Chigier, N. A., Combustion Aerodynamics (Applied Science Publishers, Ltd., London, 1974).

[5] Gaydon, A. G. and Wolfhard, H. G., Flames, Their Structure, Radiation, and Temperature (Chapman and Ha11, Ltd., London, 1953).

[6] Eckbreth, A. C., Bonczyk, P. A., and Verdieck, J. F., Technical Report on Review of Laser Raman and Fluorescence Techniques for Practical Combustion Diagnostics, R72952665-6, United Technologies Research Center, East Hartford, CT (1977).

[7] Hartley, P. L., ed., The Role of Physics in Combustion, American Physical Society Summer Study Report (1976).

[8] Goulard, R., ed., Combustion Measurements in Jet Propulsion Systems, Project SQUID Workshop Proceedings, December 1975. 
[9] AFOSR, Compilation of Abstracts--1977 AFOSR Contractors Meeting on Air Breathing Combustion Dynamics, Purdue University, West Lafayette, IN (1977).

[10] Regnier, P. R. and Taran, J. P. E., Appl. Phys. Lett. 23, 240 (1973).

[11] Moya, F., Druet, S., Pealat, M., and Taran, J. P., Flame Investigation by Coherent Anti-Stokes Raman Scattering, AIAA Paper 76-29, AIAA 14th Aerospace Science Meeting, Washington, D.C., January 1976.

[12] Lapp, M. , Laser Raman Probe for Flame Temperature, SQUID Report GE-1-PU, Purdue University, West Lafayette, IN (1973).

[13] Robbin, F., Comparison of Density and Temperature Measurement Using Raman Scattering and Rayleigh Scattering, from reference [8].

[14] Stephenson, D. A. and Arman, W. R., Combust. Flame, 31, 85 (1978).

[15] Strickler, W., Combust. Flame, 27, 133 (1976).

[16] Setche11, R. E., Analysis of Flame Emission by Laser Raman Spectroscopy, Paper WSS/CI 74-6, Western States Section, The Combustion Institute, Pullman, WA (1974).

[17] Pitz, R. W., Cattolica, R., Robben, F., and Talbot, L., Combust. Flame, 27, 313 (1976)

[18] Frank, P. and Krause, L., Spectroscopic Diagnostics of Hydrogen-Oxygen Flame, Translation of DLR-FB-74-65.

[19] Snelleman, W. , A Flame As a Standard of Temperature, Ph.D. Thesis, University of Utrec Utrecht, The Netherlands (1965).

[20] Reif, I., Fassel, V. A., and Kniseley, R. N., Spectrochim. Acta 28B, 105 (1973).

[21] Ibid. 29B, 79 (1974).

[22] Browner, R. and Winefordner, J. D., Anal. Chem. 44, 247 (1972).

[23] Omenetto, N., Browner, R. , Winefordner, J., Rossi, G., and Benetti, P., Anal. Chem. 44, 1683 (1972).

[24] Haraguchi, H., Smith, B., Weeks, S., Johnson, D. J., and Winefordner, J. D., Appl. Spectrosc. 31, 156 (1977).

[25] Omenetto, N. and Winefordner, J. D., Appl. Spectrosc. 26, 555 (1972).

[26] Omenetto, N. and Winefordner, J. D., Chapter on Atomic fluorescence spectroscopy with laser excitation, in Lasers in Analytical Chemistry, N. Omenetto, ed. (John Wiley, 1978), in press.

[27] Boutilier, G. D., Blackburn, M. B., Mermet, J. M., Weeks, S. J., Haraguchi, H., Winefordner, J. D., and Omenetto, N., Appl. Optics, in press.

[28] Haraguchi, H. and Winefordner, J. D., Appl. Spectrosc. 31, 195 (1977).

[29] Haraguchi, H., and Winefordner, J. D., Appl. Spectrosc. 31, 330 (1977).

[30] Haraguchi, H., Weeks, S., and Winefordner, J. D., Can. J. Spectrosc. 22, 61 (1977).

[31] Winefordner, J. D., Svoboda, V., and Cline, L. J., CRC Crit. Rev. Anal. Chem. I, 233 (1970). 


\section{Discussion}

Comment (Daily): I want to tout this method. I think this is really a fantastic method.

One of the things that the speaker did not point out and in fact is probably the most important thing about this technique, is that if you go through the equations that he alluded to, you find out interestingly enough that all quenching and collisional parameters drop out. That means that you no longer have this quenching problem and you can apply this to any part of the flame under any kind of temperature or pressure conditions and still get a measurement. The only thing you have to do is measure the ratio of those two fluorescent signals. To me that is really intriguing.

The other thing is that this is the only method that we have been able to discover that comes close to duplicating the low level of uncertainty for a temperature measurement that you can get from line reversal, which is the other best way that we have ever found for measuring temperature.

It is very reasonable to expect less than one percent uncertainty in the final temperature measurement. So, I am a voter for this.

Response (Bradshaw): Thank you. I would like to point out though that as far as quenching is concerned, if you do not measure the Stokes direct line fluorescence and the anti-Stokes direct line fluorescence is on a time scale that is less than any changes in the quenching observed in the flame, you will get an error at that point.

I mean quenching will affect you. If you do it in a simultaneous manner, as in very fast modulation with two dyelasers, where you are on a very fast time scale, where flame conditions will not change in your analysis volume, then it is independent of quenching.

But, the later data that we showed was done on a very slow time scale, we were changing filters and changing wave lengths on the monochrometer, and the data were affected by quenching. You have to do it on a fast time scale if you want to be not affected. 
National Bureau of Standards Special Publication 561, Proceedings of the 10th Materials Research Symposium on Characterization of High Temperature Vapors and Gases held at NBS, Gaithersburg, Maryland, September 18-22, 1978. Issued October 1979.

A Comparison of Spectroscopic Flame Temperature Measurements: $\mathrm{Na}-\mathrm{D}$ Line Reversal, Rotational and Vibrational Raman, and $\mathrm{OH}$ Absorption

M. C. Drake, ${ }^{1}$ L. H. Grabner, and J. W. Hastie ${ }^{2}$

National Bureau of Standards

Washington, D.C. 20234

Different spectroscopic methods are used for the measurement of flame temperature. This report compares measurement of temperature profiles for atmospheric premixed $\mathrm{H}_{2} / \mathrm{O}_{2} / \mathrm{N}_{2}$ laminar flames using the methods of: (1) Raman scattering $\left(\mathrm{N}_{2}\right.$, vibrational and rotational temperatures), (2) line reversal (D-1ine of $\mathrm{Na}$, electronic temperature), and (3) line ratio ( $\mathrm{OH}$, rotational temperature). Two separate burner designs are considered. To obtain the most accurate comparison possible, all measurements were made with the same burners and auxiliary gas-feed system. The theory for each technique is summarized and the sensitivity, accuracy, and convenience are compared. Necessary corrections are discussed. In general, the results indicate very good agreement (better than 98 percent) between the various methods for certain combinations of flame composition and burner type.

\section{Introduction}

\subsection{Status of flame temperature measurement}

Temperature, and its spatial and temporal distribution, is perhaps the most important fundamental quantity for characterization of a high temperature vapor or gaseous system. This is especially the case for atmospheric pressure flames, where both laboratory and industrial systems are included. For instance, temperature can be used as a rating parameter for comparing the effectiveness of various control measures in combustion efficiency and flame inhibition $[1]^{3}$. More commonly, temperature is used in conjunction with species concentration data to define the kinetic or thermodynamic state of the flame $[2,3]$.

The recent development of computer-based methods of temperature calculation for both equilibrium [4] and non-equilibrium [5] flame systems, together with the availability of an

1 NRC-NBS Doctoral Research Associate, present address, General Electric Co., Research and Development Center, P. 0. Box 8, Schenectady, New York.

2 Authors listed alphabetically.

${ }^{3}$ Figures in brackets indicate the literature references at the end of this paper. 
adequate basic thermodynamic [6] and chemical kinetic data base [7,8], particularly for $\mathrm{H}_{2} / \mathrm{O}_{2} / \mathrm{N}_{2}$, has added a new dimension to temperature determination. Accurate experimental flame temperature profiles are now needed to validate these computer based models of flame processes.

On the experimental side, the use of different burners and flames by most workers is a serious impediment to interlaboratory transfer of data. It is now apparent that combustion standards are needed, particularly in the areas of burner design and flame selection. The present study is a mere beginning in this direction.

In addition to these difficulties, little is known about the accuracy of various temperature measurement methods. With the continuing development, in recent years, of novel temperature measurement techniques, e.g., Raman scattering, Coherent Antistokes Raman Spectroscopy (CARS), and two line fluorescence, the need for accurate comparison data by established techniques such as line-reversal for validation of these methods is further indicated. Evidence of this continued development of new or modified flame temperature measurement techniques is provided by the chapters presented elsewhere in this volume on the methods:

- Coherent Antistokes Raman Spectroscopy $[9,10]$,

- Raman [11,12],

- optoacoustic [13],

- two line fluorescence [14], and

- modified D-1ine reversal [15].

An obvious first candidate for a standard burner-flame system is the Padley-Sugden type burner [16] $-\mathrm{H}_{2} / \mathrm{O}_{2} / \mathrm{N}_{2}$ flame combination. Many workers have used this combination over the past twenty years or so (e.g., see ref. [17]). In practically all cases the Na-D line rever sal method was used for temperature measurement, and good interlaboratory agreement obtained. Much basic thermodynamic and kinetic data for high temperature inorganic species is based on these temperature results [17]. The present study extends these earlier investigations by including other optical temperature measurement methods (see Section 1.3), and different burner geometries.

\subsection{Thermal nature of flames}

The thermal nature of flames, that is, the distribution of combustion energy in the form of kinetic or translational energy, internal, and chemical energy, is well understood in a broad sense [17]. For atmospheric pressure flames, these degrees of freedom are usually thermalized rapidly with the internal energy states achieving a Boltzmann population distribution. However, for such flames the relaxation times associated with the redistribution of excess energy arising from the exothermic combustion process are such that regions of disequilibrium may exist, particularly near the primary reaction zone. The location and extent of this disequilibrium varies according to the flame type, burner, gas flow rate, temperature and pressure. Well known examples of the occurrence of nonequilibrium 
state-distributions include certain $\mathrm{OH}$ rotational energy levels and $\mathrm{Na}-\mathrm{D}$ line chemiluminescence near the reaction zone $(<1 \mathrm{~ms})^{4}$ of atmospheric premixed flames. Under turbulant conditions these effects may be even more significant [18]. Chemical (concentration) disequilibrium for the radicals $\mathrm{H}, \mathrm{OH}$, and 0 persists for times of several ms (or a few $\mathrm{cm}$ distance from the reaction zone) or longer, depending largely on the flame temperature.

Usually there is insufficient quantitative data available on the various flame relaxation processes, to predict a priori when or where a particular flame system will achieve a thermalized condition with respect to each, or a11, of the degrees of freedom. Hence, if a spectroscopic temperature measurement is to be used in a chemical kinetic or thermodynamic sense, the thermal condition of the internal energy needs to be established by empirical means.

Usually, the time required to achieve a thermal energy distribution increases according to the sequence:

translation < rotational < vibrational < electronic < chemical.

Generally, if one can establish thermalization for one of these energy states then higher (faster) members can be assumed to follow the same condition. Also, if the state populations are thermally distributed then measurement of these populations can be used as a basis for spectroscopic temperature measurements.

Temperature, so obtained, represents only the local thermodynamic equilibrium (LTE) condition of those states monitored. Thus, such a temperature does not necessarily correlate with the thermodynamic equilibrium temperature for the total system, where all degrees of freedom are included. If an LTE temperature is to be used to characterize a particular kinetic or thermodynamic process, all the states involved must satisfy the LTE condition.

As flames are not isolated systems, due to burner and atmospheric influences, they can only approach equilibrium and it is therefore useful to retain the term LTE when referring to flame temperature data. Also, in the absence of general information about the thermal nature of all the states present, we should qualify temperature statements with respect to the states involved in the measurement of temperature. Thus, terms such as rotational, vibrational, and electronic temperature are used, with the understanding that not all rotational states, etc., are included.

\subsection{Selection of temperature measurement methods}

Following the discussion of Section 1.2, it is clear that a definitive measurement of flame temperature should include direct observation of the relative population distribution for as many degrees of freedom as possible. For practical purposes the states of rotational, vibrational and electronic degrees of freedom are the most accessible. As these three state-types encompass an exceptionally wide range of relaxation times ( $\underline{c a} 10^{-8}-10^{-3} \mathrm{~s}$ ) a demonstration of LTE for each state with the same temperature would indicate that this characteristic temperature is valid for the whole system.

\footnotetext{
Represents flow time downstream of the reaction zone; the distance equivalent is typically several mm.
} 
Temperature measurement methods covering these three state-types and selected for the present study include:

(1) The Na-D line reversal method, which essentially measures an electronic temperature for the spectroscopic transition $3 \mathrm{~S}_{1 / 2}-3 \mathrm{P}_{3 / 2}$ at $589.0 \mathrm{~nm}$.

(2) A line ratio method where the relative intensities of the rotational lines of the $A^{2} \Sigma-X^{2} \Pi$ electronic transition ( $306.5 \mathrm{~nm}$ band) for $\mathrm{OH}$ are measured.

(3) A Raman spectroscopic method using scattering from the vibrational and rotational states of $\mathrm{N}_{2}$.

Method (1) is an established technique of known accuracy for temperature measurement in post flame regions ${ }^{5}$. However, there are limitations, among which is a possible disturbance of the chemical balance in the flame by the presence of $\mathrm{Na}$. Errors inherent in the line reversal method have been discussed in detail by Snelleman [21].

Method (2) has rarely been used with atmospheric pressure flames and its accuracy is uncertain.

Method (3) is a novel technique developed in recent years [22] and the use of rotational states has only barely begun $[23,12]$.

Each technique has relative merit, depending on the system of interest. For instance, the Raman method can be used throughout the flame whereas methods (1) and (2) are limited to the post reaction zone region ${ }^{6}$. Thus an intercomparison of temperatures obtained from these methods serves both as a test for thermal equilibrium between the various states and as a demonstration of the reliability and accuracy of methods (2) and (3) with respect to (1).

The measured temperatures may also be compared with the calculated adiabatic values, using a well established minimization of free energy multicomponent equilibrium computation procedure [4]. This technique provides an upper limit to the possible measured flame temperature (for constant stoichiometry) and is based on the ideal condition of general thermodynamic equilibrium. That is, all states are in LTE and no heat transfer to, or from, the surrounding occurs. In order that the calculated temperatures be useful, we have used burners where heat losses are small and flame temperatures closely approach the adiabatic values (to within 96 percent).

For convenience we shall refer to temperatures obtained by these four approaches as:

- reversal temperature,

- $\mathrm{OH}$ rotational temperature,

- Raman (rotational or vibrational) temperature, and

- adiabatic temperature,

where these first three terms refer only to the actual spectroscopic states involved. A fifth temperature:

- the LTE (local thermodynamic equilibrium) temperature will be referred to when the states involved are believed to be thermalized, i.e., follow a single Boltzmann

${ }^{5}$ The long established $\mathrm{Na}-\mathrm{D}$ line reversal method of temperature measurement continues to find new application, e.g., in propellant gases [19] and in magnetohydrodynamic flame gases [20]. ${ }^{6} \mathrm{~A}$ particularly useful combination is to use $\mathrm{Na}-\mathrm{D}$ line reversal data for thermocouple correction, thereby permitting a complete temperature profile to be obtained $[9,24]$ with subsequent thermocouple measurements. 
population distribution. For cases where LTE is not established, the term:

- pseudo temperature

may be used [25].

\section{The Methods}

2. $1 \mathrm{Na}-\mathrm{D}$ line reversal

This method is the oldest optical technique for measuring flame temperature [26]. A recent description may be found in the book of Gaydon and Wolfhard [27] and a critical evaluation is given in references [21] and [28]. Figure 1 shows our particular experimental arrangement. The method is popular because it is rapid, convenient, and requires no data analysis. Upon reversal, the Na-D line temperature is measured directly by a pyrometer. The following discussion of this well known method serves mainly to derive the reversal sensitivity with temperature and to indicate the physical meaning of the reversal temperature.

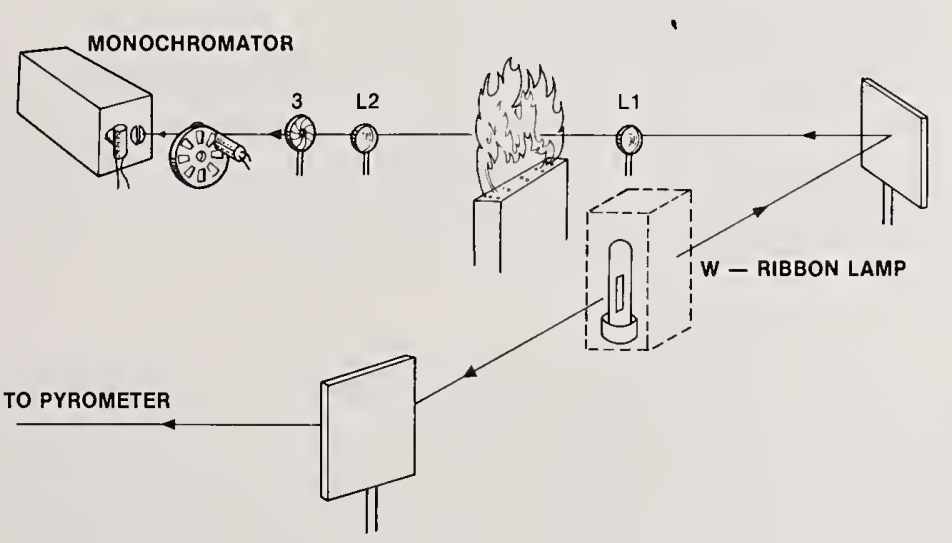

Figure 1. Na-D line reversal apparatus (isometric view). Light from $W$-ribbon lamp is focused by lens LI to give an image of the $W$-ribbon in the flame. A second lens L2 forms an image of both flame and W-ribbon on the monochromator entrance slit. Aperture stop 3, located at the position of image of $L 1$ formed by $L 2$, is adjusted so that the solid angle of the light from flame and reversing source are equal. The optics must be alined so that the image of flame and W-ribbon overlap on the monochromator entrance slit. This is best done photoelectrically. Reversal temperature is pyrometer reading at $655 \mathrm{~nm}$ uncorrected for $W$-emissivity change with $\lambda$. This change is generally assumed (and so verified) to be compensated by reflection losses from Ll, taken as 5 percent. 
The method depends on the spectrally resolved observation of one of the D-lines of a $\mathrm{Na}$ colored flame which is simultaneously irradiated in the D-line region by a reversing source, a W-ribbon lamp. In the present study, Na was introduced to the flame in the form of a spray of $\mathrm{NaNO}_{3}$ solution ( 0.01 molar). Depending on the current carried by the lamp (i.e., color temperature), the D-Tine (at $589.0 \mathrm{~nm}$ ) is observed either in absorption or emission, and the lamp current is adjusted to "reverse," i.e., until neither occurs. This is shown by the curves a of figure 2. At reversal, the radiation absorbed by the flame from the reversing source is just compensated by the Na-D line emission from the flame. If the solid angle of the reversing source and flame, as seen by the detector, are equal, then the following relationships apply [29]

$$
[1-A(\lambda, T)] \cdot I\left(\lambda, T_{R}\right)+E(\lambda, T) \gtreqless I\left(\lambda, T_{R}\right)
$$

$\lambda=$ wavelength.

$\mathrm{T}=$ temperature as measured by the pyrometer .

$A(\lambda, T)=$ absorbing power of flame at $T$ and $D$ line $\lambda$.

$E(\lambda, T)=$ emissive power of flame at $T$ and $D$ line $\lambda$.

$I\left(\lambda, T_{R}\right)=$ radiant flux from reversing source at brightness temperature $T_{R}$ of $D$ line $\lambda$.

In the relationships (1), reflection of the reversing radiation by the flame is neglected. For emission we have $(>)$, for absorption $(<)$, and at reversal (=). At reversal eq. (1) does not directly determine $T$ in terms of $T_{R}$. However, if Kirchhoff's law applies,

$$
E(\lambda, T)=A(\lambda, T) I(\lambda, T),
$$

where $I(\lambda, T)=$ radiant flux of a blackbody, and eq. (1) reduces to
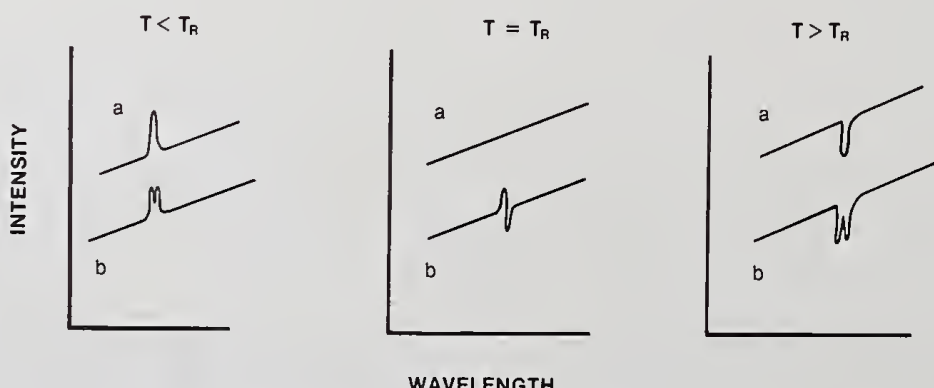

Figure 2. Reversal method. Spectrum of $\mathrm{Na}^{-} \mathrm{D}_{1}$ line (schematic). When brightness temperature of $W$-ribbon 1 amp $T<T_{R}, T=T_{R}$, and $T>T_{R} . \quad T_{R}$ is the reversal temperature. In series (a) the concentration of $\mathrm{Na}$ in the nebulizing solution is $0.01 \mathrm{M}$, in (b) $0.1 \mathrm{M}$. Burner: rectangular with either $0(2)$ or $U(4)$ composition. 


$$
I(\lambda, T)=I\left(\lambda, T_{R}\right)
$$

which is satisfied only if $T=T_{R}$. $T_{R}$ is the brightness temperature (pyrometer reading) at the $D-1$ ine $\lambda$ and not the temperature of the reversing source (1amp), except if the source is a blackbody, which is usually not the case.

It should be noted that the Na-D line method contains no criterion for local thermodynamic equilibrium (LTE). Even if the line of another element, such as $L i$, is found to reverse at the same temperature as the $\mathrm{Na}-\mathrm{D}$ line this condition is necessary but not sufficient that $T_{R}$ is a LTE temperature. However, it has been our experience that Na-D line emission intensities follow a Boltzmann distribution with temperature and with the correct energy for thermal excitation to the $3 \mathrm{P}_{3 / 2}$ level. This has been observed for a large number of $\mathrm{H}_{2} / \mathrm{O}_{2} / \mathrm{N}_{2}$ flames over the temperature range 1800-2500 $\mathrm{K}$ and at distances of $210-30 \mathrm{~mm}$ from the reaction zone and suggests LTE for the internal degrees of freedom present.

With the reasonable assumption that Kirchhoff's law applies here, a temperature sensitivity can be defined. Re-arranging relationships (1):

$$
I\left(\lambda, T_{R}\right)+A(\lambda, T)\left[I(\lambda, T)-I\left(\lambda, T_{R}\right)\right] \stackrel{\gtrless}{<} I\left(\lambda, T_{R}\right)
$$

Close to reversal, the expression in the square brackets is

$$
\left[\frac{d I(\lambda, T)}{d T}\right]_{T_{R}} d T \text {, where } d T=T-T_{R}
$$

We shall define the derivative as the temperature sensitivity. $I(\lambda, T)$ is Planck's function. The value of the derivative as a function of $T$ is tabulated in reference [30]. A sensitivity plot for the temperature range of interest to the present study is given in figure 3 . It should also be noted that the signal increases inversely with the spectral slit width of the monochromator until this is equal to the Doppler width of the Na-D line $(\sim 0.005 \mathrm{~nm}$ at $2000 \mathrm{~K})$. This is because the integrated absorption is invariant with spectral slit width $[31,32]$. Errors in the method of 1 ine-reversal have been extensively discussed by Snelleman [21].

Finally, we wish to call attention to the physical significance of $T_{R}$ measured by the reversal method. Kohn [33] has shown that

$$
\frac{N_{m}}{N_{n}}=\frac{g_{m}}{g_{n}} \exp \left(-h_{\nu} / k T_{R}^{n m}\right),
$$

where $g_{m}$ and $g_{n}$ are the degeneracy of the states involved in the transition hu, with populations $N_{m}$ and $N_{n}$. The superscript $n m$ is affixed to $T_{R}$, to emphasize that, in general, $T_{R}$ depends on the transition except for conditions of LTE. 


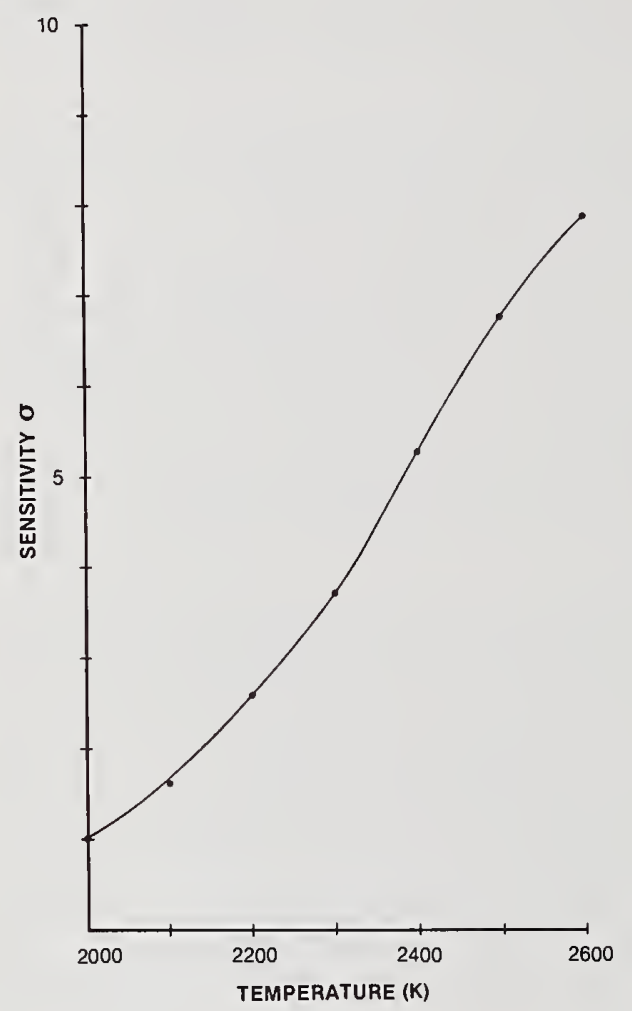

Figure 3. Temperature sensitivity of reversal method defined by equation (2) $\mathrm{dI}(\lambda, \mathrm{T}) / \mathrm{dT}$ vs $\mathrm{T}$ at $\lambda=589 \mathrm{~nm}$. The temperature sensitivity $\sigma=\frac{\mathrm{dI}}{\mathrm{dT}} /\left(\frac{\mathrm{dI}}{\mathrm{dT}}\right)_{\mathrm{T}_{0}}$ is normalized to $\mathrm{T}_{0}=2000 \mathrm{~K}$.

In applying the $\mathrm{Na}-\mathrm{D}$ line reversal method, one has to empirically determine the appropriate range of $\mathrm{flame} \mathrm{Na}$ concentration where reversal occurs [24]. Typical D-1ine spectra are given in figure 2 where the effects of too great a $\mathrm{Na}$ concentration are indicated (curves b). In the present study the temperature sensitivity of the reversal was within several Ke1vins. Reproducibility was limited by the human eye response to the pyrometer, which was typically in the range 5-10 K.

\section{$2.2 \mathrm{OH}$-rotational line ratio}

\subsubsection{Method}

A schematic of the apparatus used to obtain $\mathrm{OH}$-absorption spectra, and hence, flame temperatures, is given in figure 4 . Figure 5 shows a typical $\mathrm{OH}$ absorption spectrum in the region of 307-309 $\mathrm{nm}$ which is a particularly useful region for temperature measurements, as will be discussed in Section 4. The general theory of the method is as follows. 


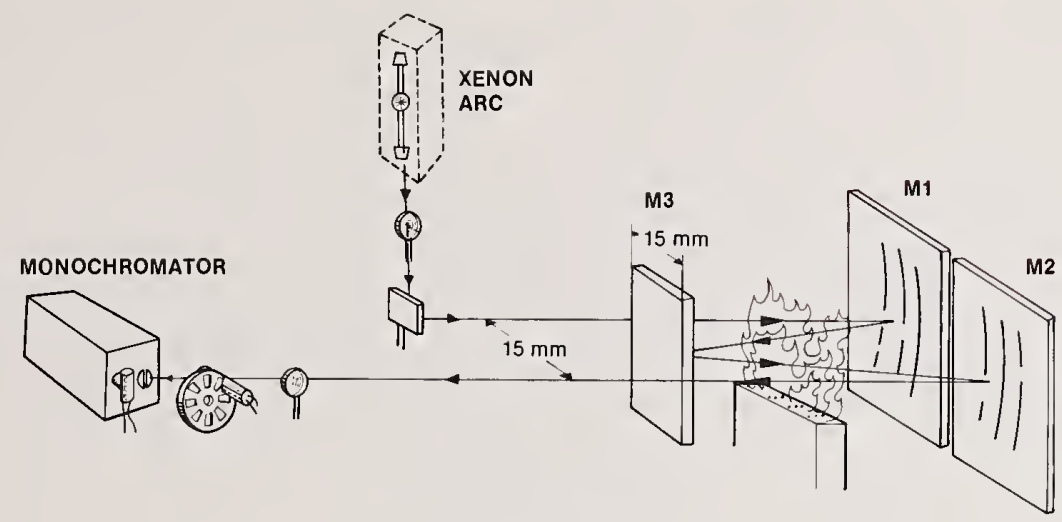

Figure 4. Apparatus for $\mathrm{OH}$ absorption measurements (isometric view). White multi-reflection system consists of three concave mirrors each of focal length $f=22.9 \mathrm{~cm}$. $M 1$ and $M 2$ are separated by distance $2 f$ from $M 3$ which is cut to $15 \mathrm{~mm}$ width to minimize beam spread through flame. Xe-arc light is focused onto M3. The number of traversals is changed by adjustment of the dihedral angle between $M 1$ and $M 2$ from 4, shown in the figure, to 8 , or 12, or any multiple of 4. Two traversals use only M2 and M3. For a single traversal the White system cannot be used, and Xe source-lens-flame-lens-monochromator are set up collinearly.

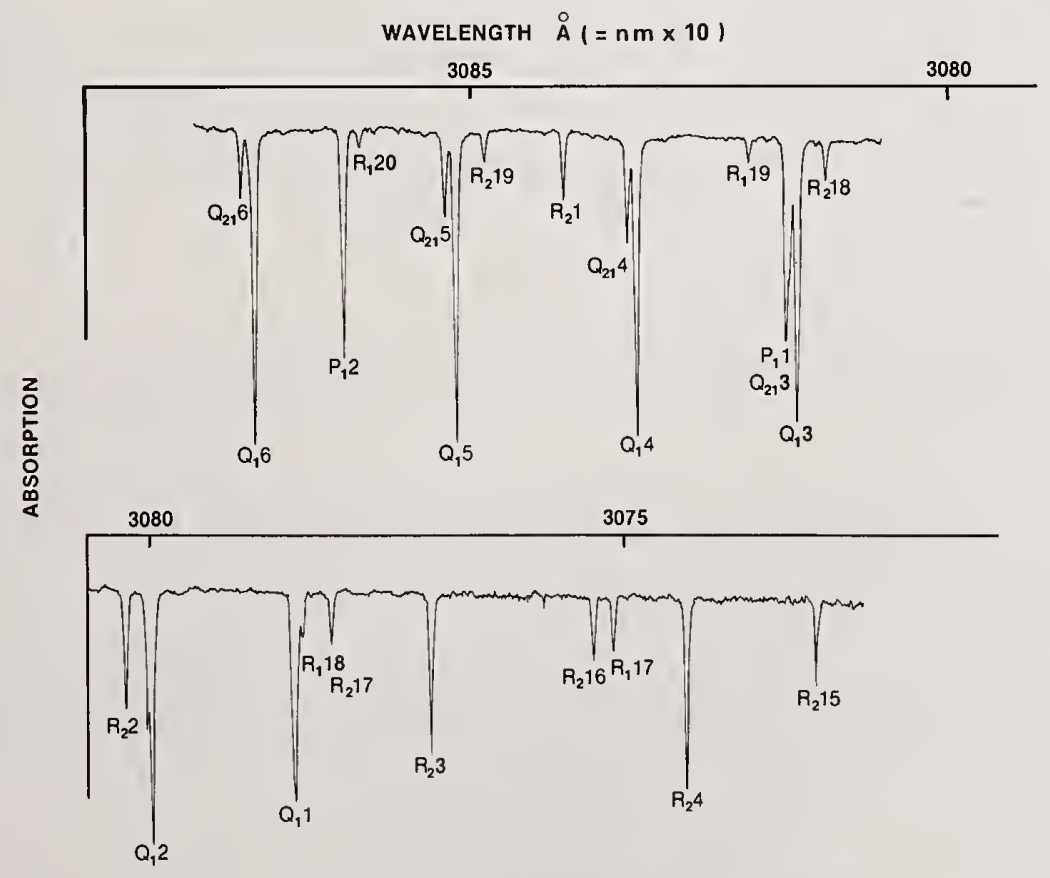

Figure 5. OH rotational absorption spectrum. Lines are identified by standard spectroscopic notation. For an atlas, see reference [34]. For $R_{2} 4$ line, $\left.I_{\text {abs }}=\left[\left(I_{v}^{\circ}-I_{v}\right) / I_{v}^{\circ}\right)\right]_{\text {peak }}=0.54$. Rectangular burner, 0 (2) composition $\mathrm{H}_{2} / \mathrm{O}_{2} / \mathrm{N}_{2}=3.4 / 1 / 2$, and two passes of Xe-arc radiation through flame. 
In this method, the intensity of two lines is measured in absorption, although emission can be used in some instances, and the temperature is calculated from their ratio. This temtemperature has thermodynamic significance if the population of the two states has a thermal (Boltzmann) distribution. The following comments are mainly methodological and apply only to absorption. Similar arguments can be made for the case of emission.

The absorption is measured by an absorption-coefficient $\alpha_{v}$ defined by Lambert's law. An index is affixed to indicate a functional dependence on the wave number $v$ of the radiation. In quantum theory the coefficient is described by Einstein's phenomenological theory of the interaction of radiation with matter. The connection between the two is provided by:

$$
\int \alpha_{\nu} d v=\frac{h v}{c} B_{m n} N_{n},
$$

where $\mathrm{B}_{\mathrm{mn}}=$ Einstein probability for absorption and $\mathrm{N}_{\mathrm{n}}=$ population of upper state. This relationship was developed by Füchtbauer [35] and Ladenberg [36]. In equation (3) there is no restriction on the state of the system. If a Boltzmann distribution of the states under consideration is assumed:

$$
N_{i}=g_{i} \exp \left(-E_{i} / k T\right) / \Sigma g_{i} \exp \left(-E_{i} / k T\right) \quad,
$$

where $g_{i}=$ statistical weight,

$E_{i}=$ energy, and

$k=$ Boltzmann constant, and

the system is restricted to a state of local thermodynamic equilibrium. Temperature can then be calculated using equation (3) if the ratio of $\int \alpha_{\nu} d_{\nu}$ is measured for two lines, and if the $B_{n m}$ of the corresponding transitions are known. For example, for two lines in absorption, we have:

$$
\frac{\left[\int \alpha_{v} d v\right]_{1}}{\left[\int \alpha_{v} d v\right]_{2}}=\frac{\left(v \cdot B_{m n} \cdot N_{n}\right)_{1}}{\left(v \cdot B_{m n} \cdot N_{n}\right)_{2}}=\frac{\left(v \cdot B_{m n} \cdot g_{n}\right)_{1}}{\left(v \cdot B_{m n} \cdot g_{n}\right)_{2}} \cdot \exp \left[-\left(E_{n 1}-E_{n 2}\right) / k T\right]
$$

The $\mathrm{B}_{\mathrm{nm}}$ have been calculated for the transitions of $\mathrm{OH}$ by Learner [37] and tabulated in the convenient form, $(g B) \nu \equiv P \nu$, for absorption, and for emission, where $P$ is a weighted probability factor.

Thus, only two lines are needed in the line ratio method for temperature determination. A necessary, but not sufficient, condition that the temperature thus obtained refers to LTE is that a plot (sometimes known as a Boltzmann plot) of

$$
\log \frac{\left[\int \alpha_{v} d v\right]_{n}}{P v} \text { vs } E_{n}
$$


be linear. For such a case the temperature may be derived from the slope of the line, as will be shown in Section 4 . There are cases known where such plots have been found to yield two straight lines [38], indicating a different effective temperature for two sets of states.

As in the Na-D line reversal method, the line ratio approach does not contain a criterion of how well the measured temperature approximates the thermodynamic equilibrium value. Coincidence of the 'reversal' and the 'line ratio' temperatures (where greatly different energy states are involved) is good, though not conclusive, evidence that the temperatures have thermodynamic significance.

If $\ell \int \alpha_{\nu} d \nu$ is to be measured, for optical path length $\ell$, the line must be "optically thin," i.e., $\alpha_{v} \ell<<1$. Otherwise $\int\left(1-e^{-\alpha_{\nu} l}\right) d v$ is measured in absorption. From equation (3) it can be shown that this quantity, representing an optical thickness condition, cannot be related to the temperature. The optical thickness of an absorption line of a flame species, $\mathrm{OH}$ for example, can be checked by measuring absorption as a function of the number of radiation passes through the flame. This is conveniently accomplished by a white [39] multi-reflection system shown in figure 4. If a flame is optically thick for a particular absorption line, but not too thick, the fractional absorption for various numbers of passes (i.e., $\ell$ ) can be extrapolated to zero passes $(\ell=0)$ to yield $\ell \int \alpha_{\nu} d v$. Examples of this procedure are given in Section 4.2 .

\subsubsection{Sensitivity}

Assuming $\alpha_{v} \ell<<1$ and a state of thermodynamic equilibrium, the temperature sensitivity can be given as

$$
\frac{d}{d T} \frac{\left(\int \alpha_{\nu} d v\right)_{1}}{\left(\int \alpha_{\nu} d v\right)_{2}} \equiv \frac{d}{d T}\left[\frac{\alpha_{1}}{\alpha_{2}}\right] .
$$
Using equation (3) for absorption ratios $r=\frac{\alpha_{1}}{\alpha_{2}}$ and energy difference between lower
(upper) and upper (lower) states of $E_{1}-E_{2}$

$$
\frac{d r}{d T}=r \frac{E_{1}-E_{2}}{k T} \cdot \frac{1}{T}
$$

Thus, the relative error, $\frac{\Delta T}{T}$, due to error, $\Delta r$, in the measurement of the absorption ratio of the two lines is

$$
\frac{\Delta T}{T}=\frac{k T}{E_{1}-E_{2}} \cdot \frac{\Delta r}{r},
$$

an expression given by Broida [38]. 
Values of $r$ can be measured by the ratio of the peak intensity of the lines since

$$
\int \alpha_{v} d v \simeq\left[\left(I_{v}^{0}-I_{v}\right) / I_{v}^{0}\right]_{\text {peak }} \cdot \Delta v_{s l i t}
$$

is invariant with the spectral slit width $\Delta v_{\text {slit }}[28,29]$, which is usually constant over the scanned spectral range. It is advantageous to maximize the peak intensity of a line by setting $\Delta v_{\text {slit }} \simeq \Delta v_{D}$, the Doppler width of the line $(\sim 0.005 \mathrm{~nm}$ for $\mathrm{OH}$ at $2000 \mathrm{~K})$. This minimizes the fractional error with which the peak intensity can be measured and thus $\Delta r$ of equation (4) which is the sum of these errors for two lines. The same conclusions can be drawn if emission instead of absorption lines are used.

For the flames used in this report the $\mathrm{OH}$ radical can be detected in absorption but not by its thermal emission, except near the reaction zone where chemiluminescence occurs. This is in contrast to the high oscillator strength $\mathrm{D}$-lines of $\mathrm{Na}$ for which thermal emission is strong. A contributing factor here is the wavelength dependence of the emissivity (Planck's radiation law). In order to demonstrate this dependence we sha11 assume (arbitrarily) that, at $2200 \mathrm{~K}$, the concentrations of $\mathrm{OH}$ and $\mathrm{Na}$ are such as to give equal absorption coefficients $\alpha_{v}$ at $300 \mathrm{~nm}$ and $590 \mathrm{~nm}$, respectively. The application of Kirchhoff's relationship then shows that $\varepsilon(590 \mathrm{~nm}) / \varepsilon(300 \mathrm{~nm}) \sim 10^{3}$ for emission. Thus, the detection of thermal emission from $\mathrm{OH}$ is indeed unfavorable compared with that from the Na-D lines.

\subsection{Raman scattering}

The application of pure rotational and vibrational-rotational Raman intensities to flame temperature measurements is reasonably well established [40-43] and has been discussed elsewhere in this volume by Drake and Rosenblatt [12]. Raman temperature measurements typically rely on scattering from the major flame species, e.g., $\mathrm{N}_{2}, \mathrm{O}_{2}$, or $\mathrm{H}_{2}$. The apparatus used for the present study is shown schematically in figure 6 .

\subsubsection{Rotational Raman}

Determining temperatures from rotational Raman scattering is similar to the line ratio method discussed for $\mathrm{OH}$ absorption. In both cases measured relative intensities are converted to relative energy level populations. If only two rotational Raman lines are used, the sensitivity is given by equation (4). It is important to measure intensities of rotational transitions from low to high rotational states to maximize the $E_{1}-E_{2}$ term in equation (4). The most accurate temperatures are calculated from measurement of many, rather than just two, transitions.

In this study, Raman scattering from many individual rotational transitions in $\mathrm{N}_{2}$ is easily observed and provides reliable temperatures, particularly below $2000 \mathrm{~K}$ [12]. A typical spectrum from which temperature data were obtained is shown in figure 7 . Both Stokes and anti-Stokes regions were routinely scanned and analyzed, but the anti-Stokes spectrum is not shown since it is essentially a mirror image of the Stokes case. Since 


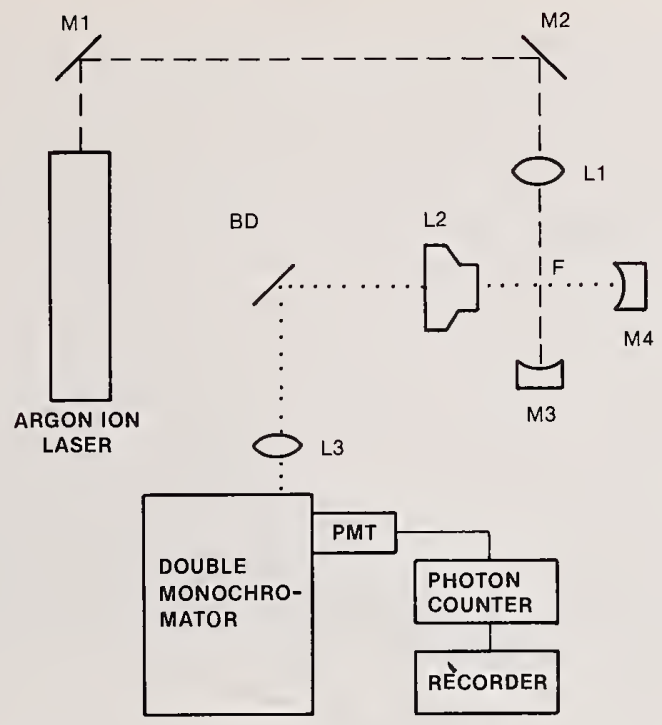

Figure 6. Schematic (in plan view) of Raman spectroscopic apparatus. Output from a $12 \mathrm{~W}$ argon ion laser (Coherent Radiation) is focused by $\mathrm{L} 1$ (60 $\mathrm{mm}$ focal length) into the cylindrical sample volume $(\sim 0.1 \mathrm{~mm}$ diameter $\times 1.5 \mathrm{~mm}$ length--in the same direction as the flame gas flow) of the flame $(F)$. The flame burns in a vertical direction and the vertical spatial resolution is $1.5 \mathrm{~mm}$. The mirror $\mathrm{M} 3$ reflects the laser beam back into the sample area creating an external resonating cavity (gain factor 5-7) between $\mathrm{M} 3$ and the output mirror of the laser without appreciably increasing the size of the sampled volume. The lens ( $L 2(\sim f / 1.0,25 \mathrm{~mm}$ working distance) collects light scattered at right angles to the incident laser beam. A two mirror beam detector (BD) and lens L3 focus the scattered light onto a Spex double monochromator which incorporates a thermoelectrically cooled photomultiplier tube (ITT FW130) and photon counting electronics. The mirror M4 effectively doubles the signal intensity by directing light scattered opposite to the collection lens back along the collection optical axis.

a large number (typically 100) of intensities can be measured, rotational Raman temperature measurements are statistically precise $( \pm 1$ percent for $\pm \sigma$, where $\sigma$ is the standard deviation).

In figure 7 , notice in addition to the major peaks of alternating intensity, small sidepeaks appear on the low frequency shift side of the major peaks. This is particularly noticeable in the $300-400 \mathrm{~cm}^{-1}$ region. These side peaks are the result of pure rotational Raman scattering from vibrationally excited $\mathrm{N}_{2}$ molecules which naturally become more prominent in hotter flames. For example, figure 8 shows a section of the Stokes rotational Raman spectrum from a hotter $\mathrm{H}_{2} / \mathrm{O}_{2} / \mathrm{N}_{2}$ flame where the side peaks are much more pronounced. The separation of the side peaks from the main peaks in figure 8 increases with the rotational quantum number of the transition involved. This is in quantitative agreement with separations calculated from known energy levels of $\mathrm{N}_{2}$ (see for example figure 10 in reference [12]). 


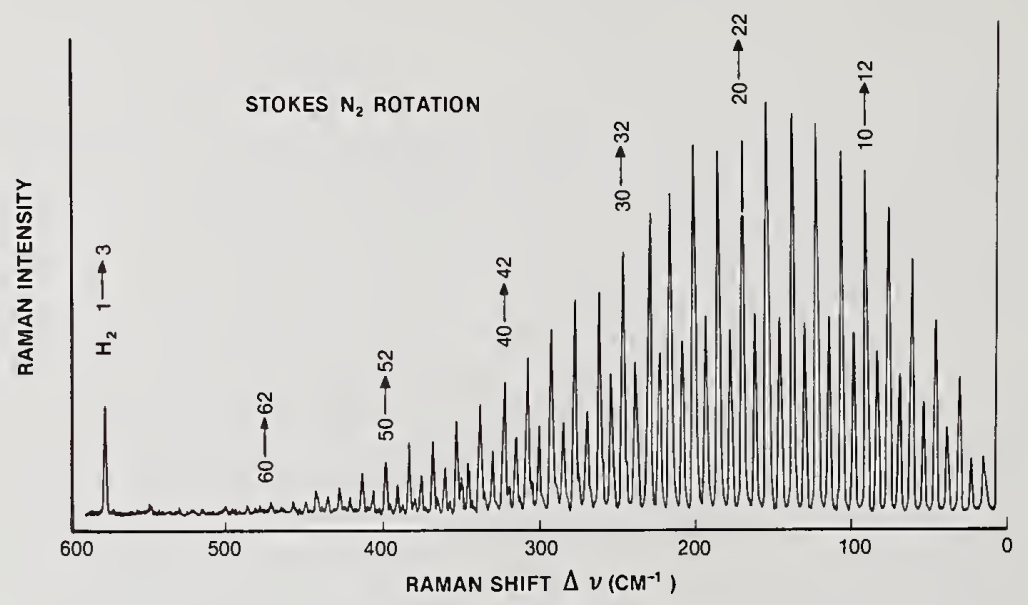

Figure 7. Stokes rotational Raman scattering from flame $\mathrm{N}_{2}$ and $\mathrm{H}_{2}$. The only $\mathrm{H}_{2}$ feature shown is near $600 \mathrm{~cm}^{-1}$. Labels indicate the rotational quantum numbers of the states involved in the transition. Spectrum obtained for the U(4) flame at a location, relative to the face of the rectangular burner, of $z=30, y=2$, and $x=0 \mathrm{~mm}$ (see fig. 11 coordinate system). Temperature (rotational) corresponds to $2113 \mathrm{~K}$ (see fig. 12).

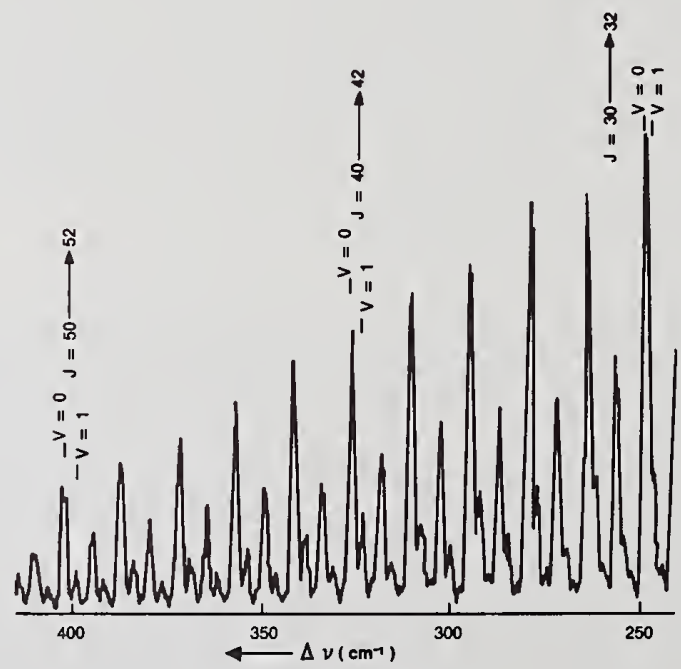

Figure 8. Segment of the Stokes rotational Raman Spectrum from an 0(2) torch flame showing the presence of hot bands $(v=1)$. Temperature (rotational) corresponds to $2450 \mathrm{~K}$. 
The effects of scattering from vibrationally excited molecules must be accounted for in temperature determinations from pure rotational Raman spectra. Above about $2000 \mathrm{~K}$, the overlapping contribution of pure rotational Raman transitions from vibrationally excited $\mathrm{N}_{2}$ molecules necessitates a large correction $(>100 \mathrm{~K}$ ) in the temperature calculation. Although this correction could be made precisely if the spectrometer slit function was accurately known, in practice additional uncertainty is introduced because the correction is very sensitive to the shape and width of this function. 0ther corrections necessary for accurate temperature determinations from rotational Raman spectra, i.e., centrifugal distortion corrections to the line strength, are relatively small for $\mathrm{N}_{2}$ [12]. The statistical uncertainties in the calculated temperature ( \pm 1 percent in the best cases) do not include the additional uncertainties in the magnitude of the correction terms which may be a large source of uncertainty, particularly for temperatures greater than $2000 \mathrm{~K}$.

\subsubsection{Vibrational Raman}

More commonly, for the measurement of temperature, vibrational rather than rotational Raman intensities are used. Since individual rotational transitions within the vibrationalrotational $Q$ branch of $\mathrm{N}_{2}$ cannot be resolved at high temperature in conventional Raman measurements, computer generated profiles are required. At least three methods of determining the flame temperature from vibrational Raman scattering intensities are possible. These are:

(1) Stokes/anti-Stokes intensity ratios,

(2) comparison of the entire Stokes profile with computer generated profiles, and

(3) determination of the Stokes intensity ratios for transitions from the ground state and from vibrationally excited states $[12,40]$.

Previous studies have found that the various vibrational Raman methods give flame temperatures which agree with each other and with radiation corrected thermocouple measurements (within 2 percent for the best cases) for premixed flames of $\mathrm{H}_{2}$-air [22], $\mathrm{CH}_{4}$-air [42], and $\mathrm{NH}_{3}-\mathrm{O}_{2}$ [42].

In the present study, Stokes vibrational Raman scattering profiles from $\mathrm{N}_{2}$ are scanned. Results are similar to spectra shown in figure 11 in reference [12]. Ratios are calculated from intensities measured for the $v=1 \rightarrow 2$ peak and the $v=0 \rightarrow 1$ peak and for the $v=2 \rightarrow 3$ peak and the $v=0 \rightarrow 1$ peak. These measured ratios are converted to a vibrational temperature by comparison with intensity ratios obtained from computer band profile calculations shown in figure 9. A trapezoidal spectrometer slit function, determined experimentally from the shape of the $\mathrm{J}=1 \rightarrow 3$ rotational Raman peak from room temperature $\mathrm{H}_{2}$, was used in the computer calculation. The band profile calculations are similar to those of Setchell [43] and Lapp and Penney $[22,40]$ who include the isotropic part of the $Q(\Delta J=0)$ branch which is the dominant part of the vibrational-rotational Raman profile. However, the present calculations also include contributions from, (1) the anisotropic part of the $Q$ branch, (2) $0(\Delta J=-2)$ and $S(\Delta J=+2)$ branch transitions, and (3) corrections to the line strength due to centrifugal distortion (discussed in detail in reference [12]). These additional 
correction terms have a negligible ( $<10 \mathrm{~K}$ at $2000 \mathrm{~K}$ ) effect on the calculated intensity ratios. Uncertainties in vibrational temperatures $(\Delta T)$, measured from peak intensity ratio are estimated by the uncertainty in measuring the intensity ratio $(\Delta r)$ multiplied by $\left.(d T / d r)\right|_{r}$ determined from figure 9. From photon statistics the minimum uncertainty in an intensity measurement equals $\sqrt{I}$ where $I$ is the number of detected photons so.

$$
\Delta r \geq \sqrt{\mathrm{I}(v=1 \rightarrow 2)} / \mathrm{I}(v=0 \rightarrow 1)
$$

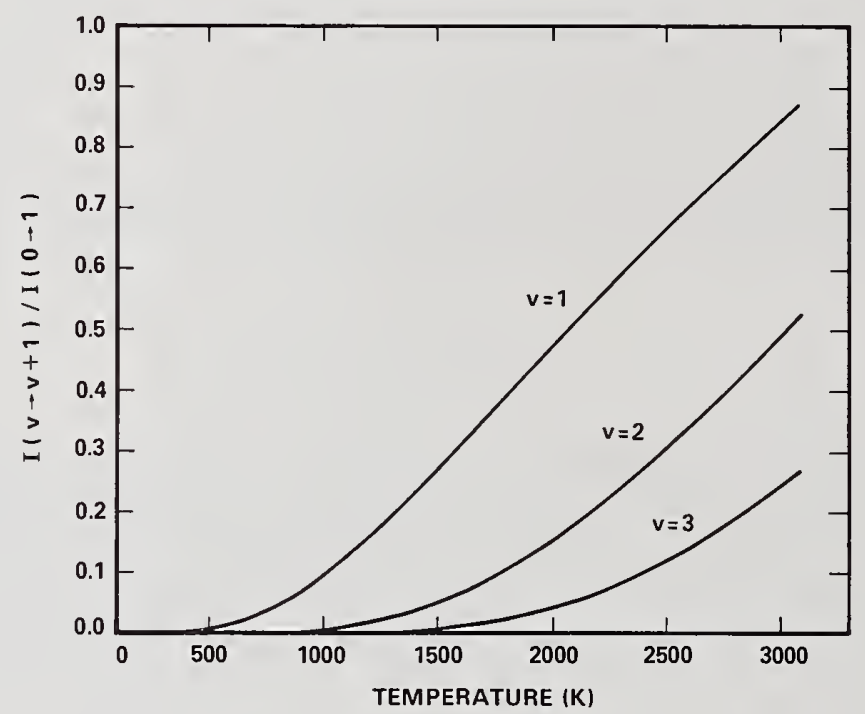

Figure 9. Computer calculation of the $\mathrm{N}_{2}$ Stokes intensity ratios for the $v \rightarrow v+1$ transition compared to $v=0 \rightarrow v=1$. An experimentally determined slit function was used in the calculation.

\subsection{Adiabatic temperature computation}

Calculated adiabatic flame temperatures are computed using the free energy minimization algorithm implemented by Gordon and McBride (GB) [4].

The calculations are posed as "assigned" enthalpy-pressure problems since, by definition, the term adiabatic implies no net heat transfer. The calculations are specified using the mixed burner-input gas (ideal mixing) heats of formation as the enthalpy to be assigned for reactants. Where the reactant mixture gases are in the data base, the program is reques: to calculate the initial heats at laboratory initial temperature and pressure. Where the initial gases are not present in the data base and are not expected to be product species, heats of formation at laboratory conditions are supplied. In this case, selected thermodynamic data are prepared for inclusion in the data base using GB equations (90-92) (see [4]) via a fit to $C_{p} / R$ data. It should be noted that in the syntax of this program, assigned enthalpies are defined by GB equation (89), 


$$
\Delta H_{\mathrm{T}}^{\circ}=\left(\Delta H_{\mathrm{f}}^{\circ}\right)_{298.15}+\left(H_{\mathrm{T}}^{\circ}-\mathrm{H}_{298.15}^{\circ}\right)
$$

and for assigned enthalpy-pressure problems, the product heat is set equal to the reactant heat.

Calculations varying the laboratory pressure by \pm 10 percent showed the expected insignificant effect of small errors in knowledge of the exact pressure (expected error $< \pm 1$ percent) of the reactants just below the reaction zone. For the $\mathrm{H}_{2} / \mathrm{O}_{2} / \mathrm{N}_{2}$ system the thermodynamic data for reactants, products and intermediate species are sufficiently accurate that the uncertainty in calculated temperature should not exceed one degree Kelvin. Calculations were also made to verify the insignificant cooling effect of the small amounts of added $\mathrm{H}_{2} \mathrm{O}$ in the $\mathrm{Na}-\mathrm{D}$ line reversal method.

\section{Burners}

Two water-cooled (but non-quenching) burners, of differing geometry, were used to generate laminar premixed $\mathrm{H}_{2} / \mathrm{O}_{2} / \mathrm{N}_{2}$ flames at atmospheric pressure. Both burners were constructed after the fashion of that used by Padley and Sugden [16], and subsequently by many others $[2,17]$. Bundles of stainless steel capillary tubing, or hypodermic needles, are the essential feature of this burner-type. Stiff columns of flame gas, over a distance of at least $50 \mathrm{~mm}$, are provided by such burners. For optical convenience and minimization of convection effects, the burners were mounted vertically. The low heat losses associated with this type of burner allow flames to be supported under near-adiabatic conditions.

\subsection{Cylindrical burner}

For comparison with literature data one of the burners was designed and constructed to be basically very similar to the Padley-Sugden burner [16]. This burner type produces well shielded circular cross section pseudo-flat premixed flames. The inner flame has a $0.7 \mathrm{~cm}$ cross section and the concentric shield flame produces an overall cross section of $1.1 \mathrm{~cm}$. Additional details of construction, characteristic properties, kinematic control, and the auxiliary gas flow control system will be given elsewhere in connection with a discussion of species concentration profiles [44]; see also reference [45].

\subsection{Rectangular burner}

A second burner of rectangular configuration was designed for use with the white multi-reflection system, used to make optical thickness corrections in the $\mathrm{OH}$ absorption measurements. In the White cell (fig. 4), there is an inherent spread (18 $\mathrm{mm}$ ) in the lateral dimension of the light beams passed by the cell. In order to confine this spread to a well defined cross section of the flame, with an essentially constant temperature, a rectangular capillary burner was constructed similar to the design of Padley and Sugden and modelled after that of Adams, et al. [46]. The capillary tube bundles are supported by a water-cooled brass burner body. The rectangular central flame section (cross section 
$3.6 \mathrm{~mm} \times 22 \mathrm{~mm}$ ) contains 90 stainless steel tubes $(0.028$ in o.d., 0.016 in i.d.) and the encircling shield flame section $(5.2 \mathrm{~mm} \times 25 \mathrm{~mm}$ ) contains 148 tubes. It should be noted that the cross sectional area for the central flame is twice that for the corresponding dimension of the cylindrical burner. Since air entrainment was considered more probable with this long narrow burner, a rectangular slot ( 0.005 in wide) with outer dimensions of $10 \mathrm{~mm} \times 30 \mathrm{~mm}$, provided an additional shield of argon around the flame. Experience has shown such a shield to be unnecessary with the cylindrical burners. The rectangular burner design had the additional advantage for Raman measurements of permitting short focal length low f-number collection, lenses to be used for efficient light collection.

For both burners, the same gas flow and kinematic control systems were used. Gas flow were controlled by pressure regulators and measured by calibrated flow rotameters. The central and shield burner gas compositions were identical and adjusted so that the linear flow velocities were the same. The argon flow rate was adjusted (arbitrarily) to fifty percent of the value where turbulence was observed.

\section{Results}

Flame temperature profiles have been obtained using the optical methods discussed in Section 2 for the combinations of flames and burners listed in table 1 .

Table 1. Combinations of Measurement Technique and Burner-Flame Composition Used

$$
\begin{aligned}
& \text { flame } \\
& \mathrm{H}_{2} / \mathrm{O}_{2} / \mathrm{N}_{2}
\end{aligned}
$$

burner

$$
4 / 1 / 4^{\mathrm{a}}
$$

$u(4)^{b}$
$3.4 / 1 / 2^{a}$

$0(2)^{b}$

$\begin{array}{ll}\text { cylindrical } & \text { Na-D line } \\ \text { none } & e^{e^{c}}\end{array}$

Raman

rectangular ${ }^{d}$

Na-D line

Na-D line

$\mathrm{OH}$-absorption

$\mathrm{OH}$-absorption

Raman

Raman

\footnotetext{
Mole ratios of initial gas mixture (NTP); gas flows were 141.5 and $94.3 \mathrm{~cm}^{3} / \mathrm{s}$ for the $O(2)$ and $U(4)$ flames, respectively, at NTP.

" "Standard" notation in common use, e.g., see Miller [47] and elsewhere in this volume [2].

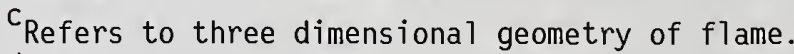

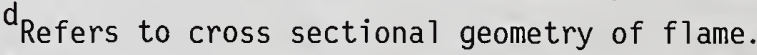

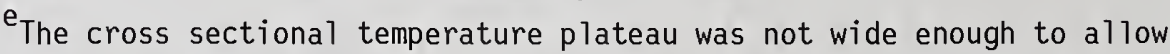
use of multibeam passing for $\mathrm{OH}$-absorption measurement of temperature.
} 


\subsection{Cylindrical burner}

For the 0 (2) flame-cylindrical burner combination, a high background luminosity and relatively unfavorable scattered light collection optics prevented useful high precision Raman temperature data from being obtained. Also, the relatively narrow cross sectional temperature plateau meant that the $\mathrm{OH}$-absorption method could not be applied to this especially optically thick flame. Thus no useful comparisons between methods could be obtained for this flame-burner combination.

Longitudinal temperature profiles (i.e., along the $z$ coordinate of fig. 10) were obtained by passing the light or laser beams through the flame along the $x$ axis (see fig. 10) and adjusting the motor driven micrometer burner drive along the $z$ axis. Application of the Raman and Na-D line reversal methods to temperature profile determinations were relatively straightforward. However, this was not the case for the line-ratio method using optical absorption by the $\mathrm{OH}$ radical as the condition for optical thinness $\left(\alpha_{\nu} l<1\right)$ was not met, except for very weak transitions. The optical thickness was checked and extrapolated to $\alpha_{v} \ell<1$ using the White multi-reflection system.

The longitudinal temperature profile, for the $U(4)$ flame, obtained by the vibrational Raman method is compared with that obtained by the Na-D line reversal technique

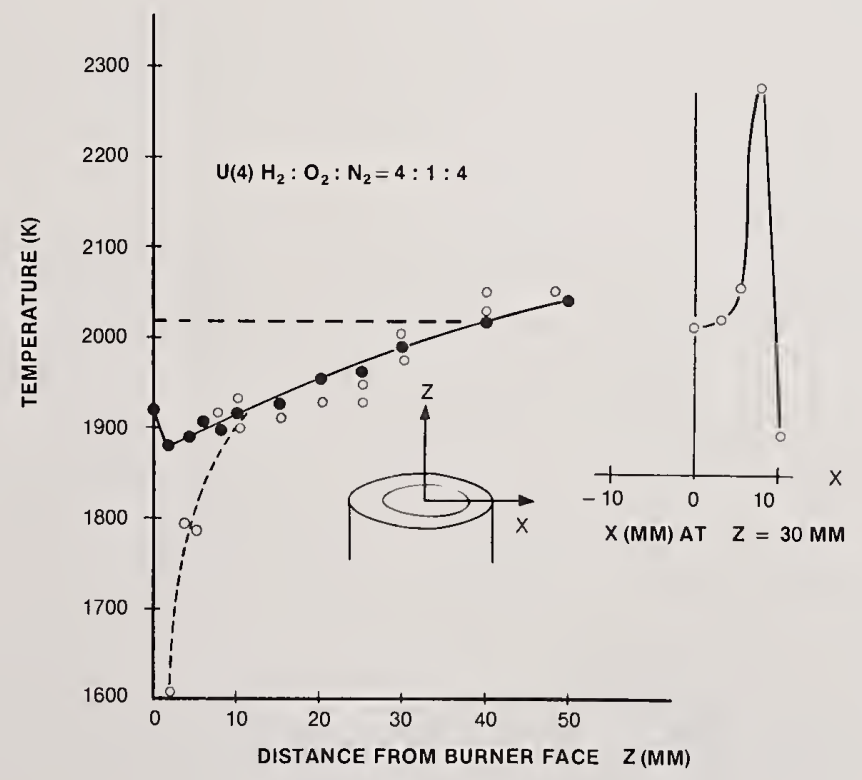

Figure 10. Longitudinal ( $z$ ) and transverse $(x)$ temperature profiles, for $y=0 \mathrm{~mm}$, using $\mathrm{Na}-\mathrm{D}_{1}$ line reversal (closed circles) and vibrational Raman techniques (open circles). Burner: cylindrical. Broken horizontal line indicates calculated adiabatic temperature. Flame gas compositions (initial at STP) are given as mole ratios. Calculated linear hot gas velocity equals $1.5 \times 10^{3} \mathrm{~cm} / \mathrm{s}$. 
in figure 10. The calculated adiabatic temperature and a transverse Raman temperature profile are also shown. Note (in fig. 10) that in the outer flame regions ( $z=50 \mathrm{~mm}$ or $x=8 \mathrm{~mm}$ ) the observed temperatures exceed the maximum allowed adiabatic value for the initial flame stoichiometry indicated.

Previous information on transverse temperature profiles for $\mathrm{H}_{2} / \mathrm{O}_{2} / \mathrm{N}_{2}$ flames using the Padley-Sugden type burner is limited to the recent thermocouple measurements of Hayhurst and Kittelson [48]. Evidence of a several hundred degree temperature rise at the outer flame edge due to afterburning was noted for fuel-rich flames. Leaner flames, on the other hand, showed a tendency to decrease in temperature along the radial axis due to the cooling effect of air entrainment. Under these non-isothermal conditions, line of sight methods will yield temperatures intermediate of the highest and lowest values along the optical path [28].

\subsection{Rectangular burner}

A summary of the data obtained using the various methods is given in figure 11. In addition to the vibrational Raman data (fig. 11), rotational Raman spectra and temperatures were obtained, at $z=30, y=2 \mathrm{~mm}$, for the $U(4)$ flame as shown in figure 7 . To obtain similar data for the 0(2) flame, a small torch burner was used (see fig. 8). Temperatures were determined indirectly from measured rotational Raman intensities using the slopes of the linear least square data fits, such as those given in figure 12. Stokes and anti-Stoke data points are plotted on the same graph and any deviation from a Boltzmann distribution 0 rotational states would be evident since both data sets would simultaneously deviate from $t$ straight line.

Vibrational Raman spectra were used to obtain the flame temperature profiles for the $\mathrm{U}(4) \mathrm{flame}$ shown in figure 11 . Note the large temperature gradient $(45 \mathrm{~K} / \mathrm{mm})$ which exists across the burner (along the $y$ axis) with the temperature maximum $4 \mathrm{~mm}$ from the center. A somewhat lower gradient $(\sim 38 \mathrm{~K} / \mathrm{mm})$ was found for the cylindrical burner (see fig. 10), with the central $10 \mathrm{~mm}$ having a nearly flat temperature profile $(\sim 10 \mathrm{~K} / \mathrm{mm})$. The temperatur determined from the rotational $(2113 \pm 22 \mathrm{~K}$ ) and the vibrational (2096 $\pm 40 \mathrm{~K}$ ) Raman spectr) at $z=30 \mathrm{~mm}$ and $y=2 \mathrm{~mm}$ are in good agreement with each other. For the U(4) flame data in figure 11 , seven $\mathrm{N}_{2}$ Stokes vibrational Raman spectra were also obtained at the location, $z=30, y=0 \mathrm{~mm}$. The vibrational Raman temperatures were determined from measured peak intensity ratios for $v=1 \rightarrow 2$ compared to $v=0 \rightarrow 1$, and $v=2 \rightarrow 3$ compared to $v=0 \rightarrow 1$ in the $\mathrm{N}_{2}$ Stokes vibrational Raman profile. The seven temperature values ranged from 2006 to $2043 \mathrm{~K}$ with an uncertainty of $\sim \pm 40 \mathrm{~K}$. Only the three median values are shown in figure 11 .

Raman measurements on the less fuel rich 0(2) composition were more difficult, particularly for the vibrational Raman, because of the increased flame luminosity. At $30 \mathrm{~mm}$ above the burner face the temperatures measured were $2500 \pm 150 \mathrm{~K}$ from vibrational and $2450 \pm 40 \mathrm{~K}$ from rotational Raman spectra. The lower uncertainties in the rotational temperature data result from the reduced background luminosity associated with the use of smaller slit widths $\left(\sim 2 \mathrm{~cm}^{-1}\right.$ versus $\sim 5 \mathrm{~cm}^{-1}$ resolution for vibrational data). These stated 
uncertainties are statistical only. True uncertainties, particularly for rotational temperatures, are larger and must include uncertainties in temperature corrections for vibrationally excited molecules [41].

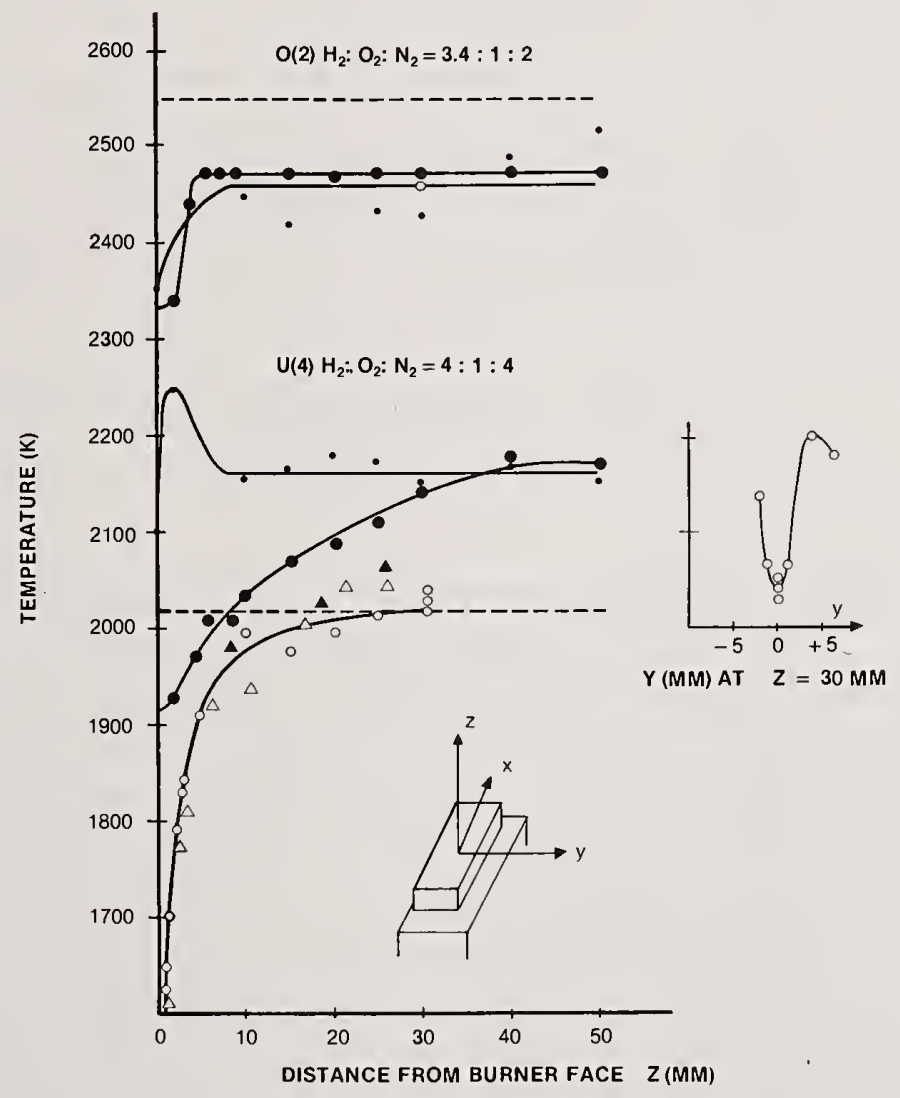

Figure 11. Longitudinal $(z)$ and transverse $(y)$ temperature profiles, at $x=0 \mathrm{~mm}$, for $U(4)$ and $0(2)$ flames using $\mathrm{Na}-\mathrm{D}_{1}$ line reversal (large closed circles), vibrational Raman (open circles) and $\mathrm{OH}$ absorption (small closed circles). Broken horizontal line indicates calculated adiabatic temperature. Burner: rectangular; argon shield on. Flame burns in $z$ direction. Flame gas compositions are given as mole ratios of initial gas mixture. Calculated linear hot gas velocities $=0.75 \times 10^{3} \mathrm{~cm} / \mathrm{s}[U(4)]$ and $0.96 \times 10^{3} \mathrm{~cm} / \mathrm{s}[0(2)]$. Data from figure 10 , normalized to the same time scale, are indicated by closed triangles ( $\mathrm{Na}-\mathrm{D}$ line) and open triangles (Raman). 


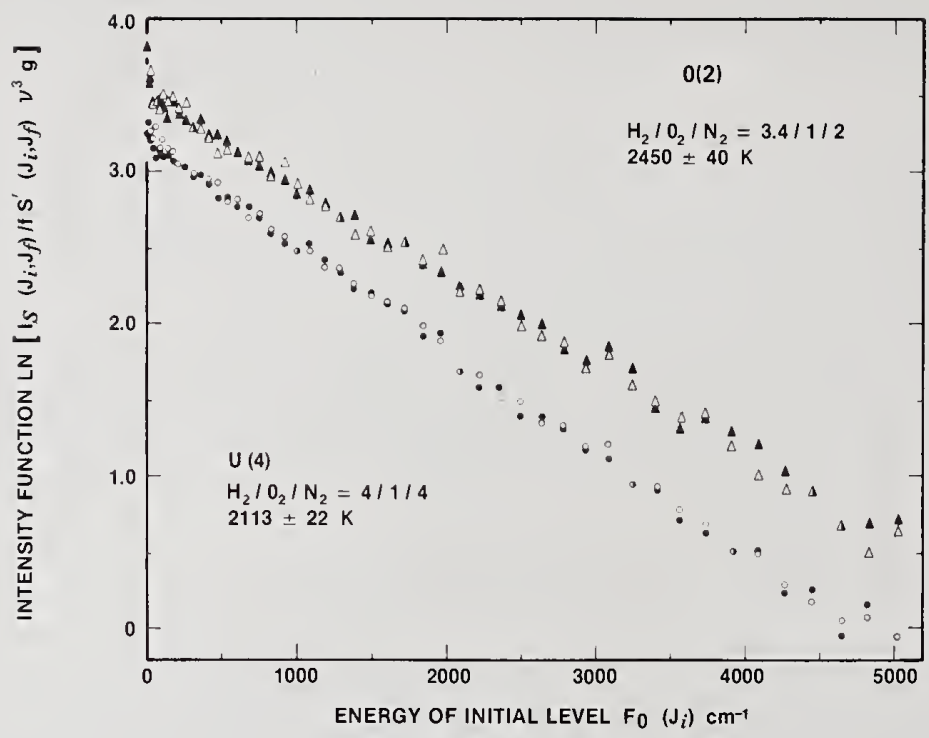

Figure 12. Temperature determination plot (Boltzmann-type) from rotational Raman intensities. $I_{s}$ are relative Raman intensities, measured from spectra such as that shown in figure 7 , and corrected for contributions from scattering by vibrationally excited $\mathrm{N}_{2}$ molecules; $f \mathrm{~S}^{\prime}\left(\mathrm{J}_{j}, \mathrm{~J}_{f}\right)$ is the rotational Raman 1 ine strength corrected for centrifugal distortion effects; is the frequency of the scattered light; $g$ is the nuclear degeneracy factor; and $F_{0}\left(J_{j}\right)$ is the energy of the initial energy level. The temperature is inversely proportional to the slope. The uncertainties listed are \pm two standard deviations including statistical uncertainty only. Large temperature corrections are required to correct for contributions from transitions from vibrationally excited $\mathrm{N}_{2}$ molecules. For the $0(2)$ flame, $T=2450 \pm 40 \mathrm{~K}(2262 \pm 42 \mathrm{~K}$ without correction). For the U(4) flame, $T=2113 \pm 22 \mathrm{~K}(2082 \pm 22 \mathrm{~K}$ without correction). The required experimental slit function was determined by measuring the line shape of the $\mathrm{H}_{2}(\mathrm{~J}=1 \rightarrow 3)$ line at room temperature. The slit function is approximately trapezoidal with $\mathrm{f} \mathrm{wh} \mathrm{m}=2.05 \mathrm{~cm}^{-1}$ and top $=0.76 \mathrm{~cm}^{-1}$. Flame location $z=30$ and $y=0 \mathrm{~mm}$ for $0(2)$ and $z=30$ and $y=2 \mathrm{~mm}$ for $U(4)$ flame.

For the line ratio method of temperature measurement, a suitable band for measurement of the $\mathrm{OH}$ absorption spectrum is that in the region of $306.4 \mathrm{~nm}$ (see fig. 5). A plot of the optical density of the absorption lines versus the number of radiation passes through the flame (fig. 13) shows that many of the lines are not optically thin. Extrapolation of the fractional absorption $I_{\text {abs }}$ to $\alpha_{\nu} \ell<<1$, shown in figure 13 , yields values of $I_{\text {abs }}$ which can be converted by the theory (see Section 2.2) to a temperature. Alternatively, the data can be expressed in logarithmic form, as shown in figure 14 . Both sets of curves (figs. 13 and 14 ) yield the same values of $I_{\text {abs }}$ and hence temperature. The self 
consistency of the extrapolation procedure and the thermal nature (Boltzmann distribution pertains) of the selected rotational states is demonstrated by the linear relationships given in figure 15 for both the $U(4)$ and $0(2)$ flames. Another alternative extrapolation procedure for obtaining corrected values of $\mathrm{I}_{\text {abs }}$ is from a growth curve (van der Held plot) of the type shown in figure 16. The extrapolated $I_{\text {abs }}$ values obtained from the growth curves also agree with those using the procedure in figure 13.

Except for data at the $z=30 \mathrm{~mm}$ location (see fig. 11), where the many line Boltzmann-type plots of figure 15 were used, temperatures were obtained using the corrected intensity ratios for the $R_{2} 4$ and $R_{2} 15$ lines (see fig. 5 ). As is shown in figure 13 , these lines satisfy a condition of relatively low degree of optical thickness. For the $U(4)$ and $0(2)$ flames with one pass, optical thickness temperature corrections of $\sim 0$ and $\sim 19 \mathrm{~K}$ are required, respectively. We estimate from eq. (4) that the error in the temperature measurement using these lines is $40 \mathrm{~K}$ for the $U(4)$ and $60 \mathrm{~K}$ for the $O(2)$ flame compositions.

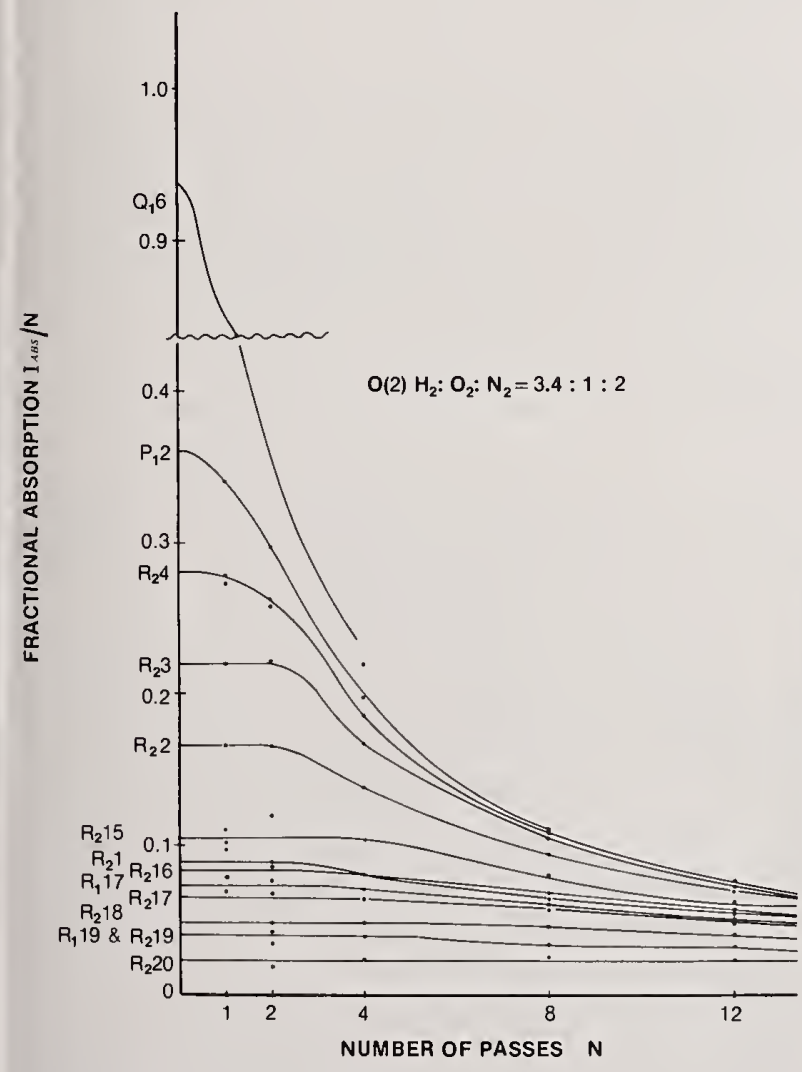

Figure 13. $\mathrm{I}_{\mathrm{abs}} / \mathrm{n}$ vs $\mathrm{n}$, where $\mathrm{I}_{\text {abs }}=$ $\left[\left(I_{\nu}^{0}-I_{v}\right) / I_{\nu}^{0}\right]_{\text {peak }}$ and $n$ is the number of traversals through the flame via the White multi-reflection system at $30 \mathrm{~mm}$ from face of rectangular burner. As $\mathrm{n} \rightarrow 0$ the slope must approach zero. Lines are identified in figure 5 .

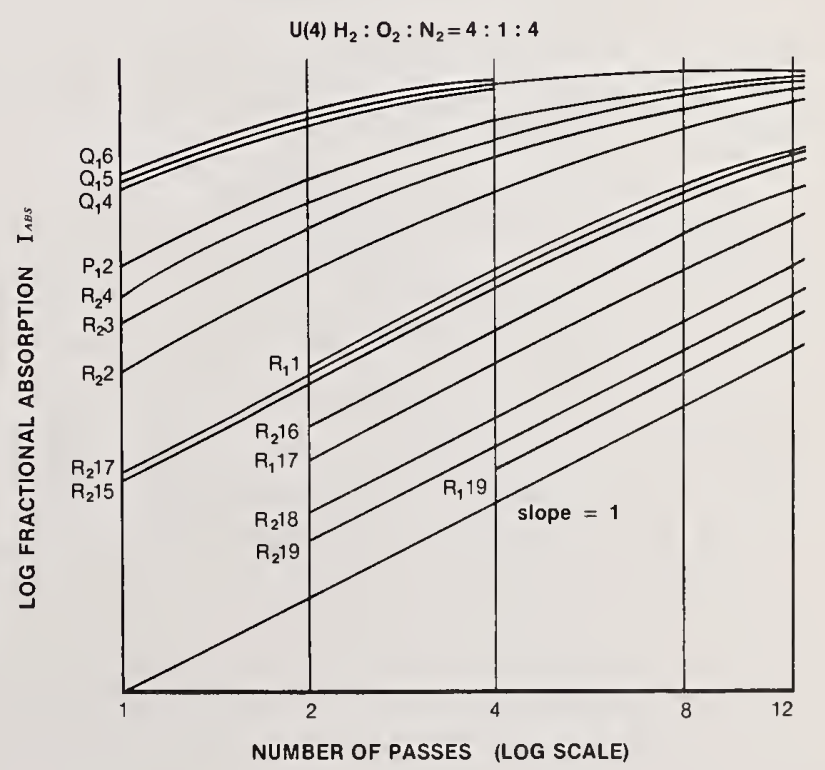

Figure 14. Experimental growth curves (van der Held plots) of absorption lines shown in figure 5 versus number of traversals through flame at $30 \mathrm{~mm}$ from the face of rectangular burner. When $\alpha_{v} \ell<<1$ (optically thin) the slope of each curve approaches the value 1. $\left.I_{\text {abs }}=\left[\left(I_{\nu}^{0}-I_{\nu}\right) / I_{\nu}^{0}\right)\right]_{\text {peak }}$ 


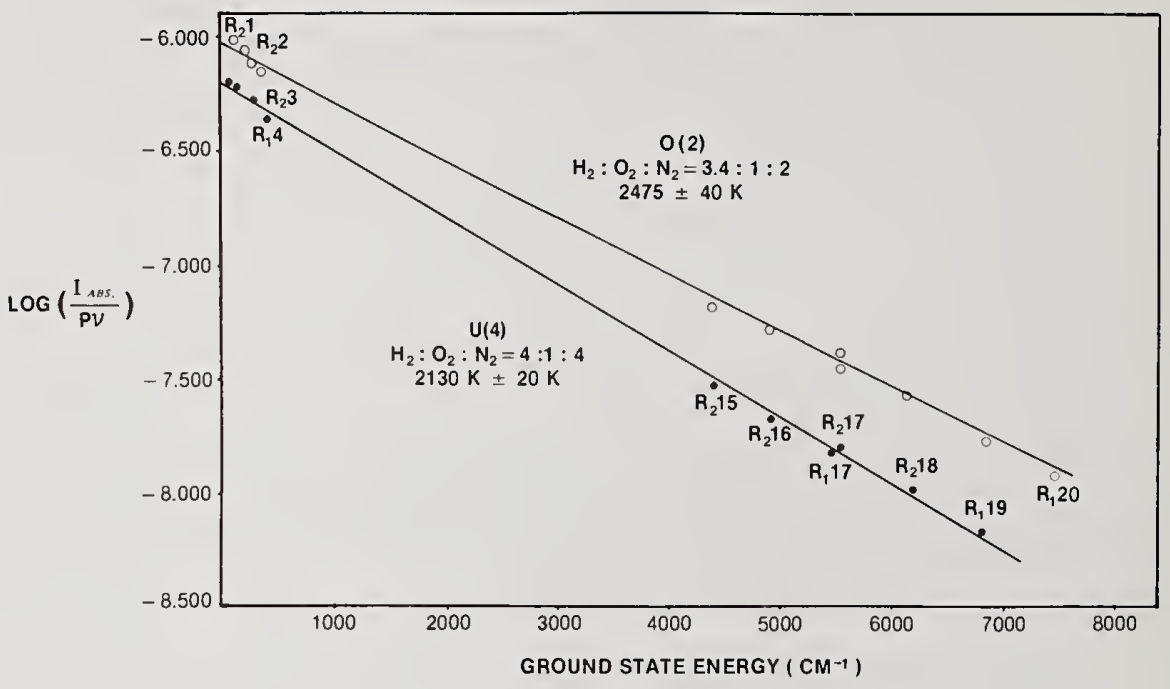

Figure 15. $\log \left[\left(I_{a b s} / n\right) / P \nu\right]_{n \rightarrow 0} \vee s E_{n}$ is energy of initial state of a transition of wave numberv $\left(\mathrm{cm}^{-1}\right)$. $I_{\text {abs }}=\left[\left(\mathrm{I}_{v}^{0}-\mathrm{I}_{v}\right) / \mathrm{I}_{v}^{0}\right]_{\text {peak }}$. Lines are linear least squares fits to data points obtained at the $z=30 \mathrm{~mm}$ position. Data were calculated from extrapolated $\left(I_{\text {abs }} / n\right)_{n \rightarrow 0}$ taken from figure $13 ; P \equiv g_{n} B_{n m}$ where $B_{n m}$ is Einstein probability for absorption; and $E_{n}$ for the labelled transitions are tabulated in reference [37]. The temperature is calculated from the slope $(-2.303 \mathrm{kT})^{-1}$ of the 1 ines where $\mathrm{k}=0.697 \mathrm{~cm}^{-1} / \mathrm{K}$. Rectangular burner data.

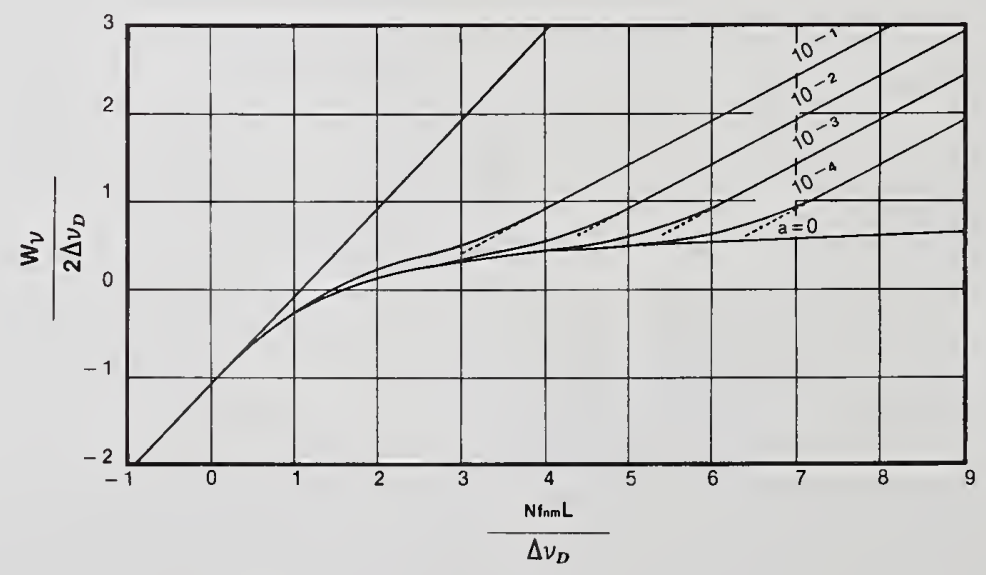

Figure 16. Growth curve (hypothetical) for exponential absorption [49]. $\left.W=\int\left[\left(I_{v}^{\circ}-I_{v}\right) / I_{v}^{0}\right] d v=\left[\left(I_{v}^{\circ}-I_{v}\right) / I_{v}^{0}\right]_{\text {peak }} \cdot \Delta v_{s}\right]$ it $v s f_{n m} \cdot N$ is the number of absorbing "centers" in the initial state $E_{n}$ contained in a $1 \mathrm{~cm}^{2}$ column of length $\ell . f_{n m}$ is the oscillator strength of the transition $n \rightarrow m$. $a=$ damping constant $\gamma /$ Doppler width $\Delta \nu_{D}$. Straight line of slope 1 is region in which $\alpha \ell<<1$ (optically thin). For $\mathrm{OH}$ at $2000 \mathrm{~K}$, a $\sim 10^{-2}$ (collisional broadening neglected). 


\section{Discussion}

\subsection{Cylindrical burner}

Except for the region close to the burner face, the agreement between the results of the various methods is strong evidence that the rotational, vibrational, and electronic temperatures measured, respectively, by the rotational Raman, vibrational Raman, and the Na-D line reversal methods, each represent a thermal equilibrium state (see fig. 10). Close to the burner face, chemiluminescent excitation of $\mathrm{Na}$ atoms by radical recombination [50] leads to an erroneously high temperature determination, particularly for the U(4) flame where the ratio of excess to equilibrium radical concentration is known to be greater. Disagreement between population temperatures for the $\mathrm{Na}-\mathrm{D}$ line and $\mathrm{OH}\left(\mathrm{X}^{2} \Pi\right)$ states are known and have been attributed to possible non-equilibrium state conditions in an atmospheric methane-oxygen flame, particularly under turbulant conditions [18]. The transverse temperature profile (fig. 10) indicates that the flame is non-isothermal along the transverse axis. This condition should not significantly affect the Raman data as they are essentially 'point' source results. The reversal method, on the other hand, measures some transverse line of sight temperature average of the region encountered by the passage of radiation from the reversing source through the flame [28]. Thus, the temperatures measured by the two methods may not be the same. The excellent agreement obtained here is probably due to the presence of a relatively large isothermal central flame region $(\sim 10 \mathrm{~mm})$ as compared with the thinner, non-isothermal outer region.

\subsection{Rectangular burner}

The Raman temperature data (fig. 11) for the U(4) flame are identical (within experimental uncertainty) to those obtained with the cylindrical burner (fig. 10), when allowance is made for the different gas velocities in both cases. These data are also quite consistent with the calculated adiabatic temperature. However, the $\mathrm{Na}-\mathrm{D}$ line reversal temperatures are appreciably different to the Raman results and the corresponding cylindrical burner data. As these data (at $z>10 \mathrm{~mm}$ ) lie well above the adiabatic limit, either the system, with respect to the Na-D line transition, is not at local thermal equilibrium or the average composition across the flame (along $y$ axis) is closer to a stoichiometric (hotter) mixture than the initial U(4) composition. Arguments in favor of the latter condition will be presented in Section 6.

Note (fig. 11) that the $\mathrm{OH}$-absorption temperature data are similar to the Na-D line results at $z \geq 30 \mathrm{~mm}$ but deviate at $z \leq 30 \mathrm{~mm}$. This difference can be attributed to the greater influence of the shield flame properties on the $\mathrm{OH}$ temperature relative to the $\mathrm{Na}-\mathrm{D}$ line data. At $\mathrm{z}<30 \mathrm{~mm}$ most of the $\mathrm{Na}$ is still in the inner flame, but at larger distances radial diffusion of $\mathrm{Na}$ to the hotter (Raman evidence) outer flame will cause an increase in the reversal temperature.

In contrast to the $U(4)$ flame results, the $0(2)$ flame data show good agreement between the three temperature measurement techniques and are consistent with the adiabatic limit. A 
transverse Raman temperature profile (along the $y$-axis) was not obtained for the 0 (2) flame due to interference from the relatively high background luminosity.

\subsection{Burner comparisons}

It is well established $[2,16,47]$ that the $z=30 \mathrm{~mm}$ position for flames supported by Padley-Sugden-type burners (and with similar flow velocities) is a convenient comparison point. This position is sufficiently far removed from the reaction zone to be free of chemiluminescence and other radical-controlled non-equilibrium effects, but is not far enough to be significantly influenced by air-entrainment. Table 2 shows a summary of our data and an inter-comparison with literature data at the $z=30 \mathrm{~mm}$, or an equivalent (with respect to flow time), flame position. The Na-D line data of Miller [47] is typical of tha obtained by other workers of the Sugden school. Note that our corresponding data are in excellent agreement. With the exceptions already noted, the intra-comparison for the various techniques and burners is also very good and indicative of the reliability of the exper mental approach.

Table 2. Flame Temperature Comparisons ${ }^{a}$

\begin{tabular}{|c|c|c|c|c|c|c|}
\hline $\begin{array}{c}\text { Initial Mole } \\
\text { Ratio }\end{array}$ & $\begin{array}{l}\text { Argon } \\
\text { Shield }\end{array}$ & $\begin{array}{l}\text { Na-D Line } \\
\text { Reversal }\end{array}$ & OH Absorption & $\begin{array}{c}\text { Vibrational } \\
\text { Raman }\end{array}$ & $\begin{array}{l}\text { Rotational } \\
\text { Raman }\end{array}$ & $\begin{array}{l}\text { Calculated } \\
\text { Adiabatic }\end{array}$ \\
\hline $\mathrm{H}_{2} / \mathrm{O}_{2} / \mathrm{N}_{2}$ & & & & & & \\
\hline \multirow[t]{4}{*}{$4.0 / 1.0 / 4.0$} & no & $2100 \pm 20$ & & $2146 \pm 40$ & & \\
\hline & yes & $2060 \pm 20$ & $2160 \pm 40$ & $1980 \pm 40$ & $\mathrm{~g}$ & 2019 \\
\hline & -- & $1990 \pm 20^{C}$ & & $2010 \pm 40^{C}$ & & \\
\hline & -- & $2020^{d}$ & & & & \\
\hline \multirow[t]{2}{*}{$3.4 / 1.0 / 2.0$} & yes & $2470 \pm 20$ & $2425 \pm 60$ & $2500 \pm 150^{b}$ & $f$ & 2545 \\
\hline & -- & $2475^{d}$ & & & & \\
\hline
\end{tabular}

\footnotetext{
a Temperature in degrees $\mathrm{K}$ measured at a height of $30 \mathrm{~mm}$ (cylindrical burner) or $15 \mathrm{~mm}$ (rectangular burner) above the burner face. Rotational Raman uncertainties are given as two standard deviations.

Data obtained at $z=30 \mathrm{~mm}, y=0 \mathrm{~mm}$ but value should be similar at $z=15 \mathrm{~mm}$ (see fig. 11).

${ }^{C}$ Data underlined are for the cylindrical burner, all other data refer to the rectangular burner.

dData obtained by W. Miller [47] using an almost identical burner and gas flow condition. ${ }^{\mathrm{e}}$ These data should be accurate to within a degree.

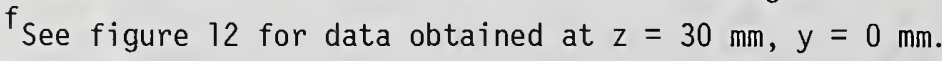

${ }^{g}$ A comparison of rotational and vibrational Raman temperatures was made at $z=30 \mathrm{~mm}$, $y=2 \mathrm{~mm}$, where $T=2113 \pm 22$ and $2096 \pm 40$, respectively.
} 


\section{Conclusions and Summary}

Temperatures of atmospheric pressure premixed $\mathrm{H}_{2} / \mathrm{O}_{2} / \mathrm{N}_{2}$ flames have been measured by $\mathrm{Na}-\mathrm{D}$ line reversal, $\mathrm{OH}$ absorption, and Raman scattering (vibrational and rotational) techniques. With a cylindrical burner of design very similar to the widely used Padley-Sugden burner [16], longitudinal temperature profiles (along the direction of gas flow) measured by the $\mathrm{Na}-\mathrm{D}$ line and Raman scattering methods agreed well within experimental error ( $\pm 50 \mathrm{~K}$ ) and with previous $\mathrm{Na}-\mathrm{D}$ line temperature measurements [47] for distances of 5-50 $\mathrm{mm}$ above the burner surface. For distances less than $5 \mathrm{~mm}$ the Na-D line temperatures are excessively high due to well known chemiluminescent effects.

Temperature measurements on $U(4)$ and $0(2)$ flame compositions, using a rectangular burner, were in serious disagreement for the former but not the latter flame. The discrepancies can be traced to the effects of air entrainment and subsequent combustion of $\mathrm{H}_{2}$ in this flame. The rectangular burner produced a thin sheet of flame with a larger circumference-to-area ratio than for the cylindrical burner, making the rectangular burner more susceptible to air entrainment effects. That the 0 (2) flame apparently did not suffer from this difficulty may be explained by the lower excess $\mathrm{H}_{2}$ present in this case. However, the extent of this entrainment difficulty for various flame compositions cannot be determined a priori. This indicates that caution must be exercised in the selection, and empirical validation, of new burner-flame composition combinations if the results are to be used for temperature and chemical diagnosis.

A summary of the experimental evidence for significant air entrainment with the rectangular burner is as follows:

- Raman temperature transverse profiles, measured at a constant height (30 mm) above the burner, indicate that the outer part of the flame is 150-200 K hotter than the flame center.

- In the center of the flame, and at a distance of $30 \mathrm{~mm}$ above the burner, temperatures measured by $\mathrm{line-of-sight} \mathrm{techniques} \mathrm{(} \mathrm{Na}-\mathrm{D}$ line reversal and $\mathrm{OH}$ absorption) are higher by $\sim 150 \mathrm{~K}$ than Raman temperature measurements.

- Flame temperatures at $30 \mathrm{~mm}$ above the burner increased when the argon shield flow, which normally provides some protection against air entrainment, was removed. The Raman temperature increased by $125 \mathrm{~K}$ and the $\mathrm{Na}-\mathrm{D}$ line value by $42 \mathrm{~K}$.

- Measured temperatures using Na-D line reversal or $\mathrm{OH}$ absorption are above the calculated adiabatic flame temperature (2019 K). Similarly, temperatures determined from Raman scattering are above the calculated adiabatic temperatures near the outer parts of the flame or in the center of the flame without the argon shield flow.

- The temperature measured by the three techniques on the cylindrical burner are in agreement within experimental error. However, as is evident by the Raman temperature transverse profile at $30 \mathrm{~mm}$ above the burner, the temperatures are again non-uniform, the outer part of the flame being much hotter.

The major difference in burner performance is that the cylindrical burner provides a central region of essentially constant temperature over a lateral distance of $\sim 10 \mathrm{~mm}$. Thus 
the presence of a narrow high temperature outer region has relatively little effect on the $\mathrm{Na}-\mathrm{D}$ line or $\mathrm{OH}$ temperature measurements. The flame centers (along $\mathrm{z}$ axis) for both burner types had essentially identical temperature profiles (on a flow time basis) as determined by the Raman technique.

We should emphasize that the temperature measurement methods of (1) Raman, (2) Na-D line, and (3) $\mathrm{OH}$ absorption, with shielded flames, each refer to a different flame element. Method (1) gives essentially point source information, method (2) data on the whole lateral section of the seeded inner flame, and method (3) data on the combined lateral sections of the inner and shield flames. Thus, if these lateral sections are non-isothermal, each method will yield different temperature information.

Comparison of the various optical temperature methods indicates that the Na-D line reversal technique is the best method for measuring temperatures where the optical paths through the inner flame are isothermal. This method has the greatest precision (limited by the reproducibility of reading an optical pyrometer), is faster and easier, and requires less expensive equipment than Raman techniques. However, when "point" temperature measurements are required, as in flames with large amounts of air entrainment, Raman measurement are required since line of sight measurements give only an integrated average temperature.

We wish to acknowledge Dr. W. J. Miller for the loan of his Padley-Sugden-type burner, which we reproduced, and for useful discussions on the Na-D line reversal method. Dr. D. W. Bonnell assisted with the calculation of adiabatic flame temperatures and with the design of burners and gas feed systems. Mr. A. Sessoms assisted with data taking and maintenance of the apparatus. Suggestions by Dr. Gary Mallard for improving the manuscript are also appreciated.

\section{References}

[1] Reuther, J. J., Measures of effectiveness and mechanisms for chemical and physical inhibition of quenched adiabatic premixed flames, this volume.

[2] Miller, Jr., W. J., The use of flames as media for the study of ion-molecule thermochemistry, this volume.

[3] Jensen, D. E., Chemical processes in high Reynolds number turbulent diffusion flames, this volume.

[4] Gordon, S. and McBride, B. J., NASA SP-273, NTIS Document No. 7137775 (1971).

[5] Creighton, J. R. and Lund, C. M., Modeling study of flame structure in low-pressure, laminar, premixed methane flames, this volume.

[6] JANAF, Joint Army Navy Air Force Thermochemical Tables, 2nd ed., NSRDS-NBS 37 (1971) U.S. Government Printing Office, Washington, D.C.

[7] Baulch, D. L., Drysdale, D. D., Horne, D. G., and Lloyd, A. C., Evaluated kinetic data for high temperature reactions, Vol. I., Homogeneous Gas Phase Reactions of the $\underline{H}_{2} \frac{10}{2}$ System (Butterworths, London, 1972). 
[8] Ratajczak, E. and Trotman-Dickenson, A. F., Supplementary Tables of Bimolecular Gas Reactions (Publications Department, UWIST, Cardiff, Wales, 1970).

[9] Eckbreth, A. C. and Hall, R. J., CARS diagnostic investigations of flames, this volume.

[10] McDonald, J. R., Baronavski, A. P., Pasternack, L., Lamont, S., and Harvey, A. B., New optical diagnostic techniques for temperature and species concentration measurements in flames, this volume.

[11] Roquemore, W. M. and Yaney, P. P., Comparison of thermocouple, gas sampling and Raman measured temperatures in an afterburning turbojet engine plane, this volume.

[12] Drake, M. C. and Rosenblatt, G. M. , Raman spectroscopy in high temperature chemistry, this volume.

[13] Anderson, W. R., Allen, Jr., J. E., Fansler, T. D., and Crosley, D. R., Laser generated opto-acoustic pulses in an atmospheric pressure flame, this volume.

[14] Bradshaw, J., Bower, J., Weeks, S., Fujiwara, K., Omenetto, N., Haraguchi, H., and Winefordner, J. D., Application of the two line atomic fluorescence technique to the temporal measurement of small volume flame temperatures, this volume.

[15] Self, S. A., Diagnostic techniques in combustion MHD flows, this volume.

[16] Padley, P. J. and Sugden, T. M., Proc. Roy. Soc. A248, 235 (1958).

[17] Hastie, J. W., High Temperature Vapors: Science and Technology (Academic Press, New York, 1975).

[18] Fissan, H. J., Combust. Flame, 17, 355 (1971); ibid, 19, 11 (1972).

[19] Klingenberg, G. and Mach, H., Combust. Flame, 27, 163 (1976).

[20] Daily, J. W. and Kruger, C. H., J. Quant. Spectrosc. Radiat. Transfer, 17, 327 (1977).

[21] Snelleman, W., Combust. Flame, 11, 453 (1967).

[22] Lapp, M. , Goldman, L. M., and Penney, C. M., Science, 175, 112 (1972).

[23] Striker, W., Combust. Flame, 27, 113 (1976).

[24] Ahrich, R., Vovelle, C., and Delbourgo, R., Combust. Flame, 32, 171 (1978).

[25] Bennett, J. G., and Pirani, M., J. Inst. Fue1, 12, No 64, SI (1939).

[26] Fery, C., Compt. Rend. 137, 909 (1903).

[27] Gaydon, A. G. and Wolfhard, H. G., Flames. Their Structure, Radiation and Temperature, 3rd ed., p. 239. (Chapman and Ha11, Ltd., London, 1970).

[28] Sasaki, Y., Jap. J. App 1. Phys. $\underline{5}, 439$ (1966).

[29] Definitions, Terminology and Symbols of Radiation Quantities are as in Max Planck, Theory of Heat Radiation, 2nd ed. (1913); (Dover Publications, New York, 1959).

[30] Czerny, M. and Walther, A., Tables of the Fractional Functions for the Planck Radiation Law (Springer-Verlag, Berlin, 1961).

[31] Nielsen, J., Thornton, V., and Dale, E. B., Rev. Mod. Phys. 16, 307 (1944).

[32] De Prima, C. R. and Penner, S. S., J. Chem. Phys. 23, 757 (1955).

[33] Kohn, H., Phys. Z. $\underline{29}, 49$ (1928).

[34] Bass, A. M. and Broida, H. P., NBS Circ. 41 (1953).

[35] Fuchtbauer, C., Phys. Z. 21, 322 (1920).

[36] Ladenburg, R., Z. Phys. 4, 451 (1921).

[37] Learner, R. C. M., Proc. Roy. Soc. A269, 311 (1962). 
[38] Broida, H. P., Temperature. Its Measurement and Control in Science and Industry, Vol. II, p. 269 (Reinhold Publ. Co., New York, 1955).

[39] White, J. U., J. Opt. Soc. Amer. 32, 285 (1942).

[40] Lapp, M. and Penney, C. M., in Adv. in Infrared and Raman Spectrosc. R. J. H. Clark and R. E. Hester, eds., Vol. 3, chap. 6 (Heyden and Sons, Ltd., London, 1977).

[41] Drake, M. C. and Rosenblatt, G. M., Combust. Flame, 33, 179 (1978).

[42] Schoenung, S. M. and Mitche11, R. E., Sandia Report SAND 77-8722 (0ct. 1977).

[43] Setche11, R. E., AIAA Paper 76-28 (1976).

[44] Hastie, J. W. and Bonne11, D., Species Concentration Profiles for Premixed $\mathrm{H}_{2} / \mathrm{O}_{2} / \mathrm{N}_{2}$ Flames, determined by Molecular Beam Mass Spectrometry, to be published (1979).

[45] Hastie, J. W. and McBee, C., Mechanistic Studies of Triphenylphosphine 0xide-Poly (Ethyleneterephthalate) and Related Flame Retardant Systems, NBSIR 75-741 (1975).

[46] Adams, K. M., Browner, R. F., Dagna11, R. M., and West, T. S., Anal. Chem. 42, 939 (1970).

[47] Miller, W. J., J. Chem. Phys. 57, 2354 (1972).

[48] Hayhurst, A. N. and Kittelson, D. B., Combust. Flame, 28, 301 (1977).

[49] Unsold, A., Physik der Sternatomospharen (Springer-Verlag, Berlin, 1938).

[50] Carabetta, R. and Kaskan, W., Eleventh Symp. (Intl.) Combust. (The Combustion Inst.), p. 331 (1967).

\section{Discussion}

Comment (Bradshaw): It is extremely important when doing comparisons between seeded and non-seeded techniques that great care be taken in ensuring that the seed species remain in a radially homogeneous volume such as that in a shielded flame where an isotropic environment is maintained around the inner seeded flame over the entire height measurement regime.

This will enable comparisons between seeded and non-seeded techniques without the problems associated with edge effects on 1 ine of sight seeded techniques.

Response (Hastie): This point is covered in the written version of this presentation. In actual fact no disparity was found between seeded and non-seeded data, e.g. compare the line reversal and $\mathrm{OH}$-absorption data. Where disparate data did occur, this was between the integrated path length methods (reversal and $\mathrm{OH}$ ) and the point source Raman technique. 
National Bureau of Standards Special Publication 561, Proceedings of the 10th Materials Research Symposium on Characterization of High Temperature Vapors and Gases held at NBS, Gaithersburg, Maryland, September 18-22, 1978. Issued October 1979.

\section{OPTICAL DIAGNOSTICS IN CVD REACTORS ${ }^{1}$}

G. H. Miller, A. J. Mulac, and P. J. Hargis, Jr. Sandia Laboratories, Laser Applications and Spectroscopy Division, Albuquerque, NM 87185

The application of spontaneous rotational Raman and fluorescence spectroscopy to linear CVD reactors is discussed. $\mathrm{N}_{2}$ rotational temperature profiles were obtained above a susceptor at 600,800 , and $950^{\circ} \mathrm{C}$. The gas flow conditions and susceptor temperatures investigated cover the transition regime between dominance of the flow by inertial or by buoyancy effects. Fundamental data needed to carry out fluorescence measurements of CVD gases and intermediates are being obtained. $\mathrm{NH}$ free radicals for these studies were produced by the $266 \mathrm{~nm}$ photodissociation of $\mathrm{NH}_{3}$.

\section{Introduction}

In this paper we discuss optical diagnostics applicable to the gas phase aspects of chemical vapor deposition (CVD). Two areas for which optical diagnostic techniques are particularly advantageous have been singled out. These are temperature measurements obtained by spontaneous rotational Raman spectroscopy in a flowing gas system and measurement of the concentration of minor species by a fluorescence technique. We present experimental methods for making the required measurements and furnish some preliminary results from our experiments.

There are a number of types of CVD systems which differ in: 1) compound deposited and constituents used to achieve that compound; 2) geometry of the cell; 3) energy input mechanism; and 4) pressure regime. In addition, each system has various parameters, controllable over some range. These include flow rate, pressure, percentage of the various constituents, and energy input rate. Since it is not the purpose of this paper to review the vast body of CVD technology, we concentrate on one system and note that the same diagnostic techniques, with appropriate modifications, are applicable to other systems. Figure 1 shows schematically the system chosen, which is a linear flow cell in which the energy is supplied by means of a heated graphite block (susceptor). In an operational system this block would support the substrate on which the deposition would occur. The

${ }^{1}$ This work was supported by the United States Department of Energy. 
susceptor is heated by an $r$ induction furnace. The gas mixture flows longitudinally over the heated susceptor, thereby acquiring energy. The chemical reaction occurs on the surface and/or in the gas phase above the surface with the result that deposition takes place on the substrate as well as on other surfaces in the cell. Our objective is to investigate the deposition of $\mathrm{Si}_{3} \mathrm{~N}_{4}$ on a silicon substrate covered with a thin film of $\mathrm{SiO}_{2}$. In this case the gas mixture consists of $\mathrm{N}_{2}, \mathrm{NH}_{3}$, and $\mathrm{SiH}_{4}$ in the approximate ratio 40:1:0.01. The total pressure is roughly one atmosphere, and the flow velocity is of the order of $1 \mathrm{~cm} / \mathrm{s}$. The susceptor temperature is in the vicinity of $750^{\circ} \mathrm{C}$. The reaction rate is extremely sensitive to this temperature [1] ${ }^{2}$. This high sensitivity to surface temperature indicated the desirability of obtaining a three-dimensional temperature map above the susceptor, particularly in the immediate vicinity of the surface. In the past these measurements have been made in CVD reactors by several techniques. Ban [2] and Cochet, et a1. [3] have made such measurements using a thermocouple probe. However, measurements made with physical probes are suspect because the temperature distribution and the flow field may be influenced by the probe. Sedgwick, et al. [4], have measured the temperature without perturbing the flow by using rotational-vibrational Raman spectra. This also allowed them to achieve excellent spatial resolution. They used the ratio of the integrated Stokes and anti-Stokes $\mathrm{N}_{2} \mathrm{Q}$-branch spectra. It is objectionable to use this procedure in the CVD temperature regime due to the extremely small anti-Stokes intensity. $A$ better procedure for extracting temperature and density from vibrational Raman Q-branch spectra has been described by Hill, et al. [5].

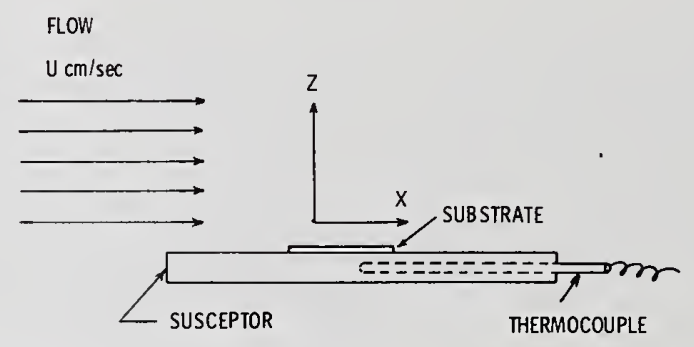

Figure 1. Schematic diagram of Tinear CVD.

\subsection{Temperature measurements}

The temperature measurement method described herein is also an application of spontaneous Raman scattering. Here the pure rotational spectrum is used for the temperature measurement [6]. This method has the following advantages. First, since S-branch $(\Delta \mathrm{J}=+2)$ spectra are used the line spacing is large enough so that the individual lines can be resolved. This simplifies measurement and the analysis. Second, because of the low rotational transition energies that are involved, both the Stokes and anti-Stokes spectra can

${ }^{2}$ Figures in brackets indicate the literature references at the end of this paper. 
be measured. This gives two independent temperature determinations, thereby providing a convenient check on both the measurements and the effects of blackbody radiation from the hot susceptor. The blackbody background makes the temperature obtained from the antiStokes spectrum too low and that from the Stokes spectrum too high. The background correction should bring the two more into agreement. Finally, the problem of interference from fluorescence is different from that in vibrational Raman (Sedgwick and Smith [4] noted an interference took place in a mixture containing chlorinated silane). For laser-induced fluorescence the anti-Stokes spectrum should be free of interference. Further, since only a few of the rotational transitions are required to provide a temperature, it may be possible that one can choose lines that have no interference.

Spontaneous Raman scattering measurements require sophisticated instrumentation including a laser, double monochromator, photomultiplier, and counting system. The cell must be provided with optical windows for the laser light and the Raman-scattered light. It is necessary to move the cell rather than the optical system to achieve spatial scanning due to the critical optical alinement. Variations in the index of refraction in either the laser path or the viewing path could produce systematic temperature variations and/or contribute additional noise. The system developed for this work will be described in Section 2 and some of the data obtained will be presented.

\subsection{Concentration measurements}

Any understanding of the CVD reaction requires knowledge of the concentration of some of the minor species. These include not only species which are normally stable and are admitted at a low rate (low concentration reactants and impurities) but also radicals which might be produced at a high rate but which have a very short life. Traditionally such concentrations have been determined mass spectrometrically $[2,3]$. This requires that some type of movable probe be inserted in the sensitive region. The flow intercepted by this probe is piped into the mass spectrometer ion source and the species are analyzed. The problems associated with this technique are apparent. The flow is perturbed and perhaps with it the local temperature and/or the reaction process. More important, reactive species may have undergone significant concentration changes between the region of interest and the ion source, so that the abundance obtained cannot be related to what is present in the flow.

As an alternative to mass spectroscopy, we are implementing an optical fluorescence system. Selected species are optically excited by means of a tunable laser and the fluorescence radiation is collected and measured. The advantages of such a system are the same as those in the Raman spectroscopy temperature measurement. There is no structural perturbation placed in the flow. The measurement is made in situ, so that no changes occur due to the transporting of the species to another apparatus. The region sampled can be small and well defined. The sensitivity is dependent on the molecule involved, but is generally good. Following are some of the difficulties and restrictions in the use of this method. 1) The absorption and emission spectra of the species of interest must be known. In 
addition there must be a radiative state of the species which can be excited by an available laser. 2) The absorption cross sections and the radiative lifetimes for the transitions of interest must be known. 3) The collisional deactivation cross sections for the excited states of interest and the major constituents must be known. 4) Generally only one molecular species can be excited by a particular laser beam. 5) 0perationally the cell must be such that the laser beam can be introduced and the fluorescence viewed.

For the species of interest, very little is known about the spectra and the collisional deactivation probabilities. It has thus been necessary to carry on a program to determine these values. This program, along with the results obtained thus far, is described in Section 3.

\section{Temperature Measurements by Rotational Raman Spectroscopy}

\section{1 Method and apparatus}

For a diatomic molecule, the counting rate, $\dot{n}$, for the rotational Raman line having a lower state, $\mathrm{J}$, is given by [7]:

$$
\dot{n}(J)=\frac{\eta_{v}^{3} S(J) \rho f}{Q_{r}} \exp \left[-\frac{B h c}{k T} u\left(J_{i}\right)\right] \text {. }
$$

Here $\nu$ is the frequency of the Raman-shifted radiation, $\rho$ is the density of the scattering gas, $f$ is the laser photon flux, $B$ is the rotational constant of the molecule, $T$ is the Kelvin temperature, and $h, c$, and $k$ are the usual constants ( $P$ lanck, velocity of light in a vacuum, and Boltzmann, respectively). $Q_{r}$ is the rotational partition function and is approximately $\frac{k T}{B h c}$. The line strength, $S(J)$, is given by:

$$
S(J)=\frac{3(J+1)(J+2)}{2(2 J+3)}
$$

and $u\left(J_{j}\right)$, is given approximately by:

$$
u\left(J_{i}\right)=J_{i}\left(J_{i}+1\right)
$$

In the last expression, $J_{j}$ refers to the initial state. The constant, $\eta$, includes all of the constant factors involved in producing, collecting, transmitting, and detecting the Raman-scattered light. To obtain the temperature, the above equation is rewritten in the following form:

$$
\ln \left[\frac{\dot{n}(J)}{S(J)}\left(\frac{\nu_{0}}{\nu(J)}\right)^{3}\right]=-\frac{B h c}{k T} J_{i}\left(J_{i}+1\right)+c \text {, }
$$


where $\nu_{0}$ is the laser frequency. The slope from a linear fit of the logarithms to the corresponding values of $\mathrm{J}_{i}\left(\mathrm{~J}_{i}+1\right)$ can then be used to obtain the temperature. Although we have been concerned only with the temperature measurement, the density also could be obtained from the same data. The constant $C$ is given by:

$$
c=\ln \frac{\eta f_{v_{0}}^{3}}{Q_{r}} \rho .
$$

One can determine the product, $\eta f$, if a data run is made at known density, noting that $Q_{r}$ is the negative reciprocal of the slope.

Since the slope is used for temperature determination, the lines with the extreme $J$-values are the most important. Consequently we chose to make measurements on two lines having small $\mathrm{J}$ and two with large $\mathrm{J}$ and ignored those in between. Any time which one might devote to counting on lines of intermediate $J$ would be better spent in improving the statistics for the extreme J-valued lines.

The experimental arrangement is shown in figure 2. The light source was a $\mathrm{CW}$ argon ion laser operating at $488 \mathrm{~nm}$ wavelength and at an output power of 1 watt. The output beam diameter was expanded by a factor of two to $2.5 \mathrm{~mm}$. This permitted beam propagation over a long distance without objectionable diffraction effects. Turning mirrors were used to adjust the beam position and direction in a multipass 1 ight system [8] within which the CVD cell was located. This multipass system reflected the laser beam back through the scattering volume many times so that the photon flux, $f$, was greatly enhanced. Typically, gains of about 20 over the singlepass flux were obtained. There is a problem in using multipass systems in this environment. When the gas flow became turbulent the paths of the light beams in the multipass cell were disturbed by index of refraction fluctuations. The long beam path $(\sim 50 \mathrm{~m})$ resulted in large fluctuations in the count rate. The optical system was adjusted so that the beam passed through a minimum diameter in the viewing region at each transit. It is estimated that this diameter was several hundred microns.

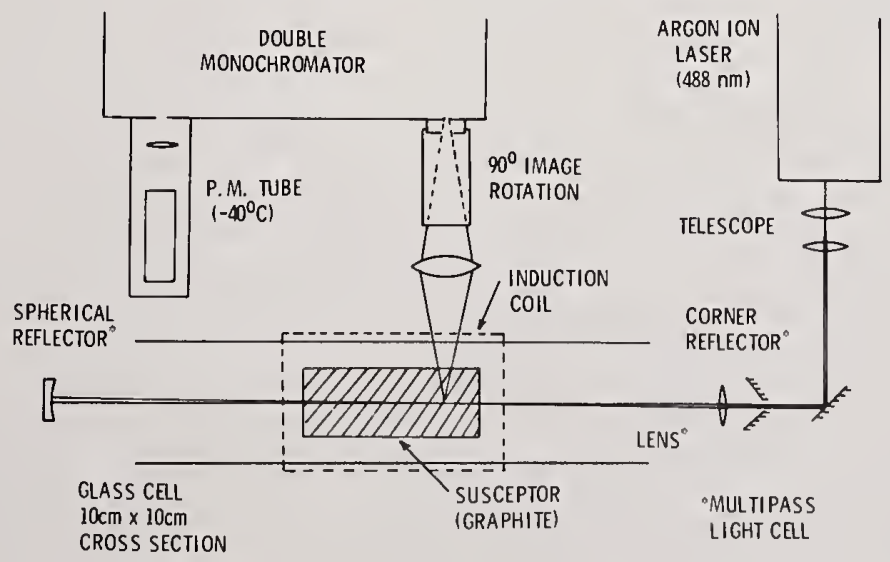

Figure 2. Spontaneous rotational Raman experimental arrangement. 
The Raman scattered light was collected and focused on the entrance slit of the double monochromator by an f/4 lens. The optical system had a magnification of two and filled the monochromator optics. Because the source was horizontal and the entrance slit vertical, a $90^{\circ}$ image rotator was placed in the light path. The scattering volume was a cylinder approximately 100 microns in diameter and $5 \mathrm{~mm}$ long. The monochromator setting was controlled by a stepping motor, which required 500 pulses to advance the grating setting by one nanometer. In this experiment the procedure was to advance the monochromator setting rapidly ( 100 pulses per second) from one rotational line of interest to the next. The counts from a line were integrated for a prescribed period and the monochromator was then advanced to the next line. This process was repeated through four anti-Stokes and four Stokes lines with a background measurement for each spectrum. Unequal entrance and exit slit widths ( $150 \times 250$ microns) were used in order to achieve a trapezoidal slit function. This enabled us to tolerate the irregularities inherent in the monochromator drive. The flat top of the slit function corresponded to $0.04 \mathrm{~nm}$. It was, therefore, necessary to achieve an initial monochromator setting with a precision better than this. This was accomplished by making a high resolution scan of an atomic Xe line at the start of each run. The transmitted light was detected by a photomultiplier tube. This tube had a small photocathode and was cooled to $-40{ }^{\circ} \mathrm{C}$ to minimize dark current. The output pulses were processed by an amplifier-discriminator, and were recorded by a multichannel scaler controlled by a microprocessor.

The microprocessor: a) supplied the required number of pulses to advance the monochromator to the next position; b) advanced the multichannel scaler to the next channel; c) opened a gate permitting the pulses from the discriminator to reach the multichannel scaler; and d) closed the gate after a specified counting time and returned to (a).

The CVD cell is shown in figure 3 and consisted of a pyrex tube, square in cross section, $10 \mathrm{~cm}$ on a side. The overall length was $78 \mathrm{~cm}$. The graphite susceptor was $12.7 \mathrm{~cm} \times 5 \mathrm{~cm} \times 1.3 \mathrm{~cm}$, and was located approximately in the center of the cell. The susceptor had a chromel-alumel thermocouple imbedded in a well along the longitudinal axis. A horizontal quartz platform extended from the susceptor to the sides of the cell over the susceptor length and all the way across the cell from the front edge of the susceptor to a distance $15 \mathrm{~cm}$ in front. The top of the susceptor was flush with the quartz platform. The space underneath the quartz and susceptor was blocked so that the gas was constrained to flow above the susceptor. The gas was admitted and removed through four equally-spaced fittings in the front and rear flanges, respectively. These were directed radially toward the axis. Polished quartz flats, antireflective coated on both sides at $488 \mathrm{~nm}$, were mounted on the end flanges. The loss was negligible when these windows were inserted in the multipass light cell. The Raman signal was transmitted through the pyrex wall. Even though the wall exhibited much striation and was of poor optical quality, the effect on the signal was small. The susceptor was heated by rf induction. A pancake excitation coil, constructed of copper tubing, was mounted to the 
underside of the cell in the region of the susceptor. The rf leads were flexible so that the cell could be moved to change the region sampled. The cell was mounted on a threeaxis translator which permitted a region $10 \mathrm{~cm}$ long, $5 \mathrm{~cm}$ high, and $5 \mathrm{~cm}$ wide to be scanned.

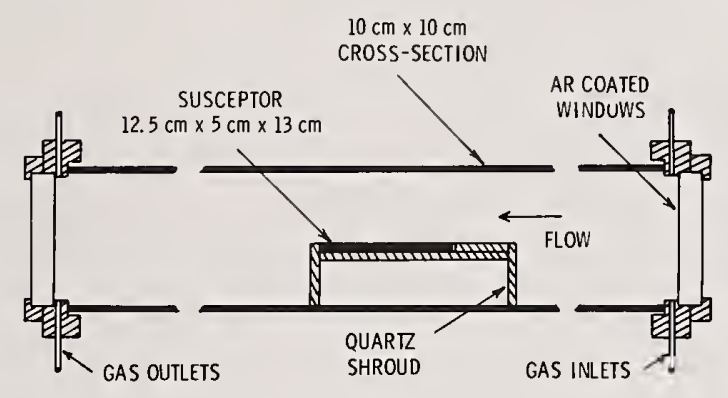

Figure 3. Details of the CVD cell.

\subsection{Rotational Raman temperature results}

Vertical temperature profiles on the susceptor centerline along the flow direction have been obtained for several susceptor temperatures and nitrogen flow rates. The nominal susceptor temperatures used throughout are thermocouple readings from the center of the susceptor. Susceptor temperatures of 600,800 , and $950{ }^{\circ} \mathrm{C}$ have been examined with $\mathrm{N}_{2}$ flow rates of $0.6,3$, and $15 \mathrm{l} / \mathrm{min}$ which correspond to $0.2,1$ and $5 \mathrm{~cm} / \mathrm{s}$ flow velocities in the reactor.

Figures 4 and 5 show centerline temperature profiles at $x=0.64 \mathrm{~cm}, 6.35 \mathrm{~cm}$, and $11.43 \mathrm{~cm}$ where the upstream edge of the susceptor is at $x=0.0$. These were obtained for flow velocities of $0.2 \mathrm{~cm} / \mathrm{s}$ and $1 \mathrm{~cm} / \mathrm{s}$ at a nominal $800^{\circ} \mathrm{C}$ susceptor temperature. Both leading-edge temperature profiles exhibit the largest temperature gradients as the cold gas first interacts with the surface. The temperature profile at the center $(x=6.35 \mathrm{~cm})$ for the $0.2 \mathrm{~cm} / \mathrm{s}$ flow velocity exhibits considerable heating of the gas above the susceptor whereas the trailing edge profile returns to a large gradient. The $1 \mathrm{~cm} / \mathrm{s}$ data exhibit a different character with the center and trailing edge profiles quite similar, much like an ordinary boundary layer. This change with flow rate is a manifestation of the interaction of forced convection and natural convection present in this flow. We will discuss this flow below. The data points shown represent the average of temperature determinations from the anti-Stokes spectrum and the Stokes spectrum obtained successively.

Figure 6 is a more detailed plot of the center-temperature profile for a $950{ }^{\circ} \mathrm{C}$ susceptor temperature and $0.2 \mathrm{~cm} / \mathrm{s} \mathrm{N}_{2}$ flow velocity. The length of the bars on the data points indicates the differences between Stokes and anti-Stokes temperature values used to obtain the average. These differences are very much larger than the statistical uncertainty in the data and show no systematic character such as Stokes temperature consistently being higher than that from the anti-Stokes, etc. Our temperature 


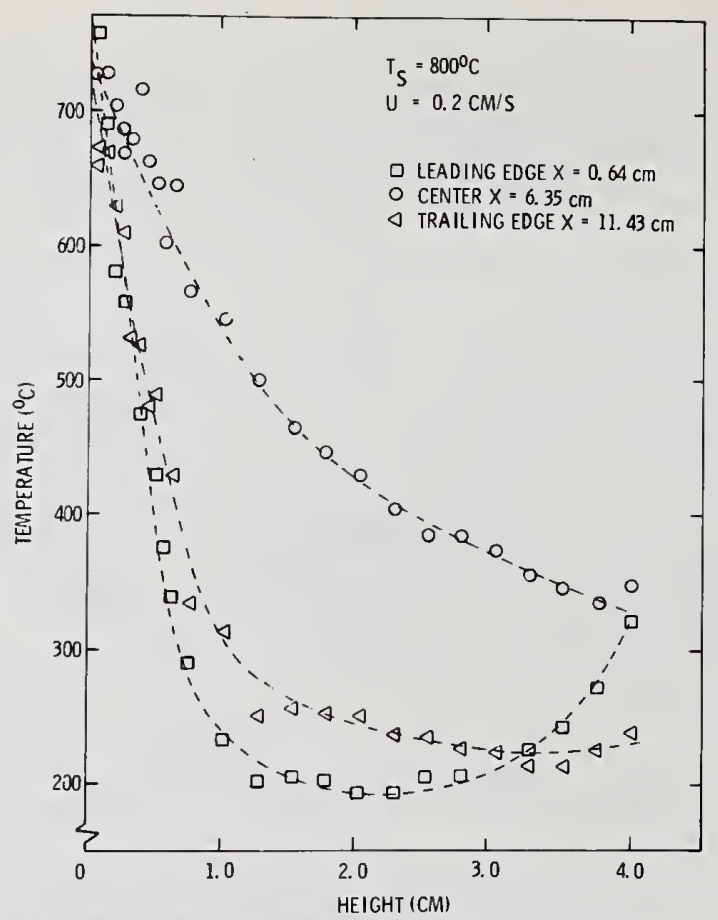

Figure 4. Centerline temperature profiles at $x=0.64,6.35$, and $11.43 \mathrm{~cm}$; $0.2 \mathrm{~cm} / \mathrm{s}$ flow velocity; and $800{ }^{\circ} \mathrm{C}$ susceptor temperature. The curves drawn through the data points show the trend of the data.

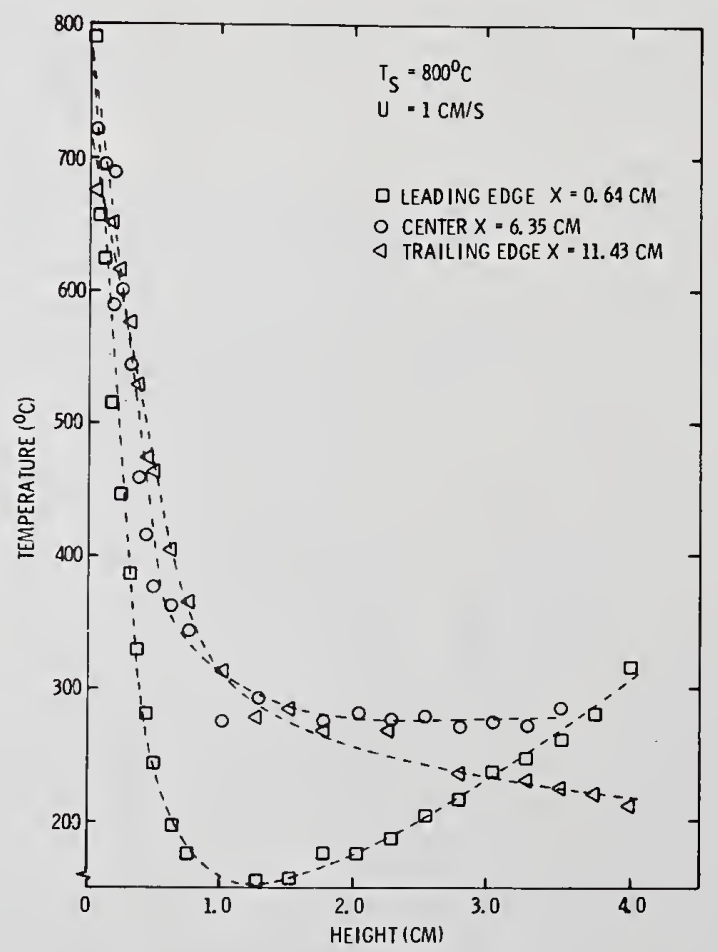

Figure 5. Centerline temperature profiles at $x=0.64,6.35$, and $11.43 \mathrm{~cm}$; $1.0 \mathrm{~cm} / \mathrm{s}$ flow velocity; and $800{ }^{\circ} \mathrm{C}$ susceptor temperature. The curves drawn through the data points show the trend of the data. 
determinations from Stokes and anti-Stokes spectra for flowing room-temperature nitrogen with no susceptor heating consistently agreed to within 1 percent. This is approximately the statistical uncertainty $\left(\sqrt{n_{j}}\right)$ for this measurement. These temperature differences at high susceptor temperature are a further manifestation of the combined forced and natural convection flow. The straight line plotted on the expanded scale on figure 6 is a leastsquares fit to the data close to the surface. The solid portion of the line indicates the points included in the fit. This fit was used to determine the temperature gradient in the vicinity of the surface. A tabulation of all the centerline temperature gradients and extrapolated surface temperature as a function of position, flow velocity, and nominal susceptor temperature is given in table 1. Temperature profiles taken near the sides of the susceptor did not differ significantly from those on the centerline.

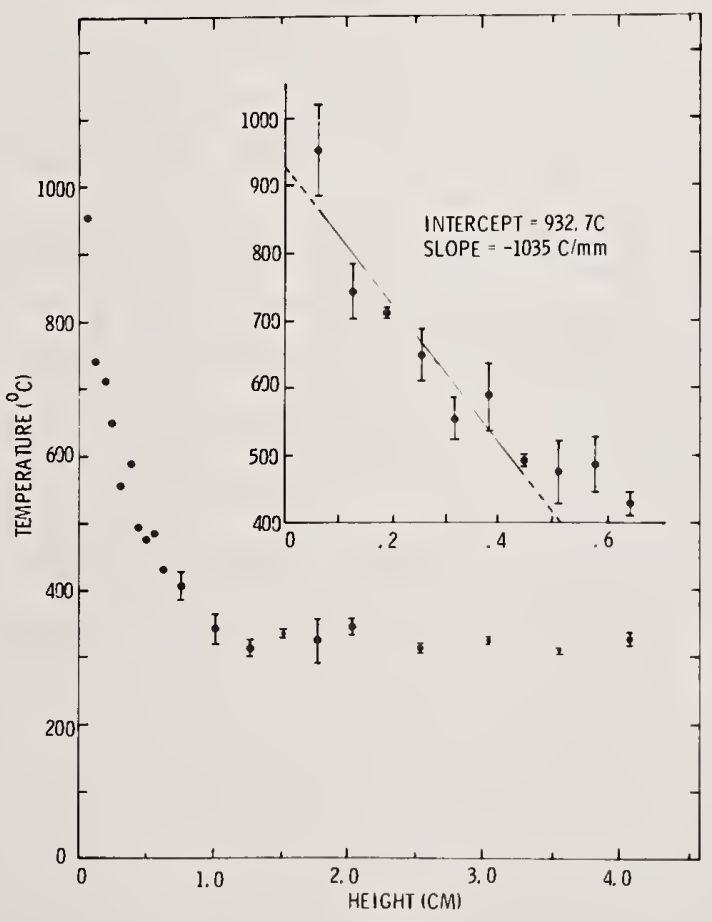

Figure 6. Detailed centerline temperature profile at $x=6.35 \mathrm{~cm}, 1.0 \mathrm{~cm} / \mathrm{s}$ flow velocity, and $950^{\circ} \mathrm{C}$ susceptor temperature.

Combined forced and natural convection $[9,10]$ over a heated horizontal flat plate in a linear duct is characterized by two dimensionless groups. Reynolds number, the ratio of inertial forces to viscous forces in forced convection, is given by:

$$
N_{R}=\frac{U L}{V}
$$


where $U$ is the free stream velocity, $L$ is a characteristic length, and $v$ is the kinematic viscosity. The Grashof number characterizes natural convection and is given by:

$$
N_{G}=\frac{L^{3} g \beta \Delta T}{v^{2}} .
$$

Table 1

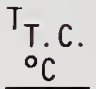

600

600

600

600

600

600

600

600

600

800

800

800

800

800

800

950

950

950

950

950

950

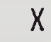

$\mathrm{Cm}$

0.64

6. 35

11.43

0.64

6.35

11.43

0.64

6.35

11.43

0.64

6.35

11.43

0.64

6. 35

11.43

0.64

6. 35

11.43

0.64

6.35

11.43

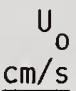

5.0

5.0

5.0

1.0

1.0

1.0

0.2

0.2

0.2

1.0

1.0

1.0

0.2

0.2

0.2

1.0

1.0

1.0

0.2

0.2

0.2

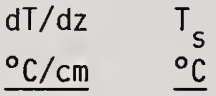

$94.8 \quad 556$

$76.7 \quad 537$

$59.3 \quad 567$

$79.6 \quad 536$

$68.4 \quad 584$

$42.6 \quad 527$

$68.3 \quad 574$

$43.2 \quad 591$

$48.9 \quad 556$

$123.1 \quad 792$

$83.8 \quad 796$

$49.4 \quad 719$

$73.6 \quad 770$

$15.2 \quad 737$

$48.2 \quad 715$

$105.9 \quad 757$

103.5933

$76.3 \quad 910$

$63.7 \quad 757$

$24.6 \quad 892$

$91.4 \quad 916$ 
In this expression, $g$ is the acceleration of gravity, $\beta\left(=\frac{1}{T}\right)$ is the thermal expansion coefficient, and $\Delta T$ is the temperature difference over the characteristic length. If the Grashof number equals the square of the Reynolds number, the viscous, inertial, and buoyancy forces in the flow are of equal importance.

As a flat plate, such as a susceptor, is heated the gas above the surface is heated and rises due to buoyancy forces. At low temperatures stable convection cells, analogous to Benard cells in the liquid boiling problem, are formed. At higher plate temperatures the steady convection cells break down into unsteady chaotic motion [9]. When a steady horizontal flow is impressed over the heated plate at very low Reynolds $\left(N_{R} \ll \sqrt{N_{G}}\right)$ number the buoyancy dominates and the above description is still valid. However, if the Reynolds number is increased (e.g., increased velocity) the vorticity generated by the buoyancy combines with the forced flow to give a spiral character to the flow. This has been observed in many CVD reactors $[2,4,11]$ by means of smoke flow visualization. The sense (clockwise, counterclockwise) of the spiral flow has been observed to depend on flow velocity [11]. Finally as $N_{R}^{2}$ approaches and becomes larger than $N_{G}$ the spirals disappear and the influence of buoyancy forces is confined to a layer analogous to a viscous boundary layer.

Table 2 gives $N_{G}$ and $N_{G} / N_{R}^{2}$ for the experimental conditions presented here. The parameters indicate that natural convection (buoyancy) dominates the flow field for $U=0.2 \mathrm{~cm} / \mathrm{s}$. However, at $U=5 \mathrm{~cm} / \mathrm{s}$ the forced convection dominates and the buoyancy effects are confined to a layer next to the hot susceptor. This is illustrated in figure 7 , which shows the temperature profiles at the center of the susceptor $\left(600^{\circ} \mathrm{C}\right)$ for $0.2,1.0$, and $5 \mathrm{~cm} / \mathrm{s}$ flow velocities. The differences in Stokes and anti-Stokes temperature determinations clearly decrease as $N_{G} / N_{R}^{2}$ becomes smaller. The unstable natural convection is being confined to a layer along the surface.

Table 2

\begin{tabular}{llccc} 
& $\frac{0.2 \mathrm{~cm}}{\mathrm{~s}}$ & $\frac{1.0 \mathrm{~cm}}{\mathrm{~s}}$ & $\frac{5 \mathrm{~cm}}{\mathrm{~s}}$ \\
\hline $\mathrm{T}^{\circ} \mathrm{C}$ & $\stackrel{\mathrm{N}_{\mathrm{G}}}{600}$ & $\underline{\mathrm{N}_{\mathrm{G}} / \mathrm{N}_{\mathrm{R}}^{2}}$ & $\underline{\mathrm{N}_{\mathrm{G}} / \mathrm{N}_{\mathrm{R}}^{2}}$ & $\underline{\mathrm{N}_{\mathrm{G}} / \mathrm{N}_{\mathrm{R}}^{2}}$ \\
800 & $5.46[14]$ & 26.0 & 1.06 & 0.04 \\
950 & $3.05[14]$ & 14.7 & 0.59 & 0.02 \\
\hline
\end{tabular}

No attempt has been made to quantify the turbulence which produces the temperature fluctuations observed above. The reason is that the fluctuations are strongly amplified by the multipass optical system. The laser beam experiences index of refraction variations along the entire length of the CVD cell on each pass. Because of this problem, density values determined from Raman spectra are of questionable value for multipass systems in turbulent flow regimes such as in this type of CVD reactor. 


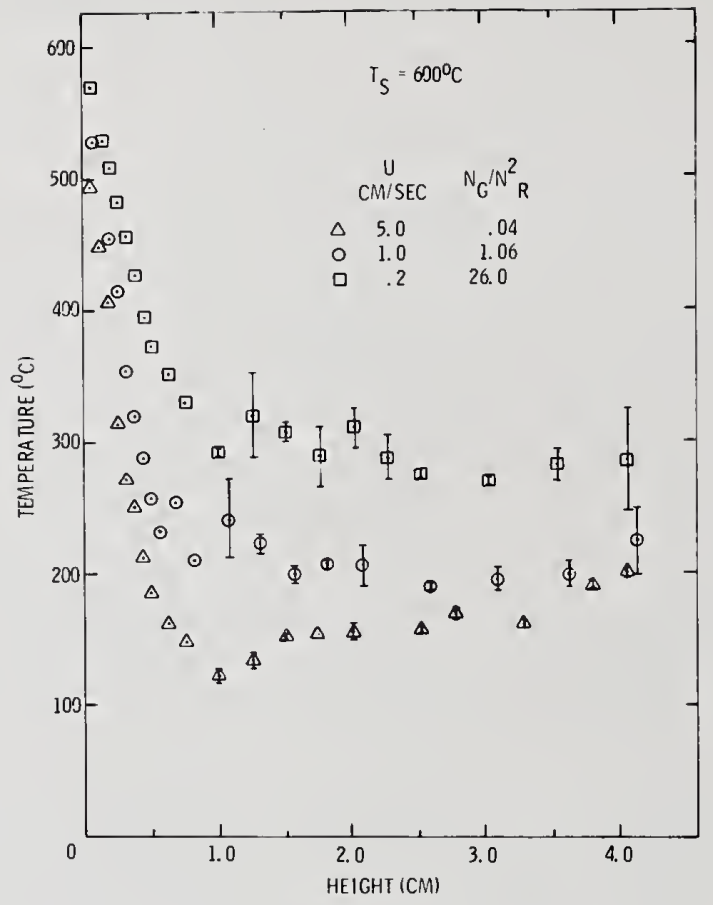

Figure 7. Temperature profiles at the center of the susceptor for $0.2,1.0$, and $5 \mathrm{~cm} / \mathrm{s}$ flow velocities and $600{ }^{\circ} \mathrm{C}$ susceptor temperature.

The flow conditions examined in this study to date represent a partial simulation of the conditions present in a bell-jar type CVD reactor. Unfortunately, these conditions do not overlap other available temperature data in linear reactors; therefore, direct comparisons cannot be made.

\section{Concentration Measurements}

Free radicals which are produced by the reaction of CVD gases are expected to play an important role in the reactions which lead to the deposition of solid films [12]. Due to the low concentration of some of the CVD gases in the carrier gas stream, diagnostic techniques having high sensitivity are required to detect the free radicals.

Pulsed laser-induced fluorescence is one of the most sensitive spectroscopic techniques for detecting trace concentrations of atomic species [13]. This high sensitivity is also expected for the detection of molecular species. In this technique, a pulsed laser is tuned to a characteristic absorption line of the molecular species of interest. The ground-state molecules which absorb radiation are excited to a higher electronic state. The excited molecules subsequently lose energy by collisions with other molecules (quenching) or by the emission of optical radiation (fluorescence). Since the intensity of the emitted fluorescent radiation is proportional to the concentration of the molecular species, 
the fluorescent intensity can be used to monitor changes in the concentration of the molecular species of interest.

In practice, fluorescence measurements are carried out by using an optical system to focus the laser radiation into a volume of interest. The fluorescent radiation excited by this beam is collected by a second optical system whose field of view overlaps the focal volume of the laser beam. The two optical systems are generally oriented normal to each other so that concentration measurements are made with good spatial resolution. In addition, due to the short duration ( $\sim 7 \mathrm{~ns}$ ) of the laser pulses used to excite the molecules, these measurements can be made with a temporal resolution of about 10 ns. Fluorescence measurements are thus characterized by high sensitivity and good spatial and temporal resolution. Limitations of this measurement technique were discussed in the introduction.

The most severe of these restrictions is the unknown effect of molecular collisions on the fluorescent intensity. Even though this effect is minimized by using high-power pulsed tunable lasers to saturate the fluorescent transition [14], absolute concentrations are still difficult to obtain using this technique. However, relative concentration measurements can generally be made.

At the present time, the required information on absorption and fluorescence spectra and on the effects of collisions is not known with sufficient accuracy to allow the study of free radicals in the CVD reaction. In order to obtain this spectroscopic information, we have developed an apparatus which uses repetitively-pulsed high-power UV lasers to produce free radicals by UV photodissociation [15]. We have chosen this technique since it provides a reliable source of free radicals and can be applied to all of the CVD gases of interest.

A schematic diagram of the experimental apparatus is shown in figure 8 . Free radicals are produced in a stainless-steel reaction vessel which can be evacuated and filled with appropriate gases (e.g., $\mathrm{NH}_{3}, \mathrm{SiH}_{4}$, etc.). UV radiation is coupled into the reaction vessel through sapphire or magnesium fluoride windows, depending on the wavelength. The UV light used to dissociate the gases is provided by either a rare-gas-halogen excimer laser; which can be operated at $193 \mathrm{~nm}, 248 \mathrm{~nm}$, or $350 \mathrm{~nm}$; or a Nd:YAG laser whose output at $1064 \mathrm{~nm}$ can be frequency tripled or quadrupled to obtain light at $355 \mathrm{~nm}$ or $266 \mathrm{~nm}$. The absorption spectrum of the free radicals produced in the reaction vessel is obtained by using either a broadband dye laser or a pulsed xenon flashlamp as the light source. A narrowband dye laser is used to excite the free radicals and a scanning monochromator is used to analyze the resulting fluorescence spectrum. The effect of molecular collisions is studied by introducing known amounts of a CVD carrier gas (e.g., $\mathrm{N}_{2}$ or $\mathrm{H}_{2}$ ) into the reaction vessel and measuring its effect on both the intensity and lifetime of the fluorescence. The pulsed lasers and xenon flashlamp are temporally synchronized (to $5 \mathrm{~ns}$ ) and spatially overlapped (to $0.1 \mathrm{~mm}$ ) at the center of the reaction vessel. Pulse-digitizing electronics are used to record single-pulse absorption and fluorescence spectra.

In our first experiment we have produced $\mathrm{NH}$ free radicals by the $266 \mathrm{~nm}$ photodissociation of $\mathrm{NH}_{3}$. Although single-photon absorption of $266 \mathrm{~nm}$ radiation by $\mathrm{NH}_{3}$ does not occur [16], multiphoton absorption of this radiation can take place. The Nd:YAG laser was 
operated at a pulse repetition rate of $10 \mathrm{pps}$. The pulse obtained at $266 \mathrm{~nm}$ had an energy of $20 \mathrm{~mJ}$ and a time duration of $12 \mathrm{~ns}$ FWHM. This light was focused into the reaction vessel by an $\mathrm{f} / 9$ lens resulting in a fluence of $\sim 3 \mathrm{~J} / \mathrm{cm}^{2}$ at the center of the cell. The resulting emission was collected by an $\mathrm{f} / 7$ lens and imaged onto the slit of a $1 \mathrm{~m}$ monochromator which was used to scan the spectrum. The dispersed radiation was detected by a photomultiplier tube and recorded with a boxcar integrator.

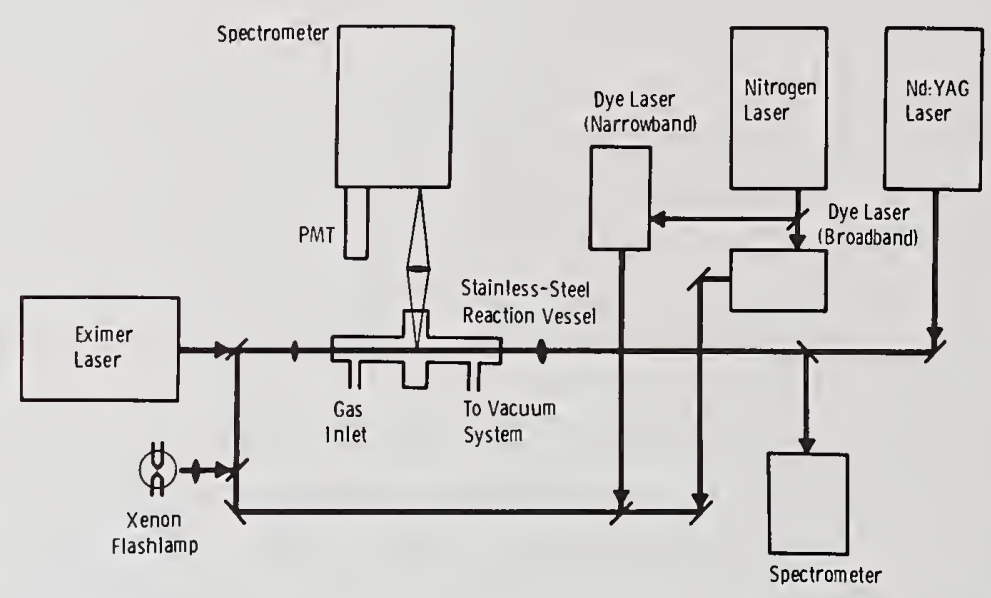

Figure 8. Schematic diagram of the photodissociation experiment.

Figure 9 shows the emission spectrum between $330 \mathrm{~nm}$ and $340 \mathrm{~nm}$ when 10 Torr of $\mathrm{NH}_{3}$ was irradiated by the $266 \mathrm{~nm}$ light. The peak near $336 \mathrm{~nm}$ is the $Q$ branch of the $(0,0)$ band of the $\mathrm{NH}\left(\mathrm{A}^{3} \Pi-X^{3} \Sigma^{+}\right)$transition [17]. The peaks on either side of the $Q$ branch were identified as the $P$ and $R$ branches for this transition. At an $\mathrm{NH}_{3}$ pressure of 100 Torr, emission from the $Q$ branch of the $(1,1)$ band of this transition was also observed. An Fe-Ne hollow cathode lamp was used to calibrate the spectrum. Since the $336 \mathrm{~nm}$ emission occurred simultaneously with the $266 \mathrm{~nm}$ laser pulse, $\mathrm{NH}\left(\mathrm{A}^{3} \Pi\right)$ is expected to be a primary photodissociation product. The reaction $\mathrm{NH}_{3}\left(\hat{X}^{\top} A_{1}\right)+7.6$ e.v. $\rightarrow \mathrm{NH}\left(A^{3} \Pi\right)+H_{2}\left(X_{1} \Sigma_{g}^{+}\right)$is the least energetic mechanism leading to $\mathrm{NH}\left(A^{3} \Pi\right)$. Since a $266 \mathrm{~nm}$ photon only has an energy of $4.8 \mathrm{eV}$, a minimum of two photons must be absorbed by $\mathrm{NH}_{3}$ to give rise to the observed $\mathrm{NH}$ emission signal.

The time dependence of the $Q$ branch emission at $336 \mathrm{~nm}$ was measured by using a transient digitizer. Because of the very weak emission signal, 100 photomultiplier output pulses had to be recorded and averaged. Figure 10 shows the averaged signal. A time duration of $(90 \pm 10)$ ns was measured from the dotted curve drawn through the data. This is much less than the measured radiative lifetime of $455 \mathrm{~ns}$ [18]. Since the quenching rate of $\mathrm{NH}\left(\mathrm{A}^{3} \Pi\right)$ by $\mathrm{NH}_{3}$ has not been measured it is not known whether our reduced lifetime is due to quenching or secondary reactions involving $\mathrm{NH}\left(\mathrm{A}^{3} \Pi\right)$. Further work is in progress to clarify this. 


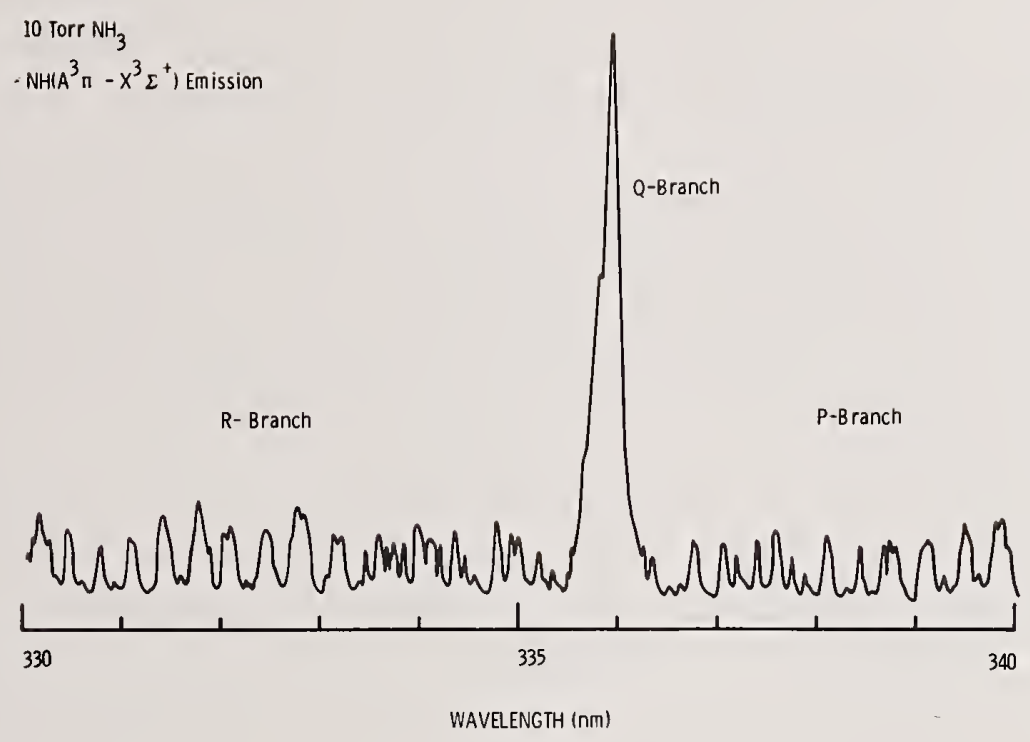

Figure 9. NH emission spectrum between $330 \mathrm{~nm}$ and $340 \mathrm{~nm}$.

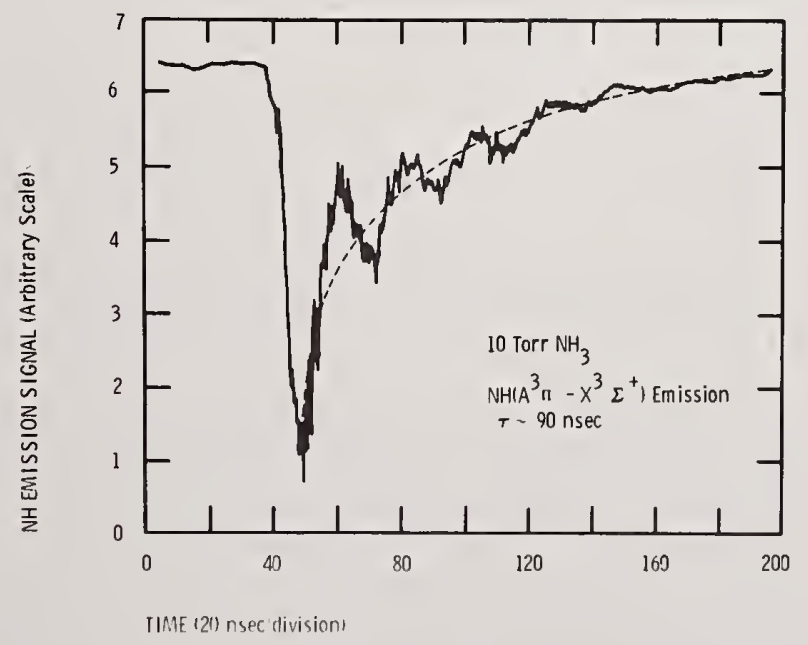

Figure 10. Time dependence of the $\mathrm{NH}\left(\mathrm{A}^{3} \Pi\right)$ emission at $336 \mathrm{~nm}$. 


\section{Summary}

In this paper we have discussed two optical diagnostic techniques applicable to CVD reactors. Both of these are non-perturbing and provide excellent spatial resolution. Spontaneous rotational Raman spectroscopy has been used to make temperature measurements.

Three-dimensional temperature maps were obtained in a linear CVD cell with a horizontal flow of $\mathrm{N}_{2}$ over a hot graphite susceptor. The temperature of the susceptor and the gas flow rate were varied. The time required for the measurements was reduced by the use of a multipass light cell having a gain of approximately 20 . The anomalies in the vertical temperature profiles were found to be manifestations of the combined forced and natural convection present in horizontal linear thermal CVD reactors. The flow conditions and susceptor temperatures investigated here cover a transition regime between dominance of the flow by inertial or by buoyancy effects.

We have indicated the lack of fundamental data needed to carry out fluorescence measurements of CVD gases and intermediates. In our initial experiment we have produced NH free radicals by the $266 \mathrm{~nm}$ photodissociation of $\mathrm{NH}_{3}$. This technique is applicable to all the CVD gases.

We are pleased to recognize at this time the valuable assistance of Tom Cabe and Michael Johnson in the construction and operation of the apparatus. We are indebted to Gary Bergeson for the construction of the microprocessor. We appreciate helpful discussions with A. Wayne Johnson and Victor A. Wells.

\section{References}

[1] Wells, V. A., private communication.

[2] Ban, V. S., J. Electrochem. Soc.: Solid State Science and Technology, 125, No. 2, 317 (1978).

[3] Cochet, G., Mellottee, H., and Delbourgo, R., J. Electrochem. Soc. 125, 487 (1978).

[4] Sedgwick, T. 0. and Smith, J. E., Jr., J. Electrochem. Soc.: Solid State Science and Technology, 123, No. 2, 254 (1976).

[5] Hill, R. A., Mulac, A. J., Aeschliman, D. P., and Flower, W. L., Temperature from rotational-vibrational Raman Q-branches, accepted for publication in J. Quant. Spectrosc. Radiat. Transfer.

[6] Hi11, R. A., Peterson, C. W., Mulac, A. J., and Smith, D. R., J. Quant. Spectrosc. Radiat. Transfer, 16, 953-962 (1976).

[7] Herzberg, G., Spectra of Diatomic Molecules, chap. III (Van Nostrand Reinhold Co., NY, 1950).

[8] Hill, R. A., Mulac, A. J., and Hackett, C. E., Applied Optics, 16, 2004 (1977). 
[9] Prandt1, L., Essentials of Fluid Dynamics (Hafner Publishing Co., NY, 1952).

[10] Schlicting, H., Boundary Layer Theory (McGraw-Hill Book Co., Inc., NY, 1960).

[11] Takahashi, R., Koga, Y., and Sugawara, K., J. Electrochem. Soc.: Solid State Science and Technology, 119, No. 10, 1406 (1972).

[12] Lin, Sin-Shong, J. Electrochem. Soc. 124, 1945 (1977).

[13] Hohimer, J. P. and Hargis, P. J., Appl. Phys. Lett. 30, 344 (1977); Analytica Chim. Acta, 97, 43 (1978).

[14] Piepmeier, E. H., Spectrochim. Acta, 27B, 431, 445 (1972).

[15] McNesby, J. R. and Okabe, H., in Advances in Photochemistry, Vol. 3, p. 157 (Interscience, NY, 1964).

[16] Douglas, A. E., Discussions Faraday Soc. 35, 158 (1963).

[17] Lents, J. M. , J. Quant. Spectrosc. Radiat. Transf. 13, 277 (1973).

[18] Smith, W. H., J. Chem. Phys. 51, 520 (1969).

\section{Discussion}

Comment (McDonald): If you will use your Eximer laser you can generate overwhelming amounts of $\mathrm{NH}$. We have been doing those experiments.

Response (Miller): That is right. At the time that this measurement was made, the Eximer laser was just in the process of being put into operation. So, it was not available at that time. We are now using it and you are right. It makes lots more.

Comment (McDonald): It is also probable that the production of $\mathrm{NH}_{2}$ is going to really dominate over the production of $\mathrm{NH}$ that you are looking at there also.

Response (Miller): That is an interesting part, because using the quadrupled neodymium-yag laser, we just don't see any $\mathrm{NH}_{2}$ at all.

Response (McDonald): It quenches so fast at the pressures you are looking at, that essentially all of the fluorescence will be lost due to quenching. Its cross-section is several hundred times higher than what you are looking at here.

Question (Rosenblatt): For a jealous academic do you think you could just estimate how much it costs to set up that very impressive facility that we saw?

Response (Miller): The major cost in the facility--which one were you referring to, the first or the second?

Clarification (Rosenblatt): The second. 
Response (Miller): With the second facility, the major cost was in the lasers. The electronics, I really don't know how much the electronics cost. So, if you look at the cost of a neodymium-yag, of an Eximer laser, of several narrow band dyelasers, that represents really the major cost of the system and you could probably just about double that for the electronics. So, I guess you are probably talking about something in the neighborhood of $100 \mathrm{~K}(\$)$.

(Editor's note): Much mirth ensued over this conservative estimate. The consensus opinion was that the figure should be around $400 \mathrm{~K}(\$)$. 
National Bureau of Standards Special Publication 561, Proceedings of the 10th Materials Research Symposium on Characterization of High Temperature Vapors and Gases held at NBS, Gaithersburg, Maryland, September 18-22, 1978. Issued 0ctober 1979.

\title{
LASER-GENERATED OPTO-ACOUSTIC PULSES IN AN ATMOSPHERIC PRESSURE FLAME
}

\author{
William R. Anderson, ${ }^{1}$ John E. Allen, Jr. ${ }^{1}$ \\ Todd D. Fansler, and David R. Crosley \\ Ballistic Research Laboratory \\ Aberdeen Proving Ground, MD 21005
}

\section{Introduction}

Recent developments in lasers and laser spectroscopy have led to a variety of techniques now beginning to be applied to the measurement of both species concentrations and population distributions (temperatures) in gaseous systems at high temperature--especially flames. Prominent among these techniques is laser-excited-fluorescence, in which a dye laser is tuned to match an absorption line of some species of interest; a photomultiplier detects the light emitted from the resulting excited state. The result is a probe for molecular ground states combining extremely high sensitivity with the spatial and temporal resolution afforded by laser methods. Tuning the laser across an absorption band allows one to map out population distributions from the intensity variation of the emitted radiation; even with modest bandwidth etalons, one may easily obtain resolution comparable to that of relatively large spectrometers.

Laser-excited fluorescence, as well as most of the other new laser probe methods, relies on the scattering (emission) of light as the detection mode. Although this is responsible for the high sensitivity of the method under many conditions, there may be other situations in which it is less suitable. In particular, high collisional quenching rates, at atmospheric pressure and above, greatly reduce the fraction of laser-excited states decaying by photon emission.

We describe here the observation and characterization of another means of detecting the occurrence of selective absorption of tuned laser radiation. This is the production of a pulsed opto-acoustic effect--pulsed sound waves--within a flame operating at atmospheric pressure, following deposition of the laser energy into an electronic transition [1] ${ }^{2}$. The phenomenon itself depends on the rapid collisional conversion of electronic to translational energy (through the intermediary of molecular vibration), and the sigrial is thus not reduced at high pressure. The experiments have been carried out on alkalis ( $\mathrm{Na}$ and $\mathrm{Li}$ ) seeded into a flame. The results have provided directly useful information as well as projections concerning the sensitivity of the technique.

${ }^{1}$ NAS-NRC Resident Research Associates.

${ }^{2}$ Figures in brackets indicate the literature references at the end of this paper. 
The opto-acoustic effect in general, viz., the conversion of optical to acoustical energy through absorption and collisional quenching, has long been known as a means of sensitively detecting the absorption of radiation [2]. What is newly demonstrated here is the nature of its occurrence following pulsed excitation, and the existence of the effect under in situ conditions on a 1-atm pressure flame burner (in contrast to the typical mode of operation using specially constructed cells at low pressure).

In addition to exploiting its detection capabilities, we have also utilized the effect to perform measurements of the speed of sound within the flame on a spatially resolved basis. These data provide information on the translational motion of the flame gases. This aspect may prove especially useful for environments too hostile to admit thermocouple probes or processes too rapid to obtain usable thermocouple response.

The actual mode of formation of the pressure wave--the fluid dynamics aspects--is not investigated in any detail in these experiments. Observations have been made of the form of the pressure wave produced; it appears to be dictated by density gradients in the flame and surrounding atmosphere. Some modification of the experiment might permit the waveform to be used to extract bulk energy transfer rates, but we have not pursued that end.

Most of the experiments to be described were carried out pumping the 3P state of $\mathrm{Na}$. We have been unable to detect opto-acoustic pulse formation when pumping the 4P level, and we conclude that the net effective conversion of electronic to translational energy, at least over the salient time scale, is less efficient for this level. In conjunction with this experiment, we also describe the observation of two-photon excited fluorescence from $\mathrm{Na}$ in the flame [3] and its use to map out energy transfer pathways among excited states including the $4 \mathrm{P}$ level [4].

\section{Experimental Details and Results}

\subsection{Apparatus}

The experimental arrangement is depicted in figure 1. The flame is an approximately stoichiometric mixture of $\mathrm{C}_{2} \mathrm{H}_{2}$ and air, and the slot burner is a standard type used for atomic absorption analysis. $\mathrm{Na}$ is introduced by aspiration of $\mathrm{NaI}$ solution; many of the experiments were carried out at a Na concentration of the order of $10^{10}-10^{11}$ atoms cm ${ }^{-3}$, as determined from both absorption and absolute fluorescence measurements. Additionally, a few experiments have been run using $\mathrm{Li}$ as the seed atom, or with $\mathrm{Na}$ in a $\mathrm{CH}_{4} / \mathrm{air}$ flame.

The laser used is a Chromatix CMX-4, a commercially available flashlamp-pumped tunable dye laser having a pulse duration of $1 \mu \mathrm{s}$. Although the laser is capable of delivering 10 $\mathrm{mJ} / \mathrm{pulse}$ in the region of the $\mathrm{Na}$ resonance 1 ines, it was operated typically at $<1 \mathrm{~mJ} / \mathrm{pulse}$. The bandwidth is nominally $3 \mathrm{~cm}^{-1}$ and can be narrowed to $0.16 \mathrm{~cm}^{-1}$ by insertion of an intracavity etalon. The laser beam (a few $\mathrm{mm}$ in diameter) is directed into the flame. Upon tuning to one of the $\mathrm{Na}$ resonance lines, the pulsed pressure wave is produced and propagates outward from the region illuminated by the laser. This sound wave is detected by a calibrated condenser microphone typically located some $4 \mathrm{~cm}$ from the flame. The amplified 
microphone signal is fed to an oscilloscope, and also to a dual-channel boxcar (gated) integrator for averaging over successive pulses. Both of these are triggered by a signal from the laser flashlamp circuitry.

The laser power is usually monitored by a thermopile located behind the flame (at high Na concentrations, this served as a monitor of the amount of absorption). Not shown in figure 1 is a spectrometer, with associated optics and photomultiplier, which was used to measure the fluorescence from the $\mathrm{Na}^{--p a r t i c u l a r l y}$ in those experiments involving ultraviolet and two-photon excitation.

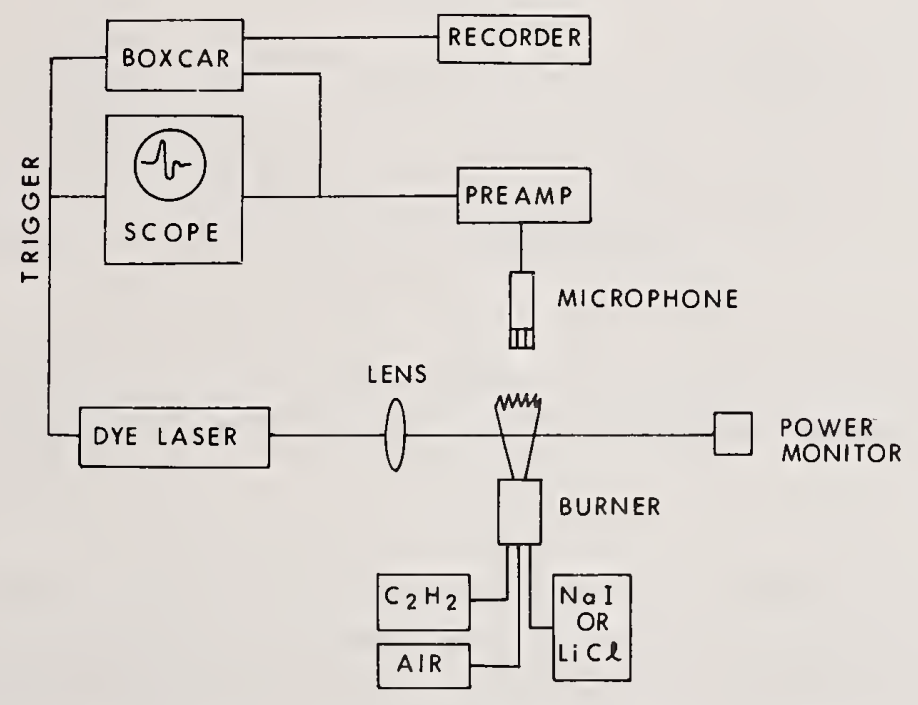

Figure 1. Experimental arrangement for observation of opto-acoustic pulse. The flame is seeded with alkali on an aspirator burner, and the tunable laser is directed into the secondary combustion zone. A microphone detects the pulsed pressure waves; after preamplification, the signal is sent to an oscilloscope and boxcar integrator triggered by the laser.

\subsection{Qualitative observations}

When the laser is tuned to one of the resonance lines, the pulsed pressure wave is produced. At relatively high sodium density, so that a hundred microjoules or more of laser energy are absorbed, this wave is readily audible to an observer located within a few meters of the burner. In fact, the normal procedure for coarse manual tuning of the laser is to locate the resonance lines by listening for the sound wave.

As measured by the microphone and displayed on the oscilloscope, the waveform shows a sharp rise as the sound wave reaches the detector, followed by a series of oscillations typically diminishing in magnitude. Figure 2 is a tracing of a scope photo showing four individual single-shot pressure waves. The time axis is $20 \mu \mathrm{s}$ per division, and the laser is fired at the extreme left-hand side of the scope trace. The four different waveforms 
result from four different arrangements of the beam-flame-microphone geometry (see below); for a fixed geometry, the waveform was very reproducible from pulse to pulse. The two boxcar gates were set to correspond to the first minimum and first maximum of the waveform; the difference between these values was output to the recorder and constitutes the "microphone signal" used below to denote the magnitude of the pressure wave.

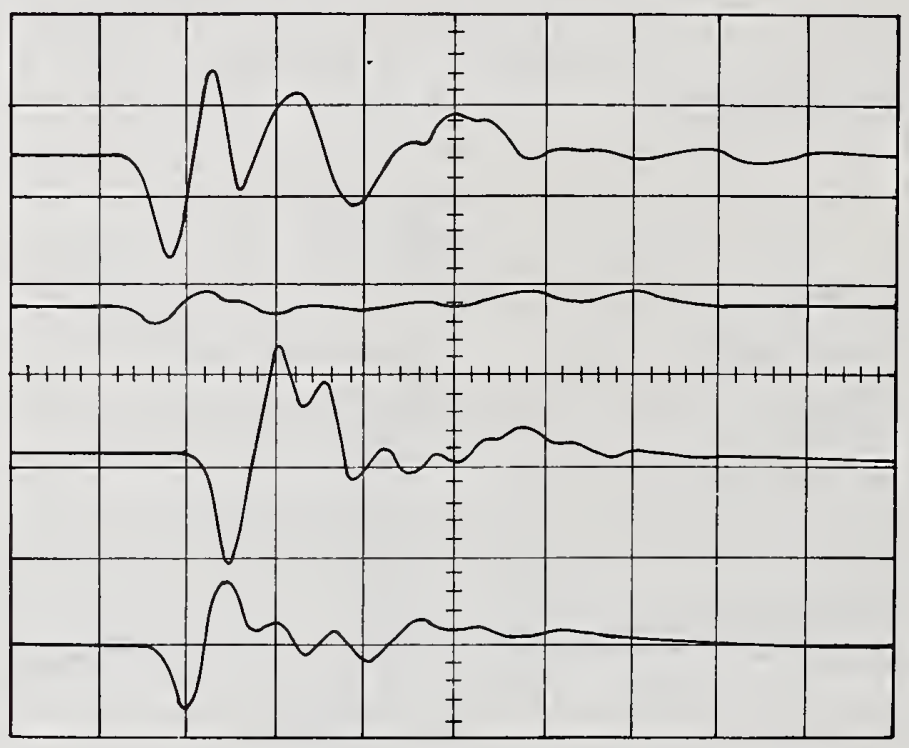

Figure 2. Four individual pulsed pressure waves, traced from a scope photograph. Each results from a single shot; the waveform is quite reproducible from shot to shot. The differences in shape result from differences in beam/flame/microphone geometry for each shot. The horizontal scale is $20 \mu \mathrm{s}$ per $\mathrm{cm}$, and the laser is fired at the left hand end of the scope trace.

Figure 3 demonstrates that the signal is due to resonant absorption by the $\mathrm{Na}$, and not to some other means of energy deposition, such as laser-induced breakdown of the flame gases. The laser is operated with a bandwidth of $0.16 \mathrm{~cm}^{-1}$, and automatically scanned through the region containing both doublet components. The spikes marked 'etalon reset' are artifacts of the scanning sequence.

\subsection{Magnitude of the pressure wave}

From the information directly available from the experiment, we can only speculate on the mode of formation of the pressure wave. Collisional quenching of the excited state transfers the Na electronic energy into vibrational levels of the flame gases (as demonstrated by an experiment on electronic-to-vibrational-to-electronic energy transfer between $\mathrm{Na}$ and $\mathrm{L} i$ in the same flame [4]). This energy is then rapidly converted to translational energy, resulting in translational heating of the region illuminated by the laser beam. 
Because sound travels only about $1 \mathrm{~mm}$ during the $1 \mu \mathrm{s}$ heating time, and the laser beam is typically a little larger than this, we envision the energy deposition as resulting in a slightly hotter region where the laser beam has passed through the flame. A small shock wave may be initially formed; if so, it soon degrades into a pressure wave, and propagates outward through the flame.

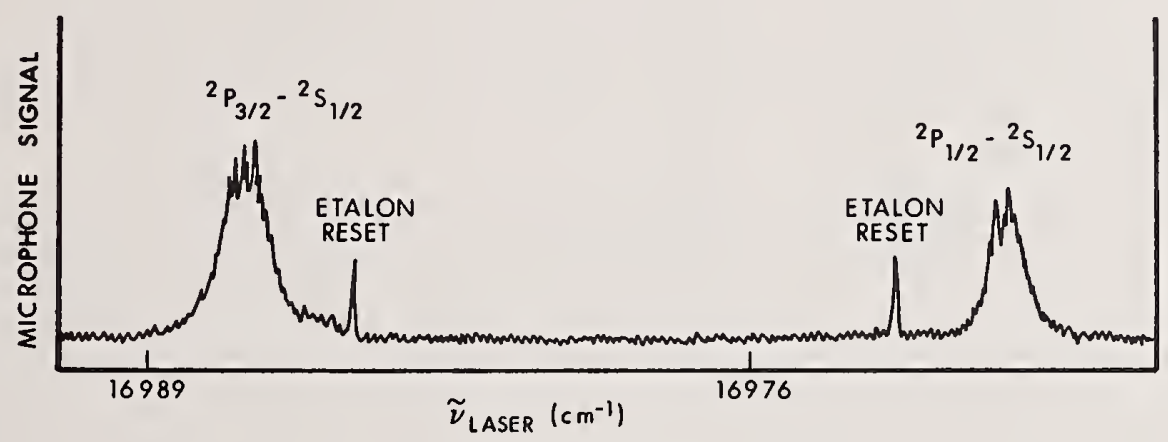

Figure 3. Microphone signal vs laser tuning. The laser bandwidth is $\sim 0.16 \mathrm{~cm}^{-1}$. The spikes marked 'etalon reset' arise from the automatic scanning operation, and are artifacts.

We describe a particular, though typical, experiment to measure the absolute laser energy deposited and the resulting pressure wave amplitude. The $\mathrm{Na}$ absorbs $0.22 \mathrm{~mJ}$ from a single laser pulse as the beam traverses a $1 \mathrm{~cm}$ path through the flame, with a diameter of $15 \mathrm{~mm}$. Over 99 percent of the excited states are quenched by collisions under our conditions, so this is the amount of energy transferred into translation.

The flame gases are assumed diatomic here, with a density appropriate to 1 atm and the flame temperature (from speed of sound measurements described below) of $2300 \mathrm{~K}$. The irradiated volume of $1.75 \mathrm{~cm}^{3}$, thus, has a heat capacity of $0.34 \mathrm{~mJ} /$ degree and is heated $0.64 \mathrm{~K}$. This is truly negligible perturbation compared with the temperature of the flame gases themselves.

A pressure rise of 0.22 Torr within the heated volume is calculated using the ideal gas law. At the microphone, the amplitude of the resulting pressure wave will be lower, since the amplitude falls off inversely with distance from the source (as verified by experiment). For the geometry used here, an amplitude of 0.044 Torr would result at the microphone. The measured value is 0.13 Torr. In view of concerns about the homogeneity of the laser beam over its spatial profile, some uncertainties in the measurement of the path length, and especially the lack of knowledge concerning the actual mode of formation of the pressure wave, we consider this to be reasonable agreement, confirming our simple physical picture. 


\subsection{Form of the pressure wave}

The waveform of the sound wave is quite complex (see fig. 2), and we have examined it extensively, although no quantitative information could be extracted. As noted above, it is quite reproducible from pulse to pulse for a fixed geometry. The characteristics of the waveform could be varied by introducing disturbances, especially turbulence, into the flame; this particularly influenced the smaller oscillations following the first two extrema. This implies that the form is dictated by reflections and/or interference from density gradients within the flame and the flame-air interface, though no analysis was attempted. Perhaps in a more carefully controlled experiment probing those gradients, useful information could be obtained from the waveform.

It was originally hoped that the waveform might provide information on the bulk energy transfer rates within the flame. Now, a sound wave having a frequency of the order of the inverse of some relaxation time (e.g., a vibrational energy transfer rate) within the system will undergo attenuation and phase shifts due to that relaxation process. Vibrational relaxation times at $1 \mathrm{~atm}$ are of the order of the laser pulse duration. The laser pulse thus is expected to produce a spectrum of sound frequencies, and it was hoped that a Fourier analysis of the opto-acoustic waveform might yield information on these relaxation times within the flame. This approach was not successful, due to the dominance of the density gradient effects and the bandwidth $(140 \mathrm{kHz})$ of our fastest microphone. "Air" mixtures of $\mathrm{N}_{2} / \mathrm{O}_{2}, \mathrm{Ar} / \mathrm{O}_{2}$, and $\mathrm{CO}_{2} / \mathrm{O}_{2}$ were tried in this series of experiments, but no differences could be discerned. With a faster microphone, it remains possible that this will be a useful application of this technique.

\section{Applications As A Probe}

\section{1 Concentration measurements}

It has already been demonstrated that the formation of the opto-acoustic pulses introduces a negligible perturbation into the flame gases, insofar as either the chemical kinetics or gas dynamics is concerned. Here we consider the use of the technique as a sensitive probe of selective absorption of laser radiation, i.e., as an alternative or complement to laser excited fluorescence.

Our simple picture of E-V-T transfer and local translational heating suggests that the pressure wave amplitude should be proportional to the laser power and to the concentration of the $\mathrm{Na}$ atoms. Figure 4 shows the dependence of the microphone signal on these two parameters. The power dependence is measured at a sufficiently low power density so as to be in the linear absorption region, that is, we do not approach optical saturation in figure 4 a. In figure $4 \mathrm{~b}$, the microphone signal is plotted against the concentration of the NaI solution aspirated into the flame. The nonlinear curve-of-growth form of the plot reflects the effect of increasing optical thickness. The results shown here demonstrate that we may scale our observed signals to lower $\mathrm{Na}$ concentrations and, with due regard to optical saturation, to higher laser power. 


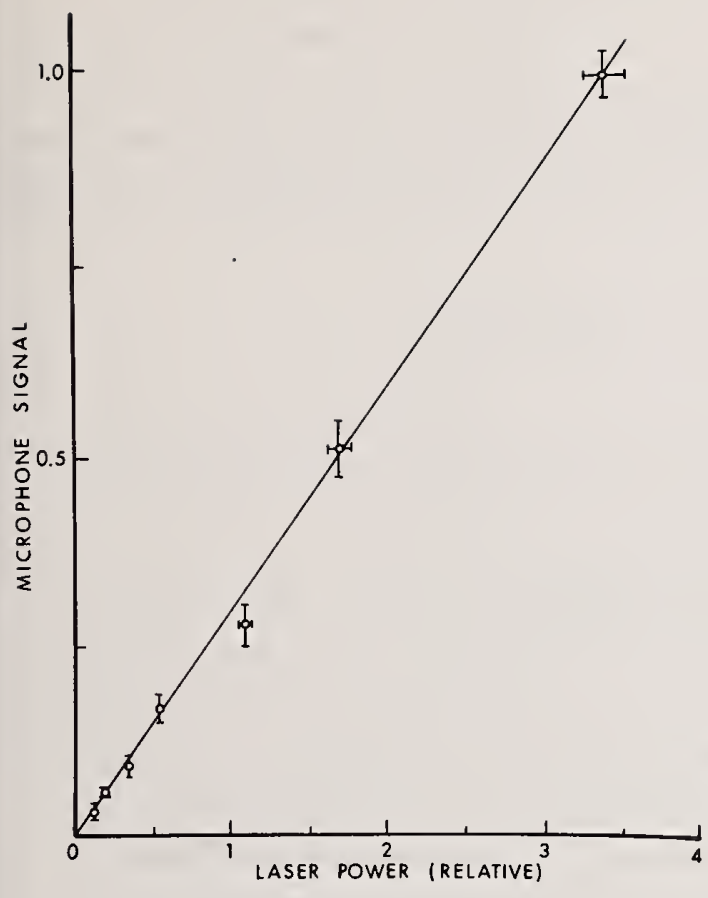

(a)

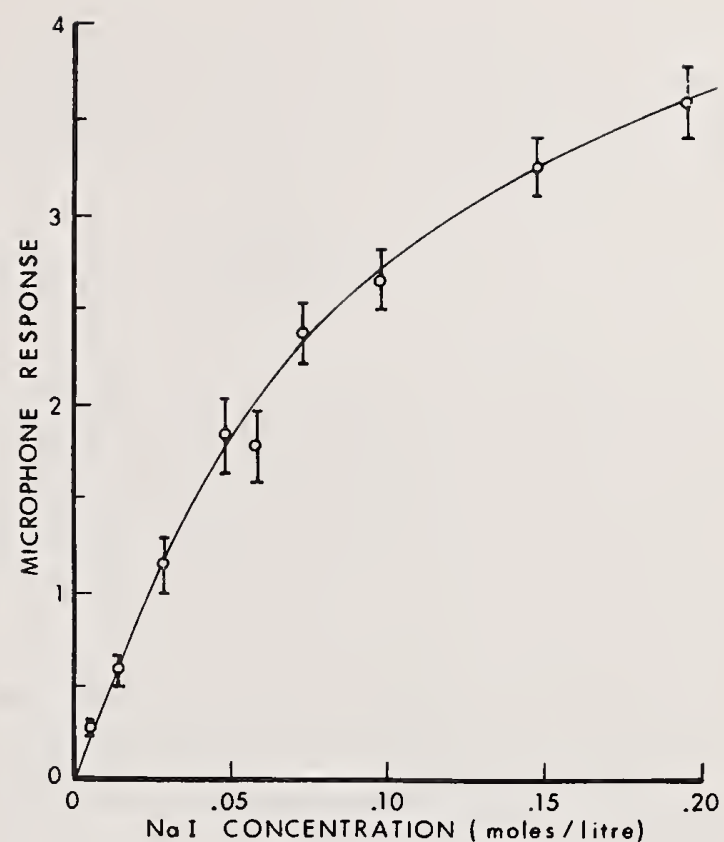

(b)

Figure 4. Behavior of the opto-acoustic signal pertinent to scaling the detection sensitivity: (a) dependence on laser power, at power densities much lower than saturation values; (b) dependence on $\mathrm{NaI}$ concentration; presumably the $\mathrm{Na}$ concentration in the flame follows the solute concentration.

From these guidelines, we may project from our observed signal sizes and background noise a detectability limit for $\mathrm{Na}$. We estimate that with a time constant of about $1 \mathrm{~s}$ (corresponding to averaging over 30 laser pulses), a signal-to-noise ratio of about 2:1 should be obtainable for Na densities of the order of $10^{7}$ atoms per $\mathrm{cm}^{3}$ in our 1 atm flame. Although this is not as low a limit as one can obtain using laser-excited fluorescence for this species, opto-acoustic pulses nonetheless do constitute a sensitive detection technique. It should be emphasized that we have undertaken no effort to shield the microphone from extraneous sources (pumps, etc.) of background noise; perhaps with two microphones and differencing techniques, the noise could be considerably reduced.

Although at higher total number densities, the quantum yield of fluorescence is reduced due to the higher frequency of collisions, the opto-acoustic signal should remain essentially constant. Consequently, this may be the method of choice for some experiments carried out at high pressure.

In addition, there may be some systems which exhibit selective absorption of laser radiation but which do not readily fluoresce. For example, a triatomic or larger molecule may undergo rapid intersystem crossing into a state of different multiplicity from the ground state before fluorescing. Collisions then would remove the energy of excitation 
before any long-lived phosphorescence would occur. However, the collisional transfer would still result in the formation of a pressure wave upon conversion into translational energy. Another molecular situation might be a case in which the excited molecular state predissociates; pressure waves would be produced upon resonant tuning provided that the absorption lines are still relatively sharp, and not all of the absorbed photon energy goes into breaking the chemical bond.

\subsection{Temperature measurements: speed of sound}

The pulsed sound wave formation offers the ability to measure the speed of sound $u_{s}$ within the flame; $u_{s}$ can then be related to the translational temperature, providing an important means of characterizing the flame.

To measure the speed of sound in situ, the laser beam is fired into the flame at a particular point, as illustrated in figure 5. The scope (or boxcar) is triggered at the time of the laser pulse, so that the arrival time of the pressure wave at the microphone can be accurately measured. The laser beam is then moved within the flame (a $1 \mathrm{~cm}$ shift is indicated in the figure). The difference in arrival times for the different points, together with the distance between them, furnishes the speed of sound within the flame. (Although the measurements carried out were made by physically moving the beam, a better method would be to split the beam into two or three components and obtain the information from one laser pulse, thus adding time resolution.)

This demonstration experiment was carried out with a rather crude measurement of the laser beam position, which is the dominant cause of the estimated error. The result is $u_{s}=$ $(9.7 \pm 0.5) \times 10^{4} \mathrm{~cm} \mathrm{~s}^{-1}$. From the stoichiometric composition of the initial flame gases, and reasonable assumptions on the degree of combustion, average values were calculated for $\bar{M}$ (molecular weight) and $\bar{\gamma}$ (heat capacity ratio). Since most of the flame gas is made up of $\mathrm{N}_{2}$, this is not a critical pair of numbers. These values are then used to calculate the temperature from the relationship, $u_{s}=(\bar{\gamma} R T / \bar{M})^{1 / 2}$. The result is $2280 \pm 230 \mathrm{~K}$, which may be compared to the adiabatic flame temperature for this flame of $2545 \mathrm{~K}$.

The translational temperature is a most important flame characteristic, from the standpoint of both the gas dynamics and the chemical kinetics. The opto-acoustic pulse method offers the possibility of measuring the closely related speed of sound on a basis of high spatial and temporal resolution. No perturbation such as a thermocouple need be introduced into the flame, and extremely high temperatures (which are hostile to non-optical probes) appear to pose no fundamental difficulties. Such speed of sound measurements could well form the unique contribution of this method as a combustion diagnostic tool.

of course, by carrying out an excitation scan over a series of molecular absorption lines or bands, using opto-acoustic pulses or fluorescence for detection, one can measure population distributions corresponding to the temperatures of internal degrees of freedom as we11. 


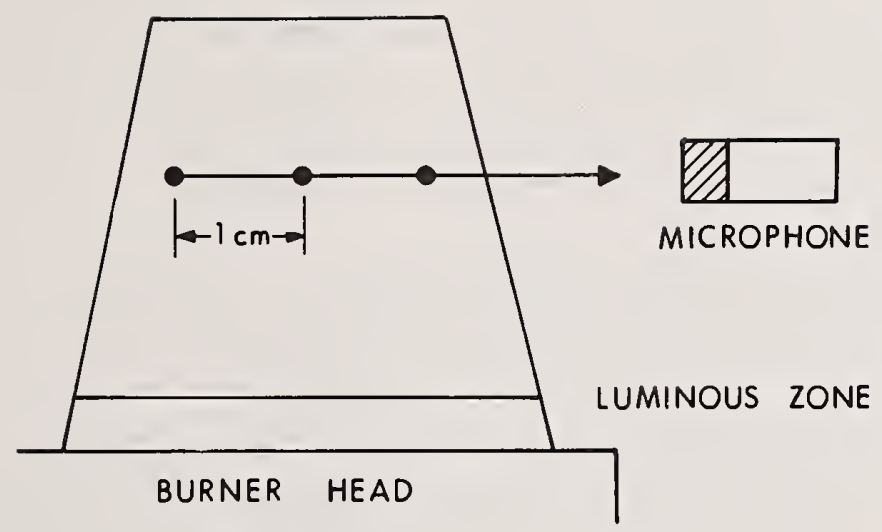

Figure 5. Schematic illustration of the measurement of the speed of sound. On three different laser pulses, the laser beam is fired into the flame at three different positions, and the arrival time of the pressure pulse at the microphone is measured.

\section{Quenching and Related Measurements}

A series of experiments has been carried out in order to probe energy transfer and quenching collisions of electronically excited $\mathrm{Na}$ within the flame. These involve detection of both opto-acoustic pulses and fluorescence, the latter by means of a scanable monochromator. They fall, in general, into two categories: the measurement of population ratios of highly excited levels populated using two-photon or ultraviolet excitation, and measurements on the $3 \mathrm{P}$ level as the laser drives the 3S-3P transition toward optical saturation.

\section{1 Highly excited states}

These experiments involve the series of energy levels $3 \mathrm{D}, 4 \mathrm{P}, 5 \mathrm{~S}$, and $4 \mathrm{D}$ lying in the range $30-35,000 \mathrm{~cm}^{-1}$ above the ground state. Of these, only the $4 \mathrm{P}$ is readily accessible by a one-photon transition from the ground state. Quadrupole transitions, in principle, connect the $3 S$ with the $3 D$ and $4 D$ states, but one-photon 3S-5S transitions of a 11 orders are rigorously forbidden. Thus, two-photon excitation is the only practical means of selectively populating these states. 
Excitation to the $4 \mathrm{P}$ level is provided by frequency doubling the laser to the resonance wavelength of $3303 \AA$. Although the oscillator strength of this transition is low, we can operate at high enough $\mathrm{Na}$ density to observe measureable excitation, as confirmed by fluorescence from the 4P level itself, as well as from other levels populated by collision (see below). Using the amount of energy absorbed, we may estimate the size of the expected optoacoustic pulse signal in a manner similar to that outlined above for the $3 P$ level. The anticipated value is $18 \mathrm{mTorr}$ at the microphone, but no signal is observed above the background noise level of $0.2 \mathrm{mTorr}$.

We find the absence of an opto-acoustic signal from this higher-lying level quite surprising. One possibility is that there exists a markedly different rate either for direct quenching of the 4P level itself, or for the rate of subsequent $V$ to $T$ transfer in the flame gases. If the energy were to be trapped in internal modes, it might not form as sharply defined an initially heated region and thus might yield a sound wave much reduced in amplitude. Another possibility is that the $4 \mathrm{P}$ level is removed rapidly by chemical reaction, so that little of the electronic energy is converted into translational energy. Although this latter phenomenon has been seen to exist in flames for the $3 P$ level [5], it would have to be much faster than observed there in order to compete with collisional quenching in our system.

Further information about relative quench and energy transfer rates is obtained from observations using two-photon excitation. Here, the laser is tuned to the wavelength corresponding to half the energy difference between the $3 S$ state and the 3D, 5S, or 4D state. This is, to our knowledge, the first observation of multiphoton excitation within a flame [3], and the technique has useful diagnostic potential beyond the applications to the present experiment.

We observe emission from all four high-lying states when the laser is tuned to excite each one of them, in turn. Ratios of the populations of these levels are obtained from ratios of the fluorescence intensities and inserted into steady-state rate equations describing the collisional energy transfer. By invoking detailed balancing to relate upward and downward transfer rates between pairs of states, we determine all ten transfer rates among the four levels as well as the total quenching rates out of each state, normalized to the value of one of them. The results [4] are collected in table 1.

It may be seen from the results of the table that the 4P level does indeed possess a collisional loss rate (at least in this flame) which is considerably smaller than that of the other nearby levels. On the other hand, the absolute quench rate still remains high, faster than one quenching collision per ns. This value is obtained from our measurements on the 3P level (see below) together with quenching cross-sections for $\mathrm{N}_{2}$ obtained from low pressure experiments on both the $3 \mathrm{P}^{6}$ and $4 \mathrm{P}^{7}$ levels. Consequently, there would appear to be no energy bottleneck attributable to at least the $E$ to $V$ aspect of the energy transfer. 
Table 1. Energy transfer and total loss rates for highly excited states.

Total Loss

$\begin{array}{lr}Q(4 d) & 3.4 \\ Q(5 s) & 3.4 \\ Q(4 p) & 1^{a} \\ Q(3 d) & 2.9\end{array}$

$\begin{array}{lr}\text { Downward } & \text { Transfer } \\ k(4 d \rightarrow 5 s) & 0.49 \\ k(4 d \rightarrow 4 p) & 0.36 \\ k(4 d \rightarrow 3 d) & 1.70 \\ k(5 s \rightarrow 4 p) & 0.22 \\ k(5 s \rightarrow 3 d) & 1.05 \\ k(4 p \rightarrow 3 d) & 0.42\end{array}$

$\begin{array}{ll}\text { Upward Transfer } & \\ k(5 s \rightarrow 4 d)=2.33 \quad k(4 d \rightarrow 5 s) & 1.14 \\ k(4 p \rightarrow 4 d)=0.148 \quad k(4 d \rightarrow 4 p) & 0.053 \\ k(3 d \rightarrow 4 d)=0.048 \quad k(4 d \rightarrow 3 d) & 0.082 \\ k(4 p \rightarrow 5 s)=0.064 \quad k(5 s \rightarrow 4 p) & 0.014 \\ k(3 d \rightarrow 5 s)=0.021 \quad k(5 s \rightarrow 3 d) & 0.022 \\ k(3 d \rightarrow 4 p)=0.323 \quad k(4 p \rightarrow 3 d) & 0.14\end{array}$

${ }^{a} A 1 l$ values normalized to $Q(4 p)=1$.

${ }^{\mathrm{b}}$ Constrained by detailed balancing, at $2545 \mathrm{~K}$, to ratios indicated.

If the quenching of the $4 \mathrm{P}$ level returned the Na predominantly to the ground ( $3 \mathrm{~S}$ ) state, then about twice as much energy would be transferred to the vibrational levels of the flame gases as occurs in a $3 \mathrm{P}$ to $3 \mathrm{~S}$ quenching collision. This would populate a different set of vibrational levels in the flame gases, and conceivably one could have a different mode of $V$ to $T$ transfer and slower formation of the pressure wave. However, the results of table 1 argue against this possibility as well. It is seen from the state-to-state transfer rates that half of the transfer out of the $4 \mathrm{P}$ leaves the $\mathrm{Na}$ atom in the $5 \mathrm{~S}, 4 \mathrm{D}$, or $3 \mathrm{D}$ level (mostly the 3D). Much of the remainder is likely to terminate on the 3P, as fluorescence is observed originating from that level as well. (In fact, the results of table 1 show altogether convincing evidence of step-wise relaxation in the flame, as opposed to a direct return to the ground state. There is a smooth decrease of energy transfer probability with energy defect. This fact has important implications for treating, in a microscopic way, the occurrence of non-equilibrium populations, especially of reactive species, within high temperature systems.) As a result, it appears that a similar range of vibrational levels in the flame gases participate in the $E \rightarrow V \rightarrow T$ transfer out of the $4 P$ level and the $3 P$ level. 
The small value of $Q(4 P)$ from table 1 , which necessarily includes reactive collisions as well, and the size of $k(4 p \rightarrow 3 d)$ suggest that reaction of the $4 P$ level does not occur rapidly. We thus do not understand why the $4 \mathrm{P}$ level excitation does not produce optoacoustic pulses. Perhaps the answer lies in more detailed understanding of the dynamical formation of the pressure wave.

\subsection{Quenching of the 3P level}

When the laser is operated at sufficiently high power to approach optical saturation of the transition, the stimulated emission constitutes a non-negligible contribution to the rate of removal of excited atoms, in comparison with spontaneous radiation and quenching. A measurement of the excited state number density (obtained from fluorescence or opto-acoustic signal) vs laser power allows one to extract the quenching rate. Such operation near saturation was first proposed by Daily [8], and has been applied to the $C_{2}$ molecule [9]. The quench rate of the $3 \mathrm{P}$ level was measured in the flame using the opto-acoustic pulses and a slightly different analysis technique [1]. We have carried out a more extensive series of investigations [4] which include the effects of collisional energy transfer within the $3^{2} \mathrm{P}_{1 / 2}$ and $3^{2} \mathrm{P}_{3 / 2}$ levels as well as quenching of each level.

For a three level system, the steady state equations for the populations of the two excited levels may be written as

$$
\begin{aligned}
B_{13} I N_{1}+T_{23} N_{2} & =\left(B_{31} I+A_{31}+T_{32}+Q_{31}\right) N_{3} \\
T_{32} N_{3} & =\left(A_{21}+Q_{21}+T_{23}\right) N_{2}
\end{aligned}
$$

in the case where level 3 is pumped by the laser, level 2 is populated by collision, and level 1 is the ground state. The terms used are defined in table 2. The experimental value of the population ratio of levels 2 and 3 is obtained from measurement of the fluorescence intensities (denoted $S_{2}$ and $S_{3}$ ) from both doublet components resulting from excitation of one of them. Absolute quench rates for each upper level are obtained from the slope-tointercept ratio of plots of the inverse of the fluorescence from the level vs the inverse of the laser intensity. Furthermore, the opto-acoustic signal $M \propto Q_{31} N_{3}+Q_{21} N_{2}$ may be treated similarly; an example of such a plot is shown in figure 6 . For near thermal equilibration of the doublet levels, the rate equations predict that the plot of $\mathrm{S}_{3}^{-1} \mathrm{vs} \mathrm{I}^{-1}$ should exhibit a slope-to-intercept ratio $2 / 3$ that of a plot of $\mathrm{M}^{-1} \mathrm{Vs} \mathrm{I}^{-1}$. The experimental result over a series of runs was $0.65 \pm 0.09$, indicating that the basic approach of the equations is correct. 
Table 2

laser spectral power density

$\mathrm{N}_{1}$

ground state population $\left(3^{2} S\right)$

$\mathrm{N}_{2}, \mathrm{~N}_{3}$

excited state populations $\left(3^{2} \mathrm{P}_{3 / 2}, 3^{2} \mathrm{P}_{1 / 2}\right)$

$A_{31}, A_{21}$

Einstein coefficients for spontaneous emission, levels $3 \rightarrow 1$ and $2 \rightarrow 1$

$\mathrm{B}_{31}$

Einstein coefficient for stimulated emission, leve $13 \rightarrow 1$

${ }^{B} 13$

Einstein coefficient for absorption, level $1 \rightarrow 3$

$Q_{31}, Q_{21} \quad$ collisional quench rates, levels $3 \rightarrow 1$ and $2 \rightarrow 1$

$T_{32}$

energy transfer rate, leve $13 \rightarrow 2$

$\mathrm{T}_{23}$

energy transfer rate, level $2 \rightarrow 3$

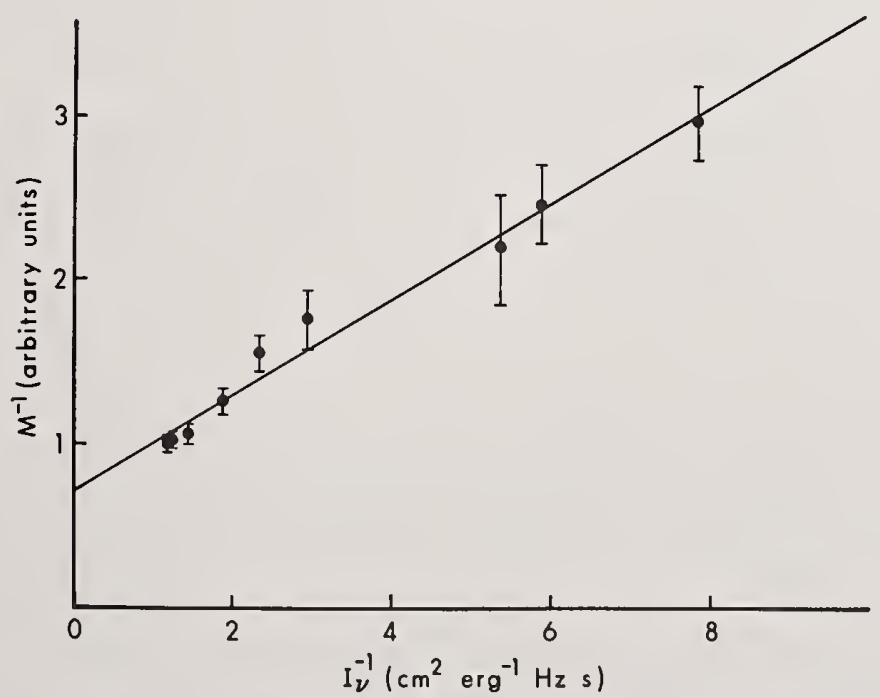

Figure 6. Plot of the inverse of the microphone signal (in arbitrary units) vs the inverse of the laser spectral power density (in absolute units). From the slope to intercept ratio the quenching rate can be obtained. The error bars are from estimates of noise in the recorder output. 
We find that the results [4] can be interpreted only in terms of different quench rates for the two doublet components, such that the ${ }^{2} \mathrm{P}_{3 / 2}$ component is quenched more rapidly by about a factor of three. This rather surprising result has received some confirmation from low pressure experiments on Cs [10], but not on $\mathrm{Rb}[11]$; no information is apparently available for $\mathrm{Na}$. The unequal quench rates clearly have significant implications concerning proper choice of which Na line to monitor during experiments on atomic or laser-excited fluorescence, and other experiments on $\mathrm{Na}$ emission.

The use of laser excited fluorescence requires, in order to obtain absolute concentrations, knowledge of the quenching rate. The use of saturation techniques is a most promising way to overcome this problem in real systems. We have carried out a series of measurements [4] probing the quench rate at different positions in our flame. At fixed $\mathrm{Na}$ concentration, we have found a smooth fall-off of quench rate with height above the burner. However, subsequent experiments over a large range in $\mathrm{Na}$ density (a factor of $10^{4}$ ) have shown some disturbing variation with $\mathrm{Na}$ concentration. (Both $\mathrm{Na}-\mathrm{Na}$ collisions, and a simple picture of self-absorption or optical thickness, can be ruled out as the source of this effect.) In addition, the variation of signal with laser power over a very wide range does not show a simple behavior. The results of our studies indicate that the important problem of the approach to optical saturation in a strongly quenched multi-level system requires significantly more experimental work for a detailed interpretation.

\section{Conclusions}

We have described a new type of opto-acoustic effect--the production of pulsed sound waves in an atmospheric pressure flame following deposition of a laser pulse into electronic energy levels. The experiments on $\mathrm{Na}$ demonstrate that good signal to noise ratios can be obtained at sub-ppb levels of the seeded atoms, using a commercially available laser of modest power. (We add that opto-acoustic signals have also been observed by pumping $\mathrm{Na}$ in the $\mathrm{C}_{2} \mathrm{H}_{2}$-air flame using a dye laser pumped by a $\mathrm{N}_{2}$ laser; a more quantitative study may reveal further details concerning the formation of the pressure wave, since the dye laser pulse is in this case only several ns long as opposed to $1 \mu$ s for the flashlamp-pumped laser.)

The opto-acoustic pulse signal thus forms a sensitive detector of selective absorption of laser radiation. There appears to be no fundamental reason why the technique should not be applicable to other species, including in particular molecular absorption systems. We remain mystified, however, by the lack of opto-acoustic signal from the $\mathrm{Na} 4 \mathrm{P}$ excitation, which suggests that the situation is not entirely straightforward and should be approached on a case-by-case basis. Nonetheless, we feel that there is promise for the technique simply as a detection mode, particularly for species which absorb laser radiation but do not fluoresce readily (either due to high pressure or the rapid crossing into a nonfluorescing state).

The form of the pressure pulse produced, if it yields to analysis, holds information on density gradients within the flame and perhaps on relaxation phenomena within the flame 
gases. For the investigations of quenching in $\mathrm{Na}$ under conditions approaching optical saturation, detection via opto-acoustic pulses provides a measure of the excited state number density under conditions in which fluorescence detection is hampered by selfabsorption problems. It may thus be a useful complement to fluorescence in experiments designed to probe behavior near saturation over a wide range of $\mathrm{Na}$ density.

Finally, and perhaps most important, the method affords the ability to produce a pulsed soundwave locally within the flame. This has provided rapid and spatially resolved measurements of the speed of sound within the combusting system, and could form a useful tool to generate sound waves for an investigation of the noise produced by flames.

\section{References}

[1] Allen, Jr., J. E., Anderson, W. R., and Crosley, D. R., Optics Letters, 1, 118 (1977).

[2] Y. H. Pao (ed.), Optoacoustic Spectroscopy and Detection (Academic Press, New York, 1977).

[3] Fansler, T. D., Colonna-Romano, L. M., and Crosley, D. R., to be published.

[4] Allen, Jr., J. E., Anderson, W. R., Crosley, D. R., and Fansler, T. D., 17th Symposium (International) on Combustion, Leeds, England (Aug. 1978).

[5] Muller, III, C. H., Schofield, K., and Steinberg, M., American Chemical Society Meeting, Anaheim, CA (March 1978).

[6] Hooymayers, H. P. and Alkemade, C., J. Quant. Spect. Radiat. Transfer, $\underline{6}, 847$ (1966).

[7] Gallagher, T. F., Cooke, W. E., and Edelstein, S. A., Phys. Rev. A 17, 125 (1978).

[8] Daily, J. W., App 1. Opt. 16, 568 (1977).

[9] Baronavski, A. P. and McDonald, J. R., J. Chem. Phys. 66, 3300 (1977); Appl. Opt. 16, 1897 (1977).

[10] McGil1is, D. A. and Krause, L., Can. J. Phys. 46, 1051 (1968).

[11] Siara, I. N. and Krause, L., Can. J. Phys. 51, 257 (1973).

\section{Discussion}

Comment (Daily): I just wanted to say I think it is really neat the fun things we can do and still make a living. The opto-acoustic stuff is really great. I think it is a great solution hunting a problem, and one of the problems that occurs to me, particularly in combustion sources, is that often you can't get at what you want to measure by emissive techniques because the species are not emissive.

There seems to be a real potential for looking at some of those dark species 1 ike $\mathrm{H}_{2}$ and $\mathrm{HCO}$, and methoxys, and these kinds of species in flames. I think it really needs to be looked at carefully.

Response (Crosley): It is clear that the opto-acoustic effect is hardly anything new. The point here is that by using a pulse laser, just as one has with fluoresence in looking at the light emission, that one gets a signal which overcomes the background for that short period of time that the laser is on. 
In other words, even that one degree Kelvin over a micro-second, or several micro-seconds time interval gives you an effective temperature for the generation of noise, whatever one might call that, which is much greater than the flame background as opposed to say using a $\mathrm{CW}$ laser and chopping it, and doing ordinary opto-acoustic spectroscopy.

Comment (Hastie): It is kind of interesting that yesterday we had a lot of discussion about high pressure sampling mass spectrometry, and today we are into these pulse laser methods.

It is possible to couple the two techniques. A few years ago we did a very quick experiment, which is essentially an opto-acoustic experiment except that we used the high pressure sampling mass spectrometer instead of a microphone to monitor this pulse character of the gases and flames that we had injected a modulated laser beam into [ref:

Hastie, J. W., Int. J. Mass Spectrom. Ion Phys. 16, 89 (1975)].

There are other ways of looking at this opto-acoustic effect in flames and there may be some advantages to using a mass spectrometer as opposed to a microphone in some cases.

Response (Crosley): A mass spectrometer is obviously very sensitive. I was very surprised to find out even how sensitive ones ears were, until I went and looked it up. You can hear like ten to the minus fifth microns if it is quiet, I quess.

Question (Rosenblatt): You made a very convincing case about how well understood this phenomenon is, and there is one thing you didn't discuss that $I$ am curious about, and that is it appeared that the frequency of the amplitude fluctuations in your microphone signal was pretty constant in the experiments you showed, even when the nature of those amplitudes was varying widely.

I wondered if you could explain what determines that frequency? You have implied that perhaps it had something to do with density gradients in the sample.

Response (Crosley): We didn't pursue this in any kind of quantitative detail, Targely because I don't know enough about either microphones or the flow dynamics.

A lot of what you saw in those traces, in terms of that recurrent frequency, was at least in part a rippling which was set up by the microphone in there. Superimposed upon that were other frequencies which from a very qualitative standpoint were connected with the flow conditions by introducing turbulence into the flame. That is to say, we took an air hose and blew at it. You can vary these things all over the place and you get lots of changes occurring.

This also comes from talking with some people that know a lot more about it than I do. They suggest as we11, that is what is causing some of the structure we are seeing. But, beyond that I have no comment on it.

Question (Rosenblatt): Are there any marked effects seen due to a change in focusing of the Taser? 
Response (Crosley): None that cannot be explained by virtue of the increased power density. due to bringing the thing closer to saturation.

If you dump the laser beam in slowly, or if you dump it into a very small region, a really tiny region, I mean really focus it down, then the energy that you are dumping in is going to have a chance to spread out further, and sort of ooze out a bit without forming a sharp pressure front.

This is more on a time scale type thing than it is on a distance scale. But, there can be some dependence, I think, on that, but we have not been able to find that in the midst of the other aspects, some unknown, of increasing the spectral power density and coming closer to saturation. 

National Bureau of Standards Special Publication 561, Proceedings of the 10th Materials Research Symposium on Characterization of High Temperature Vapors and Gases held at NBS, Gaithersburg, Maryland, September 18-22, 1978. Issued October 1979.

\author{
MEASUREMENT OF ROTATIONAL DISTRIBUTIONS WITHIN \\ $\mathrm{OH}\left(\mathrm{A}^{2} \Sigma^{+}, V^{\prime}=0\right)$ IN FLAMES
}

Mark A. DeWilde and David R. Crosley

Ballistic Research Laboratory

Aberdeen Proving Ground, MD 21005

\title{
1. Introduction
}

Historically, spectroscopic probes using absorption and emission of radiation have provided a large part of our knowledge concerning species and conditions present in high temperature systems such as flames $[1]^{1}$. Even with the advent of new laser-based probe methods, absorption and emission spectroscopy remains a viable (and relatively inexpensive) tool, and information concerning the formation and fate of electronically excited states of course must rely on emission spectra.

We describe here a photon counting experiment which we have used for absorption and emission measurements on the $A^{2} \Sigma^{+}-x^{2} \Pi_{i}$ system of the important $O H$ radical, present in flames of methane or ethane in air at atmospheric pressure. The data analysis scheme uses as input the intensities of several rotational branches. The techniques can also be applied to spectra of other diatomics, such as $\mathrm{CH}, \mathrm{NH}$, or $\mathrm{CN}$, whose spectroscopy is relatively well known, and the experiment should be readily adaptable to on-line control and data acquisition using a mini-or microcomputer.

\section{Experimental Apparatus}

Lean mixtures of methane or ethane with air are burned on a flat flame burner. The burner, constructed along the designs of Bothe and Spaulding [2], is topped with a sintered bronze disk of $6.4 \mathrm{~cm}$ diameter. At the operating pressure of $1 \mathrm{~atm}$, the luminous, primary reaction zone floats $\leq 1.5 \mathrm{~mm}$ above the burner surface. The flame is operated with the burner surface parallel or perpendicular to the spectrometer slit; a 1-4 mm Hartman diaphragm, along with baffles at the flame, provide spatial resolution of a $6 \mathrm{~mm}$ wide $\times 3 \mathrm{~mm}$ high column through the flame.

The image of the flame is focused onto the slit for emission measurements. For absorption measurements, the beams (see below) from a tungsten halide lamp are focused on the slit. Large ( $78 \mathrm{~mm}$ diam), short focal length quartz lenses are used with an iris limiting the aperture to the central portion of the lens to reduce spherical aberration.

1Figures in brackets indicate the literature references at the end of this paper. 
The spectrometer is an in-house modified McPherson. Maintaining proper alinement of the spectrometer has presented a continuing problem; a slight mismatch with narrow slits causes severe loss of signal and degradation of resolution. Using a 1200 groove/mm grating blazed at $7500 \AA$ we obtain half-widths of $\sim 0.04 \AA$ in third order.

The detector is a magnetically shielded EMI 9789QA photomultiplier cooled by the controlled boiloff from liquid nitrogen and operated at a typical voltage of $1350 \mathrm{~V}$. The output pulses from the photomultiplier form the input to a wideband preamplifier. This preamp (used with NIM power supplies) is constructed around a 702 wideband dc amplifier chip, and is illustrated in part of figure 1. With some adjustment of the compensating resistors and capacitors to prevent ringing, this inexpensive $(<\$ 5)$ circuit performs admirably, offering a gain of 100 at a bandwidth of $\geq 30 \mathrm{MHz}$. This is followed by a $10 \mathrm{MHz}$ linear amplifier (gain of 3 ), which is used basically for impedance match.
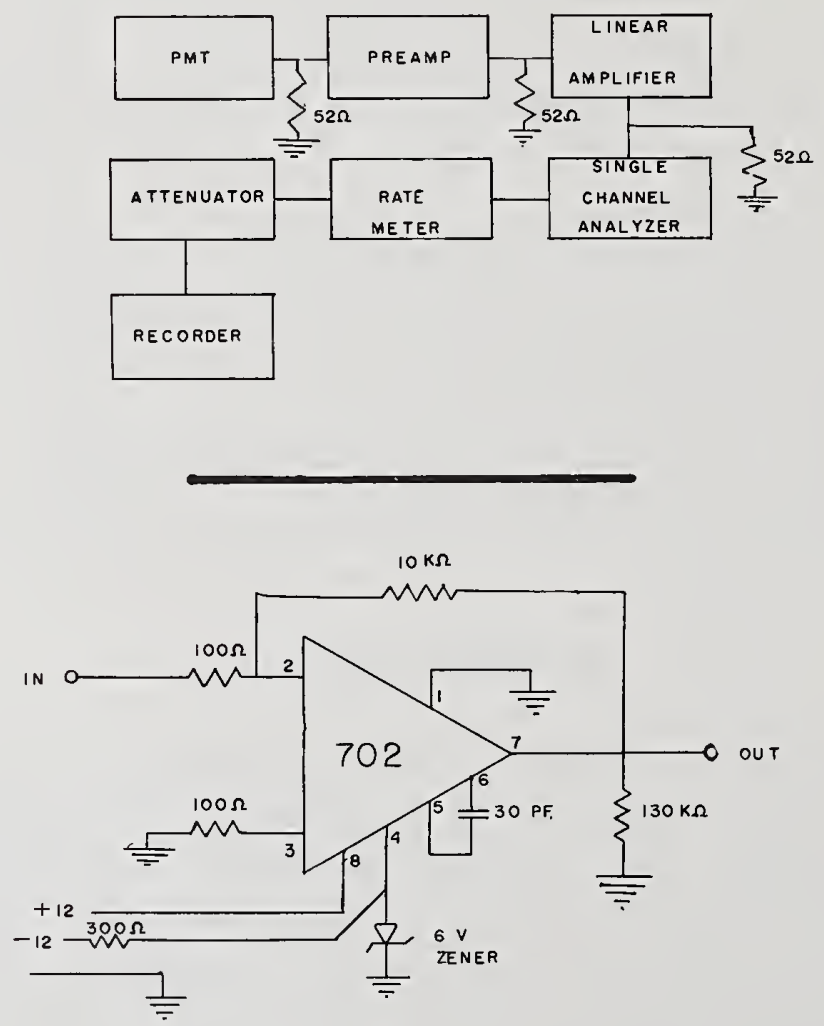

Figure 1. Electronic schematic for emission measurements (see text). The $30 \mathrm{MHz}$, gain 100 amplifier is shown in detail below. 


\section{1 Emission measurements}

Figure 1 shows the photon counting electronics utilized for the emission measurements. The fast preamp and linear amplifier form pulses from each photon detected by the photomultiplier. Since the pulse spread time of the 9789 is $\sim 4$ ns, this permits count rates well in excess of $1 \mathrm{MHz}$ with negligible overlap between pulses. The pulses are then fed to a single channel analyzer (SCA) with upper and lower discriminator levels on the input. The lower is set so as to block dark current originating from the dynode chain; this tailoring results in dark count levels of $<0.5 \mathrm{~s}^{-1}$. The upper removes high voltage spikes such as radiofrequency pickup. The SCA also shapes the pulses, whose rate is monitored by $1 \mathrm{MHz}$ ratemeter. The analog ratemeter output is then recorded. Selectable time constants are available within the ratemeter and at the recorder input.

The ratemeter forms the limiting upper count rate available with this setup. Occasionally, a logarithmic ratemeter and second recorder are used in parallel for monitoring purposes. Typical signal levels for strong lines are at count rates of $1000 \mathrm{~s}^{-1}$ for a viewed region of $1 \mathrm{~mm} \times 4 \mathrm{~mm}$ within the primary zone of the flame at maximum resolution. Count rates greater than $100 \mathrm{kHz}$ are accommodated by attenuating optical filters or operation in a standard current-readout mode (using a chopper and lock-in amplifier to discriminate against dark current).

\subsection{Absorption measurements}

The absorption measurements are made using the output from a $1 \mathrm{KW}$ tungsten halide lamp. The optical and electronic schematic is outlined in figure 2. The lamp housing was modified so as to permit extraction of two beams using front surface mirrors. A standard light chopper was modified (two holes at $90^{\circ}$ ) to permit passage of alternately the probe beam and the reference beam, at a chopping frequency of typically $80 \mathrm{~Hz}$. The probe beam traverses the $\mathrm{flame}$, and the two beams are recombined with a beam splitter. A Corning 754 filter, at the iris diaphragm, removes visible light, while the beams are focused on the slit by an f/0.75 lens. The spatial resolution of the absorption measurements is controlled by baffles in the light path; to date they have only been made at a resolution of $\sim 0.5 \times 1.5 \mathrm{~cm}$.

The reference beam is balanced to the probe beam, for no absorption, by insertion of an attenuating filter or iris. An alternative method was to use a single beam from the lamp, first split and then recombined with a matched pair of beam splitters made by coating quartz with a thin film of aluminum according to the recipe in Strong [3]. Placement of the twohole chopper between lamp and flame assures that any flame emission falls continuously on the slit with either arrangement. The photomultiplier, preamp and linear amplifier are the same as in the emission experiment, though the output is now fed to two parallel SCA-ratemeter channels (see fig. 2). 
The reference signal from the chopper is a square wave; used as a gate to a pulse inhibitor, it allows input of pulses into one of the SCA's (channel B) only when the probe beam is incident on the slit. A level shifter using the reference signal operates as an inverter to raise the lower discriminator level from the baseline section of the other SCA in order to accept only pulses from the reference beam (channel A). The output from the corresponding rate meters, after smoothing with a time constant, is converted into fractional absorption. This is carried out using a small analog computer, whose output is read on a chart recorder.

Typical absorption measurement operating conditions yield $\sim 1000$ counts per second in each channel with $<200$ counts per second of flame emission. With a $3-5$ time constant, the two channels automatically balance to better than 0.2 percent in the presence of emission, permitting absorption measurements at relatively low optical density.

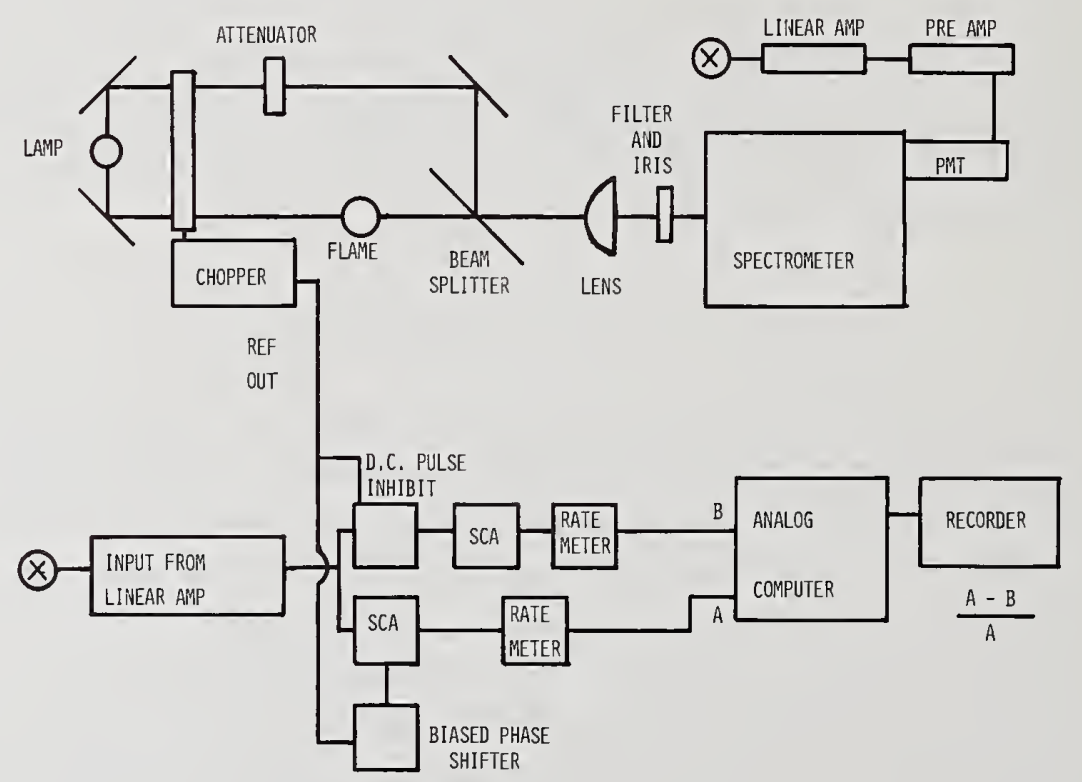

Figure 2. Optical and electronic schematic for absorption measurements. The chopper housing has two windows $90^{\circ}$ apart and uses a two-blade wheel. See text for further description.

\section{Data Analysis and Results}

A small portion of an emission spectrum, near the head of the $(0,0)$ band, is shown in figure 3. This is taken in the primary zone of an ethane/air flame. Emission is seen (in the full scan) from levels as high as $N^{\prime}=30$, requiring coverage to past $3200 \AA$ to include the Q-branches. Many of the individual rotational lines are resolved with $0.04 \AA$ linewidths here, although, as seen from figure 3 , some are overlapped. The data analysis 
procedure used as input the intensities of all the rotational lines which provide reliable data. We typically measure $~ 120$ separately identifiable spectral features in order to fit the populations of $\sim 50-55$ levels.

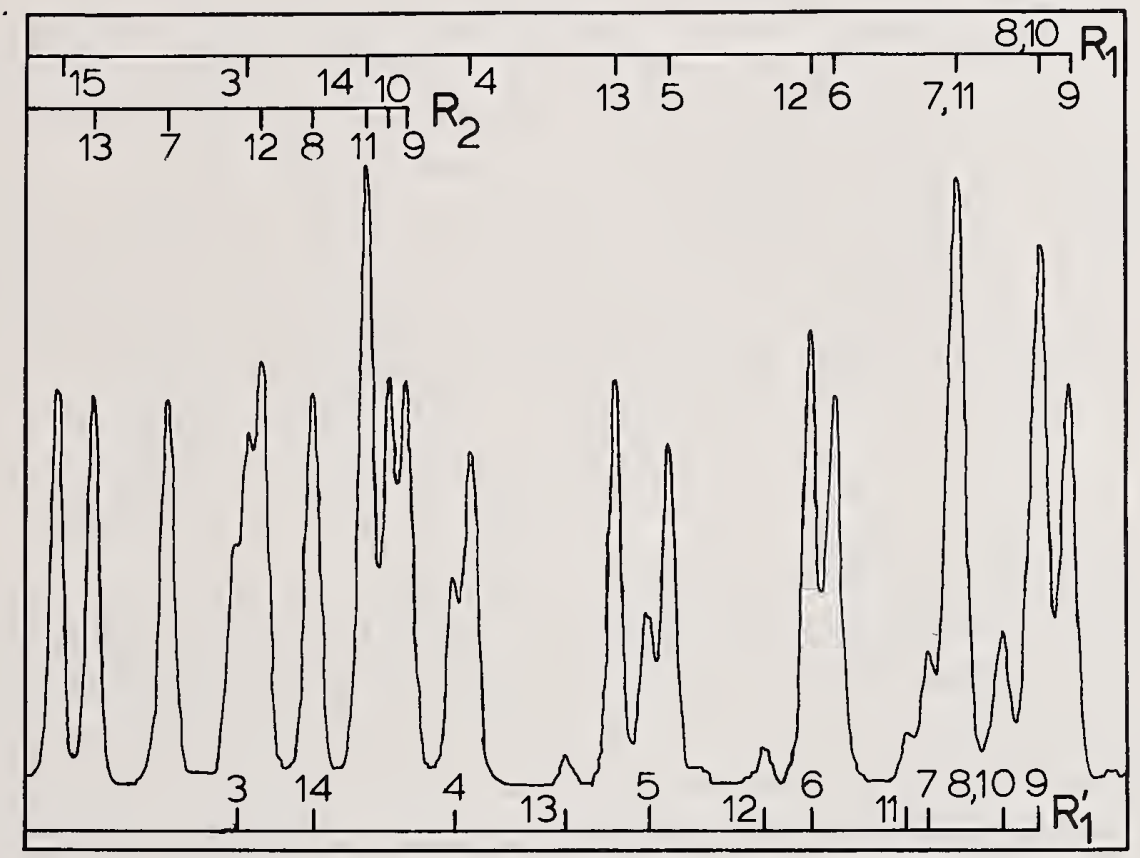

Figure 3. A small portion (3064 to $3070 \AA$ ) near the $(0,0)$ band head, of the emission spectrum from the primary reaction zone of an ethane/air flame.

The data analysis technique used is a least-squares fit of the intensities to the level populations, using matrix computations easily handled by a computer. This program was originally set up for the analysis of spectral data in a study [5] of energy transfer within the A-state. In reference [5], this was applied to data taken at significantly lower resolution than that shown in figure 3 , and yielded reliable populations from lines containing much overlap and blending. While we here operate at higher resolution, largely to overcome overlap with lines of the $(1,1)$ band not important in [5], the procedure, in general, wil] be outlined.

From the spectra are extracted, manually, numerical magnitudes of a number of spectral "features." These are definable, reproducible peaks or obvious shoulders; at the resolution used for the scan of figure 3 they are almost all peaks.

Now the intensity at any given wavelength, $I(\lambda)$, may be expressed as 


$$
I(\lambda)=\sum_{i, j} N_{j} S_{i, j} f\left(\lambda-\lambda_{j}\right)
$$

Here, $N_{i}$ is the population of level $i$ and $S_{i j}$ is the relative transition probability [6] of the $j$ th rotational branch emitted by level $i$. The rotational line is centered at wavelength $\lambda_{j}$; the fraction of light passed by the monochromator at wavelength $\lambda$ is given by the bandpass function $f\left(\lambda-\lambda_{j}\right)$. For low resolution measurements [5], a triangular bandpass function was appropriate. In the present work, a numerical analysis of lineshapes in terms of Voigt integrals [7] indicated a complex function, so when needed the function $f$ was determined by direct graphical comparison to unblended lines.

The intensity of the kth feature can then be expressed by eq. (1), in terms of one or more rotational level populations. In matrix notation $\vec{I}=E \vec{N}$, where $F_{k i}$ is the matrix element $s_{i j} f\left(\lambda_{k}-\lambda_{j}\right)$ for the kth feature containing the $j$ th branch emitted by level $i$. The least squares solution to this overdetermined set of equations is

$$
\vec{N}=\left(E^{t} E\right)^{-1} E^{t} \overrightarrow{1}
$$

where $t$ denotes transpose and -1 signifies inversion.

The features are numbered consecutively in order of increasing wavelength, and the populations are labeled in order of ascending energy. The wavelengths of those features containing more than one line is not measured, but rather calculated from the known transition wavelengths [4] and estimated populations. Often, the wavelength of the feature is that of its strongest component, although in some cases no dominant transition exists and an average value is assigned. In these cases, subsequent adjustment was necessary in order to correct for poor initial estimates, although the average value after this adjustment was then usable for the same flame and slit width conditions. Some of the features are problematic because they contain many strong transitions of the $(0,0)$ band, e.g., the $Q_{2}$ head, or because of severe overlap with a congested region of the $(1,1)$ band. Weighting factors are included in the fitting procedure; usually the $Q_{2}$ head in particular is weighted at zero.

The matrices used are large $(120 \times 55)$ but sparse (over 95 percent of the matrix elements are zero), and the program was more efficiently run on the computer by writing specific element-by-element programs for handling the multiplication and transposition (the matrix to be inverted is smaller and treated with a standard package).

The results may be gaged by the smoothness of the Boltzmann plot (see below), and by the ability of the fitted populations to reproduce the measured intensities to within an average deviation of $\sim 6$ percent, which is approximately the level of noise due to flame instabilities in the experiments to date. It should be noted that the lower resolution spectra of [5], with about a fourth of the levels and features used here, were commonly fitted to within 1 percent.

A Boltzmann plot for the secondary zone of an ethane/air flame is shown in figure 4. Here, for plotting purposes, the populations of the two fine structure components for a given rotational level have been combined in each point; this reduces the apparent scatter 
but does not alter the fitted line. The high rotational levels correspond to a temperature of $2700 \mathrm{~K}$; a line corresponding to $1400 \mathrm{~K}$, which is the temperature measured with a thermocouple, is also included.

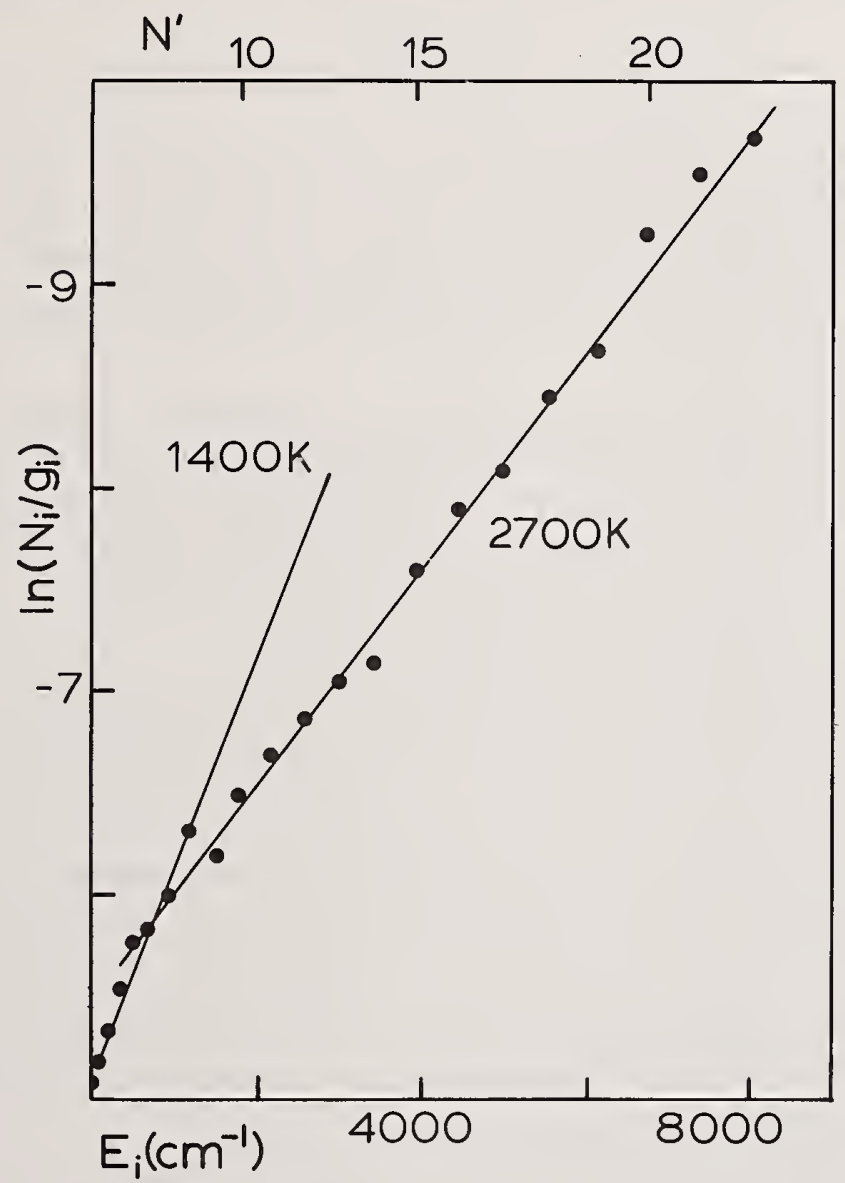

Figure 4. Boltzmann plot of the rotational level populations in the secondary combustion zone of an ethane/air flame. Across the top are the values of rotational quantum number $N$ corresponding to the energies.

The higher temperature for the high rotational levels may be indicative of a chemiluminescent reaction forming the $\mathrm{OH}$. Much hotter temperatures for these high-lying rotational levels were also observed in the primary zone for both ethane/air and methane/air $\mathrm{fl}$ ames. For the latter, a temperature of $8500 \pm 500 \mathrm{~K}$ was obtained. This phenomenon has been observed before $[1,8]$ for methane/air flames and was there considered in terms of formation through a chemiluminescent reaction with subsequent removal of the $\mathrm{OH}$ from the $\mathrm{A}^{-}$ state (by quenching) prior to rotational thermalization.

In several of our emission scans, it is obvious that self-absorption of the emitted radiation is present. The preliminary absorption scans indicate about 25 percent absorption for the strongest lines through the entire path length of the flame in the secondary zone, and as much as 75 percent absorption in the primary zone. Consequently, the data must be 
examined within this framework. We have not yet implemented the program to include selfabsorption; this could be done by a computer search and subsequent analysis [9] using the iso-intensity method [4].

\section{Summary}

This experiment has been undertaken primarily as an application of modern pulsecounting technology--such as is widely used in nuclear physics--to absorption and emission spectroscopy of a high temperature system. Also envisioned is the ready adaptation of these techniques to on-line control and data acquisition by a small computer such as an 8080-based microprocessor.

Integral to this end is the use of the fast pre-amp built around the inexpensive 702 chip, and a liberal use of SCA's with discriminator level inputs. Analog equipment has been used in large part due to its availability in the laboratory here, although count rates of $100 \mathrm{kHz}$ and less can be easily accommodated digitally by a computer. Inclusion of a third channel, and appropriate changes in the timing circuitry of figure 2 , should permit simultaneous recording of the absorption and emission spectra.

Incorporation of the analysis program directly into the computer is feasible if very reproducible slit settings are obtained over a long period of time. Here, the adjustable slits of the McPherson are probably inadequate and should be replaced by fixed slits fabricated by etching a quartz sheet sputter-coated with niobium (niobium films are almost indestructible). For a molecular band system with well characterized spectra such as the $\mathrm{OH}$ studied here, a pattern-recognition program (counting the peaks) could be built into the computer for the purpose of assigning the lines. This would be preferable to a reliance on the linearity of the spectrometer drivescrew and an assignment of peaks by wavelength obtained from, say, the number of pulses into a stepper motor.

We have not yet concentrated on the data in terms of the analysis needed to take into account self-absorption in this long path length, optically dense flame. The absorption scans clearly demonstrate the necessity of this, and it is hoped that the analysis can be easily and directly incorporated into the analysis programs.

\section{References}

[1] Gaydon, A. G., The Spectroscopy of Flames, 2nd Ed. (Chapman and Ha11, London, 1974).

[2] Botha, J. P., and Spaulding, D., Proc. Roy. Soc. A225, 71 (1959).

[3] Strong, J., Procedures in Experimental Physics, (Prentice-Ha11, Englewood Cliffs, NJ, 1938).

[4] Dieke, G. and Crosswhite, H. M., JQSRT 2, 97 (1962); see also Bass, A. M., and Broida, H. P., NBS Circular 541 (1953).

[5] Lenge1, R. K., and Crosley, D. R., J. Chem. Phys. 67, 2085 (1977); 68, 5309 (1978).

[6] Chidsey, I. L., and Crosley, D. R., this volume. 
[7] Kuhn, H. G., Atomic Spectra, 2nd Ed. (Academic Press, New York, 1969).

[8] Broida, H. P., J. Chem. Phys. 19, 1383 (1951).

[9] Shuter, K. E., J. Chem. Phys. 18, 1466 (1950); see also Broida, H. P., NBS Circular 523,23 (1954). 



\section{ROTATIONAL DEPENDENCE OF TRANSITION PROBABILITIES \\ IN THE OH A-X BAND SYSTEM}

Irving L. Chidsey and David R. Crosley

Ballistic Research Laboratory

Aberdeen Proving Ground, MD 21005

\section{Introduction}

Spectroscopic observations furnish a nonintrusive thermometer for use in gaseous systems over a wide range of temperature. From measurements of the relative intensities of lines or bands of some molecular electronic transition, can be obtained the population distribution over a specified set of states. Under the presumption that thermal equilibrium does obtain, this distribution may be used to calculate a temperature for the internal degree of freedom corresponding to that particular set of states. When thermal equilibrium is not the case--as in a set of product states formed from some exoergic, perhaps chemiluminescent, reaction--then the population distribution itself is often of direct interest.

The techniques traditionally employed for this purpose have been absorption and emission measurements, providing vibrational and/or rotational population distributions for ground and excited electronic states, respectively. In the past few years, the method of laser-excited fluorescence (LEF) has been seen to furnish a very sensitive means of carrying out what is, essentially, an absorption measurement on a variety of (typically free radical) transient species. In addition to the high sensitivity, LEF also offers high spectral resolution and excellent spatial and temporal resolution.

Obtaining accurate population distributions from any of these three methods requires, in turn, accurate values of the electronic transition probability, i.e., that quantity connecting the intensity of a given line or band with the population of the level (or set of levels) responsible for its occurence.

In this paper, we consider the specific problem of the accurate determination of transition probabilities as a function of vibrational and rotational levels for the $A^{2} \Sigma^{+}-X^{2} \Pi_{i}$ system of the $\mathrm{OH}$ radical. Such a study is important for two reasons. First, $\mathrm{OH}$ is a nearly ubiquitous molecule in any high temperature system containing hydrogen and oxygen, and is a key participant in many reaction networks involved in combustion and pollutant chemistry. It is easily monitored in emission, and has been studied relatively widely in absorption as we11. In particular, it is a choice candidate for study by LEF, due both to its importance and the fact that the $A-X$ system falls serendipitously in the wavelength region corresponding 
to the frequency-doubled output of some of the most efficient laser dyes. Second, the $\mathrm{OH}$ molecule is unusual in that the transition probability depends markedly on rotational level, a consequence of the strong variation in the electronic transition moment with internuclear distance coupled with the high degree of centrifugal distortion in this light molecule.

The form of the electronic transition moment is a key part of these calculations. Earlier studies were performed on $\mathrm{OH}[1]^{1}$ and $\mathrm{OD}[2]$, referred to as $\mathrm{I}$ and II, respectively, which established this form from a combination of experimental measurements, a critical review of the literature, and theoretical calculations. These papers, which deal with vibrational band intensities, are summarized below. However, the experimental data available are not in themselves accurate enough to determine the rotational dependence of the transition probabilities directly from measurement. Thus, the transition moment established from vibrational band intensities in I and II, with one modification, are used to calculate this rotational dependence using accurate wavefunctions for the two electronic states involved. These calculations are described, and a few selected results are presented. The full set of transition probabilities will be published separately as a report [3].

We conclude the introduction with a set of equations defining the quantities involved. Single and double primes denote, respectively, the excited $(A)$ and ground $(X)$ states; $v$ and $J$ refer to vibrational and rotational quantum numbers.

For a dipole operator $M_{e}$ and electronic wavefunctions $\psi_{e}$, the electronic transition moment $R_{e}$ is given by

$$
R_{e}(r)=\int_{0}^{\infty} \psi_{e}^{\prime}\left(r, r_{e}\right) M_{e}\left(r, r_{e}\right) \psi_{e}^{\prime \prime}\left(r, r_{e}\right) d r_{e}
$$

Here $r$ is the internuclear distance, and $r$ signifies all electron coordinates. The transition probability for two vibrational-rotational levels is

$$
p_{v^{\prime \prime} J^{\prime \prime}}^{v^{\prime} J^{\prime}}=\left|\int_{0}^{\infty} \psi_{v^{\prime} J^{\prime}}(r) R_{e}(r) \psi_{v^{\prime \prime} J^{\prime \prime}}(r) d r\right| 2
$$

If $R_{e}$ can be expressed as a linear function of $r, p$ can be easily written in terms of the Franck-Condon factor

$$
q_{v^{\prime \prime} J^{\prime \prime}}^{v^{\prime} J^{\prime}}=\left|\int_{0}^{\infty} \psi_{v^{\prime} J^{\prime}}(r) \psi_{v^{\prime \prime} J^{\prime \prime}}(r) d r\right|^{2}
$$

and the $r$-centroid

$$
\bar{r}_{v^{\prime \prime} J^{\prime \prime}}=\int_{0}^{\infty} \psi_{v^{\prime} J^{\prime}}(r) r \psi_{v^{\prime \prime} J^{\prime \prime}}(r) d r / \int_{0}^{\infty} \psi_{v^{\prime} J^{\prime}}(r) \psi_{v^{\prime \prime} J^{\prime \prime}}(r) d r .
$$

\footnotetext{
${ }^{1}$ Figures in brackets indicate the literature references at the end of this paper.
} 
The Einstein A-coefficient, or rate of emission $\left(s^{-1}\right)$ from the $v^{\prime} J^{\prime}$ level to the $v^{\prime \prime} J$ "level, is

$$
A_{v^{\prime \prime} J^{\prime \prime}}^{v^{\prime} J^{\prime}}=\frac{64 \pi^{4}}{3 h c^{3}} p_{v^{\prime \prime} J^{\prime \prime}}^{v^{\prime} J^{\prime}} S_{J^{\prime} J^{\prime \prime}}\left(v_{v^{\prime \prime} J^{\prime \prime}}^{v^{\prime} J^{\prime}}\right)^{3} /\left(2 J^{\prime}+1\right)
$$

where $\nu$ is the frequency of the transition. $S_{J^{\prime} J^{\prime \prime}}$ is the rotational line strength, here normalized such that $\Sigma_{J^{\prime \prime}} S_{J^{\prime} J^{\prime \prime}}=2 J^{\prime}+1$. It is an angular momentum factor denoting that portion of all emission from the $J^{\prime}$ level which goes into the particular $J^{\prime}, J^{\prime \prime}$ branch. The lifetime of the excited level, $\tau_{v^{\prime} J^{\prime}}$, is the reciprocal of the sum of all Einstein coefficients for that level:

$$
\tau_{v^{\prime} J^{\prime}}=\left(\Sigma_{v^{\prime \prime} J^{\prime \prime}} A_{v^{\prime \prime} J^{\prime \prime}}^{\left.v^{\prime}\right)^{\prime}}\right.
$$

For absorption from the $v^{\prime \prime} J "$ level, one must consider the Einstein B-coefficient

$$
B_{v^{\prime} J^{\prime}}^{V^{\prime \prime} J^{\prime \prime}}=\frac{1}{4 \pi} \frac{2 J^{\prime}+1}{2 J^{\prime \prime}+1} \frac{c^{2}}{2 h v^{3}} A_{v^{\prime \prime} J^{\prime \prime}}^{v^{\prime} J^{\prime}}=\frac{8 \pi^{3}}{3 h^{2} c} p_{v^{\prime \prime} J^{\prime \prime}}^{V^{\prime} J^{\prime}} s_{J^{\prime} J^{\prime \prime}} /\left(2 J^{\prime \prime}+1\right) .
$$

A comment on the units of $B$ is in order. They are $\mathrm{cm}^{2} \mathrm{erg}^{-1} \mathrm{~s}^{-1}$. An incident spectral power density per unit frequency interval in units of erg $\mathrm{cm}^{-2} \mathrm{~s}^{-1} \mathrm{~Hz}^{-1}$, when multiplied by $B$, yields the rate of absorption per second (i.e., the number of absorptions per second per molecule).

\section{Vibrational Band Intensities}

The first series of measurements on intensities in the $A-X$ system of $O H$ was a part of the classic work by Dieke and Crosswhite [4] (referred to as DC) on this molecule. From these data have been suggested two functional forms for $R_{e}$. The first, due to Shuler [5], was a transition moment linear in $r$ :

$$
\mathrm{R}_{\mathrm{e}}=c(1-\rho r)
$$

where $c$ is a constant. Using Morse oscillator wavefunctions, Shuler fitted to the DC data a value for $\rho$ of $0.75 \AA^{-1}$. Learner $[6,7]$ later suggested that a preferable form of $R_{e}$ was one exponential in internuclear distance:

$$
R_{e}=c^{\prime} e^{-\alpha r}
$$

His preference was based on the fact that the linear transition moment provided very poor agreement with the measured $D C$ intensity ratio of the $(1,1)$ and $(1,0)$ bands, and on a more 
recent absorption oscillator strength measurement [8] of the $(1,0)$ and $(0,0)$ ratio. The disagreement between ratios of band intensities, among the several experimental and theoretical studies, range over more than a factor of two for some key bands. This is clearly an untenable situation if these intensity values are used to obtain vibrational temperatures or population distributions.

In I and II, a pulsed, tunable, frequency-doubled dye laser was used to populate individual $V^{\prime}, J^{\prime}$ levels of the $A^{2} \Sigma^{+}$state, and the fluorescence intensities of transitions to various $v^{\prime \prime}$ levels were measured. For $\mathrm{OH}, 10$ bands were measured, providing seven independent ratios with no necessary presumptions concerning the upper state vibrational temperature. For $0 D$, six such ratios were obtained. From these, a linear moment was fitted to the experimenta data, with the resulting choice for $\rho$ the same as that of Shuler. The results exhibited considerably better agreement with the predictions of the linear moment compared to the predictions of the exponential moment.

The earlier data mentioned above, as well as the results of several other studies, were examined critically as an overall comparison between the two forms of $R_{e}$. The $(1,0)$ band intensity measurement of $D C$ was seen to be inconsistent with their other measurements; they themselves had expressed doubts as to its accuracy. In addition, the oscillator strength measurement [8] appeared out of line with other values for this quantity, though it was not clear why this was so. The overall conclusion of I was that a linear transition moment did a much preferable job of fitting the vibrational transition probability data, and that the predicted values were within limits imposed by experimental error bars. In II, the same conclusion was reached considering only the $O D$ data; this is valid independent confirmation since $q$ and $\bar{r}$ vary with isotope but $R_{e}$ should not.

One fundamental difficulty with a linear transition moment is that it unphysically passes through zero at a value of $r=\rho^{-1}=1.33 \AA$. This did not significantly affect the calculation of the vibrational transition probabilities, since the important bands effectively "sample" a range of $r$ much less than this value. An ab initio calculation by Henneker and Popkie [9] (referred to as HP) of the wavefunctions $\psi_{e}$ in eq. (1) resulted in an $R_{e}$ which was linear at small $r$ and tailed smoothly to zero at larger $r$. This yielded good agreement with the relative vibrational transition probabilities, and this general form is adopted for the calculations described below.

The data on the rotational dependence of the transition probabilities were also examined in $\mathrm{I}$ and II. Experimental results exist primarily over the range of $\mathrm{J}$ between 0.5 and 10.5, and the variation is too small to discern any preference for a particular form of $R_{e}$. Over this limited range, the transition probability could be represented by a form linear in $J^{\prime}\left(J^{\prime}+1\right)$ :

$$
p_{v^{\prime \prime} J^{\prime \prime}}^{v^{\prime} J^{\prime}}=c^{\prime \prime}\left[1-\beta J^{\prime}\left(J^{\prime}+1\right)\right]
$$

where $\beta$ varies with vibrational band and rotational branch. The linear moment calculations in I furnished a value of $\beta=3 \times 10^{-4}$, for the $(0,0)$ band of $0 \mathrm{H}$. Using Coxon's values [10] for $q_{v^{\prime \prime}}^{v^{\prime} J^{\prime \prime}}$ and $\bar{r}_{v^{\prime \prime} J^{\prime \prime}}$ for $0 D, \beta$ values for a variety of bands were obtained in II. However, it was clear that eq. (10) did not hold true over the full range of $\mathrm{J}$ often observed in emission 
experiments, and that a full set of calculated transition probabilities would be desirable. In addition, for high values of $\mathrm{J}$, the region of internuclear distance near and beyond 1.33 . A cannot be neglected.

\section{Calculation of the Transition Probabilities}

\subsection{The functional form of the transition moment}

We adopt the following approach to the electronic transition moment. The vibrational band intensity information both reported and reviewed in I and II confirms the linear form, eq. (8) with $\rho=0.75 \AA^{-1}$, for values of $r<1.25 \AA$. This is valid since the most accurate experimental data exist for the stronger bands at low values of $\mathrm{J}$; there is relatively little contribution to the integral of eq. (2) for $r$ greater than this value.

The HP calculations [9] also exhibit a linear form for $R_{e}$ in this region and good agreement with the experimental data of $I$. At larger values of $r$ the HP form for $R_{e}$ smoothly tails toward zero, with an apparent functional form resembling a hyperbola (the results of [9] are presented in numerical form). This is consistent with the fact that the $A-X$ transition is allowed in the united atom limit but forbidden in the separated atom limit. The success of the HP ab initio form at low $r$ compared with experiment engenders confidence in this form at larger $r$ where no such comparison can be made.

Accordingly, we have fitted the ab initio $R_{e}$, using parameters compatible with the RKR wavefunctions described below, to a hyperbolic functional form. The asymptotes are $R_{e}^{\ell}=0$ at large $r$ and $R_{e}{ }^{S}=c(1-\rho r)$ at small $r$. The total functional form may then be written

$$
R_{e}(r)=\frac{1}{2} c(1-\rho r)-\frac{1}{2} c\left[(1-\rho r)^{2}+a\right]^{1 / 2} .
$$

This is obtained by solving the equation

$$
\left[R_{e}(r)-R_{e}^{l}\right]\left[R_{e}(r)-R_{e}^{s}\right]=b
$$

and fitting to the HP results at an intermediate point to obtain the constant a. This functional form exhibits good agreement with the HP numerical results over the entire range of internuclear distance, using this fit at one point and two asymptotes.

\subsection{Determination of parameters in equation (11)}

As mentioned above, the experimental data on which to base a fit of eq. (11) consists of vibrational band intensity ratios at low $\mathrm{J}$. Such ratios for $\mathrm{J}=0.5$ were computed using a range of values of both $\rho$ and $a$. A search for a minimization of the unweighted sum of squares of the deviation between predicted and experimental values, as a function of both $\rho$ and $a$, provided an initial guide to this fit. The final choice, however, was dictated by weighting the fitted ratios according to our judgment of the quality of the experimental data. The rationale for this judgement is discussed in detail in I and II. Certain of the band intensity ratios are more dependent upon the value of $R_{e}(r)$ at small $r$, and are thus 
more sensitive to the value of $\rho$ than to the value of a. These were used primarily to choose essentially by inspection of the numerical results, $\rho=0.756 \AA^{-1}$. 0ther ratios involving larger $\bar{r}$ values and hence more sensitive to $a$, were used with this value of $\rho$ to select $a=$ 0.004 .

This (rather judgmental) approach results in what we feel is the best two-parameter fit to the A-X transition moment, in view of all the information available. (It should be recalled that, given a linear form at low $r$, a two-parameter form is demanded by a physical picture.)

3.3 The wavefunctions and transition probabilities $p_{v^{\prime \prime}}^{v^{\prime} J^{\prime \prime}}$

The wavefunctions used in eq. (2) were obtained from the RKR (Rydberg-Klein-Rees) method of inverting spectral constant data to obtain diatomic potential energy curves, and numerical integration of the pertinent Schrödinger equation. Modern computational programs devised for this purpose [11] have made the RKR method a standard procedure. We have utilized the programs RKR/FCF developed and made available to us by Professor R. N. Zare.

These two programs were combined into one so that the rotational level could be varied in a loop fashion, and calculations made for $P, Q$, and $R$ branches $\left(\Delta J \equiv J^{\prime}-J^{\prime \prime}=-1,0\right.$ and +1 , respectively) over a wide range of $v$ and $J$. Input Dunham coefficients were taken from Kinsey [12] for $\mathrm{OH}$ and Coxon [10] for $0 \mathrm{D}$. The program calculated $\mathrm{q}$ and $\bar{r}$ from eqs. (3) and (4), respectively, for insertion into the integrated form of eq. (8), and carried out the direct numerical integration of eq. (2) for the $R_{e}(r)$ given in eq. (11).

Checks of the program indicated proper operation. Comparisons of $q$ and $\bar{r}$ for $J=0$ were made with the Morse calculations of I and II, and Coxon's J-dependent RKR results [10] for $0 D$. $\quad v^{\prime}$ ranged from 0 to $3, v^{\prime \prime}$ from 0 to 4 ; the maximum value of $J$ was dictated by the approach of the total energy to the A-state dissociation limit, at which point the integration routines failed. The limits of integration were 0.3 to $5.3 \mathrm{~A}$, well covering the range of significant contribution in eq. (2).

\subsection{Rotational line strengths}

The rotational line strengths $S_{J^{\prime}} J^{\prime \prime}$ were calculated using (corrected forms of) eq. (13) in DC. The spin-orbit coupling constant was calculated as a function of rotational level [13], using the DC spectral constants, for use in the line strength calculations.

\subsection{Transition probability results}

The results of the preceding two sections were then combined to yield relative Einstein $A$ and $B$ coefficients (eqs. (5) and (7)) for direct use in analyses of intensity measurements. The relative lifetime of each excited level was calculated using eq. (6) for comparison with experimental data. The frequencies $v_{v^{\prime \prime}}^{v^{\prime} J^{\prime \prime}}$ needed for these calculations were taken from table 13 of DC when available, and otherwise computed from the spectral constants; they are of sufficient accuracy for the current purposes. 
These results may be put on an absolute foundation, using the radiative lifetime measurements of German [14], although this is not necessary for temperature determinations. The full set of results will be published separately [3]. Table 1 lists some selected and representative relative values of the quantity $p_{v^{\prime \prime} J^{\prime \prime}}^{v^{\prime}} \nu^{3}$ for the $(0,0),(0,1)$, and $(1,1)$ bands (that is, the Einstein coefficients divided by rotational line strengths). The $v^{3}$ value used is computed for the transitions to ${ }^{2} \Pi_{3 / 2}$; explicit computation of the ${ }^{2} \Pi_{1 / 2}$ transitions alter these figures zero or one digits in the last significant figure.

Table 1. Selected values of $p^{3}$, normalized to the value for $\mathrm{J}^{\prime}=2.5, \mathrm{~J}^{\prime \prime}=1.5$ of the $(0,0)$ band.

\begin{tabular}{|c|c|c|c|c|c|c|c|c|c|}
\hline BAND & & 0,0 & & & 1,0 & & & 1,1 & \\
\hline BRANCH & R & $Q$ & $P$ & $\mathrm{R}$ & $Q$ & $P$ & $\mathrm{R}$ & Q & $\mathrm{P}$ \\
\hline$J^{\prime \prime}=1.5$ & 1000 & 997 & 994 & 335 & 329 & 325 & 567 & 569 & 571 \\
\hline 10.5 & 906 & 894 & 882 & 346 & 321 & 298 & 481 & 492 & 500 \\
\hline 20.5 & 647 & 640 & 629 & 327 & 292 & 259 & 284 & 306 & 324 \\
\hline 25.5 & 480 & 479 & 475 & 294 & 262 & 231 & 174 & 198 & 219 \\
\hline
\end{tabular}

In considering the results in table 1 , the following remark is in order. In general (though not for all bands) the transition probabilities $p_{v^{\prime \prime}}^{v^{\prime}} J^{\prime}$ for a given $J^{\prime \prime}$ are ordered such that the value for the P-branch is larger than that for the Q-branch, which is in turn larger than that for the R-branch. On the other hand, the values of table 1 contain as well the $\nu^{3}$ factor (see eq. (5)), which has the opposite ordering effect.

\section{Discussion}

The physical reason for the rotational dependence of the transition probabilities in the $\mathrm{OH}$ A-X system is the large amount of centrifugal distortion in this light molecule. As $\mathrm{J}$ increases, the effective average internuclear distance in both states increases, so that the effective average transition moment within any given band decreases. In addition, the amount of vibrational overlap (the Franck-Condon factor) is also altered, although the magnitude and sign of its variation with $\mathrm{J}$ depends on the vibrational levels involved.

The oscillator strength measurements as a function of $\mathrm{J}$ in the $(0,0)$ band [8] were the first direct experimental confirmation of this dependence expected on theoretical grounds [6] as well as by an examination [15] of rotational temperatures in $\mathrm{OH}$. The 1 inear dependence of $p_{v^{\prime \prime} J^{\prime \prime}}^{v^{\prime}}$ on $J(J+1)$, as in eq. (8), was suggested in [8], and examined in $I$ in the light of a linear $R_{e}$ and the existence of further experimental data. In II, Coxon's $q$ and $\bar{r}$ values [10] together with the linear $R_{e}$ were used to calculate $\beta$ values for a number of bands for the $0 D$ isotope. This was done through computation of the $p$ values and a fit to eq. (8), even though the variation was not quite linear. Nonetheless, the observed trends--that $\beta$ increases as $\Delta v$ $=v^{\prime}-v^{\prime \prime}$ decreases, and, for a given $\Delta v$, increases as $v^{\prime}$ increases--were useful and consistent with the physical picture. 
A comparison of the anticipated $\beta$ values for $\mathrm{OH}$ with the results of the present calculations show again the expected trends. However, we have been unable to fit our results to a simple functional dependence on $\mathrm{J}(\mathrm{J}+1)$. Over a limited range, $0<\mathrm{J}<15$, eq. (8) is good to within a few percent and $\beta$ values have been calculated [3]. This is of some advantage in that a simple correction to the observed temperature could then be applied (see I). On the other hand, since the analysis of $\mathrm{OH}$ spectral data will likely be undertaken using machine computation, it seems to us nearly as simple to use the direct numerical results in a table look-up manner.

Using the exponential moment, eq. (9), Anketell and Learner [7] (referred to as $\mathrm{AL}$ ) calculated transition probabilities as a function of $\mathrm{v}$ and $\mathrm{J}$ using Morse-Pekeris wavefunctions. This work has been widely utilized in subsequent investigations. In I and II, it was concluded that this form of $R_{e}$ poorly described some of the important vibrational transition probabilities. However, the variation in $\bar{r}$ with $\mathrm{J}$ over a single band is considerably less than the differences in $\bar{r}$ from band to band. Hence, the relative values of $p_{v^{\prime \prime}}^{v^{\prime \prime}} J^{\prime \prime}$ varying with $\mathrm{J}$ over an individual band, calculated using the exponential moment, should be more accurate. In table 2 we compare some selected values from our calculation with those of $\mathrm{AL}$; listed are ratios for $\mathrm{p}$ with a given $\mathrm{J}$ to the value of $\mathrm{p}$ for $\mathrm{J}=1.5$ in the same band.

It may be seen from table 2 that, for $\mathrm{J}<20$, our results and those of AL differ by amounts of the order of ten percent or less. Thus, rotational temperatures deduced using the transition probabilities of $A L$ do not suffer from much systematic error. In this connection, the major point in favor of our calculations over those of $A L$, when one is considering only the rotational dependence of $p$, is the firmer basis of our form of $R_{e}(r)$.

We reiterate the philosophy of the current calculation: the linear form of $R_{e}(r)$ at small $r$ is ascertained by comparison with the better-determined vibrational transition probabilities at low J, and a hyperbolic "tail" is added following the ab initio form and as demanded from a realistic physical picture. In table 3 we compare the results for $\mathrm{J}=0$ of the linear moment calculations of $I$ with the $\mathrm{J}=0$ hyperbolic moment calculations, against the "best" experimental values of the listed ratios (see I). If anything, there is slight improvement using the hyperbolic moment, though the experimental error bars render improper any preference on this basis alone.

\section{Summary}

It is our opinion that these calculations represent the best currently available values for $\mathrm{OH} \mathrm{A-X}$ transition probabilities. Only measurements of relatively high accuracy $( \pm 5 \%$ ) at values of $v$ and $J$ appreciably sampling large values of $r(>1.3 \AA$ ) will improve the picture-examples would be the $A(2,3) / A(2,4)$ ratio or lifetimes for $J>20$, at low pressure.

To obtain these results, we have folded together three modern developments in molecular spectroscopy. The RKR method of calculation of vibrational-rotational wavefunctions has been combined with a transition moment whose form is dictated by ab initio calculations. The primary bank of data by means of which these theoretical results are evaluated and fitted is obtained from laser excited fluorescence. Through such an approach, one can develop for other important molecular band systems a firm data base for the measurement of temperatures and population distributions. 


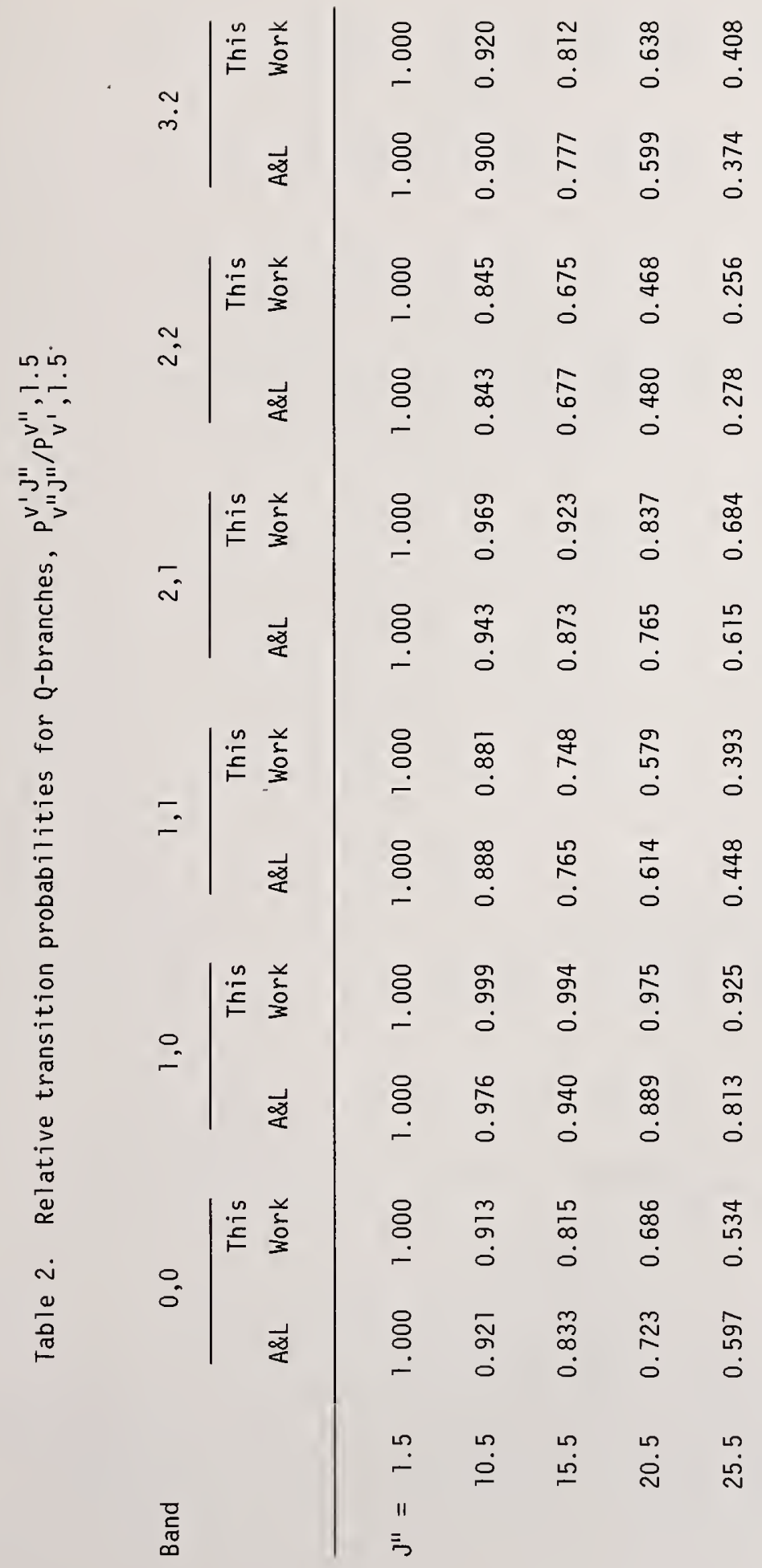


Table 3. Selected ratios of transition probabilities.

Ratio Linear Moment ${ }^{a}$ Hyperbolic Moment ${ }^{b}$ Experiment Reference

$\begin{array}{llccr}\mathrm{A}_{11} / \mathrm{A}_{10} & 1.75 & 1.73 & 1.58 \pm 0.08 & 1 \\ \mathrm{~A}_{10} / \mathrm{A}_{00} & 0.33 & 0.33 & 0.30 \pm 0.04 & 13 \\ \mathrm{~A}_{21} / \mathrm{A}_{22} & 1.73 & 1.67 & 0.36 & 13 \\ \mathrm{\tau}_{0} / \mathrm{\tau}_{1} & 0.92 & 0.90 & 1.79 \pm 0.07 & 1 \\ \mathrm{~A}_{23} / \mathrm{A}_{22} & 0.005 & 0.020 & 0.012 \pm 0.002 & 17 \\ \mathrm{~A}_{20} / \mathrm{A}_{22} & 0.22 & 0.26 & 0.23 \pm 0.01 & 1 \\ \mathrm{~A}_{01} / \mathrm{A}_{00} & 0.0040 & 0.0038 & 0.0037 \pm 0.0004 & 1 \\ \mathrm{~A}_{12} / \mathrm{A}_{11} & 0.0067 & 0.0091 & 0.0104 \pm 0.0002 & 1 \\ \mathrm{~A}_{23} / \mathrm{A}_{11} & & 2.2 & 2.0 \pm 1.1 & 1\end{array}$

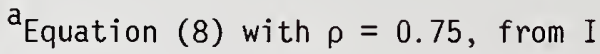

${ }^{b}$ Equation (11) with $\rho=0.756, a=0.004$
}

We thank Dr. R. N. Zare for providing us a copy of his RKR and FCF programs.

\section{References}

[1] Crosley, D. R. and Lengel, R. K., JQSRT 15, 579 (1975); (referred to as I).

[2] Crosley, D. R. and Lengel, R. K., JQSRT 17, 59 (1977); (referred to as II).

[3] Chidsey, I. L. and Crosley, D. R., Calculated Rotational Transition Probabilities for the A-X System of $\mathrm{OH}$, Ballistic Research Laboratory Report, to be published.

[4] Dieke, G. H. and Crosswhite, H. M., JQSRT 2, 97 (1962); (referred to as DC). 
[5] Shuter, K. E., J. Chem. Phys. 18, 1221 (1950).

[6] Learner, R. C. M. , Proc. Roy. Soc. A269, 311 (1962).

[7] Ankete11, J. and Learner, R. C. M., Proc. Roy. Soc. A301, 355 (1967); (referred to as $A L)$.

[8] Ankete11, J. and Pery-Thorne, A., Proc. Roy. Soc. A301, 343 (1967).

[9] Henneker, W. H. and Popkie, H. E., J. Chem. Phys. 54, 1763 (1971); (referred to as HP).

[10] Coxon, J. A., J. Mo1. Spec. 58, 1 (1975).

[11] Zare, R. N., J. Chem. Phys. 50, 1934 (1964).

[12] Silver, J. A., Dimpf1, W. L., Brophy, J. H., and Kinsey, J. L., J. Chem. Phys. 65, 1811 (1976).

[13] Klein, L., JQSRT 13, 581 (1973).

[14] German, K. R., J. Chem. Phys. 62, 2584 (1975).

[15] Learner, R. C. M. and Gaydon, A. G. , Nature, 183, 242 (1959).

[16] Rouse, P. E. and Engleman, Jr., R., JQSRT 13, 1503 (1973).

[17] German, K. R., J. Chem. Phys. 63, 5252 (1975). 



\section{RATE PROCESSES IN GASES--HOMOGENEOUS SYSTEMS}



National Bureau of Standards Special Publication 561, Proceedings of the 10th Materials Research Symposium on Characterization of High Temperature Vapors and Gases held at NBS, Gaithersburg, Maryland, September 18-22, 1978. Issued 0ctober 1979.

\title{
CHEMICAL PROCESSES IN HIGH REYNOLDS NUMBER TURBULENT DIFFUSION FLAMES
}

\author{
D. E. Jensen
}

Propellants, Explosives, and Rocket Motor Establishment Westcott, Aylesbury, Buckinghamshire, England

A theoretical method of calculating characteristic properties of turbulent diffusion flames is outlined, and its application to the particular case of rocket exhaust flames, where Reynolds numbers of $10^{5}-10^{6}$ are commonplace, is illustrated. The computer program based on the metholology described accepts any number of nonequilibrium chemical reactions for which kinetic, as well as thermochemical data, are required as input. Examples are given of the ways in which such data may be obtained and used. Some needs for further research are briefly indicated. It is important that fundamental research on characterization of individual chemical processes and development of computational techniques for practical flames should proceed in parallel, with continual feedback, and not in mutual isolation.

\section{Introduction}

Properties of turbulent diffusion flames of practical interest include: radiations at infrared, visible, and ultraviolet wavelengths; electrical characteristics dependent upon free electron content; mean and fluctuating refractive index fields; and smoke (condensed particle) and gas-phase pollutant emissions. Calculation of these and other flame properties requires knowledge of the flame structure, including the spatial distributions of temperature, pressure, and concentrations of various chemical species. This paper begins with a brief description of a general theoretical technique for characterizing flame structures. Two special advantages offered by this technique are that it is capable of dealing with upstream diffusion and with flame shock waves. The technique is explicitly designed for application to low altitude, axisymmetric rocket exhaust flames, where Reynolds numbers are often as high as $10^{5}-10^{6}$, but may readily be applied, after appropriate modifications, in other combustion contexts where coupling between chemical reactions and turbulent mixing processes is strong. A wide range of chemical inputs is required for these applications, and the main body of the paper is concerned with characterization of the thermochemistry and kinetics of relevant chemical reactions. Some likely directions for future research on chemical processes of interest are also briefly indicated. The implicit theme of the paper is that fundamental research on characterization of individual 
chemical processes and development of techniques for computing properties of practical flames should proceed in parallel, with continual feedback, and not in mutual isolation.

\section{Computational Technique}

In a typical rocket exhaust flame, fuel-rich gases (often containing 30-50 percent of carbon monoxide and hydrogen) from a conical nozzle at temperatures between 700 and $2500 \mathrm{~K}$ and velocities $\approx 2 \mathrm{~km} \mathrm{~s}^{-1}$ mix with a moving stream of air, creating a turbulent diffusion flame. The structure of such a flame is calculated in three stages, one each for the combustion chamber, nozzle, and external flame jet or "plume." The last, and most difficult, stage concerns us here.

A diagrammatic representation of the main regions of the exhaust flame is given in figure 1. Structural features of the (steady-state) exhaust flame required include distributions of the time-mean chemical composition, temperature, pressure, velocity, and turbulence length scale, and the RMS fluctuating components of intensive variables. These are to be calculated from the nozzle exit conditions and rocket velocity for any given altitude from an appropriate input of chemical reaction mechanism and turbulence mixing model parameters. Simplifying assumptions remaining in the technique presentiy used (which is futty described in ref. $[1]^{1}$ ) are that the flow is axisymmetric and that thermal and velocity lags of any condensed particles present are negligibly small (the latter is a reasonable assumption for particles of radius below about $1 \mu \mathrm{m}$ ); further work designed to make these assumptions unnecessary is currently in progress.

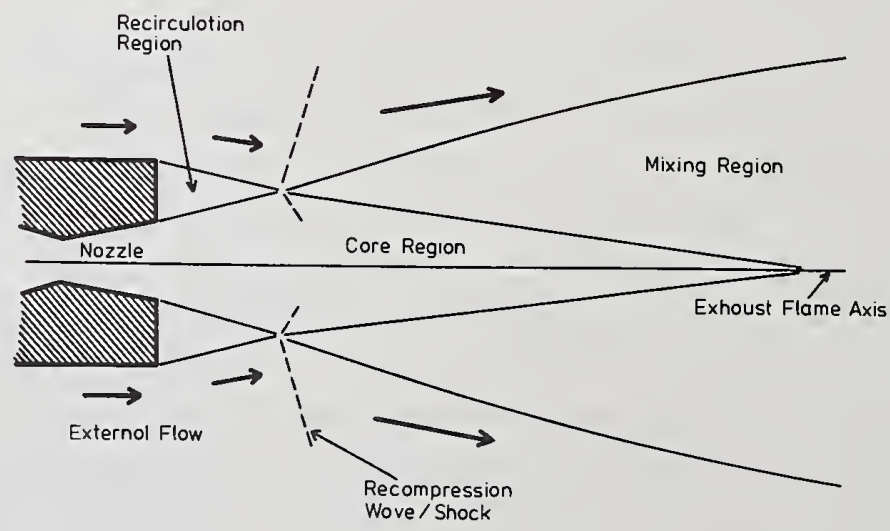

Figure 1. Diagrammatic representation of main features of the structure of a rocket exhaust flame.

${ }^{1}$ Figures in brackets indicate the literature references at the end of this paper. 


\subsection{Governing equations}

With axial and radial coordinates $x$ and $r$, respectively, the empirical partial differential equations governing turbulent shear layer flow in a free jet may be written as follows:

(a) conservation of the axial component of momentum,

$$
\begin{aligned}
\rho u \frac{\partial u}{\partial x}+\rho v \frac{\partial u}{\partial r} & =\frac{\partial}{\partial x}\left(\mu \frac{\partial u}{\partial x}\right)+\frac{1}{r} \frac{\partial}{\partial r}\left(r \mu \frac{\partial u}{\partial r}\right) \\
& -\frac{d p}{d x}+\frac{\partial}{\partial x}\left(\mu \frac{\partial u}{\partial x}\right)+\frac{1}{r} \frac{\partial}{\partial r}\left(r \mu \frac{\partial v}{\partial x}\right) ;
\end{aligned}
$$

(b) conservation of the radial component of momentum,

$$
\begin{aligned}
\rho u \frac{\partial v}{\partial x}+\rho v \frac{\partial v}{\partial r}=\frac{\partial}{\partial x}\left(\mu \frac{\partial v}{\partial x}\right) & +\frac{1}{r} \frac{\partial}{\partial r}\left(r \mu \frac{\partial v}{\partial r}\right)-\frac{d p}{d r}-\frac{2 \mu v}{r^{2}} \\
& +\frac{\partial}{\partial x}\left(\mu \frac{\partial u}{\partial r}\right)+\frac{1}{r} \frac{\partial}{\partial r}\left(r \mu \frac{\partial v}{\partial r}\right) ;
\end{aligned}
$$

(c) conservation of energy,

$$
\begin{aligned}
\rho u \frac{\partial H}{\partial x}+\rho v \frac{\partial H}{\partial r}=\frac{\partial}{\partial x}\left(\frac{\mu}{\sigma_{H}} \frac{\partial H}{\partial x}\right) & +\frac{1}{r} \frac{\partial}{\partial r}\left(\frac{r \mu}{\sigma_{H}} \frac{\partial H}{\partial r}\right)+\frac{1}{r} \frac{\partial}{\partial r}\left\{r \mu\left(1-\frac{1}{\sigma_{H}}\right) \frac{\partial\left(1 / 2 u^{2}\right)}{\partial r}\right\} \\
& +\frac{1}{r} \frac{\partial}{\partial r}\left\{r \mu\left(1-\frac{1}{\sigma_{k}}\right) \frac{\partial k}{\partial r}\right\} ;
\end{aligned}
$$

(d) conservation of chemical species,

$$
\rho u \frac{\partial F_{i}}{\partial x}+\rho v \frac{\partial F_{i}}{\partial r}=\frac{\partial}{\partial x}\left(\frac{\mu}{\sigma_{m}} \frac{\partial F_{i}}{\partial x}\right)+\frac{1}{r} \frac{\partial}{\partial r}\left(\frac{r \mu}{\sigma_{m}} \frac{\partial F_{i}}{\partial r}\right)+\dot{w}_{i} .
$$

In these equations, $u$ and $v$ are axial and radial components of velocity respectively, $\rho$ is the density, $p$ the pressure, $H$ the total (stagnation) enthalpy, $F_{i}$ the mass fraction of species $i, \dot{w}_{i}$ the mass rate of production of this species due to chemical reaction, $\mu$ the turbulence eddy viscosity coefficient, $\sigma_{H}$ the ratio of rate of enthalpy exchange to rate of momentum exchange (i.e. the enthalpy exchange coefficient ratio or turbulence Prandt 7 number) and $\sigma_{m}$ the turbulence Schmidt number. The eddy viscosity coefficient is described in terms of the turbulence kinetic energy $k=1 / 2\left(u^{\prime 2}+v^{\prime 2}\right)$ and the eddy energy dissipation rate $\varepsilon$ via the equation

$$
\mu=\rho k^{2} C_{D} \varepsilon^{-1} \text {, }
$$


where $C_{D}$ is an empirical constant and $u^{\prime}$ and $v^{\prime}$ are temporally fluctuating components of the axial and radial velocities, respectively. Transport equations for $k$ and $\varepsilon$ may be written in the forms $[1,2]$

$$
\begin{aligned}
\rho u \frac{\partial k}{\partial x}+\rho v \frac{\partial k}{\partial r}=\frac{\partial}{\partial x}\left(\frac{\mu}{\sigma_{k}} \frac{\partial k}{\partial x}\right) & +\frac{1}{r} \frac{\partial}{\partial r}\left(\frac{r \mu}{\sigma_{k}} \frac{\partial k}{\partial r}\right)+\mu\left\{2\left[\left(\frac{\partial u}{\partial x}\right)^{2}+\left(\frac{\partial v}{\partial r}\right)^{2}\right]\right. \\
& \left.+\left(\frac{\partial u}{\partial r}+\frac{\partial v}{\partial x}\right)^{2}+\frac{2 v^{2}}{r^{2}}\right\}-\rho \varepsilon
\end{aligned}
$$

and

$$
\begin{aligned}
\rho \mathrm{u} \frac{\partial \varepsilon}{\partial x}+\rho v \frac{\partial \varepsilon}{\partial r}=\frac{\partial}{\partial x}\left(\frac{\mu}{\sigma_{\varepsilon}} \frac{\partial \varepsilon}{\partial x}\right) & +\frac{1}{r} \frac{\partial}{\partial r}\left(\frac{r \mu}{\sigma_{\varepsilon}} \frac{\partial \varepsilon}{\partial r}\right)+c_{1} \frac{\varepsilon \mu}{k}\left\{2\left[\left(\frac{\partial u}{\partial x}\right)^{2}+\left(\frac{\partial v}{\partial r}\right)^{2}\right]\right. \\
& \left.+\left(\frac{\partial u}{\partial r}+\frac{\partial v}{\partial x}\right)^{2}+\frac{2 v^{2}}{r^{2}}\right\}-\frac{c_{2} \rho \varepsilon^{2}}{k},
\end{aligned}
$$

where $C_{1}$ and $C_{2}$ are empirical coefficients and $\sigma_{k}$ and $\sigma_{\varepsilon}$ are exchange coefficient ratios for $k$ and $\varepsilon$, respectively. A further equation describes the transport of the mean square fluctuating component $g$ of a scalar $f[1-3]$ :

$$
\begin{aligned}
\rho u \frac{\partial g}{\partial x}+\rho v \frac{\partial g}{\partial r}=\frac{\partial}{\partial x}\left(\frac{\mu}{\sigma_{g}} \frac{\partial g}{\partial r}\right)+\frac{1}{r} \frac{\partial}{\partial r}\left(\frac{r \mu}{\sigma_{g}} \frac{\partial g}{\partial r}\right) & +c_{g 1} \mu\left\{\left(\frac{\partial f}{\partial x}\right)^{2}+\left(\frac{\partial \vec{f}}{\partial r}\right)^{2}\right\} \\
& -c_{g 2} \rho \varepsilon k^{-1} g,
\end{aligned}
$$

where $\bar{f}$ is the local mean value of $f, g$ the local mean value of $(f-\bar{f})^{2}$ and $c_{g l}$ and $c_{g 2}$ are empirical coefficients.

Two additional equations are needed to permit calculation of the unknowns $u, v, \rho, p$, $H, k, \varepsilon, F_{i}$, and $g$, and derivatives like $\mu$ and the turbulence length scale $\left(=C_{D} k^{1.5} \varepsilon^{-1}\right)$. The first used in the ideal gas equation of state

$$
\mathrm{pM}=\rho \mathrm{R} \overline{\mathrm{T}}
$$

where $M$ is the mixture mean molecular weight, $R$ is the gas constant, and the local mean temperature $\bar{T}$ is a function of enthalpy, velocity, turbulence kinetic energy, and mixture composition. The second equation represents the continuity condition applied to a local elementary fluid volume and is

$$
\int_{S} \rho \mathrm{Uds}=0 \text {, }
$$


where $U$ is the local total mean velocity, and the integration is carried out over the elementary volume surface area $S$.

The main flow equations (1)-(4) and (6)-(8) are a set of simultaneous partial differential equations of the elliptic general form

$$
\rho u \frac{\partial \phi}{\partial x}+\rho v \frac{\partial \phi}{\partial r}=\frac{\partial}{\partial x}\left(\frac{\mu}{\sigma_{\phi}} \frac{\partial \phi}{\partial x}\right)+\frac{1}{r} \frac{\partial}{\partial r}\left(\frac{\mu r}{\sigma_{\phi}} \frac{\partial \phi}{\partial r}\right)+S_{\phi}
$$

where $S_{\phi}$ is the local rate of production of variable $\phi$. The elliptic form of the equations necessitates an iterative approach to their solution. An arbitrary "guessed" initial flow field structure is converged towards a final structure via successive iterative simultaneous solution of the governing equations and eqs. (9) and (10) over all points in a flow field grid by means of the finite difference method of reference [1], carefully specified boundary conditions $[1,4]$ being employed. Calculation of the pressure distribution requires certain additional procedures $[1,5]$, which allow pressure and velocity fields to be corrected until the continuity relationship is satisfied for each local volume element. In cases where axial transport is governed largely by convection rather than diffusion (recirculation effects being negligible), or where there are no radial pressure gradients (and no shocks), simplified forms of the governing equations may be used [1,6], with consequent savings in computational time. Recommended coefficients for the turbulence model are given in references [1] and [2].

\subsection{Chemical reactions}

Effects of chemical reactions are incorporated by way of the term $S_{\phi}$ in eq. (11), which, when $\phi$ refers to a particular chemical species, represents the net rate of mass production of that species due to chemical reaction. The (two-way) reactions are represented by

$$
\sum_{i} A_{i j} X_{i}-\sum_{i} B_{i j} X_{i}=0
$$

where $A_{i j}$ and $B_{i j}$ are the numbers of molecules of species $X_{i}$ taking part in one unit of the $j$ th reaction, on the reactants and products sides, respectively. The net reaction rate is then the difference between forward and reverse rates:

Rate $_{j}=k_{f j} N^{\sum^{i} A_{i j}{ }^{-1}} \prod_{i}\left(\frac{F_{i}^{\rho}}{M_{i}}\right)^{A_{i j}}-k_{r j} N^{\sum^{i} B_{i j}-1} \prod_{i}\left(\frac{F_{i} \rho}{M_{i}}\right)^{B_{i j}} k m o l e m^{-3} s^{-1}$

and

$$
\dot{w}_{i}=M_{i} \sum_{j} R a t e, \quad \mathrm{~kg} \mathrm{~m}^{-3} \mathrm{~s}^{-1},
$$


where $\mathrm{N}=6.023 \times 10^{20}, \rho$ is the $\mathrm{kg} \mathrm{m}^{-3}, \mathrm{M}_{i}$ is the species molecular weight and the forward

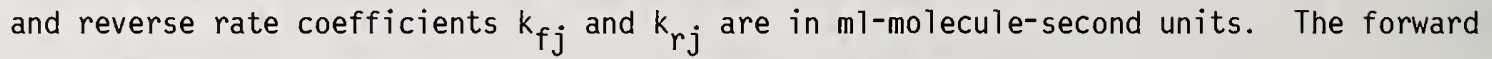
rate coefficients are conveniently expressed in the form

$$
k_{f}=z \bar{T}^{-n} \exp (-E / \bar{T})
$$

Reverse rate coefficients are derived from

$$
k_{r j}=k_{f j} k_{c j}^{-1}
$$

where $K_{c j}$, the equilibrium constant of reaction $j$, is given by

$$
\ln K_{c j}=-\Delta G_{j}^{0} / R \bar{T}+\sum_{i}\left(A_{i j}-B_{i j}\right) \ln R \bar{T}
$$

and $\Delta G_{j}^{0}$ is the standard Gibbs free energy change for the reaction. The constant pressure specific heat $C_{p}$ of each species is expressed as a ninth-order polynomial in temperature:

$$
c_{p}=\sum_{n=-4}^{n=+4} a_{n} \bar{T}^{n}
$$

Standard species enthalpies $h^{0}$, entropies $S^{0}$, and Gibbs free energies are then found from

$$
\begin{gathered}
h^{0}=\int_{\bar{T}} c_{p} d \bar{T}+\left(c_{h}-a_{-1}\right), \\
s^{0}=\int_{\bar{T}} \frac{C p}{\bar{T}} d \bar{T}+c_{s}
\end{gathered}
$$

and

$$
G^{0}=h^{0}-\bar{T} S^{0}
$$

where $C_{h}$ and $C_{s}$ are known integration constants. The source term for the ith species is thus calculated (with appropriate linearization procedures in the finite difference formulation [1]) via addition of the rates of all reactions involving that species.

It is implicit in the above that no allowance is made for perturbations of reaction rates stemming from temporal fluctuations in local temperature and concentrations: Rate ${ }_{j}$ is calculated from time-mean $\bar{T}$ and $F_{i}$. Because many chemical reactions in rocket exhaust flames have relatively low activation energies, effects of temperature fluctuations on reaction rates are often relatively small, and even for higher activation energy reactions 
the ability of the technique, to provide RMS values of temperature fluctuations does permit one to assess the magnitudes of perturbations of reaction rate terms stemming from these fluctuations. For some applications, however (and especially those where high activation energy reactions are important--such as in formation of oxides of nitrogen or in collisional ionization of alkali metals), this treatment is quantitatively inadequate. Proper resolution of this difficulty, and removal of other deficiencies inherent in a purely Eulerian approach to the flow field computation, must await development of an approach incorporating

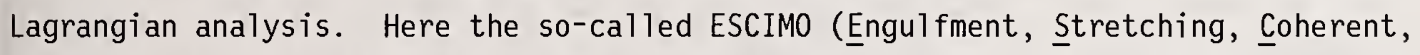
Interdiffusion, Moving Observer) model recently outlined by Spalding [7] appears promising, although much further work will be needed before application to detailed flame calculations becomes feasible.

\section{Chemical Processes}

The theoretical technique described above is able to cope with any arbitrary set of chemical reactions. What reactions are to be included in any particular calculation depends upon the nature of the flame under consideration and the structure properties required. It is desirable that the number of chemical species and reactions treated should be always be kept close to the minimum consistent with adequately realistic calculation of required properties, in order that computer run times and storage requirements should be minimized.

Examples of the use of the technique, and of its success in accounting for direct experimental observations, are given in references [1] and [6]. Attention is now turned to some examples of chemical processes occurring in flames which are of practical, as well as fundamental, interest.

\section{1 Combustion inhibition}

The reactions occurring in hydrogen and carbon monoxide combustion are now fairly well characterized. They include the chain branching steps

$$
\mathrm{H}+\mathrm{O}_{2} \rightarrow \mathrm{OH}+\mathrm{O}
$$

and

$$
\mathrm{O}+\mathrm{H}_{2} \rightarrow \mathrm{OH}+\mathrm{H}
$$

linear propagation reactions like

$$
\mathrm{OH}+\mathrm{H}_{2} \rightarrow \mathrm{H}_{2} \mathrm{O}+\mathrm{H}
$$


and

$$
\mathrm{CO}+\mathrm{OH} \rightarrow \mathrm{CO}_{2}+\mathrm{H}
$$

and such chain termination steps as

$$
\mathrm{H}+\mathrm{OH}+\mathrm{M} \rightarrow \mathrm{H}_{2} \mathrm{O}+\mathrm{M}
$$

and

$$
\mathrm{H}+\mathrm{H}+\mathrm{M} \rightarrow \mathrm{H}_{2}+\mathrm{M} \text {. }
$$

(The notation for numbering of reactions follows that of reference [8], to which considerable reference is made in the following pages.) Evidence is now accumulating [9-14] that various metals can significantly accelerate radical removal (chain termination), even at mole fractions as low as $10^{-6}$ (see, for example, fig. 2), and can thus substantially modify the combustion process. Some possible implications of this work for suppression of hydrogen/carbon monoxide combustion in rocket exhaust flames have been discussed by Jensen and Webb [15].

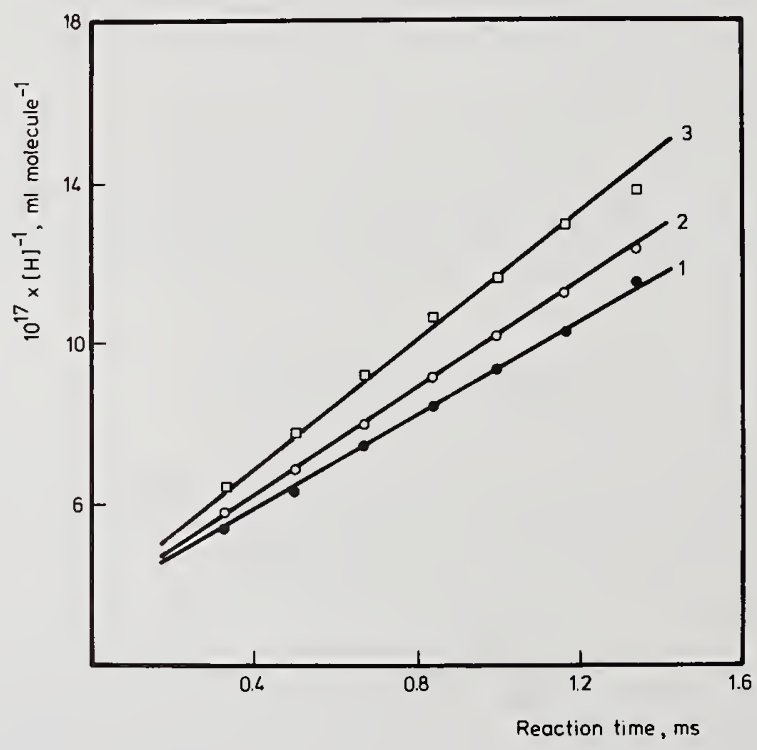

Figure 2. Acceleration of hydrogen atom recombination by calcium and barium in a premixed laminar $\mathrm{H}_{2} / \mathrm{N}_{2} / \mathrm{O}_{2}$ flame. $\mathrm{H}_{2} / \mathrm{N}_{2} / \mathrm{O}_{2}=4 / 5 / 1$. Temperature $=1900 \mathrm{~K}$. Line 1 , closed circles, no additive. Line 2 , open circles, total calcium mole fraction $=2.5 \times 10^{-6}$. Line 3 , open squares, total barium mole fraction $=2.5 \times 10^{-6}$ Reaction time $=0$ at the flame primary reaction zones: measurements made in burned gases. From reference [16]. 
The alkaline earth metals calcium, strontium, and barium are of particular interest in this context. Cotton and Jenkins [9] suggested that the following cyclic (catalytic) sequence of relatively rapid bimolecular reactions was responsible for causing observed accelerated recombination of $\mathrm{H}$ and $\mathrm{OH}$ radicals in fuel-rich, pre-mixed, laminar $\mathrm{H}_{2} / \mathrm{O}_{2} / \mathrm{N}_{2}$ flames containing barium:

$$
\begin{gathered}
\mathrm{BaOH}+\mathrm{H} \rightarrow \mathrm{BaO}+\mathrm{H}_{2} ; \\
\mathrm{BaO}+\mathrm{H}_{2} \mathrm{O} \rightarrow \mathrm{Ba}(\mathrm{OH})_{2} ; \\
\mathrm{Ba}(\mathrm{OH})_{2}+\mathrm{H} \rightarrow \mathrm{BaOH}+\mathrm{H}_{2} \mathrm{O} .
\end{gathered}
$$

Analogous mechanisms were put forward for strontium and calcium. More recent work [16] confirms that this type of sequence provides a probable interpretation of observations for strontium and barium and offers rate coefficients for the reactions involved, but suggests that the mechanism for calcium may be more complicated. Although these flame studies of accelerated radical removal have proved valuable in indicating the magnitudes of catalytic effects and affording enough information (from measurements at different flame temperatures and compositions) for semi-quantitative kinetic mechanisms to be inferred, it is likely that future accurate characterization of the reactions involved will come from studies of the individual reactions in isolation, perhaps in molecular beam or hot flow tube environments.

Alkali metals have also been used as combustion inhibitors in a variety of practical contexts, and a qualitative interpretation of a mode of action of potassium in fuel-rich flames has been given by Rosser, Inami, and Wise [17] and Friedman and Levy [18] in terms of the reaction sequence

and

$$
\mathrm{KOH}+\mathrm{H} \rightarrow \mathrm{K}+\mathrm{H}_{2} \mathrm{O}
$$

$$
\mathrm{K}+\mathrm{OH}+\mathrm{M} \rightarrow \mathrm{KOH}+\mathrm{M} \text {. }
$$

Jensen, Jones, and Mace [19] have recently studied the effects of $K$ and $\mathrm{KOH}$ on radical recombination in laminar, pre-mixed, fuel-rich $\mathrm{H}_{2} / \mathrm{O}_{2} / \mathrm{N}_{2}$ flames, potassium being supplied to these flames at higher concentrations (mole fractions up to $10^{-3}$ ) than have previously been reliably obtainable in the gas phase via use of an additive supply system containing volatile potassium di-divaloylmethane, $\left[\left(\mathrm{CH}_{3}\right)_{3} \mathrm{CCOCHCOC}\left(\mathrm{CH}_{3}\right)_{3}\right] \mathrm{K}$. Preliminary evaluation of these (photometric) results suggests that reactions (-D6) and (F3) do indeed account for the observations, with rate coefficients within the limits specified in reference [8]; it is expected that more precise values for these rate coefficients will emerge from the detailed analysis of results now in progress. Quantitative characterization of this mechanism is especially important in view of the demonstrated capacity of potassium salts to suppress rocket exhaust combustion in certain practical situations [20]. 


\subsection{Ionization processes}

Because many high energy rocket propellants are based on ammonium perchlorate and contain alkali metal impurities, ionization processes in flames containing both alkali metal and chlorine are of considerable relevance to the worker concerned with characterizing the electrical properties of rocket exhausts (see, for example, ref. [21]). For potassium, at least three reactions are significant:

$$
\begin{gathered}
K+M \rightarrow K^{+}+e^{-}+M, \\
K+C l \rightarrow K^{+}+\mathrm{Cl}^{-},
\end{gathered}
$$

and

$$
\mathrm{KCl}+\mathrm{M} \rightarrow \mathrm{K}^{+}+\mathrm{Cl}^{-}+\mathrm{M} .
$$

For reaction (-G3), a number of studies in laboratory pre-mixed laminar flames has been made, all consistent with the recommended rate coefficient [8] $\left.\mathrm{k}_{\mathrm{G} 3}=4 \times 10^{-24} \mathrm{~T}^{-1} \mathrm{~m}\right]^{2}$ molecule $\mathrm{s}^{-2} \mathrm{~s}^{-1}$. Reactions $(-\mathrm{H} 3)$ and $(-\mathrm{H} 9)$ have recently been the subject of a careful mass-spectrometric flame investigation by Burdett and Hayhurst $[22,23]$; the rate coefficients $\mathrm{k}_{\mathrm{H} 3}=1.7 \times 10^{-9} \mathrm{~T}^{-0.5} \mathrm{ml}$ molecule $\mathrm{s}^{-1}$ and $\mathrm{k}_{\mathrm{H} 9}=1.6 \times 10^{-15} \mathrm{~T}^{-3.5} \exp (-2400 / \mathrm{T}) \mathrm{ml}^{2}$ molecule $\mathrm{e}^{-2} \mathrm{~s}^{-1}$ obtained fall nicely into line with theoretical expectations $[8,23]$. Similar results are available for lithium and sodium $[22,23]$.

Detachment of electrons from $\mathrm{Cl}^{-}$is also of kinetic importance in flames, the reaction

$$
\mathrm{Cl}^{-}+\mathrm{H} \rightarrow \mathrm{HCl}+\mathrm{e}^{-}
$$

being of especial interest. Despite several investigations, the rate coefficient of this reaction is still the subject of some dispute [8] and is in need of yet further study. The reader interested in the methods by which attempts are made to characterize both this and other flame ionization reactions (they include mass-spectrometry, microwave techniques, electrostatic probing, and photometric methods) may find references [8] and [24] offer useful guidance to the literature.

\subsection{Equilibrium and disequilibrium of flame reactions}

In flames where concentrations of free radicals like $[\mathrm{H}]$ and $[\mathrm{OH}]$ depart from equilibrium, it is useful to be able to distinguish between reactions of additives which are likely to be balanced (i.e., for which the quotient $\{[C][D] \ldots\} /\{[A][B] \ldots\}$ of a reaction $A+B \ldots \rightarrow C+D \ldots$ is equal to the equilibrium constant $K)$ and those which are probably

unbalanced. As an example, consider the reactions

$$
X+H Y \stackrel{\rightarrow}{\leftarrow} X Y+H
$$


and

$$
X+Y+M \stackrel{*}{\leftarrow} X Y+M,
$$

where $X$ is a metal atom, metal-containing radical or free electron, $Y$ is a free radical (e.g. $\mathrm{H}, \mathrm{OH}$ or $\mathrm{Cl}$ ), $\mathrm{M}$ is a collision partner, and, in contrast to the situation of Section 3.1, $[X]$ and $[X Y]$ are too small to affect $[H]$ or $[Y]$. If these are the only reactions influencing the ratio $[X Y] /[X] \equiv \Psi$, and $[\mathrm{HY}],[\mathrm{H}],[\mathrm{Y}]$, and $[\mathrm{M}]$ are relatively insensitive to reaction time, $\Psi$ is given by [25]

$\psi=\left\{\left(1+\Psi_{0}\right) \gamma\left(\gamma-\eta \Psi_{0}\right)^{-1}-\exp [-(\gamma+\eta) t]\right\}\left\{\exp [-(\gamma+\eta) t]+\left(1+\psi_{0}\right) \eta\left(\gamma-\eta \Psi_{0}\right)^{-1}\right\}^{-1}$,

where $\Psi=\Psi_{0}$ at time $t=0, \gamma \equiv k_{A}[H Y]+k_{B}[Y][M]$ and $\eta \equiv k_{-A}[H]+k_{-B}[M]$. Inspection of eq. (22) reveals that $\Psi$ tends to $\Psi_{0}=\gamma / \eta$ as $t$ tends to infinity. If $k_{A}[H Y] \gg k_{B}[Y][M]$ and $k_{-A}[H] \gg k_{-B}[M], \Psi_{\infty}$ takes the value $\Psi_{\infty A}=K_{A}[H Y] /[H]$. This applies to high stability product compounds or negative ions, Sugden's case [26] of domination by bimolecular exchange. If, on the other hand, $k_{B}[Y][M] \gg k_{A}[H Y]$ and $k_{-B}[M] \gg k_{-A}[H], \Psi_{\infty}$ takes the value $\Psi_{\infty} B=K_{B}[Y]$; this corresponds to Sugden's case [26] of domination of termolecular recombination/dissociation and arises for low stability product compounds and ions. In practice, intermediate cases are also important [25]. Equation (22) may also be written in the form

$$
\exp [-(\gamma+\eta) t]=\left(1+\Psi_{0}\right)(1+\Psi)^{-1}\left(\Psi_{\infty}-\Psi\right)\left(\Psi_{\infty}-\Psi_{0}\right)^{-1}
$$

which may conveniently be used to calculate the reaction time necessary for close approach of $\Psi$ to $\Psi_{\infty}$. Thus, criteria are available for competing reactions of types (A) and (B) for determining (a) the rate of approach of $\psi$ to $\Psi_{\infty}$ and (b) the value of $\Psi_{\infty}$, which may correspond to balance of reaction (A), balance of reaction (B), or some intermediate case.

Such criteria are of central importance in laboratory flame characterizations of additives undergoing simple sets of competing reactions $[25,26]$ and have been widely invoked in this connection. They also provide useful guidance to the selection of reactions for inclusion in calculations on practical flames.

When a flame additive gives rise to several different species, however, and each species undergoes various chemical reactions with flame molecules, these simple criteria are in general no longer applicable. For example, calcium forms $\mathrm{Ca}, \mathrm{Ca} 0, \mathrm{Ca}(\mathrm{OH})_{2}$, and $\mathrm{CaOH}$ in flames, and the ratio $\left[\mathrm{Ca}(\mathrm{OH})_{2}\right] /[\mathrm{CaOH}]$ is no longer determined only by a competition between

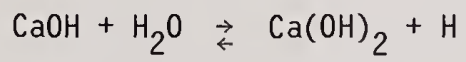


and

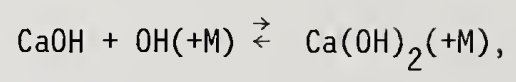

but by a mixture of reactions of oxide and hydroxides [16]. It is not legitimate to assume that reaction $(-\mathrm{S} 6)$ is balanced, despite the high stability of $\mathrm{Ca}(\mathrm{OH})_{2}$, and attempts to measure the relative heats of formation of $\mathrm{CaOH}$ and $\mathrm{Ca}(\mathrm{OH})_{2}$ which are based on the assumption of balance are subject to error [16].

In general, then, it is important to weigh up explicitly contributions from all likely reactions of a given additive species before deciding whether any one reaction involving this species may reasonably be assumed to be balanced, even for conditions where the additive does not significantly affect the main flame chemistry itself. Particular caution must be exercised when attempts are made to characterize reaction enthalpy changes by second-law methods, inasmuch as the degree of possible departure from balance of a reaction studied may vary considerably with temperature. Either an assumption of balance must be unequivocally tested by experiment or, at the very least, probable rates of all reactions of species involved should be calculated from best available values of rate coefficients before any such assumption is made.

\subsection{Calculation of line radiations from flames}

Calculations of spectral distributions of radiations from turbulent flames present challenging problems in fluid dynamics, chemistry, and radiative transport, even for such strong and clearly defined emissions as those of the sodium atom first resonance doublet (Na D-lines). Such calculations nicely illustrate the need for fundamental studies of individual processes and development of computational techniques for practical flames to proceed in parallel. The rocket motor exit plane conditions of table 1 and the computational method of Section 2 have recently been employed to provide a calculated $D-1$ ine spectral distribution for the turbulent exhaust diffusion flame resulting from the mixing and combustion of the gases leaving the motor with the atmosphere [27]. First, the spatial distribution of $\left[\mathrm{Na}\left({ }^{2} \mathrm{P}_{1 / 2}\right)\right],\left[\mathrm{Na}\left({ }^{2} \mathrm{P}_{3 / 2}\right)\right]$, and $\left[\mathrm{Na}\left({ }^{2} \mathrm{~S}_{1 / 2}\right)\right]$ in the flame is computed by this method, the reaction mechanism of table 2 being used. Then, an analysis of the radiation transport process (with inputs of absolute line strengths and Lorentz and Doppler broadening parameters) is employed to generate the radiance/wavelength profile (fig. 3 ). The shape of the profile obtained is sensitive to uncertainties in: (i) certain of the constants in the turbulence model (changes which affect the flame temperature distribution, which in turn affect local values of $\left[\mathrm{Na}\left({ }^{2} \mathrm{P}_{1 / 2}\right)\right] /\left[\mathrm{Na}\left({ }^{2} \mathrm{~S}_{1 / 2}\right)\right]$ and $\left[\mathrm{Na}\left({ }^{2} \mathrm{P}_{3 / 2}\right)\right] /\left[\mathrm{Na}\left({ }^{2} \mathrm{~S}_{1 / 2}\right)\right]$; (ii) the rate coefficients and thermochemistry of some of the reactions forming the compounds $\mathrm{NaOH}$ and $\mathrm{NaO}_{2}$--particularly high proportions of sodium are present as compounds in the outer, cooler, fuel-lean regions of the flame, and less sodium is therefore present here as free $\mathrm{Na}$ atoms (if no compound formation occurred here, self-reversal of the two doublet components, already conspicuous in figure 3 , would be even more pronounced); and (iii) the 
chosen line broadening parameters. In view of all these uncertainties, the theoretically calculated and experimentally measured profiles of figure 3 may be regarded as being in adequate agreement: it is not difficult to force the calculated profile to fit that measured by varying input parameters within their probably uncertainty bounds (cf. refs. [6] and [8]). No more precise calculation of the theoretical profile, and consequently no more stringent test of the model formulation, will be possible until the basic input parameters are more precisely known.

Table 1. Exit conditions of rocket motor Species concentrations, molecule $\mathrm{ml}^{-1}$ :

$\begin{array}{llll}\mathrm{N}_{2} & 2.12 \times 10^{18} & \mathrm{NaOH} & 5.53 \times 10^{14} \\ \mathrm{H}_{2} & 1.66 \times 10^{18} & \mathrm{Na}\left({ }^{2} \mathrm{~S}_{1 / 2}\right) & 6.22 \times 10^{14} \\ \mathrm{CO} & 5.65 \times 10^{18} & \mathrm{NaO}_{2} & 2.87 \times 10^{10} \\ \mathrm{H}_{2} \mathrm{O} & 4.01 \times 10^{18} & \mathrm{Na}\left({ }^{2} \mathrm{P}_{3 / 2}\right) & 4.24 \times 10^{7} \\ { }_{2} & 4.16 \times 10^{18} & \mathrm{Na}\left({ }^{2} \mathrm{P}_{1 / 2}\right) & 2.14 \times 10^{7} \\ \mathrm{CO}_{2} & 2.62 \times 10^{18} & \mathrm{SnO} & 2.21 \times 10^{16} \\ \mathrm{OH}^{2} & 3.21 \times 10^{14} & \mathrm{Ca} & 5.96 \times 10^{16} \\ \mathrm{H} & 5.99 \times 10^{15} & \mathrm{PbO} & 3.92 \times 10^{16} \\ 0 & 9.44 \times 10^{11} & & \\ \mathrm{HO}_{2} & 5.94 \times 10^{9} & & \end{array}$

Temperature: $1450 \mathrm{~K}$. Velocity: $2.21 \mathrm{~km} \mathrm{~s}^{-1}$. Exit radius: $57.4 \mathrm{~mm}$. Exit pressure: $3.23 \times 10^{5} \mathrm{Nm}^{-2}$. Ambient pressure $1.01 \times 10^{5} \mathrm{Nm}^{-2}$. Mean molecular weight 26.06. Rocket velocity $=0$. Altitude $=$ sea level. Sno, Ca and PbO are treated, for the specific purpose of the calculation of Section 3.4 only, as chemically inert.

For the particular flame considered here, results of calculations in which rate coefficients of reactions N13-N24 are varied demonstrate that collisional excitation of $\mathrm{Na}\left({ }^{2} \mathrm{~S}_{1 / 2}\right)$ to $\mathrm{Na}\left({ }^{2} \mathrm{P}_{1 / 2}\right)$ and $\mathrm{Na}\left({ }^{2} \mathrm{P}_{3 / 2}\right)$ dominates over chemi-excitation in determining the spectral radiance profile. This flame reaches temperatures we 11 in excess of $2000 \mathrm{~K}$ in its hotter regions, however, as a result of the burning of $\mathrm{H}_{2}$ and $\mathrm{CO}$ in the exhaust. In cooler flames, where ratios of actual/equilibrium concentrations of $[\mathrm{H}],[\mathrm{OH}]$, and $[\mathrm{O}]$ are much higher, chemi-excitation may be expected to compete much more successfully with collisional excitation. For these circumstances, more precise determinations of the kinetics of such reactions as N13-N18, which presently carry large uncertainty factors [8], 


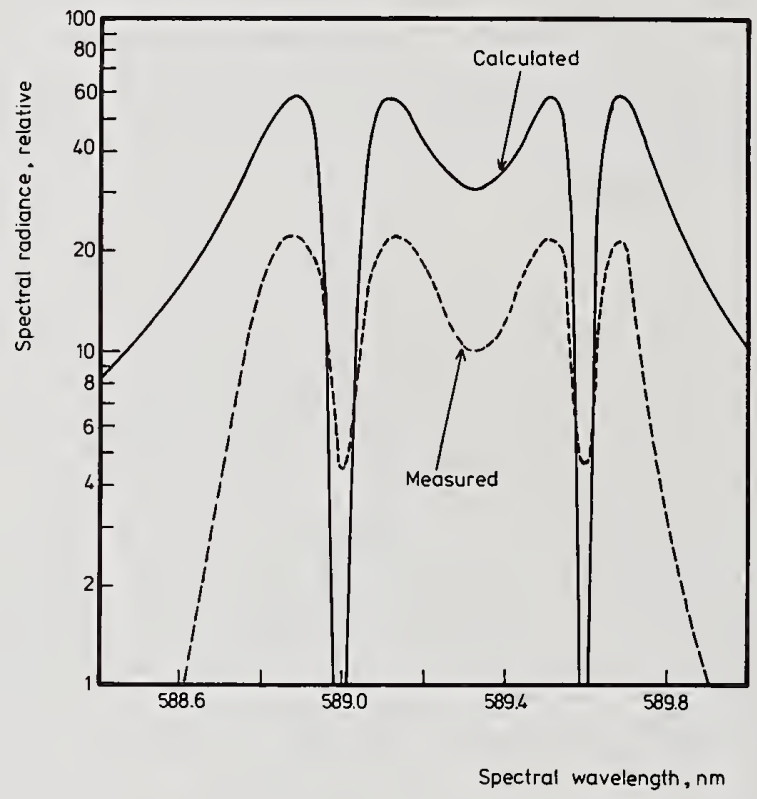

Figure 3. Calculated (continuous line) and measured (broken line) spectral distributions of $\mathrm{Na} \mathrm{D}-\mathrm{li}$ ne radiation from an exhaust flame under static sea level conditions. Range = $100 \mathrm{~m}$. Flame viewed along line of sight at $90^{\circ}$ to axis. Calculated and measured distributions are in the same (relative) ordinate units. Each doublet component shows strong self-reversal (from reference [27]).

will be needed. Shock tubes may well be found to provide suitable experimental environments for this characterization, although further flame studies would certainly also be helpful.

In broad summary, the value of these calculations of spectral radiances (and of other properties) lies not only in their ability to provide useful answers (and associated uncertainty bounds) for flames of practical interest without recourse to experiment, but also in the means they provide of identifying key property-controlling processes and indicating needs for further research on such processes.

\subsection{Kinetics of condensation processes}

A topic in chemical kinetics currently receiving increased attention is that of characterizing processes of condensation of material from gas to liquid or solid phases. An adequate treatment of condensation must take account of nucleation, coagulation, and growth processes, as well as gas phase reactions. The approach to analys is of soot formation from gaseous hydrocarbons described in reference [28] allows for all these processes. 
Table 2. Reaction mechanism for calculation of $\mathrm{Na} \mathrm{D}$-line intensities from rocket exhaust flame

Number

\begin{tabular}{|c|c|}
\hline A1 & $0+0+M \rightarrow 0_{2}+M$ \\
\hline A2 & $\mathrm{O}+\mathrm{H}+\mathrm{M} \rightarrow \mathrm{OH}+\mathrm{M}$ \\
\hline A3 & $\mathrm{H}+\mathrm{H}+\mathrm{M} \rightarrow \mathrm{H}_{2}+\mathrm{M}$ \\
\hline A4 & $\mathrm{H}+\mathrm{OH}+\mathrm{M} \rightarrow \mathrm{H}_{2}^{2} \mathrm{O}+\mathrm{M}$ \\
\hline A5 & $\mathrm{CO}+\mathrm{O}+\mathrm{M} \rightarrow \mathrm{CO}_{2}+\mathrm{M}$ \\
\hline B1 & $\mathrm{OH}+\mathrm{H}_{2} \rightarrow \mathrm{H}_{2} \mathrm{O}+\mathrm{H}$ \\
\hline B2 & $\mathrm{O}+\mathrm{H}_{2} \rightarrow \mathrm{OH}+\mathrm{H}$ \\
\hline B3 & $\mathrm{H}+\mathrm{O}_{2} \rightarrow \mathrm{OH}+\mathrm{O}$ \\
\hline B4 & $\mathrm{CO}+\mathrm{OH} \rightarrow \mathrm{CO}_{2}+\mathrm{H}$ \\
\hline B5 & $\mathrm{OH}+\mathrm{OH} \rightarrow \mathrm{H}_{2}^{2} \mathrm{O}+\mathrm{O}$ \\
\hline B6 & $\mathrm{CO}+\mathrm{O}_{2} \rightarrow \mathrm{CO}_{2}^{2}+\mathrm{O}$ \\
\hline D5 & $\mathrm{Na}+\mathrm{H}_{2} \mathrm{O} \rightarrow \mathrm{NaOH}+\mathrm{H}$ \\
\hline $\mathrm{F} 2$ & $\mathrm{Na}+\mathrm{OH}+\mathrm{M} \rightarrow \mathrm{NaOH}+\mathrm{M}$ \\
\hline N2 & $\mathrm{Na}+\mathrm{O}_{2}+\mathrm{M} \rightarrow \mathrm{NaO}_{2}+\mathrm{M}$ \\
\hline N8 & $\mathrm{NaO}_{2}+\mathrm{H}_{2} \rightarrow \mathrm{NaOH}+\mathrm{OH}$ \\
\hline N11 & $\mathrm{NaO}_{2}^{2}+\mathrm{OH} \rightarrow \mathrm{NaOH}+\mathrm{O}_{2}$ \\
\hline N13 & $\mathrm{Na}+\mathrm{H}+\mathrm{OH} \rightarrow \mathrm{Na}\left({ }^{2} \mathrm{P}_{1 / 2}\right)+\mathrm{H}_{2} \mathrm{O}$ \\
\hline N14 & $\mathrm{Na}+\mathrm{H}+\mathrm{OH} \rightarrow \mathrm{Na}\left({ }^{2} \mathrm{P}_{3 / 2}\right)+\mathrm{H}_{2} \mathrm{O}$ \\
\hline N15 & $\mathrm{Na}+\mathrm{H}+\mathrm{H} \rightarrow \mathrm{Na}\left({ }^{2} \mathrm{P}_{1 / 2}\right)+\mathrm{H}_{2}$ \\
\hline N16 & $\mathrm{Na}+\mathrm{H}+\mathrm{H} \rightarrow \mathrm{Na}\left({ }^{2} \mathrm{P}_{3 / 2}\right)+\mathrm{H}_{2}^{2}$ \\
\hline N17 & $\mathrm{Na}+\mathrm{O}+\mathrm{O} \rightarrow \mathrm{Na}\left({ }^{2} \mathrm{P}_{1 / 2}\right)+0_{2}^{2}$ \\
\hline N18 & $\mathrm{Na}+\mathrm{O}+0 \rightarrow \mathrm{Na}\left({ }^{2} \mathrm{P}_{3 / 2}\right)+0_{2}^{2}$ \\
\hline N19 & $\mathrm{Na}\left({ }^{2} \mathrm{~S}_{1 / 2}\right)+\mathrm{M} \rightarrow \mathrm{Na}\left({ }^{2} \mathrm{P}_{1 / 2}\right)+\mathrm{M}^{2}$ \\
\hline N20 & $\mathrm{Na}\left({ }^{2} \mathrm{~S}_{1 / 2}\right)+\mathrm{M} \rightarrow \mathrm{Na}\left({ }^{2} \mathrm{P}_{3 / 2}\right)+\mathrm{M}$ \\
\hline N24 & $\mathrm{Na}\left({ }^{2} \mathrm{P}_{1 / 2}\right)+\mathrm{M} \rightarrow \mathrm{Na}\left({ }^{2} \mathrm{P}_{3 / 2}\right)+\mathrm{M}$ \\
\hline N38 & $\mathrm{Na}+\mathrm{HO}_{2} \rightarrow \mathrm{NaO}_{2}+\mathrm{H}$ \\
\hline P1 & $\mathrm{H}+\mathrm{O}_{2}+\mathrm{M} \rightarrow \mathrm{HO}_{2}+\mathrm{M}$ \\
\hline P3 & $\mathrm{H}+\mathrm{HO}_{2} \rightarrow \mathrm{OH}+\mathrm{OH}$ \\
\hline P4 & $\mathrm{H}+\mathrm{HO}_{2} \rightarrow \mathrm{H}_{2}+\mathrm{O}_{2}$ \\
\hline P5 & $\mathrm{H}_{2}+\mathrm{HO}_{2} \rightarrow \mathrm{H}_{2} \mathrm{O}+\mathrm{OH}$ \\
\hline P6 & $\mathrm{CO}+\mathrm{HO}_{2} \rightarrow \mathrm{CO}_{2}+\mathrm{OH}$ \\
\hline P7 & $\mathrm{O}+\mathrm{HO}_{2} \rightarrow \mathrm{O}_{2}+\mathrm{OH}$ \\
\hline P8 & $\mathrm{OH}+\mathrm{HO}_{2} \rightarrow \mathrm{H}_{2} \mathrm{O}+\mathrm{O}_{2}$ \\
\hline
\end{tabular}

\section{Rate Coefficient}

$3 \times 10^{-34} \exp (900 / \mathrm{T})$

$1 \times 10^{-29} \mathrm{~T}^{-1}$

$3 \times 18^{-30} \mathrm{~T}^{-1}$

$1 \times 10^{-25} \mathrm{~T}^{-2}$

$7 \times 10^{-33} \exp (-2200 / \mathrm{T})$

$1.9 \times 10^{-15} \mathrm{~T}^{1.3} \exp (-1825 / \mathrm{T})$

$3 \times 10^{-14} \mathrm{~T} \exp (-4480 / \mathrm{T})$

$2.4 \times 10^{-10} \exp (-8250 / \mathrm{T})$

$2.8 \times 10^{-17} \mathrm{~T}^{1.3} \exp (330 / \mathrm{T})$

$1.0 \times 10^{-11} \exp (-550 / \mathrm{T})$

$4.2 \times 10^{-12} \exp (-24000 / \mathrm{T})$

$8 \times 10^{-11} \exp (-22000 / \mathrm{T})$

$$
5 \times 10^{-28} \mathrm{~T}^{-1}
$$

$1 \times 10^{-30} \mathrm{~T}^{-1}$

$3 \times 10^{-12} \exp (-10000 / \mathrm{T})$

$2 \times 10^{-11}$

$1 \times 10^{-31}$

$1 \times 10^{-31}$

$1 \times 10^{-31}$

$1 \times 10^{-31}$

$5 \times 10^{-30}$

$5 \times 10^{-30}$

$1.2 \times 10^{-11} T^{0.5} \exp (-24400 / T)$

$2.4 \times 10^{-11} T^{0.5} \exp (-24400 / \mathrm{T})$

$3 \times 10^{-10}$

$1 \times 10^{-11} \exp (-1000 / \mathrm{T})$

$2 \times 10^{-32} \exp (500 / \mathrm{T})$

$4 \times 10^{-10} \exp (-950 / \mathrm{T})$

$4 \times 10^{-11} \exp (-350 / \mathrm{T})$

$1 \times 10^{-12} \exp (-9400 / \mathrm{T})$

$2.5 \times 10^{-10} \exp (-11900 / \mathrm{T})$

$8 \times 10^{-11} \exp (-500 / \mathrm{T})$

$5 \times 10^{-11}$

Rate coefficients, in ml-molecule-second units, from ref. 8 . Where the electronic state of atomic sodium is unspecified, it may be assumed to be ${ }^{2} \mathrm{~S}_{1 / 2}$. 
An analogous approach to the particular case of iron atom condensation has been suggested in reference [29]. The treatment applies to isothermal conditions but should be readily capable of extension to a general nonisothermal flame and, of course, to other condensing species. The structure of the model (which has progressed since ref. [29] was written) will now be briefly outlined and its application illustrated; a full description is to be published elsewhere [30].

\subsubsection{Gas phase reactions}

Gas phase species formed by iron in combustion systems include $\mathrm{Fe}, \mathrm{FeO}, \mathrm{FeOH}$, and $\mathrm{Fe}(\mathrm{OH})_{2}[12]$. The variations of vapor pressures of condensed $\mathrm{Fe}, \mathrm{FeO}$, and $\mathrm{Fe}(\mathrm{OH})_{2}$ (no data are available for $\mathrm{FeOH}$ ) with temperature are shown in figure 4 . The species most likely to condense in flames at temperatures $\approx 1200 \mathrm{~K}$ is $\mathrm{Fe}^{--a}$ though the vapor pressure of $\mathrm{Fe}$ (condensed) is higher than that of $\mathrm{FeO}$ (condensed), $[\mathrm{Fe}(\mathrm{g})]$ at small reaction times is much higher than $[\mathrm{Fe} O(\mathrm{~g})]$. The reversible gas phase reactions of table 3 are designed [30] to account for the proportions of total gas phase iron present in the forms of free atoms and compounds. None of the rate coefficients is known with an uncertainty factor $<10$.

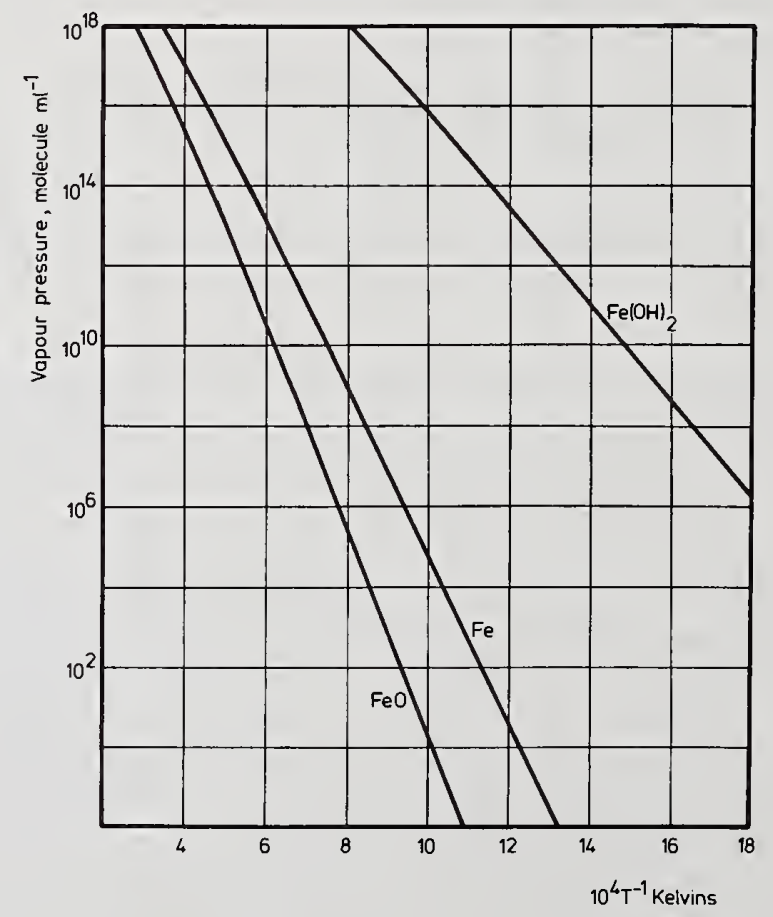

Figure 4. Vapor pressures of $\mathrm{Fe}, \mathrm{FeO}$ and $\mathrm{Fe}(\mathrm{OH})_{2}$ as functions of reciprocal temperature (data from reference $[41]$ ). 


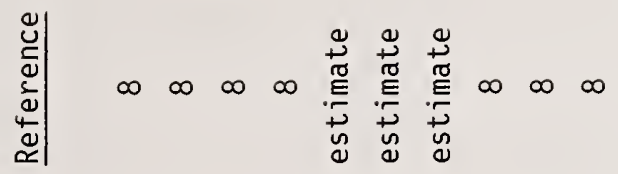

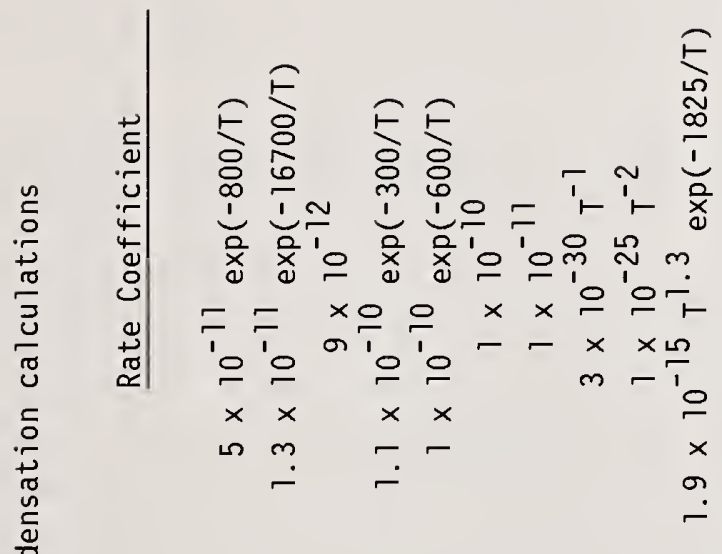

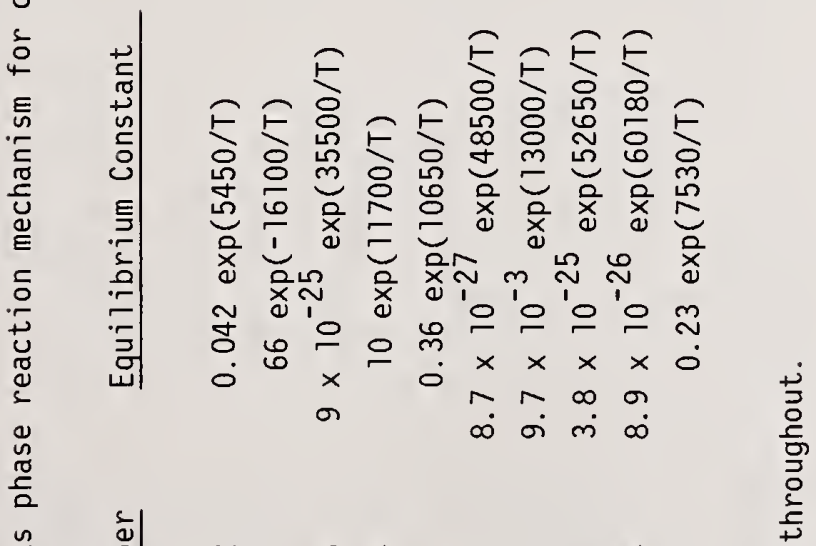

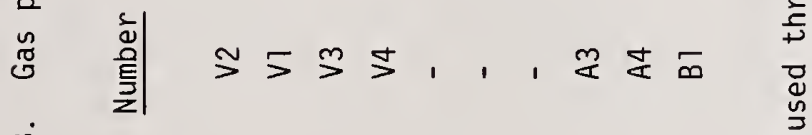

尚

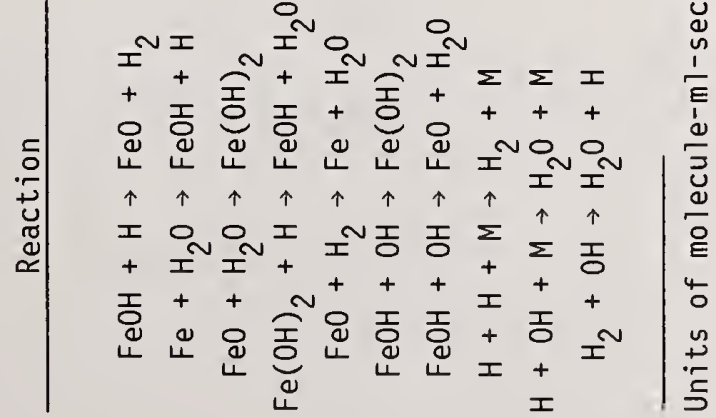




\subsubsection{Coagulation process}

The essentially continuous distribution of iron particle radi $i$ is replaced by a discrete distribution with specified particle size ranges and averaged rates of conversion from one size range to another. The radii of the fifteen (spherical) particle types selected for the present purpose are given in table 4 , together with the number of iron atoms per particle, calculated from the density of bulk phase iron.

Table 4. Iron particle radi $i$ and growth rate terms

\begin{tabular}{|c|c|c|c|}
\hline Species & $\underline{\text { Radius, } \mathrm{nm}}$ & $\begin{array}{l}\text { Iron Atoms } \\
\text { per Particle }\end{array}$ & $\mathrm{K}_{1}, \mathrm{ml}$ molecule $\mathrm{s}^{-1}, 1200 \mathrm{~K}$ \\
\hline$P_{A}$ & 0.2 & 3 & $5 \times 10^{-71}$ \\
\hline$P_{B}^{H}$ & 0.3 & 10 & $1.9 \times 10^{-10}$ \\
\hline$P_{C}$ & 0.6 & 77 & $7.6 \times 10^{-10}$ \\
\hline$P_{D}$ & 1.2 & 610 & $3.1 \times 10^{-9}$ \\
\hline$P_{E}$ & 2.4 & $4.91 \times 10^{3}$ & $1.22 \times 10^{-8}$ \\
\hline$P_{F}$ & 4.8 & $3.93 \times 10^{4}$ & $4.88 \times 10^{-8}$ \\
\hline$P_{G}$ & 9.6 & $3.14 \times 10^{5}$ & $1.95 \times 10^{-7}$ \\
\hline$P_{H}$ & 19.2 & $2.51 \times 10^{6}$ & $7.82 \times 10^{-7}$ \\
\hline$P_{I}$ & 38.4 & $2.01 \times 10^{7}$ & $3.13 \times 10^{-6}$ \\
\hline$P_{j}$ & 76.8 & $1.61 \times 10^{8}$ & $1.25 \times 10^{-5}$ \\
\hline$P_{K}$ & 154 & $1.29 \times 10^{9}$ & $5.00 \times 10^{-5}$ \\
\hline$P_{1}^{n}$ & 307 & $1.03 \times 10^{10}$ & $2.00 \times 10^{-4}$ \\
\hline$P_{M}$ & 614 & $8.23 \times 10^{10}$ & $8.00 \times 10^{-4}$ \\
\hline$P_{N}$ & 1230 & $6.59 \times 10^{11}$ & $3.20 \times 10^{-3}$ \\
\hline$P_{0}^{N}$ & 2460 & $5.27 \times 10^{12}$ & \\
\hline
\end{tabular}

A11 particles except the largest (selected as being so large as to play no significant part in the kinetic scheme) are permitted to coagulate irreversibly with all others via inclusion of rate terms of the type

$$
P_{i}+P_{j} \rightarrow P_{j+1},
$$
where the radius $r_{p_{i}}$ of particle $P_{i}$ is not greater than $r_{p_{j}}$ and $i$ and $j$ run alphabetically
from $A$ to 0 . For $i \neq j$,

$$
-d\left[P_{i}\right] / d t=\kappa_{\alpha i j}\left[P_{i}\right]\left[P_{j}\right]
$$


and

$$
-d\left[P_{j}\right] / d t=d\left[P_{j+1}\right] / d t=\kappa_{\beta i j}\left[P_{j}\right]\left[P_{j}\right]
$$

where $\left(n_{j+1}-n_{j}\right) \kappa_{\beta i j}=n_{i} k_{\alpha i j}$ and the $i$ th particle contains $n_{i}$ iron atoms. For $i=j$,

$$
-d\left[P_{i}\right] / d t=2 \kappa_{\alpha i j}\left[P_{i}\right]^{2} ; d\left[P_{i+1}\right] / d t=2 \kappa_{\beta i j}\left[P_{j}\right]^{2} ;
$$

and $n_{i+1} K_{\beta i}=n_{j} k_{\alpha i j}$. Values of $k_{\alpha}$ are given in table 5. They are based on two main assumptions: (i) that the unit concentration collision rate $v$ for two particles of radius $r_{1}$ and $r_{2}$, respectively, is given by

$$
v=(6 \mathrm{kT} \bar{v} / \mathrm{m})^{1 / 2}\left\{\left(r_{1}^{3}+r_{2}^{3}\right)\left(r_{1}+r_{2}\right)^{4} r_{1}^{-3} r_{2}^{-3}\right\}^{1 / 2}
$$

where $\bar{v}$ is the specific (atomic iron) condensed phase volume of $1.18 \times 10^{-23} \mathrm{ml}$ and $\mathrm{m}$ is the atomic mass of iron $\left(9.27 \times 10^{-23} \mathrm{~g}\right)$; and $(i i)$ that the sticking probability for two colliding particles is unity. This leads to $\kappa_{\alpha}$ and $\kappa_{\beta}$ values for the larger particles which are considerably greater than would be consistent with the Smoluchowski theory [31,32]; under conditions where coagulation rates of these larger particles are of kinetic importance (as they are not for the case considered below), proper allowance must be made for the

\begin{tabular}{|c|c|c|c|c|c|c|c|c|c|c|c|c|c|c|c|}
\hline & $P_{A}$ & $P_{B_{B}}$ & $\mathrm{Pe}$ & $P_{0}$ & $P_{E}$ & $P_{E}$ & $P_{s}$ & $P_{u}$ & $p_{1}$ & $p$ & $P_{x}$ & $p_{1}$ & $p_{x}$ & $p$ & $p$ \\
\hline$p_{p_{B}}$ & $2.9(-10)$ & $3.6(-10)$ & $8.2(-10)$ & $2.5(-9)$ & $8.5(-9)$ & $3.1(-8)$ & $1.2(-7)$ & $4,7(-7)$ & $1.9(-6)$ & $75(-6)$ & $30(-5)$ & $2(-4)$ & $48(-4)$ & $9(-3)$ & \\
\hline $\mathrm{P}_{B}$ & - & $3.5(-10)$ & $5.9(-10)$ & $1.6(-9)$ & $5.0(-9)$ & $1.8(-8)$ & $6.7(-8)$ & $2.6(-7)$ & $1.0(-6)$ & 4. $1(-6)$ & $1.6(-5)$ & $6.5(-5)$ & $2.6(-4)$ & $1.0(-3)$ & - \\
\hline $\mathrm{P}_{\mathrm{c}}$ & - & - & $4.9(-10)$ & $8.3(-10)$ & $2.2(-9)$ & $7.1(-9)$ & $2.5(-8)$ & $9.5(-8)$ & $3.7(-7)$ & $1.5(-6)$ & $5.8(-6)$ & $2.3(-5)$ & $9.2(-5)$ & $3.7(-4)$ & - \\
\hline$P_{0}$ & - & - & - & $7.0(-10)$ & $1.2(-9)$ & 3.1(-9) & $1.0(-8)$ & $3.6(-8)$ & $1.3(-7)$ & $5.2(-7)$ & $2.1(-6)$ & $8.1(-6)$ & $3.2(-5)$ & $1.3(-4)$ & - \\
\hline $\mathrm{P}_{\mathrm{E}}$ & - & - & - & - & $9.9(-10)$ & $1.7(-9)$ & $4.4(-9)$ & $1.4(-8)$ & $5.0(-8)$ & $1.9(-7)$ & $7.4(-7)$ & $2.9(-6)$ & $1.2(-5)$ & $4.4(-5)$ & - \\
\hline$P_{F}$ & - & - & - & - & - & $1.4(-9)$ & $2.4(-9)$ & $6.2(-9)$ & $2.0(-8)$ & $7.1(-8)$ & $2.6(-7)$ & $1.0(-6)$ & $4.1(-6)$ & $1.6(-5)$ & - \\
\hline$P_{G}$ & - & - & - & - & - & - & $2.0(-9)$ & $3.2(-9)$ & $8.8(-9)$ & $2.8(-8)$ & $1.0(-7)$ & $3.8(-7)$ & $1.5(-6)$ & $5.8(-6)$ & - \\
\hline$P_{H}$ & - & - & - & - & - & - & - & $2.8(-9)$ & $4.7(-9)$ & $1.2(-8)$ & $4.0(-8)$ & $1.4(-7)$ & $5.4(-7)$ & $2.1(-6)$ & - \\
\hline$p_{1}$ & - & - & - & - & - & - & - & - & $3.9(-9)$ & $6.7(-9)$ & $1.8(-8)$ & $5.7(-8)$ & $2.0(-7)$ & $7.6(-7)$ & - \\
\hline$P_{J}$ & - & - & - & - & - & - & - & - & - & $5.6(-9)$ & $9.4(-9)$ & $2.5(-8)$ & $8.0(-8)$ & $2.9(-7)$ & - \\
\hline$P_{k}$ & - & - & - & - & - & - & - & - & - & - & $7.9(-9)$ & $1.3(-8)$ & $3.5(-8)$ & 1. $1(-7)$ & - \\
\hline$P_{L}$ & - & - & - & - & - & - & - & - & - & - & - & $1.1(-8)$ & $1.9(-8)$ & $5.0(-8)$ & - \\
\hline$P_{M}$ & - & - & - & - & - & - & - & - & - & - & - & - & $1.6(-8)$ & $2.8(-8)$ & - \\
\hline$P_{N}$ & - & - & - & - & - & - & - & - & - & - & - & - & - & $2.2(-8)$ & - \\
\hline$P_{0}$ & - & - & - & - & - & - & - & - & - & - & - & - & - & - & - \\
\hline
\end{tabular}
fact that eq. (28) gives correct collision rates only for $r \ll \lambda$ (the mean free path, here $\approx 300 \mathrm{~nm}$ ).

Values tabulated are those of $k_{\text {all }}$ and $K_{\text {alf }}\left(m l\right.$ particle $\left.{ }^{-1}, s^{-1}\right)$, as defined in the text, at $1200 \mathrm{~K}$. For $P_{E}+p_{J} \rightarrow p_{K}$, for example, $-d\left[P_{E}\right] / d t=1.9 \times 10^{-7}\left[P_{E}\right]\left[P_{J}\right]$ particle $\mathrm{ml}^{-1} s^{-1}$, and $-d\left[P_{J}\right] / d t=d\left[P_{K}\right] / d t=1.9 \times 10^{-7} n_{E}\left(n_{K}-n_{J}\right)^{-1}\left[P_{E}\right]\left[P_{J}\right]$ part clcle $\mathrm{ml}^{-1} \mathrm{~s}^{-1} \cdot A(-B) \equiv A \times 10^{-B}$. 


\subsubsection{Growth}

The growth process comprises collisions of atomic iron with the particle surfaces and assimilation of this iron into the particles. A series of growth terms analogous to those for coagulation may be written: for the irreversible conversion

$$
\begin{gathered}
P_{i}+F e \rightarrow P_{i+1} \\
-d\left[P_{i}\right] / d t=d\left[P_{i+1}\right] / d t=k_{2 i}\left[P_{j}\right][F e]
\end{gathered}
$$

and

$$
-d[\mathrm{Fe}] / \mathrm{dt}=\kappa_{1 i}\left[\mathrm{P}_{i}\right][\mathrm{Fe}]
$$

where $\left(n_{i+1}-n_{j}\right) \kappa_{2 i}=k_{1 j}$. Values of $\kappa_{1 j}$ at $1200 k$ are listed in table 4 . These are based on the assumption that every collision of an iron atom at a particle surface results in assimilation of the atom into the particle. Collision rates are again calculated from a simple gas kinetic theory expression:

$$
v^{\prime}=(8 \mathrm{kT} / \pi \mathrm{q})^{1 / 2} \pi r_{i}^{2}
$$

where $q$ is the reduced mass for the colliding species. The neglect of diffusion terms does not introduce significant error in the present context, but under other conditions it may be necessary to take explicit account of such terms. Growth steps involving $\mathrm{Fe}_{2}$ were also considered, but were found to be of little kinetic importance in the present case.

\subsubsection{Link reactions}

The description of condensation is formally completed via inclusion of steps linking the smallest particle $P_{A}$ (treated as "condensed" despite the obvious inappropriateness of attempting to assign to it any solid or liquid phase property) with gas phase atomic iron. These steps are [30]

$$
\mathrm{Fe}+\mathrm{Fe}+\mathrm{M} \rightarrow \mathrm{Fe}_{2}+\mathrm{M}
$$

$\left(k_{33}=1 \times 10^{-32} \mathrm{ml}^{2}\right.$ molecule $\mathrm{e}^{-2} \mathrm{~s}^{-1}$ at $\left.1200 \mathrm{~K}\right)$,

$$
\mathrm{Fe}+\mathrm{Fe}_{2}+\mathrm{M} \rightarrow \mathrm{P}_{\mathrm{A}}+\mathrm{M}
$$

$\left(k_{34,1200}=3 \times 10^{-32} \mathrm{ml}^{2}\right.$ molecule $\left.\mathrm{s}^{-2}\right)$, and

$$
\mathrm{Fe}_{2}+\mathrm{Fe}_{2} \rightarrow \mathrm{P}_{\mathrm{A}}
$$


$\left(-\mathrm{d}\left[\mathrm{Fe}_{2}\right] / \mathrm{dt}=2 \mathrm{k}_{35}\left[\mathrm{Fe}_{2}\right]^{2} ; \mathrm{d}\left[\mathrm{P}_{\mathrm{A}}\right] / \mathrm{dt}=4 / 3 \mathrm{k}_{35}\left[\mathrm{Fe}_{2}\right]^{2} ; \mathrm{k}_{35}=3 \times 10^{-10} \mathrm{ml}\right.$ molecule $\mathrm{s}^{-1}$ at $1200 \mathrm{~K}$ ). These kinetic parameters (and the selected growth rate terms) are nicely consistent [30] with the recent thorough shock tube measurements of Bauer and colleagues [3337]. There are no immediate fundamental objections to treating $\mathrm{Fe}_{2}$ and $\mathrm{Fe}_{3}\left(\mathrm{alias} \mathrm{P}_{\mathrm{A}}\right.$ ) as viable centers for homogeneous nucleation $[28,38]$. The link steps, like the growth and coagulation steps, are formally assumed, to a first approximation, to be irreversible. Removal of this assumption will become feasible only when reliable thermochemical data for small molecules of type $\mathrm{Fe}_{\mathrm{n}}$ become available; mass spectrometric and effusion cell techniques will probably be the best sources of such data.

\subsubsection{Use of condensation model}

A computer program which solves simultaneously the rate equations governing gas phase reactions, link reactions, coagulation, and growth has been written $[28,39]$. This was used to calculate the condensation rate of iron under particular conditions at $1200 \mathrm{~K}$ (a typical rocket motor nozzle exit temperature) from the following initial ( $t=0$ seconds) concentrations of gaseous species, which correspond to the calculated exit composition of this rocket motor: $[\mathrm{Fe}]=9.54 \times 10^{14},[\mathrm{FeO}]=6.9 \times 10^{13},[\mathrm{FeOH}]=9.04 \times 10^{15},\left[\mathrm{Fe}(\mathrm{OH})_{2}\right]$ $=1.0 \times 10^{15},\left[\mathrm{H}_{2}\right]=8.5 \times 10^{17},\left[\mathrm{H}_{2} \mathrm{O}\right]=1.4 \times 10^{18},[\mathrm{H}]=5.9 \times 10^{14},[\mathrm{OH}]=7.1 \times 10^{12^{2}}$, $[\mathrm{M}]=6.08 \times 10^{18^{2}}$ molecule $\mathrm{ml}^{-1} ;$ all other species concentrations $=0$. Species concentrations are shown as functions of reaction time in figure 5 . With the standard values of input parameters, the condensation time $t_{c}$ for $F e$ (arbitrarily taken to be the time at which $\left[\mathrm{Fe}_{\mathrm{p}}\right] \equiv \sum_{i} \mathrm{n}_{i}\left[\mathrm{P}_{i}\right]$ becomes equal to $[\mathrm{Fe}]$ ) is about $8 \times 10^{-4}$ seconds. Figures 6 and 7 show the effects of varying input rate parameters upon $\left[\mathrm{Fe}_{p}\right] /$ time profiles. It is clear that $t_{c}$ is subject to substantial uncertainties imposed by the imprecision with which gas phase rate coefficients, link reaction rate coefficients, and growth rate terms are known.

In addition to calculations in which these rate parameters were varied, two further calculations were made. In the first, the initial concentration of $P_{K}$ (particle radius $0.154 \mu \mathrm{m}$ ) was set equal to $5 \times 10^{7}$ particle $\mathrm{ml}^{-1}$ instead of zero, and $5 \times 10^{7} \mathrm{n}_{\mathrm{K}}=6.45$ $\times 10^{16}$ atoms $\mathrm{ml}^{-1}$ was subtracted from $\sum_{i} n_{i}\left[\mathrm{P}_{i}\right]$ in the computation of $\left[\mathrm{Fe}_{p}\right]$ at all reaction times. The object was to assess roughly the likely influence of heterogeneous nucleation terms, the initial concentration and particle size of condensed material being approximately equivalent to that to be expected if a particulate mass fraction of about 0.02 were produced from a propellant and acted as a source of such nucleation. The rate of condensation (fig. 7, line 2) is indeed expected to increase significantly in this case. In the second calculation, $\mathrm{FeOH}(\mathrm{g})$ rather than $\mathrm{Fe}(\mathrm{g})$ was allowed to be the growth species via inclusion of rate terms for steps of the type

$$
P_{i}+\mathrm{FeOH} \rightarrow \mathrm{P}_{\mathrm{i}+1}+\mathrm{OH}
$$



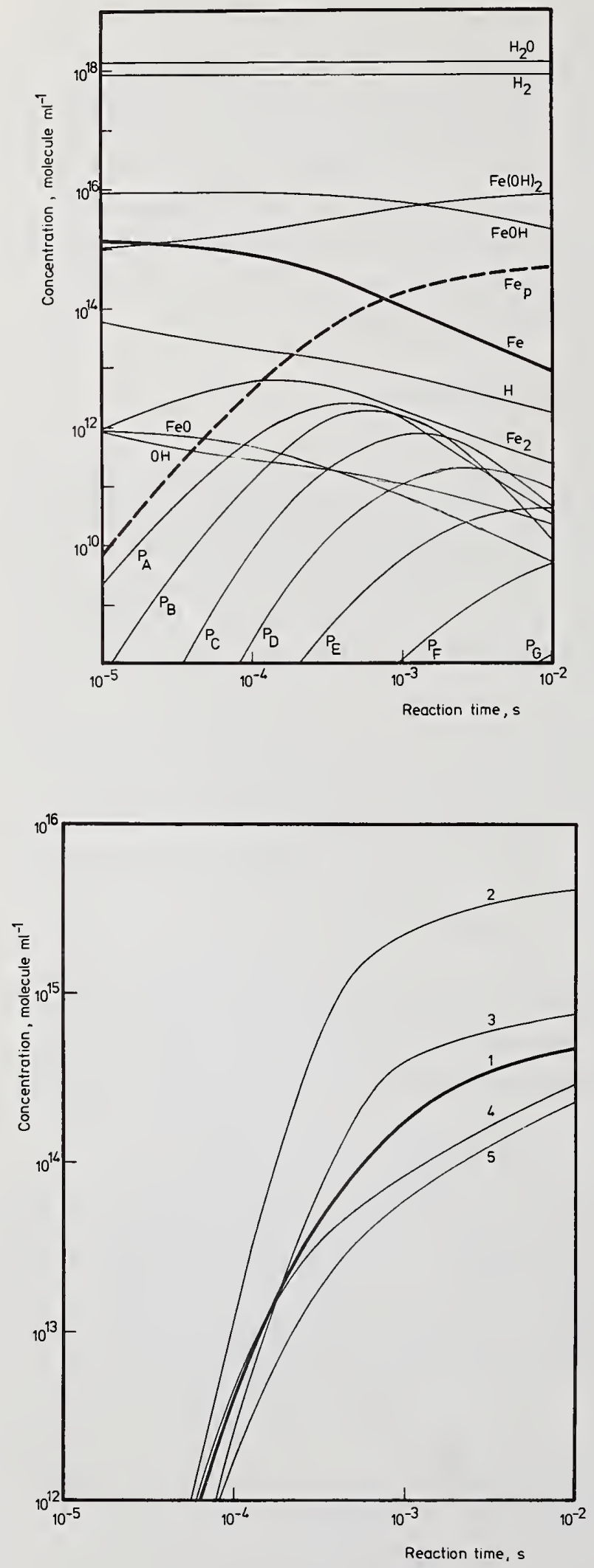

Figure 5. Calculated species concentrations as functions of reaction time for standard input parameters in iron condensation mode 1 . Temperature $=1200 \mathrm{~K}$. $\left[\mathrm{Fe}_{\mathrm{p}}\right] \equiv \sum_{i} \mathrm{n}_{i}\left[\mathrm{P}_{j}\right]$.
Figure 6. $\left[\mathrm{Fe}_{\mathrm{p}}\right] \equiv \sum_{i} \mathrm{n}_{i}\left[\mathrm{P}_{i}\right]$ as function of reaction time at $1200 \mathrm{~K}$. Line 1, standard input. Lines 2-5 correspond to the following individual changes in rate parameters: 2 , rate coefficient of $\mathrm{FeO}+\mathrm{H}_{2} \mathrm{O} \rightarrow \mathrm{Fe}(\mathrm{OH})_{2}$ reduced by factor of $10 ; 3$, rate coefficient of $\mathrm{FeO}+\mathrm{H}_{2} \rightarrow \mathrm{Fe}+\mathrm{H}_{2} \mathrm{O}$ reduced by factor of $10 ; 4$, rate coefficient of $\mathrm{Fe}(\mathrm{OH})_{2}+\mathrm{H} \rightarrow \mathrm{FeOH}$ $+\mathrm{H}_{2} \mathrm{O}$ reduced by factor of $10 ; 5$, rate coefficient of $\mathrm{FeOH}+\mathrm{H} \rightarrow \mathrm{Fe} 0$ $+\mathrm{H}_{2}$ reduced by factor of 10 . The calculated values of $\left[\mathrm{Fe}_{\mathrm{p}}\right]$ are relatively insensitive to changes in rate coefficients of $\mathrm{Fe}+\mathrm{H}_{2} \mathrm{O} \rightarrow \mathrm{FeOH}+\mathrm{H}, \mathrm{FeOH}+$ $\mathrm{OH} \rightarrow \mathrm{FeO}+\mathrm{H}_{2} \mathrm{O}$, and $\mathrm{FeOH}+\mathrm{OH} \rightarrow \mathrm{Fe}(\mathrm{OH})_{2}$. Thermochemical data input unchanged throughout. 


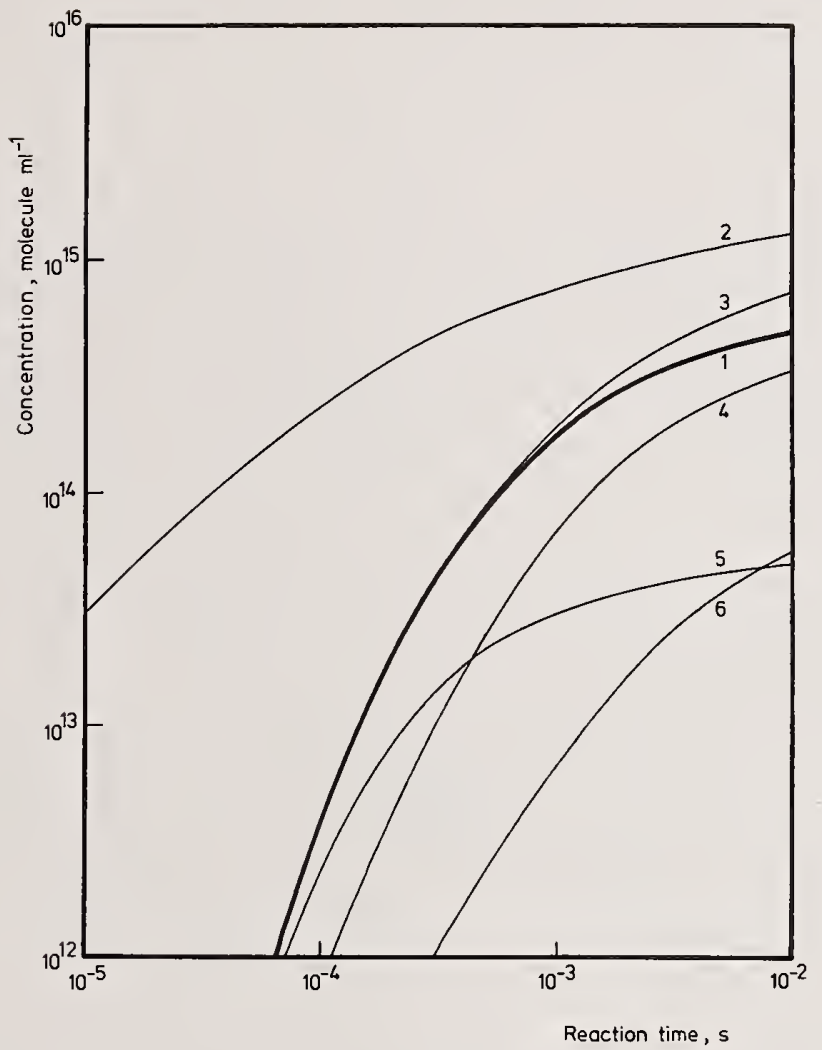

Figure 7. $\left[\mathrm{Fe}_{\mathrm{p}}\right] \equiv \sum_{i} \mathrm{n}_{i}\left[\mathrm{P}_{i}\right]$ as function of reaction time at $1200 \mathrm{~K}$. Line 1 , standard input. Lines 2-6 correspond to the following changes in individual rate parameters or initial concentrations: 2 , initial concentration of $P_{K}$ changed to allow test for effect of heterogeneous nucleation (see text); 3 , all coagulation rate terms reduced by factor of $10 ; 4, \mathrm{Fe}_{2}+\mathrm{Fe}_{2} \rightarrow \mathrm{P}_{\mathrm{A}}$ rate terms reduced by factor of $10 ; 5$, all growth rate terms reduced by factor of $10 ; 6$, rate coefficient of $\mathrm{Fe}+\mathrm{Fe}+\mathrm{M} \rightarrow \mathrm{Fe}_{2}+\mathrm{M}$ reduced by factor of 10 . Calculated values of $\left[\mathrm{Fe}_{\mathrm{p}}\right]$ are relatively insensitive to changes in growth rate terms for $\mathrm{Fe}_{2}$ or to changes in the rate coefficient of $\mathrm{Fe}+\mathrm{Fe}_{2}+M \rightarrow P_{A}+M$.

which is slightly exothermal $[40,41]\left(\Delta \mathrm{H}_{1200}^{0} \approx-30 \mathrm{~kJ}\right.$ mole $\left.{ }^{-1}\right)$. As expected, the rate of condensation calculated again increased markedly when the input rates of these steps corresponded to the collision frequencies. Similar increases would be expected if $\mathrm{Fe}(\mathrm{OH})_{2}$ were formally to be treated as a growth species. These additional calculations further illustrate the uncertainties involved in calculations of condensation rates for these conditions.

It is instructive to compare $t_{c}$ with the approximate time of residence $t_{r}$ occupied by molecules after leaving the rocket nozzle in reaching a location in the exhaust where secondary burning of $\mathrm{CO}$ and $\mathrm{H}_{2}$ either occurs or is prevented $[15,29]$ ( $t_{r}$ is generally $\gg$ the nozzle residence time). Calculated axial velocity and temperature distributions for 
the exhaust flames of three rocket motors, differing principally in that they have widely disparate thrust levels (Motor 1, $0.31 \mathrm{kN}$; Motor 2, $31 \mathrm{kN}$; Motor 3, $300 \mathrm{kN}$ ), are shown in figure 8. Inspection of these distributions shows that $t_{r}$ is roughly $2 \times 10^{-4} \mathrm{~s}$ for Motor 1, $2 \times 10^{-3} \mathrm{~s}$ for Motor 2, and $5 \times 10^{-3} \mathrm{~s}$ for Motor 3 . (A11 three exhausts do have secondary combustion present, but $t_{r}$ would not differ greatly from these values even if it were absent.) For Motor $1, t_{c}>t_{r}$, but for Motor 3 the opposite is true. The input mole fraction of iron compounds corresponds to about 0.3 percent by weight of the propellant gas flow. In view of the uncertainties in $t_{c}$, it is not possible to draw any firm conclusion as to whether significant depletion of gas phase iron-containing species will occur as a result of condensation for any one of these exhausts, although it is certainly true that the likelihood of such depletion increases with size of motor.

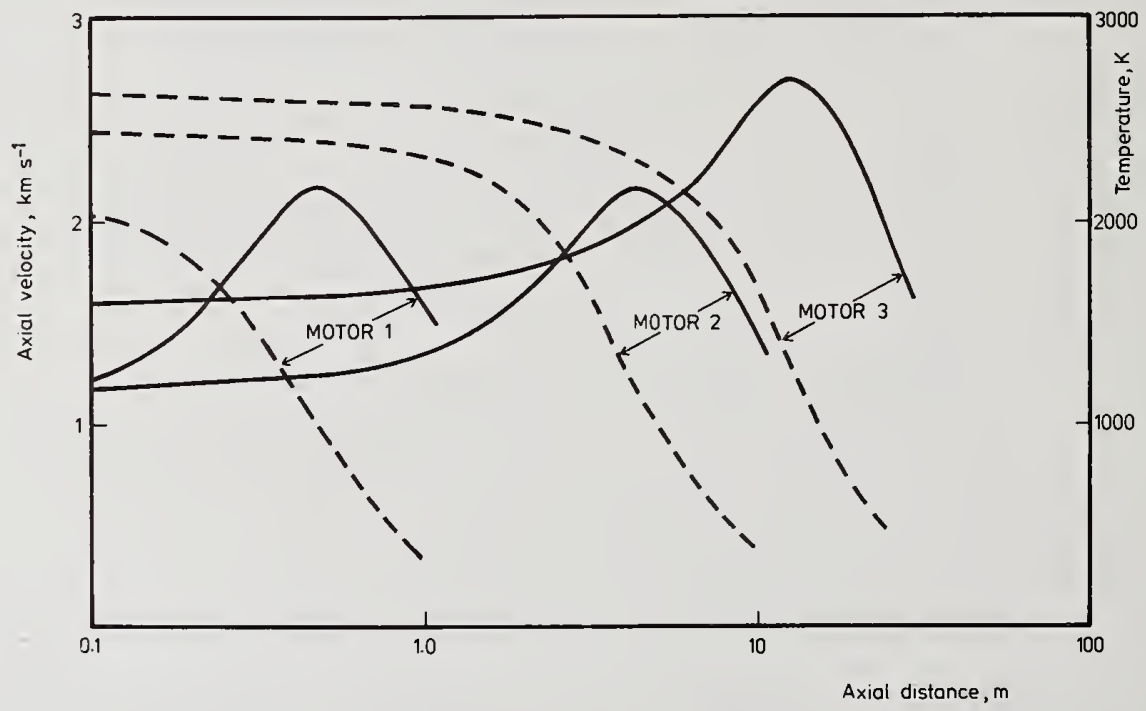

Figure 8. Calculated variation of axial velocity component (broken lines) and temperature (continuous lines) with axial distance downstream of the nozzle exit for three rocket motor exhaust flames.

The above calculations have clearly been greatly over-simplified in that coupling between the reactions directly involved in the condensation process and other flame reaction and mixing processes has not been taken into account. Current work at Westcott is aimed at achieving just such a coupling via incorporation of the condensation model into that of Section 2. It is likely, however, that the broad conclusion reached when a combined model is used will remain the same: uncertainties in input rate parameters are at present too great for quantitative assessments of effects of condensation of metals in many rocket exhaust flames to be made; $t_{c}$ will be neither much greater nor much smaller than $t_{r}$ in many cases. Much further research on various aspects of the condensation process will be necessary before quantitatively reliable characteristics of flames containing condensible 
materials can be calculated. Nevertheless, the simple condensation model offered has al ready proved useful for identifying rate-controlling processes in typical exhaust flames and may well provide less frustrating answers when applied in other practical contexts!

\section{Conclusions}

A theoretical method for characterizing turbulent flame structures has been outlined. The computer model based on the methodology described will accept any input of gas phase chemical reactions, for which thermochemical and kinetic parameters must be specified. Some examples of the ways in which such parameters may be obtained from experiment and are I used have been indicated. A preliminary account of a method for calculating condensation rates in flames or other environments has been provided.

Computational method of characterizing practical flames are primarily used to provide information inaccessible to experiment, but are also valuable for identifying ill-defined key fundamental rate processes of interest and hence for indicating needs for further research. Because results of such research will in turn eventually be fed back into computations in order that more accurate practical information should be generated, it is important that development of computational techniques and fundamental research proceed in parallel, with continual feedback, and not in mutual isolation.

Crown Copyright, Her Majesty's Stationery Office, London, 1978.

\section{References}

[1] Jensen, D. E., Spalding, D. B., Tatche11, D. G., and Wilson, A. S., Computation of structures of flames with recirculating flow and radial pressure gradients, Combustion, and Flame (1978), submitted for publication.

[2] Launder, B. E. and Spalding, D. B., in Mathematical Models of Turbulence (Academic Press, London and New York, 1972).

[3] Spalding, D. B., Chem. Eng. Sci. 26, 75 (1971).

[4] Spalding, D. B., Imperial College, London, Dept. Mech. Eng. Report HTS/69/23.

[5] Caretto, L. S., Gosman, A. D., Patankar, S. V., and Spalding, D. B., Proc. 3rd Intl. Conf. on Num. Meth. in Fluid Mech., Springer-Verlag Lecture Notes in Physics, Vol. 11, No. 19, p. 60 (1973).

[6] Jensen, D. E. and Wilson, A. S., Comb. Flame, 25, 43 (1975).

[7] Spalding, D. B., Imperial College, London, Dept. Mech. Eng. Report HTS/76/13.

[8] Jensen, D. E. and Jones, G. A., Comb. Flame, 32, 1 (1978).

[9] Cotton, D. H. and Jenkins, D. R., Trans. Faraday Soc. 67, 730 (1971).

[10] Bulewicz, E. M. and Padley, P. J., 13th Int1. Symp. Comb., p. 73 (The Combustion Institute, Pittsburgh, 1971). 
[11] Bulewicz, E. M. and Padley, P. J., Trans. Faraday Soc. 67, 2337 (1971).

[12] Jensen, D. E. and Jones, G. A., J. Chem. Phys. 60, 3421 (1974).

[13] Jensen, D. E. and Jones, G. A., J. Chem. Soc. Faraday Trans. 1, 71, 149 (1975).

[14] Jensen, D. E. and Jones, G. A., J. Chem. Soc. Faraday Trans. 1, 72, 2618 (1976).

[15] Jensen, D. E. and Webb, B. C., AIAA J. 14, 947 (1976).

[16] Jensen, D. E. and Jones, G. A., Proc. Roy. Soc. (London), in press.

[17] Rosser, W. A., Inami, S. H., and Wise, H., Comb. Flame, 7, 107 (1963).

[18] Friedman, R. and Levy, H., Comb. Flame, 7, 195 (1963).

[19] Jensen, D. E., Jones, G. A., and Mace, A. C. H., to be published.

[20] McHale, E. T., Comb. Flame, 24, 277 (1975).

[21] Jensen, D. E. and Pergament, H. S., Comb. Flame, 17, 115 (1971).

[22] Burdett, N. A. and Hayhurst, A. N., Chem. Phys. Lett. 48 , 95 (1977).

[23] Burdett, N. A. and Hayhurst, A. N., private communication (1978).

[24] Jensen, D. E. and Travers, B. E. L., IEEE Trans. Plasma Sci. PS-2, 34 (1974).

[25] Jensen, D. E., Comb. Flame, 18, 217 (1972).

[26] Sugden, T. M., Trans. Faraday Soc. 52, 1465 (1956).

[27] Jensen, D. E., Ridout, J. M. , and Webb, B. C., unpublished MOD work.

[28] Jensen, D. E., Proc. Roy. Soc. (London), A338, 375 (1974).

[29] Jensen, D. E., in Proceedings of the Symposium on High Temperature Metal Halide Chemistry, p. 443 (The Electrochemical Society, Princeton, N.J., 1978).

[30] Jensen, D. E., to be published.

[31] Smoluchowski, M., Z. Phys. Chem. 29, 129 (1918).

[32] Whytlaw-Gray, R. and Patterson, H. S., in Smoke: A Study of Aerial Disperse Systems (Arnold, London, 1932).

[33] Kung, R. T. V. and Bauer, S. H., in Shock Tube Research, J. L. Stollery, A. G. Gaydon, and P. R. Owen, eds. paper 61 (Chapman and Hal1, London, 1971).

[34] Freund, H. J. and Bauer, S. H., J. Phys. Chem. 81, 994 (1977).

[35] Frurip, D. J. and Bauer, S. H., J. Phys. Chem. 81, 1001 (1977).

[36] Frurip, D. J. and Bauer, S. H., J. Phys. Chem. 81, 1007 (1977).

[37] Bauer, S. H. and Frurip, D. J., J. Phys. Chem. 81, 1015 (1977).

[38] Homer, J. B., in Shock Tube Research, J. L. Stollery, A. G. Gaydon, and P. R. Owen, eds., paper 62 (Chapman and Ha11, London, 1971).

[39] Allen, K. and Jensen, D. E., MOD unpublished work.

[40] Jensen, D. E. and Jones, G. A., J. Chem. Soc. Faraday Trans. 1, 69, 1448 (1973).

[41] JANAF Thermochemical Tables, NBS NSRDS No. 37 (1971).

\section{Discussion}

Question (John Hastie): You showed data on the potassium hydroxide species and said that Dave Jensen had verified the mechanism for the action of potassium on catalyzing hydrogen atom recombination. In connection with those studies, did you get any new information about the likely heat of formation of the potassium hydroxide molecule? 
Response (Webb): I don't have the answer to that, John, but it is going to be written up, and should be appearing within the next few months. I think they (Jensen et al.) are just finishing their final interpretation of the work at the moment.

Comment (Hastié): I know some people looked at the JANAF tables and have been concerned about the inaccuracy in the heat of formation of potassium hydroxide, and any new information on that molecule would be useful, particularly for those who worry about potassium concentrations in MHD gases and that sort of thing.

Question (Rosner): You mentioned your concern with turbulence properties of the rocket plume and also your concern with the nucleation of compounds and elements such as the iron-containing compounds, and I am wondering if you have considered, or perhaps have some results on the possibly significant effects of turbulence on the rates of condensation?

The reason $I$ ask is that the condensation rate should be an extremely sensitive and highly non-linear function of the local super saturations that are produced.

And if these are indeed fluctuating because of the turbulence, I would assume that the steady-state predicted rate of nucleation would in fact be strongly influenced by the local turbulence.

I wonder if you have any results of this condensation model that might in fact include such effects?

Clarification (Webb): Can I just get it clear? Are you talking about effects that arise as a result of turbulent temperature fluctuations, or of turbulent species concentration fluctuations?

Response (Rosner): Both. Both would contribute to the super-saturation fluctuations which are ultimately involved in the nucleation kinetics aspect.

Response (Webb): This model does not allow for perturbation of the rates of chemical reactions as the result of turbulent temperature fluctuations.

The modelers have told me that really this treatment will have to await the development of a Lagrangian type model, which is, as I am told, some years off yet.

With respect to the other possible mechanism for influence of turbulent fluctuations on nucleation, we don't know yet because the work where we are incorporating the condensation model into the plume model has not yet been completed. 
National Bureau of Standards Special Publication 561, Proceedings of the 10th Materials Research Symposium on Characterization of High Temperature Vapors and Gases held at NBS, Gaithersburg, Maryland, September 18-22, 1978. Issued October 1979.

\title{
MODELING STUDY OF FLAME STRUCTURE IN LOW-PRESSURE, LAMINAR, PRE-MIXED METHANE FLAMES
}

\author{
John R. Creighton and Carl M. Lund \\ Lawrence Livermore Laboratory \\ University of California \\ Livermore, CA 94550
}

\begin{abstract}
Low pressure, laminar, methane flames were modeled numerically using a fully implicit, one-dimensional flame code with detailed chemical kinetics and adaptive gridding. Calculated major species profiles were in good agreement with experiment; and most radical and intermediate species showed fair to good agreement. The calculated flame velocity was about half of experimental and was insensitive to variation of species diffusion coefficients and chemical reaction rate constants except decomposition of HCO. Quadrupling that rate constant improved the calculated flame velocity, as well as radical species profiles. Insight was gained into the physical and chemical processes governing flame propagation.
\end{abstract}

\section{Introduction}

With all our state-of-the-art instruments we must use mathematical analysis to obtain chemical reaction rate data in high temperature gases, except in the simplest cases. We can measure the rate of change of some species, and if the reaction scheme is quite simple the reaction rate is proportional to this rate of change. If the chemical reaction scheme is somewhat more complicated, we may still be able to write rate equations which can be solved in closed mathematical form, especially if we use approximations such as steadystate or partial equilibrium. When we get to reaction schemes as complicated as the oxidation of hydrocarbons we must resort to numerical calculations. Several groups now routinely do such calculations using reaction schemes of 20 to 200 elementary reactions. These calculations sometimes indicate appropriate approximations which permit closed form mathematical solutions.

In order to mathematically model practical combustion systems, such as a laminar, pre-mixed, flame, we must couple fluid mechanics to the chemistry. This generally requires numerical solution, even for simple chemistry, because the Navier-Stokes equations are inherently non-linear, requiring numerical solution in all but a few special cases. The low flow velocities in a laminar flame and the thin reaction zone introduce numerical 
difficulties. We describe a new computer code in this paper which addresses these numerical difficulties.

We have used this code to mathematically simulate two experiments with low pressure, pre-mixed, laminar methane/oxygen flames $[1,2]^{1}$. These state-of-the-art experiments were chosen because species concentration profiles of radicals and other intermediate species were measured, along with the major reactants and products. This provides a more rigorous test of the computer code and the assumed chemical reaction mechanism. With about fifty rate constants, it may be possible to vary enough of them to match the major species profiles without a correct reaction mechanism. If we can also match radical and intermediate species profiles, we can have more confidence in our reaction mechanism.

When we have done a calculation which agrees with experiment, we may use it in several ways. We may use the code and reaction mechanism to predict, or do design calculations, under conditions not too different from those of the experiments. With some caution as to the validity of the results we may also predict the course of the reaction under conditions far from those of the experiments. This might be done if we wished to predict what would happen under conditions not accessible to state-of-the-art experimentation. The calculation might then represent the best available prediction. We can also use the code and reaction mechanism to investigate the sensitivity of the results to changes in the value of selected parameters. We report the results of such studies in this paper. Finally, we may use the calculations to give us general principles and insight into the physical and chemical processes which govern the behavior of the system. A complicated computer calculation is much like an experiment in the sense that it must be interpreted. Without interpretation there is too much detail for the human mind to comprehend. There is considerable emphasis in this paper on interpretation and simple conceptual models of the chemical and physical processes occurring in a laminar, pre-mixed, methane/oxygen flame.

\section{Review of the Chemical Reactions in Shock Tubes and Turbulent Flow Reactors}

Before discussing the flame calculations, it is useful to review the chemical reactions which occur in shock tubes and turbulent flow reactors because the chemistry can generally be understood separate from the fluid dynamics. In this case, if we consider a small (Lagrangian) element moving at the mean flow velocity, the chemistry is independent of the conditions in adjacent elements. This allows us to calculate the chemistry using a single zone, in contrast to the multiple zones required for a flame calculation. The absence of fluid mechanical effects also aids in understanding which effects are primarily chemical. We have modeled the shock tube primarily to study the induction period at temperatures in the range of 1000 to $2000 \mathrm{~K}$ [3] and we have modeled the turbulent flow reactor to study the fuel consumption phase at temperatures in the neighborhood of $1000 \mathrm{~K}$ [4].

\footnotetext{
${ }^{1}$ Figures in brackets indicate the literature references at the end of this paper.
} 
The chemistry early in the induction phase of methane oxidation is shown schematically in figure 1. Figure 1 shows how branching reactions provide a feedback of radicals which causes an exponential increase in radical concentrations. The figure also shows that most of the radicals are in steady-state in the sense that the rates of creation and consumption

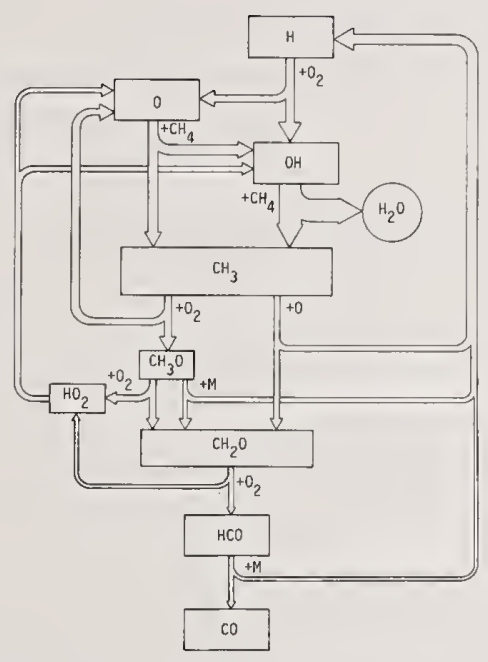

Figure 1. Flow chart of the principal reactions during the induction period of methane oxidation in a shock tube, with about 1 percent of the methane consumed. Radicals and intermediates are represented as boxes, stable products as ovals or circles. The width of the arrows is proportional to the reaction rate. Initial reactants and third bodies are indicated beside the arrows, as in the $\mathrm{H}^{+\mathrm{O}_{2}} \rightarrow \mathrm{O}+\mathrm{OH}$ reaction at the top of the figure.

approximately balance. The obvious exception is $\mathrm{CH}_{3}$. Examination of the figure suggests that the rate controlling reaction (bottleneck) is ${ }^{2}$

$$
\mathrm{CH}_{3}+\mathrm{O}_{2} \rightarrow \mathrm{CH}_{3}+\mathrm{O}
$$

This can be verified by writing rate equations for the reactions in figure 1 and solving them, using the steady-state approximation for radicals other than $\mathrm{CH}_{3}$. This gives the rate equation

$$
\mathrm{d}\left[\mathrm{CH}_{3}\right] / \mathrm{dt}=4 \mathrm{k}_{37}\left[\mathrm{O}_{2}\right]\left[\mathrm{CH}_{3}\right]
$$

${ }^{2}$ Reaction numbers correspond to those in table 1. 
with the solution

$$
\left[\mathrm{CH}_{3}\right]=\left[\mathrm{CH}_{3}\right]_{0} \exp \left(4 \mathrm{k}_{37}\left[\mathrm{O}_{2}\right] \mathrm{t}\right) .
$$

This shows that the $\mathrm{CH}_{3}$ concentration rises exponentially in time; and the mathematics shows that other radical concentrations are proportional to $\left[\mathrm{CH}_{3}\right]$, but two to four orders of magnitude smaller. We can eliminate the unknown constant, $\left[\mathrm{CH}_{3}\right]_{0}$, by adding the decomposition reaction

$$
\mathrm{CH}_{4}+\mathrm{M} \rightarrow \mathrm{CH}_{3}+\mathrm{H}+\mathrm{M}
$$

which gives

$$
\left[\mathrm{CH}_{3}\right]=\frac{\mathrm{k}_{27}[\mathrm{M}]\left[\mathrm{CH}_{4}\right]}{\mathrm{k}_{37}\left[\mathrm{O}_{2}\right]}\left\{\exp \left(4 \mathrm{k}_{37}\left[\mathrm{O}_{2}\right] \mathrm{t}\right)-1\right\} .
$$

Reaction 27, although its rate is very smal1, provides the initial radicals to start the feedback process. Once we know $\left[\mathrm{CH}_{3}\right]$ as a function of time, we can calculate the fuel consumption and induction time. The induction times calculated in this way agree with experimental shock tube data except at high pressures where three-body recombination becomes important [3].

At certain temperatures, pressures, and dilutions the reaction scheme of figure 1 may change somewhat, but the description of the induction process remains qualitatively the same. Also, the reaction scheme used for the calculations may not represent the true chemistry exactly, but the qualitative picture remains valid.

The fuel consumption phase of methane oxidation is controlled by a somewhat different set of reactions as shown in figure 2. Not only are more reactions important, but reactions involving two radicals (or intermediate species) become important, for example

$$
\mathrm{CH}_{3}+\mathrm{O} \rightarrow \mathrm{CH}_{2} \mathrm{O}+\mathrm{H}
$$

and

$$
\mathrm{CH}_{2} \mathrm{O}+\mathrm{OH} \rightarrow \mathrm{CHO}+\mathrm{H}_{2} \mathrm{O} \text {. }
$$

This causes the rate equations to become non-linear and generally impossible to solve, though they might be solved in special circumstances. In spite of the mathematical difficulties we can gain some qualitative understanding of the reaction mechanism. 


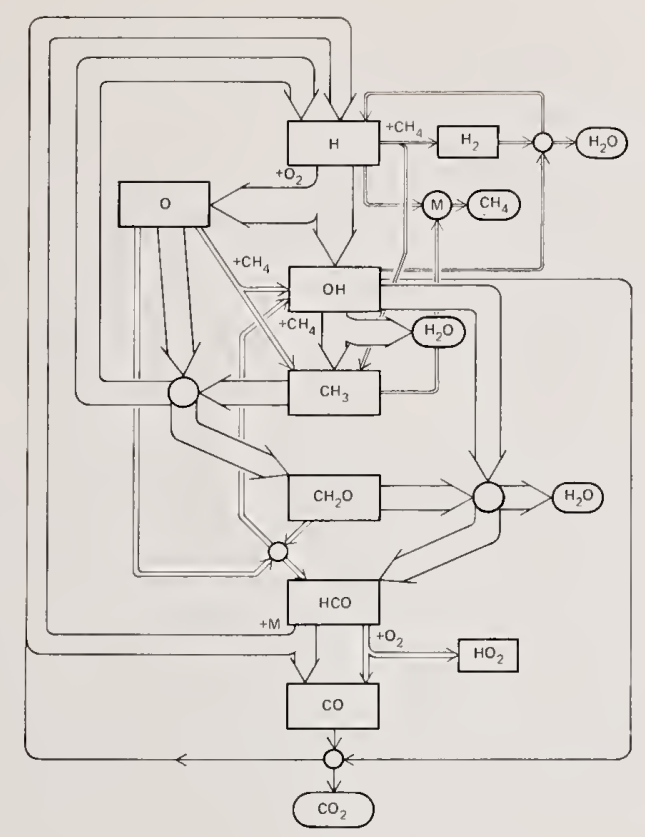

Figure 2. Flow chart of the principal reactions when about 50 percent of the methane has been consumed. Reactions involving two radicals are indicated by arrows meeting at a circle; other conventions are the same as figure 1. Not shown are reactions which consume $\mathrm{HO}_{2}$, these can be summarized by $3 \mathrm{HO}_{2}+\mathrm{O}+\mathrm{H}+\mathrm{CH}_{3} \rightarrow 3 \mathrm{OH}+\mathrm{O}_{2}+\mathrm{CH}_{2} \mathrm{O}+\mathrm{H}_{2} \mathrm{O}$.

First, we note that a reaction rate is the product of a rate constant and one to three species concentrations. The rate constant is a function of temperature, but otherwise can be viewed as a fixed parameter. Reaction rates vary because the species concentrations vary. If one of the species is a reactant, its concentration will change slowly. Radicals and other intermediates are present in such small amounts that their concentrations can change rapidly, so the concentration of radicals and other intermediates determines most reaction rates.

Second, we look at some implications of the steady-state. For simplicity consider a radical which is created by a single reaction and consumed by one other reaction. The rate of consumption is proportional to the concentration of the radical and in steady-state the concentration of the radical will be at a level which makes the rate of consumption equal to the rate of creaction. Further, in most cases the rate of creation will depend on the concentration of another radical which is also in steady-state. In this case the concentrations of the two radicals will be proportional. The constant of proportionality will be a function of the rate constants and the other reactants participating in the two reactions. 
When there are several reactions creating or consuming a radical, the concentration is a function of all of the rate constants and reactant species involved; but, at least in principle, we can find an algebraic relationship for the concentrations of all radicals which are in steady-state. This relationship will be a function of temperature because of the temperature dependence of the rate constants. It will also depend on the concentration of some or $\mathrm{a} 11$ of the reactants and products. (Reverse reactions must be treated as separate reactions because they depend on species concentrations different from those of the forward reactions. )

Some additional aspects of the steady-state are illustrated by figure 3 , which shows the history of several radical concentrations for an atmospheric pressure methane-air mixture initially at $1000 \mathrm{~K}$. The induction period, with exponentially increasing concentrations, lasts about 100 milliseconds. After that there is a plateau in radical concentrations, which is characteristic of all flammable methane-air mixtures at atmospheric pressure, or above. The plateau is due to reactions between two radicals. The branching reactions which lead to exponential growth during the induction period depend on the concentration of a single radical reacting with a reactant whose concentration is approximately constant. Such reactions depend linearly on the radical concentrations. Reactions of two radicals depend quadratically on the radical concentrations. Many of these, such as recombination reactions, destroy radicals. At low concentrations the linear terms dominate and radical concentrations increase. At sufficiently high concentrations the linear and quadratic terms balance, giving a steady-state. This picture is further illustrated by calculations with initial radical concentrations well above steady-state. The calculations show that the radical concentrations drop to the steady-state value in microseconds. Not only do the concentrations drop to steady-state, but the ratio of the various radical concentrations achieves steady-state, no matter which radical is injected. The injected radical reacts immediately with reactants and is "converted" to the appropriate distribution of radicals. The distribution and steady-state level depends primarily on temperature, and varies an order of magnitude for a $100 \mathrm{~K}$ change in temperature for the three radicals, $\mathrm{H}, 0$, and $\mathrm{OH}$. The change for $\mathrm{CH}_{3}$ is considerably smaller. The rise in concentrations at late times in figure 3 appears to result mainly from the temperature rise resulting from the slow reaction at steady-state concentrations. Calculations where the temperature is forced to be constant show orders of magnitude smaller increases. A more detailed understanding as to what controls the steady-state concentrations is needed, but it is clear that the curves of figure 3 show essentially a classic thermal explosion.

\section{Description of the One-Dimensional Flame Propagation Code}

The tool used for the calculations presented here was a second generation, onedimensional flame propagation code, HCT, developed at LLL [5]. It is available to other researchers. The code solves a detailed chemical kinetics mechanism which is coupled to a complete set of fluid mechanical equations. This permits us to calculate a one-dimensional, propagating, pre-mixed flame, treating the chemistry in the same detail as in the case of a shock tube or turbulent flow reactor, where one considers only a single spatial zone. The 


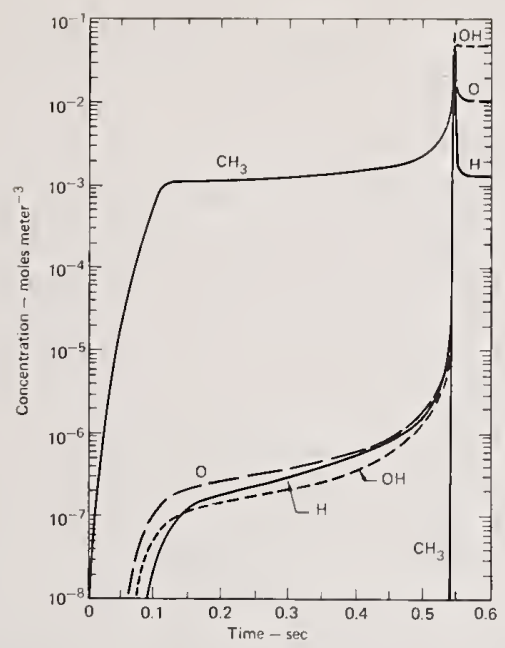

Figure 3. Calculated radical concentrations vs time for a methane-air mixture, $\emptyset=0.5$, initially at $1000 \mathrm{~K}$ and atmospheric pressure.

code can also be used to calculate a steady flame, such as a flat burner, because the steady flame is equivalent to a propagating flame when viewed in a frame that moves with the gas. This second generation code has several features rarely found in earlier codes.

Most earlier codes are "operator split." In an "operator split" code one first calculates the fluid motion, then separately calculates the chemistry; the two changes are then merged at the end of the time step. In reality, the fluid mechanics and chemistry interact, but for short time steps the error is small and the computational advantages are believed to outweigh this small error. The HCT code calculates the chemistry and fluid mechanics simultaneously, including the interactions, and uses special techniques to deal with the resulting complexity.

The numerical method is implicit in both chemistry and fluid mechanics. An explicit numerical method uses the values of the physical variables at the beginning of a time step to evaluate the time derivatives in the differential equation. The derivatives are then used to get new values of the variables representing the solution at the end of the time step. An implicit numerical method uses the values of the variables at the end of the time step to evaluate the derivatives. To obtain these values, a set of algebraic equations must be solved by iteration. Explicit methods are obviously simpler and faster, but develop catastrophic instabilities of solution if the time step becomes larger than the fastest time constant in the set of equations. Implicit methods can be devised which eliminate or reduce this instability, allowing much larger time steps. For some problems the implicit time step is large enough to compensate for the extra calculational effort. Calculating detailed chemical kinetics mechanisms is a case in point. The time constant for many radical concentrations is a fraction of a microsecond, but once steady-state is achieved an implicit numerical method can take time steps of milliseconds or seconds. 
Virtually all flame codes solve the chemistry by implicit methods, but some use explici methods for the fluid dynamics. It is generally advantageous to use implicit methods for the fluid dynamics in a flame code. The time step in an explicit method must be less than the sound transit time across the smallest zone. The time step for an implicit method is approximately the zone thickness divided by the mean flow velocity, and is typically two to three orders of magnitude larger than the explicit time step.

In practice, the size of the implicit time step depends on the method of iteration used to obtain the new values. Newton's iteration is generally superior to simpler schemes. This involves inverting a matrix, but, if done efficiently, is cost effective. In an operator split code one must invert an $\mathrm{N} \times \mathrm{N}$ matrix for each spatial zone in order to solve the chemistry for $N$ chemical species. Coupling the three fluid mechanical variable results in a $(N+3) \times(N+3)$ matrix for each zone, but the mathematics is made more complex because the variables in one zone are coupled to those in the two neighboring zones. The coupling is represented by another $(N+3) \times(N+3)$ matrix. If we consider the matrix associated with a zone to be an element of a larger matrix, these elements lie on the diagonal and the coupling matrices lie just above and below the diagonal, i.e., a "block tri-diagonal" matrix. The implicit method requires that this entire matrix be inverted. Fortunately a very efficient "block tri-diagonal" solver [6] can do this inversion with about three times the computational effort required for the operator split method. We have found that the fully coupled method uses larger time steps which largely compensate for the additional effort. The time step in the operator-split code is limited by the errors due to ignoring the coupling between chemistry and fluid mechanics.

In addition to coupling the chemistry and fluid mechanics, we have taken several measures to reduce errors due to "numerical diffusion." A fluid mechanics code, of necessity, has finite spatial zones. Discontinuities must be spread over several zones to obtain stable solutions, even though the physical process may take place over a distance less than one zone width. A laminar flame is a typical example. To obtain a stable numerical solution averaging over several zones must be introduced. This alters the solution in the same manner as diffusion, but since it is a purely numerical effect it is referred to as "numerical diffusion." In many flame codes numerical diffusion exceeds the molecular diffusion being modeled in a laminar flame.

Numerical diffusion can be eliminated by doing the calculation in a Lagrangian reference frame. In a Lagrangian frame zone boundaries are drawn around elements of mass, and these move with the average flow. However, after a number of time steps the zones can be severely distorted, so the problem is re-mapped onto an Eulerian grid, which is fixed in the laboratory frame. The re-map will introduce numerical diffusion, which can be minimized by careful design. In particular, numerical diffusion is minimized by placing a number of fine zones in the flame front and using coarse zones in regions where gradients are sma11, i.e., "adaptive zoning."

The HCT code uses an adaptive zoning scheme which places fine zones in regions of large temperature gradient, i.e., in the flame front. The scheme uses a minimization algorithm to limit the temperature change across any zone. The algorithm can easily be modified to 
minimize the change in additional variables, such as radical concentrations. It is difficult to obtain a precise estimate of the numerical diffusion, but we have done test calculations which show that it is much smaller than the molecular diffusion in a laminar flame.

In summary, the HCT code eliminates the possible errors introduced by operator splitting and reduces the errors due to numerical diffusion that can arise in less sophisticated codes. By careful attention to computational efficiency this has been achieved without a significant increase in running time, and without inconvenience to the user.

\section{Calculation of Flame Structure and Variation of Parameters}

\section{1 Initial values of parameters}

In our first flame calculation we chose to model the low pressure methane-oxygen flame of Peeters and Mahnen [1]. All comparisons with experiment in this paper refer to this work, but we did make semi-quantitative comparisons with the similar methane-air flame of Biordi, Lazzara, and Papp [2]. These were chosen because the experimenters had measured the concentration profiles of most of the radicals, as well as reactants and products. Comparison of calculated and experimental radical concentrations help us to evaluate the chemical reaction scheme which is used as input in the calculations. As noted above, radical concentrations self-adjust to match the rates of creation and consumption. An incorrect rate constant will cause the calculated radical concentration to be incorrect in order to balance the rates. Comparison with experimental radical concentrations is a better test of the reaction mechanism than comparison of only the reactant and product concentrations. This will be illustrated when we discuss the results.

For input to the code we used the 45 reaction mechanism given in table 1 . This mechanism had been verified earlier by comparison with the turbulent flow reactor and with shock tubes [4], except that the reactions creating and oxidizing $\mathrm{C}_{2} \mathrm{H}_{6}$ were omitted for the flame calculations. This part of the mechanism was known to be incomplete, and the recombination of $\mathrm{CH}_{3}$ to form $\mathrm{C}_{2} \mathrm{H}_{6}$ was judged to be unimportant at the low pressure, 0.025 atmospheres. A single calculation which included the ethane mechanism showed some differences in the details of the reaction scheme, but did not change the general picture. The ethane mechanism will be important for atmospheric pressure flames, and should be investigated in detail when we have a reasonably complete mechanism.

Initial species concentrations were obtained from reports of the experimental work [1]. The initial temperature was taken to be the temperature of the water-cooled flame holder. This was initially assumed to be $300 \mathrm{~K}$, since no value was reported. The calculations indicate that a higher temperature should have been chosen, as discussed later.

The species diffusion coefficients are calculated in the HCT code from the formula, $D_{i}=d_{i} t^{1 / 2} / x_{\text {tot }}$, where $x_{\text {tot }}$ is the total number of moles per cc. The values of $D_{j}$ are input parameters, and may be different for each species $i$. The exponents of $T$ and $X_{\text {tot }}$ also may be altered in the input, but were not for these calculations. An identical expression is used for the thermal conductivity, $\lambda$. In the first calculation we used the 
Table 1. Reactions and rate constants used for the flame constants. Rate constants are in the form $k=A T^{n} \exp \left(-E_{a} / R T\right)$ in units of $\mathrm{cm}^{3}$, moles and seconds. References for rate constants may be found in [4]. Reverse rates are calculated from equilibrium constants using JANAF thermodynamic data [11].

\begin{tabular}{|c|c|c|c|c|}
\hline No. & Reaction & $\log A$ & $n$ & $E_{\mathrm{a}}$, kcal \\
\hline 1 & $\mathrm{HCO}+\mathrm{OH}=\mathrm{CO}+\mathrm{H}_{2} \mathrm{O}$ & 14.0 & 0 & 0 \\
\hline 2 & $\mathrm{OH}+\mathrm{CO}=\mathrm{CO}_{2}+\mathrm{H}$ & 7.18 & 1.3 & -0.765 \\
\hline 3 & $\mathrm{H}+\mathrm{O}_{2}=\mathrm{OH}+\mathrm{O}$ & 14.34 & 0 & 16.79 \\
\hline 4 & $\mathrm{H}_{2}+\mathrm{O}=\mathrm{OH}+\mathrm{H}$ & 10.26 & 1 & 8.9 \\
\hline 5 & $\mathrm{O}+\mathrm{H}_{2} \mathrm{O}=\mathrm{OH}+\mathrm{OH}$ & 13.83 & 0 & 18.36 \\
\hline 6 & $\mathrm{H}+\mathrm{H}_{2} \mathrm{O}=\mathrm{OH}+\mathrm{H}_{2}$ & 13.97 & 0 & 20.37 \\
\hline 7 & $\mathrm{HCO}+\mathrm{M}=\mathrm{H}+\mathrm{CO}+\mathrm{M}$ & 14.16 & 0 & 19.0 \\
\hline 8 & $\mathrm{O}+\mathrm{H}+\mathrm{M}=\mathrm{OH}+\mathrm{M}$ & 16.00 & 0 & 0 \\
\hline 9 & $\mathrm{O}+\mathrm{O}+\mathrm{M}=\mathrm{O}_{2}+\mathrm{M}$ & 15.70 & .0 .25 & 0 \\
\hline 10 & $\mathrm{H}_{2}+\mathrm{M}=\mathrm{H}+\mathrm{H}+\mathrm{M}$ & 14.34 & 0 & 96.0 \\
\hline 11 & $\mathrm{O}_{2}+\mathrm{H}_{2}=\mathrm{OH}+\mathrm{OH}$ & 14.90 & 0 & 44.7 \\
\hline 12 & $\mathrm{H}_{2}^{2} \mathrm{O}+\mathrm{M}=\mathrm{H}+\mathrm{OH}+\mathrm{M}$ & 16.34 & 0 & 105.1 \\
\hline 13 & $\mathrm{CO}+\mathrm{O}+\mathrm{M}=\mathrm{CO}_{2}+\mathrm{M}$ & 15.77 & 0 & 4.10 \\
\hline 14 & $\mathrm{CO}+\mathrm{O}_{2}=\mathrm{CO}_{2}+\mathrm{O}$ & 11.50 & 0 & 37.6 \\
\hline 15 & $\mathrm{HCO}+\mathrm{H}=\mathrm{CO}^{2}+\mathrm{H}_{2}$ & 14.30 & 0 & 0 \\
\hline 16 & $\mathrm{HCO}+\mathrm{O}=\mathrm{CO}+\mathrm{OH}$ & 14.00 & 0 & 0 \\
\hline 17 & $\mathrm{H}+\mathrm{O}_{2}+\mathrm{M}=\mathrm{HO}_{2}+\mathrm{M}$ & 15.22 & 0 & -1.00 \\
\hline 18 & $\mathrm{O}+\mathrm{OH}+\mathrm{M}=\mathrm{HO}_{2}+\mathrm{M}$ & 17.00 & 0 & 0 \\
\hline 19 & $\mathrm{HO}_{2}+\mathrm{O}=\mathrm{O}_{2}+\mathrm{OH}$ & 13.70 & 0 & 1.0 \\
\hline 20 & $\mathrm{HCO}+\mathrm{O}_{2}=\mathrm{CO}+\mathrm{HO}_{2}$ & 12.52 & 0 & 7.00 \\
\hline 21 & $\mathrm{H}+\mathrm{HO}_{2}^{2}=\mathrm{OH}+\mathrm{OH}^{2}$ & 14.40 & 0 & 1.90 \\
\hline 22 & $\mathrm{H}+\mathrm{HO}_{2}^{2}=\mathrm{H}_{2}+\mathrm{O}_{2}$ & 13.40 & 0 & 0.70 \\
\hline 23 & $\mathrm{OH}+\mathrm{HO}_{2}=\mathrm{H}_{2} \mathrm{O}+\mathrm{O}_{2}$ & 13.70 & 0 & 1.00 \\
\hline 24 & $\mathrm{HO}_{2}+\mathrm{HO}_{2}=\mathrm{H}_{2} \mathrm{O}_{2}+\mathrm{O}_{2}$ & 13.00 & 0 & 1.00 \\
\hline 25 & $\mathrm{H}_{2} \mathrm{O}_{2}+\mathrm{M}=\mathrm{OH}+\mathrm{OH}+\mathrm{M}$ & 17.08 & 0 & 45.50 \\
\hline 26 & $\mathrm{H}+\mathrm{H}_{2} \mathrm{O}_{2}=\mathrm{HO}_{2}+\mathrm{H}_{2}$ & 12.23 & 0 & 3.78 \\
\hline 27 & $\mathrm{CH}_{4}+\mathrm{M}=\mathrm{CH}_{3}+\mathrm{H}+\mathrm{M}$ & $17.3 \mathrm{C}$ & 0 & 88.40 \\
\hline 28 & $\mathrm{CH}_{4}+\mathrm{H}=\mathrm{CH}_{1}+\mathrm{H}_{2}$ & 14.10 & 0 & 11.90 \\
\hline 29 & $\mathrm{CH}_{4}+\mathrm{OH}=\mathrm{CH}_{3}+\mathrm{H}_{2} \mathrm{O}$ & 12.50 & 0 & 3.77 \\
\hline 30 & $\mathrm{CH}_{4}+\mathrm{O}=\mathrm{CH}_{3}+\mathrm{OH}^{2}$ & 13.30 & 0 & 9.20 \\
\hline 31 & $\mathrm{CH}_{2} \mathrm{O}+\mathrm{M}=\mathrm{HCO}+\mathrm{H}+\mathrm{M}$ & 16.70 & 0 & 72.00 \\
\hline 32 & $\mathrm{CH}_{2} \mathrm{O}+\mathrm{OH}=\mathrm{HCO}+\mathrm{H}_{2} \mathrm{O}$ & 14.70 & 0 & 6.30 \\
\hline 33 & $\mathrm{CH}_{2} \mathrm{O}+\mathrm{H}=\mathrm{HCO}+\mathrm{H}_{2}$ & 13.10 & 0 & 3.76 \\
\hline 34 & $\mathrm{CH}_{2} \mathrm{O}+\mathrm{O}=\mathrm{HCO}+\mathrm{OH}$ & 13.70 & 0 & 4.60 \\
\hline 35 & $\mathrm{CH}_{3}+\mathrm{OH}=\mathrm{CH}_{2} \mathrm{O}+\mathrm{H}_{2}$ & 12.60 & 0 & 0.00 \\
\hline 36 & $\mathrm{CH}_{3}+\mathrm{O}=\mathrm{CH}_{2} \mathrm{O}+\mathrm{H}$ & 14.10 & 0 & 2.00 \\
\hline 37 & $\mathrm{CH}_{3}+\mathrm{O}_{2}=\mathrm{CH}_{3} \mathrm{O}+\mathrm{O}$ & 13.40 & 0 & 29.00 \\
\hline 38 & $\mathrm{CH}_{2} \mathrm{O}+\mathrm{CH}_{3}=\mathrm{HCO}+\mathrm{CH}_{4}$ & 10.00 & 0.5 & 6.00 \\
\hline 39 & $\mathrm{HCO}+\mathrm{CH}_{3}=\mathrm{CH}_{4}+\mathrm{CO}$ & 11.50 & 0.5 & 0.00 \\
\hline 40 & $\mathrm{CH}_{3} \mathrm{O}+\mathrm{M}=\mathrm{CH}_{2} \mathrm{O}+\mathrm{H}+\mathrm{M}$ & 13.70 & 0 & 21.00 \\
\hline 41 & $\mathrm{HCO}+\mathrm{HO}_{2}=\mathrm{CH}_{2} \mathrm{O}+\mathrm{O}_{2}$ & 14.00 & 0 & 3.00 \\
\hline 42 & $\mathrm{CH}_{3} \mathrm{O}+\mathrm{O}_{2}^{2}=\mathrm{CH}, \mathrm{O}+\mathrm{HO}_{2}^{2}$ & 12.00 & 0 & 6.00 \\
\hline 43 & $\mathrm{CH}_{3}+\mathrm{HO}_{2}=\mathrm{CH}_{4}+\mathrm{O}_{2}$ & 12.00 & 0 & 0.40 \\
\hline 44 & $\mathrm{CH}_{4}+\mathrm{HO}_{2}=\mathrm{CH}_{3}+\mathrm{H}_{2} \mathrm{O}_{2}$ & 13.30 & 0 & 18.00 \\
\hline 45 & $\mathrm{CH}_{2} \mathrm{O}+\mathrm{HO}^{2}$ & 12.00 & 0 & 8.00 \\
\hline
\end{tabular}


conventional assumption of unity Lewis number, with all $d_{j}=4.6 \times 10^{-7}$ (in units involving centimeters, moles, seconds and Kelvins) [7].

To be rigorous we would need binary diffusion coefficients for each species diffusing through every other species. Calculation of these coefficients is a considerable task. We elected, instead, to use a simpler prescription initially and perform a sensitivity analysis. Calculations showed that the results were relatively insensitive to the values of the species diffusion coefficients.

\subsection{Calculation of the nominal case}

The first calculation gave a flame thickness in good agreement with experiment, as shown by the temperature profile in figure 4. (Because we are calculating a propagating flame, there is freedom in matching the calculated position with the experimental. The two were matched by arbitrarily making the temperature curves approximately coincide near 1500 K.) The profiles for reactants and products were in reasonable agreement as shown in figure 5. The small displacement in position indicates that the calculated reaction occurs at a somewhat higher temperature than the experiment, but the matching slopes indicate agreement of the reaction rates. Some of the radical concentration profiles, such as $\mathrm{CH}_{3}, 0$, and $\mathrm{OH}$ showed good to fair agreement with experiment as shown in figures 68. Others such as $\mathrm{H}, \mathrm{H}_{2}$, and $\mathrm{CH}_{2} \mathrm{O}$ showed agreement in general shape, but the calculated concentrations were about a factor of two low. Some species such as $\mathrm{C}_{2} \mathrm{H}_{6}$ and $\mathrm{CH}_{3} \mathrm{OH}$ were observed experimentally in trace amounts, but no reactions for their creation were included in the reaction mechanism. This was not considered to be a significant omission, but should be checked with further calculations. One group of experimenters observed a signal in their mass spectrometer which they believed to be $\mathrm{CH}_{3} \mathrm{O}_{2}$ [1]. One would expect this species to be created by the reaction

$$
\mathrm{CH}_{3}+\mathrm{O}_{2}+\mathrm{M} \rightarrow \mathrm{CH}_{3} \mathrm{O}_{2}+\mathrm{M}
$$

with further decomposition to $\mathrm{CH}_{3} \mathrm{O}$ and $\mathrm{O}$. The species, $\mathrm{CH}_{3} \mathrm{O}_{2}$ was not included in our reaction mechanism, but $\mathrm{CH}_{3} \mathrm{O}$ was, so its calculated concentration was compared with the experimental curve for $\mathrm{CH}_{3} \mathrm{O}_{2}$. The calculated curve was an order of magnitude low and occurred at higher temperature. Since the reactions involving $\mathrm{CH}_{3} \mathrm{O}$ did not play a major role, this was not considered a serious error. However, this is worthy of further study, assuming that the experimental identification of $\mathrm{CH}_{3} \mathrm{O}_{2}$ is correct. The calculated curve for $\mathrm{HO}_{2}$ is also $10 \mathrm{~W}$ and peaks at a lower temperature than the experimental curve. None of the variations done later had a substantial effect on this. It is likely that we omitted a reaction involving $\mathrm{HO}_{2}$, which is not surprising considering the lack of knowledge of $\mathrm{HO}_{2}$ chemistry. The reactions involving $\mathrm{HO}_{2}$ do play a significant role in methane oxidation, and while the discrepancy is not crucial, it is more significant than the other radicals just mentioned. 


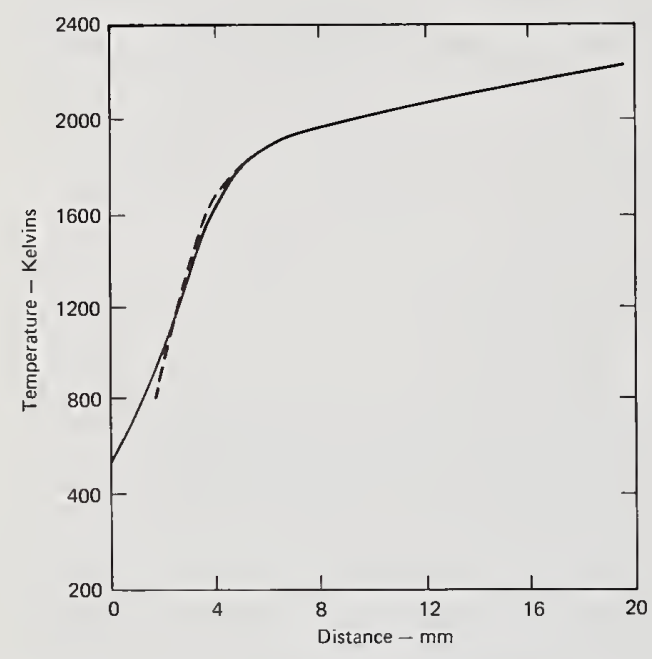

Figure 4. Comparison of calculated (solid line) temperature profile with experiment [1] (dashed line), for a 1/40 atmosphere methane-oxygen flame. Nominal values of initial parameters were used.

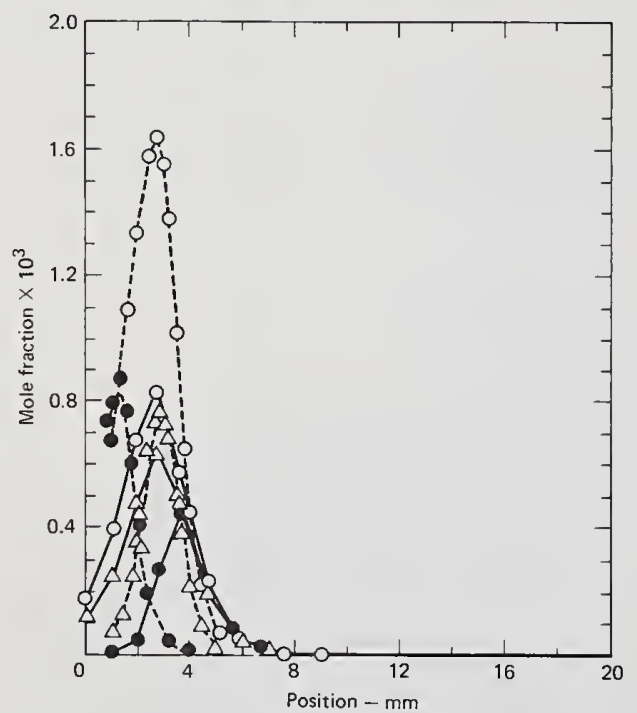

Figure 6. Comparison of calculated (solid Figure 7. Comparison of calculated (solid lines) and experimental [1] (dashed lines) species mole fraction profiles for $\mathrm{CH}_{2} \mathrm{O}$ (o) and $0.5 \times \mathrm{CH}_{3}(\Delta)$. Also shown is the experimental profile for $100 \mathrm{CH}_{3} \mathrm{O}_{2}$ (O) and the calculated profile for $100 \times \mathrm{CH}_{3} \mathrm{O}(\boldsymbol{e})$, as discussed above.

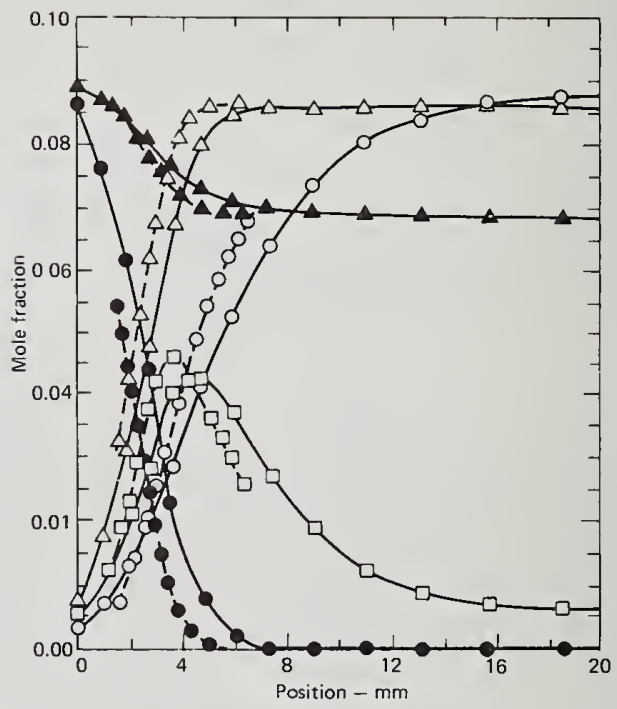

Figure 5. Comparison of calculated (solid lines) and experimental [1] (dashed lines) species mole fraction profiles for $\mathrm{CH}_{4}(\mathbf{O})$, $\mathrm{H}_{2} \mathrm{O}(\Delta), 0.1 \times \mathrm{O}_{2}(\Delta), \mathrm{O}(\mathrm{E})$, and $\mathrm{CO}_{2}(\mathrm{O})$.

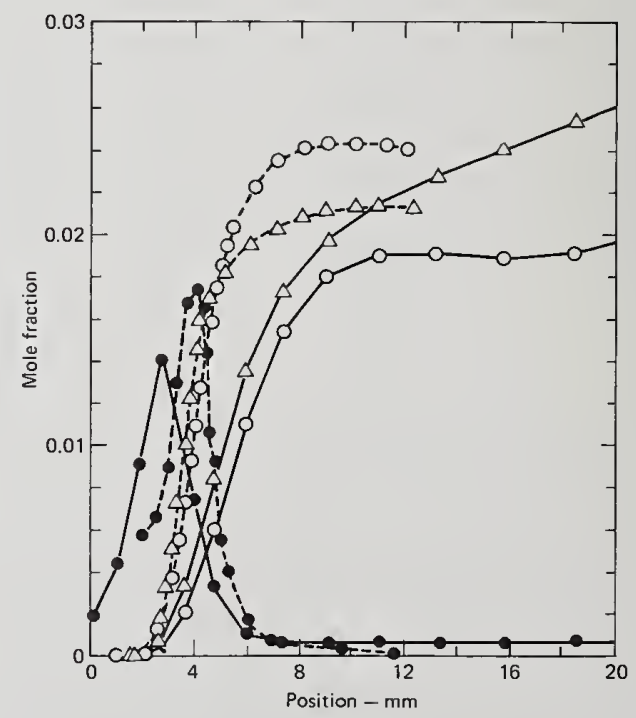




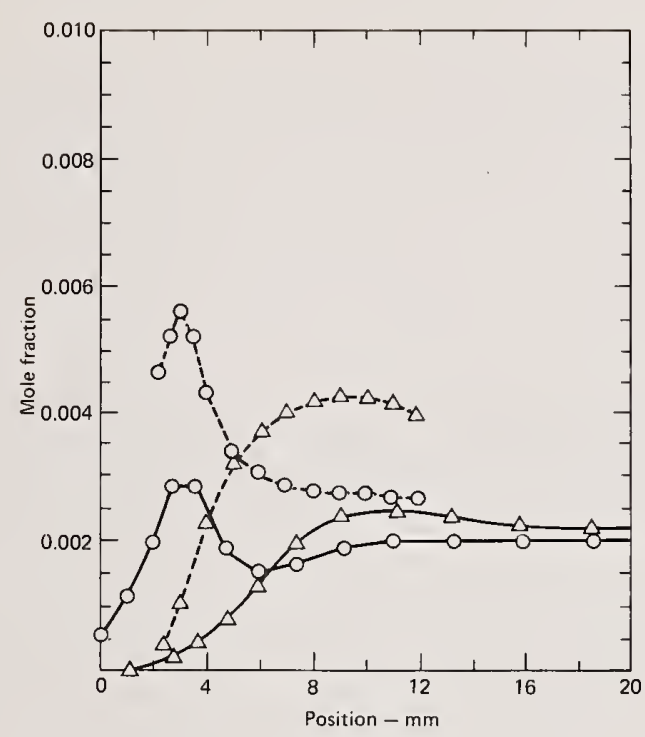

Figure 8. Comparison of calculated (solid lines) and experimental [1] (dashed lines) species mole fraction profiles for $\mathrm{H}_{2}(0)$ and $\mathrm{H}(\Delta)$, using nominal input parameters.

The most serious disagreement between the calculation and experiment was the calculated flame velocity of $30 \mathrm{~cm} / \mathrm{s}$ compared to the experimental $67 \mathrm{~cm} / \mathrm{s}$. Input parameters were varied in a number of calculations in an attempt to raise the calculated flame velocity. Many of the variations were motivated by dimensional arguments regarding flame speed and thickness. It can be shown that [8]

$$
v_{0}=\left(1 / \rho_{0}\right) \sqrt{w\left(\lambda / C_{p}\right)}
$$

and

$$
\delta=\sqrt{\lambda\left(C_{p} w\right)}
$$

where $v_{0}$ is the laminar flame velocity; $\delta$ is the flame thickness; $w$ is the mass of reactant mixture reacting per unit time in a unit volume; $\lambda$ is the thermal conductivity; $C_{p}$ is the specific heat at constant pressure; and $\rho_{0}$ is the density of the unburned reactant mixture. The density, $\rho_{0}$, and the specific heat are accurately known, so we are only free to vary the thermal conductivity and the reaction rate. Neither the velocity nor the thickness of the flame depend explicitly on the species diffusion coefficients. The reaction rate depends explicitly on the rate constants, but also depends on species concentrations which may depend in turn on diffusion coefficients. 


\subsection{Variation of thermal conductivity}

In the first attempt to get better agreement for the flame velocity the thermal conductivity was increased a factor of four, with species diffusivities remaining at the nominal value. (This is not physically realistic; and, since it changes the Lewis number, it also destroys the similarity of the solutions. Nevertheless, it allows us to separate two effects.) Both the flame velocity and thickness increased as expected, but the velocity did not quite double as expected. Examination of the computer results showed that the reaction rate had been reduced, apparently due to lower temperatures caused by increased thermal conduction. The better agreement obtained using the nominal value of the thermal conductivity indicates that the nominal value is approximately correct.

\subsection{Variation of species diffusivities}

Although species diffusivities do not appear explicitly in the above expressions for flame velocity and thickness, they were altered to determine indirect effects and to determine the sensitivity of the species profiles to the diffusion coefficients. In the first variation all diffusion coefficients had the same value, but four times nominal. (The thermal conductivity was reset to the nominal value.) There was a small but significant increase in the flame velocity, and the flame thickness was correct, but agreement of calculated and experimental radical profiles was worse. Examination of the computer results showed that the increased velocity was due to a faster burning rate. In the second variation all species diffusivities were reset to the nominal value, except those for $\mathrm{H}$ and $\mathrm{H}_{2}$, which were four times nominal. This approximates physical reality since the coefficients should be inversely proportional to the square root of the molecular weights. The results of the calculation were quite similar to the original, nominal, case. On the basis of these calculations we concluded that the sensitivity of the flame velocity and structure to variations in species diffusion coefficients is fairly small.

\subsection{Variation of initial temperature}

Calculations with elevated initial temperatures showed that the flame velocity increased rapidly with increasing initial temperature. An initial temperature of nearly $500 \mathrm{~K}$ would give a correct flame velocity while leaving the thickness and species profiles essentially unchanged. Smoot [9] obtained similar results. This agreement with experiment seemed artificial to us. The experiment used a water-cooled burner and it is difficult to imagine an initial gas temperature much above the boiling point of water, $373 \mathrm{~K}$; so we proceeded to study the effects of changing chemical rate constants. 


\subsection{Variation of chemical rate constants}

With 45 chemical rate constants it was necessary to alter them in a strategic order. A total of eleven rate constants were altered, the remaining ones were not changed because their contribution to the overall reaction rate was judged to be insignificant.

The first change was to eliminate reaction 11 ,

$$
\mathrm{H}_{2}+\mathrm{O}_{2} \rightarrow 2 \mathrm{OH} \text {, }
$$

by setting the rate constant effectively to zero. This was done because the reaction was judged to be an improbable four-center reaction. Eliminating the reaction had no significant effect on the calculation, and the rate constant remained low for all succeeding calculations.

The reactions most likely to affect the overall reaction rate are the chain branching reactions,

$$
\begin{array}{r}
\mathrm{H}+\mathrm{O}_{2} \rightarrow \mathrm{O}+\mathrm{OH} \\
\mathrm{CH}_{4}+\mathrm{O} \rightarrow \mathrm{CH}_{3}+\mathrm{OH}
\end{array}
$$

Surprisingly, changing these rate constants did not affect the flame velocity or overall reaction rate, although changing the rate constants did change the fuel consumption rate in flow reactor calculations. We do not yet understand the lack of sensitivity in the flame calculations, but the major difference is the large radical concentrations due to diffusion in the flame.

The rate constant for the reaction

$$
\mathrm{CH}_{3}+\mathrm{O} \rightarrow \mathrm{CH}_{2} \mathrm{O}+\mathrm{H}
$$

was the next one changed. This is a reaction between two radicals and is expected to have a significant effect on the flame velocity. The rate constants of the remaining major reactions were then varied. The reactions are:

$$
\begin{array}{ll}
\mathrm{CH}_{4}+\mathrm{H} & \rightarrow \mathrm{CH}_{3}+\mathrm{H}_{2} \\
\mathrm{CH}_{4}+\mathrm{OH} & \rightarrow \mathrm{CH}_{3}+\mathrm{H}_{2} \mathrm{O} \\
\mathrm{HCO}+\mathrm{OH} & \rightarrow \mathrm{CO}+\mathrm{H}_{2} \mathrm{O} \\
\mathrm{H}+\mathrm{H}_{2} \mathrm{O} & \rightarrow \mathrm{OH}+\mathrm{H}_{2} \\
\mathrm{HCO}+\mathrm{M} & \rightarrow \mathrm{H}+\mathrm{CO}^{2} \mathrm{M} \\
\mathrm{CH}_{2} \mathrm{O}+\mathrm{OH} & \rightarrow \mathrm{HCO}+\mathrm{H}_{2} \mathrm{O} \\
\mathrm{HCO}+\mathrm{O}_{2} & \rightarrow \mathrm{CO}+\mathrm{HO}_{2}
\end{array}
$$


Of these, only reactions 7 and 20 had a significant effect on the flame velocity. When the rate constant for reaction 7 was increased four times the flame velocity increased 40 percent. Examination of the computer results showed that the increase was due to a doubling of the overall reaction rate. The 40 percent increase in velocity is consistent with this. Further calculations showed that the flame velocity and overall reaction rate depend on the ratio of the rate constants for reactions 7 and 20 , rather than the absolute values. These reactions were also important in determining the overall reaction rate in flow reactor calculations.

Quadrupling the rate constant of reaction 7 approximately doubled the concentration of $H$, giving much better agreement with experiment as shown in figure 9 . We took this as evidence that the appropriate way to get the correct calculated flame velocity is to adjust the rate constants of reactions 7 and 20 . There is considerable uncertainty about the value of both rate constants. The nominal value for reaction 20 was obtained by fitting the rate of consumption in calculations of the turbulent flow reactor [4]. The nominal value for reaction 7 was double the recommended value given by Benson and 0 'Neal [10], but their value is an estimate. With appropriate values of the rate constants for reactions 7 and 20 and a temperature a little below the boiling point of water we should be able to simultaneously match the experimental flame velocity and thickness, as well as the more important radical profiles. Unfortunately, there was insufficient time to do this calculation.

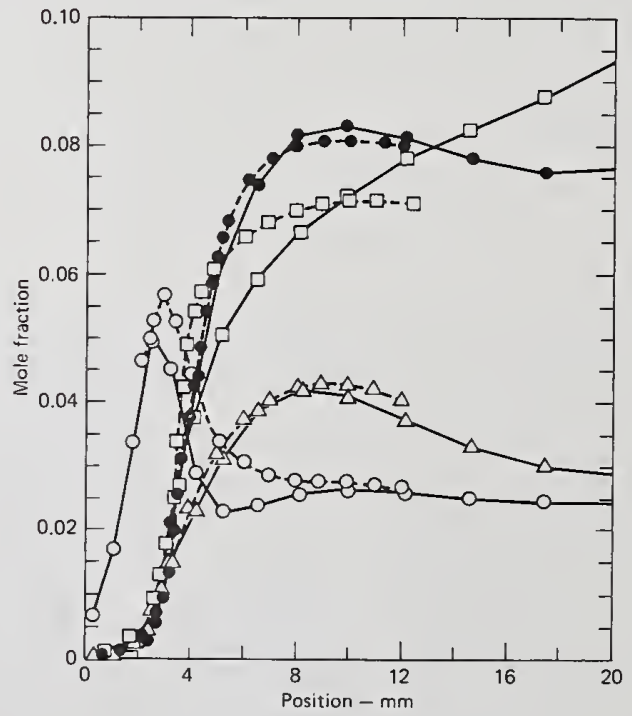

Figure 9. Comparison of calculated (solid lines) and experimental [1] (dashed lines) species mole fraction profiles for $\mathrm{H}_{2}(0), H(\Delta)$, $\mathrm{OH}(\square)$, and $\mathrm{O}(\mathbf{O})$, with reaction rate constant 7 set to four times the nominal value, and all other input variables at nominal value. 
It is clear that the doubled overall reaction rate is due to the doubled $\mathrm{H}$ atom concentration. This then doubles the rate of reaction 3 , and the entire chain of reactions oxidizing $\mathrm{CH}_{4}$ to $\mathrm{CO}$. The $\mathrm{H}$ atom concentration is increased because a larger fraction of the hydrogen in $\mathrm{HCO}$ is routed into the chain branching path, rather than being diverted to $\mathrm{HO}_{2}$. The reactions of $\mathrm{HO}_{2}$ transform some of it to other radicals, but they also result in a net reduction of the radical pool. This explanation is not completely satisfying but represents our current understanding.

In summary, some of the results of varying input parameters were expected, but others were somewhat surprising. The flame velocity and thickness both increased with increased thermal conductivity, as expected from dimensional arguments. Large changes in species diffusion coefficients had little effect on flame velocity and thickness. The effect on species profiles was larger, but not as large as one might expect. Species diffusion coefficients do not appear explicitly in the dimensional arguments, so this lack of sensitivity is not too surprising. The lack of sensitivity to most reaction rate constants was surprising. The flame was insensitive to several rate constants that controlled the methane consumption rate in the flow reactor. This insensitivity appears to be related to diffusion of radicals, but is not yet clearly understood. Nor do we entirely understand why the ratio of rate constants for $\mathrm{HCO}$ consumption has such a large effect.

\section{Discussion}

\section{1 Diffusion of radicals eliminates the induction period}

Examination of the computer results shows that there is no induction period in a laminar pre-mixed flame. Radicals from the flame and post-flame regions diffuse ahead of the flame creating radical concentrations generally in excess of the steady-state value. This is readily seen in figure 10 , which shows the contributions of chemistry, diffusion, and expansion to the rate of change in $\mathrm{CH}_{3}$ concentration. The net rate of change is also shown. For reference, the temperature, concentration, and mole fraction are also plotted to an arbitrary scale. (Note that the chemical rate is itself a net rate: the algebraic sum of all of the chemical reaction rates for that species. Some of the individual rates represent creation and others consumption; since these tend to cancel, the net chemical rate is usually less than the larger individual rates.) Ahead of the flame, diffusion is the main source of radicals and figure 10 shows that most of these radicals are being consumed by the chemistry, but with a small net increase in radical concentration. Even far ahead of the flame, where diffusion is too small to be seen in figure 10 , radicals arrive by diffusion and are consumed by the chemistry. Because radicals are furnished by diffusion, the schematic diagram of the reaction is nearly identical to that of figure 2, and not at all like the induction reactions of figure 1. Diffusion provides radical concentrations large enough for radical-radical reactions to occur. 


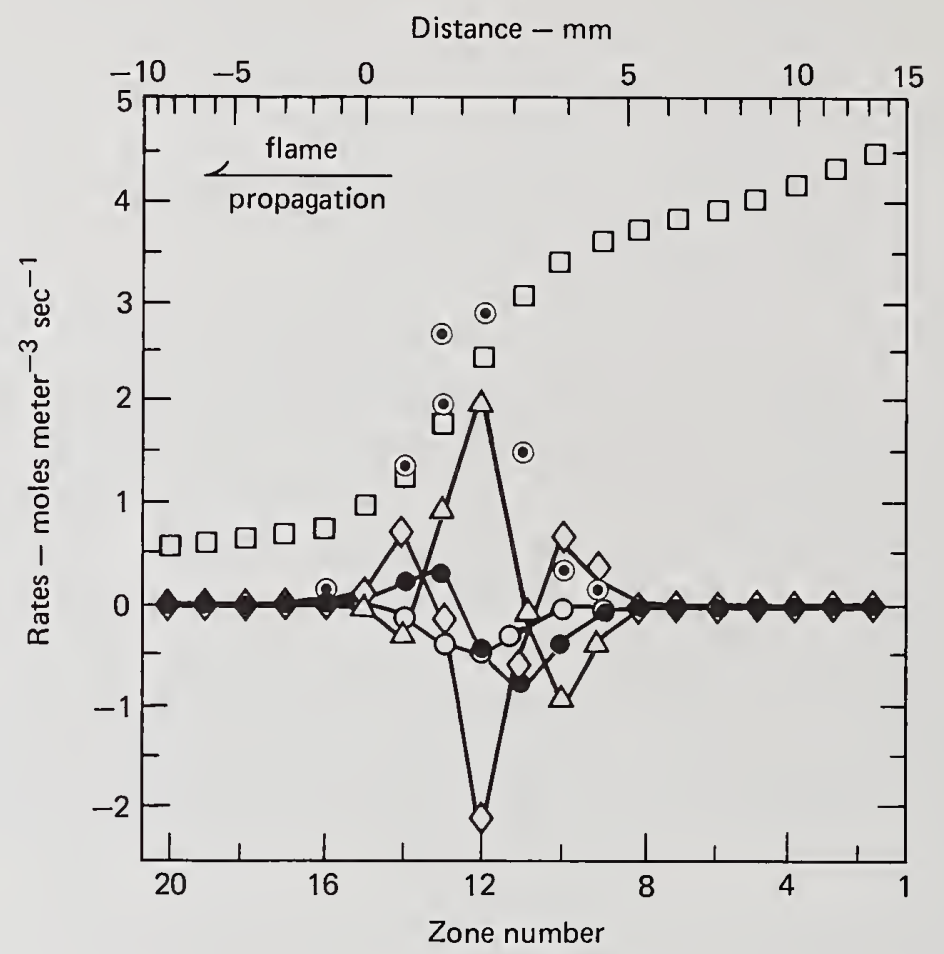

Figure 10. Contributions of various rates to the change in $\mathrm{CH}_{3}$ concentration in each zone. A distance scale is shown at the top for comparison. The net rate $(O)$ is the algebraic sum of rates due to chemical reaction $(\Delta)$, diffusion $(\diamond)$ and hydrodynamic expansion $(0)$. For reference, the species mole fraction $(\nabla)$ and concentration $(\odot)$, in $\mathrm{mol} / \mathrm{m}^{3}$, are plotted to arbitrary vertical scale as is the temperature $(\square)$.

The source of the radicals is clear. It is well known that the concentration of $\mathrm{OH}$ is well above equilibrium for the temperature in the post-flame region. These high concentrations are due to the chain reaction which oxidizes $\mathrm{CO}$ to $\mathrm{CO}_{2}$, and represents the steady-state concentration required by this reaction [3]. In the absence of diffusion the $\mathrm{OH}$ concentration in the fuel consumption zone would be several orders of magnitude lower because the $\mathrm{OH}$ reacts much faster with hydrocarbons (or any $\mathrm{CH}$ bond) than with $\mathrm{CO}$. In the flame the level of $\mathrm{OH}$ is high throughout the fuel consumption region because of diffusion, as shown in figure 11. The same thing applies to the 0 atom concentration, and the rate curve is nearly identical to figure 11. The rates for $\mathrm{H}$ atom are similar, but an order of magnitude lower because of the lower steady-state concentration. 
Comparison of figures 10 and 11 show that the curves for $\mathrm{OH}$ and $\mathrm{CH}_{3}$ are quite different. In particular, chemical consumption of $\mathrm{OH}$ peaks in the zone where chemical creation of $\mathrm{CH}_{3}$ is maximum. This is to be expected because it is the reactions of $\mathrm{OH}, \mathrm{O}$ and $\mathrm{H}$ with $\mathrm{CH}_{4}$ that creates $\mathrm{CH}_{3}$. The curves for $\mathrm{CH}_{2} \mathrm{O}$ and $\mathrm{HCO}$ are similar to $\mathrm{CH}_{3}$ for the same reasons.

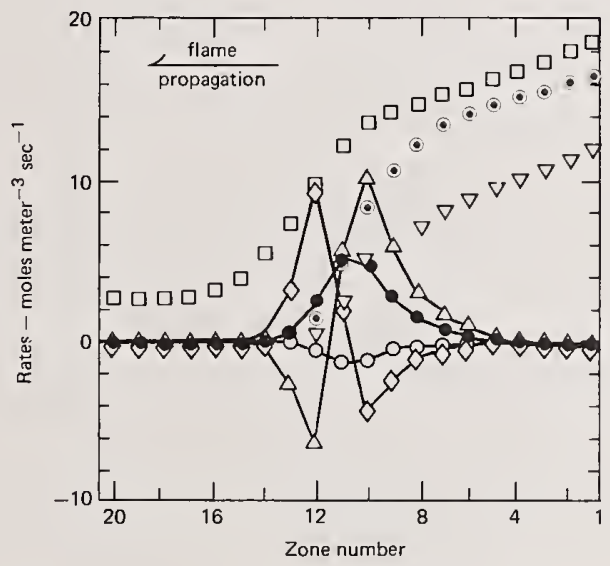

Figure 11. Contributions of various rates to the change in $\mathrm{OH}$ concentration in each zone. The net rate $(\bullet)$ is the algebraic sum of rates due to chemical reaction $(\Delta)$, diffusion $(\diamond)$ and hydrodynamic expansion $(0)$. For reference, the species mole fraction $(\nabla)$ and concentration $(\odot)$, in $\mathrm{mol} / \mathrm{m}^{3}$, are plotted to arbitrary vertical scale as is the temperature ( $\square$ ).

\subsection{Diffusion and chemical rates are opposed}

Examination of figures 10 and 11 reveals another important phenomenon in the 1 aminar, pre-mixed flame. The diffusion and chemical rates are in the opposite direction for all species, except for brief intervals when the curves are crossing. A few minutes thought shows that this is an essential feature. Consider a system where one species initially has a spatially uniform concentration. Because there is no concentration gradient, there is no diffusion. If we raise the rate of creation due to chemistry at one point, the concentration will rise, creating a gradient, and result in diffusing the species away from that location. The reverse will occur if the chemistry begins to consume the species. Even if we were to start with a non-uniform distribution, the chemistry and diffusion would pull in opposite directions to try to minimize the non-uniformity. The effect of the two opposing rates is a net rate of creation or consumption which is some fraction of the smaller rate, but the two rates do not entirely cancel. The opposition of chemistry and diffusion will be seen in all laminar, pre-mixed flames and is not a peculiarity of the methane/oxygen system.

The close coupling between diffusion and chemistry limits the degree to which we can simplify our mental pictures of a laminar, pre-mixed flame. We cannot consider the chemistry while neglecting diffusion; we have already seen, for example, that diffusion eliminates 
the induction period. We also have seen that the rate of diffusion depends on the gradients created by the chemical reaction rate. This has mathematical implications as well. We could calculate the chemistry in the turbulent flow reactor by calculating the time history of the reaction in a moving volume element, neglecting the interaction with other elements. The close-coupling of diffusion and chemistry in the flame means that we must consider the chemistry in additional elements downstream. This converts the problem from an initial value problem to a boundary value problem, which is generally harder to solve in closed form. This reduces the likelihood of finding simple, closed form, models similar to that for the induction period in a shock tube.

The intimate coupling of diffusion and chemistry also raises questions about numerical operator-splitting techniques. If the chemical and diffusion operators are split, we may introduce large errors.

\section{3 Flame propagation also depends on thermal conduction}

The diffusion of radicals has been discussed at length, but thermal conduction appears to be equally important. The influence of the thermal conductivity on flame velocity is clear evidence of its importance. Figure 12 shows the contributions of thermal conduction and chemical reactions to the generation of heat. Ahead of the flame the gas is heated primarily by thermal conduction, although the chemical reactions of diffused radicals provide some heat. Examination of the computer printouts shows that about a third of the fuel is consumed and that the temperature has risen several hundred Kelvins before the chemical release of heat becomes dominant. As the chemistry becomes dominant heat is conducted out of those zones to cooler zones, as indicated by the negative rates of thermal conduction shown in figure 12 . Chemical heat release is large during both the methane consumption and $\mathrm{CO}$ oxidation phases of the reaction. Hydrodynamic expansion of the gas results in a small amount of cooling, as can be seen by examining figure 12.

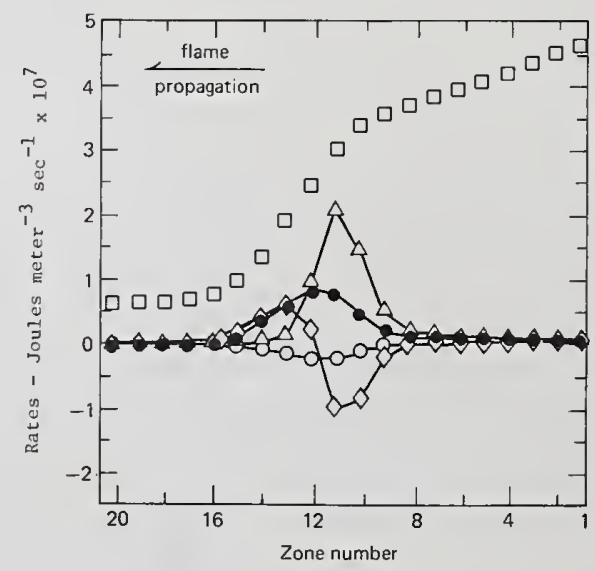

Figure 12. Contributions of thermal conduction $(\diamond)$ chemical reaction $(\Delta)$ and hydrodynamic expansion (0) rates to the net heat release rate (0) in each zone. The temperature $(\square)$ also is plotted with an arbitrary vertical scale. 
It is difficult to separate the effects of thermal conduction from chemistry because they are coupled. However, there is evidence which indicates how thermal conduction affects flame propagation.

In the flow reactor or shock tube, the radical concentration reached a plateau, and the reaction rate, which was proportional to the concentration, increased rapidly with temperature. A similar phenomenon may occur in front of the flame. Presumably, the radicals which diffuse ahead of the $f 1$ ame are consumed so long as the radical concentration exceeds that of the plateau at the local temperature. As the temperature increases, the $\mathrm{plateau}$ concentration, and the reaction rate, rises until the chemistry produces heat and additional radicals at a rate which exceeds thermal conduction and species diffusion. Once that point is reached the reaction goes to completion, similar to a thermal explosion. This picture is consistent with the observed increase in flame velocity when the initial temperature is raised. Since less heating by conduction is required, the flame propagates faster. Additional calculations are required to justify or modify this picture. In any case, thermal conduction is an essential part of the flame propagation mechanism.

\section{Conclusion}

Some of the results of these calculations are specific to methane flames, but others appear to be more general. The species profiles, of course, are specific to methane oxidation, and show that the initial reaction mechanism is fairly good. The agreement was improved by changing the ratio of the rate constants for reactions 7 and 20 . Agreement between the calculated and experimental flame velocity improved, as did the species profiles for $\mathrm{H}_{2} \mathrm{H}_{2}, \mathrm{O}$, and $\mathrm{OH}$. On the other hand, some changes need to be made with respect to reactions involving $\mathrm{HO}_{2}$, as well as the reactions involving $\mathrm{CH}_{3} \mathrm{O}_{2}$ and $\mathrm{CH}_{3} \mathrm{O}$. The reaction mechanism would also be improved by adding the formation and subsequent oxidation of $\mathrm{C}_{2} \mathrm{H}_{6}$, as well as $\mathrm{CH}_{3} \mathrm{OH}$. The lack of sensitivity of the overall reaction rate to changes in most reaction rate constants was surprising, and is not well understood. This may be true also of other hydrocarbon flames, but the required calculations have not been done.

The relationship between diffusion and chemistry seems to be general, and should apply to all hydrocarbon/oxygen flames. The fact that chemical and diffusion rates are generally opposed was not unknown, but has not received much emphasis. This opposition derives from the basic nature of the diffusion process, and should be common to all flames. Similarly, we would not expect to see induction chemistry in any hydrocarbon/oxygen flame. Al1 hydrocarbons oxidize first to $\mathrm{CO}$, and large steady-state concentrations of $\mathrm{OH}$ and $\mathrm{O}$ are required to oxidize the $\mathrm{CO}$ to $\mathrm{CO}_{2}$. Diffusion of $\mathrm{OH}$ and 0 into the pre-flame region will eliminate induction chemistry. The relative insensitivity of the overall reaction rate to changes in the species diffusion coefficients also may be a general feature of hydrocarbon flames, but we cannot be sure until we understand the insensitivity better, or until we have done calculations for other hydrocarbons. 
The increase of flame velocity with increased thermal conductivity was known beforehand to be a general feature of flames. So was the increase in flame velocity with increased initial temperature. Calculations of the chemistry in shock tubes and flow reactors has given us some insight into the mechanism of flame propagation, but we do not have the complete picture yet.

In short, the work reported here has given us some insight into the physical and chemical mechanism governing flame propagation, but it has also raised several new questions.

This work is performed under the auspices of the United States Department of Energy under Contract No. W7405-Eng-48.

\section{References}

[1] Peeters, J. and Mahnen, G., Fourteenth Symposium (Internationa1) on Combustion, The Combustion Institute, Pittsburgh, PA, p. 133 (1973).

[2] Biordi, J. C., Lazzara, C. P., and Papp, J. F., Fifteenth Symposium (Internationa1) on Combustion, The Combustion Institute, Pittsburgh, PA, p. 917 (1974).

[3] Creighton, J. R., J. Phys. Chem. 81, 2520 (1977).

[4] Westbrook, C. K., Creighton, J., Lund, C., and Dryer, F. L., J. Phys. Chem. 81, 2542, (1977).

[5] Lund, C. M., Univ. of California, Lawrence Livermore Lab. Report UCRL-52504. Inquiries regarding the code should be addressed to $\mathrm{Dr}$. Lund.

[6] Hindmarsh, A. C., Univ. of California, Lawrence Livermore Lab. Report UCID-30150 (1977).

[7] Handbook of Tables for Applied Engineering Science (The Chemical Rubber Co., Cleveland, $\mathrm{OH}, 1970)$, p. 29.

[8] Williams, F. A., Combustion Theory (Addison-Wesley, Reading, MA, 1963), p. 98.

[9] Smoot, L. D., Hecker, W. C., and Williams, G. A., Combustion and Flame, 26, 323 (1976).

[10] Benson, S. W. and O'Neal, H. E., Kinetic Data on Gas Phase Unimolecular Reactions, U.S. Govt. NSRDS-NBS21, Washington, D.C., p. 587 (1970).

[11] JANAF Thermochemical Tables, U.S. Govt. NSRDS-NBS37, Washington, D.C. (1971).

\section{Discussion}

Question (Tsang): Not being a modeler, I am often puzzled because the modelers trot out new models every year. Could you tell me some differences between your model and $\mathrm{Dr}$. Spaulding's version, or Dixon-Lewis' and so forth. 
Response (Creighton): I have some of the same problems you do figuring out what is different between the various models. Sometimes it isn't entirely explicit, and another problem is that if we have enough parameters we can fit a camel, if we vary them all.

I tried to be fairly explicit about what I have and haven't done. For example, Smoots model, I think his chemistry is somewhat different than mine, but he obtained good agreement on the flame velocity with the Biordi flame experiment because he varied the initial temperature. His initial temperature, like the one I would need, would have to be around 500 Kelvin to get agreement.

Question (Tsang): Have you tried running your model on simpler flame systems, like the hydrogen-bromine systems or hydrogen-oxygen systems? Wouldn't that be a simpler process, we know the kinetics of the hydrogen-oxygen, hydrogen-bromine systems much better, therefore you don't have the liberty of playing around with the rate constants as much, and the fluid dynamics would, of course, then stand out clearly in those systems. In those systems the older models do give you the proper flame velocities. I thought the problems for those simple systems were solved.

Response (Creighton): No, I haven't. It probably would be a good idea to do it. I don't have time within the constraints of my funding.

(Editor's note): The question of how this method relates to that of Spalding and others has been addressed in a recent report by C. M. Lund, "HCT - A general computer program for calculating time-dependent phenomena involving one-dimensional hydrodynamics, transport, and detailed chemical kinetics," UCRL-52504, Aug. 1978.

Question (Hastie): Unfortunately, I couldn't tell very well which were experimental and which were calculated curves in the slides. Did you show temperature profiles amongst those data? If so, did the calculated and experimental profiles agree?

Response (Creighton): Yes. The agreement there was very good.

Question (Hastie): I am a little unsure about how you incorporate heat losses to the burner. These are very non-adiabatic flames, and I don't know how you can match your temperature profiles with experimental ones without knowing something about the amount of heat loss to the burner.

Response (Creighton): That was ignored in the model and we start looking at temperatures out from the burner face. I think, his (Peeters) earliest reported temperatures are something like 800 Kelvin. So, I don't know what happens in close to the burner. I don't know what losses there are. 
Question (Hastie): These calculations: do you expect that you will have any difficulty in adapting them to one atmosphere conditions where the three body reactions will be much more important and knowledge of the chaperon efficiencies will be important?

Response (Creighton): I have the three body reactions in the model. I think the thing that bothered me most about adapting them to one atmosphere is that for this one-fortieth of an atmosphere system, the radical concentrations have been measured, but I can get results at one atmosphere. It is pretty easy usually to get agreement with the major species profiles, and I might be able to get agreement and be totally wrong. That bothers me.

I might have the radical concentrations wrong. I might have the wrong reactions at one atmosphere. One of my real concerns is, how do I validate this thing at one atmosphere when the radical concentrations aren't known.

(Editor's note): While it is true that radical concentration data are lacking for 1 atm hydrocarbon flames, there is sufficient data available for the recombination zone of $\mathrm{H}_{2}-\mathrm{O}_{2}-\mathrm{N}_{2}$ flames to test many aspects (but not all since pre-reaction zone data are lacking) of the model at atmospheric pressure; e.g. see Chapter 5 in High Temperature Vapors, J. W. Hastie (Academic Press), 1975.

Question (Lin): I wonder if you could say a few words about $\mathrm{CH}_{3}$ plus $0_{2}$ reactions which have been questioned recently by Dave Golden and coworkers.

Response (Creighton): The rate I have in there is the rate Brokaw used. I am familiar with Dave Golden's questions. Apparently this rate is just a little bit below the upper limit he sets for the reaction rate.

There may, in fact, be some other way that the carbon oxygen bond is formed, I am not sure. This one happens to fit a lot of reaction mechanisms, that is all you can say for it.

Comment (Fontijn): There is, of course, as much disagreement there. It isn't only Golden's work, there are other room temperature measurements that say that doesn't occur. It is a very important question, I think. In combustion, what does this particular reaction do? Several people I have talked to at the combustion symposium recently in Leeds plan to study it. We also plan to look at it.

Comment (Gardiner): I have some further comment regarding Wing Tsang's suggestion that modeling the hydrogen-oxygen or hydrogen-bromine flames might be useful in helping to understand the Creighton-Lund model.

I think this is actually not the case. The $\mathrm{H}_{2}-\mathrm{O}_{2}$ flame is quite different in character from the methane-oxygen flame studied by Creighton and Lund. 
In $\mathrm{H}_{2}-\mathrm{O}_{2}$, one has a rapid creation after the induction zone of a partial equilibrium mix of atoms and radicals, which then deliver the flame heat by recombination reactions. There is no hang up in essentially methyl radicals as in the case of the $\mathrm{CH}_{4}-\mathrm{O}_{2} \mathrm{flame}$, and the participation of transport occurs in a quite different way in those two flames.

Further, Tsang suggested that the $\mathrm{H}_{2}-\mathrm{O}_{2}$ chemistry is well known. Well some of it is, but perháps the critical part for a flame calculation is not. That would be the reactions of $\mathrm{HO}_{2}$ at low temperatures. Those rate constants are really not all that well known. 
National Bureau of Standards Special Publication 561, Proceedings of the 10th Materials Research Symposium on Characterization of High Temperature Vapors and Gases held at NBS, Gaithersburg, Maryland, September 18-22, 1978. Issued October 1979.

\title{
PREDICTIVE MODELING OF DIBORANE OXIDATION
}

\author{
W. M. Shaub and M. C. Lin \\ Chemistry Division \\ Naval Research Laboratory \\ Washington, DC 20375
}

\begin{abstract}
A predictive mechanism for oxidation of diborane has been developed and compared with some experimental data. The objective of this work is to assess the feasibility of using the predictive modeling approach to theoretically acquiring diagnostics useful for understanding some aspects of alternate fuel combustion systems. The limitations of the method and nature of the information which one may hope to extrapolate from such studies are discussed. It is recommended that this approach be further investigated, as it may prove useful in determining and subsequently characterizing some physical phenomena (e.g., induction period, detonations, product distributions) which under some circumstances may be relatively insensitive to many of the precise details of reactive channels and their associated rate parameters.
\end{abstract}

\section{Introduction}

In recent times heavily industrialized, technologically advanced societies have been faced with rapidly declining natural gas and petroleum based resources and have consequently begun to refocus attention on alternate fuel technologies. Particularly in this country, the impending shortages and the uncertainty of foreign fuel imports has defined a problem of great urgency. As chemists, the demands of an advanced technology-dependent society dictate that we make a strong commitment to develop an extensive program for understanding the chemistry of coal, oil shale, and biomass based fuel resources. Ideally, we would like to know as much as possible about the costs, storability, toxicity, volumetric heating, and combustion properties of the fuels which may ultimately be derived from these alternate resources (e.g., hydrogen, alcohols, aromatics, etc.). While it is highly desirable to perform the extensive experiments required for acquisition of thermodynamic and kinetic diagnostics necessary for complete and accurate specification of the combustion characteristics of each candidate fuel, the temporal immediacy of the impending fuel crisis dictates 
that, if possible, methods for rapid screening of the gross characteristics of these fuels ought to be developed as soon as possible. We report here our investigations of a potentially useful method for rapidly screening candidate fuels via a predictive modeling algorithm which is conceptually easy to apply and which seeks to establish a physically meaningful basis for trial fuel combustion mechanisms.

\section{Predictive Modeling - Methodology}

The methodology for development of a physically rational predictive mechanism follows, by analogy, the detailed mechanism construction algorithm developed by Shaub [1] ${ }^{1}$, Shaub and Bauer [2], and Engleman [3,4,5]. However, the predictive mechanism seeks to define aspects of fuel performance for fuels which have not yet been experimentally characterized. Briefly, construction of a predictive mechanism for modeling the combustion of any candidate fuel proceeds via three steps [2]:

(1) Specify a 11 reactants, products, radicals, and reactive intermediates which may participate in the combustion process and their associated thermochemical parameters (which may have to be estimated), e.g., $\left(G_{f}^{\circ}-H_{0}^{\circ}\right) / R T$ and $\left(H_{f}^{\circ}-H_{0}^{\circ}\right) / R T$ which may be usefully represented in polynomial form [6].

(2) Systematically, via a physically rational algorithm, identify important reaction steps to be included in the predictive mechanism.

(3) Assign rate constants for each forward reaction step included in the proposed mechanism. For consistency between thermodynamic and kinetic data bases, the reverse rate constants may be computed from knowledge of the forward rate constants and the thermodynamic equilibrium constants.

\section{Diborane Combustion - Reaction Mechanism Construction}

To test the utility of the predictive modeling approach, we have chosen to consider the oxidation of diborane for the following reasons:

(1) It is sparsely characterized both thermodynamically and kinetically and should therefore provide a strong test of the method.

(2) We avoid for the present the problem of cost overrides with our existing on-line, Gear-type computer programs, since there are presumably not as many reaction steps to consider as with some of the more complex organic fuels.

(3) There are some experimental data with which comparisons may be made.

As per (1) above, we list in table 1 the species considered in the oxidation of diborane, together with heats of formation (either taken from the literature or estimated from bond energy considerations). Utilizing this set of candidate species, we then generated via a simple computer program [7] all possible uni-, bi-, and ter-molecular (third-body) reaction possibilities, together with calculated heats of reaction at various

\footnotetext{
${ }^{1}$ Figures in brackets indicate the literature references at the end of this paper.
} 
temperatures. A systematic species and reaction step algorithm was used as in reference [2]. to establish a physically reasonable predictive mechanism consisting of 53 reaction steps as shown in table 2. It is to be stressed that this mechanism is not all inclusive and is intended only as a tool to identify some bulk physical properties of the reacting system, elucidate the main features of the mechanism, and predict final product distributions at reaction times several orders of magnitude beyond the delay induction period. With specific reference to our study we note that:

(a) Reaction steps involving $\mathrm{HO}_{2}$ were only added once the mechanism was constructed. These steps do not figure prominently in the overall mechanism and were added as a test of the hydrogen afterburning scheme.

(b) Several third-body reactions have been inserted to determine their importance under high pressure conditions and to characterize termination phases of the oxidation process. Again, they do not perturb substantially the calculated induction times. Generally, third-body reactions were not included.

(c) We have assumed that the product species $\mathrm{B}_{2} \mathrm{O}_{3}$ and $\mathrm{HBO}_{2}$ are thermodynamically stable once formed.

(d) We have rejected $\mathrm{BOH}$ as a candidate species as our thermodynamic estimates led us to conclude that $\mathrm{HBO}$ formation is probably more likely.

(e) Reaction steps endoergic in excess of $35 \mathrm{kcal}$ were eliminated.

(f) Equilibrium calculations were performed (see fig. 1) utilizing the species data set in table 1 [8]. These calculations have been performed with argon included as a diluent in anticipation of later comparisons with some key experimental data. Briefly, we found that the major species anticipated at equilibrium over the indicated parameter ranges (see figure legends) are $\mathrm{H}_{2}, \mathrm{HBO}_{2}$, and $\mathrm{H}_{2} \mathrm{O} . \mathrm{B}_{2} \mathrm{O}_{3}$ mole fraction levels are noticeably lower than for $\mathrm{HBO}_{2} . \mathrm{O}_{2}$ is, as expected, prominent under fuel-lean conditions. We also note, although not shown here, that the equilibrium chemistry is fairly independent of temperature over the pressure range $0.1<P<5 \mathrm{~atm}$. A further consideration of moderately important species (mole fraction range $<10^{-4}$ to $\sim 10^{-7}$ ) has led us to assume that for characterizing the main features of the reaction mechanism, those steps retained should have at least one reactant species from the equilibrium based set: $\mathrm{H}_{2}, \mathrm{O}_{2}, \mathrm{BO}, \mathrm{O}, \mathrm{BO}, \mathrm{OH}$, $\mathrm{H}_{2} \mathrm{O}$, and $\mathrm{H}$. $\quad\left(\mathrm{B}_{2} \mathrm{H}_{6}\right.$ was included in this set also, as it is an initial reactant. $\mathrm{BH}_{3}$ was added to this set as an initial decomposition product of $\mathrm{B}_{2} \mathrm{H}_{6}$.)

(g) We assume that $\mathrm{B}_{2} \mathrm{H}_{5}$, once formed, is immediately decomposed by any subsequent collision.

(h) The reader is referred to references [1,2] for a discussion of other useful reaction step elimination procedures and bases for elimination scheme exceptions which were employed here in a similar manner.

An examination of the proposed mechanism as shown in table 2 reveals five basic features:

$$
\begin{aligned}
& \text { Initiation (pyrolysis) } \\
& \qquad \mathrm{B}_{2} \mathrm{H}_{6} \rightarrow \mathrm{BH}_{3}+\mathrm{BH}_{3}
\end{aligned}
$$




$$
\begin{aligned}
& \text { Chain (boron species oxidation) } \\
& \mathrm{O}_{2}+\mathrm{BH}_{3} \rightarrow \mathrm{H}_{2} \mathrm{BO}+\mathrm{OH} \\
& \mathrm{OH}+\mathrm{H}_{2} \mathrm{BO} \rightarrow \mathrm{H}_{2} \mathrm{O}+\mathrm{HBO} \\
& \text { etc. } \\
& \text { Chain (hydrolysis) } \\
& \mathrm{BH}_{3}+\mathrm{H}_{2} \mathrm{O} \rightarrow \mathrm{H}_{2} \mathrm{BOH}+\mathrm{H}_{2} \\
& \mathrm{OH}+\mathrm{H}_{2} \mathrm{BOH} \rightarrow \mathrm{H}_{2} \mathrm{BO}+\mathrm{H}_{2} \mathrm{O} \\
& \text { etc. } \\
& \text { Chain (hydrogen afterburning) } \\
& \mathrm{H}+\mathrm{OH} \rightarrow \mathrm{H}_{2} \mathrm{O} \\
& \mathrm{OH}+\mathrm{OH} \rightarrow \mathrm{H}_{2} \mathrm{O}+\mathrm{O} \\
& \text { etc. } \\
& \text { Termination (product formation) } \\
& \mathrm{OH}+\mathrm{HBO} \rightarrow \mathrm{HBO}_{2}+\mathrm{H} \\
& \mathrm{HBO}+\mathrm{BO}_{2} \rightarrow \mathrm{B}_{2} \mathrm{O}_{3}+\mathrm{H}
\end{aligned}
$$

Each reaction step was assigned a rate constant of the Arrhenius form. Attempts were made to assign values to these parameters by estimation methods which have been previously utilized for hydrocarbon combustion and pyrolysis kinetics [9]. It was found that extrapolative application of these methods to diborane kinetics led to extremely inconsistent estimates of parameter values. Ultimate assignment of rate parameters as indicated in table 2 was performed by (1) constraining parameter values to generally fit within observed ranges [10] for bimolecular reactions and by (2) generally linearly scaling activation energies with reaction exoergicity as is often done in hydrocarbon combustion correlation schemes [11]. With regards to (2), application of this scaling was generally within the constraint introduced by (1). In the case of endoergic reactions, generally the reverse exoergic parameters were assigned, and parameters for the endoergic step were computed through the thermodynamic equilibrium constant.

\section{Diborane Combustion - Computer Analysis}

A computer analysis of the predicted mechanism for a range of initial conditions was performed to establish the probable temporal levels for each species and additionally to 

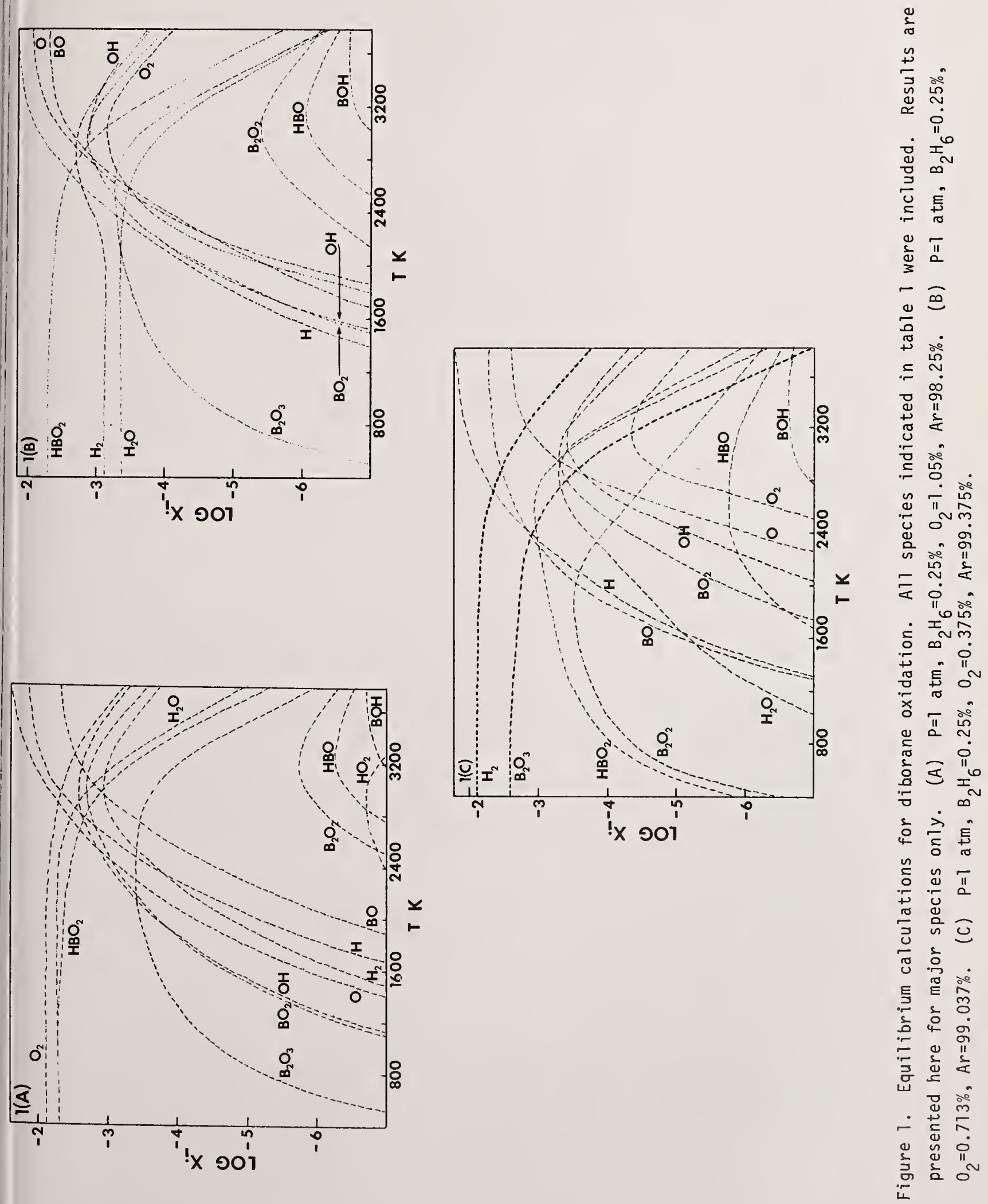
Table 1. Gas phase species initially considered ${ }^{*}$.

\begin{tabular}{|c|c|c|c|}
\hline Specie & 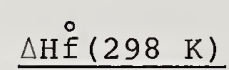 & & $\stackrel{\circ}{\mathrm{H} \dot{f}}(1700 \mathrm{~K})$ \\
\hline $\mathrm{BH}$ & 105.8 & & 103.7 \\
\hline $\mathrm{HBO}$ & -20.0 & & -21.8 \\
\hline $\mathrm{HBOH}$ & $-17(15)^{1}$ & $\mathrm{a}$ & $-24.5 a$ \\
\hline $\mathrm{H}_{2} \mathrm{BO}$ & - & & $-25.9 a$ \\
\hline $\mathrm{H}_{2} \mathrm{BOH}$ & $-70(1)^{1}$ & $\mathrm{a}$ & $-82.6 a$ \\
\hline $\mathrm{OBOH}$ & -134.0 & & -137.1 \\
\hline $\mathrm{BH}_{2}$ & 48.0 & & 46.0 \\
\hline $\mathrm{BH}_{3}$ & 25.5 & & 21.1 \\
\hline BO & 10.7 & & 8.2 \\
\hline $\mathrm{BO}_{2}$ & -75.3 & & -75.8 \\
\hline $\mathrm{B}_{2} \mathrm{H}_{5}$ & $50.8^{2}$ & a & 52.1 a \\
\hline $\mathrm{B}_{2} \mathrm{H}_{6}$ & 9.8 & & 6.0 \\
\hline $\mathrm{B}_{2} \mathrm{O}_{2}$ & -109.0 & & -111.0 \\
\hline $\mathrm{B}_{2} \mathrm{O}_{3}$ & -199.1 & & -201.2 \\
\hline $\mathrm{H}$ & 52.1 & & 54.0 \\
\hline $\mathrm{OH}$ & 9.3 & & 9.0 \\
\hline $\mathrm{H}_{2} \mathrm{O}$ & -57.8 & & -60.0 \\
\hline 0 & 59.6 & & 60.9 \\
\hline $\mathrm{O}_{2}$ & 0.0 & & 0.0 \\
\hline $\mathrm{H}_{2}$ & 0.0 & & 0.0 \\
\hline $\mathrm{BOH}$ & $9(18)^{1}$ & $\mathrm{a}$ & $6.6 \mathrm{a}$ \\
\hline
\end{tabular}

* All data refers to gas phase species; $\Delta \mathrm{H} \stackrel{\circ}{\mathrm{f}}$ units: $\mathrm{kcal} / \mathrm{mol}$

1 G. K. Anderson, PhD Thesis, Cornell University, Ithaca, New York, 1975.

2 Reference 13 of this report was used in making estimate.

a Estimated value, values in parenthesis are \pm uncertainty limits. 


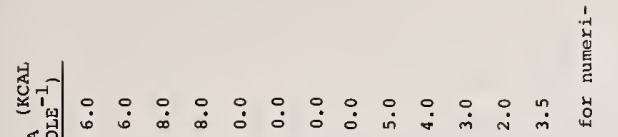

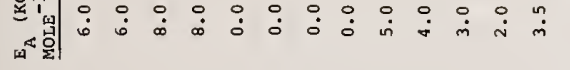

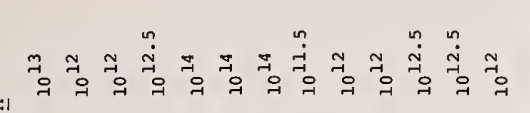
A1 2 ,

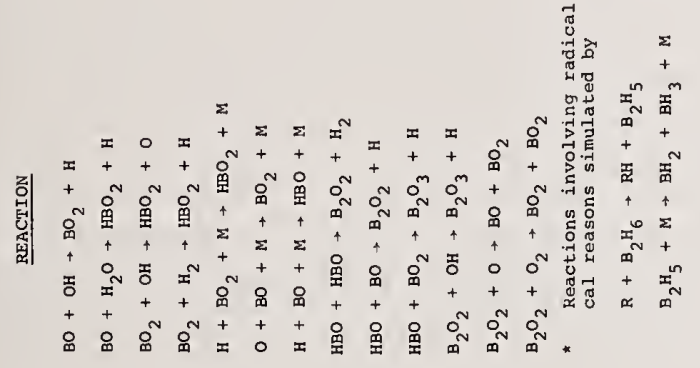

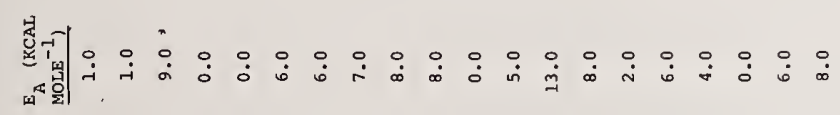

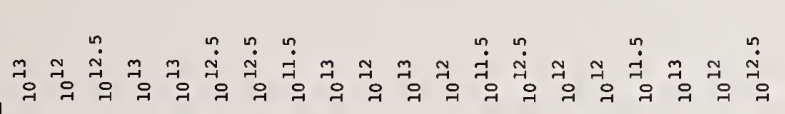

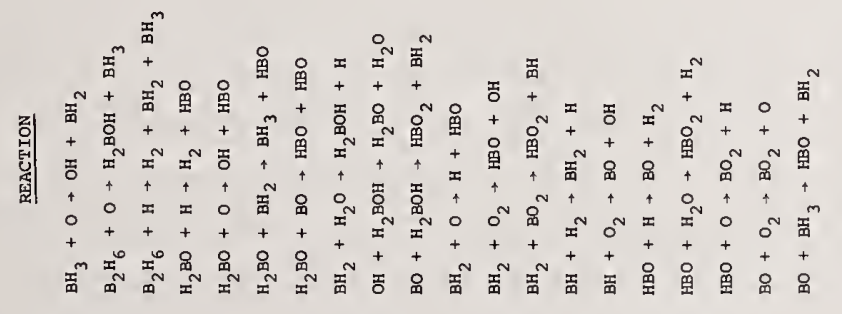

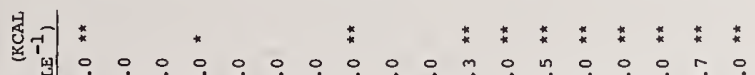

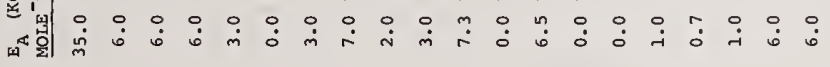

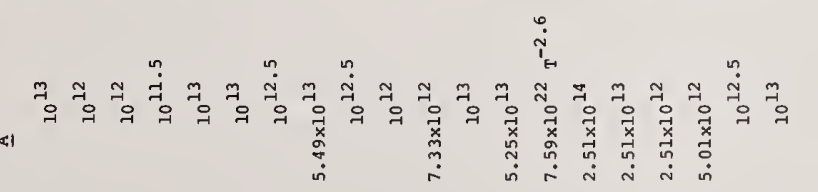

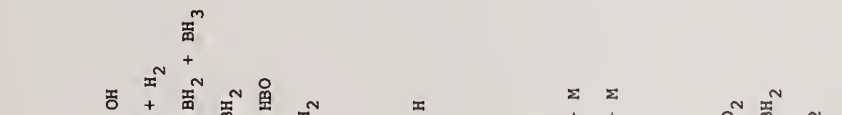

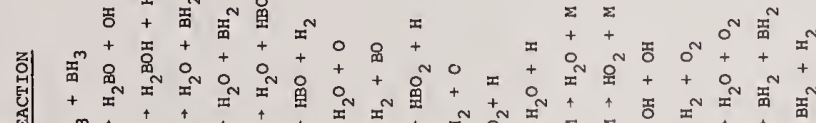
迹苗

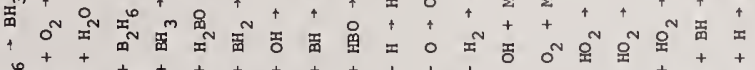

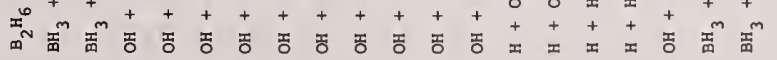

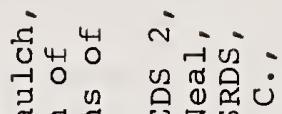

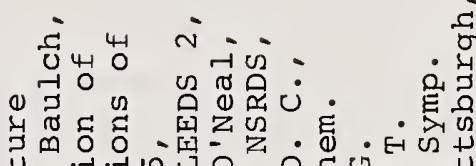
मै.

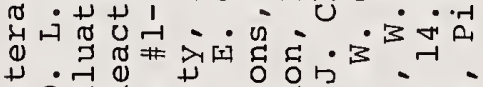

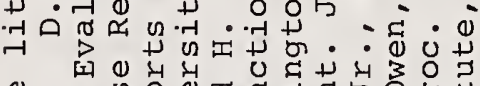

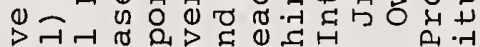
ब्द c. U. 药茂 मे (1) 廿 . ช

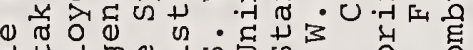

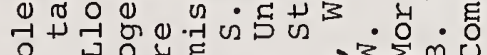

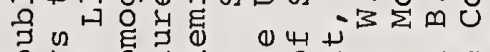
ช ช ๘

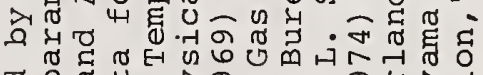
ठ का

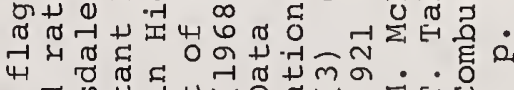

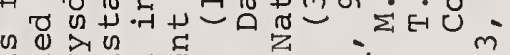

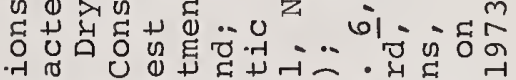

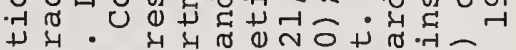
U艹

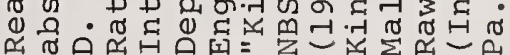


generate temperature/time histories. Figure 2 summarizes the computer concentration profiles for one typical set of initial reaction conditions which were defined to ultimately permit comparison with experimentally measured delay induction times as reported by Skinner for diborane combustion over a range of equivalence ratios. It is clear from these figures that the burning rate is as previously reported [12-14], very fast. These displays should be compared for example to those for acetylene combustion under similar conditions as reported by Shaub and Bauer [2]. It is clear that the time required for substantial exoergic energy release should be on the order of tens of microseconds. A slight adjustment (factor of two) of the pyrolysis and initial oxidation step rate constants produces no dramatic perturbations in the delay induction times. We define the delay induction time as the time required for $\partial T / \partial t(K / s)$ to become positive (indicating that the reaction system is becoming self-driving due to control by exoergic steps). We note that a decrease of four orders of magnitude in the rate for the diborane pyrolysis step lengthens the predicted delay induction periods by two orders of magnitude. Figure 3 illustrates an extrapolated comparison of the predicted delay induction period for diborane combustion with delay induction periods reported by Skinner. The agreement is quite good. Extreme numerical stiffness in the set of differential equations to be solved for the indicated chemistry precluded direct comparison of predicted induction delay times with experimentally acquired diagnostics even when utilizing a Gear-type numerical integration program. Exclusion of reaction steps including $\mathrm{B}_{2} \mathrm{O}_{2}$ and $\mathrm{B}_{2} \mathrm{O}_{3}$ species does not significantly perturb the predicted delay induction times. In table 3 we have listed all species present at substantial levels at the end of the induction delay period for various temperatures and equivalence ratios. It is clear that the delay induction time also marks the onset of a highly branched mechanism as seen by the buildup of many radicals and substantial pyrolysis of $\mathrm{B}_{2} \mathrm{H}_{6}$. Note also that as observed experimentally by Skinner, this reaction mechanism predicts a weak dependence of the delay induction period upon equivalence ratio. Additionally, our analysis leads us to conclude that molecular $\mathrm{H}_{2}$, atomic hydrogen and atomic oxygen do not play a significant role prior to the end of the delay induction period.

In figure 4 we present predicted $\mathrm{H}_{2} / \mathrm{H}_{2} \mathrm{O}$ product distributions generated after long $(1000 \mu s)$ reaction times at $2000 \mathrm{~K}$. Qualitatively, the predicted behavior agrees well with experimental observations $[13,14]$. It should be emphasized that these distributions cannot be predicted in the absence of the included borane hydrolysis steps. There is strong evidence [15] that borane hydrolysis plays a major role in the overall kinetics scheme.

\section{Prospects for General Application of the Predictive Method}

Results regarding matching of product distributions as reported in reference [2] together with qualitative reproduction of $\mathrm{H}_{2} / \mathrm{H}_{2} \mathrm{O}$ product distributions for various equivalence ratios reported in this paper are reasonable in comparison to available 
Table 3. Predicted species mole fraction ratios and major species for various conditions

\begin{tabular}{|c|c|c|c|c|c|c|}
\hline $\mathrm{T}(\mathrm{K})$ & $\begin{array}{l}\text { Equivalence } \\
\text { Ratio }(\gamma) \\
\end{array}$ & $\mathrm{BH}_{3} / \mathrm{B}_{2} \mathrm{H}_{6}$ & $\mathrm{OH} / \mathrm{BH}_{3}$ & $\mathrm{H}_{2} \mathrm{O} / \mathrm{OH}$ & $\mathrm{x}_{\mathrm{i}}>10^{-}$ & \\
\hline \multirow[t]{3}{*}{3000} & 2.0 & 5.51 & $2.86 \times 10^{-2}$ & 0.413 & & \\
\hline & 1.0 & 3.31 & $4.66 \times 10^{-2}$ & 0.258 & \multicolumn{2}{|c|}{ ALL CASES: ** } \\
\hline & 0.5 & 2.01 & $7.19 \times 10^{-2}$ & 0.149 & $\mathrm{~B}_{2} \mathrm{H}_{6}$ & $\mathrm{BH}_{3}$ \\
\hline \multirow[t]{3}{*}{2000} & 2.0 & 0.68 & $6.65 \times 10^{-2}$ & 0.489 & $\mathrm{O}_{2}$ & $\mathrm{H}_{2} \mathrm{O}$ \\
\hline & 1.0 & 0.43 & $1.05 \times 10^{-2}$ & 0.239 & $\mathrm{OH}$ & $\mathrm{H}_{2} \mathrm{BO}$ \\
\hline & 0.5 & 0.26 & 0.155 & 0.120 & $\mathrm{H}_{2} \mathrm{BOH}$ & $\mathrm{H}_{2}$ \\
\hline \multirow[t]{3}{*}{1000} & 2.0 & 0.026 & $1.40 \times 10^{-2}$ & 6.10 & $\mathrm{BH}_{2}$ & HBO \\
\hline & 1.0 & 0.018 & $2.73 \times 10^{-2}$ & 3.21 & 0 & BO \\
\hline & 0.5 & 0.012 & $5.04 \times 10^{-2}$ & 1.73 & $\mathrm{H}$ & \\
\hline
\end{tabular}

* Prediction is at delay induction time defined as time required for $\partial \mathrm{T} / \partial t>0$ to be achieved.

** $\mathrm{X}_{\mathrm{H}}, \mathrm{X}_{\mathrm{H}_{2}}, \mathrm{x}_{0}, \mathrm{x}_{\mathrm{BO}} \ll 10^{-7} 1000 \mathrm{~K}$
$\mathrm{x}_{\mathrm{H}}$
$\sim 10^{-8}$ for $2000 \mathrm{~K}$
$\mathrm{x}_{\mathrm{H}}$
$\sim 10^{-8}$ for $3000 \mathrm{~K}$ when $\gamma=0.5$ 

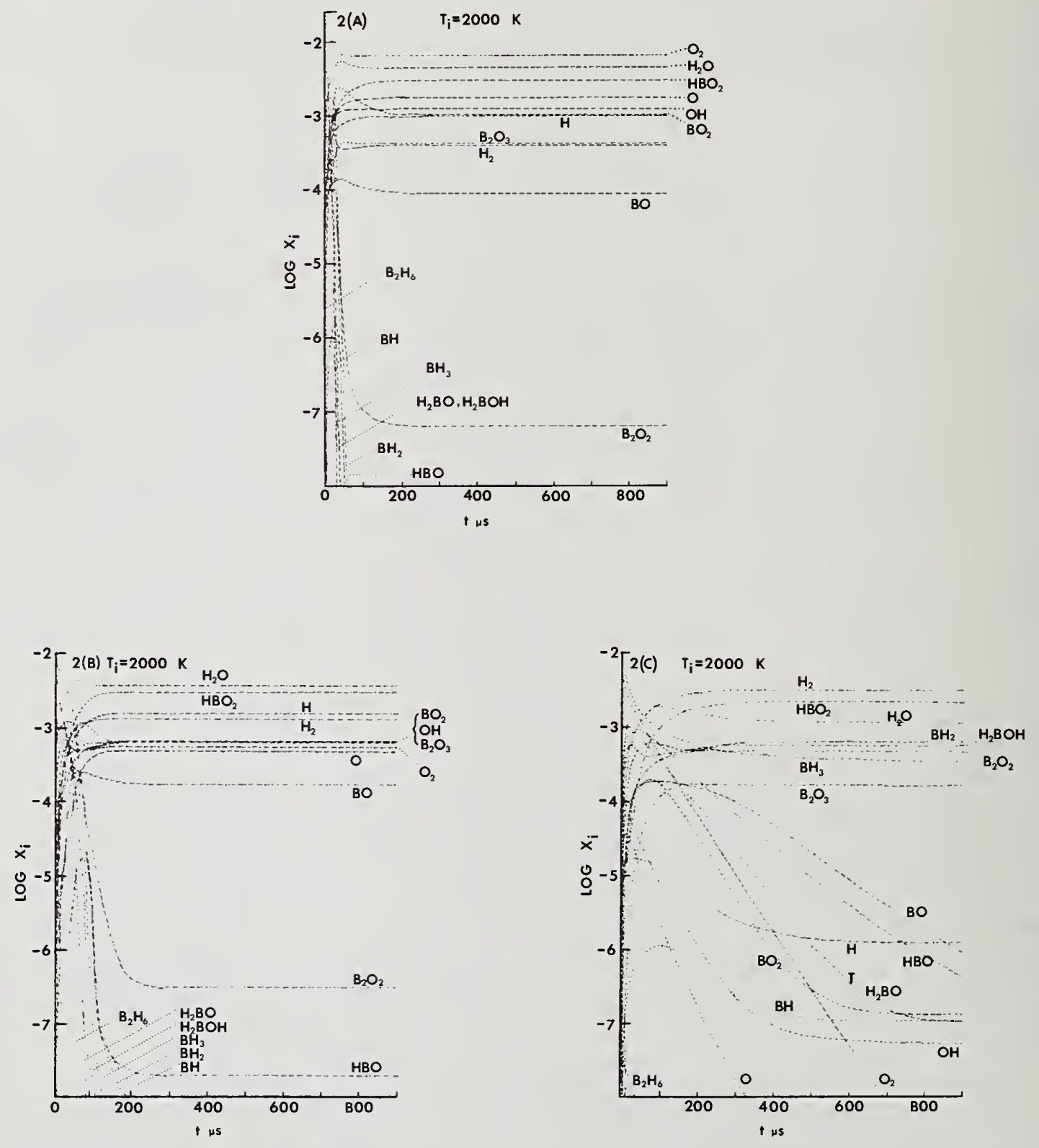

Figure 2. Kinetic plots for diborane oxidation at constant volume and an initial pressure of $5 \mathrm{~atm}$.

(A) Fuel lean, $\mathrm{B}_{2} \mathrm{H}_{6}=0.25 \%, \mathrm{O}_{2}=1.50 \%, A r=98.25 \%$

(B) Stoichiometric, $\mathrm{B}_{2} \mathrm{H}_{6}=0.25 \%, 0,=0.75 \%, A r=99.0 \%$

(C) Fuel rich, $\mathrm{B}_{2} \mathrm{H}_{6}=0.25 \%, \mathrm{O}_{2}=0.375 \%, \mathrm{Ar}=99.375 \%$ 


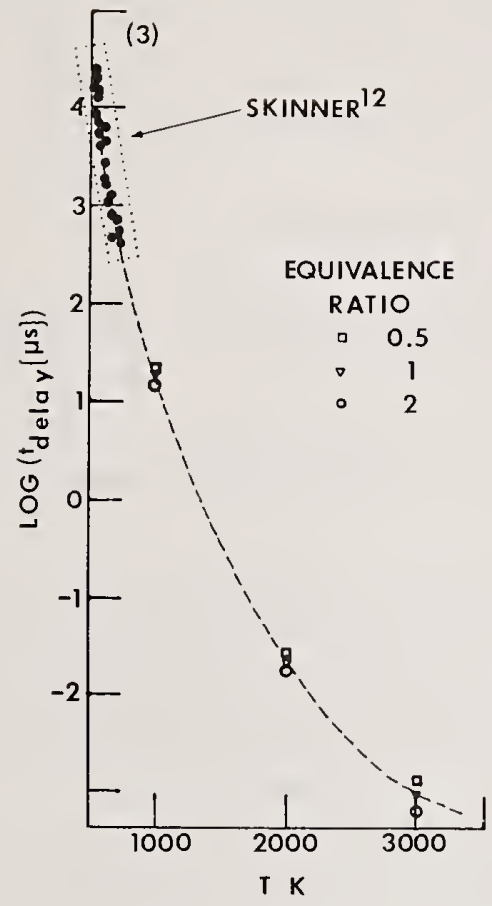

Figure 3. Plot of delay induction times vs temperature showing comparison of computed profiles with results reported by Skinner and Snyder [12].

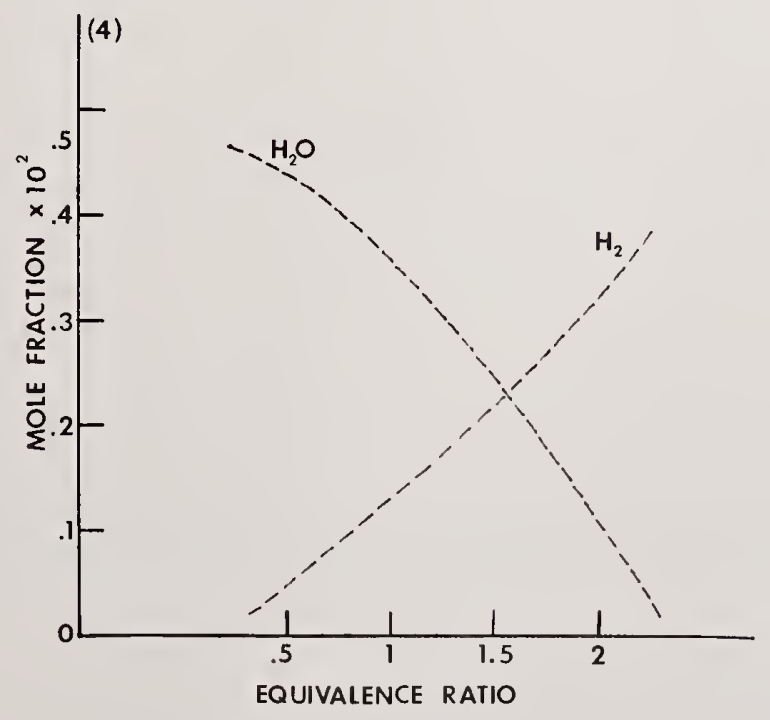

Figure 4. Plot of $\mathrm{H}_{2} / \mathrm{H}_{2} \mathrm{O}$ distributions at $1000 \mu \mathrm{s}$. Initial conditions as per figure 2. 
experimental data. This leads us to suggest that for highly complex combustion systems where there are many reactive channels leading to product formation, it may be possible to use this method to estimate product distributions at time intervals substantially longer than the delay induction period. That is, there appears to be an insensitivity to the precise details of each reactive channel if the system is complex. On the other hand, for simple mechanisms involving perhaps only one or two paths for product formation, a detailed knowledge of reaction channels is certainly important.

We suggest that in dealing with highly complex systems where practically all rate constants are unknown, it is dangerous to attempt adjustment of rate constants beyond an initial guess. When there are too many experimentally uncharacterized reactive channels, attempting a good fit by rate constant adjustment may obfuscate identification of general reaction features.

It appears possible to obtain reasonable agreement with experimentally reported delay induction times for complex systems for the case of the delay induction period defined as the time required for $\partial \mathrm{T} / \partial \mathrm{t}$ to become positive. We recommend defining the delay induction period in this manner as it appears to be a general property of the overall reaction chemistry of complex systems and not channel specific.

In spite of the fact that construction of the predictive mechanism is a "detailed" approach, it appears that a systematic and physically reasonable algorithm ultimately capable of computer programmed control can greatly reduce the time required to establish a trial mechanism for a complex reactive system. Future work should be undertaken to further quantify and standardize details and utilization of the elimination algorithm.

In the case of high molecular weight starting fuels, it is conceivable that the ultimate predicted reaction mechanism for numerical simulation may involve hundreds of reaction steps in combinations which are extremely stiff. However, it is expected that the use of a more recent approach developed by Boris and co-workers $[16,17]$ may reduce considerably the time required for such laborious simulation.

As indicated in this paper, predictive modeling can also serve to identify general features (e.g. borane hydrolysis, hydrogen afterburning, etc.) of reaction chemistry which probably are important to include in any proposed mechanism. However, it is rather difficult to unequivocably identify all key reaction steps. As mentioned previously, it is meaningless to adjust rate constants for specific reaction steps while most of the details of a complex mechanism are experimentally uncharacterized. Again we note that, in general, the fewer the possible number of reactive channels for formation or destruction of any particular species, the more sensitive the mechanism is to the assigned thermochemical and kinetic parameters for those specific steps. If such important channels are predicted, kineticists should perform experiments to establish rate parameters for these steps.

In summary, it appears that some features of the combustion of alternative fuels may be rapidly screened by predictive modeling techniques when the urgency for information precludes the allotment of sufficient time for extensive experimentation. The features 
which appear to be accessible by this approach may include the delay induction time, bulk energy release rates, and product distributions. We recommend that additional studies of this approach be undertaken to further establish the validity of the method and to determine if other reaction features are accessible by this method.

The authors gratefully thank Dr. A. B. Harvey for his keen interest and support of this work.

\section{References}

[1] Shaub, W. M., Ph.D. Thesis, Corne11 University, Ithaca, NY (August 1975).

[2] Shaub, W. M. and Bauer, S. H., Combustion and Flame, 32, 35 (1978).

[3] Engleman, V. S., J. Phys. Chem. 81, 2320 (1977).

[4] Engleman, V. S., Survey and evaluation of kinetic data on reactions in methane/air combustion, Environmental Protection Agency Report No. EPA-600/2-76-003 (January 1976).

[5] Engleman, V. S., 173rd Meeting ACS, Physical Chemistry Division, Abstr. \#111 (March 1977).

[6] Gordon, S. and McBride, B. J., NASA Report \#SP-273, NASA (1971) Washington, D. C. (see for example the discussions of thermofit coefficients contained therein). The thermochemical properties of some species considered in this report were estimated.

[7] The computer program for generating reaction possibilities is available on request. Contact W. M. Shaub, Code 6110 , U.S. Naval Research Laboratory, Washington, D.C. 20375.

[8] Polynomical coefficients for some of these species have been estimated.

[9] Rosenstock, H. M., et a1., NBS Report NBSIR 75-910 (and references contained therein), National Bureau of Standards, Gaithersburg, MD (October 1975).

[10] See for example, Golden, D. M. Estimation of rate constants of elementary processes a review of the state-of-the-art, 14th Symposium on Combustion (The Combustion Institute, 1973), p. 121.

[11] See for example, Semenoff, N. N. , Some problems in chemical kinetics and reactivity, Chap. 1 (Pergammon Press, Princeton, NJ, 1978).

[12] Skinner, G. B. and Snyder, A. D., Mechanism and chemical inhibition of the diborane-oxygen reaction, Heterogeneous Combustion, H. G. Wolfhard, I. Glassman, L. Green, Jr., eds. (Academic Press, NY, 1974), p. 345.

[13] Carabine, M. D., and Norrish, R. G. W., Proc. Roy. Soc. 296, 1 (London, 1967).

[14] Berl, W. G., A brief review of the combustion of boron hydrides, Heterogeneous Combustion, H. G. Wolfhard, I. Glassman, and L. Green, Jr., eds. (and references contained therein) (Academic Press, NY, 1964) p. 312.

[15] Poling, E. L. and Simons, H. P., Ind. Eng. Chem. 50, 1695 (1958); see also reference 13. 
[16] Oran, E., Young, T., and Boris, J., Application of time-dependent numerical methods to be description of reactive shocks, paper submitted for publication in the Seventeenth Symposium (International) on Combustion.

[17] Young, T. R., Boris, J. P., J. Phys. Chem. 81, 2424 (1977).

\section{Discussion}

Question (Gann): Did you calculate an adiabatic flame temperature for this system?

Response (Shaub): This was strictly a homogeneous zero dimension calculation, chemistry only with the energy fed back in. We are not playing with flames; we are not coupling any fluid dynamics.

Question (Gann): Right, but you can get a reaction temperature if you just let the thing go?

Response (Shaub): Well, since it was highly diluted argon, the temperature more or less hovered about the initial temperature specified, although there was in some cases a 200 degrees Kelvin deviation.

Question (McDonald): I understand that changing one individual reaction in a highly branched system like this may not be of importance, but say the first two steps that are involved, the production of $\mathrm{BH}_{3}$, and then the reaction of $\mathrm{BH}_{3}$ plus $0_{2}$; you have assumed that these things go a certain way.

If the mechanism is wrong and these things don't go that way, how is this going to affect the overall model?

Response (Shaub): I understand the point. In fact, the real difference in activation energy is the difference between the pyrolysis and then everything else.

Whether or not you play around with most of the other steps, say $\mathrm{BH}_{3}$ plus $0_{2}$ does not even give $\mathrm{OH}$, but something else, well all the activation energies for those processes are considerably less than the pyrolysis step, which is up around $35 \mathrm{kcals}$ per mole. So, it is really not that much of a perturbation, again in terms of the bulk physical properties.

On the other hand, because of this insensitivity it does not help you to establish what the correct mechanism is; it only helps you to build a working merhanism for making engineering type predictions about system behavior.

Question (McDonald): Even the fact that, that is a primary step, if its mechanism is wrong does it not affect the overall total energy release rates, or the induction times? 
Response (Shaub): They are not substantially affected if you are satisfied with gross approximations.

Question (Tsang): You say the induction problem is independent of the initiation process?

Response (Shaub): The induction period is independent of the concentration of oxygen present. It waits only on the pyrolysis of the diborane.

Question (Tsang): On the pyrolysis of the diborane, the two $\mathrm{BH}_{3}$ must be controlling your induction process, isn't it? And in that case it is very effective.

Response (Shaub): Yes, it is. In the case of the pyrolysis of diborane it is very important. But, it is not so important as you would think again because of the difference between the activation energy for that step and all the others. So, a considerable uncertainty, say calling diborane pyrolysis at 42 kcals per mole instead of 35 , or 25 , isn't really going to make that much of a difference.

It is because it is so much greater than all of the other steps that you have some range of flexibility in making these gross predictions.

Question (Tsang): What temperature are you making these calculations at now?

Response (Shaub): This is at 2000 degrees Kelvin. I have gone from 1000 to 3000 .

Question (Tsang): Say you worked at 1500 degrees: are you finding a change of 15 kilocalories in the activation energy has no effect?

Response (Shaub): Well, as I mentioned in one of the points, four orders of magnitude of variation in the rate constant for that pyrolysis step only perturbed a predicted delay induction period by two orders of magnitude.

It is not negligible, but if you are satisfied with gross approximations --you see our ultimate effort here is to scale relative fuel performance. We don't care about absolute numbers. We just want to know how this fuel is going to behave relative to another, or how this fuel mixture is going to behave relative to another.

So, we have a sort of leniency there. If you want it highly accurate, absolute values, then we are certainly in trouble.

Comment (Shaub): In consideration of the paper presented and in reference also to earlier studies, it is interesting to speculate as to why in many cases there is apparently often observed, an insensitivity toward rate constant adjustment. Along these lines of thought I should like to make two suggestions: 
1. When the system is highly exoergic and highly branched, the net total rate of production or consumption of energy at any given point in time during the course of reaction often may far exceed the energy produced or consumed by any specific reaction step channel. Under these conditions, one might expect an apparent insensitivity to specific channel rate constant adjustment. One only needs to "be in the ball park" so to speak. Clearly, this postulate cannot apply to highly linear chain mechanisms, where the total energy nearly passes through only one or two channels, which act as bottlenecks. The conclusion is that one apparently needs to specify rate constants accurately when systems are linear (or nearly linear) in total or during some phases of the combustion process, but not during highly branched phases. Additionally, this also implies it will often be difficult to assign rate constants based on inferences made from kinetic modeling schemes.

2. After the initial high energy release phase which follows the induction period, often, the bimolecular exoergic reaction steps will go into a very rapid local psuedoequilibrium, thus becoming more sensitive to the thermodynamics (assuming one has calculated "reverse" rate constants through the "forward" rate constants and thermodynamic equilibrium constants) and less sensitive to the kinetics. This means two things: (a) a sensitivity to the accuracy of kinetic rate constant parameters is diminished in this time domain; (b) since thermodynamic parameters are usually better known than kinetic parameters, one may expect a reasonable reproduction of long time period (psuedo local equilibrium domain) product distributions. In this paper, see figures $2(a-c)$ for chemistry at about $800 \mathrm{~ms}$ to get a feel for this effect. The above comments regarding linear vs branched chain mechanisms are applicable here also.

In summary, while this method will never replace good fundamental experimentation, it may serve as a directional or feedback instrument for defining critical aspects of some chemical processes which should be further characterized via laboratory work, and thus accelerate our understanding of combustion processes which occur at high temperatures and pressures. 
National Bureau of Standards Special Publication 561, Proceedings of the 10th Materials Research Symposium on Characterization of High Temperature Vapors and Gases held at NBS, Gaithersburg, Maryland, September 18-22, 1978. Issued October 1979.

\title{
NEW COMPUTATIONAL ALGORITHMS FOR CHEMICAL KINETICS
}

\author{
David T. Pratt
}

The University of Michigan

Ann Arbor, MI 48104

This paper describes the development of a new algorithm for multicomponent, one-dimensional chemical kinetics calculations. The new algorithm, derived from a variety of sources, is single-step, implicit, and uses second derivative or Jacobian information both for accuracy and convergence of the integration algorithm. Comparison on a test case with the Bittker-Scullin NASA code GCKP showed the new algorithm to be nearly ten times faster for comparable accuracy, and potentially faster yet for cases where only moderate accuracy is required.

\section{Background}

Currently available computer codes for integration of the differential equations of homogeneous, gas-phase chemical kinetics were developed primarily for use in interpreting rate data derived from shock tube or plug-flow reactor experiments, and in prediction of finite-rate chemistry effects on rocket motor performance. Typically, these codes $[1,2,3]^{1}$ are variable-order, multistep methods, designed primarily for accuracy and stability. These codes are generally difficult to implement for the non-specialist, and require large storage and long execution times for a typical kinetics problem.

Recent interest in prediction of reacting laminar and turbulent flows has revealed a need for integration codes which are single-step (to avoid massive storage in multidimensional flow net computations), fast, and stable. Accuracy is less important, as the basic flow computation techniques $[4,5]$ have relatively low accuracy. For steady flows, a chemical-kinetic algorithm CREK [6] has been shown to be fast, moderately accurate, and stable $[7,8]$. However, for unsteady flows, such as anticipated Lagrangian treatments of reacting turbulent flows [9] and for coalescence/dispersion or "Monte Carlo" modeling [10], a time-dependent batch integrator is required to have many of the characteristics of CREK (single-step, fast and stable) but with greater accuracy. The present paper describes how such an algorithm, called ODCREK, is being developed.

${ }^{1}$ Figures in brackets indicate the literature references at the end of this paper. 


\section{Introduction ${ }^{2}$}

The starting point for the present development is the steady-state, zero-dimensional, chemical kinetics algorithm CREK, which has been described in detail elsewhere [5,6]. Of immediate interest are the characteristics of CREK as a one-dimensional integrator. As presently formulated, CREK solves a system of coupled, non-linear algebraic equations of the form

$$
\sigma_{i}=\sigma_{i}^{*}-\tau r_{i}
$$

where the superscript $\left(^{*}\right)$ denotes values of the mole number $\sigma_{i}^{*}$ at inlet to the computational cell of interest, $\sigma_{i}$ is the stationary-state value at the cell, $r_{j}$ is the mass-specific rate of disappearance of species $i$, and $\tau$ is the convective/diffusive time constant for the cell of interest. (Equation (1) is also the species-i conservation equation for a perfectly stirred reactor with mean residence time $\tau$.)

Considering now the set of species conservation equations for transient batch reaction,

$$
\frac{d \sigma_{i}}{d t}=-r_{i}
$$

it is apparent that eq. (1) can be regarded as a finite-difference representation of eq. (2), with superscript $\left({ }^{*}\right)$ now denoting values at the beginning of a time step of length $\tau$. Equation (1) thus represents an implicit Euler approximation [11] for the solution of eq. (2) over the time interval $\tau$.

CREK has in fact been used as a one-dimensional integrator, with characteristically rapid convergence over a coarse time grid. Of course, accuracy suffers considerably when coarse time steps are taken, because eq. (1) approximates the rate term $r_{j}$ as a constant over the interval $\tau$, thus leading to only first-order accuracy of the result [11].

It occurred to the present authors that, since eq. (1) is solved by a "self-adjusting" accelerated Newton-Raphson scheme [5], the required Jacobian (matrix of partial derivatives of the Newton-Raphson correction equation) could be used to improve the accuracy of eq. (1) as an approximation to eq. (2). For example, fourth order accuracy should be obtainable by fitting $r_{i}$ over the interval $\tau$, not by a constant as in eq. (1), but by a cubic equation. In multistep methods, the four bits of information needed to achieve fourth-order accuracy are provided by four values of the functional evaluated at three past time steps and at the current one. However, cubic splines can be fit over a single step, by using the values of the functional and of its first derivatives at only the most recent time step and at the time step of current interest; i.e., two bits of information are evaluated at each of two points.

${ }^{2}$ Nomenclature used is summarized in the Appendix. 
A little algebra shows that, with a single-step cubic spline fit for $r_{j}$, eq. (2) can be approximated over $\tau$ by

$$
\sigma_{i}=\sigma_{i}^{*}-\frac{\tau}{2}\left[r_{i}+r_{i}^{*}\right]+\frac{\tau^{2}}{12}\left[\dot{r}_{i}-\dot{r}_{i}^{*}\right]
$$

where

$$
\dot{r}_{i} \equiv \sum_{k=1}^{N S} \frac{\partial r_{i}}{\partial \sigma_{k}} r_{k}
$$

and where the superscript $\left({ }^{*}\right)$ refers to values calculated at the beginning of the time step, as before.

The significance of the proposed cubic spline fit to $r_{i}$, as summarized in eqs. (3) and (4), is that accuracy can apparently be increased from first- to fourth-order with negligible increase in execution time, because the Jacobian elements required in the eq. (4) terms are already calculated for Newton-Raphson convergence of eq. (1), and the algorithm can be easily modified to converge eq. (3) in the same matter.

Equations (3) and (4) are essentially the method "Liniger-Willoughby \#3" [12], as discussed by Lapidus and Seinfeld [11], with only minor exceptions.

Lapidus and Seinfeld [11] discuss a variety of single-step methods for solution of stiff systems of differential equations, and point out a related method which is somewhat superior to Liniger-Willoughby \#3, namely "Liniger-Willoughby \#1." This is the method which has been adapted for ODCREK, and which will now be developed in some detail.

It is instructive to return briefly to eq. (3) before proceeding further. Note that the second term on the right-hand side of eq. (3) represents a trapezoidal approximation to $r_{i}$ over the interval $\tau$, with the remaining terms appearing as small corrections. If only the trapezoidal approximation were used, accuracy would be improved to second-order, but possibly at the expense of some of the stability properties of the implicit Euler approximation [11]. The cubic approximation, eq. (3), can be improved on by fitting $r_{i}$ over the interval by an exponential function, as suggested by Liniger and Wi1loughby [12]. An exponential requires only two bits of information to fit, as does the straight line or trapezoid, and therefore leads apparently to only second-order accuracy. However, the form of the exponential function is particularly well suited for temperature-dependent chemical rate equations, and the accuracy should be considerably better than second-order in this particular application. In fact, it has been shown that exponential fitting leads to accuracy which is at least fourth-order and sometimes as great as sixth-order, while preserving all the desirable characteristics of A-stability (reliable convergence to the steady-state solution) and freedom from oscillations characteristic of the implicit Euler approximation [13]. 
Following Liniger and Willoughby, the solution to eq. (2) over the interval $\tau$ is first approximated by a "tunable trapezoid,"

$$
\sigma_{i}=\sigma_{i}^{*}-\tau\left[\left(1-w_{i}\right) r_{i}+w_{i} r_{i}^{*}\right]
$$

where the "tuning" or integrating factor $W_{i}$ is to be determined by exponential fitting of $r_{j}$ over the interval $\tau$, as follows:

If $r_{i}$ varies exponentially with time over $\tau$, so does $\sigma_{i}$, as it is the integral of $r_{j}$; therefore

$$
\frac{d \sigma_{i}}{d t}=-r_{i}=c_{i} \sigma_{i}
$$

Brandon [13] defines a diagonal transition matrix $z_{i}$ for the eigenvalue $c_{j}$ over the integration interval $\tau$ so that eq. (6) is given by

$$
-\frac{d \sigma_{i}}{d t} \equiv r_{i}=\left(\frac{z_{i}}{\tau}\right) \sigma_{i}
$$

With the substitution of eq. (7) into eq. (5), algebraic manipulation leads to the identity

$$
w_{i}=\frac{1}{z_{i}}-\frac{1}{\left(e^{z_{i}}-1\right)}
$$

If $r_{i}$ does indeed vary exponentially over $\tau$, then $z_{i}$ is given precisely by eq. (7). However, for arbitrary, non-exponential variation of $r_{i}$, Brandon [13] suggests an approximation to $z_{i}$ over the interval $\tau$, as follows:

$$
z_{i} \cong \frac{\tau}{2} \sum_{k=1}^{N S}\left[\left(\begin{array}{ll}
\frac{\partial r_{i}}{\partial \sigma_{k}} & \frac{r_{k}}{r_{i}}
\end{array}{ }^{(0)}+\left(\frac{\partial r_{i}}{\partial \sigma_{k}} \frac{r_{k}}{r_{i}}\right)^{(s)}\right]\right. \text {, }
$$

where the superscripts (o) and (s) refer to values evaluated at the zero-th and current (or last) iteration during convergence of eq. (5).

In the present application, a Newton-Raphson functional was defined for convergence of eq. (5),

$$
f_{i} \equiv \frac{\sigma_{i}-\sigma_{i}^{*}}{\left(1-w_{i}\right)_{\tau}}+\frac{w_{i}}{\left(1-w_{i}\right)} r_{i}^{*}+r_{i}, \quad i=1, N S \text {, }
$$


where the mass-specific rate of disappearance of $i^{\text {th }}$ species is given by

$$
r_{i}=\frac{1}{\rho} \sum_{j=1}^{J J}\left(\alpha_{i j}^{\prime}-a_{i j}^{\prime \prime}\right)\left(R_{j}-R_{-j}\right), i=1, N S
$$

where $\rho$ is the mixture gas density $\rho=P / R T \sigma_{m}$ (see notation), and where $R_{j}$ and $R_{-j}$, the forward and reverse rates of the $j^{\text {th }}$ reaction $(j=1, J j)$, are given by a modified Arrhenius expression.

$$
R_{j}=10^{B} j_{T}^{N} j_{\exp \left(-T_{j} / T\right)\left(\rho \sigma_{m}\right)^{\bar{\alpha}}} \prod_{k=1}^{N S}\left(\rho \sigma_{k}\right)^{\alpha}{ }_{k j}^{\prime}
$$

and

$$
R_{-j}=10^{B}-j_{T}^{N}{ }^{N} j_{\exp \left(-T_{-j} / T\right)\left(\rho \sigma_{m}\right)^{\alpha} j} \prod_{k=1}^{N S}\left(\rho \sigma_{k}\right)^{\alpha_{k j}^{\prime \prime}}
$$

Following Gordon and McBride [14], a wise choice of correction variables for solving eq. (10) are the natural logarithms of the NS mole numbers $\left(\sigma_{i}, i=1, N S\right)$, of the sum of the mole numbers,

$$
\sigma_{\mathrm{m}} \equiv \sum_{\mathrm{k}=1}^{\mathrm{NS}} \sigma_{\mathrm{k}} \text {, }
$$

and of the temperature $T$.

The correction equation for eq. (10) may now be written as

$$
\begin{gathered}
\sum_{k-1}^{N S} \frac{\partial f_{i}}{\partial \log \sigma_{k}} \Delta \log \sigma_{k}+\frac{\partial f_{i}}{\partial \log \sigma_{m}} \Delta \log \sigma_{m} \\
\quad+\frac{\partial f_{i}}{\partial \log T} \Delta \log T=-f_{i}, \quad i=1, N S .
\end{gathered}
$$


Noting that the integrating factors $W_{j}$ are considered constant during each iteration, partial derivatives of the species $-i$ functional $f_{i}$ of eq. (10) are

$$
\begin{gathered}
\frac{\partial f_{i}}{\partial \log \sigma_{k}}=\frac{\delta_{i k}}{\left(1-w_{i}\right) \tau}+\frac{1}{\rho} \sum_{j=1}^{J J}\left(\alpha_{i j}^{\prime}-\alpha_{i j}^{\prime \prime}\right)\left(R_{j} \alpha_{k j}^{\prime}-R_{-j} \alpha_{k j}^{\prime \prime}\right), \quad i=1, N S \\
\frac{\partial f_{i}}{\partial \log \sigma_{m}}=\frac{1}{\rho} \sum_{j=1}^{J J}\left(\alpha_{i j}^{\prime}-\alpha_{i j}^{\prime \prime}\right)\left(R_{-j} n_{j}^{\prime \prime}-R_{j} n_{j}^{\prime}\right)+r_{i},
\end{gathered}
$$

and

$$
\begin{array}{r}
\frac{\partial f_{i}}{\partial \log T}=\frac{1}{\rho} \sum_{j=1}^{J J}\left(\alpha_{i j}^{\prime}-\alpha_{i j}^{\prime \prime}\right)\left[R_{j}\left\{N_{j}+\frac{T j}{T}-\bar{\alpha}_{j}-n_{j}^{\prime}\right\}\right. \\
\left.-R_{-j}\left\{N_{-j}+\frac{T-j}{T}-\bar{a}_{j}-n_{j}^{\prime \prime}\right\}\right]+r_{i} .
\end{array}
$$

Alternative treatments of the rate expressions, eqs. (12) and (13) are possible. For example, if it is desired to construct the reverse rate data from the relation

$$
k_{-j}=\frac{k_{j}[R T]_{j}^{\left(n^{\prime \prime}-n_{j}^{\prime}\right)}}{k_{j}^{P}},
$$

where $K_{j}^{p}$ is the equilibrium constant for reaction $j$,

$$
K_{j}^{P} \equiv \exp \left[\sum_{i=1}^{N S}\left(a_{i j}^{1}-a_{i j}^{\prime \prime}\right) \frac{g_{1}^{0}}{R T}\right]
$$

then the reverse rate $R_{-j}$ is given by

$$
R_{-j}=10^{B_{j}} T^{N} j \exp \left[-\frac{l^{T} j}{T}-\sum_{j=1}^{N S}\left(a_{i j}^{\prime}-a_{i j}^{\prime \prime}\right) \frac{g_{j}^{0}}{R T}\right][R T]^{\left(n_{j}^{\prime \prime}-n_{j}^{\prime}\right)}
$$




$$
\left(\rho \sigma_{m}\right)^{\bar{a}_{j}} \prod_{k=1}^{N S}\left(\rho \sigma_{k}\right)^{a_{i j}^{\prime \prime}}
$$

and the partial derivative coefficient of $\Delta \log T$ in eq. (18) becomes

$$
\begin{gathered}
\frac{\partial f_{i}}{\partial \log T}=\frac{1}{\rho} \sum_{j=1}^{J J}\left(a_{i j}^{\prime}-a_{i j}^{\prime \prime}\right)\left[\left(R_{j}-R_{-j}\right)\left\{N_{j}+\frac{T j}{T}-\bar{a}_{j}-n_{j}^{\prime}\right\}\right. \\
\left.-R_{-j} \sum_{i=1}^{N S}\left(a_{i j}^{\prime}-a_{i j}^{\prime \prime}\right) \frac{h_{i}}{R T}\right] .
\end{gathered}
$$

The functional for mixture reciprocal mole number, $\sigma_{m}$, is simply

$$
f_{m} \equiv \sum_{k=1}^{N S} \sigma_{k}-\sigma_{m}
$$

and the corresponding correction equation is

$$
\sum_{k=1}^{N S} \sigma_{k} \Delta \log \sigma_{k}-\sigma_{m} \Delta \log \sigma_{m}-\sigma_{m}-\sum_{k=1}^{N S} \sigma_{k} .
$$

The functional for temperature is the equation for conservation of thermal energy for adiabatic batch reaction,

$$
f_{T} \equiv \frac{H^{*}}{R T}-\sum_{i=1}^{N S} \frac{\sigma_{i} h_{i}}{R T}
$$

and the corresponding correction equation

$$
\sum_{k=1}^{N S} \frac{h_{k} \sigma_{k}}{R T} \Delta \log \sigma_{k}+\sum_{i=1}^{N S} \frac{{ }^{C} p_{i}}{R} \sigma_{i} \Delta \log T
$$




$$
=\sum_{i=1}^{N S} \frac{\sigma_{i} h_{i}}{R T}-\sum_{i=1}^{N S} \frac{h_{k}^{*} \sigma_{i}^{*}}{R T}
$$

The partial derivatives required in eq. (9) for the approximated diagonal transition matrix elements $Z_{i}$ are given by

$$
\frac{\partial r_{i}}{\partial \sigma_{k}}=\frac{1}{\sigma_{k}} \frac{\partial r_{i}}{\partial \log \sigma_{k}}=\frac{1}{\left(\rho \sigma_{k}\right)} \sum_{j=1}^{J J}\left(\alpha_{i j}^{\prime}-\alpha_{i j}^{\prime \prime}\right)\left(R_{j} \alpha_{k j}^{\prime}-R_{-j} \alpha_{k j}^{\prime \prime}\right)
$$

which may be seen to be related to the second term on the right-hand side of eq. (16).

In order to preserve the absolute A-stability of the implicit formulation and to avoid numerical singularities, the integrating factors for eq. (8) are actually calculated in the code as

$$
w_{i}=\left\{\begin{array}{l}
0.5, z_{i} \leq 0.015 \\
\frac{1}{z_{i}} \frac{1}{\left(e^{z_{i}}-1\right)}, z_{i}>0.015 .
\end{array}\right.
$$

The evaluation of the Jacobian for eq. (10) in eqs. (15), (20) and (22) follow the efficient coding techniques of CREK [6]. As the Jacobian elements contain the expressions required for evaluation of the integrating factors $W_{i}$ (which are regarded as constants during each iteration), little additional computation is required beyond that required by CREK.

\section{Convergence, Accuracy, and Step-Size Control}

Experience to date with the new algorithm has shown it to be remarkably stable and accurate. For all practical purposes, the limiting factor in its use appears to be resolution, rather than stability or accuracy. of course, execution time is directly proportional to the desired resolution, independent of other considerations.

Stability is insured simply by using a modified version of the same "self-adjusting" under-relaxation parameter used in CREK. This technique, originally due to Gordon and McBride [14], calculates under-relaxation parameters designed to assure that, for any iteration,

(i) Major species (mole fraction $>10^{-8}$ ) may not increase by more than a factor $\exp (2)$.

(ii) Minor species (mole fraction $\leq 10^{-8}$ ) may not increase so as to exceed $10^{-4}$ mole fraction.

In the present application, under-relaxation parameters so determined are applied not to mole number and temperature Newton-Raphson corrections at a fixed step size, but rather to the time step size $\tau$ itself. 
In practice, the above criteria insure stability, but do not insure adequate resolution. Therefore, an additional constraint was added:

(iii) The temperature may not change by more than $20^{\circ} \mathrm{C}$.

With this additional constraint, automatic step-size control was easily achieved, and resulted in between 2 and 5 iterations per time step on the test problem considered.

During the development of the present algorithm, both fixed and automatic step-size control were investigated. For fixed step-size integration, the self-adjusting underrelaxation feature was used to under-relax the step size, refining the assigned, fixed step into as many substeps as required to maintain stability of the convergence scheme. An alternate method was explored, using the under-relaxation parameter defined in the preceding paragraph to under-relax the Newton-Raphson corrections at the fixed time step. However, the existence of non-unique solutions [5] led to stably converged, but incorrect, results.

\section{Results of Test Calculation}

As a test case for accuracy and speed, both the new algorithm and the NASA onedimensional code GCKP [1] were utilized to calculate the ignition and subsequent combustion of a stoichiometric mixture of pyrolized (to $\mathrm{H}_{2}$ and $\mathrm{CO}$ ) methane and air, at ten atmospheres and initial temperature of 1000 kelvin. This case was considered by Marteney [15].

Standard or default values of integration parameters for GCKP were utilized for the comparative calculation, except that three values of relative error were employed; these are summarized in table 1 . Also summarized in table 1 are the resulting number of steps, and the total elapsed execution time. All calculations were performed on the University of Utah Computer Center's UNIVAC 1108 computer.

Table 1. Parameters for comparative calculation of ignition and combustion of stoichiometric, pyrolized $\mathrm{CH}_{4}$ /air at $10 \mathrm{~atm}, 1000 \mathrm{~K}$ initial temperature ${ }^{3}$.

$$
\begin{aligned}
& \text { Initial stepsize(s) } \\
& \text { Relative error } \varepsilon \\
& \text { Ignition time }(s) \\
& \quad\left(\Delta T=+1^{\circ} \mathrm{C}\right)
\end{aligned}
$$

Stop calculation (s)

Temperature $(K)$ at

$$
T=1.0-3 \mathrm{~s}
$$

Number of steps

Average iterations/step

Execution time (s)

\begin{tabular}{lcc} 
GCKP $($ Ref. $[1])$ & \\
\hline $1.0 E-6$ & $1.0 E-6$ & $1.0 E-6$ \\
$1.0 E-4$ & $1.0 E-3$ & $1.0 E-2$ \\
$8.1 E-6$ & $8.1 E-6$ & $8.0 E-6$
\end{tabular}

ODCREK

$1.0 \mathrm{E}-6$

see text

8. $3 \mathrm{E}-6$

$\begin{array}{rrrr}1.0 \mathrm{E}-3 & 1.0 \mathrm{E}-3 & 1.0 \mathrm{E}-3 & 1.0 \mathrm{E}-3 \\ 2623 & 2617 & 2607 & 2628 \\ & & & \\ 1592 & 1054 & 704 & 114 \\ 3 & 3 & 3 & 3 \\ 145 & 108 & 76 & 15\end{array}$

${ }^{3}$ Editor's note: From this point on, computer notation is used for exponents, e.g. 1.0E-6 = $1.0 \times 10^{-6}$. 
Table 2 shows temperature and composition calculated by GCKP with assigned maximum relative error $\varepsilon=1 . E-4$. This solution, the most accurate obtained, was used as a standar, of comparison in the absence of an exact solution.

The first column in table 2 presents immediate post-ignition values; at this point, th temperature and most mole fraction profiles are changing rapidly, so that comparisons among solutions are the severest possible. The remaining three columns in table 2 were selected to show development of post-ignition profiles. The last column shows profiles at a time when all profiles are within an order of magnitude of their steady-state or equilibrium values.

Table 2. Temperature (K) and composition (mole fraction) variation with time. Calculated with GCKP [1], relative error $\varepsilon=1.0 \mathrm{E}-4$.

\begin{tabular}{cllll}
$\frac{\text { Time }(\mathrm{s})}{\mathrm{T}}$ & $\frac{1.0 \mathrm{E}-5}{1043}$ & $\frac{5.0 \mathrm{E}-5}{2404}$ & $\frac{1.0 \mathrm{E}-4}{2507}$ & $\frac{1.0 \mathrm{E}-3}{2623}$ \\
$\mathrm{CO}$ & $8.228 \mathrm{E}-2$ & $3.094 \mathrm{E}-2$ & $2.545 \mathrm{E}-2$ & $1.732 \mathrm{E}-2$ \\
$\mathrm{CO}_{2}$ & $1.096 \mathrm{E}-3$ & $6.111 \mathrm{E}-2$ & $6.734 \mathrm{E}-2$ & $7.634 \mathrm{E}-2$ \\
$\mathrm{H}$ & $1.453 \mathrm{E}-2$ & $5.826 \mathrm{E}-3$ & $3.100 \mathrm{E}-3$ & $1.059 \mathrm{E}-3$ \\
$\mathrm{H}_{2}$ & $1.396 \mathrm{E}-1$ & $1.358 \mathrm{E}-2$ & $1.024 \mathrm{E}-2$ & $6.276 \mathrm{E}-3$ \\
$\mathrm{H}_{2} \mathrm{O}$ & $1.960 \mathrm{E}-2$ & $1.612 \mathrm{E}-1$ & $1.684 \mathrm{E}-1$ & $1.769 \mathrm{E}-1$ \\
$\mathrm{~N}$ & $2.108 \mathrm{E}-16$ & $3.168 \mathrm{E}-7$ & $3.973 \mathrm{E}-7$ & $3.257 \mathrm{E}-7$ \\
$\mathrm{NO}$ & $1.078 \mathrm{E}-15$ & $6.257 \mathrm{E}-5$ & $1.926 \mathrm{E}-4$ & $1.579 \mathrm{E}-3$ \\
$\mathrm{~N}_{2}$ & $6.269 \mathrm{E}-1$ & $6.921 \mathrm{E}-1$ & $6.976 \mathrm{E}-1$ & $7.035 \mathrm{E}-1$ \\
0 & $2.034 \mathrm{E}-3$ & $3.717 \mathrm{E}-3$ & $2.147 \mathrm{E}-3$ & $7.796 \mathrm{E}-4$ \\
$\mathrm{OH}$ & $5.506 \mathrm{E}-4$ & $1.284 \mathrm{E}-2$ & $1.071 \mathrm{E}-2$ & $7.134 \mathrm{E}-3$ \\
$\mathrm{O}_{2}$ & $1.134 \mathrm{E}-1$ & $1.861 \mathrm{E}-2$ & $1.477 \mathrm{E}-2$ & $9.083 \mathrm{E}-3$ \\
\hline
\end{tabular}

Table 3 gives percent difference from "most accurate" values of table 2. At time 1.0-5 s, table 3 shows the ODCREK has the greatest temperature difference. This is consistent with the longer ignition time shown in table 1. However, the RMS and maximum differences in mole fractions are still less than GCKP with relative error $\varepsilon=0.01$. This result may be spurious, however, because ODCREK is presently formulated to treat mole numbers less than 1.0-2.0 as equal to 1.0-20; thus predicted minor species mole numbers necessarily differ between GCKP and ODCREK during the early pre-ignition period. 
Table 3. Percent difference from solutions in table 2, for solutions obtained with GCKP with relative errors of $\varepsilon=0.001$ and

0.002 , and with ODCREK

\begin{tabular}{|c|c|c|c|c|c|}
\hline Time (s) & & $1.05-5$ & $5.0-5$ & $1.0-4$ & $1.0-3$ \\
\hline & $\varepsilon=0.001$ & 0.24 & -0.35 & -0.40 & -0.23 \\
\hline \multirow[t]{3}{*}{ Temperature } & $\varepsilon=0.01$ & 1.92 & -0.85 & -0.90 & -0.61 \\
\hline & ODCREK & -2.88 & 0.17 & 0.24 & 0.19 \\
\hline & $\varepsilon=0.001$ & 4.41 & 2.21 & 2.11 & 1.76 \\
\hline \multirow{3}{*}{$\begin{array}{l}\text { Mole fraction } \\
\text { (RMS) }\end{array}$} & $\varepsilon=0.01$ & 41.66 & 5.12 & 5.33 & 4.13 \\
\hline & ODCREK & 34.64 & 0.83 & 1.46 & 2.35 \\
\hline & $\varepsilon=0.001$ & 12.43 & -6.25 & -6.02 & -5.32 \\
\hline \multirow{2}{*}{$\begin{array}{l}\text { Mole fraction } \\
\text { (Maximum) }\end{array}$} & $\varepsilon=0.01$ & 123.90 & -14.40 & -14.28 & -13.02 \\
\hline & ODCREK & -80.66 & 2.16 & 3.89 & 3.93 \\
\hline
\end{tabular}

At longer, post-ignition times, table 3 shows that ODCREK is usually superior to the lower-relative error GCKP results, for RMS difference. Perhaps more significant, where interest in pollutant formation is concerned, is that ODCREK is always superior with respect to maximum difference (almost always $\mathrm{N}$ or $\mathrm{NO}$ in the present computations).

\section{Discussion of Results}

Since GCKP with $\varepsilon=0.0001$ was assumed as the standard for comparison, ODCREK is by definition inferior. However, the close agreement for all species over the entire problem time span (with the exception of the possibly spurious immediate post-ignition results) suggests that ODCREK is in fact of comparable accuracy. The excellent agreement of ODCREK at time 1.0-3 s demonstrates the inherent A-stability of single-step methods [11]. Therefore, the fewer number of steps required, and the resulting order-of-magnitude reduction in execution time, is significant. A further two- to five-fold reduction may be anticipated simply by using more efficient matrix inversion techniques; a simple pivotal Gaussian reduction scheme is presently used in both CREK and ODCREK.

On reviewing the analysis of Liniger and Willoughby [12], it appears that the original cubic spline fit should be re-examined. With exponential fitting to a "tunable cubic" in the same manner as a trapezoid was exponentially-fitted in the present algorithm, it may be possible to further improve accuracy without sacrificing the inherent stability of the trapezaidal approximation. 
Another interesting prospect is the possibility of replacing the temperature $T$ with the time step $\tau$ as independent variable; that is, assigning a fixed increment in $T$ and solving the system of equations for the correct value of $\tau$ (and the corresponding set of mole numbers $\sigma_{k}$ ) which satisfies the new temperature. In this case, $\Delta \log \tau$ replaces $\Delta \log$ $T$ as a correction variable, and corresponding changes in the correction equations are required. In the $i^{\text {th }}$ species correction equation, eq. (15), the last term on the left-hand side becomes

$$
\frac{\partial f_{i}}{\partial \log \tau} \Delta \log \tau,
$$

and the corresponding eq. (18) becomes simply

$$
\frac{\partial f_{i}}{\partial \log \tau}=\frac{\sigma_{i}^{*}-\sigma_{i}}{\left(1-W_{i}\right) \tau}
$$

This formulation allows for great economy in determining an ignition delay or induction time for a given kinetic mechanism. In effect, one simply asks "what is the time interval required for the temperature to rise (say) one degree Celsius?" Of course, an estimate $\tau^{(0)}$ must still be made, and possibly under-relaxation of the correction equations may be required until the correction $\Delta \log \tau$ is iteratively reduced to zero (i.e., the correct value of $\tau$ achieved).

\section{Summary and Conclusions}

A new class of high order, single-step algorithms for integration of batch finiterate, homogeneous kinetics has been described, which appears to offer the following advantages over traditional multi-step methods:

1. Speed. At least comparable, possibly faster or given accuracy and resolution. This follows from the computationally efficient use of the Jacobian matrix both for accuracy and convergence.

2. Accuracy. Variable, up to sixth-order accurate. Permits any degree of accuracy while retaining good convergence properties.

3. Resolution. Extremely flexible with respect to automatic step-size control, by specifying desired resolution of either temperature or species mole number profiles.

In addition, only with a single-step method such as ODCREK is it possible to solve directly for the induction or ignition delay time directly, in a single step. 
While ODCREK was developed to meet the peculiar needs of coalescence/dispersion modeling lof turbulent reacting flows, its power and flexibility merits consideration as a robust, al1-purpose integrator for homogeneous gas-phase chemical kinetics.

Patti L. Case performed the comparison runs with GCKP, and provided valuable technical discussion during the development of ODCREK. Discussions with Bowen, Hindmarsh, and Brandon are gratefully acknowledged.

This work was sponsored by the Kistler Glass Fund of the University of Utah, and by the National Science Foundation, Grant No. ENG-7684533.

\section{References}

[1] Bittker, D. A. and Scullin, V. J., General chemical kinetics computer program for static and flow reactions, NASA TN D-6586 (1972).

[2] Nickerson, G. R., Frey, H. M., and Coats, D. E., Generalized Kinetic Analysis Program, Dynamic Science Inc. (1971).

[3] Dickinson, R. P. and Gelinas, R. J., GENKIN-I, a general atmospheric code for atmospheric applications, Lawrence Livermore Laboratories Report, UCID-16577 (1974).

[4] Young, T. R. and Boris, J. P., A numerical technique for solving stiff ordinary differential equations associated with reactive flow problems, NRL Memorandum Report 2611 (1973).

[5] Pratt, D. T., Calculation of chemically reacting flows with complex chemistry, in Studies in Convection-Vol II, B. F. Launder, ed. (Academic Press, 1976).

[6] Pratt, D. T. and Wormeck, J. J., CREK - a computer program for calculation of combustion reaction equilibrium and kinetics in laminar or turbulent flows, Report WSU-TEL-76-1, Washington State University (1976).

[7] Pratt, D. T., Stock, D. E., and Wormeck, J. J., Non-premixed combustion in a simple shear flow with finite-rate chemistry, Paper No. WSS/CI 75-1, Western States Section/The Combustion Institute (1975).

[8] Wormeck, J. J. and Pratt, D. T., Computer modelling of combustion in a longwell jet-stirred reactor, in Sixteenth Symposium (International) on Combustion (The Combustion Institute, 1977).

[9] Spalding, D. B., A general theory of turbulent combustion. the Lagrangian aspects, AIAA Paper No. 77-141 (1977).

[10] Pratt, D. T., Coalescence/Dispersion Modeling of Gas Turbine Combustors, Project SQUID Workshop on Gas Turbine Combustion, Purdue University, May 31-June 1, 1978.

[11] Lapidus, L. and Seinfeld, J. H., in Numerical Solution of Ordinary Differential Equations (Academic Press, 1971).

[12] Liniger, W., and Willoughby, R. A., Efficient numerical integration of stiff systems of ordinary differential equations, IBM Research Report RL-1970 (1967). 
[13] Brandon, D. M., Jr., A new single-step implicit integration algorithm with A-stability and improved accuracy, Simulation, 17-29 (July 1974).

[14] Gordon, S. and McBride, B., Computer program for calculation of complex chemical equilibrium compositions, NASA SP-273 (1971).

[15] Marteney, P. J., Formation of nitrogen oxides in hydrocarbon-air combustion, Comb. Sci. and Tech. I, No. 6 (1970).

\section{Appendix: Nomenclature}

$B_{j}, B_{-j} \quad$ Exponent in modified Arrhenius pre-exponential factor

$b_{i} \quad k g$-atom number of element $i$ in mixture

$c_{p_{i}} \quad$ Constant-pressure specific heat capacity of ideal gas species $i$

$g_{k}^{0} \quad h_{k}-T_{s_{k}^{0}}^{0}$ one-atmosphere ideal-gas partial molal specific Gibbs function of species $k$

$g_{k} \quad h_{k}-T_{s_{k}}^{0}+R T\left[\log \left(\sigma_{k} / \sigma_{m}\right)+\log \left(P / P_{0}\right)\right]:$ ideal-gas partial molal specific Gibbs function of species $k$

$h_{k} \quad$ Ideal-gas enthalpy of species $k$

JJ The number of distinct chemical reactions in the kinetic mechanism under consideration

$n_{j}^{\prime}, n_{j}^{\prime \prime} \quad n_{j}^{\prime}=\sum_{i=1}^{N S} a_{i j}^{\prime} ; n_{j}^{\prime \prime}=\sum_{i=1}^{N S} a_{i j}^{\prime \prime}:$ molecularity of forward and reverse reactions

j, dimensionless

$\mathrm{N}_{j}, \mathrm{~N}_{-j} \quad$ Exponent of modified Arrhenius pre-exponential factor for forward and reverse reactions $j$

NS The number of distinct chemical species under consideration

P Pressure

$P_{0} \quad$ Pressure of one standard atmosphere: $101,325.0 \mathrm{~N} \mathrm{~m}^{-2}$

$\mathrm{R} \quad$ Universal gas constant: $8,314.3 \mathrm{~J} /(\mathrm{kg}-\mathrm{mol})(\mathrm{K})$

$R_{j}, R_{-j} \quad$ Rate of forward and reverse reactions $j$, respectively

$s_{i}^{0} \quad$ One-atmosphere ideal gas specific entropy of species $i$

$s_{i} \quad$ Ideal gas specific entropy of species $i$ 
Activation temperature (activation energy divided by gas constant) for reaction $j$ in exponential term of modified Arrhenius rate "constant"

$a_{i j}^{\prime}, a_{i j}^{\prime \prime} \quad$ Stoichiometric coefficients for species $i$ : number of $(\mathrm{kg} \cdot \mathrm{moles})_{i}$ in reaction $j$ as a reactant or as a product, respectively Third-body stoichiometric coefficient in reaction $j$; either 0 or 1 Kronecker delta Convergence interval criterion Under-relaxation factor Mole number of species $i$ in mixture NS

$=\sum \sigma_{i}:$ reciprocal of mixture mean molecular weight $i=1$

Mixture ideal-gas mass density, $P /\left(R T \sigma_{m}\right)$

$x_{j}, x_{-j}$ Contact index of forward and reverse reactions $j$ 

National Bureau of Standards Special Publication 561, Proceedings of the 10th Materials Research Symposium on Characterization of High Temperature Vapors and Gases held at NBS, Gaithersburg, Maryland, September 18-22, 1978. Issued October 1979

\title{
MEASURES OF EFFECTIVENESS AND MECHANISMS FOR CHEMICAL AND PHYSICAL INHIBITION OF QUENCHED AND ADIABATIC PREMIXED FLAMES
}

\author{
James J. Reuther ${ }^{1}$ \\ Fuels and Combustion Laboratory \\ Fuel Science Section \\ The Pennsylvania State University \\ University Park, PA 16802
}

This paper is a selective survey of various aspects of homogeneous gas phase flame inhibition. Reviewed are: (a) the criteria which, when observed, indicate that flame inhibition is taking place; (b) how phenomenological changes in physical macrostructure can be used to evaluate inhibitor effectiveness; and (c) how these measures of inhibition, either separately, or in conjunction with chemical microstructure data, can be used to elucidate the mechanisms by which flame inhibitors operate. Chemical and physical inhibition of quenched and adiabatic premixed hydrocarbon/oxidant flames have been characterized in terms of the concept of self-inhibition. Published data are used in this analysis.

\section{Introduction}

The development of techniques for suppressing and preventing unwanted combustion is becoming an increasingly important scientific challenge in today's energy conscious world. In anticipation of extensive handling and use of alternate, coal-derived synfuels, which will pose new problems as far as safety, now is the time for a review of the extent to which the science of conventional fuel combustion/inhibition is understood.

The literature concerning flame inhibition research is extensive, and many surveys have been published $[1-9]^{2}$. Some reviewers have restricted their reports to the assemblage and summary of the facts [1-4,6]. Others, having done the same, used the review article as a vehicle to introduce, and then document, new ideas on various aspects of flame inhibition [5,7-9]. This paper is patterned after the latter type. It is a selective survey which addresses some of the unanswered questions and controversies that exist in flame inhibition research today. The review concerns only the gas-phase inhibition of flames which are already propagating, i.e., ignition effects are not considered.

${ }^{1}$ Assistant Professor of Fuel Science.

${ }^{2}$ Figures in brackets indicate the literature references at the end of this paper. 
The data used in this study were taken entirely from the literature. These published data were re-analyzed in terms of the recently introduced concept of self-inhibition [10]. The method has been extended to include fuel self-inhibition in adiabatic premixed flames [11]. It has also been applied to fuel-lean quenched and adiabatic flames. This has allowed the characterization of oxidant self-inhibition. The concept of self-inhibition is universal in its applicability to gas-phase flame inhibition research, since it can be employed to evaluate both chemical (fuel self-inhibition) flame suppression processes. The general theory of self-inhibition allows much of the data gathered on fuel- and oxidant-rich flames to be used in the investigation of flame inhibition phenomena. On the practical side, this concept points out that studies with non-fuel and non-oxidant inhibitors should be performed only on stoichiometric flames. On the scientific side, the results obtained via the self-inhibition analysis provide evidence which can be used to help resolve some controversial aspects of flame suppression. Throughout this selective review, attention is restricted only toward homogeneous gas-phase inhibition of laminar, quenched and adiabatic premixed flames.

The objective of most flame inhibition research has been to screen various chemical substances in order to measure their ability to inhibit flame propagation. One must study the chemistry of clean, uninhibited flames in order to be able to understand the effects of agents on the combustion process. Since many of these studies have been on non-stoichiometri clean flames, there is a wealth of literature data amenable for analysis in terms of fuel and/or oxidant self-inhibition. Re-evaluation of these published data allows new associations to be made among various types of inhibition measures which could not be made previously. The application of fuel self-inhibition to one set of literature data permits the formulation and demonstration of a new measure of inhibition applicable to constanttemperature quenched flames. This new criterion for characterizing inhibition allows one to reach a subtle, yet significant, conclusion regarding the validity of using non-adiabatic flames as tools in investigations which are directed toward deciphering the chemistry and physics of adiabatic combustion.

After characterizing criteria and measures of inhibition, and how they are useful in ranking inhibitors in terms of effectiveness, the next effort was to explore the extent to which these numerical indices were informative at revealing the mode, i.e., chemical or physical, of action for each inhibitor. The pressure dependence of inhibitor effectiveness also provides evidence regarding the mode of action. When an inhibitor's effectiveness exhibits a pressure dependence, one has information on the specific chemical mechanism which is responsible for the inhibition. The results of this particular investigation point out that these approaches have been under-utilized in flame inhibition research. The lack of attention to these methods has resulted in a major information gap for some of the more practical flame inhibitors, such as $\mathrm{CO}_{2}$ and $\mathrm{H}_{2} \mathrm{O}$.

Finally, where numerical indices fail to provide useful information regarding mechanisms, use is made of flame microstructure and kinetics data in order to identify the channels through which these molecules act to disrupt flame propagation chemistry. This aspect of the investigation dealt with the role of ions in the inhibition of flames by halogenated 
hydrocarbons, which is a controversial matter. It was found that ions do not play a major role in the inhibition mechanism.

\section{Measures of Inhibition}

The chemistry and physics of clean, uninhibited flames can be altered by adding small amounts of certain gases to the unburned gas mixture. If continued addition of one of these materials eventually produces a mixture in which no flame will propagate, the additive is referred to as an extinguishant. When less than the amount of agent required to cause extinction is added to the flame, the resulting phenomenon is called inhibition [8]. In order to characterize the extent to which the flame's propagation process is inhibited upon addition of an agent, one traditionally measures the change in a physical property, e.g. burning velocity, between that in the clean, uninhibited flame and that in the flame which has been doped with agent. The magnitude of the change in burning velocity is used as a measure of inhibitor effectiveness.

A survey of flame inhibition literature reveals that several measures have been developed for the evaluation of the effectiveness of a molecule to inhibit flame propagation $[1,2,8]$. Since laboratory flames can be stabilized for study under either adiabatic [12] or non-adiabatic (quenched) conditions [13], measures of inhibition effectiveness had to be developed for each. All measures of inhibition discussed in this paper will be denoted by the symbol (I). For classification purposes, each symbol (I) will have a superscript and a subscript associated with it. The superscript will denote whether the measure is applicable to either an adiabatic (A) or a quenched $(Q)$ flame. The subscript represents the physical parameter, either burning velocity $(V)$ or maximum flame temperature $(T)$, which is observed to undergo a change upon inhibition. In most inhibition studies, while one of these two parameters is changing, the other is either maintained or assumed to be constant. To i1lustrate this nomenclature, I $I_{T}^{Q}$ represents the temperature change measure of inhibition applicable to constant-velocity quenched flames.

Flame produced by Bunsen burners are generally assumed to be adiabatic, i.e., there is no heat loss to the surroundings [12]. The combustion process occurs at the highest possible temperature and burning-velocity that the initial unburnt gas thermochemistry will permit. The classical criterion for the inhibition of premixed adiabatic flames is the reduction in burning-velocity upon addition of agent [2]. It is generally assumed that this phenomenon takes place at constant temperature, i.e., the adiabatic temperature [14]. The depression of burning-velocity can be characterized in two ways: (a) on the basis of relative reduction in burning-velocity, $V_{i} / V_{0}$, the ratio of the inhibited flame burningvelocity $\left(V_{i}\right)$ to the clean flame burning velocity $\left(V_{0}\right)$ per mole percent inhibitor, [I] $[2,14]$; or $(b)$ by the fractional reduction in burning-velocity, $\left(V_{0}-V_{i}\right) / V_{0}$, per mole percent inhibitor [8]. These measures permitted the comparison of the effectiveness of one inhibitor with another in the same flame system. It did not, however, permit one to reliably compare the effectiveness of one inhibitor in two flames with different fuels. Early screening studies found that an inhibitor would suppress the oxidation of some fuels greatly, 
but others not to the same degree $[2,8,9]$. Because of this situation, Fristrom and Sawyer suggested that the fractional reduction in burning-velocity per unit inhibitor be normalized to the stoichiometric oxygen concentration [8]. This normalized adiabatic measure of inhibition is given by:

$$
I_{V}^{A}=\frac{\left[0_{2}\right]_{s} \Delta V}{[I] V_{0}}
$$

This normalization process permits one to compare results derived from inhibition studies using various experimental techniques and flame systems having different chemistries (i.e., fuels). The dimensionless index given in eq. (1) will be used extensively in this paper to evaluate the effectiveness of inhibitors in adiabatic premixed flames.

The other type of flame available for study in the combustion laboratory is the nonadiabatic, or quenched, flame [13]. Whereas the temperature of an adiabatic flame is a unique function of the state of the unburned gas, the final temperature of a flame burning on a cooled porous-metal burner can, within limits, be fixed independent of the properties of the unburned gas. An excellent description of flame stabilization on quenched burners, adapted from Botha and Spalding [15], is given by Biordi, et al. [16]. A quenched flame burns at maximum temperatures less than the adiabatic flame temperature and at velocities less than the adiabatic burning-velocity. Only in the last two decades has this quenched burner been available to researchers studying the structure of flames [13] and only since 1963 has it been used in studies of flame inhibition [17].

Recently, Kaskan and coworkers introduced a new measure of inhibition applicable to these quenched premixed flames [18]. They found that for constant-velocity quenched flames, inhibition was characterized by a rise in maximum flame temperature. The numerical index for evaluating the effectiveness of inhibitors in quenched flames is given by:

$$
I_{T}^{Q}=\frac{T_{i}^{M A X}-T_{0}^{M A X}}{[I]}
$$

where $T_{0}^{M A X}$ is the clean flame maximum temperature and $T_{i}^{M A X}$ is the inhibited flame maximum temperature. This new measure has been used to demonstrate gas phase inhibition by $\mathrm{CH}_{3} \mathrm{Br}$ [19] and $\mathrm{CF}_{3} \mathrm{Br}$ [20]. Most recently, the reality of this quenched measure was used to demonstrate the concept of fuel self-inhibition in low-pressure, premixed, quenched and fuel-rich constant-velocity hydrocarbon/air flames [10]. In this phenomenon, stoichiometric hydrocarbon/air flames were considered to be the clean, uninhibited flames. During the course of research on nonequilibrium flame chemistry and dissociation, it was observed that as the percentage fuel-richness was increased above stoichiometric, the maximum flame temperature increased above that of the stoichiometric hydrocarbon/air flame. This behavior was recognized as inhibition. Since no other change was made to the constant-velocity quenched flames except the addition of more fuel, it was recognized that the excess fuel, i.e., that above the stoichiometric requirement, was acting to inhibit the flame propagation. Thus, 
the phenomenon was called self-inhibition. Associating the observance of the temperaturerise effect to self-inhibition by hydrocarbon fuel was intuitively sound since it was known that continued increase in the percentage fuel-richness would, eventually, lead to total inhibition, or more properly, extinction of the flame (the fuel-rich flammability limit).

One of the purposes of a recent paper by Biordi, et al. [20], was to correlate the burning-velocity-reduction criterion for inhibition of constant-(adiabatic) temperature flames with the temperature-rise criterion for inhibition of constant-burning-velocity quenched flames. Biordi and coworkers pointed out the fact that Hayes and Kaskan [19] failed to make this same comparison between inhibition measures for $\mathrm{CH}_{3} \mathrm{Br}$ inhibited methane/ air flames, even though all the necessary data were available. Neither Biordi's nor Kaskan's group, however, evaluated $I_{V}^{A}$ and $I_{T}^{Q}$ as per eqs. (1) and (2), respectively. These indices have been calculated for both $\mathrm{CF}_{3} \mathrm{Br}$ and $\mathrm{CH}_{3} \mathrm{Br}$. Table 1 contains the results.

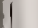

$\begin{array}{lcccccc}\text { Inhibitor } & \text { Flame } & \mathrm{I}_{\mathrm{T}}^{\mathrm{Q}} & \mathrm{I}_{\mathrm{V}}^{\mathrm{A}} & \text { Pressure } & \text { Data sources } \\ & \text { system } & (\mathrm{K}) & & \text { (atm) } & \text { Q } & \mathrm{A} \\ & & & & & & \\ \mathrm{CF}_{3} \mathrm{Br} & \mathrm{CH}_{4} / \mathrm{Air} & - & 18.1 & 1.00 & - & 14 \\ \mathrm{CF}_{3} \mathrm{Br} & \mathrm{CH}_{4} / 0_{2} / \mathrm{Ar} & 156 & 6.3 & 0.04 & 16,20 & 20 \\ \mathrm{CH}_{3} \mathrm{Br} & \mathrm{CH}_{4} / \mathrm{Air} & 226 & 13.0 & 1.00 & 19 & 14\end{array}$

To date, $\mathrm{CF}_{3} \mathrm{Br}$ and $\mathrm{CH}_{3} \mathrm{Br}$ are the only gas phase inhibitors which have the data necessary to permit the comparison between these two inhibition criteria. Biordi, et al., were attempting to link quenched and adiabatic inhibition phenomena as a first step in the formulation of a unified theory of flame suppression. Continued efforts in this direction were halted at this point because of a lack of appropriate data. It is here that the concept of self-inhibition demonstrates its usefulness.

In the paper introducing the concept of hydrocarbon self-inhibition in quenched flames, no attempts were made to relate this phenomenon to adiabatic inhibition [10]. Hydrocarbons were, however, ranked according to their own non-adiabatic combustion. This is illustrated in figure 1. Hydrocarbon self-inhibitor effectiveness can be evaluated by use of the following expression:

$$
I_{T}^{Q}=\frac{T_{\phi>1.0}^{\operatorname{MAX}}-T_{\phi=1.00}^{\operatorname{MAX}}}{[H C]_{\phi>1.0}-[H C]_{\phi=1.00}}=\frac{\Delta T}{[H C]}=K .
$$

Throughout this paper, the equivalence ratio, $\phi$, is defined by: 


$$
\phi=\frac{([\text { Fue }]] /[\text { Air }]) \text { ACTUAL }}{([\text { Fuel }] /[\text { Air }]) \text { STOICHIOMETRIC }}
$$

Numerical values for $I_{T}^{Q}$ are listed in table 2 and, represent the slopes of the straight lines in figure 1. The larger the I $I_{T}^{Q}$ value, the more effective a hydrocarbon is as a selfinhibitor. The decreasing order of effectiveness among hydrocarbons as self-inhibitors is given by the following:

$$
\mathrm{C}_{3} \mathrm{H}_{8}>\mathrm{C}_{2} \mathrm{H}_{6}>\mathrm{C}_{2} \mathrm{H}_{4}>\mathrm{CH}_{4} \quad .
$$

After completing the study of self-inhibited quenched flames, one obvious question remained: did self-inhibition occur in adiabatic hydrocarbon/air flames? To answer this question, one had only to consult tabulations of adiabatic burning-velocities of hydro-

\begin{tabular}{|c|c|c|c|c|c|c|}
\hline $\begin{array}{l}\text { Self- } \\
\text { inhibitor }\end{array}$ & $\begin{array}{l}\text { Flame } \\
\text { system }\end{array}$ & $\begin{array}{c}I_{T}^{Q} \\
(K)\end{array}$ & $I_{V}^{A}$ & $\begin{array}{c}\text { Pressure } \\
(\mathrm{atm})\end{array}$ & $\begin{array}{c}\text { Data } \\
Q\end{array}$ & $\begin{array}{c}\text { sources } \\
\text { A }\end{array}$ \\
\hline $\mathrm{CH}_{4}$ & $\mathrm{CH}_{4} / \mathrm{Air}$ & - & 1.62 & 1.00 & - & $27-22$ \\
\hline $\mathrm{CH}_{4}$ & $\mathrm{CH}_{4} / \mathrm{Air}$ & 50 & - & 0.50 & 23 & - \\
\hline $\mathrm{CH}_{4}$ & $\mathrm{CH}_{4} / \mathrm{Air}$ & 30 & - & 0.25 & 10 & - \\
\hline $\mathrm{CH}_{4}$ & $\mathrm{CH}_{4} / \mathrm{Air}$ & 19 & - & 0.08 & 24 & - \\
\hline $\mathrm{CH}_{4}$ & $\mathrm{CH}_{4} / \mathrm{O}_{2}$ & - & 0.99 & 1.00 & - & 24,25 \\
\hline $\mathrm{CH}_{4}$ & $\mathrm{CH}_{4} / \mathrm{O}_{2}$ & - & 0.50 & 0.50 & - & 24 \\
\hline $\mathrm{C}_{2} \mathrm{H}_{4}$ & $\mathrm{C}_{2} \mathrm{H}_{4} / \mathrm{Air}$ & - & 2.36 & 1.00 & - & 26 \\
\hline $\mathrm{C}_{2} \mathrm{H}_{4}$ & $\mathrm{C}_{2} \mathrm{H}_{4} / \mathrm{Air}$ & - & 1.65 & 1.00 & - & 27 \\
\hline $\mathrm{C}_{2} \mathrm{H}_{4}$ & $\mathrm{C}_{2} \mathrm{H}_{4} / \mathrm{Air}$ & - & 0.88 & 0.50 & - & 27 \\
\hline $\mathrm{C}_{2} \mathrm{H}_{4}$ & $\mathrm{C}_{2} \mathrm{H}_{4} / \mathrm{Air}$ & 40 & 0.54 & 0.25 & 10 & 27 \\
\hline $\mathrm{C}_{2} \mathrm{H}_{6}$ & $\mathrm{C}_{2} \mathrm{H}_{6} / \mathrm{Air}$ & - & 2.83 & 1.00 & - & 25 \\
\hline $\mathrm{C}_{2} \mathrm{H}_{6}$ & $\mathrm{C}_{2} \mathrm{H}_{6} / \mathrm{Air}$ & 47 & - & 0.25 & 10 & - \\
\hline $\mathrm{C}_{3} \mathrm{H}_{8}$ & $\mathrm{C}_{3} \mathrm{H}_{8} / \mathrm{Air}$ & 462 & 6.12 & 1.00 & 28 & 25,29 \\
\hline $\mathrm{C}_{3} \mathrm{H}_{8}$ & $\mathrm{C}_{3} \mathrm{H}_{8} / \mathrm{Air}$ & 62 & 5.53 & 0.25 & 10 & 29 \\
\hline $\mathrm{C}_{3} \mathrm{H}_{8}$ & $\mathrm{C}_{3} \mathrm{H}_{8} / \mathrm{Air}$ & 47 & 4.47 & 0.10 & 10 & 29 \\
\hline
\end{tabular}
carbon/oxidant flames as a function of equivalence ratio $[21,22,25]$ to arrive at the answer. 


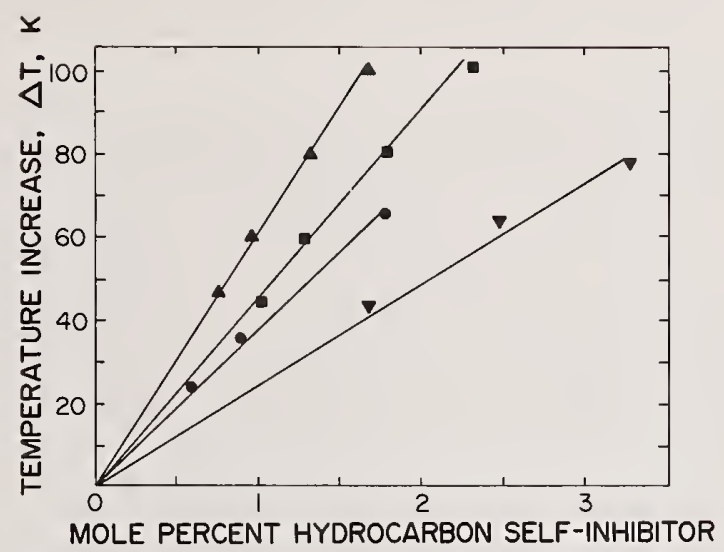

Figure 1. Quenched measure, IQ . Ranking of effectiveness for hydrocarbon selfinhibitors in quenched flames. $\nabla \mathrm{CH}_{4}$ /air flames; $\mathrm{C}_{2} \mathrm{H}_{4} /$ air flames; $-\mathrm{C}_{2} \mathrm{H}_{6} / \mathrm{air}$ flames; $\mathrm{C}_{3} \mathrm{H}_{8}$ /air flames. $P=0.25$ atm (ref. [10]).

Adiabatic burning-velocities for hydrocarbon/oxidant flames have a maximum value centering around stoichiometric. The burning-velocity decreases from this maximum as these flames are made fuel-rich. Thus, the criterion for adiabatic self-inhibition is observed. In order to calculate self-inhibitor effectiveness in terms of $I_{V}^{A}$, one has to assume that the stoichiometric hydrocarbon/oxidant flame is the clean, uninhibited flame. The fact that the maximum adiabatic burning-velocity is centered around stoichiometric supports this assumption. Inspection of hydrocarbon/oxidant adiabatic burning-velocity data vs equivalence ratio indicates that for data analysis purposes, this assumption is a reasonable one. For example, some papers report the maximum adiabatic burning-velocity of a methane/air flame at $\phi=1.0[21,22]$ while another reports it at $\phi=1.1$ [25]. The assumption that there is zero self-inhibitor concentration at the equivalence ratio where the maximum burning-velocity occurs, instead of at $\phi=1.0$, has a negligible effect on the outcome of the results of this study.

With these uncertainties in burning-velocity data in mind, it is appropriate to comment on why the data in papers listed in table 2 were selected for analysis. Instead of an exhaustive critical review, two simple rules were followed in selecting data: (a) only burning-velocity data recommended in published critical reviews were used; and (b) when no such reviews were available, the most recently published data were assumed to be the (best) most reliable. It is also safe to assume that the data gathered in one laboratory is internally consistent, i.e., its behavior versus pressure will produce a reliable trend. $I_{V}^{A}$ coefficients for hydrocarbons as self-inhibitors are presented in table 2 . The larger the value of $I_{V}^{A}$, the more effective a hydrocarbon is at suppressing its own combustion. The results in table 2 show that the normalized burning-velocity criterion for innibition of (constant temperature) adiabatic flames, $I_{V}^{A}$, produces the same ranking of 
effectiveness for hydrocarbon self-inhibitors as does the temperature-rise criterion of inhibition for constant burning-velocity quenched flames, $I_{T}^{Q}$. The ranking is given in eq. (5).

It has been well established that the flame suppression effectiveness of an inhibitor decreases as pressure is reduced $[20,24,30,31]$. This behavior has also been found for propane self-inhibition in quenched flames [10]. Figure 2 illustrates that trend. Data in figure 2 at 0.10 and 0.25 atm were taken from Kaskan and Reuther [10], while the 1.00 atm data were taken from a paper published before the concept of self-inhibition was introduced [28]. This latter set of data represents one of many sets of data from inhibition studies on quenched flames which have never been interpreted in terms of the Kaskan I criterion [32].

Figure 2 demonstrates that the effectiveness of hydrocarbon self-inhibitors in quenched flames is pressure dependent. By replotting some literature data [29], one can show that this is the case for adiabatic hydrocarbon self-inhibition as well. This is given in figure 3. It is evident that the adiabatic self-inhibitor effectiveness of propane decreases as pressure is reduced. By averaging the data over the concentration range given in figure 3 , one can evaluate $I_{V}^{A}$ for propane self-inhibition in adiabatic flames as a function of pressure. The results are listed in table 2; literature data also permit this evaluation to be performed for methane [24,25] and ethylene [27] adiabatic self-inhibition. The average uncertainty in the $I_{V}^{A}$ values tabulated in table 2 is about \pm 10 percent. Although $I_{V}^{A}$ results may vary from study to study at one given pressure (e.g., $\mathrm{C}_{2} \mathrm{H}_{4}$ at $\mathrm{P}=1 \mathrm{~atm}$ ), the important trend with regard to pressure is always the same. To summarize, the quenched and the adiabatic measures of inhibition yield the same ordering of effectiveness (eq. 5) for hydrocarbons as self-inhibitors, regardless of pressure.

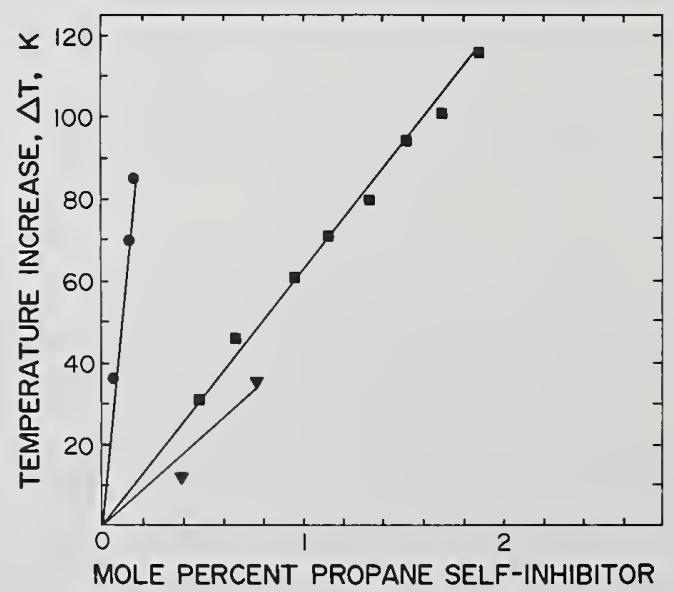

Figure 2. Quenched measure, $I_{T}^{Q}$. Pressure dependence of propane as a self-inhibitor in quenched $\mathrm{C}_{3} \mathrm{H}_{8}$ /air flames. $\nabla P=0.10 \mathrm{~atm}$ and $P=0.025 \mathrm{~atm}$ (ref. [10]); - $P=1.00 \mathrm{~atm}$ (ref. [28]). 


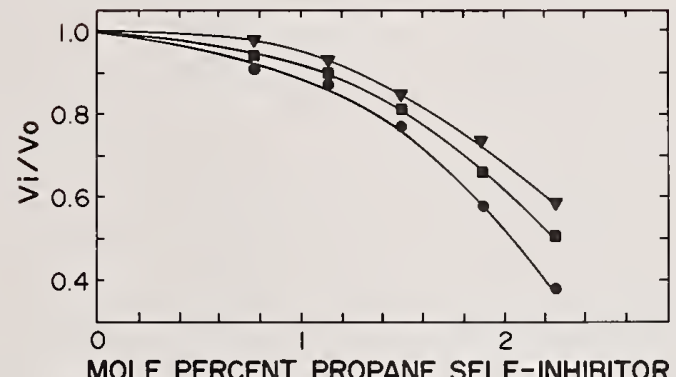

Figure 3. Adiabatic measure, $V_{j} / V_{0}$. Pressure dependence of propane as a selfinhibitor in adiabatic $\mathrm{C}_{3} \mathrm{H}_{8} /$ air flames. $\nabla P=0.10 \mathrm{~atm} ; \quad P=0.25 \mathrm{~atm} ; \quad P=1.00$ atm (ref. [29]).

It should be noted that all the $I_{V}^{A}$ have been evaluated by averaging data over the same range of self-inhibitor concentration given in figure 3 , i.e., at about $\sim 10-40$ percent of the range of between stoichiometric and the fuel-rich flammability limit [25]. The principles of self-inhibition were meant only to describe the chemical behavior of flames doped with this relatively low hydrocarbon self-inhibitor concentration. As Fristrom and Sawyer pointed out [8], in the limit of total inhibition, i.e., extinction, $\Delta V / V_{0}$ in eq. (1) goes to unity and the inhibition index, $I_{V}^{A}$, becomes an extinction index, $I_{E}^{A}$, which is given by the following:

$$
I_{E}^{A}=\frac{\left[0_{2}\right]_{S}}{[I]}
$$

The self-inhibitor concentration at extinction is determined by subtracting the stoichiometric fuel concentration from the fuel concentration at the rich-flammability limit. Values for hydrogen self-extinction coefficients, $I_{E}^{A}$, have been calculated for atmospheric pressure flames from literature data [33], and are listed in table 3 . For reference, $I_{V}^{A}$ coefficients are also tabulated. For the fuel-rich flammability limit to be accountable by the principles of self-inhibition, it would be necessary for the self-extinction coefficients to equal the self-inhibition coefficients. Such is not the case. This exercise only proves again that the chemical processes in near limit flames are very complex. 
Table 3

\begin{tabular}{|c|c|c|c|}
\hline Self-inhibitor & & Inhibition & Extinctio \\
\hline $\begin{array}{c}\text { or } \\
\text { self-extinguishant }\end{array}$ & $\begin{array}{l}\text { Flame } \\
\text { system }\end{array}$ & $\begin{array}{l}\text { index } \\
I_{V}^{A}\end{array}$ & $\begin{array}{c}\text { index } \\
I_{E}^{A}\end{array}$ \\
\hline $\mathrm{CH}_{4}$ & $\mathrm{CH}_{4} / \mathrm{Air}$ & 1.62 & 3.44 \\
\hline $\mathrm{C}_{2} \mathrm{H}_{4}$ & $\mathrm{C}_{2} \mathrm{H}_{4} / \mathrm{Air}$ & 2.36 & 0.77 \\
\hline $\mathrm{C}_{2} \mathrm{H}_{6}$ & $\mathrm{C}_{2} \mathrm{H}_{6} / \mathrm{Air}$ & 2.83 & 2.94 \\
\hline $\mathrm{C}_{3} \mathrm{H}_{8}$ & $\mathrm{C}_{3} \mathrm{H}_{8} / \mathrm{Air}$ & 6.12 & 3.65 \\
\hline
\end{tabular}

Comparisons between $I_{V}^{A}$ and $I_{T}^{Q}$ are not as meaningful as they could be since one is comparing inhibition indices which employ a different parameter changing upon inhibition. If one could incorporate the same phenomenological physical change into measures of inhibition applicable to both adiabatic and quenched flames, one would have a basis for direct comparison of inhibitor effectiveness in these two types of flames. As previously mentioned, the properties of a quenched flame are a maximum temperature and mass burningvelocity less than that achievable under adiabatic conditions. These two parameters are dependent on each other [13]. The measure of inhibition applicable to quenched flames was the temperature-rise in constant-velocity combustion. A fundamental question is what effect does an inhibitor have on a constant-temperature quenched flame, i.e., how is its burningvelocity altered upon inhibition? There are no reports of conventional flame inhibition research using these experimental conditions. There are literature data on fuel-rich propane/oxygen/nitrogen flames, however, which, when re-evaluated in terms of fuel selfinhibition, provide an answer to this question. Durie, et al. [34], studied $\mathrm{H}$-atom recombination in a series of premixed, $\mathrm{C}_{3} \mathrm{H}_{8} / \mathrm{O}_{2} / \mathrm{N}_{2}$ isothermal $(2110 \mathrm{~K}$ ) flames over a range of equivalence ratios from stoichiometric to 40 percent fuel-rich. As the fuel-richness of the flame was increased from stoichiometric, the unburned gas flow velocity had to be increased in order to maintain a constant maximum flame temperature $(2110 \mathrm{~K})$. This phenomenological change represents a new criterion for fuel self-inhibition, and defines a new measure. The relative basis for this new measure is the analogue to the temperature-rise effect in constant-velocity quenched flames, ${ }_{T}^{Q}$ : inhibition in constant-temperature quenched flames is characterized by a velocity-rise, $\mathrm{I}_{V^{-}}^{Q}$ In figure 4 , adopted from Kaskan and coworkers [18], a comparison is made between the temperature-rise criterion, $I_{T}^{Q}$, and the velocity-rise criterion, $I_{V}^{Q}$, for self-inhibited constant-velocity and constant-temperature quenched propane/oxygen/nitrogen flames, respectively. The locus of clean flames (stoichiometric $=$ no self-inhibitor) is given by the straight line. Velocity- and temperaturerises should originate from this line. The literature data [34] for the new velocity-rise measure do, while literature data for the temperature-rise [10] do not. This is because the 
latter $I_{T}^{Q}$ data were for self-inhibited 0.25 atm flames [10], while the stoichiometric burningvelocity versus temperature data were from atmospheric pressure flames [13]. The trends are correct, however.

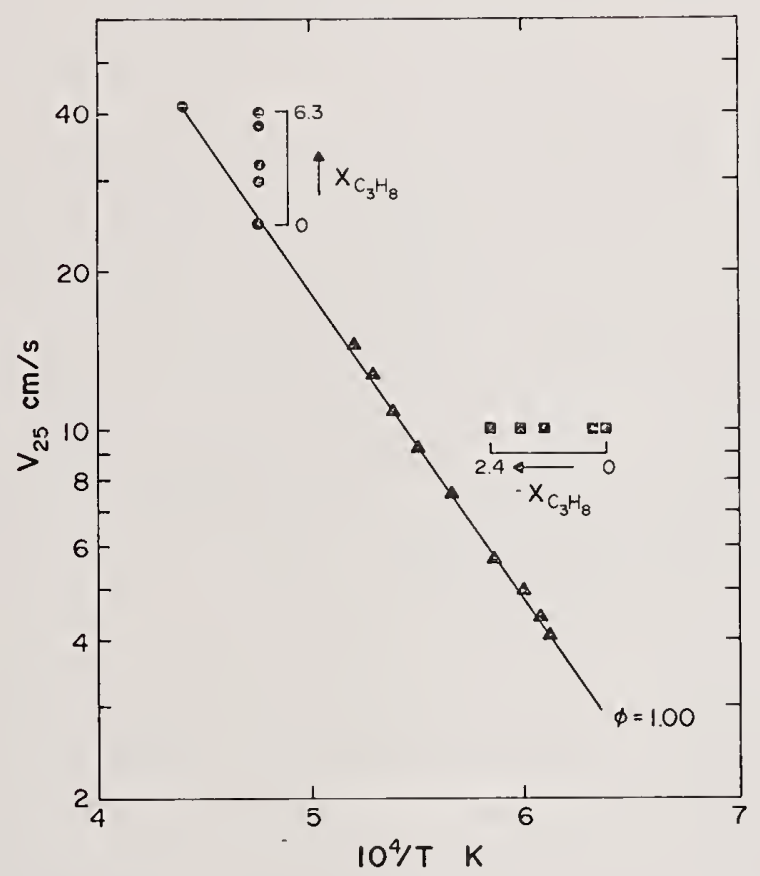

Figure 4. Quenched measures. Comparison of temperature-rise, $I_{T}^{Q}$, and velocityrise, $I_{V}^{Q}$, upon self-inhibition of constant-velocity and constant-temperature quenched $\mathrm{C}_{3} \mathrm{H}_{8} / \mathrm{O}_{2} / \mathrm{N}_{2}$ flames res.. Mass burning velocity, $V_{25}$ (at $\mathrm{T}=25^{\circ} \mathrm{C}$ ), as a function of reciprocal maximum flame temperature, T. - Locus of quenched, clean flames as determined by data $\boldsymbol{\Delta}$ on stoichiometric $(\phi=1.00)$ $\mathrm{C}_{3} \mathrm{H}_{8} / \mathrm{O}_{2} / \mathrm{N}_{2}$ flames at $\mathrm{P}=1.00 \mathrm{~atm}$ (ref. [13]). $\theta$ (at $\sim 40 \mathrm{~cm} / \mathrm{s}$ ) adiabatic flame temperature and adiabatic burning-velocity at $\phi=1.00$. $\square I_{T}^{Q}$ for propane self-inhibition in $\mathrm{C}_{3} \mathrm{H}_{8} / \mathrm{O}_{2} / \mathrm{N}_{2}$ flames at $P=0.25 \mathrm{~atm}$ (ref. [10]). - IQ for propane self-inhibition in $\mathrm{C}_{3} \mathrm{H}_{8} / \mathrm{O}_{2} / \mathrm{N}_{2}$ flames at $1.00 \mathrm{~atm}$ (ref. [34]). Subplots indicate mole percent, $\mathrm{X}_{\mathrm{C}_{3}} \mathrm{H}_{8}$, of propane self-inhibitor acting to produce the phenomenological change which characterizes the quenched flame inhibitor measures.

This new measure of inhibition for quenched flames is very useful because it allows a more direct correlation between adiabatic, $I_{V}^{A}$, and quenched, $I_{V}^{Q}$, measures, since both are now expressed in terms of a velocity change upon inhibition. These relative velocity changes, $V_{i} / V_{0}$, for adiabatic and non-adiabatic self-inhibited propane/oxygen/nitrogen flames are given in figure 5 . One can calculate the quenched-flame complement of $I_{V}^{A}$ in eq. (1) by the following: 


$$
I_{V}^{Q}=\frac{\left[0_{2}\right]_{s}\left(V_{i}-V_{0}\right)}{V_{0}[I]}
$$

The results are $I_{V}^{Q}=8.05$ and $I_{V}^{A}=6.12$. One interpretation of this information is that it tells us that, in flame inhibition research, one can take advantage of the favorable geometry and stability offered by quenched flat-flame burners without fear that the non-adiabaticity of the system is dramatically altering the results. In only one other study has a comparison been made between the quenched and adiabatic, chemical and physical microstructure of the same fuel/oxidant flame [35]. Smoot found that a significant part of the difference in the adiabatic equilibrium temperature and non-adiabatic outlet flame temperature was due to lack of complete reaction, and not entirely to heat loss [35]. Comparing $I_{V}^{Q}$ with $I_{V}^{A}$ is another waj of characterizing this effect because burning-velocity is actually an overall combustion rate [13]. $I_{V}^{Q}$ is greater than $I_{V}^{A}$ because the quenched flame is already partially inhibited by the effect of non-adiabaticity, which has slowed down the overall combustion process. History bears out the fact that this effect, although observable, is negligible. It certainly has not hindered combustion scientists from learning a great deal about flame chemistry through the use of quenched burners!

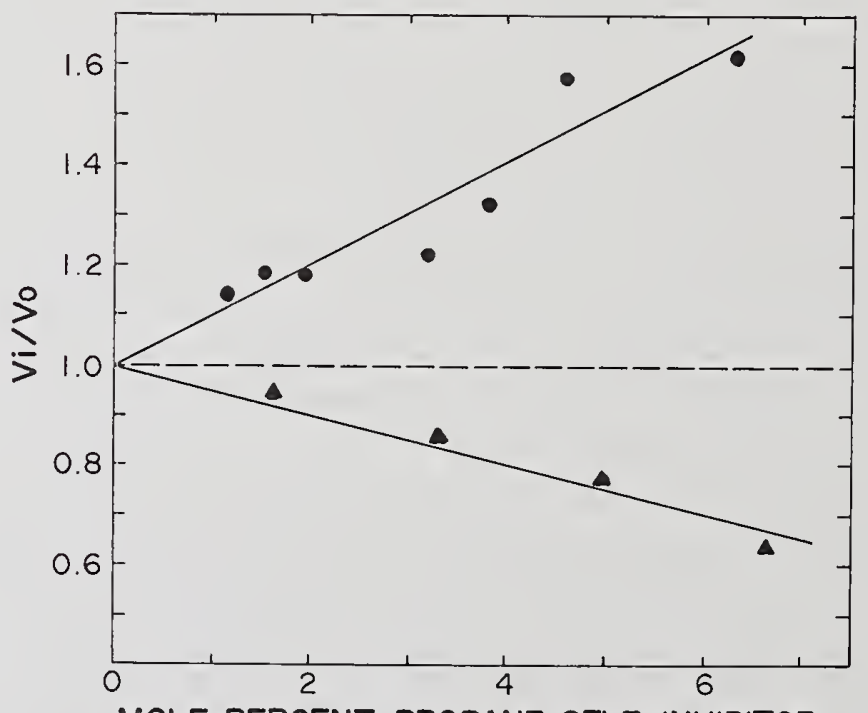

MOLE PERCENT PROPANE SELF-INHIBITOR

Figure 5. Quenched and adiabatic measures, $V_{i} / V_{0}$. Direct comparison of adiabatic and quenched measures of inhibition using the relative velocity change as an index of inhibition. Propane self-inhibition in $\mathrm{C}_{3} \mathrm{H}_{8} / \mathrm{O}_{2} / \mathrm{N}_{2}$ flames. $P=1.00 \mathrm{~atm}$. - Quenched flame velocity-rise data, $I_{V}^{Q}$ (ref. [34]). $\Delta$ Adiabatic flame velocity-reduction data, $I_{V}^{A}$ (ref. [25]). 
With regard to this new measure of inhibition, $I_{V}^{Q}$, one may be puzzled about how the burning-velocity can increase in a series of constant-temperature flames. The answer will be reserved until the following section, which explores whether measures of inhibition can provide more information about the inhibition process than just a ranking of effectiveness.

\section{Modes of Inhibition}

Early flame inhibition research discovered that some inhibitors were more effective than others, no matter what measure of inhibition was used $[1,2,8,9]$. The objective of much modern flame inhibition research is to discover why this is so.

From 1950 [1] until 1974 [36-38], it was generally accepted that there were two separate modes of flame inhibition, one chemical and the other physical. For molecules which fragment and become chemically involved in flames, such as halogenated hydrocarbons and hydrocarbon self-inhibitors, inhibition is thought to proceed because reactions important to flame propagation are disrupted upon addition of agent. For species which have been assumed to be chemically inert in flames, such as nitrogen, carbon dioxide, water, and the noble gases, inhibition is thought to occur because the physics of the flame, i.e., flame temperature, is altered. Previous studies found that a greater percent by volume of inert additive, compared to a chemically active one, was required to inhibit or extinguish a flame. For example, the $\mathrm{I}_{\mathrm{V}}^{\mathrm{A}}$ for $\mathrm{CH}_{3} \mathrm{Br}$ [14], $\mathrm{H}_{2} \mathrm{O}$ [39] and $\mathrm{N}_{2}$ [39] inhibition of atmospheric methane flames are $13.0,0.64$, and 0.48 , respectively. This relative degree of effectiveness led to the hypothesis of two separate modes. In a series of publications, which began in 1974, Larsen challenged the traditional concept of chemical and physical inhibition. Having reevaluated flammability peak data (a measure of total inhibition, or extinction, defined as the concentration of an agent which represents the minimum concentration of agent required to prevent flame propagation through any and all fuel/oxidant mixtures) on a weight [36] and heat capacity $[37,38]$ basis, Larsen discovered that chemical and physical additives were nearly equal in their ability to suppress flames. Without elaborating on the details of Larsen's index, $k C p$, and how it was calculated, suffice it to say that the effectiveness coefficient for all inhibitors, chemically active or inert, fell in the range of $80 \pm 3$ percent [38]. This finding led Larsen to proclaim that all postulated chemical reaction mechanisms for flame suppression by chemically active agents were unfounded. Larsen contended that any inhibitor's effectiveness was adequately described by its thermal dilution mechanism and, therefore, the primary mode of all inhibition was physical [38].

At the time, Larsen's findings appeared to be significant, and therefore, offered a formidable challenge to flame inhibition researchers, this author included, who had supported or contributed to the concept of inhibition via chemical processes. Larsen's position was immediately challenged by Dehn and a lively discussion resulted [38]. The controversy was ignored until a Combustion Institute meeting in November 1977, when researchers from two different laboratories argued in support of the existence of two modes of inhibition $[11,40]$. One paper began by stating that Larsen had not provided all the valid and convincing evidence with which to void postulation of chemically induced inhibition [11]. 
The paper presented important cases where the Larsen $k C p$ calculation failed to produce the $80 \pm 3$ value for the index when applied to literature data. These exceptions, which were for conventional inhibitors $\left(\mathrm{CO}_{2}\right.$ and $\left.\mathrm{N}_{2}\right)$, are listed in table 4 . The flammability peak had never been evaluated via the Larsen method in terms of the concept of self-inhibition. This has been done, and more exceptions to Larsens $80 \pm 3$ percent rule were found. They are also listed in table 4.

\begin{tabular}{cccc|}
\hline & & Table 4 \\
Inhibitor & $\begin{array}{c}\text { Flame } \\
\text { system }\end{array}$ & $\begin{array}{c}\text { Larsen index } \\
k C p \%\end{array}$ & Data source \\
& & & \\
$N_{2}$ & $\mathrm{H}_{2} / \mathrm{Air}$ & 90 & 33 \\
$\mathrm{CO}_{2}$ & $\mathrm{H}_{2} / \mathrm{Air}$ & 87 & 33 \\
$\mathrm{CH}_{4}$ & $\mathrm{CH}_{4} / 0_{2}$ & & 25 \\
$\mathrm{C}_{2} \mathrm{H}_{4}$ & $\mathrm{C}_{2} \mathrm{H}_{4} / \mathrm{O}_{2}$ & 47 & 25 \\
$\mathrm{C}_{2} \mathrm{H}_{6}$ & $\mathrm{C}_{2} \mathrm{H}_{6} / 0_{2}$ & 80 & 25 \\
$\mathrm{C}_{3} \mathrm{H}_{8}$ & $\mathrm{C}_{3} \mathrm{H}_{8} / \mathrm{O}_{2}$ & 70 & 25 \\
\hline
\end{tabular}

Since exceptions do not break rules, it was essential that Larsen's unified theory for physical inhibition be challenged on more fundamental principles. It soon became evident that the Larsen criterion could not satisfactorily account for one important phenomenological aspect of flame inhibition: some molecules have an effectiveness which is pressure dependent [11]. As indicated previously, many researchers have found that the flame suppressing effectiveness for certain chemically active inhibitors decreases as pressure is reduced $[20,24,30,31]$. Additional cases, in terms of conventional and self-inhibitors are listed in tables 1 and 2 . The Larsen postulate cannot account for this behavior, as one would not expect the thermal dilution capacity of a molecule, and hence, its inhibitor effectiveness, to diminish as pressure is reduced.

Sheinson and coworkers have provided new data which supports the conclusion that flame suppression is achieved via both chemical and physical alteration of combustion [40]. They have also developed a method for quantifying the two effects.

As should be evident from the discussion above, there never seemed to be any reason to doubt that chemically active flame suppressants could derive their high inhibitory efficiency from their ability to disrupt chemical reactions essential to flame propagation. Because of their high effectiveness, chemically active materials have been studied fairly extensively [1-9]. Inert diluents, on the other hand, have been important from a scientific point of view only because they provide a comparison which helps to separate physical effects from chemical effects. From the practical point of view, no research has ever been needed to 
prove that physical diluents 1 ike $\mathrm{CO}_{2}$ and $\mathrm{H}_{2} \mathrm{O}$ work effectively in practical fire extinguishants.

There have been some reports dealing with the effect of physical inhibitors on adiabatic burning-velocities in flames. In review papers, $I_{V}^{A}$ data have been evaluated for them $[8,9]$. Not all the experimental data have, however, been evaluated in terms of this index of inhibition [39,41-43]. These new $I_{V}^{A}$ data are given in table 5. All were determined with atmospheric stoichiometric flames. The values of $\mathrm{I}_{\mathrm{V}}^{A}$ for $\mathrm{H}_{2} \mathrm{O}$ are quite scattered, in spite of the fact that they were calculated from the normalized adiabatic measure of inhibition [8]. This uncertainty is most probably the result of experimental errors, since experiments using water vapor are difficult [41]. On the other hand, these data may indicate that the Fristrom and Sawyer normalized figure of merit for adiabatic inhibitor effectiveness [8] may not permit comparison of results from differently fueled flames.

Table 5

$\begin{array}{cccc}\text { Inhibitor } & \begin{array}{r}\mathrm{Flame} \\ \text { system }\end{array} & \mathrm{I}_{\mathrm{V}}^{\mathrm{A}} & \text { Data source } \\ & \mathrm{CH}_{4} / \mathrm{Air} & 0.64 & 39 \\ \mathrm{H}_{2} \mathrm{O} & \mathrm{C}_{2} \mathrm{H}_{4} / \mathrm{Air} & 0.21 & 41 \\ \mathrm{H}_{2} \mathrm{O} & \mathrm{C}_{3} \mathrm{H}_{8} / \mathrm{Air} & 0.96 & 42 \\ \mathrm{H}_{2} \mathrm{O} & \mathrm{C}_{3} \mathrm{H}_{8} / \mathrm{Air} & 0.30 & 41 \\ \mathrm{H}_{2} \mathrm{O} & \mathrm{CH}_{4} / \mathrm{Air} & 0.48 & 39 \\ \mathrm{~N}_{2} & \mathrm{C}_{6} \mathrm{H}_{14} / \mathrm{Air} & 0.86 & 43 \\ \mathrm{CO}_{2} & & \end{array}$

The concept of self-inhibition allows the continuation of this investigation into inhibition by physical, inert agents. If it were not for this concept, further analysis would cease because of the paucity of data. As previously mentioned, the maximum burningvelocity of an adiabatic hydrocarbon/air flame is centered about stoichiometric [25]. Addition of excess air above the stoichiometric concentration causes a reduction in burningvelocity [25]. This is the criterion for adiabatic inhibition [8]. Since no other change is made to the flame except the addition of more oxidant, this phenomenon is called oxidant self-inhibition. It is the complement of fuel self-inhibition. For oxidant selfinhibition, it is assumed that the stoichiometric fuel/oxygen flame is the clean, uninhibited flame. Continued increase in the percentage oxygen richness leads to total inhibition, or extinction (the oxidant-rich flammability limit). Since air is a mixture of nitrogen and oxygen, one can only evaluate the oxidant self-inhibitor effectiveness for the mixture of these inert gases. The individual effectiveness of oxygen and nitrogen can, however, be determined separately. For oxygen, one uses data which characterizes the 
burning-velocity reduction upon addition of excess oxygen to a hydrocarbon/oxygen flame. The effectiveness of nitrogen as a physical inhibitor can be evaluated by considering the reduction in adiabatic burning velocity when the stoichiometric oxidant is changed from oxygen to air. If the "air $\mathrm{N}_{2}$ " component (79 percent) is replaced by some inert gas other than nitrogen [13], then the inhibitor effectiveness for that gas can be evaluated as well. The appropriate criteria for both adiabatic and quenched oxidant self-inhibition have been observed [16,21-25,44-48]. Data have been evaluated in terms of the adiabatic measure, $I_{V}^{A}$, and are listed in table 4 . The data on the temperature-rise upon air selfinhibition of a constant-velocity quenched atmospheric methane/air flame yields an I ${ }_{T}^{Q}$ of $82 \mathrm{~K}[23]$.

In order to appreciate the significance of the new results reported in tables $1-3,5$ and 6 , one must now correlate the phenomenological changes in physical macrostructure to detailed changes in chemical microstructure.

\section{Mechanisms for Chemical and Physical Inhibition}

For species which are assumed to be chemically inert in flames, such as $\mathrm{N}_{2}, \mathrm{CO}_{2}, \mathrm{H}_{2} \mathrm{O}$, or the noble gases, inhibition is thought to proceed via a thermal dilution mechanism. Because of its high heat capacity, the physical diluent acts as an inert heat sink in the flame and, therefore, reduces the flame temperature. When the flame temperature is lowered, the chemical reaction rate is reduced through decreases in both the specific reaction rate and in the radical concentrations [8].

\section{Table 6}

\begin{tabular}{|c|c|c|c|c|}
\hline Inhibitor & $\begin{array}{r}\text { Flame } \\
\text { system }\end{array}$ & $I_{V}^{A}$ & $\begin{array}{l}\text { Pressure } \\
\text { atm }\end{array}$ & Data sources \\
\hline $\mathrm{N}_{2}$ & $\mathrm{H}_{2} / \mathrm{O}_{2}$ & 0.53 & 1.00 & $44-47$ \\
\hline $\mathrm{N}_{2}$ & $\mathrm{H}_{2} / \mathrm{O}_{2}$ & 0.45 & 0.09 & 48 \\
\hline $\mathrm{Ar}$ & $\mathrm{H}_{2} / \mathrm{O}_{2}$ & 0.49 & 1.00 & $44-45$ \\
\hline Ar & $\mathrm{H}_{2} / \mathrm{O}_{2}$ & 0.42 & 0.09 & 48 \\
\hline $\mathrm{O}_{2}$ & $\mathrm{CH}_{4} / \mathrm{O}_{2}$ & 0.78 & 1.00 & 24 \\
\hline $0_{2}$ & $\mathrm{CH}_{4} / \mathrm{O}_{2}$ & 0.69 & 0.50 & 24 \\
\hline Ar & $\mathrm{CH}_{4} / \mathrm{O}_{2}$ & 0.67 & 1.00 & 45 \\
\hline Ar & $\mathrm{CH}_{4} / \mathrm{O}_{2}$ & 0.44 & 0.04 & 16,25 \\
\hline Air & $\mathrm{CH}_{4} / \mathrm{Air}$ & 1.54 & 1.00 & 21,22 \\
\hline Air & $\mathrm{C}_{3} \mathrm{H}_{8} / \mathrm{Air}$ & 5.78 & 1.00 & 25 \\
\hline
\end{tabular}


For molecules which fragment and become chemically involved in flames, such as hydrocarbon self-inhibitors or halons, inhibition is believed to occur because the fragments of the parent inhibitor interfere with the normal, clean flame, chemical reactions responsible for propagation of the combustion. Inhibition is believed to proceed via mechanisms which, compared to clean flames, result in either enhanced radical recombination rates in the postflame-gas zone [10a] or retardation of radical production rates in the pre-flame or flame zone [10b], or both. The inhibitor (or fragment) may react with a radical in a chainbranching step or may compete for a crucial radical in a non-branching step [5].

In the past, measures of inhibition, particularly $I_{V}^{A}$, have been used to classify inhibitors according to mode of action $[1,8,9]$. Hastie [9], states that an $I_{V}^{A}$ figure of merit greater than unity can only be explained in terms of chemical effects; an $I_{V}^{A}$ coefficient less than unity is explainable in terms of a physical effect such as cooling [9]. Until now, there were no violations to this empirically based "rule" [8,9]. Air has a self-inhibitor effectiveness in adiabatic flames greater than unity, as listed in table 6. By comparing results in table 2 with that in table 6 , one finds that air self-inhibitors are nearly as effective as fuel self-inhibitors. The data on air self-inhibition indicate that there may be a minor chemical component to the suppression mechanism by this mixture of inert gases. The stimulus for this present study was the fact that analogous behavior had been reported for two other physical-type, inert diluents, $\mathrm{CO}_{2}$ and $\mathrm{H}_{2} \mathrm{O}$. Recent papers have reported that the inhibitor effectiveness of $\mathrm{H}_{2} \mathrm{O}[40,41]$ and $\mathrm{CO}_{2}$ [40] could not be explained solely on their thermal dilution capacity. The next section discusses whether or not the new results reported in tables 5 and 6 are of any value in resolving these contradictions.

The numerical values of $\mathrm{I}_{\mathrm{V}}^{\mathrm{A}}$ for $\mathrm{H}_{2} \mathrm{O}, \mathrm{CO}_{2}, \mathrm{~N}_{2}, \mathrm{Ar}$, and $\mathrm{O}_{2}$ listed in tables 5 and 6 indicate, by the Hastie "rule" [9], that these gases act as thermal diluents in suppressing flames. However, the pressure dependence of inhibitor effectiveness for $\mathrm{N}_{2}, \mathrm{Ar}$, and $\mathrm{O}_{2}$ indicates otherwise. These results, in table 6 , demonstrate that the inhibitor effectiveness for these molecules decreases as pressure is reduced. Since one would not expect the thermal dilution capacity to diminish as pressure is reduced, some other mechanism for inhibition, one dependent on pressure, must be in operation. The simple process of elimination indicates that this mechanism must be chemical in nature. Chemical inhibition mechanisms consist of bimolecular and/or termolecular steps $[5,8,10]$. If the inhibition process were a bimolecular event, as in the interference of a chain-branching step [8], one would expect very little or no pressure dependence. If termolecular processes are dominant in the inhibition mechanism, the effectiveness should decrease as pressure is reduced because of the fact that three-body collisions would become less frequent. Hence, the observation of a pressure dependent inhibitor effectiveness for $\mathrm{N}_{2}, A r$, and $\mathrm{O}_{2}$ is convincing evidence that these molecules induce inhibition, in part, via a chemical mechanism. The results in table 6 demonstrate for the first time that these inert inhibitors have a chemical component in their flame suppression mechanism, i.e., they act to enhance rates of radical recombination, as compared to those rates in clean flames. 
Chaperon efficiencies are useful in comparing the abilities of these molecules to act in a chemical mode. For atmospheric flames, the order of decreasing effectiveness for $\mathrm{N}_{2}$, $0_{2}$, and $\mathrm{Ar}$ is similar to that trend given by available third-body efficiencies [49].

The results in table 6 are unique. They represent the only attempt at determining the pressure dependence of the inhibitor effectiveness of physical-type suppressants in premixed flames. It should be noted that the concept of oxidant self-inhibition applied to data from various laboratories provided this information. This behavior should be confirmed by direct measurements.

The question of whether or not $\mathrm{CO}_{2}$ and $\mathrm{H}_{2} \mathrm{O}$ have a chemical component to their inhibition mechanism appears to be still unanswerable at this point since the concept of selfinhibition can be of no help. Flame inhibition specialists should not, however, be too surprised at the recent findings of Sheinson, et al. [40], and Müller-Dethlefs and Schlader [41] for $\mathrm{CO}_{2}$ and $\mathrm{H}_{2} \mathrm{O}$, respectively. If they are, it is because of the fact that reviews on the subject of the inhibitory action of $\mathrm{CO}_{2}$ and $\mathrm{H}_{2} \mathrm{O}$ have often not been careful enough in using the lables "inert" and "physical class" inhibitor [5,8,9,36-38]. To illustrate this point, some early (and fairly recent) research which appears to have been forgotten or overlooked will be surveyed. First, the case for $\mathrm{CO}_{2}$.

In 1950, Arthur [50] concluded that $\mathrm{CO}_{2}$ molecules were able to assist in the removal of $\mathrm{H}$-atoms via both bimolecular and termolecular events. More than 25 years ago, Wheatley and Linnett [51] concluded that $\mathrm{CO}_{2}$ could interfere with the propagation of combustion reactions and, thereby, retard the burning-velocities of acetylene/air flames. In 1956, Kaskan [13] found anomalous behavior for $\mathrm{CO}_{2}$ in hydrogen/air flames which he suggested was evidence in favor of disqualifying $\mathrm{CO}_{2}$ as an inert gas. This background information makes the Sheinson and coworkers' results [40] all the less surprising.

$\mathrm{H}_{2} \mathrm{O}$ has a similar history. The early research of Garner, et al., [42], prompted Friedman and Levy [26] to state in their review paper that "...it seems permissable to class the widely used extinguishing agent, $\mathrm{H}_{2} \mathrm{O}$, as a chemical agent." Twice since this 1958 review have other researchers $[39,52]$ provided evidence which supported the idea that $\mathrm{H}_{2} \mathrm{O}$ was not "inert" in flames. Thus, the recent results [41] which indicate that $\mathrm{H}_{2} \mathrm{O}$ does not act strictly as an inert heat sink are not unique.

To the best of this author's knowledge, no experiments have ever been performed to straightforwardly measure the inhibitor effectiveness of $\mathrm{CO}_{2}$ and $\mathrm{H}_{2} \mathrm{O}$ as a function of pressure in premixed flames. These experiments are planned for the near future for this laboratory. These experiments should provide valuable information on the subject of inhibition by practical extinguishants. It appears that some valuable information on $\mathrm{CO}_{2}$ has been overlooked, information which would allow one to give indirect evidence in favor of the capacity of $\mathrm{CO}_{2}$ to act in the chemical inhibition mode. The information can be extracted from published stoichiometric hydrogen/air quenched flame data [13]. These data are listed in table 7 . When the nitrogen in the combustion air is replaced by $\mathrm{CO}_{2}$, there is an observable increase in the "apparent" activation energy for the global $\mathrm{H}_{2} / \mathrm{O}_{2}$ combustion process. This increase is not observed when the air nitrogen is replaced by argon or helium. The addition of $\mathrm{CO}_{2}$ clearly affects the chemistry. 
Table 7

$$
\mathrm{H}_{2} \text { /air flames } \quad P=1 \text { ATM; } \phi=1.00
$$

$$
\begin{array}{cc}
\text { "Air"* } & \text { Apparent global activation energy } \\
\left(\mathrm{O}_{2}+\mathrm{diluent}\right) & (\mathrm{kcal} / \mathrm{mol})
\end{array}
$$

$\begin{array}{ll}\mathrm{He} & 44 \\ \mathrm{CO}_{2} & 59 \\ \mathrm{~N}_{2} & 47 \\ \mathrm{Ar} & 44\end{array}$

$$
\text { *"Air" }=0.21\left[\mathrm{O}_{2}\right]+0.79[\mathrm{Diluent}]
$$

No firm conclusions can be drawn from this literature survey-except that researchers should be cautious when describing the mode of inhibition for $\mathrm{CO}_{2}$ and $\mathrm{H}_{2} \mathrm{O}$. Since these two molecules are by far the most widely used fire extinguishants, new research on their inhibition effectiveness is warranted.

From the preceding exercise, it is apparent that indices of inhibition and their pressure dependence do not provide all the information necessary to understand the mechanisms of flame inhibition. A case is point is the velocity-rise criterion for the inhibition of constant-temperature quenched flames, given in figures 4 and 5 . In order to understand why this phenomenon is observed, one has to examine both the chemical (species concentration) and physical (temperature) ${ }^{3}$ microstructure profiles for the clean and the inhibited flames.

Although the data of Durie and coworkers [34] permitted the demonstration of the new measure of inhibition applicable to quenched flames, $I_{V}^{Q}$, they are not sufficient to explain this effect. A survey of the literature reveals that the velocity-rise effect upon increased fuel input to constant-temperature quenched flames had been discussed by Fenimore and Jones [53]. Using chemical microstructure profiles, these researchers concluded that this effect was taking place because the excess fuel was delaying the normal overall flame reactions in the pre-flame gas zone. This delay causes the radical species profiles to shift to new positions with respect to the temperature profiles. Having the maximum radical concentration occur at a higher temperature causes the maximum overall reaction rate to increase, even though the maximum flame temperature is held constant. The increase in local reaction rate causes the burning-velocity to increase. This velocity-rise upon addition of excess fuel in constant-temperature flames is a criterion for inhibition. Continued increase in fuel-richness extinguishes the combustion.

${ }^{3}$ Editor's note: Temperature profiles are also affected by chemical inhibition. 
Detailed microstructure profiles are also needed to understand the concept of selfinhibition. In the original paper [10], an attempt was made to explain fuel self-inhibition on the basis of dissociation efficiency and radical recombination. As constant-velocity quenched flames were made more fuel-rich, the net dissociation efficiency $N_{N E T-D}$, decreased. Net dissociation efficiency per input fuel molecule is given by the following expression $[8,55]$ :

$$
N_{\text {NET-D }}=\frac{([\mathrm{OH}]+[\mathrm{H}]+2[0])_{\text {MAX }}}{[\mathrm{HC}]}
$$

or, is the total maximum radical pool concentration (numerator) per input fuel concentration, [HC]. Continued fuel self-inhibition leads to a lower total maximum radical pool concentration, and eventually, extinction.

Recent computer modeling data by Smoot and coworkers [54] permit the demonstration of the same dissociation efficiency reduction upon oxidant self-inhibition. In order to determine the dissociation efficiency in fuel-lean flames, one has to modify the form of the total maximum radical pool concentration term according to the requirements imposed by stoichiometry in dissociation-recombination reactions [56]. Dissociation efficiency in oxidant-rich flames is given by $[57,58]$ :

$$
\mathrm{N}_{\text {NET-D }}=\frac{\left([\mathrm{OH}]+2[\mathrm{O}]+3[\mathrm{H}]+2\left[\mathrm{H}_{2}\right]+2[\mathrm{CO}]\right)_{\text {MAX }}}{[\mathrm{HC}]}
$$

Table 8 contains the results of using the Smoot, et al. composition data in eqs. (8) and (9). Dissociation efficiencies are normalized to that in the clean, uninhibited (stoichiometric) atmospheric pressure methane/air flame. Upon oxidant or fuel self-inhibition, the dissociation efficiency decreases. The Smoot, et al., data [54] were checked for accuracy by computing a limiting equivalence ratio [10] for the atmospheric pressure methane/air flames. The result is in line with those determined at $0.25 \mathrm{~atm}$ [10].

Dissociation efficiency is itself, a sensitive measure of inhibition [10]. In the original study [10], an attempt was made to determine the chemical mechanism by which fuel-inhibitors reduced dissociation efficiency, thereby derailing the clean flame combustion mechanism. Chemical inhibition is believed to proceed via mechanisms which, compared to clean flames, result in either enhanced radical removal rates in the postflame-gas zone [10a] or retardation of radical of radical production rates in the preflame zones [10b], or both. Since hydrocarbon self-inhibitor effectiveness was reduced when pressure was reduced, it was believed that fuel self-inhibition was the result of enhanced radical recombination rates. The important elementary reactions are given in the following set:

$$
\begin{aligned}
& H+H+M=O H+O \\
& H+O H+M=H_{2} O+M \\
& H+O+M=O H+M
\end{aligned}
$$


Table 8

$\begin{array}{cccc}\begin{array}{c}\text { Equivalence } \\ \text { ratio }\end{array} & \begin{array}{c}\text { Self-inhibitor } \\ \text { [Air] }\end{array} & \text { [Fuel] } & \begin{array}{c}\text { Dissociation } \\ \text { efficiency }\end{array} \\ 1.00 & - & 0 & 100 \\ 1.24 & - & 2.1 & 88 \\ 1.42 & - & 3.5 & 59 \\ 1.68 & - & 5.5 & 24\end{array}$

$\begin{array}{lccc}1.00 & 0 & - & 100 \\ 0.72 & 2.5 & - & 56 \\ 0.50 & 4.5 & - & 27\end{array}$

Numerical analysis, however, indicated that hydrocarbon self-inhibition could not be accounted for solely in terms of enhanced radical recombination rates [10a]. This finding suggested that a more fundamental process was being disrupted upon inhibition. As an alternative to (R1)-(R3), Schott [10b] associated the root cause of the observed inhibition by excess fue 1 with the kinetics of attaining the minimum extent of dissociation necessary for the formation of the radical pool, in preference to an effect upon the kinetics of removal of such a pool. The pressure effect, previously associated with three-body association kinetics, could be accommodated in this new causal mechanism of self-inhibition by the pressure-dependent diffusional mobility of the radical pool. Schott suggested that the associative sequestering of radicals as the unreactive $\mathrm{HO}_{2}$ species might be the most important elementary step in the self-inhibition mechanism. Schott's hypothesis is based on the following set of reactions:

$$
\begin{aligned}
& \mathrm{H}+\mathrm{O}_{2}+\mathrm{M}=\mathrm{HO}_{2}+\mathrm{M} \\
& \mathrm{HO}_{2}+\mathrm{H}=\mathrm{H}_{2}+\mathrm{O}_{2} \\
& \mathrm{HO}_{2}+\mathrm{OH}=\mathrm{H}_{2} \mathrm{O}+\mathrm{O}_{2} \\
& \mathrm{HO}_{2}+\mathrm{O}=\mathrm{OH}+\mathrm{O}_{2}
\end{aligned}
$$

Reactions (R4)-(R7) certainly affect the total maximum radical pool concentration, since they inactivate the most important components of the pool, $\mathrm{H}, \mathrm{OH}$, and 0 [10]. The set of reactions (R1)-(R3) operate in the post-flame-gas zone. The Schott set of elementary reactions would be important in the relatively cool pre-flame zone, where the $\mathrm{O}_{2}$ concentration is the largest. The Smoot, et al. [54], computer modeling data on $\mathrm{CH}_{4} /$ air flames support the Schott mechanism. Compared to clean, stoichiometric flames, flames doped with hydrocarbon self-inhibitor have a two-times higher $\left[\mathrm{HO}_{2}\right]$ and a four-fold lower dissociation 
efficiency. The Smoot, et al., data do not support the $\mathrm{HO}_{2}$ mechanism for oxidant selfinhibition. This indicates that fuel and oxidant self-inhibitors do not suppress combustion by exactly the same mechanism. This is what is expected since even though both types of self-inhibitors act in both modes, fuel self-inhibitors have a dominant chemical component while oxidants have a dominant physical component. This is supported by the results in tables 1,2 , and 6 which demonstrate that fuel self-inhibitor effectiveness is more strongly dependent on pressure than oxidant self-inhibitors are.

The fact that inhibition is still observed in low-pressure flames where chaperons have forfeited most of their ability to enhance radical recombination is indirect evidence that gaseous inhibitors may also act to disrupt bimolecular elementary reactions. The bimolecular reactions most likely to be interfered with are those which make up the radical-pool production and maintenance mechanism [10]:

$$
\begin{aligned}
& \mathrm{OH}+\mathrm{H}_{2}=\mathrm{H}_{2} \mathrm{O}+\mathrm{H} \\
& \mathrm{O}_{2}+\mathrm{H}=\mathrm{OH}+\mathrm{O} \\
& \mathrm{O}+\mathrm{H}_{2}=\mathrm{OH}+\mathrm{H} \\
& \mathrm{CO}+\mathrm{H}_{2} \mathrm{O}=\mathrm{CO}_{2}+\mathrm{H}_{2}
\end{aligned}
$$

Further indirect support for this comes from literature data available on "apparent" activation energies for the global kinetics of adiabatic [59] and non-adiabatic [13] combustion. The adiabatic data are theoretical in nature in that they were determined by computer modeling the performance of a well-stirred spherical combustor using propane/air [59]. The quenched flame global activation energies were determined experimentally by plotting the log of mass burning-velocity versus reciprocal temperature [13]. The burningvelocity is proportional to the overall combustion rate, so that plots, like that of figure 4, are essentially Arrenihus-type graphs. Table 9 demonstrates the effect of self-inhibitors on the global activation energy of the stoichiometric combustion process.

Table 9

Equivalence

Ratio

1.0

1.1

1.3
Self-inhibition

[Air] [Fuel]

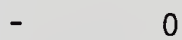

$-\quad 0.4$
$-\quad 10$

$\begin{array}{ll}- & 1.1\end{array}$
Global activation energy Theory [59] Experiment [13]
1.0
0
29
0.9
0.4
36
54
0.8
0.8
44 
Although the magnitude of effectiveness for an inhibitor is diminished upon reduction of pressure, inhibition is still observable in low-pressure flames [20]. Note that this study provides strong evidence in favor of the idea that all inhibitors have both physical and chemical components to their flame suppression mechanism, with one dominating. One wonders whether the chemical component could not be reduced to the point where its magnitude would be negligible. This could be accomplished by lowering the pressure to the point where chaperons have forfeited most, if not all, of their ability. This would leave only the thermal dilution capacity of the molecule as its means for inducing inhibition. Evidence for this conversion from chemical plus physical type to physical type is given for $\mathrm{C}_{2} \mathrm{H}_{4}$ self-inhibition in table 2. As pressure is reduced from one atmosphere to 0.50 , the self-inhibitor effectiveness, $I_{V}^{A}$, for $\mathrm{C}_{2} \mathrm{H}_{4}$ goes from greater than unity to less than unity. If the Hastie criterion is correct [9], this trend indicates that at $0.5 \mathrm{~atm}, \mathrm{C}_{2} \mathrm{H}_{4}$ has lost its ability to chemically interfere with combustion and can now induce inhibition only by acting as an inert heat sink. Data on other hydrocarbon self-inhibitors do not exhibit this property.

As previously discussed, the inhibitor effectiveness of $\mathrm{CO}_{2}$ and $\mathrm{H}_{2} \mathrm{O}$ cannot be explained solely on their thermal dilution capacity. Referring to reactions ( $R 8)-(R 11)$, the reactions responsible for radical-pool production and maintenance, one realizes that $\mathrm{CO}_{2}$ and $\mathrm{H}_{2} \mathrm{O}$ are the principle participants in the formation of the partial-equilibrium-state [10]. Altering their concentration by direct addition could account for both the decrease in dissociation efficiency and their ability to be more effective at inhibition than their thermal dilution capacity would predict. Reduction in radical pool concentrations would cause the observed decrease in the adiabatic burning-velocity. These observations are supported by the data in table 7 [13].

As mentioned, this review is being used as a vehicle to introduce, then document, this author's ideas on various aspects of flame inhibition. With this in mind, the review will switch its attention from self-inhibition to the role of negative halogen ions in flame inhibition. This is a controversial matter. The following is an exercise undertaken with the objective of resolving this controversy.

Table 1 indicates that certain halogenated hydrocarbons, e.g., $\mathrm{CH}_{3} \mathrm{Br}$ and $\mathrm{CF}_{3} \mathrm{Br}$, are very efficient flame inhibitors. Current research projects are directed toward elucidating the chemical mechanisms operating in flames inhibited by these materials $[16,60]$.

One of the proposed mechanisms of flame inhibition by halogenated hydrocarbons, first formulated by Mills [61], is that low energy electrons become attached to the halogen fragment of the inhibitor molecule through a dissociative process, such as

$$
R X+e^{-} \stackrel{k_{12}}{\longrightarrow} X^{-}+R
$$


where:

$$
\begin{aligned}
& \mathrm{RX}=\text { halogenated hydrocarbon } \\
& \mathrm{X}^{-}=\text {negative halogen ion } \\
& \mathrm{R}^{-}=\text {radical } \\
& \mathrm{e}^{-}=\text {electron }
\end{aligned}
$$

Mills stated that (R12) was the important first step, and that after the negative halogen ion was generated, inhibition could proceed by radical scavenging via an associative detachment process, given as:

$$
\mathrm{X}^{-}+\mathrm{H} \stackrel{\mathrm{k}}{\longrightarrow} \mathrm{HX}+\mathrm{e}^{-} .
$$

Inhibition by radical scavenging has been proposed by Fenimore and Jones [17], and is the most widely-accepted concept of how inhibition is achieved [8-10].

Mills incorporated semi-quantitative analysis into his arguments, advocating (RI2) and (RI3) as the reaction sequence. This author was skeptical of one of the vital assumptions Mills used in support of the role of the negative halogen ion. The objection stems from Mills' treatment of inhibition in $\mathrm{H}_{2} / \mathrm{O}_{2}$ and $\mathrm{CO} / \mathrm{O}_{2}$ flames [61]. Bulewicz and Padley found that no measurable trace of ionization occurred in $\mathrm{H}_{2} / \mathrm{O}_{2}$ and $\mathrm{CO} / \mathrm{O}_{2}$ flames [62]. Mills stated that the reason such flames can still be inhibited by halogenated hydrocarbons is that the necessary free electrons are generated by the ionization of hydrocarbon impurities in the gas mixture. This argument seemed implausible in view of the fact that most research gases contain very few impurities of any kind. For example, in Palmer and Seery's study of $\mathrm{CO} / 0_{2}$ flames inhibited by halogens, most of the research gases contained less than 0.1 percent impurity [63]. One would be safe in assuming that not all of this impurity was due to trace hydrocarbons; most of the impurity was water [63]. Sti11, appreciable inhibition was observed when halogens were added to the $\mathrm{CO} / \mathrm{O}_{2}$ flames [63].

Mills seemed to share this skepticism, but went on to state that the electron concentration of the order of $10^{11}$ electrons $/ \mathrm{cm}^{3}$ were not insignificantly smal1 [61].

The contention that Mills' semi-quantitative arguments are not convincing is shared by Spence and McHale, who recently produced experimental evidence in support of the position that negative halogen ions play no significant role in inhibition [64]. They measured the collision cross-section for the dissociative electron detachment process given in (R12). They then showed that no correlation existed between decreasing cross-section and decreasing inhibitor effectiveness. Data illustrating this comparison are given in table 10 . From the lack of any correlation, Spence and McHale concluded that the ionic mechanism, (R12) and (RI3), did not play a role in flame inhibition by halogenated hydrocarbons. 
Agent $\quad \begin{array}{ll}\sigma \text { at } 1200 \mathrm{~K} & \left(10^{-16} \mathrm{~cm}^{2}\right)\end{array} \quad$ Peak $\% \quad$ by $\sigma$ Ranking by Peak $\%$

\begin{tabular}{lrrrr}
$\mathrm{CH}_{2}{ }^{\mathrm{Br}}{ }_{2}$ & 61 & 5.2 & 8 & 1 \\
$\mathrm{CH}_{3} \mathrm{Br}$ & 80 & 9.7 & 7 & 4 \\
$\mathrm{CF}_{3} \mathrm{Br}$ & 98 & 6.1 & 6 & 2 \\
$\mathrm{CHCl}_{3}$ & 115 & 17.5 & 5 & 7 \\
$\mathrm{CH}_{3} \mathrm{I}$ & 135 & 6.1 & 4 & 2 \\
$\mathrm{CCl}_{4}$ & 182 & 11.5 & 3 & 5 \\
$\mathrm{CFCl}_{3}$ & 219 & $15 \pm 3$ (est) & 2 & 6 \\
$\mathrm{SF}_{6}$ & 246 & 20.5 & 1 & 8 \\
\hline
\end{tabular}

Spence and McHale supported their position by using available data to calculate and to compare the rates of the coupled ionic reactions, (RI2) and (RI3), with the rate of the neutral inhibition process, given as follows:

$$
\mathrm{RX}+\mathrm{H} \stackrel{\mathrm{k} 14}{\longrightarrow} \mathrm{R}+\mathrm{HX}
$$

Using, as an example, $\mathrm{CF}_{3} \mathrm{Br}$-inhibited atmospheric methane/air flames at $1200 \mathrm{~K}$, they calculated that:

$$
\frac{\text { Rate }(R 14)}{\text { Rate }(R 12)}=3 \times 10^{2}
$$

From this result, they concluded that the ionic mechanism cannot compete with the neutral inhibition process and, therefore, negative halogen ions play no significant role in inhibition.

Additional data are available [17] on a different flame system $\left(\mathrm{H}_{2} / \mathrm{O}_{2} / \mathrm{Ar}\right)$ inhibited by a different inhibitor $\left(\mathrm{CH}_{3} \mathrm{Br}\right)$, which can be used in their calculation to reinforce their conclusion. In accordance with Spence and McHale, it is assumed that Rate (R12) is governed by the steady-state electron density in the flame, and that the $\left[X^{-}\right]$is also in steadystate. From these assumptions, the following expression is obtained: 


$$
\left[e^{-}\right]=\frac{k_{13^{[H]}\left[X^{-}\right]}}{k_{12}[R X]}
$$

where:

$$
k_{i}=\text { rate constant for reaction } i
$$

The specific rate constant for associative detachment, $k_{13}$, is given by Spence and McHale as $1.2 \times 10^{15} \mathrm{~cm}^{3} / \mathrm{mol}-\mathrm{s}$. Using the Fenimore and Jones [17] data on $0.1 \mathrm{~atm} \mathrm{H}_{2} / \mathrm{O}_{2}$ flames at $1800 \mathrm{~K}, \mathrm{k}_{12}$ can be calculated from eq. (7) of Spence and McHale, which is as follows:

$$
k_{12}=\sigma \overline{v N}
$$

where:

$\sigma=$ collision cross-section for dissociative electron attachment,

$\bar{v}=$ average velocity of an electron at temperature $T$, and

$N=$ Avogadro's number.

The $\mathrm{CH}_{3} \mathrm{Br}$ value for $\sigma$ at $1800 \mathrm{~K}$ was taken from figure 1 in Spence and McHale as $9 \times 10^{-17}$ $\mathrm{cm}^{2}$. The average velocity of an electron at $1800 \mathrm{~K}$ was computed to be $2.6 \times 10^{7} \mathrm{~cm} / \mathrm{s}$ [65]. The value of [H] was taken from Fenimore and Jones [17] as $2.4 \times 10^{15}$ atoms $/ \mathrm{cm}^{3}$. Substitution of these numbers into (11) and (12) gave:

$$
\left[\mathrm{e}^{-}\right]=1.7 \times 10^{-3}\left[\mathrm{x}^{-}\right]
$$

Bulewicz and Padley [62] found no measurable trace of ionization in $\mathrm{H}_{2} / \mathrm{O}_{2}$ flames. From Mills [61], it is assumed that the ions responsible for the $\mathrm{CH}_{3} \mathrm{Br}$ inhibition in the Fenimore and Jones $\mathrm{H}_{2} / \mathrm{O}_{2}$ flames were generated from hydrocarbon impurities in the unburned $\mathrm{H}_{2}$ and $\mathrm{O}_{2}$ gases. Peeters, et al. showed that the ion production from such impurities to be directly proportional to hydrocarbon impurity concentration [66]. For this calculation, the hydrocarboi impurity concentration in the Fenimore and Jones $\mathrm{H}_{2} / \mathrm{O}_{2}$ gases is assumed to be 5 mole percent methane. Why this liberal and unrealistic impurity concentration was chosen will become evident at the end of the calculation. Peeters, et al. stated that the ion yield in methanedoped $\mathrm{H}_{2} / \mathrm{O}_{2}$ flames was $3.83 \times 10^{-6}$ ions $/ \mathrm{CH}_{4}$ molecule [66]. Thus, if the hydrocarbon impurity in the Fenimore and Jones flames was 5 percent $\mathrm{CH}_{4},\left[\mathrm{X}^{-}\right]=7 \times 10^{10}$ ions $/ \mathrm{cm}^{3}$. Substituting this $\left[X^{-}\right]$value into (13) yields the free electron density as $1.2 \times 10^{8}$ electrons $/ \mathrm{cm}^{3}$.

As in Spence and McHale, the effectiveness of the ionic mechanism compared to the neutral mechanism can be calculated from the following: 


$$
\frac{\text { Rate }(\mathrm{R} 14)}{\operatorname{Rate}(\mathrm{R} 12)}=\frac{\mathrm{k}_{14}[\mathrm{H}]}{\mathrm{k}_{12}\left[\mathrm{e}^{-}\right]}
$$

The value for $\mathrm{k}_{14}$ was calculated at $1800 \mathrm{~K}$ to be $3.3 \times 10^{13} \mathrm{~cm}^{3} / \mathrm{s}-\mathrm{mol}$ [67]. Substitution of all applicable numbers into (14) shows that for the Fenimore and Jones $\mathrm{CH}_{3} \mathrm{Br}$-inhibited $\mathrm{H}_{2} / \mathrm{O}_{2}$ flames, doped with 5 percent methane impurity:

$$
\frac{\text { Rate }(R 14)}{\text { Rate }(R 12)}=4.6 \times 10^{3}
$$

This result is greater than an order of magnitude higher than what Spence and McHale calculated for $\mathrm{CF}_{3} \mathrm{Br}$-inhibited atmospheric methane/air flames (see eq. (10)). Thus, this calculation shows, more dramatically, that Spence and McHale were correct in conclusions that the ionic mechanism plays no role in inhibition. It also gives quantitative evidence illustrating the implausibility of Mills' claim that observed inhibition in $\mathrm{H}_{2} / \mathrm{O}_{2}$ flames is a result of hydrocarbon impurities in the gases [61]. Most experimentalists would use research gases with a hydrocarbon impurity at least an order of magnitude less than the 5 percent value assumed in the calculation. Such realistic values would result in an even larger value of (15), further favoring the neutral mechanism.

Mandl has recently challenged Spence and McHale on their statement that ion chemistry is unimportant in flame inhibition [60]. Mandl supports his position by claiming that the rates of ( $R 13$ ) and ( $R 14$ ) should be compared, instead of (R12) and (R14), if one wants a direct comparison between the effect of ions and inhibitors on flame-free-radical concentrations. Using the following:

$$
\frac{\text { Rate }(\mathrm{R} 13)}{\operatorname{Rate}(\mathrm{R} 14)}=\frac{\mathrm{k}_{13}\left[\mathrm{X}^{-}\right][\mathrm{H}]}{\mathrm{k}_{14}[\mathrm{RX}][\mathrm{H}]}
$$

and making general estimates of numerical data, Mandl finds that:

$$
\frac{\text { Rate }(R 13)}{\text { Rate }(\text { R14) }} \gtrsim 1
$$

From this result he concludes that one cannot claim than inhibition via an ionic mechanism is unreasonable [60].

Using the data just gathered to produce a second example of the Spence and McHale argument, one can challenge Mandl's position, using his own calculation to do so. By substituting these data into (16), one obtains:

$$
\frac{\text { Rate }(R 13)}{\text { Rate }(\text { R } 14)}=2.2 \times 10^{-4}
$$


This shows that Mandl's estimates were unrealistic. The 5 percent $\mathrm{CH}_{4}$ impurity assumed in this calculation was also unrealistic, but, reduction of it to a more realistic value only makes (18) smaller. A smaller (18) means that the ionic mechanism is even more insignificant compared to the neutral mechanism.

The role of negative ions in inhibition is a controversial topic. The spence and McHale result, that the collision cross-section and the inhibitor effectiveness did not correlate, warrants further scrutiny. Spence and McHale based their ordering of inhibitor effectiveness on flame inhibitor peak percentages from one study only, that of Spence and Schulz [68]. A review of the inhibition literature shows that by using one measure of inhibition from one data source, this ordering may not have been unique, as different researchers have found different rankings of inhibitor effectiveness $[42,69,70]$. Table 11 illustrates how these other studies have ranked inhibitor effectiveness, and what exchanges exist compared to the Spence and McHale ordering. Although the ordering is altered from Spence and McHale's original listing, the non-correlation between collision cross-section and inhibitor effectiveness is maintained.

From the information given in this section, the controversy over whether negative halogen ions play a role in inhibition seems to have been resolved.

Table 11

Inhibitor

Collision cross

Ranking of inhibitor effectiveness

selection rank

ref. [64]

ref. [64] ref. [70] ref. [42] ref. [69]

$\begin{array}{ll}\mathrm{CCl}_{4} & 1 \\ \mathrm{CH}_{3} \mathrm{I} & 2 \\ \mathrm{CHCl}_{3} & 3 \\ \mathrm{CF}_{3} \mathrm{Br} & 4 \\ \mathrm{CH}_{2} \mathrm{Br}_{2} & 5 \\ \mathrm{CH}_{3} \mathrm{Br} & 6\end{array}$

5. Summary and Conclusions

The concept of self-inhibition by excess fuel and oxidant has permitted the application of what is known about fuel-rich and oxidant-rich combustion chemistry to the understanding of measures of effectiveness and mechanisms for chemical and physical inhibition of quenched and adiabatic premixed flames. 
A literature review of flame inhibition research indicated a paucity of experimental data on the inhibitor effectiveness of $\mathrm{CO}_{2}$ and $\mathrm{H}_{2} \mathrm{O}$. New research on these practical fire extinguishants is warranted in order to bridge this information gap.

All inhibitors appear to possess both a chemical and a physical component to their inhibition capacity, with one dominating.

Exactly how clean flame combustion chemistry is disrupted upon inhibition is beginning to be understood in terms of bulk approximations of the flame microstructure. These include radical pool concepts and dissociation efficiencies.

Flame inhibition by halogenated hydrocarbons proceeds via a neutral mechanism. Ions do not play a significant role.

The research was supported by the National Science Foundation and the Army Research Office.

\section{References}

[1] Fryburg, G., Review of literature pertinent to fire-extinguishing agents and to basic mechanisms involved in their action, NACA Technical Note 2102, Lewis Flight Propulsion Laboratory, Cleveland, $\mathrm{OH}$ (May 1950).

[2] (a) Friedman, R. and Levy, J., Survey of Fundamental Knowledge of Mechanisms of Action of Flame Extinguishing Agents, Wright Air Development Center Technical Report 56-568 (Jan. 1957); (b) ibid., Supplement I, Sept. 1958; (c) ibid., Supplement II (Apr. 1959).

[3] Berl, W., Survey of current fire research activities, Fire Research Abstracts Revs. $\underline{3}, 113$ (1961).

[4] Friedman, R., Survey of chemical inhibition in flames, Fire Research Abstracts Revs. 3, 128 (1961).

[5] Fristrom, R., Combustion suppression, Fire Research Abstracts Revs. 9, 125 (1967).

[6] McHale, E., Survey of vapor phase chemical agents for combustion suppression, Fire Research Abstracts Revs. 11, 90 (1969).

[7] Crietz, E., Literature survey of the chemistry of flame inhibition, J. Research Nat1. Bur. Standards 74A, 521 (1970).

[8] Fristrom, R. and Sawyer, R., Flame Inhibition Chemistry, AGARD Conf. Proc. on Aircraft Fuels, Lubricants and Fire Safety, AGARD-CP-84-71, Paper \#12, NATO, The Hague, Netherlands (May 1971).

[9] Hastie, J., Molecular basis of flame inhibition, J. Research Nat1. Bur. Standards $\underline{77 A}, 733$ (1973); and Chemical Inhibition in Flames, High Temperature Vapors, pp. 332357 (Academic Press, NY, 1975). 
[10] (a) Kaskan, W. and Reuther, J., Sixteenth Symposium (International) on Combustion, p. 1083, The Combustion Institute, 1977; (b) Comments by G. L. Schott.

[11] Reuther, J., Comments on chemical and physical inhibition of quenched and adiabatic premixed flames, Paper IV-31, presented at Eastern States Section/The Combustion Institute Fall Technical Meeting, United Technologies Research Center, East Hartford, CT, Nov. 1977.

[12] Fristrom, R. and Westenberg, A., Flame Structure (McGraw-Hi11, NY, 1965).

[13] Kaskan, W., Sixth Symposium (International) on Combustion, p. 134 (Reinhold, 1957).

[14] Rosser, W., Wise, H., and Miller, J., Seventh Symposium (International) on Combustion, p. 175 (Butterworths, 1959).

[15] Botha, J. and Spalding, D., Proc. Roy. Soc. (London) A225, 71 (1954).

[16] Biordi, J., Lazzara, C., and Papp, J., The effect of $\mathrm{CF}_{3} \mathrm{Br}$ on radical concentration profiles in methane flames, Paper \#9, p. 256, Halogenated Fire Suppressants, R. Gann, ed., American Chemical Society Symposium Series 16 (1975).

[17] Fenimore, C. and Jones, G., Combustion and Flame, 2, 323 (1963).

[18] Iya, K., Wollowitz, S. and Kaskan, W., Combustion and Flame, 22, 415 (1974).

[19] Hayes, K. and Kaskan, W., Combustion and Flame, 24, 405 (1975).

[20] Biordi, J., Lazzara, C., and Papp, J., Combustion and Flame, 24, 401 (1975).

[21] Andrews, G. and Bradley, D., Combustion and Flame, 18, 133 (1972).

[22] Andrews, G. and Bradley, D., Combustion and Flame, 19, 275 (1972).

[23] Bulau, J. and Kaskan, W. (unpublished results, 1974).

[24] Bonne, U., Jost, W., and Wagner, H., Fire Research Abstracts Revs. 4, 6 (1962).

[25] Zabetakis, M., Flammability characteristics of combustible gases and vapors, Bureau of Mines Bulletin 627 (1965).

[26] Scholte, T. and Vaags, P., Combustion and Flame, $\underline{3}, 511$ (1959).

[27] Wheatley, P. and Linnett, J., Trans. Faraday Soc. $\underline{45}, 1152$ (1949).

[28] Powna11, C. and Simmons, R., Thirteenth Symposium (International) on Combustion, p. 585, The Combustion Institute (1971).

[29] Kueh1, D., Eighth Symposium (International) on Combustion, p. 510 (Wi11iams \& Wilkins, 1962).

[30] Miller, W., Combustion and Flame, 13, 210 (1969).

[31] Homann, K. and Poss, R., Combustion and Flame, 18, 300 (1972).

[32] Reuther, J. (unpublished results, 1976).

[33] Coward, H. and Jones, G., Limits of flammability of gases and vapors, Bureau of Mines Bulletin 503 (1957).

[34] Dune, R., Johnson, G., and Smith, M., Combustion and Flame, 17, 197 (1971).

[35] Smoot, L., Combustion and Flame, 31, 325 (1978).

[36] Larsen, E., J. Fire and Flammability/Fire Retardant Chemistry, 1, 4 (1974).

[37] Larsen, E., J. Fire and Flammability/Fire Retardant Chemistry, 2, 5 (1975).

[38] Larsen, E., Halogenated fire extinguishants: flame suppression by a physical mechanism? Paper \#13, p. 376, Halogenated Fire Suppressants, R. Gann, ed., American Chemical Society Symposium Series 16 (1975). 
[39] Babkin, V. and V'yun, A., Combustion, Explosions, and Shockwaves, ㄱ, 339 (1971).

[40] Sheinson, R., Hahn, J., Geary, K., and Williams, R., Quantification of physical fire suppression of heptane pool fires, Paper IV-30, presented at Eastern States Section/ The Combustion Institute Fall Technical Meeting, United Technologies Research Center, East Hartford, CT, Nov. 1977.

[41] Müller-Dethlefs, K. and Schlader, A., Combustion and Flame, 27, 205 (1976).

[42] Garner, F., Long, R., Graham, A. and Badakhshan, A., Sixth Symposium (International) on Combustion, p. 802 (Reinhold, 1957).

[43] Lask, G. and Wagner, H., Eighth Symposium (International) on Combustion, p. 432 (Williams and Wilkins, 1962).

[44] Agnew, J. and Graiff, L., Combustion and Flame, 5, 209 (1961).

[45] Morgan, G. and Kane, W., Fourth Symposium (International) on Combustion, p. 313 (Wil1iams and Wilkins, 1953).

[46] Van Tiggelen, A. and Deckers, J., Sixth Symposium (International) on Combustion, $\mathrm{p}$. 61 (Reinhold, 1957).

[47] Scholte, T. and Vaags, P., Combustion and Flame, 3, 495 (1959).

[48] Gray, P., Holland, S., and Smith, D., Combustion and Flame, 14, 361 (1970).

[49] Bitlker, D. and Scullin, V., General chemical kinetics computer program for static and flow reactions, with applications to combustion and shock tube kinetics, NASA TN D6586 (1972).

[50] Arthur, J., Nature, 165, 557 (1950).

[51] Wheatley, P. and Linnett, J., Trans. Faraday Soc. 48, 338 (1932).

[52] Kueh1, D., Am. Rocket Soc. J. 32, 1724 (1962).

[53] Fenimore, C. and Jones, G., Combustion and Flame, $\underline{5}, 135$ (1961).

[54] Smoot, L., Hecker, W., and Williams, G., Combustion and Flame, 26, 323 (1976).

[55] Bulau, J. and Kaskan, W., J. Chem. Phys. 62, 3605 (1975).

[56] Kaskan, W. and Schott, G., Combustion and Flame, $\underline{6}, 73$ (1962).

[57] Peeters, J. and Mahnen, G., Fourteenth Symposium (International) on Combustion, p. 133, The Combustion Institute, 1973.

[58] Peeters, J. and Mahnen, G., Combustion Institute European Symposium, p. 53 (Academic Press, NY, 1973).

[59] Kretschmer, D. and Odgers, J., J. Eng. and Power, Trans ASME, 173 (1972).

[60] McHale, E. and Mandl, A., The role of ions and electrons in flame inhibition by halogenated hydrocarbons: two views, Paper \#14, p. 403, Halogenated Fire Suppressants,

R. Gann, ed., American Chemical Society Symposium Series 16 (1975).

[61] Mi11s, R., Combustion and Flame, 12, 513 (1968).

[62] Bulewicz, E. and Padley, P., Ninth Symposium (International) on Combustion, p. 638 (Academic Press, NY, 1963).

[63] Palmer, H. and Seery, D., Combustion and Flame, 4, 213 (1960).

[64] Spence, D. and McHale, E., Combustion and Flame, 24, 211 (1975).

[65] Moore, W., Physical Chemistry, 3rd ed. (Prentice-Hal1, NJ, 1962).

[66] Peeters, J., Vinkier, C., and Van Tiggelen, A., 0xid. and Comb. Revs. $\underline{4}, 93$ (1969). 
[67] Trotman-Dickenson, A. and Milne, G., Table of Bimolecular Gas Reactions, NSRDS-NBS 9 , $12,1967$.

[68] Spence, D. and Schultz, G., J. Chem. Phys. 58, 1800 (1973).

[69] Belles, F. and O'Neal, C., Sixth Symposium (International) on Combustion, p. 806 (Reinhold, 1957).

[70] Lee, T., J. Phys. Chem. 67, 360 (1963).

\section{Discussion}

Question (Fristrom): I would like to ask a question about the oxygen self inhibition. You indicated that you consider this to be a physical inhibitor.

It seems to be that oxygen really is intimately tied up with the flame chemistry, and one should consider it as a chemical inhibitor, and I was wondering could you say something about the relation between nitrogen and oxygen? Does nitrogen also show the same trend?

Response (Reuther): The same trend is the case for oxygen, nitrogen, and argon. So, the same effect is observed for both oxygen and nitrogen, and both of them show a pressure dependence of their inhibitor effectiveness and everything is the same for both.

I would like to point out that water is also usually considered an inert-type inhibitor, and it is only recently that it is found that its inhibitor effectiveness cannot be accounted for in terms of a thermal dilution mechanism.

This is something that had been pointed out almost 30 years ago, that it did have an ability to disrupt flame chemistry, and it has been forgotten over the years. So, this is another purpose of this survey, to try to update all flame inhibition specialists on what is actually known already, and to avoid some confusion.

Comment (Gann): On that point I might add that there is a paper by Feugier, which will not be presented here, but will be in the proceedings. That deals with the water question in much more detail.

Question (Tsang): When you mentioned that the water was disrupting the flame chemistry, are you suggesting the usual reactions that people put in the hydrogen-oxygen flame or are they missing steps in it involving water?

Response (Ruether): Not that they are missing, but the normal propagation of those reactions are being changed by the prior addition of water. One step in particular is the formation reaction of $\mathrm{HO}_{2}$. That molecule is intimately tied into schemes involving self inhibition in fuel rich flames. I didn't have time to go into it.

Since I could not account for the self inhibition in fuel rich flames on the basis of enhanced radical recombination, I couldn't do any more except say that the enhanced radical recombination rates didn't correlate very well with self inhibition. 
Gary Schott has pointed out that the $\mathrm{HO}_{2}$ molecule can get around that problem and explain the rest of the effect (see paper by Reuther in Proc. 16th Symp. (Int.) Comb.). It is a very important set of reactions which can be verified on some recent work by Smoot in his computer modeling study, where he shows that as the dissociation efficiency in a fuel rich flame decreases, the $\mathrm{HO}_{2}$ molecule concentration increases, which is exactly what you would expect. So, it is starting to fall into place. 

National Bureau of Standards Special Publication 561, Proceedings of the 10th Materials Research Symposium on Characterization of High Temperature Vapors and Gases held at NBS, Gaithersburg, Maryland, September 18-22, 1978. Issued October 1979.

\section{INFLUENCE OF WATER VAPOR ON HYDROGEN COMBUSTION}

\section{A. Feugier}

Institut Francais Du Petrole

92502 Rueil Malmaison, France

\section{Introduction}

In countries where great efforts are being made to develop nuclear energy, hydrogen is considered to be a particularly interesting "vector" for storing electricity. Indeed, hydrogen produced during slack hours, e.g. by the electrolysis of water, would then be consumed to produce electricity during peak hours. This would be done by combustion in turbines of an $\mathrm{H}_{2}-\mathrm{O}_{2}$ mixture diluted by water vapor. As a result, understanding the properties of such a system appears to be of importance.

The kinetics of the $\mathrm{H}_{2}-\mathrm{O}_{2}$ reaction is relatively well understood, largely thanks to the works done by R. R. Baldwin and G. Dixon-Lewis. However, the use of water vapor as a diluent had to be experimentally investigated, and at the same time we felt that it would be interesting to take advantage of all published kinetic data to work out a model capable of predicting the properties of the general $\mathrm{H}_{2}-\mathrm{O}_{2}-\mathrm{H}_{2} \mathrm{O}-\mathrm{N}_{2}$ system. The aim of this paper is, for such a system, to compare the calculated and measured values of the auto-ignition delays, the burning velocities and the flammability limits.

\section{Auto-ignition}

The kinetic flow diagram takes 28 elementary steps into consideration, involving $\mathrm{H}_{2}-\mathrm{O}_{2}-\mathrm{H}_{2} \mathrm{O}-\mathrm{H}_{2} \mathrm{O}_{2}-\mathrm{H}-\mathrm{OH}-\mathrm{O}$ and $\mathrm{HO}_{2}$. It includes:

a) an initiation reaction: $\mathrm{H}_{2}+\mathrm{O}_{2} \longrightarrow \mathrm{H}+\mathrm{HO}_{2}$,

b) two branching reactions: $\mathrm{H}+\mathrm{O}_{2} \stackrel{\mathrm{I}}{\longrightarrow} \mathrm{OH}+\mathrm{O}$ and $\mathrm{H}_{2} \mathrm{O}_{2}+\mathrm{M} \stackrel{2}{\longrightarrow} 2 \mathrm{OH}+\mathrm{M}$,

c) propagation reactions such as: $\mathrm{H}+\mathrm{O}_{2}+\mathrm{M} \stackrel{3}{\longrightarrow} \mathrm{HO}_{2}+\mathrm{M}, \mathrm{HO}_{2}+\mathrm{H}_{2} \stackrel{4}{\longrightarrow} \mathrm{H}_{2} \mathrm{O}_{2}+\mathrm{H}$, $\mathrm{H}_{2}+\mathrm{O} \stackrel{5}{\longrightarrow} \mathrm{H}+\mathrm{OH}, \mathrm{H}_{2}+\mathrm{OH} \stackrel{6}{\longrightarrow} \mathrm{H}_{2} \mathrm{O}+\mathrm{H}$, etc...

d) recombination reactions such as: $\mathrm{H}+\mathrm{H}+\mathrm{M} \longrightarrow \mathrm{H}_{2}+\mathrm{M}$, etc.

The delays are determined by numerically integrating nine differential equations:

a) eight rate equations: $d x_{i} / d t=$ formation rate - disappearance rate $\left(X_{i}=\right.$ mole fraction),

b) one equation: $C_{p} d T / d t=\Sigma V_{j} \times Q_{j}$, in which $C_{p}$ is the molar specific heat of the 
mixture, $T$ is the temperature, and $V_{j}$ and $Q_{j}$ are respectively the reaction rate and the liberated heat of reaction $j$. Calculations were done between 700 and $1500{ }^{\circ} \mathrm{C}$ and between 1 and $40 \mathrm{~atm}$. The rate constants $\left(\mathrm{s}^{-1}\right)$ are taken from the Leeds compilation.

For part of the delay $t$ :

$$
x_{i}=k_{i} \exp (\alpha t)
$$

in which $\mathrm{i}=\mathrm{H}_{2} \mathrm{O}, \mathrm{H}_{2} \mathrm{O}_{2}, \mathrm{H}, \mathrm{OH}, \mathrm{O}$ and $\mathrm{HO}_{2} ; \alpha$ has exactly the same value for each species (fig. 1). Under such conditions, the determinant treatment used by Brokaw is valid [1] ${ }^{1}$. It is applied to a scheme including reactions $1,3,4,5$, and 6 . By also including reaction 2 (always neglected in literature), such a treatment gives a quintic equation, which can be further simplified in some cases; at a given pressure, it reduces to a cubic or a second degree equation for relatively high temperatures or low temperatures respectively.

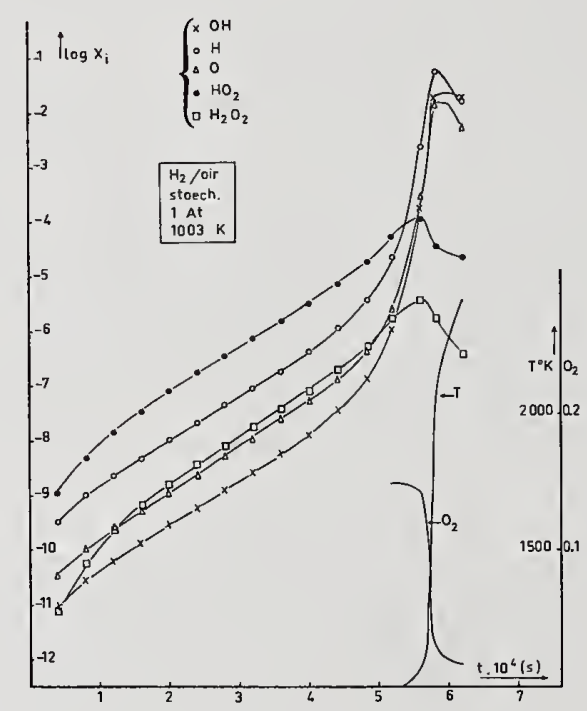

Figure 1. Species concentration and temperature profiles as a function of time at 1 atm pressure.

However, eq. (1) is valid solely during a time $t$ that is different from delay $\theta$. Hence, it is dangerous to correlate $\alpha$ and $\theta$ to determine $\theta$, as suggested by some authors. The difference between $t$ and $\theta$ may be great, and this means that some of the 2 nd order reactions are not negligible. For instance, in figure 2 , eq. (1) is not valid after $t=$ $2 \times 10^{-3}$ s because recombination reactions between $\mathrm{HO}_{2}$ radicals cannot be neglected.

${ }^{1}$ Figures in brackets indicate literature references at the end of this paper. 


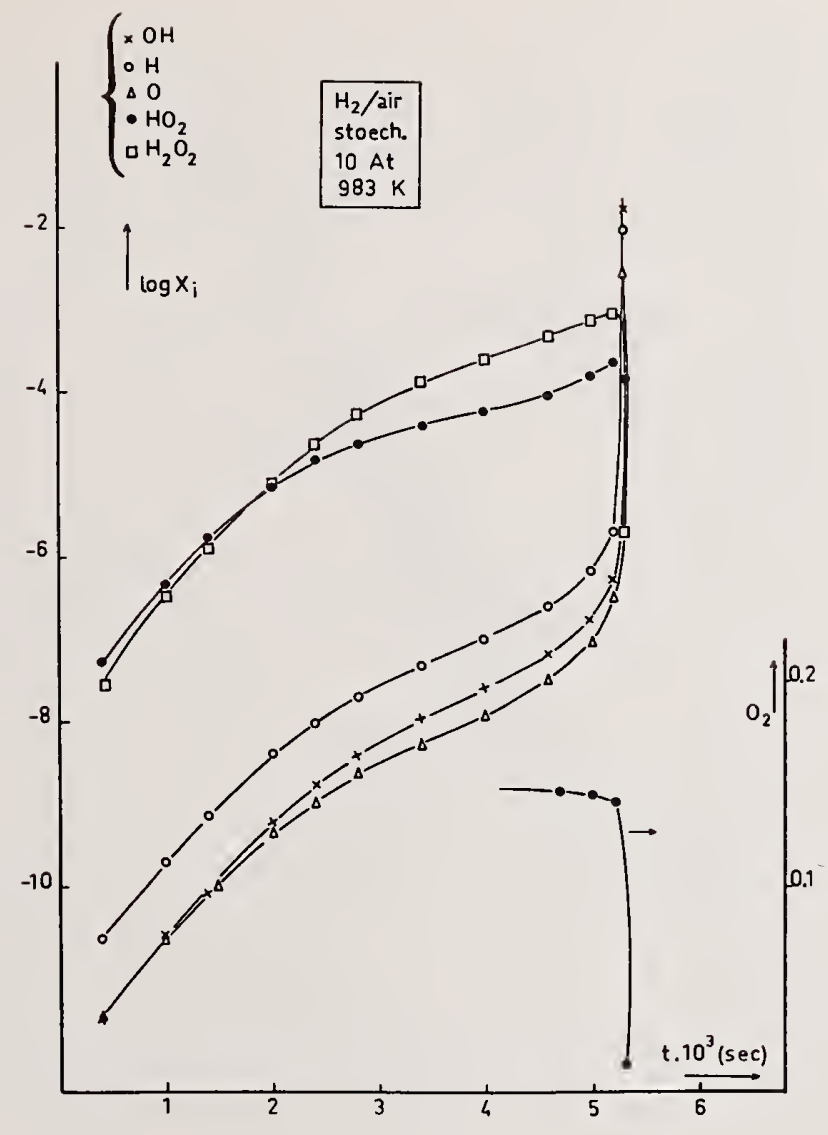

Figure 2. Species concentration profiles as a function of time at 10 atm pressure.

Whatever the case may be, it is always very abrupt increases in the concentration of $\mathrm{H}, \mathrm{OH}$ and 0 that cause auto-ignition (figs. 1 and 2).

From $\log \theta=f(1 / T)$ curves ( $f i g .3)$, at a given pressure, a short-delay zone $\left(2 k_{1}>k_{3} M\right)$ and a long-delay zone $\left(k_{3} M>2 k_{1}\right)$ can be distinguished. The separation between them occurs over a short temperature interval, with the inflection point corresponding to $2 \mathrm{k}_{1}=\mathrm{k}_{3} \mathrm{M}$. The corresponding temperatures are: $991 \mathrm{~K}$ (1 atm) $1180 \mathrm{~K}$ (5 $\mathrm{atm}), 1283 \mathrm{~K}(10 \mathrm{~atm}), 1403 \mathrm{~K}(20 \mathrm{~atm})$, and $1550 \mathrm{~K}(40 \mathrm{~atm})$.

When $2 k_{1}>k_{3} M, \theta$ is inversely proportional to $P$ and the temperature coefficient is equal to $17,000 \mathrm{cal} / \mathrm{mol}$. By varying the rate constants of the most important reactions it can be deduced that the predominant reaction is the branching stage 1 . It was also predicted that: $\theta=k\left(\mathrm{X}_{\mathrm{H}_{2}}\right)^{-0.35}\left(\mathrm{X}_{\mathrm{O}_{2}}\right)^{-0.60}$. These findings compare closely with the experimental data found by various authors, e.g. [2] and [3]. 


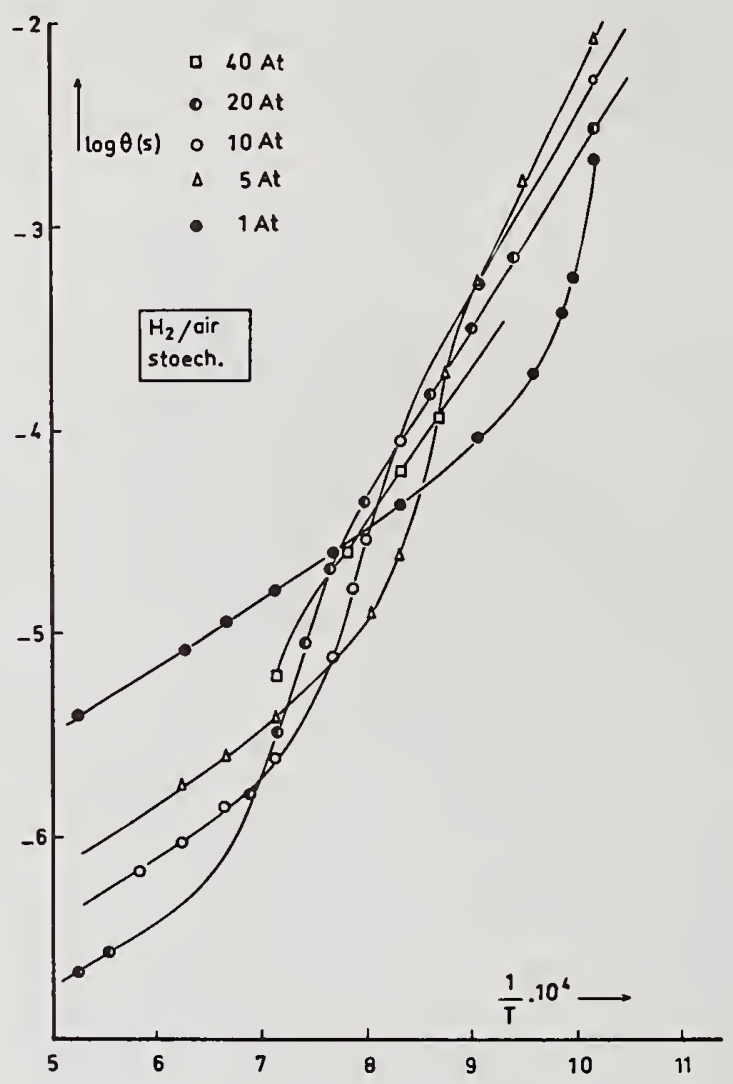

Figure 3. Dependence of auto-ignition delay time $\theta(s)$ with temperature $(K)$ and pressure (atm).

When $k_{3} M>2 k_{1}$, the temperature coefficient is higher than in the previous case. It also varies slightly with $P(35 \mathrm{kcal} / \mathrm{mol}$ at $40 \mathrm{~atm}$ and $46 \mathrm{kcal} / \mathrm{mol}$ at $5 \mathrm{~atm})$ so that a simple relation cannot be proposed between $\theta, P$ and $T$. A different mechanism occurs in this region where reactions with $\mathrm{HO}_{2}$ and $\mathrm{H}_{2} \mathrm{O}_{2}$ play an important part, as we found by varying the rate constants of the most important reactions. Here we determined: $\theta=k$ $\left(\mathrm{X}_{\mathrm{H}_{2}}\right)^{-0.70}\left(\mathrm{X}_{\mathrm{O}_{2}}\right)^{-0.05}$. The value of 0.70 compares favorably with that found by other authors [1].

Using only chemical kinetics does not predict auto-ignition limits.

The substitution of part or all of the $\mathrm{N}_{2}$ by $\mathrm{H}_{2} \mathrm{O}$ in stoichiometric $\mathrm{H}_{2}$-air mixtures leads to the following observations: As previously, for part of the delay $t, x_{i}=k_{j} \exp$ $(\alpha t) ; \alpha$ has the same value for each species, but only over a time range which is generally different from the delay value $\theta$ (fig. 4). 


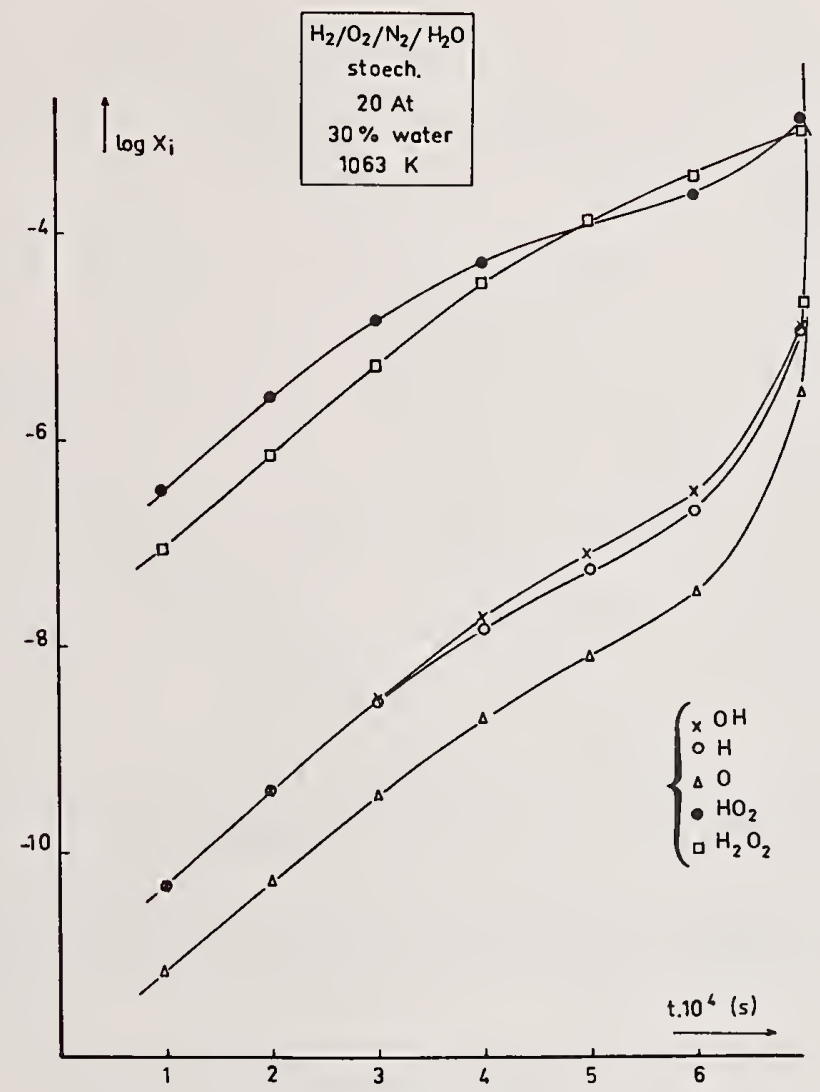

Figure 4. Dependence of species concentration on time at 20 atm and in the presence of 30 percent water.

Figure 5 gives an example of the influence of water vapor on auto-ignition delay time. Because the inflection point of $\log \theta=f(1 / T)$ curves is displaced toward the highest temperatures on account of the $2 k_{1}=k_{3} M$ condition (greatly affected by the presence of $\left.\mathrm{H}_{2} \mathrm{O}\right), \mathrm{H}_{2} \mathrm{O}$ has an inhibiting effect in the intermediate temperature zone. It can be shown that $\mathrm{H}_{2} \mathrm{O}$ acts mainly through its great effectiveness in reactions with a third body $M$ (mainly reaction 3 ), however, with a contribution opposite from the $\mathrm{HO}_{2}+\mathrm{H}_{2} \mathrm{O} \longrightarrow \mathrm{H}_{2} \mathrm{O}_{2}+$ $\mathrm{OH}$ reaction in the intermediate temperature zone. The importance of this latter reaction passes through a maximum versus temperature, for a given pressure; on the other hand it increases with pressure, for a given temperature. At relatively high temperatures, more precisely when $2 k_{1} / k_{3} M>7$, the effect of water is negligible. Hence, the reaction $\mathrm{H}+\mathrm{H}_{2} \mathrm{O} \longrightarrow \mathrm{H}_{2}+\mathrm{OH}$, for example, is negligible. At relatively low temperatures, more precisely when $k_{3} M / 2 k_{1}>40$, the effect of water is very slight. 


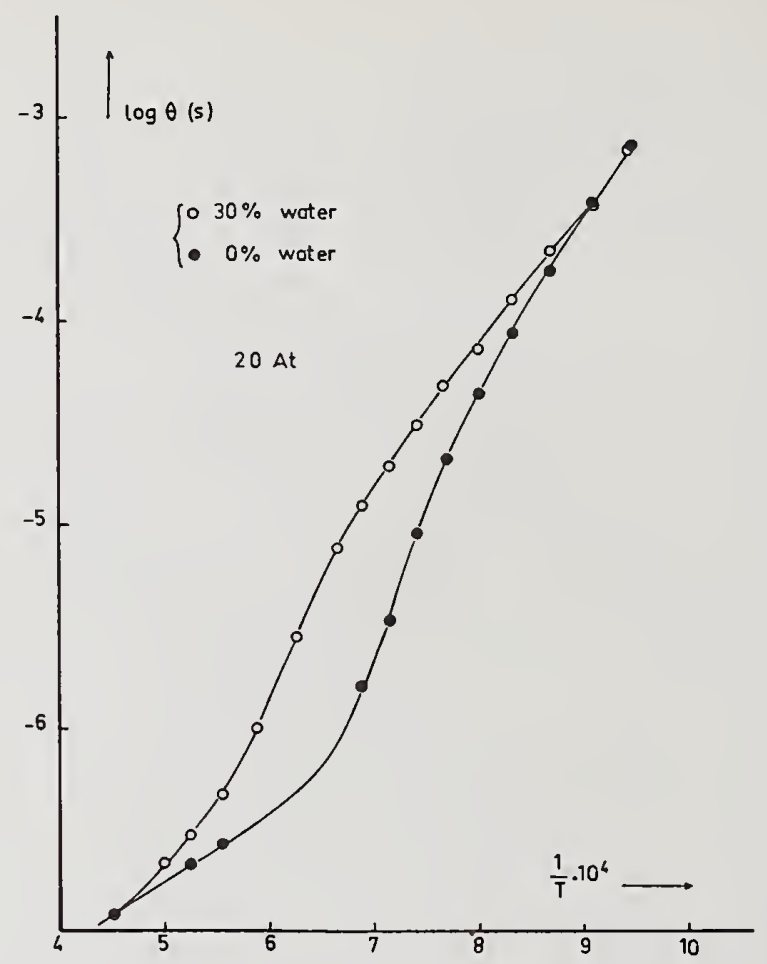

Figure 5. Effect of $\mathrm{H}_{2} \mathrm{O}$ on auto-ignition delay time at various temperatures and $20 \mathrm{~atm}$ pressure.

\section{Burning Velocities}

The burner method was used to experimentally determine the burning velocities $V_{0}$ for a number of $\mathrm{H}_{2}-\mathrm{O}_{2}-\mathrm{N}_{2}-\mathrm{H}_{2} \mathrm{O}$ flames between fuel-air equivalence ratios of 0.70 and 2.00 . As shown in figure 6 , an inhibiting effect of $\mathrm{H}_{2} \mathrm{O}$ was revealed.

The same kinetic model with 28 reactions was used with the following initial conditions: composition, $P=1 \mathrm{~atm}, T=$ mean temperature of the flame front $=T_{0}+\sigma\left(T_{f}-T_{0}\right)$, with $T_{0}=$ initial gas temperature, $T_{f}=$ adiabatic temperature of burned gases, and $\sigma=$ mean temperature coefficient [4]. For all the mixtures used, we could determine the profile of the different species, particularly of the hydrogen atoms. As shown in figure 7 , we observed a good and single linear relation between $V_{0}(\exp )$ and $\left(X_{H}\right)_{\max }(c a l c)$ over a large range of $V_{0}$ (from 40 to $250 \mathrm{~cm} / \mathrm{s}$ ), as was obtained by Fristrom under different conditions [5]. It can be shown that $\mathrm{H}_{2} \mathrm{O}$ does not act solely indirectly by lowering $\mathrm{T}_{f}$, but that it also acts directly by reactions with a third body, $M$, mainly by reaction 3 whose influence increases as $T_{f}$ decreases $^{2}$. It can also be seen ( $f i g .8$ ) that if the calculated values of $\left(X_{H}\right)$ max (which are directly proportional to $\mathrm{V}_{0}$ ) are plotted as a function of $\mathrm{X}_{\mathrm{H}_{2}}$ in $\mathrm{H}_{2}$-air mixtures, good agreement is obtained with the experimental data deduced from studies of other authors [6].

${ }^{2}$ Editor's note: Reuther, elsewhere in this volume makes a similar observation of this effect. 


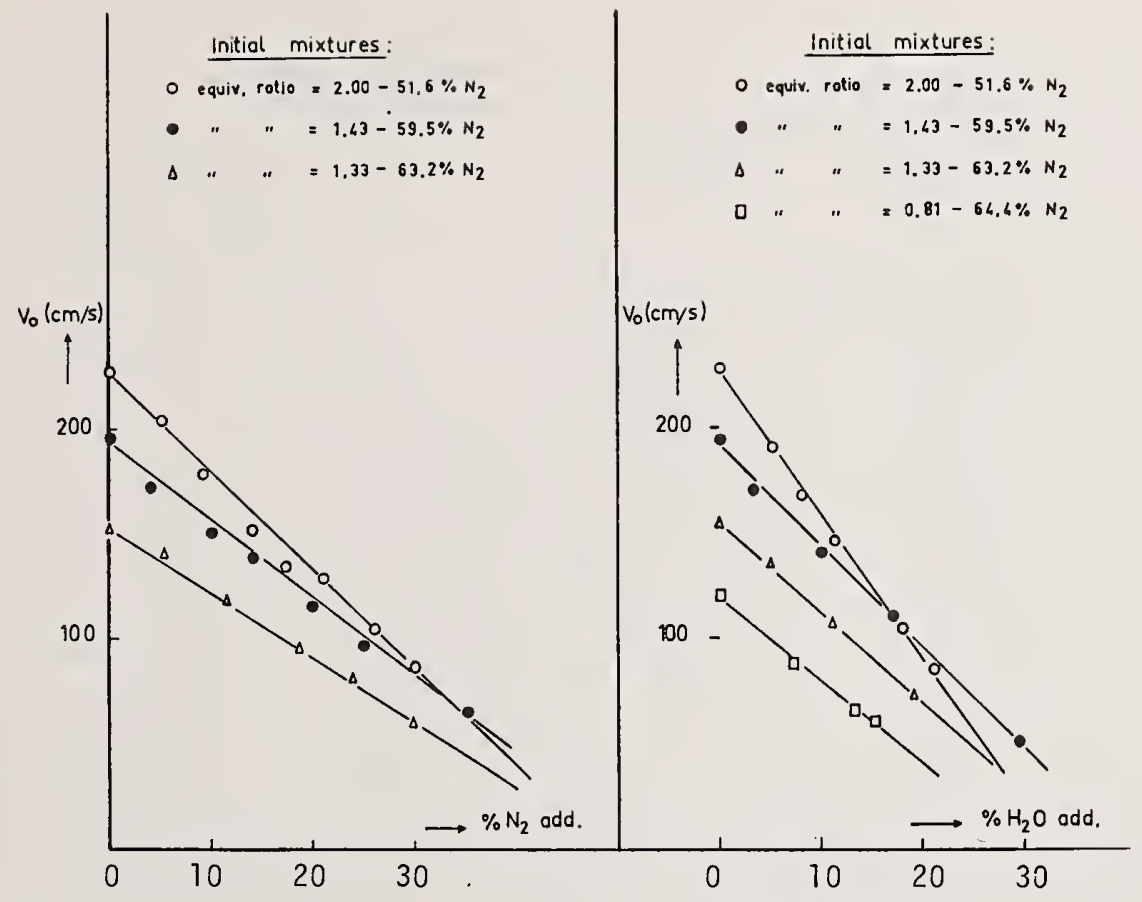

Figure 6. Effect of added $\mathrm{N}_{2}$ and $\mathrm{H}_{2} \mathrm{O}$ on experimental burning velocities.

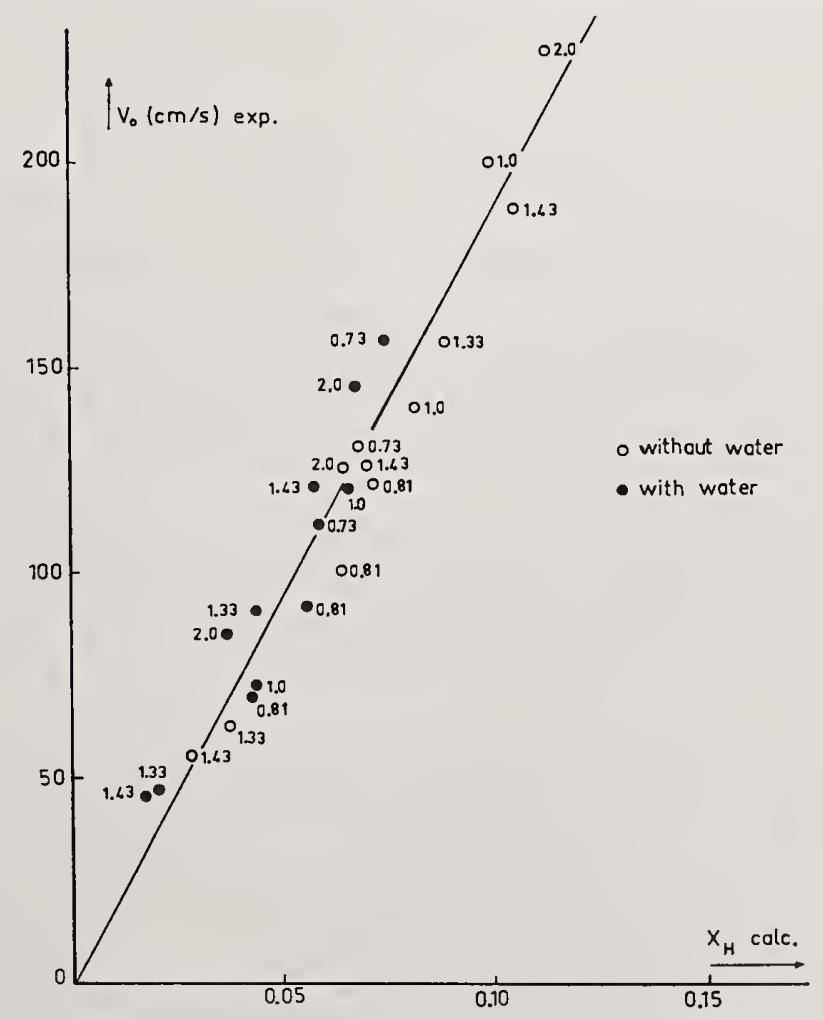

Figure 7. Correlation between burning velocity and $\mathrm{H}$-atom concentration. 


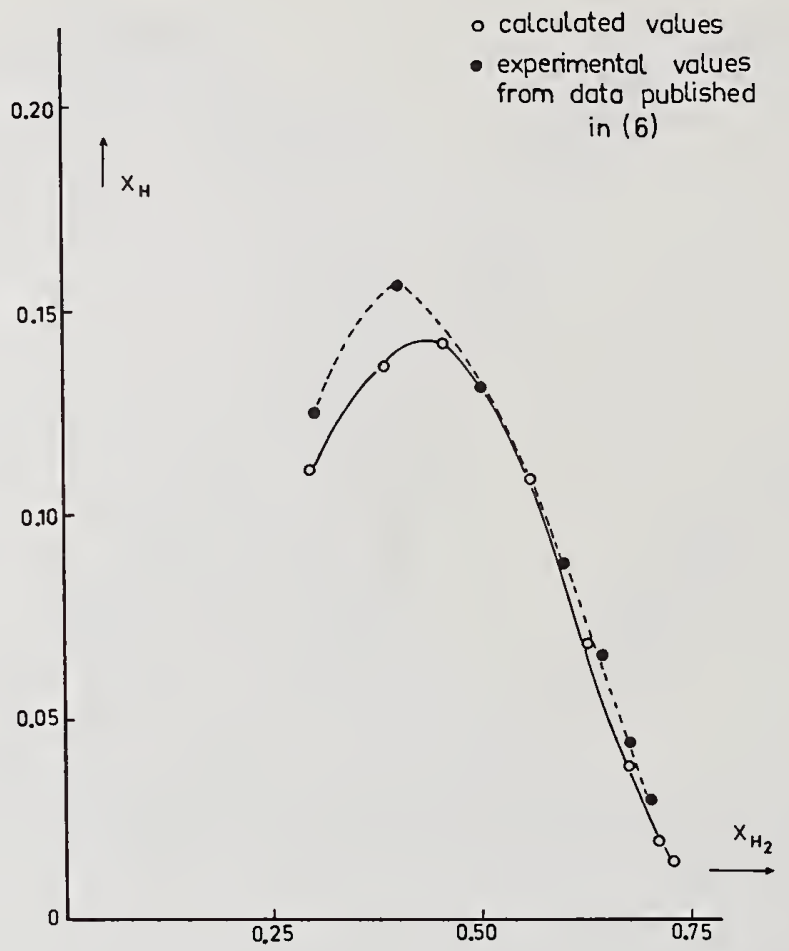

Figure 8. Comparison of calculated and experimental H-atom concentrations.

\section{Flammability Limits}

We experimentally determined the flammability limits of a great many $\mathrm{H}_{2}-\mathrm{O}_{2}-\mathrm{N}_{2}-\mathrm{H}_{2} \mathrm{O}$ mixtures at $T=388 \mathrm{~K}$ between $1 \mathrm{~atm}$ and $8 \mathrm{~atm}$ (by isothermal compression) in an electrically heated cylindrical vessel. A high-energy spark plug ( 5 joules) was used, and the measurement of the end pressure indicated both whether combustion had taken place and the completion of combustion. By restricting the range of flammability (fig. 9 for instance) $\mathrm{H}_{2} \mathrm{O}$ was again found to have an inhibiting effect. For example, for $\mathrm{H}_{2}-\mathrm{O}_{2}-\mathrm{N}_{2}$ mixtures at 1 atm with $\mathrm{N}_{2} / \mathrm{O}_{2}=3.76=$ and $\mathrm{N}_{2} / 0_{2}=8.00, \mathrm{~L}_{\mathrm{u}}$ (upper $1 \mathrm{imit}$ ) $=75$ percent and 53 percent respectively, while for $\mathrm{H}_{2}-\mathrm{O}_{2}-\mathrm{H}_{2} \mathrm{O}$ mixtures with the same $\mathrm{H}_{2}{ }^{0 / 0}$ ratios, $L_{u}=71$ percent and 38 percent, respectively.

The following experimental relations allow the calculation of the upper limits $L_{s}$ for different water vapor contents $M^{\prime}$ (expressed in percent) in the mixture:

$$
\begin{aligned}
& L_{s}=94.75-1.036 M^{\prime} \text { for } N_{2} / 0_{2}=0 \\
& L_{s}=84.25-1.09 M^{\prime} \text { for } N_{2} / 0_{2}=2 \\
& L_{s}=75.00-1.15 M^{\prime} \text { for } N_{2} / 0_{2}=3.76 \\
& L_{s}=63.25-1.28 M^{\prime} \text { for } N_{2} / 0_{2}=6.00
\end{aligned}
$$




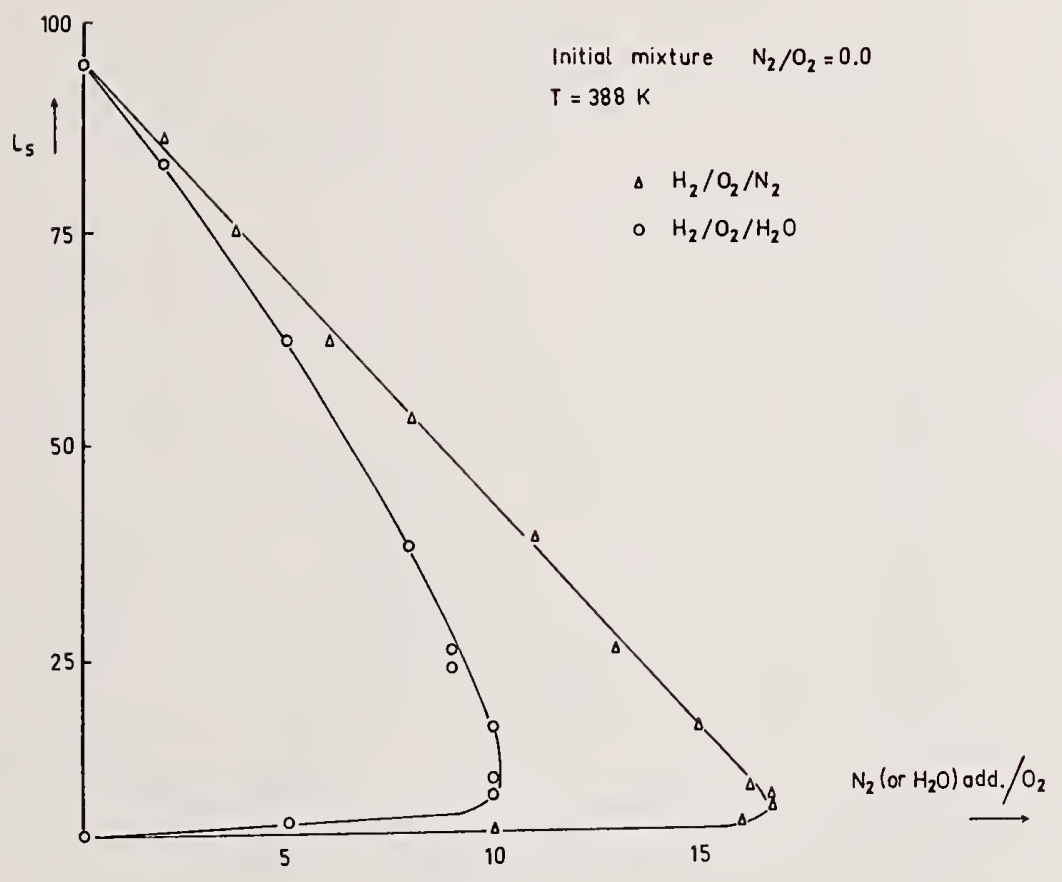

Figure 9. Effect of added $\mathrm{H}_{2} \mathrm{O}$ on flammability limits.

By plotting the calculated values of $\left(\mathrm{X}_{\mathrm{H}}\right)_{\max }$ (proportional to $\mathrm{V}_{\mathrm{O}}$ ) as a function of $\mathrm{X}_{\mathrm{H}_{2}}$ for mixtures having a given ratio $\left(\mathrm{uN}_{2}+\mathrm{vH}_{2} \mathrm{O}\right) / \mathrm{O}_{2}$ it was found (fig. 10) for all the mixtures analyzed that $L_{u(\exp )}=X_{H_{2}}$ when the $V_{0}$ value is relatively low. The extrapolation of curves with $V_{0}=0$ would give slightly higher values of $L_{u}$, but such an extrapolation is probably risky because it was experimentally found that combustion was only about 80 percent complete near the limits. Consequently, the true value $\mathrm{T}_{f}$ is smaller than the adiabatic value used in our model.

On the other hand, because of preferential diffusion phenomena, combustion is very incomplete near the lower limits $\mathrm{L}_{e}$, as experimentally observed. Therefore, we cannot interpret easily the values of $\mathrm{L}_{e}$, but fortunately they slightly vary with the dilution. At the same time, the effect of pressure on $L_{u}$ is satisfactorily interpreted with our model. 


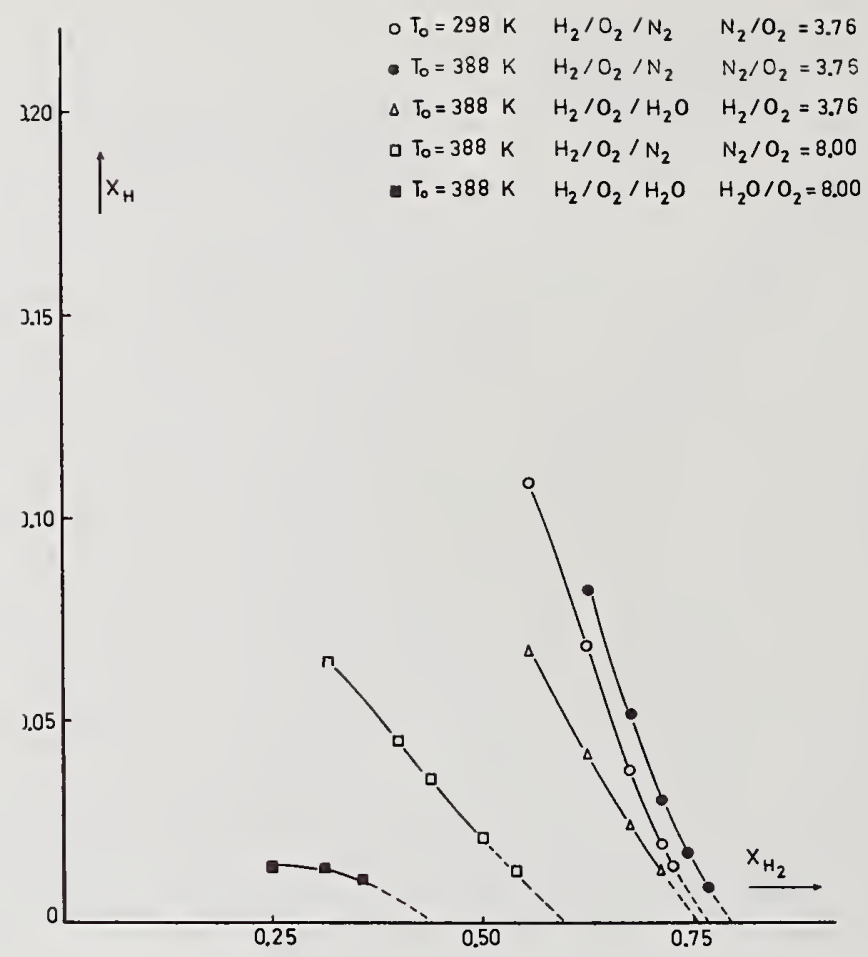

Figure 10. Dependence of $\mathrm{H}$-atom concentration on initial temperature, $\mathrm{N}_{2}, \mathrm{H}_{2}$, and $\mathrm{H}_{2} \mathrm{O}$ concentration.

\section{Appendix} $\left.s^{-1}\right)$.

Rate constants of the principal reactions used in the kinetic model (expressed in

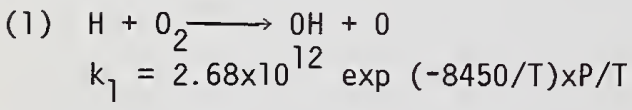

(2) $\mathrm{H}_{2} \mathrm{O}_{2}+\mathrm{M} \longrightarrow \mathrm{OH}+\mathrm{OH}+\mathrm{M}$

$k_{2}=1.82 \times 10^{15}\left[1+X_{H_{2}}+3 X_{H_{2}} \mathrm{O}\right] \quad \exp (-22,900 / \mathrm{T}) \times \mathrm{P} / \mathrm{T}$

(3) $\mathrm{H}+\mathrm{O}_{2}+\mathrm{M} \longrightarrow \mathrm{HO}_{2}+\mathrm{M}$

$k_{3}=1.108 \times 10^{11}\left[4+6 \mathrm{X}_{\mathrm{H}_{2}}+60 \mathrm{X}_{\mathrm{H}_{2} \mathrm{O}}\right] \exp (500 / \mathrm{T}) \times \mathrm{P}^{2} / \mathrm{T}^{2}$

(4) $\mathrm{HO}_{2}+\mathrm{H}_{2} \longrightarrow \mathrm{H}_{2} \mathrm{O}_{2}+\mathrm{H}$

$\mathrm{k}_{4}=1.78 \times 10^{10^{2}} \exp (-9400 / \mathrm{T}) \times \mathrm{P} / \mathrm{T}$ 
(5) $\mathrm{H}_{2}+\mathrm{O} \longrightarrow \mathrm{H}+\mathrm{OH}$

$k_{5}=2.19 \times 10^{8} \quad \exp (-4480 / \mathrm{T}) \times \mathrm{P} / \mathrm{T}$

(6) $\mathrm{H}_{2}+\mathrm{OH} \longrightarrow \mathrm{H}_{2} \mathrm{O}+\mathrm{H}$

$\mathrm{k}_{6}=2.68 \times 10^{11^{2}} \exp (-2590 / \mathrm{T}) \times \mathrm{P} / \mathrm{T}$

(7) $\mathrm{HO}_{2}+\mathrm{H}_{2} \mathrm{O} \longrightarrow \mathrm{H}_{2} \mathrm{O}_{2}+\mathrm{OH}$

$\mathrm{k}_{7}=3.41 \times 10^{11} \quad \exp (-16,500 / \mathrm{T}) \times \mathrm{P} / \mathrm{T}$

(8) $\mathrm{H}+\mathrm{H}_{2} \mathrm{O} \longrightarrow \mathrm{H}_{2}+\mathrm{OH}$

$k_{8}=1.13 \times 10^{12^{2}} \exp (-10,250 / \mathrm{T}) \times \mathrm{P} / \mathrm{T}$

(9) $\mathrm{H}+\mathrm{H}+\mathrm{M} \longrightarrow \mathrm{H}_{2}+\mathrm{M}$

$k_{9}=3.54 \times 10^{11}\left[1+4 X_{H_{2}}\right] \times P^{2} / T^{2}$

(10) $\mathrm{HO}_{2}+\mathrm{HO}_{2} \longrightarrow \mathrm{H}_{2} \mathrm{O}_{2}+\mathrm{O}_{2}$

$\mathrm{k}_{10}=1.80 \times 10^{10} \times \mathrm{P} / \mathrm{T}$

\section{References}

[1] Brokaw, R. S., 10th Symposium on Combustion, The Combustion Institute, Pittsburgh, PA, 1965 , p. 269.

[2] Voevodsky, V. V. and Soloukhin, R. I., ibid, 1965, p. 279.

[3] Asaba, T., Gardiner, W. C., and Stubbeman, R. F., ibid, 1965, p. 295.

[4] Van Tiggelen, A., et al., Oxydationset Combustions, Tome I, Editions Technip, Paris (1968), p. 480.

[5] Brown, N. J., Fristrom, R. M., and Sawyer, R. F., Combustion and Flame, 23, 269 (1974).

[6] Gunther, R. and Janisch, G., Combustion and Flame, 19, 49 (1972). 
National Bureau of Standards Special Publication 561, Proceedings of the 10th Materials Research Symposium on Characterization of High Temperature Vapors and Gases held at NBS, Gaithersburg, Maryland, September 18-22, 1978. Issued October 1979.

\title{
INHIBITION ON THE PROPAGATION OF FLAME
}

\author{
Tadaaki Inomata, Satiko Okazaki, \\ Takao Moriwaki, and Mototaro Suzuki \\ Department of Chemistry \\ Faculty of Science and Technology \\ Sophia University \\ Tokyo, Japan
}

\section{Introduction}

Though the inhibition of halides on hydrocarbon flames were investigated using various burners, the conclusions did not agree between one another and the mechanisms of inhibition were not yet unanimously established.

Miller, et al. [1] ${ }^{1}$ have reported the flame velocities of methane-air gas mixtures to be related linearly with the molar fraction of $\mathrm{CHBr}_{3}$ and the inhibition effects were less in chlorides than in other halogen compounds. Similar tendencies are also reported in the case of an ethylene-air system [2]. Meanwhile, Heap and Edmondson [3] have reported the flame inhibition of methane and $\mathrm{CH}_{3} \mathrm{Br}$ flames to be augmented with the content of methane but not linearly with the concentration of $\mathrm{CH}_{3} \mathrm{Br}$. This slow down of velocity was less with increased concentration of $\mathrm{CH}_{3} \mathrm{Br}$. The same tendency was observed in the propane-air mixture [4].

In the investigations in the past, the main agreements were where the inhibitions were large in the fuel rich mixture and the trends of inhibition are iodide > bromide $>$ chloride. The mechanism of inhibition is, for the present, attributed to the decrease of the active agents $\mathrm{H}, \mathrm{OH}$, and 0 , but the elementary processes are not yet clearly decided.

In the case where the inhibitor was the halogen acid, the reaction

$$
\mathrm{HX}+\mathrm{H} \rightarrow \mathrm{H}_{2}+\mathrm{X}
$$

was more rapid than the chain branching

$$
\mathrm{H}+\mathrm{O}_{2} \rightarrow \mathrm{OH}+\mathrm{H}
$$

and these reactions were said to be responsible for the inhibition of flame [5], but they were still insufficient to fully describe the phenomena $[6,7]$. Butlin and Simmons supported the mechanism of (1) and (2) in rich mixtures but not for lean mixtures [8].

\footnotetext{
${ }^{1}$ Figures in brackets indicate the literature references at the end of this paper.
} 
In the case of hydrocarbon halides, hydrogen halide was created as an intermediate and inhibition occurred according to the reactions (1) and (2) $[1,8,9]$ or the active agents might react directly with the hydrocarbon halide [10-14]. It was suggested that the inhibition had taken place in the pre-ignition zone and the inhibitors were said to be consumed in this region $[10,13]$. The reports of inhibition in the propagating flame (reaction zone) were almost none.

In this report, the propagating velocity of the flame, the relative intensity of emission of $\mathrm{OH}, \mathrm{CH}$, and $\mathrm{C}_{2}$, and the infrared absorption spectra of the combustion products were compared, and the mechanism of inhibition was investigated.

\section{Experimental}

The initial gas mixtures were 7-12 volume percent $\mathrm{CH}_{4}$-air and 1-5 volume percent $\mathrm{CH}_{2} \mathrm{Cl}_{2}$, $\mathrm{CHCl}_{3}, \mathrm{CCl}_{4}$, and $\mathrm{Ar}$ and initial pressures of $100-300 \mathrm{mmHg}$ were used. The combustion tube was measured by photography using a rotating disk with a hole of $2 \mathrm{~cm}$ and a velocity of $1500 \mathrm{rpm}$. With this method within the region of $45 \mathrm{~cm}$ from the beginning, the combustion velocity of the flame cannot be measured accurately, the data were used for distances of greater than $50 \mathrm{~cm}$ from the ignition.

At the place of $78 \mathrm{~cm}$ from the ignition point, where the flame stabilizes enough, the light was taken out, divided in two with a half mirror and measured simultaneously. The measured wavelengths were $\mathrm{OH}(306.4 \mathrm{~nm}), \mathrm{CH}(431.5 \mathrm{~nm})$, and $\mathrm{C}_{2}(516.5 \mathrm{~nm})$ with monochrometer or interference filter. The products were analyzed with an infrared spectrophotometer.

\section{Results and Discussion}

\section{1 Flame velocity studies}

Before $45 \mathrm{~cm}$ from the ignition point, the flame propagates with rapid repeating of the acceleration and deceleration, but after that the wave front is half elliptic and the flame is laminar with a smooth repeating of small changes of velocity. The results are given in figure 1. In figure 1 , the ratios of the flame velocities without any inhibitor to those with inhibitor are given with respect to the location of the flame in the tube. The ratio increases with the decrease of suppression as the curve goes farther. The curve of the flame velocity without any inhibitor decreases as the curve goes further from the ignition point linearly, but the curve with inhibitor tends to a constant velocity as the curve goes on.

The inhibition effect changes with distance from the ignition point. At $78 \mathrm{~cm}$ from the ignition point, the flame becomes laminar and steady and the fluctuation of flame velocity is smal1. Therefore, this point was taken as a standard point of flame velocity and emission intensity.

In figures $2 a, b$, and $c$, the curves for the initial pressure of gas mixture versus $v / v_{0}$, where $v_{0}$ is the flame velocity of the gas mixture without inhibitor, and $v$ is with inhibitor, are shown. As the concentration of methane increases, the dependence of the inhibitor on the initial pressure is larger, though the effects vary according to the nature of the inhibitor. 
In the case of $\mathrm{CH}_{2} \mathrm{Cl}_{2}$ and $\mathrm{CHCl}_{3}$, large effects are seen in methane rich mixtures, but for $\mathrm{CCl}_{4}$ in a methane lean system the effect is large. That the effect of inhibitor increases a the methane concentration increases [3], is not always true. In all initial gas pressures and in more than 12.0 percent $\mathrm{CH}_{4}$, the effect is $\mathrm{CCl}_{4}<\mathrm{CHCl}_{3}<\mathrm{CH}_{2} \mathrm{Cl}_{2}$ and in less than 10.0 percent $\mathrm{CH}_{4}, \mathrm{CH}_{2} \mathrm{Cl}_{2}<\mathrm{CHCl}_{3}<\mathrm{CCl}_{4}$, i.e. the direction is quite opposite. In the mixture with 11.4 percent $\mathrm{CH}_{4}$, the direction is intermediate.

$\mathrm{Cl}$ atoms are effective for the suppression of the flame, therefore, $\mathrm{CCl}_{4}$ will naturally be the most effective. The opposite direction of effectiveness observed with more than 12.0 percent $\mathrm{CH}_{4}$ is due probably to the fact that the $\mathrm{Cl}$ atom then contributes to the chain branching. In fact, in the flame, the $\mathrm{Cl}$ atom will react with $\mathrm{H}_{2}$

$$
\mathrm{Cl}+\mathrm{H}_{2} \rightarrow \mathrm{HCl}+\mathrm{H}
$$

as has been proposed by Butlin and Simmons [8], and in a methane rich gas mixture this reaction cannot be ignored. With the addition of inhibitor, the temperature of the flame becomes higher than that of uninhibited flame [13]. Thus, in case of adding $\mathrm{CCl}_{4}$ to the 12.0 percent methane mixture, it is difficult to ignite the gas mixture at first but after the ignition, because of the high temperature in the flame, the reaction (1) will tend to reverse.

As is reported elsewhere [10-14], the reaction (1) suppresses the flame, but in the case of chloride the reverse reaction $(-1)$ will occur. This type of reaction is strongly exothermic; e.g. ,

$$
\mathrm{H}+\mathrm{CH}_{2} \mathrm{Cl}_{2} \rightarrow \mathrm{HCl}+\mathrm{CH}_{2} \mathrm{Cl}+24 \mathrm{kCal} \text {. }
$$

The reports of Butlin and Simmons [8] and of Levy, et al. [9] in which after combustion the halide will take the form of an acid as in (1) is not adequate explanation for inhibition in the present investigation. As is shown in figure $3, v / v_{0}$ tends to a definite value as the concentration of the chloride is increased in the initial gas mixture. Rosser, et al. [1] indicated that the decomposition of inhibitor occurred first and then the suppressive reaction followed. But the result of figure 3 contradicts this. This is also supported by the analysis of combustion products (table 1). Rosser, et al. [1] suggested that the inhibitors were all consumed in the pre-ignition zone and would not be found in the combustion products. But in the present investigation, inhibitors were found in the combustion products. Apparently some of the inhibitor was not consumed by the flame.

Table 1. Combustion products detected by infrared absorption spectra. Uninhibited flame Inhibited flame

$$
\mathrm{CO}, \mathrm{CO}_{2}, \mathrm{H}_{2} \mathrm{O} \quad \mathrm{CO}, \mathrm{CO}_{2}, \mathrm{H}_{2} \mathrm{O}, \mathrm{HCl} \text {, inhibitor }
$$



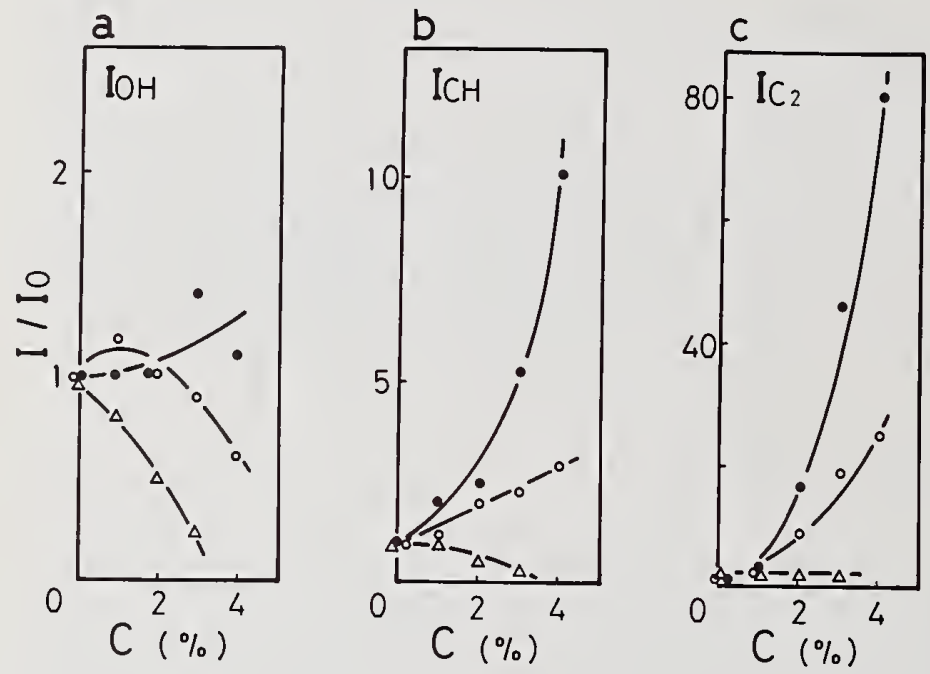

Figure 4. Ratio of intensities of emission spectra in uninhibited $\left(I_{0}\right)$ and inhibited (I) flames. $\mathrm{C}$ is volume percentage of $\mathrm{CH}_{2} \mathrm{Cl}_{2}$. - 6.8 percent; $0,9.1$ percent; $\Delta, 11.4$ percent $\mathrm{CH}_{4}$.

In figure 5, the ratios of intensities of two types of radicals where the one inhibitor is zero and the other inhibitor is present are shown. In the reactive gas mixture, two reactions are present [17]

$$
\begin{gathered}
\mathrm{C}+\mathrm{CH} \rightarrow \mathrm{C}_{2}{ }^{*}+\mathrm{H} \\
\mathrm{C}_{2}+\mathrm{OH} \rightarrow \mathrm{CH}^{\star}+\mathrm{CO} .
\end{gathered}
$$

Therefore

$$
\begin{array}{ll}
\mathrm{I}_{\mathrm{OH}} \propto[\mathrm{CH}]\left[\mathrm{O}_{2}\right] & {[\text { from }(5)]} \\
\mathrm{I}_{\mathrm{C}_{2}} \propto[\mathrm{C}][\mathrm{CH}] & {[\text { from }(6)]} \\
\mathrm{I}_{\mathrm{CH}} \propto\left[\mathrm{C}_{2}\right][\mathrm{OH}] & {[\text { from }(7)]}
\end{array}
$$

and from (I) and (II)

$$
\frac{\mathrm{I}_{\mathrm{OH}} / \mathrm{I} \mathrm{C}_{2}}{\left(\mathrm{I}_{\mathrm{OH}}\right)_{0} /\left(\mathrm{I}_{\mathrm{C}_{2}}\right)_{\mathrm{O}}} \propto \frac{\left[\mathrm{C}_{0}\left[\mathrm{O}_{2}\right]\right.}{[\mathrm{C}]\left[\mathrm{O}_{2}\right]_{0}}
$$

where $I_{0}$ is the intensity of the flame emission without inhibitor. 


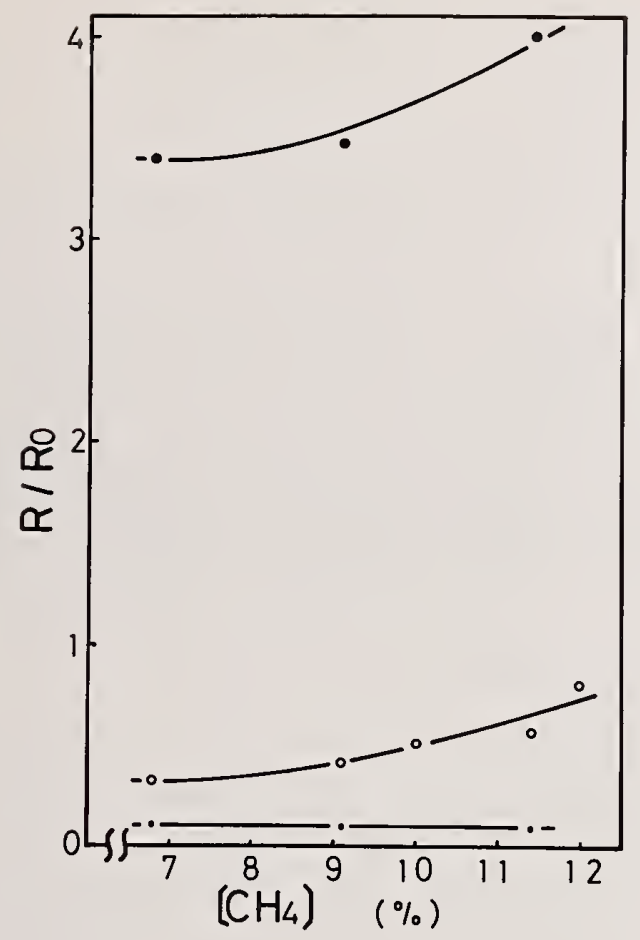

Figure 5. $R$ is the ratio of intensities of emission spectra of any two species in inhibited flame. $R_{0}$ is the case of uninhibited flame. $R$ are: $\bullet, \mathrm{I}_{\mathrm{C}_{2}} / \mathrm{I}_{\mathrm{CH}} ; \mathrm{O}, \mathrm{I}_{\mathrm{OH}} / \mathrm{I}_{\mathrm{CH}} ; \bullet$, $\mathrm{I}_{\mathrm{OH}} / \mathrm{I}_{\mathrm{C}_{2}}$

Here the methane concentration is constant, then $\left[\mathrm{O}_{2}\right]=\left[\mathrm{O}_{2}\right]_{0}$ and left side of expression (IV) is proportional to the $[\mathrm{C}]_{0} /[\mathrm{C}]$. As is clear from figure 5 , the left side of expression (IV) has a constant value and is not proportional to the methane concentration, hence $[\mathrm{C}]_{0} /[\mathrm{C}]$ must also have a constant value and not be proportional to the methane concentration.

In figure $6, \mathrm{I}_{\mathrm{C}_{2}} /\left(\mathrm{I}_{\mathrm{C}_{2}}\right)_{0}$ will decrease as the methane concentration increases. From the expression (II)

$$
\frac{\mathrm{I}_{\mathrm{C}_{2}}}{\left(\mathrm{I}_{\left.\mathrm{C}_{2}\right)_{0}}\right.} \propto \frac{[\mathrm{C}][\mathrm{CH}]}{[\mathrm{C}]_{0}[\mathrm{CH}]_{0}}
$$

and as the methane concentration increases $[\mathrm{CH}] /[\mathrm{CH}]_{0}$ decreases accordingly. Therefore, the increase of inhibitor or the increase of methane concentration, or in other words, the more the suppression effect, the more $[\mathrm{CH}]$ decreases.

In figure 7 , the intensity ratios of emission of radical are shown. From (I) and (II),

$$
\frac{\mathrm{I}_{\mathrm{OH}}}{\mathrm{I}_{\mathrm{C}_{2}}} \propto \frac{\left[\mathrm{O}_{2}\right]}{[\mathrm{C}]}
$$



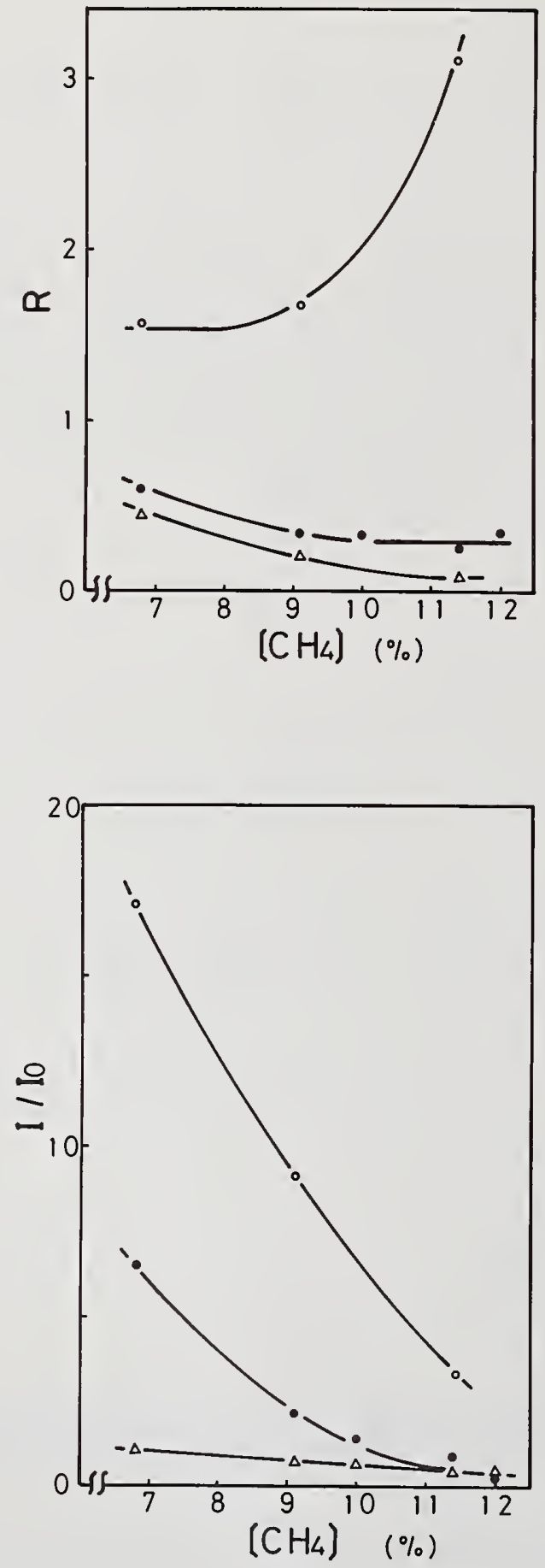

Figure 6 . The relation of methane concentration and the ratio of emission intensity. The initial pressure is $200 \mathrm{mmHg}$ and the inhibitor is 2 percent $\mathrm{CH}_{2} \mathrm{Cl}_{2}$. $\mathrm{O}, \mathrm{I}_{\mathrm{C}_{2}} ; \bullet, \mathrm{I}_{\mathrm{CH}} ; \Delta, \mathrm{I}_{\mathrm{OH}}$.
Figure 7. Ratio of the emission intensities of any two species: $\mathrm{O}, \mathrm{I}_{\mathrm{C}_{2}} / \mathrm{I}_{\mathrm{CH}} ; \bigcirc, \mathrm{I}_{\mathrm{OH}^{\prime}}$ $\mathrm{I}_{\mathrm{CH}} ; \Delta, \mathrm{I}_{\mathrm{OH}} / \mathrm{I}_{\mathrm{C}_{2}}$. Inhibitor is 2 percent $\mathrm{CH}_{2} \mathrm{Cl}_{2}$ and the initial pressure is 200 $\mathrm{mmHg}$.

In this expression, though $\left[\mathrm{O}_{2}\right]$ increases, this alone cannot explain the remarkable change of the emission intensity. Thus, the concentration of [C] may increase along with the increase of the concentration of methane. The suppressed flame is characterized by the decrease of $[\mathrm{CH}]$ and increase of $[\mathrm{C}]$. 
Now from (II) and (III)

$$
\frac{{ }^{\mathrm{I}_{2}}}{\mathrm{I}_{\mathrm{CH}}} \propto \frac{[\mathrm{C}][\mathrm{CH}]}{\left[\mathrm{C}_{2}\right][\mathrm{OH}]}
$$

and the precursor of $C_{2}$ is $C$, from (VII)

$$
\frac{\mathrm{I}_{2}}{\mathrm{I}_{\mathrm{CH}}} \propto \frac{[\mathrm{CH}]}{[\mathrm{OH}]} .
$$

Because the left side of this expression increases with the increase of methane concentration (fig. 7) and $[\mathrm{CH}]$ decreases (eq. $\mathrm{V}$ ), $[\mathrm{OH}]$ decreases with the increase of methane contration. The oxidation of methane is [18]

$$
\begin{gathered}
\mathrm{CH}_{4}+\beta(\mathrm{H}, \mathrm{OH}, \mathrm{O}) \rightarrow \mathrm{CH}_{3}+\mathrm{HB} \\
\mathrm{H}+\mathrm{O}_{2} \rightarrow \mathrm{OH}+\mathrm{O} \\
\mathrm{OH}+\mathrm{CO} \rightarrow \mathrm{CO}_{2}+\mathrm{H} .
\end{gathered}
$$

The diffusion velocity is said to be unimportant in flame inhibition [1]; the suppression of a flame prevents the reactions (2), (8), and (9). If the suppression is in effect through the $H$ radical reaction, then (2) and ( 8 ) will be suppressed by (3) and as no methane is observed in the end product the suppression is effected mainly through (2). Moreover, if the $\mathrm{OH}$ radical reaction is effective in the suppression reaction, then (8) and (9) will be suppressed by

$$
\begin{gathered}
\mathrm{OH}+\mathrm{HCl} \rightarrow \mathrm{H}_{2} \mathrm{O}+\mathrm{Cl} \\
\mathrm{OH}+\mathrm{CH}_{2} \mathrm{Cl}_{2} \rightarrow \mathrm{ClOH}+\mathrm{CH}_{2} \mathrm{Cl} .
\end{gathered}
$$

The activation energy of reaction (3) is $6 \mathrm{kcal} \mathrm{mol}^{-1}$ and is smaller than $(8)\left(13 \mathrm{kcal} \mathrm{mol}^{-1}\right)$ and (2) $\left(17 \mathrm{kcal} \mathrm{mol}^{-1}\right)$ [19] and is therefore more favorable for the suppression of $\mathrm{H}$ atoms. The reaction (11) has a high activation energy and (10) is more favorable for $\mathrm{OH}$ suppression. The rate constant of the reaction (10), at flame temperature, ten times greater than that of the reaction (9) and about half reaction (8) [10]. Therefore the suppression of the $\mathrm{OH}$ radical through reaction (10) cannot be neglected, but the suppression through reaction (3) will be more favorable.

In figure $8, \mathrm{I}_{\mathrm{C}_{2}} / \mathrm{I}_{\mathrm{CH}}$ is shown vs the flame velocities. Note that the ratio is only dependent on the inhibitors and $\mathrm{I}_{\mathrm{C}_{2}} / \mathrm{I} \mathrm{CH}$ is therefore an important factor in flame inhibition. 
The fact that the argon, which is just a diluent, results in a horizontal line indicates that the suppression by the inhibitors is more important than a diluent effect. In the presence of inhibitors, $\mathrm{I}_{\mathrm{C}_{2}} / \mathrm{I}_{\mathrm{CH}}$ increases as the flame velocities decrease. $\mathrm{I}_{\mathrm{C}_{2}} / \mathrm{I}_{\mathrm{CH}}$ is proportional

to the value of $[\mathrm{CH}] /[\mathrm{OH}]$ (VII), thus the decrease of $[\mathrm{OH}]$ means that the reaction (2) is suppressed and the $[\mathrm{H}]$ concentration decreases in the suppressed $\mathrm{flame}$. The fact that $\mathrm{OH}$ created in the reaction (2) is consumed in the suppressed reaction and the chain propagation is prevented can explain the experimental facts only in part, in addition one has to include reaction (3) as a main inhibition reaction.

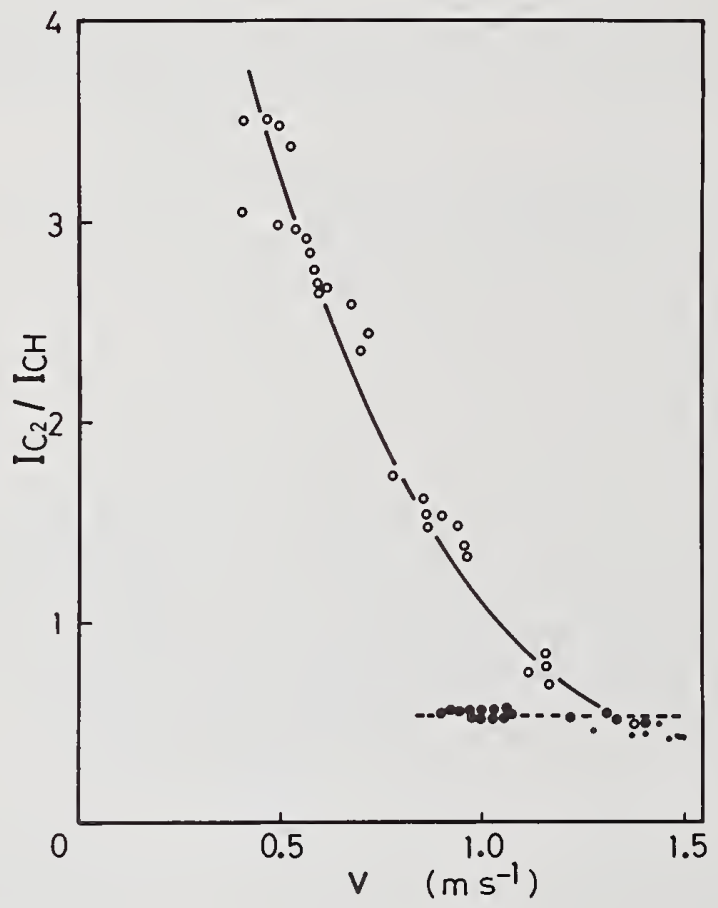

Figure 8. The relation of $\mathrm{I}_{\mathrm{C}_{2}} / \mathrm{I}_{\mathrm{CH}}$ and flame velocity (v) in 9.1 percent $\mathrm{CH}_{4}$. Initial pressures are 100-300 $\mathrm{mmHg}$ and concentration of inhibitors are 0-4 percent. $\mathrm{O} ; \mathrm{CH}_{2} \mathrm{Cl}_{2}, \mathrm{CHCl}_{3}$, and $\mathrm{CCl}_{4}$ : 0 ; $\mathrm{Ar}: \bullet$; no additive (inhibitors and argon).

\section{Summary}

The inhibition effects on flames by the chloro methane additives in fuel lean mixtures indicate: $\mathrm{CCl}_{4}>\mathrm{CHCl}_{3}>\mathrm{CH}_{2} \mathrm{Cl}_{2}$ and in fuel rich mixtures $\mathrm{CH}_{2} \mathrm{Cl}_{2}>\mathrm{CHCl}_{3}>\mathrm{CCl}_{4}$. In the propagating flame, the inhibition is carried out through a reaction mainly of the type $H+R X$ $\rightarrow \mathrm{HCl}+\mathrm{R}$. But the contribution of $\mathrm{OH}$ to the suppression cannot be ignored. In the suppressed flame, the value of $[\mathrm{C}]$ increases and $[\mathrm{CH}]$ and $[\mathrm{OH}]$ decrease. Moreover, $\mathrm{I}_{\mathrm{C}_{2}} / \mathrm{I}_{\mathrm{CH}}$ increases as the flame velocities decrease and this is a good criterion for flame inhibition. 


\section{References}

[1] Rosser, W. A., Wise, H., and Miller, J., Seventh Symposium (International) on Combustion, 175 (1959).

[2] Simmons, R. F. and Wolfhard, H. G., Trans. Faraday Soc. 51, 1211 (1955).

[3] Edmondson, H. and Heap, M. P., Combust. Flame, 13, 472 (1969).

[4] Garner, F. H., Long, R., Graham, A. J., and Badakhshan, A., Sixth Symposium (International) on Combustion, 802 (1957).

[5] Wilson, W. E., Jr., Donovan, J. T., and Fristrom, R. M., Twelfth Symposium (International) on Combustion, 929 (1969).

[6] Dixon-Lewis, G. and Simpson, R. J., Sixteenth Symposium (International) on Combustion, 1111 (1976).

[7] Day, M. J., Stamp, D. V., Thompson, K., and Dixon-Lewis, G., Thirteenth Symposium (International) on Combustion, 705 (1971).

[8] Butlin, R. N. and Simmons, R. F., Combust. Flame, 12, 447 (1968).

[9] Levy, A. J., Droege, J. W., Tighe, J. J., and Foster, J. F., Eighth Symposium (International) on Combustion, 524 (1962).

[10] Wilson, W. E., Jr., Tenth Symposium (International) on Combustion, 47 (1965).

[11] Burdon, M., Burgoyne, J. H., and Weinberg, F. J., Fifth Symposium (International) on Combustion, 647 (1955).

[12] Fenimore, C. P. and Jones, G. W., Combust. Flame, ㄱ, 323 (1963).

[13] Biordi, J. C., Lazzara, C. P., and Papp, J. F., Fourteenth Symposium (International) on Combustion, 367 (1973).

[14] Biordi, J. C., Lazzara, C. P., and Papp, J. F., Fifteenth Symposium (International) on Combustion, 917 (1975).

[15] Gaydon, A. G. and Wolfhard, H. G., Fourth Symposium (International) on Combustion, 211 (1953).

[16] Gaydon, A. G., The Spectroscopy of Flames (Chapman and Ha11, London, 1957).

[17] Bradley, J. N., Flame and Combustion Phenomena (Methuen \& Co. Ltd., London, 1969).

[18] Shchelkin, K. I. and Troshin, Ya. K., preprint for Ninth Symposium (International) on Combustion (1962).

[19] Semenov, N. N., Some Problems in Chemical Kinetics and Reactivity (Princeton University Press, New Jersey). 

National Bureau of Standards Special Publication 561, Proceedings of the 10th Materials Research Symposium on Characterization of High Temperature Vapors and Gases held at NBS, Gaithersburg, Maryland, September 18-22, 1978. Issued October 1979.

MEASUREMENTS OF SOME ELEMENTARY HYDROCARBON REACTIONS AT HIGH TEMPERATURES

\author{
P. Roth \\ University of Duisburg \\ Fachgebiet Strömungstechnik \\ West Germany \\ and \\ T. H. Just \\ DFVLR Stuttgart \\ Inst. f. Phys. Chemie d.V. \\ West Germany
}

1. Introduction

In order to understand the oxidation and combustion of hydrocarbons, accurate kinetic data are needed for the elementary reactions which sustain the overall process. There are a lot of measurements in flames and behind shock waves. Because of the complexity of the systems, the interpretation of measured concentrations, density profiles, or ignition delay times is sometimes quite difficult. The whole kinetic process is determined by a series of elementary reactions steps and must be mathematically described by a complex overall mechanism. The data reduction to simple kinetic constants is not beyond doubt.

Our concept in studying hydrocarbon reactions is to use very low concentrations of reactants under high temperature conditions. The number of reactions which determine the system is low and, therefore, the interpretation of the data is more clear. For this purpose we need an extremely sensitive experimental detection technique and we use the atomic resonance absorption spectrophotometry (ARAS) in shock tube experiments. Very low initial reactants concentrations of the order of 5-100 ppm are needed for $\mathrm{H}$ or 0 atoms to generate sufficient absorption signals. In particular, this method promises new and substantial results when applied to the investigation of the primary step and the first subsequent reactions of the thermal decay or the oxidation of small molecules.

The first application of the ARAS-technique in shock tube experiments has been done by Myerson and Watt $[1,2]^{1}$. They studied the dissociation reactions of $\mathrm{H}_{2}$ and $\mathrm{O}_{2}$. Roth and Just [3-6] used this method for more complicated reactions of simple hydrocarbons. By the direct measurement of the time dependent $\mathrm{H}$-atom concentration in the reaction zone behind shock waves in mixtures of $\mathrm{Ar}$ with small concentrations of $\mathrm{CH}_{4}, \mathrm{HCN}, \mathrm{C}_{2} \mathrm{H}_{6}$ or $\mathrm{C}_{2} \mathrm{H}_{4}$, the rate constants of the first kinetic decomposition steps of the molecules have been measured. In mixtures of Ar with small concentrations of $\mathrm{CH}_{4}$ and $\mathrm{N}_{2} \mathrm{O}$, the rate coefficient of the

${ }^{1}$ Figures in brackets indicate the literature references at the end of this paper. 
radical reaction $\mathrm{O}+\mathrm{CH}_{4}$ could be determined by measurements of the time dependent 0 - and $\mathrm{H}$-atom concentration behind reflected shock waves.

\section{Experimental}

\section{1 Apparatus}

A complete description of the experimental apparatus and the optical setup have been given in some detail in reference [6]. The shock tube with $75 \mathrm{~mm}$ i.d. is an UHV-apparatus with a very low leak plus outgassing rate. A careful experimental procedure allowed us to evacuate it by liquid-air-cooled mercury diffusion pumps or turbomolecular pumps down to about $5.10^{-8}$ mbar. The mechanical pumps were connected via molecular sieves, which prevent back streaming of oil vapor. The residual gas has been monitored by a quadrupole massspectrometer. The gas mixing system, the storage bottle for the mixture, and all valves and tubing could be heated to about $300{ }^{\circ} \mathrm{C}$; the shock tube was unheated.

The optical arrangement is shown in figure 1 and consisted of a microwave-excited discharge lamp for the emission of the $\mathrm{H}_{\alpha}$-doublet at $121.5 \mathrm{~nm}$ or the $0 \mathrm{I}$-triplet at $130.5 \mathrm{~nm}$, a $1 \mathrm{~m}$ McPherson vacuum UV monochromator and a special solar blind photomultiplier as detector. The opto-electrical setup has a signal to noise ratio of 10:1 and a time constant of about 20 $\mu \mathrm{s}$.

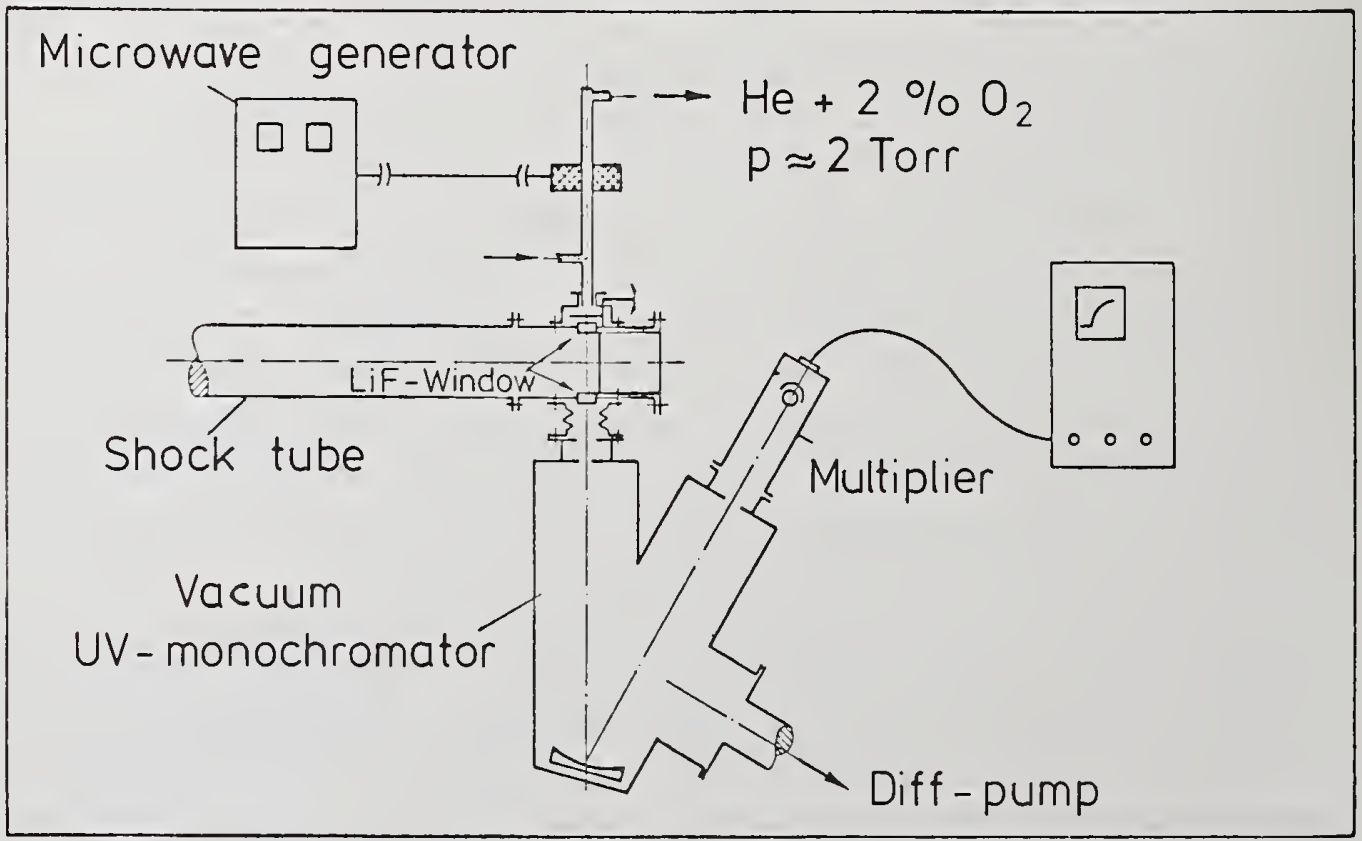

Figure 1. Optical arrangement. 
Al1 gases used were of ultrahigh purity and are highly diluted in argon. The carrier gas $\operatorname{Ar}(99.997 \%)$ was further purified by means of selected molecular sieves. Test runs in Ar showed almost negligible absorption signals by $\mathrm{H}$ - and 0 -atoms behind reflected shock waves at $T_{S}<2700 \mathrm{~K}$.

Shock velocities were determined in the standard manner. Four thin-film resistances at distances of $30 \mathrm{~cm}$ from each other triggered three $10 \mathrm{MHz}$ electronic counters.

\subsection{Calibration}

The radiation of the resonance lamp is affected by self-absorption and other processes. Therefore a simple relation between measured absorption and 0-atom or $\mathrm{H}$-atom concentration does not exist. Calibration measurements with well known atom concentrations has been performed.

Figure $2 \mathrm{a}$ shows an oscilloscope record of the time dependent absorption of 0 -atoms behind a reflected shock wave in Ar with $3 \mathrm{ppm} \mathrm{N} \mathrm{N}_{2} \mathrm{O}$. Partial equilibrium concentration of 0 -atoms is established after $200-300 \mu \mathrm{s}$. At temperatures $\mathrm{T}_{\mathrm{s}}>1600 \mathrm{~K}$ the equilibrium $\mathrm{N}_{2} \mathrm{O} \underset{\leftarrow}{\rightarrow} \mathrm{N}_{2}+0$ is completely displaced to $\mathrm{N}_{2}$ and $\mathrm{O}$ and the concentration of 0 -atoms can be computed. All calibration data are shown in figure 3. The circles are the partial equilibrium measurements; the triangles are from kinetic decomposition measurements using rate coefficients.

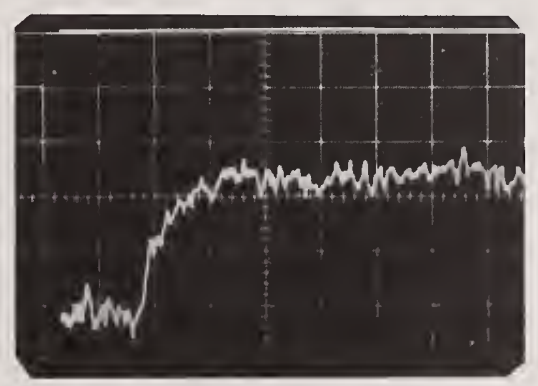

$$
\begin{gathered}
3 \mathrm{ppm} \mathrm{N} 2_{2} \mathrm{O} A \mathrm{Ar} \quad \mathrm{T}_{\mathrm{s}}=2210 \mathrm{~K} \\
\mathrm{p}_{\mathrm{S}}=1.85 \mathrm{bar}
\end{gathered}
$$

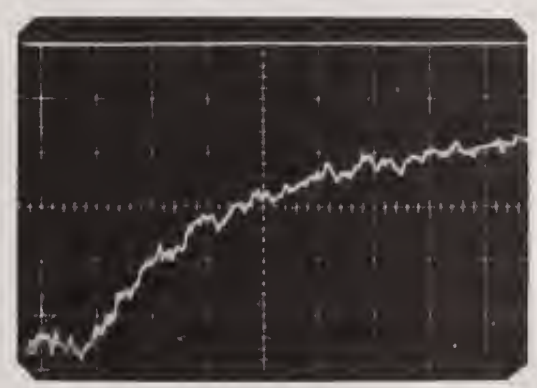

(b) $\begin{gathered}1000 \mathrm{ppm} \mathrm{H}_{2} / \mathrm{Ar} \quad \mathrm{T}_{\mathrm{s}}=2260 \mathrm{~K} \\ \mathrm{p}_{\mathrm{s}}=1.77 \mathrm{bar}\end{gathered}$

Figure 2. Examples of calibration measurements. 
The calibration of $\mathrm{H}$-atoms is based entirely on measurements using the wel1-known rate coefficient for $\mathrm{H}_{2}$ dissociation in $\mathrm{Ar}$ or on measurements with mixtures of $\mathrm{N}_{2} \mathrm{O} / \mathrm{H}_{2} / \mathrm{Ar}$ using the rate coefficients of the $\mathrm{H}_{2}$ oxidation steps. Figure $2 \mathrm{~b}$ shows the time-dependent absorption of $\mathrm{H}$-atoms behind a reflected shock wave in $\mathrm{Ar}$ with $1000 \mathrm{ppm} \mathrm{H}_{2}$. The required rate coefficient for $\mathrm{H}_{2}$ dissociation was taken from Baulch, et al. [7]. All calibration dató are shown in figure 4. A weak temperature dependence was observed. The absolute accuracy of all measured $\mathrm{H}$-atom concentrations and their interpretation in terms of rate coefficients depend, of course, on the accuracy of the kinetic calibration data.
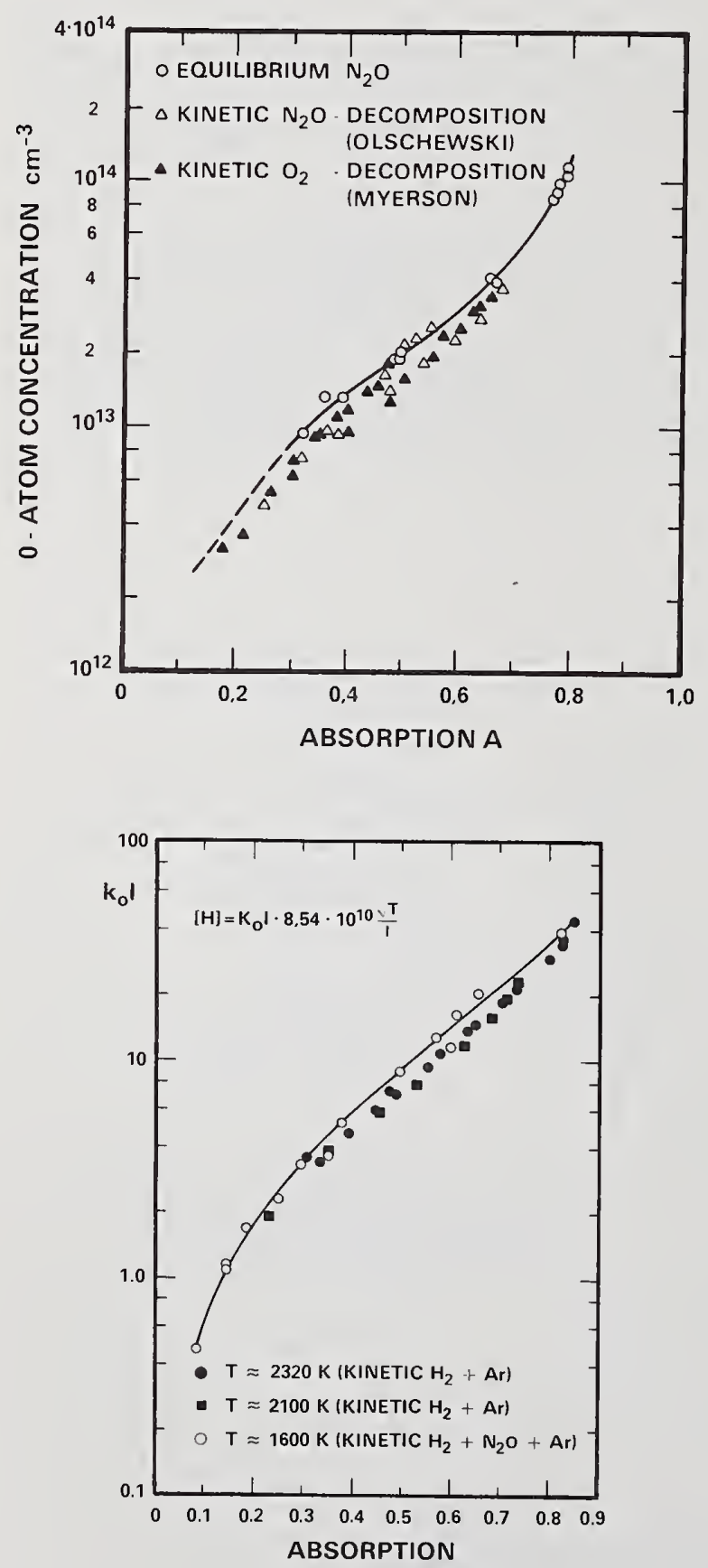

Figure 3. Measured calibration curve for 0 -atoms.
Figure 4. Measured calibration curve for H-atoms. 


\section{Results and Discussion}

A11 measurements were performed behind reflected shock waves at about $0.4 \mathrm{~cm}$ from the end plate of the shock tube.

\section{1 Results for decomposition of $\mathrm{HCN}$}

The thermal decomposition of $\mathrm{HCN}$ with relative concentrations of $125-500 \mathrm{ppm}$ in argon has been investigated in the temperature range of $2200 \leq T \leq 2700 \mathrm{~K}$ at densities of about $6.10^{18} \mathrm{~cm}^{-3}$. The measured time dependent $\mathrm{H}$-atom profiles were absolutely linear. In no case could an induction time be observed. Figure 5 shows a plot of the gradient $[\dot{\mathrm{H}}] /[\mathrm{HCN}][\mathrm{Ar}]$ against reciprocal temperature. The linear increase of the H-atom concentration can be interpreted as a simple dissociation reaction

$$
\mathrm{HCN}+\mathrm{M} \stackrel{\mathrm{k}}{\longrightarrow} \mathrm{H}+\mathrm{CN}+\mathrm{M} \text {. }
$$

In our case secondary reactions of $\mathrm{H}$-atoms and $\mathrm{CN}$-radicals with $\mathrm{HCN}$ do not influence the overall pyrolytic decomposition. The production of $\mathrm{H}$-atoms is described by the equation

$$
[\dot{\mathrm{H}}]=k[\mathrm{HCN}][\mathrm{M}]
$$

and the measured gradient $[\dot{\mathrm{H}}] /[\mathrm{HCN}][\mathrm{Ar}]$ of figure 5 is equal to the rate coefficient

$$
k=9.5 \cdot 10^{-8} \exp (-59060 / \mathrm{T}) \mathrm{cm}^{3} \mathrm{~s}^{-1} \text { molecule } \mathrm{e}^{-1} \text {. }
$$

This can be very well explained by collision theory as the coefficient of the low pressure decomposition of $\mathrm{HCN}$.

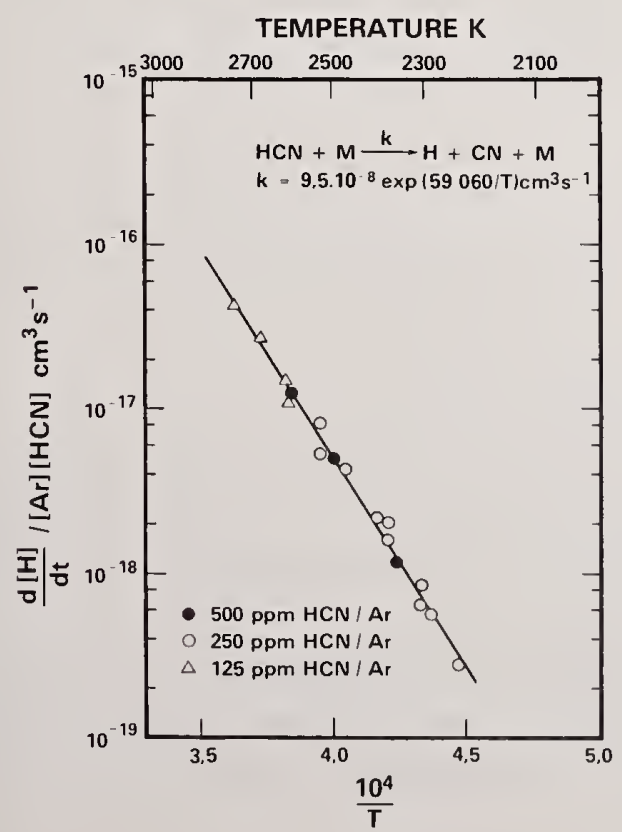

Figure 5. Arrhenius plot of the rate coefficient for the decomposition of $\mathrm{HCN}$. 


\subsection{Results for decomposition of $\mathrm{CH}_{4}$.}

The thermal decomposition of $\mathrm{CH}_{4}$ has been studied in the same manner as $\mathrm{HCN}$. By the direct measurement of the time-dependent $\mathrm{H}$-atom concentration behind reflected shock waves in argon with $\mathrm{CH}_{4}$ concentrations of $5-200 \mathrm{ppm}$ and densities of about $6 \cdot 10^{18} \mathrm{~cm}^{-3}$, the rate coefficients of the first reaction steps have been measured.

For low initial concentrations of $\mathrm{CH}_{4}(5-10 \mathrm{ppm})$, the experiments show an immediate linear increase of the $\mathrm{H}$-atom concentration with time. The rate $[\dot{H}]$ obtained from the linear part of the $\mathrm{H}$-atom profiles was divided by $\left[\mathrm{CH}_{4}\right]_{0}$ and $[\mathrm{Ar}]$ and set equal to $\mathrm{k}_{1}$ corresponding to the reaction

$$
\mathrm{CH}_{4}+\mathrm{M} \stackrel{\mathrm{K}}{\longrightarrow} \mathrm{CH}_{3}+\mathrm{H}+\mathrm{M}
$$

These $k_{1}$ data are shown in figure 6 . A variation of pressure does not influence the rate coefficient

$$
\mathrm{k}_{1}=7.85 \cdot 10^{-7} \exp (-46800 / \mathrm{T}) \mathrm{cm}^{3} \mathrm{~s}^{-1} \text { molecule }{ }^{-1}
$$

TEMPERATURE $K$

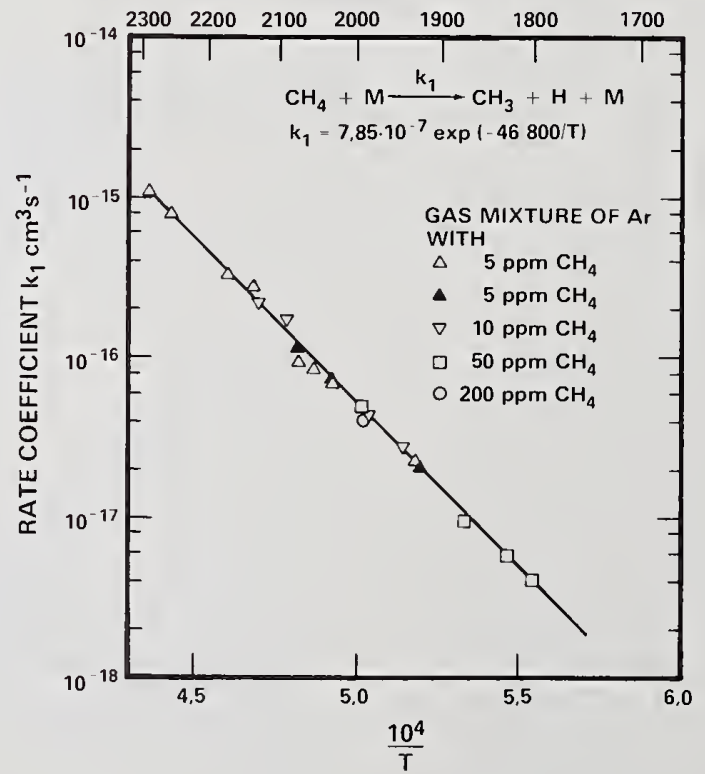

Figure 6. Arrhenius plot of the rate coefficient for $\mathrm{CH}_{4}+\mathrm{M} \longrightarrow \mathrm{CH}_{3}+\mathrm{H}+\mathrm{M}$.

At higher initial $\mathrm{CH}_{4}$ concentrations of 50 and $200 \mathrm{ppm}$ and temperatures $\mathrm{T}_{\mathrm{s}}<2000 \mathrm{~K}$, the measured $\mathrm{H}$-atom profiles are curved and after some $100 \mu \mathrm{s}$ a quasi stationary value could be observed. This experimental fact is shown in figure 7 . The measured reciprocal steady state 
H-atom concentration multiplied by the total density is plotted against reciprocal temperature. The least-squares line through the data is

$$
{\frac{[\mathrm{M}]}{[\mathrm{H}]_{\text {stat }}}}=1.54 \cdot 10^{-3} \exp (+39180 / \mathrm{T}) .
$$

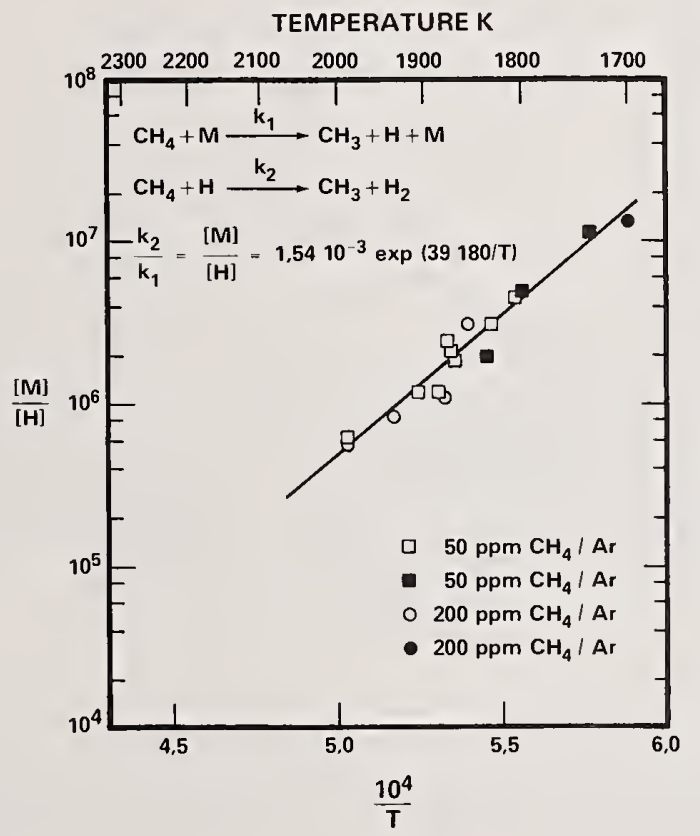

Figure 7. Arrhenius plot of measured steady state $\mathrm{H}$-atom concentration and kinetic interpretation.

A pressure dependence could not be observed. The experimental evidence indicates that the first pyrolytic decomposition step of $\mathrm{CH}_{4}$ is followed by reactions of the products with methane. Very important is the reaction of $\mathrm{H}$-atoms with $\mathrm{CH}_{4}$ as pointed out in reference [8]

$$
\mathrm{H}+\mathrm{CH}_{4} \stackrel{\mathrm{K}_{2}}{\longrightarrow} \mathrm{H}_{2}+\mathrm{CH}_{3}
$$

Because of the low $\mathrm{CH}_{4}$ concentrations further reactions could be neglected. With the steady state condition for $\mathrm{H}$-atoms the observed concentration ratio $[\mathrm{M}] /[\mathrm{H}]_{\text {stat }}$ must be interpreted as the ratio of $k_{2} / k_{1}$.

$$
\mathrm{k}_{2}=1.2 \cdot 10^{-9} \exp (-7580 / \mathrm{T}) \mathrm{cm}^{3} \mathrm{~s}^{-1} \text { molecule } \mathrm{m}^{-1} \text {. }
$$


Figure 8 shows measured rate coefficients for $k_{2}[9-12]$ and a computation of Clark and Dove [13] where the non-Arrhenius behavior of the reaction is clear.

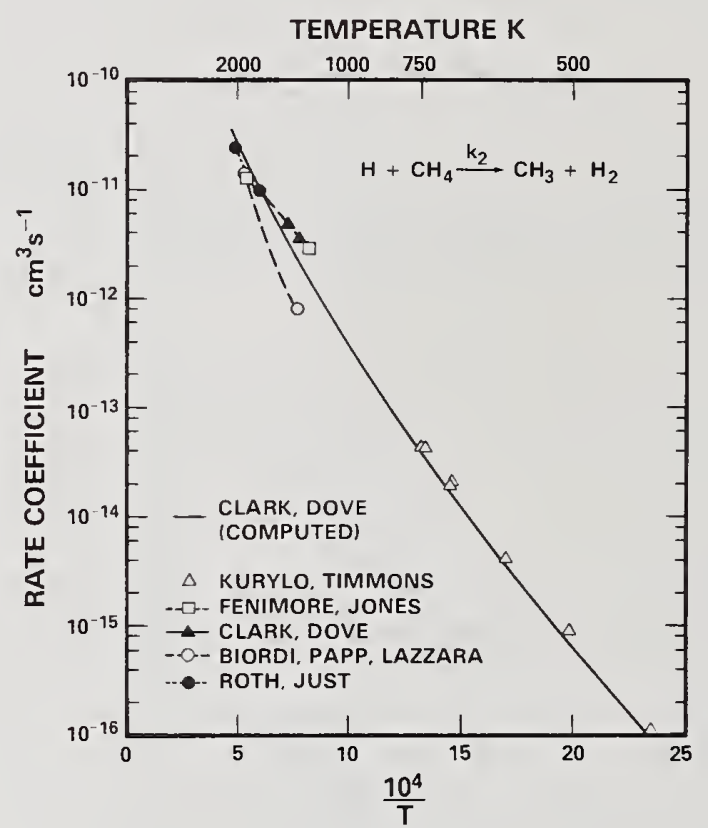

Figure 8. Temperature dependence of the rate coefficient $\mathrm{k}_{2}$ for the reaction $\mathrm{H}+\mathrm{CH}_{4} \longrightarrow \mathrm{H}_{2}+\mathrm{CH}_{3}$.

\subsection{Results for the reaction $\mathrm{O}+\mathrm{CH}_{4}$}

In five different gas mixtures $10 / 5,20 / 10,25 / 25,15 / 100$ and $15 / 150 \mathrm{ppm}, \mathrm{CH}_{4} / \mathrm{ppm} \mathrm{N}_{2} \mathrm{O}$ highly diluted in argon, measurements of $\mathrm{O}$ - and $\mathrm{H}$-atom absorption have been performed. We obtained two kinds of 0 -atom absorption signals.

At high temperatures and low $\mathrm{N}_{2} \mathrm{O}$ concentrations the production of 0 -atoms from $\mathrm{N}_{2} \mathrm{O}$ begins immediately after shock arrival and the absorption increases. The produced atoms react with $\mathrm{CH}_{4}$ and the absorption signal reaches a maximum. It decreases because of the consumption of $\mathrm{N}_{2} \mathrm{O}$ and the decrease of 0 -atom production (see fig. 9). The absorption of $\mathrm{H}$-atoms increases rapidly. The atoms are products of the thermal decomposition of $\mathrm{CH}_{4}$ and of secondary reactions between $\mathrm{CH}_{3}$ and 0 -atoms.

At lower temperatures (but above $1500 \mathrm{~K}$ ) high $\mathrm{N}_{2} \mathrm{O}$ concentrations of $100 \mathrm{ppm}$ are needed to produce sufficient 0 -atoms. After shock arrival a discontinuity can be observed because of the quasi continuous absorption of $\mathrm{N}_{2} \mathrm{O}$ at $130.5 \mathrm{~nm}$. It is followed by the resonance absorption of 0 -atoms which reach a maximum value. Under these conditions the concentration of $\mathrm{N}_{2} \mathrm{O}$ remains nearly constant. 


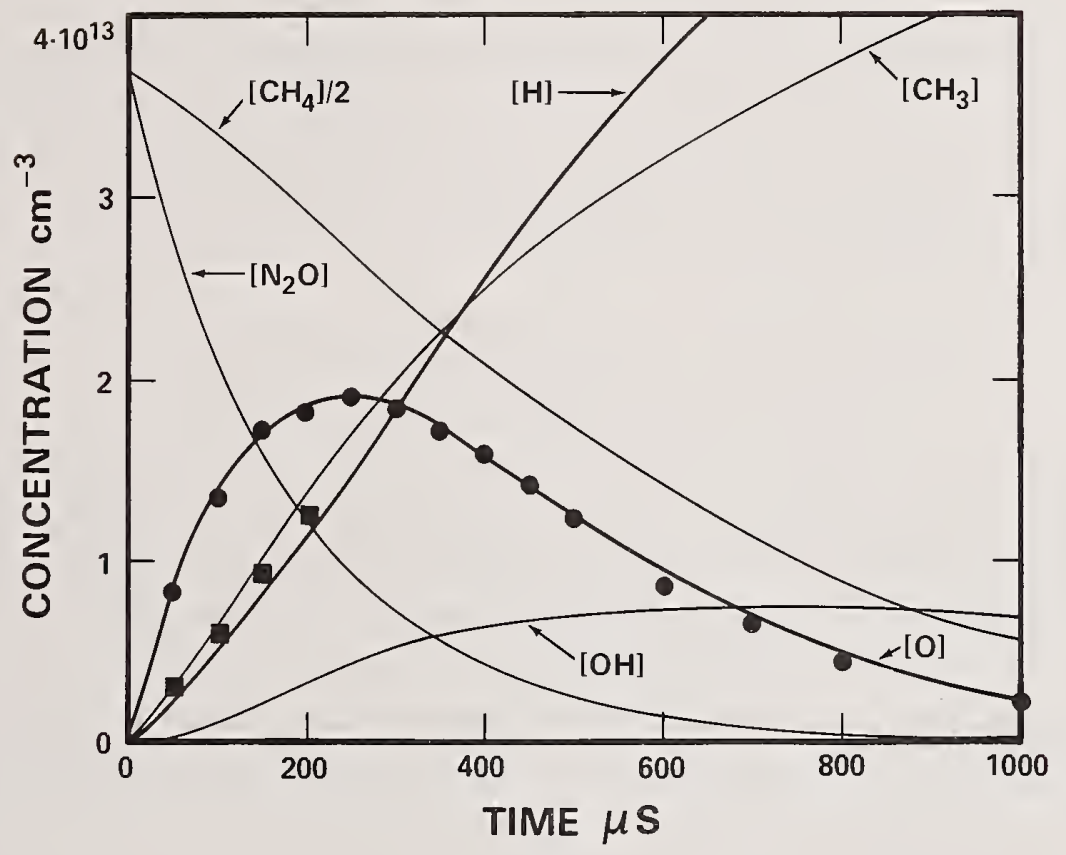

Figure 9. Measured $\mathrm{H}$ - and 0 -atom profiles and fitted curves by analog computer simulation: $10 / 5 \mathrm{ppm} \quad \mathrm{CH}_{4} / \mathrm{N}_{2} \mathrm{O} / \mathrm{Ar}, \mathrm{T}_{\mathrm{s}}=2055 \mathrm{~K}, \mathrm{p}_{\mathrm{s}}=2.09$ bar.

The whole kinetic mechanism is basically determined by the competition of the reactions

$$
\begin{aligned}
& \mathrm{N}_{2} \mathrm{O}+\mathrm{M} \stackrel{\mathrm{k}_{1}}{\longrightarrow} \mathrm{N}_{2}+\mathrm{O}+\mathrm{M} \\
& \mathrm{CH}_{4}+\mathrm{O} \stackrel{\mathrm{k}_{2}}{\longrightarrow} \mathrm{CH}_{3}+\mathrm{OH} .
\end{aligned}
$$

The measured $\mathrm{H}$-atom concentrations indicate that further reactions of the radicals $\mathrm{CH}_{3}$ and $\mathrm{OH}$ take place,

$$
\begin{aligned}
& \mathrm{CH}_{3}+\mathrm{O} \stackrel{\mathrm{K}_{3}}{\longrightarrow} \mathrm{CH}_{2} \mathrm{O}+\mathrm{H} \\
& \mathrm{CH}_{4}+\mathrm{OH} \stackrel{\mathrm{K}_{4}}{\longrightarrow} \mathrm{CH}_{3}+\mathrm{H}_{2} \mathrm{O} .
\end{aligned}
$$

At temperatures above $1900 \mathrm{~K}$ the influence of the known pyrolytic decomposition of $\mathrm{CH}_{4}$ cannot be neglected, 


$$
\begin{aligned}
& \mathrm{CH}_{4}+\mathrm{M} \longrightarrow \mathrm{CH}_{3}+\mathrm{H}+\mathrm{M} \\
& \mathrm{CH}_{4}+\mathrm{H} \longrightarrow \mathrm{CH}_{3}+\mathrm{H}_{2}
\end{aligned}
$$

The differential equation system following from the above reaction mechanism has been programmed on an analog computer and simulated concentration profiles compared with the experiments. Parameter studies showed the very important influence of reactions 1 and 2 with reference to the 0 -atom profiles. From the $\mathrm{H}$-atom measurements one can estimate the influence of reaction 3 . With a rate coefficient

$$
\mathrm{k}_{3}=1.5 \cdot 10^{-10} \exp (-1000 / \mathrm{T}) \mathrm{cm}^{3} \mathrm{~s}^{-1} \text { molecule } \mathrm{e}^{-1}
$$

which is a factor of 1.5 smaller than the value reported by Peeters and Mahnen [14] an adaptation of measured and computed $\mathrm{H}$-atom profiles is possible. With the fixed rate coefficients and the literature value of the unimportant coefficient $k_{4}$ one can adjust the computed 0 -atom profiles to the measured concentration profiles by variation of the rate coefficients $k_{1}$ and $k_{2}$ (see individual measurement fig. 9). The determined value of $k_{1}$ is very similar to the earlier experiments of 0lschewski, et al. [15]. The result for the chain branching reaction (2) is shown in figure 10 . The least-squares line through the data gives,

$$
\mathrm{k}_{2}=6.8 \cdot 10^{-10} \exp (-7030 / \mathrm{T}) \mathrm{cm}^{3} \mathrm{~s}^{-1} \text { molecule } \mathrm{e}^{-1}
$$

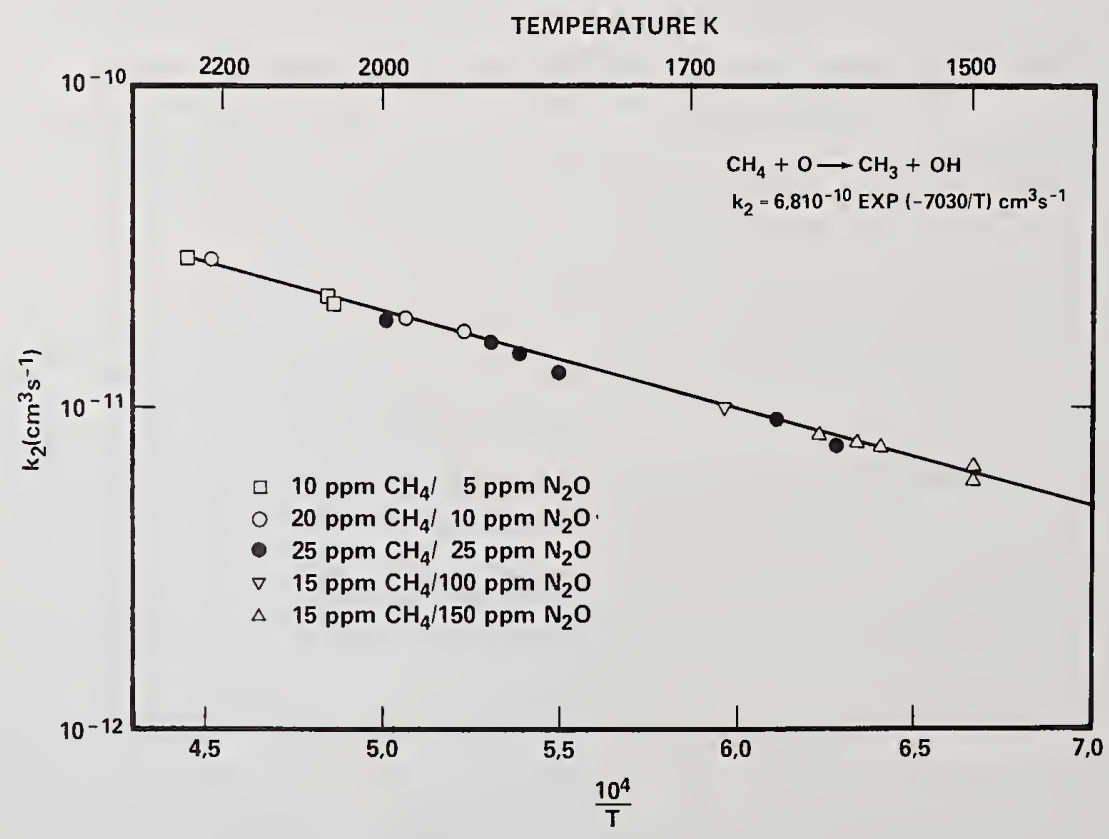

Figure 10. Temperature dependence of the rate coefficient for the reaction $\mathrm{O}+\mathrm{CH}_{4} \longrightarrow \mathrm{OH}+\mathrm{CH}_{3}$. 
The activation temperature of reaction (2) is much higher than the values of the low temperature experiments of Wong and Potter [16] and Westenberg and de Haas [17]. The measured coefficient, $k_{2}$, is very similar to the value of Brabbs and Brokaw [18]. The non-Arrhenius behavior of $k_{2}$ is obvious from the comparison of low temperature (1iterature) and high temperature data. In the whole temperature range from $300 \mathrm{~K}$ to $2200 \mathrm{~K}$, one can describe $k_{2}$ by an analytical expression

$$
\mathrm{k}_{2}=1.94 \cdot 10^{-17} \mathrm{~T}^{2.075} \exp (-3840 / \mathrm{T}) \mathrm{cm}^{3} \mathrm{~s}^{-1} \text { molecule } \mathrm{e}^{-1} \text {. }
$$

The temperature dependence of the pre-exponential factor seems to be quite reasonable.

\subsection{Results for decomposition of $\mathrm{C}_{2} \mathrm{H}_{6}$}

The thermal decomposition of $\mathrm{C}_{2} \mathrm{H}_{6}$ has been studied by measuring the time dependent $\mathrm{H}$-atom concentration in the reaction zone behind shock waves in mixtures of argon with small concentrations of $1-50 \mathrm{ppm} \mathrm{C}_{2} \mathrm{H}_{6}$. In the temperature range $1450 \leq T_{S} \leq 2100 \mathrm{~K}$ experiments have been performed at pressures of $0.5,2.0$ and 4.2 bar.

These measurements show the potentiality and the limit of the high temperature ARAStechnique with respect to hydrocarbon reactions. The production and consumption of $\mathrm{H}$-atoms is an important but not the only significant step in the overall mechanism. Therefore, the kinetic interpretation of measured $\mathrm{H}$-atom concentrations is more complex. There are characteristic differences in the measured profiles as a function of temperature

(a) at low temperatures $\mathrm{T}_{\mathrm{S}}<1600 \mathrm{~K}$, the $\mathrm{H}$-atom profiles increase parabolically,

(b) in the range $T_{s}>1600 \mathrm{~K}$, a parabolic curvature is followed by a linear increase, and

(c) at temperatures $T_{S}>1680 \mathrm{~K}$, an immediate linear increase of $\mathrm{H}$-atom concentration with time has been observed.

A first summary of a 11 measurements is given in the Arrhenius plot (fig. 11). The gradient $[\dot{\mathrm{H}}]_{500}$ at $t=500 \mu \mathrm{s}$ divided by $8\left[\mathrm{C}_{2} \mathrm{H}_{6}\right]_{0}^{2}$ is plotted against reciprocal temperature. Two different regimes are distinguishable and the overall decomposition process must be determined by two or more important reactions:

$$
\begin{aligned}
& \mathrm{C}_{2} \mathrm{H}_{6} \stackrel{\mathrm{K}_{1}}{\longrightarrow} \mathrm{CH}_{3}+\mathrm{CH}_{3} \\
& \mathrm{C}_{2} \mathrm{H}_{6}+\mathrm{CH}_{3} \stackrel{\mathrm{K}_{2} \longrightarrow}{\longrightarrow} \mathrm{C}_{2} \mathrm{H}_{5}+\mathrm{CH}_{4} \\
& \mathrm{CH}_{3}+\mathrm{CH}_{3} \stackrel{\mathrm{k}_{3}}{\longrightarrow} \mathrm{C}_{2} \mathrm{H}_{5}+\mathrm{H} \\
& \mathrm{C}_{2} \mathrm{H}_{5} \stackrel{\mathrm{K}_{4}}{\longrightarrow} \mathrm{C}_{2} \mathrm{H}_{4}+\mathrm{H} .
\end{aligned}
$$




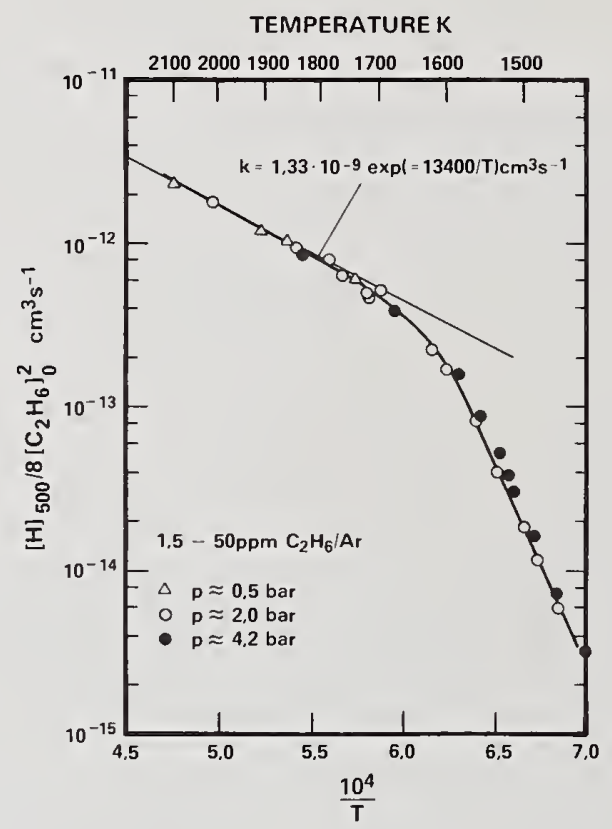

Figure 11. Pyrolytic decomposition of $\mathrm{C}_{2} \mathrm{H}_{6}$. Arrhenius plot of measured H-atom gradient.

In the computer analysis further reactions are included but their influence with respect to the $\mathrm{H}$-atom profiles is unimportant. The production of $\mathrm{H}$-atoms is restricted to reactions (3) and (4). Reaction (4) is very fast in comparison with the other reactions. The radical $\mathrm{C}_{2} \mathrm{H}_{5}$ in reactions (2) and (3) may be replaced, therefore, by $\mathrm{C}_{2} \mathrm{H}_{4}+\mathrm{H}$ and the kinetic mechanism is basically determined by the competition of the reactions (1), (2) and (3). With the assumption that the concentrations of $\mathrm{C}_{2} \mathrm{H}_{6}$ and $\mathrm{CH}_{3}$ are chiefly determined by the reaction (1), an analytical solution can be deduced.

$$
\begin{aligned}
\frac{[\dot{\mathrm{H}}]}{8\left[\mathrm{C}_{2} \mathrm{H}_{6}\right]_{0}} & =\frac{\mathrm{k}_{2}}{4} \exp \left(-\mathrm{k}_{1} \mathrm{t}\right)\left[1-\exp \left(-\mathrm{k}_{1} \mathrm{t}\right)\right] \\
& +\mathrm{k}_{3}\left[1-\exp \left(-\mathrm{k}_{1} \mathrm{t}\right)\right]^{2} .
\end{aligned}
$$

At high values of $k_{1} t>3$ the expression is equal to $k_{3}$. This is in agreement with the experimental fact of linear increase of $\mathrm{H}$-atom concentration for $\mathrm{T}>1680 \mathrm{~K}$. The rate coefficient

$$
\mathrm{k}_{3}=1.33 \cdot 10^{-9} \exp (-13400 / \mathrm{T}) \mathrm{cm}^{3} \mathrm{~s}^{-1} \text { molecule } \mathrm{e}^{-1}
$$


is equal to the expression $[\dot{\mathrm{H}}] / 8\left[\mathrm{C}_{2} \mathrm{H}_{6}\right]_{0}^{2}$. The decomposition of $\mathrm{C}_{2} \mathrm{H}_{6}$ into $\mathrm{CH}_{3}$ is very fast under these conditions and $\mathrm{CH}_{3}$ may be regarded as an initial concentration.

The interpretation of the measured $\mathrm{H}$-atom profiles for lower temperatures is more difficult. Only a relative estimation of $k_{1}$ and $k_{2}$ is possible. Under the present conditions the rate coefficient $k_{1}$ is in the fall-off region and following Burcat, et al. [19] and Glänzer, et al. [20] equals,

$$
\mathrm{k}_{1}=2.0 \cdot 10^{15} \exp (-42000 / \mathrm{T}) \mathrm{s}^{-1} .
$$

The computer simulation of the complete reaction mechanism yielded to the rate coefficient

$$
\mathrm{k}_{2}=8.3 \cdot 10^{11} \exp (-9800 / \mathrm{T}) \mathrm{cm}^{3} \mathrm{~s}^{-1} \mathrm{molecule}^{-1}
$$

All computed $\mathrm{H}$-atom profiles were found to be in very good agreement with the measured time dependent $\mathrm{H}$-atom concentrations during the thermal decomposition of $\mathrm{C}_{2} \mathrm{H}_{6}$.

\section{Conclusion}

The direct concentration measurement of $\mathrm{H}$ - and 0 -atoms in high temperature hydrocarbon reaction systems has been shown to be a promising addition to shock tube technology. Because of the high sensitivity, measurements in highly diluted reaction systems are possible. The interpretation of elementary reaction rate coefficients is also facilitated.

\section{References}

[1] Myerson, A. L. and Watt, W. S., J. Chem. Phys. 49, 225 (1968).

[2] Watt, W. S. and Myerson, A. L., J. Chem. Phys. 51, 1638 (1969).

[3] Roth, P. and Just, Th., Ber. d. Bunsenges. Phys. Chem. 79, 682 (1975).

[4] Roth, P. and Just, Th., Ber. d. Bunsenges. Phys. Chem. 80, 171 (1976).

[5] Just. Th., Roth, P., and Damm, R., 16th Symp. (Int1.) on Combustion, p. 961 (The Combustion Institute, 1976).

[6] Roth, P. and Just, Th., Ber. d. Bunsenges. Phys. Chem. 81, 572 (1977).

[7] Baulch, D. L., Drysdale, D. D., Horne, D. G., and Lloyd, A. C., Evaluated kinetic data for high temperature reactions, Vol. 1 (Butterworths, London, 1972).

[8] Hartig, R., Troe, J., and Wagner, H. Gg., 13th Symp. (Intl.) on Combustion, 147 (1970).

[9] Kurylo, M. J. and Timmons, R. B., J. Chem. Physics, 50, 5076 (1969).

[10] Fenimore, C. P. and Jones, G. W. , J. Phys. Chem. 65, 2200 (1961).

[11] Clark, T. C. and Dove, J. E., Canad. J. Chem. 51, 2155 (1973).

[12] Biordi, J. C., Papp, J. F., and Lazzara, C. P., J. Chem. Phys. 61, 741 (1974).

[13] Clark, T. C. and Dove, J. E., Canad. J. Chem. 51, 2147 (1973).

[14] Peeters, J. and Mahnen, G., 14th Symp. (Int1.) on Combustion P., 133 (1972). 
[15] 01 schewski, H. A., Troe, J., and Wagner, H. G., Ber. d. Bunsenges. Phys. Chem. 70, 450 (1966).

[16] Wong, E. L. and Potter, A. E., Canad. J. Chem. 45, 367 (1967).

[17] Westenberg, A. A. and de Haas, N., J. Chem. Physics, 50, 2512 (1969).

[18] Brabbs, T. A. and Brokaw, R. S., 15th Symp. (Int1.) on Combustion (1974).

[19] Burcat, A., Skinner, G. B., Crossley, R. W., and Scheller, K., Int1. J. of Chem. Kinetics, $\underline{5}, 345$ (1973).

[20] Glänzer, K., Quack, M., and Troe, J., Chem. Phys. Letters, 39, 304 (1976).

\section{Discussion}

Question (Gann): I have a question on the first reaction you studied, the 0 atom plus methane reaction. You describe that as distinctly non-Arrhenius behavior. It seems--if you take Kurylo's data and tilt it just a very small amount, then in fact one can draw a fairly good straight line through the low and high temperature data.

To do so you have to disregard or pay less attention to the Biordi data, but that is taken from flame slopes, and that is approximate.

Response (Roth): Yes. I think one can approximate the high temperature measurements by straight lines, but not the behavior of the whole temperature range by a straight line.

Comment (Gann): The Kurylo data is the low temperature data. And, as I say, you can put a fairly good line through the data, at least on the plot, if you allow for a slight uncertainty in their activation energy.

Comment (Tsang): Regarding the curvature, I think we could expect curvature ---.

Question (Gardiner): On your calibration graph for $\mathrm{H}$ atoms, you found a substantially different answer using the $\mathrm{N}_{2} \mathrm{O}$ method for the calibration.

It appeared to be perhaps 30 or 40 percent higher than for the previously used methods. Which calibration do you now use in reducing the data, and how do you feel about that descrepancy?

Response (Roth): Yes. We used the system $\mathrm{H}_{2}$ plus $\mathrm{N}_{2} \mathrm{O}$ under low temperature conditions near $1600 \mathrm{~K}$, and we used the calibration with the $\mathrm{O}_{2}$ dissociation at very high temperatures of about $2500 \mathrm{~K}$.

There must be a difference between these two calibration procedures, and we can explain it by the temperature shift of the $\mathrm{H}$ atom profile.

Question (Gardiner): I see. So, the several methods of getting the sensitivity actually give very similar results about the effective absorption coefficient?

Response (Roth): I think so. 
National Bureau of Standards Special Publication 561, Proceedings of the 10th Materials Research Symposium on Characterization of High Temperature Vapors and Gases held at NBS, Gaithersburg, Maryland, September 18-22, 1978. Issued October 1979.

THE WALL-LESS REACTOR. A TECHNIQUE FOR THE STUDY OF GAS PHASE OXIDATIONS AND PYROLYSES OF HYDROCARBONS

\author{
Jay E. Taylor \\ Department of Chemistry \\ Kent State University \\ Kent, $\mathrm{OH} 44242$
}

That surface effects may have a significant influence on many gas phase reactions has been long recognized. The evidences are variations in the rate constants and/or reaction products upon adding surface. Often, surface effects take a more subtle form in that they are responsible for inconsistencies on similar studies made by a number of investigators. To demonstrate, a partial summary of the pyrolyses of butane is shown in table I. It is suspected that the differences in these results are partially, or even largely, due to varying surface effects.

Table 1

\begin{tabular}{|c|c|c|c|c|}
\hline${ }^{\text {Temp. Range }}{ }^{\circ} \mathrm{C}$ & Order & $\begin{array}{l}\text { Eact. } \\
\text { kcal/ } \\
\text { mole }\end{array}$ & $\log A$ & Investigators \\
\hline $530-625$ & 1 & 73.9 & 17.0 & Paul \& Marek (1936) \\
\hline $700-900$ & 1 & 45.6 & 9.93 & Sandler \& Chung (1961) \\
\hline $\begin{array}{l}430-530 \\
10-15 \mathrm{~mm}\end{array}$ & $3 / 2$ & 58.3 & 14.88 & Purnell \& Quinn (1962) \\
\hline $\begin{array}{l}520-590 \\
30-600 \mathrm{~mm}\end{array}$ & $3 / 2$ & 59.9 & 15.51 & Sagert \& Laidler (1963) \\
\hline $460-550$ & 1 or 2 & 55.0 & 12.52 & Wang, Rinker \& Corcoran (1963) \\
\hline $630-1230$ & 1 & 41.7 & 9.51 & Wittig (1969) * \\
\hline
\end{tabular}

The processes of "packing" a reactor to identify surface effects and "conditioning" or "coating" a reactor to standardize such effects have long been used for gas phase kinetic studies. These practices are generaliy quite unsatisfactory in that no absolute measurement of surface effects is possible, and both procedures are highly empirical. Other developments such as molecular beam, shock tube, or cool flame studies permit, with appropriate limitations, studies of reactions under partially or completely surface free conditions. They do not allow convenient or reliable comparative surface effect evaluations.

In order to provide an experimental technique whereby the products and kinetics of gas-phase reactions may be accurately studied under totally homogeneous and directly comparative surface conditions, the wall-less reactor was first developed. 
With the original design of the wall-less reactor, a micro jet of cool reactant is injected into a hot stream of inert gas $[1,2]^{1}$. The inert gas normally used is purified, oxygen-free nitrogen which flows through a 2-inch diameter quartz tube suspended in a vertically placed Lindberg tube furnace. The temperature is controlled to $\pm 1^{\circ}$ or less.

The reactant and decomposition products as formed then flow downstream, and samples are removed via a cooled sampling tube. During the time of reaction, there is no contact of reactants or products with any surface of the containing tube. Analyses of the product mixtures are carried out via gas chromatography.

Surfaces are introduced by inserting a set of rods of the desired material such that these rods are parallel to stream flow and are equally spaced throughout the tube. The rods so placed provide minimum turbulence. With this arrangement, a direct comparison of totally homogeneous versus added surfaces of varied surface to volume ratios can be made.

The three outstanding advantages of this reactor are: (1) the ability to evaluate kinetic constants without prior conditioning or coating of the reactor walls, (2) excellent reproducibility, and ( 3 ) the ease of detailed evaluation of surface effects.

Data permitting comparison of the wall-less reactor with other techniques is not generally available. However, some examples of reactions not influenced by surface and a reaction done under surface free conditions are listed in table II. Under these valid conditions, the comparison is seen to be quite good.

The primary disadvantage of the wall-less reactor is the changing concentration due to diffusion as the injected reactant flows downstream. As a result of this effect, quantitative results are possible only with first order reactions, i.e., those whose rate of reaction is independent of concentration. It should be emphasized that this limitation applies broadly to phenomenological first order reactions and is not restricted to unimolecular reactions. However, reactions with dual reactants, e.g., hydrocarbons plus oxygen,

Table II

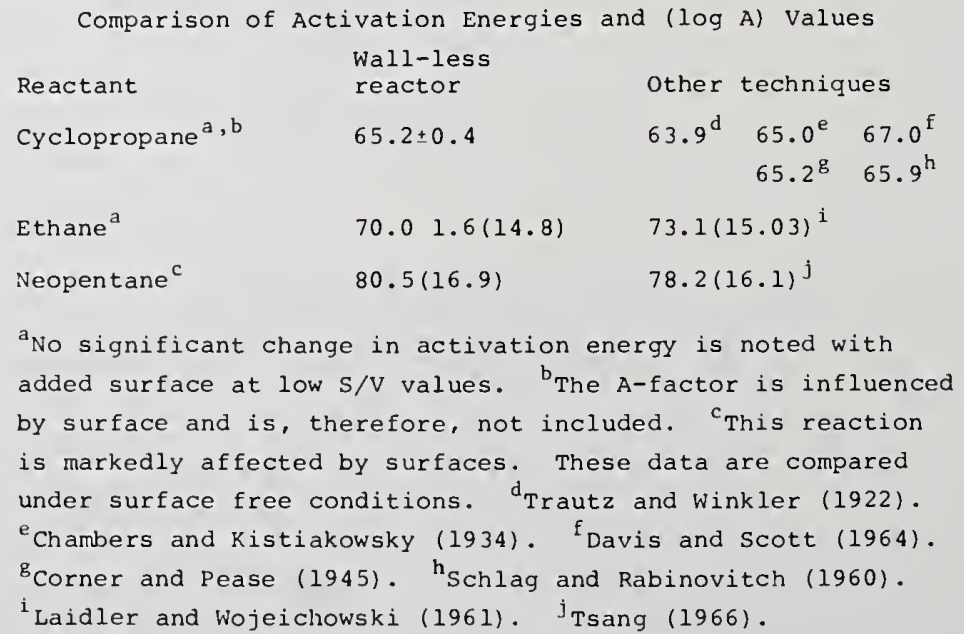

${ }^{1}$ Figures in brackets indicate the literature references at the end of this paper. 
have been studied. Although the absolute rate constants are difficult to evaluate in this case, comparative results are valid.

The wall-less reactor has been employed in the study of the pyrolyses of ethane [2], propane [3], isobutane [3], neopentane [1,4], ethylene [3], propylene [3], isobutylene [3], cyclopropane [5], the tetramethyl derivatives of silicon, germanium, and tin [6], and 2,2,3,3-tetramethylbutane [7].

A11 of the above compounds decompose under homogeneous conditions by a first order process. In every instance, the homogeneous data gave excellent reproducibility although the surface data were often erratic.

The reactions of oxygen with many of these compounds have been examined. Since oxygen is introduced with the carrier gas (nitrogen) and the reactant hydrocarbon diffuses into the nitrogen stream, the ratio of oxygen to reactant varies in a complex manner as reaction time progresses. The data with oxygen are, therefore, as previously indicated, semiquantitative in nature.

Data on the pyrolysis of ethane and isobutane, with and without oxygen, and on neopentane are selected to illustrate the effects of oxygen and of surface on these reactions, see table III. With ethane alone, there is a negligible surface effect, but, upon adding oxygen to give an appreciable increase in rate, a pronounced surface effect is observed. Isobutane shows the impact of both oxygen and surface.

Table III

Selected Pyrolyses of Hydrocarbons using the Wall-less Reactor

\begin{tabular}{|c|c|c|c|c|c|c|}
\hline Hydrocarbon & $\begin{array}{l}\mathrm{s} / \mathrm{v}^{\mathrm{a}} \\
\mathrm{cm}\end{array}$ & $\begin{array}{l}\text { Rate } \\
\text { const. at } \\
\sec ^{-1}\end{array}$ & ${ }^{\circ} \mathrm{C}$ & $\begin{array}{l}\mathrm{E}_{\text {act }} \\
\text { kcal/ } \\
\text { mole }\end{array}$ & $\log A$ & $\begin{array}{l}\text { Products }{ }^{b} \\
\text { (in order of abundance) }\end{array}$ \\
\hline \multicolumn{2}{|l|}{ Ethane ${ }^{c}$} & 0.437 & 742 & 71.8 & 14.8 & $\mathrm{C}_{2} \mathrm{H}_{4} \gg \mathrm{CH}_{4}(18)^{d}$ \\
\hline \multirow{2}{*}{$\begin{array}{l}\text { Ethane }+\mathrm{O}_{2} \mathrm{e} \\
\text { (moderate conv.) }\end{array}$} & none & 2.44 & 742 & --- & --- & \multirow{2}{*}{$\mathrm{C}_{2} \mathrm{H}_{4} \gg \mathrm{CH}_{4} \quad(38)^{d}$} \\
\hline & 1.2 & 1.34 & 742 & --- & -- & \\
\hline \multirow{2}{*}{$\begin{array}{l}\text { Ethane }+\mathrm{O}_{2}{ }^{e} \\
\text { (very low conv.) }\end{array}$} & none & $\begin{array}{l}0.258 \text { conv. } \\
\text { in } 0.22 \mathrm{sec}\end{array}$ & 630 & --- & --- & \multirow[t]{2}{*}{$\mathrm{C}_{2} \mathrm{H}_{4}$} \\
\hline & 1.2 & $\begin{array}{l}0.558 \text { conv. } \\
\text { in } 0.20 \mathrm{sec}\end{array}$ & 630 & --- & -- & \\
\hline \multirow{2}{*}{ Isobutane } & none & 0.16 & 723 & 56.3 & 11.7 & $i-\mathrm{C}_{4} \mathrm{H}_{8}>\mathrm{C}_{3} \mathrm{H}_{6}>>\mathrm{CH}_{4}>\mathrm{C}_{2} \mathrm{H}_{4}-6$ \\
\hline & 0.7 & 0.38 & 719 & 50.7 & 10.7 & $\mathrm{C}_{3} \mathrm{H}_{6}>i-\mathrm{C}_{4} \mathrm{H}_{8}>>\mathrm{CH}_{4}>\mathrm{C}_{2} \mathrm{H}_{4}-6$ \\
\hline \multirow[t]{2}{*}{ Isobutane $+\mathrm{O}_{2}{ }^{\mathrm{e}}$} & none & 0.39 & 720 & -- & -- & $i-\mathrm{C}_{4} \mathrm{H}_{8}>>\mathrm{C}_{3} \mathrm{H}_{6}>>\mathrm{CH}_{4}>\mathrm{C}_{2} \mathrm{H}_{4}-6$ \\
\hline & 0.7 & 0.38 & 720 & --- & -- & $i-\mathrm{C}_{4} \mathrm{H}_{8} \geqq \mathrm{C}_{3} \mathrm{H}_{6}>>\mathrm{CH}_{4}>\mathrm{C}_{2} \mathrm{H}_{4}-6$ \\
\hline \multirow{3}{*}{ Neopentane ${ }^{f}$} & none & 0.029 & 695 & 80.5 & 16.9 & $i-\mathrm{C}_{4} \mathrm{H}_{8}>\mathrm{Cl}_{4} \cong \mathrm{C}_{2} \mathrm{H}_{6}>>0$ ther products \\
\hline & s.s. ${ }^{1}$ & 0.082 & 695 & $65^{8}$ & $-\cdots$ & \multirow{2}{*}{$i-\mathrm{C}_{4} \mathrm{H}_{8}>\mathrm{CH}_{4}>>C_{2} \mathrm{H}_{6}>$ other products } \\
\hline & S.s. ${ }^{1}$ & $-\infty$ & --- & $30^{h}$ & $\overline{---}$ & \\
\hline
\end{tabular}

${ }^{a}$ Surface to volume ratio. Stainless steel rods are used. biven as carbon equivalencies. ${ }^{c}$ No detectable surface effect was observed at $S / V=1.2 \mathrm{~cm}^{-1}$. ${ }^{d}$ Percent of product.

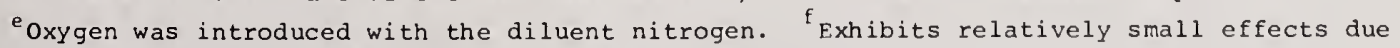
to the added oxygen. ${ }^{B}$ Above $625^{\circ} \mathrm{C}$. ${ }^{h}$ Below $625^{\circ} \mathrm{C}$. ${ }^{1}$ Stainless steel screen, $\mathrm{S} / \mathrm{V}$ not determined. 
Neopentane, on the other hand, exhibits a marked surface effect in the absence of oxygen, but is not noticeably influenced by modest additions of oxygen.

In addition, it has been observed that the pyrolysis products of propylene and isobutylene are markedly influenced by surface. Good yields of methylacetylene and allene are obtained in the total absence of surface, but with a stainless steel surface present the yields are reduced to nearly zero. Also, the conversion of ethylene to acetylene is greatly accelerated by oxygen; the addition of stainless steel surface significantly reduces that product.

One of the unusual aspects of surface effects which has been demonstrated is the lack of linearity of surface-to-volume ratio and surface effect. This is demonstrated in table IV where the surface effect for cyclopropane is shown to optimize at $S / \mathrm{V}=0.6 \mathrm{~cm}^{-1}$. The reason for this lack of linearity is not known, but it does demonstrate one of the challenging complications related to surface effects.

Table IV

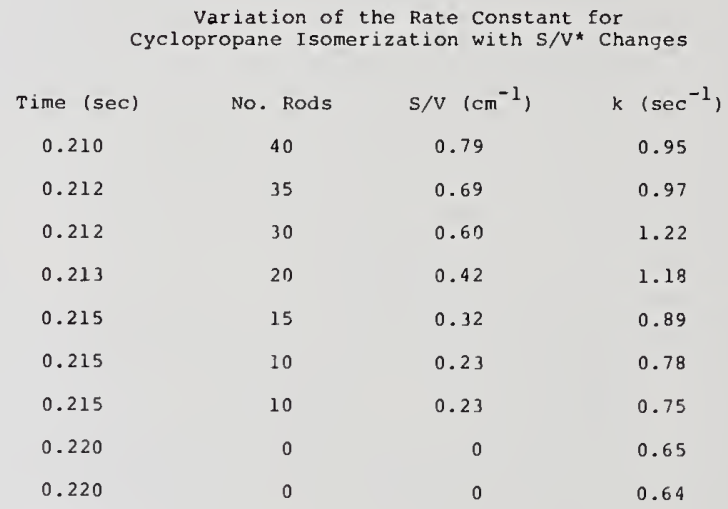

* The surface was provided by oxidized stainless steel rods equally spaced throughout the $5.2 \mathrm{~cm}$ I.D. quartz reactor tube. The data were taken at $676-680^{\circ} \mathrm{C}$ and corrected to $678{ }^{\circ} \mathrm{C}$.

These selected results demonstrate the effectiveness of the wall-less reactor in exploring the relatively virgin area of totally homogeneous reactions and of comparative surface effects. However, the limitation of precision data to first order reactions is restrictive.

To overcome this disadvantage, a new "homogeneous front" reactor has been developed. In principle, this new reactor provides an instantly and continuously formed front of mixed gases whose concentrations are laterally consistent as they flow through a large tube. The reaction occurring in the midstream is completely homogeneous, and diffusion into the midstream is counterbalanced by diffusion from the midstream. Thus, the only concentration changes are those resulting from the chemical changes under study. Since more than one reactant may be introduced and surfaces may be added in the form of evenly spaced rods placed parallel to the stream flow, this new reactor has all the advantages of the earlier wall-less reactor with none of the significant disadvantages. The sampling procedure is the same as with the wall-less reactor. 
It should be added that the new "homogeneous front" reactor permits highest efficiency, and once the reactor is set up, data with excellent reproducibility can be obtained. The reactor is brought to temperature while passing sufficient new reactant gas through it to displace the previous gases. The system is then ready for removal of samples for analysis. No conditioning other than for temperature is necessary. The limitation in accuracy is with the analytical procedures rather than the reactor technique.

The details of the reactor are shown in figure 1 . The entire reactor is enclosed in a Lindberg 8-inch tube furnace (not shown) with $\pm 1{ }^{\circ} \mathrm{C}$ temperature control. The furnace is set in an upright position for upward flow. Gases I and II are brought to temperature in the furnace prior to entering the reactor and then flow into chambers $A$ and $B$, respectively. Gas I is distributed through alternate slits (see $C^{\prime}$ ) and gas II through the other set of slits. There are a total of 42 parallel alumina separator plates 0.022 inch thick and spaced 0.062 inch. The slit width of the drawing is grossly exaggerated and conveys the intent but not the proportion of the design.
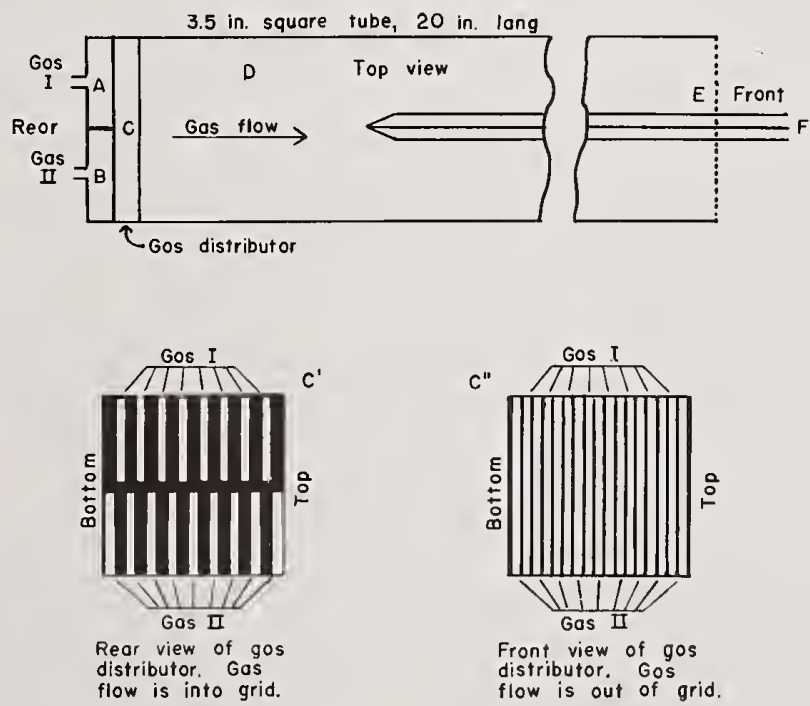

Figure 1. The "homogeneous front" reactor. The slit widths shown in $C^{\prime}$ and $C^{\prime \prime}$ are not in proportion to the actual dimensions.

The separated gases then pass through the slits of $C^{\prime}$ and $C^{\prime \prime}$ into open tube $D$ (made of 310 stainless stee 1 ); immediate mixing of gases I and II occurs. Only then does the reaction begin.

The distribution of gases $I$ and II is equalized across each slit and among all slits carrying the same gas by flow barriers (not shown) so that a small consistent back pressure is established along all of the slits. Further, the design is such that the gas flowing 
into $D$ exhibits a microturbulence and, therefore, a more rapid mixing of the merging gases, but a macroturbulence over the entire reactor is not seen.

Reaction time is determined by the cross-sectional area of the reactor and the rate of gas flow. Plug flow is established as the gases enter tube D; this, however, slowly changes to laminar flow. Since the extent of change is small with the allotted time span, correction for laminar flow is very modest.

To make observations under wall-iess or surface free conditions, the reaction time is limited to the time required for those reactants or products (which may be influenced by surface) to diffuse from the outer wall to the center stream. Depending on the nature of the reaction system as well as temperature and pressure, the allowable maximum time span can vary from 0.2 to 2 seconds.

Analysis of the products and reactants for the purpose of determining the kinetics of the reaction is possible with the sampling tube shown at $F$. This is an insulated air cooled (water cooling may also be used) probe which withdraws the gas mixture from $D$ and immediately cools it to at least $300^{\circ}$ below reaction temperature. The sampled gas is then analyzed in a gas chromatograph or mass spectrometer-gas chromatograph combination.

Highly consistent and reproducible flow characteristics are observed in this reactor providing that wind currents do not disturb the gas flow at the open end of $D$. To avoid the latter possibility, a screen is placed over this end to establish a slight back pressure and to eliminate the effects of stray breezes.

The potential applications for the "homogeneous front" reactor are extensive and can relate to almost any type of gas phase reaction. The only limitation is the factor of time. More specific applications may include studies on the influence of totally surface free conditions upon both rates and products formed with a variety of gas phase reactions and the controlled effects of added surfaces upon these same reactions.

\section{References}

[1] Taylor, J. E., Hutchings, D. A., and Frech, K. J., J. Am. Chem. Soc. 91, 2215 (1969).

[2] Taylor, J. E. and Kulich, D. M., Int. J. Chem. Kinet. ㅌ, 455 (1973).

[3] Taylor, J. E. and Kulich, D. M., ACS Symp. Ser. 1976, 32, p. 72 (Ind. Lab. Pyrolyses, Symp., 1975).

[4] Hutchings, D. A., Taylor, J. E., and Frech, K. J., J. Phys. Chem. 73, 3167 (1969).

[5] Taylor, J. E., and Milazzo, T. S., J. Phys. Chem. 82, 847 (1978).

[6] Taylor, J. E. and Milazzo, T. S., Int. J. Chem. Kinet., in press. 
National Bureau of Standards Special Publication 561, Proceedings of the 10th Materials Research Symposium on Characterization of High Temperature Vapors and Gases held at NBS, Gaithersburg, Maryland, September 18-22, 1978. Issued October 1979.

\title{
STUDIES OF THE ELEMENTARY REACTION KINETICS OF HIGH-TEMPERATURE SPECIES USING OPTICAL MEASUREMENT TECHNIQUES ${ }^{1}$
}

\author{
Arthur Fontijn \\ AeroChem Research Laboratories, Inc. \\ P.0. Box 12 \\ Princeton, NJ 08540
}

\begin{abstract}
A unique experimental method is described for providing information on the kinetics of individual elementary reactions of neutral metallic species (free atoms and diatomic radicals) as a function of temperature. This high-temperature fast-filow reactor (HTFFR) technique has allowed the study of oxidation reactions of refractory metal atom and metal oxide species from about 300-1900 K by a single experimental method, thereby overlapping the temperature domains of traditional near room temperature techniques and flames and shock tubes. The technique, which uses absorption and fluorescence measurements of the consumption of the metallic species, allows for independent variation of temperature, pressure, reactant concentrations, and reaction time. Some significant results, and an extension to measurements on chemiluminescent and electronic excitation quenching reactions are discussed. A brief comparison is made to other techniques for obtaining information on homogeneous gas-phase reactions of such metallic species. Adaptation of the heated reactor technique to determine the temperature dependence of rate coefficients of elementary reactions of hydrocarbons using hightemperature photolysis (HTP) in a static mode is discussed.
\end{abstract}

\section{Introduction}

Proper understanding, prediction, and modeling of reacting systems requires reliable knowledge of the reaction mechanisms and the rate coefficients of the individual reactions involved. Actual practical (e.g., combustion) environments are usually too complicated to provide a suitable medium for such measurements. While various techniques for obtaining

\footnotetext{
${ }^{1}$ Work supported by the Air Force Office of Scientific Research under Contracts F44620-76C-0108 (J137), F49620-77-C-0033 (J150), and the Department of Energy under Contract EG-77-C-02-4169 (J153).
} 
such kinetic data have been used over the years, these techniques have only allowed measurements over limited temperature ranges, which often do not overlap the actual temperatures of interest in practical systems. Extrapolation and interpolation over various temperature regimes is not a recommendable procedure since the simple Arrhenius-law type expression for the temperature dependence of rate coefficients $k(T)=A T \exp (-\Delta E / R T)$ is often unreliable when applied to large temperature ranges and since systematic errors due to differences in measurement techniques for different temperature regimes may unduly influence the apparent T-dependences. Thus, techniques that span wide temperature ranges are needed.

To begin to fill this void in diagnostic capability we have developed the hightemperature fast-flow reactor (HTFFR) technique. This development has opened up the 1000$1900 \mathrm{~K}$ regime for study by the same flow tube technique previously used in $\approx 100-1000 \mathrm{~K}$ experiments. Thus a single technique can now be used, in principle, to span the $\approx 100^{-}$ $1900 \mathrm{~K}$ temperature range, thereby overlapping the temperature domains of more traditional flow tube work and that of high temperature techniques such as flames and shock tubes. (We have thus far covered the 300-1900 K regime in our measurements.) The HTFFR technique, like other flow tube methods, allows for independent control of reaction times, pressure, reactant concentrations, and temperature; it was originally developed to study the homogeneou gas-phase kinetics of isolated individual free metal atom oxidation reactions, principally of refractory metal species for which no suitable experimental techniques existed at any tempera ture. It has now also been applied to kinetic measurements on free radical metal monoxide oxidation reactions and on chemiluminescent metal atom oxidation reactions.

We have recently prepared a very extensive review on the HTFFR technique and its achievements $[1]^{2}$. This review, which also describes and discusses the other methods presently in use for kinetic observations on metal atom reactions, may be consulted for further details. The question of achievable accuracy in heated flow tube measurements is additionally discussed in reference [2].

\section{Apparatus}

Schematics of an HTFFR are given in figures 1 and 2. This HTFFR consists of a $2.5 \mathrm{~cm}$ i.d. alumina reactor tube contained in a $25 \mathrm{~cm}$ i.d., $95 \mathrm{~cm}$ long vacuum chamber. Optical observations are made through ports in the reactor and vacuum furnace jacket. The metal to be studied is vaporized and entrained in a stream of inert carrier gas. 0xidant is introduced into this gas stream through an axially movable inlet. Excess oxidizer is used to allow pseudo-first order measurements to be made. The reactor is heated in three contiguous, independently controlled, heating zones, using Pt-40 percent Rh resistance wire. The advantage of this arrangement is that the metal to be vaporized can be placed in the upstream heating zone which is kept at the vaporization temperature, while the reaction zone

\footnotetext{
${ }^{2}$ Figures in brackets indicate the literature references at the end of the paper.
} 
temperature can be independently controlled. The minimum reaction zone temperature which can be achieved by the reactor of figure 1 is determined by the heat required to vaporize the metal and is $\approx 700$ to $800 \mathrm{~K}$ for refractory species such as $\mathrm{Sn}$ and $\mathrm{A}$.. These species have been studied [3] at $300 \mathrm{~K}$ using the modular reactor shown in figure 3 . This low temperature is achieved via a modification of the method whereby a $30 \mathrm{~cm}$ long HTFFR is used to generate a metal atom/inert gas mixture, which then flows through a cooled zone in which the rate coefficients are measured. The reactor of figure 4 has been used [4] to achieve temperatures from about $400 \mathrm{~K}$ and up. In it, the metal atom generating section is followed by a short $(\approx 10 \mathrm{~cm})$ non-insulated copper section where additional carrier gas at room temperature may be introduced (as in the reactor of fig. 3). The metal atom/inert gas mixture then flows into the independently heated reaction tube section.

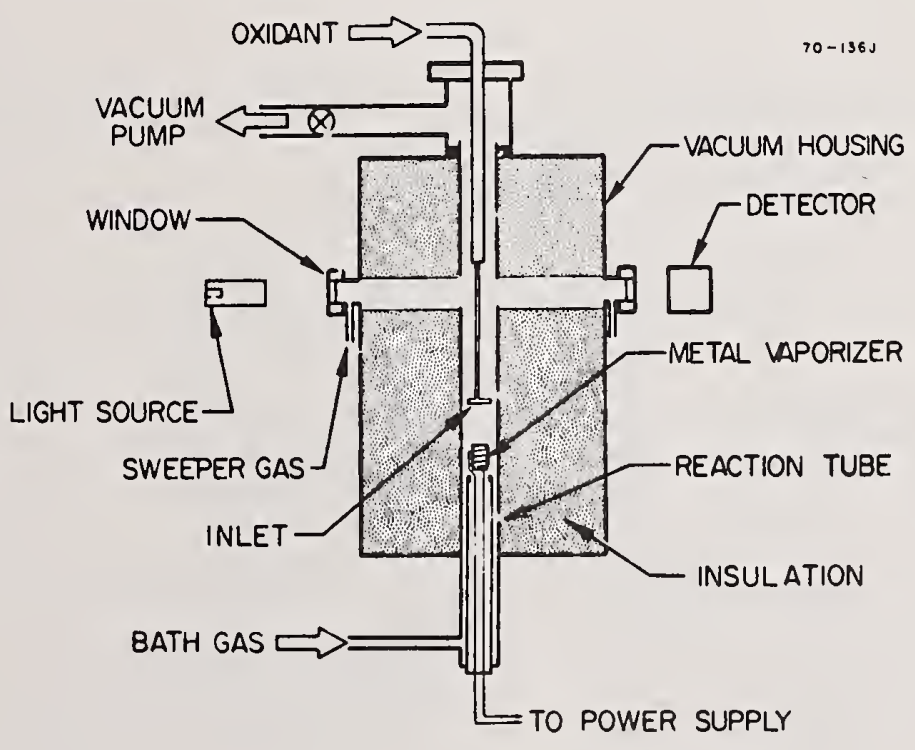

Figure 1. Schematic of a high-temperature fast-flow reactor (HTFFR).

\section{Overall Rate Coefficients of Elementary Reactions}

Overall rate coefficients ${ }^{3}, k$, are obtained from the observed variation in the relative metal atom concentration, $[\mathrm{Me}]_{\mathrm{re}]}$, as a function of reaction time, $t$, oxidizer concentration, $[0 x]$, total pressure, $P$, and temperature, T. Flow velocity, $\bar{v}$, is varied to ascertain that the measured $\mathrm{k}$ is independent of this parameter. Both absorption and fluorescence are

\footnotetext{
${ }^{3}$ The overall rate coefficients pertain to metal atom consumption without regard to the excitation states of the products formed. Investigations of excited product states are discussed in Section 4.
} 


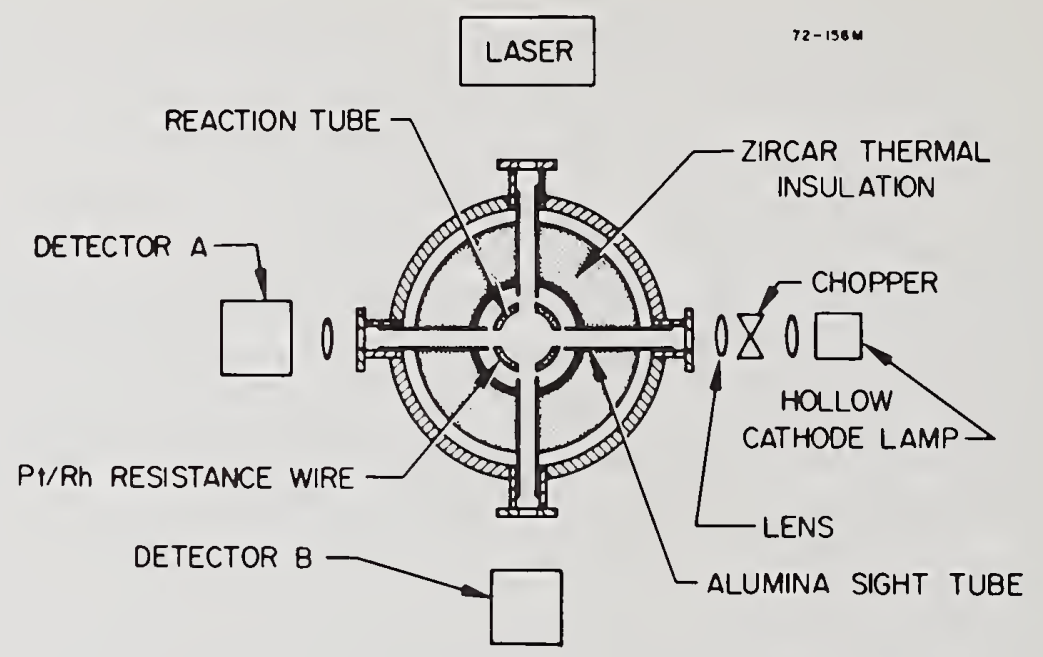

Figure 2. Cross section of the observation plane of an HTFFR. Detector A for atomic absorption and laser-induced fluorescence measurements. Detector B for atomic fluorescence measurements.

T2-8se

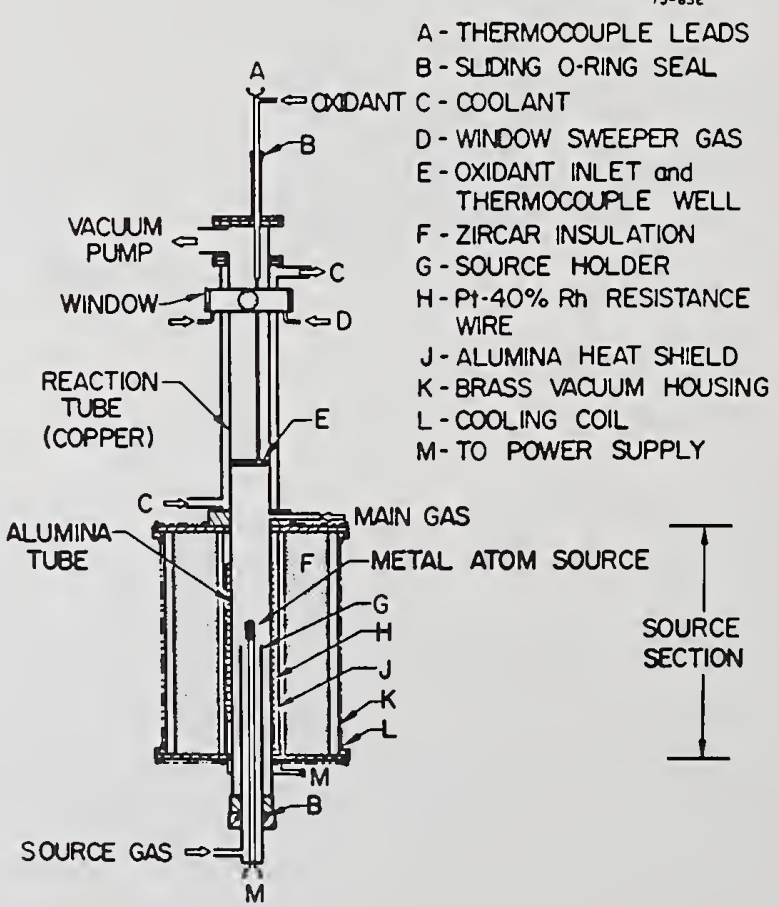

Figure 3. Schematic of modular HTFFR for $\approx 300 \mathrm{~K}$ measurements. 
used to measure $[\mathrm{Me}]_{\mathrm{rel}}$, cf. figure 2, to cover large variations (factors of 10-100) in the initial (reaction time zero) absolute metal atom concentrations. In an extension of the technique, laser induced fluorescence is used and metal monoxide reactions are studied $[3,5]$.

As examples of results obtained by the technique, we briefly discuss here two sets of reactions. The first set concerns a number of $A 1$ reactions, the temperature-dependence of the rate coefficients of which clearly demonstrates that Arrhenius-law type behavior may often not be assumed. These are the reactions $[3,4]$ :

$$
\begin{aligned}
& \mathrm{Al}+\mathrm{O}_{2} \rightarrow \mathrm{AlO}+0 \\
& \mathrm{Al0}+\mathrm{O}_{2} \rightarrow \mathrm{AlO}_{2}+0 \\
& \mathrm{Al}+\mathrm{CO}_{2} \rightarrow \mathrm{AlO}+\mathrm{CO} .
\end{aligned}
$$

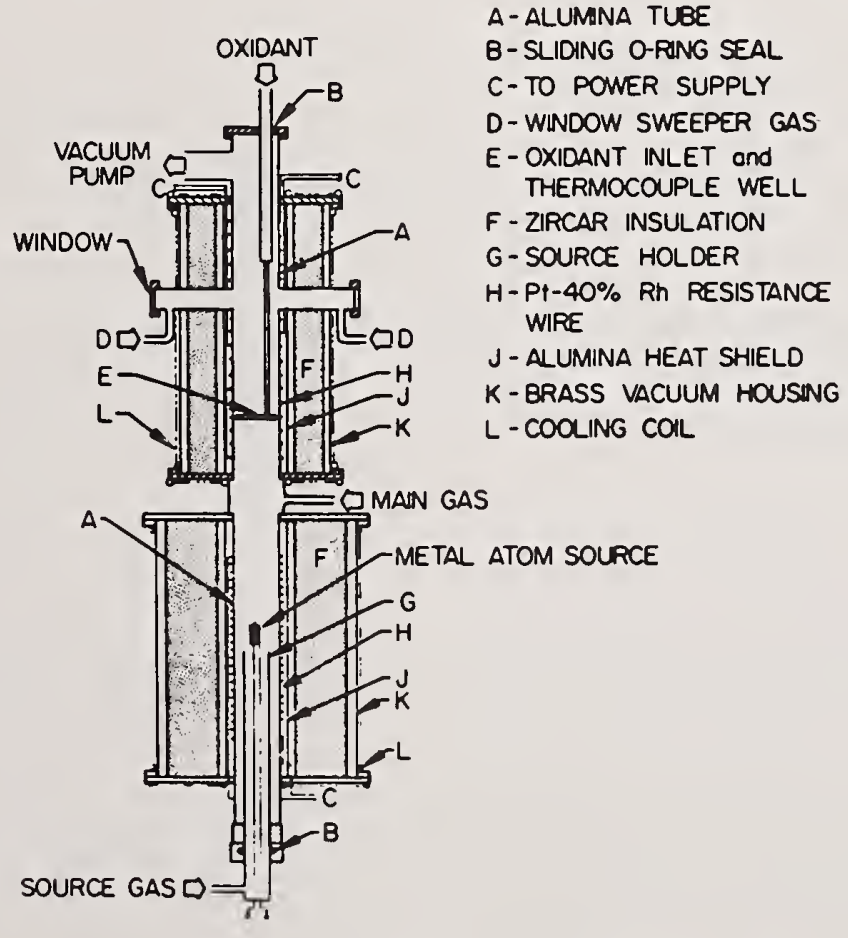

Figure 4. Schematic of modular HTFFR for the intermediate temperature range $(\approx 400-1400 \mathrm{~K})$.

The first two reactions have temperature independent rate coefficients: $k_{1}=(3.4 \pm 2.2) \times$ $10^{-11} \mathrm{ml}$ molecule $\mathrm{s}^{-1}$ and $k_{2}=(4.8 \pm 3.1) \times 10^{-13} \mathrm{ml}$ molecule $\mathrm{s}^{-1}$ over the 300 to 1700 and 300 to $1400 \mathrm{~K}$ ranges, respectively. Reaction (3) gives a strongly curved Arrhenius plot, figure 5. There is some doubt regarding the values of $D(A T-0)$ and $D(0-A 10)$, especially the latter. The lack of an activation energy of reactions (1) and (2) suggests a 
$\Delta \mathrm{H} \leq 0 \mathrm{kcal} \mathrm{mol}{ }^{-1}$. Thus, in addition to basic kinetic data, HTFFR measurements can also yield thermochemical information. On the basis of the most commonly accepted value in the present literature $D(A)-0)=122 \mathrm{kcal} \mathrm{mol}^{-1}$ and reaction (3) is $4 \mathrm{kcal} \mathrm{mol}^{-1}$ endothermic; however, HTFFR measurements currently in progress on the $\mathrm{Al} / \mathrm{SO}_{2}$ reaction suggest an $\mathrm{AlO}$ bond energy at least $4 \mathrm{kcal} \mathrm{mol}^{-1}$ larger, in which case $\Delta \mathrm{H}_{3} \leq 0 \mathrm{kcal} \mathrm{mol}{ }^{1}$.

While such $k(T)$ behavior can be explained in terms of modern kinetics theory, it cannot be predicted and is, in fact, somewhat unexpected. This points to the need to obtain more data of this nature to allow advances in theory and predictive capability. Reactions (1) and (2) may be showing the effects of an intermediate bound complex [6]. In general terms, the effect of such complex formation can be described by:

$$
\begin{gathered}
A+B C \underset{(-\overrightarrow{4})}{(4)} A \cdot B C \stackrel{(5)}{\rightarrow} A B+C \\
k_{a p p}=k_{4} k_{5} /\left(k_{-4}+k_{5}\right)
\end{gathered}
$$

where the app(arent) indicates the observed quantities. If $k_{-4} \gg k_{5}, k_{a p p}=k_{4} k_{5}$ and the apparent activation energy $E_{\text {app }}=\Delta H_{4}+E_{5}$. Hence since for such an association-dissociation process $\Delta \mathrm{H}_{4}<0$, if $\Delta \mathrm{H}_{4} \geq \mathrm{E}_{5}, \mathrm{E}_{\text {app }} \leq 0$. While transition state theory considerations can lead to curved Arrhenius plots, the curvature shown in figure 5 for $\mathrm{T}>750 \mathrm{~K}$ cannot be described on this basis alone. The opening of a second reaction channel for reaction (3) has to be assumed; the likely candidates are [4]: production of AlO in its lowest electronically excited state $A 10\left(A^{2} \Pi\right)$ and preferential reaction of $A 1$ with $\mathrm{CO}_{2}$ in bending modes. Another interesting series of reactions is that of two Group IVA element reactions with $\mathrm{N}_{2} \mathrm{O}$ :

$$
\begin{array}{ll}
\mathrm{Sn}+\mathrm{N}_{2} \mathrm{O} \rightarrow \mathrm{SnO}+\mathrm{N}_{2} & \Delta \mathrm{H}=-92 \mathrm{kcal} \mathrm{mol}^{-1} \\
\mathrm{Ge}+\mathrm{N}_{2} \mathrm{O} \rightarrow \mathrm{GeO}+\mathrm{N}_{2} & \Delta \mathrm{H}=-117 \mathrm{kcal} \mathrm{mol}^{-1}
\end{array}
$$

for which the following rate coefficients ${ }^{4}$ were obtained for $5 n$ [7]:

$$
\begin{aligned}
& \operatorname{Sn}\left({ }^{3} P_{0}\right): \quad k_{6}=(8.9 \pm 4.0) \times 10^{-13} \exp [(-2260 \pm 180) / T] ; \\
& \operatorname{Sn}\left({ }^{3} P_{1}\right): \quad k_{6} \approx 9 \times 10^{-13} \text { at } 350 \mathrm{~K} ;
\end{aligned}
$$

and for Ge [8]

${ }^{4}$ Since only metal atom disappearance measurements are made, it should be realized that the measurements for $\mathrm{J}=1,2$ states do not distinguish between chemical reaction and physical quenching. 


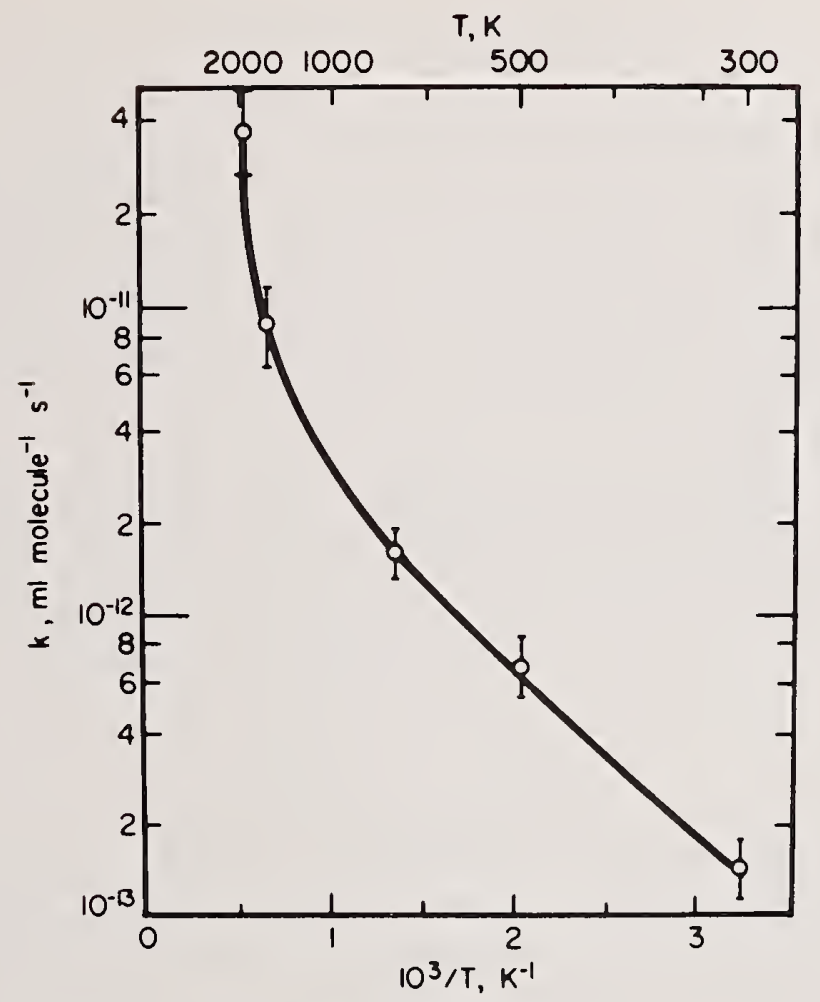

Figure 5. Rate coefficient of the $\mathrm{Al}+\mathrm{CO}_{2} \rightarrow \mathrm{A} 10+\mathrm{CO}$ reaction from 300 to $1900 \mathrm{~K}$.

$$
\begin{array}{ll}
\operatorname{Ge}\left({ }^{3} P_{0}\right): & k_{7}=(1.7 \pm 0.3) \times 10^{-11} \exp [(-490 \pm 50) / T] \\
\operatorname{Ge}\left({ }^{3} P_{1}\right): & k_{7}=(8.4 \pm 1.3) \times 10^{-12} \exp [(100 \pm 40) / T] \\
\operatorname{Ge}\left({ }^{3} P_{2}\right): & k_{7}=(1.2 \pm 0.3) \times 10^{-11} \exp [(-170 \pm 100) / T]
\end{array}
$$

all in ml molecule $\mathrm{e}^{-1} \mathrm{~s}^{-1}$. These $\mathrm{k}(\mathrm{T})$ measurements pertain to the 450 to $950 \mathrm{~K}$ range; the latter represents approximately the highest temperature at which $\mathrm{N}_{2} \mathrm{O}$ is thermally stable in the HTFFR. Over this temperature range, no deviation from Arrhenius behavior is observed reactions (6) and (7). It is speculated that reactions (6) and (7) proceed with higher probability for the spin-orbit excited states than for $\mathrm{J}=0$, with the differences in activation energies of the two strongly exothermic processes (6) and (7) reflecting the difference in spacing between $\mathrm{Sn}$ and Ge spin orbit states (for $\mathrm{J}=1$ excitation, 4.8 and $1.6 \mathrm{kcal}$ $\mathrm{mol}^{-1}$, respectively). It should be pointed out that reactions (6) and (7) violate spin conservation for formation of the singlet ground state monoxides, but not for triplet excited states which will be discussed in the next section. 


\section{Chemiluminescent Reactions}

Many metal atom oxidation reactions are high yield chemiluminescent processes, leading to intense radiation in the visible and ultraviolet. To study their kinetics, HTFFRs offer advantages similar to those for the overall (all product states) oxidation reactions discussed, i.e., those of independent control of individual reactant and quencher concentrations, pressure, temperature, and reaction time interval observed. In addition to spectral observations and overall rate coefficients, the quantities of concern are photon yields and quenching rate coefficients.

Photon yield $\phi$ is defined as the fraction of reaction events leading to emission of a photon. A related quantity, often confused with $\Phi$, is the rate coefficient for light emission $k_{h v}$ which is defined by (written for the special case of metal atom oxidation reactions):

$$
I=d[h v] / d t=k_{h v}[M e][0 x]
$$

where $I$ is the rate of light emission per unit volume. Hence,

$$
\mathrm{k}_{\mathrm{h} \nu}=\phi \mathrm{k}_{\text {overall }} \text {. }
$$

It follows that the measurement of $\Phi$ requires the measurement of absolute intensity and absolute metal atom concentrations. The former is obtained by comparison to the 0/NO standard chemiluminescent reaction [9] observed under identical geometry with the HTFFR at room temperature. [Me] is determined by absorption measurements using published gf values for the elements of interest. These $\Phi$ measurements are discussed in greater detail in references [1] and [7]. As a check on the [Me] absorption measurements, we have, for the case of [Sn], developed a chemiluminescent titration technique [10] which has yielded $\Phi$ data in reasonable agreement with those based on absorption measurements. Similar titrations should, in turn, allow determination of gf values for other elements.

The highest $\phi$ values we have observed are for the $\mathrm{Sn} / \mathrm{N}_{2} 0$ reaction (6) for which we obtained [7] for the excitation levels $\mathrm{s}^{5}$ observed in the 450-950 $\mathrm{K}$ temperature range:

$$
\begin{aligned}
{ }_{\mathrm{Sn} 0}\left(\mathrm{a}^{3} \Sigma^{+}(1)\right) & =0.53 \pm 0.26 \text { independent of } \mathrm{T} \\
{ }_{\mathrm{Sn} 0\left(\mathrm{~b}^{3} \Pi\right)} & =(5.9 \pm 2.9) \times 10^{-1} \exp [-(1200 \pm 200) / \mathrm{T}] .
\end{aligned}
$$

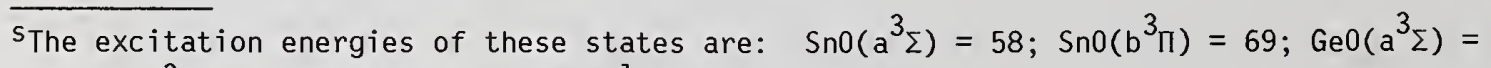
$78 ; \mathrm{Ge} 0\left(\mathrm{~b}^{3} \Pi\right)=106$, all in $\mathrm{kcal} \mathrm{mol} \mathrm{l}^{-1}$. 
The Ge0 formation reaction (7) was found to yield much lower $\$ s$ in this temperature range [8]:

$$
\begin{aligned}
& \Phi_{\mathrm{Ge} 0}\left(\mathrm{a}^{3} \Sigma\right) \approx(2.7 \pm 1.4) \times 10-2 \exp [(-2300 \pm 370) / \mathrm{T}] \\
& \Phi_{\mathrm{Ge} 0}\left(\mathrm{~b}^{3} \Pi\right) \approx(9.4 \pm 7.1) \times 10-3 \exp [(-3800 \pm 360) / \mathrm{T}] \\
& \Phi_{\mathrm{Ge} 0}\left(A^{1} \Pi\right) \approx(7.7 \pm 6.9) \times 10-2 \exp [(-5200 \pm 360) / \mathrm{T}]
\end{aligned}
$$

It may be pointed out that in the extreme case of no quenching, $\Phi$ for a given state equals its branching ratio, i.e., the fraction of reaction events that leads to production of that state.

The methods of obtaining quenching rate coefficients have been discussed in reference [11]. Basically, the standard Stern-Volmer plot

$$
\frac{\Phi_{0}}{\phi}=1+\tau_{\mathrm{rad}} k_{\mathrm{q}}[X]
$$

approach of photochemistry can be followed. However, in chemiluminescent reactions the reacting species themselves can also act as quenchant. In this work, we thus take the maximum photon yield observed as a function of reactant concentration as the $\Phi_{0}$. Some typical $\mathrm{k}_{\mathrm{q}}$ values in $\mathrm{ml}$ molecule $\mathrm{e}^{-1} \mathrm{~s}^{-1}$ obtained here are for the effective quenching of $\mathrm{Ba} 0$ emitting states in the $\mathrm{Ba} / \mathrm{N}_{2} \mathrm{O}$ reaction;

$$
\begin{aligned}
& A r \leq 3 \times 10^{-13} ; \quad N_{2}=(4.8 \pm 2.0) \times 10^{-12} ; \\
& N_{2} 0=(4.2 \pm 1.0) \times 10^{-10} ; 0_{2}=(2.5 \text { to } 7.0) \times 10^{-10}
\end{aligned}
$$

The products of the $\mathrm{Sn}_{n} / \mathrm{N}_{2} \mathrm{O}$ reaction are far less readily quenched; the $\mathrm{k}_{\mathrm{q}}$ values for $\mathrm{Sn} 0\left(\mathrm{a}^{3} \Sigma\right)$ near $900 \mathrm{~K}$ are [7]:

$$
\operatorname{Ar}, N_{2} \leq 1 \times 10^{-16} ; \quad N_{2} 0 \leq 1 \times 10^{-14} ; \quad S n \leq 1.2 \times 10^{-12} \text {, }
$$

all in ml molecule $\mathrm{e}^{-1} \mathrm{~s}^{-1}$. 


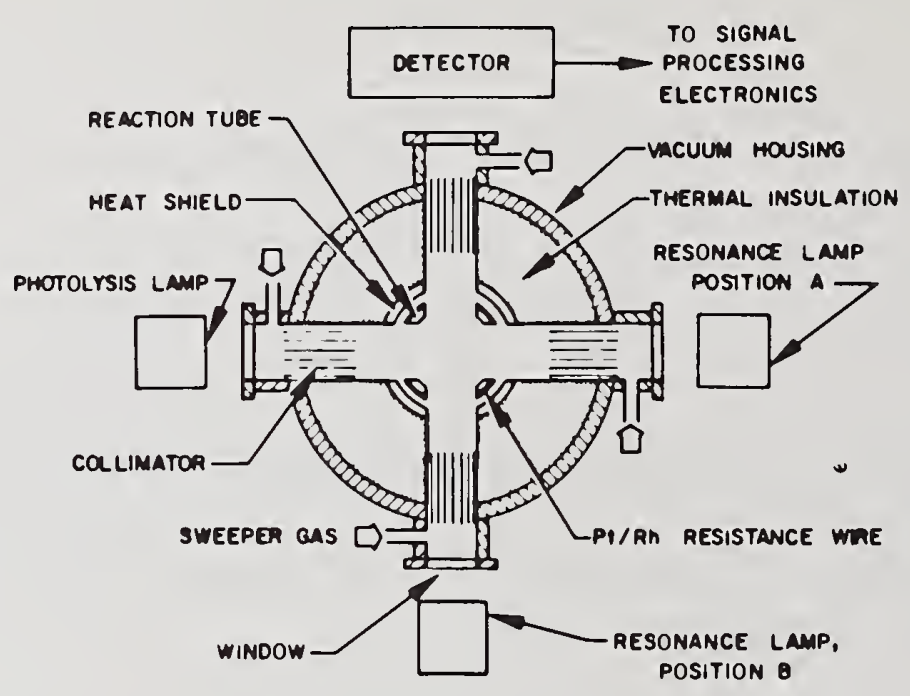

Figure 6. Cross section of HTP reactor. Resonance lamp in position A for fluorescence measurements, in position B for absorption measurements.

\section{Other Techniques for Metal Oxidation Reaction Kinetic Observations}

Other methods that are in current use for the study of metal atom reaction kinetics are discussed in some detail in reference [1]. Briefly, the major methods are: (i) beam studies, (ii) atomic diffusion flame studies, and (iii) flash photolysis resonance absorption/ fluorescence (FPRAF) techniques. The chemiluminescence of a large number of metal atom reactions has been studied by beams; much of this work has used beam-scattering gas arrangements. The particular advantage of beam techniques in the present context is that the observation of nascent product states, i.e., spectral distributions not modified by subsequent collisions, is often possible. The achievable accuracy of photon yield measurements is comparable to that of HTFFR. For observations on non-chemiluminescing product states, laser-induced fluorescence has extensively been used. Atomic diffusion flames can produce very intense spectra, since higher reactant concentrations than practical for the other techniques mentioned can be used; a rough idea of rate coefficients and photon yields can be obtained with it. Experimentally, the diffusion flame technique is the simplest of the techniques discussed, which makes it very useful for rapid rough comparisons between reaction systems; it is, however, often not possible to distinguish between primary and secondary reactions and reaction temperatures (and reactant concentrations) are often poorly known. Accurate overall rate coefficient determinations have been made by the FPRAF technique; the use of this technique has, however, been mainly restricted to near room temperature measurements. 


\section{Elementary Reactions of Hydrocarbons}

The 500-1500 $\mathrm{K}$ temperature range is one in which there is a general dearth of kinetic data [12]. It may be asked if the HTFFR technique could not be used to provide kinetic data in this temperature regime for reactions other than those of refractory species, such as metal atoms and metal monoxide radicals. Particularly because of many problems related to fossil fuel combustion and conversion, the availability of a technique for measurements of the kinetics of isolated individual reactions pertinent to these problems would be important in this temperature regime. (Preferentially, such a technique should extend to somewhat higher temperatures to provide maximum overlap with flame and shock tube studies.) Reactions of interest here would be those between free atoms and radicals, such as $0, H$, and $\mathrm{OH}$, and hydrocarbon molecules and free radicals (as well as reactions of $\mathrm{N}$ and $\mathrm{S}$ species for pollution problems). For such reactions, the HTFFR as such does not appear practical since the high temperature walls would cause rapid recombination of the atoms and radicals and tend to pyrolyze the hydrocarbon molecules. However, such problems can be avoided with the FPRAF technique ${ }^{6}$ mentioned in Section 5 if it could be adapted to this temperature regime.

To do so, Dr. W. Felder and the author have developed a FPRAF technique named HTP (high temperature photolysis) which uses essentially a short HTFFR with a doubled diameter reaction tube ( $5 \mathrm{~cm}$ i.d.) and a cooled hydrocarbon inlet to prevent pyrolysis. In the first studies with this apparatus 0 atoms are produced from $0_{2}$ with a flash lamp and their decay is followed by their resonance fluorescence pumped by a microwave discharge flow lamp [13]. Fluorescence is observed on a time scale of $10^{-4}$ to $10^{-1}$ s using a multichannel analyzer. Thus, the atoms are produced in the observation plane, figure 6 , and their in situ decay is measured. The gases flow slowly through this apparatus, but flow and diffusion losses of the 0 atoms are small compared to the reactive losses in the presence of hydrocarbons. This is a we1l-tested low temperature technique (e.g., reference [14]) and the first results obtained with the HTP technique are encouraging for its extension to cover the 300-1800 $\mathrm{K}$ regime and thus to provide $k(T)$ data for a large number of combustion reactions.

\section{References}

[1] Fontijn, A. and Felder, W., High temperature flow tubes. Generation and measurement of refractory species, in Reactive Intermediates in the Gas Phase: Generation and Monitoring, D. W. Setser, ed. chap. II. (Academic Press, New York, in press).

[2] Fontijn, A. and Felder, W., Factors affecting the accuracy of rate coefficients of metal atom oxidation reactions in heated flow tubes (HTFFRs), J. Phys. Chem. (submitted).

\footnotetext{
${ }^{6}$ The FPRAF technique is essentially a static technique with which real time observations are made, while flow tubes are steady-state techniques.
} 
[3] Fontijn, A., Felder, W., and Houghton, J. J., HTFFR kinetics studies. Temperature dependence of $\mathrm{Al}_{2} / \mathrm{O}_{2}$ and $\mathrm{AlO} / \mathrm{O}_{2}$ kinetics from 300 to $1700 / 1400 \mathrm{~K}$, in sixteenth Symposium (International) on Combustion, p.871 (The Combustion Institute, Pittsburgh, 1977).

[4] Fontijn, A. and Felder, W. HTFFR kinetic studies of $\mathrm{Al}+\mathrm{CO}_{2} \rightarrow \mathrm{AlO}+\mathrm{CO}$ from 300 to $1900 \mathrm{~K}$, a non-Arrhenius reaction, J. Chem. Phys. 67, 1561 (1977).

[5] Felder, W. and Fontijn, A., High-temperature fast-flow reactor kinetic studies. The A10/0 2 reaction near $1400 \mathrm{~K}, \mathrm{~J}$. Chem., Phys. 64, 1977 (1976).

[6] Golden, D. M., Experimental and theoretical examples of the value and limitations of transition state theory, J. Phys. Chem. (submitted).

[7] Felder, W. and Fontijn, A., HTFFR kinetic studies of $\mathrm{Sn} / \mathrm{N}_{2} \mathrm{O}$, a highly efficient chemiluminescent reaction, J. Chem. Phys. $\underline{69}, 1112$ (1978).

[8] Fontijn, A. and Felder, W. (work in progress).

[9] Fontijn, A., Meyer, C. B., and Schiff, H. I., Absolute quantum yield measurements of the $\mathrm{N} 0-0$ reaction and its use as a standard for chemiluminescent reactions, $J$. Chem. Phys. 40, 64 (1964).

[10] Fontijn, A. and Felder, W. HTFFR kinetics studies: a chemiluminescence titration method for the determination of absolute Sn concentrations, Chem. Phys. Lett. $\underline{47}, 380$ (1977).

[11] Felder, W., Gould, R. K., and Fontijn, A., HTFFR kinetic studies of the fate of excited $\mathrm{BaO}$ formed in the $\mathrm{Ba} / \mathrm{N}_{2} \mathrm{O}$ chemiluminescent reaction, J. Chem. Phys. $\underline{66}, 3256$ (1977).

[12] Baulch, D. L. and Montague, D. D., Some aspects of the evaluation of kinetic data, J. Phys. Chem. (submitted).

[13] Davis, D. and Braun, W., Intense vacuum ultraviolet atomic line sources, App1. 0pt. $\underline{7}$, 2071 (1968).

[14] Kurylo, J. J. and Braun, W., Flash photolysis resonance fluorescence study of the reaction $\mathrm{Cl}+\mathrm{O}_{3} \rightarrow \mathrm{ClO}+\mathrm{O}_{2}$ over the temperature range 213-298 K, Chem. Phys. Lett. 37, 232 (1976).

\section{Discussion}

Question (Gardiner): Could you describe briefly the comparison between the conditions of temperature, pressure, and flow speed, that can be obtained in your apparatus as opposed to the people (Irv Glassman) two miles north of you in the adiabatic flow reactor at Princeton University.

Response (Fontijn): Yes. We work under laminar flow conditions, they work under turbulent flow conditions.

They don't look at isolated reactions. They look at what they call global reactions. So they essentially come up with a scheme that can be used to explain an overall reaction.

They deduce reaction mechanisms from stable end product analysis. In our reactor, reactant consumption is used to measure kinetics. 
The measurement, the generation technique of the high temperatures here is very different. They use mainly an arc discharge to produce the high temperatures.

I am not sure what the flow conditions are that they are working under. We typically work from 3 to 100 Torr, from 10 to 100 meters a second, and 300 to 2000 Kelvin.

Actually, they are two miles south.

Question (Spencer): How do you handle the problem of stagnation of metal atoms in that tee portion of your flow tube? Is that a problem?

Response (Fontijn): No, because that is downstream from the observation zone. --- Actually we do show a window sweeper here, and we have to make measurements where we pass small flows of gas through there to prevent this sort of phenomenon, but we have found over the years that is not necessary. It doesn't make any difference.

As a matter of fact, you can---if you want to do it as a visual experiment sometime at room temperature use reactions with acetylene, which is a beautiful chemical-1uminescent reaction; you do this thing in glass and you see that the stream goes right through it. It doesn't diffuse out.

Now, the other point here is that the observation region is, of course, as such, a slightly disturbed region. I have no time to show you detailed results.

But, that region is normally outside the temperature zone and is about 100 degrees lower than the rest of the reactor because it can radiate out freely. It is not protected by the insulator.

But, the theory of this has been gone over fairly extensively. As long as you work in the pseudo first order mode it doesn't matter because you measure the difference of the arriving metal concentration as a function of distance as you move this.

So, what happens at the actual observation region is basically immaterial.

Question (Hauge): Is it true that you are measuring basically the disappearance of metal atoms and that if there were more than one reaction occurring, your rate constant would be a combination of those two rates of reaction?

Response (Fontijn): Yes. For all of these reactions I have described it seems to be impossible to write down another reaction path.

You see, the only other reaction path would be a three body reaction, but since we have changed our pressure over a factor of 10 , one would see such a thing. 
National Bureau of Standards Special Publication 561, Proceedings of the 10th Materials Research Symposium on Characterization of High Temperature Vapors and Gases held at NBS, Gaithersburg, Maryland, September 18-22, 1978. Issued 0ctober 1979.

\section{DIFFUSION OF CARBON DIOXIDE AT HIGH TEMPERATURES}

J. R. Ferron, D. L. Kerr ${ }^{1}$, and A. U. Chatwani $i^{2}$

Department of Chemical Engineering

University of Rochester

Rochester, NY 14627

Self-diffusion coefficients for carbon dioxide and binary diffusivities in $\mathrm{CO}_{2}$-argon mixtures have been measured at one atmosphere and temperatures of 300 to about $1900 \mathrm{~K}$ by a simulated point-source method. The data are in good agreement with prior measurements at the lower temperatures, for which conditions spherically-symmetric potential functions provide an adequate means for correlations and comparison with pure and mixture viscosities. The experimental results for $\mathrm{CO}_{2}$-argon, used with literature data on thermal diffusion, permit one to examine the separate contributions of elastic and inelastic effects on polyatomic diffusion. Inelastic effects are predominant in thermal diffusion of $\mathrm{CO}_{2}$-argon, and there is an observable effect on ordinary diffusion.

At the higher temperatures, diffusivities approach those of nonspherical molecular forms while viscosities retain a spherical description. It has been found feasible to model various transport properties of carbon dioxide by a Kihara-type potential having a rigid, spherocylindrical core with a soft, square-well field surrounding the core. Monte Carlo calculations of properties of the soft, spherocylindrical form are described, and the results compared with experimental data.

${ }^{1}$ Eastman Kodak Company, Rochester, New York 14650.

${ }^{2}$ DFVLR, Institut für angewandte Gasdynamik, 5000 Köln 90, West Germany. 


\section{Introduction}

At moderately low temperatures and at one atmosphere pressure, diffusion of gaseous carbon dioxide is described quite well by monatomic kinetic theory. Experimental diffusivities can be correlated precisely by a Lennard-Jones potential, and the correlation parameters are quite close to those obtained from similar treatment of experimental viscosities, thermal conductivities and second virial coefficients.

The monatomic description of diffusion begins to fail if temperatures are higher than 300-350 K. The effects are readily seen by comparison of measured diffusivities with those predicted from correlations of viscosity data. Deviations are apparently caused by the polyatomic structure of carbon dioxide, and this point of view is supported by model calculations which simulate the effects of combined rotational and translational motion during diffusion. There is further substantiation in the comparison of ordinary diffusion and thermal diffusion of carbon dioxide, especially for the system of carbon dioxide and argon in which monatomic-polyatomic interactions may be studied without the complication of significant differences of molecular mass.

The purpose of this paper is to illustrate the above arguments by descriptions of experimental methods and of model calculations which have been found to be especially useful for systems containing carbon dioxide at temperatures as high as $1900 \mathrm{~K}$. The paper includes new experimental and computational results in smoothed form; detailed numerical data will be presented elsewhere.

\section{Experimental Methods}

The experimental method applied in the present work is that of diffusion of a trace gas from a simulated point source in a steady, isothermal, one-dimensional, laminar flow of carrier gas.

Point-source and line-source techniques were used in early experimental studies of turbulent diffusion. (Hinze $[1]^{3}$ provides a full summary of the early work.) In 1957, Westenberg and Walker [2] first applied the method to the study of molecular diffusion, using nitrogen as a tracer in flows of helium, argon, and carbon dioxide. Later, Westenberg and coworkers [3-8] extended the initial work, using various binary systems at temperatures of 300-1150 K. They preheated the gases with electrical resistance heaters, and $1150 \mathrm{~K}$ was the maximum temperature which could be readily attained. They analyzed for the tracer by careful thermal conductivity methods.

Ember, Ferron, and Woh $1[9,10]$ and Pakurar and Ferron [11-13] extended the hightemperature limit to $1800-2000 \mathrm{~K}$ by using combustion to generate the carrier gas. This technique precluded experiments at temperatures below about $1000 \mathrm{~K}$, though measurements at room temperature were carried out without heating. These studies also introduced use of ratioactive tracers, ${ }^{14} \mathrm{CO}_{2}$ and tritium, making possible measurements of self-diffusivities

${ }^{3}$ Figures in brackets indicate the literature references at the end of this paper. 
of $\mathrm{CO}_{2}$ and water vapor as well as searches for concentration dependence of binary diffusivities.

The apparatus used in the present work combines resistance heating and combustion so that the full thermal range, from room temperature to flame temperature, can be utilized. The tracer has again included ${ }^{14} \mathrm{CO}_{2}$, and concentrations have been obtained by proportional counting of samples pumped continuously from the diffusion apparatus.

The experimental results referred to here are those for the self-diffusion coefficient of carbon dioxide and the binary diffusion coefficient in mixtures of carbon dioxide and argon, all at a pressure of one atmosphere.

The new self-diffusivities are for temperatures of 428-1506 K. The data may be combined with earlier measurements $[9,11]$ and with recent data at lower temperatures $[14,15]$, providing an experimental description of self-diffusion of $\mathrm{CO}_{2}$ at temperature intervals no greater than $40 \mathrm{~K}$ for most of the range $194 \mathrm{~K}$ - $1944 \mathrm{~K}$. The full data set may be correlated within \pm 3.3 percent by a Lennard-Jones potential with parameters $\sigma_{1}=2.928 \mathrm{~A}$ and $(\varepsilon / \mathrm{k})_{1}=600 \mathrm{~K}$. This is the same correlation as that obtained by Pakurar and Ferron [11] using the combinations of two sets of data at 194-363 K and 1103-1944 K.

The binary diffusivities for $\mathrm{CO}_{2}$-argon have been measured at $435-1482 \mathrm{~K}$. These new data, in combination with prior results $[13,16]$, provide seventy-two values at temperature intervals of less than $60 \mathrm{~K}$ for the range 276-1800 K. The Lennard-Jones correlation, within \pm 3.1 percent, has the parameters $\sigma_{12}=3.208 \AA$ and $(\varepsilon / \mathrm{k})_{12}=277.5 \mathrm{~K}$. This is again identical to an earlier correlation [13] based on five points at $276-317 \mathrm{~K}$ and thirty-five points at $1132-1800 \mathrm{~K}$.

\subsection{Mathematical description of the experiment}

Figure 1 illustrates some principal features of the experimental apparatus. Carrier gas is introduced at the bottom and is preheated either by combustion on the porous burner or by electrical heating in the region above the burner. Tracer gas is introduced through a fine capillary tube and is preheated in the vertical section of the capillary just before it exits into the carrier gas. The region above and just below the point of tracer flow into the carrier gas is maintained at a uniform temperature.

Diffusion in the isothermal carrier gas may be represented by

$$
U \frac{\partial C}{\partial z}=\frac{D^{*}}{r} \frac{\partial}{\partial r} \quad r \frac{\partial C}{\partial r}+D^{*} \frac{\partial^{2} C}{\partial z^{2}}
$$

where $C(r, z)$ is local, steady-state concentration of tracer at the point $(r, z)$ of the cylindrical coordinate system sketched in figure 2. The local gas velocity is $U \mathrm{~cm} / \mathrm{s}$, and $D{ }^{*} \mathrm{~cm}^{2} / \mathrm{s}$ represents the tracer diffusion coefficient. It is assumed to be a constant, as is $U$. 

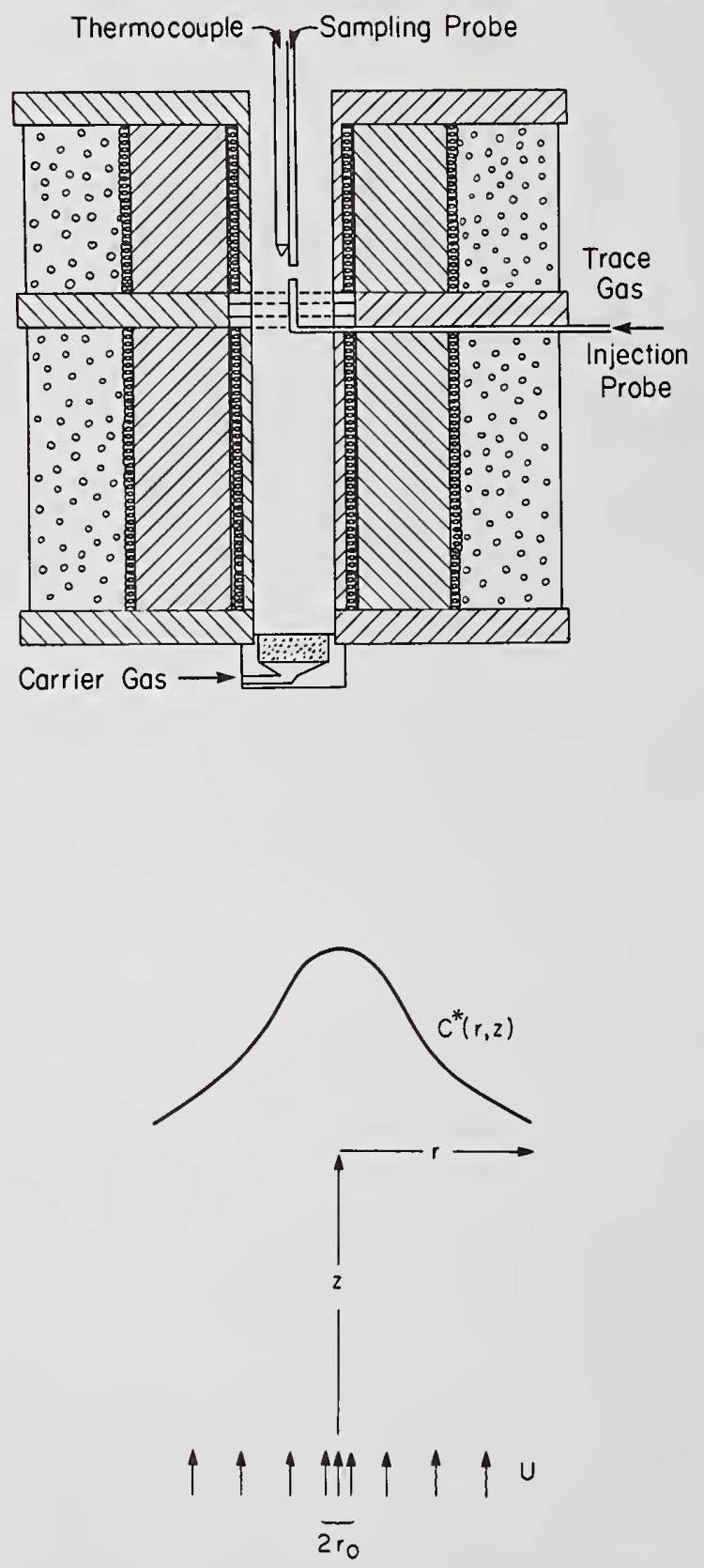

Figure 1. Apparatus for simulated point-source measurements of diffusion coefficients. Cross-hatched regions are cast alumina. Outer insulation is alumina powder.
Figure 2. Coordinate system of the simulated point source.

Boundary conditions applied to eq. (1) include stipulations that concentration, as well as the axial and radial gradients of concentration, become zero at infinite values of $z$, both positive and negative, and of $r$. The radial gradient of concentration along the axis, that is, at $r=0$, is also zero so that radial symmetry of the concentration field is preserved. 
One more condition, that describing the mechanism, concentration and amount of tracer injection, is sufficient to provide a solution for $c(r, z)$ from eq. (1).

The boundary condition of the true point source may be written as:

$$
\lim _{s \rightarrow 0}\left(-4 \pi s^{2} \frac{\partial C}{\partial s}\right)=q C_{0} / D^{*}
$$

where $s=\left(r^{2}+z^{2}\right)^{\frac{1}{2}}$ is distance from the point of injection, $q \mathrm{~cm}^{3} / \mathrm{s}$ is the volumetric flowrate at which the tracer stream is injected, and $C_{0}$ is concentration of tracer in the injected stream. With this condition, the solution for concentration becomes:

$$
C(r, z)=\left(C_{0} q / 4 \pi 0^{*}\right)\left(r^{2}+z^{2}\right)^{-\frac{1}{2}} \exp \left\{-\left(U / 2 D^{*}\right)\left[\left(r^{2}+z^{2}\right)^{\frac{1}{2}}-z\right]\right\}
$$

This result is particularly convenient for analysis of experimental data; it has been applied in all previous studies, and we use it here also.

Convenience arises in that if sampling for $c(r, z)$ is confined to the flow axis, i.e., to the line $r=0$, eq. (3) resolves to:

$$
C(0, z)=C_{0} q / 4 \pi D^{*} z
$$

Along the flow axis, then, concentration of tracer changes linearly with respect to the reciprocal of axial position, and the slope of this linear form is inversely proportional to $D^{*}$.

The true point source cannot be realized in practice. Equation 2 requires that the trace gas ooze in $4 \pi$-geometry from a source of zero size strictly by diffusion, with no convective assistance. A more realistic boundary condition may be written as:

$$
\begin{aligned}
\pi r_{0}^{2} & {\left[U C^{+}(r, 0)-D^{*} \frac{\partial C^{+}(r, 0)}{\partial z}\right] } \\
& =\pi r_{0}^{2}\left[U C^{-}(r, 0)-D^{*} \frac{\partial C^{-}(r, 0)}{\partial z}\right]+q C_{0} H\left(r_{0}-r\right) .
\end{aligned}
$$

Here $r_{0}$ is the finite radius of the tracer injection tube, $\mathrm{C}^{+}$stands for concentration in the region above the point of injection, and $\mathrm{C}^{-}$is concentration below the injection point. The quantity $\mathrm{H}\left(r_{0}-r\right)$ is the Heaviside unit step function; it has the value unity for $r \leq r_{0}$ and the value zero for $r>r_{0}$.

Equation 5 states that combined convective and diffusive flow of tracer is the same just above and just below the level of injection for radial positions beyond $r_{0}$. Within the region of injection, $r \leq r_{0}$, tracer flow just above $z=0$ is the sum of that just below $z=0$ and that which comes from the injection tube. 
Using eq. (5) instead of eq. (2) as a boundary condition for eq. (1), we obtain:

$$
C^{+}(r, z)=\frac{q C_{0}}{\pi r_{0}{ }^{2} U} \int_{0}^{\infty} \frac{J_{1}(x) J_{0}\left(x r / r_{0}\right)}{\left(1+r x^{2} / P^{2}\right)^{\frac{1}{2}}} \exp \left\{-\left(P z / 2 r_{0}\right)\left[\left(1+4 x^{2} / P^{2}\right)^{\frac{1}{2}}-1\right]\right\} d x
$$

where $J_{1}(x)$ and $J_{0}\left(x r / r_{0}\right)$ are Bessel functions of the first kind and $P=r_{0} U / D$ * is the Peclet number. Equation (6) is the concentration for non negative values of $z$; that for $c^{-}(r, z)$ is identical except that the final sign within the exponential term is positive rather than negative.

Concentration along the positive axis is:

$$
C^{+}(0, z)=\left(q C_{0} / \pi r_{0}^{2} U\right)\left\{1-\exp \left[-\left(U / 2 D^{*}\right) W(z)\right]\right\}
$$

where $w(z)=\left(z^{2}+r_{0}^{2}\right)^{\frac{1}{2}}-z$. As with eq. (4), this has a linear form which is convenient for analysis of experimental data. For this purpose, one $\operatorname{plots} 1-\left(\pi r_{0}{ }^{2} U / q C_{0}\right) C^{+}(0, z)$ as a function of $\ln W(z)$, obtaining $D^{*}$ from the intercept on semi-logarithmic graph paper.

Such analysis introduces more error than does use of eq. (4), because uncertainty in the value of gas velocity, $U$, enters twice in the use of eq. (7). Fortunately, one finds that if all levels of sampling are high enough above the point of injection so that $z$ is at least $10 r_{0}$, then eq. (7) is indistinguishable from eq. (4). Indeed, it is readily shown that the limit of eq. (7) for large values of $z$ is exactly eq. (4). Such sampling conditions are easily employed, and useful for other reasons, so that eq. (4) is the preferred basis for analysis of experimental measurements.

All of the above applies to conditions which are steady in time. A truly steady-state experiment is not practicable, however, because among other reasons, it would be so wasteful of materials, particularly of radioactive tracer when this is used. During the approach to steady state, regions below the point of injection and otherwise outside those of useful sampling accumulate small concentrations of tracer by diffusion. Solid surfaces of the apparatus would eventually adsorb tracer and become contaminated. It is necessary, therefore, to conclude an experiment well before a true steady state obtains. We are, thus obliged to look into the transient mathematical description.

To do this, we add to the left side of eq. (1) the term $\partial c / \partial t$, where $t(\mathrm{sec})$ is the elapsed time after initial admission of tracer to the isothermal system. We also add the initial condition, specifying that concentration be everywhere zero at the start. The solution is readily found. Here, we need to consider only the form along the positive axis. Provided the quantity $U t / z$ is considerably larger than unity, a normal state because $t=z / U$ is the very short time required for an element of gas to rise from the injection level to the point of sampling, the time-dependent version of eq. (7) differs only in that the right-hand side is multiplied by

$\left(\frac{1}{2}\right)\left[1+\operatorname{erf}\left(U t^{\frac{1}{2}} / 2 D^{\frac{k_{1}}{2}}\right)\right]$ 
where erf $x=\left(2 / \pi^{\frac{1}{2}}\right) \int_{0}^{x} e^{-a^{2}} d a$ is the error function. At very high temperatures, for which $D^{*}$ is as much as $4 \mathrm{~cm}^{2} / \mathrm{s}$ and taking $U=10 \mathrm{~cm} / \mathrm{s}$, a commonly used velocity, we need wait only a few seconds before the transient multiplier has become nearly unity. This brief wait thus produces essentially steady conditions, though concentrations far from the sampling region are still infinitesimal. Equation (4) remains an excellent model of useful experimental conditions.

Additional idealizations have been included in the mathematical description, of course. For example, we have taken $D^{*}$ to be constant, or essentially so, consistent with predictions of monatomic theory. It is possible to include a postulated form of the concentration dependence for polyatomic diffusion in the mathematical description. Because precision of concentration measurement probably limits overall precision of the method at this stage, possible concentration dependence is better sought through experiments at different carriergas concentrations, each such experiment being analyzed for $D^{\star}$ constant. Dependence of $D^{*}$ on tracer concentration will then appear when the temperature dependence of each data set is obtained by appropriate smoothing, usually in terms of monatomic theory and a Lennard-Jones or other two-parameter potential. Such treatment leads to the conclusion that dependence of $D^{*}$ on concentration is just outside the region of feasible experimental precision [13].

We have also assumed that flow is laminar, one-dimensional, isothermal and not influenced by effects of natural convection. Laminar conditions obtain at all points if the Reynolds numbers based on injection-tube and sampling-tube diameters are well below unity and if that based on the inside diameter of the apparatus is well below, say, 2000. Such conditions are readily attained with the capillaries used here, each of $0.5 \mathrm{~mm}$ inside diameter, and the inside diameter of the apparatus of 2 inches, provided gas velocity is no more than about $10 \mathrm{~cm} / \mathrm{s}$. Reynolds numbers for the capillaries and for the apparatus, then, are respectively $2 r_{0} \mathrm{U} / \nu$, about 0.5 on the average, and $5 \mathrm{U} / \nu$, about 50-100 normally, where $v$ is the kinematic viscosity of the carrier gas.

One-dimensional flow cannot be provided because of the interference of the finite-sized injection and sampling tubes and the nearby thermocouples. Such interference can be minimized by tapering the capillaries so that there is gradual radial adjustment of the flow near their tips and by maintaining carefully uniform temperatures so that thermocouples need not be so close that they influence the flow field significantly.

Flow and concentration fields are also affected by the velocity, $q / \pi r_{0}{ }^{2}$, at which tracer is injected. The ideal velocity with respect to the flow field is slightly greater than $U$, so that tracer flow helps to fill the gap caused by the finite thickness of the capillary tube wall and limits radial acceleration of the nearby gas flow. Even with ideal conditions, of course, there will be a slight positive error in the measured diffusion coefficient brought about by convection at the tips of injection and sampling tubes.

Equation (4) suggests that we need not be concerned about a difference between $U$ and the tracer velocity; it does not enter after we set $r=0$ in eq. (3). This is deceptive. Even though we use eq. (7) in the limit of large $z$ to obtain $D^{*}$, we recognize that eq. (7) better represents the concentration profile along the axis for small $z$ than does eq. (4). The former equation includes the ratio $q / \pi r_{0}{ }^{2} U$ explicitly and as a simple multiplier. 
Hence, the concentration at some value of $z$ will be greater than if there is a precise match between tracer and carrier velocities; the result will be a slight negative error in the measured diffusivity.

It is fortunate that ideal flow conditions, with $q / \pi r_{0}{ }^{2} U$ slightly greater than unity, produce an error which is essentially cancelled by the opposite error in the concentration field. In practice $q / \pi r_{0}{ }^{2}$ is kept within 10 percent of $U$, any error being arranged to be positive. There is no measurable effect on diffusivity in these circumstances.

Isothermal conditions may be checked by probing with several thermocouples. The apparatus does lose heat, and wall temperature must be higher than that at the point of sampling. As a result, one must account for radiation to the thermocouples, but the region of sampling is isothermal to within at least 1 percent of the absolute temperature.

Natural convection can arise for several reasons. Conduction along the injection-tube wal1, for example, may cool the wall and induce convection. This is insignificant if the injection tube is supported horizontally by the hot insulation of the apparatus and extends vertically for a distance equivalent to about 25 tube diameters. Measurements of temperatures above the tracer injection tube fail to disclose presence of the tracer gas under such circumstances, so that preheating is efficient and conduction along the injection tube is not measurable.

The relatively hot wall of the apparatus may also induce natural convection. Provided that the region of sampling involves temperature differences of the order of 1 percent of the absolute temperature or less, this region feels wall convection only through a slightly (5-10 percent at most) increased velocity.

The flow field will also be influenced by density gradients, the effect being proportional to the product of $C_{0}$ and the negative derivative of density with respect to molar concentration of tracer. This product resolves approximately to $\left(C_{0} / \rho\right)\left(M_{\text {carrier }} / M_{\text {tracer }}-1\right)$, where $\rho$ is gas molar density, $C_{0}$ molar concentration of the tracer feed, and $M$ stands for molecular weight. In its effect on flow conditions, this term is analogous to the ratio of maximum temperature difference to absolute temperature used in the paragraph before this one. If both such terms are to be of the order of 1 percent or so, $c_{0} / \rho$, the maximum mole fraction of tracer, must be small when the molecular weight difference is significant. The criterion is readily met in the present experiments as the result of the small molecular weight differences involved and because use of radioactive $\mathrm{CO}_{2}$ permits its inlet concentration to be very low.

\subsection{Apparatus and procedures}

With the apparatus of figure 1, a carrier gas mixture was fed to the bottom of the 2inch-diameter, heated, alumina tube at the core of the furnace. For operation at room temperature to about $1400 \mathrm{~K}$, with electrical preheating and no combustion, the gas consisted of pure, ordinary carbon dioxide for self-diffusion studies and of various mixtures of argon and $\mathrm{CO}_{2}$ (either 50 percent or 33 percent $\mathrm{CO}_{2}$ in the majority of experiments of this work) for binary diffusion studies. Flowrates were metered by calibrated orifices and regulated 
by micrometer valves. Recalibration of the orifices was performed frequently by the wettest-meter method. All gases were supplied by the Matheson Company and were of purities exceeding 99.5 percent.

Preheating occurred in the first ten inches of the core. The inner, hotter electrical circuits had platinum heating wire. The outer circuits were of Kanthal. Electrical control was manual.

After the preheating section flow was smoothed by four, 80-mesh, platinum-rhodium $(80,20)$ screens. The tracer injection tube, $0.5-\mathrm{mm}$ inside diameter, also of platinumrhodium and carefully sharpened at the tip to reduce radial acceleration of the flow, had its tip in the flow axis about 0.5 inch above the top screen. Volumetric flowrate of the tracer varied from 0.6 to $1.2 \mathrm{ml} / \mathrm{min}$, corresponding to 1 inear velocities of $5-10 \mathrm{~cm} / \mathrm{s}$ at injection. The injection velocity matched that of the carrier within 10 percent.

Tracer flowrate was metered and pumped by a water-driven piston mounted with 0-ring seals between two precision-bore glass tubes of different diameters, the larger on the water side. By this means, a substantial water flow, readily controlled and metered by needle valve and rotameter, provided the required steady but tiny flow of tracer gas. The apparatus has been described more fully [13].

The sampling probe, also a $0.5-\mathrm{mm}$ ID platinum-rhodium capillary tube, was mounted on a ceramic frame to provide mechanical strength. It was positioned to a precision of 0.001 inch with a three-dimensional slide rest. Sample probe and injection tube were alined in the axis visually, and the alinement was checked by the linearity of the plot of $c(0, z)$ in terms of $1 / z$. Re-alinement was necessary after each $50 \mathrm{~K}$ change in operating temperature. At the start of an experiment, the sampling probe was mounted 0.6 inch above the injection tube. Data were taken at intervals of 0.1 inch, normally to a final height of 1.1 inch above injection.

Sampling was continuous, the flow being drawn at a suction equivalent to about one inch of water through a cooler, then to a detector cell, through a water bubbler and finally to dilution in the exhaust system. The flowrate was regulated by a calibrated orifice valve, the bubbler serving as a rough visual measure. The most convenient sampling rate was about twice that of injection, though this could be increased to four times tracer injection rate without measurable effects.

The flow-through detector consisted of a thin (about $3 \mathrm{~mm}$ ) sheet of acrylic plastic sandwiched between two proportional counting cells. The plastic was cut to provide an irregular flow path with a total volume of about $2 \mathrm{ml}$. Capillary inlet and outlet tubes were cemented in the plastic. The gas sample was separated from the counting gas by aluminized mylar windows. The differential pressure between sampling gas and counting gas was carefully controlled to prevent movement of the mylar windows with resulting change of the sample volume. The proportional counters were operated at 2300 volts. Pulses were shaped and amplified by a proportional preamplifier and sent to a scaler-timer. Background was controlled to about 30 counts per minute by 2 -inch lead shielding. Scaling was in all cases sufficiently long to keep the statistical counting error below 2 percent at 95 percent confidence. The tracer gas had a nominal activity of 0.6 microcuries per milliliter. The 
measured activity, corresponding to current detector operating conditions, was obtained for each experiment.

Operation with combustion employed the porous bronze burner, sketched in figure 3 , which had been designed by Pakurar [12].

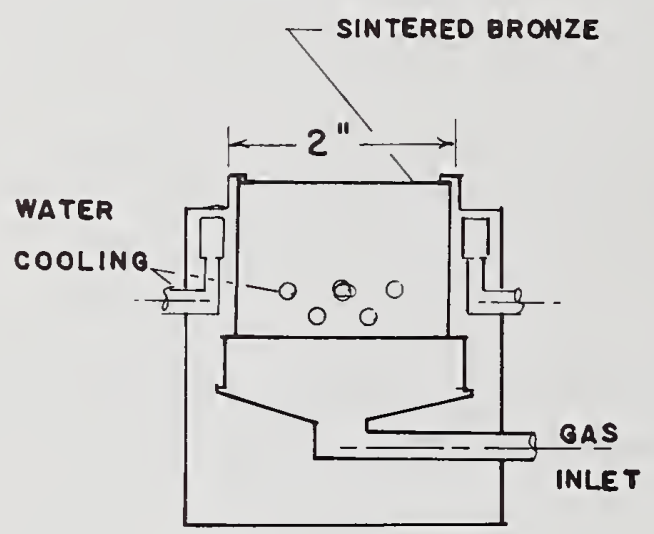

Figure 3. Porous bronze burner for combustion-heated conditions (from Pakurar [17]).

For study of self-diffusion in $\mathrm{CO}_{2}$ the inlet gas consisted of a mixture of carbon monoxide, oxygen, and $\mathrm{CO}_{2}$. A very slight deficiency of oxygen was used, and the oxygen stream was partially saturated with water before metering. These conditions produced a stable, flat flame, a few millimeters high, on the porous burner. Temperature was regulated by the amount of carbon dioxide added as diluent and by gas flowrate through the watercooled burner, the latter being the more sensitive control. Stable operation has been achieved at 1100-1944 K, principally by Pakurar [17]. The burner deteriorated rather quickly, with nonuniform velocity profiles the principal result, under conditions which yielded excessive heat transfer backward to the burner or oxidation of the metal when the gas was rich in oxygen.

Binary diffusion studies involved similar conditions, the diluent gas consisting of carbon dioxide and the second component. The tracer was always ${ }^{14} \mathrm{CO}_{2}$ for the studies emphasized here.

In all cases, exhaust gases from the apparatus and detector system were cooled by a hea exchanger, then drawn into an exhaust system, diluted carefully to safe radiation levels and discarded to the atmosphere.

The apparatus operated at slight vacuum, which, along with the barometric pressure, was recorded so that reported diffusivities could be corrected to exactly one atmosphere by use of monatomic theory.

Correction of results was also made by monatomic theory for the slight excess mass of ${ }^{14} \mathrm{CO}_{2}$, and results are reported for ${ }^{12} \mathrm{CO}_{2}$.

For safety in the use of $\mathrm{CO}$ and of radioactive $\mathrm{CO}_{2}$, the exhaust system was monitored carefully and the operators used face masks and an independent air supply. 


\subsection{Temperature measurement}

Two Pt-Rh thermocouples mounted on the sampling probe were used to measure the temperature of the carrier gas. Two thermocouples were used in order to account for the radiation transfer from the hot tube wall at $T_{W}$ to a thermocouple bead. Given two beads of different diameters, $d_{1}$ and $d_{2}$, one obtains a relation for the gas temperature, $T_{g}$, by combining the individual heat balances for beads at $T_{1}$ and $T_{2}$. Unknown emissivities and heat transfer coefficients are eliminated, producing the following relationship:

$$
T_{g}=\frac{T_{1}-\left(d_{1} / d_{2}\right) T_{2}\left(T_{1}{ }^{4}-T_{w}^{4}\right) /\left(T_{2}{ }^{4}-T_{w}{ }^{4}\right)}{1-\left(d_{1} / d_{2}\right)\left(T_{1}{ }^{4}-T_{w}{ }^{4}\right) /\left(T_{2}{ }^{4}-T_{w}{ }^{4}\right)} .
$$

Here $\mathrm{T}_{\mathrm{g}}$ is the temperature reported for a particular experiment.

Aside from the need to know the experimental temperature in order to report the temperature dependence of the measured diffusion coefficients, we also require the temperature in order to calculate the diffusion coefficient for each experiment. The axial concentration profile given by eq. (4) is written for temperature $T_{g}$, while measurements of concentrations are performed at room temperature. The measured concentration of tracer is greater by a factor $T_{g} / T_{\text {room }}$ than that within the apparatus. The working equation for calculation of the diffusion coefficient becomes:

$$
D^{*}=A T_{g}
$$

where:

$$
A=\left(q C_{0} / 4 \pi T_{\text {room }}\right) d[1 / C(0, z)] / d z
$$

The product $\mathrm{q} \mathrm{C}_{0}$ is measured at room conditions and is independent of temperature.

Uncertainty in the measured value of $\mathrm{T}_{\mathrm{g}}$, obtained from eq. (8), thus translates to an uncertainty in $D^{*}$. Fortunately, the strong dependence of $D^{*}$ on temperature decreases the effect of such uncertainty. Figure 4 illustrates this for a particular experiment involving determination of a self-diffusion coefficient. Figure $4 \mathrm{a}$ is the plot of measured activities as a function of $z$. From the slope and the product $q C_{0}$ we obtain $A=0.00105$. The dashed line of figure $4 b$ is the plot $D^{*}=A T_{g}$ of eq. (9), representing the locus of possible values of $D_{11}$ for this experiment. Equation (8) yields $T_{g}=832 \mathrm{~K}$, and the asterisk of figure $4 \mathrm{~b}$ shows the diffusivity of $0.874 \mathrm{~cm}^{2} / \mathrm{s}$ reported for the experiment. The solid line of figure $4 \mathrm{~b}$ represents the prior correlation of self-diffusion data [11]. We observe that there is a range of about $80 \mathrm{~K}$ for which eq. (9) represents $\mathrm{D}_{11}$ within \pm 5 percent of the value which would agree exactly with the correlation. 


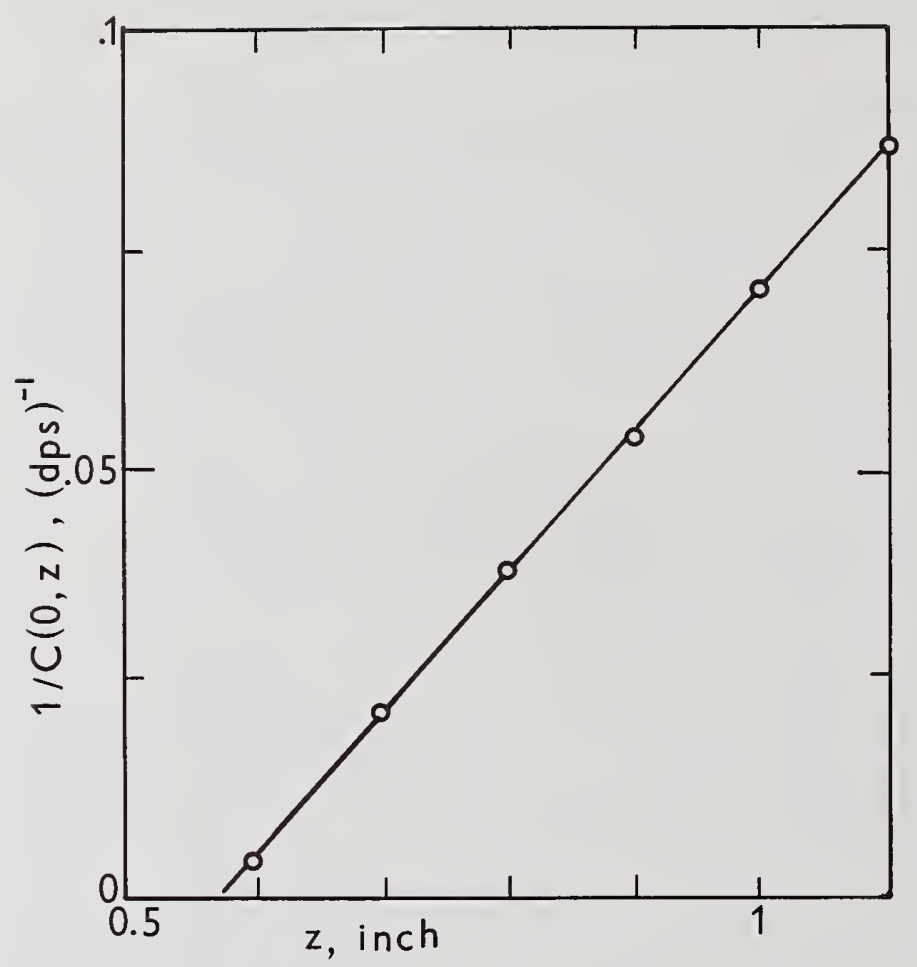

Figure 4a. Measured axial concentration (in disintegrations per second) as a function of position. Conditions are: $q=0.831$ $\mathrm{ml} / \mathrm{min}, \mathrm{C}_{0}=4280 \mathrm{dps}, \mathrm{T}_{1}=847 \mathrm{~K}, \mathrm{~T}_{2}=853 \mathrm{~K}, \mathrm{~T}_{\mathrm{W}}=894, \mathrm{~d}_{1} / \mathrm{d}_{2}=$ 0.64 .

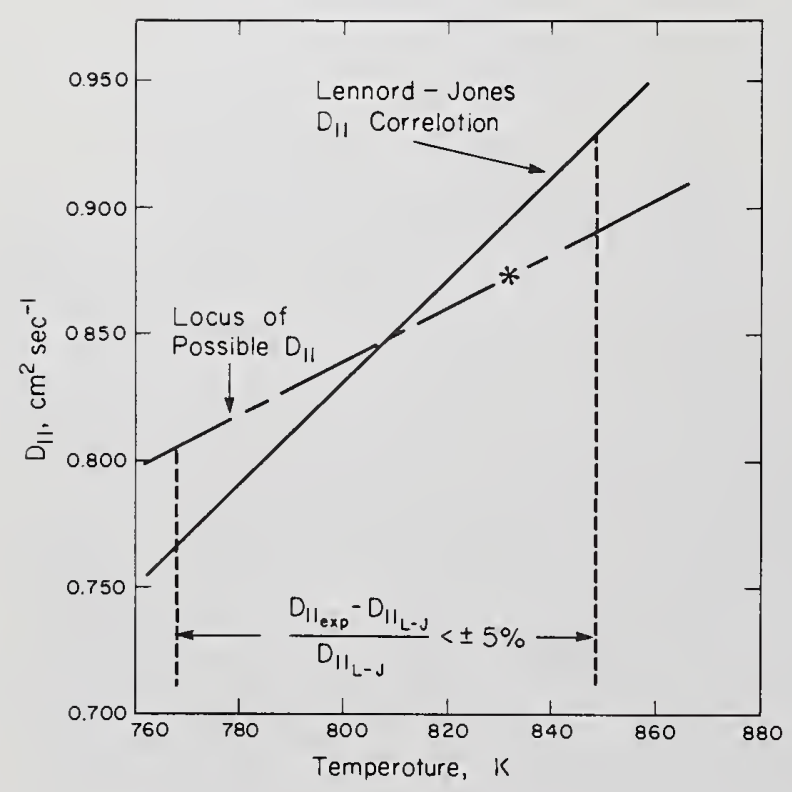

Figure $4 b$. Effects of temperature correction. 


\subsection{Use of the tracer diffusivity}

When $D^{*}$ for a self-diffusion experiment has been multiplied by $(46 / 44)^{1 / 2}$, the result is $\mathrm{D}_{11}$, the self-diffusion coefficient of ${ }^{12} \mathrm{CO}_{2}$. For the case of binary diffusion, $D^{*}$ is the result of like and unlike interactions between the tracer and the major gases of the mixture. The combined effect, for vanishingly small tracer concentration, is dependent on concentration of the binary constituents and may be represented by:

$$
1 / D_{1}^{*}=x_{1} / D_{11}+x_{2} / D_{12}
$$

sometimes called the first approximation of Blanc's law [18], where $x_{1}$ and $x_{2}$ are mole fractions of the major binary species.

When we measure $D_{1}^{*}$ and mole fraction, we may obtain $D_{12}$ from the result if the selfdiffusion coefficient, $D_{11}$, is available. Alternatively, we may measure $D_{1}^{*}$ in pure species 2 in order to obtain $\mathrm{D}_{12}$ directly.

For the present experiments with $\mathrm{CO}_{2}$-argon, we have chosen to use non-zero values of $x_{1}$ in order to investigate for measurable concentration dependence of $D_{12}$. Such dependence is very smal1, however. Sandler and Mason [18] consider the second-order version of Blanc's law and note that neglect of second-order effects results in errors of the order of 0.1 percent in calculation of $\mathrm{D}_{12}$. And, indeed, the present binary experiments can be correlated as a single set; concentration effects (at least for mixtures which are a third or half $\mathrm{CO}_{2}$ ) are not detectable within the precision of the data.

\section{Model Calculations}

It is convenient to use monatomic theory and a suitable two-parameter potential such as the Lennard-Jones 12-6 form as a means for smoothing experimental data. For nonpolar systems, there seems to be no difficulty in reproducing data within their precision by this means. The diffusivity-temperature relationship and that for at least the first derivative of diffusivity with respect to temperature are thus available in dependable form for interpolation and other purposes of numerical calculation. There is no physical information to be inferred from the success of smoothing by monatomic theory, however. It is, therefore, desirable to seek polyatomic models which can form the basis for both correlation and prediction of diffusion coefficients and other properties.

Suitable models for nonpolar gases should take into account at least three aspects of polyatomic behavior: the details of binary collision between nonspherical particles, the "softness" of collisions caused by finite molecular fields of force and the ability of polyatomic species to carry vibrational energy which is not freely exchangeable with the translational mode. Progress has been made with respect to the first two aspects, and we make use of this in the present work.

Separating the first two aspects, hard and soft nonspherical collision dynamics, from other influences is based on the argument that vibrational and electronic modes of energy 
storage are not often disturbed during molecular collisions. Thus, only translational and rotational modes need to be considered for many cases of interest.

Model calculations of this kind treat translational and rotational motion classically. One assumes that molecular shape, size, and mass distribution are retained during colitisional encounters. This suggests a rigid molecule, and the level of information gained is something like that obtained with rigid-sphere estimates of monatomic behavior. Corrections for the "softness" of elastic collisions must then be added in order that a suitable predictive theory be constructed. Secondary effects of vibration are added implicitly by the appearance of heat capacity in defining expressions for the transport coefficients.

The Chapman-Enskog procedure for solving the required form of Boltzmann's equation has most often been employed. The singlet distribution function for species $i, f_{j}^{l}$, is expanded in terms of a perturbation form, $f_{j}^{0} \phi_{j}$, added to the equilibrium distribution, $f_{j}^{0}$, resulting in a linear integral equation for $\phi_{j}$. This depends on space and time variables implicitly through dependence of number density, $n$, macroscopic velocity, $\underline{u}$, and temperature, $T$, on space and time. Solution of the linear integral equation can be sought in terms cf the form

$$
\phi_{i}=-\underline{A}_{i} \cdot \nabla \ln T-n \sum_{j} \underline{c}_{i}^{j} \cdot \underline{d}_{j}-\underline{B}_{i}: \nabla \underline{u}-D_{i} \nabla \cdot \underline{u}
$$

where $\underline{d}_{j}$ is the diffusional flux of species $j$ and where the proportionality factors $\underline{A}_{j}, \underline{\underline{B}}_{j}$, $\underline{c}_{j}^{j}$, and $D_{j}$ are functions only of the dimensionless molecular angular and linear velocities. This allows one to separate the Boltzmann equation into four different linear integral equations, one for each of the unknown proportionality factors. The linearized equations are then solved by series expansions in terms of orthogonal functions. It is convenient to use Sonine polynomials. Unknown coefficients in the expansions are determined by taking moments of the integral equations with each of the terms used in the expansion. The result is an infinite set of linear algebraic equations for the coefficients. Because convergence is rapid, the infinite set can be truncated after two or three terms depending on the level of approximation required. After the solution is obtained, it is a straightforward matter to evaluate transport properties by numerical integration $[19,20]$.

\subsection{Rigid models}

Ishida [21] made an early study of the kinetic theory of rigid nonspherical molecules, constructing the generalized Boltzmann equation and the equations of change (which are the same as those for a monatomic gas of rigid spheres except that an additional equation is obtained for conservation of angular momentum). The model was not developed to the extent of yielding the numerical values of transport properties, but the concept of the exchange rate of angular momentum was introduced and an additional transport property (bulk viscosity) related to rotational relaxation time appeared.

Transport properties have been fully developed for the rough-sphere model, introduced as early as 1894 by Bryan. Molecules are assumed to grip each other without slipping. Each 
molecule in a binary collision strains the other, and strain energy is converted into translation and rotation with the result that the relative velocity of approach is reversed on collision. The mass distribution of rough spheres is described in terms of a dimensionless moment of inertia, $K=I / \mathrm{mo}^{2}$, which varies from zero when mass is concentrated at the center, to $2 / 3$ when mass is uniformly distributed over the surface. The model allows interchange of angular and translational energies, and calculations are simplified because no angular coordinates are necessary to describe molecular orientation.

The method of Chapman and Enskog was first applied to the rough-sphere model by Pidduck [22]. He assumed the instantaneous singlet distribution function for a pure gas to depend on position, $\underline{r}$, linear velocity, $\underline{v}$, and angular velocity, $\underline{\Omega}$, that is $f^{1}=f^{l}(\underline{r}, \underline{v} \underline{\Omega}, \underline{t})$. For determination of unknown coefficients in a doubly-infinite Sonine polynomial expansion, he supposed $\underline{A}$ of eq. (11) to be proportional to $\underline{W}$, the reduced peculiar velocity, with the proportionality terms as well as the coefficient $D$ scalar functions of $\underline{W} \cdot \underline{W}$ and of the scalar square of angular velocity, $\underline{\Omega} \cdot \underline{\Omega}$. The tensor $B$ was taken as proportional to $\underline{W^{0}} \underline{W}$, the dyadic square of peculiar velocity. Calculation of transport properties by Pidduck's approximation shows that for $K=2 / 3$, viscosity is 10 percent higher and the diffusion coefficient 30 percent lower than for rigid spheres. Thermal conductivity differs by about 10 percent from the value predicted by the modified Eucken correction [23].

The rough-sphere model is not very realistic. Its special disadvantage is that deflection does not vary continuously with the impact parameter representing closest distance of approach in the absence of collision. Hence, grazing collisions produce the same deflection as head-on collisions, contrary to what is expected on physical grounds.

Another disadvantage of the rough-sphere model is the absence of true inverse encounters. Because of this, the Boltzmann equation has an unsymmetric kernel, and the successively higher approximations do not converge monotonically to the true value. Condif, Lu, and Dahler [24] studied the convergence problem using

$$
\underline{A}=A_{1} \underline{W}+A_{2} \underline{W} \cdot \underline{\Omega \Omega}+A_{3} \underline{W} \times \underline{\Omega}
$$

and retaining five polynomial terms in the expansion of $A_{1}$, three in that of $A_{2}$ and one term for $A_{3}$. Pidduck's approximation corresponds to $A_{2}=A_{3}=0$. The Kagan-Afanas'ev approximation [25], in which $A_{3}=0$ and a single expansion term of the form $A_{2} \underline{W} \cdot \underline{\Omega} \Omega$ is used, gives a spin-anisotropic effect was found to change thermal conductivity by as much as 10 percent. The effect of additional terms is an oscillating value of thermal conductivity with amplitude of about 2 percent. Similar results are found for viscosity and bulk viscosity. The rough sphere model seems to over-emphasize the importance of non-central forces because grazing collisions as well as more direct interactions result in inelastic scattering. The effect of spin anisotropy in rough spheres is thus greater than that to be expected with more realistic models such as the loaded sphere or the spherocylinder, a conjecture which has been confirmed in later investigations.

The loaded-sphere model was introduced by Jeans in 1904 [26]. The loaded sphere is rigid and smooth with mass $m$ and radius $\sigma$. Its center of gravity is shifted from the center 
of the sphere by a small distance $\xi$, along which mass is distributed uniformly, giving a moment of inertia, I, about the axis perpendicular to that of symmetry. Loaded spheres wobble about the center of mass and exchange translational and rotational energy on collision. Rotation about the axis of symmetry is unaffected by collisions (as with the rigid sphere but unlike the rough sphere).

Dahler and Sather [27] analyzed loaded spheres but neglected the effect of spin anisotropy on transport properties. Sandler [23] later studied the effects of orientation anisotropy and of spin anisotropy. He found that the spin-anisotropic term of the KaganAfanas'ev form, when added to Pidduck's approximation, increases transport properties by a maximum of 5 percent, while orientation anisotropy has an insignificant effect. This result supports the suggestion that the rough-sphere model provides an upper limit for contribution of spin anisotropy.

The spherocylinder, a cylinder of length, $L$, and radius, $\sigma$, capped at either end by a hemisphere of radius, $\sigma$, was first used by Kihara [28]. He included orientation-dependent intermolecular forces represented by the Lennard-Jones potential. He also carried out the analysis for ellipsoids and calculated the best-fit parameters for second virial coefficients using both models.

A formal kinetic theory for rigid, convex, nonspherical molecules was developed and applied by Curtiss and coworkers $[29,30]$ to calculation of transport properties of the spherocylinder. The initial work did not require that rotational velocity about the symmetry axis be unchanged in a collisional encounter or that spin anisotropy be considered. In the last paper of the series [30], the Boltzmann equation was derived rigorously from Liouville's equation, and it was found that the Boltzmann equation for rigid, symmetric-top, non-spherical molecules in which the center of mass coincides with the center of symmetry is the same as the Boltzmann equation for loaded spheres. This result is useful because it allows simultaneous treatment of both loaded-sphere and spherocylinder models. Many of the results developed for one model can be directly applied to the other.

Certain transport property calculations were performed by Sandler and Dahler [31] for pure gases composed of loaded spheres and of spherocylinders and for binary mixtures of loaded spheres and rigid spheres of equal mass and radius. Viscosity and thermal conductivity were found to depend very little on spin anisotropy. Sandler and Dahler also argued that the parameter $L / 2 \sigma$ of the spherocylinder should affect transport properties mainly by enlarging the mean projected area for collision over that for spherical particles. This area is proportional to $(1+\mathrm{L} / 2 \sigma)$, and Sandler and Dahler found calculated transport properties to vary inversely with this quantity as they expected.

Chatwani [32] extended Sandler and Dahler's analysis to the general case of binary mixtures composed of spherocylinders of unequal size and mass. Spin-anisotropic terms were retained in the Sonine polynomial expansions and the effects studied for various transport properties.

Figure 5 illustrates the calculated self-diffusion coefficient for an arbitrary, rigid spherocylinder. Sandler and Dahler's observation [31] that transport properties change with the length-to-width ratio, $\beta=L / 2 \sigma$, approximately as $1 /(1+\beta)$ is reasonably well confirmed, though there is some residual dependence on $\beta$. 


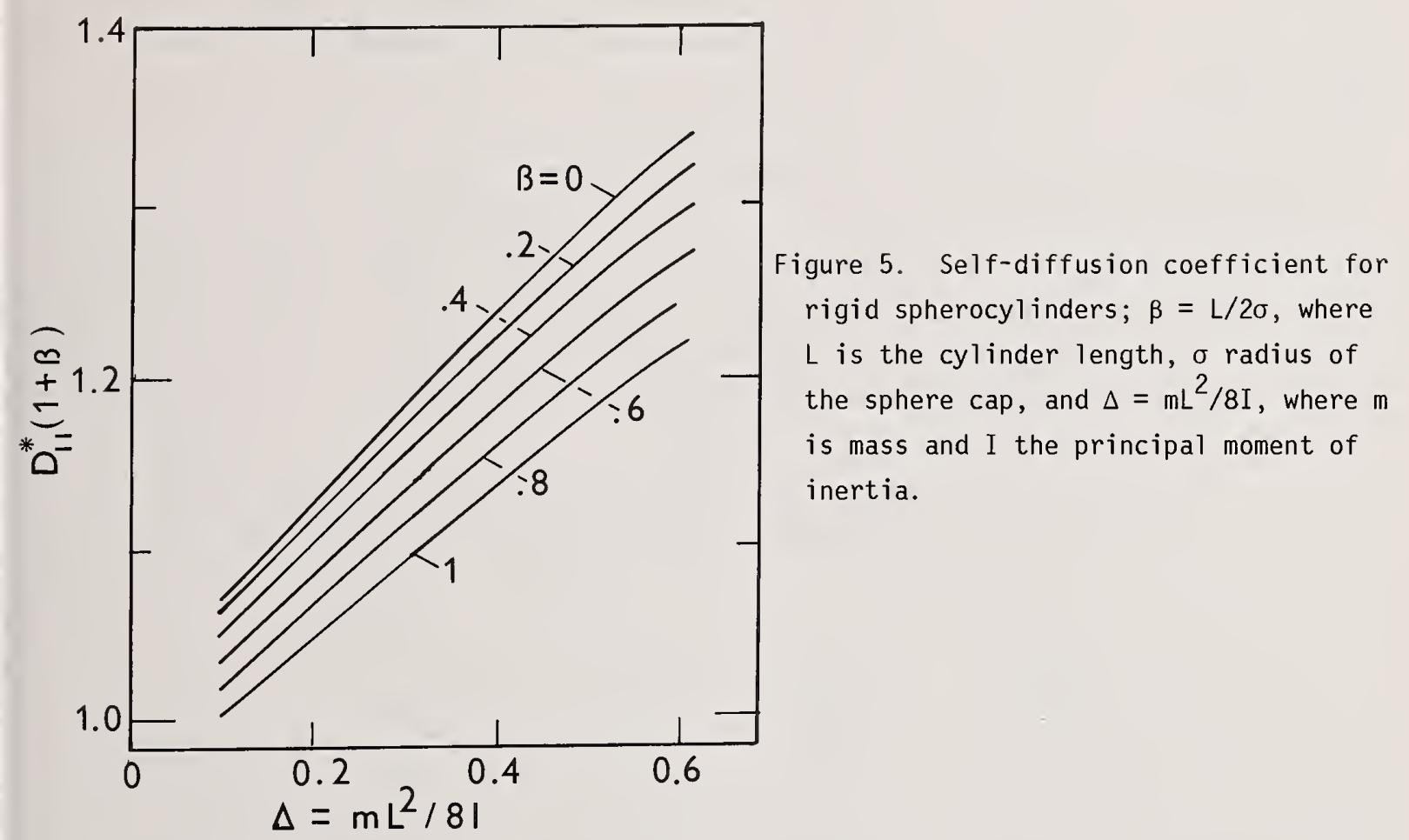

Table 1 is a comparison of some specific calculations for various properties of spherocylinders with the calculations of Curtiss and Muckenfuss [29] and of Sandler and Dahler [31]. Al1 properties except the Eucken factor are expressed as the ratio of the spherocylinder values to those of a rigid sphere with volume equal to that of the spherocylinder.

Table 1. Model calculations for spherocylinders ${ }^{a}$.

\section{Property}

Shear viscosity, $\eta^{*}$
Bulk viscosity, $\eta^{*}{ }_{B}$
Translational Thermal
Conductivity, $\lambda^{*}{ }_{\text {tr }}$

Rotational Thermal

Conductivity, $\lambda^{*}$ rot

Self-diffusivity, $D_{11}^{*}$

Euken factor, $\lambda / C_{v} n$
Ref. [32]

0.7036

0.2018

0.6280

0.3090

0.8202

1.96

$\overline{\mathrm{a}_{\Delta}=\mathrm{mL}} \mathbf{2} / 8 \mathrm{I}=0.3, \beta=\mathrm{L} / 2 \sigma=0.43$.

${ }^{b}$ The asterisk represents calculated values divided by values for a rigid sphere of volume equal to that of the spherocylinder. 
There is good agreement, as expected, with Sandler and Dahler's calculations of viscosity, bulk viscosity, and the two contributions to thermal conductivity. Chatwani confirms their relatively low values of bulk viscosity, compared to the results of Curtiss and Muckenfuss, because of the inclusion of spin anisotropic terms. The values exceed Curtiss and Muckenfuss' calculations of viscosity and self-diffusion coefficient by about 5 percent.

Further information on self-diffusion is shown in table 2. Spin anisotropy evidently has a small effect on the self-diffusion coefficient except for small $\beta^{2} / 2 \Delta$, corresponding to the limit of the degenerate loaded sphere. The nearly constant difference of 2-3 percent between Curtiss and Muckenfuss' results and the present calculations for isotropic spin are caused by inclusion of rotational velocity changes during collision in the earlier model.

Table 2. Effects of spin anisotropy on calculated self-diffusion coefficients of spherocylinders ${ }^{a}$.

\begin{tabular}{|c|c|c|c|}
\hline$\beta^{2} / 2 \Delta$ & $D_{11}^{* b}$ & $0_{11}^{*}$ & $D_{11}^{* d}$ \\
\hline 0.1 & 1.1007 & 1.1214 & 1.2189 \\
\hline .2 & 0.8730 & 0.8914 & 0.8917 \\
\hline .3 & 0.7798 & 0.7969 & 0.7972 \\
\hline .4 & 0.7278 & 0.7441 & 0.7443 \\
\hline .5 & 0.6943 & 0.7101 & 0.7101 \\
\hline
\end{tabular}

\footnotetext{
The asterisk indicates spherocylinder values divided by values for a rigid sphere of volume equal to that of the spherocylinder. Calculations are for $\beta=0.8$.

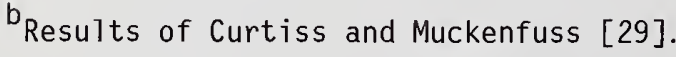

${ }^{\mathrm{C}}$ Calculations of Chatwani [32] assuming spin isotropy.

${ }^{d}$ Calculations of Chatwani [32] assuming spin anisotropy.
}

Chatwani did not consider binary systems in detail because of the many numerical conditions which must be studied. He did complete calculations for the elastic and rotational, inelastic contributions to the thermal diffusion factor and to the temperature derivative of diffusivity for binary systems with components of equal mass. These contributions involve the collision-integral ratios $6 \mathrm{C}_{i j}^{*}-5$ and $6 \tilde{C}_{i j}-5$ defined by Monchick, Sandler, and Mason [33], and in similar fashion by Alievskii and Zhadanov [34]. Computed values are shown in figure 6 for two kinds of binary systems, one for which both components are rigid and spherocylindrical and the order for which one component is a rigid sphere and the other a rigid spherocylinder. The abscissa is the reciprocal of the rotational relaxation number, $Z^{\infty}$, where the superscript of infinity signifies the rigid case expected for the limiting number at high temperature. Figure 7 relates $z^{\infty}$ to the parameters $\beta$ and $\Delta$. The curves for 
spherocylinders are based on Sandler's calculations [23]. The degenerate loaded sphere is represented in figure 6 by the case $\beta=0$.

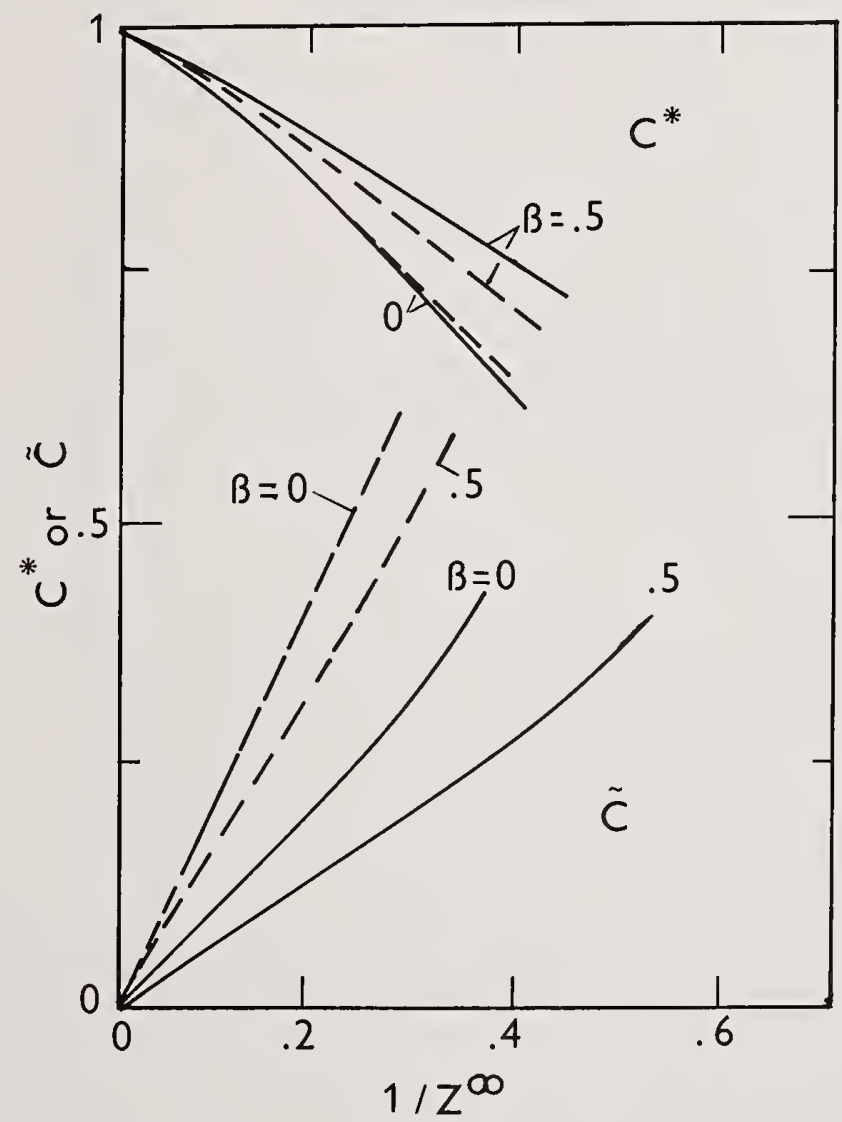

Figure 6. Elastic terms $\left(6 C^{*}-5\right)$ and inelastic terms $(6 \tilde{C}-5)$ for binary mixtures of rigid spherocylinders (solid lines) and of rigid spheres and spherocylinders (dashed lines) (from [32]).

The thermal diffusion factor is computed from

$$
\begin{aligned}
\alpha_{12} & =\left[\left(6 C_{12}^{*}-5\right) \mu_{12} / 5 n k D_{12}\right]\left(\lambda_{2, t r} / x_{2} m_{2}\right. \\
& \left.-\lambda_{1, t r} / x_{1} m_{1}\right) \\
& +\left(1 / 5 n k D_{12}\right)\left[\left(6 \tilde{C}_{21}-5\right) \lambda_{2, \mathrm{int}^{/ x_{2}}}\right. \\
& \left.-\left(6 \tilde{C}_{12}-5\right) \lambda_{1, i n t}^{\infty} / x_{1}\right]
\end{aligned}
$$




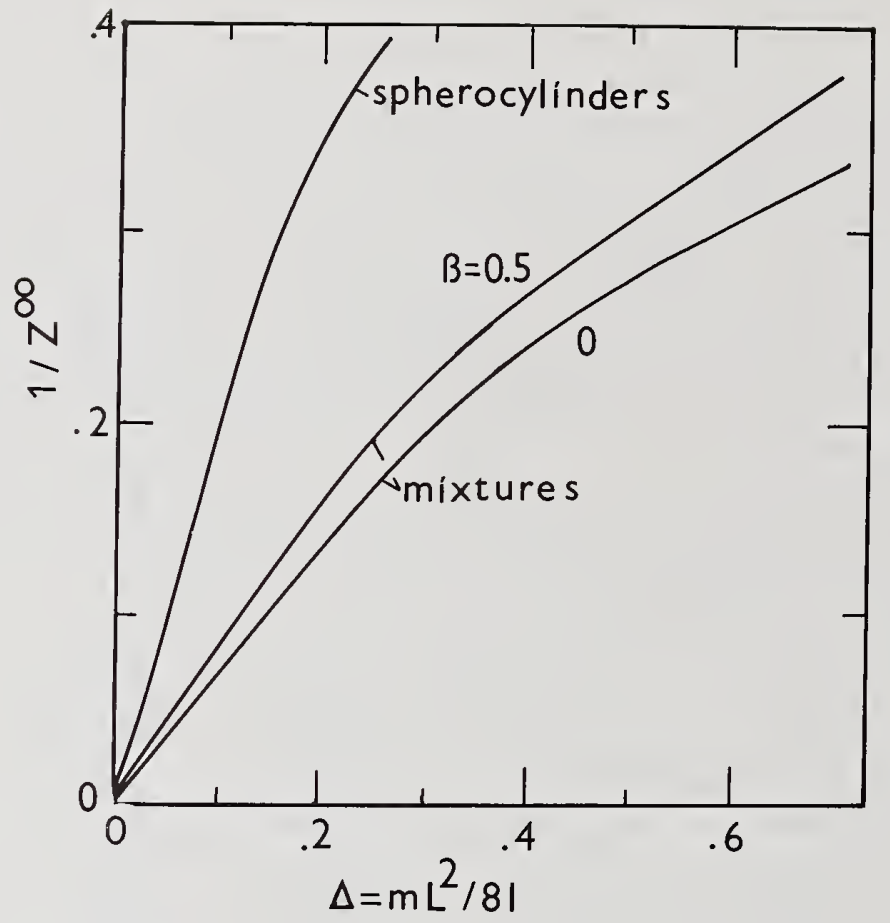

Figure 7. Rotational relaxation number for rigid spherocylinders (based on [23]) and for mixtures of rigid spheres and spherocylinders (from [32]).

The temperature derivative of binary diffusivity is obtained from

$$
\begin{gathered}
d \ln D_{12} / d \ln T=2-(1 / 2)\left(6 C_{12}^{\star}-5\right) \\
+\left(C_{1, \text { int }} / 5 k\right)\left(6 \tilde{C}_{12}-5\right)+\left(C_{2, \text { int }} / 5 k\right)\left(6 \tilde{C}_{21}-5\right)
\end{gathered}
$$

Here $\mu_{12}=\left(1 / m_{1}+1 / m_{2}\right)^{-1}$ is the reduced mass, $x_{1}$ and $x_{2}$ are mole fractions, $D_{12}$ is binary diffusivity, $\alpha_{12}$ is the thermal diffusion factor, $n$ is number density and $k$ is the Boltzmann constant. The contributions to thermal conductivity, $\lambda$, are the translational and "internal" (in this case, rotational) parts [35]; for which some values are given in table 1. The internal contributions to heat capacity at constant volume are represented by $C_{\text {int }}$. To obtain $6 \tilde{C}_{12}-5$ from figure 6 , one uses $\Delta_{1}$ and $\beta_{1}$; for $6 \tilde{C}_{21}-5$, the proper values are $\Delta_{2}$ and $\beta_{2}$.

One of the reasons for studying thermal diffusion for the system rigid spherocylinderrigid sphere was to look for an explanation of anomalous behavior of $\mathrm{CO}_{2}$-argon, which is considered later in the present paper, in the rotational contributions to thermal diffusion. 
Rotational effects turn out to be small and incapable of explaining the minimum with respect to temperature of $\alpha_{12}$ for $\mathrm{CO}_{2}$-argon or the unusual dependence on concentration.

\subsection{A soft spherocylindrical model}

Chatwani [36] went on to study a rigid spherocylindrical molecule surrounded by a finite, attractive field of uniform width and depth. This is of the type of model studied by Kihara, except that the potential is a square well. The rigid cylinder again has length, $L$, with hemispherical, rigid caps of radius, $\sigma$. A flat, attractive well of depth, $\varepsilon / k$, and of width, $\rho_{0} \sigma$, envelopes the entire rigid part.

The square-well potential was chosen in order to simplify calculations. To aid in relating results to a continuous potential, perhaps of Lennard-Jones form emanating from a central line representing the cylinder axis, the value of $\rho_{0}$ was taken as 1.83 for all calculations, as has been done in relating spherical square well and Lennard-Jones forms $[20$, p. 159].

The procedures developed by Dahler and coworkers [37] provided an invaluable guide to selection of the appropriate form of the Boltzmann equation. Major assumptions are those of a dilute gas with molecular chaos, a cutoff potential so that only molecules originally outside the range of interaction contribute to collisional properties, an isotropic distribution of orientations prior to collision and a symmetric kernel of the Boltzmann integral.

The complex geometry and multiplicity of integrations involved, precluded the use of closed-form solutions of the dynamical equations as is usual with the Chapman-Enskog method of solving the Boltzmann equation. Instead, a Monte Carlo technique was employed. This involved solving for the trajectories of many binary collisional events, initial conditions in each case being selected randomly (though with appropriate constraints). The postcollisional and pre-collisional properties provided information which could be averaged over the full set of trajectories to yield the collision integrals and transport properties.

The constraints on random selection included necessary factors such as rejection of choices which caused molecules to move so as to avoid one another completely. In addition, a variety of techniques was employed to hasten numerical convergence of the calculated integral values. Chatwani [36] discusses these in detail.

Despite such constraints, the rate of reduction of the variance of the estimate of collision integrals was quite slow. But as a result of the constraints, the averages themselves converged fairly quickly. For example, it was found that an average value of a collision integral varied considerably during collection of about 600 trajectories. At this point, the variance would be of the order of 20 percent of the average value. Collection of about 1800 more trajectories reduced the variance by about a factor of two (as is to be expected, the variance decreasing as the inverse square root of the number of events). This additional collection changed the average estimate of the collision integral by perhaps 1 percent at most, however.

Calculations were normally based on 600 trajectories. The variance was 10-20 percent for the collision integrals of diffusivity and viscosity at this point, higher for some 
other properties. Average values were not changing greatly, and the principal effect of further calculation was expected to be only a formal reduction in variance. Nonetheless, the averages after 600 trajectories are reported with the variances at that stage. In some cases, particularly that of $6 \tilde{C}-5$, the inelastic contribution to the thermal diffusion factor, the estimated error was comparable to the average itself. No attempt was made to use these data as a result.

The principal results of Chatwani's study have been published [38]. Most important for purposes of the present paper is the observation that the viscosity and diffusion coefficient respond to the nonspherical molecular form quite differently. To illustrate we must compare spherical and nonspherical models, and this, in turn, requires that the crosssections of the two forms be comparable. If $\rho_{0}$, previously set equal to 1.83 , is taken as $2^{3 / 2}-1=1.828$, we calculate that the area swept by rotation of the spherocylinder on its minor axis is greater than the projected area of a sphere of radius $2^{3 / 2} \sigma$ by the factor

$$
f_{n}(\beta)=1+\beta / 2^{\frac{1}{2}}+\beta^{2} / 8
$$

where, as before, $\beta=L / 2 \sigma$. The comparable quantity for the rigid spherocylinder is

$$
f_{c}(\beta)=(1+\beta)^{2}
$$

The first indication of different behavior may be obtained from the values for rigid spherocylinders of table 1. These have been compared to rigid spheres of equal volume. To compare in terms of equal projected area, we multiply entries of table 1 by $(1+\beta)^{2} /(1+$ $3 \beta / 2)^{2 / 3}=1.467$ for the case $\beta=0.43$ of table 1 . The results for shear viscosity are values of 1.004-1.032 and for self-diffusivity of 1.176-1.203. That is, viscosity of the rigid spherocylinder and rigid sphere are within about 3 percent of one another. Selfdiffusivity of the spherocylinder is 17-20 percent larger than that of the comparable sphere; for extreme cases represented in figure 5 , this value is above 30 percent.

Differences of this kind were noticed in experimental data for $\mathrm{CO}_{2}$ by Ember [39], and he used the calculations of Curtiss and Muckenfuss [29] to justify the unexpected enhancement in diffusion which he observed. These applications of rigid spherocylinder calculations were later exploited in predictive schemes $[9-13,17,32,40]$, never completely successfully, however, because only molecular shape had been included as a polyatomic feature. Both translational and rotational effects were present, but there was no way to account fully for temperature dependence of rotation. One could only conclude that rigid behavior would be approached at high temperature.

At the same time, however, it was quite clear that high-temperature viscosities and self-diffusivities probably could not be correlated by the same monatomic model, because viscosity follows a nearly spherical model for the high-temperature limit while selfdiffusivity more nearly follows a spherocylindrical model in the same limit.

By means of his Monte Carlo calculation, Chatwani [36] was able to obtain cross sections for the spherocylinder with square wel1. He found that, indeed, computed viscosities retain 
a nearly spherical description throughout the temperature range studied. Self-diffusivities are approximated by the spherical model at low temperatures, but there is a transition to spherocylindrical behavior at elevated temperature [38]. He studied only the case $\beta=\Delta=$ 0.5 in detail. For this case, the diffusion coefficient may be represented by the empirical form:

$$
\bar{D}=\left[0.82+0.7264 / T^{\star}+0.1185 / T^{\star 2}\right]^{-1}
$$

where $T^{*}=k T / \varepsilon$ and $\bar{D}$ is the diffusivity divided by that of a rigid sphere of projected area equal to that of the soft spherocylinder as in eq. (15).

To compare this result with experimental data is not straightforward, partly because eq. (17) represents a non-continuous potential and also because the parameters $\beta=\Delta=0.5$ used to obtain the result are not accurate for the systems we consider. (Bond lengths and moments of inertia from spectroscopic data would suggest, approximately, $\beta=0.6$ and $\Delta=0.7$ for $\mathrm{CO}_{2}$, for example.)

We can attempt some comparison, however, by calculating $D$ for the experimental data from:

$$
\bar{D}_{\text {exp }}=\sigma^{2} f_{n}(0.5) /(2.928)^{2} \Omega_{L J}^{(1,1)^{*}}(T / 600)
$$

where we have used the Lennard-Jones parameters, $2.928 \AA$ and $600 \mathrm{~K}$, which we have previously found to represent experimental self-diffusion coefficients of $\mathrm{C}_{2}$. Here $\sigma$ together with $\varepsilon / k$ used in eq. (17) represent parameters of the soft, spherocylinder model to be selected by comparing eqs. (17) and (18) numerically.

Viscosities of $\mathrm{CO}_{2}$ are correlated by the Lennard-Jones parameters $3.894 \AA$ and $210 \mathrm{~K}$ [13]. We may check these as possible spherocylinder parameters by comparing $D$ calculated from eqs. (17) and (18). Setting $\varepsilon / k=210 \mathrm{~K}$ in eq. (17) produces the average value, $\sigma=$ $2.79 \AA$, from eq. (18) for diffusivities in the range $300-1800 \mathrm{~K}$. It is not possible for the model to yield $\sigma=3.894$; the largest feasible value is $3.28 \AA$. We may also try $\varepsilon / \mathrm{k}=600 \mathrm{~K}$ as obtained from diffusion. The resulting value of $\sigma$ is 2.28. The model thus prefers values of $\sigma$ closer to that of monatomic, Lennard-Jones correlation of the self-diffusion coefficient.

Similar results are obtained for $\mathrm{CO}_{2}$-argon. For this case, the Lennard-Jones parameters from viscosity [13] are $3.665 \AA$ and $158 \mathrm{~K}$; those from binary diffusivities are $3.208 \AA$ and $277.5 \mathrm{~K}$. Equations (17) and (18) yield values for $\sigma$ of 2.5 to $2.7 \AA$ for $\varepsilon / k$ in the range $158-$ $277.5 \mathrm{~K}$. Again, the appropriate spherocylinder model is of smaller radius than the equivalent spherical model.

Parameters obtained for the soft spherocylinders are not totally unreasonable. We can conclude that including rotation and softness in the model of $\mathrm{CO}_{2}$ does permit successful correlation of data. Unfortunately, it is not possible to detect how much of the total 
inelastic effect in diffusion is accounted for by rotation. We can seek the answer in another way, however, by comparison of data for diffusion and for thermal diffusion.

\section{Diffusion and Thermal Diffusion}

The availability of extensive measurements of the binary diffusion coefficient makes possible further study of the thermal diffusion factor for carbon dioxide-argon mixtures, the two transport properties being related through the temperature derivative of the binary diffusivity, as shown by eqs. (13) and (14). For the $\mathrm{CO}_{2}$-argon system, in which we again use subscript 1 for $\mathrm{CO}_{2}$ and 2 for argon, eqs. (13) and (14) are simplified by setting $\lambda_{2}$, int and $c_{2, \text { int }}$ to zero. We conveniently obtain the quantity $2\left(2-d \ln D_{12} / d \ln T\right)$ by means of the Lennard-Jones form of $6 C_{12}^{\star}-5$, which is tabulated [20, p. 1128], using $(\varepsilon / \mathrm{k})_{12}=277.5 \mathrm{~K}$. Also $c_{1, \text { int }} / k=C_{v 1} / k-3 / 2$, where $C_{v 1}$ is the heat capacity at constant volume of carbon dioxide.

The separate contributions to the thermal conductivity may be calculated by the linearized form of the method of Monchick, Pereira, and Mason [41], though the nonlinear form is better [36]. This requires values of the rotational relaxation numbers for $1 i k e$ and unlike interactions. For purposes of a first approximation, we have taken these as equal and estimated them from Parker's formula (as well as other means; note [38]). We used $Z^{\infty}=30$, a compromise between the value of 15 often used for pure carbon dioxide and the infinite value expected for pure argon. Computed mixture thermal conductivities compare favorably in magnitude with the experimental values reported by Barua, Manna, and Mukhopadhyay [42].

Equations (13) and (14), together with experimental data for $\alpha_{12}$, make possible the evaluations of $c_{12}^{\star}$ and $\tilde{C}_{12}$ as functions of temperature and concentration. These, in turn, permit us to separate the elastic and inelastic contributions to $\alpha_{12}$ and to $D_{12}$ and, thus, to seek better understanding of these separate effects.

Experimental values of $\alpha_{12}$ are available from several sources. Here, we chose to work only with the measurements of Batabyal, Ghosh, and Barua [43] which have been obtained for a reasonable range of temperature and composition and for which values of experimental precision have been reported. The data are summarized in figure 8 , and figures 9 and 10 portray results of $\mathrm{C}_{12}^{*}$ and $\tilde{C}_{12}$ from eqs. (13) and (14).

Referring first to figure 8 , we note that the thermal diffusion factor, $\alpha_{21}$, may be either positive or negative. For low concentrations of $\mathrm{CO}_{2}$, it decreases from a fairly large value near $300 \mathrm{~K}$, monotonically with temperature; but with 50 percent or more $\mathrm{CO}_{2}$ present there is a minimum with respect to temperature. Both effects, the relatively large positive values of $\alpha_{21}$ and the minimum in $\mathrm{CO}_{2}$-rich systems, have been considered curiosities which cannot be explained by elastic theories of thermal diffusion. The explanation might be found, in part, by evaluating the elastic and inelastic contributions as shown in figure 11. The elastic contribution is seen to be small, and it clearly could not account for the behavior of $\alpha_{21}$. 

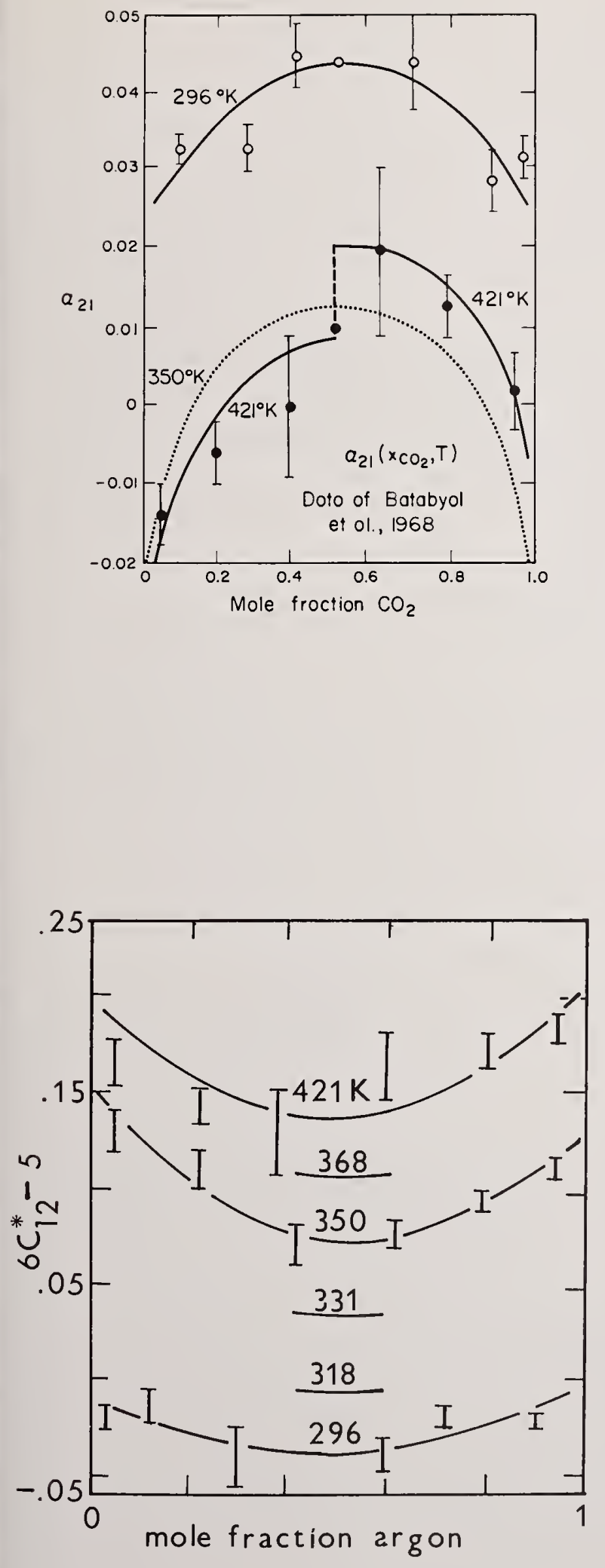

Figure 8. The thermal diffusion factor for carbon dioxide-argon mixtures. Data are from [43]. The lines are guides for estimation and have no other significance.
Figure 9. Elastic contribution $\left(6 c^{\star}-5\right)$ to thermal diffusion in $\mathrm{CO}_{2}$-argon. Calculated from eqs. (13) and (14). 

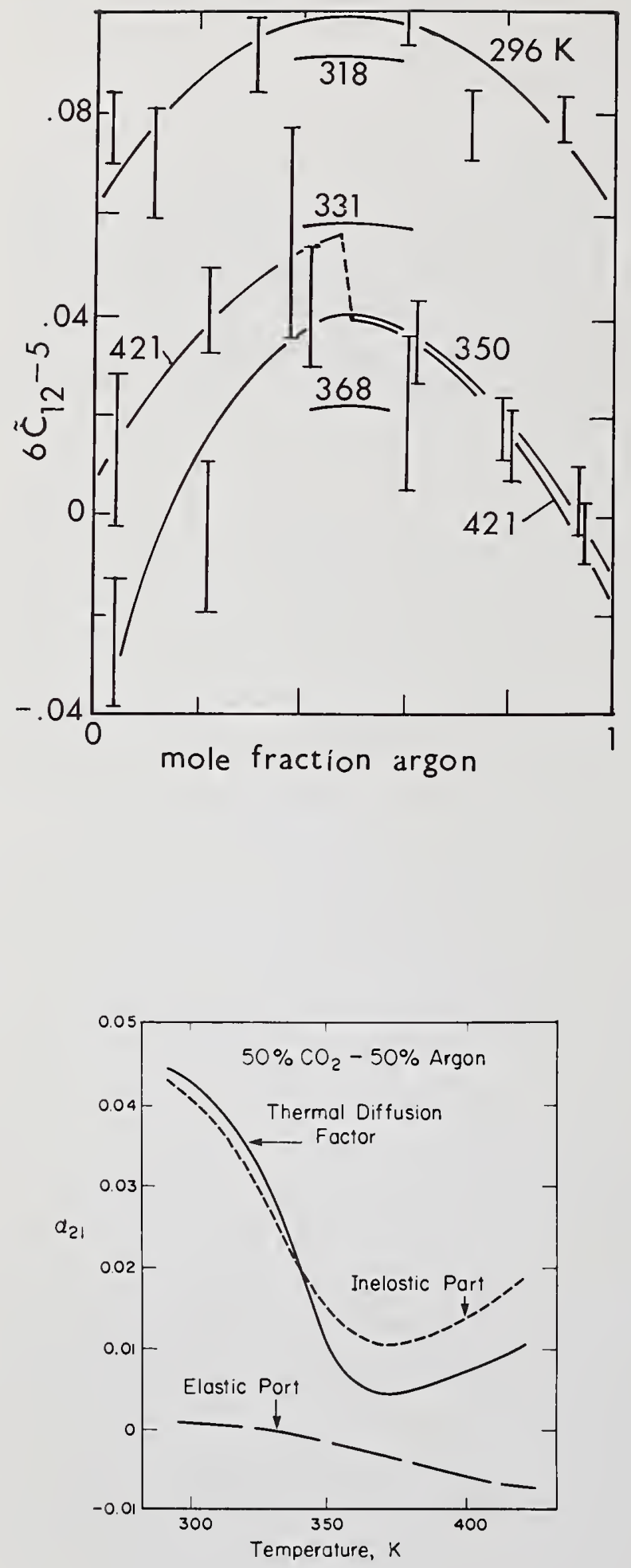

Figure 10. Inelastic contribution $(6 \tilde{C}-5)$ to thermal diffusion in $\mathrm{CO}_{2}$-argon. Calculated from eqs. (13) and (14).
Figure 11. Estimated contributions to the thermal diffusion factor of $\mathrm{CO}_{2}$-argon. 
The inelastic part, on the other hand, is quite large. The physical picture of what occurs is, perhaps, somewhat as follows. A positive value of $\alpha_{21}$ means that $\mathrm{CO}_{2}$ tends to be present at its higher concentration at the hot end of the thermal diffusion column. When steady state is attained with the average temperature at about $300 \mathrm{~K}$, the thermal diffusion factor is quite large, not because $\mathrm{CO}_{2}$ and argon differ significantly in molecular size, mass, or even shape, but because so much of the energy of $\mathrm{CO}_{2}$ is in the internal modes while argon's energy is translational. Argon is more mobile through the temperature gradient. When the temperature is increased, carbon dioxide gains translational energy and moves more freely, but inelastic absorption of energy continues to control the separation which is achieved, hence, the value of $\alpha_{21}$.

Near $350 \mathrm{~K}$, the temperature derivative of internal heat capacity is maximum (representing the point of maximum influence of the bending modes of vibration on $d C_{v} / d T$ ) and henceforth decreases with rising temperature. The inelastic part of $\alpha_{21}$ would, as a result, be expected to have a somewhat lesser effect compared to the elastic part, consistent with what we see in figure 11.

\section{Elastic and Inelastic Contributions}

to the Binary Diffusivity

We may now return to eq. (14) and to the results of figures 9 and 10 in order to evaluate the elastic and inelastic parts of the temperature derivative of binary diffusivity. These are as follows:

$$
\begin{aligned}
& \left(\mathrm{dlnD}_{12} / \mathrm{dlnT}\right)_{\text {elastic }}=2-\frac{1}{2}\left(6 \mathrm{C}_{12}^{*}-5\right) \\
& \left(\mathrm{dlnD}{ }_{12} / \mathrm{d} \operatorname{lnT}\right)_{\text {inelastic }}=-(1 / 5)\left(\mathrm{C}_{1, \text { int }} / \mathrm{R}\right)\left(6 \tilde{C}_{12}-5\right) .
\end{aligned}
$$

The calculations are plotted in figure 12. The upper lines represent the result of eq. (19) divided by temperature. The lower lines are similar values obtained from smoothed experimental measurements of $\mathrm{D}_{12}$. Concentration effects for both calculations are small and invisible at the scale of figure 12 . The sharp change in second derivative of $\mathrm{D}_{12}$ at $350 \mathrm{~K}$ indicated in figure 12 for both the elastic contribution and the experimental measurements is of interest. This may again be associated with the diminished temperature derivative of heat capacity above $350 \mathrm{~K}$.

It is clear from the smoothed lines of figure 12 that eqs. (19) and (20) may be integrated to give elastic and inelastic portions of $\mathrm{D}_{12}$ for temperatures up to about $420 \mathrm{~K}$. We note that the elastic diffusivity will exceed the measured value. From our previous discussion of relative translational mobility of carbon dioxide and argon, we can seek the explanation. Elastic carbon dioxide would diffuse more readily and have a higher value of $D_{12}$ than the actual molecule because all of its energy would be freely available for the translational mode. The measured binary diffusivity is lower because $\mathrm{CO}_{2}$ holds energy internally. 


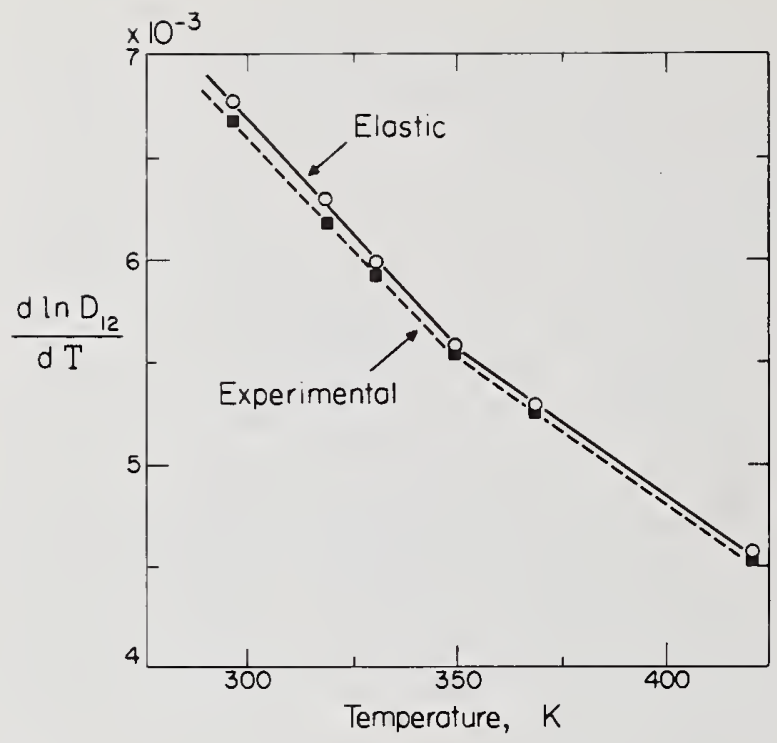

Figure 12. Estimated elastic contribution to the temperature derivative of the binary diffusion coefficient of $\mathrm{CO}_{2}$-argon and the experimental value. Points represent temperatures at which thermal diffusion data are available.

The contribution of inelastic effects to $D_{12}$ can be evaluated approximately from the integrated forms suggested above. A few calculations are shown in table 3 . These are based on the assumption that elastic and measured values of $D_{12}$ are equal at $200 \mathrm{~K}$. It is interesting to note from table 3 that the inelastic portion of $\mathrm{D}_{12}$ as a function of temperature exhibits an inflection point at $350 \mathrm{~K}$, again coincident with the temperature of maximum influence of the bending modes of vibration on the temperature derivative of heat capacity.

Table 3. Estimated inelastic contribution to the binary diffusion coefficient of carbon dioxide-argon.

\begin{tabular}{c} 
Temperature, $\mathrm{K}$ \\
\hline 200 \\
250 \\
300 \\
350 \\
400 \\
450 \\
500 \\
550 \\
600
\end{tabular}

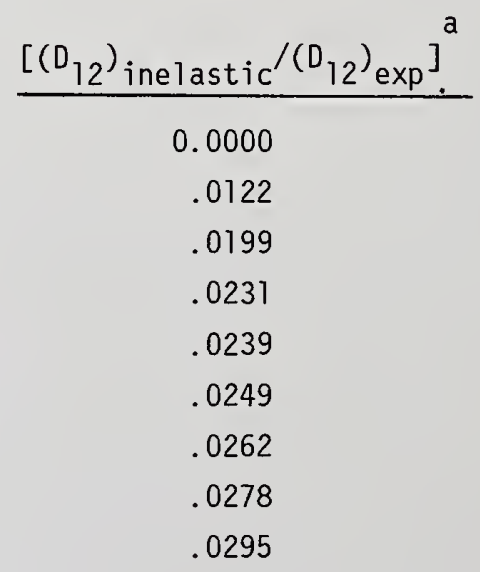

$\overline{\mathrm{a}_{\text {Based on }} \text { the assumption that at } 200 \mathrm{~K}\left(\mathrm{D}_{12}\right)_{\exp }=\left(\mathrm{D}_{12}\right) \text { elastic }}$. 


\section{Summary}

Experimental measurements and model calculations both indicate that polyatomic structure influences diffusion of carbon dioxide, especially at high temperatures. The diffusion coefficient is greater than that predicted from spherical models and monatomic theory. Rotational phenomena may be included in the model of polyatomic behavior, but rotation alone cannot account completely for the experimental observations. Comparison of diffusion and thermal diffusion discloses behavior which must be attributed to inelastic effects other than rotation. For carbon dioxide, the appearance of various unusual phenomena, presumably the result of inelastic influences, is coincident with the temperature of maximum contribution of the bending modes of vibration to the temperature derivative of the heat capacity.

The research described here was carried out at the Universities of Delaware and Rochester. Financial support of the National Science Foundation, the Office of Naval Research, and various industrial sources is gratefully acknowledged.

\section{References}

[1] Hinze, J. 0., Turbulence, p. 324-352 (McGraw-Hi11, New York, 1959).

[2] Westenberg, A. A. and Walker, R. E., J. Chem. Phys. 26, 1753 (1957).

[3] Walker, R. E. and Westenberg, A. A., J. Chem. Phys. 29, 1139 (1958).

[4] Walker, R. E. and Westenberg, A. A., J. Chem. Phys. 29, 1147 (1958).

[5] Walker, R. E. and Westenberg, A. A., J. Chem. Phys. 31, 519 (1959).

[6] Walker, R. E. and Westenberg, A. A., J. Chem. Phys. 32, 436 (1960).

[7] Walker, R. E., de Haas, N., and Westenberg, A. A., J. Chem. Phys., 32, 1314 (1960).

[8] Westenberg, A. A. and Frazier, G., J. Chem. Phys. 36, 3499 (1962).

[9] Ember, G., Ferron, J. R., and Woh1, F., J. Chem. Phys. 37, 891 (1962).

[10] Ember, G., Ferron, R. R., and Woh1, K., A. I.Ch.E. Journa1, 10, 68 (1964).

[11] Pakurar, T. A. and Ferron, J. R., J. Chem. Phys. 43, 2917 (1965).

[12] Pakurar, T. A. and Ferron, J. R., in The Performance of High-Temperature Systems, G. S. Bahn, ed., p. 89-107 (Gordon and Breach, New York, 1968).

[13] Pakurar, T. A. and Ferron, J. R., Ind. Eng. Chem. Fundamenta1s, 5, 553 (1966).

[14] Schaefer, V. K. and Reinhard, P., Z. Naturforshung, 18, 187 (1963).

[15] Swinton, F. L., in Diffusion Processes, J. N. Sherwood, A. V. Chadwick, W. M. Muir, and F. L. Swintin, eds., Vol. I, p. 53-63 (Gordon and Breach, London, 1971).

[16] Ivakin, B. A. and Suetin, P. E., Soviet Physics, Tech. Phys. 9, 866 (1964).

[17] Pakurar, T. A, Diffusivities in the carbon dioxide-nitrogenargon system, Doctoral Dissertation, University of Delaware, Newark (1965).

[18] Sandler, S. I. and Mason, E. A., J. Chem. Phys. 48, 2873 (1968). 
[19] Chapman, S. and Cowling, T. G., Mathematical Theory of Nonuniform Gases (Cambridge Univ. Press, 1959).

[20] Hirschfelder, J. G., Curtiss, C. F., and Bird, R. B., Molecular Theory of Gases and Liquids (Wiley, New York, 1954).

[21] Ishida, Y., Phys. Rev. 10, 305 (1917).

[22] Pidduck, F. B., Proc. Roy. Soc. (London), A101 (1922).

[23] Sandler, S. I., Doctoral Dissertation, Univ. of Minnesota, Minneapolis (1966).

[24] Condiff, D., Lu, W. K., and Dahler, J. S., J. Chem. Phys. 42, 3445 (1965).

[25] Kagan, Yu. and Afanas'ev, A. M. , Soviet Physics-Tech Physics, 14, 1096 (1962).

[26] Jeans, J. H., Dynamical Theory of Gases (Cambridge Univ. Press, Cambridge, 1904).

[27] Dahler, J. S. and Sather, N. F., J. Chem. Phys. 38, 2363 (1963).

[28] Kihara, T., J. Phys. Soc. Japan, $\underline{6}, 289$ (1951).

[29] Curtiss, C. F. and Muckenfuss, C., J. Chem. Phys. 26, 1619 (1957); 29, 1257 (1958).

[30] Curtiss, C. F. and Dahler, J. S., J. Chem. Phys. 38, 2352 (1963).

[31] Sandler, S. I. and Dahler, J. S., J. Chem. Phys. 43, 1750 (1965); 47, 2621 (1967).

[32] Chatwani, A. U., Spherocylinder model calculations, M. S. Thesis, Univ. of Rochester, Rochester (1972).

[33] Monchick, L., Sandler, S. I., and Mason, E. A., J. Chem. Phys. $\underline{49}$, 1178 (1968).

[34] Alievskii, M. Y. and Zhdanov, V. M., Soviet Physics, JETP, 28, 116 (1969).

[35] Mason, E. A. and Monchick, L., J. Chem. Phys. 36, 1622 (1962).

[36] Chatwani, A. U., Monte Carlo evaluation of the transport properties of polyatomic gases, Doctoral Dissertation, Univ. of Rochester, Rochester, NY (1974).

[37] Hoffman, D. K. and Dahler, J. S., in Transfer and Storage of Energy by Molecules, Burnett and North, eds. (Wiley, New York, 1969).

[38] Chatwani, A. U. and Ferron, J. R., Phys. Fluids, 20, 1438 (1977).

[39] Ember, G., Diffusivities in the $\mathrm{CO}_{2}$-water system at flame temperatures, Doctoral Dissertation, Univ. of Delaware, Newark, (1962).

[40] Kerr, D. L., A study of gaseous diffusion at elevated temperatures, Doctoral Dissertation, Univ. of Delaware, Newark (1970).

[41] Monchick, L., Pereira, A. N. G., and Mason, E. A., J. Chem. Phys. 42, 3241 (1965).

[42] Barua, A. K., Manna, A., and Mukhopadhyay, P., J. Phys. Soc. Japan, 25, 862 (1968).

[43] Batabyal, A. K., Ghosh, A. K., and Barua, A. K., J. Chem. Phys. 48, 5238 (1968).

\section{Discussion}

Question (Rosner): Would it be useful to regard these effects as the result of even pure $\mathrm{CO}_{2}$ at high temperature being the equivalent of many species, each species of which is a unique quantum state, each of which has its own cross section and energy path, and therefore you are really dealing with a multicomponent mixture with some mix of properties? Is that a useful perspective in this case? 
Response (Ferron): Well, I am sure, as you know, the thermal conductivity traditionally is that way.

Here, I wouldn't know quite what to do with it if one were going to calculate trajectories in binary collisions, calculating enough of these say to make a Monte Carlo estimate, which is what we have done with collision integrals.

With only one potential it is expensive to separate into the number of components that might be helpful, no doubt, but it would surely be tedious.

I agree with you. If we could say definitely that this is an important thing, as it seems to be, then surely it is worthwhile modeling that sort of thing by a series of species or states, or however. 



\section{RATE PROCESSES IN GASES-- HETEROGENEOUS SYSTEMS}



National Bureau of Standards Special Publication 561, Proceedings of the 10th Materials Research Symposium on Characterization of High Temperature Vapors and Gases held at NBS, Gaithersburg, Maryland, September 18-22, 1978. Issued October 1979.

\section{THE COMBUSTION OF PULVERIZED COALS - AN ASSESSMENT OF RESEARCH NEEDS}

D. R. Hardesty and J.H. Pohl

Combustion Research Division, Sandia Laboratories

Livermore, CA 94550

We are conducting a technical assessment of pulverized coal combustion problems in conventional boilers and furnaces and in advanced combustors. The assessment is being conducted in response to a request by the Division of Fossil Fuel Utilization/Department of Energy. It is designed to assist DOE in establishing goals and priorities for research related to pulverized coal combustion. This paper is an interim report on the assessment task. A general background to the subject area is provided and emphasis is given to selected research areas of special relevance to the material sciences.

\section{Introduction}

We have recently undertaken an investigation of pulverized coal combustion. This project consists of three elements: (a) an experimental study of the rates and mechanisms of combustion and formation of polluting, corrosive, slagging and fouling species for well characterized pulverized coals in a laminar flow reactor; (b) an investigation, using optical and physical diagnostics probes, of local flame structure in full scale utility boiler pulverized coal flames, and; (c) a technical assessment of pulverized coal combustion problems and research needs in conventional boilers and furnaces and in advanced combustors.

This discussion is a report on the assessment task. The assessment was requested by the Division of Fossil Fuel Utilization/U.S. Department of Energy to assist in establishing goals and priorities for research on coal combustion. Such research, in the past, has been conducted in a limited number of university, industrial and government laboratories in the United States and abroad. Studies, in general, have been directed toward improving coal combustion technology by reducing pollutant formation, boiler corrosion and fouling, while maintaining combustion efficiencies. Of immediate concern is the extent to which fundamental research on pulverizea coal combustion can contribute to improvement in these technologies.

We have completed approximately $80 \%$ of intended site visits to coal combustion research and development 1 aboratories and utility companies in the United States, Western Europe, Australia, New Zeal and and Poland $[1,2,3] .1$ Selected interim conclusions and

${ }^{1}$ Figures in brackets indicate the literature references at the end of this paper. 
recommendations, based on discussions with the principal scientists involved, are reviewed in this first report. A fully documented summary report will be available for later review.

\section{A Case for Expanded Research on Pulverized Coal Combustion}

Increased use of coal, our most abundant fossil fuel resource, will be required to meet both immediate and long-term energy demands. As indicated in figure 1, coal accounts for nearly $90 \%$ of the energy reserves of the United States.

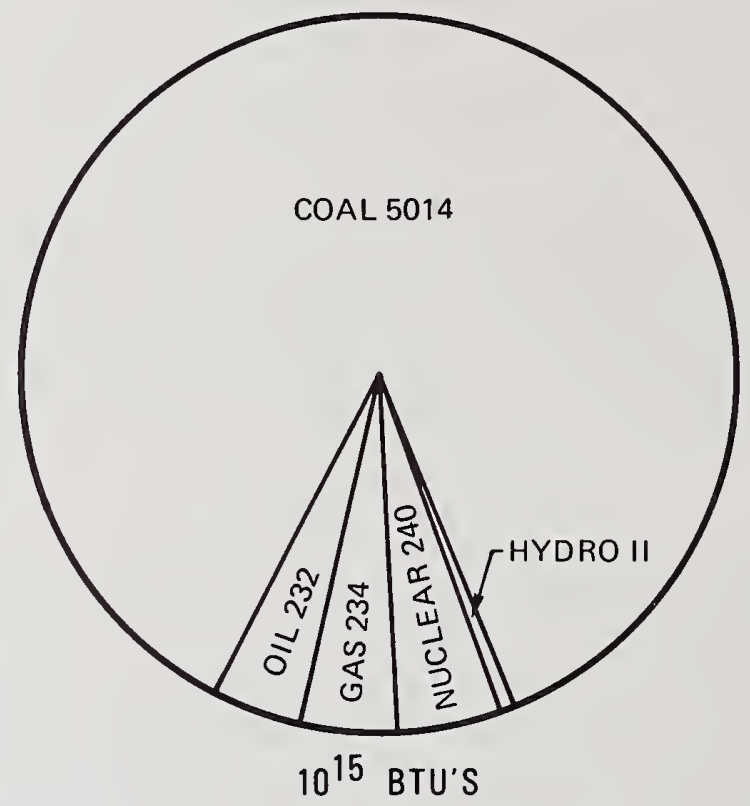

Figure 1. U.S. energy reserves.

However, estimates by IGT, shown in figure 2, indicate that current total energy consumption in the U.S. is not commensurate with these vast coal reserves.

The importance of correcting this imbalance of reserves and use of coal is emphasized by projections of fuel demands for central station generation of electricity, as shown in figure 3, and for steam raising and process heating by industry, as shown in figure 4 . A four fold increase in coal utilization in the electric utility sector is anticipated by the year 2000. Over the same period, a doubling of fossil fuel utilization by industry is predicted, with only minor contributions from coal. Substitution of coal for the premium fuels (gas and oil) in the industrial sector will have a major impact on future requirements for imported oil and domestic gas supply. 


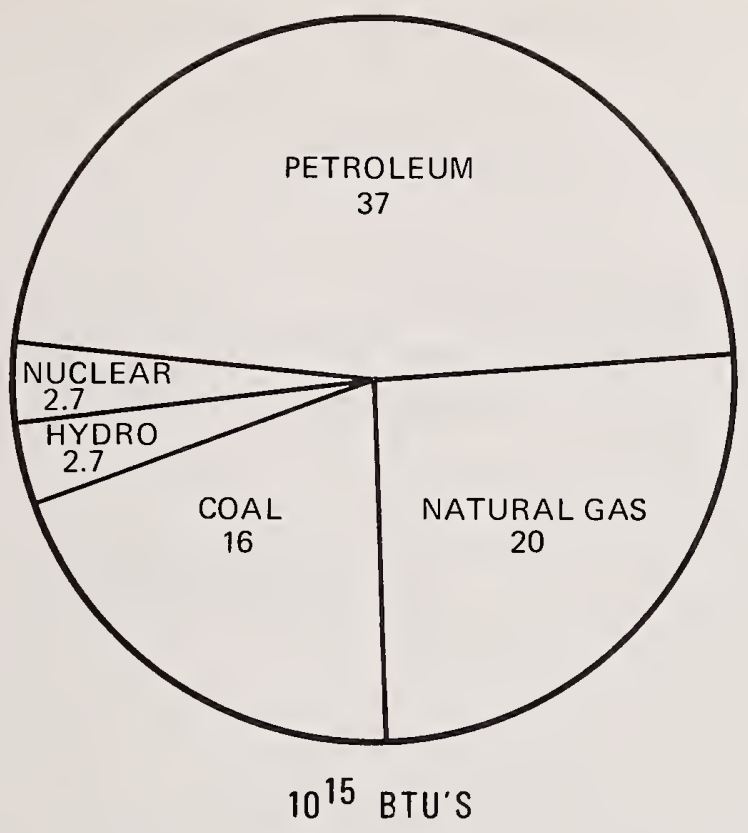

Figure 2. U.S. energy consumption in 1977 [IGT estimate].

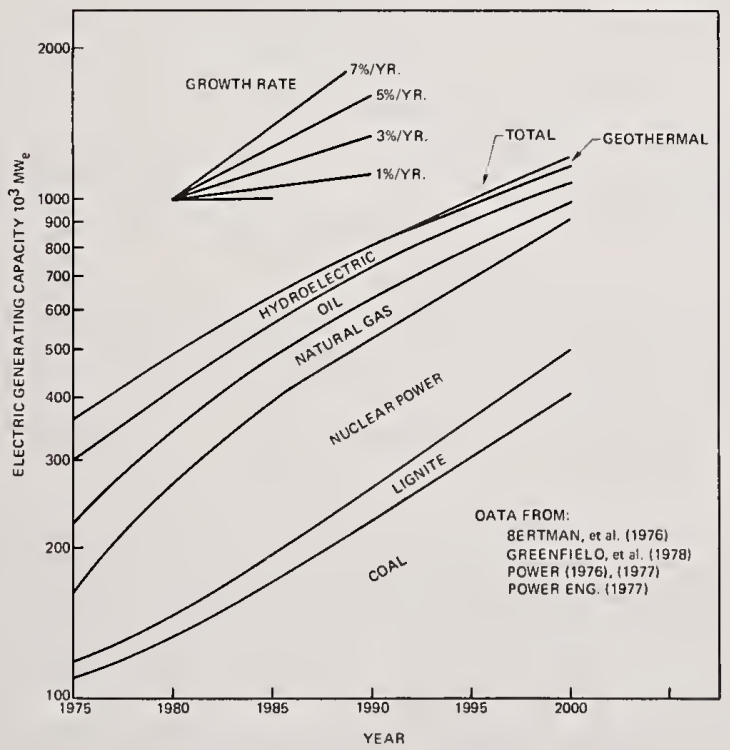

Figure 3. Forecast of electric power generation in the U.S. $[4,5,6,7]$. 


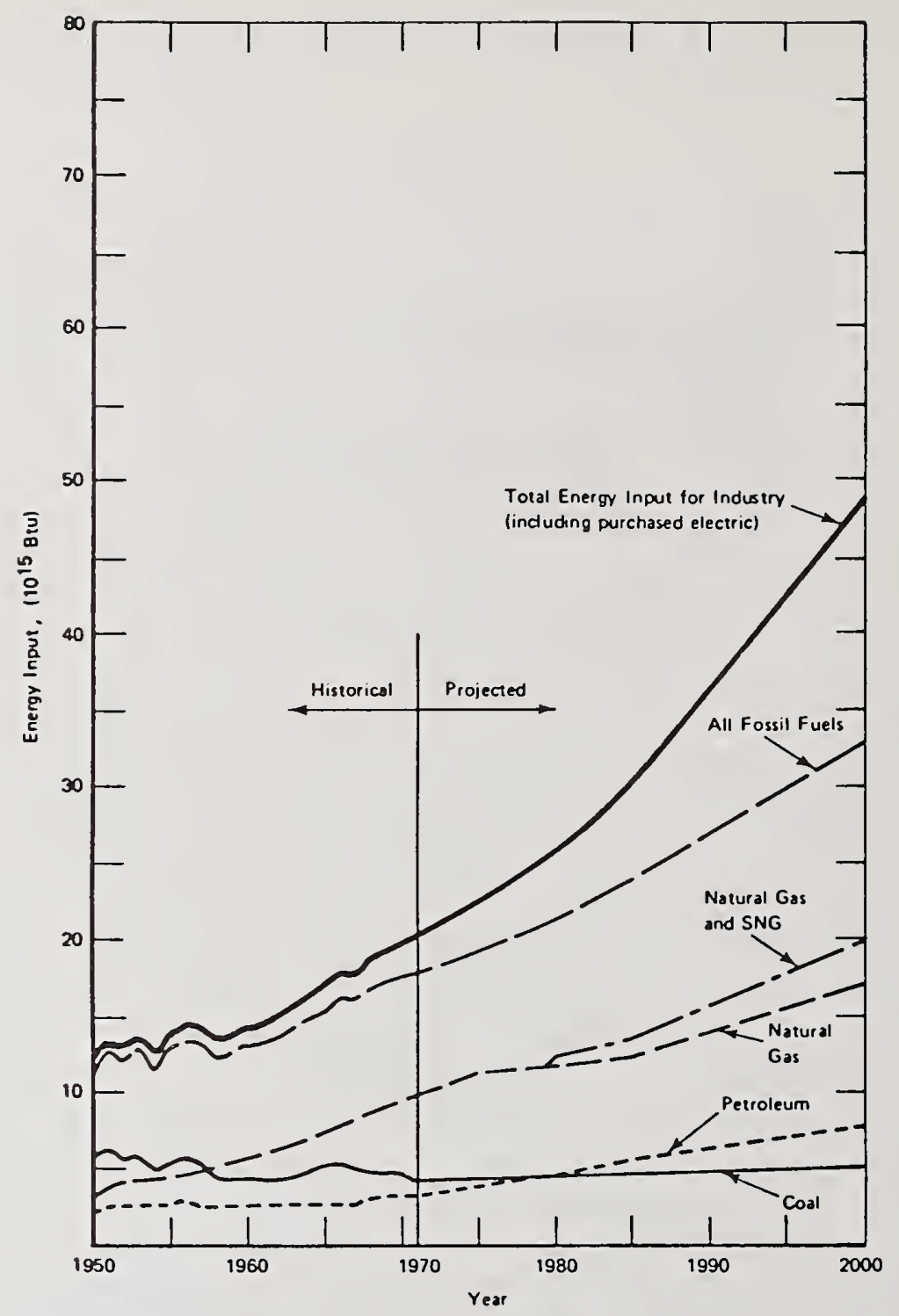

Figure 4. Forecast of energy use by industry in the U.S. [8].

The distribution of fossil fuel usage in industrial boilers, is shown in table la according to boiler capacity. Limiting cases, which illustrate the potential savings in premium fuels achieved by substitution of coal for part or all of the oil and gas used by industry, are shown in table $1 b$. 
Table 1. Industrial usage of fossil fuels [8].

a. Distribution according to boiler size

\begin{tabular}{|c|c|c|c|c|c|}
\hline Capacity & $\%$ of Total & $\%$ Dist & bution by Fuel & & Coal \\
\hline $\begin{array}{c}10^{6} \mathrm{Btu} / \mathrm{hr} \\
10^{3} \mathrm{lb} \text { steam } / \mathrm{hr} \\
\end{array}$ & $\begin{array}{l}\text { Industrial Capacity } \\
\text { (including coking) }\end{array}$ & $\begin{array}{c}0 i 1 \text { (and/or) } \\
\text { Gas } \\
\end{array}$ & $\begin{array}{c}\text { Coal (and/or) } \\
0 i 1 \text { or Gas } \\
\end{array}$ & Other & $\begin{array}{l}\% \text { of Total } \\
\text { Ind Cap } \\
\end{array}$ \\
\hline $10-16$ & 10 & 96 & 3 & 1 & 0 \\
\hline $17-100$ & 45 & 88 & 10 & 2 & 0 \\
\hline $101-250$ & 36 & 78 & 19 & 3 & 6 \\
\hline $251-500$ & 9 & 67 & 28 & 5 & 6 \\
\hline
\end{tabular}

b. Limiting cases for effect of coal substitution for premium fuels

\begin{tabular}{ccc} 
& Increase in $0 i 1$ or Gas \\
Increase in & Available for 0ther Uses \\
Coal Usage & $0 i l$ & Gas \\
$(\%$ of total coal $)$ & $(\%$ of total oil) & $(\%$ of total gas $)$ \\
\hline
\end{tabular}

61

8

31

oil and gas as boiler

fuel

II. Coal substituted for

88

11

45

oil and gas as boiler

fuel and process heat

In figure 5, the recent pattern of coal consumption in the U.S. is depicted. The inserted percentages indicate the anticipated growth rate per year in each sector to 1985. At present, central station generation of electricity accounts for approximately $70 \%$ of coal usage ( $85 \%$ of which is in pulverized form), while industry (industrial steam raising and process heat for coking operations in the steel industry) accounts for about $21 \%$ (of which about $12 \%$ is used in pulverized form). 


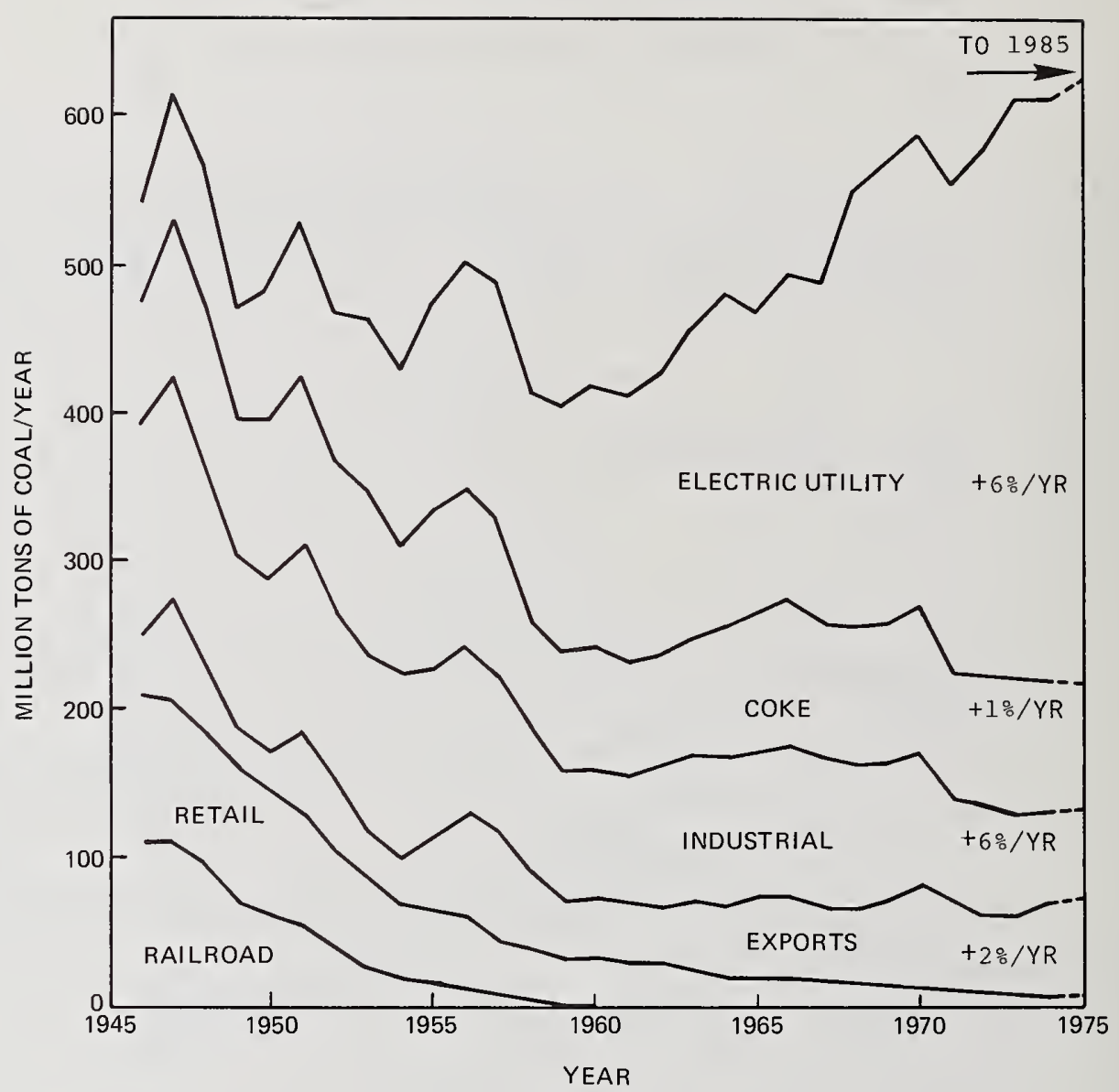

Figure 5. Coal consumption in U.S. according to end use sector [9].

We believe the foregoing description to be a reasonably conservative account of the present situation and prospects for near term coal utilization in the context of the overall U.S. energy situation. Expansion of coal utilization in the electric utility sector is necessary. Substitution of coal or coal-derived liquid and gaseous fuels in the industrial sector is desirable. At present rates of utilization, domestic supplies of natural gas and $0 i 1$ will be rapidly depleted. $0 i l$ prices have increased dramatically and further expansion of imports would be required.

Although development and demonstration of new coal conversion systems and advanced coal combustors for fluidized bed, magnetohydrodynamic, and gas turbine stationary power systems are underway (principally under DOE sponsorship), it has been conservatively estimated that these new systems will have only minor impact on power generation capacity during the near term, i.e., the next 20 years [10] (certainly, a major portion of the technical lifetimes of readers of this paper). Similar uncertainties are generally accepted with regard to the prospects for expansion of nuclear fission power, solar power, 
and advanced fuel cells. In contrast, improvement in existing technologies of steam raising and industrial process heating through the clean, direct firing of pulverized coal can have major and immediate impact in the near term and provide additional lead time and insurance for the proof of the reliabilities and economies of the advanced technologies. 2

The Division of Fossil Fuel Utilization/DOE has established a broad program of research and development related to meeting the long term objective of increased coal utilization, and in particular, to developing various technologies which fall into the so-called advanced category. Given the above near term needs and possible remedies, the objective of this assessment is simply to provide assistance in answering the question -to what extent, if any, is increased DOE support for research and development related to more conventional pulverized coal combustion technologies warranted? To further place this question in context, as indicated in figure 6 , we may note that the fraction of the present DOE program in this area is small (including work on coal beneficiation, and the combustion of slurries of oil and pulverized coal, the total is less than $0.5 \%$ of the fossil energy budget).

\footnotetext{
${ }^{2}$ Substitution, for $0 i 1$ and gas fired industrial units, of stoker furnaces, which use coal in lump form, will provide further relief. However, combustion equipment manufacturers and potential users in the United States, Australia and New Zeal and see a potentially expanded market in the near term for small pulverized coal-fired industrial boilers. In principal there is no minimum size restriction on pulverized coal-fired systems. Somewhat artificial, but nevertheless, practical limits have been placed due to the past unfavorable economics related to supplying, grinding and firing of coal in pulverized form in small units, i.e., less than $200,000 \mathrm{lbs}$ steam/hr (20 MWe).
} 

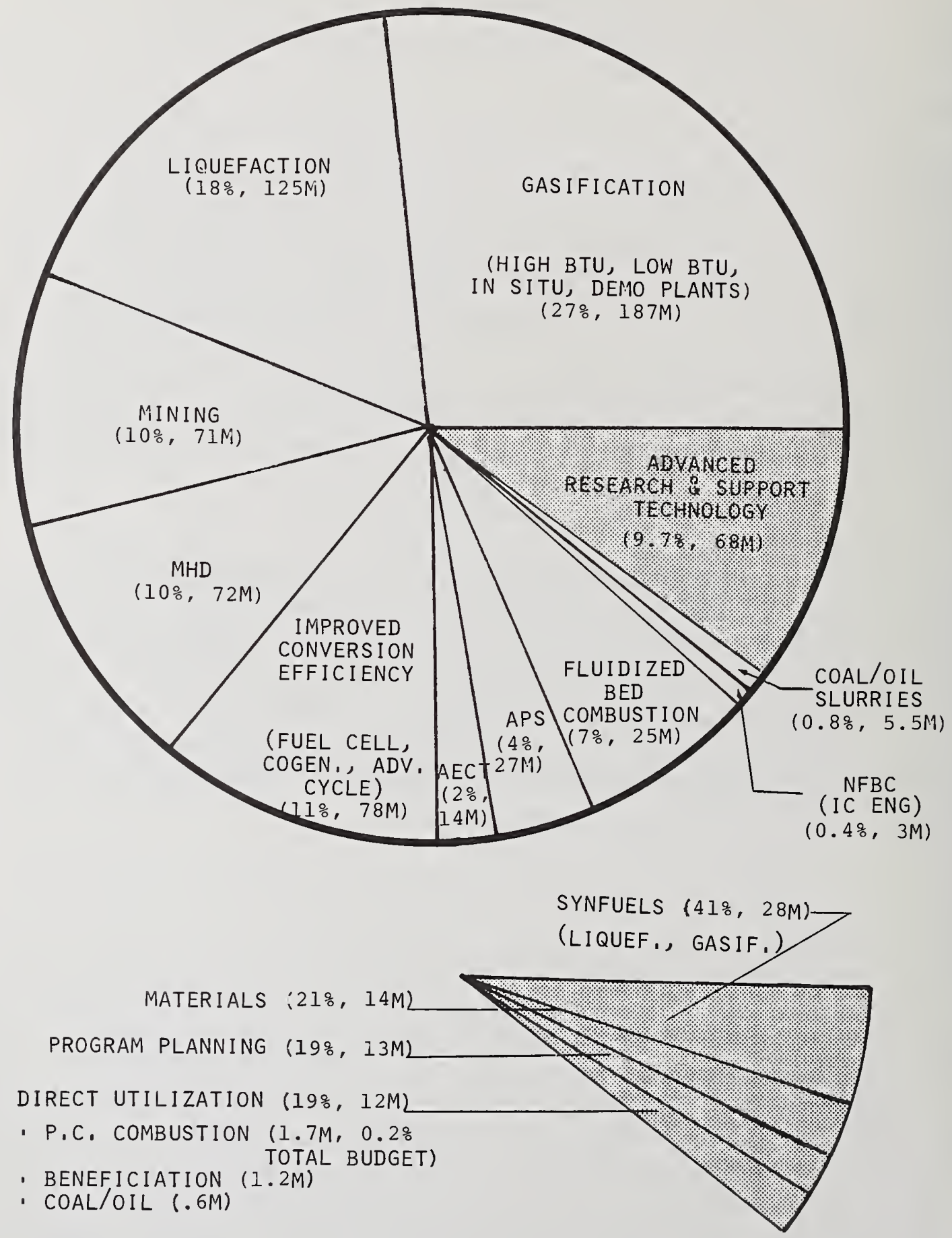

Figure 6. Proposed budget for FY79 for Fossil Fuel Utilization/DOE [11]. 
3. Limitations and Research and Development Challenges to

Increased Use of Pulverized Coals

Based on the foregoing analysis the near term reality is clear and industry has already begun to respond. A mandate for increased firing of coal in pulverized form, principally in large electric utility boilers, exists. Industry has reacted. Figure 7 , summarizes one major manufacturer's recent orders for new central station utility boilers. The shift in distribution among coal types is significant and we will return to this point. For the moment, the point is that, for this firm, no new orders for oil or gas fired plants have been placed since 1974. A recent survey [6] of all equipment manufacturers indicates that $93 \%$ of all new base load plants will be coal fired.

Total capacity million $\mathrm{lb} / \mathrm{hr}$

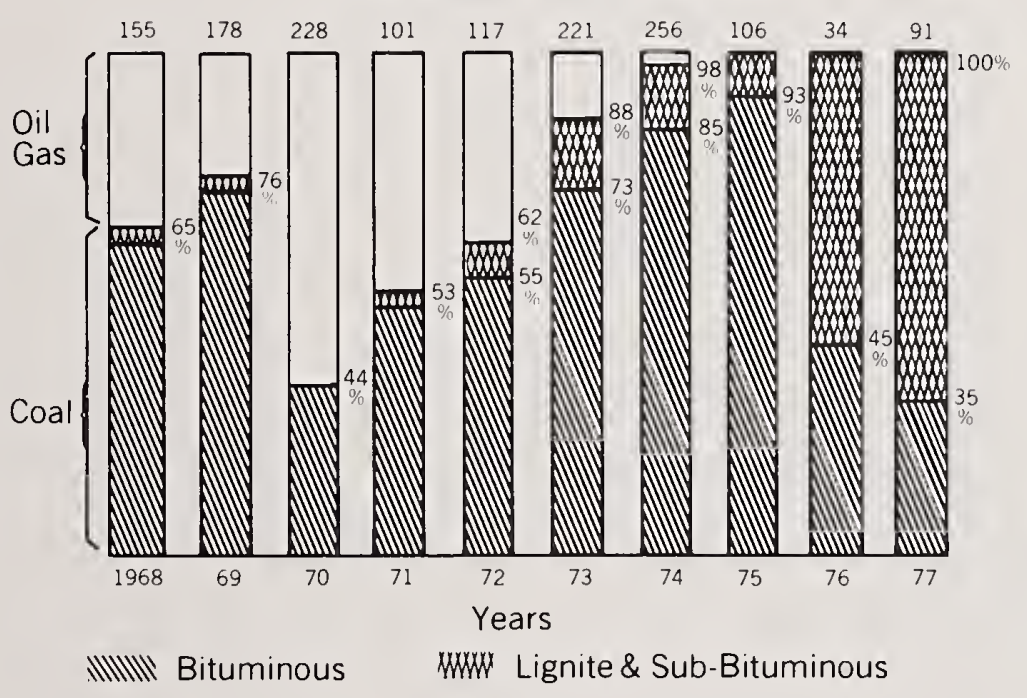

Figure 7. Distribution of electric utility boiler orders [12].

It is often stated that pulverized coal combustion is a well-established technology and that utilization aspects are largely environmentally oriented. From the exclusive standpoints of present generation combustor reliability, safety of operation, and conversion efficiency of carbon and hydrogen to $\mathrm{CO}_{2}$ and water vapor, the argument is sound. Satisfactory systems in the context of earlier demands were engineered with little need for recourse to the understanding of the particular details of the combustion processes involved. There is now, however, increased concern for predicting and restricting combustion-generated pollutant emissions while simultaneously maintaining or improving (through advanced systems) thermodynamic efficiencies in coal combustors. For most pollutants, some degree of control through combustion modification is possible or conjectured. There remain however the additional primary objectives to burn more coal to meet increasing demand and to substitute coals for premium fuels in existing or new systems. 
To meet these objectives, as well as to meet restrictions on sulfur emissions, it has been and will continue to be necessary to burn increasing amounts of coals of lower rank particularly Western lignites and subbituminous coals. These coals typically have more widely variable properties, often higher ash or moisture contents, consequently much lower heating values, and in many cases particularly undesirable ash properties. This state of affairs is transformed into a serious challenge to research and development by the simple fact that the present generation of pulverized coal combustion equipment is essentially coal specific. That is, typically, because of the complexities of the technology, equipment manufacturers have guaranteed system performance and reliability for a single baseline coal. This is not all that surprising. We might expect, for example, that serious troubles of a unique nature would be encountered if we substituted benezene for gasoline in the internal combustion engine. Major problems are frequently encountered when (feed) coal characteristics are changed in conventional pulverized coal systems.

In what follows we shall attempt to describe the general pattern of real problems, mainly derived from the imposition of new constraints, related to pulverized coal combustion. With few exceptions, these problem areas are those that have been stated in the open literature by scientists in the combustion equipment and utility industries in the U.S., Australia and Western Europe and which they discussed with us in detail in the course of our study.

At this point we may summarize these new constraints as the following additional requirements placed on pulverized coal combustion systems:

a. improved and more complete continuous control and monitoring of pollutant emissions;

b. accommodation of a wide variability in coal properties, including "new" coals, beneficiated coals, and solvent refined coals (e.g. SRC1);

c. substantial improvement in combustor/burner flexibility for control of excess air, to permit wider variation of coal/air flow rates (turndown ratio), and for expansion of pulverized coal firing in smaller industrial systems, and;

d. minimization of efficiency losses, maximization of availability, reliabiliț and safety, and minimization of costs.

Recommendations, by the various representatives from industry, of research needs, of both a fundamental, and more applied nature, are included. Because of space limitations, we have chosen to elaborate here upon only a selected few of the problem areas - those which we believe to be of special relevance to the materials scientist. 


\section{Mechanisms of Pulverized Coal Combustion}

\subsection{Pulverized coal fired boilers}

To put the subsequent discussion of research needs in perspective it is helpful to have at least a qualitative description of the macro- and micro-mechanisms of pulverized coal combustion. In figure 8 a schematic of a conventional front-wal1, pulverized coalfired utility boiler and reheat Rankine cycle is shown. Furnace cross sections are on the order of $50 \mathrm{ft} . \times 70 \mathrm{ft} .(15 \mathrm{~m} \times 21 \mathrm{~m})$; with the inlet to the convective passages at the superheater (furnace outlet) located $150 \mathrm{ft}$. $(50 \mathrm{~m})$ above the base. In figure 19 profiles of gas temperature and characteristic residence times for such a system are indicated. Crushed coal is transported by conveyer to a continuous pulverizer where final grinding takes place - typically to a specification of 60 to $90 \%$ by weight to a size less than 200 mesh $(74 \mu \mathrm{m})$. Pulverized coal is entrained in a primary air feed ( 15 to $20 \%$ of total air flow) and ducted to the burners where it is injected into the furnace and where turbulent mixing with parallel or coflowing secondary air streams (forced or induced draft) and combustion occur. Peak flame temperature $(1800$ to $2000 \mathrm{~K})$ is obtained in the lower furnace section. Steam is generated by (predominantly) radiant heat transfer from the hot product gases, char and ash to the surrounding furnace water wall tubes. Complete combustion of solid char and soot occurs during the 1 to $2 \mathrm{sec}$ furnace residence time. The products are cooled, due to heat transfer, to furnace outlet temperatures in the range of 1900 to $2600^{\circ} \mathrm{F}\left(\sim 1300\right.$ to $\left.1700^{\circ} \mathrm{K}\right)$. Further heat transfer superheats and reheats the steam in the convective passages where maximum gas velocities are on the order of 50 to $60 \mathrm{ft} / \mathrm{s}$ ( $\sim 15$ to $20 \mathrm{~m} / \mathrm{s})$. Final heat extraction occurs in the economizer where condensed water is preheated. Combustion products, cooled to temperatures above the $\mathrm{H}_{2} \mathrm{SO}_{4}$ dew point, enter various stages of emissions controls, including electrostatic precipitators and wet scrubbers prior to exhaust at the stack.

Typical efficiencies of the principal processes, for a coal fired system are:

a. combustion $\sim 99$ to $100 \%$

b. boiler (total heat input to steam/thermochemical input of coal and air) $\sim 88 \%$

c. reheat Rankine cycle $\sim 40 \%$

d. total plant $\sim 35 \%$ (product of $a \cdot b \cdot c$ ).

At this total plant efficiency, 1 MW electrical (1 MWe) corresponds to approximatley 2.9 MW thermal (MWt). For convenience, table 2 provides a reference for equivalent plant ratings assuming $1000 \mathrm{Btu} / \mathrm{lb}$ steam, boiler and plant efficiencies of 0.88 and 0.35 , and a coal heating value of $10^{4} \mathrm{Btu} / \mathrm{lb}(23 \mathrm{MJ} / \mathrm{kg})$. 


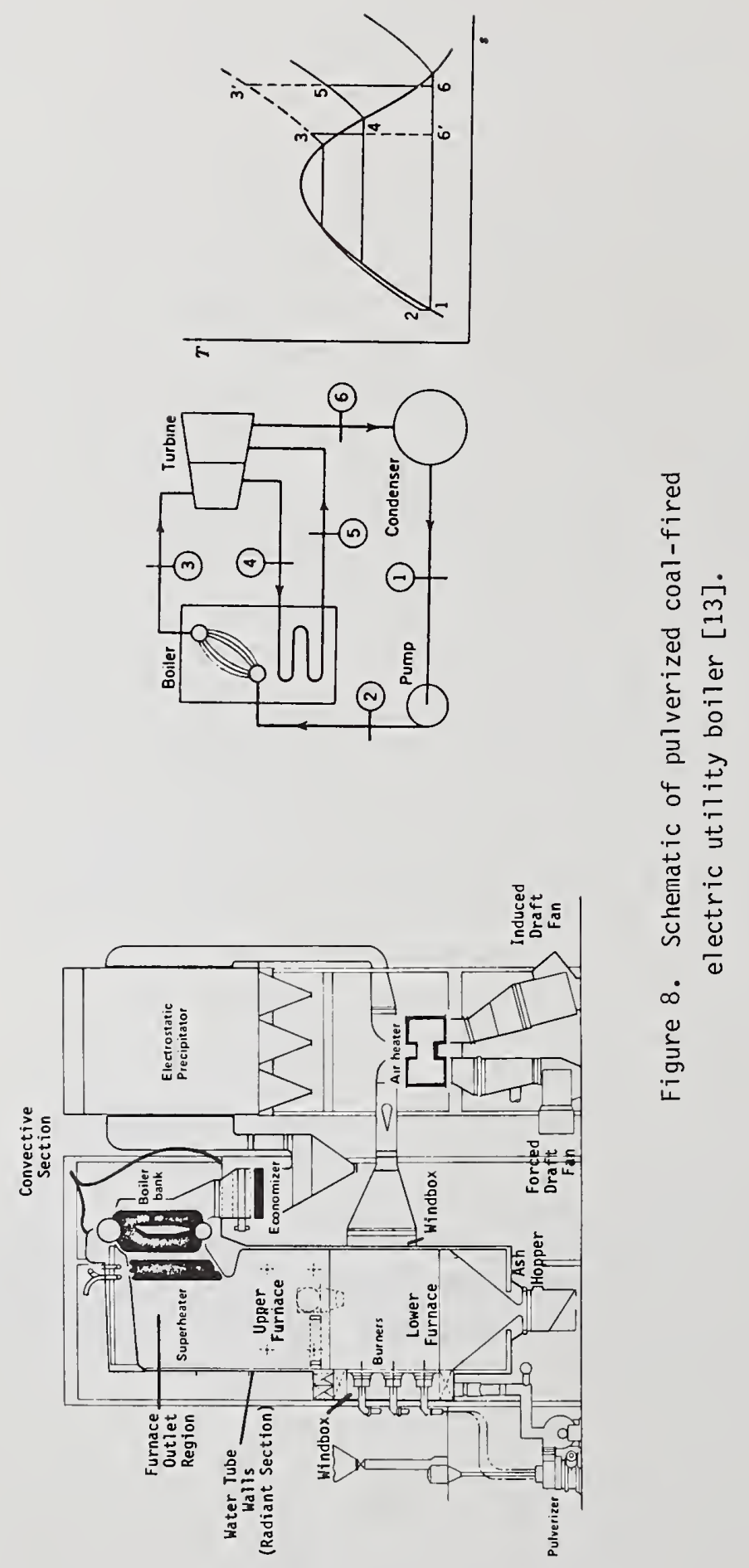


Table 2. Equivalent coal-fired plant ratings

\begin{tabular}{|c|c|c|c|c|}
\hline \multicolumn{2}{|c|}{ Power } & $\begin{array}{c}\text { Steam } \\
\text { Generation Rate }\end{array}$ & $\begin{array}{l}\text { Fuel Thermal } \\
\text { Input }\end{array}$ & $\begin{array}{c}\text { Coal Flow } \\
\text { Rate } \\
\end{array}$ \\
\hline MWe & MWt & lbs steam $/ \mathrm{hr}$ & Btu/hr & lbs coal $/ \mathrm{hr}$ \\
\hline 1 & 2.9 & $10^{4}$ & $10^{7}$ & $10^{3}$ \\
\hline 10 & 29 & $10^{5}$ & $10^{8}$ & $10^{4}$ \\
\hline 100 & 290 & $10^{6}$ & $10^{9}$ & $10^{5}$ \\
\hline 1000 & 2900 & $10^{7}$ & $10^{10}$ & $10^{6}$ \\
\hline
\end{tabular}

\subsection{Burners for pulverized coal combustion}

Systems for introducing the pulverized coal into the lower furnace and for controlling the turbulent mixing of the coal stream with air range from relatively simple to complex in design. Roughly speaking there are two classes of systems: (a) multiple ports or registers, which basically consist of lourred terminations to the coal and secondary air pipes, which cause coal and air stream impingement within the furnace and which produce what we might call a furnace-stabilized $f l$ ame and (b) burners which integrate the primary air/coal stream and secondary and tertiary air streams in individual units, such that combustion is stabilized at each burner. In figure 9, a schematic of an example of the second type of burner is shown. As indicated in the schematic, for this type of burner, flame stabilization depends upon the aerodynamics and heat and mass transfer properties within the flow field associated with the individual burner.

Self-stabilizing burners of this type have been developed at the Herman Research Laboratories in Australia [1] specifically for firing of low rank coals. It was concluded that a burner with high primary (coal plus air) and secondary (air) swirl would induce an adverse pressure gradient along the jet core, leading to prompt stagnation of the primary flow with outward radial coal dispersal, and a flame stabilizing internal, reverse flow, recirculation of hot combustion products al ong the burner centerline. As intended, mixing was dependent primarily on the pressure field induced by the $30^{\circ}$ divergent quarl and was insensitive to a wide variation in flow Reynolds number. This facilitated geometric scaling of test burners to practical sizes. In the absence of the quarl, turbulent shear stress should dominate jet mixing, even if swirl induces vortex breakdown. As a consequence, Reynolds number would have a greater effect and, hence, scal ing would have been uncertain. 


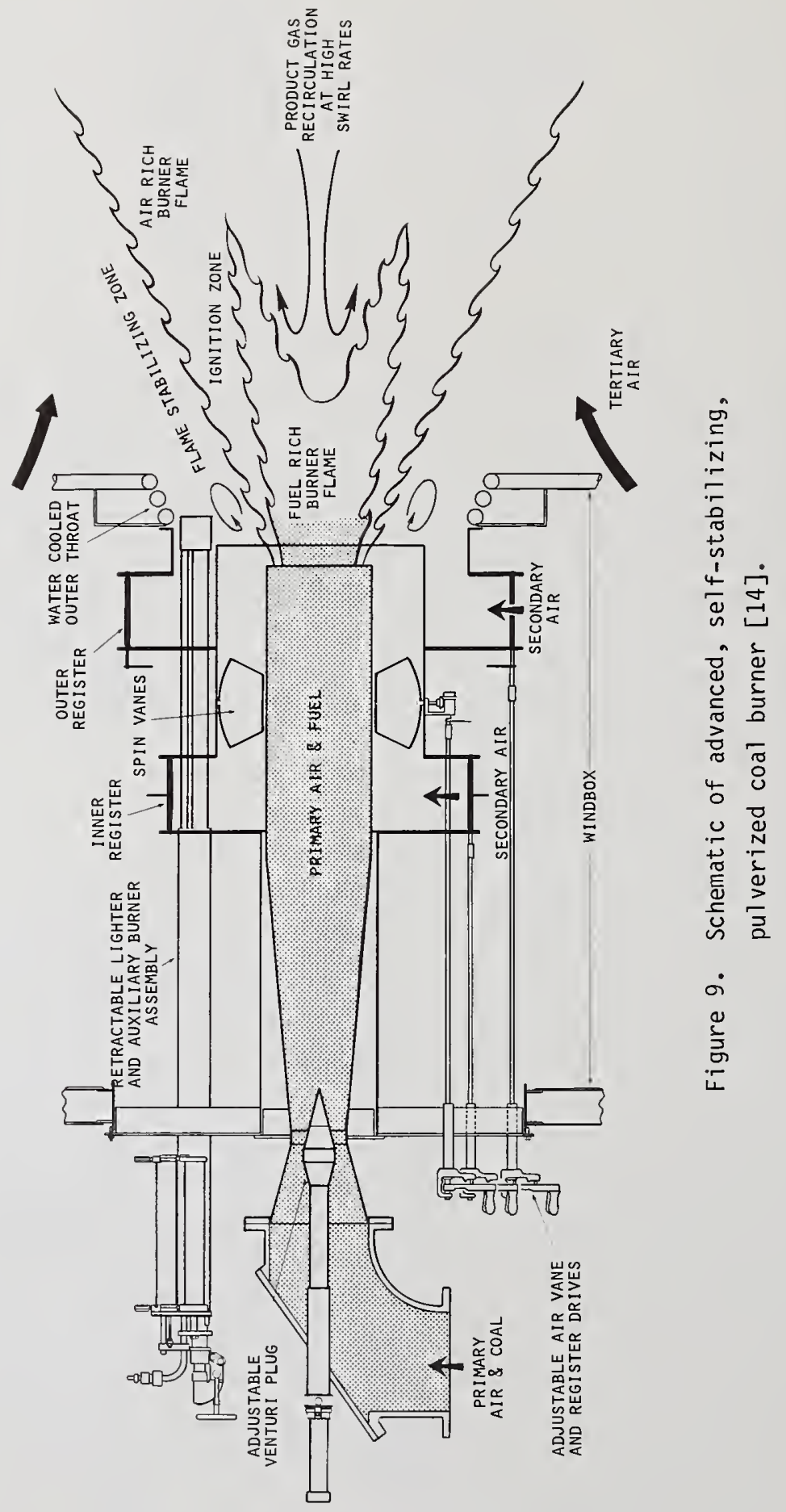




\subsection{Individual coal particle combustion}

On the microscale of an individual coal particle, the combustion process can be visualized, as indicated in figure 10 , as a sequence of rapid heat and mass transfer processes coupled with heterogeneous and homogeneous chemical reaction. While originally intended to dramatize the paths of various coal species to pollutants, the schematic is sufficiently detailed for our purposes here. As regards the formation of the principal gaseous combustion products, $\mathrm{CO}_{2}$ and $\mathrm{H}_{2} \mathrm{O}$, the combustion process can be described in terms of a rapid devolatilization step which occurs in the lower furnace on a time scale of a few to $100 \mathrm{msec}$ and a slow char burnup phase which ocsurs within the furnace enclosure on a time scale of 0.1 to $2 \mathrm{sec}$.

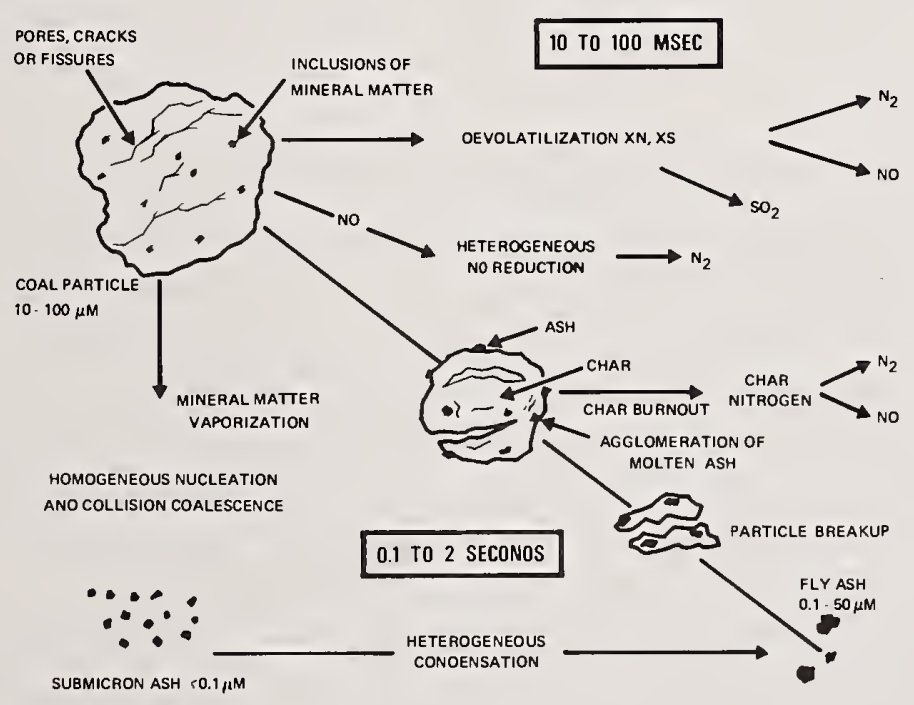

Figure 10. Schematic of mechanisms of combustion of a single pulverized coal particle [15].

There is sufficient data, much of it of a qualitative nature, to indicate that overall rates, extents of reaction, and detailed mechanisms of the oxidation of carbon and hydrogen, as well as the conversion of fuel-bound sulfur, nitrogen and mineral matter to gaseous and solid species, are strong functions of most of the principal properties of the coal particles (particle size, particle porosity or internal surface area, moisture content, and elemental chemical composition) and the surrounding gaseous environment (temperature, oxygen concentration, and total pressure). Small particles will be in Stokes flow, such that predominent heat transfer occurs via gas phase conduction and radiation from surrounding gas and nearby particles. For appreciable rates of spin or swirl imparted to the primary air/coal stream, larger particles will not follow the streamlines and convective heat transfer increases in importance. 
There is extensive data (see for example Mulcahy and Smith [16]) for the combustion, at atmospheric pressure, of chars, cokes and selected coals which indicates that the combustion process for particles in the pulverized coal regime $(<100 \mu \mathrm{m})$ is dominated by chemical kinetic reaction rates, while mass transfer processes control the burning rate of larger particles.

We don't wish to dwell on the heuristic depiction, in figure 10, of the variety of pathways for formation of pollutants from fuel-bound nitrogen, sulfur and mineral matter. Merely note that from a mechanistic view, it is believed that most of the coal hydrogen is released, along with an appreciable fraction of sulfur, during the rapid devolatilization step, that two routes (a rapid one and a slow one) to No are possible, one of which offers the prospect for homogeneous gas phase reduction of NO to $\mathrm{N}_{2}$, and several steps exist for conversion of mineral matter to fly ash. We will elaborate on the latter in a subsequent section.

\section{Summary: Specific Problem Areas Which Require Advanced Research}

Table 3 summarizes problem areas related to pulverized coal combustion, in the context of the constraints noted above. Much of this information derives from our discussions with the four principal manufacturers of pulverized coal combustion equipment in the U.S. and with electric utility and industrial coal combustion engineers in the U.S., Australia, Western Europe and Poland. During these discussions three points have been made clear. First, pulverized coal combustion technology is well-developed for obtaining maximum cycle efficiency (consistent with certain safety and reliability requirements) in the absence of the new constraints. Secondly, research at a fundamental and applied level would be helpful in developing new technologies to accommodate the new constraints. Finally, and perhaps most importantly, in their view, for the work to have any impact on practice, research results must be related to the practical systems either by demonstration of similar functional relationships between variables or by conducting the studies in situ (e.g., diagnostics measurements of local flame properties in systems no less than $1 / 3$ scale).

The categories identified in table 3 are somewhat arbitrary, however, the key point is that there is great overlap among problem areas and consequently among research and development approaches to solutions. We are not prepared at this point to define priorities among these problem areas. It is not clear that we will want to do so at the conclusion of our assessment in view of the broad scope of expressed needs. Rather, at this point we choose to elaborate upon a selected few problem areas which seem to be particularly well suited to attack by the materials scientist. In the next two sections we will treat in more detail, areas for research related to (a) the increased variability of the as-fired properties of coals and (b) the fate of mineral matter during and subsequent to pulverized coal combustion. 
Table 3. Specific problem areas for research

\begin{tabular}{|c|c|c|c|}
\hline $\begin{array}{l}\text { Continuous Control and } \\
\text { Monitoring of Emissions }\end{array}$ & Coal Properties & Combustor/Burner Flexibility & $\begin{array}{l}\text { Maximize Availability, Minimize } \\
\text { Efficiency Losses and Costs }\end{array}$ \\
\hline 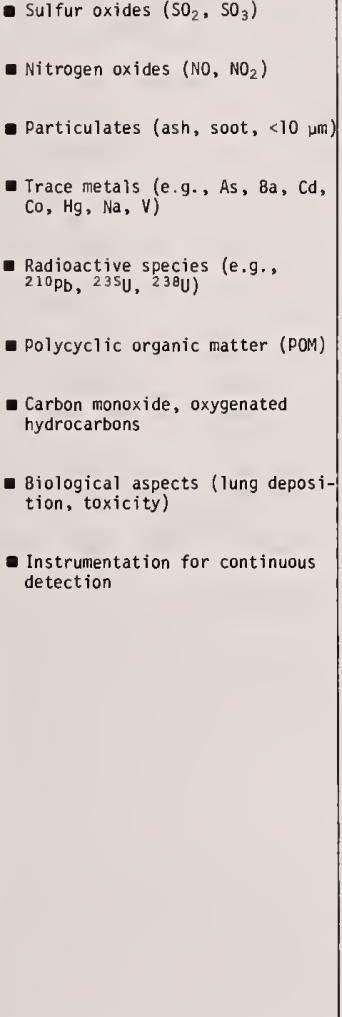 & $\begin{array}{l}\text { Wide variability of coal prop- } \\
\text { erties } \\
\text { - Metallurgicai (anthracites, } \\
\text { bituminous) } \\
\text { - Western coals (lignites, sub- } \\
\text { bituminous) } \\
\text { - Beneficiated coals } \\
\text { - SRCl } \\
\text { - Mineral matter (MM) properties } \\
\text { - Inherent components } \\
\text { - Extraneous components } \\
\text { - Ash thermochemistry } \\
\text { - Ash acidity } \\
\text { - Ash resistivity } \\
\text { - Catalytic effects } \\
\text { - Basic coal properties } \\
\text { - Rank } \\
\text { - Heating value } \\
\text { - Harticle size, porosity } \\
\text { ignition, devolatilization, } \\
\text { char burnup }\end{array}$ & $\begin{array}{l}\text { - Advanced, self-stabilizing } \\
\text { burner development } \\
\text { - Control of characteristic times } \\
\text { (mixing, combustion, pollutant } \\
\text { formation, MM conversion, pres- } \\
\text { sure drop) } \\
\text { - Variable total coal/air flow } \\
\text { rates (turndown ratio) for load } \\
\text { following } \\
\text { - Startup, shutdown } \\
\text { - Combustion stability } \\
\text { - Advanced systerns } \\
\text { - Ideally adiabatic combustor } \\
\text { - MH0 } \\
\text { - Oirect fired gas turbine } \\
\text { - Staged hot wall process } \\
\text { heaters } \\
\text { - Nonadiabatic } \\
\text { - Small industrial units }\end{array}$ & $\begin{array}{l}\text { - Engineering models of total boiler } \\
\text { - Main furnace model } \\
\text { - Corrosion, slagging, fouling } \\
\text { - Efficiencies } \\
\text { - Fluid mechanics, heat transfer } \\
\text { - Burner design } \\
\text { - Near field model } \\
\text { - Furnace stabilized versus self- } \\
\text { stabilized combustion process } \\
\text { 0iagnostics at R\&0 level } \\
\text { - Instrumentation for continuous flame } \\
\text { monitoring and control; safety } \\
\text { - Excess air minimization } \\
\text { - Enit size } \\
\text { - Availability }\end{array}$ \\
\hline
\end{tabular}

While we have not emphasized purely environmental and economic factors which are called out in table 3 , it is convenient at this point to summarize the nature of these two constraints. We should also note that while we are providing here a general background to the subject area, there are many specific areas and research needs which we will not discuss in detail in this paper. Some of these are being more or less actively investigated at the present time. For example, the EPA Combustion Research Branch, RTP has initiated R\&D programs in the area of $\mathrm{NO}_{x}$ control by combustion modifications for gas, oil and coal-fired boilers.

\subsection{Environmental factors}

Table 4 summarizes current and proposed EPA standards for control of emissions from coal fired power plants. To date no restrictions have been placed on other pollutant emissions. It is possible that additional standards will be published for such materials as trace metals and fine particulates. As noted in table 4, EPA standards are applied at present to new boilers with capacities above $250 \mathrm{MBtu} / \mathrm{hr}$ ( $25 \mathrm{MWe})$. The last column in table 4 is included to indicate some of the more stringent local standards applied by states to existing or new coal-fired boilers. 
Table 4. New source performance standards for coal fired boilers in excess of $250 \times 10^{6} \mathrm{Btu} / \mathrm{hr}$ [17].

\begin{tabular}{|c|c|c|c|c|c|c|}
\hline & Current & EPA & Goals & Goals & State & \\
\hline Pollutant & EPA & Considered & 1983 & 1988 & $\underline{\text { Restriction }}$ & Regulation* \\
\hline $\begin{array}{l}\text { Nitrogen 0xides } \\
1 \mathrm{~b} \mathrm{N0} / \mathrm{MBtu}\end{array}$ & 0.7 & 0.6 & $\begin{array}{c}0.4 \\
(0.2)\end{array}$ & 0.15 & $\begin{array}{l}\mathrm{DE} \\
\mathrm{NJ} \\
\mathrm{MA} \\
\mathrm{OH}\end{array}$ & 0.3 \\
\hline $\begin{array}{l}\text { Sulfur Oxides } \\
\text { 1b } \mathrm{SO}_{2} / \mathrm{MBtu}\end{array}$ & 1.2 & 1.2 & 0.8 & 0.4 & $\mathrm{NJ}$ & 0.3 \\
\hline $\begin{array}{l}\text { Particulates } \\
\text { lb/MBtu }\end{array}$ & 0.1 & --- & --- & --- & VT & 0.02 \\
\hline
\end{tabular}

In figures 11,12 and 13 we have combined the forecasts of increased coal utilization in electric utility plants (figure 3 ) with existing or proposed EPA standards, the assumed year of introduction of these standards, and an assumed rate of retirement of existing plants [5] (which are presumed to operate at earlier standards - higher emission levels over a plant lifetime of 30 years). This is done to demonstrate the anticipated overall levels of control for emission of particulates, nitrogen oxides and sulfur oxides from coal fired systems.

Several points are worth explanation. For particulate emissions the influence of retirement of old plants dominates, given the effectiveness of control strategies for particulate removal from stack gases on a weight basis. If we had chosen to factor in the effect of poor control, using existing technologies, for fine particulates $(<5 \mu \mathrm{m})$ and had we scaled the ordinate in terms of number of particles emitted, the curves would show a positive slope.

For $\mathrm{NO}_{2}$ emissions, two curves are shown to indicate the influence of the year of imposition of the more stringent $0.15 \mathrm{lb} \mathrm{N0} / \mathrm{MBtu}$ standard.

The 1 owest curve for $\mathrm{SO}_{2}$ emissions reflects the most stringent standard for this pollutant. According to this standard, maximum emission rate of $1.21 \mathrm{bs} \mathrm{SO}_{2} / \mathrm{MBtu}$ will be placed on high sulfur coals. For those coals which would yield emissions in excess of this 1 imit, $85 \%$ removal of sulfur from the system is required. For coals which yield lower emissions, sulfur removal is required to a level equivalent to enissions for combustion of a coal having $0.3 \%$ by wt sulfur content.

\footnotetext{
*Comparative values for states computed assuming 500 MWe utility plant boiler and state specified standard.
} 


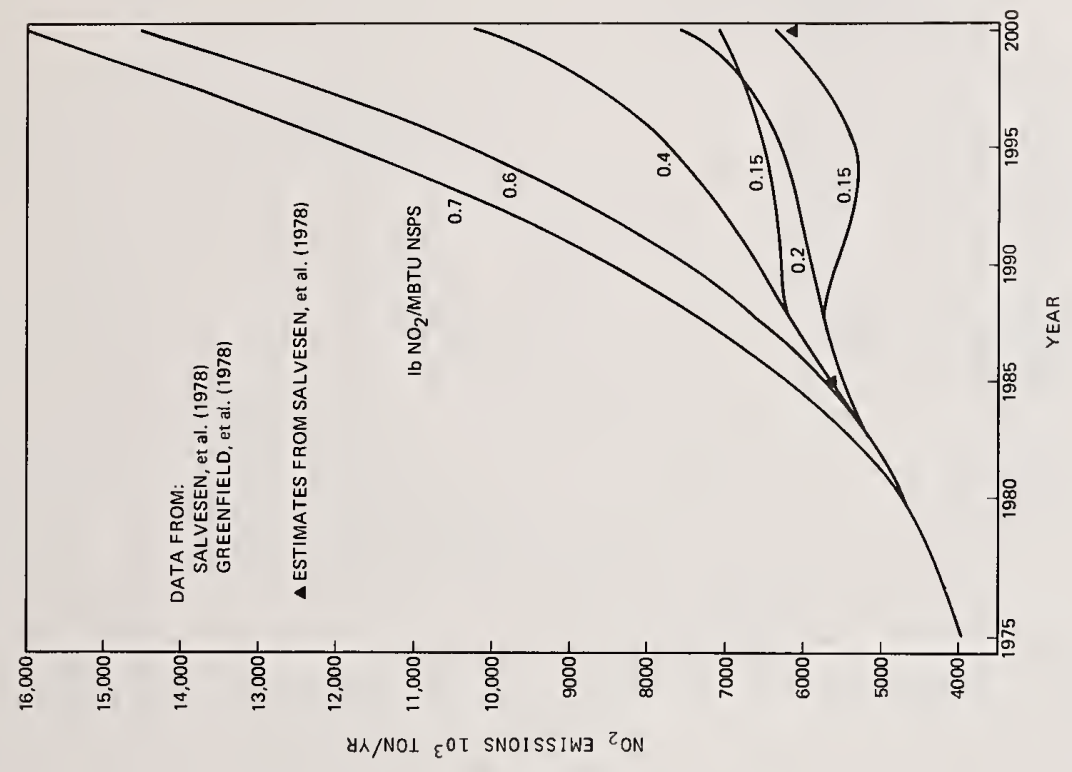

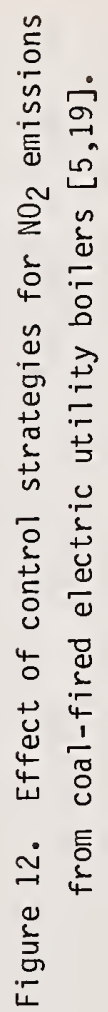

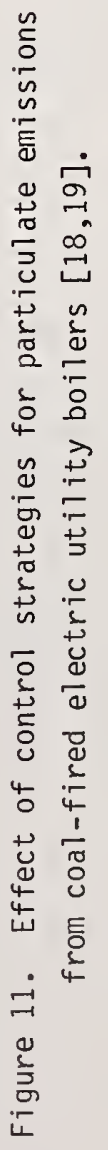




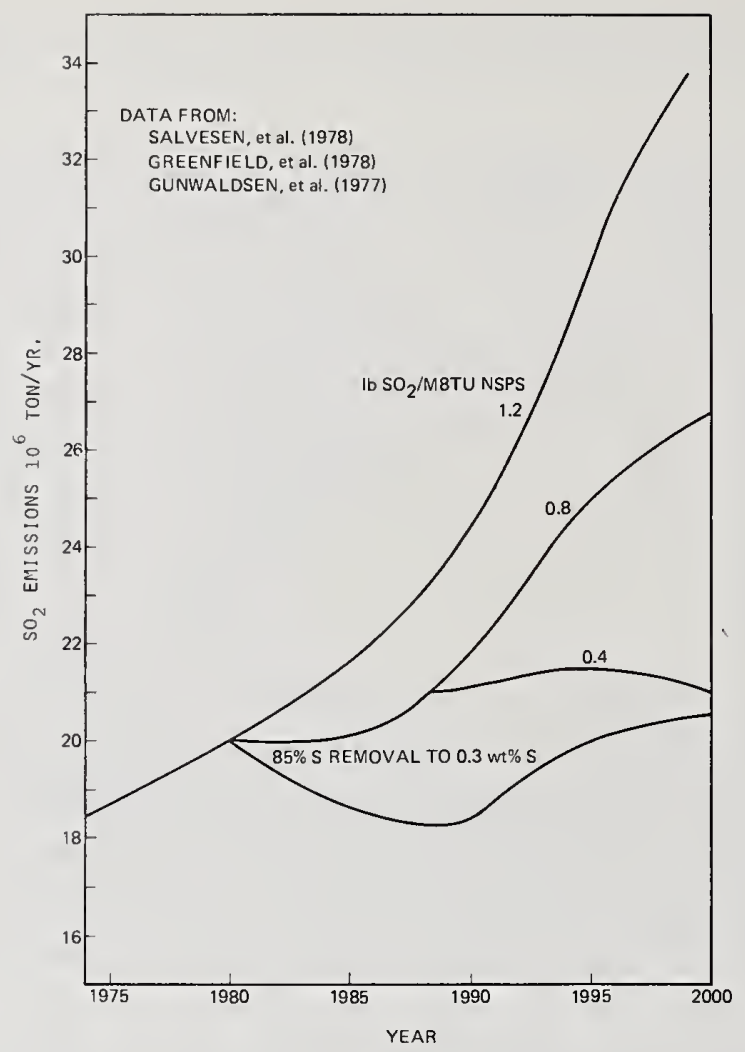

Figure 13. Effect of control strategies for $\mathrm{SO}_{2}$ emissions from coal-fired electric utility boilers $[5,19,20]$.

\subsection{Cost factors}

In figure 14 and table 5, examples are given to indicate the order of magnitude of the economics involved in pulverized coal fired utility plant operation. The point to be made is that all of the new constraints impose a premium on new technology development to minimize decreases in efficiency and plant availability. For the example utility plant (1600 MWe average capacity), a $1 \%$ decrease in boiler efficiency (which is incurred, for example, by operating the system at $3 \%$ higher excess $0_{2}$ in the stack gases than required) translates directly into a $\$ 1.8 \mathrm{M}$ annual increase in coal costs for coal priced at $\$ 1.2 / 10^{6}$ Btu (a median price at present). Research to increase the pace of and optimize that technology development will translate into large economic savings. 
ESTIMATED COST OF GENERATING ELECTRIC POWER FROM COAL-FIRED BOILERS

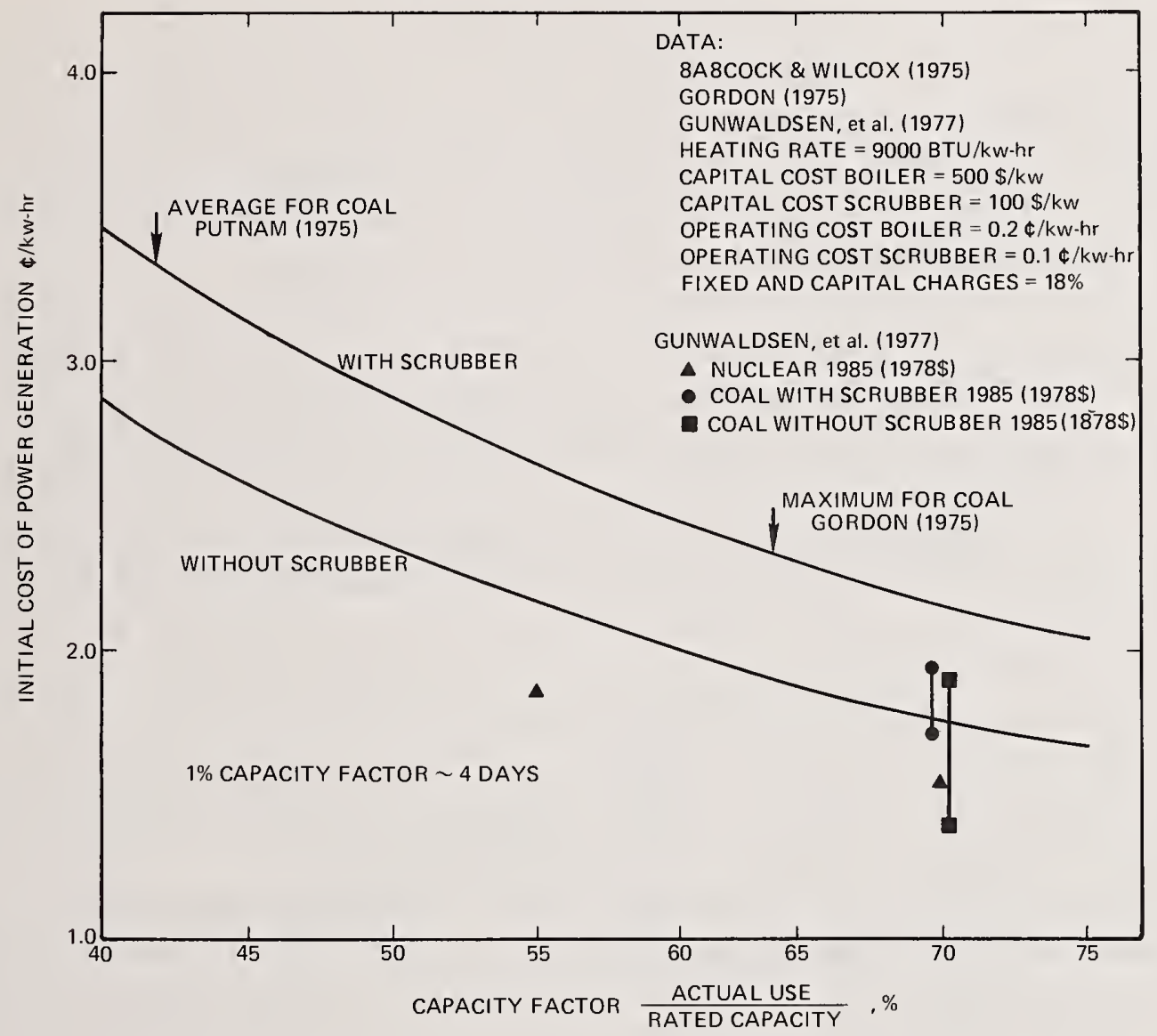

Figure 14. Estimated cost of generating electric power from coal-fired electric utility boilers $[9,20,21,22]$.

Table 5. Differential coal costs for utility boiler inefficiencies in a 1600 MWe (average daily usage rating) electric utility plant.

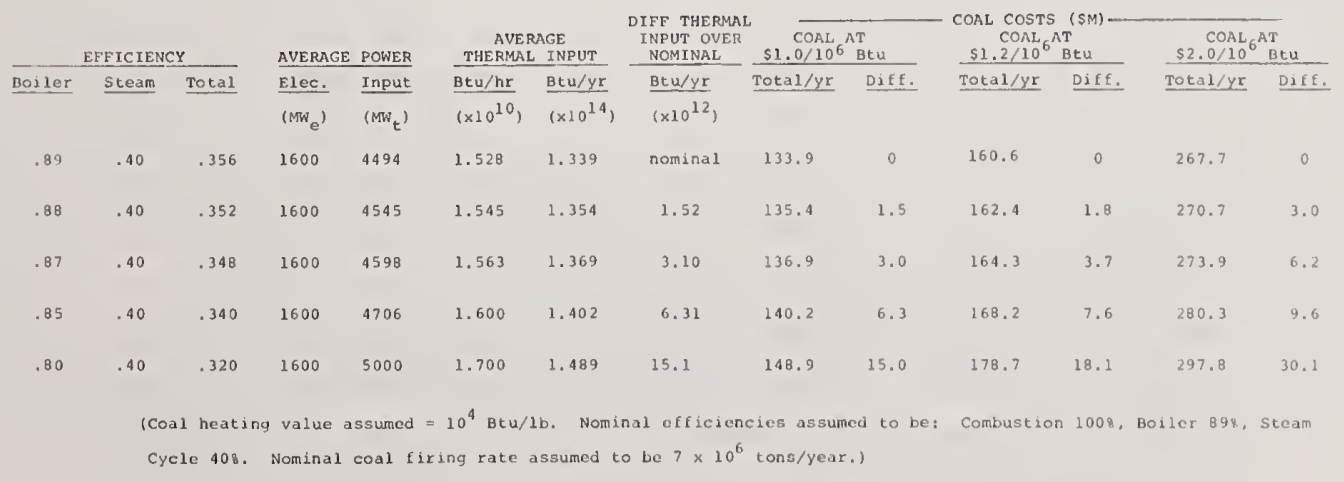

Table VI-5 Differential coal costs for Increased Boiler Inefficsency in a 1600 MW (dasly average) electric utslity plant 


\section{Influence of Coal Properties on}

\section{Pulverized Coal Combustion}

We have noted that the design of pulverized coal combustion equipment is at present largely empirical. Detailed knowledge of the properties of the particular coal which is to be fired is crucial to the design of equipment. Each manufacturer maintains a proprietary data bank of analyses on several thousand samples of coals. A single boiler is guaranteed to meet design specifications in a narrow range around one coal.

In virtually every aspect of design, an effort has been made to relate coal properties, both basic (e.g., chemical composition, heating value, ash composition, and particle size) and derived (e.g., burning indices, fouling and slagging indices, viscosity and fusion temperature of the ash, grindability), to field data on boiler performance, fouling, slagging, and emissions. Useful empirical relationships have been develped, based primarily on previous field experience, to relate derived properties to fundamental properties.

As noted in figure 7 , the imposition of the new constraints has produced a rapid shift from the use of coals, on which a great deal of field data and experience has been accumulated, to new coals - particularly Western lignites, subbituminous coals and possibly, to beneficiated coals - for which experience and data are limited. This has resulted in significant design and operational problems and resulted in marked changes in boiler and burner configurations. Empirical indices which had been applied to other coals have been found to be inadequate or misleading for many of the new coals.

In the following section, we comment further on problems introduced by variation in the composition of the mineral matter in coals as they are fired. In general, the lower heating value of these new coals and the special properties of their combustion-generated ash have required large changes in boiler size and configuration. Due to the necessity to increase coal firing rates to maintain equivalent capacity (a consequence of low heating value), the furnace size for lignites may be up to $50 \% 1$ arger than an equivalent furnace for bituminous coal firing. The size increase also satisfies the need to provide sufficient residence times for carbon burn out as well as the requirement to avoid $\mathrm{fl}$ ame (and ash) impingement on the water tube walls for the furnace. Furthernore, sufficient residence time must be permitted to reduce gas temperatures at the furnace outlet to at least $40^{\circ} \mathrm{K}$ below the ash fusion temperature.

The rapid shift to the use of coals which have wider range in heating value is noted in figure 15a. In figure 15b, the shift to much lower furnace zone heat release rates is noted. The variability of the as-fired coal properties has increased the need for coal burners which permit wide flexibility in burner operation as well as more precise control over the principal operational variables. Self-stabilizing burner designs (originally employed for control of $\mathrm{NO}_{x}$ emissions) have been adopted by several manufacturers and utilities in the U.S. and Australia for improving the ability to reliably and efficiently control the combustion so that a wider range of coals can be burned in a single boiler design. In summary these burners are being designed to meet the following objectives: 
- Control of formation and emission of oxides of nitrogen, by producing slower turbulent mixing of coal and air flows, to reduce peak flame zone temperatures and to control local oxygen concentrations in the flame zone;

- El iminate fuel rich zones near water tubes (which accompany staged firing) in the boiler, where overall reducing conditions contribute to corrosion and slagging;

- Increase burner flexibility to fire coals having variable properties, including beneficiated coals and SRC1;

- Increase the turndown ratio of individual burners to improve load following characteristics;

- Increase burner flexibility to permit a single burner design to be scaled for use in a utility or industrial boiler;

- Improve combustion stability characteristics and reliability of individual burners to reduce or eliminate requirements for auxiliary oil or gas firing during boiler start up;

- Reduce boiler inefficiency by minimizing the total excess air requirement through control of air supply to individual burners or to groups of burners fed by individual pulverizers.

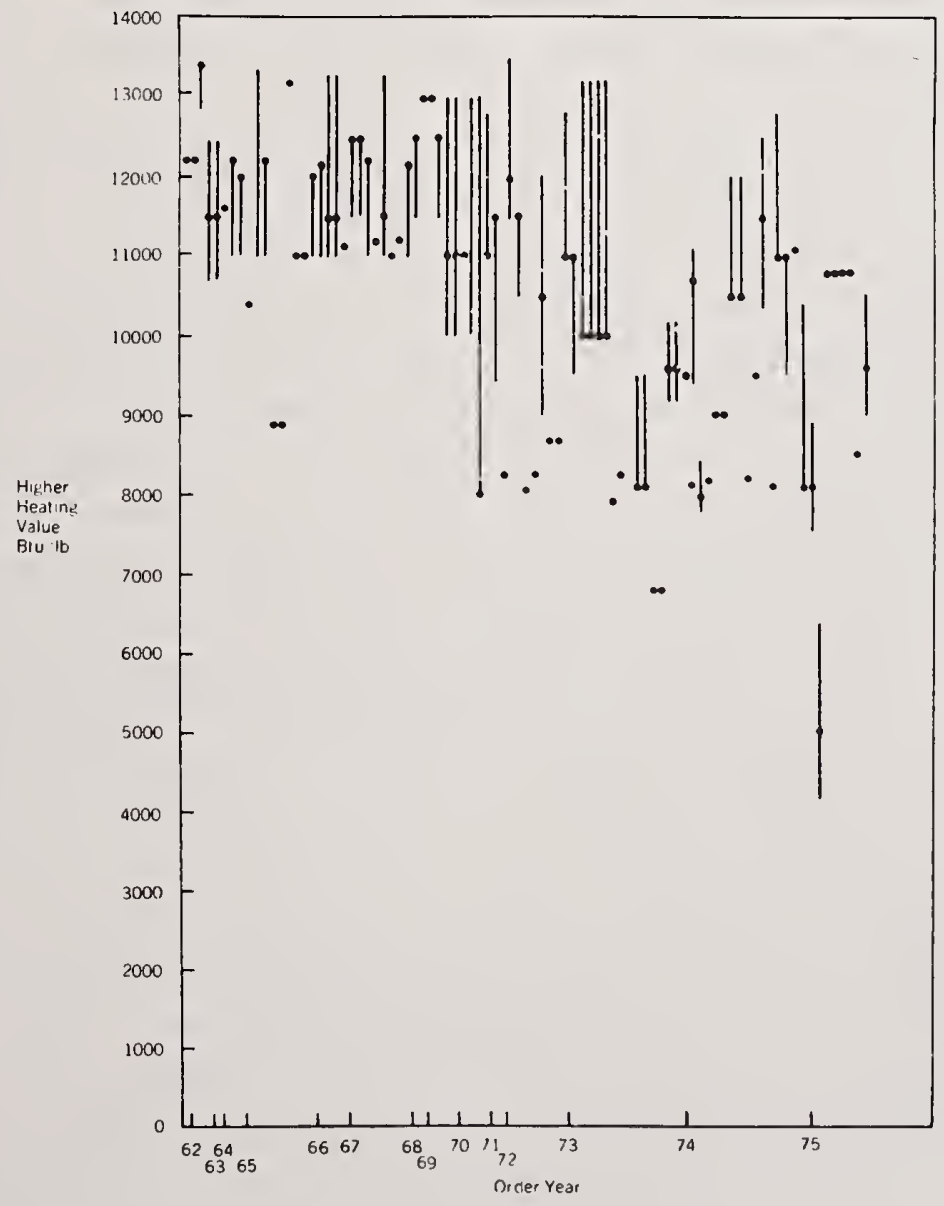

Figure 15a. Range of coal heating values for which recent electric utility boilers have been designed [12]. 


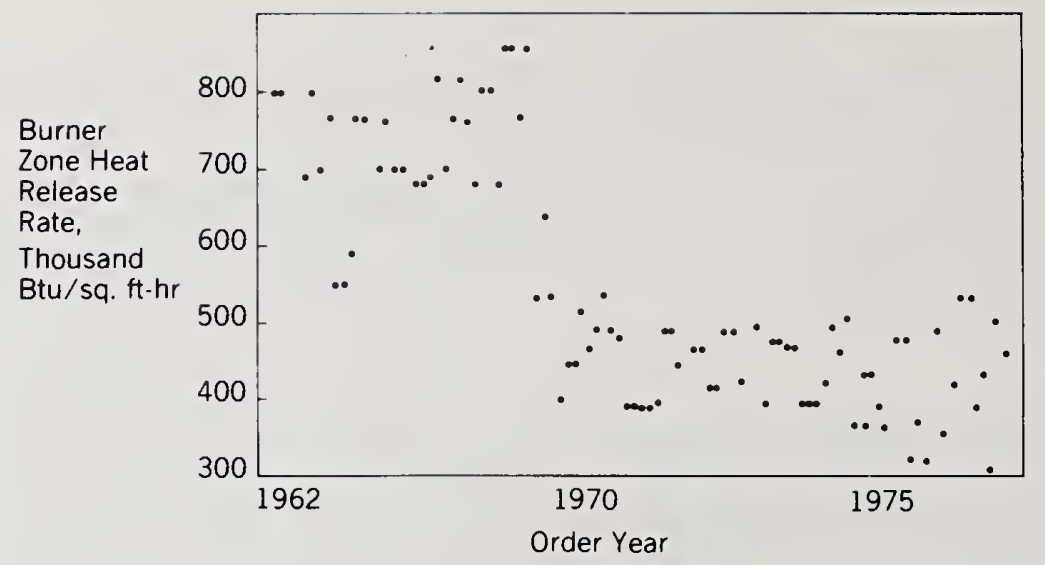

Figure 15b. Design points for heat release rate in the burner zone for recent coal-fired utility boilers [12].

Design and operation of these new systems depends critically upon the near field properties of the burner/flame environment. Basic research on improved diagnostic systems for defining the $\mathrm{fl}$ ame zone properties and the application of these diagnostic techniques to define the dependence of these properties (e.g., local temperatures, velocities, oxygen concentration, coal distribution, ignition point, and $f l$ ame stability) on burner operational variables (e.g., total flow rates of coal and air, initial velocity and mass distributions, turbulence, swirl rates, and pressure drop) during the design and full scale testing is one area where payoffs will be large. Similarly the development of improved predictive capabilities - modeling of the multi-phase combustion process - is needed.

Finally, we must emphasize that the combustion process is strongly dependent upon the chemical and physical properties of the coal. Industry has developed several empirical burning rate indices which have been used to assist in designs. At present these indices are based on thermogravimetric analyses (TGA) of individual coal samples. Typical data are shown in figure 16. Data of this type is used to qualitatively define the relative ease (ignitability, burning rate, total burn-up time) with which a particular coal may be burned in a particular boiler - when used in combination with a specification of coal particle size and previous field experience with each coal in the same boiler. The difficulty comes with the lack of field data for the new coals.

Since the TGA data are not directly related to combustion characteristics, a need exists for more precise experimental and analytical techniques to define the ignition and combustion characteristics (relevant to practice) of a wide range of coals. Ideally such techniques would permit further definition of the rates and mechanisms of conversion of trace species, including fuel-bound nitrogen, sulfur and mineral matter, to polluting and fouling products. 


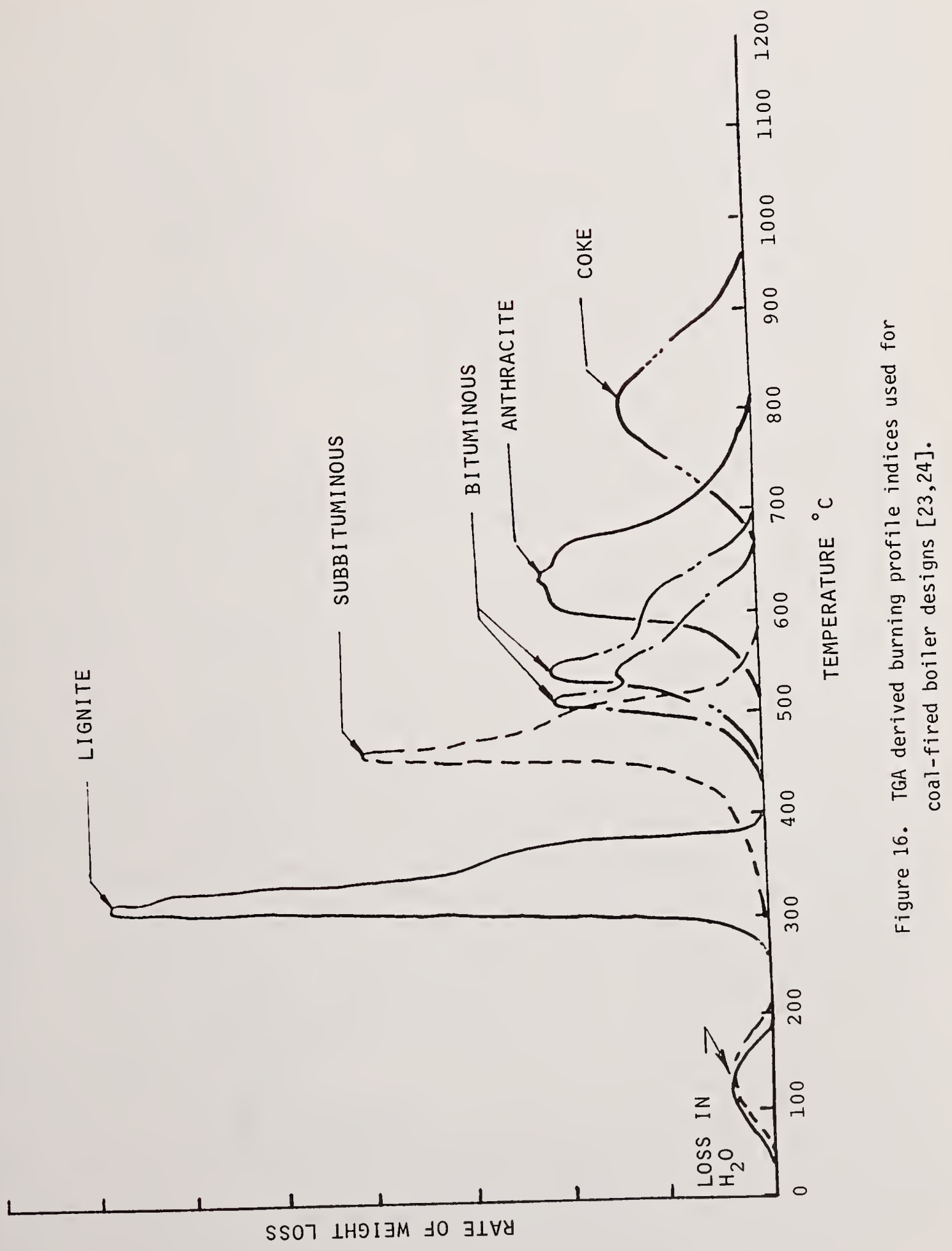



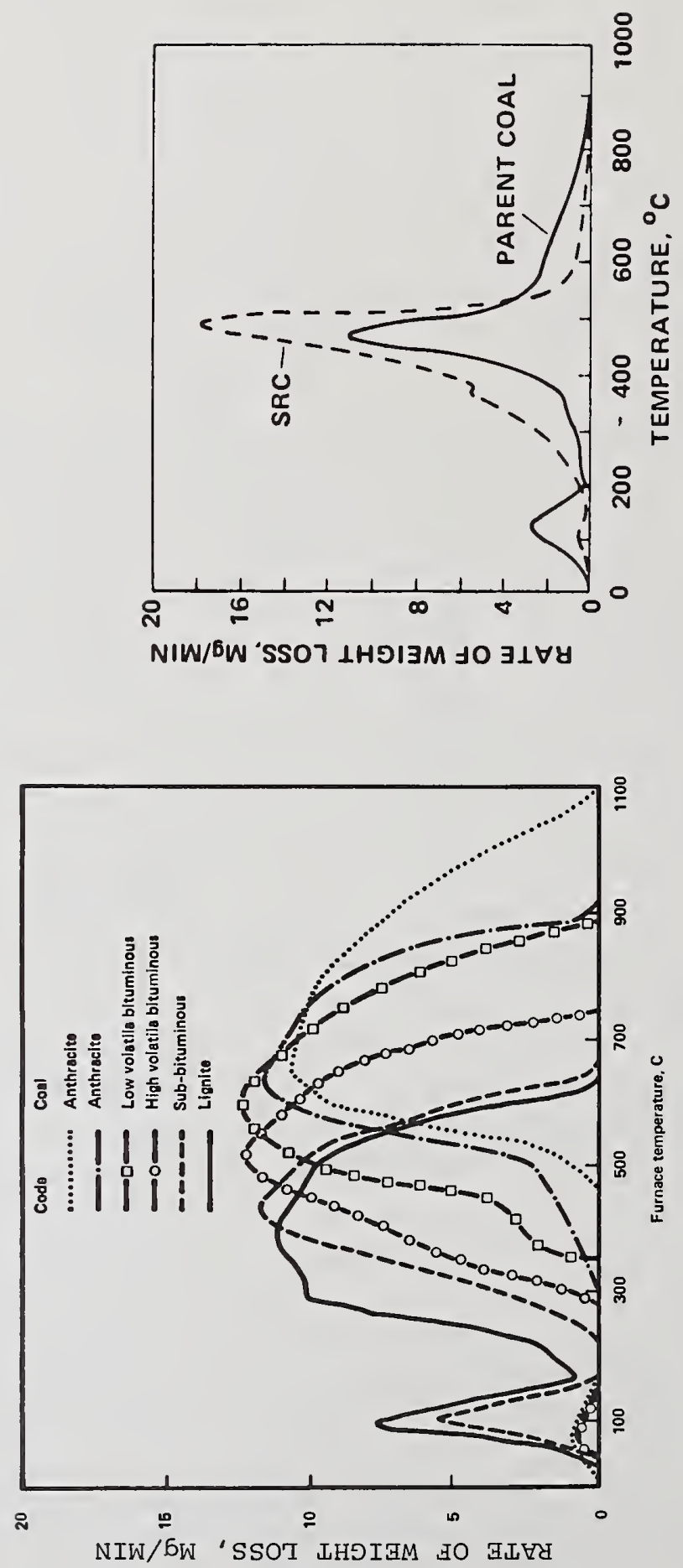

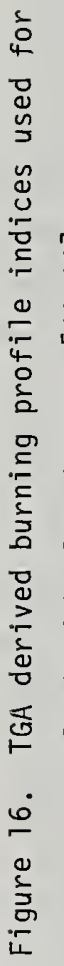




\section{The Effect of Mineral Matter in the Combustion Process}

In combustion processes ash has a well earned reputation; all bad. Mere presence of mineral matter can disrupt the processes of burning coal. The presence of ash increases the cost of transporting and grinding coal, and makes the coal more difficult to ignite and burn stably. Large quantities of ash must al so be removed from the stack and ash hoppers and disposed of. For instance, a 500 MWe $\mathrm{pl}$ ant burning a 10\% ash coal produces 200,000 tons of ash per year and this ash occupies a volume of about 300 cubic yards.

In addition to the problem of having mineral matter present in the coal, particular elements in mineral matter cause problems such as slagging, fouling, and corroding the boiler and polluting the atmosphere. While information on these processes is typically insufficient to indicate strategies to control these problems, some elements have been clearly implicated as contributors to the problems. For instance, sulfur contributes to fouling and air pollution, sodium contributes to fouling and corrosion, and mercury contributes to air pollution.

Similar to other naturally occurring materials, the mineral matter contained in coal is extremely complex. As shown in figure 17, most elements of the periodic table have been identified in coal. Those elements which are present in mineral matter in the coal are derived from four sources [25]:

1. Minerals present in the original plant material which formed the coal bed;

2. Minerals present during the initial coalification stage that have become chemically bonded to the coal matrix;

3. Minerals that have been water-borne to cracks and fissures in the coal and deposited by evaporation of water during solidification of the coal;

4. Minerals that are not included within the coal seam but which have been added to the coal during mining from the rock structures that surround the coal.

The minerals from different origins have different physical structures in the coal and behave differently during combustion. Such transformations are shown in figure 18 . Minerals chemically bound to the coal structure will be called inherent mineral matter, regardless of whether this mineral matter was inherent in the plant material or was added during transformation of the plant material into coal. Such mineral matter accounts for only $1-2 \%$ of the mineral matter present in high rank eastern coals [21]. However, inherent mineral matter can account for as much as $80 \%$ of the mineral matter contained in some lignite coals [30]. During combustion this mineral matter is released as finely dispersed particles or vapors.

Included and freed mineral matter accounts for the major portion of mineral matter in most pulverized coals currently fired in the power generating industry. Included mineral matter is material that is finely distributed in the coal seam but which is not a part of the organic structure. Such material is typically added to the coal after the first stage of coalification from mineral-containing waters. Freed mineral matter is included mineral matter that has been released from the coal matrix during grinding of the coal. 


\begin{tabular}{|c|c|c|c|c|c|c|c|c|c|c|c|c|c|c|c|c|c|}
\hline$\stackrel{H}{N A}$ & & & & & & & & & & & & & & & & & $\begin{array}{l}\text { He } \\
\text { NA }\end{array}$ \\
\hline $\begin{array}{l}\mathrm{Li} \\
4- \\
63\end{array}$ & $\begin{array}{c}\mathrm{Be} \\
0.4- \\
6 . \\
\end{array}$ & & & & & & & & & & & $\begin{array}{c}\mathrm{B} \\
1-230\end{array}$ & $\underset{\mathrm{NA}}{\mathrm{C}}$ & $\begin{array}{c}N \\
N A\end{array}$ & $\begin{array}{c}0 \\
\text { NA }\end{array}$ & $\begin{array}{l}F \\
1- \\
110 \\
\end{array}$ & $\begin{array}{l}\mathrm{Ne} \\
\mathrm{NA}\end{array}$ \\
\hline $\begin{array}{l}\mathrm{Na} \\
100- \\
6000 \\
\end{array}$ & $\begin{array}{l}\mathrm{Mg} \\
240- \\
3500 \\
\end{array}$ & & & & & & & & & & & $\begin{array}{c}A 1 \\
3000- \\
23000 \\
\end{array}$ & $\begin{array}{c}\mathrm{si} \\
5000- \\
41000 \\
\end{array}$ & $\begin{array}{r}P \\
6- \\
300 \\
\end{array}$ & $\begin{array}{c}S \\
300- \\
10000 \\
\end{array}$ & $\begin{array}{l}\mathrm{Cl} \\
10- \\
260 \\
\end{array}$ & $\begin{array}{l}\text { Ar } \\
\text { NA }\end{array}$ \\
\hline $\begin{array}{c}K \\
100- \\
6500 \\
\end{array}$ & $\begin{array}{c}\mathrm{Ca} \\
50- \\
12300 \\
\end{array}$ & $\begin{array}{l}\text { Sc } \\
3- \\
30 \\
\end{array}$ & $\begin{array}{c}T i \\
200- \\
1800 \\
\end{array}$ & $\begin{array}{l}V \\
2- \\
77 \\
\end{array}$ & $\begin{array}{l}\mathrm{Cr} \\
10- \\
400 \\
\end{array}$ & $\begin{array}{l}\mathrm{Mn} \\
5- \\
240 \\
\end{array}$ & $\begin{array}{c}\mathrm{Fe} \\
340- \\
23000 \\
\end{array}$ & $\begin{array}{l}\text { Co } \\
1- \\
90 \\
\end{array}$ & $\begin{array}{l}\mathrm{Ni} \\
2- \\
60 \\
\end{array}$ & $\begin{array}{l}\mathrm{Cu} \\
3- \\
250 \\
\end{array}$ & $\begin{array}{l}2 n \\
3- \\
80 \\
\end{array}$ & $\begin{array}{c}G a \\
0.3- \\
60 \\
\end{array}$ & \begin{tabular}{|c}
$\mathrm{Ge}$ \\
0.03 \\
10 \\
\end{tabular} & \begin{tabular}{l|l} 
As \\
$1-$ \\
60 \\
\end{tabular} & $\begin{array}{c}\mathrm{Se} \\
0.04- \\
5 \\
\end{array}$ & $\begin{array}{c}B r \\
1-23\end{array}$ & $\begin{array}{l}\mathrm{Kr} \\
\mathrm{NA}\end{array}$ \\
\hline $\begin{array}{l}\mathrm{Rb} \\
1- \\
150\end{array}$ & \begin{tabular}{|c|}
$S r$ \\
$17-$ \\
1000
\end{tabular} & $\begin{array}{l}Y \\
3- \\
25\end{array}$ & $\begin{array}{l}\mathrm{Zr} \\
28- \\
300\end{array}$ & $\begin{array}{l}\mathrm{Nb} \\
5- \\
41\end{array}$ & $\begin{array}{l}\text { Mo } \\
1- \\
10\end{array}$ & $\begin{array}{l}\text { TC } \\
\text { NA }\end{array}$ & $\begin{array}{c}\mathrm{Ru} \\
<0.1\end{array}$ & $\begin{array}{c}\mathrm{Rh} \\
<0.1\end{array}$ & $\begin{array}{c}P d \\
<0.1\end{array}$ & $\begin{array}{c}\mathrm{Ag} \\
0.1- \\
5\end{array}$ & \begin{tabular}{|c|}
$\mathrm{Cd}$ \\
0.01 \\
3
\end{tabular} & $\begin{array}{l}\text { In } \\
\text { NA }\end{array}$ & $\begin{array}{l}\text { Sn } \\
1- \\
400\end{array}$ & $\begin{array}{l}S b \\
0.1- \\
30\end{array}$ & $\begin{array}{ll}\mathrm{Te} \\
0.1- \\
0.4\end{array}$ & I & $\begin{array}{l}\text { Ye } \\
\text { NA }\end{array}$ \\
\hline $\begin{array}{c}C S \\
0.2- \\
9\end{array}$ & \begin{tabular}{l|}
$\mathrm{Ba}$ \\
$20-$ \\
1600
\end{tabular} & \begin{tabular}{|c|}
$\mathrm{La}$ \\
$0.3-$ \\
29 \\
\end{tabular} & $\begin{array}{c}\mathrm{Hf} \\
0.3- \\
4 \\
\end{array}$ & $\begin{array}{c}\mathrm{Ta} \\
0.1- \\
8\end{array}$ & $\begin{array}{l}W \\
0.1- \\
0.4 \\
\end{array}$ & $\begin{array}{c}\mathrm{Re} \\
<0.2\end{array}$ & $\begin{array}{r}0 \mathrm{~s} \\
<0.2\end{array}$ & $\begin{array}{r}\text { Ir } \\
<0.2\end{array}$ & $\begin{array}{r}P t \\
0.3\end{array}$ & $\begin{array}{r}\mathrm{Au} \\
<0.7\end{array}$ & $\begin{array}{c}\mathrm{Hg} \\
0.1- \\
0.5 \\
\end{array}$ & $\begin{array}{l}\text { TC } \\
0.1- \\
0.3 \\
\end{array}$ & $\begin{array}{l}\mathrm{Pb} \\
1- \\
60 \\
\end{array}$ & $\begin{array}{c}\mathrm{Bi} \\
0.1- \\
i . \\
\end{array}$ & $\begin{array}{l}\text { Po } \\
\text { NA }\end{array}$ & $\begin{array}{l}\text { At } \\
\text { NA }\end{array}$ & $\begin{array}{l}\mathrm{Rn} \\
\mathrm{NA}\end{array}$ \\
\hline \multirow[t]{3}{*}{$\begin{array}{l}\mathrm{Fr} \\
\mathrm{NA}\end{array}$} & $\begin{array}{l}\mathrm{Ra} \\
\mathrm{NA}\end{array}$ & $\begin{array}{l}\text { Ac } \\
N A\end{array}$ & $\begin{array}{c}(\mathrm{Rf}) \\
\mathrm{NA}\end{array}$ & $\begin{array}{c}(\mathrm{Ha}) \\
\mathrm{NA}\end{array}$ & & & & & & & & & & & & & \\
\hline & & & & $\begin{array}{l}\mathrm{Ce} \\
1- \\
30 \\
\end{array}$ & $\begin{array}{r}\mathrm{Pr} \\
1-8\end{array}$ & $\begin{array}{c}\mathrm{Nd} \\
4-36\end{array}$ & $\begin{array}{l}\text { Pm } \\
\text { NA }\end{array}$ & $\begin{array}{l}\text { Sm } \\
1-6\end{array}$ & $\begin{array}{ll}E u \\
0.1- \\
0.4 \\
\end{array}$ & $\begin{array}{c}\mathrm{Gd} \\
0.1- \\
\end{array}$ & $\begin{array}{c}T b \\
0.1- \\
2 . \\
\end{array}$ & $\begin{array}{c}\text { Dy } \\
0.1- \\
5\end{array}$ & $\begin{array}{l}\text { Ho } \\
0.1- \\
0.4 \\
\end{array}$ & $\begin{array}{l}E r \\
0.1- \\
0.4 \\
\end{array}$ & $\underset{<m}{\operatorname{Tm}}$ & $\begin{array}{c}Y b \\
0.1- \\
0.5 \\
\end{array}$ & $\begin{array}{l}\text { Lu } \\
0.1- \\
0.3 \\
\end{array}$ \\
\hline & & & & $\begin{array}{c}\text { Th } \\
0.1- \\
5\end{array}$ & $\begin{array}{l}\mathrm{Pa} \\
\mathrm{NA}\end{array}$ & $\begin{array}{l}U \\
0.1- \\
1 .\end{array}$ & $\begin{array}{l}\mathrm{Np} \\
\mathrm{NA}\end{array}$ & $\begin{array}{l}\text { Pu } \\
\text { NA }\end{array}$ & $\begin{array}{l}\text { Am } \\
\text { NA }\end{array}$ & $\begin{array}{l}\mathrm{Cm} \\
\mathrm{NA}\end{array}$ & $\begin{array}{l}\mathrm{Br} \\
\mathrm{NA}\end{array}$ & $\begin{array}{l}\text { Cf } \\
N A\end{array}$ & $\begin{array}{l}E s \\
N A\end{array}$ & $\begin{array}{l}F m \\
N A\end{array}$ & $\begin{array}{l}\mathrm{Md} \\
\mathrm{NA}\end{array}$ & $\begin{array}{l}\text { No } \\
\text { NA }\end{array}$ & $\left(\begin{array}{l}(\mathrm{LW}) \\
\mathrm{NA}\end{array}\right.$ \\
\hline
\end{tabular}

NA - Not Available

Figure 17. Concentration range of elements found in American coals $[21,26,27,28,29]$.

(units are ppm by weight in the coal)

The included and freed mineral matter is typically 1-2 $\mu \mathrm{m}$ in size [31], although other forms and sizes of included mineral matter may occur [25]. During combustion the organic material burns away and the included mineral matter softens, becomes spherical, and adheres to the coal particle surface. Less than $6 \%$ by weight of these ash spheres react with the carbon matrix to form hollow cenospheres [32]. The rest of the ash spheres remain attached to the coal surface and may agglomerate as the coal particle burns $[31,33]$. In the free stream, negligible fusion of individual freed ash particles is expected. In addition, certain volatile elements contained in both included and freed mineral matter will be vaporized either as the original compound or as a more volatile compound that has been formed by solid and gas phase reactions.

Extraneous mineral matter is contained in larger sized particles than included or freed mineral matter. These particles typically have been added to the coal from the bed materials that surround the coal seam. However, some of this material could be added from large mineral matter inclusions contained in some coal seams [25]. Since $65 \%$ of coal in the United States is cleaned at the mine, much of the extraneous ash material will be removed [21]. The portion of this material that is not removed will principally form large ash particles during the combustion process. In addition, such extraneous mineral matter can contribute to the amount of mineral matter that is evaporated. 


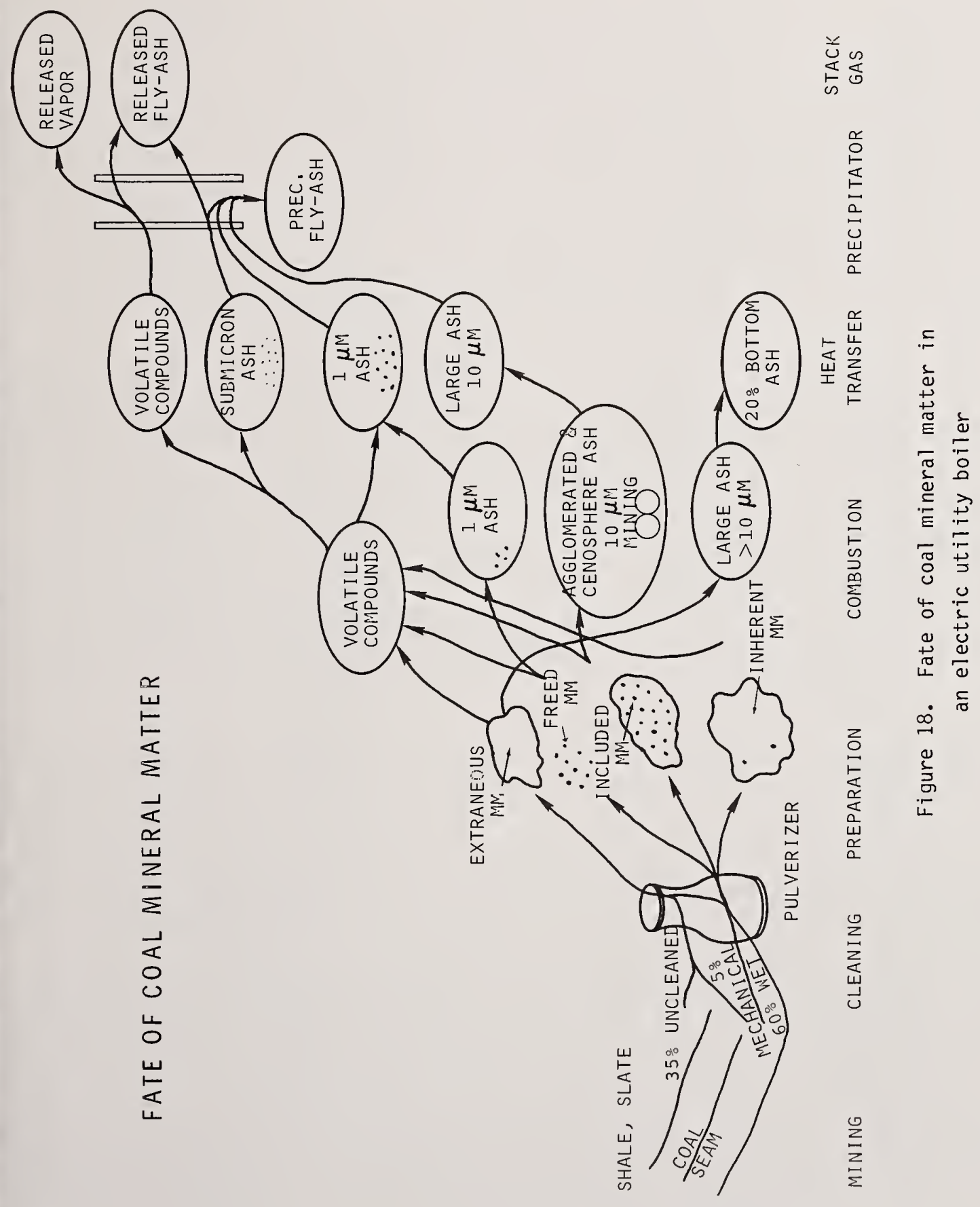


The physical behavior of these various forms of mineral matter during the combustion process is shown schematically in figure 18. Large ash particles, which are likely to appear as bottom ash or to be easily precipitated, will originate from extraneous mineral matter and included mineral matter. Cenospheres will be formed only from included ash. These large light particles should be easily removed in the precipitator. Freed ash and some included ash will form micron size particles which will largely be removed in the precipitator. All of the inherent ash and some of the other ash forms will evaporate. The material which evaporates is of greatest concern. This material can condense on tube walls and eventually lead to deposits that reduce heat transfer to the boiler surface or beneath which alkali-sulfates corrode the tubes. The vaporized materials can also condense to form fine particles or may be adsorbed on the surface of other small particles. These small particles are difficult to remove in the precipitator and hence a large portion of the vaporized material leaves the boiler either as small respirable particles or in the vapor phase.

Fortunately, only a fraction, albeit a large fraction, of the elements contained in the mineral matter of coal are important in the combustion process. The elements contained in mineral matter that are considered to be the most important in the combustion processes are listed in table 6. In addition to the important mineral elements, this table lists the expected concentration range of the elements, the most common minerals containing the elements, use of the elements by industry as design indices, and the likely action of an element in a boiler. As an example, the concentration of sulfur is shown to vary from 300 to 1000 ppm by wt. of U.S. coals, appears mainly as pyrite or organically bound to the coal matrix, is used to predict the slagging potential of coals, and contributes to slagging, fouling, and corroding of boilers, as well as polluting the atmosphere in the form of $\mathrm{SO}_{2}$ and $\mathrm{SO}_{3}$.

The purpose of any research on mineral matter is to 1) predict how a given coal will perform in a boiler based on the properties of the ash, and 2) to discover ways to control the undesirable behavior of ash. Table 7 shows the ash concentrations of some typical U.S. coals and calculated indices (based upon the chemical composition of the ash), which are commonly used in industry to predict ash behavior. Ash is not mineral matter nor is the ash prepared for these tests necessarily representative of ash at any stage during the combustion process. ASTM ash is prepared by completely burning the coal at $700-750^{\circ} \mathrm{C}$ in air. During this process the mineral matter undergoes physical and chemical changes. Since the mineral matter is burned at a temperature, time, and in an atmosphere different from the conditions in a furnace, the ASTM ash will likely be different from boiler ash. Consequently, while empirical or semi-empirical correlations of ASTM ash composition with performance of that ash in a boiler are extremely useful, such methods will typically be of 1 imited use for predicting the behavior of ashes which have compositions different from ashes whose performance is known. To illustrate this point, performance indices for the various coals are listed in table 7. To be fair, indices are calculated for ash compositions for which the indices are not recommended, but separating the ash into "types" of ash is another indication of how little is known about ash behavior. The point is that 
Table 6. Form and effect of el ements found

in U.S. coals $[21,26,27,28,29]$.

\begin{tabular}{|c|c|c|c|c|}
\hline Element & $\begin{array}{l}\text { Range of } \\
\text { Concentration, pprr? } \\
\text { in Coal } \\
\end{array}$ & Cominon Minerals in Coal & $\underline{\text { Use }}$ & Concern \\
\hline $\begin{array}{l}\text { As } \\
\text { A1 }\end{array}$ & $\begin{array}{l}1-60 \\
3000-23000\end{array}$ & $\begin{array}{l}\text { Arsenopyrite (FeAsS) } \\
\left.\text { Kaol inite ( } \mathrm{Al}_{4} \mathrm{Si}_{4} \mathrm{O}_{\mathrm{O}} \mathrm{O}\right)(\mathrm{OH})_{8} \\
\text { I11 ite-Sericite }\left(\mathrm{KAl}_{2}\left(\mathrm{AlSi}_{3} \mathrm{O}_{10}\right)(\mathrm{OH})_{2}\right.\end{array}$ & $\begin{array}{l}\text { None } \\
\text { Base/Acid Ratio }\end{array}$ & $\begin{array}{l}\text { Toxic Vapor } \\
\text { Large portion of ash }\end{array}$ \\
\hline $\begin{array}{l}\mathrm{Be} \\
\mathrm{Ca}\end{array}$ & $\begin{array}{c}0.4-6 \\
50-12300\end{array}$ & $\begin{array}{l}\text { Partially chelated } \\
\text { Organically bound, Calcite }\left(\mathrm{CaCO}_{3}\right) \\
\text { Oolomite }(\mathrm{Ca}, \mathrm{Mg})\left(\mathrm{CO}_{3}\right)_{2} \\
\text { Gypsum }\left(\mathrm{CaSO}_{4} \quad \mathrm{H}_{2} \mathrm{O}\right)\end{array}$ & $\begin{array}{l}\text { None } \\
\text { Base/Acid Ratio } \\
\text { Silica Ratio, } \\
\text { 0olomite \% }\end{array}$ & $\begin{array}{l}\text { Toxic lapor } \\
\text { Can reduce fouling and } \\
\text { corroding }\end{array}$ \\
\hline $\begin{array}{l}\mathrm{Cd} \\
\mathrm{Cl}\end{array}$ & $\begin{array}{l}0.01-3 \\
10-260\end{array}$ & $\begin{array}{l}\text { Unidentified } \\
\text { Halite (NaCl), Sylvite }(\mathrm{KCl}) \\
\text { Bischofite }\left(\mathrm{MgCl}_{2}{ }^{\left.6 \mathrm{H}_{2} \mathrm{O}\right)}\right.\end{array}$ & $\begin{array}{l}\text { None } \\
\text { Content }\end{array}$ & $\begin{array}{l}\text { Toxic Vapor } \\
\text { Low temperature corrosion }\end{array}$ \\
\hline $\begin{array}{l}\mathrm{Cr} \\
\mathrm{Fe}\end{array}$ & $\begin{array}{c}10-400 \\
340-23000\end{array}$ & $\begin{array}{l}\text { Unidentified } \\
\text { Pyrite }\left(\mathrm{FeS}_{2}\right)\end{array}$ & $\begin{array}{l}\text { None } \\
\text { Base/Acid Ratio } \\
\text { Silica Ratio, Dolomite \% }\end{array}$ & $\begin{array}{l}\text { Toxic Vapor } \\
\text { Lowers fusion temperature }\end{array}$ \\
\hline $\begin{array}{l}\mathrm{Ga} \\
\mathrm{Ge} \\
\mathrm{Hg}\end{array}$ & $\begin{array}{l}0.3-60 \\
0.03-10 \\
0.1-0.3\end{array}$ & $\begin{array}{l}\text { Unidentified } \\
\text { Unidentified } \\
\text { Unidentified }\end{array}$ & $\begin{array}{l}\text { None } \\
\text { None } \\
\text { None }\end{array}$ & $\begin{array}{l}\text { Possible toxic vapor } \\
\text { Possible toxic vapor } \\
\text { Toxic Vaporv }\end{array}$ \\
\hline k & $100-6500$ & $\begin{array}{l}\text { Organically bound } \\
\text { Illite-sericite }(\mathrm{KA}]_{2}\left(\mathrm{AlSi}_{3} \mathrm{O}_{\mathrm{O}}(\mathrm{OH})_{2}\right. \\
\left.\text { Biotite } \mathrm{K}(\mathrm{Mg}, \mathrm{Fe})_{3}\left(\mathrm{Al} \mathrm{Si}_{3} \mathrm{O}_{10}\right)_{\mathrm{COH}}\right)_{2} \\
\left.\text { Orthoclase (KAiSi }{ }_{3} \mathrm{O}_{8}\right)\end{array}$ & $\begin{array}{l}\text { Base/Acid Ratio } \\
\text { Oolomitic } \%\end{array}$ & Fouling and corroding \\
\hline $\mathrm{Mg}$ & $240-3500$ & $\begin{array}{l}\text { 0olomite }(\mathrm{Ca}, \mathrm{Mg})\left(\mathrm{CO}_{3}\right)_{2} \\
\text { Ankerite }(\mathrm{Ca}, \mathrm{Fe}, \mathrm{Mg}) \mathrm{CO}_{3} \\
\text { Organically bound }\end{array}$ & $\begin{array}{l}\text { Base/Acid Ratio } \\
\text { Silica ratio } \\
\text { 0olomitic } \%\end{array}$ & $\begin{array}{l}\text { Can reduce fouling } \\
\text { and corroding }\end{array}$ \\
\hline $\begin{array}{l}\text { Mo } \\
\mathrm{Na}\end{array}$ & $\begin{array}{l}1-10 \\
1-6000\end{array}$ & $\begin{array}{l}\text { Associated with Pyrite } \\
\text { Organically bound } \\
\text { Halite }(\mathrm{NaCl}) \\
\text { Tourmaline } \mathrm{Na}(\mathrm{Mg}, \mathrm{Fe})_{3} \mathrm{Al}_{6}\left(\mathrm{BO}_{3}\right)_{3}\left(\mathrm{Si}_{6} \mathrm{O}_{8}\right)(\mathrm{OH})_{4}\end{array}$ & $\begin{array}{l}\quad \text { None } \\
\text { Base/Acid Ratio } \\
\text { Oolomite \% } \\
\text { Fouling Potential }\end{array}$ & $\begin{array}{l}\text { Toxic Vapor } \\
\text { Fouling and corroding } \\
\text { element }\end{array}$ \\
\hline$\stackrel{\mathrm{Ni}}{\mathrm{P}}$ & $\begin{array}{l}2-60 \\
6-300\end{array}$ & $\begin{array}{l}\text { Unidentified } \\
\text { Apatite }\left(\mathrm{CaF}_{2} \quad 3 \mathrm{Ca}_{3} \mathrm{P}_{2} \mathrm{O}_{8}\right)\end{array}$ & $\begin{array}{l}\text { None } \\
\text { None }\end{array}$ & $\begin{array}{l}\text { Toxic Vapor } \\
\text { Fouling }\end{array}$ \\
\hline $\begin{array}{l}\mathrm{Pb} \\
\mathrm{Ra}\end{array}$ & $\begin{array}{c}1-60 \\
\text { Unknown }\end{array}$ & $\begin{array}{l}\text { Galena (PbS) } \\
\text { Unidentified }\end{array}$ & $\begin{array}{l}\text { None } \\
\text { None }\end{array}$ & $\begin{array}{l}\text { Toxic Vapor } \\
\text { Possible Toxic Vapor }\end{array}$ \\
\hline$S$ & $300-10000$ & Pyrite $\left(\mathrm{FeS}_{2}\right)$, organically bound & Slagging Potential & $\begin{array}{l}\text { Slagging, fouling, corroding, } \\
\text { polluting }\end{array}$ \\
\hline Sb & $0.1-30$ & Unidentified & None & Toxic Vapor \\
\hline Se & $0.04-5$ & Unidentified & None & Possible Toxic Vapor \\
\hline Si & $5000-41000$ & $\begin{array}{l}\text { Kaolinite }\left(\mathrm{Al}_{4} \mathrm{Si}_{4} \mathrm{O}_{10}\right)(\mathrm{OH})_{8} \\
\text { Illite-Sericite }\left(\mathrm{KAl}_{2}\right)\left(\mathrm{AlSi}_{3} \mathrm{O}_{10}\right)(\mathrm{OH})_{2} \\
\text { Quartz }\left(\mathrm{SiO}_{2}\right)\end{array}$ & $\begin{array}{l}\text { Base/Acid Ratio } \\
\text { Silica Ratio }\end{array}$ & Major Ash Element \\
\hline Te & $0.4-4$ & Unidentified & None & Possible Toxic Vapor \\
\hline Th & $0.1-5$ & Unidentified & None & Possible Toxic Vapor \\
\hline $\mathrm{Ti}$ & $200-1800$ & Chelated, Rutile $\left(\mathrm{TiO}_{2}\right)$ & Base/Acid Ratio & Not known \\
\hline TI & $0.1-0.3$ & Unidentified & None & Toxic Vapor \\
\hline V & $2-77$ & Chelated & None & Corroding, Toxic Vapor \\
\hline W & $0.1-0.4$ & Unidentified & None & Toxic Vapor \\
\hline $\mathrm{Zn}$ & $3-80$ & Sphalerite (ZnS) & None & Toxic Vapor \\
\hline
\end{tabular}

the different indices suggest different potential problems for the same coals. We believe that such indices should be cautiously used.

Since the time-temperature history of the mineral matter is likely to affect the behavior of such substances in boilers, a typical time-temperature profile through a 500 MWe coal-fired boiler is shown in figure 19. Points of interest are that the temperature rapidly increases to a maximum of about $2000 \mathrm{~K}$ in the $\mathrm{flame}$ region. The temperature slowly decreases as heat is transferred by radiation to the furnace walls. The temperature of the ash must be reduced below the ash fusion temperature before the ash enters tine convective heat transfer passages; if not deposits will rapidly plug these passages. The time scale superimposed on the top of the figure indicates that the times available for chemical and physical transformations in boilers are on the order of one second. 
Table 7. Ash characteristics of typical U.S. coals $[21,34,35]$.

ASH CHARACTERISTICS OF TYPICAL AMERICAN COALS

8abcock and Wilcox (197S)

Vecci, et al., (1978)

\begin{tabular}{|c|c|c|c|c|c|c|c|c|}
\hline Rank: & $\begin{array}{l}\text { Low Volatile } \\
\text { 8ituminous }\end{array}$ & \multicolumn{4}{|c|}{ High Volatile Bituminous } & $\begin{array}{c}\text { Sub- } \\
\text { bituminous }\end{array}$ & Lignite & Lignite \\
\hline \multirow{5}{*}{$\begin{array}{l}\text { Seam } \\
\text { Location } \\
\text { Ash, dry basis, \% } \\
\text { Sulfur, dry basis, } \\
\% \\
\text { Analysis of ash, } \\
\% \text { by wt }\end{array}$} & Pocahontas No. 3 & No. 9 & Pittsburgh & No. 6 & & & & \\
\hline & West Virginia & Ohio & W. Virginia & Illinois & Utah & Wyoming & Texas & No. Oakota \\
\hline & 12.3 & 14.10 & 10.87 & 17.36 & 6.6 & 6.6 & 12.8 & 11.2 \\
\hline & 0.7 & 3.30 & 3.53 & 4.17 & 0.5 & 1.0 & 1.1 & 0.8 \\
\hline & & & & & & & & \\
\hline $\begin{array}{l}\mathrm{SiO}_{2} \\
\mathrm{Al}_{2} \mathrm{O}_{3} \\
\mathrm{TiO}_{2} \\
\mathrm{Fe}_{2} \mathrm{O}_{3} \\
\mathrm{CaO} \\
\mathrm{MgO} \\
\mathrm{Na}_{2} \mathrm{O} \\
\mathrm{K}_{2} \mathrm{O}\end{array}$ & $\begin{array}{r}60.0 \\
30.0 \\
1.6 \\
4.0 \\
0.6 \\
0.6 \\
0.5 \\
1.5 \\
\end{array}$ & $\begin{array}{r}47.27 \\
22.96 \\
1.00 \\
22.81 \\
1.30 \\
0.85 \\
0.28 \\
1.97 \\
\end{array}$ & $\begin{array}{r}37.64 \\
20.11 \\
0.81 \\
29.28 \\
4.25 \\
1.25 \\
0.80 \\
1.60 \\
\end{array}$ & $\begin{array}{r}47.52 \\
17.87 \\
0.78 \\
20.13 \\
5.75 \\
1.02 \\
0.36 \\
1.77 \\
\end{array}$ & $\begin{array}{r}48.0 \\
11.5 \\
0.6 \\
7.0 \\
25.0 \\
4.0 \\
1.2 \\
0.2 \\
\end{array}$ & $\begin{array}{r}24.0 \\
20.0 \\
0.7 \\
11.0 \\
26.0 \\
4.0 \\
0.2 \\
0.5 \\
\end{array}$ & $\begin{array}{r}41.8 \\
13.6 \\
1.5 \\
6.6 \\
17.6 \\
2.5 \\
0.6 \\
0.1 \\
\end{array}$ & $\begin{array}{r}28.4 \\
11.0 \\
0.4 \\
14.0 \\
18.0 \\
5.0 \\
3.6 \\
0.7 \\
\end{array}$ \\
\hline Total & 98.8 & 98.44 & 95.74 & 95.20 & 97.5 & .86 .4 & 84.3 & 81.1 \\
\hline $\begin{array}{l}\text { Ash fusibility } \\
\text { Initial defor- } \\
\text { mation (IT) } \\
\text { temperature, F }\end{array}$ & & & & & & & & \\
\hline Reducing & $2900+$ & 2030 & 2030 & 2000 & 2060 & 1990 & 1975 & 2170 \\
\hline oxidizing & $2900+$ & 2420 & 2265 & 2300 & 2120 & 2190 & 2070 & 2170 \\
\hline $\begin{array}{l}\text { Softening tem- } \\
\text { perature, } F\end{array}$ & & & & & & & & \\
\hline Reducing & & 2450 & 2175 & 2160 & & 2180 & 2130 & 2200 \\
\hline Oxidizing & & $260 \mathrm{~S}$ & 2385 & 2430 & & 2220 & 2190 & 2210 \\
\hline $\begin{array}{l}\text { Hemispherical } \\
\text { temperature, } \\
F(\mathrm{HT})\end{array}$ & & & & & & & & \\
\hline Reducing & & 2480 & $222 S$ & 2180 & 2140 & 2250 & 21so & 2230 \\
\hline Oxidizing & & 2620 & 2450 & 2450 & 2220 & 2240 & 2210 & 2230 \\
\hline $\begin{array}{l}\text { Fluid tempera- } \\
\text { ture, } F\end{array}$ & & & & & & & & \\
\hline Reducing & & 2620 & 2370 & 2320 & 2250 & 2290 & 2240 & 2310 \\
\hline Oxidizing & & 2670 & 2540 & 2610 & 2460 & 2300 & 2290 & 2370 \\
\hline Type of Ash & eastern & eastern & eastern & eastern & lignite & lignite & lignite & lignite \\
\hline $\begin{array}{l}\text { 8ase-to-acid ratio } \\
\text { 8/A }\end{array}$ & & & & & & & & \\
\hline $\begin{array}{l}\mathrm{Fe}_{2} \mathrm{O}_{3}+\mathrm{CaO}_{3} \mathrm{MgO}+\mathrm{Na}_{2} \mathrm{O}+ \\
\mathrm{K}_{2} \mathrm{O} / \mathrm{SiO}_{2}+\mathrm{Al}_{2} \mathrm{O}_{3}+\mathrm{TiO}_{2}\end{array}$ & 0.079 & 0.382 & $0.63 \mathrm{~S}$ & 0.439 & 0.622 & 0.933 & 0.482 & 1.04 \\
\hline $\begin{array}{l}\text { Silica ratio } \\
\mathrm{SiO}_{2} \times 100 / \mathrm{SiO}_{2}+ \\
\mathrm{Fe}_{2} \mathrm{O}_{3}+\mathrm{CaO}+\mathrm{MgO}\end{array}$ & 92 & $6 S$ & S2 & 64 & 57 & 37 & 61 & 43 \\
\hline $\begin{array}{l}\text { Oolomite \% } \\
(\mathrm{CaO}+\mathrm{MgO}) \times 100 / \\
\mathrm{Fe}_{2} \mathrm{O}_{3}+\mathrm{CaO}+\mathrm{MgO}^{2} \mathrm{Na}{ }_{2} \mathrm{O}+ \\
\mathrm{K}_{2} \mathrm{O}\end{array}$ & 17 & 8 & 17 & 23 & 78 & 72 & 73 & S6 \\
\hline $\begin{array}{l}\text { Slagging potential } \\
\text { for eastern coals }\end{array}$ & low & med i um & high & medium & low & medium & low & medium \\
\hline $8 / A * \% 5$ & 0.06 & 1.26 & 2.24 & 1.83 & 0.31 & 0.93 & 0.53 & 0.83 \\
\hline $\begin{array}{l}\text { Slagging potential } \\
\text { for lignitic ash }\end{array}$ & & high & high & severe & severe & severe & severe & high \\
\hline $\max \mathrm{HT}+4 \mathrm{IT} / \mathrm{S}$ & $\cdots$ & 2148 & 2114 & 2090 & 2092 & 2042 & 2022 & 2182 \\
\hline $\begin{array}{l}\text { Fouling potential } \\
\text { for eastern coals }\end{array}$ & low & low & medium & low & high & low & medium & severe \\
\hline $\mathrm{B} / \mathrm{Ax}^{2} \mathrm{Na}_{2} \mathrm{O}$ & 0.04 & 0.11 & 0.51 & 0.16 & $0.7 \mathrm{~S}$ & 0.19 & 0.29 & 3.74 \\
\hline $\begin{array}{l}\text { Fouling potential } \\
\text { for lignite ash }\end{array}$ & low-medium & $\begin{array}{l}\text { low- } \\
\text { medium }\end{array}$ & $\begin{array}{l}\text { low- } \\
\text { medium }\end{array}$ & $\begin{array}{l}\text { low- } \\
\text { medium }\end{array}$ & $\begin{array}{l}\text { low- } \\
\text { medium }\end{array}$ & $\begin{array}{l}\text { low- } \\
\text { medium }\end{array}$ & $\begin{array}{l}\text { low- } \\
\text { medium }\end{array}$ & high \\
\hline${ }^{2} \mathrm{Na}_{2} \mathrm{O}$ & 0.5 & 0.28 & 0.80 & 0.36 & 1.2 & 0.2 & 0.6 & 3.6 \\
\hline
\end{tabular}




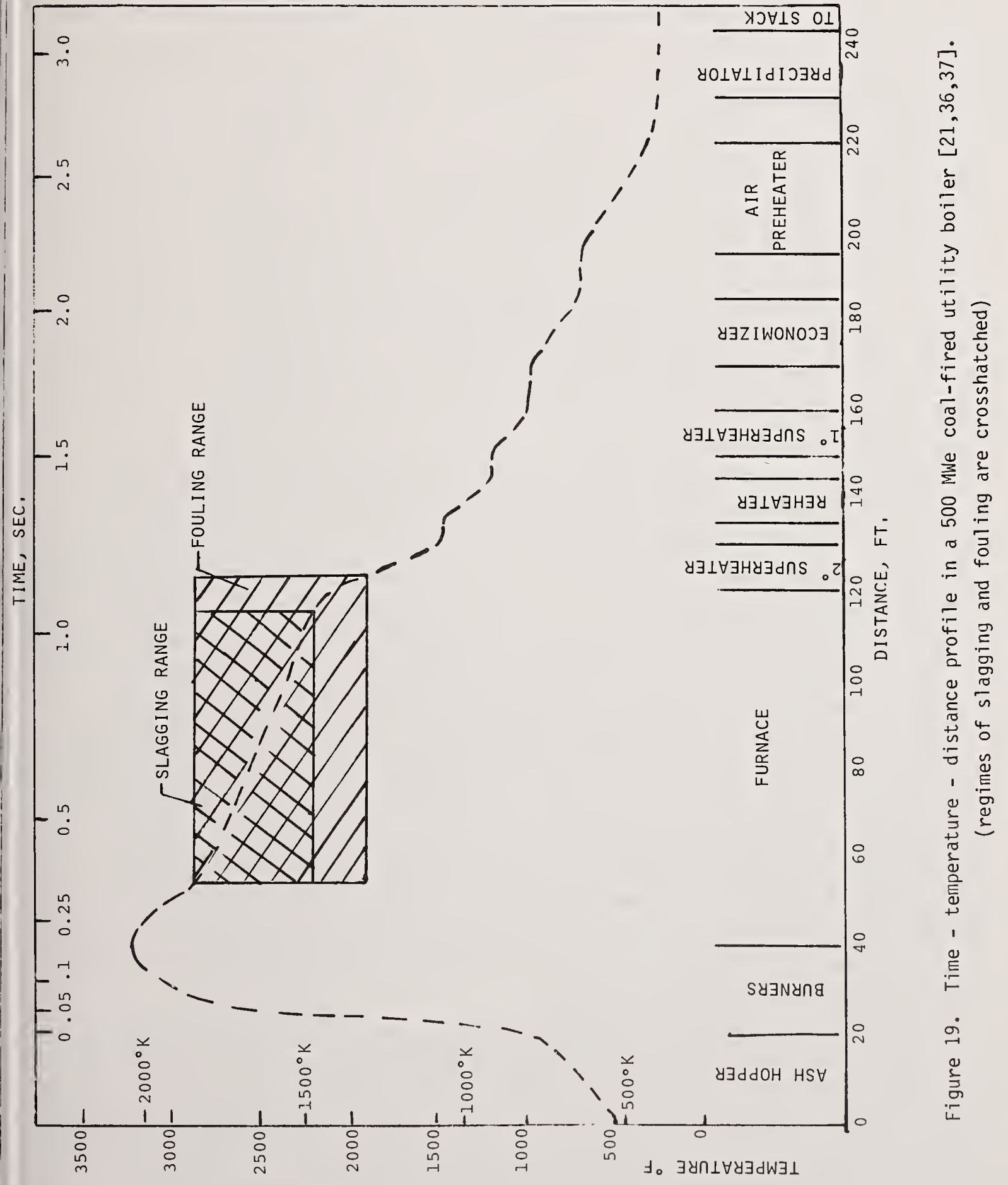


During the combustion process in a boiler many transformations of mineral matter may occur. The transformation, and temperature at which some of these equilibrium transforinations are expected to occur, are listed in figure 20. The temperatures listed for evaporation of solid compounds are the temperatures at which the compound listed will produce an equilibrium vapor concentration corresponding to evaporation of $1 \mathrm{ppm}$ of the substance from the coal. While neither space nor reader patience will allow a detailed discussion of this table, some points should be mentioned. First, many pure elements will appreciably evaporate at 1 ow temperatures, e.g. $\mathrm{Hg}(273 \mathrm{~K})$, Se $(302 \mathrm{~K})$, Cd $(430 \mathrm{~K})$, As (450 K), Sb $(494 \mathrm{~K}), \mathrm{Pb}(572 \mathrm{~K})$, and $\mathrm{Tl}(680 \mathrm{~K})$. More than $50 \%$ of all these elements are evaporated in boilers [40]. Loss of water of hydration begins at $420 \mathrm{~K}$ but continues to $870 \mathrm{~K}$. Sulfur release occurs in several stages; the first sulfur is released at $475^{\circ} \mathrm{K}$ while the last sulfur is not released until $772 \mathrm{~K}$. Carbonates are decomposed to carbon dioxide at $670 \mathrm{~K}$ and $1070 \mathrm{~K}$. Minerals start to melt at about $975 \mathrm{~K}$ but the bulk of the mineral matter in the coal remains solid at temperatures less than $1700 \mathrm{~K}$. In contrast, ash fusion temperatures are typically in the range 1300-1700 K. It is widely believed that low melting point eutectics contribute to softening of the ash materials. Investigation of low melting eutectics should, therefore, be pursued.

A method of predicting ash behavior should be developed that will permit useful estimates of the behavior of ash of widely varying composition over widely varying conditions. With such a predictive capability progress can be made in controlling the undesirable effects of ash contained in the coal. Logically, the transformations that take place, during the combustion process, in coal mineral matter depend upon the composition of the ash, the temperature-time history of the mineral matter and the environment in which the ash transformation occurs. Once a coal is selected, little can be economically done to change the composition of the ash except to minimize the day to day variations in ash composition by blending. However, the temperature-time history of the ash can be controlled to some extent by modifying the combustion process and the environment can be changed either by controlling the combustion process or by introducing an extraneous material into the boiler. For instance, higher oxygen concentrations, in specific regions of the flame have reduced the fouling produced by German brown coal [41] and addition of poultry grit $\left(\mathrm{Ca}\left(\mathrm{CO}_{3}\right)_{2}\right)$ has reduced the fouling from a Canadian lignite [42]. That such approaches have been successful on a limited basis gives hope that a better understanding of the fundamental transformations of mineral matter in a boiler can reduce the problems concerned with mineral matter in the coal.

Mineral matter in a boiler undergoes all the processes familiar to the material scientists. The action of mineral matter in boilers may be controlled by any of the following: 1) heat transfer to the ash; 2) mass transfer internally or externally to the ash; 3) liquid or solid internal reactions; 4) gas, solid or liquid reactions, and; 5) equilibria between the particle and the surrounding environments.

We will complete this section with comments on each of these areas. 


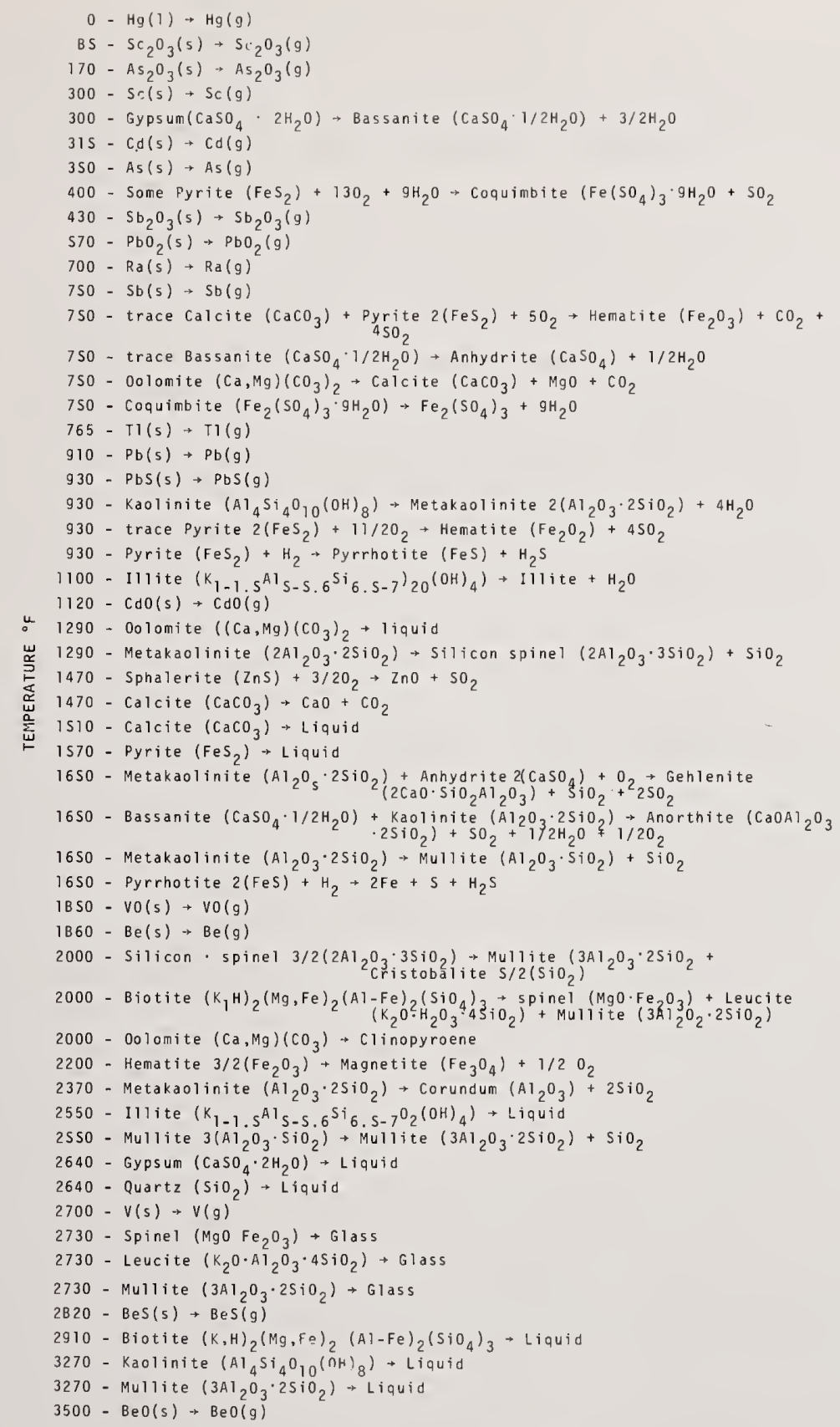

Figure 20. Equilibrium transformations of minerals contained in coal $[27,28,31,38,39]$. 


\subsection{Equilibrium considerations}

Since equilibrium control is the simplest, equilibrium will be considered first. In large volume, high temperature systems such as utility boilers, computed equilibrium concentrations show some agreement with observed compositions for many compounds [43]. Some compounds that react slowly, however, are known not to be equilibrated in boilers. However, even in the absence of complete equilibration of compounds of interest, equilibrium calculations usually supply reasonable estimates of the composition of many substances and most often will indicate the direction in which reactions are proceeding and the relative changes in compositions that occur with changes in operating conditions.

Previous equilibrium calculations for compositions in utility boilers have been hampered by poor and incomplete thermodynamic data. The data required to make ineaningful equilibrium calculations in utility boilers requires knowledge of the free energy, as a function of temperature to about 3000K, for all the important solid, liquid, and gaseous materials in the mineral matter, in the ash, and in boiler deposits. In addition, knowledge of the partial free energy of compounds in solution as a function of temperature may be required. The last is a most difficult requirenent and suitable and sufficient data may never exist. There are a number of compilations of thermodynamic data for discrete minerals and constituents [45-52]. However, the data appear to be incomplete. For the data to be useful to those interested in coal combustion, a single evaluation of thermodynamic properties of mineral, ash, and deposit compounds must be conpiled. Missing data must be supplied from experimental and computational studies. This is a service that the National Bureau of Standards has admirably supplied in the past [53].

Once sufficient thermodynamic data, of the required precision, have been assembled equilibrium compositions of the solid, gas, and possibly liquid mineral matter can be calculated, under various conditions, as the mineral matter passes through the boiler. Such calculations should indicate the regions in which minerals evaporate and condense and the equilibrium composition of wall deposits (at tube metal external surface temperatures).

Additional important thermodynamic data to boiler designers are the softening, deformation, and melting points of mineral compounds. These temperatures are routinely measured for ASTM ash and some temperatures are reported in table 7 . However, the softening temperature of the minerals which actually deposit on the tube wall is required. As a first approximation the composition of material deposited on walls can be estimated from the equilibrium calculations mentioned previously and from compositions measured for wall deposits extracted from boilers [21]. However, once the compositions of deposits have been determined, the critical temperatures of these deposits are best measured in the laboratory. Some phase diagrams al ready exist in the literature for binary mixtures [47,54-57] and for some ternary phase diagrams [55-57]. More complicated phase diagrams exist for special processes $[45,58]$ but, in general, very few phase diagrams exist that can be directly applied to mineral matter deposits in boilers. Once the composition of the complex mixtures of minerals, liquids, and gases present in wall deposits have been determined, then the important phase diagrams required for determining the behavior of mineral matter in boilers can be developed. 
The previous discussion has assumed that equilibrium considerations can be used to design strategies for controlling the undesirable behavior of mineral matter in boilers. We believe, that in general, this is true. However, some important mineral matter transformations may be controlled by heat transfer, mass transfer or chemical kinetics or by a combination of the above.

\subsection{Heat transfer considerations}

Unfortunately, very little data exists on heat transfer-controlled mineral matter transformation in boilers. In the initial phases of combustion, the coal and ash particles will be heated to around $2000 \mathrm{~K}$ in several milliseconds. At these heating rates $\left(10^{6} \mathrm{~K} /\right.$ $\mathrm{sec}$ ), transformations controlled by (relatively slow) heat transfer processes can adequately be described by equilibrium calculations. Endothermic transformations that occur near flame temperatures (in regions of high gradients) may, however, be controlled by heat transfer. To our knowledge no such processes have yet been identified.

\subsection{Mass transfer considerations}

Several mineral transformations that may occur in boilers and which are controlled by mass transfer have been identified. Control by mass transfer can take the form of restricted transport of gaseous, liquid, or solid material required for the transformation or restricted transport of the product material away for the location at which the transformation took place. Some examples of information available on mineral transformations restricted by mass transfer follow.

Iron sulfide initially decomposes in a reducing atmosphere in less than 0.5 seconds at $1375^{\circ} \mathrm{K}$ to pyrrhotite. However, loss of the second sulfide atom is slow and is apparently controlled by diffusion of the second atom to and away from the liquid surface [59]. Evaporation of sodium chloride requires less than 0.5 seconds at $1375 \mathrm{~K}$. The rate of evaporation may be augmented by reaction between the chloride and hydrogen or between sodium and silica [59]. The rate of evaporation of potassium sulfate in neutral and oxidizing atmospheres appears to be controlled by diffusion of potassium from the surface. However, in reducing atmospheres the evaporation of potassium sulfate is controlled by the rate of diffusion of potassium sulfate to the surface and the rate of diffusion of reducing gases to the surface $[60]$.

\subsection{Reaction kinetics considerations}

Similarly, little direct information is available on the kinetics of mineral matter transformations in boilers. However, what information is available indicates that such chemical reactions are rapid. A $60 \mu \mathrm{m}$ calcium carbonate particle is $75 \%$ calcined at $1500 \mathrm{~K}$ in 30 milliseconds [61]. In a boiler, this process may be heat transfer controlled [632.

Information such as that quoted above indicates that some mineral matter transfomultions occur in times less than 0.5 seconds at temperatures on the order of $1400 \mathrm{~K}$. Additional information of a qualitative nature suggest that mineral matter transformations are 
rapid compared to residence times in boilers. Analysis of ash withdrawn from a small test furnace at several sample points showed no significant changes in mineral matter composition [62]. Typical residence time between sampling ports was on the order of $0.1-0.5$ seconds.

To adequately develop strategies to control the behavior of mineral matter in boilers, information on the chemical transformation of mineral matter in boilers must be obtained. Limited information indicates that many of the transformations are sufficiently rapid such that equilibrium calculations yield useful information for formulating control strategies. Unfortunately, much of the relevant thermodynamic data does not appear to be available and what data is available is scattered in the literature. The first step is then to compile, evaluate, and supplement the thermodynamic data relevant to mineral matter in coal. Secondly, equilibrium calculations and analysis of boiler deposits will specify the systems for which phase diagrams need to be developed. Lastly, control of important transformations by heat transfer, mass transer, or chemical kinetics should be investigated. The results from such programs will contribute to understanding how the combustion process affects the transformation of mineral matter, how the mineral matter affects the boiler and the form of emitted pollutants. With this type of data boiler designs can be based on fundamental coal/ash characteristics with less dependence upon extensive empirical data. Further, such information will permit an evaluation of the influence of modifications of the combustion process (including adding extraneous material to the coal or air strearns) for controlling the composition and other chemical and physical properties of the ash.

\section{Conclusions}

In conclusion, coal is a vast energy resource of the United States that has been underused in the past. Much greater use is required in the future. During this century (and possibly much longer) most of this use will be in the form of direct firing of pulverized coal in electric utility and industrial boilers. New constraints have been imposed on coal use, and particularly on pulverized coal burning technologies. These constraints are related to various factors involving environment, coal supply, and coal quality. Reliable and acceptable pulverized coal combustion, on the scale contemplated, will require a concentrated joint effort of government, research scientists and engineers, boiler manufacturers, and utility companies. The problems facing this group are many. We have provided a general summary of most of these and attempted to place in context research needs.

We have focussed our comments in two areas in this paper, the influence of coal properties on coal burning and the effect of mineral matter on the performance of and emission of pollutants from coal-fired utility boilers. Other topics such as $\mathrm{NO}_{\mathrm{x}}$, $\mathrm{SO}_{\mathrm{X}}$, and fine particulates control will be treated elsewhere. These are already the subjects of much investigation.

Unfortunately, very little information is available on the performance of Inineral matter in boilers. We have reviewed what information is available and outlined a direction 
of research which we feel confident will lead to boiler designs and operations which will minimize the effects of mineral matter on the performance of boilers and on the quality of the atmosphere. We see a real need to compile and supplement thermodynamic data available on mineral matter at both $10 \mathrm{w}$ and high temperatures $(<3000 \mathrm{~K})$. This data will be used in conjunction with analysis of boiler deposits to select materials and to develop phase diagrams for both the mineral matter and construction materials in critical regions of the boiler. Consideration must be given to evaluating, by diagnostic and computational means, departures from equilibrium mineral matter that may affect boiler design and strategies for control of mineral matter behavior in the boiler. 
[1] Hardesty, D. R., "Coal Combustion Research in Australia and New Zealand", Sandia Technical Report, SAND78-8210, March 1978.

[2] Pohl, J. H., "Research on Pulverized Coal Burning in Europe," Sandia Technical Report, in press.

[3] Hardesty, D. R. and Van Dyke, D. P., "Site Visits to Pulverized Coal-Fired Utility Plants in Colorado and New Mexico, Sandia Technical Report, SAND78-8259, August 1978.

[4] Bertman, L., Cox, D., Lamb, G., Morris, J., Pfeffer, J., Rivas, M., "Benefit-cost evaluation of the ERDA fossil energy combustion and advanced power development program," FE-2453-1 (1976).

[5] Greenfield, S. M., Peyton, T. 0., Anderson, G. E., Attaway, L. D., Hillyer, M. J., MacBride, D. W., "Preliminary evaluation of potential NOX control strategies for the electric power industry," EPRI FP-715, vol. 1 (1978).

[6] "Annual plant design survey," Power, 1976, 1977.

[7] "New generating plants," Power Engineering, May 1977.

[8] Dupree, W. C. and West, J. A., "United States Energy Through the Year 2000," U.S. Dept. Interior, USGP0 2400-00775, Dec. 1972.

[9] Gordon, R. L., U.S. Coal and the Electric Power Industry, The John Hopkins University Press, Baltimore, MD (1975).

[10] Essenhigh, R. H., "Future Fuel Supplies for Industry: Bases for Choice," presented at ASHRAE Semiannual Meeting, Chicago, 1977.

[11] Fossil Energy Research and Development Program of the Department of Energy, FY 1979, DOE/ET-0013 (78), March 1978.

[12] Durrant, 0. W., "Design, Operation Control and Modeling of Pulverized Coal Fired Boilers," Babcock \& Wilcox Technical Rept., BR-1082, Feb. 1977.

[13] Duzy, A. F. and Rudd, A. H., "Steam Generator Design Considerations for Western Fuels Babcock \& Wilcox Technical Report, BR-939,TP1-10, 1971.

[14] Barsin, J. A. and Campobenedetto, E. J., "NOX Emissions Control in Pulverized Coal Fired Steam Generators," Babcock \& Wilcox Technical Report, BR-1118, Apri1 1978.

[15] Flagan, R. C., "Ash Particle Formation in Pulverized Coal Combustion," Paper No. 77-4 Western States Section, Spring Meeting Combustion Institute, Seattle, WA (1977).

[16] Mulcahy, M. F. R. and Smith, I. W., "Kinetics of combustion of pulverized fuel: A review of theory and experiment," Rev. Pure and Appl. Chem. 19, 81 (1969).

[17] "Know your state emission regulations," Power, July and August (1973).

[18] Waterl and, L. R., Mason, H. B., Evans, R. M., Salvesen, K. G., and Wolfe, K. J., "Environmental assessment of sttionary source $\mathrm{NO}_{\mathrm{X}}$ control technologies, first annual report," EPA-600/7-78-046 (1978).

[19] Salvesen, K. G., Wolfe, K. J., Chu, E., and Herther, M. A., "Emission characterization of stationary $\mathrm{NO}_{\mathrm{x}}$ sources: Volume I. Results," EPA-600/7-78-1202 (1978).

[20] Gunwaldsen, D., Bhagat, N., and Beller, M., "A study of potential coal utilization 1985-2000," NTIS, BNL-50771 (1977). 
[21] Steam/Its Generation and Use, 38th edition, The Babcock and Wilcox Company, New York (1975).

[22] Putnam, A. A., Kropp, E. L., and Bavrett, R. E., "Evaluation of national boiler inventory," NTIS PB-248 100 (1975).

[23] Wagoner, C. L. and Winegartner, E. C., "Further development of the burning profile," J. Power Eng., p. 119, April 1973.

[24] Bryers, R. W., Biswas, . K., and Taylor, T. E., "Fuel Evaluation Using Differential Thermal Techniques," presented at 68th Ann. Mtg. AICHE Los Angeles, Nov. 1975.

[25] Stach, E., Mackowsky, M., Teichmuller, M., Taylor, G., Chandra, D., Teichmul ler, R., Stach's Textbook of Coal Petrology, Translated by D. G. Murchison, G. Taylor and F. Zierke, Gebruder Borntraeger, Berl in (1975).

[26] Sharkey, A. G., Jr., Kessler, T., and Friedel, R. A., "Trace elements in coal dust by spark-source mass spectrometry," in S. P. Babu, ed., Trace Elements in Fuel, Advances in Chemistry Series 141, p. 48, ACS Washington, D. C. (1975).

[27] Gluskoter, H. J., "Mineral matter and trace elements in coal," S. P. Babu, ed., Trace Elements in Fuel, Advances in Chemistry Series 141, p. 1, ACS, Washington, D.C. (1975).

[28] O'Gorman, J. V. and Walker, P. L. Jr., "Mineral matter characteristics of some American coals," Fuel, 50, pp. 135-151 (1971).

[29] Ode, W. H., "Coal analysis and mineral matter," Lowing, H. H., ed. Chemistry of Coal Utilization, Supplementary Volume, pp. 202-231, John Wiley and Sons Inc., New York (1963).

[30] Miller, R. N. and P. H. Given, "Variations in organic constituents of some low rank goals," in Bryers, R. W., Ash Deposits and Corrosion Due to Impurities in Combustion Gases, pp. 39-50, Hemisphere Publishing Corporation, Washington (1978).

[31] Padia, A. S., "The behavior of ash in pulverized coal under simulated combustion conditions," SC.D. Thesis, Massachusetts Institute of Technology (1976).

[32] Raask, E., "Cenospheres in pulverized-fuel ash," J. Inst. Fuel, (43), pp. 339-344 (1968).

[33] Raask, E., "Slag-coal interface phenomena," Trans. ASME, J. Eng Power, 88, pp. 40-44 (1966).

[34] Gray, R. J. and Moore, G. F., "Burning the sub-bituminous coals of Montana and Wyoming in large utility boilers," ASME Paper No. 74-WA/FU-1 (1974).

[35] Vecci, S. J., Wagoner, C. L., 01 son, G. B., "Fuel and ash characterization and its effect on the design of industrial boilers," presented to Anerican Power Conference, Chicago, IL, April (1978).

[36] Field, M. A., Gill, D. W., Morgan, B. B., and Hawksley, P. G. W., Combustion of Pulverized Coal, The British Coal Utilisation Research Association, Leatherhead, Engl and (1967).

[37] Moore, G. F. and Ehrler, R. F., "Western coals - 1aboratory characterization and field evaluations of cleaning requirements," ASME Paper No. 73-WA/FU-1 (1973). 
[38] Mitchell, R. S. and Gluskoter, H. S., "mineralogy of ash of some American coals: variations with temperature and source," Fuel, $\underline{55}, 90$ (1976).

[39] Bryers, R. W. and Taylor, T. E., "An examination of the relationship between ash chemistry and ash fusion temperatures in various coal size and gravity fractions using polynomial regression analysis," ASME Paper No. 75-Wa/CD-3 (1975).

[40] Vaughan, B. E., Abel, K. H., Cataldo, D. A., Hales, J. M., Hane, C. E., Rancitelli, L. A., Routson, R. C., Wildung, R. E., Wolf, E. G., "Review of potential impact on health and environmental quality from metals entering the environment as a result of coal utilization," Battelle Memorial Institute, Columbus, OH (1975).

[41] Hein, K. R. G., "Problems associated with the use of brown coal for electricity generation in Germany," Proceedings of the Fifth Members Conference, International Flame Research Foundation, pp. 269-287, Noorduijkerhout, Holland, May (1978).

[42] Baker, B. K., and Gardiner, W. H., "Modification of ash behavior in lignite fired boilers," Gronhoud, G. H. and Kube, W. R., ed., Technology and Use of Lignite, GFERC/IC-7718, pp. 74-81 (1977).

[43] Feldmann, H. F. Leonardo, M. A., Simons, W. H., and Bienstock, D., "Thermodynamic, electrical, physical, and compositinoal properties of seeded coal combustion products," U.S. Bureau of Mines Bulletin 655 (1970).

[44] Stu11, D. R., "Vapor pressure of pure substances: inorganic compounds," Ind. Eng. Chem., 39, 540 (1947).

[45] Elliott, J. F. and Gleiser, M., Thermochemistry for Steel Making, Addison-Wesley Publishing Co., Inc., Reading, MA (1960).

[46] Nesineyanov, An. N., Vapor Pressure of the Elements, J. I. Carasso transl., Academic Press, New York (1963).

[47] Hultgren, R., Orr, R. L., Anderson, P. D., Kelley, K. K., Selected Values of Thermodynamic Properties of Metal and Alloys, John Wiley and Sons, Inc., New York (1963).

[48] Hirugama, C., "Thermodynamic properties of solid monoxides, monosulfides, monoselendies, and monotellerides of Ge, Sn, Pb," J. Chem. Eng. Data, 9, 65 (1964).

[49] Margrave, J. L., ed., The Characterization of High-Temperature Vapors, John Wiley \& Sons, Inc., New York (1967).

[50] Touloukian, Y. S., ed., Thermophysical Properties of High Temperature Solid Materials, vol. 1,4,5, The MacMillan Company, New York (1967).

[51] Stul1, D. R. and Prophet, "JANAF thermochemical tables, wnd ed.," NSRDS-NBS 37 (1971).

[52] Adolphson, D. R., ed., "Formation of protective layers on alloys in coal processing environments," Sandia Laboratories Energy Report SAND77-8260 (1977).

[53] Rossini, F. D., Wagman, D. D., Evans, W. H., Levine, S., and Jutte, I., "Selected values of chemical thermodynamic properties, part I, tables," NBS Circular 500-Part I (1961).

[54] Hultgren, R., Desai, P. D., Hawkins, D. T., Gleiser, M., and Kelley, K. K., Selected Values of the Thermodynamic Properties of Binary Alloys, American Society for Metals, Metals Park, OH (1973). 
[55] Levin, E. M., Robbins, C. R., and McMurdie, H. F., Phase Diagrams for Ceramists, The American Ceramic Society, Inc., Columbus, OH (1964).

[56] Levin, E. M., Robbins, C. R., and McMurdie, H. F., Phase Diagrams for Ceramists, 1969 Supplement, The American Ceramic Society, Inc., Columbus, OH (1969).

[57] Levin, E. M. and McMurdie, H. F., Phase Diagrams for Ceramists, 1975 Supplement, The American Ceramic Society, Inc., Columbus, OH (1975).

[58] Reid, W. T., External Corrosion and Deposits, Boilers and Gas Turbines, American Elsevier Publishing Company, Inc., New York (1971).

[59] Hulstead, W. D. and Ruask, E., "The behavior of sulphur and chlorine compounds in pulverized-coal-fired boilers," J. Inst. Fuel, 42, pp. 344-349 (1969).

[60] Nettleton, M. A. and Raask, E., "The rate of evaporation of potassium sulfate," J. Appl. Chem, 17, pp. 18-21 (1967).

[61] TVA Report, "Removal of sulfur dioxide from stack gases," TVA Fundamental Research Branch, Monthly Project Report to EPA, Office of Air Programs (0ct. 1969).

[62] Stinespring, C. D., Zulkski, M., Mazza, M., "Chemical transformations of the minerals in eastern bituminous coals under simulated pulverized coal firing conditions," in Bryers, R. W., ed. Ash Deposits and Corrosion Due to Impurities in Combustion Gases, pp. 202-241, Hemisphere Publishing Corporation, Washington, D.C. (1978).

\section{Discussion}

(Editor's note): A question was raised concerning what temperatures are involved, and that the mineral particles are a couple of 100 degrees above the gas temperature.

Response (Hardesty): We11, the point I was making there was there is a possibility for mineral matter, that is included in the heated coal particle, going to higher temperatures because of the exothermicity of the coal particles.

At the kinds of temperatures we are talking about, our peak temperatures are on the order of 2000 degrees Kelvin and time scales are on the order of tens of milliseconds at these very high temperatures.

One of the difficulties in the field is that equilibrium calculations can be done, but so many of the processes are transport--mass transport, and heat transfer limited.

I think there is very little definition of the relative effects of these various processes at this point. And, it is just one of the problems. 

National Bureau of Standards Special Publication 561, Proceedings of the 10th Materials Research Symposium on Characterization of High Temperature Vapors and Gases held at NBS, Gaithersburg, Maryland, September 18-22, 1978. Issued October 1979.

\section{TRANSPORT, THERMODYNAMIC AND KINETIC ASPECTS OF SALT/ASH DEPOSITION RATES FROM COMBUSTION GASES}

D. E. Rosner, K. Seshadri, and J. Fernandez de la Mora

Yale University

High Temperature Chemical Reaction

Engineering Laboratory

New Haven, CT 06520

and

G. C. Fryburg, F. J. Koh1, C. A. Stearns, and G. J. Santoro

NASA Lewis Research Center

Cleveland, $\mathrm{OH} \quad 44135$

A combined experimental-theoretical attack on the problem of multicomponent deposition of condensible inorganic materials (present in combustion gases) on immersed solids (turbine blades, duct walls, heat exchanger surfaces) is outlined. With the help of seeded laboratory burner data on $\mathrm{Na}_{2} \mathrm{SO}_{4}$ and $\mathrm{B}_{2} \mathrm{O}_{3}$ deposition, a comprehensive but tractable convective diffusion deposition rate theory, based on the assumption of a multicomponent "chemically frozen" boundary layer $(\mathrm{CFBL})^{1}$, has been developed for making self-consistent salt/ash deposition rate predictions over a wide variety of environmental conditions of gas turbine and magnetogas-dynamic (MGD) interest. This CFBL theory utilizes the results of the NASA free-energy minimization LTCE computer program only at free stream and wall conditions, imposing the coupled effects of mass transport by both multicomponent Fick (concentration) and Soret (thermal) diffusion across the boundary layer. The application of our multicomponent formulation of CFBL deposition rate theory is illustrated using recent gravimetric measurements of the deposition rate of $\mathrm{Na}_{2} \mathrm{SO}_{4}$ (c) from a seeded laboratory burner on targets cooled below the prevailing "dew" point. Under the conditions of these experiments (highly undersaturated mainstream), particle formation and transport is evidently negligible; however, our CFBL theory is sufficiently general to embrace the transport of particles present in the mainstream, provided they are small enough to be considered heavy molecules. Interesting corollaries of the present CFBL theory are: (i) a "dew point" is not a

${ }^{1}$ Terminology is summarized as an Appendix at the end of this paper. 
purely thermodynamic quantity, but rather is influenced by Soret and multicomponent diffusion phenomena, as well as (gas phase and interfacial) nonequilibrium phenomena, and $(i i)$ in any particular situation there is more than one "dew point," depending upon its operational definition. We conclude with an assessment of the onset and role of boundary layer condensation processes and a summary of research needs highlighted by this program.

\section{Introduction}

Inorganic materials in fossil fuels (e.g., sulfur compounds in hydrocarbons and coal ash) and/or combustion air cause deposition and corrosion problems at surfaces immersed in combustion products. For example, "hot corrosion" is caused by $\mathrm{Na}_{2} \mathrm{SO}_{4}$ ( $\ell$ ) on gas turbine (GT) blades when the fuel contains sulfur (ca. 0.02 - 1.0 percent by element wt) and the intake air contains $\mathrm{NaCl}\left(\mathrm{s}\right.$ ) (a. $0.01-10 \mathrm{ppm}$ by wt, as in the marine environment) [1] ${ }^{2}$. Similarly, the vanadium in heavy fuel oils and the ash and sulfur content of coals used for conventional [2] or magneto-gas-dynamic power generation (see e.g., [3] result in aggressive condensates on heat exchanger surfaces and confining walls $[4,5]$. Current trends in the availability and price of fossil fuels indicate that these problems should, if anything, intensify. Moreover, whether the control strategy adopted is (a) fuel pretreatment, (b) fuel additives, (c) altered deposition conditions, and/or (d) the development of more resistant high temperature materials, an improved understanding of the kinetics, thermochemistry and dynamics of salt/ash formation and deposition will be necessary. Accordingly, our program is focused on the processes which lead to condensate formation on surfaces exposed to combustion products. Such condensed layers appear to be necessary to observe corrosive attack in GT and other applications [6].

Brown [7], Hedley, Brown and Shuttleworth [5], and Ross [8], all considered the transport of a single condensible vapor to the surface of a target by convection and Fick diffusion, using available engineering mass transfer coefficients. Two important assumptions of these analyses are: no condensation within the vapor concentration boundary layer (cf. Section 3.2) and local vapor liquid equilibrium (VLE) at the vapor/condensate interface In support of the experimental program summarized in Section 2.1 and 2.2, we have generalize these treatments to include the following additional essential features of the deposition problem in GT and MGD applications:

1. Multicomponent transport effects. At GT and MGD temperature levels, the salt which ultimately deposit are often partially dissociated, existing as vapor "precursors" [9]. Thus, many species containing the elements which ultimately appear in the condensate layer must be simultaneously considered in the vapor transport model.

\footnotetext{
${ }^{2}$ Figures in brackets indicate the literature references at the end of this paper.
} 
2. Thermal (Soret) diffusion. The inorganic salt or oxide vapors, particles, and their precursors, are typically characterized by effective molecular weights very different from that of the "host" combustion products. This gives rise to a non-negligible thermal (Soret) diffusion contribution to the species flux whenever there is a significant temperature gradient within the boundary layer ${ }^{3}$.

3. Variable property effects. When the target temperature is lower than that of the incident combustion products, gas properties (transport and thermodynamic) vary across the boundary layer, thereby affecting the dimensionless mass transfer coefficients ${ }^{4}$ in a manner somewhat different from (better known) heat transfer coefficients.

We have included these effects in a more general and accurate, but tractable scheme for predicting deposition rates (cf. Section 2.3), exploiting powerful LTCE computer codes $[9,11]$ for dealing with the intrinsically multicomponent character of this class of problems. An accurate, yet practical method for predicting dew points and deposition rates under both operating and laboratory test conditions can clearly be exploited to evaluate and/or suggest control strategies, relate laboratory test conditions to those encountered in engineering practice, and anticipate future problems associated with new operating conditions. Here, we describe (i) two types of experiments for obtaining experimental dew point and deposition rate data (Sections $2.1,2.2$ ), (ii) our present deposition rate theory (Section 2.3), and (iii) computational results and comparisons with laboratory test data (Section 2.4). Our conclusions and recommendations for future research (Section 4) follow a discussion of the treatment of particulate deposition phenomena (Section 3). Both the cases of pre-existing particles (Section 3.1) and particle formation within the boundary layer (Section 3.2) are briefly considered, and compared with the case of vapor (monomer) transport (Section 2.3).

\section{Inorganic Vapor Transport Across Combustion-Product Gas Boundary Layers}

When the combustion product temperature is high enough to preclude the existence of the offending condensed inorganic compounds, or if kinetic barriers preclude their appearance in the prevailing residences times, then condensates may form only at target surface temperatures lower than the "dew point" ( $d p)$ and the mechanism of transport at surface temperatures sufficiently close to $T_{d p}$ will be vapor diffusion across the combustion-product boundary layers.

${ }^{3}$ Both thermal diffusion and the multicomponent nature of the transport complicate imposition of the constraint of local thermochemical equilibrium (LTCE) at the vapor/liquid interface [10].

${ }^{4}$ Additionally, explicit allowance must be made for the empirically well-known effect of free stream turbulence (intensity and scale) on time-average transport rates to immersed surfaces. 


\subsection{Gravimetric experiments: experimental techniques}

Koh1, et a1. [12] have carried out atmospheric pressure experiments in which such a target is exposed to the products of a "seeded" liquid fuel/air combustor (cf. fig. 1) operated on a Jet $A-1$ fuel with an air-to-fuel weight ratio around $25 / 1$. Salt solutions were injected into the combustion chamber through a pump-fed aspirator system ${ }^{5}$. Solutions of the three different salts have been used: sea salt (see Section 2.3), $\mathrm{NaCl}$, and $\mathrm{Na}_{2} \mathrm{SO}_{4}$ with solution concentrations adjusted so that the Na concentration was roughly equivalent fo a11 three salts. Deposits were collected on $0.25 \mathrm{~mm}$ thick Pt-10 percent Rh cylinders mounte on Inconel supports. The collector, located about two jet diameters downstream of the burne exit nozzle, was rotated at $\mathrm{ca}$. $100 \mathrm{rpm}$ to ensure temperature homogeneity. Collector temperature was measured with a thermocouple peened in a hole in the support so that the couple junction made contact with the Pt cylinder. In the earliest experiments, collector temperatures were varied by simply adjusting the fuel mass flow rate into the burner. Subsequently, active (gas) cooling has been used for independent temperature control. Because deposition rates were shown to be independent of time, most runs were for about six hours to ensure sufficient deposits for subsequent chemical analysis. Deposits were leached from collectors first with water and then with 1:1 $\mathrm{HCl}$. Resulting solutions were analyzed for metal cations, $\mathrm{SO}_{4}^{=}$and $\mathrm{Cl}^{-}$.

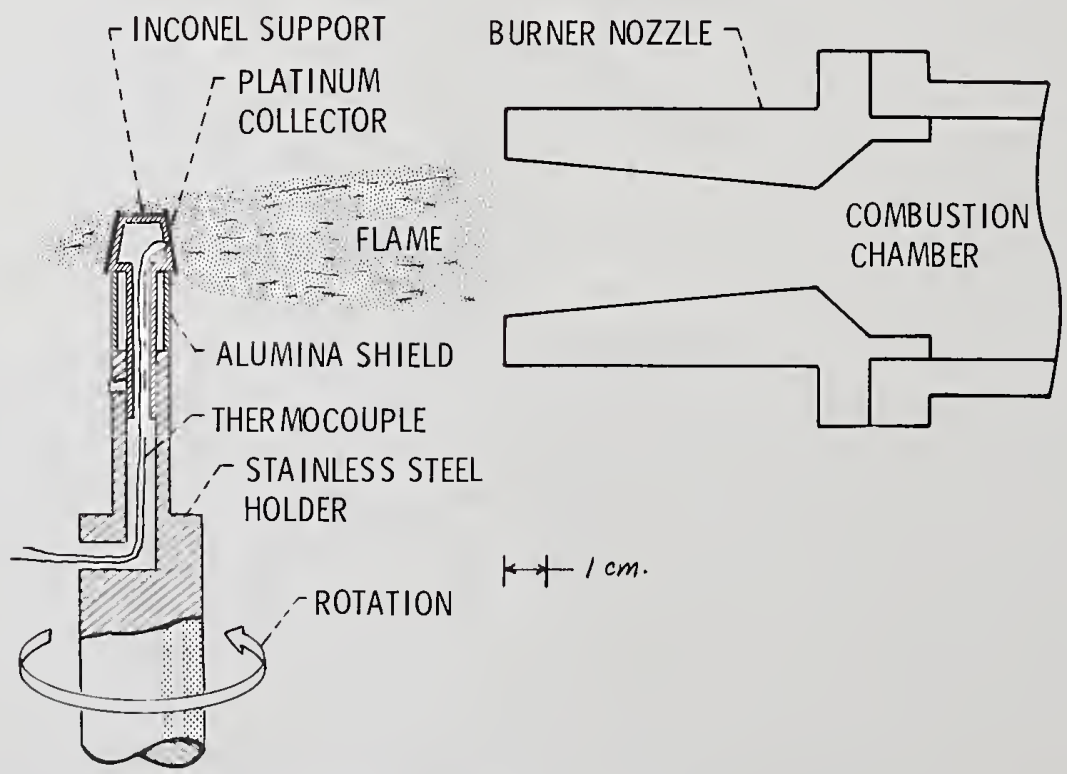

Figure 1. Schematic of burner-rig salt deposition apparatus; gravimetric technique.

${ }^{5}$ Residence time between the salt injection and collection points was about 2.2 ms. 
Typical observed $\mathrm{Na}_{2} \mathrm{SO}_{4}$ (c) deposition rates from atmospheric pressure flames doped with ca. $10.5 \mathrm{ppm} \mathrm{Na} \mathrm{SO}_{4}$ are shown in figure 2 as a function of target surface temperature. While exhibiting considerable scatter, for surface temperature above $1000 \mathrm{~K}$, the experimental trend reveals a monotonic increase in deposition rate with the temperature difference $T_{w}-T_{d p}$, where $T_{d p}$ is operationally defined by extrapolating the deposition rate curve to zero. The various predictions superimposed on figure 2 will be discussed in Section 2.4, however, it is interesting to note that in this system, $\mathrm{T}_{\mathrm{dp}}$ can be estimated to within about $10 \mathrm{~K}$ by a simple thermochemical equilibrium calculation at the mainstream chemical element ratios $[9,10]$. In general, the dew point is influenced by transport (thermal and multicomponent diffusion) and kinetic (homogeneous and heterogeneous) phenomena [13]. Also, in these experiments, the mainstream was sufficiently undersaturated and BL residence times sufficiently small that condensation did not occur within the gas boundary layer adjacent to the cooled target. However, under other experimental conditions (see e.g. [7]) boundary layer condensation can set in, lowering the net observed deposition rates sufficiently far below the dew point. A theoretical treatment of this regime has not yet been reported, but now appears within reach (see Section 3.2 and [14]). The simpler problem of deposition from a stream containing "pre-existing" particles (e.g., the mainstream gas temperature is itself below the "dew point") is considered in Section 3.1.

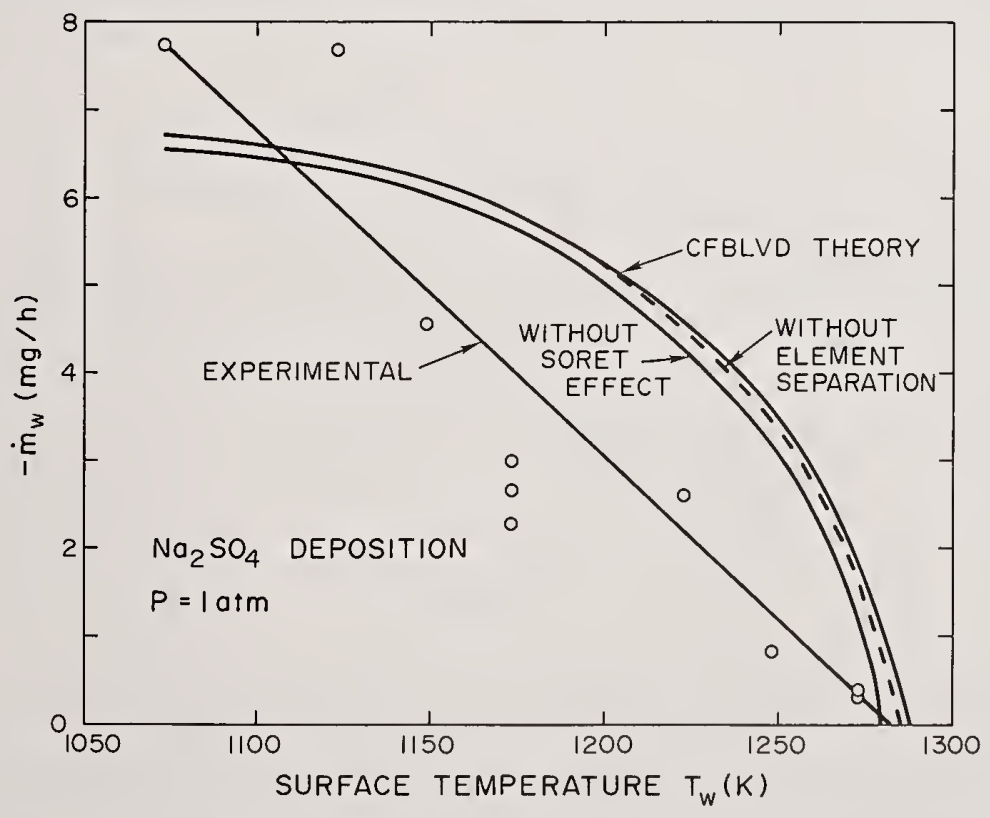

Figure 2. Predicted and experimental $\mathrm{Na}_{2} \mathrm{SO}_{4}$ deposition rate as a function of target temperature.

\subsection{Optical experiments: techniques and preliminary results}

Remote optical methods can be used for measuring dew points, and deposition rates of inorganic salts on surfaces exposed to seeded combustion gases [15]. Such measurements 
are made either by recording interference in a reflected beam of monochromatic light ${ }^{6}$, or by measuring the change in polarization upon reflection. To demonstrate the interference method, a deliberately simple experimental system was used, in which an electricaliy heated platinum ribbon was immersed in seeded flat-flame combustion product gases. To avoid experimental uncertainties involved in accurately providing and measuring steady flow rates of salt solutions into the burner, boron trichloride, a gas at STP, was used as the seed material in our preliminary experiments. Boric oxide $\left(\mathrm{B}_{2} \mathrm{O}_{3}\right)$ is formed (presumably) in the flame and its dew point and growth rate can then be measured using the interference technique. The apparatus [15] in its present form (cf. fig. 3) consists of: (a) an optical system, (b) an electrical system (for heating the target, and measuring its temperature), (c) the burner assembly, and (d) the gas feed system. Only a brief discussion of the apparatus and experimental procedure is given here.

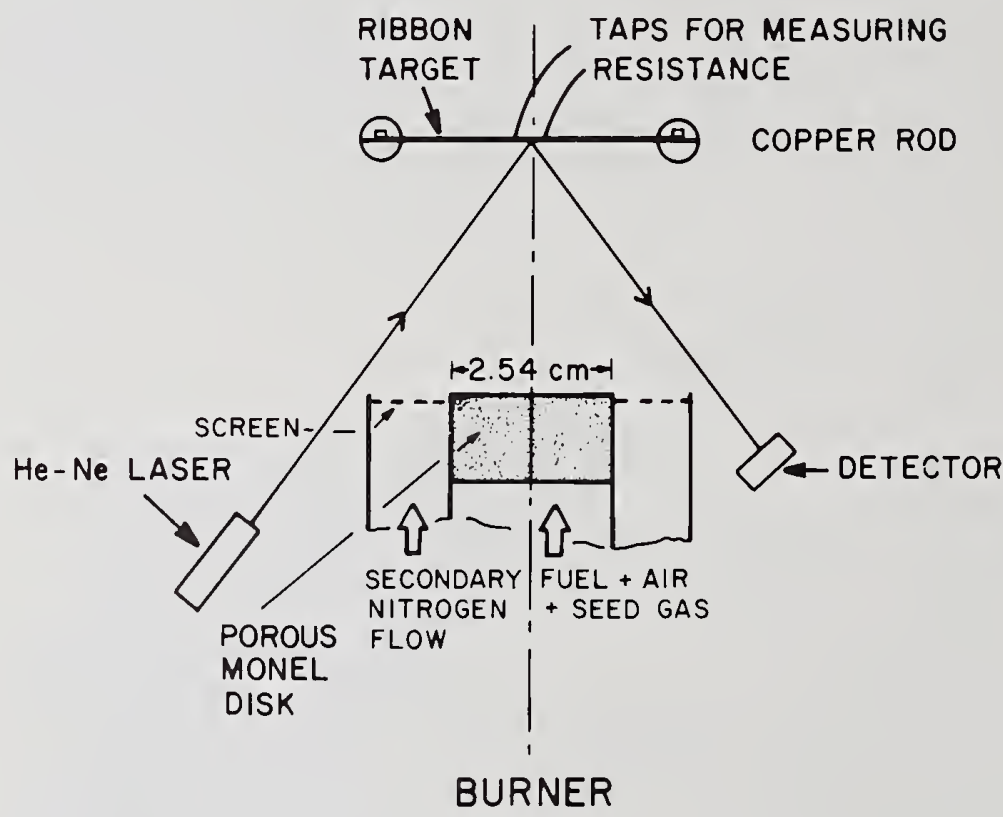

Figure 3. Schematic diagram of experimental arrangement for deposition rate measurements using the optical interference method [15].

A linearly polarized helium-neon laser is used as a source of monochromatic light $(0.6328 \mu \mathrm{m})$. The beam reflected from the target is then focused on the aperture of a photometer whose output is recorded on a strip chart potentiometer.

\footnotetext{
6McIntyre and McTaggart [16] used interference in reflected sodium lamp radiation to study solid iodide film growth on silver. Sugawara, et al. [17] exploited the interference technique to study silicon dioxide $\left(\mathrm{SiO}_{2}\right)$ growth rates on silicon wafers using $\mathrm{He}-\mathrm{Ne}$ laser radiation.
} 
The target is a platinum ribbon ( $50 \mathrm{~mm}$ long, $6 \mathrm{~mm}$ wide, $0.127 \mathrm{~mm}$ thick) held above the burner by two copper rods. The ribbon is electrically heated by connecting the copper rods to a $50 \mathrm{amp}(\max ) \mathrm{dc}$-power supply. The electrical resistance of a $4 \mathrm{~mm}$ wide central section of the ribbon is measured using two platinum "tap" wires of $0.127 \mathrm{~mm}$ diameter spotwelded to the ribbon. The temperature of the ribbon is then calculated from the known value of its electrical resistance.

The burner consists of a stainless steel tube of $2.54 \mathrm{~cm}$ inner diameter, $7.62 \mathrm{~cm}$ long. A premixed gaseous stream of fuel (propane), air, and the seed vapor is fed to the burner at the bottom of the tube. Variable area flowmeters are used to measure individual gas flow rates. To obtain a laminar flow of the premixed gases with a uniform velocity above the burner, a porous monel disk $2.54 \mathrm{~cm}$ diameter and $1.27 \mathrm{~cm}$ thick is placed at the top of the tube.

To measure boric oxide deposition rates by the interference method, the ribbon was positioned at approximately $3.8 \mathrm{~cm}$ from the surface of the burner. Flow rates of air and propane were adjusted to obtain a blue, stable, flat-flame. Initially, the electrical current flowing through the ribbon was maintained high enough for the temperature of the ribbon to be a few hundred degrees above the dew point of boric oxide at the anticipated $\mathrm{BCl}_{3}$-seed level. The seed gas is then turned on, at which point the flame was visually observed to turn green. The heating current to the ribbon is then reduced to bring the

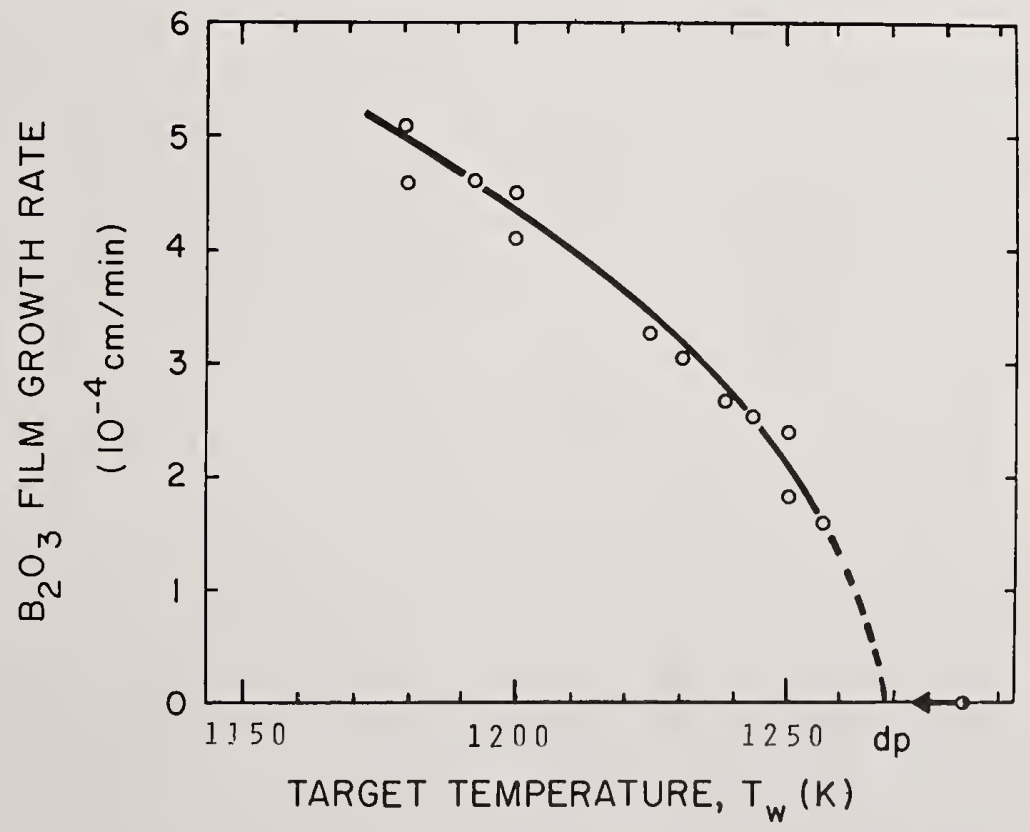

Figure 4. $\mathrm{B}_{2} \mathrm{O}_{3}$ deposition rates inferred using the interference method [15] $\left(\mathrm{BCl}_{3}\right.$-seeded propane-air flame, equivalence ratio $=0.812$, moles $\mathrm{BCl}_{3} /$ moles fuel $=1.70 \times 10^{-2}$, ribbon $3.8 \mathrm{~cm}$ above burner). 
ribbon temperature below the $\mathrm{B}_{2} \mathrm{O}_{3}$-dew point. A sharp decrease in the intensity of the reflected light is observed at the instant of oxide condensation. As the oxide film grows, the light reflected from the outer surface of the growing oxide layer and from the ribbon surface undergoes constructive and destructive interference causing a modulated intensity in the collected beam. The corresponding film growth rate is calculated from the spacing between intensity maxima, using the known chart recorder speed. The ribbon heating current is then increased to evaporate the oxide layer, and the experiment repeated to obtain the growth rate at a different target temperature. The composition of the $\mathrm{film}$ was analyzed on a Hewlett-Packard Mode1 5950 ESCA Spectrometer system, and confirmed to be boric oxide. Our preliminary results at one $\mathrm{BCl}_{3}$-seed level are shown in figure 4 . The dew point of boric oxide, defined as the temperature of the target $^{7}$ at which the growth rate of boric oxide extrapolates to zero, is evidently about $1270 \mathrm{~K}$.

\subsection{CFBL theory of dew points and deposition rates}

To quantitatively describe deposition phenomena such as shown in Sections 2.1 and 2.2, we make the simplifying assumption that only a negligible amount of chemical reaction and/or condensation occurs within the mass transfer gaseous boundary layer adjacent to the target surface. This is the tractable limiting case of a single phase "chemically frozen" boundary layer (CFBL) within which convection, turbulence, concentration (Fick) diffusion and thermal (Soret) diffusion participate in species mass transport to/from the surface. While the specific numerical examples cited below pertain to sodium sulfate $\mathrm{Ca}_{2} \mathrm{SO}_{4}(\ell)$ deposition, the theory embraces other inorganic compounds and their precursors, including mixtures (e.g., "sea salt") 8 and particles small enough to be considered heavy "molecules." As discussed in detail elsewhere [10], we exploit the fact that the inorganic molecules participating in the deposition process are present in only trace amounts. For each such species $i$, present in local mass fraction $w_{j}$, the flux law for boundary layer mass transport can be simplified to:

$$
\vec{j}_{i}^{\prime \prime}=-D_{i} \rho\left(\nabla w_{i}+\alpha_{i} w_{i} \nabla \ell n T\right)
$$

\footnotetext{
${ }^{7}$ Uncertainties in target temperature measurement are expected to be less than $\pm 10 \mathrm{~K}$, and the inaccuracies in obtaining the growth rate are expected to be less than \pm 5 percent. Deposition rates reported here are based on the room temperature refractive index of $\mathrm{B}_{2} \mathrm{O}_{3}(1.458)$.

${ }^{8} \mathrm{On}$ a weight basis a typical analysis of sea salt [12] is: $\mathrm{NaCl}$ (68.78\%), $\mathrm{MgCl}_{2}(14.57 \%)$, $\mathrm{Na}_{2} \mathrm{SO}_{4}(11.46 \%), \mathrm{CaCl}_{2}(3.25 \%), \mathrm{KCl}(1.93 \%)$.
} 
where the two scalar transport properties $D_{i}$ (Fick diffusion coefficient) and $\alpha_{i}$ (dimensionless thermal (Soret) diffusion factor) are specific to each species-host mixture combination ${ }^{9}$. This flux equation applies equally well to the Brownian and thermophoretic (Soret) migration of particles, provided they are small enough to be treated as heavy molecular species.

In the absence of turbulence and thermal diffusion, the form of eq. (1) leads directly to the introduction of dimensionless mass transfer coefficients $\mathrm{Nu}_{\mathrm{m}, i}$ (local Nusselt numbers for mass transport) defined such that each species diffusional flux at the "wall" (vapor side of the vapor/condensate interface) can be written in the separable algebraic form

$$
-j "{ }_{i, w}\left(C F B L, \alpha_{i}=0\right)=\frac{\left(D_{i} \rho\right) e}{x} \cdot N u_{m, i}\left(x / L, R e, S c_{i}, \ldots\right) \cdot\left(w_{i, e}-w_{i, w}\right)
$$

where the subscripts $e$ and $w$, respectively, pertain to local conditions at the outer and inner "edges" of the gas boundary layer (fig. 5)10. Inclusion of thermal diffusion into the species $i$ mass conservation equation introduces three effects $[18,19]$ :

El - a convection-like (thermophoretic) transport term, which produces a "suction like" augmentation of the mass transfer coefficient $\mathrm{Nu}, i$,

E2 - a "source-like" term analogous to that which would appear in the presence of the homogeneous reaction of species $i$, and

E3 - a non-Fick contribution to the species $i$ flux at the wall (whenever $w_{i, w}$ is nonzero).

In what follows effects $\mathrm{l}$ and 2 on $\mathrm{Nu}_{\mathrm{m}, j}$ are lumped in the factor $\mathrm{F}_{j}$ (Soret). When the target is colder than the free stream (the case of interest here), E2 is usually negligible [19] and $F_{i}$ (Soret) is expected to depend predominantly on the dimensionless group:

$$
\left.\tau_{i} \equiv \alpha_{i, w}\left(L_{i, w}\right)^{0.4}\left[\left(T_{e}-T_{w}\right) / T_{W}\right] \text { (thermophoretic parameter }\right)^{11}
$$

${ }^{9} 0$ ften (owing to small fuel/air ratios in GT-combustion), the host mixture can be considered to be merely "hot air," treated as a single ("lumped") species with properties close to that of $\mathrm{O}_{2}(\mathrm{~g})$ and $\mathrm{N}_{2}(\mathrm{~g})$.

${ }^{10} \mathrm{~A} 11$ remaining subscripts and symbols are defined in the Appendix.

${ }^{11}$ When the Mach number is not negligible, $T_{e}$ in eq. (3) should be replaced by the "recovery" temperature [18]. In the particle limit ( $S C \gg 1$ ) the $L_{i, w}$ dependence of $\tau_{i} i s$ altered and E2 is no longer negligible. 
and the Lewis number $L_{i}$. Then, in the absence of free stream turbulence effects, each species diffusive flux to the wall can be written ${ }^{12}$

$$
\begin{aligned}
-j{ }^{\prime \prime}{ }_{i, w} & =\frac{\left(D_{i} \rho\right)}{x} \cdot F_{i}(\text { Soret }) \cdot N u_{m, i}\left[\left(w_{i, e}-w_{i, w}\right)\right. \\
& \left.+\tau_{i} \cdot \frac{F(\text { ncp })}{F_{i} \text { (Soret) }} \cdot w_{i, w}\right]
\end{aligned}
$$

where $\mathrm{Nu}_{\mathrm{m}, \mathrm{i}}$ includes the effects of variable properties but not thermal diffusion, and $F(n c p)$ is a correction factor for variable (non-constant) properties ${ }^{13}$.

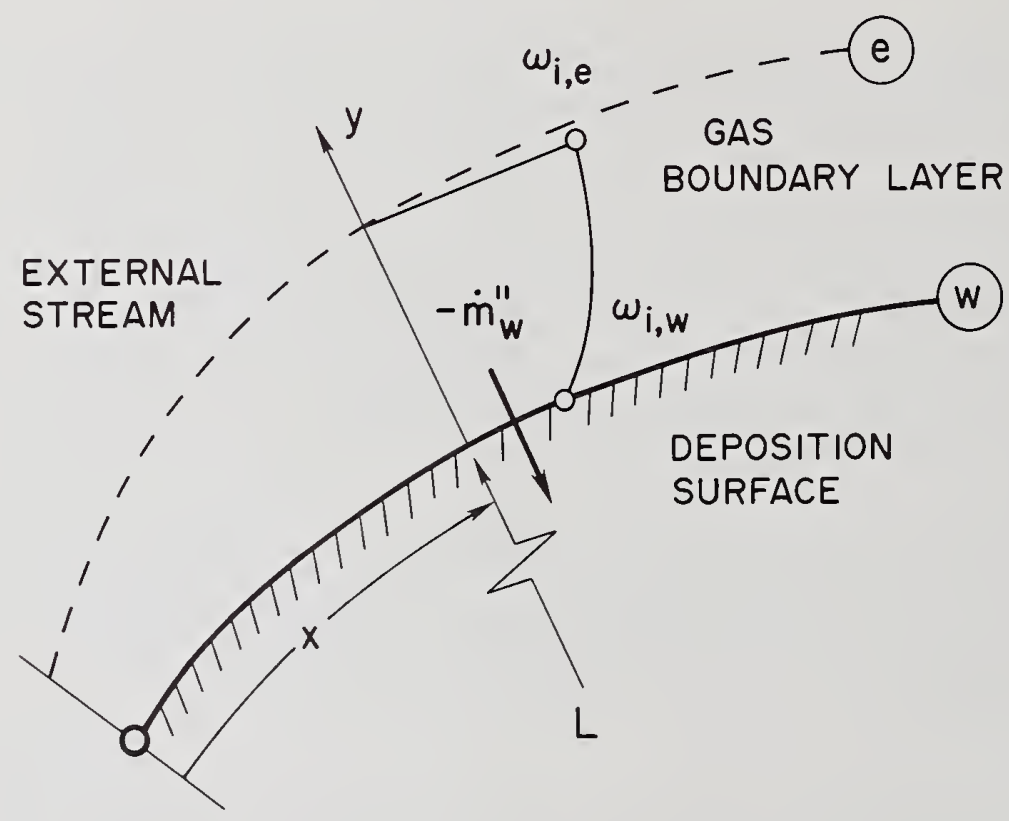

Figure 5. Diffusion boundary layer configuration and nomenclature.

\footnotetext{
${ }^{12}$ For the calculations made here (cf. fig. 2), we have used the preliminary estimate $F_{i}($ Soret $) \approx \tau_{i} /\left[1-\exp \left(-\tau_{j}\right)\right]$.

${ }^{13}$ Variable properties do not have the same effect on $\mathrm{Nu}_{\mathrm{m}, j}$ as on the corresponding heat transfer coefficient $\mathrm{Nu}_{h}$. Results for $\mathrm{Nu}_{m}$ have recently been computed for stagnation line boundary layer flows [19].
} 
Apart from turbulence within the boundary layer, which influences the prevailing values of $\mathrm{Nu}_{\mathrm{m}, i}$, free stream turbulence (level and scale) can augment the time-averaged transport rates to/from stationary surfaces. Perimeter-mean heat transfer measurements for cylinders (diam. d) in cross-flow can be correlated by introducing an augmentation factor $F($ turb $)[10,12]$. In the absence of more detailed information on the local effects of free stream turbulence on each species $i$, this factor is applied to the predicted perimeter-mean and time-mean transport rate of each species $i^{14}$. The trace species mass fractions $w_{i, e}$ at the outer edge of the boundary layer are rarely known via direct sampling measurements and may be influenced by the kinetics of vapor phase reactions involved in the formation of condensible species precursors. Thus, even in situations where $\mathrm{Na}_{2} \mathrm{SO}_{4}(l)$ is the ultimate deposit, sodium in the external stream can be distributed among the vapor species $\mathrm{NaCl}, \mathrm{Na}, \mathrm{NaOH}, \mathrm{NaSO}_{2}, \mathrm{NaSO}_{3}, \mathrm{Na}_{2} \mathrm{SO}_{4}, \mathrm{NaO}$, etc. Chemically, the simplest assumption that can be made, and fortunately one which is often realistic, is that local thermochemical equilibrium (LTCE) is achieved at station $e$ in the vicinity of the stagnation line (point). In such cases, the species compositions $w_{i, e}$ (or their corresponding mole fractions $y_{i, e}$ ) are those that would be expected from LTCE at the stagnation pressure, stagnation temperature and overall elemental composition, the latter usually being close to (or even equal to) the elemental compositions entering the combustor. All other flow quantities at stations e can then be estimated as in conventional inviscid gas dynamic calculations (e.g., around GT-blades) for the "carrier" gas.

Perhaps more interesting is the situation at the gas/condensate interface, for even if LTCE is imposed at stations $\underline{w}$, the local element mass fractions of the inorganic constituents need not be (and generally will not be) equal to those characterizing the external stream. Both thermal diffusion and multicomponent diffusion will contribute to this element "segregation," 15 hence a self-consistent LTCE calculation at station w requires simultaneous consideration of the transport laws and the LTCE condition [10]. For example, in the case of deposition of a single pure compound; e.g., $\mathrm{Na}_{2} \mathrm{SO}_{4}(\ell)$ below its "dew point," the following two constraints are simultaneously imposed:

Cl - Vapor-Liquid Equilibrium (VLE). The vapor mixture composition at station $\underline{w}$ should be compatible with the thermochemical co-existence of $\mathrm{Na}_{2} \mathrm{SO}_{4}(\mathrm{c})$ at temperature $T_{W}$ and pressure $p$.

${ }^{14}$ More generally, eq. (4) should be multiplied by a factor $F_{i}$ (turb) at the prevailing streamwise location prior to perimeter-averaging.

${ }^{15}$ Quite analogous to additive segregation in other "seeded" systems with heat and mass transport; e.g., halogen cycle lamps [20] and metal halide-augmented discharge lamps [21]. 
$\mathrm{C} 2$ - $\mathrm{Na} / \mathrm{S}$ Flux-Ratio. Since $\mathrm{Na}_{2} \mathrm{SO}_{4}$ (c) is being deposited, the molar fluxes of Na and $\underline{S}$ must stand in the ratio $2: 1$ at the prevailing vapor compositions.

In practice, these two conditions are satisfied by searching along the VLE-constraint for the point at which the total $\mathrm{Na}$ and $\mathrm{S}$ element mass fluxes stand in the ratio 1.434:1. In the more complex case of mixed (multicomponent) condensates (see e.g., the experimental results of Durie [22]), the VLE constraint is based on equality of chemical potential of each chemical species $i$ in both vapor and condensed phase, and trace element fluxes have to stand in the same ratios as those prevailing in the co-existing condensate.

A useful expression for the total CFBL mass deposition flux $-\dot{m}_{w^{\prime \prime}}$ (CFBL) can be obtained by noting that for each element $k$, we can write the "jump" condition $[23,24]$ :

$$
-\dot{m}_{w}^{\prime \prime}\left(w^{c}(k)-w_{(k), w}\right)=j^{\prime \prime}{ }_{(k)}-j_{(k), w}^{\prime \prime}
$$

where superscript $\underline{c}$ designates the condensate side of the vapor/condensate interface. Neglecting element diffusion within the condensate, and solving for total mass deposition rate, $-\dot{m}_{w}^{\prime \prime}$, we obtain:

$$
-\dot{m}_{w}^{\prime \prime}=\frac{-j^{\prime \prime}(k), w}{w_{(k)}^{c}-{ }^{w}(k), w} .
$$

In the sodium sulfate case considered in Section $2.1, w_{(\mathrm{Na})}^{c}=0.3237$ and $w_{(\mathrm{Na}), \mathrm{w}}$ is of the order of $10^{-5}$, hence eq. (6) for the element $\mathrm{Na}$ specializes to $-\dot{m}_{w}^{\prime \prime} \approx-j_{(\mathrm{Na}), w}^{\prime \prime} /(0.3237)$ [12]. of course, to apply CFBL deposition rate theory, engineering estimates of the thermodynamic and transport properties of the individual species and prevailing local gas mixtures are required (see e.g., $[25,26]$ ). For this purpose, we assume that transport properties for each vapor species depend on local pressure and temperature in accord with the predictions of first-order Chapman-Enskog (CE) theory with estimated Lennard-Jones (LJ) 12:6 interaction parameters [26]. In this connection, it is interesting to note that while for trace species $i$ the (dimensionless) thermal diffusion factor is independent of the mole fraction $y_{i}$, it is given by rather cumbersome expressions [27]. To facilitate repetitive computations, it was, therefore, assumed that the "carrier" gas for each such species $i$ was pure hot air and each CE-calculated $\alpha_{j}(T)$ was curve-fit using the two-parameter law 


$$
\alpha=\alpha_{\infty}+\alpha_{-1} T^{-1}=\alpha_{\infty} \cdot\left[1+\frac{\left(\alpha_{-1} / \alpha_{\infty}\right)}{T}\right]
$$

(e.g., for $\mathrm{Na}_{2} \mathrm{SO}_{4}(\mathrm{~g})$ in air, we estimated $\alpha_{\infty}=0.7828$ and $\left(\alpha_{-1} / \alpha_{\infty}\right)=-476.2 \mathrm{~K}$ ). When the diffusing "species" are small particles, we have developed simple formulae to interpolate between transport property results applicable to the extremes of particles small or large compared to the prevailing gas mixture mean-free-path. The above-mentioned "diffusional" theory is then used only under conditions such that the local inertial stopping distance of the particle is small compared to the prevailing particle diffusion boundary layer thickness (see Section 3.1).

Combining the above-mentioned information allows rational predictions of the distribution of condensate flux on targets with prescribed surface temperature distributions. For isothermal surfaces, often only the perimeter-mean deposition rate is required, in which case eq. (4) is applied with the average transfer coefficients $\bar{N} u_{m, i}$ and the reference target dimension $L$ (replacing $\mathrm{Nu}_{m, j}(x)$ and $x$, respectively). The dew point defined by $-\dot{m}_{w}^{\prime \prime}\left(T_{d p}\right)=0$ is, in general, different from that predicted on the basis of thermodynamics and mainstream element ratios alone (see Section 2.4). Indeed, neither of these values correspond exactly to the "dew point" defined by asking when a clean (condensate-free) surface will first exhibit a stable condensate [13]. Calculations of these differences are in progress using the above-mentioned CFBL formalism, as well as an alternative theory based on assuming the existence of LTCE everywhere within the boundary layer (not just at stations $\underline{e}$ and $\underline{w}$ ) [28].

\subsection{Comparisons between theory and experiment}

Returning to the $\mathrm{Na}_{2} \mathrm{SO}_{4}(\mathrm{c})$ deposition rates from atmospheric pressure flames doped with ca. $10.5 \mathrm{ppm} \mathrm{Na}_{2} \mathrm{SO}_{4}$ (fig. 2), CFBL theory predictions are seen to be reasonably successful far below the dew point, but apparently CFBL theory over-predicts deposition rates in its vicinity (ca. $100 \mathrm{~K}$ ). Observe that, (i) only in this range does the inclusion of element segregation noticeably influence the CFBL deposition rate prediction, and (ii) both thermal diffusion and element separation become quite important sufficiently close to the above-mentioned dew point ${ }^{16}$. While condensation within the boundary layer

${ }^{16}$ Indeed, these phenomena are associated with dew point "shifts" of approximately $9 \mathrm{~K}$ and $3 \mathrm{~K}$, respectively, revealing that the "dew point" is by no means purely "thermodynamic" quantity [13]. 
can be precluded as the cause of CFBL over-predictions near $T_{d p}$ (Section 3.2), homogeneous chemical reactions among the trace species transporting $\underline{\mathrm{Na}}$ and $\underline{\mathrm{S}}$ may become important. However, pending ongoing improvements in the characterization and precision of such deposition experiments, speculation on such refinements in CFBL theory would clearly be premature.

The expense of high pressure test facilities provides ample incentive to understand the pressure dependence of deposition rate and hot corrosion phenomena in GT-applications. Typical CFBL predictions at two temperatures $(1073 \mathrm{~K}, 1173 \mathrm{~K}$ ) below the 1 atm dew point are shown in figure 6 on logarithmic coordinates ${ }^{17}$. As expected, the sensitivity of deposition rate to pressure is greatest at the higher target temperature, until pressure levels are reached such that even this temperature is low compared to the prevailing dew point (at which the dependence is almost $\sim p$ at very high Reynolds number). In contrast, for surface temperatures well below the $p=1$ atm dew point, $-\dot{m}_{w}^{\prime \prime}$ increases more like the square root of the pressure (ca. $\mathrm{p}^{0.55}$ ) near $1 \mathrm{~atm}$. The validity of such CFBL predictions will be tested using experimental results from a pressurized turbine combustor/deposition rig.

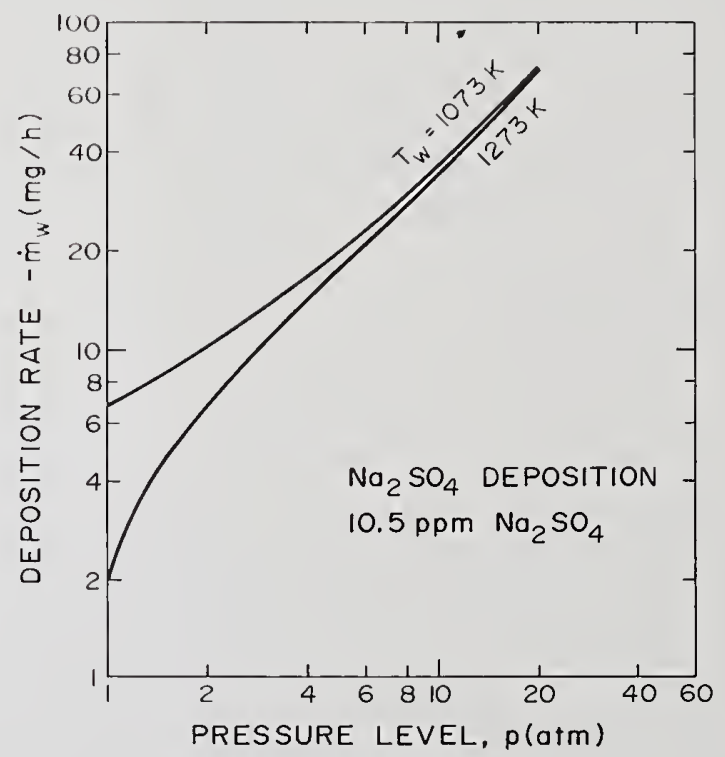

Figure 6. Predicted pressure dependence of the $\mathrm{Na}_{2} \mathrm{SO}_{4}$ deposition rate at two target temperatures.

${ }^{17}$ In making these predictions, we assumed that all input (fuel + air) mass flows increased linearly with pressure. At the prevailing fuel-air ratios, there was a negligible effect on combustion gas temperatures. 
For $\mathrm{B}_{2} \mathrm{O}_{3}$-deposition, detailed comparisons between our results (fig. 4) and $\mathrm{CFBL}$ theory are in progress ${ }^{18}$. However, it is interesting to note that whereas in flames doped with sodium sulfate, the dew point could be estimated to within $10 \mathrm{~K}$ by a simple LTCEcalculation at the mainstream chemical element ratios; in the corresponding $\mathrm{BCl}_{3}$-seeded case, a similar LTCE calculation does not predict a dew point for boric oxide condensation even at $300 \mathrm{~K}$ ! Such "complete" equilibrium thermochemical calculations show that the formation of $\mathrm{B}_{2} \mathrm{O}_{3}(\mathrm{c})$ is "prevented" by the formation of metaboric acid $\left(\mathrm{HBO}_{2}(\mathrm{~g})\right.$ ) and its trimer. The fact that experimentally $\mathrm{B}_{2} \mathrm{O}_{3}(\mathrm{c})$ appears below $1270 \mathrm{~K}$ corroborates our earlier assertion that the "dew point" is by no means a pure thermodynamic quantity, but can be affected by transport phenomena and kinetics limitations [13]. In the present case the kinetic limitations may be: (1) the gas phase hydrolys is of boron oxides, or (2) the gasification of $\mathrm{B}_{2} \mathrm{O}_{3}(\mathrm{l})$ by hydrogen-containing species (e.g., $\mathrm{B}_{2} \mathrm{O}_{3}(\mathrm{l})+\mathrm{H}_{2} \mathrm{O}(\mathrm{g}) \rightarrow 2 \mathrm{HBO}_{2}(\mathrm{~g})$, etc.). Indeed, a calculated dew point close to that experimentally observed can be obtained by assuming that: (1) the mainstream is in LTCE, (2) the boundary layer is chemically frozen, and (3) only "partial" chemical equilibrium exists at the surface of the target. Specifically, we assumed that mainstream $\mathrm{HBO}_{2}(\mathrm{~g})$ was inert, and precluded the formation of $\mathrm{HBO}_{2}(\mathrm{~g})$ and its trimer at the surface. The dew point so predicted was found to be approximately $160 \mathrm{~K}$ higher than the experimental dew point, perhaps implying that the above-mentioned gasification reaction is not completely negligible at the prevailing target temperatures. We plan to investigate the heterogeneous limitation via experimental studies.

\section{Mainstream and Boundary Layer Condensation Effects}

The formation of a condensate "aerosol," either in the mainstream or within the thermal boundary layers, influences the deposition rate because: (i) the effective values of Fick and Soret diffusivity are altered from their vapor-values, and (ii) sufficiently large particles will move differently than the host gas mixture, allowing them to "inertially" or "thermophoretically" impact by crossing gas streamlines.

\subsection{Mainstream condensation-deposition rate behavior of "pre-existing" particles/droplets}

Useful perspective on the simplest case of "pre-existing" particles can be obtained by considering a hypothetical flow situation of fixed inorganic content (mass fraction) and first asking how the deposition rate would vary if all of the inorganic material were

${ }^{18}$ These calculations require an estimate of the Nusselt numbers for the transport of vapor species containing boron across the concentration boundary layer at the prevailing Reynolds number (approximately $\approx 15$ ) and estimates of property values of all relevant vapor species. 
present in particles of a single size, containing g monomer units ${ }^{19}$ (see fig. 7 and [29]). Up to some value of $g$, written $g_{\star \star}$, our CFBL deposition rate theory is applicable since, under the prevailing boundary layer flow conditions, such particles behave like heavy ("macro-") molecules. If thermal diffusion (thermophoresis) were absent (e.g., $\Delta T / T_{W}=0$ ), the deposition rates would be less than the corresponding monomer (vapor) value, as shown. Particles above the value $g_{*}$, corresponding to the prevailing critical Stokes (impaction) parameter, can inertially impact on the target. Indeed, at sufficiently large g all upstream particles within the projected area of the target impact, causing the deposition rate to exceed the corresponding monomer value by a factor of the order of (Re $)^{1 / 2}$ if these particles or droplets can "stick." Inspection of figure 7 immediately suggests the strateg: of controlling particle nucleation/growth/coagulation such that the mainstream predominantl? contains particles with $g$-values in the range $g_{* *} \leq \mathrm{g} \leq \mathrm{g}_{*}$, thereby minimizing the deposition rate. Expressions for $\dot{\mathrm{m}}_{\mathrm{min}} / \dot{\mathrm{m}}_{1}$ in this transition size range have been derived [29], and we find that the presence of thermal diffusion significantly modifies (and can even eliminate) this predicted minimum deposition rate. Turning again to the large particles $\left(g \gg g_{*}\right.$ ), deposition rate reductions can be obtained only by achieving reflection or reentrainment, conditions usually feasible only if the condensate is a solid at the moment of impact. Of course, in general, the "available" inorganic material is distributed among large numbers of size classes so that values such as shown on figure 7 would have to be

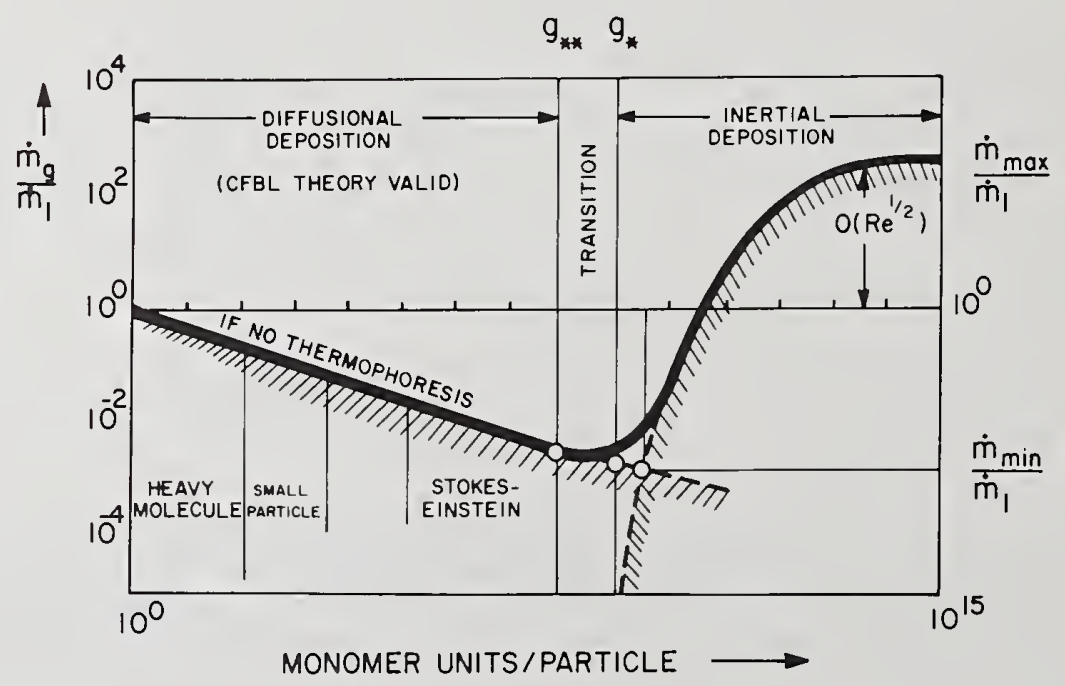

Figure 7. Schematic of deposition rate behavior for pre-existing particles (g-mers).

${ }^{19}$ If $R_{1}$ is the monomer radius, then $R_{g} \approx g^{1 / 3} R_{1}$. 
"weighted" in accord with the mass fraction of material in each size class [30]. Results of this type, as well as the corresponding implications for the spatial distribution of deposition ${ }^{20}$, will be presented elsewhere.

\subsection{Boundary layer condensation-onset condition and deposition rate behavior}

If vapor condensation occurs within the boundary layer, the CFBL vapor deposition (VD) model breaks down. The mechanism of trace impurity transport to the wall changes, and deposition rates fall rapidly as the target is cooled. Early experimental evidence of such behavior is availble [7]--it may be regarded as the counterpart of enhanced vaporization rates due to fog formation in the thermal boundary layer near an evaporating condensed phase $[31,32,33]$. However, in the condensation case no rational method has been reported to predict the effect of fog formation on the mass deposition rate.

Two different "barriers" can prevent boundary layer nucleation: (BI) thermodynamic (i.e., the local supersaturation has to be sufficient to produce the required number of nuclei for an appreciable nucleation and growth vapor sink); and (B2) kinetic (i.e., the number of collisions between trace vapor species, and between nuclei and vapor molecules, has to be sufficient to allow nucleation and growth during the limited residence time available within the boundary layer). Both increasing the mainstream supersaturation (via higher inorganic impurity levels and/or lower temperature) and reducing the wall temperature contribute to remove BI. Barrier 2 is overcome at sufficiently high impurity levels and residence times. Both barriers probably play a role in the $\mathrm{Na}_{2} \mathrm{SO}_{4}$-deposition rate experiments reported by Kohl, et al. [12], where no reduction in the deposition rates are apparent even $200 \mathrm{~K}$ below the observed dew point (fig. 2).

The crossing of Barrier 1 can be predicted by comparing the diffusion term and the sink term in the vapor mass transport equation, exploiting the facts that: ( $i$ ) the nucleation rate is negligible outside a very narrow zone (nucleation "peak"); and (ii) the Brownian diffusivity of the condensation nuclei is also negligible. In this way, a simple criterion [14] can be developed giving a locus in the temperature-supersaturation (T-s) plane, which divides the fog-free CFBLVD region from the regime in which the CFBLVD model fails. Interestingly enough, this approach preserves the validity of the critical supersaturation method [32] and eliminates the previous arbitrariness in the relevant value of $\mathrm{J}_{\text {crit }}$ [33]. Thus, under the conditions of the experiments of Kohl, et al. [12], we find that the relevant $s(T)$ locus (assuming all $\mathrm{Na}$ in the gas phase in the form of $\mathrm{Na}_{2} \mathrm{SO}_{4}$ ) lies very close to the locus of constant nucleation rate $\mathrm{J}_{\mathrm{crit}}=10^{15} \mathrm{~cm}^{-3} \mathrm{~s}^{-1}$ (cf. fig. 8) predicted by formally applying classical nucleation theory [34]. The method of Rosner and Epstein [31] with this environment-dependent "effective" scrit (T)-locus, then rules out boundary layer condensation under these conditions (cf. fig. 2). Unfortunately, more positive confirmation of this model is not available using the data of Brown [7] because information on the prevailing mainstream temperature is lacking.

${ }^{20}$ The impaction mechanism ( $\mathrm{g} \gg \mathrm{g}_{*}$ ) yields deposition on the "windward" (pressure) surfaces of objects, but surface flows can sometimes obscure this fact. 


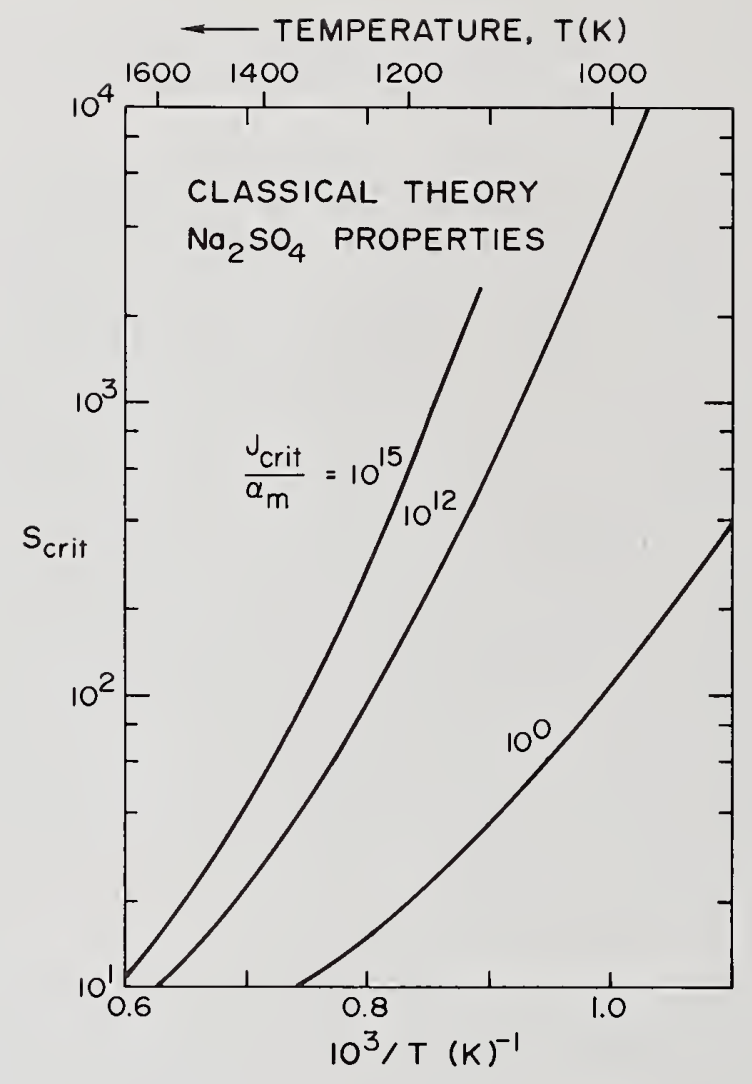

Figure 8. Predicted critical supersaturations for $\mathrm{Na}_{2} \mathrm{SO}_{4}(\mathrm{~g})$ homogeneous nucleation.

An asymptotic analysis of this CFBLVD "breakdown" region, enabling rational predictions of deposition rates at surface temperatures below that of boundary layer condensation onset is under development [35] and will be reported elsewhere. However, by considering the limiting case of $\mathrm{s}_{\text {crit }}=1$, it can easily be shown that the BL condensation onset wall temperature is itself significantly below the dew point--at about $1200 \mathrm{~K}$ for the case shown in figure $2[10]$. Thus, boundary layer condensation cannot be the cause of CFBL vapor deposition rate over-prediction in the immediate vicinity of the dew point (cf. Section 2.4)

\section{Conclusions--Research Needs and Future Work}

\section{1 Research needs}

Many research needs connected with the general GT/MGD/boiler deposition problem discussed herein have become apparent to us in the course of the present program and are indeed implicit or explicit in the above mentioned sections. Among those which currently 
command our attention are:

- Basic phase equilibrium data and practical computer models of multielement VLE, VSE to allow deposition rate predictions for solution condensates (cf. Section 2.3).

- Methods for estimating transport parameters (e.g., Lennard-Jones potential) for vapor species having no characterized stable condensed phase.

- Direct measurements of the transport properties of inorganic vapor molecules to develop/confirm correlations for prediction methods.

- Boundary layer calculations and/or measurements to better define the effects of variable properties and thermophoresis on mass transfer coefficients.

- A self-consistent particle transport theory for the transition range between diffusional and inertial deposition (cf. fig. 7).

- A transport theory for the effects of boundary layer condensation on deposition rates (cf. Section 3.2).

- A transport theory for the effects of homogeneous and heterogeneous nonequilibrium phenomena on dew point and deposition rates.

- Precise dew point and deposition rate data using optical (interference and polarization) techniques (cf. Section 2.2).

- Direct kinetic data on the mechanism of sulfate salt formation in combustors; elementary step rate constants for relevant vapor phase chemical reactions.

- Useful homogeneous nucleation rate theory for condensates formed by reaction from vapor precursors of different stoichiometry.

- Techniques for, and direct measurements of, individual inorganic vapor and particulate concentrations in combustion gas environments.

- Synthesis and exploitation of above information to suggest practical strategies for reducing fouling and/or corrosion phenomena to acceptable limits.

While much past effort has gone into reviewing the (inadequate) "state-of-the-art," in the future, more effort will have to go into advancing it in those areas of greatest technological significance.

\subsection{Summary}

Using laboratory burner data, a comprehensive but tractable convective diffusion deposition theory, based on the assumption of a multicomponent "chemically frozen" boundary layer (CFBL), has been developed for making self-consistent salt/ash deposition rate predictions over a wide variety of environmental conditions of gas turbine and magneto-gas-dynamic interest. This CFBL theory (Section 2.3 [10] utilizes the results of the NASA free-energy minimization LTCE computer program, but only at both free stream and wall conditions, imposing the coupled effects of net mass transport by both multicomponent Fick (concentration) and Soret (thermal) diffusion across the boundary layer. To illustrate the application of our multicomponent formulation of CFBL deposition rate theory, we have considered (Section 2) recent gravimetric and optical measurements of the deposition rate of 
$\mathrm{Na}_{2} \mathrm{SO}_{4}$ and $\mathrm{B}_{2} \mathrm{O}_{3}$ from seeded laboratory burners to targets cooled below the prevailing "dew" point. The applications of our methods to readily predict deposition rates under less accessible or extreme operating conditions is illustrated via predictions of the dependence of deposition rate on pressure level. Insight into the domain of validity of CFBL depositior rate theory and fruitful extensions thereof, have been provided by estimates of the effects of pre-existing condensate aerosols (Section 3.1) and vapor nucleation within the thermal boundary layer (Section 3.2). While additional input information (kinetic, thermodynamic, and transport properties; cf. Section 4.1) and laboratory deposition rate data of improved precision are being obtained, we hope the present program provides a sufficiently general, rational framework for future predictions of salt/ash deposition rates, and, ultimately, the evaluation of control strategies.

This research program is supported in part by NASA grants NSG-3107 and NSG-3169 at Yale University, Department of Engineering and Applied Science. It is a pleasure to acknowledge helpful discussions with Drs. P. C. Nordine, R. Srivastava, and R. Israel.

\section{References}

[1] Stringer, J., Hot corrosion of high-temperature alloys, Ann. Rev. of Materials Science, ㄱ, R. A. Huggins, ed. (1977).

[2] Boll, R. H. and Patel, H. C., The role of chemical thermodynamics in analyzing gas-side problems in boilers. ASME Paper 60-WA-182 (1960).

[3] Heywood, J. B. and Womack, G. J., Open-Cycle MHD Power Generation (Pergamon Press, Oxford, U.K., 1969).

[4] Halstead, W. D. and Raask, E., The behavior of sulfur and chlorine compounds in pulverized coal fired boilers, J. Inst. Fuel, 344 (1969).

[5] Hedley, A. B., Brown, T. D., and Shuttleworth, A., Vanadium pentoxide deposition from combustion gases, Trans. American Soc. Mech. Engrs. (A), J. Engineering for Power, 88, 173 (1966).

[6] DeCrescente, M. A. and Bornstein, M. S., Formation and reactivity thermodynamics of sodium sulfate with gas turbine alloys, Corrosion, 24, 127 (1969).

[7] Brown, T. D., Deposition of sodium sulphate from combustion gases, J. Inst. Fuel, 39, 378 (1966).

[8] Ross, K., Condensation of sulphuric acid from flue gas on a cooled cylinder, Inst. of Fuel Journal, 38, 273 (1965).

[9] Koh1, F. J., Stearns, C. A., and Fryburg, G. C., Sodium sulfate: vaporization thermodynamics and role in corrosive flames, in Metal-Flue-Gas Reactions and Processes, Z. A. Foroul is and W. W. Smeltzer, eds., p. 649 (The Electrochem. Soc., Princeton, N.J., 1975). 
[10] Rosner, D. E., Chen, B.-K, Fryburg, G. C., and Kohl, F. J., Chemically frozen multicomponent boundary layer theory of salt and/or ash deposition rates from combustion gases, Comb. Sci. and Tech. (1979), in press.

[11] Gordon, S. and McBride, B. J., Computer program for calculation of complex chemical equilibrium compositions, rocket performance, incident and reflected shocks, and Chapman-Jouguet detonations, NASA Report SR-273 (1971).

[12] Koh1, F. J., Santoro, G. J., Stearns, C. A., Fryburg, G. C., and Rosner, D. E., Theoretical and experimental studies of the deposition of $\mathrm{Na}_{2} \mathrm{SO}_{4}$ from seeded combustion gases, NASA TMX-73683 Technical Paper presented at the Symposium on Corrosion Problems Involving Volatile Corrosion Products, sponsored by the Electrochemical Society, Philadelphia, PA, May 8-13, 1977; J. Electrochem. Soc. (1979), in press.

[13] Rosner, D. E. and Seshadri, K., Dew point nonuniqueness in multicomponent, nonisothermal systems (1979), in preparation.

[14] Rosner, D. E. and Fernandez de la Mora, J., A simple criterion for the influence of homogeneous nucleation on boundary layer mass transport (1979), in preparation.

[15] Seshadri, K. and Rosner, D. E., Optical methods of dew point and deposition rate measurement in salt/ash - containing combustion gases - $1 . \mathrm{B}_{2} \mathrm{O}_{3}(l)$ deposition rates by the interference method, submitted to AIChEJ (1979).

[16] McIntyre, R. J. and McTaggart, F. K., Comparison of the reactions of atomic and molecular halogens with silver, J. Phys. Chem., 74, 866 (1970).

[17] Suguwara, K., Yoshini, T., Okuyama, H., and Shirasu, T., Monitoring of CVD film thickness by laser system, in Proceedings of the Fourth International Conference on Chemical Vapor Deposition, Boston, MA, October 1973, p. 205 (The Electrochemical Society, Princeton, NJ, 1973).

[18] Rosner, D. E., Thermal (Soret) diffusion effects on interfacial mass transport rates, J. Physicochemical Hydrodynamics (Pergamon Press) (1979), in press.

[19] Srivastava, R. and Rosner, D. E., A new approach to the correlation of boundary layer mass transfer rates with thermal diffusion and/or variable properties, accepted for publication in IJHMT (1978).

[20] Coaton, J. R. and Phillips, N. J., The influence of convection and thermal diffusion on halogen separation in vertical burning linear tungsten-halogen lamps, J. Phys. B., Atom. Molec. Physics, 4, 248 (1971).

[21] Fischer, E., Axial segregation of additives in mercury-metal halide arcs, J. Appl. Phys., 47, 2954 (1976).

[22] Durie, R. A., Milne, J. W., and Smith, M. Y., The deposition of salts from hydrocarbon flames containing sodium and sulphur species, Comb. and Flame, $\underline{30}, 221$ (1977).

[23] Rosner, D. E., Environmental effects of gas/metal reactions at elevated temperatures, AGARD Materials Panel Conference Proceedings No. 120 on High Temperature Corrosion of Aerospace Alloys, J. Stringer, R. I. Jaffee, and T. F. Kearns, eds., p. 129 (1972). 
[24] Rosner, D. E., Energy, mass and momentum transport - the treatment of jump conditions at phase boundaries and fluid-dynamic discontinuities, Graduate issue of, Chemical Engineering Education, $\underline{X},[4], 190$ (1976).

[25] Westenberg, A. A., A critical survey of the major methods for measuring and calculating dilute gas transport properties, Vol. 3, Advances in Heat Transfer, T. F. Irvine, Jr. and J. P. Hartnett, eds. p. 253 (1966).

[26] Svehla, R. A., Estimated viscosities and thermal conductivities of gases at high temperatures, NASA Technical Report R-132 (1962).

[27] Hirschfelder, J. 0., Curtiss, C. F., and Bird, R. B., Molecular Theory of Gases and Liquids (J. Wiley and Sons, New York, 1954).

[28] Rosner, D. E., Seshadri, K., and Fernandez de la Mora, J., Local thermochemical equilibrium multicomponent boundary layer theory of salt deposition (Fall 1978), in preparation.

[29] Rosner, D. E. and Israel, R., Effect of thermophoresis on the minimum attainable diffusional deposition rate before the onset of inertial impaction (1978), in preparation.

[30] Rosner, D. E. and Srivastava, R., Brownian and thermophoretic deposition of particle size distributions (Fall 1978), in preparation.

[31] Rosner, D. E. and Epstein, M., Fog formation conditions near cool surfaces, J. Colloid Interface Sci., 28, 60 (1968).

[32] Rosner, D. E., Enhancement of diffusion-limited vaporization rates by condensation within the thermal boundary layer. 1. The critical supersaturation model, Int. J. Heat Mass Transfer, 10, 1267 (1967).

[33] Epstein, M. and Rosner, D., Enhancement of diffusion-limited vaporization rates by condensation within the thermal boundary layer. 2. Comparison of homogeneous nucleation theory with the critical supersaturation model, Int. J. Heat Mass Transfer, 13, 1393 (1970).

[34] Katz, J. L., Condensation of a supersaturated vapor. I. The homogeneous nucleation of the n-alkanes, J. Chem. Phys., $\underline{52}, 4733$ (1970).

[35] Fernandez de la Mora, J. and Rosner, D., Effects of heterogeneous nucleation, particle growth and transport on laminar boundary layer mass transport (1979), in preparation.

\section{Appendix}

\section{Nomenclature}

$c_{p} \quad$ specific heat at constant pressure

d diameter of cylindrical target (cm)

$D_{i-m i x}$ Fick diffusion coefficient for species $i$; eq. (1)

$F_{j}$ (Soret) correction factor for Soret diffusion; eq. (4)

$F$ (turb) correction factor for free stream turbulence

$F(n c p) \quad$ correction factor for non-constant properties; eq. (4) 


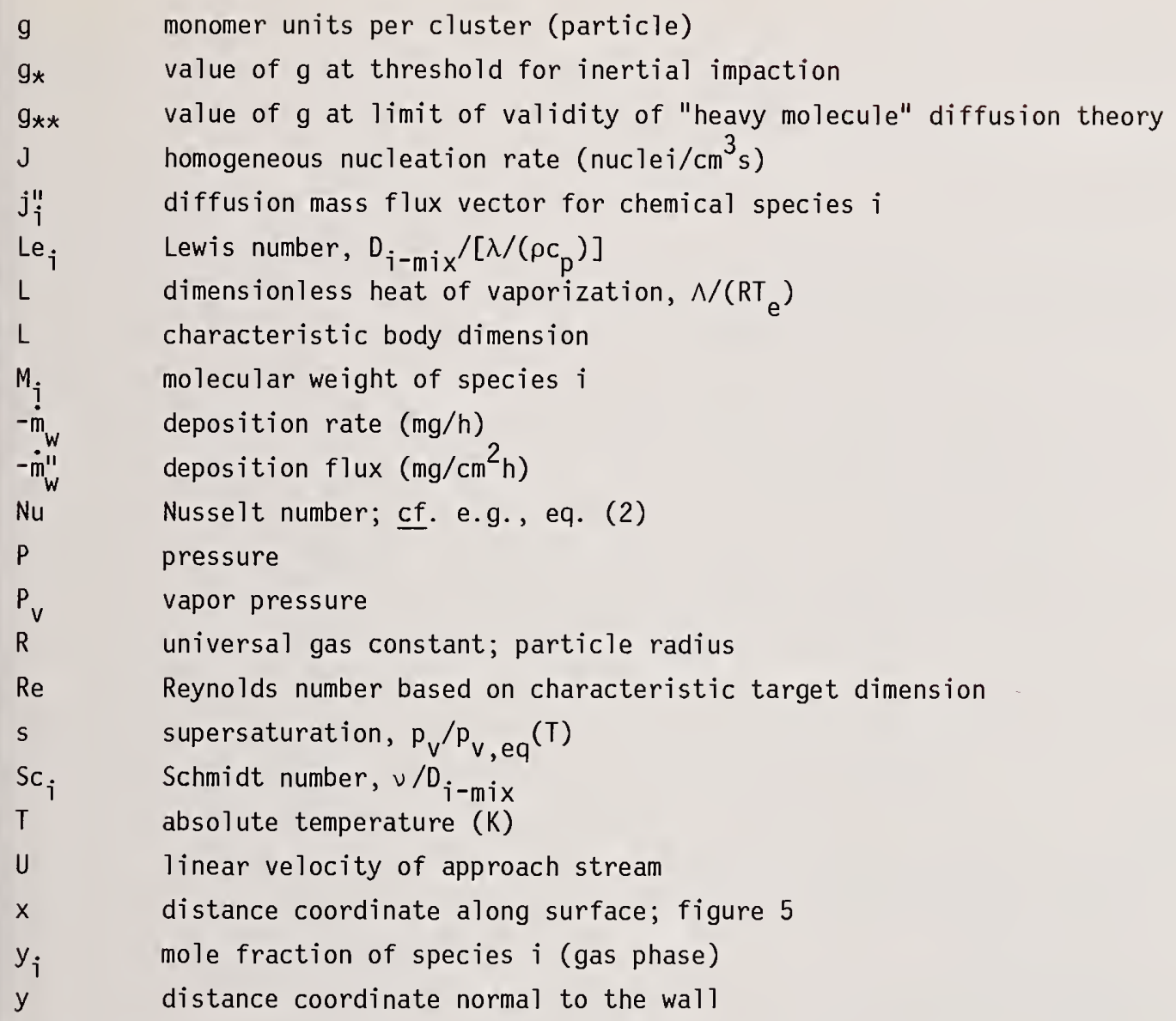

\section{Subscripts} cp constant property

crit pertaining to critical condition for nucleation onset 


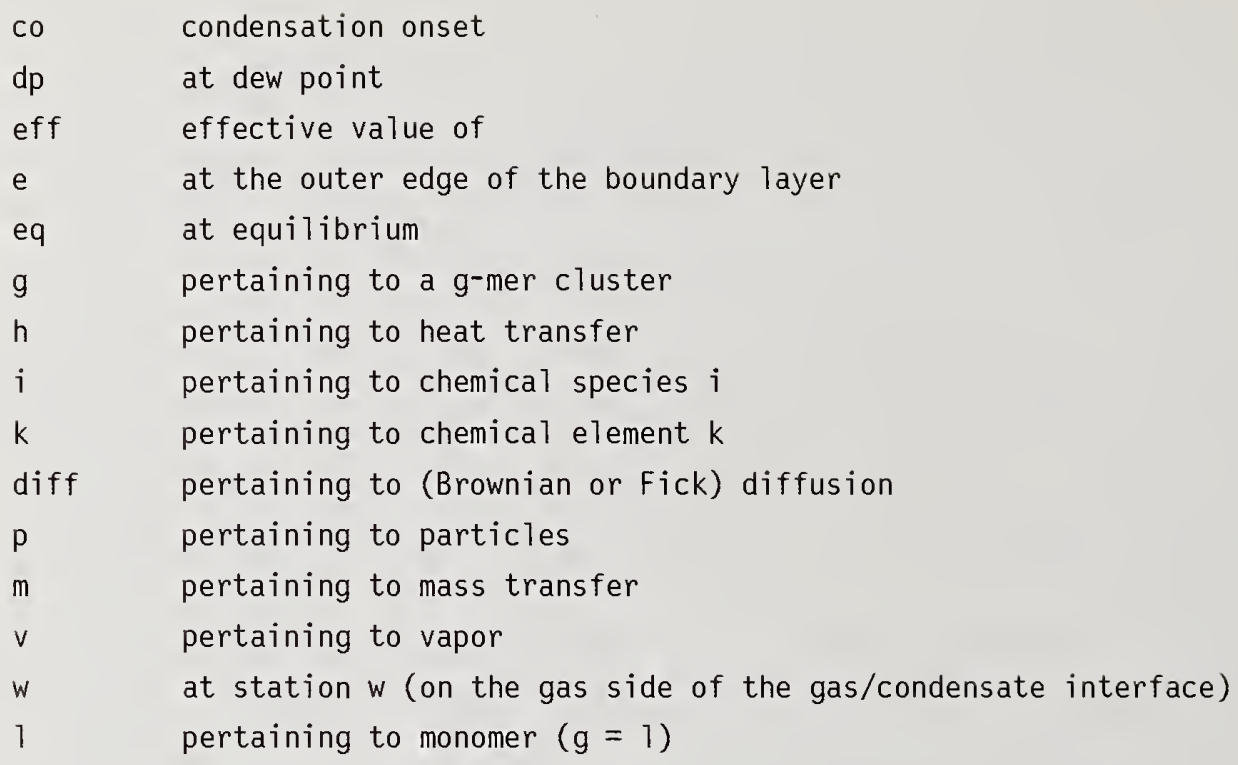

\section{Superscript}

c pertaining to condensed phase

\section{Miscellaneous}

$\begin{array}{ll}\Delta & \text { change in (across boundary layer) } \\ \nabla & \text { spatial gradient operator (grad) } \\ \text { BL } & \text { boundary layer } \\ \text { CF } & \text { chemically frozen } \\ \text { GT } & \text { gas turbine } \\ \text { LTCE } & \text { local thermochemical equilibrium } \\ \text { MGD } & \text { magneto-gas-dynamic } \\ \text { VD } & \text { vapor deposition } \\ \text { VLE } & \text { vapor/liquid equilibrium } \\ \text { VSE } & \text { vapor/solid equilibrium } \\ \text { CO } & \text { condensation onset } \\ \text { CE } & \text { Chapman-Enskog } \\ \text { LJ } & \text { Lennard-Jones } \\ \left({ }^{-}\right) & \text {perimeter-mean value } \\ \text { ppm } & \text { parts per million (by mass) }\end{array}$




\section{Discussion}

Question (Spencer): In the actual combustor, is there any evidence that there might be charged particles causing extra deposition on the turbine blades?

Response (Rosner): Frankly, I haven't pursued that aspect of charge induced capture, so I can't really answer that directly.

There are certainly many situations where charging effects are exploited to increase capture efficiencies, and I suppose one could change the sign and perhaps adopt such a thing as a control strategy.

We have been focused for the time being on transport due to turbulence, transport due to thick diffusion, brownian-type diffusion, and transport due to thermophoretic effects.

Indeed, there is even in certain kinds of furnaces what might be called photophoresis, an actual driving of particles away from their region of radiative flux.

We are also rather concerned with cases where the condensation process may actually occur within the boundary layer itself and not at the surface or in the mainstream.

Indeed that introduces a whole class of boundary layer transport processes which are rather subtle, but nevertheless we feel we have a way of making predictions of when that would occur, and what the effects on the deposition rate would be.

One of the interesting things about that is, there are two ways to go. If one went to very high temperature surfaces one might prevent condensation. It also is true that if one goes to very cold surfaces, one can get the condensation to occur away from the surface and then not capture that condensation.

This is well known to physical chemists who try to capture by cold fingers condensible vapors in flow systems. If you really have a very cold, cold finger you may not capture the material you are after, and this would be the analogue of that. We are interested in pursuing exactly when that would happen and what the deposition rates would be under those conditions as well.

But, I think the area of charging is probably an important one that you can't say will generally be important, but I am sure that there are conditions where charging effects can be exploited or should be included in a deposition rate theory.

Question (Beattie): I wanted to ask you about injecting sodium chloride, and then collecting on a cold plate and carrying out analyses. I wondered whether the condensate was 99 percent sodium sulfate, or 90 percent or 50 percent?

Response (Rosner): In some of those experiments there were contaminants beyond the sodium that was introduced in the salt solution.

For example, there were always a certain number of materials that probably came off the nozzle walls. Kohl perhaps you could comment, there were always a few percent of such contaminants in the condensates, is that not the case? 
Response - Kohl: Yes, except for trace metal impurities, which come from the combustor line and nozzle, $\mathrm{Na}_{2} \mathrm{SO}_{4}$ (c) is the only deposit collected on the platinum targets. Extensive test failed to reveal any detectable chloride. Equilibrium thermodynamic calculations predict fo our conditions that the $\mathrm{NaCl}$ (c) phase should not appear as a deposit in the temperature rang of our experiments. Furthermore, the calculation indicates that at lower target temperature the next phase to deposit will be $\mathrm{Na}_{2} \mathrm{CO}_{3}(\mathrm{c})$.

Because of the high level of $\mathrm{NaCl}(\mathrm{g})$ present in the gas stream, some $\mathrm{NaCl}(1)$ should be dissolved in the $\mathrm{Na}_{2} \mathrm{SO}_{4}$ (c) deposit. This situation results from phenomena explainable by Raoult's Law. We have considered this aspect [C. A. Stearns, F. J. Koh1, G. C. Fryburg and R. A. Miller: "High Temperature Metal Halide Chemistry," D. L. Hildenbrand and D. D. Cubicciotti, eds., the Electrochemical Society Softbound Symposium Series, Vol. 78-1, Princeton, N.J. 1978, pp. 555-573] and performed tests and calculations which showed this to be a negligible effect. 
National Bureau of Standards Special Publication 561, Proceedings of the 10th Materials Research Symposium on Characterization of High Temperature Vapors and Gases held at NBS, Gaithersburg, Maryland, September 18-22, 1978. Issued 0ctober 1979.

THE MODELING OF MODERN HIGH PRESSURE ARC LAMPS

\author{
R. J. Zollweg \\ Westinghouse $R$ \& D Center \\ Pittsburgh, PA 15235
}

Numerical models used to describe physical phenomena in high pressure arc lamps are reviewed together with more approximate methods of interest for routine measurements. Experimental techniques unique to lamps are briefly discussed.

The radiation efficiency, electrical characteristics and stability of high pressure arcs are strongly influenced by the arc temperature profile. Modeling of such arcs has progressed from the historic channel model approximation to more accurate continuum models. Away from the electrodes, the arc column can usually be accurately described by the steady-state energy balance model. By adding time-dependent terms, this mode 1 can be extended to arcs powered by alternating current. Convection velocities in symmetrical, vertical, cylindrical arcs can be determined in two-dimensions by a convection model which simultaneously solves five equations: energy balance, axial and radial momentum balances, continuity and $0 \mathrm{hm}$ 's Law. Especially for mercury plus metal halide arcs, the large radial temperature gradients lead to radial diffusion demixing. This coupled with convection leads to significant axial segregation in vertical arcs.

The physical material properties of the discharge medium used in these models are: the electrical and thermal conductivities, the net radiative emission, density, specific heat, and viscosity. These, in turn, depend upon the compositions of the medium and interaction cross sections which are needed for a wide temperature range $(\sim 1000-6000 \mathrm{~K})$. Characterization of the discharge medium is most reliable in the arc core region where all species are normally dissociated and atomic spectral methods can be used. The vaporization process from mixtures of metal halides often involves the evaporation of complex molecules and is dependent upon the condensed phase composition. 


\section{Introduction}

High pressure arc lamps in extensive commercial use today include the high pressure mercury vapor discharge, the high pressure sodium discharge $(\mathrm{Na}, \mathrm{Hg}, \mathrm{Xe})$, and mercury plus metal halide additive discharges. The metal halides are often combined to improve the source color, color rendition and efficacy, and include such blends as: (1) sodium and scandium iodides, often with thorium iodide in addition; (2) sodium, thallium and indium iodides; (3) tin iodide often with the chloride; and (4) thallium and dysprosium iodides, often with other rare earth iodides as we11. Many other metal or metal halide additives have been shown experimentally to have attractive characteristics for general illumination as well as specialized applications, i.e., underwater illumination, laser pumping, photochemical processing, but have not yet achieved widespread application.

The selection of additive pressures and combinations for optimum satisfaction of application requirements, i.e., efficiency, color and color rendition, lamp life and, maintenance of lumen efficiency during life, is a fascinating subject. But, this will not be treated in the present review. We should recognize, however, that the electrical characteristics of the discharge and the atomic and molecular radiation from the additives will depend upon the additive densities, their distribution in the discharge, and the temperature and temperature profile of the arc. The additive pressures depend upon the condensed phase composition at the lowest temperature reservoir part of the arc tube wall and also upon physical phenomena which take place within the discharge which cause additive segregation. When mixtures of metal halides are used, these evaporate as complex or individual iodides which dissociate in the discharge to atoms, ions, and lower iodides which are the sources of radiation. The physical material properties of the discharge medium depend upon the species composition of the discharge medium and its temperature. In turn, the "arc discharge" characteristics, e.g., its temperature profile, electrical and radiative characteristics, depend upon interrelationships between the physical material properties of the discharge medium and their relative temperature dependences.

Most of the physical processes involved and the foundations for modeling high pressure arc discharges have been published in the two books by Elenbaas $[1,2]^{1}$ and that by Waymouth [3]. This review will emphasize progress since that time when the modeling has become considerably more quantitative and additional processes, associated with the more complex vapor systems, have been treated. Collections of papers, some of which deal with modeling of high pressure arcs are given in references [4, 5, and 6].

First, we shall discuss the theoretical numerical modeling of high pressure arcs. This characterization of the "discharge properties" will be seen to result from cooperative interactions of the physical properties of the discharge medium, such as the electrical and thermal conductivity, radiation, enthalphy, and viscosity of the mixture of species forming the discharge medium. These physical properties arise, of course, from the medium composition.

${ }^{1}$ Figures in brackets indicate the literature references at the end of this paper. 
Next, we shall mention some approximate and semi-empirical models that are useful for general comparison of different arcs or for routine measurement purposes. We shall then discuss briefly some of the experimental techniques that have been used in the arc lamp field to characterize the composition of the discharge medium as well as the arc characteristics and boundary conditions. Because of the complexities involved and uncertainties of thermochemical data and interaction cross sections, approximations have to be made to deduce the physical material properties from the vapor composition. Some recommended data sources will be identified.

\section{Theoretical Numerical Modeling}

Following Elenbaas [1], most models of high pressure arc lamps have accepted the concept of local thermodynamic equilibrium (LTE) as a useful approximation, which greatly simplifies the theoretical treatment. Recent work by deGroot [7] and by Waszink [8] on high pressure sodium lamps and by Fromm, Seehawer, and Wagner [9] on lamps containing thallium iodide have shown that departures from LTE do exist in the outer parts of certain arcs where strong absorption takes place. However, these departures are usually evident only in small details, and serve primarily to show that LTE is, indeed, a good approximation for the most important characteristics of arc lamps.

\section{1 The channel model}

Elenbaas [1] used a channel model to simplify the characterization of the high pressure. mercury lamp. In this approach, the real arc, which in this case has approximately a parabolic temperature profile, is replaced by a conducting arc channel of radius $r_{\text {eff }}$ with uniform material properties corresponding to a temperature $T$ eff. With suitable choice of $r_{\text {eff }}$ and $T_{\text {eff }}$, this channel model arc has electrical and radiative characteristics closely approximating the real arc.

\subsection{The steady-state dc arc}

With mercury arcs containing other additives, especially when metal iodides are present, the arc temperature profile can be significantly altered, which also changes the electrical and radiative properties of the discharge. Then, the arc temperature profile is an important parameter in the characterization of the arc discharge. Lowke [10] developed a numerical relaxation method to solve explicitly the Heller-Elenbaas energy balance equation

$$
\sigma E^{2}+\operatorname{div}(k \operatorname{grad} T)-u=\rho C_{p} \frac{\Delta T}{\Delta t}
$$

for the steady-state (dc) wall-stabilized arc with a current I 


$$
I=2 \pi E \int_{0}^{R} \sigma r d r
$$

and suitable boundary conditions.

In eqs. (1) and (2), $E$ is the axial electrical field strength, $T$ is the temperature, and the material properties are the electrical conductivity $\sigma$, thermal conductivity $k$, net radiant power per unit volume $u$, density $\rho$ and specific heat $C_{p}$ which are all functions of temperature. $\Delta t$ is a time step associated with the relaxation process and $R$ is the radius of the confining wall of the arc tube. The electrical and thermal conductivities can be calculated from the composition of the discharge medium by methods discussed below using known, calculated, or estimated cross-sections for all collision processes. The net radiant power $u$ leaving the volume element is the difference between the radiant power emitted and that absorbed within the volume element. The absorbed radiation includes that which originates from other portions of the arc that is transported to the volume element under consideration and thus involves a complex radiative transport calculation. Various approximations have been used to solve this problem $[8,10,11,12]$.

In doing the iterative numerical calculation by the relaxation method, one starts with an approximate initial temperature distribution defined at a set of grid positions and uses eq. (2) to calculate the electric field $E$ for a given arc current. With this $E$ and approximate temperature distribution, the left-hand side of eq. (1) will not, in general, be zero. By putting the right-hand side of (1) equal to $\rho C_{p} \frac{\Delta T}{\Delta t}$, the temperature at each grid position can be incremented according to the value of the left-hand side of (1) for that position with a time step $\Delta t / \rho C_{p}$. This process continues until both (2) and (1) are satisfied for all grid positions. Figure 1 shows temperature profiles calculated in this way (solid curves) for mercury arcs of approximately $3 \mathrm{~atm}$ pressure at four different arc currents (powers) compared with experimental measurements (symbols).

\subsection{The ac arc}

However, most commercial lamps are operated from ac power sources with inductive ballasts. The steady-state model was extended [13] to cover this case by adding to eqs. (1) and (2) the circuit equation

$$
V_{0} \cos \omega t=L \frac{d I}{d t}+I R+E h
$$

where $V_{0}$ is the applied line voltage, $w=2 \pi n$ where $n$ is the ac frequency, $L$ and $R$ are equivalent series inductance and resistance, respectively, and $h$ is the arc length. The current $I$ and electric field $E$ are now functions of time $t$. The numerical calculation proceeds as previously with suitable boundary conditions and an initial approximate temperature profile and current I. 


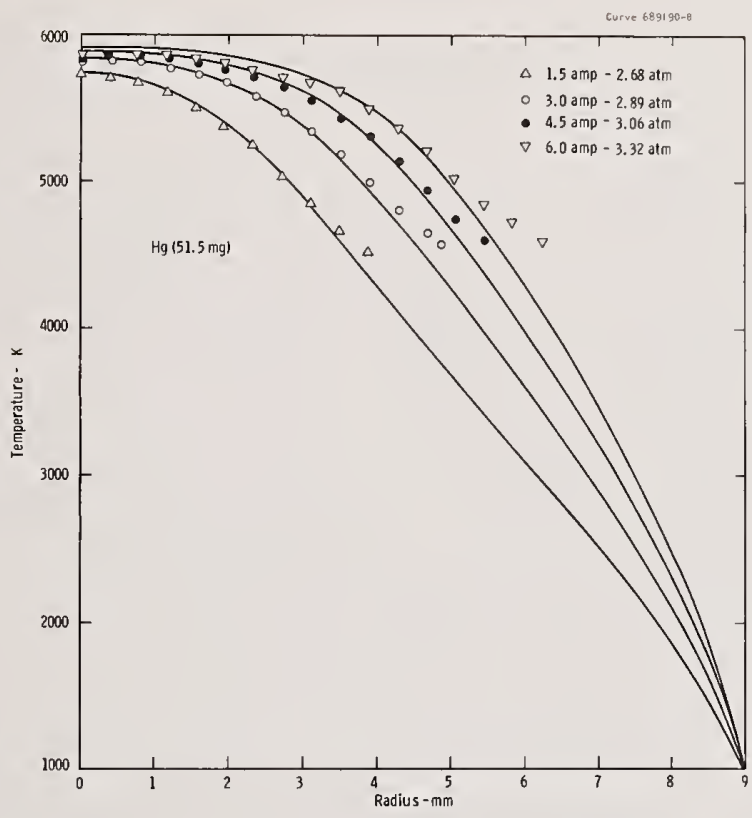

Figure 1. Comparison of steady-state calculated arc temperature profiles for mercury arcs of approximately $3 \mathrm{~atm}$ pressure (solid curves) with experimental measurements (symbols) at four current (power) loadings.

The three eqs. (1), (2), and (3) must now be satisfied at each time step of the ac cycle. The temperature profile is incremented according to eq. (1) and the current according to eq. (3). The calculation proceeds until the E-I characteristics are repetitive for each ac cycle. Both the central axis temperatures and the temperature profiles change with time during the ac cycle. Figure 2 shows a comparison of experimental and calculated electrical characteristics for a 1-atm mercury arc obtained by Lowke, et al. [13]. deGroot, et al. [14] have obtained similar results for high pressure sodium arcs. Both the steady-state and the ac arc calculations discussed above assume that the arc is cylindrically symmetric for its entire length, only approximately taking into account electrode sheath effects and neglecting convection effects. LTE is also assumed for the entire ac cycle which is not necessarily true for all arc vapors [13]. Thus, the calculated results do not always accurately reproduce the voltage maximum at arc re-ignition each half cycle.

Although for simple vapor systems, it is possible to calculate the material properties to reasonable accuracy, that accuracy is often inadequate to predict arc temperature profiles as accurately as they can be measured experimentally. Thus, it has been found useful to use model calculations in connection with experimentally measured profiles to test the consistency of arc material parameters [15]. 


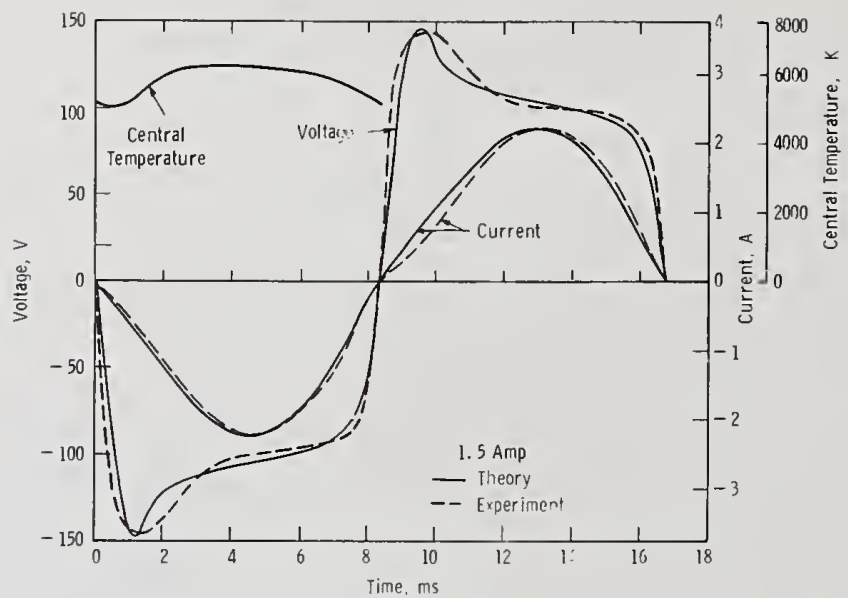

Figure 2. Comparison of the time dependence of measured lamp current and voltage for a 1-atm, 1.5-A mercury arc with those calculated with the ac arc model.

\subsection{Convection models for vertical arcs}

At high mercury pressures, the temperature differences within the arc tube cause buoyancy forces which lead to significant convection currents. For vertical arcs, this leads to constriction of the arc near the lower electrode and a conical appearance of the arc in that region. Such constriction may also lead to arc instability $[15,16]$. When additives are present, the combination of convection and diffusion processes may lead to segregation of additives as will be discussed below.

Lowke [17] developed a model to calculate convection in steady-state, verticallyoriented, cylindrically-symmetric arcs in LTE. This model was applied to wall-stabilized mercury arcs by Zollweg [15]. This two-dimensional model for an arc in a cylindrical enclosure satisfies a set of five equations:

(1) Ohm's Law, eq. (2) above;

(2) the mass continuity equation,

$$
\operatorname{div}(\overrightarrow{\rho V})=0
$$

(3) the steady-state energy-balance equation which now includes convection terms;

$$
\rho c_{p} \frac{\Delta T}{\Delta t_{2}}=\sigma E^{2}+\operatorname{div}(\operatorname{kgrad} T)-u-\rho C_{p} \vec{V} \cdot \operatorname{grad} T ;
$$


(4) the axial momentum equation (Navier-Stokes);

$$
\begin{aligned}
\rho \frac{\Delta V_{z}}{\Delta t_{3}} & =\rho g-\frac{\partial p}{\partial z}-\rho V_{r} \frac{\Delta V_{z}}{\partial r}-\rho V_{z} \frac{\Delta V_{z}}{\partial z} \\
& +\frac{1}{r} \frac{\partial}{\partial r}\left(r \eta \frac{\partial V_{z}}{\partial r}\right)+\frac{\partial}{\partial z} \eta \frac{\partial V_{z}}{\partial z} ;
\end{aligned}
$$

and (5) the radial momentum equation

$$
\begin{aligned}
\frac{\partial p}{\partial r} & =-\rho V_{r} \frac{\partial V_{r}}{\partial r}-\rho V_{z} \frac{\partial V_{r}}{\partial z}+\frac{1}{r} \frac{\partial}{\partial r}\left(r \eta \frac{\partial V_{r}}{\partial r}\right) \\
& +\frac{\partial}{\partial z} \eta \frac{\partial V_{r}}{\partial z}-\frac{\eta V_{r}}{r^{2}}-\vec{J} \times \vec{B} .
\end{aligned}
$$

In these equations, $\vec{V}$ is the stream velocity with components $V_{r}$ and $V_{z}, p$ is the pressure, $g$ is the gravitational acceleration, and $\eta$ is the viscosity. This set of equations with suitable boundary conditions at the arc axis and along the containing walls can be solved numerically in iterative fashion as were the models discussed earlier. The additional material parameters of the vapor, i.e., the density $\rho$, the specific heat $c_{p}$, and the viscosity are now needed as functions of temperature. Figures 3 and 4 show how convection can change the temperature profiles of 13-atm mercury arcs near the lower electrode.

In lighter gases non-LTE effects are more likely $[18,19]$. Scott, et a1. [20] have developed a model to calculate nonequilibrium flow conditions in the constricted hydrogen arc while Clark, et al. reported similar results for the constricted argon arc [21]. Incropera [22] has reviewed the procedures of these authors in modeling the laminar cascade arc.

\subsection{Radial and axial segregation of species}

The radial and axial segregation of additives in a mercury arc is often apparent by simply observing an image or colored photograph of the arc [23]. Radial demixing was identified some years ago to result from diffusion processes in large radial temperature gradients in $\mathrm{SF}_{6}$ cascade arcs. Frie [24] developed a procedure to calculate the change in vapor composition with temperature. There are two major causes of radial demixing: dissociation processes, and (2) ionization processes. When dissociation occurs within the arc column, the diffusion rates for molecules inward is often less than that of the dissociated atoms outward which causes a greater concentration of the total elements (in 


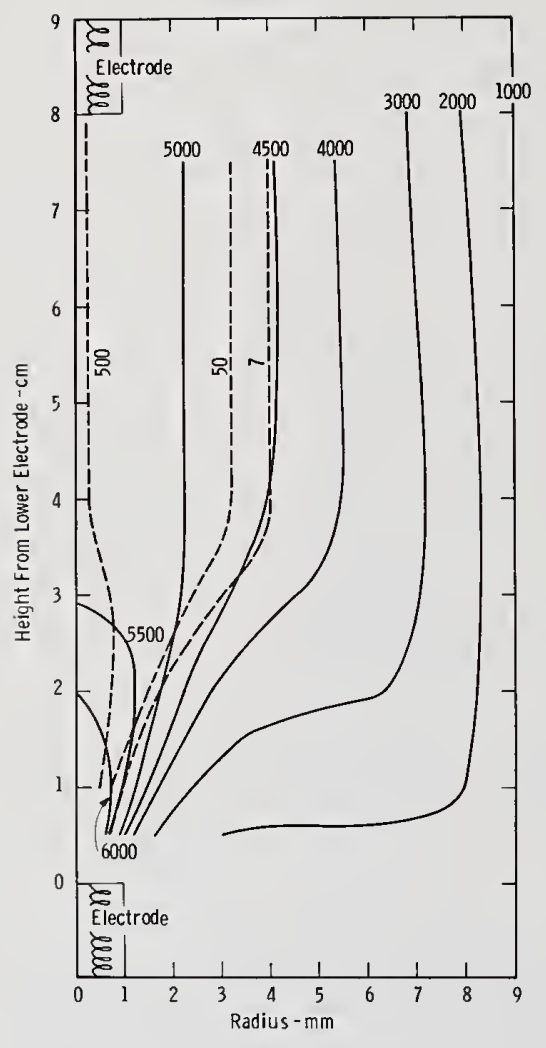

Figure 3. Isotherms calculated by the vertical convection model are shown for a 13-atm mercury arc at $1.47 \mathrm{~A}$ $(592 \mathrm{~W})$ by the solid curves with temperatures indicated in degrees $\mathrm{K}$. The dashed curves are approximate contours of equal radiation as viewed by an observer side-on of relative intensities 500,50 , and 7 , respectively.

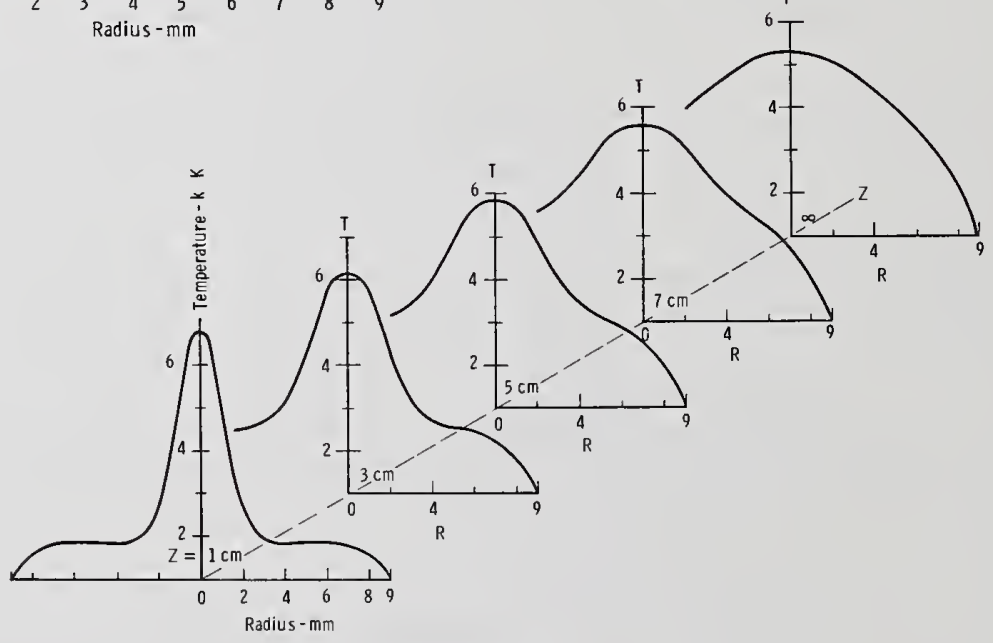

Figure 4. Theoretically calculated radial temperature profiles for a 13-atm vertical mercury arc with a current of $1 \mathrm{~A}$ at various axial positions. The lower electrode tip is $Z=0$ and the upper at $Z=8 \mathrm{~cm}$. The profile for $Z=\infty$ was obtained without convection and would be approximately correct for the midplane of a considerably longer arc. 
atomic plus molecular form) in the outer part of the arc. Near the core of the arc where ionization occurs, the lighter electrons diffuse outward most rapidly creating an ambipolar electric field which enhances the outward diffusion of positive ions. This process leads to a depletion of additives in the arc core which is especially significant if a large fraction of that species is ionized. Somewhat surprisingly, the theoretical model by Frie [24] of radial demixing resulting from such diffusion processes, as well as a conceptually simpler version developed recently by Liebermann, et a1. [25], shows that the vapor compositions depend upon temperature but are independent of the temperature gradient and geometry of the arc. That is because it is assumed that the diffusion processes have continued unti 1 equilibrium is achieved, that LTE exists throughout, and gas flow and convection effects are neglected. Frie's demixing calculations for $\mathrm{SF}_{6}$ were shown to give reasonable agreement with the experimental measurements of Motschmann [26]. For mercury arcs containing sodium and scandium iodides, Zollweg and Liebermann [27] have found that the measured sodium and scandium densities in the arc core are in rather good agreement with the radial demixing model. However, the neglect of free convection makes the calculated demixing slightly larger than that observed experimentally.

When radial demixing and convection both take place, there may be a large axial additive segregation for vertically operated arcs [23,27]. Fischer [28] has succeeded in developing a model combining diffusion and convection with results in reasonably good agreement with experiment. The set of equations satisfied by Fischer for the steady-state arc include: the axial momentum equation, (2) the steady-state condition for each species in the plasma, (3) the multicomponent diffusion equation, (4) the total and elemental mass continuity equations, and (5) the mass action equations for chemical equilibrium assuming LTE, together with suitable boundary conditions. The energy balance equation is not used since it is assumed that the arc temperature profile is independent of axial position, in spite of the observed axial segregation. Other simplifications used by Fischer to obtain numerical results for this very complex problem are the neglect of radial convection and ambipolar field terms and the assumption that mercury terms are usually dominant. The degree of axial segregation is found to vary rather strongly with the mercury pressure in the arc tube, since this has a strong influence on convection velocities. In some additive arcs, the temperature profile does change significantly with axial position. For such cases, it would be necessary to extend Fischer's model and also satisfy the energy balance equation.

\subsection{Phase dependent segregation}

Most of the models discussed previously are directly applicable only to steady-state arcs with zero time-averaged axial electric fields (so that electrophoretic segregation of additives is negligible). Most commercial lamps, however, are operated on alternating current with time-averaged radiation, efficiency, and color of most interest. deGroot [29] found that time-averaged quantities for ac arcs were usually in close agreement with similar quantities for dc arcs of the same power. Some authors have measured phase-resolved quantities using boxcar integrators [30] and interpreted the results in terms of instantaneous physical quantities [31]. Such quantities should then be integrated over the ac 
cycle to obtain the more usefu] time-averaged quantities. Work [32] has found that near the electrodes certain additive species, e.g., $\mathrm{Na}$, can be significantly depleted during certain parts of the ac cycle. This apparently results from the electrophoretic effect of the applied axial electric field in removing a species with high fractional ionization. Figure shows oscilloscope photographs confirming this effect for one of our experimental lamps containing mercury and sodium and scandium iodides. The lower trace e shows the ac lamp voltage waveform, positive when the upper electrode is positive. The current waveform (not shown) is a slightly distorted sine wave with current zero corresponding to the zero voltage times. The trace $d$ shows the $\mathrm{Na}$ resonance line $589 \mathrm{~nm}$ radiation viewed at a position $3.0 \mathrm{~cm}$ below the upper electrode while immediately above, trace $c$ shows that radiation $0.75 \mathrm{~cm}$ belo the upper electrode. Above that are the $\mathrm{Na} 616.1 \mathrm{~nm}$ line trace $\mathrm{b}$ and the $\mathrm{Hg} 577 \mathrm{~nm}$ line trace a, also $0.75 \mathrm{~cm}$ below the upper electrode. Note that, although the mercury radiation is nearly symmetrical with the ac cycle, the sodium radiation near the upper electrode is reduced when the upper electrode is positive. This effect is more evident in viewing the $\mathrm{Na}$ resonance line because that line is self-absorbed and the sodium density is evidently depleted faster in the hot core where partially ionized than in the colder outer region responsible for most of the absorption. The $\mathrm{Sc}$ and $\mathrm{Sc}^{+}$waveforms are nearly symmetrical at the additive pressures in this lamp. To model such complicated lamp behavior would require time-dependent solutions of the energy balance, momentum, and diffusion models mentioned above and is probably not economically justifiable at this time.
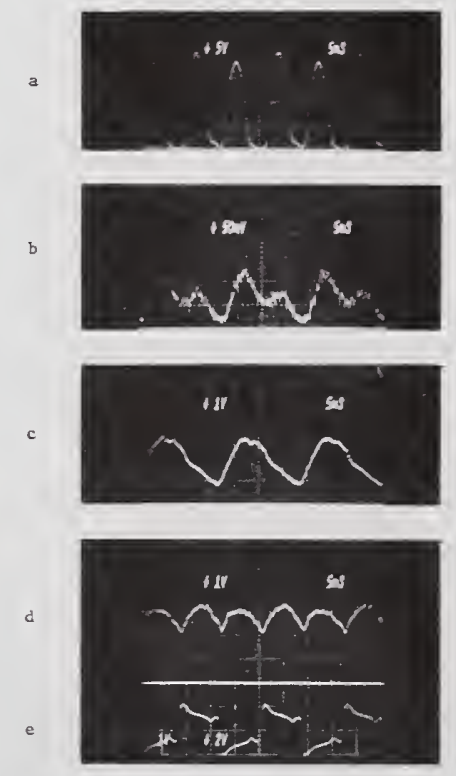

Figure 5. 0scilloscope photographs-the ac voltage waveform $e$, sodium radiation viewed from the side of the arc tube $\mathrm{b}-\mathrm{d}$ and the mercury $577 \mathrm{~nm}$ radiation a. Although the mercury radiation is nearly symmetrical on both halves of the ac cycle, the sodium radiation near the upper electrode is not as shown in trace b $616.1 \mathrm{~nm}$ and in trace c $589 \mathrm{~nm}$.

\subsection{Summary of numerical models to characterize the arc discharge}

Table 1 gives a summary of the various numerical models that have been used for cylindrical lamp arcs in LTE. On the left side in each case are the equations satisfied and or the right are the results obtained. For all of the models, the material properties are 
Table 1. Summary of numerical models for cylindrical LTE arcs.

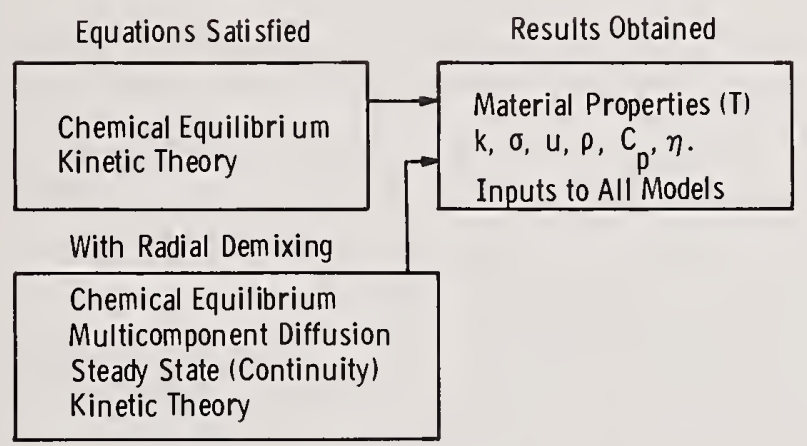

STEADY-STATE (dC) ARC

\begin{tabular}{|c|c|}
\hline Energy Balance & Temperature Profile $T(r)$ \\
\hline Ohm's Law & \\
\hline
\end{tabular}

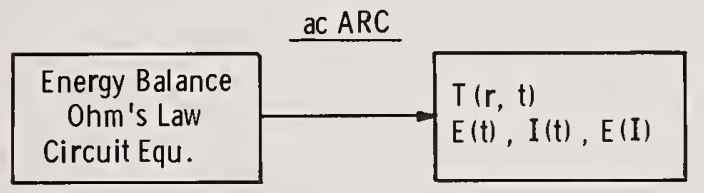

\section{VERTICAL CONVECTION MODEL (dC)}

\begin{tabular}{|l|l|}
\hline $\begin{array}{l}\text { Energy Balance } \\
\text { Ohm's Law } \\
\text { Axial Momentum } \\
\text { Radial Momentum } \\
\text { Continuity (Steady State) }\end{array}$ & $\rightarrow \begin{array}{l}T(r, z) \\
\text { Axial Velocity }(r, z) \\
\text { Radial Velocity }(r, z) \\
\text { Pressure }(r, z)\end{array}$ \\
\hline
\end{tabular}

RADIAL AND AXIAL SEGREGATION FOR VERTICAL ADDITIVE ARCS (rigorous Soln).

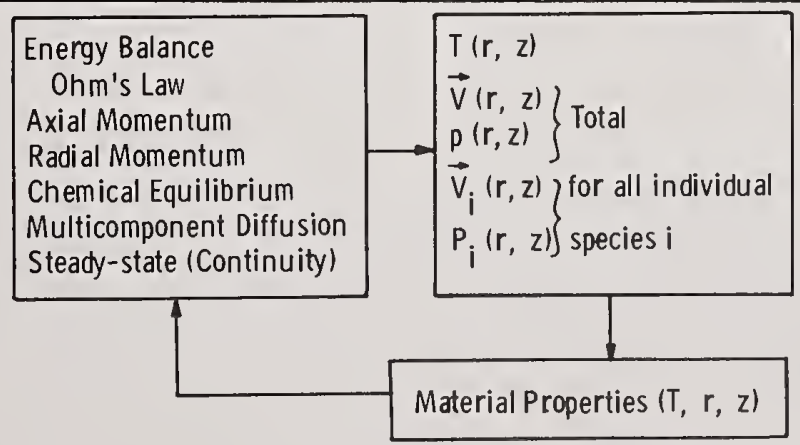


needed as a function of temperature. These depend, therefore, on the results of the chemical equilibrium calculation. When several components are present, diffusion should be included because of large radial temperature gradients. To do this, the chemical equilibrium (mass action) equations plus the multicomponent diffusion equations are satisfied in steady state.

Where convection and segregation effects are not important, the electrical and radiative properties of the arc depend upon the temperature profile of the arc which results primarily from energy balance considerations. The vapor material properties needed for the steadystate arc energy balance calculations are the electrical and thermal conductivities and the net radiative emission. For ac calculations, the density and specific heat of the arc vapor are also needed as well as the circuit parameters.

For laminar convection calculations, the density, specific heat, and viscosity are needed in addition to the parameters affecting the temperature profile, i.e., electrical and thermal conductivities and net radiative emission. The momentum equations are now satisfied in addition to the energy balance, $0 \mathrm{hm}^{\prime} \mathrm{s}$ Law, and continuity equations. The temperature and pressure are now obtained as functions of both $r$ and $z$ for vertical arcs as are the axial and radial stream velocities.

A rigorous calculation of axial segregation would combine the convection model (energy and momentum equations) with the diffusion and chemical equilibrium equations used in the radial segregation model. From such a calculation, in addition to the temperature $T$, pressure $p$, and stream velocities $\vec{V}$ as functions of $r$ and $z$, we would obtain the velocities of the individual species $\vec{V}_{j}$ and partial pressures $p_{j}$ also as functions of position. The vapor material properties will become properties of position as well as temperature. This complication can be handled by a feedback loop in the calculations as shown. To model this behavior for an ac arc would require the addition of the circuit equation and time dependent solutions throughout--a major undertaking.

\subsection{Vapor material properties}

To discuss the calculation of all the needed material properties for lamp arcs would lead us too far astray. However, since mercury plays an important or dominant role in most arc lamps and because of confusion regarding its properties, a short digression may be desirable. For any vapor, the chemical equilibrium composition may first be obtained at each appropriate temperature by minimizing the free energy $[33,27]$ using thermochemical data from the JANAF Tables [34] or elsewhere. Thermochemical data for species not listed there can be estimated by methods discussed in reference [34]. Interaction cross sections for heavy species can be obtained using model potentials [13] and used to determine the transport properties. The electrical conductivity of mercury, however, depends strongly on electron scattering by neutral mercury atoms. Scattering cross sections are most accurately determined by electron drift-velocity and diffusion measurements. The electron driftvelocity measurements of McCutchen [35] in mercury have recently been confirmed by Nakamura and Lucas [36]. However, the method used by McCutchen to deduce the electron-neutral mercury cross section from his drift-velocity measurements was incorrect. Thus, when McCutchen's 
cross sections are used with rigorous methods, they do not reproduce the measured driftvelocities. Rockwood [37] deduced a more consistent electron-mercury cross section that is in good agreement with the more recent value [36]. The agreement with arc lamp measurements suggests that electrical conductivities calculated using the Rockwood or Nakamura-Lucas cross sections should be correct to within 10-20 percent. Accuracy, or at least selfconsistency, in the set of material parameters to this level is needed in order to calculate temperature profiles to the desired accuracy and to the accuracy of measured values. It should also be emphasized that rigorous methods need to be employed in calculating the electrical conductivity from the densities and cross section and should include electronelectron and electron-ion scattering as well as electron-neutral scattering [38]. Errors in conductivities as large as 50 percent may result from the approximations sometimes employed.

Net radiative emission from arc vapors taking into account absorption has been calculated in several different approximations. Lowke [39] and Liebermann and Lowke [40] used radiative transfer approaches to calculate the net emission from isothermal cylinders of sodium and $\mathrm{SF}_{6}$ vapors, respectively. They assume that the spectral lines are of Lorentian shape, which is not rigorously correct far from line centers. Zollweg and Liebermann [12] used the escape parameter concept of Holstein [41] to determine what fraction of the atomic line radiation would escape from an isothermal cylindrical volume. This method can be used with a large number of self-absorbed lines; however, it does not explicitly allow for spectral overlap between nearby self-absorbed lines or between such lines and continuous radiation, nor does it predict negative emission values for the outer cold portion of the arc. Waszink [8] (1975) has extended the escape parameter approach making use of the formulation of vanTrigt and vanLaren [42]. Waszink discusses the considerable complications and the remaining approximations.

\section{Approximate and Semi-Empirical Models}

The several complexities of arc lamps and of numerical modeling have led to a need for: (1) simpler overall pictures of the energy distribution in arcs, and (2) for simpler models which can be routinely used in lamp manufacture and maintenance evaluation. Jack and Koedam [43] measured the overall radiative characteristics of five different commercial arc lamps as functions of lamp power and arrived at approximate energy distributions between electrodes, ultraviolet, infrared, and visible radiation and nonradiative losses. Keeffe [44] reported similar data for the mercury plus sodium and scandium iodide lamp.

As stated earlier, the temperature profiles of metal iodide lamps may be far from parabolic. Keeffe, et al. [31] have introduced an approximation to characterize the temperature profile by

$$
T(r)=T\left(R_{1}\right)+\left[T(0)-T\left(R_{1}\right)\right]\left[\frac{1-\left(r / R_{1}\right)^{2}}{1-\alpha\left(r / R_{1}\right)^{3}}\right]
$$


where $\alpha$ is an adjustable parameter and $R_{1}$ may be some location in the arc or perhaps the arc wal1. If the arc temperature profile can be satisfactorily characterized by such a one- or two-parameter expression, then the entire temperature profile and axis temperature can be determined from a single absolute side-on measurement of an optically thin line of mercury, such as the $577 \mathrm{~nm}$ line. The densities of other radiative species can then also be found from similar absolute intensity measurements of optically thin lines. Although quantitative errors are likely to result, the simplicity of this approach makes it attractive for routine, non-destructive monitoring of factory lamps and their changes during life [45].

In addition to the modeling of high pressure sodium arc lamps mentioned above $[7,8$, 10,29], mention should be made of the semi-empirical model of Waymouth [46]. This model uses empirical constants to correct for several simplifying assumptions but is successful in yielding calculated lumen-per-watt values within 5 percent of those measured over a range of lamp parameters.

\section{Experimental Methods for Lamp Arcs}

The temperature and species composition of lamp arcs have been determined primarily by traditional methods of optical spectroscopy. Temperature profiles have often been obtained by absolute intensity measurements of optically thin mercury lines viewed side-on and subsequently Abel-inverted. The mercury pressure and the atomic transition probability of the atomic line must be known. The mercury 577.0 and $491.6 \mathrm{~nm}$ lines have frequently been used, but published atomic transition probabilities vary widely [47]. It should be noted that the value for the $577 \mathrm{~nm}$ line quoted by Pilz and Seehawer [47] has now been adjusted downward [9] to $0.25 \times 10^{8} \mathrm{~s}^{-1}$, near that of Jean, et a1. [47] $\left(0.27 \times 10^{8} \mathrm{~s}^{-1}\right)$. Similar absolute intensity measurements of additives can be used, knowing the temperature, to deduce additive densities. Suitable lines and atomic transition probabilities for $\mathrm{Na}, \mathrm{Sc}, \mathrm{Sc}^{+}$, and iodine have been identified [27,32]. Such measurements may be either phase-resolved or timeaveraged (with a small error). Because of radial demixing and axial segregation, it is not usually reliable to depend upon weak impurity lines for temperature measurements.

For high pressure sodium lamps, there are no good optically thin lines. The method developed by Bartels [48] which makes use of self-absorbed, non-resonant atomic lines has been employed by several authors $[49,50,9]$ to measure arc temperature profiles. The selfreversal width of the sodium resonance lines has been found to be a convenient measure of the sodium pressure in high pressure sodium lamps [51,29]. Quantitative use of this technique requires a knowledge of the atomic spectral line profiles near the reversal maxima and approximate knowledge of the arc temperature profile. deGroot and vanVliet [52] recently extended this technique to measure mercury pressures from the red wing reversal of the 253.7 nm line. Commercial high pressure sodium lamps contain both sodium and mercury and the outer envelope does not transmit the $253.7 \mathrm{~nm}$ 1ine. However, when both sodium and mercury are present, the red wing of the sodium resonance line is significantly modified while the blue wing is largely unchanged as illustrated in figure 6 . This permits the sodium resonance line to be used as a nondestructive diagnostic for both sodium and mercury pressures [53] which are prone to change during lamp life. 


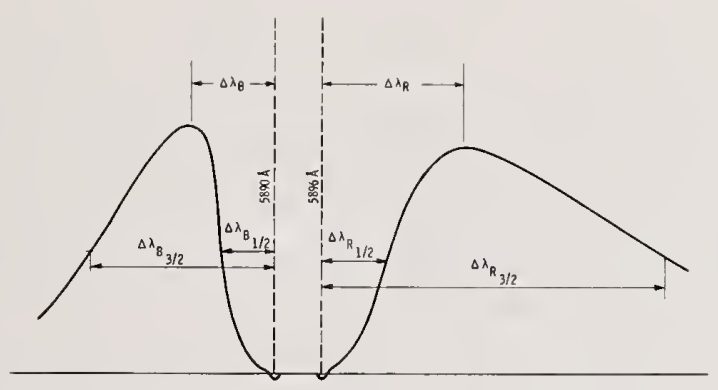

Figure 6. Illustrates the sodium D line reversal from a high pressure sodium lamp when both $\mathrm{Na}$ and $\mathrm{Hg}$ are present. The quantities indicated can be used for diagnostic purposes.

In a number of metal halide lamps it is necessary to deal with molecular radiation $[54,55,56]$ or absorption $[57,58]$ often for molecular species, e.g. HgI, ScI, SnI, that have not heretofore been studied quantitatively.

Emission spectroscopy is often of little value in the outer mantle region of high pressure discharges. Vriens and Adriaansz have investigated Rayleigh and Raman scattering techniques $[59,60]$ which can be used for this region. More recently, they have investigated near resonant dye laser scattering and fluorescence as a diagnostic technique for the sodium density in both low and high pressure sodium discharges [61,62]. Light scattering from imperfections in the arc tubes of commercial lamps can often be a serious problem with these techniques.

The wall temperature of the quartz or alumina arc tubes can be important to the energy balance, to possible light absorption near the wall, and to possible condensation of vapor species. The wall temperatures can be measured by standard infrared pyrometry using suitable infrared transmitting windows [63]. deGroot [7] developed a unique technique for measuring the wall temperature of high pressure sodium lamps enclosed in standard glass outer envelopes. This method is based on measurement of radiation from the optically thick sodium lines immediately after turning off the arc. The temperature of the sodium gas layer measured in this way is very close to the inside wall temperature.

It was mentioned earlier that interactions between additives frequently result in enhanced vaporization of desirable radiative species for lamps through the formation of complex molecules in the vapor phase. Recent results in this area, together with references to the literature and discussions of experimental techniques, were recentiy reviewed by Rehder and Wilson [64] and by Hirayama, et al. [65] ${ }^{2}$.

'Editor's note: Several other chapters in this volume deal with the subject of complex halide vapor species; see Papatheodorou, Schafer, Gruen and Øye, and Orlovskii. 


\section{Status and Conclusions}

In this review I have tried to indicate the present status of numerical modeling of modern high pressure arc lamps together with a few more approximate methods of interest for routine measurements and some experimental techniques uniquely related to lamp problems. Present numerical models can describe many arc lamp phenomena reliably, provided that accurate material parameters can be obtained. Improvements in material parameters, in scattering cross sections, in thermochemical data, in the net radiative emission, and radiative transfer areas are still needed. Better information on continuum radiation mechanisms, spectral line broadening, and molecular radiation would be useful.

Present numerical models are generally limited to one or two dimensions and to the description of a limited range of physical phenomena. Thus, they cannot be expected to describe the stability limit between cylindrical and helical arc forms (helical instability) since this would involve azimuthal $\theta$ as well as radial $r$ and axial $z$ terms in the several equations. The ability to develop models which are more complete in their description of all relevent physical phenomena is developing rapidly, and such more complete models should be available in the next few years. This progress is somewhat limited today by computer core size and expense. Models of the electrode region should also be expected in the next few years.

Our knowledge of the arc mantle region is quite limited. A number of subiodides may exist in this region and be the source of molecular radiation and significant contributions to the thermal conductivity. Further progress is also expected in understanding the considerable interactions between additives and the contributions of complex halides to the vaporization process.

The effects of impurities on long term lamp behavior also deserve considerable attention. Even in small concentrations, impurities can contribute to wall darkening and possible halogen cycle behavior.

The author is deeply indebted to J. J. Lowke who developed many of the numerical models we have used and instructed us in their use. To R. W. Liebermann who developed the technique to calculate chemical equilibria, material transport properties, theoretical net emission coefficients, and radial demixing in additive arcs. D. K. McLain has made significant modifications in the convection model. Finally, I wish to thank F. M. Ryan for the oscilloscope photos shown in figure 5 .

\section{References}

[1] Elenbaas, W., The High Pressure Mercury Vapor Discharge (North-Holland, Amsterdam, 1951). 
[2] Elenbaas, W., ed., High Pressure Mercury Vapor Lamps and Their Applications (Philips Technical Library, Eindhoven, 1965).

[3] Waymouth, J. F., Electric Discharge Lamps (The M.I.T. Press, Cambridge, Mass, 1971).

[4] Proceedings of the IEEE, Special Issue on Atomic and Molecular Plasmas (Apri1 1971).

[5] Philips Technical Review, Special Issue on Light, Vol. 35, Nos. 11 and 12 (1975).

[6] Proceedings of the Symposium of High Temperature Metal Halide Chemistry, D. L.

Hildenbrand and D. D. Cubicciotti, eds., Proceedings, Vol. 78-1 (The Electrochemical Soc. Inc., Princeton, N. J., 1978).

[7] deGroot, J. J., Proc. 2nd IEE Conf. on Gas Discharges, p. 124 (London, 1972).

[8] Waszink, J. H., J. Phys. D6, 1000 (1973); J. of App1. Phys. 46, 3139 (1975).

[9] Fromm, D. C., Seehawer, J., and Wagner, W. J., reference [6], p. 61.

[10] Lowke, J. J., J. Quant. Spectrosc. Radiat. Transfer, 9, 839 (1969).

[11] Lowke, J. J., J. Quant. Spectrosc. Radiat. Transfer, 14, 111 (1974).

[12] Zol1weg, R. J. and Liebermann, R. W., Bu11. Am. Phys. Soc. 19, 164 (1974).

[13] Lowke, J. J., Zollweg, R. J., and Liebermann, R. W., J. Appl. Phys. 46, 650 (1975).

[14] deGroot, J. J. and vanVliet, J. A. J. M. , J. Physics, D8, 651 (1975), (dc); deGroot, J. J., vanVliet, J. A. J. M., and Waszink, J. H., Philips Tech. Rev. 35, 334 (1975) (ac).

[15] Zollweg, R. J., J. Appl. Phys. 49, 1077 (1978).

[16] Reference [6], p. 347.

[17] Lowke, J. J., Twelfth Int. Conf. on Phen. in Ionized Gases, Eindhoven, 1975, Pt. 1, p. 144 (unpublished). Submitted for publication in J. Appl. Phys. (1978).

[18] Emmons, H. W. , Phys. Fluids, 10, 1125 (1967).

[19] Uhlenbusch, J., Fischer, E., and Hackman, J., Zeits. f. Physik, 238, 404 (1970).

[20] Scott, R. K. and Incropera, F. P., AIAA Journa1, 11, 1714 (1973).

[21] Clark, K. J. and Incropera, F. P., AIAA Journal, 10, 17 (1972).

[22] Incropera, F. P., IEEE Trans. Plasma Science, PS-1, No. 3 (September 1973).

[23] Zollweg, R. J., Journal of IES, $\underline{5}, 12$ (1975).

[24] Frie, W., Zeits. f. Physik, 201, 269 (1967).

[25] Liebermann, R. W., Rogoff, G. L, and Vine, J., Bul1. Amer. Phys. Soc. 21, 153 (1976).

[26] Motschmann, H. , Zeitschrift fur Physik, 200, 93 (1967).

[27] Zollweg. R. J. and Liebermann, R. W. , reference [6], p. 52.

[28] Fischer, E., J. Appl. Phys. 47, 2954 (1976).

[29] deGroot, J. J., Thesis, Technical University, Eindhoven (1974).

[30] Patterson, P. L., J. Opt. Soc. Amer. 62, 627 (1972).

[31] Keeffe, W. M., Morris, J. C., and Walter, W., J. I11. Eng. Soc. to be published (1978).

[32] Work, D. E., J. I11. Eng. Soc. 7, 118 (1978).

[33] See, for example, Frost, L. S. and Liebermann, R. W., Proc. IEEE, 59, 474 (1971).

[34] Stu11, D. R. and Prophet, H., JANAF Thermochemical Tables, 2nd Ed., NSRDS-NBS No. 37

U.S. Government Printing Office, Washington, DC, 1971).

[35] McCutchen, C. W., Phys. Rev. 112, 1848 (1958). 
[36] Nakamura, Y. and Lucas, J., J. Phys. D11, 225, 337 (1978).

[37] Rockwood, S. D., Phys. Rev. A8, 2348 (1973).

[38] Rockwood, S. D., J. App1. Phys. 45, 5229 (1974).

[39] Lowke, J. J., J. Quant. Spectrosc. and Radiation Transfer, 14, 111 (1974).

[40] Liebermann, R. W. and Lowke, J. J., J. Quant. Spectrosc. Radiat. Transfer, 16, 253 (1976).

[41] Holstein, T., Phys. Rev. 72, 1212 (1947); 83, 1159 (1951).

[42] vanTrigt, C. and vanLaren, J. B., J. Phys. D6, 1247 (1973).

[43] Jack, A. G. and Koedam, M., J. I11. Eng. Soc. 3, 323 (1974).

[44] Keeffe, W. M. , J. I11. Eng. Soc. 4, 260 (1975).

[45] Keeffe, W. M. , Bamberg, W. I., and Waymouth, J. F., J. I11. Eng. Soc. 5 , 200 (1976).

[46] Waymouth, J. F., J. I11. Eng. Soc. 6 , 131 (1977).

[47] Pilz, W. and Seehawer, J., Proc. 12th Int. Conf. on Phen. in Ion. Gases, Eindhoven, Pt. I, p. 146 (1975).

[48] See, for example, Zwicker, H., in Plasma Diagnostics, W. Lochte-Holtgreven, ed., p. 214 (North-Holland, 1968).

[49] deGroot, J. J. and Jack, A. G., J. Quant. Spectrosc. Radiat. Transfer, 13, 615 (1973).

[50] Ozaki, N., J. Quant. Spectrosc. Radiation Transfer, 11, 1111 (1971).

[51] Jen, Teh-Sen, Hoyaux, M. F., and Frost, L. S., J. Quant. Spectrosc. Radiation Transfer, 9, 487 (1969).

[52] deGroot, J. J., and vanVliet, J. A. J. M., Bul1. Amer. Phys. Soc. 23, 133 (1978).

[53] Jacobs, C. A. J., Sprengers, L., deVaan, R. L. C., J. I11. Eng. Soc. I, 125 (1978).

[54] Zol1weg, R. J., Lowke, J. J., and Liebermann, R. W., J. App1. Phys. 46, 3828 (1975).

[55] Kramer, J., McNei11, W., and Haugsjaa, P., reference [6], p. 348.

[56] Pilz, W. and Popp, H. -P., Proc. 11th Int. Conf. on Phenomena in Ionized Gases, p. 409 (Prague, Czechoslovakia, 1973).

[57] Maya, J., reference [6], p. 106.

[58] Drop, P. C., Fischer, E., Oostvogels, F., and Wesselink, G. A., Philips Technical Review, 35, 345 (1975).

[59] Vriens, L. and Adriaansz, M., Philips Technical Review, 35, 344 (1975); Philips Res. Repts. 31, 193 (1976).

[60] Vriens, L. and Adriaansz, M., J. Appl. Phys. 45, 4422 (1974); $\underline{46}, 3146$ (1975).

[61] Vriens, L. and Adriaansz, M. , App1. Phys. 11, 253 (1976).

[62] Vriens, L., J. App1. Phys. $\underline{48}, 653$ (1977).

[63] See, for example, Rickman, J. D., Jr., J. App1. Phys. 48, 3733 (1977).

[64] Rehder, L. and Wilson, I., reference [6], p. 71.

[65] Hirayama, C., Liu, C. S., and Zollweg, R. J., reference [6], p. 95. 


\section{Discussion}

Question (Wormhoudt): You showed, calculated and experimental temperature profiles in the arc, and I was curious as to how the experimental temperature profiles were done?

Response (Zollweg): Okay. They were time averaged measurements actually of an AC arc, and one can argue about how accurate those are compared to $D C$, but they were done by side-on measurements of a 5770 angstrom mercury line, and the data subsequently Abel inverted using absolute intensity measurements using the atomic oscillator strengths for that line.

Question (Hastie): A related question. I noticed in the outer part of the arc for that particular comparison of experimental and calculated temperature profiles, there tended to be a disagreement between the experiment and calculation. Was there an explanation for that?

Response (Zollweg): Well, in the process of Abell inversion--you are dealing with the derivative of the intensity. When you are dealing with the region where those points are deviating, our intensity was down to about one percent of what it was at the center, and there are problems of scatter -- allowing for the proper amount of scatter of light from the quartz envelope and these kinds of problems. So, I think it is experimental error.

Question (Spencer): I have two questions. On that sodium depletion work that you showed, is that symmetrical, do you have the same type of phenomenon occurring on the bottom electrode?

Response (Zollweg): We didn't make the measurement at the bottom.

(Editor's note): An anonymous comment was made to the effect that it is probably not geometrically symmetrical.

Question (Spencer): The other question I had, have you tried to model the AC reignition spike?

Response (Zollweg): We have not in detail tried to model the reignition spike. We have seen in things like sodium lamps that we come quite close, but there are some small differences in that.

Now, our model includes only the bulk of the discharge, it doesn't include electrode effects. So, that any effects of electrodes may distort that, and we think that some of the differences are may be in that. 
Now, we did try to model a one-atmosphere argon arc. This was published in the paper on AC arcs. There we predicted with LTE (local thermodynamic equilibrium) a much higher reignition spike than was actually measured. We take that as evidence that in a one-atmosphere argon arc, one doesn't have LTE during the reignition period.

Question (Eddy): I was wondering if on the convection model you included any effects of the anode and cathode jet, or were they either not important or not included?

Response (Zollweg): We didn't include anything of that sort and we purposely didn't emphasize the electrode region because we included in the model only axial electric fields.

Another term that we neglected, which is about a five percent effect is the thermal energy carried by electrons to the electrodes. It is a fairly small effect but it could be put in. We have not done that as yet. We intend to. 
National Bureau of Standards Special Publication 561, Proceedings of the 10th Materials Research Symposium on Characterization of High Temperature Vapors and Gases held at NBS, Gaithersburg, Maryland, September 18-22, 1978. Issued October 1979.

\title{
THE FUTURE OF GAS-TRANSPORT REACTIONS
}

\author{
V. P. Orlovskii \\ Kurnakov Institute General and Inorganic Chemistry \\ Academy of Sciences \\ Moscow, U.S.S.R.
}

The extensive use of gas-transport reactions for scientific and practical aims is to a great extent due to the work of $H$. Schafer and coworkers. In the well known monograph $[1]^{1}$, he summarized his own results and data obtained by other authors and laid down the theoretical foundation of this method.

Numerous studies of substance transfer through the gaseous phase via chemical transport reactions and the promising possibilities of this method for preparation of single crystal films and single crystals of semiconductors, dielectrics, and metals have stimulated research in this area ${ }^{2}$.

Investigations of crystal and $f i 1 \mathrm{~m}$ growth from the gaseous phase have provided answers to fundamental problems in the theory of crystal growth. However, analysis of the mechanisms of crystal growth from the gaseous phase shows that a secondary role has been assigned to chemical transport reactions, although they considerably affect the crystallochemical, structural, and morphological properties of the crystal. Elucidation of the true mechanism of transport reactions, which takes into account formation of intermediate gas-transport complexes and the distribution of gas phase composition at the growing edge, is essential for physico-chemical and mathematical simulation of crystallization processes.

The substantial effect of gas phase composition on the processes of crystal growth and the variety of forms of growth depending on crystallization temperature have been reported in $[2,3]$. No quantitative theory explaining these effects has yet been proposed, but an approach to these phenomena is emerging. As an example, one may cite reference [4] where the dependence of $\mathrm{HoPO}_{4}$ crystal habitus on crystallization temperature was studied. This dependence is evidently associated with variation of the gas phase composition and adsorption.

This is clearly seen by calculating and plotting (fig. 1) the temperature dependence of vapor phase composition of reaction (1) responsible for transport of $\mathrm{LnPO}_{4}$.

${ }^{1}$ Figures in brackets indicate the literature references at the end of this paper.

2Editor's note: For a detailed discussion of these and other applications see Hastie, J. W., High Temperature Vapors: Science and Technology, p. 91-211, Academic Press, NY (1975). 


$$
\mathrm{LnPO}_{4(\mathrm{~s})}+3 \mathrm{PX}_{3(\mathrm{~g})}+3 \mathrm{X}_{2(\mathrm{~g})}=\operatorname{LnX} 3(\mathrm{~g})+4 \mathrm{POX} 3(\mathrm{~g})
$$

(where $L n=$ rare earth elements and $X=C 1, B r$ ). In the temperature range $1200-1400 \mathrm{~K}$, the relative equilibrium partial pressures of gaseous components vary considerably and a unique ratio corresponds to each crystallization temperature. Variation of crystal form during growth may also occur on account of adsorption of admixtures or gaseous reaction components.

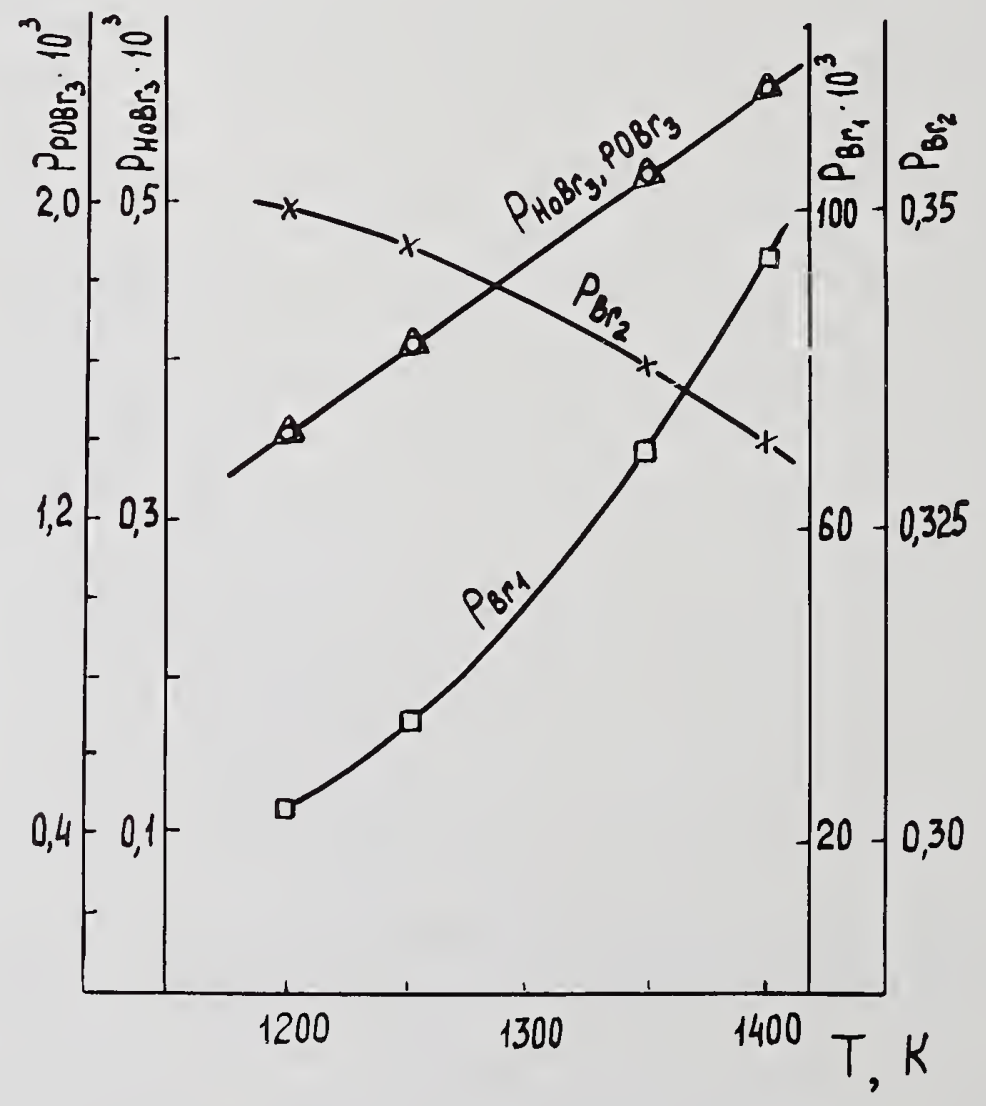

Figure 1. Temperature dependence of equilibrium partial pressures of gas phase components. 
According to a mass spectrometric study of reaction (1), the vapor composition is rather complex [5], therefore, adsorption of a gaseous component by the growing crystal is possible. In the thermodynamic analys is of the $\mathrm{LnPO}_{4} / \mathrm{PBr}_{3} / \mathrm{Br}_{2}$ system, the partial pressures of $\mathrm{P}_{4} \mathrm{O}_{10}(\mathrm{~g}), \mathrm{O}_{2}$ and $\mathrm{PBr}_{5(\mathrm{~g})}$ were discarded on account of their negligible values which do not affect the reaction equilibrium [6]. However, in conditions of real crystallization, the small partial pressures of components adsorbing on different edges of the crystal may substantially affect the form of growth. Unfortunately, direct study of the variation of gas phase composition with crystallization conditions is experimentally impossible at present. Hence, the actual molecular composition of the gaseous phase in the source and crystallization zones cannot be monitored during growth and this hinders fundamental studies of the mechanisms of single crystal growth from gaseous phase.

On account of the above-mentioned, investigation of the qualitative and quantitative composition of the gaseous phase presents considerable interest.

Mass spectrometry is the most comprehensive method of determining gas phase composition. It encounters, however, certain restrictions when applied to reactions of transfer and crystallization of solid phase. The upper pressure limit in the Knudsen chamber is determined by the minimal size of the aperture. For a $0.1 \mathrm{~mm}$ aperture the pressure is 1 imited to below $\sim 10^{-3}$ atm, whereas in transport reactions, pressures are in the range of $10^{-2}-40 \mathrm{~atm}$. Using special devices, the upper limit of mass spectrometry may be extended to $10 \mathrm{~atm}$, which made it possible to examine transfer of beryllium silicate [7], lanthanum orthophosphate [5], and carbon [8].

Optical spectroscopy is a convenient method for studying gas phase composition at high temperatures where suitable spectroscopic transitions exist. Examination of absorption and luminescence spectra provides information about the chemistry of the reaction, valence states, nature of chemical bonds and the structural environment of ions and molecules.

The following regions of the electromagnetic spectrum are the most informative:

1. $150,000-20,000 \mathrm{~cm}^{-1}$ - Near ultraviolet and blue. Analysis of electronic states of anionic groups $\mathrm{P}-0, \mathrm{Mo}-0, \mathrm{~A}-0$, etc. Absorption spectra.

2. $20,000-6000 \mathrm{~cm}^{-1}$ - Visible region and near ir spectrum. Analysis of electronic states of unfilled $d$ and $f$ shells. Absorption and luminescence spectra.

3. $8000-100 \mathrm{~cm}^{-1}$ - ir region. Analysis of vibrational states. Absorption spectra.

4. Raman scattering - Analysis of electronic and vibrational on vibrational and states. Electronic states on laser frequencies.

Several studies have been published on the optical spectra of gaseous coordination compounds, $\mathrm{NdAl}_{3} \mathrm{Cl}_{12}[9], \mathrm{CrAl}_{2} \mathrm{Cl}_{8}[10]$, and $\mathrm{TlCl}_{3} \cdot \mathrm{AlCl}_{3}[11]$. In our laboratory, we are investigating the luminescence spectra of $\mathrm{LnX}_{3}-\mathrm{Al}(\mathrm{Fe}) \mathrm{X}_{3}$ systems in order to determine the mechanism of crystallization of rare earth halides.

A prerequisite for growing perfect crystals and films is phase stability of the reaction mixture. Along with the fundamental question of occurrence of chemical gastransport reactions in the case of two condensed phases, this problem is related to 
preparation of single crystals of most high-melting compounds by the reaction, one of the products of which, depending on experimental conditions, may be in different phases. As shown in [12], phase stability may be disturbed at relatively high pressures of one of the gaseous components. Thus, condensation of one of the components, for instance $\mathrm{LnCl}_{3}$, may change the direction of $\mathrm{LnPO}_{4}$ transfer in reaction (1). It should be noted that in our opinion the statement that transfer does not occur in the case of two condensed phases is erroneous. This especially concerns gas-transport reactions where the partial pressure of components are comparable with saturated vapor pressures over liquid products in the zone of the reversed transport reaction. Experimental verification of $\mathrm{LnPO}_{4}$ transport by different transporting compounds $\left(\mathrm{PCl}_{3}+\mathrm{Cl}_{2}\right.$ and $\left.\mathrm{PBr}_{3}+\mathrm{Br}_{2}\right)$ confirmed the theoretical concepts we discussed in [12].

It should be noted that the transfer reactions with one of the components being a solid or liquid metal halide are a particular case of a more general phenomenon. These problems have not been considered in the theory of transport reactions, although a large number of systems, in particular $\mathrm{Me}_{\mathrm{m}} \mathrm{X}_{\mathrm{n}}-\mathrm{I}_{2}(\mathrm{X}=\mathrm{Se}, \mathrm{S}, \mathrm{Te})$, have been studied experimentally. The use of the gas-transport reaction method is not restricted to growing films and single crystals, but covers a wide area, including determination of thermodynamic characteristics, diffusion coefficients of gas mixtures, etc.

In most cases, determination of thermodynamic characteristics presents considerable difficulties, causing the experimentator to use indirect methods while being aware of their advantages and shortcomings.

The problems of employing gas-transport reactions for estimation of thermodynamic characteristics of inorganic compounds has been discussed in $[1,13,14]$. This method may also be used to decide between controversial values of $\Delta H_{f 298.15}^{\circ}$ and $S_{298.15}^{\circ}$ for the same substances.

In [15] the method of chemical transport reactions is used to estimate thermodynamic properties of rare earth tribromides.

The reliability of enthalpy of formation and entropy values for gaseous rare earth trihalides, including tribromides, may be estimated from experimentally determined quantities of transported rare earth orthophosphates by reaction (1). The divergence in $\Delta H_{\text {f298. }}^{\circ} 15$ values for $\mathrm{LnBr}_{3(\mathrm{~g})}$ (up to $30 \mathrm{kcal} / \mathrm{mol}$ ) is explained by the use of different data for calculating $\Delta \mathrm{H}_{\mathrm{f} 298.15}^{\circ}$ of solid rare earth tribromides [16-18].

The substantial divergence in $\Delta \mathrm{H}_{\mathrm{f} 298.15}^{\circ}$ and $\mathrm{S}_{298.15}^{\circ}$ values for $\mathrm{LnBr}_{3}$ affects the value of $1 \mathrm{~g} \mathrm{Kp}$ which characterizes the efficiency of the gas-transport reaction (amount of transported solid phase).

As an example, let us consider determination of thermodynamic properties of lanthanum orthophosphate and tribromide.

Table 1 shows that values of $\log \mathrm{Kp}$ for the reaction

$$
\mathrm{LaPO}_{4(\mathrm{~s})}+3 \mathrm{PBr}_{3(\mathrm{~g})}+3 \mathrm{Br}_{2(\mathrm{~g})}=\mathrm{LaBr}_{3(\mathrm{~g})}+4 \mathrm{POBr}_{3(\mathrm{~g})}
$$


calculated from different initial data on $\Delta \mathrm{H}_{\mathrm{f} 298.15}^{\circ}$ and $\mathrm{S}_{298.15}^{\circ}$ and from temperature dependence of heat capacity of lanthanum orthophosphate and tribromide [19-21]. The values of $\Delta \mathrm{H}_{\mathrm{f} 298.15}^{\circ} \mathrm{S}_{298.15}^{\circ}$ and $\mathrm{Cp}$ for gaseous $\mathrm{BBr}_{3}, \mathrm{Br}_{2}$, and $\mathrm{POBr}_{3}$ taken from reference tables $[22,23]$ coincide.

Table 1. Equilibrium constants of reaction (1).

Log $\mathrm{Kp} \mathrm{LaPO}_{4}$ $1200 \mathrm{~K} \quad 1300 \mathrm{~K} \quad 1400 \mathrm{~K} \quad \Delta \mathrm{H}_{\mathrm{f} 298.15}^{\circ} \mathrm{S}_{298.15}^{\circ}$
Ref.

$\mathrm{LaBr}_{3}$ Ref.

$\begin{array}{cccccccccc}1 & -12.0 & -11.2 & -10.5 & 474 & 32 & {[14]} & 115.3 & 106.4 & \text { [6] } \\ 2 & -10.5 & -9.6 & -8.9 & 471 & 29 & {[5]} & 115 & 104 & {[15]} \\ 3 & -7.8 & -7.4 & -7.1 & 471 & 29 & {[5]} & 146 & 90.2 & \text { [16] }\end{array}$

The theoretical dependence of mass transfer rate on concentration of the transport agent or total pressure of gaseous components are shown in figure 2 .

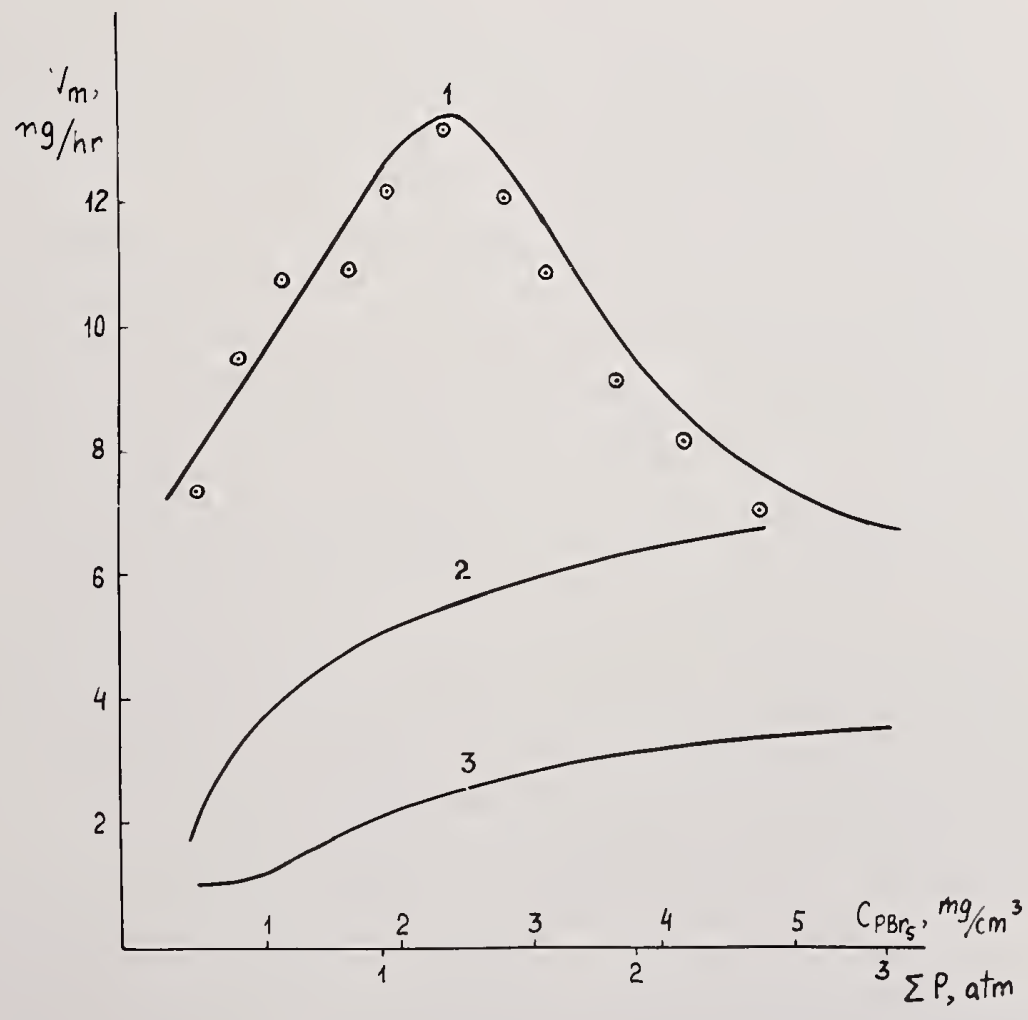

Figure 2. Dependence of $\mathrm{LaPO}_{4}$ mass transfer rate on initial $\mathrm{PBr}_{5}$ concentration and total pressure of gaseous components. 1, 2, and 3 are calculated according to data [16-19], [17-19], and [6], respectively. 
Calculations were carried out according to $\log \mathrm{Kp}$ values listed in table 1 . Comparison of these dependences shows that the maxima correspond to different values of total pressure.

From the experimentally determined dependence of $V_{m}$ on $\Sigma P$, it was found that transfer is maximal at $\Sigma P=1.3 \mathrm{~atm}$. This result agrees with the mass transfer rate calculated from values $-7.8,-7.4,-7.0$ at 1200,1300 and $1400 \mathrm{~K}$, respectively. Therefore, the values of enthalpy of formation and entropy of lanthanum tribromide used for calculating these $1 \mathrm{~g} \mathrm{Kp}$ are the most reliable. The shape of the experimental $\mathrm{V}_{\mathrm{m}} \mathrm{vs}_{\mathrm{PBr}_{5}}$ curve coincides with that calculated for other cerium group orthophosphates (Nd, Eu, Gd). Such agreement is observed only when the values of $\Delta H_{f 298.15}^{\circ}$ and $S_{298.15}^{\circ}$ for tribromides obtained in [16] were taken for calculation of the mass transfer rate, and it is these values that should be used in thermodynamic calculations.

Estimation of $\Delta H_{T}^{\circ}$ and $S_{T}^{\circ}$ is made easier when the number of initial species is small and, consequently, from comparison of mass transfer rate vs total pressure dependences, a quite definite conclusion can be drawn. This is clearly seen for $\mathrm{LnPO}_{4} / \mathrm{PBr}_{3} / \mathrm{Br}_{2}$ systems where $L n$ is a rare earth element of the cerium group. It should be noted that thermochemical characteristics of rare earth tribromides listed in table 2 should be used in thermodynamic calculations.

Experimental methods of determining diffusion coefficients are usually very tedious and theoretical calculations are frequently hindered by the absence of initial data.

In [24], a method of determining diffusion coefficients in reaction (2) is discussed, which is based on the concepts of chemical transport reactions:

1. Chemical transport occurs according to a reversible heterogeneous reaction resulting in the solid phase being transferred to the gaseous phase. A change of equilibrium conditions, e.g., temperature, leads to formation of a solid phase. This means that the solid phase is the only condensed phase. In the case of another condensed phase, for example, $\operatorname{LnX}_{3}(1 i q)$, conditions are chosen from the P-T diagram at which a liquid phase is not formed.

2. The solid substance is at equilibrium with the gaseous phase.

3. The surface reaction rates in the system "solid substance-gas" are sufficiently high and do not limit the process (the rate of substance transfer is independent of experiment duration and the surface area of the initial substance).

4. The slowest step determining the overall rate of the process is diffusion (the amount of transferred substance is independent of the angle of the furnace and is proportional to the cross section of the ampoule).

Knowing the yield of the transport reaction and its thermodynamic parameters and also the law governing gas motion (transport of gaseous products occurs by diffusion), it is quite easy to determine diffusion coefficients of gas mixtures from formulae given in [1]. If the reaction proceeds with variation of the molar quantities of gaseous products, the calculation of diffusion coefficients is carried out according to formula given in [25]. 
Table 2. Standard enthalpy of formation and entropy of gaseous rare earth tribromides [16].

\begin{tabular}{|c|c|c|}
\hline ompound & $\begin{array}{l}-\Delta H_{\mathrm{f} 298.15}^{\circ} \\
\mathrm{kcal} / \mathrm{mol}\end{array}$ & $\begin{array}{l}\mathrm{S}_{298.15}^{\circ} \\
\quad \mathrm{cal} / \mathrm{deg} \mathrm{mol}\end{array}$ \\
\hline
\end{tabular}

\begin{tabular}{|c|c|c|}
\hline $\mathrm{LaBr}_{3}$ & 146 & 90.2 \\
\hline $\mathrm{CeBr}_{3}$ & 146 & 90.3 \\
\hline $\mathrm{PrBr}_{3}$ & 147 & 90.3 \\
\hline $\mathrm{NdBr}_{3}$ & 141 & 90.4 \\
\hline $\mathrm{PmBr}_{3}$ & 142 & 90.5 \\
\hline $\mathrm{SmBr}_{3}$ & 142 & 90.6 \\
\hline $\mathrm{EuBr}_{3}$ & 121 & 90.7 \\
\hline $\mathrm{GdBr}_{3}$ & 141 & 90.8 \\
\hline $\mathrm{ThBr}_{3}$ & 137 & 90.9 \\
\hline $\mathrm{DyBr}_{3}$ & 136 & 91.0 \\
\hline $\mathrm{HoBr}_{3}$ & 136 & 91.0 \\
\hline $\mathrm{ErBr}_{3}$ & 135 & 91.1 \\
\hline $\mathrm{TmBr}_{3}$ & 134 & 91.2 \\
\hline $\mathrm{YbBr}_{3}$ & 125 & 91.3 \\
\hline $\mathrm{LuBr}_{3}$ & 132 & 91.3 \\
\hline $\mathrm{ScBr}_{3}$ & 130 & 84.9 \\
\hline $\mathrm{YBr}_{3}$ & 133 & 88.1 \\
\hline
\end{tabular}

Using this method, the diffusion coefficients of gaseous mixtures $\mathrm{LnBr}_{3}, \mathrm{PBr}_{3}, \mathrm{Br}_{2}, \mathrm{Br}$, and $\mathrm{PoBr}_{3}$ were estimated.

A promising application of gas transport reactions is the preparation of single crystals and films of high melting compounds in non-isothermal plasmas.

Reactions with extreme equilibrium positions ( $K \gg 1$ or $K \ll 1$ ) cannot be used for materials transport, as discussed by Schafer [1]. Also in many gas-transport reactions (in closed systems), the diffusion rate considerably exceeds in absolute value the rate of the heterogeneous reaction. Therefore, the yield of the transported substance will be smaller than the one calculated in the assumption that transfer is determined by diffusion. An example of such reactions is transfer of carbon and nitrides, where the $\mathrm{CO}$ and $\mathrm{N}_{2}$ transport molecules are very stable.

The advantage of a non-isothermal plasma as compared with temperature gradients is that at relatively low temperature $\left(400-1000{ }^{\circ} \mathrm{C}\right.$ ) relatively high concentrations of active 
molecules and free radicals, including $\mathrm{CO}^{*}$ and $\mathrm{N}^{*}$ may be obtained. This method does not require complex equipment. A discharge is maintained by generators with different frequencies using external electrodes. The external temperature gradient along the discharge tube is created by a tube resistance furnace.

Several works have been published on transfer of As, Se, and Te [26], Ge and Si [27] in hydrogen plasma, of carbon, aluminum, and titanium nitrides in nitrogen plasma $[28,29]$ and of iodine pentoxide in oxygen plasma [30].

Study of chemical transport reactions of high-melting compounds in non-isothermal plasma presents considerable interest for practical applications. From the point of view 01 theory, there are still many problems to be solved, first of all the relationship between reaction conditions and plasma parameters (excitation, temperature of neutral gas, etc.).

Chemical transport reactions may also be used to study complexation in the gaseous phase. Literature on this subject is scarce, although it is of considerable practical and scientific importance and is of interest to scientists studying the chemistry of vapor state. The most studied is the role played by halide complexes during transfer of solid halides of copper, silver, gold, ruthenium, rhodium, palladium, osmium, iridium platinum, etc. A comprehensive survey of this problem is given in [31,32]. A11 the observed processes of transfer of solid metal halides are endothermic reactions. The low-volatile metal halide is transferred from the "hotter" to the "cooler" zone and crystallizes in the latter. $\mathrm{AlCl}_{3}, \mathrm{FeCl}_{3}, \mathrm{GaCl}_{3}, \mathrm{NbCl}_{5}$, etc., are used as complexing agents.

From calculation of $\log \mathrm{Kp}$ of reactions (1) and (2) at 1200,1300 , and $1400 \mathrm{~K}$, it would seem that the transport properties of the $\mathrm{LnPO}_{4} / \mathrm{PCl}_{3} / \mathrm{Cl}_{2}$ system are better than of the $\mathrm{LnPO}_{4} / \mathrm{PBr}_{3} / \mathrm{Br}_{2}$ system. But, already at low temperature and initial pressure of $\mathrm{PCl}_{5}$, a second condensed phase is formed, $\mathrm{LnCl}_{3}(\mathrm{liq})$, in the zone of the direct reaction and the direction of transfer is changed $\left(T_{1} \rightarrow T_{2}\right.$, where $\left.T_{2} \rightarrow T_{1}\right)$. Bonding of condensed rare earth trichlorides into gaseous complexes $\mathrm{LnAl}_{3} \mathrm{Cl}_{12}$ and $\mathrm{LnAl}_{4} \mathrm{Cl}_{15}$ [5] makes it possible to carry out reaction (1) in the required direction in high yield and to reduce substantially the crystallization temperature of rare earth orthophosphates.

Let us now consider the following reactions:

$$
\begin{gathered}
\mathrm{LnPO}_{4}(\mathrm{~s})+3 \mathrm{PCl}_{3}(\mathrm{~g})+3 \mathrm{Cl}_{2}(\mathrm{~g})=\mathrm{LnCl}_{3}(\mathrm{~s}, 1 \mathrm{iq})+4 \mathrm{POCl}_{3}(\mathrm{~g}) ; \Delta \mathrm{H}_{1}^{\circ}<0 \\
\mathrm{LnCl}_{3}(\mathrm{~s}, 1 \mathrm{iq})+4 \mathrm{AlCl}_{3}(\mathrm{~g})=\mathrm{LnAl}_{4} \mathrm{Cl}_{15}(\mathrm{~g}) ; \Delta \mathrm{H}_{\mathrm{II}}^{\circ}>0 \\
\mathrm{LnPO}_{4}(\mathrm{~s})+4 \mathrm{AlCl}_{3}(\mathrm{~g})+3 \mathrm{PCl}_{3}(\mathrm{~g})+3 \mathrm{Cl}_{2}(\mathrm{~g})= \\
\mathrm{LnAl}_{4} \mathrm{Cl}_{15}(\mathrm{~g})+4 \mathrm{POCl}_{3}(\mathrm{~g}) ; \mathrm{H}_{\mathrm{III}}^{\circ}=\mathrm{H}_{\mathrm{I}}^{\circ}+\mathrm{H}_{\mathrm{II}}^{\circ}
\end{gathered}
$$

Depending on the ratio between $\Delta \mathrm{H}_{\mathrm{I}}^{\circ}$ and $\Delta \mathrm{H}_{\mathrm{II}}^{\circ}, \mathrm{LnPO}_{4(\mathrm{~s})}$ will be transferred either to zone $\mathrm{T}_{2}$ or to $\mathrm{T}_{1}$. Qualitative thermodynamic considerations lead to the following conclusions. $\Delta H^{\circ}$ of reaction (3) is higher in absolute value than $\Delta H^{\circ}$ of reaction (4) and, therefore, $\mathrm{LnPO}_{4}$ should be transported from $\mathrm{T}_{1}$ to $\mathrm{T}_{2}$ (hotter). 
Strictly speaking, (3) is not a transport reaction, but the combination of (3) and (4). gives a typical transport reaction, proceeding with variation of the molar quantities of gaseous components $(\Delta n=-3)$. For negative values of $\Delta n$ an exothermic reaction is thermodynamically more favourable $\left(\Delta H^{\circ}<0\right)$ and an increase in temperature evidently shifts the equilibrium of reaction (5) towards formation of the gaseous complex, favouring transfer from $T_{1}$ and $T_{2}$.

Application of these complexation concepts to systems with a liquid or a solid metal halide shows that crystallization of solid substances ( $\mathrm{LnPO}_{4}$ for instance) may be carried out at temperatures $\left(\sim 600^{\circ} \mathrm{C}\right.$ ) lower than at which gaseous metal halides exist (for instance, metal trichlorides). Study of these problems, however, has only just begun.

In conclusion, it should be noted that this paper does not cover such important aspects as the use of chemical gas-transport reactions for synthesis of new compounds, study of phase diagrams, acceleration of reactions between solid phases, and automation of crystallization from gaseous phase.

\section{References}

[1] Schafer, H., "himicheskie Transportnye reaktsii (Chemical Transport Reactions), Mir, Moscow (1964).

[2] Kristallizatsiya iz Gazovoi Fasy (Crystallization from Gaseous Phase), Mir, Moscow, p. 11 (1965).

[3] Bulakh, B. M. and Sheftal, N. N., Rost Kristallov (Growth of Crystals), X. Nauka, Moscow, p. 98 (1974).

[4] Orlovskii, V. P., Khalikov, B. S., Bulgakov, V. I., and Kurbanov, Kh. M., Zh. Neorganicheskie Materialy, 11, 494 (1975).

[5] Orlovskii, V. P., Ionkina, E. A., and Agafonov, I. L., Inorg. Mat. USSR, 8,1701 (1972).

[6] Schafer, H., Orlovskii, V., and Wiemeyer, M., Z. Anorg. Allg. Chem., 390, 13 (1972).

[7] Novoselova, A. V., Orlova, Yu. V., Sobolev, B. N., and Sidorov, L. I., Dokl AN SSSR, 159,1338 (1964).

[8] Gingerich, K. A., J. Crystal Growth, 9, 31 (1971).

[9] Øye, H. A. and Gruen, D. M., J. Am. Chem. Soc. 91, 2229 (1969).

[10] Aits, M. and Schafer, H., Z. Anorg. Al1g. Chem. 408, 37 (1974).

[11] Hessler, J. P., Wagner, Jr., F., Williams, C. W., and Carna11, W. T., J. Appl. Phys. 48, 8, 3260 (1977).

[12] Orlovskii, V. P., Belyaevskaya, T. V., Bulgakov, V. I., and Khalikov, B. S., Zh. Neorganicheskie Materialy, 13, 1484 (1977).

[13] Schafer, H. and Etzel, K., Z. Anorg. Allg. Chem. 301, 137 (1959).

[14] Oppermann, H., Z. Anorg. Chem. 383, 285 (1971).

[15] Orlovski,, V. P., Belyaevskaya, T. V., and Bugakov, V. I., Zh. Neorg. Khim. 22, 2354 (1977). 
[16] Polyachonok, 0. G., Doctor's Thesis, Leningrad, LGU (1972).

[17] Krestov, G. A., Termokhimiya soedinenii redkozemelnykh $i$ aktinidnykh elementov (Thermochemistry of Compounds of Rare Earth and Actinide Elements), Moscow, Atomizdat (1972).

[18] Wiks, K. E. and Block, F. E., Termodinamicheskie svoistva 65 elementov, ikh okislov, galogenidov, karbidov i nitridov (Thermodynamic Properties of 65 Elements, Their Oxides, Halides, Carbides and Nitrides), Moscow, Metallurgiya (1965).

[19] Tananaev, I. V., Orlovskii, V. P., Kurbanov, Kh. M., Khalikov, B. S., Osmanov, Sh. O., and Bugakov, V. I., Dok1. AN Tad. SSR, 17, 42 (1974).

[20] Tsagareishvili, D. Sh., Gvelisiani, G. G., Orlovsky, V. P., Belyevskaya, T. V., and Repko, V. P., Zh. Neorganicheskie Materialy, $\underline{8}, 1790$ (1972).

[21] Orlovskii, V. P., Kurbanov, Kh. M., Khalikov, B. S., Bugakov, B. I., and Tananaev, I. V., Zh. Neorganicheskie Materialy, 10, 671 (1974).

[22] Termicheskie konstanty veschestv (Thermal Constants of Substances), Handbook, V. P. Glushkov, ed., vyp. 1,2,3, Moscow, VINITI (1968).

[23] JANAF Thermochemical Tables, 2nd ed., NSRDS-NBS 37, U.S. Gov't. Printing Office, Washington, DC (1971).

[24] Orlovskii, V. P., Belyaevskaya, T. V., Bugakov, V. I., and Khalikov, B. S., Zh. Neorganicheskie Materialy, 13, 1489 (1977).

[25] Schafer, H., Z. Anorg. Chem. 400, 242 (1973).

[26] Jnq, S. W. and Chiang, V. S., J. Electrochem. Soc. 113, 192 (1966).

[27] Veprek, S. and Marecek, V., Sol. State Electron. 11, 683 (1958).

[28] Veprek, S., Brende], C., and Schafer, H., J. Crystal Growth, 9, 226 (1971).

[29] Veprek, S., J. Crystal Growth, 17, 101 (1972).

[30] Veprek, S. and Haupman, Z., Z. Anorg. A11g. Chem. 359, 313 (1968).

[31] Schafer, H., Binneuris, M., Domke, W., and Karbovski, J., Z. Anorg. A11g. Chem. 403, 116 (1974).

[32] Schafer, H. and Trenke1, M., Z. Anorg. A1lg. Chem. 426, 113 (1976). 
National Bureau of Standards Special Publication 561, Proceedings of the 10th Materials Research Symposium on Characterization of High Temperature Vapors and Gases held at NBS, Gaithersburg, Maryland, September 18-22, 1978. Issued October 1979.

\title{
NEW MOLECULES INVOLVED IN CHEMICAL TRANSPORT REACTIONS: GASEOUS HALIDE COMPLEXES
}

\author{
Harald Schäfer \\ Westfalische Wilhelms-Universitat, Anorganisch-Chemisches Institut \\ 44 Munster (Westf.), Germany
}

1. Short Introduction to the Principles of Chemical Transport

If a closed vessel containing a solid and a gaseous transport agent is heated in a temperature gradient and the solid migrates, not by its own vapor pressure, but by means of a reversible reaction into a defined temperature region within the ampoule, then this effect is called chemical transport [1] ${ }^{1}$. Usually, this situation can be treated by a model which considers diffusion between two spaces with the temperatures $T_{1}$ and $T_{2}$ where the equilibrium between the solid and the gas phase is established. That means that diffusion is the rate limiting process. With very few exceptions this is true for most systems at total pressures between $0.01 \mathrm{~atm}$ to a few atmospheres. Only this situation will be considered.

With respect to uncertainties of the thermodynamic data, some simplifications concerning the gas mobility are possible:

- Even with complex compositions of the gas phase an average diffusion coefficient is used.

0 One of the problems is the bulk movement of the gas for the cases where the transport reaction proceeds with a change in mole number. This streaming is, for stoichiometric reasons, proportional to the diffusion rate. The influence of this effect can be neglected, if one uses a gas species present in a low concentration to express the concentration gradient [1].

- Generally (especially in cases where the gas phase is of complex nature) it is recommended to make use of the so called solubility $\lambda$ of the solid in the gas phase to calculate the concentration gradient [2].

- A useful preliminary estimation of the transport properties of a system is obtained by considering the thermodynamic aspects only.

- Generally, it can be assumed that the chemical transport is large, if the transport equilibrium is not too far from $\log \mathrm{Kp}=0$.

A description of chemical transport reactions with solid sulfides [3] and oxides [4] has been given in 1971 and 1972. Since that time, new progress has been made by different authors which will be included in the following text.

${ }^{1}$ Figures in brackets indicate the literature references at the end of this paper. 


\section{Novel Transport Agents}

The main process of chemical transport reactions may be given in terms of the following equation:

$$
\text { solid }+ \text { gaseous transport agent }=\text { gaseous products } .
$$

Therefore, a large number of transport agents can be found depending on temperature and thermodynamics. Most of the transport agents used up to 1972 are summarized in [4]. Some interesting additional systems are given in tables 1 and 2 .

Table 1. Some novel transport systems.

\begin{tabular}{llll|} 
solid & \multicolumn{1}{c}{ transport agent } & \multicolumn{1}{c}{ gaseous products } & references \\
\hline Oxides & $\mathrm{TeCl}_{4}\left(\mathrm{TeCl}_{2}+\mathrm{Cl}_{2}\right)$ & chlorides, $\mathrm{TeOCl}_{2}\left(\mathrm{TeO}_{2}\right)$ & [5-8] \\
$\mathrm{Fe}, \mathrm{Co}, \mathrm{Ni}$ & $\mathrm{GaCl}_{3}\left(\mathrm{or} \mathrm{GaI}_{3}\right) \mathrm{Ga}_{2} \mathrm{X}_{6}$ & $\mathrm{GaX}$, halides, halide complexes & [9] \\
$\mathrm{Ni}$ & $\mathrm{InI}_{3}$ & $\mathrm{InI}, \mathrm{NiI}_{2}$, complexes & {$[10]$} \\
$\mathrm{Ge}$ & $\mathrm{GaCl}_{3}, \mathrm{Ga}_{2} \mathrm{Cl}_{6}$ & $\mathrm{GaCl}, \mathrm{Ga}_{2} \mathrm{Cl}_{4}, \mathrm{GeCl}_{2}, \mathrm{GeCl}_{4}$ & {$[11]$} \\
& & $\left(\mathrm{GeGaCl}{ }_{5}\right.$ ? $)\left(\mathrm{GeCl}_{3}\right.$ ?) & \\
$\mathrm{FeS}$ & $\mathrm{GeI}_{2}$ & $\mathrm{FeI}_{2}, \mathrm{GeS}$ & [12] \\
$\mathrm{MCl}_{2}$ & $\mathrm{Al}_{2} \mathrm{Cl}_{6}$ & complexes as $\mathrm{MAl}_{2} \mathrm{Cl}_{8}$ & see section 4 \\
\hline
\end{tabular}

Table 2. Two combined transport agents.

\begin{tabular}{llll} 
solid & transport agents & gaseous products & references \\
\hline $\mathrm{Pt}$ or $\mathrm{PtCl}_{2}$ & $\mathrm{CO}+\mathrm{Cl}_{2}$ & $\mathrm{Pt}_{2}(\mathrm{CO})_{2} \mathrm{Cl}_{2}$ & {$[13]$} \\
$\mathrm{LaPO}_{4}$ & $\mathrm{Br}_{2}+\mathrm{PBr}_{3}$ & $\mathrm{LaBr}_{3}, \mathrm{POBr}_{3}$ & {$[14]$} \\
$\mathrm{UO}_{2}$ & $\mathrm{Br}_{2}+\mathrm{S}_{2}$ & $\mathrm{UBr}_{4}+\mathrm{SO}_{2}$ & {$[15]$} \\
$0 \times$ ides & $\mathrm{I}_{2}+\mathrm{S}_{2}$ & iodides $+\mathrm{SO}_{2}$ & {$[16]$} \\
$\mathrm{SnO}_{2}$ & $\mathrm{I}_{2}+\mathrm{S}_{2}$ & iodides $+\mathrm{SO}_{2}$ & {$[17]$} \\
$\mathrm{V}_{2} \mathrm{O}_{3}$ & $\mathrm{I}_{2}+\mathrm{S}_{2}$ & iodides $+\mathrm{SO}_{2}$ & {$[18]$} \\
$\mathrm{Fe}_{2} \mathrm{O}_{3}$ & $\mathrm{HCl}+\mathrm{GaCl}_{3}$ & $\mathrm{Fe}_{2} \mathrm{Cl}_{6}, \mathrm{FeGaCl}_{6}, \mathrm{H}_{2} \mathrm{O}$ & {$[19]$} \\
$\mathrm{CdCr}_{2} \mathrm{~S}_{4}$ & $\mathrm{Cl}_{2}+\mathrm{AlCl}_{3}$ & chlorides, $\mathrm{S}_{2}$, complexes & {$[20]$} \\
$\mathrm{CdCr}_{2}(\mathrm{~S}, \mathrm{Se})_{4}$ & $\mathrm{I}_{2}+\mathrm{AlCl}_{3}$ & iodides, $\mathrm{S}_{2}, \mathrm{Se}_{2}$, complexes & {$[20]$} \\
\hline
\end{tabular}

\section{Unexpected Transport Observations}

Unexpected transport observations may be an indication that unknown molecules are involved. However, in such cases one has to exclude carefully the possibility that impurities--especially water even when present in trace amounts--are responsible for the transport effect. One should remember also that migration of the solid into the low 
temperature region of the ampoule may occur either by chemical transport or sublimation, whereas migration into the high temperature zone must necessarily be chemical transport.

\section{1 Examp les}

The transport of Ge in a quartz ampoule which has not been previously baked out may be caused by traces of halogen or water [21] and the same may be true for the transport of $\mathrm{GaP}$ in a stream of $\mathrm{H}_{2}$ [22].

An important and well known example is the tungsten cycle in iodine lamps. It is dependent upon the presence of small amounts of oxygen or water introduced as an impurity, because gaseous $\mathrm{WO}_{2} \mathrm{I}_{2}$ is the effective species [23]. The analogue is true for the transport of $\mathrm{MoS}_{2}$ and $\mathrm{WS}_{2}$ [24] as well as for $\operatorname{Re}$ and $\mathrm{ReS}_{2}$ [25] by means of iodine $\left(+\mathrm{H}_{2} 0\right)$.

Another example is the chemical transport of Fe0Cl. When an ampoule contains only $\mathrm{Fe}_{2} \mathrm{O}_{3}$ and an excess of $\mathrm{FeCl}_{3}$ at $350 \rightarrow 325{ }^{\circ} \mathrm{C}$, the formation of $\mathrm{FeOCl}$ occurs, but no chemical transport is possible. If, however, the ampoule also contains traces of water, then $\mathrm{HCl}$ is formed and $\mathrm{FeOCl}$ is transported into the low temperature region [26]. Similar water effects (formation of hydrogen halide by hydrolysis) may be involved, e.g., in chemical transport observations with the systems $\mathrm{ZnS} / \mathrm{ZnI}{ }_{2}, \mathrm{~g}$ [27], $\mathrm{Cr}_{2} \mathrm{~S}_{3} / \mathrm{AlCl}_{3}, \mathrm{~g}$ [28] sulfides $/ \mathrm{ATCl}_{3}, \mathrm{~g}$ [29], $\mathrm{Cu}_{x} \mathrm{Hg}_{7-x} \mathrm{Cr}_{2} \mathrm{Se}_{4} / \mathrm{AlCl}_{3}, \mathrm{~g}$ [30] and especially in the migration of $\mathrm{GaOCl}, \mathrm{GaOBr}, \mathrm{GaOI}$ into the hot (!) region of an ampoule [31].

An interesting observation is the chemical transport of $\mathrm{Ti}_{2} \mathrm{O}_{3}$ with $\mathrm{TiCl}_{4}$ as transport $\left(1100 \rightarrow 850^{\circ} \mathrm{C}\right)$ [32] and similarly the transport of $\mathrm{TiOCT}$ in the presence of $\mathrm{TiCl}_{3}, \mathrm{~g}$ $\left(650 \rightarrow 550^{\circ} \mathrm{C}\right)$ [33] and of $\mathrm{TiOBr}$ besides $\mathrm{TiBr}_{3}, \mathrm{~g}$ [34]. Here the question arises in which way the oxygen may be transported. Are there gaseous oxide halides present, in spite of the fact that at $850^{\circ} \mathrm{C}$ no weight loss could be found when a stream of $\mathrm{TiCl}_{4}, \mathrm{~g}$ (30 Torr; 24h) passed over $\mathrm{TiO}_{2}$ [35]? Or, are traces of water again the reason for the formation of hydrogen halide, which acts as a transport agent?

These examples make it clear that unexpected chemical transport observations may stimulate further investigations, with the aim of finding out what kind of unknown species may be present in the gaseous phase.

\section{Gaseous Halide Complexes}

We regard here only a part of this large family of molecules with halide double or triple bridges, which has gained increasing interest during the last few years. Main investigators are Novikov, Sidorov, Dewing, Gruen, Øye, Papatheodorou, Emmenegger and the author (for references see [36] and the review of Hastie ${ }^{2}$ ).

Especially suitable as transport agents are volatile trihalides such as $\mathrm{AlX}_{3}, \mathrm{GaX}_{3}$, InX $_{3}, \mathrm{FeX}_{3}$ or their dimers. With metal dihalides for example, they form gaseous complexes of the types $\mathrm{MATCl}_{5}, \mathrm{MAT}_{2} \mathrm{Cl}_{8}, \mathrm{MAI}_{3} \mathrm{Cl}_{11}$ and $\mathrm{MAT}_{4} \mathrm{Cl}_{14}$ with the probable structures:

2Editor's note: Hastie, J. W., High Temperature Vapors: Science and Technology, p. 126-148 (Academic Press, NY, 1975). 
$\mathrm{Cl}-\mathrm{M}_{\mathrm{Cl}}^{-\mathrm{Cl}} \underset{\mathrm{Al}}{-\mathrm{Cl}}$

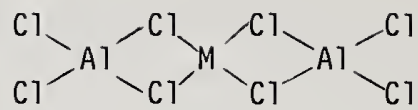

or
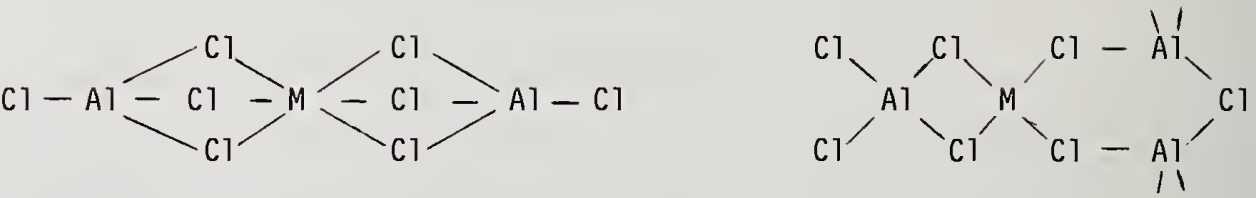<smiles>C[Al](C)Cl</smiles>

The periodic table (table 3 ) shows the elements of which the chlorides are known to form gas complexes with trichlorides. Bromides and iodides act in a similar way. In the following, we will not consider the chemistry of these complexes in detail [36]. We are looking only for their ability to support chemical transport reactions. A survey along with rules for the complex stability are given in [36]. Generally, this stability is relatively large at temperatures of about $300{ }^{\circ} \mathrm{C}$ and diminishes on going to higher temperatures so that the concentration of the complexes is small at $\geqq 1000{ }^{\circ} \mathrm{C}$.

Table 3. Elements of which the chlorides form gas complexes with $\mathrm{AlCl}_{3}$ (or $\mathrm{GaCl}_{3}, \mathrm{InCl}_{3}, \mathrm{FeCl}_{3}$ ).

Li Be

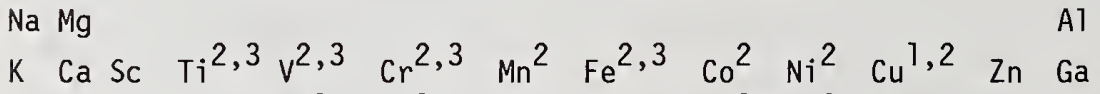

$\mathrm{Rb} \mathrm{Sr} \mathrm{Y} \mathrm{(Zr)} \mathrm{Nb}^{3} \quad \mathrm{Mo}^{3} \quad \mathrm{Ru} \quad \mathrm{Rh}^{3} \quad \mathrm{Pd}^{2}$ Ag $\mathrm{Cd}$ In $\mathrm{Sn}^{2} \mathrm{Sb}^{3}$

Cs Ba La (Hf) (Ta)

Ir $\mathrm{Pt}^{2} \mathrm{Au}^{3} \quad \mathrm{Hg}^{2} \mathrm{Tl} \quad \mathrm{Pb} \quad \mathrm{Bi}^{1,3}$

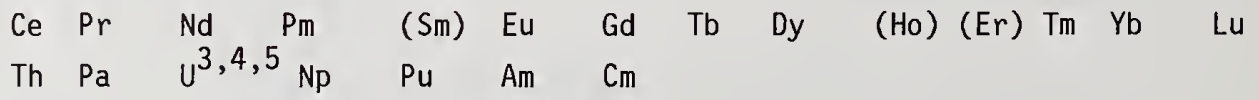

$\mathrm{ACl}, \mathrm{s}+1 / 2 \mathrm{Al}_{2} \mathrm{Cl}_{6}, \mathrm{~g} \quad(\mathrm{~A}=\mathrm{Alkali})$

The main gaseous reaction product is considered to be $\mathrm{AACl}_{4}$, besides small concentrations of $\mathrm{A}_{2} \mathrm{AlCl}_{5}$ and $\mathrm{A}_{2} \mathrm{Al}_{2} \mathrm{Cl}_{8}$. The first observation of such a molecule $\left(\mathrm{NaAlCl}_{4}, \mathrm{~g}\right)$ was made by W. Fischer [37]; see also [38]. In these cases, the gaseous complexes have a relatively high stability. They may represent the main constituent of the gas phase. Because the solid or liquid is also rather stable, $\mathrm{AAlCl}_{4}$ can be distilled. However, no back reaction with deposition of $A C 1, s$ occurs, which means that no chemical transport of $\mathrm{ACl}$ itself is possible. Combined with other compounds, $\mathrm{ACl}-\mathrm{AlCl}_{3}$ complexes may play a role in chemical transport reactions. 
In the reaction of $\mathrm{CuCl}, s$ with $\mathrm{Al}_{2} \mathrm{Cl}_{6}, \mathrm{~g}$ it was assumed originally that $\mathrm{CuAlCl}_{4}, \mathrm{~g}$ is the product [39]. A mass spectroscopic investigation, however, showed, that $\mathrm{Cu}_{3} \mathrm{AlCl}_{6}$ and $\mathrm{Cu}_{2} \mathrm{Al}_{2} \mathrm{Cl}_{8}$ are the predominant gaseous complexes [40]. Probably the latter have cube structures as it is known for $\mathrm{Cu}_{4} \mathrm{Cl}_{4}[41]$.

$\mathrm{MCl}_{2}, \mathrm{~s}+\mathrm{Al}_{2} \mathrm{Cl}_{6}, \mathrm{~g}(\mathrm{M}=\mathrm{Mg}, \mathrm{Ca}, \mathrm{Cr}, \mathrm{Mn}, \mathrm{Fe}, \mathrm{Co}, \mathrm{Ni}, \mathrm{Cu}, \mathrm{Pd}, \mathrm{Pt})$

$\mathrm{MAl}_{2} \mathrm{Cl}_{8}, \mathrm{~g}$ and, at higher temperatures, $\mathrm{MAlCl}_{5}$ are the principal complexes under usual transport conditions [42]. The following general scheme is found:

$$
\begin{gathered}
\mathrm{MAl}_{4}{ }^{\mathrm{Cl}}{ }_{14}<\mathrm{MAl}_{3} \mathrm{Cl} 1_{11}<\mathrm{MAl}_{2} \mathrm{Cl}_{8}<\mathrm{MATCl}_{5} \\
\text { where } \mathrm{T} \text { increases } \\
\text { or } \mathrm{P}\left(\mathrm{Al}_{2} \mathrm{Cl}_{6}\right) \text { decreases }
\end{gathered}
$$

With $\mathrm{PdCl}_{2}$, s besides the main complex $\mathrm{PdAl}_{2} \mathrm{Cl}_{8}$ a lesser amount of $\mathrm{Pd}_{2} \mathrm{Al}_{2}{ }_{2} \mathrm{Cl}_{10}, \mathrm{~g}$ [43] could be observed mass spectrometrically.

With $\mathrm{TiCl}_{2}$ and $\mathrm{VCl}_{2}$ the vapor species $\mathrm{TiAl}_{3} \mathrm{Cl}_{11}$ [44] and $\mathrm{VAl}_{3} \mathrm{Cl}_{11}$ [45] respectively, have been described.

The chemical transport can be described in most cases by

$$
\mathrm{MCl}_{2}, \mathrm{~s}+\mathrm{Al}_{2} \mathrm{Cl}_{6}, \mathrm{~g}=\mathrm{MAl}_{2} \mathrm{Cl}_{8}, \mathrm{~g}\left(400 \rightarrow 350{ }^{\circ} \mathrm{C}\right)
$$

In this way, crystals of the dichlorides can be prepared starting with powders of the dehydrated dichlorides and $\mathrm{Al}_{2} \mathrm{Cl}_{6}$ as transport agent or performing a synthesis with excess metal $+\mathrm{Cl}_{2}+\mathrm{Al}_{2} \mathrm{Cl}_{6}$. The same is true for the bromides.

The synthesis of $\mathrm{CrCl}_{2}$ deserves special mention: if one reacts an excess of $\mathrm{Cr}$ with $\mathrm{Cl}_{2}$ in a closed tube in a temperature gradient $450 \rightarrow 350^{\circ} \mathrm{C}$ one observes the deposition of $\mathrm{CrCl}_{3}$ only. But, when the same reaction is carried out with addition of $\mathrm{Al}_{2} \mathrm{Cl}_{6}$ one obtains well formed $\mathrm{CrCl}_{2}$ crystals. In a similar way $\mathrm{CrBr}_{2}$ and $\mathrm{CrI}_{2}$ can be prepared [46]. Larger amounts of $\mathrm{CrCl}_{2}$ were prepared starting with $\mathrm{CrCl}_{3}+\mathrm{Cr}$ (excess $+\mathrm{Al}_{2} \mathrm{Cl}_{6}+\mathrm{HCl}$ or $\mathrm{NH}_{4} \mathrm{Cl}, 450$ $\rightarrow 350{ }^{\circ} \mathrm{C}$ [46]. In this way $\mathrm{HCl}\left(+\mathrm{H}_{2}\right)$ transports the redox equivalents between $\mathrm{Cr}$ and $\mathrm{CrCl}_{3}$. $\mathrm{Al}_{2} \mathrm{Cl}_{6}$ transports $\mathrm{CrCl}_{2}$ into the $350^{\circ}$ zone.

The complex formation between $\mathrm{CuCl}_{2}$ and $\mathrm{FeCl}_{3}\left(\mathrm{Fe}_{2} \mathrm{Cl}_{6}\right)$ plays a role in the chlorination of copper-iron-ores. By disregarding the gaseous complex one would expect the volatilization of $\mathrm{FeCl}_{3}$ and a residue containing $\mathrm{CuCl}_{2}$. Contrary to this expectation $\mathrm{CuCl}_{2}$ is volatilized in the form of $\mathrm{CuCl}_{2} / \mathrm{FeCl}_{3}$ complexes [47]. Similarly, the separation of alkali chlorides, $\mathrm{MgCl}_{2}$ and $\mathrm{CaCl}_{2}$ from $\mathrm{Al}_{2} \mathrm{Cl}_{6}$ fails because complexes are volatilized together with $\mathrm{Al}_{2}{ }_{2}{ }_{6} 6[48,49]$.

Complexes of the same type exist with $\mathrm{GaCl}_{3}$ or $\mathrm{InCl}_{3}$ as complexing and transport agents. The relatively low dimerization enthalpy of $\mathrm{GaCl}_{3}$ leads in some cases (with $\mathrm{CaCl}_{2}, \mathrm{CrCl}_{2}$, $\mathrm{CoCl}_{2}, \mathrm{CuCl}_{2}, \mathrm{PdCl}_{2}$ ) to an inversion of the transport direction. Under special conditions $(P, T)$ transport occurs into the high temperature region by means of the exothermic reaction [50] 


$$
\mathrm{MCl}_{2}, \mathrm{~s}+2 \mathrm{GaCl}_{3}, \mathrm{~g}=\mathrm{MGa}_{2} \mathrm{Cl}_{8}, \mathrm{~g}
$$

$\mathrm{MCl}_{3}, \mathrm{~s}+\mathrm{Al}_{2} \mathrm{Cl}_{6}, \mathrm{~g}$

Gaseous complexes are known for $\mathrm{ScCl}_{3}[51], \mathrm{TiCl}_{3}[44], \mathrm{VCl}_{3}[45], \mathrm{CrCl}_{3}[52,53]$, $\mathrm{MoCl}_{3}[51], \mathrm{FeCl}_{3}[36], \mathrm{YCl}_{3}[51], \mathrm{LaCl}_{3}[51]$ and $\mathrm{LnCl}_{3}[54,55]$.

The formulae $\mathrm{TiAlCl}_{6}, \mathrm{VAlCl}_{6}, \mathrm{VAl}_{2} \mathrm{Cl}_{9}, \mathrm{CrAl}_{3} \mathrm{Cl}_{12}, \mathrm{NdAl}_{3} \mathrm{Cl}_{12}$ and $\mathrm{NdAl}_{4} \mathrm{Cl}_{15}$ are demonstrated or probable. Most of these systems are suitable for chemical transport, e.g., for purification and for crystal growth of the trichlorides. With the lanthanide chlorides and also the chlorides of post uranium elements, separations by gas chromatography have been made [54].

$\mathrm{MCl}_{4}, \mathrm{~s}+\mathrm{Al}_{2} \mathrm{Cl}_{6}, \mathrm{~g}$

$\mathrm{UAl}_{2}{ }_{2} \mathrm{Cl}_{10}[56]$ and the similar $\mathrm{ThAl}_{2}{ }_{2} \mathrm{Cl}_{10}$ are known. In both cases the tetrachlorides are transportable by means of $\mathrm{Al}_{2} \mathrm{Cl}_{6}, \mathrm{~g}[51]$.

\section{Pentahalides}

The gaseous complex $\mathrm{UCl}_{5} \cdot \mathrm{AlCl}_{3}=\mathrm{UAlCl}_{8}$ has been reported [56]. However, pentaand hexahalides have relatively high vapor pressures, so that chemical transport is not of importance.

\section{Transport of Metals}

Up to now, the study of transport reactions which involve gaseous halide complexes has been restricted to chlorides (halides) as solid phasès. However, gas complexes can be constituents of the gas phase in equilibrium with other solids also, which can be seen from the following examples.

An important step in the twenties was the development by van Arkel of the purification of a number of metals by iodine transport, via deposition onto a glowing wire. We observed that even noble metals ( $\mathrm{Pd}, \mathrm{Pt}, \mathrm{Au}$ ), the gaseous iodides of which are not stable enough for transport into the high temperature region of a transport system, can be transported in this way if a suitable complexing agent is added [57]:

transport direction of the metal
$400 \rightarrow 600{ }^{\circ} \mathrm{C}$
$300 \rightarrow 350{ }^{\circ} \mathrm{C}$
$500 \rightarrow 700{ }^{\circ} \mathrm{C}$
$400 \rightarrow 900{ }^{\circ} \mathrm{C}$

$$
\begin{aligned}
& \mathrm{Cu}+\mathrm{HCl}+\mathrm{Al}_{2} \mathrm{Cl}_{6}, \mathrm{~g}=\text { complex }+\mathrm{H}_{2} \\
& \mathrm{Au}+\mathrm{I}_{2}+\mathrm{Al}_{2} \mathrm{I}_{6}, \mathrm{~g}=\text { complex } \\
& \mathrm{Au}+\mathrm{Cl}_{2}+\mathrm{Fe}_{2} \mathrm{Cl}_{6}, \mathrm{~g}=\text { complex } \\
& \mathrm{Pd}+\mathrm{Cl}_{2}+\mathrm{Fe}_{2} \mathrm{Cl}_{6}=\mathrm{PdFe}_{2} \mathrm{Cl}_{8}, \mathrm{~g}
\end{aligned}
$$

The transport of $\mathrm{Fe}, \mathrm{Co}, \mathrm{Ni}$, and $\mathrm{Ge}$ (table 1) proceeds with $\mathrm{GaCl}_{3}, \mathrm{GaI}_{3}$ and $\mathrm{InI}_{3}$ as halogen sources as well as complexing agents. Under special conditions the complex formation causes inversion of the transport direction, that is chemical transport into the high temperature zone $[10]$. 
Transport of $\mathrm{Fe}_{2} \mathrm{O}_{3}$

The high stability of $\mathrm{Al}_{2} \mathrm{O}_{3}$ excludes the use of $\mathrm{Al}_{2} \mathrm{Cl}_{6}$ as a complexing agent for the chemical transport of oxides. However, in such a case one can replace $\mathrm{Al}_{2} \mathrm{Cl}_{6}$ by $\mathrm{Ga}_{2} \mathrm{Cl}_{6}$. The combination of the equibibria (7) and (8) leads to a substantial $\mathrm{Fe}_{2} \mathrm{O}_{3}$ transport into the high temperature region $\left(327 \rightarrow 427^{\circ} \mathrm{C}\right)$ [58].

$$
\begin{aligned}
& \mathrm{Fe}_{2} \mathrm{O}_{3}+6 \mathrm{HCl}=3 \mathrm{H}_{2} \mathrm{O}, \mathrm{g}+\mathrm{Fe}_{2} \mathrm{Cl}_{6}, \mathrm{~g} \\
& \mathrm{Fe}_{2} \mathrm{Cl}_{6}, \mathrm{~g}+\mathrm{Ga}_{2} \mathrm{Cl}_{6}, \mathrm{~g}=2 \mathrm{FeGaCl}_{6}, \mathrm{~g}
\end{aligned}
$$

In this special case, because of the similarity of the ionic radii of $\mathrm{Fe}^{3+}$ and $\mathrm{Ga}^{3+}$, some Ga was also introduced into the transported solid.

Synthesis of $\mathrm{HgCr}_{2} \mathrm{Se}_{4}$

By heating $\mathrm{Hg}+\mathrm{Cr}+\mathrm{Se}\left(600^{\circ} \mathrm{C}\right)$ one obtains $\mathrm{HgSe}+\mathrm{Cr}$. The addition of $\mathrm{HgCl}_{2}$ as a transport agent leads to solid $\mathrm{CrCl}_{3}$. The addition of $\mathrm{HgCl}_{2}+\mathrm{Al}_{2} \mathrm{Cl}_{6}$ results in the formation of nice crystals of $\mathrm{HgCr}_{2} \mathrm{Se}_{4}$. In this case $\mathrm{Al}_{2} \mathrm{Cl}_{6}$ forms a gaseous complex with $\mathrm{CrCl}_{3}$, which means that the gas phase acts as a solvent and, therefore, promotes the formation of $\mathrm{HgCr}_{2} \mathrm{Se}_{4}$ [51].

\section{Final Remarks}

Chemical transport to date has been mainly used to grow crystals, but in the future should be extended to other applications, e.g., for syntheses or thermodynamic investigations. The new family of gaseous halide complexes represents an important enrichment in the area of chemical transport reactions.

\section{References}

[1] Schäfer, H., Chemical Transport Reactions, Weinheim 1962, New York 1964, Moskau 1964.

[2] Schäfer, H., Z. Anorg. Allg. Chem. 400, 242 (1973).

[3] Schäfer, H., J. Cryst. Growth, 9, 17 (1971).

[4] Schäfer, H., Nat. Bur. Stand. Spec. Publ. 364, Solid State Chem. (1972).

[5] Niemyski, T. and Piekarczyk, W. , J. Cryst. Growth, 1, 177 (1967).

[6] Schäfer, H., Z. Anorg. Allg. Chem. 435, 5 (1977).

[7] Bando, Y., Nagasawa, K., and Mitarb, H., Oppermann U. Mitarb., Referiert in (252).

[8] Oppermann, H., Z. Anorg. Allg. Chem. 434, 239 (1977).

[9] Schäfer, H. and Nowitzki, J., Z. Anorg. Allg. Chem. 435, 49 (1977).

[10] Schäfer, H. and Nowitzki, J., Z. Anorg. Allg. Chem. 439, 80 (1978).

[11] Schäfer, H. and Trenkel, M., Z. Anorg. Allg. Chem., in press.

[12] Krabbes, G., Oppermann, H., and Wolf, E., Z. Anorg. Allg. Chem. 423, 212 (1976). 
[13] Schäfer, H. and Wiese, U., J. Less-Common Metals, 24, 55 (1971).

[14] Schäfer, H., Orlovskii, V. P., and Wiemeyer, M., Z. Anorg. Al1g. Chem. 390, 13 (1972).

[15] Naito, K., Kamegashira, N., and Nomura, Y., J. Cryst. Growth, 8 , 219 (1971).

[16] Nitsche, R., J. Phys. Chem. Solids 1967, Supp1. 1, 215; Internat. Conf. on Crystal Growth, Boston (1966).

[17] Matsumoto, K., Kaneko, S., and Takagi, K., J. Cryst. Growth, 40, 291 (1977).

[18] Pickardt, J., Reuter, B., and Söchtig, J., Z. Anorg. A11g. Chem. 401, 21 (1973).

[19] Schäfer, H. and Trenke1, M., Z. Anorg. A11g. Chem. 426, 113 (1976).

[20] Pickardt, J., Riede1, E., and Reuter, B., Z. Anorg. Al1g. Chem. 373, 15 (1970).

[21] Pritchard, C., J. Electrochem. Soc. 109, 993 (1962).

[22] Miche1, J. and Fahmy, D., J. Mater. Sci. 2, 299 (1967).

[23] Dettingmeijer, J. H., Tillack, J., and Schäfer, H., Z. Anorg. A1lg. Chem. 369, 161 (1969).

[24] Schäfer, H., Grofe, T., and Trenke1, M., J. Solid State Chem. $\underline{8}, 14$ (1973).

[25] Schäfer, H., Bode, M., and Trenke1, M., Z. Anorg. A11g. Chem. 400, 253 (1973).

[26] Schäfer, H. and Trenkel, M., unpublished work (1977).

[27] Aotsu, S., Takahashi, M., and Fuj.isaki, H., Tohoku Daigaku Kagaku Keisoku Kenkyusho Hokoko 24, 109 (1976).

[28] Lutz, H. D. and von Lovász, C., Angew. Chem. 80, 562 (1968).

[29] Lutz, H. D. , Bertram, K. H., Wrobe1, G., and Ridder, M., Monatshefte Chem. 105, 849 (1974).

[30] Okanska - Kozloska, I., Jelonek, M., and Drzazga, Z., Z. Anorg. A11g. Chem. 436 , 265 (1977).

[31] Hahn, H. and Katscher, H., Z. Anorg. Al1g. Chem. 321, 85 (1963).

[32] Fourcaudot, G., Dumas, J., Devenyi, J., and Mercier, J., J. Cryst. Growth, 40, 257 (1977).

[33] Schäfer, H., Wartenpfuhl, F., and Weise, E., Z. Anorg. Allg. Chem. 295, 268 (1958).

[34] von Schnering, H. G., Collin, M., and Hassheider, Z. Anorg. A1lg. Chem. 387, 137 (1972).

[35] Farber, M. and Darne11, A. J., J. Chem. Phys. 23, 1460 (1955).

[36] Schäfer, H. , Angew. Chem. 88, 775 (1976), Angew. Chem. Internat. Edit. in English, 15, 713 (1976).

[37] Fischer, W. , Angew. Chem. 61, 336 (1949).

[38] Novikov, G. I. and Gavryuchenkov, F. G., Russ. Chem. Reviews, 36, 156 (1967).

[39] Laughl in, W. C. and Gregory, N. W., J. Phys. Chem. 80, 127 (1976).

[40] Schäfer, H. and Rabeneck, H., Z. Anorg. Allg. Chem. in press.

[41] Cesaro, S. N., Coffari, E., and Spoliti, M., Inorg. Chim. Acta, 6, 513 (1972).

[42] Binnewies, M., Z. Anorg. Allg. Chem. 437, 25 (1977).

[43] Binnewies, M, Flörke, U., and Schäfer, H., unpublished work (1977/78).

[44] Sørlie, M. and Øye, H. A., preprint 1978.

[45] Anundskas, A. and Øye, H. A., J. Inorg. Nucl. Chem. 37, 1609 (1975). 
[46] Schäfer, H. and Laumanns, R., Z. Anorg. Allg. Chem. 436, 47 (1977).

[47] Spreckelmeyer, B. W., D. A. S. 2216807 (1972).

[48] Grothe, H. and Piel, C. A., Z. Electrochem. Angew. Physic. Chem. 54, 210, 216 (1950).

[49] Dewing, E. W. , Meta11. Trans. 1, 2169 (1970).

[50] Schäfer, H. and Trenke1, M., Z. Anorg. Allg. Chem. 437, 10 (1977).

[51] Schäfer, H., Binnewies, M., Domke, W., and Karbinski, J., Z. Anorg. Al1g. Chem. 403, 116 (1974).

[52] Lascelles, K. and Schäfer, H., Z. Anorg. A11g. Chem. 382, 249 (1971).

[53] Aits, M. and Schäfer, H., J. Less-Common Metals, 57, 219 (1978).

[54] Zvarova, T. S. and Zvara, I., J. Chromatogr. 44, 604 (1969); 49, 290 (1970).

[55] Øye, H. A. and Gruen, D. M., J. Amer. Chem. Soc. 91, 2229 (1969).

[56] Gruen, D. M. and McBeth, R. L., Inorg. Chem. $\underline{8}, 2625$ (1969).

[57] Schäfer, H. and Trenke1, M., Z. Anorg. Allg. Chem. 414, 137 (1975).

[58] Schäfer, H. and Trenke1, M., Z. Anorg. Al1g. Chem. 426, 113 (1976). 
National Bureau of Standards Special Publication 561, Proceedings of the 10th Materials Research Symposium on Characterization of High Temperature Vapors and Gases held at NBS, Gaithersburg, Maryland, September 18-22, 1978. Issued October 1979.

\title{
METAL HALIDE--GROUP III HALIDE VAPOR COMPLEXES WITH EMPHASIS ON ALUMINIUM CHLORIDE ${ }^{1}$
}

\author{
Harald A. Øye \\ Institutt for uorganisk kjemi \\ Norges tekniske høgskole \\ Universitetet $i$ Trondheim \\ 7034 Trondheim-NTH, Norway \\ and \\ Dieter M. Gruen \\ Chemistry Division \\ Argonne National Laboratory \\ Argonne, IL 60439
}

The thermodynamics of the presently known gas complexation reactions between metal halides and group III halides are treated in a self-consistent manner. By focusing on aluminum chloride as a complexing agent, certain systematic trends are revealed. The partial pressures of the gaseous complexes display flat maxima near $800 \mathrm{~K}$ whenever the complex molecules involve more than one molecule of $\mathrm{AlCl}_{3}$. Increasing the aluminum chloride pressure from 1 atm to $10 \mathrm{~atm}$ decreases somewhat the differences in the partial pressures among the various gaseous complexes which span two to three orders of magnitude.

The methods developed for characterizing the complexes, and their structures as well as some applications of gas complexation are discussed.

\footnotetext{
${ }^{1}$ Work performed under the auspices of the Office of Basic Energy Sciences of the U.S. Department of Energy.
} 


\section{Introduction}

The trivalent group IIIA chlorides, bromides, and iodides are characterized by having relatively low boiling or sublimation temperatures, as illustrated by the boiling or sublimation temperatures (in degrees centigrade) of $\mathrm{BCl}_{3}: 18$ (b.p.), $\mathrm{AlCl}_{3}: 183$ (s.p.), $\mathrm{GaCl}_{3}: 201$ (b.p.), $\mathrm{InCl}_{3}: 418$ (s.p.). It has long been recognized that these compounds are Lewis acids. In particular, $\mathrm{AlCl}_{3}$ has been used extensively in organic synthesis due to its Lewis catalytic activity.

High volatility at moderate temperature combined with halide acceptor ability makes these halides useful as volatilization agents for less volatile halides in the temperature range $200-1000{ }^{\circ} \mathrm{C}$.

The stability and volatility of $\mathrm{NaAlCl}_{4}$ in the gas phase was recognized by Fischer and Grothe [1] ${ }^{2}$ in 1949, and Grothe [2] remarked that $\mathrm{MgCl}_{2}$ seemed to be transported in the gas phase when binary mixtures of $\mathrm{AlCl}_{3}$ and $\mathrm{MgCl}_{2}$ were prepared. Dewing found high volatilities for reactions between divalent chlorides with $\mathrm{AlCl}_{3}$ and $\mathrm{FeCl}_{3}[3,4]$. A dramatic example of volatility enhancement is the formation of $\mathrm{NdAl}_{3} \mathrm{Cl}_{12}(\mathrm{~g})$ and $\mathrm{NdAl}_{4} \mathrm{Cl}_{15}(\mathrm{~g})$ leading to an increase by a factor of $\sim 10^{13}$ in the partial pressure of $\mathrm{NdCl}_{3}$ in 1 atm $\mathrm{Al}_{2} \mathrm{Cl}_{6}$ at $600 \mathrm{~K}$, relative to pure $\mathrm{NdCl}_{3}$ at the same temperature $[5,6]$.

Several review articles have appeared which have treated gas complexation by IIIA halides, notably by Novikov and Gavryuchenkov [7], Hastie $[8,9]$, Emmenegger [10], and Schäfer [11].

The present paper is not an exhaustive review of the literature. Rather, the emphasis is on experimental methods of characterization, correlations and the evaluation of thermodynamic quantities.

Gas complexation between a IIIA halide and another halide may be formulated generally as follows:

$$
m M X_{y}(s, 1 \text { or } g)+n A_{2} X_{6}(g)\left(\text { or } 2 n A X_{3}(g)\right)=M_{m} A_{2 n} X_{6 n+m y}(g)
$$

Such equilibria may be studied by a variety of techniques depending on whether or not $M X_{y}$ is present as a condensed phase and depending as well on the magnitude of the gas pressure and the temperature. In the following discussion, the advantages and disadvantages of the different techniques, and the conclusions to be drawn from pertinent examples will be detailed. Then, a thermodynamic treatment of the results will be presented. Finally, the structures of the gas complexes, the stabilities of condensed versus gaseous phases, and potential applications of the gas complexes will be discussed.

\footnotetext{
${ }^{2}$ Figures in brackets indicate the literature references at the end of this paper.
} 


\section{Experimental Methods}

Nearly every method used for determination of vapour pressures and structures of hightemperature vapours may be employed in the study of gas complexation with IIIA metal halides. The book edited by Margrave [12] may be consulted as a general reference. In the following, the methods that up to now have proved particularly useful for determination of gas complexation with IIIA metal halides will be examined.

\subsection{Transpiration techniques}

A general discussion of the transpiration method is given by Merten and Be11 [13]. The method requires the salt $M X_{y}$ to be in a condensed state and the vapour pressure to be from a few Torr up to 1 atm. The complexing gas $A_{2} X_{6}$ in a pure or dilute state is passed over the sample at the temperature, $T$, of investigation. The gas is saturated with the vapour of the sample and the vapour is later condensed out in a cold trap. The experiment gives information on the quantity of $\mathrm{MX}_{\mathrm{y}}$ in the vapour phase per mol $\mathrm{A}_{2} \mathrm{X}_{6}$.

With some assumptions which will be discussed later, it is possible to calculate the pressure of the gas complex $M-A-X^{3}$ as a function of $P_{A_{2}} X_{6}$ and hence determine the equilibrium constant for eq. (1). A typical example of an application of this method is the study of gas complexation between $\mathrm{CaCl}_{2}, \mathrm{CoCl}_{2}, \mathrm{MgCl}_{2}, \mathrm{MnCl}_{2}, \mathrm{NiCl}_{2}$ and $\mathrm{CdCl}_{2}$ with $\mathrm{Al}_{2} \mathrm{Cl}_{6}$ and $\mathrm{Fe}_{2} \mathrm{Cl}_{6}$ gas [4].

\subsection{Near IR-UV visible absorption spectroscopy}

Many of the gas complexes encountered contain metal ions with unfilled d- or f- shells which absorb light in the wavelength region 25000-1800 $\AA$ suitable for measurements by readily available spectrophotometers. In order to discriminate against black body radiation from the sample, it is advantageous to use spectrophotometers employing phase sensitive detection of electromagnetic radiation, as for example Cary $\mathrm{H}$ spectrophotometers.

The spectroscopic method can, in principle, give information not only about the pressure of the gaseous complexes, but also about their structures and the coordination geometry around $\mathrm{M}^{\mathrm{y}^{+}}$. Dye and Gruen [6] have applied this technique to this type of gas complexation. Usually, the salt $M X_{y}$ to be studied is enclosed in an evacuated optical cuvette together with the appropriate IIIA halide. On heating, the IIIA halide will evaporate and form complexes with $M X_{y}$. The technique is based on first establishing the molar absorptivity of $M^{{ }^{+}}$in experiments where a small amount of $M X_{y}$ is completely evaporated through gas complexation. (Usually the pressure of $M X_{y}$ is much smaller than $M-A-X$ and can be neglected. )

${ }^{3}$ Short notation for gaseous molecule $M_{m} A_{2 n}{ }^{X} 6 n+m y$ or mixtures of such gas molecules. 
The molar absorptivity of $\mathrm{M}^{\mathrm{y}^{+}}$in the gas complex at certain wavelengths, $\varepsilon_{j}$, is then given according to Beer's law:

$$
\varepsilon_{i}=\frac{A_{j} V M}{w T}
$$

where $A_{i}=$ the optical density at wave number $i, V=$ volume, $M=$ molecular weight of $M X_{y}$, $w=$ weighed in amount of $M X_{y}$, and $1=$ optical pathlength. $\varepsilon_{i}$ should be determined as a function of temperature and $A_{2} X_{6}$ pressure, but up till now the data have only permitted one to establish $\varepsilon_{j}$ as a function of temperature.

Experiments with excess $M X_{y}$ (saturated vapour) gives then the "atomic" 4 vapour pressure $\mathrm{P}^{\mathrm{a}}$ of the gas complexes by a combination of Beer's law with the law of ideal gases:

$$
P^{a}=\frac{A_{i} R \bar{T}}{\varepsilon_{i}{ }^{1}}
$$

where $R$ is the gas constant and $\bar{T}$ is the average temperature (for experimental reasons, a small temperature gradient is set up over the cell in order to prevent condensation on the windows). $\mathrm{P}^{\mathrm{a}}$ and $\varepsilon_{j}$ must be determined from corresponding conditions, i.e., the same temperature if $\varepsilon_{j}$ is temperature dependent. The pressure of the complexing gas $A_{2} X_{6}$ is usually determined from the weighed in amount correcting for dissociation and gas complexation.

Finally, the state of the condensed state must be established, i.e., whether it is a liquid or a solid with defined stoichiometry. The thermodynamics are simplest when the condensed phase is a solid compound. The work on gas complexation between $\mathrm{NdCl}_{3}$ and $\mathrm{Al}_{2} \mathrm{Cl}_{6}[6]$ gives further details on the method.

\subsection{Static pressure technique}

The static pressures of heterogeneous or homogeneous mixtures containing gas complexes may be measured by a variety of techniques. Typical methods make use of spiral, membrane or liquid manometers or of quasi-static techniques such as boiling point and dew point methods. Vapour density measurements will also give similar information. A review of many methods is given by Clopper, et al. [14]. In general, these have, however, found little use in the study of $M-A-X$ gas complexes probably because the vapour pressure of $M-A-X$ usually is much smaller than the pressure of $A_{2} X_{6}$ or $A_{3}$.

${ }^{4 P^{a}}=\sum_{i} m_{i}\left[P\left(M_{m_{i}} A_{2 n_{i}} X_{6 n_{i}}+m_{i} y\right)\right]$. If the gas complexes, however, contain only one atom (which usually is the case), then $P$ is equal to the total pressure of the gas complexes present. 
Information about gas complexation between alkali- and aluminum chlorides have, however; been obtained. Fischer [1] reports on formation of $\mathrm{NaAlCl}_{4}(\mathrm{~g})$ by vapour pressure measurements, but details are not given. Galitskii [15] made use of the transpiration method combined with determination of the total vapour pressure for $\mathrm{NaCl}_{-\mathrm{AlCl}_{3}}$ melts in order to determine the thermodynamics of formation of $\mathrm{NaAlCl}_{4}(\mathrm{~g})$. In order to obtain the necessary information, he also made use of the vapour above the melt condensed out in two different steps by the transpiration procedure.

Linga, et a1. [16] used a theoretically modified boiling point method for determination of total pressure of $\mathrm{AlKCl}^{-\mathrm{AlCl}_{3}}$ melts $(\mathrm{AlK}=\mathrm{Li}, \mathrm{Na}, \mathrm{K})$. By making full use of the data and introducing a model for the melt, information on gas complexation was obtained.

\section{4 Mass spectrometry}

Mass spectrometry has been widely used for the study of gas complexation. The review by Novikov and Gavryuchenkov [7] gives many examples. Another useful variation of the method is the work by Sidorov and Shol'ts [17] where the liquid activities and the gas complexation reaction are determined by mass spectroscopy and Knudsen effusion experiments using the socalled isothermal evaporation method.

of particular interest in connection with $\mathrm{AlCl}_{3}$-containing complexes is the recent work by Binnewies [18] which uses a double oven technique. $\mathrm{AlCl}_{3}$ is evaporated at a low temperature of $\approx 100{ }^{\circ} \mathrm{C}$, and swept over the $\mathrm{MCl}_{\mathrm{y}}$ salt to be studied at for instance $350{ }^{\circ} \mathrm{C}$. The chemically transported gas complex is then focussed on the ion source of a mass spectrometer through an effusion hole in the crucible. This method has been applied to a variety of divalent chlorides $[18,19,20]$.

The sources of the different ions are established and making the simplifying assumption that the pressure of a certain parent molecule will be proportional to ion intensities of the daughters, equilibrium constants in reasonable agreement with previous investigations are obtained. The temperature dependence of the equilibrium constant is calculated by assuming an entropy of reaction.

\subsection{Structural methods}

In addition to stoichiometry and thermodynamic information, it is highly desirable to obtain structural information on the gas complexes. Information has been obtained through the absorption spectrocopic technique discussed above. Some spectra give very clear-cut information about coordination, but in other instances, the spectra are not detailed enough for an unambiguous interpretation. The short-comings in some instances may be illustrated by the study of $\mathrm{CoAl}_{2} \mathrm{Cl}_{8}(\mathrm{~g})$ and $\mathrm{COGa}_{2} \mathrm{Cl}_{8}(\mathrm{~g})$ which give closely similar spectra. The system has been investigated by four different groups, but a concensus with respect to the interpretation of the results has not yet been obtained [21-24]. 
Electron diffraction techniques have not yet been applied to the present gas complexes. Recently some resonance Raman spectra were obtained by Emmenegger, et a1. [25] and by Papatheodorou and Capote [26]. ${ }^{5}$ A square planar coordination was proposed for $\mathrm{PdAl}_{2} \mathrm{Cl}_{8}$ in agreement with absorption spectroscopic measurements. The study of $\mathrm{Cu}-\mathrm{Al}-\mathrm{Cl}$ complexes reveals, however, that utmost care must be used in the interpretation. The threecoordination of $\mathrm{Cu}^{2+}$ in $\mathrm{CuAl}_{2}{ }_{2} \mathrm{Cl}_{8}$ suggested by Emmenegger, et al. [25] appears to be due to $\mathrm{CuAlCl}_{5}[26]$ as the laser beam creates a spectroscopic temperature which is higher than the thermodynamic one.

\section{Thermodynamics of Gas Complexation}

In principle, gas complexation is most directly studied by investigating the gaseous equilibrium

$$
m M X_{Y}(g)+n A_{2} X_{6}(g)=M_{m} A_{2 n} X_{6 n+m y}(g)
$$

without any condensed phase being present. By measuring three variables: the total gas pressure, the composition and the vapour density, the stoichiometry of the gas molecules and the thermodynamics of equilibrium (4) can, in principle, be unambiguously determined. In spite of the straightforward thermodynamics, this is not a commonly used approach for the determination of chloro-aluminate gas complexes. One principal disadvantage of the method is that it requires all pressures to be of the same order of magnitude. Nonetheless, the approach has been carried out by Novikov and Kotova who studied the $\mathrm{HgAlCl}_{5}$ gas complex [27].

A direct measurement of the relative pressures of the three components has been obtained by mass spectrometry [18-20]. Although the method is informative and rapid, it involves certain approximations (see above) which may lead to only moderate accuracy. Gas complexes with more than two Al atoms per complex molecule are not easily amenable to measurements since low pressures are a necessary condition in the mass spectrometer.

Most studies involve the presence of $M X_{y}$ as a condensed phase. The simplest case is when $M X_{y}$ is present as a pure solid (or a pure solid compound with $A_{2} X_{6}$ ). Then the activity of $M X_{y}$ is constant and only two variables need to be known, i.e., $P_{M-A-X}{ }$ and $P_{A_{2}} X_{6}$.

The thermodynamic treatment when $M X_{y}(s)$ is present will be illustrated. For simplicity, only one type of gaseous species is assumed present. Undissociated $A_{2} X_{6}$ is considered to be the complexing agent.

$$
m M X_{Y}(s)+n A_{2} X_{6}(g)=M_{m} A_{2 n} X_{6 n+m y}(g) .
$$

(The treatment is easily extended to more than one gaseous species, see [6], as $n$ will then usually become a non-half integer and vary with temperature.)

5ditors note: See also more recent work by Papatheodorou described elsewhere in this volume. 
Using the absorption spectroscopic method as an example (see above), the "atomic" gas pressure $P^{a}=m \cdot P\left(M_{m} A_{2 n} X_{6 n+m y}\right)$ is determined from a characteristic absorption peak. $P_{A_{2}} X_{6}$ can be determined from the weighed in amount of $\mathrm{AX}_{3}$ correcting for dissociation and complex formation [6].

By performing two different experiments and varying the temperature, one has the following equation:

$$
\frac{P_{M-A-X}^{a^{\prime}}}{\frac{m}{P_{A_{2} X_{6}}^{\prime n}}}=\frac{\frac{P_{M-A-X}^{a^{\prime \prime}}}{m}}{P_{A_{2}^{\prime \prime} X_{6}}^{n}}=K_{5}=\exp \quad\left\{-\frac{\Delta H_{5}^{o}}{R T}+\frac{\Delta S_{5}^{o}}{R}\right\} .
$$

The two experiments provide, in principle, enough data to determine $n$ (stoichiometry with respect to $A 1$ ) and $\Delta H_{5}^{\circ}$ and $\Delta S_{5}^{\circ}$. The reliability of the analysis is, of course, improved by doing more than two experiments and performing a least squares fit of the data. The thermodynamic analysis will predict correctly the amount of $M$ in the gas phase but $m$ (stoichiometry of $M$ in the gas complex) cannot be determined and is generally included in K. Mass spectroscopic measurements have not yet succeeded in characterizing gas complexes with $m \neq 1$ [18-20].

In the transpiration method $P_{M-A-X}+P_{A_{2} X_{6}}=P_{\text {tot }}$ and the total amount of $M, A$, and $X$ are determined. If the gas volume or gas density is also measured, the exact stoichiometry ( $m$ and $n$ ) and the equilibrium thermodynamic functions can be specified. Usually, however, the gas volume is not known [4].

One has then:

$$
\begin{gathered}
\text { Ratio of } M / A=\frac{n_{M}}{n_{A}}=\frac{{ }^{m} P_{M-A-X}}{{ }_{A_{2} X_{6}}+2{ }^{2 n} P_{M-A}-X} \\
P_{M-A-X}+P_{A_{2} X_{6}}=P_{\text {tot }}
\end{gathered}
$$

These two equations can be solved with respect to $P_{M-A-X}$ and $P_{A_{2}} X_{6}$ as functions of $n_{M} / n_{A}$, $P_{\text {tot }}, \mathrm{n}$ and $\mathrm{m}$.

By insertion into the equation for the equilibrium reaction (5), results parallel to the spectroscopic method are obtained by varying the total pressure and temperature. Again, the correct amount of $M$ in the gas phase can be predicted, but the value of $m$ has to be assumed since the activity of $A X_{y}$ is not varied. 
More care must be applied when equilibria with a liquid phase with varying composition is present, so that a varying activity of the component in the liquid phase enters the equilibrium constant

$$
\begin{gathered}
m M X_{y}(1)+{ }_{n A} X_{6}(g)=M_{m} A_{2 n} X_{6 n+m y}(g) \\
K_{9}=\frac{P_{M-A-X}}{a_{M X_{y}} P_{A_{2} X_{6}}^{n}}
\end{gathered}
$$

If the composition and the corresponding activity of the liquid phase is known, such a study will have the extra advantage that the coefficients m can also be ascertained (being usually $1)$, by varying the activity of $M X_{y}$.

Galitskii [15] performed a thermodynamic analysis of transpiration and total vapour

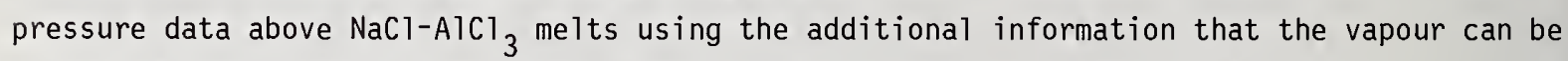
condensed out in two separate steps. He obtained plausible results for the reaction

$$
\mathrm{NaCl}(\mathrm{g})+\mathrm{AlCl}_{3}(\mathrm{~g})=\mathrm{NaAlCl}_{4}(\mathrm{~g})
$$

but it is difficult to follow the published calculations. Furthermore, it remains unclear how the changing composition of the liquid is taken into account.

Linga, et a1. [16] studied the total vapour pressure above $\mathrm{MCl}^{-\mathrm{AlCl}_{3}}(\mathrm{M}=\mathrm{Li}, \mathrm{Na}, \mathrm{K})$ melts as function of temperature in the concentration range $0<\mathrm{X}_{\mathrm{AlCl}_{3}} \leq 0.50$. The detailed data allowed a model to be set up. The liquid was assumed to be an asymmetric regular solution of $\mathrm{AlCl}_{4}^{-}$and $\mathrm{Cl}^{-}$and the data was used to determine $\Delta \mathrm{H}^{\circ}$ and $\Delta \mathrm{S}^{\circ}$ for the two reactions

$$
\begin{aligned}
& \operatorname{MATCl}_{4}(1)=\operatorname{MCl}(1)+\operatorname{AlCl}_{3}(1) \\
& \operatorname{MACl}_{4}(1)=\operatorname{MACl}_{4}(g)
\end{aligned}
$$

By combination with data from JANAF [28], the standard enthalpy and entropy for the reaction

$$
\operatorname{MCl}(g)+\mathrm{AlCl}_{3}(g)=\mathrm{MAlCl}_{4}(g)
$$

was obtained in good agreement with Fischer's [1] and Galitskii's [15] results.

The $\mathrm{AlkAlCl}_{4}$ gas complexation is an example of substantial amounts of gas complexes in equilibrium with a liquid phase at elevated temperatures. Gas complexation in equilibrium with liquid phases at low temperatures $\simeq 500 \mathrm{~K}$ are often of a very special kind due to the properties of $M X_{y}-\mathrm{AX}_{3}$ melts. We will again use $\mathrm{AlCl}_{3}$ as an example, but the arguments are also valid for melts containing other aluminum, gallium, indium, and ferric halides. 
Pure molten $\mathrm{AlCl}_{3}$ consists of doubly bridged $\mathrm{Al}_{2} \mathrm{Cl}_{6}$ molecules. Successive additions of a metal chloride $\mathrm{MCl}_{\mathrm{y}}$ whose cation has a relatively low ionic strength (which usually is also the condition for giving a stable liquid phase) results in the sequential formation of the ions $\mathrm{Al}_{3} \mathrm{Cl}_{10}^{-}, \mathrm{Al}_{2} \mathrm{Cl}_{7}^{-}$, and $\mathrm{AlCl}_{4}^{-}$[29]. When, however, the composition corresponding to

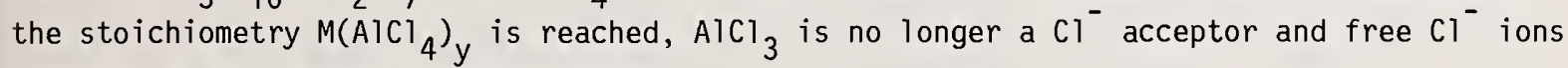
are formed upon further addition of $\mathrm{MCl}_{\mathrm{y}}$.

The equilibrium constant

$$
2 \mathrm{AlCl}_{4}^{-}=\mathrm{Al}_{2} \mathrm{Cl}_{6}+2 \mathrm{Cl}^{-}
$$

is usually very small ( $\mathrm{K}=2.8 \cdot 10^{-16}$ for $\mathrm{NaAlCl}_{4}$ at $\left.175{ }^{\circ} \mathrm{C}[30,31]\right)$, implying a drastic drop

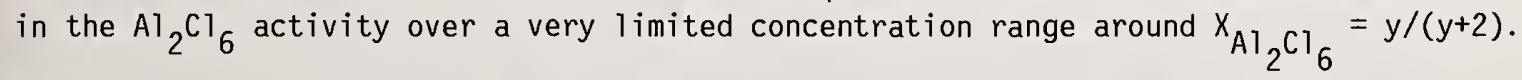

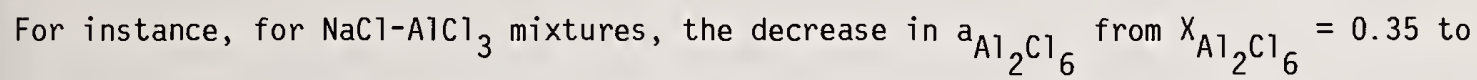
$\mathrm{X}_{\mathrm{Al}_{2}{ }_{2} \mathrm{Cl}_{6}}=0.315$ is about $10^{-8.5}$. This means that the melt composition will be nearly constant over an equally large variation in $\mathrm{P}_{\mathrm{Al}_{2} \mathrm{Cl}_{6}}$. Expressed more dramatically, a $\mathrm{MCl}_{y}{ }^{-}$

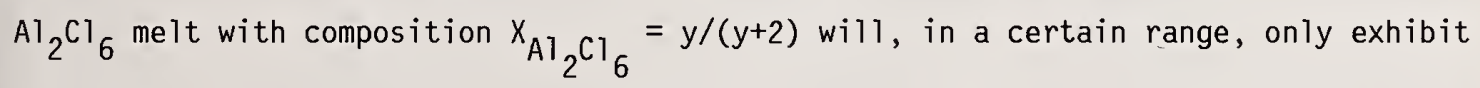
negligible concentration changes in spite of a large variation of the $\mathrm{Al}_{2}{ }_{2} l_{6}$ pressure. The Gibbs-Duhem equation can then be integrated at constant $T$ assuming constant composition: $\mathrm{X}_{\mathrm{Al}_{2}{ }_{2} \mathrm{Cl}_{6}}=\mathrm{y} /(\mathrm{y}+2)$.

$$
\mathrm{dlna}_{\mathrm{MCl}_{\mathrm{y}}}=-\frac{\mathrm{X}_{\mathrm{Al}_{2} \mathrm{Cl}}}{1-\mathrm{X}_{\mathrm{Al}}{ }_{2} \mathrm{Cl}_{6}} \mathrm{dln \textrm {A } _ { \mathrm { Al } } { } _ { 2 } \mathrm { Cl } _ { 6 }}=-\int \frac{\mathrm{y}}{2} \mathrm{dlnP}_{\mathrm{Al}_{2}{ }_{2} \mathrm{Cl}_{6}}
$$

and

$$
\mathrm{a}_{\mathrm{MCl}]_{\mathrm{y}}}=\mathrm{C} \cdot\left(\mathrm{P}_{\mathrm{Al}_{2} \mathrm{Cl}_{6}} / \mathrm{P}_{\mathrm{Al}}^{\mathrm{O}}{ }_{2} \mathrm{Cl}_{6}\right)^{-\mathrm{y} / 2}
$$

where $\mathrm{P}_{\mathrm{Al}_{2} \mathrm{Cl}_{6}}^{\mathrm{O}}$ is the vapour pressure of $\mathrm{Al}_{2} \mathrm{Cl}_{6}$ above pure $\mathrm{AlCl}_{3}$ and $\mathrm{C}$ is a constant to be determined by the choice of the standard state.

If we again make the simplifying assumption that only one type of gas complex is present:

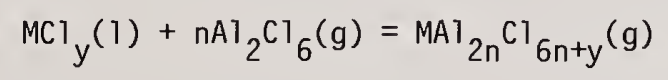


the equilibrium constant for this reaction will be

$$
\begin{aligned}
& K_{18}=\frac{P_{M-A 1-C 1}}{0^{y / 2} n-y / 2} \\
& \mathrm{a}_{\mathrm{MCl} 1_{y}} \cdot \mathrm{P}_{\mathrm{Al}}^{\mathrm{n}}{ }_{2} \mathrm{C}^{\mathrm{r}}{ }_{0} \quad \mathrm{C} \cdot \mathrm{P}_{\mathrm{Al}}{ }_{2} \mathrm{Cl}_{6} \cdot \mathrm{P}_{\mathrm{Al}}{ }_{2} \mathrm{Cl}_{6}
\end{aligned}
$$

If for instance, the gas complex is $\mathrm{MAl}_{3}{ }_{3} 1_{9+y}$, then $\mathrm{P}_{\mathrm{M}-\mathrm{Al}-\mathrm{Cl}} / \mathrm{P}_{\mathrm{Al}}^{0.5}{ }_{2} \mathrm{Cl}_{6}$ will be at constant temperature, i.e., the pressure of the gas complex varies with the square root of the $\mathrm{Al}_{2} \mathrm{Cl}_{6}$ pressure.

Sørlie and øye [31,32] made use of this property of chloroaluminate melts in the study of gas complexes in equilibrium with $\mathrm{EuCl}_{2}-\mathrm{AlCl}_{3}$ and $\mathrm{TiCl}_{2}-\mathrm{AlCl}_{3}$ melts. The analysis was most detailed in the case of the $\mathrm{EuCl}_{2}-\mathrm{AlCl}_{3}$ system. More than one gas complex was present and the equation

$$
P_{\text {tot }} \text { (gas complex) }=C \cdot P_{A 1_{2}{ }_{2}{ }^{O} l_{6}} \sum_{i} K_{i} P_{A 1}^{n_{i}^{n}-y / 2}{ }_{2}{ }^{C l_{6}}
$$

had to be used instead of eq. (19).

Data from experiments in $\mathrm{EuCl}_{2}$ or $\mathrm{TiCl}_{2}$ solid phases were presented enabling one to evaluate the integration constant $\mathrm{C}$. Such data were used to calculate $\mathrm{K}_{21}$ for the equilibrium

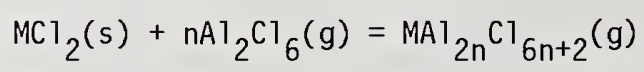

\section{Presentation of Data for Aluminium Chloride Gas Complexes}

An overview illustration of gas complexation is provided by table 1 and figures 1 and 2 which give a survey of known $\mathrm{AlCl}_{3}$ gas complexation reactions for chlorides in the condensed state. Table 1 lists $\Delta H_{22}^{\circ}$ and $\Delta \mathrm{S}_{22}^{\circ}$ for the reaction:

$$
M C 1_{y}(s)+n A 1_{2} C_{6}(g)=M A 1_{2 n}{ }^{C 1}{ }_{6 n+y}(g)
$$

In some instances, $\mathrm{AlkCl}$ and $\mathrm{EuCl}_{2}$, for example, the reaction is more complex than indicated by eq. (22) because the condensed phase is a liquid of unknown composition. For comparison $\Delta H^{\circ}$ and $\Delta S^{\circ}$ of vaporization of the pure solid is given in table 1 as well as $P_{M X}$ at

$600 \mathrm{~K}$. The coordination around the complexed ion $\mathrm{M}^{\mathrm{y}}$ is given if known from spectroscopic studies. The standard enthalpies and entropies are listed at the average temperatures of 


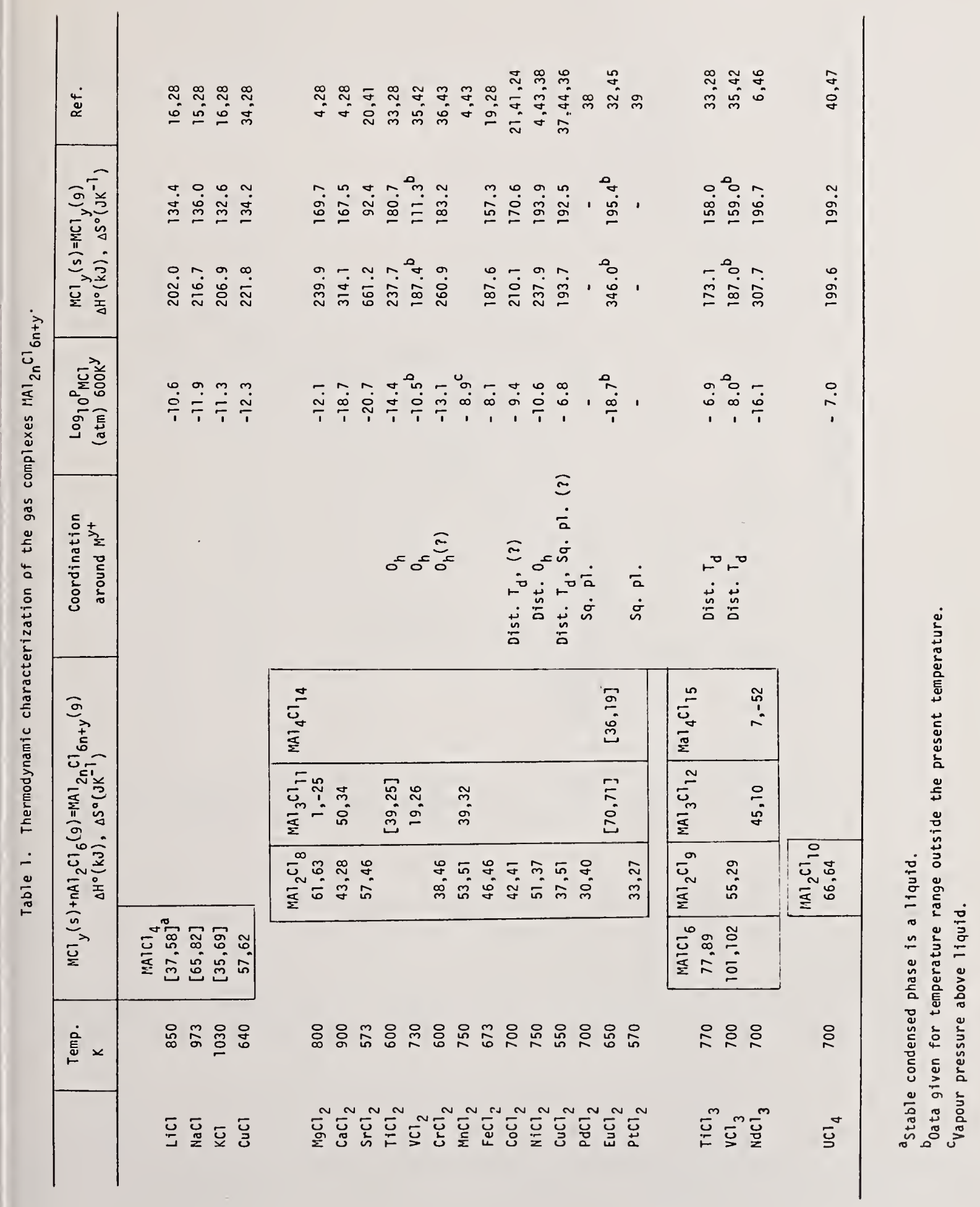


the gas complexation studies. For conversion of data to other temperatures Schäfer [11] proposed $\Delta \mathrm{C}_{\mathrm{p}}^{\circ}$ between $-6 \mathrm{JK}^{-1}$ and $14 \mathrm{JK}^{-1}$ be applied dependent on the gas complex formed. However, we have chosen not to apply this correction term in constructing figures 1 and 2 due to the limited temperature span of the measurements and the uncertainty in $\Delta H^{\circ}, \Delta S^{\circ}$ as well as $\Delta C_{p}^{\circ}$.

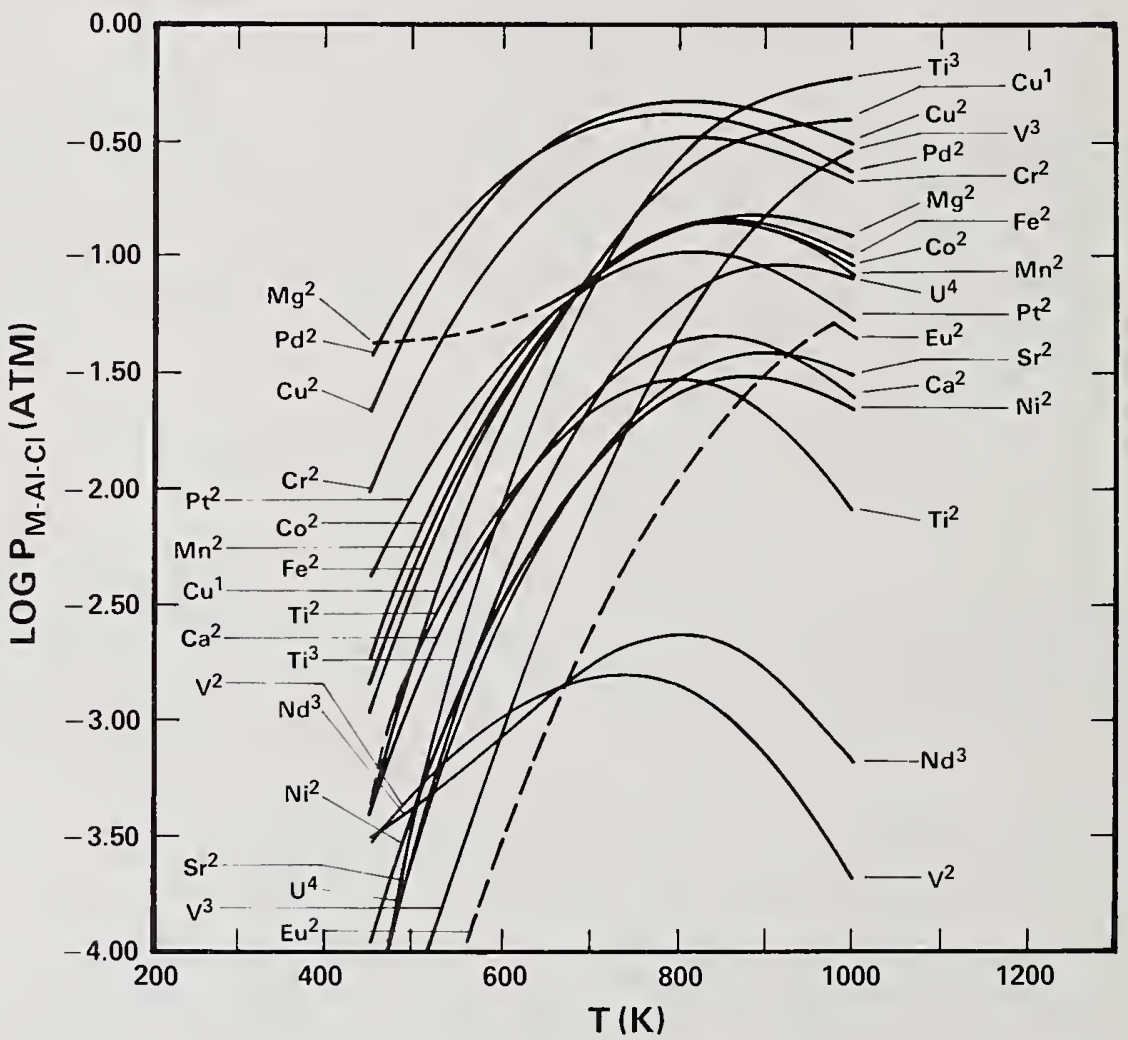

Figure 1. $\mathrm{M}-\mathrm{Al}-\mathrm{Cl}$ gas complexes in equilibrium with $\mathrm{MCl}_{y}(\mathrm{~s}), \mathrm{Al}_{2} \mathrm{Cl}_{6}(\mathrm{~g})$, and $\mathrm{AlCl}_{3}(\mathrm{~g})$. Total pressure $1 \mathrm{~atm}$. Data from table 1.

Figures 1 and 2 give the pressure of complex $\mathrm{P}_{\mathrm{M}-\mathrm{Al}-\mathrm{Cl}}$ in the presence of $\mathrm{Al}_{2} \mathrm{Cl}_{6}(\mathrm{~g})$ and $\mathrm{AlCl}_{3}(\mathrm{~g})$ at 1 and $10 \mathrm{~atm}$ total pressure, respectively. The dissociation of $\mathrm{Al}_{2} \mathrm{Cl}_{6}$ to $\mathrm{AlCl}_{3}$ is taken into consideration.

In some instances $\left(\mathrm{EuCl}_{2}, \mathrm{TiCl}_{2}\right)$, the partial pressure of the complexes refer to equilibria involving not only $\mathrm{MCl}_{\mathrm{y}}(\mathrm{s})$ (eq. 22), but also above liquid phase of unknown composition in which case dotted lines are shown. Data have been calculated directly from values given in publication $[32,33]$ and not from derived data given in table 1 . 


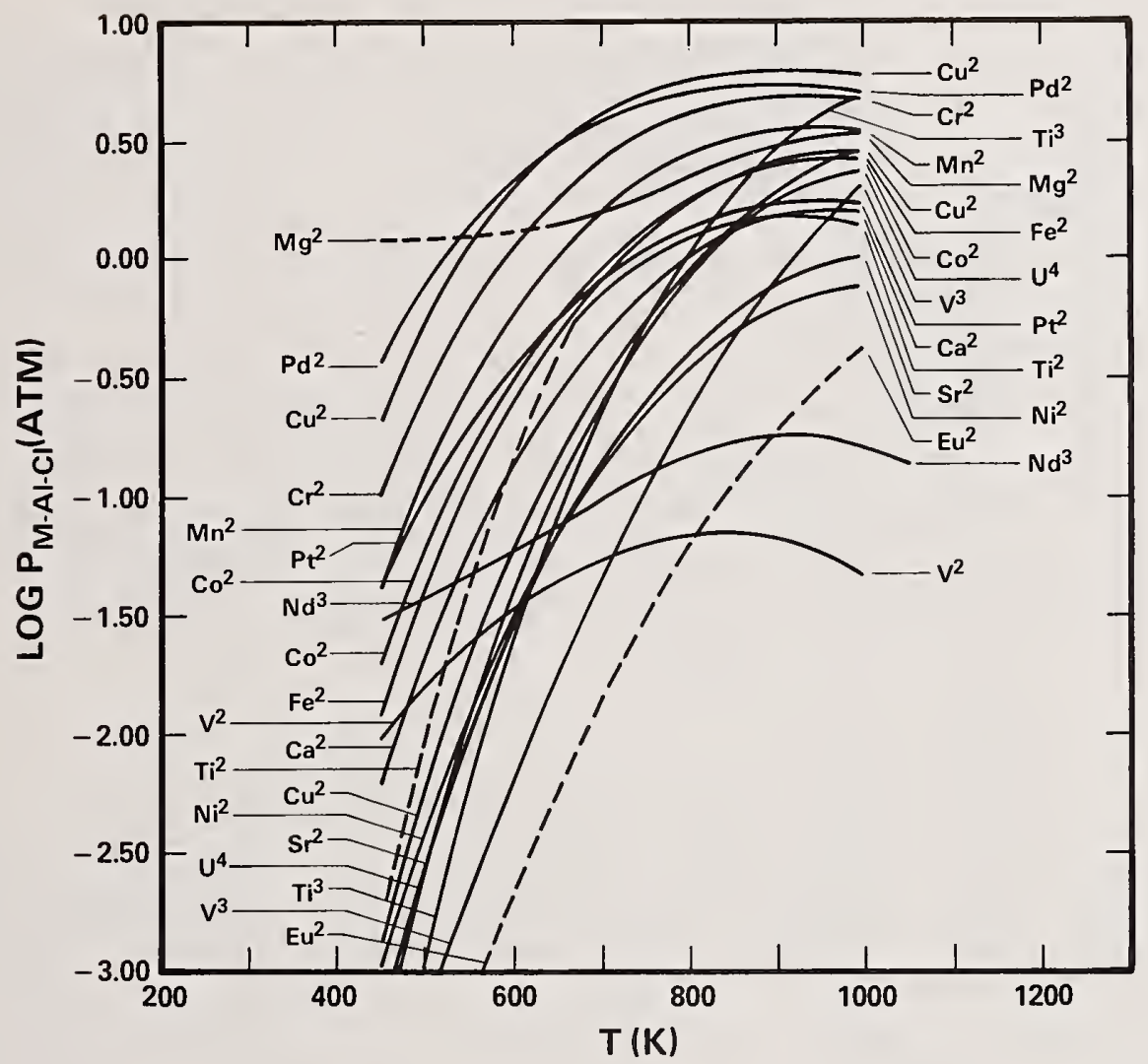

Figure 2. $M-A 1-C l$ gas complexes in equilibrium with $\mathrm{MCl}_{y}(\mathrm{~s}), \mathrm{Al}_{2} \mathrm{Cl}_{6}(\mathrm{~g})$, and $\mathrm{AlCl}_{3}(\mathrm{~g})$. Total pressure $10 \mathrm{~atm}$. Data from table 1.

From figure 1, it can be seen that the partial pressures of the complexes display two general types of behavior. The larger group shows a maximum in partial pressure between 700 to $900 \mathrm{~K}$. In a few cases, the partial pressures rise monotonically with temperature. The difference between the two groups is simply a consequence of the stoichiometry of the gas complexes. When two or more $\mathrm{Al}$-atoms are present in the gas complexes as in $\mathrm{MAl}_{2} \mathrm{Cl}_{8}$, $\mathrm{MAl}_{3} \mathrm{Cl}{ }_{11}, \mathrm{MAl}_{4} \mathrm{Cl}{ }_{14}, \mathrm{MAl}_{2} \mathrm{Cl}_{9}, \mathrm{MAl}_{3} \mathrm{Cl}_{12}, \mathrm{MAl}_{4} \mathrm{Cl}_{15}$, and $\mathrm{MAl}_{2} \mathrm{Cl}_{10}$, then the dissociation reaction

$$
\mathrm{Al}_{2} \mathrm{Cl}_{6}(\mathrm{~g})=2 \mathrm{AlCl}_{3}(\mathrm{~g}) \quad \mathrm{K}=1 \text { for } \mathrm{T}=883 \mathrm{~K}
$$

results in a diminishing complex pressure above a certain temperature. This is not the case if only one $A 1$-atom is present in the gas complex as in $\mathrm{MAICl}_{4}(\mathrm{~g})$ and $\mathrm{MAICl}_{6}(\mathrm{~g})$. 
Another observation is that the partial pressures of the different complexes show a lower degree of variability when the total pressure is 10 atm than at 1 atm. This behavior may be rationalized on the basis of bond properties of the gas complexes, to be discussed more fully below. The volatilities of the compounds $M X_{y}$, of course, differ by many orders of magnitude in the absence of gas complexing agents but in their presence, the differences are greatly diminished. The effect becomes more pronounced with increasing $\mathrm{Al}_{2} \mathrm{Cl}_{6}$ pressure, for example.

Figures 3 and 4 illustrate the gas complexation reactions in equilibrium with $\mathrm{AlkCl-}$ $\mathrm{AlCl}_{3}$ melts. Figure 3, which is taken from ref. [16] gives the pressure of $\mathrm{LiAlCl}_{4}, \mathrm{AlCl}_{3}$, and $\mathrm{Al}_{2} \mathrm{Cl}_{6}$ in equilibrium with $\mathrm{LiCl}-\mathrm{AlCl}_{3}$ melts at $919 \mathrm{~K}$. It is seen that $\mathrm{LiAlCl}_{4}$ is the major constituent of the gas. In figure 4 [48], the vapour and melt composition at the boiling point $\left(P_{\text {tot }}=1 \mathrm{~atm}\right.$ ) is given as a function of temperature for $\mathrm{AlKCl}^{-\mathrm{AlCl}_{3}}$ mixtures. For the $\mathrm{Li}$ system, $\mathrm{LiAlCl}_{4}$ becomes the major species at about $1000 \mathrm{~K}$, continuing to increase and then decreasing slightly with increasing temperature. For the $\mathrm{NaCl}$ and $\mathrm{KCl}$ system, higher temperatures are needed in order to make $\mathrm{AlkAlCl}_{4}$ the major species.

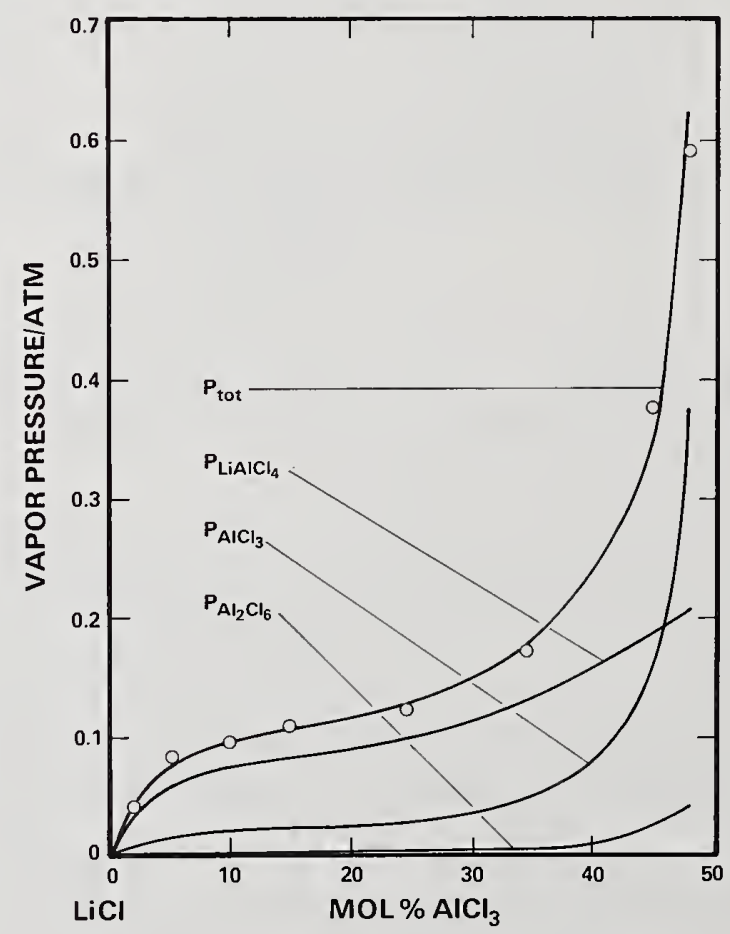

Figure 3. Total pressure, $\mathrm{P}_{\mathrm{LiAlCl}}, \mathrm{P}_{\mathrm{AlCl}_{3}}, \mathrm{P}_{\mathrm{Al}_{2} \mathrm{Cl}_{6}}$ above $\mathrm{LiCl}-\mathrm{AlCl}_{3}$ melts at $919 \mathrm{~K}$. 
$\mathrm{MOL} \% \mathrm{AlCl}_{3}$

$ळ 0000000$ 0 0 ᄂ

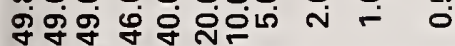

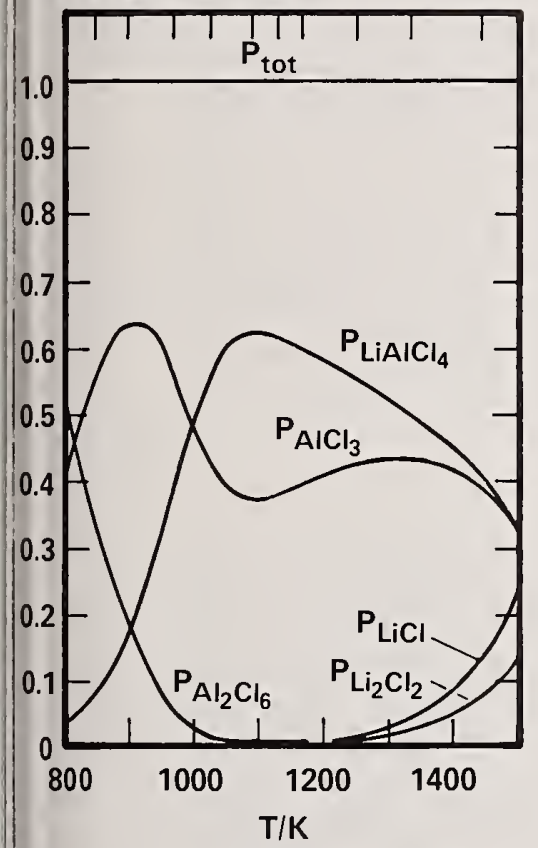

$\mathrm{LiCl}-\mathrm{AlCl}_{3}$
$\mathrm{MOL} \% \mathrm{AlCl}_{3}$

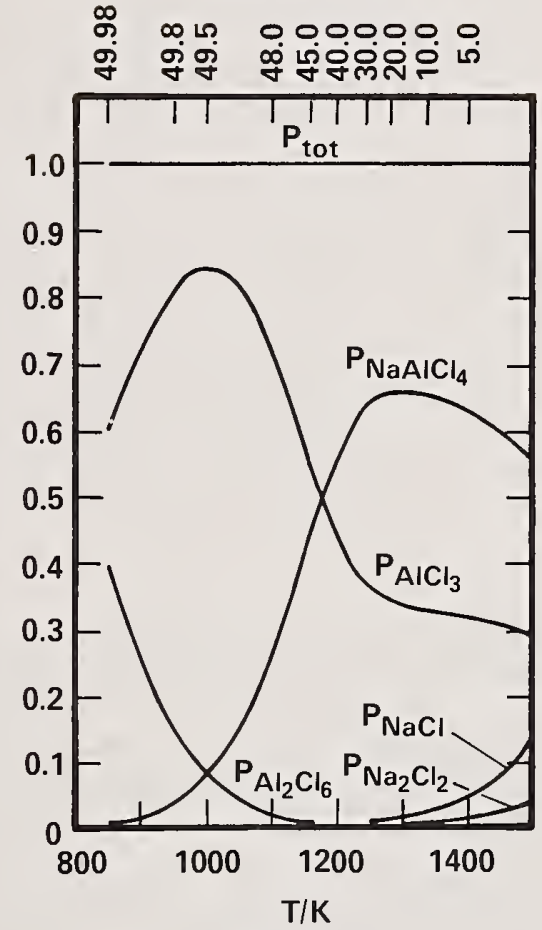

$\mathrm{NaCl}-\mathrm{AlCl}_{3}$
$\mathrm{MOL} \% \mathrm{AlCl}_{3}$

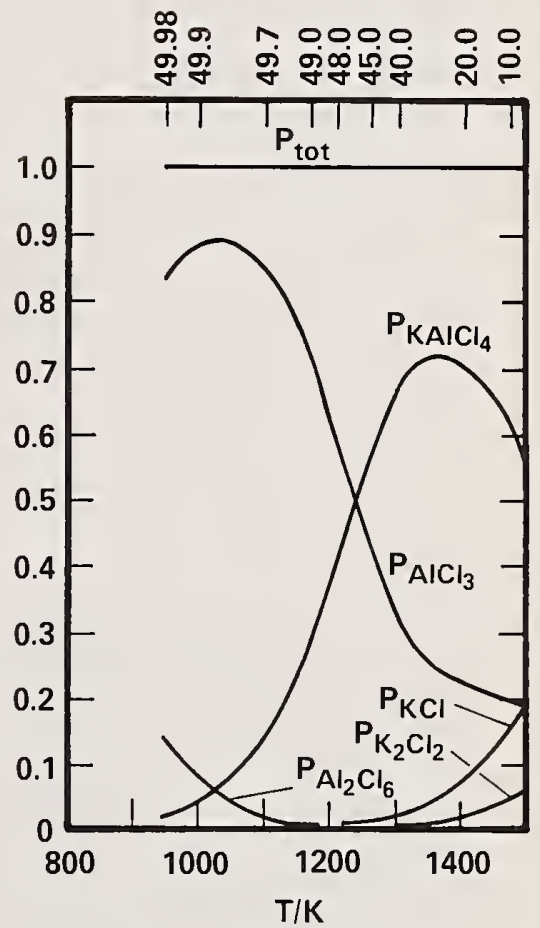

$\mathrm{KCl}-\mathrm{AlCl}_{3}$

Figure 4. Pressure of $\mathrm{AlKAlCl}_{4}, \mathrm{AlCl}_{3}, \mathrm{Al}_{2} \mathrm{Cl}_{6}$, and $\mathrm{AlKCl}$ above $\mathrm{A}_{\mathrm{K} C \mathrm{KC}-\mathrm{AlCl}_{3}}$ melts at their boiling point $\left(\mathrm{P}_{\text {tot }}=1 \mathrm{~atm}\right)$. Corresponding temperature and composition are given.

Only the data for gas complexation with $\mathrm{AlCl}_{3}$ are given here. The review papers [7-11] mentioned earlier may be referred to for further information on these and other systems. Since those papers were written, there has also been information on $\mathrm{MAlCl}_{5}[18-20], \mathrm{MInCl}_{5}$ and $\mathrm{MIn}_{2} \mathrm{Cl}_{8}$ gas complexes $[49,50,51]$, and complexes involving $\mathrm{Fe}_{2} \mathrm{Cl}_{6}, \mathrm{Al}_{2} \mathrm{Br}_{6}$, and $\mathrm{Ga}_{2} \mathrm{Cl}_{6}[37]$ as ligands.

\section{Structure of M-AT-Cl Gas Complexes}

As seen from table 1, some information has been obtained concerning the coordination of the metal ion $\mathrm{M}^{\mathrm{y}^{+}}$. The ligand field spectra which give the clearest information are those of $\mathrm{TiAl}_{3} \mathrm{Cl}_{17}(\mathrm{~g})$ [33] and $\mathrm{VAl}_{3} \mathrm{Cl}_{11}(\mathrm{~g})$ [35], both showing nearly perfect octahedral coordination, and of $\mathrm{PtAl}_{2} \mathrm{Cl}_{8}[39]$ and $\mathrm{PdAl}_{2} \mathrm{Cl}_{8}$ [38], both interpreted as close to square planar coordination. The other ligand field spectra do not point to a highly symmetric coordination $\left(0_{h}, T_{d}, D_{4 h}\right)$. 
An unusual feature of many of these gas complexes are their high molecular weight $\left(\mathrm{EuAl}_{4} \mathrm{Cl}_{14}\right.$ and $\left.\mathrm{NaAl}_{4} \mathrm{Cl}_{15}\right)$ and the variety of stoichiometries. It is probable that $\mathrm{Al}$ attempts to retain its stable 4 coordination and the molecules $\mathrm{MAICl}_{4}, \mathrm{MAlCl}_{6}, \mathrm{MAl}_{2} \mathrm{Cl}_{8}$, $\mathrm{MAl}_{3} \mathrm{Cl}_{12}$ can be understood in terms of coordination of $\mathrm{AlCl}_{4}^{-}$groups to $\mathrm{M}^{\mathrm{y}^{+}}$. However, $\mathrm{MAlCl}_{5}$ requires a $\mathrm{MCl}\left(\mathrm{AlCl}_{4}\right)$ coordination, EuAl ${ }_{4} \mathrm{Cl}_{14}$ coordination of $\mathrm{Al}_{2} \mathrm{Cl}_{7}^{-}$groups, while $\mathrm{EuAl}_{3} \mathrm{Cl}_{12}$ and $\mathrm{NdAl}_{4} \mathrm{Cl}_{15}$ involve a mixture of $\mathrm{AlCl}_{4}^{-}$and $\mathrm{Al}_{2} \mathrm{Cl}_{7}^{-}$groups. The complex $\mathrm{UAl}_{2}{ }_{2} \mathrm{Cl}_{10}$ most probably is described by the structure $\mathrm{UCl}_{2}\left(\mathrm{AlCl}_{4}\right)_{2}[40]$. There are several examples indicating that the less symmetric species are more abundant in the temperature and pressure range studied: $\mathrm{TiAl}_{3} \mathrm{Cl}_{11}>\mathrm{TiAl}_{2} \mathrm{Cl}_{8}, \mathrm{VAl}_{3} \mathrm{Cl}_{11}>\mathrm{VAl}_{2} \mathrm{Cl}_{8}, \mathrm{EuAl}_{3} \mathrm{Cl}_{11}>\mathrm{EuAl}_{4} \mathrm{Cl}_{14}$.

In addition to the Lewis acidity and $\mathrm{Cl}^{-}$acceptor property of $\mathrm{Al}_{2} \mathrm{Cl}_{6}$, the driving force for the complexation reaction appears to be the retention of a high coordination number leading to a multiplicity of ionic bonds around the metal ion $\mathrm{M}^{\mathrm{y}^{+}}$in the gas phase. Quite the contrary seems to be the case for evaporation of $\mathrm{MCl}_{\mathrm{y}}$ [5] in an inert atmosphere

$$
M C 1_{y}(s)=M C 1_{y}(g)
$$

since the $\mathrm{MCl}_{y}$ gas molecule usually has a lower coordination number than for $\mathrm{M}^{\mathrm{y}^{+}}$and has a more covalent bond character than the ionic $\mathrm{MCl}_{\mathrm{y}}(\mathrm{s})$ crystal where coordination numbers of 6 or 9 are commonly found. This may also explain the much smaller differences in the volatilities in a series of $\mathrm{MCl}_{y}$ solids in $\mathrm{Al}_{2}{ }_{2} \mathrm{l}_{6}$ atmospheres (fig. 1) than in inert atmospheres (table 1).

When comparing coordination and stoichiometry, one should be aware that both the coordination number and number of Al-atoms in the complex are expected to decrease with increasing temperature. Valid comparisons should hence be carried out only at comparable temperatures. However, this is not always possible due to the different stability ranges of the gaseous complexes and widely varying vapour pressures of the different $\mathrm{MCl}_{\mathrm{y}}$ solid compounds. Nonetheless, most studies have been carried out in the region around $650 \mathrm{~K}$, and it is interesting that octahedral site stabilizations energies [52] can give some guidance with respect to coordination numbers. Thus, $\mathrm{v}^{2+}, \mathrm{Cr}^{2+}, \mathrm{Ni}^{2+}, \mathrm{Cr}^{3+}$ [53], with high octahedral site stabilization energies all have octahedral or near octahedral coordination. On the other hand, $\mathrm{Ti}^{3+}, \mathrm{V}^{3+}$, and probably $\mathrm{Co}^{2+}$ with low octahedral site stabilization energies are tetrahedral or nearly tetrahedrally coordinated. There is one noteable exception, $\mathrm{Ti}^{2+}$, which with a very low octahedral site stabilization energy gives a spectrum characteristic of a nearly perfect octahedron in the complex $\mathrm{TiAl}_{3} \mathrm{Cl}_{11}(\mathrm{~g})$. Probably, at least a coordination number 6 is needed in order to bind a ligand as large as $\mathrm{Al}_{3} \mathrm{Cl}_{11}^{2-}$, but it is still surprising that the ligand is not $\mathrm{Al}_{2} \mathrm{Cl}_{8}^{2-}$.

In conclusion, the structures of the gaseous complexes are probably best described in terms of ionically bound $\mathrm{AlCl}_{4}^{-}$and $\mathrm{Al}_{2} \mathrm{Cl}_{7}^{-}$groups. The (assumed) presence of $\mathrm{Al}_{2} \mathrm{Cl}_{7}^{-}$ groups is particularly interesting since they may provide an extra degree of stability due to favorable charge distribution in the metal-ligand bonds. 


\section{Stability of the Condensed Phase in Equilibrium with a Highly} Volatile Chloride

If one considers the system $M X_{y}-A_{2} X_{6}$ (or $A X_{3}$ ) where $A_{2} X_{6}$ or $A X_{3}$ is a highly volatile halide which forms a gaseous complex with $M X_{y}$, the condensed phase may be $M X_{y}(s)$, $M A_{2 n} X_{6 n+y}(s)$ or $M X_{y}-A_{2} X_{6}(1)$. Excluding the presence of a solid complex compound and considering the reaction

$$
\left(M X_{y}-A_{2} X_{6}\right)(1)=M X_{y}(s)+A_{2} X_{6}(g)
$$

leads to the enthalpy of equilibrium (25) given by

$$
\Delta H^{\circ}(25)=-\Delta H_{\text {mix }}^{\circ}(1)-\Delta H_{\text {fus }}^{\circ}\left(M X_{y}\right)+\Delta H_{\text {vap }}^{\circ}\left(A_{2} X_{6}\right)
$$

where:

$$
\Delta H_{\text {mix }}^{\circ}(1)=\text { Enthalpy of mixing of liquid } M X_{y} \text { and liquid } A_{2} X_{6} \text {. }
$$

Due to the strong interaction between $\mathrm{A}_{2} \mathrm{X}_{6}$ and $M X_{y}$ which manifests itself through the formation of gaseous complexes, $\Delta H_{\operatorname{mix}}^{\circ}(1)$ is expected to be negative and because $\Delta H_{\text {vap }}^{\circ}\left(A_{2} X_{6}\right)>$ $\Delta H_{\text {fus }}^{\circ}\left(M X_{y}\right)$, the reaction enthalpy $\Delta H^{\circ}(25)>0$. Equations $(25,26)$ show that a solid phase is stabilized with decreasing pressure of $\mathrm{A}_{2} \mathrm{X}_{6}$ and increasing temperature provided the temperature is below $T_{\text {fus }}$ for $M X_{y}$. Examples of this phenomenon are found in the transformation from a liquid mixture to a pure solid for $\mathrm{NdCl}_{3}$ at around $600 \mathrm{~K}$ and 6 atm total pressure [6], and for $\mathrm{EuCl}_{2}$ [32] at around $740 \mathrm{~K}$ and $0.05 \mathrm{~atm}$ pressure. The presence of a liquid phase rather than the pure solid has also been observed for $\mathrm{CdCl}_{2}(673 \mathrm{~K})[4], \mathrm{CaCl}_{2}$ (873$773 \mathrm{~K}), \mathrm{BaCl}_{2}(873-773 \mathrm{~K})$ and $\mathrm{PbCl}_{2}(773-673 \mathrm{~K})$ [54].

The mixing process or the formation of a solid complex is an acceptor-donor reaction where $A_{2} X_{6}$ acts as a $X^{-}$acceptor while $M X_{y}$ acts as a $X^{-}$donor. Hence $\Delta H_{\text {mix }}(1)$ will be more negative the lower the charge and the greater the size of $\mathrm{M}^{\mathrm{y}^{+}}$. Low charge and large size will then make $\Delta H^{\circ}(25)$ more positive and result in a stabilization of the liquid phase with respect to the solid. This has the consequence that for salts such as the alkali halides, the gas complexes will, at moderate or high pressures, always be in equilibrium with a $1 i^{-}$ quid phase (see for instance [16]).

Melt mixtures of the donor-acceptor system $M X_{y}-A_{2} X_{6}$ may be classified as acidic or basic depending on whether the melt mixture contains more or less $A_{2} X_{6}$ than would correspond to the composition $M\left(A_{4}\right)_{y}$. As previously noted, very large activity changes, often with consequent drastic changes in the melting temperatures, take place around this composition. The basic melts containing excess $x^{-}$are ionic and have high melting points, particularly if the melting point of $\mathrm{MX}_{\mathrm{y}}$ is high. Acidic melts are partly covalent and are frequently liquid at temperatures of $100-200{ }^{\circ} \mathrm{C}$. 
Acidic melts, are generally stable under their own vapour pressures. In the low temperature range $\left(100-300^{\circ} \mathrm{C}\right.$ ) some systems do, however, show a liquid immiscibility gap in the range $0.80<\mathrm{X} \mathrm{AlCl}_{3}<1.00$ (see for instance phase diagrams by Kendall, et al. [55]).

For mixtures with alkali halides and other monovalent halides, a liquid phase can usually also be established in basic melts at high temperatures without generating excessive pressures of $A_{2} X_{6}$ or $A X_{3}$, and pure solid $M X_{y}$ is usually not stable in equilibrium with $A_{2} X_{6}$ gas. The vapour pressure along the liquidus 1 ine, however, has a maximum when changing the composition from $\mathrm{AlkAlCl}_{4}$ to more basic melts. This is due to the increase in the liquidus temperature which is not fully compensated by the lowering of the $\mathrm{AlCl}_{3}$ activity. At about $\mathrm{X}_{\mathrm{AlCl}_{3}}=0.15$, the total vapour pressure along the $\mathrm{LiCl}-\mathrm{AlCl}_{3}$ liquidus has a maximum of $0.0076 \mathrm{~atm}$, while for the $\mathrm{KCl}-\mathrm{AlCl}_{3}$ liquidus the maximum is at 0.00085 atm [16]. This effect is still more pronounced for some mixtures of divalent chlorides with $\mathrm{AlCl}_{3}$. Attempts to make basic mixtures of $\mathrm{MnCl}_{2}$ and $\mathrm{MgCl}_{2}$ with $\mathrm{AlCl}_{3}$ result in bursting of the quartz ampoules in which they were sealed due to excessive pressure [56]. Moderate pressures $(0.5-5 \mathrm{~atm})$ and temperature $\left(\simeq 300^{\circ} \mathrm{C}\right)$ result in the presence of a liquid with composition close to $M\left(A_{4}\right)_{y}$ or solid $M X_{y}$.

Many of the complex solid compounds have a low stability and only complex compounds with large monovalent cations are stable above $250{ }^{\circ} \mathrm{C}$. The melting points are: $\mathrm{CsAlCl}_{4}$ : $378{ }^{\circ} \mathrm{C}, \mathrm{RbAlCl}_{4}: 336{ }^{\circ} \mathrm{C}, \mathrm{TlAlCl}_{4}: 308{ }^{\circ} \mathrm{C}, \mathrm{NH}_{4} \mathrm{AlCl}_{4}: 303{ }^{\circ} \mathrm{C}, \mathrm{InAlCl}_{4}: 274{ }^{\circ} \mathrm{C}, \mathrm{KAlCl}_{4}:$ $250{ }^{\circ} \mathrm{C}$ (Levin, et al. [57]). Chloride complexes of the form $\mathrm{M}\left(\mathrm{Al}_{2} \mathrm{Cl}_{7}\right)$ y have only been found to be stable for large cations such as $\mathrm{Te}_{4}\left(\mathrm{Al}_{2} \mathrm{Cl}_{7}\right)_{2}$ (Couch, et al. [58]). Some complexes between $\mathrm{AlCl}_{3}$ and divalent chlorides have also been characterized, i.e., $\mathrm{Co}\left(\mathrm{AlCl}_{4}\right)_{2}$

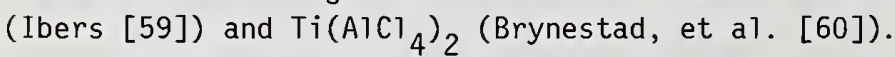

In conclusion, it should be noted that the gas phase above a mixture between $M X_{y}$ and a highly volatile $\mathrm{AX}_{3}$ compound will tend to have a gas composition with $\mathrm{AX}_{3}$ in excess relative to the composition $M\left(A X_{4}\right)_{y}$. This is so even for very basic $A l k C l-A l C l_{3}$ melts. For instance, the vapour phase above $\mathrm{NaCl}-\mathrm{AlCl}_{3}$ with 10 mol percent $\mathrm{AlCl}_{3}$ will contain $\mathrm{AlCl}_{3}$ and $\mathrm{AlKAlCl}_{4}$ in the ratio 0.28 at $1100 \mathrm{~K}$. The implication of this is that there always will be $\mathrm{AlCl}_{3}$ in the gas phase available for gas complexation with, for instance, constituents in the melt.

\section{Application and Future Research}

Hastie has discussed certain aspects of the application of gas complexation in ore transformation, extractive metallurgy, metal refining, chemical synthesis, industrial fume emission and metal halide lamps [9]. Other potentially important fields of application are in heterogeneous catalysis, nuclear fuel refining, and high power gas lasers based on rare-earth gas complexes [61].

The unfolding phenomena of gas complexation are in a sense providing "wings to metals," by enabling reactions to be carried out at much lower temperatures than otherwise possible. 
Often, the enhanced metal halide partial pressures due to gas complexation are a prerequisite for a given process. An additional advantage is the "reversibility" encountered when lowering the temperature in that the components can again be separated into their constituents. This property makes it possible, for instance, to achieve gas chromatographic separations of metal halides $[62,63]$. In other cases, gas complexation can result in industrial fumes or high-temperature corrosion and ways must be found to prevent such reactions. It is, however, important that the phenomena always be properly recognized and that gas complexation reactions are included in process equilibrium models when present.

Fundamental research has already come a long way in achieving an understanding of the basic chemistry of gas complexation. The thermodynamic results derived from absorption spectroscopic studies, are largely self consistent and agreement among different laboratories is satisfactory $[21,22,23,37]$. Notable exceptions exist as with the data for $\mathrm{CaCl}_{2}$ [4], where additional work seems warranted. Due to the high enthalpy of vaporization, one would expect the majority complex to contain at least three Al-atoms to offset the unfavorable energetics. Perhaps a liquid phase was present during the studies on $\mathrm{CaCl}_{2}$ so that the true stoichiometry is higher than that reported as in the case of $\mathrm{EuCl}_{2}$ [32]. Only moderate agreement between mass spectrometric and absorption spectroscopic $[18,19,20]$ studies has been achieved so far indicating that further work is required here. It is of particular interest to know the extent to which low molecular weight species such as the divalent chloride complex $\mathrm{MACl}_{5}$ are present. Such species are especially important with $\mathrm{InCl}_{3}[50]$, but have also been identified by mass spectrometry. The presence of such species may dominate the vaporization processes above $1000 \mathrm{~K}$. According to Binnewies [20] $\mathrm{P}_{\mathrm{MAICl}_{5}} \simeq \mathrm{P}_{\mathrm{MAl}_{2}{ }_{2} \mathrm{Cl}_{8}}$ at about $950 \mathrm{~K}$ for a total pressure of $1 \mathrm{~atm}$. It would be of interest to have this idea confirmed by thermodynamic studies in a higher pressure range than can be used in mass spectrometers.

In spite of information about coordination of the $\mathrm{M}^{\mathrm{y}+}$ ion from ligand field spectra and some recent Raman studies, there still is too little data about the structure and bond properties of this very interesting class of compounds. It appears to be experimentally difficult to obtain Raman vibration spectra of the gaseous complexes and the observation of resonance Raman spectra would be highly desirable [26]. Indeed, the application of a variety of spectroscopic techniques to studies of the structures of these intriguing gaseous molecules would appear to be a fertile field of research for molecular spectroscopy.

The assistance of Mr. Robert McBeth and Mr. Børge Knapstad is gratefully acknowledged. 
[1] Fischer, W., Angew. Chem. 61, 336 (1949).

[2] Grothe, H., Z. Elektrochem. Angew. Phys. Chem. 54, 216 (1950).

[3] Dewing, E. W. , Nature, 214, 483 (1967).

[4] Dewing, E. W. Met. Trans. 1, 2169 (1970).

[5] Gruen, D. M. and Øye, H. A., Inorg. Nucl. Chem. Letters, $\underline{3}, 453$ (1967).

[6] Øye, H. A. and Gruen, D. M., J. Am. Chem. Soc. 9l, 2229 (1969).

[7] Novikov, G. I. and Gavryuchenkov, F. G., Russ. Chem. Rev. 36, 156 (1967).

[8] Hastie, J. W., in Advances in Molten Salt Chemistry, J. Braunstein, G. Mamantov, and G. P. Smith, eds., Vol. 1 (Plenum Press, N.Y., 1971), p. 225.

[9] Hastie, J. W., High Temperature Vapors (Academic Press, N.Y., 1975).

[10] Emmenegger, F. P., J. Crystal Growth, 17, 31 (1972).

[11] Schäfer, H., Angew. Chem. 88, 775 (1976).

[12] Margrave, J. L., (ed.), The Characterization of High-Temperature Vapors (J. Wiley, N.Y., 1967).

[13] Merten, U. and Be11, W. E., in The Characterization of High-Temperature Vapors, J.L. Margrave, ed. (J. Wiley, N.Y., 1967), p. 91.

[14] Clopper, P. R., Altman, R. L., and Margrave, J. L., in The Characterization of High-Temperature Vapors, J.L. Margrave, ed. (J. Wiley, N.Y., 1967), p. 48.

[15] Galitskii, N. V., Russ. J. Inorg. Chem. 13, 1607 (1968).

[16] Linga, H., Motzfeldt, K., and Øye, H. A., Ber. Bunsenges. Phys. Chem. 82, 568 (1978).

[17] Sidorov, L. N. and Shol'ts, V. B., Int. J. Mass Spectrom. Ion Phys. 8 , 437 (1972).

[18] Binnewies, M., Z. Anorg. A11g. Chem. 435,156 (1977).

[19] Binnewies, M., Z. Anorg. A1lg. Chem. 437, 19 (1977).

[20] Binnewies, M. , Z. Anorg. Allg. Chem. $\underline{437}, 25$ (1977).

[21] Papatheodorou, G. N., Z. Anorg. Allg. Chem. 411, 153 (1975).

[22] Thistlethwaite, P. J. and Ciach, S., Inorg. Chem. 14, 1430 (1975).

[23] Dell'Anna, A. and Emmenegger, F. P., Helv. Chim Acta, 58, 1145 (1975).

[24] Anundskas, A., Mahgoub, A. E., and Øye, H. A., Acta Chem. Scand. A30, 193 (1976).

[25] Emmenegger, F. P., Rohrbasser, C., and Schläpfer, C. W., Inorg. Nucl. Chem. Letters, 12, 127 (1976).

[26] Papatheorodou, G. N., and Capote, M. A., in Proc. of the Symp. on High Temperature Metal Halide Chemistry, D. L. Hildebrand and D. D. Cubicciotti, eds. (The Electrochemical Soc. Proc. 78-1, Princeton, N.J., 1978), p. 334.

[27] Novikov, G. I., and Kotova, E. S., Russ. J. Phys. Chem. 47, 277 (1973).

[28] JANAF Thermochemical Tables, Nat1. Stand. Ref. Data Ser., Nat1. Bur. Stand., No. 37 (1971).

[29] Rytter, E., Øye, H. A., Cyvin, S. J., Cyvin, B. N., and Klaeboe, P., J. Inorg. Nucl. Chem. 35, 1185 (1973).

[30] Torsi, G. and Mamantov, G., Inorg. Chem. 10, 1900 (1971).

[31] Fannin, Jr., A. A., King, L. A., and Seegmiller, D. W., J. Electrochem. Soc. 119, 801 (1972). 
[32] Sørlie, M. and Øye, H. A., J. Inorg. Nucl. Chem. 40,493 (1978).

[33] Sørlie, M. and Øye, H. A., Inorg. Chem. 17, 0000 (1978).

[34] Laughlin, W. C. and Gregory, N. W., J. Phys. Chem. 80, 127 (1976).

[35] Anundskas, A. and Øye, H. A., J. Inorg. Nucl. Chem. 37, 1609 (1975).

[36] Aits, M. and Schäfer, H., Z. Anorg. A1lg. Chem. 408, 37 (1974).

[37] Emmenegger, F. P., Inorg. Chem. 16, 343 (1977).

[38] Papatheodorou, G. N., J. Phys. Chem. 77, 472 (1973).

[39] Papatheodorou, G. N., Inorg. Chem. 12, 1899 (1973).

[40] Gruen, D. M. and McBeth, R. L., Inorg. Chem. 8, 2625 (1969).

[41] JANAF Thermochemical Tables, 1975 Supp1. J. Phys. Chem. Ref. Data, 4, 1 (1975).

[42] McCarley, R. E. and Roddy, J. W., Inorg. Chem. 3, 60 (1964).

[43] Kubaschewski, 0., Evans, E. L., and Alcock, C. B., Metallurgical Thermochemistry, 4th

Ed. (Pergamon Press, 0xford, 1967).

[44] Hammer, R. R. and Gregory, N. W., J. Phys. Chem. 68, 3229 (1964).

[45] Hariharan, A. V. and Eick, H. A., High Temp. Sci. 4, 91 (1972).

[46] Shimazaki, E. and Niwa, K., Z. Anorg. Allg. Chem. 314, 21 (1962).

[47] Katz, J. J. and Rabinowitch, E., The Chemistry of Uranium, NNES, VIII 5 (McGraw-Hill

Book Co., Inc., New York, N.Y., 1951), p. 476.

[48] Linga, H., Motzfeldt, K., and Øye, H. A., to be published.

[49] Dienstbach, F. and Emmenegger, F., Helv. Chim. Acta 60, 166 (1977).

[50] Dienstbach, F. and Emmenegger, F., Inorg. Chem. 16, 2957 (1977).

[51] Capote, M. A., Kucera, G. H., and Papatheodorou, G. N., in Proc. of the Symp. on High Temperature Metal Halide Chemistry, D.L. Hildebrand and D.D. Cubicciotti, eds. (The Electrochemical Proc. 78-1, Princeton, N.J., 1978), p. 367.

[52] Gruen, D. M. and McBeth, R. L., Pure and Appl. Chem. $\underline{6}, 23$ (1963).

[53] Aits, M. and Schäfer, H., J. Less Common Metals, 57, 219 (1978).

[54] Schäfer, H., Z. Anorg. Allg. Chem. 403, 116 (1974).

[55] Kenda11, J., Crittenden, E. D., and Miller, H. K., J. Am. Chem. Soc. 45, 963 (1923).

[56] Bues, W., El-Sayed, L., and Øye, H. A., Acta Chem. Scand. A31, 461 (1977).

[57] Levin, E. M., Robbins, C. R., and McMurdie, H. F., Phase Diagrams for Ceramists

(The American Chem. Soc., Columbus, Ohio, 1964, 1969, 1975).

[58] Couch, T. W., Lokken, D. A., and Corbett, J. D., Inorg. Chem. 11, 357 (1972).

[59] Ibers, J. A., Acta Cryst. 15, 967 (1962).

[60] Brynestad, J., Winbush, S. V., Yake1, H. L., and Smith, G. P., Inorg. Nucl. Chem. Letters, $\underline{6}, 889$ (1970).

[61] Hessler, J. P., Wagner, Jr., F., Williams, C. W., and Carnall, W. T., J. Appl. Phys. 48, 3260 (1977).

[62] Zvarova, T. S. and Zvara, I., J. Chromatog. 44, 604 (1969).

[63] Zvarova, T. S. and Zvara, I., J. Chromatog. $\underline{49}, 290$ (1970). 
National Bureau of Standards Special Publication 561, Proceedings of the 10th Materials Research Symposium on Characterization of High Temperature Vapors and Gases held at NBS, Gaithersburg, Maryland, September 18-22, 1978. Issued October 1979.

FORMATION OF CHROMATES FROM THE REACTION OF ALKALI CHLORIDES WITH $\mathrm{Cr}_{2} \mathrm{O}_{3}$ AND OXYGEN

C. Hirayama and C. Y. Lin

Westinghouse Research \& Development Center

Pittsburgh, PA 15235

The vapor transport of chromium from $\mathrm{Cr}_{2} \mathrm{O}_{3}$ has been studied by the reaction of the oxide with $\mathrm{NaCl}(\mathrm{g})$ in dry and wet oxygen streams. Although $\mathrm{Na}_{2} \mathrm{CrO}_{4}$ condenses as the downstream product, this salt is not formed at the oxide surface. The mass spectra and vapor pressure of $\mathrm{Na}_{2} \mathrm{CrO}_{4}$ (c) have also been measured at $670^{\circ}$ to $785^{\circ} \mathrm{C}$.

\section{Introduction}

In an oxidizing atmosphere, the corrosion protection of superalloys is effected by the formation of an adherent chromium oxide $\left(\mathrm{Cr}_{2} \mathrm{O}_{3}\right)$ layer. However, this oxide disintegrates when it reacts with impurities, such as a chloride salt or vanadium oxide. The mechanisms for the removal of the protective $\mathrm{Cr}_{2} \mathrm{O}_{3}$ are generally believed to be the formation of low melting and volatile chromium compounds, which are removed in the gas stream.

In most turbine systems, especially in those fired with coal gas, small concentrations of chlorides are always present. The principal chloride salt present in the atmosphere is $\mathrm{NaCl}$. The presence of this salt is known to enhance the corrosion rate of superalloys. In spite of the extensive literature on the $\mathrm{NaCl}$ corrosion of superalloys, there is still no clear resolution for the mechanism of this process. One mechanism suggests that the $\mathrm{NaCl}$ vapor reacts with the $\mathrm{Cr}_{2} \mathrm{O}_{3}$, with the formation of volatile chromium oxychlorides and solid $\mathrm{Na}_{2} \mathrm{CrO}_{4}[1,2]^{1}$. It also has been suggested that $\mathrm{Na}_{2} \mathrm{CrO}_{4}$ (c) is formed by the reaction of $\mathrm{Cr}_{2} \mathrm{O}_{3}$ (c) with $\mathrm{NaCl}(\mathrm{g})$ and oxygen, and that the $\mathrm{Na}_{2} \mathrm{CrO}_{4}$ is subsequently vaporized from the metal surface $[2,3]$. More recently, the high pressure mass spectrometric studies of Stearns and coworkers refute the above mechanisms $[4,5]$. These investigators have observed complex vapor species of the type $(\mathrm{NaCl})_{x} \mathrm{CrO}_{3}(\mathrm{~g})$ when $\mathrm{Cr}_{2} \mathrm{O}_{3}(\mathrm{c})$ is reacted with $\mathrm{NaCl}(\mathrm{g})$ and oxygen at high temperature, according to the reaction

$$
\begin{gathered}
\mathrm{Cr}_{2} \mathrm{O}_{3}(\mathrm{c})+3 / 2 \mathrm{O}_{2}(\mathrm{~g})+\mathrm{xNaCl}(\mathrm{g}) \rightarrow(\mathrm{NaCl}) \mathrm{CrO}_{3}(\mathrm{~g})+\mathrm{CrO}_{3}(\mathrm{~g}) . \\
x=1,2,3, \ldots
\end{gathered}
$$

${ }^{1}$ Figures in brackets indicate the literature references at the end of this paper. 
The studies to date generally have been made by passing $\mathrm{NaCl}(\mathrm{g})$ in a gas stream over a superalloy surface. The exact mechanism for the vaporization of the $\mathrm{Cr}_{2} \mathrm{O}_{3}$ from the superalloy surface is no doubt a complex one, in view of the complex nature of the atmosphere in which the alloy is utilized.

Initially, we investigated the reaction of $\mathrm{NaCl}(\mathrm{g})$ with $\mathrm{Cr}_{2} \mathrm{O}_{3}(\mathrm{c})$, rather than the reaction of the $\operatorname{NaCl}(\mathrm{g})$ with the oxide scale on the alloy surface. Part of the chemistry of these initial studies was later confirmed by reactions with the oxide scale on the alloy surface. Here, we report on the reaction of $\mathrm{Cr}_{2} \mathrm{O}_{3}(\mathrm{c})$ and some superalloys with $\mathrm{NaCl}(\mathrm{g})$ entrained in both a dry and a wet $\mathrm{O}_{2}$ stream. We also report on the mass spectra and vapor pressure of $\mathrm{Na}_{2} \mathrm{CrO}_{4}$. The latter measurements support the contention of Stearns and coworkers, who conclude that the formation and vaporization of $\mathrm{Na}_{2} \mathrm{CrO}_{4}(\mathrm{c})$ at the alloy surface is not involved in the mechanism for $\mathrm{Cr}_{2} \mathrm{O}_{3}$ removal [5].

\section{Experimenta1}

\section{1 Vapor transport reactions}

The reactions involving $\mathrm{Cr}_{2} \mathrm{O}_{3}$, the chromates, or the superalloys as shown in tables 1 and 2 were studied mostly in the reactor shown in figure 1 . The apparatus consisted of a fused silica tube of $2 \mathrm{~cm}$ inside diameter, and a fused silica insert, which served as the source of $\mathrm{NaCl}$. The length of the insert was varied to regulate the $\mathrm{NaCl}$ vapor pressure. The pure oxygen, wet oxygen, or nitrogen was introduced through the sidearm. The flow velocity of the gas through the reactor was approximately $30 \mathrm{~cm} / \mathrm{min}$. The exit gas from the reactor was bubbled through oil or into a solution of starch-iodide. The sample and salt temperatures were controlled and measured with chromel-alumel thermocouples.

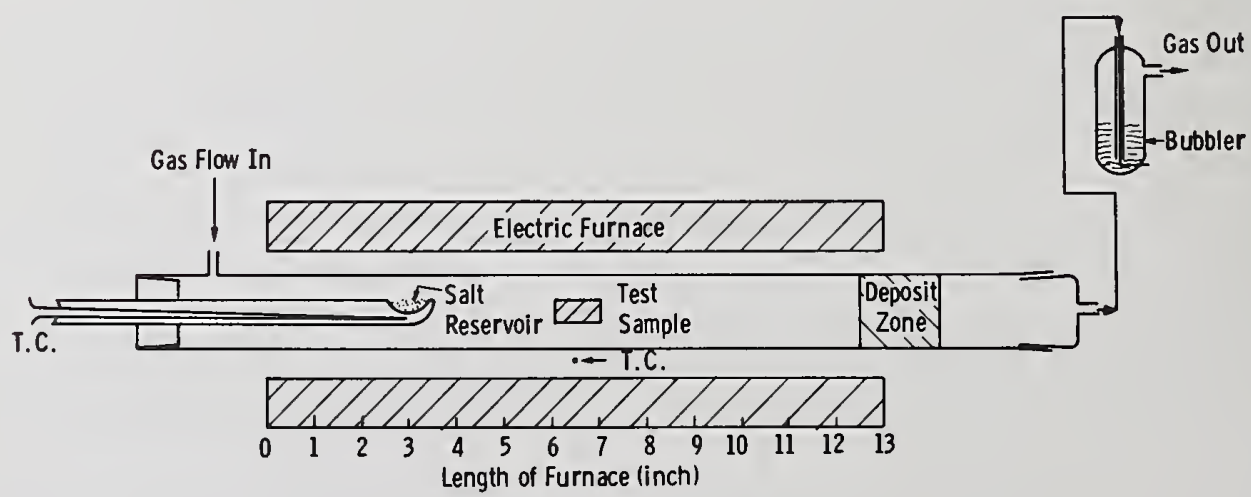

Figure 1. Fused silica vapor transport reactor. 
All of the chemicals were of reagent grade, and the superalloys were Inconel 750 (International Nickel Co.), Hastelloy C and X (Cabot Corp.), and Udimet 500 (Allegheny Lud1 um).

In one set of experiments, the $\mathrm{Cr}_{2} \mathrm{O}_{3}$ and mixtures of $\mathrm{Cr}_{2} \mathrm{O}_{3}$ with 5 percent and 10 percent of $\mathrm{NaCl}$ or $\mathrm{Na}_{2} \mathrm{O}$ were heated in a stream of nitrogen or oxygen at $800^{\circ}$ to $1000{ }^{\circ} \mathrm{C}$. The vaporization of $\mathrm{Na}_{2} \mathrm{CrO}_{4}$ and $\mathrm{K}_{2} \mathrm{CrO}_{4}$ was also determined in a stream of $\mathrm{O}_{2}$ at $800^{\circ}$ to $950{ }^{\circ} \mathrm{C}$. In another set of experiments, the $\mathrm{Cr}_{2} \mathrm{O}_{3}$ and the superalloys were heated at $800^{\circ}$ to $950^{\circ} \mathrm{C}$ in streams of oxygen, wet oxygen, and nitrogen, with and without $\mathrm{NaCl}(\mathrm{g})$ in the gas stream. In those runs where $\mathrm{NaCl}(\mathrm{g})$ was entrained in the gas stream, the salt reservoir temperature was generally maintained at $750^{\circ} \mathrm{C}$. Extrapolation of the vapor pressure data of Topor [6] for $\mathrm{NaCl}$ yields a $\mathrm{NaCl}(\mathrm{g})$ concentration of approximately 130 $\mathrm{ppm}$ by volume in the gas stream. In some runs, the concentration of $\mathrm{NaCl}(\mathrm{g})$ was $<50 \mathrm{ppm}$. In those runs where wet oxygen was used as the gas stream, the oxygen was bubbled through water to yield 12 volume percent $\mathrm{H}_{2} \mathrm{O}(\mathrm{g})$ in the stream.

The superalloys were heated at $800^{\circ}$ and at $950{ }^{\circ} \mathrm{C}$ in the oxygen stream with and without $\mathrm{NaCl}$ (g) entrainment. The $\mathrm{NaCl}$ concentration in these runs was $130 \mathrm{ppm}$.

The compositions of the condensates which resulted from the vapor transport reactions were ascertained primarily by $x$-ray diffraction analysis. The compositions of the sample residues also were determined by $x$-ray diffraction analysis.

\subsection{Mass spectrometry of $\mathrm{Na}_{2} \mathrm{CrO}_{4}$}

The mass spectra were measured on a Bendix time-of-flight spectrometer, Model 12-101, with a Knudsen cell attachment supplied by Bendix. The tungsten Knudsen cell had internal, sample chamber dimensions of 0.5 in. i.d. $\times 0.5$ in. depth. A tungsten foil disk of 0.002 in. thickness, with a $0.030 \mathrm{in.}$ diameter hole in the center, was firmly placed over the sample chamber of the cell. A Pt-Pt, 10 percent Rh thermocouple was placed in contact with the bottom of the crucible for temperature measurements. A run was also made by lining the tungsten cell with sapphire. For this purpose, a sapphire cylinder of 0.5 in. diameter $x$ $0.5 \mathrm{in}$. length was sandwiched between two sapphire disks of slightly less than $0.5 \mathrm{in}$. diameter $\times 0.05 \mathrm{in}$. thick; the disk on the top had a $0.05 \mathrm{in}$. diameter hole in the center which acted as the effusion orifice.

For each sample, the anhydrous $\mathrm{Na}_{2} \mathrm{CrO}_{4}$ was loaded into the Knudsen cel1, then heated for several hours at approximately $500{ }^{\circ} \mathrm{C}$ under the cell chamber vacuum of $10^{-5}-10^{-6}$ Torr. The sample was then heated while the mass spectrum was continuously scanned at electron accelerating voltages of 20 to $36 \mathrm{eV}$. The spectra were measured at $20 \mathrm{eV}$ to record the volatile species emanating from the Knudsen cell. The appearance potentials were determined by the method of linear extrapolation. 


\subsection{Vapor pressure measurement}

The vapor pressure measurements were made on samples of $\mathrm{Na}_{2} \mathrm{CrO}_{4}$ which were first fused in an alumina crucible in air to remove any trace of adsorbed water. Initial measurements in a tantalum Knudsen cell gave irreproducible vapor pressures, probably because of the reaction of the tantalum with the oxygen which evolved as a dissociative product from the $\mathrm{Na}_{2} \mathrm{CrO}_{4}$. The vapor pressure was subsequently measured by effusing the sample from a cylindrical Knudsen cell which was constructed completely of fused silica. The dimensions of the cell were $12 \mathrm{~mm}$ i.d. $\times 20 \mathrm{~mm}$ long. The effusion orifice on the flat surface on the top of the cell had dimensions of $1.25 \mathrm{~mm}$ diameter $\times 1 \mathrm{~mm}$ long. A Clausing factor of 0.625 was calculated for these dimensions. For the vapor pressure measurements, the loaded silica cell was placed within a graphite basket which was suspended by a fine tantalum wire from the Cahn microbalance (model RH). Details of the apparatus for the effusion rate measurements have been described previously [7]. The temperature of the sample during the measurements was controlled to within $\pm 3{ }^{\circ} \mathrm{C}$.

\section{Results}

\section{1 Vapor transport reactions}

Table 1 summarizes the observations when $\mathrm{Na}_{2} \mathrm{CrO}_{4}, \mathrm{~K}_{2} \mathrm{CrO}_{4}, \mathrm{Cr}_{2} \mathrm{O}_{3}$, and mixtures of $\mathrm{Cr}_{2} \mathrm{O}_{3}$ with $\mathrm{NaCl}$ were heated in a stream of nitrogen or oxygen. When the $\mathrm{Cr}_{2} \mathrm{O}_{3}-\mathrm{NaCl}$ mixtures were heated in nitrogen, there was no reaction except for the vaporization of the $\mathrm{NaCl}$ out of the mixture. In an oxygen atmosphere, on the other hand, there was always a reaction which produced $\mathrm{Na}_{2} \mathrm{CrO}_{4}$. Most of the chromate was deposited in the downstream section of the reaction tube, but there was also a small quantity of the chromate detected in the $\mathrm{Cr}_{2} \mathrm{O}_{3}$ residue. When the $\mathrm{Cr}_{2} \mathrm{O}_{3}-\mathrm{KCl}$ mixtures were heated in oxygen and in nitrogen, they showed similar reactions to the $\mathrm{Cr}_{2} \mathrm{O}_{3}-\mathrm{NaCl}$ mixtures.

Table 1. Test for vapor transport of chromium in various reactions.

\begin{tabular}{|c|c|c|c|c|}
\hline $\begin{array}{l}\text { Test } \\
\text { sample } \\
\end{array}$ & $\begin{array}{l}\text { Reactant } \\
\text { gas }\end{array}$ & $\begin{array}{l}\text { Temp. } \\
{ }^{\circ} \mathrm{C}\end{array}$ & $\begin{array}{l}\text { Reaction } \\
\text { time, hr } \\
\end{array}$ & $\begin{array}{l}\text { Downstream } \\
\text { deposit }\end{array}$ \\
\hline $\mathrm{Cr}_{2} \mathrm{O}_{3}$ & $\mathrm{O}_{2}$ & 1000 & 72 & None \\
\hline $\mathrm{Cr}_{2} \mathrm{O}_{3}+10 \% \mathrm{NaCl}$ & $\mathrm{N}_{2}$ & 950 & 20 & $\mathrm{NaCl}$ \\
\hline $\mathrm{Cr}_{2} \mathrm{O}_{3}+10 \% \mathrm{NaCl}$ & $\mathrm{O}_{2}$ & 800 & 20 & $\mathrm{Na}_{2} \mathrm{CrO}_{4}+\mathrm{NaCl}$ \\
\hline $\mathrm{Cr}_{2} \mathrm{O}_{3}+10 \% \mathrm{NaCl}$ & $\mathrm{O}_{2}$ & 950 & 20 & $\mathrm{Na}_{2} \mathrm{CrO}_{4}+\mathrm{NaCl}$ \\
\hline $\mathrm{Na}_{2} \mathrm{CrO}_{4}$ & $\mathrm{O}_{2}$ & 900 & 2 & None \\
\hline $\mathrm{K}_{2} \mathrm{CrO}_{4}$ & $\mathrm{O}_{2}$ & 900 & 2 & $\mathrm{~K}_{2} \mathrm{CrO}_{4}$ \\
\hline
\end{tabular}


When $\mathrm{Na}_{2} \mathrm{CrO}_{4}, \mathrm{~K}_{2} \mathrm{CrO}_{4}$, and $\mathrm{Cr}_{2} \mathrm{O}_{3}$ were heated in oxygen at the temperatures shown in table 1 , only the $\mathrm{K}_{2} \mathrm{CrO}_{4}$ was observed to vaporize and condense in the downstream section of the tube. These observations show that the volatility of $\mathrm{Cr}_{2} \mathrm{O}_{3}$ and $\mathrm{Na}_{2} \mathrm{CrO}_{4}$ in one atmosphere of oxygen is extremely low at the experimental temperatures.

Table 2 summarizes the observations when $\mathrm{Cr}_{2} \mathrm{O}_{3}$ and the superalloys were heated in oxygen or nitrogen which was doped with approximately $130 \mathrm{ppm}$ of the alkali chloride vapor, or with approximately $130 \mathrm{ppm}$ of $\mathrm{NaCl}(\mathrm{g})$ plus approximately 12 percent- $\mathrm{H}_{2} \mathrm{O}(\mathrm{g})$ in the oxygen stream. The chromate was observed only in the condensate in the downstream section; no chromate was detected in the sample residue in the hot zone. In the runs where the $\mathrm{Cr}_{2} \mathrm{O}_{3}$ was heated in the $\mathrm{NaCl}(\mathrm{g})$-entrained stream, chlorine gas was also evolved, as ascertained by the starch-iodide test of the effluent gas. The overall reaction in these runs may be represented by

$$
4 \mathrm{NaCl}(\mathrm{g})+\mathrm{Cr}_{2} \mathrm{O}_{3}(\mathrm{c})+5 / 2 \mathrm{O}_{2}(\mathrm{~g}) \rightarrow 2 \mathrm{Na}_{2} \mathrm{CrO}_{4}(\mathrm{c})+2 \mathrm{Cl}_{2}(\mathrm{~g})
$$

where the species on the right are the final products in the cold zone. When $\mathrm{H}_{2} \mathrm{O}(\mathrm{g})$ was also entrained with the $\mathrm{NaCl}(\mathrm{g})$ in the oxygen stream, there was no chlorine in the product. In the latter atmosphere, $\mathrm{HCl}$ was the product in the effluent gas. The $\mathrm{HCl}$ condensed out as hydrochloric acid at the cold end of the reaction tube. The overall reaction for these runs may be represented by

$$
4 \mathrm{NaCl}(\mathrm{g})+\mathrm{Cr}_{2} \mathrm{O}_{3}(\mathrm{c})+3 / 2 \mathrm{O}_{2}+2 \mathrm{H}_{2} \mathrm{O}(\mathrm{g}) \rightarrow 2 \mathrm{Na}_{2} \mathrm{CrO}_{4}(\mathrm{c})+4 \mathrm{HCl}(\mathrm{g}) .
$$

\begin{tabular}{|c|c|c|c|}
\hline $\begin{array}{c}\text { Test } \\
\text { sample } \\
\end{array}$ & $\begin{array}{c}\text { Reactant } \\
\text { gas }\end{array}$ & Temp. , ${ }^{\circ} \mathrm{C}$ & $\begin{array}{c}\text { Downstream } \\
\text { deposit }\end{array}$ \\
\hline $\mathrm{Cr}_{2} \mathrm{O}_{3}$ & $0_{2}$ & 1000 & None \\
\hline $\mathrm{Cr}_{2} \mathrm{O}_{3}$ & $\mathrm{O}_{2}+\operatorname{NaCl}(\mathrm{g})$ & 950 & $\mathrm{Na}_{2} \mathrm{CrO}_{4}+\mathrm{NaCl}$ \\
\hline $\mathrm{Cr}_{2} \mathrm{O}_{3}$ & $0_{2}+\mathrm{KCl}(\mathrm{g})$ & 950 & $\mathrm{~K}_{2} \mathrm{CrO}_{4}+\mathrm{KCl}$ \\
\hline $\mathrm{Cr}_{2} \mathrm{O}_{3}$ & $\mathrm{O}_{2}+\mathrm{NaCl}(\mathrm{g})+\mathrm{H}_{2} \mathrm{O}(\mathrm{g})$ & 910 & $\mathrm{Na}_{2} \mathrm{CrO}_{4}+\mathrm{NaCl}$ \\
\hline $\mathrm{Cr}_{2} \mathrm{O}_{3}$ & $\mathrm{~N}_{2}+\mathrm{NaCl}(\mathrm{g})$ & 950 & $\mathrm{NaCl}$ \\
\hline Inconel 750 & $\mathrm{O}_{2}$ & 950 & None \\
\hline Incone 1750 & $\mathrm{O}_{2}+\mathrm{NaCl}(\mathrm{g})$ & 950 & $\mathrm{Na}_{2} \mathrm{CrO}_{4}+\mathrm{NaCl}$ \\
\hline Hastelloy C & $0_{2}$ & 950 & None \\
\hline Hastelloy C & $\mathrm{O}_{2}+\mathrm{NaCl}(\mathrm{g})$ & 950 & $\mathrm{Na}_{2} \mathrm{CrO}_{4}+\mathrm{NaCl}$ \\
\hline Udimet 500 & $0_{2}+\operatorname{NaCl}(g)$ & 950 & $\mathrm{Na}_{2} \mathrm{CrO}_{4}+\mathrm{NaCl}$ \\
\hline
\end{tabular}

Table 2. Test for vapor transport of chromium in various reactions.

In comparing the reactions where the $\mathrm{Cr}_{2} \mathrm{O}_{3}$ was reacted with oxygen and the alkali chlorides, we note that there was no alkali chromate in the $\mathrm{Cr}_{2} \mathrm{O}_{3}$ residue when the reaction 
was effected with the alkali chloride vapor at low concentrations in the gas stream. In contrast, some alkali chromate was formed in the $\mathrm{Cr}_{2} \mathrm{O}_{3}$ residue when the molten chloride was in contact with $\mathrm{Cr}_{2} \mathrm{O}_{3}$ in the mixtures of table 1 .

The reaction of oxygen and of $\mathrm{NaCl}(\mathrm{g})$-entrained oxygen with the superalloys showed results similar to those observed by other workers [8]. In pure oxygen, the stable oxide film was adherent; whereas the oxide film spalled and tended to flake off when the superalloy was heated in a stream of $\mathrm{NaCl}(\mathrm{g})$-entrained oxygen.

\subsection{Mass spectra of $\mathrm{Na}_{2} \mathrm{CrO}_{4}$}

The mass spectra of the vapors over $\mathrm{Na}_{2} \mathrm{CrO}_{4}$ were determined up to a temperature of $1000{ }^{\circ} \mathrm{C}$. The only mass spectral ions observed were $\mathrm{Na}^{+}$and $\mathrm{O}_{2}^{+}$. Similar results were obtained from the tungsten and the sapphire lined crucibles. The first appearance of the $\mathrm{Na}^{+}$was at $670{ }^{\circ} \mathrm{C}$. The temperature dependence of the ion intensity was recorded only for $\mathrm{Na}^{+}$because of the high $\mathrm{O}_{2}^{+}$background in our instrument. The enthalpy of vaporization for the sodium, estimated from the mass spectrometric data, was $108 \pm 10 \mathrm{kcal} / \mathrm{mol}$ at $998 \mathrm{~K}$. The appearance potential determined for the $\mathrm{Na}^{+}$ion was $5.0 \pm 0.5 \mathrm{eV}$; this value compares favorably with the ionization potential of $5.14 \mathrm{eV}$ for $\mathrm{Na}$ [9].

Stearns and coworkers have reported that their Knudsen cell studies show at least 50 percent $\mathrm{Na}_{2} \mathrm{CrO}_{4}$ in the vapor phase [5]. Our mass spectrometric measurements show that $\mathrm{Na}_{2} \mathrm{CrO}_{4}$ vaporizes by a dissociative process as follows:

$$
\mathrm{Na}_{2} \mathrm{CrO}_{4}(\mathrm{c}) \rightarrow 1 / 2 \mathrm{Cr}_{2} \mathrm{O}_{3}(\mathrm{c})+2 \mathrm{Na}(\mathrm{g})+5 / 4 \mathrm{O}_{2}(\mathrm{~g})
$$

The dissociation does not yield $\mathrm{Na}_{2} \mathrm{O}(\mathrm{g})$ because $\mathrm{Na}_{2} \mathrm{O}(\mathrm{c})$ vaporizes primarily according to $\mathrm{Na}_{2} \mathrm{O}(\mathrm{c}) \rightarrow 2 \mathrm{Na}(\mathrm{g})+1 / 2 \mathrm{O}_{2}(\mathrm{~g})[10]$.

We note that the dissociation of $\mathrm{Na}_{2} \mathrm{CrO}_{4}$ is strongly suppressed when the salt is heated up to $900{ }^{\circ} \mathrm{C}$ in one atmosphere of oxygen. However, we have observed that reaction (4) takes place readily when the $\mathrm{Na}_{2} \mathrm{CrO}_{4}$ is heated above $800{ }^{\circ} \mathrm{C}$ in a stream of inert gas, such as argon.

\subsection{Vapor pressure of $\mathrm{Na}_{2} \mathrm{CrO}_{4}$}

Figure 2 shows the total vapor pressure data based on the rate of weight loss for $\mathrm{Na}_{2} \mathrm{CrO}_{4}(\mathrm{c})$, according to reaction (4). The least squares treatment of the data gave the equation,

$$
\log \mathrm{p}=21.03-25384 / \mathrm{T}
$$

where $\mathrm{p}$ is in Torr for temperatures of 1016 to $1057 \mathrm{~K}$. The vapor pressure of the liquid phase could not be determined reproducibly because of the excessive tendency for the 
molten salt to creep onto the wall and into the orifice. The enthalpy for the vaporization . of sodium from $\mathrm{Na}_{2} \mathrm{CrO}_{4}(\mathrm{c})$, calculated from eq. (5), is $116 \pm 5 \mathrm{kcal} / \mathrm{mol}$ at $1036 \mathrm{~K}$. This value compares satisfactorily with the mass spectrometric value of $108 \pm 10 \mathrm{kcal} / \mathrm{mol}$.

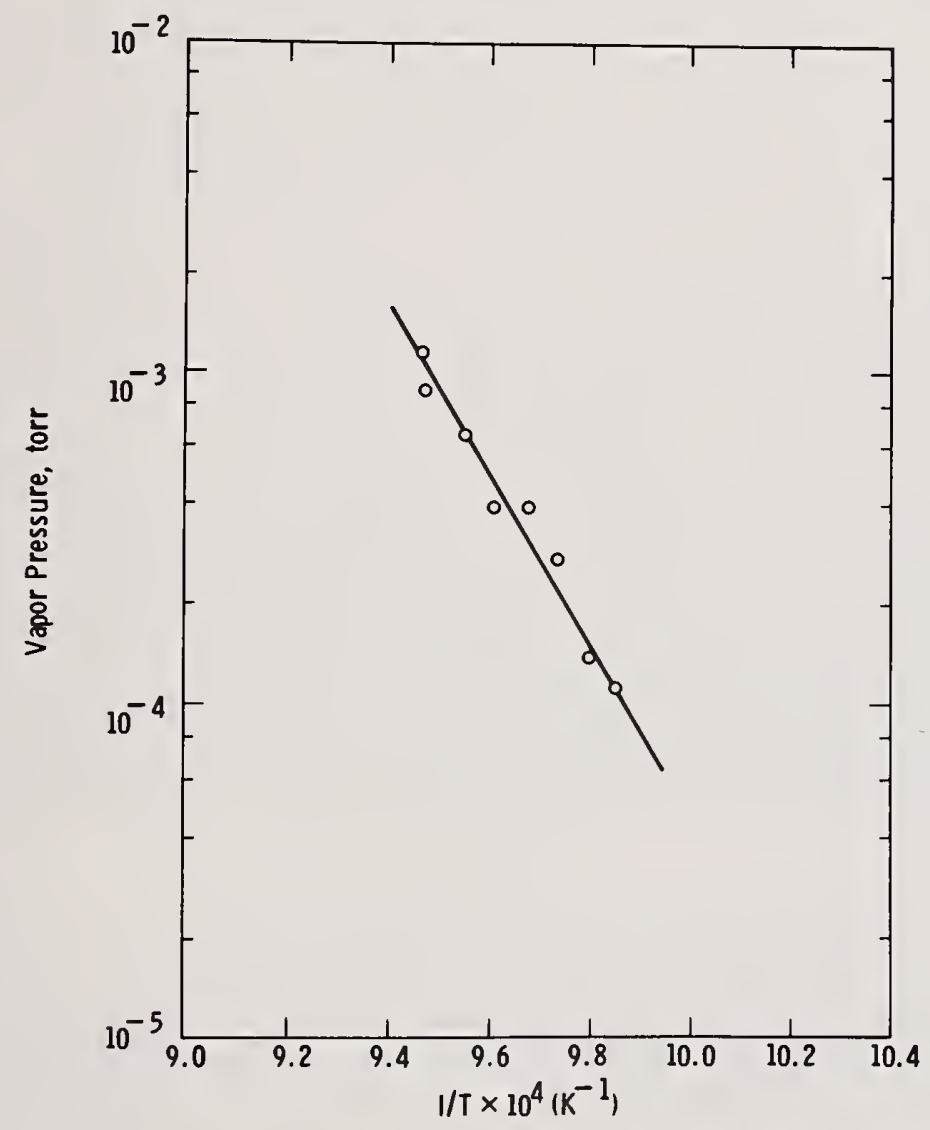

Figure 2. Vapor pressure of $\mathrm{Na}_{2} \mathrm{CrO}_{4}(\mathrm{c})$.

Afonskii earlier reported on the Knudsen cell vapor pressure of $\mathrm{Na}_{2} \mathrm{CrO}_{4}$ (c) [11]. He assumed congruent vaporization of the chromate and obtained an enthalpy of sublimation of $23.2 \mathrm{kcal} / \mathrm{mol}$. Our present measurements supersede Afonskij's data.

\section{Discussion}

The observations reported herein show that the formation of $\mathrm{Na}_{2} \mathrm{CrO}_{4}$ on the $\mathrm{Cr}_{2} \mathrm{O}_{3}$ surface is not involved as an important reaction for the removal of chromium from a superalloy surface. In a turbine atmosphere, the concentration of $\mathrm{NaCl}(\mathrm{g})$ is generally only a few parts per million. At these concentrations, there is no condensation of this salt on the hot alloy surface for low $\mathrm{NaCl}(\mathrm{g})$ concentrations. Previous studies which invoked the formation of $\mathrm{Na}_{2} \mathrm{CrO}_{4}$ on the oxidized surface were based on the formation of this salt when a macroscopic quantity of molten $\mathrm{NaCl}$ was in contact with the hot, oxidized 
metal surface [2]. These conditions are unrealistic to that of an actual gas turbine system. As we have shown, $\mathrm{Na}_{2} \mathrm{CrO}_{4}$ does not vaporize congruently, but rather it dissociates according to reaction (4). This reaction shows that the formation of the $\mathrm{Na}_{2} \mathrm{CrO}_{4}$ does not result in the vapor transport of chromium. Therefore, the vapor transport of chromium in a $\mathrm{NaCl}(\mathrm{g})$-entrained oxygen stream, with downstream deposition of $\mathrm{Na}_{2} \mathrm{CrO}_{4}$, must involve another mechanism. It has been reported that the presence of both $\mathrm{NaCl}(\mathrm{g})$ and $\mathrm{H}_{2} \mathrm{O}(\mathrm{g})$ in the oxygen stream enhances the chromium removal from the superalloy surface [4]. In this atmosphere, there is also the formation of $\mathrm{Na}_{2} \mathrm{CrO}_{4}$ as a downstream product.

Stearns and coworkers, based on their high-pressure mass spectroscopic studies, conclude that $\mathrm{Na}_{2} \mathrm{CrO}_{4}$ is not formed in the reaction between $\mathrm{Cr}_{2} \mathrm{O}_{3}(\mathrm{c}), \mathrm{O}_{2}(\mathrm{~g})$, and $\mathrm{NaCl}(\mathrm{g})$ [5]. They contend that the main reaction for chromium removal is reaction (1). Our studies confirm the conclusion of Stearns and coworkers with regard to the absence of $\mathrm{Na}_{2} \mathrm{CrO}_{4}$ on the superalloy surface. As shown by these workers, the initial formation of the complex vapor species, $(\mathrm{NaCl})_{x} \mathrm{CrO}_{3}(\mathrm{~g})$ and $(\mathrm{NaOH}){ }_{x} \mathrm{CrO}_{3}(\mathrm{~g})$, may well be the mechanism for the removal of chromium from the superalloy surface. However, our studies herein show conclusively that $\mathrm{Na}_{2} \mathrm{CrO}_{4}$ is the product which condenses downstream when $\mathrm{Cr}_{2} \mathrm{O}_{3}$ (and superalloys) reacts with an oxygen stream which is entrained with $\mathrm{NaCl}(\mathrm{g})$ and $\mathrm{H}_{2} \mathrm{O}(\mathrm{g})$. $\mathrm{Re}^{-}$ actions (2) and (3) are overall results. Stearns and coworkers state that the $(\mathrm{NaCl})_{x} \mathrm{CrO}_{3}(\mathrm{~g})$ is not formed in the vapor phase, but rather it is formed at the $\mathrm{Cr}_{2} \mathrm{O}_{3}$ surface. The downstream formation of $\mathrm{Na}_{2} \mathrm{CrO}_{4}(\mathrm{c})$ and $\mathrm{Cl}_{2}(\mathrm{~g})$ must still be accounted for. If the reaction, such as

$$
2(\mathrm{NaCl})_{2} \cdot \mathrm{CrO}_{3}(\mathrm{~g})+\mathrm{O}_{2} \rightarrow 2 \mathrm{Na}_{2} \mathrm{CrO}_{4}(\mathrm{c})+2 \mathrm{Cl}_{2}
$$

occurs at the downstream reactor surface, we can account for the condensation of the $\mathrm{Na}_{2} \mathrm{CrO}_{4}$ and the effluent $\mathrm{Cl}_{2}(\mathrm{~g})$. However, this reaction is only a conjecture at present. In the case where both $\mathrm{NaCl}(\mathrm{g})$ and $\mathrm{H}_{2} \mathrm{O}(\mathrm{g})$ are present in the oxygen stream, the first vapor phase reaction will be $\mathrm{NaCl}_{(\mathrm{g})}+\mathrm{H}_{2} \mathrm{O}(\mathrm{g}) \rightarrow \mathrm{Na}(\mathrm{OH})(\mathrm{g})+\mathrm{HCl}(\mathrm{g})$. The subsequent formation of $(\mathrm{NaOH}) \times{ } \cdot \mathrm{CrO}_{3}(\mathrm{~g})$, as suggested by Stearns et al. [4], could result in the condensation of $\mathrm{Na}_{2} \mathrm{CrO}_{4}$; for example, by the reaction

$$
2(\mathrm{NaOH})_{2} \mathrm{CrO}_{3}(\mathrm{~g}) \rightarrow 2 \mathrm{Na}_{2} \mathrm{CrO}_{4}+2 \mathrm{H}_{2} \mathrm{O}
$$

Further studies are needed to resolve the formation of $\mathrm{Na}_{2} \mathrm{CrO}_{4}$ as a product of chromium removal from $\mathrm{Cr}_{2} \mathrm{O}_{3}$ from the relatively simple systems reported herein.

\section{Conclusions}

Sodium chromate is not formed at a superalloy surface when the metal is exposed to an oxygen atmosphere entrained with $\mathrm{H}_{2} \mathrm{O}(\mathrm{g})$ and a low concentration of $\mathrm{NaCl}$. It has been shown that $\mathrm{Na}_{2} \mathrm{CrO}_{4}$ does not evaporate congruently, and the vaporization of this salt cannot be involved in the vapor transport of chromium. 
[1] Alexander, P. A., in The Mechanism of Corrosion by Fuel Impurities, H. R. Johnson and D. J. Littler, eds. (Butterworths, 1963), p. 571.

[2] Johnson, D. M., Whittle, D. P., and Stringer, J., Corrosion Sci. 15, 721 (1975).

[3] Hossain, M. K. and Saunders, S. R. J., 0xid. of Metals, 12, 1 (1978).

[4] Fryburg, G. C., Miller, R. A., Kohl, F. J., and Stearns, C. A., J. Electrochem. Soc. $\underline{124}, 1738$ (1977).

[5] Stearns, C. A., Koh1, F. J., and Fryburg, G. C., NASA-TM-X-73476 (1976); also presented at Symposium on Properties of High Temperature Alloys, Las Vegas, Nevada, 0ct. 17-22, 1976.

[6] Topor, L., J. Chem. Thermodynamics, 4, 739 (1972).

[7] Hirayama, C., Rome, J. F., and Camp. F. E., J. Chem. Eng. Data, 20, 1 (1975).

[8] For example, see References 2 and 3.

[9] Franklin, J. L., Dillard, J. G., Rosenstock, H. M., Herron, J. X., Draxl, K., and Field, F. H., Nat. Stand. Ref. Data Ser., Nat. Bur. Stand., No. 26 (1969).

[10] Hildenbrand, D. L. and Murad, E., J. Chem. Phys. 53, 3403 (1970).

[11] Afonskii, N. S., Russ. J. Inorg. Chem. , 1373 (1962) (English translation).

\section{Discussion}

Question (Behrens): In your experiments with sodium chromate, you said it vaporizes incongruently. Have you found any problems in reproducing your vapor pressure measurement? That is, after a long time do you find that your vapor pressures tend to fall off?

Response (Hirayama): We did in the liquid range, yes. With the solid chromate, we can reproduce our results. When we were using a tantalum Knudsen cell, we had this problem. I thought that perhaps this was due to reaction of the oxygen with the tantalum. Again, that probably may not be the reason. But when we went to a fused silica Knudsen cell, we could reproduce our results.

Question (Behrens): Well, what I had in mind was that we find in some of the work that we have done on metal carbides that vaporize incongruently, you get very severe--I guess people at NBS have previously called it macrolayering. That is, you get a large layer on the top of the sample in the Knudsen cell which impedes or hinders equilibrium being established in the cell.

Now, we found this with things like aluminum carbide and aluminum silicon carbide. We could reproduce our vapor pressure initially and then after a certain amount of material is vaporized, the vapor pressures tend to fall off and become a bit lower with time. When we take the Knudsen cell out of the mass spectrometer we can see a one or two millimeter layer of residue formed on the surface.

I was wondering if you had this same type of behavior with this system? 
Response (Hirayama): We haven't observed that. Maybe it is because when we run our measurements on the micro balance we don't evaporate that much of the material.

Comment (Koh1): We see exactly what Bob Behrens describes with sodium chromate even when we take sodium chromate, put it in a platinum cell and vaporize from the solid phase below the melting point. At the beginning of the experiment we see a reasonably large signal for the sodium chromate molecule ion and the sodium ion. So we think there are two processes taking place: a reaction to the molecule and a decomposition reaction which goes to sodium gas, oxygen gas, and $\mathrm{Cr}_{2} \mathrm{O}_{3}$ condensed, which after a period of time covers the surface of whatever particles we have there so that our molecule pressures drop off--or the molecular ion intensities that we observe drop off as a function of time. After hours, at reasonably high temperatures, all we have left are sodium atoms in the vapor.

\section{Clarification (Hirayama): Have you ever observed sodium chromate in the mass spec.?}

Response (Koh1): Yes, that is certainly a point that is in direct opposition to what we see because we do observe it both in the Knudsen cell mass spectrometer from a vacuum and when we vaporize sodium chromate under one atmosphere of oxygen in the high pressure sampler. We can see, in that case, surprisingly, mainly sodium dichromate molecule and not sodium chromate

Comment (written) (Stearns and Koh1): Our Knudsen cel1 results for the vaporization of $\mathrm{Na}_{2} \mathrm{CrO}_{4}(\mathrm{c})$ differ from the findings reported here by $\mathrm{Dr}$. Hirayama. We definitely see $\mathrm{Na}_{2} \mathrm{CrO}_{4}(\mathrm{~g})$ as the major species involved in the vaporization. For vaporization in a vacuum from a Pt-20 percent Rh Knudsen cell at $1224 \mathrm{~K}$, the relative intensities of the major observed ions at $30 \mathrm{eV}$ ionizing energy are $\mathrm{Na}_{2} \mathrm{CrO}_{4}{ }^{+}=100, \mathrm{Na}^{+}=9.5, \mathrm{O}_{2}{ }^{+}=1.5, \mathrm{Na}_{2} \mathrm{CrO}_{3}{ }^{+}=0.5$, $\mathrm{Na}_{2} \mathrm{O}^{+}=0.6$, and $\mathrm{NaCrO}_{3}{ }^{+}=0.5$. These results lead to the conclusion that the vaporization reaction:

$$
\mathrm{Na}_{2} \mathrm{CrO}_{4}(\mathrm{c})=\mathrm{Na}_{2} \mathrm{CrO}_{4}(\mathrm{~g})
$$

is a more important reaction in vacuum than the decompsition reaction:

$$
\mathrm{Na}_{2} \mathrm{CrO}_{4}(\mathrm{c})=2 \mathrm{Na}(\mathrm{g})+\frac{5}{4} \mathrm{O}_{2}(\mathrm{~g})+\frac{1}{2} \mathrm{Cr}_{2} \mathrm{O}_{3}(\mathrm{c}) \text {. }
$$

It must be noted, however, that we observe evidence that reaction (2) takes place to some extent in vacuum. This evidence is the fact that $\mathrm{Cr}_{2} \mathrm{O}_{3}(\mathrm{c})$ covers the sample in the cell at the end of several hours of heating.

Additionally, we have examined the vaporization of $\mathrm{Na}_{2} \mathrm{CrO}_{4}(\mathrm{c})$ in one atmosphere of slowly flowing oxygen. High pressure mass spectrometry revealed that the only $\mathrm{Cr}$-containing ion under these conditions was $\mathrm{Na}_{2} \mathrm{Cr}_{2} \mathrm{O}_{7}^{+}$.

We have previously established [G. C. Fryburg, R. A. Miller, F. J. Koh1, and C. A. Stearns, J. Electrochem. Soc. $124,1738,(1977)]$ that $\left(\mathrm{NaCl}_{1,2,3}\left(\mathrm{CrO}_{3}\right)\right.$ (g) complex molecules are responsible for the transport of $\mathrm{Cr}$ from $\mathrm{Cr}_{2} \mathrm{O}_{3}(\mathrm{c})$ or $\mathrm{Cr}^{-}$containing alloys heated in the presence of $\mathrm{O}_{2}(\mathrm{~g})$ and $\mathrm{NaCl}(\mathrm{g})$. However, upon deposition of these molecules $\mathrm{Na}_{2} \mathrm{CrO}_{4}(\mathrm{c})$ and either $\mathrm{Cl}_{2}(\mathrm{~g})$ or $\mathrm{HCl}_{(} \mathrm{g}$ ) (with $\mathrm{H}_{2} \mathrm{O}$ ) are formed. 
Comment (written) (Krause): When the authors used the transpiration method to test for the vapor transport of chromium in the various reactions which they reported in tables 1 and 2 , they would have provided more meaningful results by observing the mass of downstream deposit and the reaction time for all the reactions, in addition to the volume rate of carrier gas. Even if the experiments were designed to provide a rough comparison of the chromium transport among the reactions, a report of the mass flow of downstream deposit relative to the volume flow of carrier gas would be more explicit than the authors' indication of a deposit having been observed.

It is not clear that the authors' statement, that no $\mathrm{Na}_{2} \mathrm{CrO}_{4}$ is formed at the oxide surface, is meant to apply only to the low concentration of $\mathrm{NaCl}(\mathrm{g})$ used. Actually, when the authors mixed $\mathrm{Cr}_{2} \mathrm{O}_{3}(\mathrm{c})$ and $\mathrm{NaCl}(\mathrm{c})$, they did detect a small quantity of $\mathrm{Na}_{2} \mathrm{CrO}_{4}$ in the residue. This detection, or the lack of it in the other experiments, however, must have been difficult in that the authors ascertained the residue composition, I understand, by $x$-ray powder diffraction which is generally valid for identifying components greater than 5 mole percent.

Some doubt exists over whether reaction 4 is predominant in vaporizing $\mathrm{Na}_{2} \mathrm{CrO}_{4}$, as the authors have supposed from their mass spectral identification. Using published $\Delta \mathrm{H}_{\mathrm{f}}{ }^{\circ}$ values of $-320.8,-272.4$, and $25.6 \mathrm{kcal} / \mathrm{mol}$ at $25{ }^{\circ} \mathrm{C}$ for $\mathrm{Na}_{2} \mathrm{CrO}_{4}(\mathrm{c}), \mathrm{Cr}_{2} \mathrm{O}_{3}(\mathrm{c})$, and $\mathrm{Na}(\mathrm{g})$, respectively, gives $235.8 \mathrm{kcal} / \mathrm{mol}$ for $\Delta \mathrm{H}^{\circ}$ of reaction 4 . The authors' data give 377 $\mathrm{kcal} / \mathrm{mol}$. This difference between their result and the literature is too great to be reasonable. 

National Bureau of Standards Special Publication 561, Proceedings of the 10th Materials Research Symposium on Characterization of High Temperature Vapors and Gases held at NBS, Gaithersburg, Maryland, September 18-22, 1978. Issued 0ctober 1979.

\title{
CATALYTIC REACTIONS STUDIED BY A MATRIX ISOLATION TECHNIQUE: \\ DECOMPOSITION OF HCOOH ON Pt
}

\author{
D. E. Tevault ${ }^{1}$ and M. C. Lin \\ Chemistry Division \\ Naval Research Laboratory \\ Washington, D.C. 20375
}

\begin{abstract}
A matrix isolation technique has been used to investigate the kinetics and mechanism of $\mathrm{HCOOH}$ decomposition on Pt. Different mixtures of $\mathrm{HCOOH}(0.1-1.0 \%)$ in $\mathrm{Ar}$ were passed through a heated Pt screen and trapped on a 10-K CsI window. Progress of the reaction was monitored by observing the infrared spectra of the resulting solid matrices at different time intervals. $\mathrm{Be}^{-}$ sides the known decomposition products, $\mathrm{H}_{2} \mathrm{O}, \mathrm{CO}, \mathrm{CO}_{2}$, and presumably $\mathrm{H}_{2}$, new infrared absorptions have been found which are attributed to the $\mathrm{O}=\mathrm{C}-\mathrm{OH}$ radical. No absorptions due to $\mathrm{H}_{2} \mathrm{CO}$ or $\mathrm{HCO}$ were detected. The presence of the $\mathrm{OCOH}$ radical was further supported by the results of additional experiments using $\mathrm{HCOOD}$ and $\mathrm{DCOOH}$. The measured rates of $\mathrm{CO}_{2}$ formation at different temperatures between 420 and $730 \mathrm{~K}$ gave rise to an activation energy of $3.5 \pm 0.2 \mathrm{kcal} / \mathrm{mol}$ for the adsorption process. The pretreatment of the $\mathrm{Pt}$ catalyst with $\mathrm{H}_{2}$ was found to enhance the rate of $\mathrm{CO}_{2}$ formation. This indicates the important effect of adsorbed $\mathrm{H}$ atoms on $\mathrm{CO}_{2}$ production.
\end{abstract}

\section{Introduction}

Although numerous studies have been made on the decomposition of formic acid on various metals and metal oxides, controversies still exist regarding the kinetics and mechanisms leading to the formation of various products $[1,2,3]^{2}$. This stems partly from the complex nature of gas-surface interactions and reactions, and partly from the lack of effective diagnostic tools, not only for the gas-catalyst interfaces but also for various product species leaving catalyst surfaces.

\footnotetext{
${ }^{\mathbf{1} N R C / N R L}$ postdoctoral research associate.

${ }^{2}$ Figures in brackets indicate literature references at the end of this paper.
} 
Take the formation of free radicals in catalytic reactions, for example. Although a number of ESR measurements have previously demonstrated the presence of free radicals on catalytic surfaces [4,5], very few examples of the escape of those free radical species from the surface during catalytic reactions have been unambiguously identified. In one experiment carried out by Shalya and co-workers on the oxidation of $\mathrm{H}_{2}$ on Pt [6], ESR signals were detected in products trapped on the wall of a liquid nitrogen-cooled tube. This suggests that free radicals may have escaped from the Pt surface and perhaps taken part in homogeneous gas phase reactions. In this simple experiment, however, the identity of the ESR-active species was not characterized.

In this work, we have employed a matrix isolation technique to study the kinetics and mechanisms of heterogeneous catalytic reactions. The decomposition of formic acid on $\mathrm{Pt}$ was selected to test the effectiveness of the technique. We have found from this study that the on-line matrix trapping and IR spectral analysis of the decomposition products not only provided the information on the kinetics of stable products $\left(\mathrm{CO}_{2}, \mathrm{CO}, \mathrm{H}_{2} \mathrm{O}\right)$ but also revealed, for the first time, the production of a significant amount of hydroxy carbonyl $(0=\mathrm{C}-\mathrm{O}-\mathrm{H})$ radicals from the decomposition reaction.

\section{Experimental}

The decomposition of formic acid $(\mathrm{HCOOH})$ catalyzed by a heated platinum wire has been investigated using the matrix isolation technique in conjunction with infrared spectroscopy. Matrix experiments have been performed by flowing Ar-diluted $\mathrm{HCOOH}$ mixtures in the gas phase through a platinum wire screen (Alfa-Ventron, $>99.99 \%$ and $0.5 \mathrm{~mm} \mathrm{diam} \times 100 \mathrm{~cm}$ long) immediately prior to condensation onto a $10 \mathrm{~K}$ CsI window.

The CsI window was maintained at $10 \mathrm{~K}$ by a Model CS-202W Displex closed cycle helium refrigerator (Air Products and Chemicals, Inc.) whose cold station was tightly clamped to the CsI window [7]. Good thermal contact between the cryostat cold station and optical window was provided by indium gaskets. Matrix temperatures in the $10-35 \mathrm{~K}$ region were monitored using the output of a chromel vs Au-Fe ( 0.07 atom\%) thermocouple attached to the cold station. The cold station was surrounded by a thin stainless steel-coated copper radiation shield with appropriate holes provided for the gas deposition and spectroscopy. The outer vacuum shroud was made of stainless steel and had three $35-\mathrm{mm}$ diameter ports at the same height as the inner window. Two of the ports on opposite sides of the shroud were sealed with $50 \mathrm{~mm}$ diameter CSI windows using Viton " 0 " rings. The third port was on a face perpendicular to the two outer CsI windows and was used for the gas deposition and catalyst assembly to be described later.

Gas samples were prepared on an all stainless steel-Teflon vacuum line. The pumping system consisted of a $5-\mathrm{cm}$ oil diffusion pump (CVC Products, Inc., Model PMCS-2C) in conjunction with a rotary pump and a liquid nitrogen cold trap. Ar/ $\mathrm{HCOOH}$ mixtures in ratios of 100/1 to 1000/1 were prepared using standard techniques and stored for up to 10 days in a two-liter stainless steel container. $\mathrm{HCOOH}(\geq 97 \%$, Aldrich Chemical Co.), DCOOH (99\%, Merck and Co., Inc.) and $\mathrm{HCOOD}$ (98\%, Merck and Co., Inc.) were degassed at $196 \mathrm{~K}$ using repeated 
freeze-pump-thaw cycles. Argon (Matheson, 99.9995\%) was stored at 30 psi or greater and used without further purification. The reactant mixtures were passed through the catalysis chamber depicted in figure 1 and condensed onto the $10 \mathrm{~K} \mathrm{CSI}$ window. The argon- $\mathrm{HCOOH}$ mixtures were leaked into the deposition line through a needle valve at a constant rate of about $2.7 \mathrm{~m} \mathrm{~mol} / \mathrm{hr}$, creating a pressure behind the nozzle of about $0.75 \mathrm{Torr}$. The nozzle is a rectangular brass block with ten $100 \mu \mathrm{m}$ diameter holes drilled in the front face. The steady state pressure inside the catalytic chamber during deposition was measured to be $1 \times 10^{-3}$ Torr using a capacitance manometer. This gives a $\mathrm{HCOOH}$ pressure of $10^{-5}$ to $10^{-6}$ Torr depending on the dilution ratio. The gases then flow through the platinum catalyst screen which is coiled around two grooved ceramic rods. The wires are attached to two brass electrodes which are insulated from the aluminum back flange by glass sleeves. The glass sleeves, electrodes, Pyrex vacuum shroud, and aluminum front flange were sealed with epoxy.

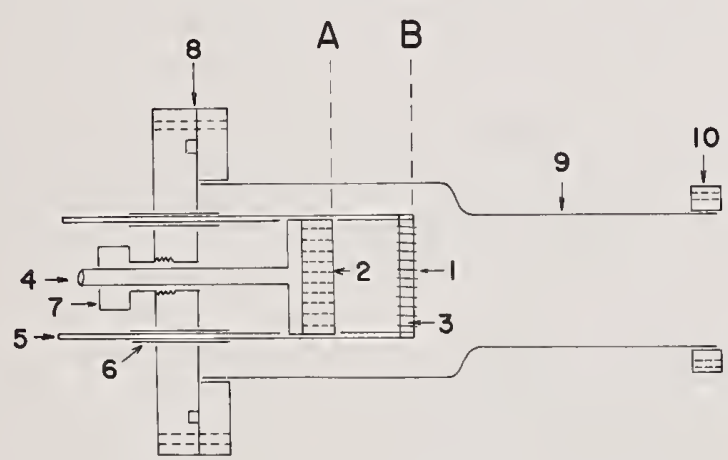

SIDE

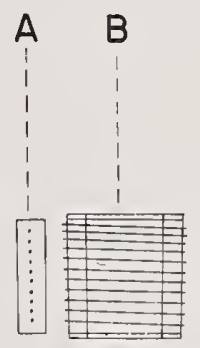

FRONT

\section{$\stackrel{\longmapsto}{\mathrm{cm}}$}

Figure 1. Catalysis apparatus. (1) $0.5 \mathrm{~mm}$ diameter $99.99 \%$ platinum wire, ca. $80 \mathrm{~cm}$ length. (2) Brass deposition nozzle with 10 equally spaced $100 \mu \mathrm{m}$ diameter holes. Inset $\underline{A}$ shows front surface of the nozzle. (3) Grooved ceramic rods around which Pt wire catalyst is coiled. Inset $\underline{B}$ shows front view of catalyst and ceramic support. (4) Brass deposition line. (5) Brass electrode. (6) Glass insulating sleeve. (7) Cajon fitting. (8) Aluminum back flange. (9) Pyrex vacuum shroud. (10) Aluminum front flange.

Experiments were performed when the vacuum line, catalyst chamber, and matrix dewar had been evacuated to $2 \times 10^{-6}$ Torr. The vacuum system was then pressurized to 5 Torr with $0_{2}$ (Matheson, Extra Dry, 99.6\%) and the catalyst heated to ca. $1100 \mathrm{~K}$ for one hour to burn away any impurities on its surface. After this initial burning, the system was evacuated, and the spectroscopic window cooled to $10 \mathrm{~K}$ over a 45-minute period. When the window reached $10 \mathrm{~K}$, the catalyst was heated to $1200 \mathrm{~K}$ for 5 minutes to remove any traces to volatile impurities from the Pt surface. The catalyst was then cooled to the desired temperature which was monitored by precisely measuring the current passing through the 
catalyst and the corresponding voltage drop. Using these experimental parameters, the resistance of the catalyst was calculated. The resistance of the catalyst at room temperature was also accurately measured in a similar manner. The temperature of the wire could then be determined by calculating $R(T) / R(0)$ [8], the ratio of the resistance at that temperature to the resistance at $0{ }^{\circ} \mathrm{C}$, which was derived from the value measured at $25{ }^{\circ} \mathrm{C}$.

Deposition times were generally 2 to 6 hours. In order to make time-resolved product yield measurements, the CsI window was maintained at a $45^{\circ}$ angle to the direction of the gas deposition and to the direction of the infrared spectrometer beam. The arrangement of the spectrometer, catalyst, and matrix window is shown in figure 2.

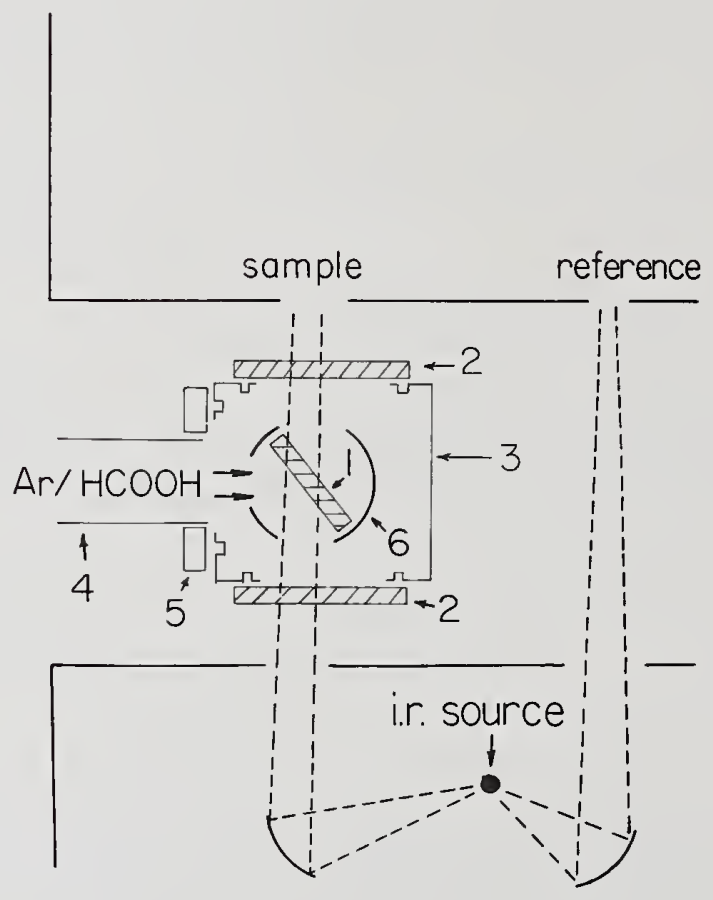

Figure 2. Sample chamber. (1) $10 \mathrm{~K}$ CsI substrate window. (2) Outer CsI optics.

(3) Stainless steel vacuum shroud. (4) Catalyst vacuum shroud (see fig. 1).

(5) Aluminum front flange connecting catalyst chamber to cryostat. (6) $40 \mathrm{~K}$ stainless steel radiation shield. After passing through the catalyst, the $\mathrm{Ar} / \mathrm{HCOOH}$ mixture is deposited onto the cryogenic salt window. Infrared spectra were recorded during and after sample deposition.

Survey scans were recorded in the 250 to $4000 \mathrm{~cm}^{-1}$ spectral region during and after sample deposition on a Perkin-Elmer Model 457 infrared spectrophotometer. Decomposition of $\mathrm{HCOOH}$ was monitored by scanning the strong $2349 \mathrm{~cm}^{-1}$ [9] absorption of $\mathrm{CO}_{2}$ at intervals as short as 90 seconds. 
Several auxiliary experiments were formed in which the catalyst was purified as described above. When the desired catalyst temperature was reached, the catalyst was pretreated with 2.5 to $10 \mu$ moles of $\mathrm{H}_{2}$ (Matheson, prepurified 99.95\%) in excess argon, followed by deposition of $\mathrm{Ar} / \mathrm{HCOOH}=100 / 1$ samples. The effect of the pretreatment was determined by measuring the $\mathrm{CO}_{2}$ production rate as before.

\section{Results}

\section{1 Spectral analysis}

Figure 3 compares typical spectra obtained when Ar/HCOOH samples were deposited (a) without the catalyst, (b) with the Pt catalyst held at room temperature, and (c) with the catalyst heated to $700 \mathrm{~K}$. It was noted during the course of the present work that the relative intensities of a number of bands in spectra taken from samples deposited without the catalyst were strongly dependent on the matrix dilution ratios. Table 1 details spectra observed in these experiments and gives the relative concentration dependence of each band. Several samples were annealed to 32 to $35 \mathrm{~K}$ and recooled to $10 \mathrm{~K}$. The qualitative spectral intensity changes effected by this treatment are also shown in table 1. Bands favored in intensity by dilution generally decrease with diffusion

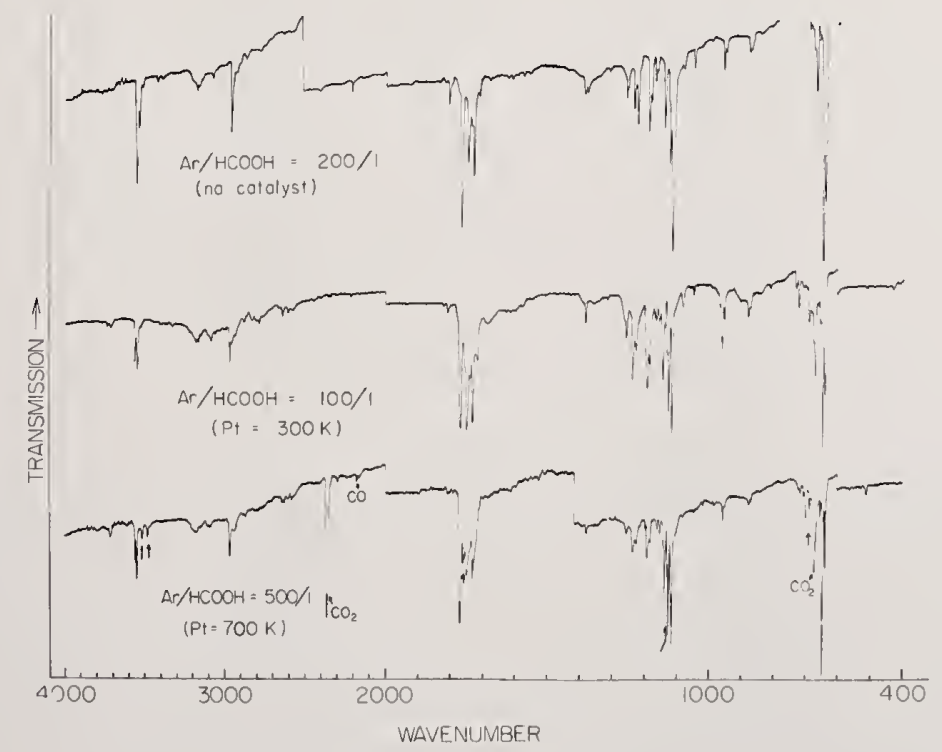

Figure 3. Survey spectra in the 400 to $4000 \mathrm{~cm}^{-1}$ region produced by (top) $\mathrm{Ar} / \mathrm{HCOOH}=200 / 1$ sample deposited without catalyst, (middle) $\mathrm{Ar} / \mathrm{HCOOH}=$ $100 / 1$ deposited through Pt catalyst at $300 \mathrm{~K}$, and (bottom) $\mathrm{Ar} / \mathrm{HCOOH}=$ $500 / 1$ deposited through catalyst at $700 \mathrm{~K}$. Arrows denote decomposition products. 
Table 1. Frequencies, concentration dependence, and diffusion behavior of the more intense infrared absorptions observed for $\mathrm{HCOOH}$ isolated in solid argon at $10 \mathrm{~K}$ without catalyst.

\begin{tabular}{|c|c|c|c|}
\hline Frequency $\mathrm{cm}^{-1}$ & $\begin{array}{l}\text { Relative intensity } \\
\text { with dilution } \\
\end{array}$ & $\begin{array}{l}\text { Diffusion behavior } \\
10 \mathrm{~K} \rightarrow 32 \mathrm{~K} \rightarrow 10 \mathrm{~K} \\
\end{array}$ & Assignment \\
\hline 3550 & Inc. & Dec. & M \\
\hline 3540 & Inc. & Dec. & M \\
\hline 3180 & $W$ & Inc. & $P$ \\
\hline 3160 & $W$ & Inc. & $P$ \\
\hline 3080 & $W$ & Inc. & $P$ \\
\hline 2960 & Inc. & Dec. & $M$ \\
\hline 2950 & Dec. & Inc. & P \\
\hline 2930 & $W$ & Inc. & $P$ \\
\hline 2640 & Inc. & Dec. & M \\
\hline 2600 & Inc. & Dec. & $M$ \\
\hline 2210 & Inc. & Dec. & M \\
\hline 1810 & -- & Dec. & $M$ \\
\hline 1770 & Inc. & Dec. & M \\
\hline 1745 & Dec. & Inc. & $P$ \\
\hline 1730 & Dec. & Inc. & $P$ \\
\hline 1715 & Dec. & Inc. & $P$ \\
\hline 1380 & $W$ & Dec. & $M$ \\
\hline 1255 & $W$ & Inc. & P \\
\hline 1235 & Inc. & Dec. & M \\
\hline 1225 & Inc. & Inc. & - \\
\hline 1190 & Inc. & Dec. & $M$ \\
\hline 1182 & -- & Inc. & - \\
\hline 1160 & -- & Dec. & $P$ \\
\hline 1150 & Dec. & Inc. & M \\
\hline 1140 & Inc. & Dec. & M \\
\hline 1125 & Inc. & Dec. & M \\
\hline 1115 & Inc. & Dec. & M \\
\hline 960 & Inc. & Dec. & $M$ \\
\hline 950 & Dec. & Inc. & p \\
\hline 880 & Inc. & Dec. & M \\
\hline 870 & Inc. & Dec. & $M$ \\
\hline 735 & Dec. & Inc. & $P$ \\
\hline 725 & Dec. & Inc. & $P$ \\
\hline 690 & Inc. & Dec. & M \\
\hline 670 & Inc. & Dec. & M \\
\hline 650 & Inc. & Dec. & M \\
\hline 640 & Inc. & Dec. & M \\
\hline
\end{tabular}

Inc. - Increased

Dec. - Decreased

W - Weak; dilution behavior not certain

M - Monomer

P - Polymer 
and are assigned to isolated $\mathrm{HCOOH}$, while the bands which were favored at the higher $\mathrm{HCOOH}$ concentrations and with matrix diffusion are assigned to polymeric $\mathrm{HCOOH}$, most likely the stable $[10,11]$ hydrogen-bonded dimer

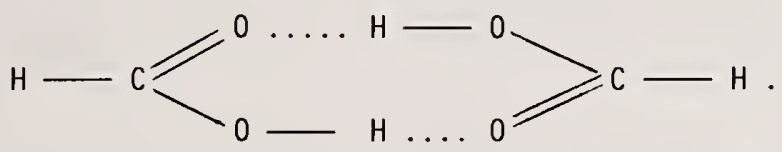

Infrared spectra produced with the Pt screen held at ambient temperatures are depicted by the middle trace of figure 3 . The spectra produced in this manner were only slightly different from those produced without a catalyst. The most obvious spectral change occurs near $3550 \mathrm{~cm}^{-1}$ where the strong $3553 \mathrm{~cm}^{-1}$ and weak $3542 \mathrm{~cm}^{-1}$ bands are changed into a doublet with the lower wavenumber feature slightly more intense. Other differences include the change in relative intensity of several bands, notably in the 1100 to $1300 \mathrm{~cm}^{-1}$ spectral region. Additionally, a trace of $\mathrm{H}_{2} \mathrm{O}$, presumably from $\mathrm{HCOOH}$ samples, was indicated by a broad, weak absorption centered at about $3730 \mathrm{~cm}^{-1}$. The most probable explanation of the splitting of the $3553 \mathrm{~cm}^{-1}$ band (and other less obvious spectral changes) is that formic acid has been isomerized from the cis- to the trans- form by the catalyst. The $\mathrm{cis}-\mathrm{HCOOH}$ species

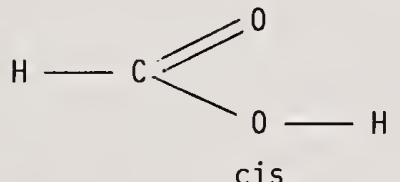

is more stable than the trans form

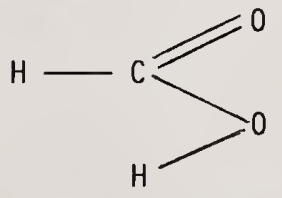

\section{trans}

by about $2 \mathrm{kcal} / \mathrm{mol}$ [12] at room temperature and, consequently, is the predominant species in the gas phase at equilibrium. Once formed, however, the trans form has a relatively high energy barrier for rotation back to the cis isomer (estimated at between 9 and 15 $\mathrm{kcal} / \mathrm{mol}$ ) and is expected to be a long-lived species in the gas phase $[12,13]$.

When the catalyst was heated to temperatures in the 420 to $730 \mathrm{~K}$ range, several totally new features due to formic acid decomposition were observed, as shown in the lower trace of figure 3 [14]. The most easily recognizable new bands are caused by $\mathrm{CO}_{2}$ at 2350 and $665 \mathrm{~cm}^{-1}, \mathrm{CO}$ at $2140 \mathrm{~cm}^{-1}$, the $\mathrm{CO} \cdot \mathrm{H}_{2} \mathrm{O}$ complex at $2150 \mathrm{~cm}^{-1}$, and $\mathrm{H}_{2} \mathrm{O}$ at $3730 \mathrm{~cm}^{-1}$. Also, several previously unidentified new infrared features resulted from the decomposition of $\mathrm{HCOOH}$ on the hot catalyst.

Experiments were carried out with the deuterium-enriched species $\mathrm{OCOOH}$ and HCOOD in order to learn the identity of the species giving rise to the new infrared features. Spectra obtained for $\mathrm{DCOOH}$ in two experiments using $300 \mathrm{~K}$ and $680 \mathrm{~K} \mathrm{Pt}$ temperatures are illustrated in figure 4. Similar results for $\mathrm{HCOOD}$ are shown in figure 5 . The isotopic 
behavior of several features could not be ascertained due to overlap with the parent formic acid absorptions, and is quite likely that more decomposition product absorptions are unobserved due to similar overlaps. However, it is clear from table 2 that most of the new bands shift in frequency in HCOOD experiments, but not in $D C O O H$ experiments. The moderately intense 3510 and $3480 \mathrm{~cm}^{-1}$ bands in the $0-H$ stretching region were observed

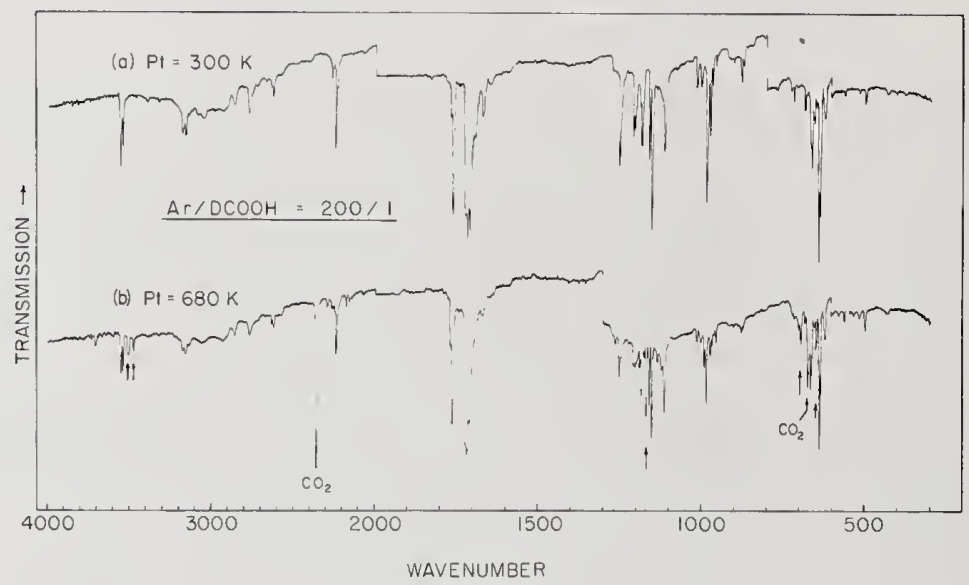

Figure 4. Survey spectra in the 400 to $4000 \mathrm{~cm}^{-1}$ region produced by (top) Ar/DCOOH $=200 / 1$ deposited through Pt catalyst at $300 \mathrm{~K}$ and (bottom) $\mathrm{Ar} / \mathrm{DCOOH}=200 / 1 \mathrm{de}-$ posited through catalyst at $680 \mathrm{~K}$. Arrows denote decomposition products.

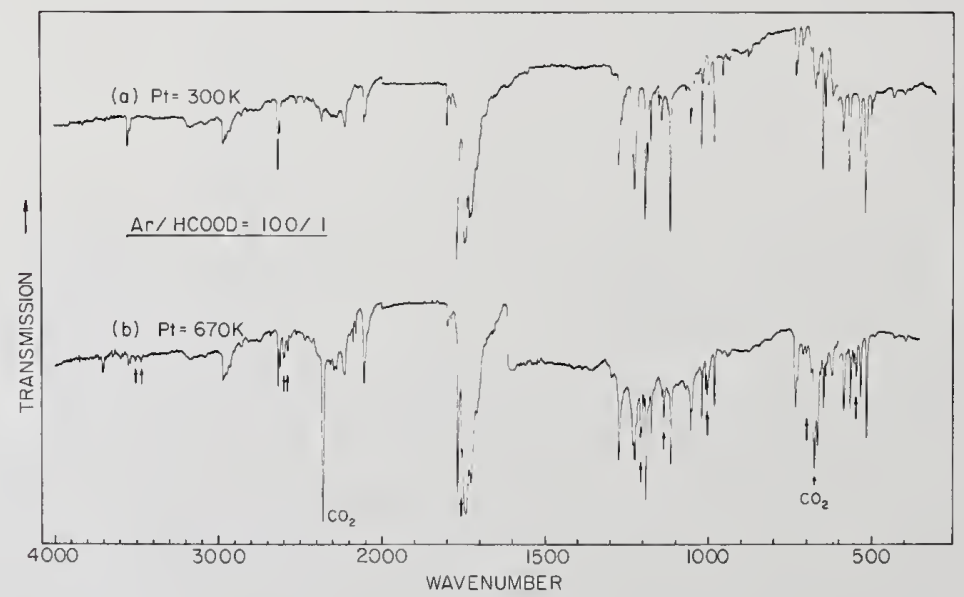

Figure 5. Survey spectra in the 400 to $4000 \mathrm{~cm}^{-1}$ region produced by (top) Ar/HCOOD $(\sim 80 \% \mathrm{D})=100 / 1$ deposited through Pt catalyst at $300 \mathrm{~K}$ and (bottom) $\mathrm{Ar} / \mathrm{HCOOD}=$ $100 / 1$, deposited through catalyst at $670 \mathrm{~K}$. Arrows denote decomposition products. 
Table 2. New infrared absorptions $\left(\mathrm{cm}^{-1}\right.$ ) due to products (except $\mathrm{CO}, \mathrm{CO}_{2}$ and $\mathrm{H}_{2} \mathrm{O}$ ) of formic acid decomposition on $\mathrm{Pt}$ and the reaction of $\mathrm{H}$ atoms with formic acid trapped in Ar matrices at $10 \mathrm{~K}$.

\begin{tabular}{ccc}
$\mathrm{HCOOH}$ & $\underline{\mathrm{HCOOD}}$ & $\underline{\mathrm{DCOOH}}$ \\
\cline { 2 - 3 } & & \\
- & 545 & - \\
- & 555 & - \\
$(0)$ & - & 650 \\
700 & 700 & 700 \\
- & 1005 & $(0)$ \\
$1135^{*}$ & $1135^{\star}$ & - \\
- & - & $1172^{*}$ \\
- & 1205 & $(0)$ \\
1755 & 1750 & $(0)$ \\
- & 2575 & - \\
- & 2600 & - \\
3480 & - & 3480 \\
3510 & - & 3510
\end{tabular}

(0) denoted band not observed due to overlap with parent absorption.

* denotes bands which cannot be assigned to $\mathrm{OCOH}$.

unshifted in $\mathrm{DCOOH}$ experiments, but red-shifted to 2600 and $2575 \mathrm{~cm}^{-1}$, respectively, with HCOOD. Furthermore, it was noted that the deuterium-enrichment in the HCOOD was actually about 80 percent and, as a result, vestiges of the 3510 and $3480 \mathrm{~cm}^{-1}$ bands were also present. No intermediate new features between 2600 and $3480 \mathrm{~cm}^{-1}$ were observed. The large deuterium shift confirms that these absorptions are due to $\mathrm{O}-\mathrm{H}$ stretching vibrations.

The second new feature was found at $1755 \mathrm{~cm}^{-1}$, in the carbonyl stretching region. This band shifted to $1750 \mathrm{~cm}^{-1}$ in the $\mathrm{HCOOD}$ experiment, but this region was obscured by a strong parent absorption in the $\mathrm{DCOOH}$ experiments.

The new band appearing at $700 \mathrm{~cm}^{-1}$ in $\mathrm{HCOOH}$ experiments was also unshifted in $\mathrm{DCOOH}$ experiments, but shifted to give a pair of new bands at 555 and $545 \mathrm{~cm}^{-1}$ in $\mathrm{HCOOD}$ decomposition experiments. This spectral region is appropriate for an $0 \mathrm{CO}$ in-plane bending vibration or an $0 \mathrm{COH}$ out-of-plane torsional vibration [12]. The $150 \mathrm{~cm}^{-1}$ isotopic shift supports the assignment of this feature to a torsional mode.

As seen in table 2, a number of new bands were observed in one isotopic experiment but obscured by strong overlapping parent molecule absorptions upon isotopic substitution, making definitive characterization impossible. This was not the case for the band which appeared at $1135 \mathrm{~cm}^{-1}$ in $\mathrm{HCOOH}$ and was essentially unshifted in $\mathrm{HCOOD}$ experiments. This band was not observed in $\mathrm{DCOOH}$ experiments. Conversely, the $1172 \mathrm{~cm}^{-1}$ band appearing in $\mathrm{DCOOH}$ experiments was not observed in either $\mathrm{HCOOH}$ or $\mathrm{HCOOD}$ work. 


\subsection{Kinetic studies}

\subsubsection{Concentration dependence}

Four experiments were performed in succession at $\mathrm{HCOOH}$ concentrations of $1 \%, 0.5 \%, 0.2 \%$ and $0.1 \%$ in argon using a constant catalyst temperature of $625 \mathrm{~K}$. The infrared spectra resulting from the more dilute samples were essentially the same as those from the more concentrated experiments except for the lower intensities of bands attributed to $\mathrm{HCOOH}$ aggregates at higher dilution ratios (see table 1). It was also noticed that the induction period for $\mathrm{CO}_{2}$ appearance increased markedly with dilution ratio.

\subsubsection{Rate measurements}

In order to better understand the mechanism of the decomposition reaction, experiments have been performed using $\mathrm{Ar} / \mathrm{HCOOH}=100 / 1$ mixtures at several catalyst temperatures between 420 and $730 \mathrm{~K}$. The intensity of the $2350 \mathrm{~cm}^{-1} \mathrm{CO}_{2}$ peak, which is the major product of the $\mathrm{HCOOH}$ decomposition, was monitored at different time intervals during deposition. Figure 6 shows the results of two experiments conducted at catalyst temperatures of 420 and $700 \mathrm{~K}$. The upper plot shows the rate of $\mathrm{CO}_{2}$ production versus time for these two temperatures, while the lower plot contrasts the total yields versus reaction time. The induction period is clearly observable in the (upper) $420 \mathrm{~K}$ experiment rate plot as the

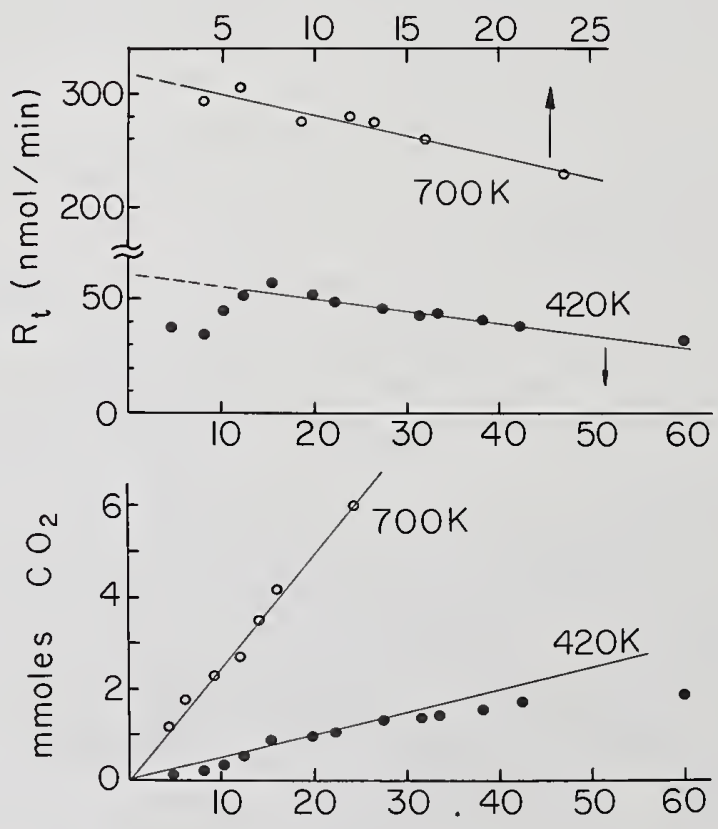

Figure 6. (Top) Graph of rate of $\mathrm{CO}_{2}$ production versus time for $\mathrm{Ar} / \mathrm{HCOOH}=100 / 1$ samples at Pt catalyst temperatures of 420 and $700 \mathrm{~K}$. (Bottom) Graph of total $\mathrm{CO}_{2}$ versus time in the same two experiments. 
initial rapid rise, which is then followed by a linear decay in the rate due to catalyst poisoning. The induction time in the $420 \mathrm{~K}$ experiment is seen in the lower graph of figure 6 as an initial lag in the $\mathrm{CO}_{2}$ yield followed by a linear growth in $\mathrm{CO}_{2}$ yield. The induction period was much less noticeable in the higher temperature experiments as illustrated by the $700 \mathrm{~K}$ plots in figure 6 . The higher reaction rates in the higher temperature experiments cause the induction period to be concluded in times comparable to the scanning interval.

In each experiment, the linear portion of the rate versus time graph was extrapolated back to $t_{0}$, the time that the deposition was begun. The extrapolated intercept gives $R_{0}$, the rate of $\mathrm{CO}_{2}$ production at the beginning of the deposition, i.e., with a clean platinum surface. The initial rates were plotted against the reciprocal absolute temperature, as shown in figure 7 . The activation energy for $\mathrm{CO}_{2}$ production calculated from the Arrhenius plot by means of the least squares method was $3.5 \pm 0.2 \mathrm{kcal} / \mathrm{mol}$. Rate data from $\mathrm{DCOOH}$ and $H C O O D$ experiments indicate that the activation energy and decomposition rate do not change significantly with deuterium substitution.

The decrease in $\mathrm{CO}_{2}$ production rate with time is attributed to poisoning of the catalyst by $\mathrm{HCOOH}$ or its decomposition products whose concentration on the catalytic surface increases during the deposition period. An attempt was made to measure the rate of poisoning (proportional to the negative slope) versus time, but it was found that the intensity of the 2350

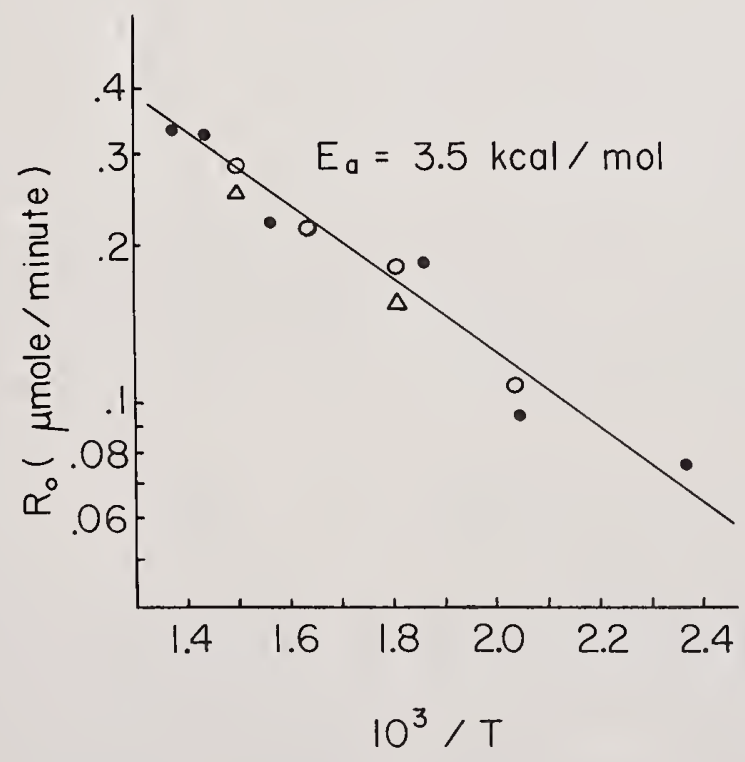

Figure 7. Arrhenius plot of initial $\mathrm{CO}_{2}$ production rate $\left(\mathrm{R}_{0}\right)$ at several catalyst temperatures between 420 and $730 \mathrm{~K}$ versus $1 / T\left(\mathrm{~K}^{-1}\right)$. Activation energy derived from this plot is $3.5 \pm 0.2 \mathrm{kcal} / \mathrm{mol}$ using linear least squares plotting method using only $\mathrm{HCOOH}$ rate data (closed circles). Triangles and open circles show $\mathrm{HCOOD}$ and $\mathrm{DCOOH}$ results, respectively. 
$\mathrm{cm}^{-1}$ band was too great at higher temperatures to obtain meaningful values because of optical saturation.

Several experiments in which the "cleaned" Pt catalyst was pretreated with small quantities (ca. 2 to $10 \mu \mathrm{mol}$ ) of $\mathrm{H}_{2}$ have been performed in order to assess the role of adsorbed hydrogen atoms upon the $\mathrm{CO}_{2}$ appearance rate. Figure 8 shows the results of these studies. It was found that $\mathrm{H}_{2}$ pretreatment increases the initial $\mathrm{CO}_{2}$ production rate by shortening the induction time. Further investigation of the effect of $\mathrm{CO}, \mathrm{H}_{2}$ and $\mathrm{H}_{2} \mathrm{O}$ pretreatment is currently underway and will be reported elsewhere.

\section{Discussion}

\section{1 Production of the $\mathrm{O}=\mathrm{C}-\mathrm{OH}$ radical}

The identity of the new decomposition product can be ascertained on the basis of group frequencies as well as isotopic data. The 3510 and $3480 \mathrm{~cm}^{-1}$ bands are clearly the $\mathrm{O}-\mathrm{H}$ stretching vibrations, probably due to cis- and trans- isomers, as supported by their frequency and by their large deuterium shifts. Furthermore, the absence of intermediate features in the partially enriched $\mathrm{HCOOD}$ experiment shows that this vibration involves only one hydrogen atom. The fact that no new absorption was observed in the $0-0$ stretching region ( 2500 to $2700 \mathrm{~cm}^{-1}$ ) in $\mathrm{DCOOH}$ experiments shows that no significant exchange between the $D$ and $H$ atoms occurs on the Pt surface or later on in the gas phase prior to condensation in the matrix (because of the low pressures used).

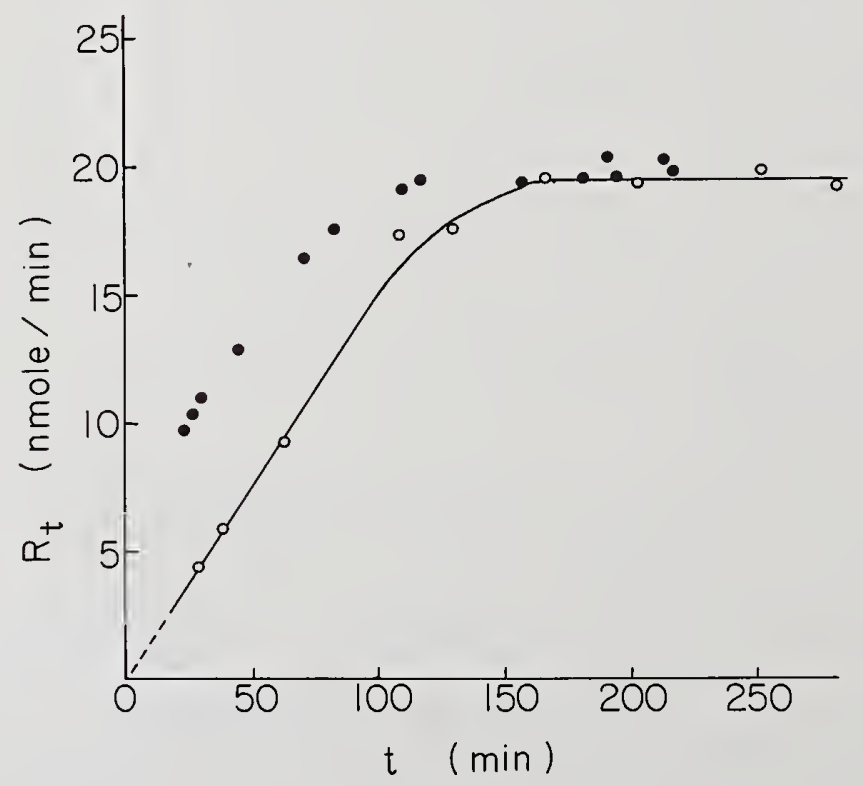

Figure 8. $\mathrm{CO}_{2}$ production rate versus time using a "clean" Pt catalyst and following pretreatment of the catalyst with $5 \mu \mathrm{mol}$ of $\mathrm{H}_{2}$ (in excess argon). $\mathrm{Ar} / \mathrm{HCOOH}=$ $1000 / 1$, and $\mathrm{Pt}=620 \mathrm{~K}$ in both experiments. (Filled circles show $\mathrm{H}_{2}$ pretreatment results). 
The new carbonyl stretch at $1755 \mathrm{~cm}^{-1}$ in $\mathrm{HCOOH}$ decomposition experiments red-shifted $\sim 5 \mathrm{~cm}^{-1}$ with $\mathrm{HCOOD}$ substitution, but the region was obscured in $\mathrm{DCOOH}$ work. Likewise, the $700 \mathrm{~cm}^{-1}$ band, characteristic of a hydrogen torsional mode, was unshifted with $\mathrm{DCOOH}$, but showed a large shift upon HCOOD substitution. The molecule most easily fitting these characteristics is the hydroxy-carbony 1 radical, $\mathrm{OCOH}$, formed by abstraction of the hydrogen atom on carbon.

The conclusion that the new bands at $3510,3480,1755$ and $700 \mathrm{~cm}^{-1}$, and their isotopic counterparts, are due to the $\mathrm{OCOH}$ radical is supported by several other observations. First, the absence of any new bands in the $\mathrm{C}-\mathrm{H}$ stretching region indicates that the new molecule does not have a carbon-hydrogen bond. This result also rules out the presence of significant amounts of other possible decomposition products such as $\mathrm{H}_{2} \mathrm{CO}$ or $\mathrm{HCO}$. Secondly, in a separate work, we have reacted hydrogen atoms with $\mathrm{HCOOH}$ in the gas phase with excess argon and condensed the resulting mixture at $10 \mathrm{~K}$, forming argon matrices [15]. The only elementary reaction which is thermochemically allowed in this system is the $\mathrm{H}+\mathrm{HCOOH}$ reaction:

$$
\mathrm{H}+\mathrm{HCOOH}^{*} \rightarrow \mathrm{H}_{2}+\mathrm{O}=\mathrm{C}-\mathrm{OH}^{*}
$$

Infrared spectra resulting from the reactions of $\mathrm{H}$ atoms and $\mathrm{HCOOH}$ were virtually identical to those of $\mathrm{HCOOH}$ decomposition on Pt including the new bands assigned to $\mathrm{OCOH}$.

$\mathrm{HCOOH}$ has also been photolyzed directly with the vacuum ultraviolet emissions from a microwave-discharge-powered windlowless argon resonance lamp, with and without a capillary array to remove plasma metastables between the discharge and the forming matrix $[16,17]$. In each case, a small amount of the $\mathrm{OCOH}$ radical was produced, as evidenced by the appearance of the 3510 and $3480 \mathrm{~cm}^{-1}$ bands, although the relative intensity of the lower wavenumber feature was clearly favored in the capillary array experiment.

The $\mathrm{O} \mathrm{COH}$ radical in the $\underline{\mathrm{cis}}$ and trans conformations had been observed in $\mathrm{CO}$ matrices following vacuum ultraviolet photolysis $(121.6 \mathrm{~nm})$ during deposition of co containing between 0.1 and 0.5 percent $\mathrm{H}_{2} \mathrm{O}$ [18]. The ${ }_{\mathrm{OD}} / \nu_{\mathrm{OH}}$ frequency ratios for the bands we assign to $\mathrm{OCOH}$ are

$$
\frac{2600}{3510}=0.741 \text { and } \frac{2575}{3480}=0.740 \text {, }
$$

which are the same as the ratios observed for cis and trans $\mathrm{OCOD} / \mathrm{OCOH}$ in $\mathrm{CO}$ matrices [18].

The IR absorption data obtained from the decomposition of $\mathrm{HCOOH}, \mathrm{DCOOH}$ and $\mathrm{HCOOD}$ strongly suggest that the $\mathrm{O} C \mathrm{H}$ radical produced in these catalytic reactions originates mainly from the direct abstraction reaction:

$$
\text { (D) } \mathrm{HCOOH}+\mathrm{M} \rightarrow \mathrm{M} . \mathrm{H}(\mathrm{D})+\mathrm{OCOH}
$$

where $M$ denotes an active site on the Pt surface. This is the first conclusive evidence of the escape of a free radical from a catalyst surface and its identity characterized. 


\subsection{Kinetics and mechanism of $\mathrm{CO}_{2}$ formation}

The decomposition of $\mathrm{HCOOH}$ on $\mathrm{Pt}$ has been extensively investigated in static systems. The principal stable products are $\mathrm{H}_{2}$ and $\mathrm{CO}_{2}$, with smaller amounts of $\mathrm{H}_{2} \mathrm{O}$ and $\mathrm{CO}$ also being produced $[1-3,19]$. This is confirmed by the results of our IR analysis. Block and Vogl had made careful investigations of the decomposition as a function of temperature at several pressures between 0.006 and 1 Torr [20]. They found that the activation energy increases with pressure and approaches a zeroth order limit of $14 \mathrm{kcal} / \mathrm{mol}$ at higher pressures (see fig. 9). They also found activation energies as low as $5.5 \mathrm{kcal} / \mathrm{mol}$ (which is in close agreement with our value of $3.5 \mathrm{kcal} / \mathrm{mol}$ ) at the low pressure limit used in that study. In our work, the steady-state partial pressure of $\mathrm{HCOOH}$ flowing through the Pt catalyst was $1 \times 10^{-5}$ Torr. Consequently, the $3.5 \mathrm{kcal} / \mathrm{mol}$ activation energy is probably very close to the low pressure limit.

On the basis of the simple Langmuir kinetic scheme, which has been shown to be valid for the $\mathrm{HCOOH}-\mathrm{Pt}$ system [20]:

$$
\mathrm{HCOOH}+\mathrm{M} \stackrel{k^{a}}{\rightarrow} M \ldots(\mathrm{HCOOH}) \stackrel{k_{d}}{\rightarrow} \mathrm{CO}_{2}+H_{2} \text {, etc. . }
$$

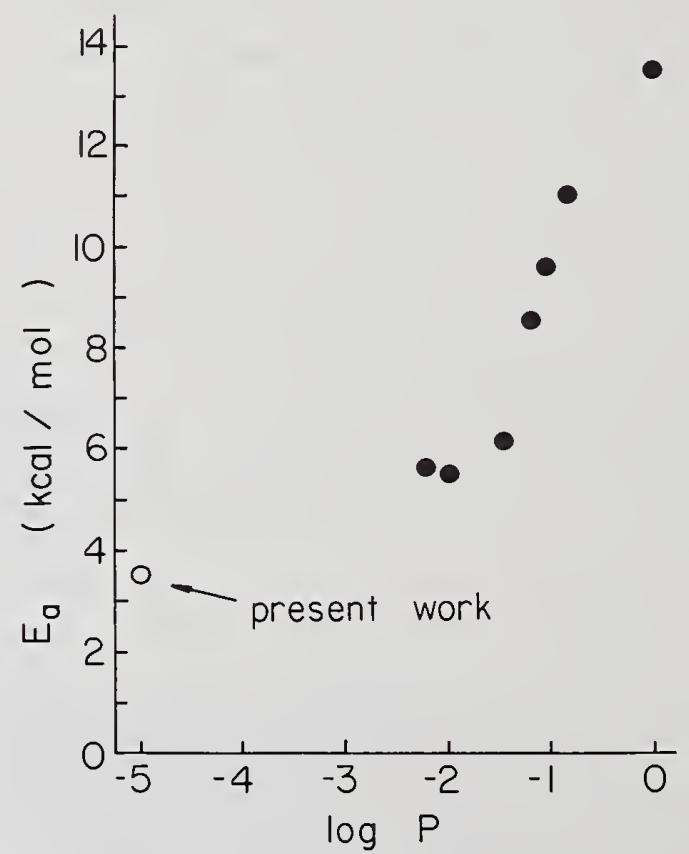

Figure 9. Graph of the activation energy for $\mathrm{HCOOH}$ decomposition versus log $P$ (Torr) using the results of the present work and the higher pressure results of reference [20]. 
The rate of product formation under steady-state conditions is given by the expression,

$$
R=\frac{k_{a} k_{d}[\mathrm{HCOOH}]}{k_{d}+k_{a}[\mathrm{HCOOH}]}
$$

where $k_{a}$ and $k_{d}$ are the rate constants for adsorption and decomposition, respectively. Under the low pressure conditions employed in our experiments,

$$
R=k_{a}[\mathrm{HCOOH}] \text {. }
$$

This first-order kinetics was observed as shown in figure 10. The activation energy $(3.5 \pm$ $0.2 \mathrm{kcal} / \mathrm{mol}$ ) determined in our experiments, therefore, corresponds to the barrier of adsorption, forming an intermediate on $\mathrm{Pt}$ such as

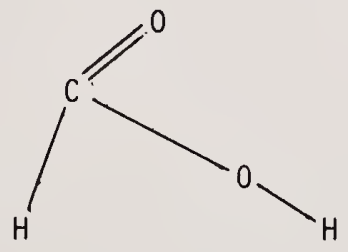

\section{7ाIIIIIIIIIIIIIII7}

or other forms with surface formate structures similar to the ones postulated for the $\mathrm{HCOOH}-\mathrm{Ni}$ system [2]. Since the pretreatment of the clean Pt surface with $\mathrm{H}_{2}$, shown in figure 8 , enhanced the rate of $\mathrm{CO}_{2}$ production, the decomposition of the above intermediate is apparently aided by the presence of adsorbed $H$ atoms, possibly via the following mechanism:

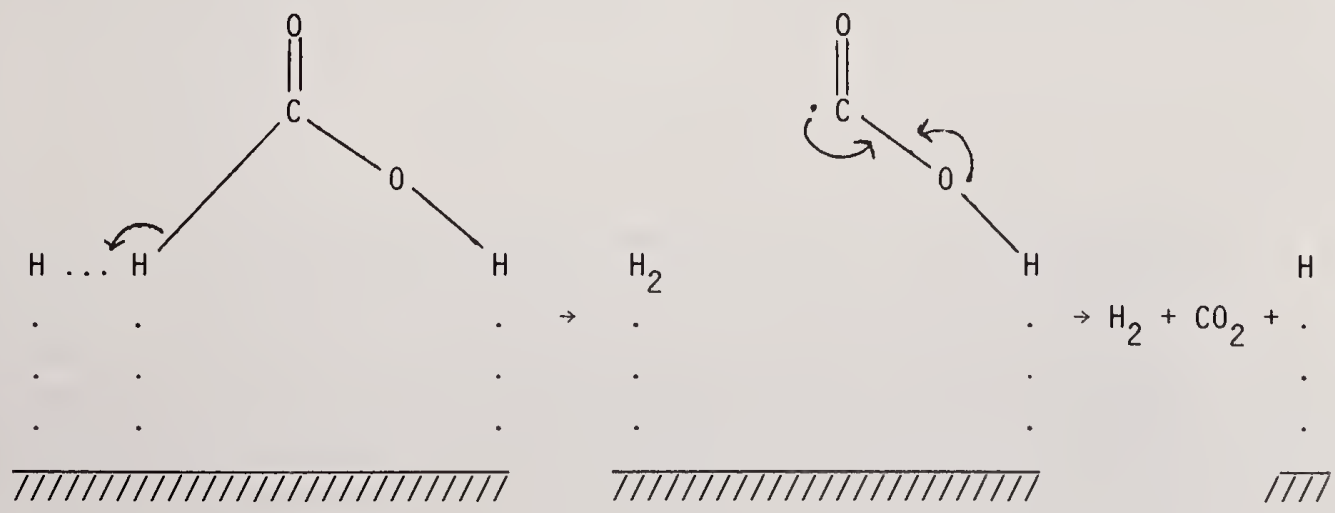




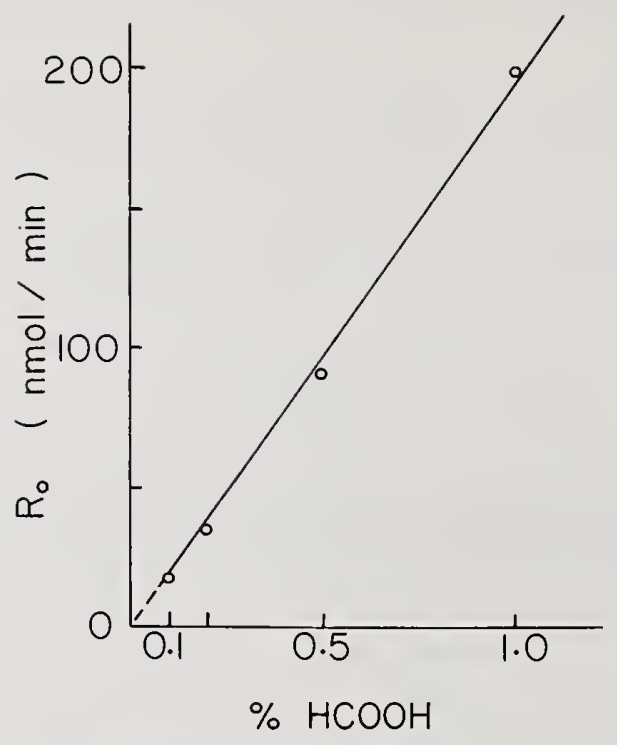

Figure 10. Graph of the initial $\mathrm{HCOOH}$ decomposition rate versus $\mathrm{HCOOH}$ concentration.

The formation of the $\mathrm{H}-\mathrm{H}$ bond according to the above scheme can obviously accelerate the decomposition process by supplying a vast amount of energy. Once the steady-state concentration of the adsorbed $\mathrm{H}$ atoms is maintained, the rate of formation of $\mathrm{CO}_{2}$ becomes constant, as indicated by the results presented in figure 8 . The observed induction times for $\mathrm{CO}_{2}$ production at lower temperatures and/or concentration can therefore be accounted for by the catalytic effect of the adsorbed $H$ atoms. A similar effect may also aid the formation of the $\mathrm{OCOH}$ radical, viz.,

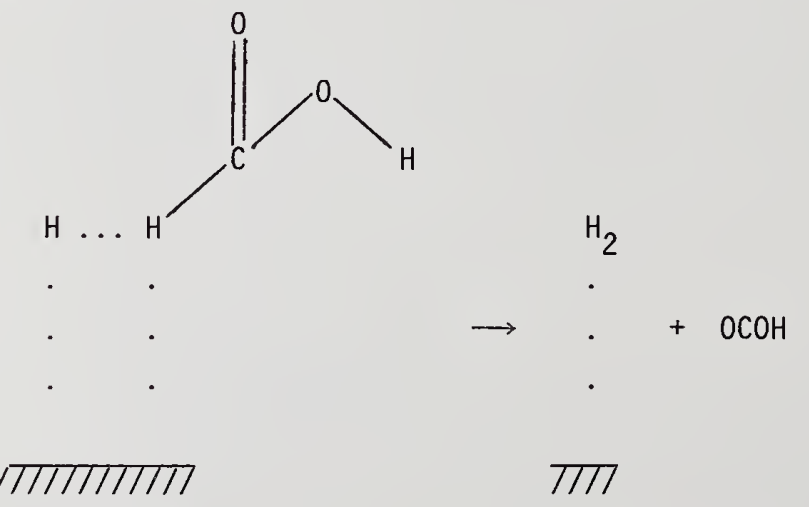

although such an enhancement effect has not yet been examined experimentally. Since there is no exchange between the $\mathrm{H}$ atoms in the $\mathrm{CH}$ and $\mathrm{OH}$ groups, it quite likely that the $\mathrm{OCOH}$ radical may be produced from a different intermediate (as shown above) than the one that leads to $\mathrm{CO}_{2}$ formation.

The ratio of $\mathrm{CO}_{2}$ to $\mathrm{CO}$ found in the present work was approximately 25:1 as determined by comparing the relative intensities of the ${ }^{13} \mathrm{CO}_{2}$ peak at $2285 \mathrm{~cm}^{-1}$ to the intensities of 
the peaks of $\mathrm{CO}$ and $\mathrm{CO} \cdot \mathrm{H}_{2} \mathrm{O}$. The extinction coefficients of $\mathrm{CO}$ and $\mathrm{CO}_{2}$ in argon were determined separately, and the extinction coefficient of the $\mathrm{CO} \cdot \mathrm{H}_{2} \mathrm{O}$ complex $\left(2149 \mathrm{~cm}^{-1}\right)$ was assumed to be the same as that of $\mathrm{CO}$. The $\mathrm{CO}_{2} / \mathrm{CO}$ ratio is in good agreement with the value determined in a similar static system in our Laboratory [21].

\section{Concluding Remarks}

The results of this work show convincingly that the matrix isolation technique can be effectively used to measure the rates of formation of stable products and, most importantly, to isolate hitherto unknown free radical species that escape from catalyst surfaces. Detailed spectroscopic studies of the products from the decomposition of $\mathrm{HCOOH}, \mathrm{OCOOH}$ and $H C O O D$ indicate that $\mathrm{OCOH}(\mathrm{D})$ radicals have been generated and escaped from the Pt surface into the gas phase. The radical was produced from the direct abstraction reaction:

$$
\mathrm{HCOOH}+\mathrm{M} \rightarrow \mathrm{M} . \mathrm{H}+\mathrm{H}+\mathrm{O}=\mathrm{C}-\mathrm{O}-\mathrm{H}
$$

The $\mathrm{OCOH}$ radical thus formed may undergo further decomposition (i.e., $\mathrm{OCOH} \rightarrow \mathrm{CO}_{2}+\mathrm{H}, \Delta \mathrm{H}^{\circ}=$ $8 \mathrm{kcal} / \mathrm{mol}$ [15]) and initiate homogeneous gas phase reactions under high pressure conditions.

The pretreatment of the "cleaned" Pt surface with $\mathrm{H}_{2}$ prior to decomposition reaction enhanced the rate of $\mathrm{CO}_{2}$ formation pronouncedly. This illustrates the important catalytic effect of the adsorbed $\mathrm{H}$ atoms on reducing the barrier for $\mathrm{CO}_{2}$ formation. Further work on the effect of pretreatment of catalysts with various molecules $\left(\mathrm{H}_{2}, \mathrm{D}_{2}, \mathrm{H}_{2} \mathrm{O}\right.$, CO, etc. $)$ will be conducted. Additionally, the effect of vibrational excitation with lasers on the rates of formation of different products, which was first demonstrated in this Laboratory using a static system [21], will also be thoroughly investigated using the matrix-catalysis technique.

The authors gratefully acknowledge Drs. M. E. Umstead and R. R. Smardzewski for their initial interest in this work.

\section{References}

[1] Bond, G. C., in Catalysis by Metals (Academic Press, NY, 1962).

[2] Mars, P., Scholten, J. J. F., and Zwietering, P., Adv. Cata1. 14, 35 (1963).

[3] Dahlberg, S. C., Fisk, G. A., and Rye, R. R., J. Catal. 36, 224 (1975).

[4] Lunsford, J. H., Adv. Catal. 22, 265 (1972).

[5] Gardner, C. L. and Casey, E. J., Catal. Rev. 9, 1 (1974).

[6] Shalya, V. V., Kashirina, R. A., Kaninskaya, R. L., and Gorokhovatski i, Ya. B., Dok1. Akad. Nauk SSSR 180, 1408 (1968). 
[7] Smardzewski, R. R. and Fox, W. B., J. Chem Phys. 60, 2980 (1974).

[8] Int. Crit. Tables 6 , 136.

[9] Perutz, R. N. and Turner, J. J., J. Chem. Soc. Farad. Trans. II, 69, 452 (1973).

[10] Coolidge, A. S., J. Am. Chem. Soc. 50, 2166 (1928).

[11] Kishida, S. and Nakamoto, K., J. Chem. Phys. 41, 1558 (1964) and references therein.

[12] Miyazawa, T. and Pitzer, K. S., J. Chem. Phys. 30, 1076 (1959).

[13] Lerner, R. G., Dailey, B. P., and Friend, J. P., J. Chem. Phys. 26, 680 (1957).

[14] Tevault, D. E., Lin, M. C., Umstead, M. E., and Smardzewski, R. R., Int. J. Chem. Kin., to appear.

[15] Tevault, D. E., Lin, M. C., and Smardzewski, R. R., to be submitted.

[16] Smardzewski, R. R., Applied Spectroscopy, 31, 332 (1977).

[17] Andrews, L., Tevault, D. E., and Smardzewski, R. R., Applied Spectroscopy, 32, 157 (1978).

[18] Milligan, D. E. and Jacox, M. E., J. Chem. Phys. 54, 927 (1971).

[19] Tingey, H. C. and Hinshelwood, C. N., J. Chem. Soc. 121, 1668 (1922).

[20] Block, J. and Vogl, J., Elektrochem., Ber. Bunsenges, Physik Chem. 63, 3 (1959).

[21] Umstead, M. E. and Lin, M. C., J. Phys. Chem., to appear. 


\section{THERMODYNAMIC DATA AND APPLICATIONS}


National Bureau of Standards Special Publication 561, Proceedings of the 10th Materials Research Symposium on Characterization of High Temperature Vapors and Gases held at NBS, Gaithersburg, Maryland, September 18-22, 1978. Issued 0ctober 1979.

\section{REFERENCE DATA FOR HIGH TEMPERATURE SYSTEMS}

L. H. Gevantman, H. J. White, Jr., and David R. Lide, Jr.

Office of Standard Reference Data

National Bureau of Standards

Washington, DC 20234

\section{Introduction}

The characterization of high-temperature systems is a complex task which frequently requires a large amount of auxiliary information. Because direct experimental measurement of properties of high-temperature species is difficult, it is often necessary to resort to calculations which provide additional information to aid in interpretation of the measurements. Thus, high temperature science is particularly dependent on good models for the systems under study and on good data to put into these models. This paper will review the current status of sources of physical and chemical reference data of interest in high temperature chemistry. It describes the data centers of the National Standard Reference Data System and the role of the National Bureau of Standards in administering this system.

\section{Thermodynamics}

A quantitative knowledge of the thermodynamics of a high-temperature system permits estimates of the equilibrium concentration of atomic and molecular species as a function of temperature and pressure. The most widely used data source for this purpose is the JANAF Thermochemical Tables, prepared at the Dow Chemical Company on a continuing basis since 1960. Financial support has been provided by the Department of Defense and, more recently, by the Department of Energy. One of the great attractions of these tables is the convenient standardized format for presenting the various thermodynamic properties as a function of temperature up to $6000 \mathrm{~K}$. The National Bureau of Standards published a consolidated volume, including about 1000 tables, in 1971 under the title "JANAF Thermochemical Tables, Second Edition" $[1]^{1}$. An additional 300 tables have appeared in supplementary collections in the Journal of Physical and Chemical Reference Data in 1974 [2] and 1975 [3], and a third supplement will appear in the same journal in late 1978.

Another important source of data on inorganic and simple organic species is the Chemical Thermodynamics Data Center at the National Bureau of Standards. The primary output of this center is standard state enthalpies, entropies, and Gibbs energies, published

${ }^{1}$ Figures in brackets indicate the literature references at the end of this paper. 
in the NBS Technical Note 270 series [4]. The group also makes a major contribution to the Bulletin of Chemical Thermodynamics [5], which provides an annual index to the published thermodynamic literature. The Chemical Thermodynamics Data Center interacts closely with the JANAF group, since internal consistency of thermodynamic tables is of paramount importance.

While these publications are familiar to most high temperature scientists in the western world, the extensive compilation activities in the Soviet Union, especially at the Institute for High Temperatures, should also be mentioned. This group produces both standard state tables [6] and tables of thermodynamic properties as a function of temperature [7]. Cooperation between the Soviet group and its U.S. counterparts has taken place for a number of years and is expected to become closer in the future.

\section{Ion Thermochemistry}

Data on ionization and appearance potentials of gaseous ions are compiled and evaluated by the Ion Energetics Data Center at NBS. Where possible, these data are combined with other thermodynamic information to yield enthalpies of formation of the ionic species. A recent publication, "Energetics of Gaseous Ions" [8], covers the literature through 1973. Ionization potentials are given for close to 2000 positive and negative ions.

\section{Chemical Kinetics}

Two data centers deal with rates of gas-phase reactions of interest in high-temperature chemistry. The Chemical Kinetics Information Center at NBS has produced several compilations: of evaluated rate constants for elementary gas phase reactions, primarily at room temperature. The program of this Center has been extended to emphasize high-temperature reactions of importance in fossil fuel combustion. A bibliography, "Chemical Kinetics of Gas Phase Combustion of Fuels" [9], appeared recently, and compilations of data are in progress.

Another kinetics group at Leeds University in the United Kingdom has specialized in rates of high temperature reactions. The most recent of their publications is "Evaluated Kinetic Data for High Temperature Reactions, Volume $3^{11}$ [10]. A further compilation from this group will be published in 1979 in the Journal of Physical and Chemical Reference Data.

Finally, mention should be made of a current project at the Aerospace Corporation which is developing a simplified data sheet format for presenting evaluated kinetics data over a wide range of temperature. The hope is to devise a format for kinetics data as popular as the JANAF format for thermodynamic data.

\section{Energy Transfer}

Collision cross sections and related data have been compiled for some years at the Electron Collision Cross Section Data Center of the Joint Institute for Laboratory 
Astrophysics of the National Bureau of Standards and the University of Colorado. Among the current interests of this Center are cross section data relevant to MHD systems. Compilation of data on collisional energy transfer between atoms and simple molecules has also been started.

\section{Spectroscopy}

Compilations of spectroscopic data and molecular constants are often useful for identifying high-temperature species and predicting their properties. Recent publications of interest in this regard include data on microwave spectra of diatomic [11] and triatomic [12] species, many of which are found only in high-temperature gaseous systems. An important forthcoming publication by Herzberg and Huber [13] will cover electronic energy levels and molecular constants of diatomic molecules. On the atomic side, the well-known NBS program on atomic energy levels and transition probabilities is continuing. The fourth volume of the Atomic Energy Levels series, covering the rare earth elements, was published in Apri1 1978 [14].

Most of the Data Centers mentioned above are components of the National Standard Reference Data System [15], which is administered by the National Bureau of Standards. The NSRDS consists of about 20 continuing data centers plus various short-term projects. Each data center has a we11-defined scope of responsibility and attempts to capture all data pertinent to this scope that are published in the world literature. The data are evaluated by experienced scientists in the particular field; after resolving discrepancies, compilations of recommended values are prepared. Uncertainty limits on the recommended values are always given, and the sources of the primary data and reasons for the choices are fully documented.

The NSRDS is coordinated by the Office of Standard Reference Data at NBS. This Office conducts an extensive publication program aimed at disseminating evaluated reference data to the scientific and technical community. The major publication is the Journal of Physical and Chemical Reference Data, published for NBS by the American Chemical Society and the American Institute of Physics. This quarterly journal contains compilations and critical reviews spanning the full range of the physical sciences. Other NSRDS outputs appear in NBS publication series distributed through the Superintendent of Documents of the U.S. Government Printing office and in other appropriate channels.

The data centers also respond to direct inquiries from the public, and in many cases are able to supply data that have not yet appeared in formal publications. A list of the current NSRDS data centers is given in the Appendix. In addition to the centers mentioned in this paper, several others deal with materials properties of interest to research workers in high temperature science. For example, the CINDAS group at Purdue University covers thermal conductivity of solids over a wide temperature range.

Finally, it should be emphasized that reference data activities are not restricted to the United States. Centralized programs have been developed in recent years in Germany, Japan, the Soviet Union, and other countries. Several international unions have active 
projects, and the International Council of Scientific Unions has established a Committee on Data for Science and Technology (CODATA) to promote and coordinate data activities in all scientific disciplines. CODATA has mounted an active program to improve dissemination of reference data, establish standards and formats, and generally promote the critical evaluation of data. One of its most successful projects has been a Task Group on Key Values in Thermodynamics, which has achieved international agreement on standard state thermodynamic properties for about 125 key compounds [16].

This paper has presented a brief summary of sources of reference data of interest in high-temperature science. Further information may be obtained from the data centers listed in the appendix or from the Office of Standard Reference Data.

\section{References}

[1] Stul1, D. R. and Prophet, H., JANAF Thermochemical Tables, Second edition, Nat. Stand. Ref. Ser., Nat. Bur. Stand. (U.S.), 37 (1971).

[2] Chase, M. W., et al., JANAF Thermochemical Tables, 1974 Supplement, J. Phys. Chem. Ref. Data 3:2, p. 311-489 (1974).

[3] Chase, M. W., et al., JANAF Thermochemical Tables, 1975 Supplement, J. Phys. Chem. Ref. Data 4:1, p. 1-175 (1975).

[4] Wagman, D. D., et al., Selected values of chemical thermodynamic properties, Nat. Bur. Stand. (U.S.), Tech. Note 270, Parts 3-7 (1968-1973).

[5] Bulletin of Chemical Thermodynamics, Robert D. Freeman, ed., Department of Chemistry, OKlahoma State University, Stillwater, OK 74074.

[6] Thermal Constants of Substances, A Handbook in Ten Parts, V. P. Glushko, ed., Institute for High Temperatures, Academy of Sciences, USSR.

[7] Thermodynamic Properties of Pure Substances, V. P. Glushko, ed., Institute for High Temperatures, Academy of Sciences, USSR.

[8] Rosenstock, H. M., Drax1, K., Steiner, B., and Herron, J. T., Energetics of gaseous ions, J. Phys. Chem. Ref. Data 6, Supplement 1 (1977).

[9] Westley, F., Chemical kinetics of the gas phase combustion of fuels (a bibliography on the rates and mechanisms of oxidation of aliphatic $C_{1}-C_{20}$ hydrocarbons and of their oxygenated derivatives), Nat. Bur. Stand. (U.S.), Spec. Publ. 449 (1976).

[10] Baulch, D. L., Drysdale, D. D., Duxbury, J., and Grant, S. J., Evaluated kinetic data for high temperature reactions, Volume 3 , Homogeneous gas phase reactions of the $\mathrm{O}_{2}-\mathrm{O}_{3}$ system, the $\mathrm{CO}-\mathrm{O}_{2}-\mathrm{H}_{2}$ system, and of sulphur-containing species (Butterworths, London, 1976).

[11] Lovas, F. J. and Tiemann, E., Microwave spectral tables, I. Diatomic molecules, J. Phys. Chem. Ref. Data 3:3 (1974), p. 609-770.

[12] Lovas, F. J., Microwave Spectral Tables, II. Triatomic molecules, J. Phys. Chem. Ref. Data 7:4 (in press).

[13] Herzberg, G. and Huber, K. P., to be published. 
[14] Martin, W. C., Zalubas, R., and Hagan, L., Atomic energy levels - the rare earth ele- . ments, the spectra of lanthanum, cerium, praseodymium, neodymium, promethium, samarium, europium, gadolinium, terbium, dysprosium, holmium, erbium, thulium, ytterbium, and lutetium, Nat. Stand. Ref. Data Ser., Nat. Bur. Stand. (U.S.), 60 (1978).

[15] Critical evaluation of data in physical sciences - A Status Report on the National Standard Reference Data System, Rossmassler, S. A., ed., Nat. Bur. Stand. (U.S.), Tech. Note 947 (May 1977).

[16] CODATA recommended key values for thermodynamics 1977, CODATA Bulletin 28, (April 1978).

\section{APPENDIX}

\section{NSRDS DATA CENTERS}

The data centers currently included in the National Standard Reference Data System are given below. Inquiries on data may be directed to all these centers.

\section{Energy and Environmental Data}

Atomic Energy Levels Data Center

Dr. W. C. Martin

Center for Radiation Research

National Bureau of Standards

Washington, D.C. 20234

Telephone: (301) 921-2011

Data Center for Atomic Transition Probabilities and Atomic Line Shapes and Shifts

Dr. W. L. Wiese

Center for Radiation Research

National Bureau of Standards

Washington, D.C. 20234

Telephone: (301) 921-2071

Direct inquiries to: Ms. Georgia Martin (301) 921-3374

Atomic Collision Cross Section Information Center

Dr. E. C. Beaty

Joint Institute for Laboratory Astrophysics

University of Colorado

Boulder, Colorado 80309

Telephone: (303) 492-7801 
Ion Energetics Data Center

Dr. H. M. Rosenstock

Center for Thermodynamics and Molecular Science

National Bureau of Standards

Washington, D.C. 20234

Telephone: (301) 921-2793

Chemical Kinetics Information Center

Dr. R. F. Hampson, Jr.

Center for Thermodynamics and Molecular Science

National Bureau of Standards

Washington, D.C. 20234

Telephone: (301) 921-2174

Radiation Chemistry Data Center

Dr. Alberta B. Ross

University of Notre Dame

Radiation Laboratory

Notre Dame, Indiana 46556

Telephone: (219) 283-6527

Molten Salts Data Center

Dr. G. J. Janz

Rensselaer Polytechnic Institute

Department of Chemistry

Troy, New York 12181

Telephone: (518) 270-6344

X-Ray and Ionizing Radiation Data Center

Mr. J. H. Hubbel1

Center for Radiation Research

National Bureau of Standards

Washington, D.C. 20234

Telephone: (301) 921-2685 
Photonuclear Data Center

Dr. E. G. Fuller

Center for Radiation Research

National Bureau of Standards

Washington, D.C. 20234

Telephone: (301) 921-2625

Table of Isotopes Center

Dr. C. M. Lederer

Lawrence Berkeley Laboratory

University of California

Berkeley, California 94720

Telephone: (415) 843-2740, ext. 5995

\section{Industrial Process Data}

Chemical Thermodynamics Data Center

Mr. D. D. Wagman

Center for Thermodynamics and Molecular Science

National Bureau of Standards

Washington, D.C. 20234

Telephone: (301) 921-2773

Aqueous Electrolyte Data Center

Dr. B. R. Staples

Center for Thermodynamics and Molecular Science

National Bureau of Standards

Washington, D.C. 20234

Telephone: (301) 921-3632

Thermodynamics Research Center

Dr. B. J. Zwolinski

Texas A \& M University

Department of Chemistry

College Station, Texas 77843

Telephone: (713) 846-8765 
Cryogenic Data Center

Mr. N. A. 01 ien

Center for Mechanical Engineering and Process Technology

National Bureau of Standards

Boulder, Colorado 80303

Telephone: (303) 499-1000, ext. 3257

Center for Information and Numerical Data Analysis and Synthesis (CINDAS)

Dr. Y. S. Touloukian

Purdue University

CINDAS

2595 Yeager Road

West Lafayette, Indiana 47906

Telephone: (317) 463-1581

To11 Free: (800) 428-7675

Direct inquiries to: $M r$. W. H. Shafer

High Pressure Data Center

Dr. Leo Merrill

574 Clark Library

Brigham Young University

Provo, Utah 84601

Telephone: (801) 374-1211, ext. 3477

Alloy Data Center

Dr. L. H. Bennett

Center for Materials Science

National Bureau of Standards

Washington, D.C. 20234

Telephone: (301) 921-2917 
Crystal Data Center

Dr. A. D. Mighel1

Center for Materials Science

National Bureau of Standards

Washington, D.C. 20234

Telephone: (301) 921-2950

Diffusion in Metals Data Center

Dr. D. B. Butrymowicz

Center for Materials Science

National Bureau of Standards

Washington, D.C. 20234

Telephone: (301) 921-3351

Superconductive Materials Data Center

Dr. B. W. Roberts

General Electric Company

Research \& Development Center

P. 0. Box 8

Schenectady, New York 12301

Telephone: (518) 385-8430

Physical Science Data

Fundamental Particle Data Center

Dr. Robert Kelly

Lawrence Berkeley Laboratory

University of California

Berkeley, California 94720

Telephone: (415) 843-2740, ext. 5885

Molecular Spectra Data Center

Dr. F. J. Lovas

Center for Thermodynamics and Molecular Science

National Bureau of Standards

Washington, D.C. 20234

Telephone: (301) 921-2021 
National Bureau of Standards Special Publication 561, Proceedings of the 10th Materials Research Symposium on Characterization of High Temperature Vapors and Gases held at NBS, Gaithersburg, Maryland, September 18-22, 1978. Issued October 1979.

\title{
EVALUATION AND COMPILATION OF THE THERMODYNAMIC PROPERTIES OF HIGH TEMPERATURE SPECIES
}

\author{
Malcolm W. Chase, Joseph R. Downey, and Alan N. Syverud \\ The Dow Chemical Company \\ Thermal Research \\ Midland, MI 48640
}

A continuous effort is being made to add new chemical species to the JANAF Thermochemical Tables and to revise existing outdated tables. The selection of species to be studied depends on current interest of the U.S. Air Force and U.S. Department of Energy. In addition to producing these tables, we are concerned with making improvements in three areas. First, prompted by user's comments, we hope to include additional information such as heats of atomization, crystal structures and more detailed transition data. Second, calculational techniques are being re-evaluated, upgraded, or extended, where necessary, and fully documented. For example, the monatomic gas partition function has been re-examined to provide a better assessment of uncertainty and a possible extension of the tables to temperatures in excess of $6000 \mathrm{~K}$. Accuracy of various forms of the diatomic gas partition function is under study. Where it is practical and appropriate, we now include higher-order corrections and, in some cases, rotation-vibration cutoff. Third, duplicate efforts are being reduced by cooperative ventures with other data centers and researchers.

All efforts to provide high quality thermochemical tables are limited by the available experimental and theoretical data. The interaction between the tables and the data will be discussed in terms of the limitations each imposes on the other.

\section{Introduction}

The JANAF Thermochemical Tables are the oldest regularly maintained and updated compilation of temperature dependent thermochemical properties of chemical species in the United States. Originally sponsored by the Department of Defense in 1959, they were later continued with Air Force funds. Current support is from the Air Force Office of Scientific Research and the Department of Energy. 
The distribution of these thermochemical tables is through quarterly supplements using mailing lists which are controlled by the Air Force Office of Scientific Research (AFOSR) and the Department of Energy (DOE). These tables are also published in the open literature in the NSRDS-NBS series with two-year supplements in the Journal of Physical and Chemical reference data $[1]^{1}$.

The chemical species studied in this project are chosen yearly on the basis of relevancy to current and future interests of the Air Force and the Department of Energy. These interests are monitored by Dr. Joseph F. Masi (Directorate of Aerospace Sciences, AFOSR and Mr. Francis E. Spencer (PETC/Combustion Division, DOE). The chosen species may be new (i.e., not previously studied by the JANAF staff) or old (i.e., the existing table is to be revised).

The JANAF Thermochemical Tables are documented such that each table contains the following information: (1) a tabulation of temperature dependent values (at $100 \mathrm{~K}$ intervals and $298.15 \mathrm{~K}$ ) of $\mathrm{CP}^{\circ}, \mathrm{S}^{\circ},-\left(\mathrm{G}^{\circ}-\mathrm{H}_{298}^{\circ}\right) / \mathrm{T}, \mathrm{H}^{\circ}-\mathrm{H}_{298}^{\circ}, \Delta \mathrm{Hf} \mathrm{f}^{\circ}, \Delta \mathrm{Gf}^{\circ}$, and 1 og $\mathrm{Kp}$, and (2) a text which describes the critical evaluation of the adopted values and itemizes input data used in the calculations. Also, the method of calculation is stated if it differs from the traditional JANAF technique. Based on users comments, we have modified the previous format of the text to include some additional information. For example, we now prominently display the dissociation energy for diatomic gases and the heat of atomization for polyatomic gases. In the future, we anticipate adding crystal structure data and more detailed data at phase transitions. We are also considering the use of temperature increments smaller than $100 \mathrm{~K}$ in cases such as liquid sulfur which has a lambda-type anomaly in $C \mathrm{p}^{\circ}$. Since many users need to interpolate the values in our tables (perhaps using an equation) tabulated values at intervals less than $100 \mathrm{~K}$ would be useful for temperature ranges where the temperaturedependent functions change very rapidly and/or nonuniformly.

The quality and the useful range of each thermochemical table depend not only on the available experimental and theoretical data but also on the methods used to calculate the functions $C \mathrm{CP}^{\circ}, \mathrm{S}^{\circ}, \mathrm{H}^{\circ}$, and $-\left(\mathrm{G}^{\circ}-\mathrm{H}^{\circ}\right) / T$. In our study of chemical species, we decide on the degree of sophistication of treatment based on our interpretation of the "goodness" and "extent" of available data. Existence of sufficiently good data for a diatomic gas, for example, may justify use of a direct summation treatment instead of the approximation of Mayer and Mayer. The more sophisticated treatment should give a significant increase in accuracy in order to justify the extra time and effort. We must judge from the available data whether this will be the case.

When the study is completed, we compile a list of "missing data" which, if available, would significantly reduce the uncertainty in the table. Our aim is to promote experimental thermochemical and spectroscopic studies in those areas which are unknown or uncertain instead of those which are well known.

To illustrate the problems in examining chemical species, we will discuss in outline form the interaction between the interpretation of data, the form of the partition function used, and the "inadequate" data. We will do this for monatomic and diatomic gases: in

${ }^{1}$ Figures in brackets indicate the literature references at the end of this paper. 
particular, $\mathrm{He}(\mathrm{g}), \mathrm{Na}(\mathrm{g}), \operatorname{SiCl}(\mathrm{g}), \mathrm{H}_{2}{ }^{+}(\mathrm{g})$, and $\mathrm{ClF}(\mathrm{g})$. Additional problems arise for polyatomic gases and for condensed phases but these will not be discussed at this time. This presentation is given in narrative form with pertinent references listed in the bibliography.

\section{Monatomic Gases}

Our efforts during the past year have dealt with problems which would arise if the tables for monatomic gases were extended to higher temperatures (e.g., T>6000 K), and the formulation of more definitive procedures for producing the tables in their present format $(T<6000 \mathrm{~K})$. Since there is a total lack of experimental thermochemical data for high temperature monatomic gases, statistical mechanics must be used to calculate the thermochemical properties. The problem of finding a suitable "cutoff" procedure for the electronic partition function constitutes the main problem.

For an ideal gas statistical mechanics may be used to calculate the thermodynamic properties of any atom or molecule, no matter what the complexity, provided the appropriate partition function is employed. For a monatomic gas only an electronic term $\left(Q_{e}\right)$ and a translational term $\left(Q_{t r}\right)$ need be considered. If these can be accurately determined at all temperatures then the thermodynamic properties may be accurately calculated via standard formulas.

$Q_{t r}$ is usually derived from a particle in a box procedure, the only assumptions being the replacement of a summation of closely spaced levels by an integration and the applicability of Boltzmann (classical) statistics. These assumptions are invalid when the gas density becomes very high (examples are the interior of stars and the electron gas in metals). At temperatures near absolute zero the classical calculation of $Q_{t r}$ will also break down. In addition to the nonapplicability of Boltzmann statistics at these temperatures, the thermal energy is such that only a small number of translational levels are accessible and the integration is no longer a valid approximation. We shall not be concerned with these deviations from classical behavior since they occur below $10 \mathrm{~K}$ for all atomic and molecular systems under standard conditions (except the electron gas ${ }^{2}$ ). Under all other conditions, classical statistics will be obeyed and $Q_{\text {tr }}$ may be accurately calculated.

${ }^{2}$ For the electron gas these "low-temperature" deviations extend to $\sim 1200 \mathrm{~K}$ at one atmosphere pressure due to the low mass of the electron. The use of classical (rather than quantum) statistics leads to a bias of $\sim 0.7 \mathrm{kcal} / \mathrm{mol}$ in the values of $\left(\mathrm{H}_{0}^{\circ}-\mathrm{H}_{298}^{\circ}\right)$ for the electron gas. At this time, we should note that the electron and any ion lead to two problems or misunderstandings. First, there is the $0.7 \mathrm{kcal} / \mathrm{mol}$ bias just mentioned and second, in the use of heat of formation for ions some conventions do not include the enthalpy of the (classical) electron. 
In the JANAF Thermochemical Tables we normally use observed values for the energy in the electronic partition function.

$$
Q_{e}=\sum_{n} g_{n} \exp \left(-\varepsilon_{n} / k T\right)
$$

For a hydrogen-like atom, the rapid initial increase of $\varepsilon_{n}$ with principal quantum number, $n$, usually leads to the statement that all levels other than the ground state may be neglected since the exponential term becomes vanishingly smal1. However, the exponential term remains finite and this, coupled with the fact that quantum mechanics predicts an infinite number of energy levels, seemingly leads to infinite values of $Q_{e}$.

Strickler has shown that the way out of this paradox is to realize that for large values of $\mathrm{n}$ the wave functions will not be hydrogenic, but will be described by the container due to the large volume of a single atom. Since this is essentially a particle in a box problem, there will then be a finite number of energy levels resulting in a finite $Q_{e}$. Since there is a finite number of energy levels, a reasonable approach would be to simply cut off the summation process for $Q_{e}$ at some point.

Several cut-off procedures have been used in the past and they generally fall into these categories:

- no dependence on temperature and pressure (JANAF)

- dependence on temperature only (IP lowering)

- dependence on temperature and pressure (Bethe)

- dependence on density of charged particles (plasmas).

The first category includes using the ground electronic level only or summing over some fixed number of levels which is usually arbitrary (to $n=5$ for example). These methods generally fail at high temperatures for obvious reasons. The present JANAF method of summing over all observed levels falls in this category (no $P$ or $T$ dependence).

Methods in the second category generally use a quantity called the "ionization potential lowering" which is a function of temperature only. The theoretical basis of this method is that the effective ionization potential will be lowered due to collisions with surrounding atoms such that an outer electron with a binding energy less that kT will generally be released (ionization) in a collision. It should be noted that at high enough temperatures all levels other than the ground state will be eliminated from the sum. The method where the summation is halted at IP-kT will be referred to later as the TEMPER method.

The methods dependent on both $P$ and $T$ are mainly due to the assumption that each atom may occupy only a limited volume in space. These methods are often referred to as excluded volume methods and are, essentially, particle in a box methods. Fermi and Bethe both derived cut-off formulas based on these assumptions. The method due to Bethe assumes hydrogenic orbits and limits the molecular volume to the molar volume divided by Avogadro's number. Bethe's criterion (at 1 atm pressure) then reduces to:

$$
n_{\max }=2.461 T^{1 / 6}(T=K) \text {. }
$$


Fermi's criterion has a more complex formulation but gives nearly the same values for $n_{\text {max }}$. The Bethe formulation is easy to implement though care must be taken to prevent discontinuities since $n_{\max }$ is allowed to change in integer increments only. This method of limiting the sum will be referred to later as the BETHE method.

The last general method of limiting the summation uses methods involving ionization potential lowering, the amount of which is a function of electron and ionized particle densities. These methods are not further considered here since they apply to a plasma only and cannot be used for a single species as required in the JANAF Thermochemical Tables.

In addition to the cut-off method some decision must be made whether to include predicted, but unobserved, energy levels which lie below the cut-off point. If they are to be included some method of filling in the missing levels must be found. Several authors have shown that only a rough approximation of the energies of upper levels is needed so a simple method of calculating how many levels are missing can be combined with a rough approximation of their energy. The missing levels for any value of $n$ are then predicted to lie at the energy of the highest known level for that value of $n$. This approximation becomes more accurate as $n$ increases, since the atoms behave in a more hydrogenic manner. This method will be referred to as the FILL procedure.

The various thermodynamic functions depend on the partition function $Q$ and its temperature derivatives. The dependence of each property on $Q$, and therefore on the cut-off and $f i 11$ methods involved in $Q_{e}$, is different and must be evaluated separately. One expects $C_{p}^{0} / R$ to show the most radical behavior since it involves both first and second derivatives of $Q$. In order to illustrate the effect of the various cut-off procedures we calculated the various thermodynamic properties of $\mathrm{Na}(\mathrm{g})$ and $\mathrm{He}(\mathrm{g})$. The alkali metals have very low ionization potentials so this represents a worst-case approach whereas the rare gases have high ionization potentials and represent a best-case approach.

The labels on the plots are as follows:

ALLN - using all observed levels. This is the present JANAF method.

ALLN-FILL - using all observed levels and filling in missing levels using FILL option

TEMPER - summation over observed levels is cut-off at IP-kT

BETHE-FILL - summation over levels using BETHE cut-off and the FILL option circles - using ground state only

dashed line - translational contribution only.

The effects of the various cut-off procedures on the thermodynamic properties of sodium are shown in figures 1 and 2. A quick reference to these figures confirms that the largest effect is for the heat capacity where differences exceeding 1000 percent are noted near $5000 \mathrm{~K}$. The least sensitive property is the free energy function, $-\left(G^{\circ}-H_{0}^{\circ}\right) / R T$, and this is fortunate since this term is important in equilibrium calculations. The largest deviations in the thermal properties arise when using the ALLN-FILL procedure and this will become more severe as energy levels are discovered to higher $n$ values. For these reasons this procedure has not been used in the fast and will not be considered for future use since it obviously overestimates the contribution of upper states. 
As shown in figures 1 and 2 the TEMPER method yields the least deviation from the classical (ground state only) case. This is because more levels are eliminated from the summation as the temperature increases. In fact at high enough temperatures all levels are cut off except the ground state. The main objective to the TEMPER method is a philosophical one since this method leads to a narrower distribution of electrons over a set of energy levels with increasing temperature which is the opposite of what is expected from fundamental principles (Boltzmann distribution).

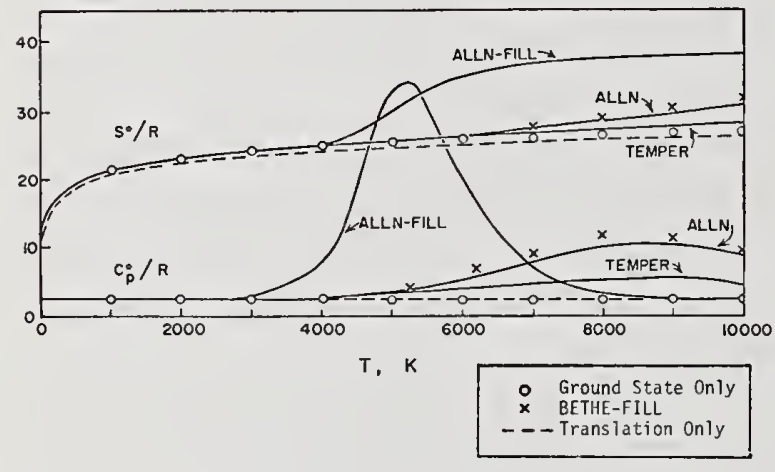

Figure 1. Heat capacity and entropy for sodium gas.

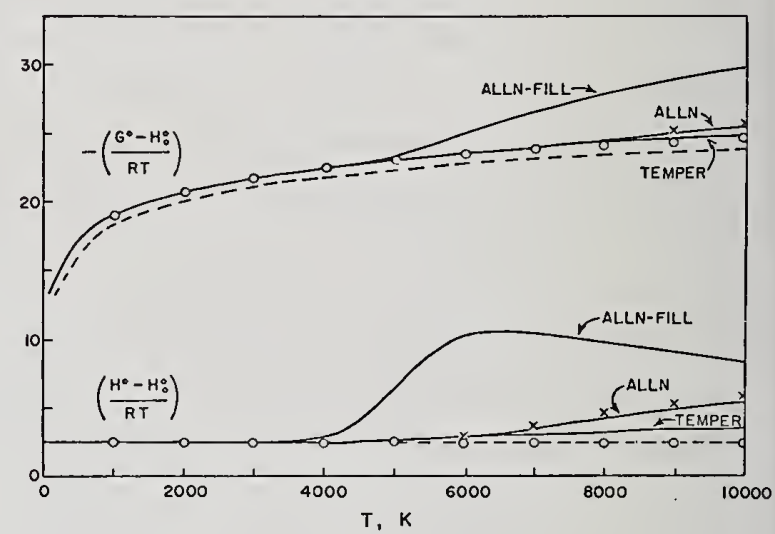

Figure 2. Free energy function and enthalpy for sodium gas.

The BETHE-FILL method results in thermodynamic properties slightly higher than for the ALLN method for sodium as illustrated in figures 1 and 2.

If $\mathrm{Na}(\mathrm{g})$ represents a worst-case approach then $\mathrm{He}(\mathrm{g})$ represents a best-case approach since it has the highest ionization potential of any neutral atom. Its thermodynamic properties are illustrated in figures 3 and 4 and the same general behavior of the various procedures is observed. The big difference is the temperature at which the various cut-off procedures have an effect. Neglecting the ALLN-FILL method, $\mathrm{Na}(\mathrm{g})$ shows deviations beginning at $\sim 4000 \mathrm{~K}$ while $\mathrm{He}(\mathrm{g})$ does not show deviations until $\sim 20000 \mathrm{~K}$.

Although we are interested primarily in the calculation of properties of individual species it seems of interest to mention some of the salient properties of plasmas, i.e., mixtures of atoms, ions, and electrons in thermal equilibrium. The reason for this is that at temperatures high enough for significant contributions from excited states in $Q_{e}$ to be important, one begins to get significant amounts of ionization, e.g. $\mathrm{Na}(\mathrm{g}) \rightarrow \mathrm{Na}^{+}(\mathrm{g})+\mathrm{e}^{-}(\mathrm{g})$. Presumably the primary use of JANAF tables at such temperatures would be to calculate equilibrium properties of these systems. 


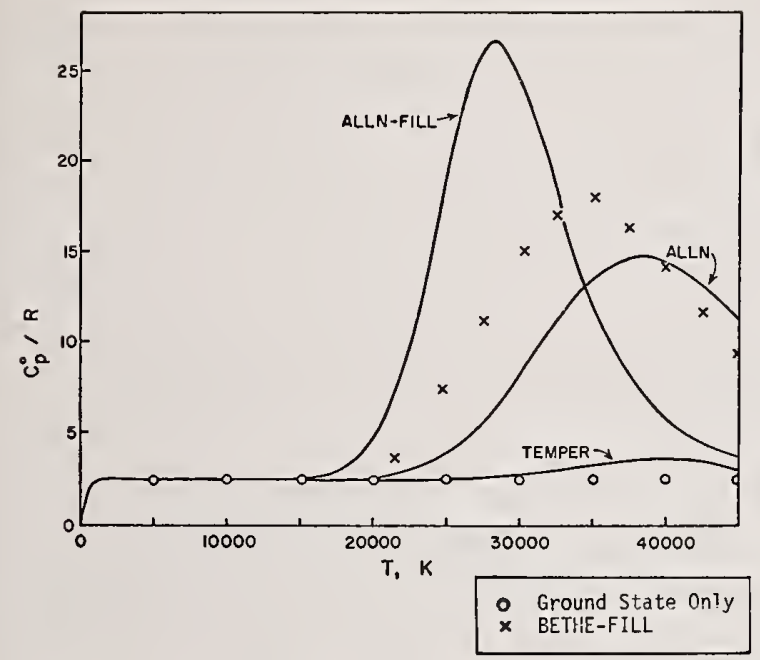

Figure 3. Heat capacity of helium gas.

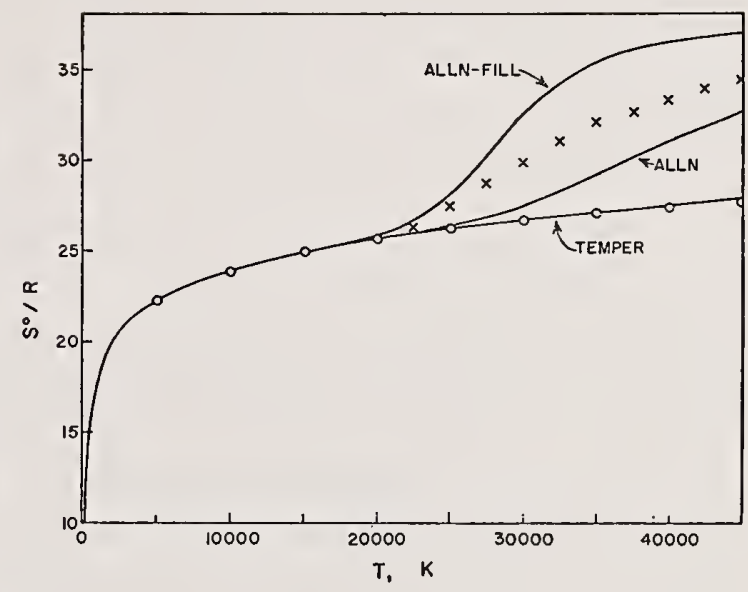

Figure 4. Entropy of helium gas.

Several investigators have shown that the equilibrium properties of these plasmas are virtually independent of the cut-off procedure as long as the same procedure is consistently used. Therefore, al though the cut-off and FILL procedures have a large effect on the thermodynamic properties of individual species there is only a small effect on equilibrium properties in plasmas.

In dealing with real systems such as plasmas one is always faced with assigning a portion of the interaction to standard states and another portion to an equation of state. This is not unlike treating dissociation in molecular systems where one may treat the system as a perfect solution of three components or as a non-ideal solution of one or two components with any other contributions accounted for by the equation of state. In either the atomic or molecular cases the choice and division of components is arbitrary to some extent, but the important thing is that consistency is maintained so that all of the energy levels are counted once and once only. In order to maintain consistency the same cut-off procedure must be used for all species or a large bias in the equilibrium properties of plasmas may result.

There is no absolute means of determining which cut-off method is correct based on measured data since appropriate experiments at these high temperatures do not seem feasible in the near future. Therefore, it seems desirable to incorporate some statement concerning at what temperature the cut-off criteria becomes important and some measure of the uncertainty due to it. The free energy function $\left[-\left(G^{\circ}-H_{298}^{\circ}\right) / T\right]$ seems to be preferable since it relates the free energy of formation at any temperature to the heat of formation at $298.15 \mathrm{~K}$ and, therefore, enters directly into equilibrium calculations. The temperature at which the difference in the free energy function, calculated via the BETHE-FILL and TEMPER procedures, reaches $0.1 \mathrm{cal} /(\mathrm{mol} \mathrm{K})$ will be tabulated on future JANAF tables as a measure of uncertainty due to the cut-off procedures. For $\mathrm{Na}(\mathrm{g})$ this is $\sim 5100 \mathrm{~K}$ and for $\mathrm{He}(\mathrm{g})$ it is $\sim 24,400 \mathrm{~K}$. 
These represent essentially the worst and best cases, respectively, for neutral atoms. The ionization potentials for positively charged ions are generally quite high and their properties will be valid to even higher temperatures before uncertainties due to cut-off methods arise. The cut-off procedure used will also be clearly stated on each monatomic gas phase table.

\section{Diatomic Gases}

Although limited $\mathrm{Cp}^{\circ}$ data are sometimes available for diatomic gases, we normally use the partition function from statistical mechanics in order to calculate thermodynamic functions. Since the start of the JANAF project, there has been a great increase in the availability of experimental and theoretical energy level data for diatomics. Thus, the accuracy of calculated thermodynamic functions for many species is now affected more by procedural limitations than by data limitations. This prompted us to review and update our procedures for diatomics. Some results from this process are shown later.

Evaluation of the "true" diatomic partition function is subject to the same limitations given previously for monatomics: namely, the need to approximate missing energy level data and to use cut-off procedures for high levels. These limitations now extend to the whole rovibronic (rotation-vibration-electronic) spectrum, not just to the electronic spectrum.

Rotation-vibration contributions introduce two new complications, one concerned with cut-off procedures and the other with approximations to the rovibronic partition function. The first difference is that theory requires there to be a finite number of rotationvibration levels for electronic states which dissociate to neutral atoms. Cut-off procedures should meet this requirement. It is not met by the approximate procedures which are often used as shortcuts for direct summation of the terms in the partition function. Approximate procedures may include zero-order, first-order or higher-order corrections to approximate the observed and extrapolated rotation-vibration levels. This combination of extrapolation and improper cut-off can lead to serious bias at high temperature in certain cases. A low-temperature bias occurs when approximate procedures fail to account for unequal statistical weights and multiplet splitting in the rovibronic levels of the ground state.

The original JANAF procedure used a form of the Mayer and Mayer approximation for the rovibronic partition function. This approximation uses first-order corrections to $w_{e}$ for anharmonicity $\left(\omega_{e} x_{e}\right)$ and to $B_{e}$ for rotation-vibration interaction $\left(\propto_{e}\right)$ and rotational distortion $\left(D_{e}\right)$. The method neglects rotational splitting and does not allow rotationvibration cut-off.

At first the JANAF Thermochemical Tables considered only the electronic ground state. Later calculations considered excited states but used ground state rovibronic constants for all states. Often the excited states were poorly characterized so that this was a reasonable first step in accounting for the contribution of excited states. The next improvement involved the use of the actual rotation-vibration constants for each excited state. 
Using $\mathrm{SiCl}$ as an example, we have calculated the thermodynamic functions from these approximations. In the following table we have summarized the entropy at 298.15, 3000, and $6000 \mathrm{~K}$. These calculations used the observed data for $\mathrm{SiCl}$ and in one case an estimated ${ }^{4} \Sigma^{-}$electronic state at $26000 \mathrm{~cm}^{-1}$. The ground state is ${ }^{2} \Pi$ with a splitting of $207.2 \mathrm{~cm}^{-1}$. Note that the $S_{298}^{\circ}$ values are all the same except when ground-state splitting is ignored. Because the first excited state lies above $20000 \mathrm{~cm}^{-1}$, it has no significant contribution below $3000 \mathrm{~K}$. At higher temperatures the differences in the calculated entropy are noticeable and are comparable to the small contribution of the "missing" ${ }_{\Sigma}{ }^{-}$state.

\section{Entropy of $\mathrm{SiCl}$ in $\mathrm{cal} /(\mathrm{mol} \mathrm{K})$}

\section{Method of Approximation}

ground state only, $g=4$

split ground state; two levels, each with

$$
\mathrm{g}=2
$$

al1 observed states, but using ground state constants

a11 observed states using appropriate rovibronic constants

all observed states and estimated ${ }^{4} \Sigma^{-}$
Temperature, $\mathrm{K}$

$\underline{298.15} \quad \underline{3000} \quad \underline{6000}$

$57.038 \quad 77.471 \quad 83.946$

$56.817 \quad 77.469 \quad 83.946$

$\begin{array}{lll}56.817 & 77.469 & 83.981\end{array}$

$56.817 \quad 77.469 \quad 84.018$

$56.817 \quad 77.470 \quad 84.046$

We decide which approximation to use after appraisal of all avaliable data. For $\mathrm{SiCl}$, the split-ground-state calculation is sufficient up to $3000 \mathrm{~K}$. At higher temperatures it is desirable to consider use of a better approximation and inclusion of "missing" states. In this case the "missing" data can be inferred by examining all four silicon halides for trends in electronic energies and molecular constants. We expect such trends in properties for like electronic states arising from analogous molecular orbital configurations (i.e., smooth trends in electronic energy or in energy separations, $w_{e}, B_{e}, w_{e} x_{e} / w_{e}, \alpha_{e} / B_{e}$, etc). Such comparisons suggest that a ${ }^{4} \Sigma^{-}$electronic state should exist at $26000 \pm 3000 \mathrm{~cm}^{-1}$ for $\mathrm{SiCl}$.

Effects due to missing electronic data can be large at much lower temperatures. This is illustrated by the alkaline earth sulfides. For these species the expected low-lying excited states have not yet been observed, making it necessary to estimate their energy levels by comparison with the alkaline earth oxides. 0xide data emphasize that metal-atom substitution does not lead to monotonic changes in energies and thermodynamic effects of low-lying states. MgS, by analogy with MgO, is likely to be the extreme case. Data for 
MgO suggest that the ${ }^{3} \Pi$ and ${ }^{1} \Pi$ excited states of MgS should be near 2000 and $3000 \mathrm{~cm}^{-1}$. These estimates would change $S^{\circ}$ by $0.01 \mathrm{cal} /(\mathrm{mol} \mathrm{K}$ ) at $298 \mathrm{~K}$ and $2.7 \mathrm{cal} /(\mathrm{mol} \mathrm{K})$ at $1000 \mathrm{~K}$. Our approximate calculational methods are useful shortcuts for many diatomics, but they can be inadequate in two cases. These involve electronic states with shallow potentialenergy wells (i.e., low dissociation energies) and those in which the rotational energies deviate seriously from the simple formula for $l_{\Sigma}$ states.

The latter case is $i l l u s t r a t e d$ by rotational splitting in a multiplet ground state. Neglect of the splitting causes no error at high temperature but a significant error at low temperature; e.g., the bias in $\mathrm{S}_{298}^{\circ}$ is $\sim 0.2 \mathrm{cal} /(\mathrm{mol} \mathrm{K})$ for $\mathrm{SiCl}$. Our usual approximation is to treat the split levels as separate electronic states with appropriate statistical weights. This approximation is adequate unless the splitting is large. It is then necessary to use the correct energy-level equation for the specific ground state, preferably in the method of direct summation.

The problem caused by an electronic state with a low dissociation energy is illustrated by CIF. The ground state of CIF is ${ }^{1} \Sigma$ and there is a shallow ${ }^{3} \Pi$ state at $18721 \mathrm{~cm}^{-1}$ (well depth of $\sim 3000 \mathrm{~cm}^{-1}$ ). We made approximate calculations by several methods using the available data $\left(w_{e}, w_{e} x_{e}, B_{e}\right.$ and $\propto_{e}$ ) for these states. The resulting heat capacities (fig. 5) start to diverge and increase rapidly near $3000 \mathrm{~K}$. The more exact calculation by direct summation gives values which decrease above $3000 \mathrm{~K}$. Heat capacity values in cal/(mol K) at $6000 \mathrm{~K}$ are 8.4 (direct summation), 15.1 (rigid-rotator harmonic oscillator), 21.7 (JANAF approximation) and 39.2 (NRRA02 method in the NASA program of McBride and Gordon). The higher values are biased due to the neglect of rotation-vibration cutoff for the excited state. At this extreme temperature, it is true that the simpler the approximation, the less the bias. If approximate methods must be used, the bias can be minimized by omitting the excited state. Direct summation is the preferred method.

Approximate methods can fail at a surprisingly low temperature if $D_{0}^{\circ}$ is small in the ground state. The ion $\mathrm{H}_{2}^{+}$is a useful example even though, with $\mathrm{D}_{0}^{\circ}=21380 \mathrm{~cm}^{-1}(61.1$ $\mathrm{kcal} / \mathrm{mol}$ ), it is not an extreme case. Figure 6 compares $\mathrm{Cp}^{\circ}$ from the usual JANAF approximation with two summation calculations: an exact one based on all rotation-vibration levels and an approximate one based only on the spectroscopic constants $\left(\omega_{e}, \omega_{e} x_{e}, B_{e}\right.$ and $\left.\alpha_{e}\right)$ from the JANAF approximation. Deviations start even below $1500 \mathrm{~K}$.

The exact summation calculation can be done only for those species for which all pertinent rovibronic energies are known. This includes $\mathrm{H}_{2}, \mathrm{H}_{2}^{+}$, their isotopic forms, and a few other diatomics accurately known from theory. Even for these, there is the question of whether to include quasi-bound levels above $D_{0}^{\circ}$.

Our approximate summation for $\mathrm{H}_{2}^{+}$simulates the more typical case in which the observed data include only the lower rotation-vibration levels (i.e., <5 percent of all levels in the ground state). The resulting spectroscopic constants often yield inaccurate predictions of high energy levels. If these spectroscopic constants are combined with an arbitrary cut-off procedure, $\mathrm{Cp}^{\circ}$ can be quite sensitive to minor perturbations in the input. For example, we obtained a three-fold increase in $\mathrm{Cp}^{\circ}$ at $3000 \mathrm{~K}$ when the cutoff for $\mathrm{H}_{2}^{+}$was changed from $18871 \mathrm{~cm}^{-1}$ (the maximum in $\mathrm{G}_{0}(\mathrm{v})$ ) to $21380 \mathrm{~cm}^{-1}\left(\mathrm{D}_{0}^{\circ}\right)$. Obviously, such approximate summations must be done with care if they are to be useful. 
The dual problems of extrapolation and cutoff of rotation-vibration levels were recognized long ago by Woolley. Both problems can be solved by representing $D_{0}^{\circ}$ and the observed levels with a realistic potential energy curve. This curve then yields mutually consistent results for both the high energy levels and the limiting curve of dissociation. This approach has been developed by Woolley et al., Gurvich et al., Feber and Herrick, and others. Such an approach is essential if direct summation is used frequently.

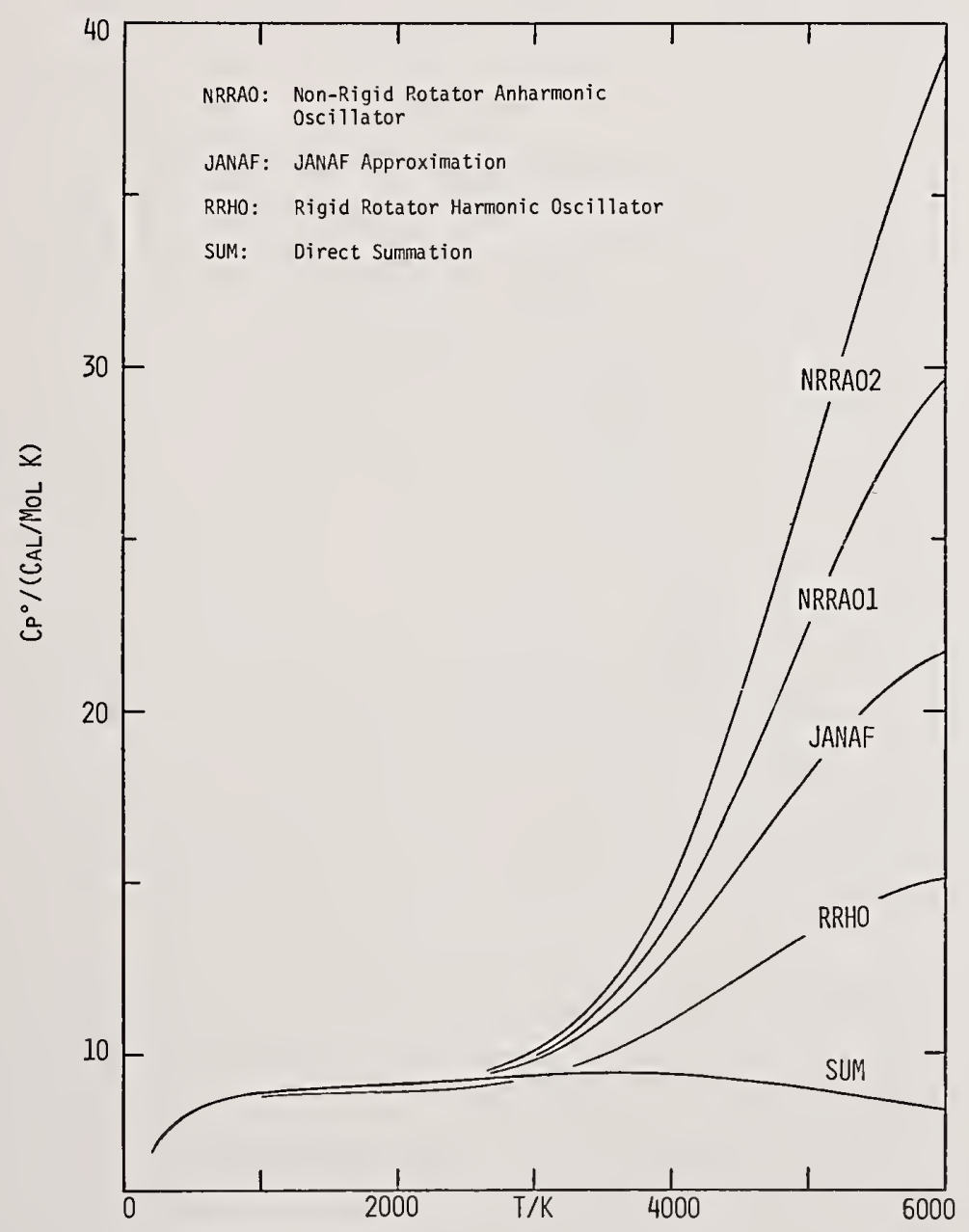

Figure 5. Approximate and summation calculations for $\mathrm{Cp}^{\circ}$ of $\mathrm{ClF}$.

\section{Cooperative Ventures}

In the preparation of JANAF Thermochemical Tables we rely on others: experimentalists, theorists, and specialists who collect or evaluate data. Cooperation with these people can reduce duplication of effort and shorten the time needed to prepare a table. 
There are two areas where such cooperation has been of great assistance to the JANAF group. These are: (1) retrieval and evaluation of literature data and (2) methods for calculation and reduction of data. Another area of current interest is direct cooperation with other groups in the actual production of thermochemical tables.

Literature search and retrieval is an obvious area for mutual cooperation since there are several individuals and groups which maintain current surveys of parts of the

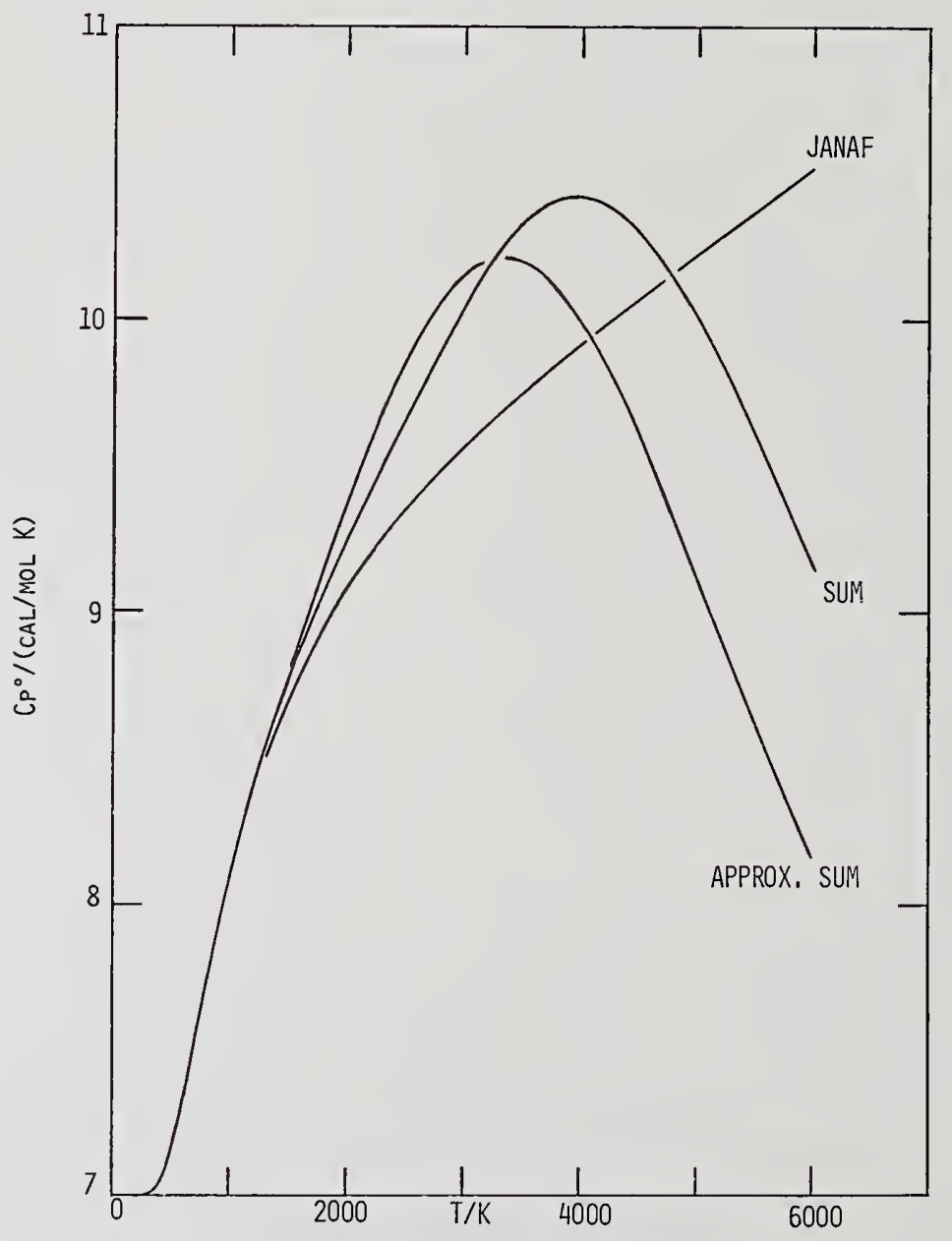

Figure 6. Approximate and summation calculations for $\mathrm{Cp}^{\circ}$ of $\mathrm{H}_{2}^{+}$.

literature pertinent to JANAF work. Many of these same groups, particularly the data centers, are also involved in critical evaluation of pertinent data. We frequently accept the evaluation of these groups, thus reducing duplication of effort in both literature retrieval and critical evaluation. Some groups with which we have had contacts are: $D$. D. Wagman et al. (Chemical Thermodynamics Data Center, NBS), H. M. Rosenstock et al. (Ion 
Energetics Data Center, NBS), W. C. Martin et al. (Atomic Energy Levels Data Center, NBS), R. Hultgren (Berkeley), and L. Brewer (Berkeley).

In the area of data reduction methods and calculational procedures we have wide leeway for interaction with other groups. This includes not only the exchange of computer programs to handle calculations of increasing complexity but also discussion of calculational procedures with various experts. For example, two of us (M. W. Chase and A. N. Syverud) recently participated in a discussion session on diatomic gas calculational methods organized by S. Abramowitz (NBS). This session was attended by U.S. personnel interested in these calculations and was a direct outgrowth of problems with ClF mentioned earlier. We have also had discussions with J. L. Haas, Jr. (U.S. Geological Survey) on multiple regression methods of correlating thermochemical data.

Finally, we should mention the efforts underway to provide an international set of high temperature thermochemical tables. A CODATA task group chaired by $H$. J. White (NBS) is currently investigating means of reaching this goal. This cooperative venture is only in a formative stage but it is proposed that the resulting network will involve personnel from NBS, Dow (JANAF), and the Institute of High Temperatures (Moscow) as well as other groups. This should be a significant step in reducing the large amount of duplicate effort of these groups.

\section{Conclusions}

The uncertainty and useful range of thermochemical tables for ideal gases is limited not only by the accuracy of the existing data but also by calculational procedures and estimates for missing data. These limitations may appear at any temperature but are usually most serious at high temperature. Pertinence of these limitations to specific thermodynamic applications depends on answers to the following questions. For atomic and diatomic gases, what are the effects due to:

(1) expected but unobserved electronic levels,

(2) cutoff procedure and its relation to real gas applications, and

(3) classical approximation for the electron gas and its relation to ionic systems? For diatomic gases, what are the effects due to the calculational treatment of:

(1) observed rotation-vibration levels (including splitting),

(2) expected but unobserved rotation-vibration levels, and

(3) electronic states with shallow potential energy curves?

The JANAF Thermochemical Tables Project is sponsored by the Air Force Office of Scientific Research and the Department of Energy. The cooperation, direction, and encouragement of our program monitors, Dr. Joseph F. Masi and Mr. Francis E. Spencer, are greatly appreciated. 
We thank William H. Evans (NBS) and Bonnie J. McBride and Sanford Gordon (NASA) for providing us with computer programs.

We thank Jerry L. Curnutt, Richard A. McDonald, and Edward A. Valenzuela for their help in the preparation of this presentation. Thanks also are extended to Betty Clark and Dana Donley for their help in the final stages of typing.

\section{References}

[1] Stu11, D. R. and Prophet, H., NSRDS-NBS-37, June 1971; Chase, M. W., et al., J. Phys. Chem. Ref. Data, $\underline{3}, 311$ (1974); ibid, $\underline{4}, 1$ (1975); ibid, $\underline{7}, 793$ (1978).

[2] McBride, B. J. and Gordon, S., Fortran IV program for calculation of thermodynamic data NASA TN D-4097 (1967).

[3] Strickler, S. J., Electronic partition function paradox, J. Chem. Ed. $\underline{43}, 364$ (1966).

[4] McChesney, M., Equilibrium shock-wave calculations in inert gas, multiply ionized Debye Huckel plasmas, Can. J. Phys. $\underline{42}, 2473$ (1964).

[5] Downey, J. R., Jr., Calculation of thermodynamic properties of ideal gases at high temperatures; monatomic gases, AFOSR-TR-78-0960 (March 1978).

[6] Mayer, J. E. and Mayer, M. G., Statistical Mechanics (John Wiley and Sons, Inc., New York, 1940), 2nd Edition (1977).

[7] Frankiss, S. G. and Green, J. H. S., Statistical methods for calculating thermodynamic functions, in Chemical Thermodynamics, Vol. 1, pp. 268-316, The Chemical Society, Londol (1973).

[8] Wooley, H. W., High rotational levels and the partition function for hydrogen, J. Chem. Phys. 9, 470 (1941).

[9] Wooley, H. W., Scott, R. B., and Brickwedde, F. G., Compilation of thermal properties of hydrogen in its various isotopic and ortho-para modifications, J. Res. Nat. Bur. Stand. 41,379 (1948).

[10] Gurvich, L. V., Khachkuruzov, G. A., et al., Thermodynamic Properties of Individual Substances, Moscow, 1962; Reports AD-659660, AD-659659, and AD-659679 (1967) (English translation available from NTIS).

[11] Feber, R. C. and Herrick, C. C., An improved calculation of the ideal gas thermodynamic functions of selected diatomic molecules, U.S. Atomic Energy Comm., Report LA-3597, 70 pp. (1966).

[12] Gorbov, S. I., Gurvich, L. V., Iorish, V. S., and Yungman, V. S., Calculation of the thermodynamic functions of ideal diatomic gases by direct summation - lithium and sodiun hydrides, High Temperature, 13 (3), 465 (1973).

\section{Discussion}

Question (Lincoln): Are you in a position yet where you can indicate on individual tables the temperature at which these problems (phase transitions) come in? 
Response (Chase): We have tried to do it on the discussion side.

Question (Lincoln): You suggest that you may be extending the tables beyond $6000 \mathrm{~K}$. What are your plans?

Response (Chase): Well, the work we did on the monatomic gases was for that specific reason, to see what we would have to do for our existing tables calculational procedure to extend molecular gases to higher temperatures $10,000 \mathrm{~K}$. We are discussing the general rationale about taking some of the ions of monatomic gases up to high temperature and then probably some of the simple diatomics.

Question (Kohl): Would you like to comment in public on the new format and the inclusion of transition points for condensed phases?

Response (Chase): There is an awful lot information that we generate when producing a table, and a lot of that was never put on tables before, but right now, we are starting to add on gas phase tables in the upper left hand corner, if you will, we are adding in the dissociation energy or the heat of atomization. We are going to add on the tabulation side, data for heat capacity, free energy, entropy and enthalpy at each transition point. That will appear right on the tabulation side, and in doing that, we will probably do away with our lines, dotted lines, and that sort of thing.

For those areas like with sulfur, which has a lambda point, probably not on the tabulation side, but on the reverse side, we will give a temperature/heat capacity table, with enough data points so you will know what the shape of the heat capacity curve is through the lambda point. Leo Brewer wants us to add in crystallographic data and we may do that if it is readily available, but that gets pretty time consuming for a questionable species.

Question (Hastie): This is probably more of a general question; and anybody in the audience might want to comment. It seems in extending the tables to high temperatures, it requires some chemical basis for doing so. For example, some people would find it hard to understand why CIF should extend up 6000 Kelvin. It is hard to imagine conditions where a system would contain CIF at such temperatures. How do you decide on which systems to extend to higher temperatures and what temperature to cut off the calculations at?

Response (Chase): Of course, all our gas phase tables automatically go to $6000 \mathrm{~K}$, whereas in fact, your point is right, that many of those species can't possibly exist up to that high temperature, but it was an arbitrary decision made early to extend them that high.

The species that you would extend at even higher temperatures would be those that would be specifically requested by the Air Force. In other words, we wouldn't extend them all to the high temperatures. It is just the few that the Air Force would like. It is true also that some of the other ones we probably should cut back to lower temperatures, but we haven't. 
Question (Horton): I have been wondering whether you have been beginning to worry about the fact that in about 1980, there is going to be a change in the International Practical Temperature Scale, and about the fact that the standard states for ideal gases may be in trouble, because of the redefinition of pressure units. I don't know whether they are going to allow us to keep the atmosphere. And there has been a suggestion also that the reference temperature be shifted to $300 \mathrm{~K}$, instead of $298.15 \mathrm{~K}$. Have these questions bothered you at a)1?

Response (Chase): Yes, we have been involved in many discussions as to whether we should stay with one atmosphere or not. The Bureau (NBS) has told us that when they publish any more of our work now, we have to do it in SI units.

The business of shifting to 300 , I think, is more of a problem. We have been very involved internally, we are converting all our programs over to dimensional-less quantities and arbitrary pressure. So, no matter what you guys decide to do, and that is a lot to finish, when you go to print the table you can convert to any pressure or thermochemical units that you want.

We published a third edition (of JANAF), which is supposedly in the works. That will al1 be 1975 fundamental constants and SI units. We will still have $298 \mathrm{~K}$ as the reference. A11 of that is dependent, of course, that CODATA doesn't come out with new fundamental constants in the meantime.

The International Practical Temperature Scale, we have been trying to convert all data to the ' 68 scale. But that causes problems: even now a lot of people don't say what temperature scale they used when they did their work. So, any further conversions are a problem. 
National Bureau of Standards Special Publication 561, Proceedings of the 10th Materials Research Symposium on Characterization of High Temperature Vapors and Gases held at NBS, Gaithersburg, Maryland, September 18-22, 1978. Issued October 1979.

\title{
DIMENSIONAL ANALYSIS OF PARTITION FUNCTIONS FOR IONIC MOLECULES: THERMODYNAMIC PROPERTIES OF VAPORS
}

\author{
David J. Frurip and Milton Blander \\ Chemical Engineering Division \\ Argonne National Laboratory \\ Argonne, IL 60439
}

\begin{abstract}
A dimensional analysis of the classical partition functions of vapors of ionic molecules leads to expressions for the relative values of thermodynamic properties for molecules having one type of cation and one type of anion. Relative values of standard entropies, $\mathrm{S}^{\mathrm{O}}$, and of free energy functions, $\left(G^{0}-H_{0}^{0}\right) / T$, can be calculated with a knowledge of the cation-anion distance, the atomic masses, and the electronic partition function. The theory is not expected to be valid for energies and enthalpies. Comparisons of the theoretical predictions with data on $S^{0}$ and $\left(G^{0}-H_{0}^{0}\right) / T$ for a large number of diatomic molecules and their associated dimers demonstrate the accuracy of this simple calculation. Covalent molecules fit the expressions derived for ionic molecules surprisingly well indicating that the equations might be more general than implied by the model. The model should be most useful for polyatomic molecules, $C_{c_{A}}{ }_{a}$, where $c$ and/or $a$ is greater than unity. The theory minimizes the amount of spectroscopic and structural information necessary to calculate some of the thermodynamic properties since functions such as $S^{0}$ and $\left(G^{0}-H_{0}^{0}\right) / T$ can be calculated for molecules of a given type if the corresponding data for one member of the class are wel1 known.
\end{abstract}

\section{Introduction}

From a dimensional analysis of the classical partition functions for ionic molecules, we have derived simple relations for the relative values of the thermodynamic properties of gaseous ionic molecules in terms of cation-anion distance, ion masses, and the electronic partition function. Because of the simplicity of the model ionic pair potentials used, the results can only be applied to calculations of relative values of entropies and of free energy functions and not for energies and enthalpies. 
In the standard method, the calculation of entropies and free energy functions for gaseous species requires information on molecular geometry, fundamental vibrational frequencies as well as vibrational anharmonicities, and vibration-rotation interactions. For ionic molecules, low vibrational frequencies are often encountered leading to large anharmonicities, to large and uncertain contributions to the vibrational partition functions, and to experimental difficulties in measuring the parameters necessary for thermodynamic calculations. Our results circumvent the need for detailed information on all molecules of a given class and greatly simplify the calculation of the entropy and the free energy functions at temperatures which are high enough for the classical partition function to be valid.

\section{Dimensional Analysis}

The dimensional analysis follows a method utilized previously for the analysis of association constants of alkali halides $[1]^{1}$. We consider the partition function for an ionic molecule $\mathrm{C}_{\mathrm{C}} \mathrm{A}_{\mathrm{a}}$

$$
Q=K Z q_{e}
$$

where $Q$ is the classical partition function corrected for electronic contributions by inclusion of the electronic partition function $q_{e}, k$ is the kinetic partition function $\left(2 \pi m_{c}^{c} m_{A}^{a} k T / h^{2}\right)^{3 / 2}$, and $z$ is the configurational integral

$$
Z=\int \cdots \int \exp (-U / k T) \prod_{C} d \tau \prod_{a} \prod_{j} \tau_{j}
$$

The parameters $m$ are atomic masses; $U$ is the total potential energy of all the cations, $C$; and the anions, $A$, in a given configuration, $d \tau_{j}$ and $d \tau_{j}$ are volume elements containing cations and anions, respectively, in this configuration; and the integral is over all possible configurations of the cations and anions in the volume $V$. If, as is usual, we take the total potential as the sum of pair potentials between the ions, we can write

$$
u=\sum^{c} \sum^{a} u_{i j}+\sum_{i<j^{\prime}}^{c-1} \sum_{i j}^{c} u_{j<j^{\prime}}^{a-1} \sum_{j j^{-}}^{a}
$$

where the sums are taken such that each pair is counted but once. Our derivation now rests on a comparison of the partition function $Q$ for a given salt, $C_{c} A_{a}$, in a volume $V$ with that of a test salt $D_{c} B_{a}$ in a volume $V_{0}$. The test salt partition function is denoted as $Q_{0}$. For each configuration of the ions of the salt $C_{c} A_{a}$ in the volume $V$, there is a geometrically similar configuration of the test salt, $D_{C} B_{a}$ in the volume $V_{0}$. The ratio of the volumes $V_{0} / V$

${ }^{1}$ Figures in brackets indicate the literature references at the end of this paper. 
is chosen so that it is equal to $\left(d_{0} / d\right)^{3}=g^{3}$ where $d_{0}$ is a size parameter for the test salt, $d$ is a size parameter for the given salt, and $g$ is a non-dimensional ratio, $\left(d_{0} / d\right)$. These size parameters are characteristic of cation-anion separations. The cation-anion pair potential, can be scaled if it has the form $g u_{0}(g r)$. For example, for a simple form $u=$ $\left(A / r^{n}\right)-\left(e^{2} / r\right)$ it is simple to demonstrate this functional form [1]. Even for a more complex potential function as, e.g., $u=\left(A / r^{n}\right)-\left(e^{2} / r\right)-\left(\alpha / r^{6}\right)$, where $\alpha$ is a dispersion energy coefficient, it can be shown that for a particular set of salts with values of $\alpha$ such that $\left(\alpha / d^{5}\right)$ is a constant, this functional form is obeyed. Since $\alpha$ generally increases monotonically with size, this condition does not deviate greatly from a realistic potential. Thus, our derivation applies to a hypothetical class of ionic salts in which the coefficients of different terms in the pair potential (such as $\alpha$ ) are related to the size parameter.

For the cation-cation or anion-anion pair potentials, only the coulombic term, $e^{2} / r$, has the form $\mathrm{gu}_{0}(\mathrm{gr})$. All other terms such as the core repulsions do not fit this form. However, the coulombic repulsions between ions of like charge lead to high potential energies for configurations in which two anions or two cations are very close to each other and, consequently, these configurations have a very small weight and thus make a negligibly small contribution to the configurational integral. Consequently, in the most probable configurations, ions of like charge are relatively far apart and contributions of the repulsive part of the pair potential (and of other parts of the pair potential which can be represented as a high inverse power of $r$ ) will be very small [1]. Consequently, the coulombic part of $u_{i j}$, and $u_{j j}$, is the most important for these configurations and, therefore, we may write for the total potential in the most significant configurations

$$
U=g U_{0}(g r)
$$

It has been shown, using the above approximation, that [2]

$$
Z(T, V)=g^{-3(c+a)} Z_{0}\left(T / g, g^{3} V\right)=g^{3(1-c-a)} Z_{0}(T / g, V)
$$

The logarithm of the right hand term in eq. (5) can be expanded in a power series in $(g-1)$ to obtain $[1,2]$

$$
1 n Z_{0}(T / g, V)=1 n Z_{0}(T, V)-\frac{E_{0}}{R T}(g-1)+\frac{C_{V}}{2 R}(g-1)^{2}+\ldots
$$

Combining eqs. (1), (5), and (6) we obtain the relation

$$
\begin{aligned}
\ln Q-\frac{3}{2} \operatorname{cln} m_{C}-\frac{3}{2} \operatorname{aln} m_{A} & -3(\eta-1) \ln d=\alpha_{0}-\frac{E_{0}}{R T}(g-1)+\frac{C_{V}}{2 R}(g-1)^{2} \\
& +\ln q_{e}+\ldots
\end{aligned}
$$


where $q_{e}$ is the electronic partition function and $\alpha_{0}$ is dependent on the properties of the test salt. If we ignore the small second order term in eq. (7), and since $\mathrm{gE}_{0}=\mathrm{E}$, we show that

$$
\frac{S^{0}}{R}(T, V)=\sigma_{0}(T, V)+\frac{3}{2} c \ln m_{C}+\frac{3}{2} a \ln m_{A}+3(\eta-1) \ln d+\ln q_{e}
$$

where $\sigma_{0}$ depends on the properties of the test salt and $\eta$ is defined as the sum of $a+c$. Differences in $C_{V}$ for different salts with the model potential are small so that to a very good approximation

$$
-\left(\frac{G^{0}(T, V)-H_{0}^{0}}{R T}\right)=\gamma_{0}(T, V)+\frac{3}{2} \operatorname{cln} m_{C}+\frac{3}{2} a \ln m_{A}+3(\eta-1) \ln d+\ln q_{e}
$$

where $H_{0}^{0}$ is the enthalpy at a standard temperature and $\gamma_{0}$ is a constant dependent on the properties of the test salt. If the molecule has no low lying electronic states, then $q_{e}$ is simply equal to the ground state degeneracy. Equations (8) and (9) provide a relationship between the entropies and between the free energy functions of ionic salts. Because the model potentials cannot lead to accurate energies for real ionic molecules, we restrict our study to the above two functions. One can rationalize this by analysis of the configurational integral for a real salt by considering that a realistic potential is a perturbation of the model potential. If the model potential is chosen so that it does not differ greatly from a pair potential which accurately describes real salts, then the differences between individual terms in the "real" and model potentials are relatively small. In such a case, the largest contributions to the differences in the free energies will be in the energy term and not in the entropy. Consequently, one might expect real salts to obey eqs. (8) and (9). In the following section, we will test this for a number of salts and show it to be valid for the cases we examine.

\section{Comparisons With Data}

We have compared eqs. (8) and (9) with available data at 298, 1000, and $5000 \mathrm{~K}[3,4,5]$ on entropies and free energy functions of a large number of diatomic molecules including alkali halides, diatomic oxides, and diatomic nitrides ${ }^{2}$. The latter two groups are not considered to be strictly ionic. These are given in figure 1 and table 1 . Equations (8) and (9) predict a linear relationship between the free energy function or entropy and the term $\ln \left[d^{3}\left(m_{A} m_{C}\right)^{3 / 2} q_{e}\right]$. In addition, we have compared available data on alkali halide dimers at 298, 1000, and $5000 \mathrm{~K}$ with these equations and these are shown in figure 2 and table 2.

${ }^{2} 0 \mathrm{nly}$ the free energy functions at 298 and $1000 \mathrm{~K}$ are presented for the oxides and nitrides. Also this data is presented in graphical form only due to the large number of substances tested. 
The solid lines in all the figures (except the oxide-nitride plot (fig. 2c)) are least squares linear fits to the data with unit slope. The intercepts which reflect the average values of $\gamma_{0}$ and $\sigma_{0}$ are given in the tables. In the diatomic oxide and nitride plot, the solid lines are least square fits of unit slope excluding all data at $\ln \left[d^{3}\left(m_{C} m_{A}\right)^{3 / 2} q_{e}\right]<11$. This was done to emphasize the classical validity of our model. At low temperatures, the data deviates from unit slope increasingly as the mass is decreased and thus as quantum effects become significant. The alkali halide free energy data also show this same effect in the $298.15 \mathrm{~K}$ plot. In all cases the effect is very small at the higher temperatures. The fact that the points in all the plots fall close to a line with unit slope indicates that the theory provides an excellent correlation of $S^{0}$ and $\left(G^{0}-H_{0}^{0}\right) / R T$ for these different substances. In fact, deviations from the predictions are within the range of uncertainties, except at $298 \mathrm{~K}$. Surprisingly, many non-ionic diatomic molecules follow the predicted correlation closely, indicating that the correlation may be more general than is implied by the assumptions of the theory. Further tests of the equations on a broader range of molecules are needed to test the range of applicability of the equations.

Another significant point should be made concerning the generality of the dimensional analysis for different classes of molecules. Note in the tables the remarkable constancy of $\gamma_{0}$ and $\sigma_{0}$ at the same temperature for the different types of compounds. For example, at $1000 \mathrm{~K}$, the oxides, nitrides, and alkali halides show average values for $\gamma_{0}$ of $15.96,16.26$, and 16.11 , respectively. The differences in $\gamma_{0}$ for these classes of substances are within the range of uncertainties.

\section{Discussion}

The success of the equations in predicting relative values of $S^{0}$ and $\left(G^{0}-H_{0}^{0}\right) / T$ lends confidence in their use for making predictions for substances where these quantities are unknown. For diatomic molecules, almost all substances tested follow eqs. (8) and (9) at 298, 1000 , and $5000 \mathrm{~K}$. Average values of $\sigma_{0}$ and $\gamma_{0}$ are given in tables 1 and 2 . $0 f$ the three temperatures, the fit at 298 was the least precise. This is to be expected since the molecules are then least classical and the theory should, strictly speaking, apply to classical molecules (i.e., where quantum effects are smal1). For alkali halide dimers, the theory permits us to fill in data for substances where it is not available. Table 3 contains predicted values for dimers of the seven alkali halides $\mathrm{NaI}, \mathrm{RbF}, \mathrm{RbCl}, \mathrm{RbBr}, \mathrm{RbI}$, $\mathrm{CsBr}_{\mathrm{r}}$, and $\mathrm{CsI}$ which are not given in the JANAF tables. The interionic distances were taken to be the same as in the corresponding monomer. Thus, the theory permits one to fill in gaps in data and could also be used to check the self consistency of data. It avoids the complications of doing a complete analysis of the dynamics and structures of every molecule in a class and minimizes the amount of spectroscopic data needed. For a group of similar molecules, one only needs to study the properties of one molecule in detail. Using this substance as a reference salt, one can then calculate the thermodynamic properties of unknown substances of similar type. 


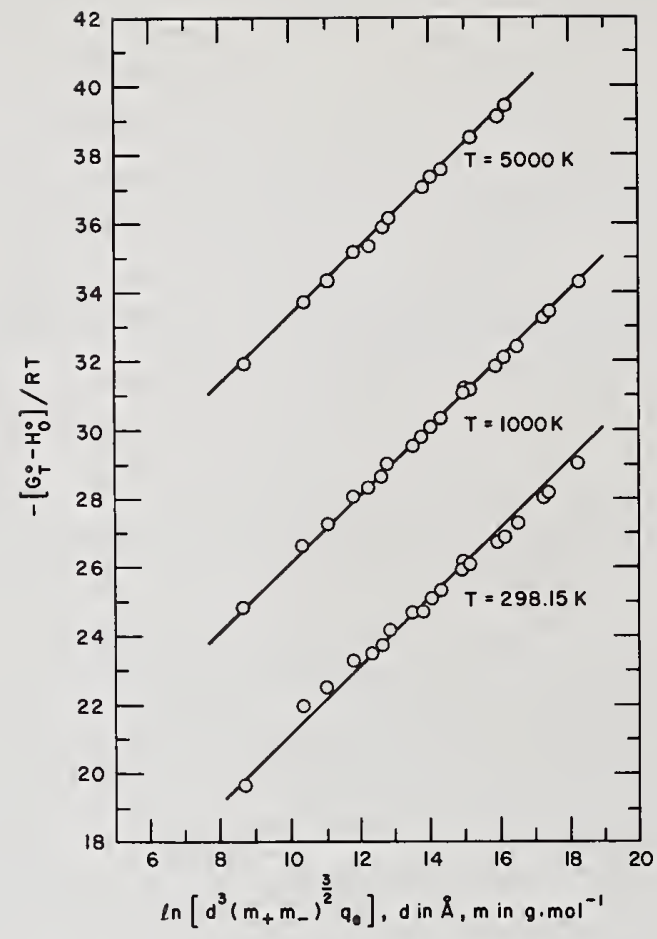

Figure 1a. The dimensionless free energy function versus $\ln \left[d^{3}\left(m_{+} m_{-}\right)^{3 / 2} q_{e}\right]$ for the alkali halide molecules at three temperatures. The plots at 298 and $1000 \mathrm{~K}$ include the bracketed data from table 1 . The solid lines are of unit slope and intercepts equal to the average $\gamma_{0}$ values from table 1.

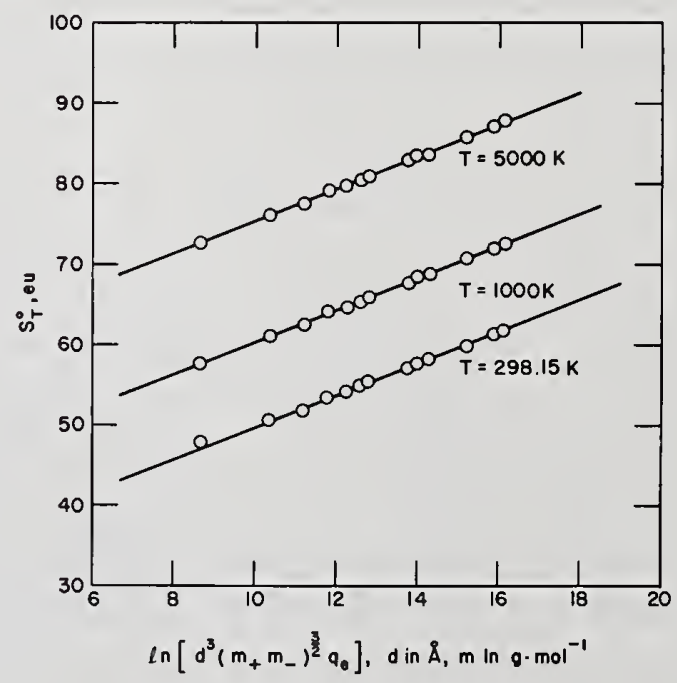

Figure 1b. The absolute entropy versus $\left.\ln \left[d^{3} m_{+} m_{-}\right)^{3 / 2} q_{e}\right]$ for the alkali halide molecules at three temperatures. The straight lines are of unit slope with intercepts equal to the average $\sigma_{0}$ values given in table 1 . 


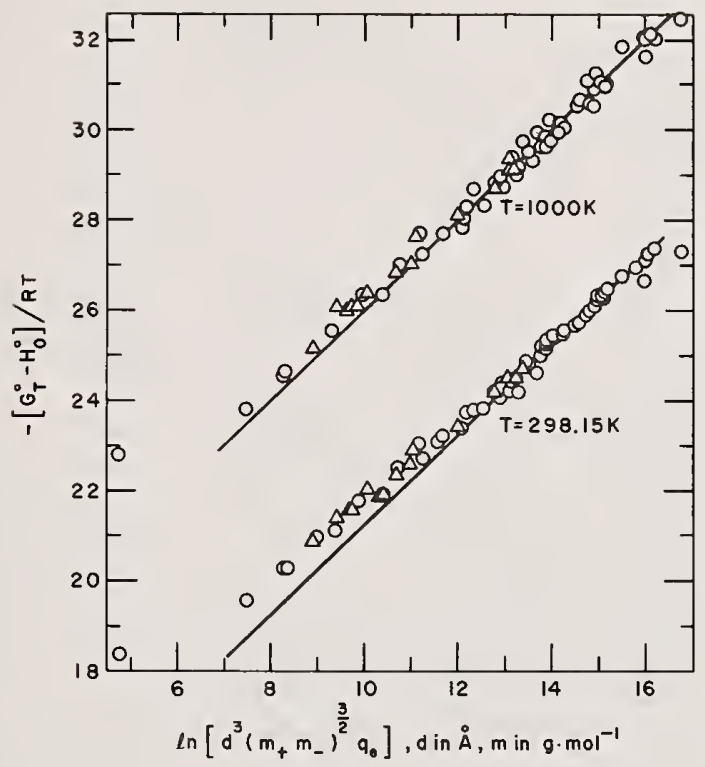

Figure 1c. The dimensionless free energy function versus $\ln \left[d^{3}\left(m_{+} m_{-}\right)^{3 / 2} q_{e}\right]$ at two temperatures for the diatomic oxides $(0)$ and nitrides $(\Delta)$. The data includes 28 oxides and 16 nitrides from the JANAF compilation [3], and 37 oxides from a compilation by Brewer and Rosenblatt [5]. The lower left-hand points in each set are for the $\mathrm{OH}$ molecule. The solid lines are of unit slope with intercepts given by the average $\gamma_{0}$ values for $\ln \left[d^{3}\left(m_{+} m_{-}\right)^{3 / 2} q_{e}\right]>11: \gamma_{0}=11.26$ at $298 \mathrm{~K}$ and $\gamma_{0}=15.98$ at $1000 \mathrm{~K}$. See text for details.

It is to be noted that it is not the molecular mass but rather the atomic masses which enter into eqs. (8) and (9). This result must come from some complex cancellations of the molecular masses from the rotational and vibrational terms. In the formulation of the dimensional analysis, the question arises therefore as to whether the complete quantum statistical approach for the free energy function and absolute entropy can be somehow manipulated to forms equivalent to eqs. (8) and (9). If so, then it is of interest to know what are the necessary approximations and assumptions involved. We will only consider the simplest case, a diatomic molecule, CA. The total quantum statistical partition function, Q (in the rigid rotor-harmonic oscillator approximation), is a product of the translational, rotational, electronic, and vibrational contributions

$$
Q=Q_{t} Q_{r} Q_{v} q_{e}
$$




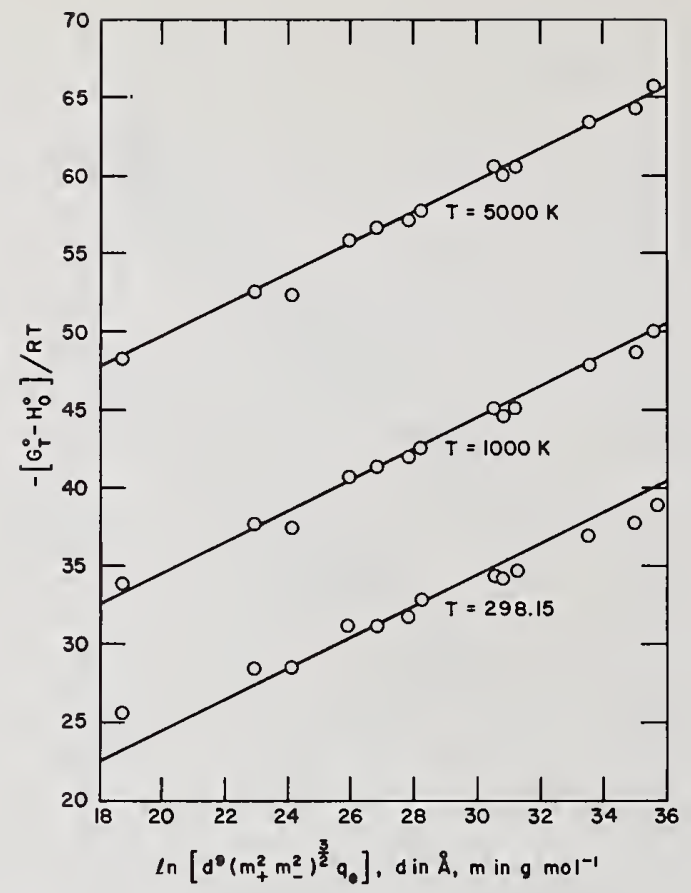

Figure 2a. The dimensionless free energy function versus $1 n\left[d^{9}\left(m_{+}^{2} m_{-}^{2}\right)^{3 / 2} q_{e}\right]$ for the dimers of the alkali halides. The solid lines are of unit slope with intercepts equal to the average $\gamma_{0}$ values of table 2 .

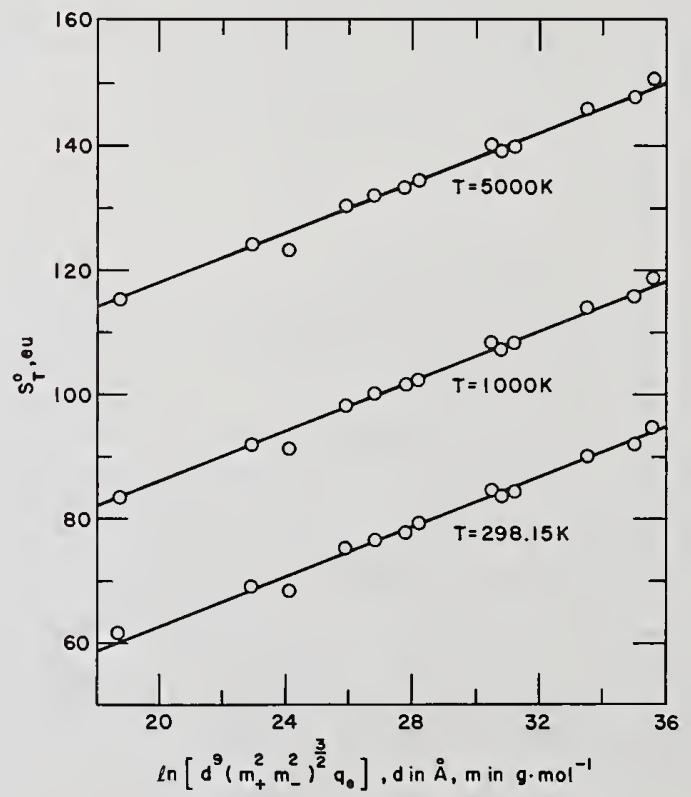

Figure $2 b$. The absolute entropy versus $\ln \left[d^{9}\left(m_{+}^{2} m_{-}^{2}\right)^{3 / 2} q_{e}\right]$ for the dimers of the alkali halides. The solid lines are of unit slope and intercepts equal to the average $\sigma_{0}$ values from table 2 . 
Table 2. Correlation of the available free energy and entropy data for the alkali halide dimers with the dimensional analysis.

\begin{tabular}{|c|c|c|c|c|c|c|c|}
\hline Species & $\begin{array}{c}\gamma_{0} \\
T=298\end{array}$ & $\begin{array}{c}\gamma_{0} \\
T=1000\end{array}$ & $\begin{array}{c}\gamma_{0} \\
T=5000\end{array}$ & $\begin{array}{c}\sigma_{0} \\
\mathrm{~T}=298\end{array}$ & $\begin{array}{c}\sigma_{0} \\
\mathrm{~T}=1000\end{array}$ & $\underbrace{}_{\substack{\sigma_{0} \\
T=5000}}$ & $\begin{array}{r}{ }^{9}\left(m^{2} m^{2}\right)^{3 / 2} q_{e}{ }^{j} \\
\text { d in } \AA, m \text { in } \\
g \cdot m^{-1}\end{array}$ \\
\hline$(\operatorname{LiF})_{2}$ & 7.0 & 15.2 & 29.6 & 12.4 & 23.2 & 39.2 & 18.7 \\
\hline$(\mathrm{LiCl})_{2}$ & 5.6 & 14.7 & 29.7 & 11.9 & 23.3 & 39.4 & 22.9 \\
\hline$(\mathrm{LiBr})_{2}$ & 5.2 & 14.7 & 29.8 & 11.9 & 23.5 & 39.6 & 25.9 \\
\hline (LiI) $_{2}$ & 4.6 & 14.4 & 29.5 & 11.6 & 23.3 & 39.3 & 28.2 \\
\hline$(\mathrm{NaF})_{2}$ & 4.3 & 13.3 & 28.1 & 10.4 & 21.8 & 37.8 & 24.1 \\
\hline$(\mathrm{NaCl})_{2}$ & 3.8 & 14.0 & 29.4 & 11.3 & 23.1 & 39.2 & 27.8 \\
\hline$(\mathrm{NaEr}){ }_{2}$ & 6.3 & 16.8 & 32.3 & 11.2 & 23.1 & 39.2 & 30.8 \\
\hline$\left.{ }^{(K F)}\right)_{2}$ & 4.3 & 14.4 & 29.8 & 11.7 & 23.5 & 39.6 & 26.8 \\
\hline$(\mathrm{KCl})_{2}$ & 3.8 & 14.5 & 30.1 & 11.9 & 23.9 & 40.0 & 30.5 \\
\hline$(\mathrm{KBr}){ }_{2}$ & 3.3 & 14.3 & 30.0 & 11.8 & 23.8 & 39.9 & 33.5 \\
\hline${ }^{(K I)}{ }_{2}$ & 3.3 & 14.5 & 30.3 & 12.0 & 24.1 & 40.2 & 35.6 \\
\hline$(\mathrm{CsF})_{2}$ & 3.4 & 13.9 & 29.3 & 11.2 & 23.1 & 39.2 & 31.2 \\
\hline$(\mathrm{CsC} 1)_{2}$ & 2.7 & 13.7 & 29.3 & 11.1 & 23.1 & 39.2 & 35.0 \\
\hline $\begin{array}{l}\text { Average } \\
\text { Values }\end{array}$ & 4.4 & 14.5 & 29.8 & 11.6 & 23.3 & 39.4 & \\
\hline
\end{tabular}

a The anion-cation distance in the dimer was taken to be equivalent to the corresponding distance in the monomer (see table 1).

Table 3. Predicted values of the thermodynamic properties of seven alkali halide dimers using the results of the dimensional analysis.

\begin{tabular}{|c|c|c|c|c|c|c|}
\hline \multirow[b]{2}{*}{ Species } & \multicolumn{3}{|c|}{$-{\frac{\left(G_{T}^{\circ}-H_{o}^{o}\right)}{R T}}^{a}$} & \multicolumn{3}{|c|}{${\frac{S_{T}^{0}}{R}}^{a}$} \\
\hline & $\mathrm{T}=298.15$ & $T=1000$ & $\mathrm{~T}=5000$ & $\mathrm{~T}=298$ & $T=1000$ & $\mathrm{~T}=5000$ \\
\hline$(\mathrm{NaI})_{2}$ & 37.3 & 47.4 & 62.7 & 44.5 & 56.2 & 72.3 \\
\hline$(\mathrm{RbF})_{2}$ & 34.0 & 44.1 & 59.4 & 41.2 & 52.9 & 69.0 \\
\hline$(\mathrm{RbCl})_{2}$ & 37.7 & 47.8 & 63.1 & 44.9 & 56.6 & 72.7 \\
\hline$(\mathrm{RbBr})_{2}$ & 40.6 & 50.7 & 66.0 & 47.8 & 59.5 & 75.6 \\
\hline$(\mathrm{RbI})_{2}$ & $4 ? .7$ & 52.8 & 68.1 & 49.9 & 61.6 & 77.7 \\
\hline$(\mathrm{CsBr})_{2}$ & 42.3 & 52.4 & 67.7 & 49.5 & 61.2 & 77.3 \\
\hline$(\mathrm{CsI})_{2}$ & 44.4 & 54.5 & 69.8 & 51.6 & 63.3 & 79.4 \\
\hline
\end{tabular}

a These values were calculated from eqs. (8) and (9) using the average $\gamma_{0}$ and $\sigma_{0}$ values given in table 2 . The electronic partition functions were assumed to be unity. 
where

$$
\begin{gathered}
Q_{t}=\frac{2 \pi\left(m_{A}+m_{B}\right) k T^{3 / 2}}{h^{2}} v, \\
Q_{r}=\frac{8 \pi^{2} I k T}{\sigma h^{2}}, \\
Q_{v}=\left(1-e^{-h \nu / k T}\right)^{-1}, \\
q_{e}=\left(g_{0}+\ldots\right),
\end{gathered}
$$

where $m$ is the atomic mass, $k$ is Boltzmann's constant, $h$ is Planck's constant, $I$ is the moment of inertia, $\sigma$ is the symmetry number $\left(\sigma=1\right.$ for $C \neq A$ ), $g_{0}$ is the electronic ground state degeneracy, and $v$ is the fundamental vibration frequency. We will assume that (1) we can neglect any effects due to anharmonicity in the vibration, and (2) there is no vibrationrotation coupling. We will further suppose that the vibration behaves classically. This will occur at small values of hv/kT so that $\left(1-e^{-h \nu / k T}\right)^{-1} \rightarrow \frac{k T}{h \nu}$. Thus, we have

$$
Q_{v} \sim \frac{k T}{h v}
$$

The vibrational frequency can be estimated by assuming a standard electrostatic attraction between $C$ and $A$ coupled with a repulsion term. The potential $U(r)$ is given by

$$
U(r)=\frac{-\varepsilon}{r}+\frac{A}{r^{n}}
$$

where $\varepsilon$ and $A$ are constants. The equilibrium atomic distance, $d$, is determined at the minimum in the potential well as

$$
d=(n A / \varepsilon)^{\frac{1}{n-1}}
$$

Expanding $u(r)$ in a Taylor series about $r=d$ and differentiating with respect to $r$, it is easily shown that the force constant, $k$, is given by

$$
\kappa=\frac{\varepsilon(n-1)}{d^{3}}
$$

The vibrational frequency is related to the force constant by 


$$
v=\left(\frac{\kappa}{\mu}\right)^{1 / 2} \frac{1}{2 \pi}
$$

where $\mu$ is the reduced mass. Combining eqs. (10)-(19) we have as a final result

$$
-\left[\frac{\mathrm{G}_{\mathrm{T}}^{0}-\mathrm{H}_{\mathrm{o}}^{0}}{\mathrm{RT}}\right]=\ln \mathrm{Q}=\left(\ln \beta \mathrm{T}^{7 / 2}\right)+\ln \left[\left(\mathrm{m}_{A} \mathrm{~m}_{B}\right)^{3 / 2} \mathrm{~d}^{7 / 2} \mathrm{q}_{\mathrm{e}}\right]
$$

and

$$
\frac{S_{T}}{R}=\left(\ln \beta T^{7 / 2}+\frac{7}{2}\right)+\ln \left[\left(m_{A} m_{B}\right)^{3 / 2} d^{7 / 2} q_{e}\right]
$$

where $\beta$ is a collection of terms given by

$$
\beta=\frac{16 \pi^{3} k^{3 / 2}}{h^{3} \varepsilon{ }^{1 / 2}(n-1)}\left(\frac{2 \pi k}{h^{2}}\right)^{3 / 2} .
$$

We see that eqs. (20) and (21) are in a form similar to eqs. (8) and (9) of the dimensional analysis. The first terms are dependent on the temperature and also on the molecular properties of the CA molecule only in the $\varepsilon$ and $n$ terms. For similar molecular bonding, one would certainly expect $\varepsilon$ and $n$ to remain essentially constant from one molecule to the next. Thus, we can identify the first terms in eqs. (20) and (21) with the universal constants $\sigma_{0}$ and $\gamma_{0}$ from the dimensional analysis. The second term in eqs. (20) and (21) is virtually identical to the dimensional approach except for the extra $1 / 2$ power of $d$.

We have shown that the quantum statistical equations for a diatomic molecule reduce approximately to the dimensional analysis result under the essential approximations of no anharmonicities and classical vibration. A similar analysis for polyatomic molecules is not as easy. There is no convenient way to determine the vibrational spectrum of a polyatomic molecule without some specific knowledge of the complex potential surface. On the other hand, the dimensional analysis circumvents this problem by allowing one to use the properties of similar molecules whose vibrational assignments have been made reliably to determine the properties of another unknown substance. The constant terms $\sigma_{0}$ and $\gamma_{0}$ can be calculated either from an equation such as eq. (22) for diatomics, or from an evaluation of the configurational integral in eq. (2) using a realistic pair potential. These constants should be essentially independent of the substance for a given stoichiometry. It should be pointed out that the dimensional analysis and the configurational integral are even valid for molecules with highly anharmonic vibrations and may be useful at very high temperatures. 
Another question arises because of corrections related to the zero point energy of the molecules involved. In this classical analysis, the molecules are at the lowest point in the potential energy curve at $0 \mathrm{~K}$, whereas in real systems they lie above it by the zero point energy $\frac{1}{2} \sum h v_{j}$, where the sum is over all vibrational states. In the final analysis, one should carefully study the influence of zero point energy corrections on the correlations given here.

\section{Conclusions}

A dimensional analysis of the configurational integral for ionic molecules leads to relations for relative values of entropies and free energy functions of molecules with the same stoichiometry. A preliminary test of these simple equations indicates their usefulness for making predictions for molecules behaving classically and for minimizing the amount of necessary spectroscopic data for such predictions.

\section{References}

[1] Blander, M., J. Chem. Phys. 41, 170 (1964).

[2] Blander, M., in Advances in Chem. Physics, Vo1. 11, I. Prigogine, ed., p. 83 (Interscience, New York, 1967).

[3] Stul1, D. R. and Prophet, H., JANAF Thermochemical Tables, NSRDS-NBS-37 (1971).

[4] Blander, M., The thermodynamic properties of alkali halide vapors, to be published in Alkali Halide Vapors: Structure, Spectra, and Reaction Dynamics, P. Davidovits and D. L. McFadden, eds. (Academic Press, New York, 1979).

[5] Brewer, L. and Rosenblatt, G. M., in Advances in High Temperature Chemistry, Vol. 2, L. Eyring, ed., p. 1 (1969).

\section{Discussion}

Question (Ogden): Could I ask you how sensitive your method is to your internuclear distance, because I noticed a ninth power dependence there. I wondered whether there was a tremendous spread, if you were to allow a variation on your diatomic distance?

Response (Frurip): I think when we first developed the model, we in fact had the wrong power of $d$. I think we were constantly using it as $d^{3}$, and it turns out that it is not very sensitive at all. 

National Bureau of Standards Special Publication 561, Proceedings of the 10th Materials Research Symposium on Characterization of High Temperature Vapors and Gases held at NBS, Gaithersburg, Maryland, September 18-22, 1978. Issued 0ctober 1979.

\title{
THERMODYNAMICS OF NON-RIGID MOLECULES AT HIGH TEMPERATURE
}

\author{
Precila Ip ${ }^{1}$ and Herbert L. Strauss \\ Department of Chemistry ${ }^{2}$ \\ University of California \\ Berkeley, CA 94720
}

At high temperatures, the average amplitude of vibration of molecules becomes large. Anharmonicity and rotation-vibration interactions contribute a greater proportion of the thermodynamic functions as the amplitudes go up. In order to determine the effect of the large amplitude motions, the free energy is written as $G=G_{\text {classical }}+G_{\text {correction, where }} G_{\text {classical }}$ includes anharmonic and rotation-vibration terms and $G_{\text {correction }}$ is the approximate correction due to quantization. The formal expressions for $G$ are written out for linear triatomic molecules and applied to $\mathrm{C}_{3}$ and $\mathrm{CO}_{2}$ as examples.

\section{Introduction}

With the continuing advance in techniques for obtaining and studying chemical species at high temperature has come increasing interest in the thermodynamic functions at high temperature. An abundance of spectroscopic information is available for many of the species of interest, and the formulas of statistical thermodynamics provide the means for calculating thermodynamic properties from this information. The classical and quantum mechanical formulas for the partition functions for rigid molecules with harmonic vibrations are we11known $[1]^{3}$. Methods for extending these formulas to molecules with non-rigidity and anharmonic vibrations have also been available for many years [2]. As data have become available at higher and higher temperatures, it has become necessary to apply these methods to polyatomic molecules and relatively little of this has been done. A good example of the importance of non-rigid and anharmonic effects is the $C_{3}$ molecule [3]. The data on the entropy of $\mathrm{C}_{3}$ between 2000 and $3000{ }^{\circ} \mathrm{C}$ give a value that is 5 to 9 eu lower than that calculated from the usual rigid molecule-harmonic oscillator formulae. Taking account of the anharmonicity and the non-rigidity of the molecule lowers the value of the entropy

${ }^{1}$ Present address: Department of Chemistry, M.I.T., Cambridge, MA 02139.

${ }^{2}$ Supported in part by the National Science Foundation.

${ }^{3}$ Figures in brackets indicate the literature references at the end of this paper. 
calculated for $C_{3}$ by about 6 eu at $2400 \mathrm{~K}$, and brings the value from the statistical calculations into approximate agreement with experiment. The $C_{3}$ anharmonicity is exceptionally large and demonstrates that large effects on the thermodynamic functions are possible.

Two different approaches are available for calculating the high temperature thermodynamic functions accurately from spectroscopic data. The first of these is to simply sum the energy levels explicitly. The second is to calculate the classical partition function and then to add quantum corrections. The first procedure uses the known spectroscopic constants more directly, but often suffers from the disadvantage that not all the energy levels are known accurately to the required high energy. Extrapolation and various expansions are then required to obtain the partition function and such a procedure has been carried out [4] successfully for $\mathrm{NH}_{3}$.

We prefer the second method for the calculation of high temperature thermodynamic functions of small molecules. In this paper, we outline the derivation of the appropriate formulae for a linear triatomic molecule, apply them to $\mathrm{C}_{3}$ and $\mathrm{CO}_{2}$, and discuss the relative effects of the terms in the formulae.

\section{Formulae and Methods of Computation}

The classical partition function for a triatomic molecule may be written in the form [3]

$$
\Phi=\frac{1}{h^{9}} \int \cdot \cdot \int \exp (-\beta H) \mathrm{dp}_{1} \underset{\sim 2}{\mathrm{dp}_{2}} \underset{\sim}{\mathrm{dp}_{3}} \underset{\sim}{\mathrm{dq}} \underset{\sim}{\mathrm{dq}_{2}} \underset{\sim}{\mathrm{dq}},
$$

where $\beta=1 / k T$, the $q^{\prime} s$ represent the Cartesian coordinates of each of the three atoms and the $\mathrm{p}^{\prime}$ 's represent the corresponding momenta. The partition function can be simplified by introducing the new coordinates

$$
\begin{aligned}
& \underset{\sim}{x}=\left(m_{1}+m_{2}+m_{3}\right)^{-1}\left(m_{1} q_{1}+m_{2} q_{2}+m_{3} q_{3}\right) \\
& \mathfrak{L}_{1}=q_{2}-q_{1} \\
& \mathfrak{q}_{2}=q_{3}-q_{2}
\end{aligned}
$$

and integrating over $\underset{\sim}{x}, \underset{\sim}{p}, \underset{\sim}{p_{2}}, \underset{\sim}{p_{3}}, \phi_{1}, \theta_{1}$ and $\phi_{2}$. This yields

$$
\Phi=\Phi_{\text {trans }} \Phi_{\text {int }}
$$

with

$$
\Phi_{\text {trans }}=\left\{\left[2 \pi\left(m_{1}+m_{2}+m_{3}\right) k T\right]^{3 / 2} / h^{3}\right\} \times \text { volume of the system }
$$

and 


$$
\begin{gathered}
\Phi_{\text {int }}=\left(\frac{2 \pi k T}{h^{2}}\right)^{3}\left(\frac{m_{1} m_{2} m_{3}}{m_{1}+m_{2}+m_{3}}\right)^{3 / 2} x \\
8 \pi^{2} \int_{0}^{\infty} r_{1}^{2} d r_{1} \int_{0}^{\infty} r_{2}{ }^{2} d r_{2} \int_{0}^{\pi} \sin \theta d \theta e^{-\beta V\left(r_{1}, r_{2}, \theta\right)}
\end{gathered}
$$

Here, the subscript has been dropped from $\theta_{2}$ and $V$ means the potential energy. The internal partition function, $\Phi_{i n t}$, can be further simplified only by making a number of assumptions. The two usual assumptions are: (1) small amplitude motion so that $r_{1} \simeq r_{1}{ }^{e q}, r_{2} \simeq r_{2}$ eq and $\theta=\theta^{\text {eq }}$ in the volume element, and (2) $v\left(r_{1}, r_{2}, \theta\right)$ just a harmonic oscillator function in its arguments. With these assumptions, the integrals in eq. (5) can be done in closed form to yield the usual result:

$$
\phi_{\text {int }}=\Phi_{\nu_{1}}\left(\Phi_{\nu_{2}}\right)^{2} \Phi_{\nu_{3}} \phi_{r}
$$

where the $\Phi_{\nu}$ are the vibrational partition functions for the four vibrations of a linear molecule, and $\Phi_{r}$ is the rotational partition function. For a nonlinear molecule, the partition function separates differently.

In the general case and particularly at high temperature, these assumptions are not valid and the integrals cannot be done except by numerical integration. often, the anharmonic terms in the potential energy prevent the separation of eq. (5) into separate onedimensional integrals, and so we have written a computer program to evaluate the threedimensional integral numerically. The program is based on the "Cartesian product formula" for the Gaussian integration of a three-dimensional rectangular solid [5]. The product formula is formed from the Gauss-Legendre rules for one-dimensional integration [6]. For the integrals that extend to infinity, the integration interval was divided into two parts: from 0 to a and from a to $\infty$. The integral in each part was done with a different coordinate transformation of the basic Gauss-Legendre formula. The point "a" was picked to provide accurate integrals using the fewest number of points.

The partition function we have written is only the classical result and quantum correction to the free energy is given by $[3,7]$

$$
G_{H}=\frac{\hbar^{2} \beta^{2}}{24 m} \int_{-\infty}^{\infty}\left(\frac{\partial V}{\partial x}\right)^{2} \exp (-\beta V) d x \int_{-\infty}^{\infty} \exp (-\beta V) d x
$$


This equation is only suitable for a one-dimensional problem and although many-dimensional correction formulas are available [7], they are difficult, and we use a different procedure for evaluating the quantum corrections. This procedure is to simply add the quantum correction for a harmonic oscillator of the appropriate frequency [1]. This correction is very simple but only approximate. It is close enough for most situations, especially at the high temperatures in which we are interested.

\section{The $\mathrm{C}_{3}$ Molecule}

The $C_{3}$ molecule has been of continuing interest as a high temperature species. Detailed spectroscopic information is available and has been used to calculate the thermodynamic functions. The bending vibration has a $0-1$ frequency of only $63 \mathrm{~cm}^{-1}$ and the vibration is very anharmonic. We have shown previously that both the anharmonicity and the rotation-vibration interaction of the bending mode have a substantial effect on the calculated value of the thermodynamic functions. We have now recalculated the thermodynamic functions taking into account the non-rigidity in the two stretching vibrations. This calculation was done because it could be used to check our new program against our old calculations and also because the accepted thermodynamics of $C_{3}$ have, once again, been questioned [8]. The new calculations were done with exactly the same spectroscopic constants as previously [3] except that the new value of $1224.5 \mathrm{~cm}^{-1}$ (instead of 1235) for the symmetric stretching frequency was used [9]. The results are summarized in table 1, which lists the thermodynamic functions for $c_{3}$ and the differences between the rigid rotor $\left(r_{1}=r_{1}^{e q}, r_{2}=r_{2}^{e q}\right.$ in the volume element of eq. (5)) and the non-rigid rotor. The non-rigid rotor has the larger partition function and thus the larger value of all the functions listed in table 1 . The rigid rotor values (non-rigid rotor minus the correction) agree with the functions we previously published [3].

\section{The $\mathrm{CO}_{2}$ Molecules}

The vibration-rotation spectra of $\mathrm{CO}_{2}$ have been analyzed in great detail [10] and the potential function is known to fourth order terms:

$$
\begin{aligned}
& V\left(r_{1}, r_{2}, \theta\right)=k_{11}\left(r_{1}{ }^{2}+r_{2}{ }^{2}\right)+k_{13} r_{1} r_{2}+k_{22} \theta^{2} \\
+ & k_{111}\left(r_{1}{ }^{3}+r_{2}^{3}\right)+k_{122}\left(r_{1}+r_{2}\right) \theta^{2}+k_{113}\left(r_{1}+r_{2}\right) r_{1} r_{2} \\
+ & k_{1111}\left(r_{1}^{4}+r_{2}^{4}\right)+k_{1113}\left(r_{1}{ }^{2}+r_{2}{ }^{2}\right) r_{1} r_{2}+k_{1133} r_{1}{ }^{2} r_{2}{ }^{2} \\
+ & k_{1122}\left(r_{1}{ }^{2}+r_{2}{ }^{2}\right) \theta^{2}+k_{1223} r_{1} r_{2} \theta^{2}+k_{2222} \theta^{4} .
\end{aligned}
$$

The values of the constants are listed in table 2. Note that the quartic terms $k_{1111}$ and $k_{2222}$ are positive which assures the convergence of the configuration integral. The 
Table 1. Thermodynamic functions of $c_{3}{ }^{a, b}$.

\begin{tabular}{rrrr} 
Temp. $(K)$ & $-\left(G^{0}-H_{0}^{0}\right) / T$ & $S^{0}$ & $C_{p}{ }^{0}$ \\
\hline 298 & 46.509 & 56.782 & 10.216 \\
1000 & 59.212 & 70.228 & 12.280 \\
2000 & 67.159 & 79.059 & 13.050 \\
3000 & 72.071 & 84.377 & 13.155 \\
4000 & 75.643 & 88.162 & 13.151 \\
5000 & 78.451 & 91.094 & 13.128 \\
6000 & 80.763 & 93.486 & 13.106
\end{tabular}

Correction for non-rigidity in the stretch modes ${ }^{c}$.

$\begin{array}{rlll}298 & 0.0009 & 0.0018 & 0.0018 \\ 1000 & 0.0030 & 0.0061 & 0.0061 \\ 2000 & 0.0061 & 0.0122 & 0.0122 \\ 3000 & 0.0091 & 0.0182 & 0.0182 \\ 4000 & 0.0122 & 0.0243 & 0.0243 \\ 5000 & 0.0152 & 0.0304 & 0.0303 \\ 6000 & 0.0182 & 0.0364 & 0.0363\end{array}$

${ }^{\mathrm{a}}$ Calories/deg-mole for $\mathrm{C}_{3}{ }^{12}$.

${ }^{\mathrm{b}}$ Quantum corrections for the two stretching modes at 2040 and $1224.5 \mathrm{~cm}^{-1}$ have been added. Reference [3] discusses quantum corrections for the bending mode.

These are not important at high temperature.

${ }^{\mathrm{C}}$ Non-rigid - rigid values.

Table 2. Structural constants of $\mathrm{CO}_{2}{ }^{\mathrm{a}}$.

Frequencies: $\mathrm{cm}^{-1}$

1354.31,

$672.85(2)$,

2396.32

Force constants ${ }^{b}$

$\begin{array}{lllll}k_{11} 8.0112 & k_{111}-18.9894 & k_{1111} & 26.2511 & \\ k_{13} 1.2613 & k_{113}-1.9545 & k_{1133} & 3.0224 & k_{1113} 3.6760 \\ k_{22} 0.3925 & & k_{2222} & 0.0461 & \\ =1.1600 & k_{122}-0.6089 & k_{1122} & 0.5037 & k_{1223} 1.8700\end{array}$

${ }^{\mathrm{a}}$ Set IV from reference [9].

bunits of mdyn. $\AA$ divided by the appropriate power of $\AA$ for each stretching coordinate and by a power of rad for the bend. 
results of the calculations are shown in table 3 . The corrections for anharmonicity are very smal1; those for non-rigidity are more substantial.

Table 3. The thermodynamic functions of $\mathrm{CO}_{2}{ }^{\mathrm{a}}$.

\begin{tabular}{rrrr} 
Temp. (K) & $-\left(G^{0}-H_{0}^{0}\right) / T$ & \multicolumn{1}{c}{$S^{0}$} & $C_{p}{ }^{0}$ \\
\hline 298 & 43.556 & 51.066 & 8.871 \\
1000 & 54.111 & 64.334 & 12.981 \\
2000 & 61.845 & 78.889 & 14.404 \\
3000 & 66.910 & 79.818 & 14.798 \\
4000 & 76.697 & 84.099 & 14.950 \\
5000 & 73.724 & 87.442 & 15.008 \\
6000 & 76.245 & 90.177 & 15.018 \\
& & & \\
298 & $E f f e c t$ of anharmonicity & \\
1000 & 0.0038 & 0.0075 & 0.0073 \\
2000 & 0.0122 & 0.0238 & 0.0217 \\
3000 & 0.0230 & 0.0427 & 0.0316 \\
4000 & 0.0318 & 0.0549 & 0.0253 \\
5000 & 0.0383 & 0.0593 & 0.0018 \\
6000 & 0.0423 & 0.0559 & -0.0364 \\
& 0.0437 & 0.0450 & -0.0860
\end{tabular}

Effect of rigidityc.

$\begin{array}{rlll}298 & 0.0092 & 0.0184 & 0.0184 \\ 1000 & 0.0309 & 0.0619 & 0.0620 \\ 2000 & 0.0618 & 0.1234 & 0.1219 \\ 3000 & 0.0924 & 0.1833 & 0.1756 \\ 4000 & 0.1223 & 0.2401 & 0.2200 \\ 5000 & 0.1512 & 0.2931 & 0.2543 \\ 6000 & 0.1790 & 0.3418 & 0.2793\end{array}$

${ }^{a} c^{12} 0_{2}^{16}$ in calories/deg-mole.

${ }^{b}$ Anharmonic value - harmonic value for rigid molecules.

${ }^{\mathrm{C}}$ Non-rigid value - rigid value.

This calculation has been carried out in a rather different fashion than that used for the JANAF Tables [11]. The vibrational frequencies used in the JANAF Tables are not the true harmonic frequencies, but rather average anharmonic frequencies. The values listed in table 3 for the anharmonic non-rigid molecule agree with the JANAF values quite well 
with the largest discrepancy being in the heat capacity which we calculate as about $0.5 \mathrm{cal} / \mathrm{deg}$ mole lower than the JANAF Table value at $6000 \mathrm{~K}$.

\section{Summary}

We have developed a computer program for the calculation of the thermodynamic functions of small molecules. The program requires detailed structural and potential function information as input and thus requires an extra stage of analysis when starting from spectroscopic data. A summary of our calculations on $\mathrm{C}_{3}$ and $\mathrm{CO}_{2}$ are shown in table 4 . The effects of both anharmonicity and non-rigidity are small for high frequency vibrational modes, but become large as the frequency goes down. If the frequency goes down sufficiently, the effects become very large as for the $c_{3}$ bending mode. We plan to calculate these effects in a few more molecules including $\mathrm{SO}_{2}$ and $\mathrm{NaOH}$ to provide a better picture of the size of these effects. Finally, we note that the accuracy of the calculations depends entirely on the accuracy of the potential functions available.

Table 4. Effects of anharmonicity and non-rigidity on thermodynamic functions ${ }^{\mathrm{a}}$.

$\begin{array}{clll}C_{3} \text { stretches, } 2040,1224 \mathrm{~cm}^{-1} & \underline{-\left(\mathrm{G}-\mathrm{H}_{\mathrm{o}}\right) / \mathrm{T}} & \underline{\mathrm{S}} & \underline{\mathrm{C}_{\mathrm{p}}} \\ 3000 \mathrm{~K} & 0.009 & 0.018 & 0.018 \\ 6000 \mathrm{~K} & 0.018 & 0.036 & 0.036\end{array}$

$\mathrm{CO}_{2}$ vibrations at $670(2), 1350,2400$

$\begin{array}{llll}3000 \mathrm{~K} & 0.124 & 0.238 & 0.193 \\ 6000 \mathrm{~K} & 0.223 & 0.387 & 0.193\end{array}$

${ }^{a}$ Calories/deg-mole. Effects of non-rigidity only for $C_{3}$.

References

[1] See standard text books and also Herschbach, D. R., Johnston, H. S., and Rapp, D., J. Chem. Phys. 31, 1652 (1959).

[2] Mayer, J. E. and Mayer, M. G., Statistical Mechanics (John Wiley and Sons, New York, 1940); Quantum Chemistry, K. S. Pitzer, ed. (Prentice Hal1, New Jersey, 1953); Lewis, G. N., Randal1, M., Pitzer, K. S., and Brewer, L., Thermodynamics (McGrawHi11, New York, 1961).

[3] Strauss, H. L. and Thiele, E., J. Chem. Phys. 46, 2477 (1967).

[4] Haar, L., Thermodynamic Properties of Ammonia as Ideal Gas, National Bureau of Standards Data Series \#19, National Bureau of Standards, Washington, D.C. (1968). 
[5] Stroud, A. H., in Mathematical Methods for Digital Computers, A. Ralston and H. S. Wilf, eds. (John Wiley, New York, 1968), Vol. II, chap. 7.

[6] Hildebrand, F. B., Introduction to Numerical Analysis (McGraw-Hi11, New York, 1974), 2nd edition.

[7] Landau, L. D. and Lifshitz, E. M. , Statistical Physics (Addison-Wesley, Reading, MA, 1958); Quantum Mechanics and Path Integrals, R. P. Feynman and A. R. Hibbs, eds. (McGraw-Hi11, New York, 1965).

[8] Whittaker, A. G., Science, 200, 763 (1978).

[9] Merer, A. J., Can. J. Phys. 45, 4103 (1967).

[10] Suzuki, I., J. Mol. Spec. 25, 497 (1968).

[11] JANAF Thermochemical Tables, 2nd edition (1971), National Bureau of Standards, NSRDS, NBS 37. 
National Bureau of Standards Special Publication 561, Proceedings of the 10th Materials Research Symposium on Characterization of High Temperature Vapors and Gases held at NBS, Gaithersburg, Maryland, September 18-22, 1978. Issued October 1979.

\title{
GRAPH THEORY PREDICTION OF THE PRESSURE SECOND VIRIAL COEFFICIENTS OF SOME HYDROCARBONS, FLUOROCARBONS AND THEIR MIXTURES
}

\author{
Witold Brostow \\ Center for Advanced Studies \\ National Polytechnic Institute \\ A. P. 14-740, Mexico 14, D. F.
}

\section{Introduction}

Let me begin this paper on a somewhat personal note. When a few years ago I started to teach a course in materials science, I found that the textbooks dealt principally with solids; liquid phases were mentioned sometimes but if so, very briefly, while gaseous phases were hardly named at all. Generally, the omission of liquids and gases was not even justified. The only "comment on this which I found was by Barrett, Nix and Tetelman [1]1: "In the most general sense the field of materials science treats all classes of materials (solids, liquids, and gases), but in this text we shall limit our attention to the most common class of engineering materials--solids."

Under the circumstances, rather than follow existing texts, I developed my own introductory course in materials science. The starting point was the assumption, that gases and liquids have equal rights with solid materials. A textbook has resulted from this course [2]. While writing my book, however, I had an impression of proclaiming a heresy. After al1, the overwhelming majority of research on materials does involve the solid state. I welcomed very much the topic chosen by the National Bureau of Standards for the present Symposium: even if I am still a heretic, at least I am far from being alone.

Among various properties of interest of gaseous phases, volumetric properties play a very important role. There are at least three reasons for this. First, the knowledge of volumetric relationships enables proper storage, transport, and handling of natural gas, various substitute combustion gases, and of so-called technical gases. Second, the same knowledge is necessary for proper operation of chemical reactors used in industrial processes involving gaseous phases. Third, volumetric properties are related to molecular interactions, in particular to the binary interaction potential $u(R)$, where $u$ is the interaction energy of a pair of molecules separated by a distance $R$. In fact, properties of gaseous phases are used more often to evaluate $u(R)$ than are properties of condensed phases. Once interaction potentials are known, the process can be inverted: one can predict macroscopic volumetric

\footnotetext{
${ }^{1}$ Figures in brackets indicate the literature references at the end of this paper.
} 
properties of gases important for industrial and other practical uses. Moreover, one is able also to say a number of things about properties of condensed phases involving the same materials.

To see the quantitative relation between the gas volume $V$ and intermolecular interactions, let us write first the so-called virial equation of state:

$$
P V=N k T+B P+\ldots
$$

where $P$ is the macroscopic pressure, $N$ - the number of particles, $k$ - the Boltzmann constant, $T$ - the thermodynamic temperature, and $B$ the second pressure virial coefficient. The word "pressure" should better be included, since there is an increased interest nowadays in the so-called dielectric virial coefficients [3-5]. The third, fourth, etc., virial coefficients are omitted from (1).

There are many equations "competitive" to eq. (1), particularly the van der Waals equation and its numerous modifications. Equation (1), however, is the only one which can be directly related to the interaction potential $u(R)$ by

$$
B(T)=-2 \pi N \int_{0}^{\infty}\left(e^{\frac{-u(R)}{k T}}-1\right) R^{2} d R
$$

Equation (2) has been written for a pure component. Extensions to mixtures will be treated later on. It is possible (see e.g., [2]) to transform (2) into an equivalent form

$$
B(T)=\frac{-2 \pi N}{3 k T} \int_{0}^{\infty} \frac{d u(R)}{d R} e^{\frac{-u(R)}{k T}} R^{3} d R
$$

As indicated in eqs. (2) and (3), B is a function of temperature only. According to eq. (1), the problem of evaluating the volume of a gaseous system at a given temperature and pressure consists in finding $B$. This is true unless high pressures are involved; for external pressures producing gas densities comparable to liquid densities, higher pressure virial coefficients have to be included. Given both the fundamental and practical importance of the second coefficient $B$, in this paper, we discuss a new method of correlating and predicting $B$ for gaseous materials consisting of chain molecules. Among other things, such molecules are constituents of natural gas as well as of various substitute combustion gases.

\section{Molecules and Graphs}

Prompted by industrial needs, there have been earlier attempts to connect B to interaction potentials, notably by Kaul and Prausnitz [6], who used the so called 
rectangular well $u(R)$ potential. Such attempts were based on an assumption of spherical force fields; clearly, along with an increase in the chain length this assumption becomes progressively worse.

Under the circumstances, the following research plan appears indicated, in fact as a natural extension of the work done before. First, to cut mentally gaseous chain molecules into segments, just as polymerists do nearly automatically for macromolecules in condensed phases. Then, instead of considering interactions between entire molecules, study interactions between segments. Next, represent intersegmental interactions in terms of their contributions to macroscopic quantities of interest, in our case in terms of the pressure second virial coefficient B. Verify the physical significance of the parameters so introduced by referring to available experimental data. Finally, propose interaction potentials $u(R)$ appropriate for intersegmental interactions.

A11 but the last stage of the proposed program have been already tackled for some hydrocarbons, fluorocarbons, and their binary mixtures [7,8]. Essential elements of the results obtained will now be reported. The basic tool used in the work is the theory of graphs.

It has been stated some years ago [9] that the one-to-one equivalence between molecules and graphs has been far from sufficiently exploited by physicists and chemists. A certain progress has been made since. In particular, a walk interaction formalism has been proposed for the prediction of equilibrium properties of liquid phases [10]. For the problem at hand, the same formalism has been extended to gases.

Before saying how the graph theory helps us to realize the program outlined above, we need to define a small number of graph theoric notions. This will be done in the following Section. More such notions have been defined by Essam and Fisher [11], in Chapter 2 of [2], and of course are discussed in textbooks of graph theory, e.g., by Harary [12].

\section{Graph Theory Definitions}

For our purposes, it is sufficient to define a graph as a set of lines between some of the pairs of points. A walk is an alternating sequence of points and lines, beginning and ending with points, in which each line is incident with the two points immediately preceding and following it. If the first point of a walk coincides with the terminal point, the walk is closed. If all $n$ points of a closed walk are distinct and $n \geq 3$, the walk is a cycle. The length of a walk is the number of occurrences of lines in it. If every pair of points in a graph can be joined by a walk, the graph is connected. A tree is a connected graph which does not contain cycles. 
Consider a graph of $r$ points, with the points distinguished from one another by labels such as $v_{1}, v_{2}, \ldots, v_{r}$. The adjacency matrix $M=\left(a_{i j}\right)$ is the $r \times r$ matrix in which each entry $a_{i j}=1$ if $v_{i}$ is adjacent with $v_{j}$; otherwise $a_{i j}=0$. Consider now a product of, say, 1 matrices. Then the $a_{i j}$ entry of $M^{\top}$ is the number of walks of length 1 from $v_{i}$ to $v_{j}$.

Not a 17 walks have physical significance. Closed walks are called uninteresting and eliminated from considerations. Further, two or more walks of unequal length can represent the same physical situation, e.g., walks ab and ababab, and then the longer one is taken as uninteresting. The exact definition of an interesting walk is given in [9]. Finally, we can have two or more interesting walks of the same length which also represent the same physical situation, e.g., the walks abcdb and abdcb, involving traveling through the same cycle in two opposite directions. Since all walks of a given length representing the same situation form an equivalence class, in each case we need to calculate the number $\Gamma_{7}$ of equivalence classes of interesting walks of length 1 in a given graph. If this appears somewhat difficult, let us note that, for such short walks as we consider here, each equivalence class of interesting walks contains exactly one element.

\section{Basic Relations}

Consider a closed system of interacting units, and label types of units present by indices such as $y$ and $y^{\prime}$. Denote the total number of units by $N$ and introduce the mole fractions $x_{y}=N_{y} / N$. Following [10], consider a class of physical properties of the system such that for each property $F$ we have

$$
F=\Sigma \Sigma^{2} y^{\prime} x_{y^{\prime}} x^{\prime} F_{y y^{\prime}}
$$

We can choose $F$ to be a quantity per mole. Parameters $F_{y y}$, represent contributions resulting from interactions of units of type $y$ on one molecule with units of type $y^{\prime}$ on another molecule. In general, the interacting units could be molecules, atoms, segments, etc. Still following [10], we take the interacting units to consist of equivalence classes of interesting walks of a given length 1 on graphs representing molecules belonging to the system.

Representation of molecules by graphs permits a treatment even more accurate than originally suggested in Section 2. For any given segment, walks of length $1=7$ which contain our segment take into account effects of all nearest neighbors that the segment has within its own molecule. Similarly, there are as many interesting walks of length $1=2$ involving a given segment as there are second nearest neighbors of the segment, and so on.

The general approach proposed in [10] contains earlier theories and models as special, simpler (that is, less accurate) cases. One reduction consists in putting $1=0$ and neglecting all walks with $1>0$; we are thus, back into conventional treatments in terms of segments. 
Another possible simplification consists in assuming for each $F_{y y^{\prime}}$ that

$$
F_{y y^{\prime}}=\frac{F_{y}+F_{y^{\prime}}}{2} .
$$

Substitution of (2) into (1) gives

$$
F=\Sigma_{y} x_{y} F_{y}
$$

where $F_{y}$ represents contributions of single units of type $y$; thus, in eq. (6) contributions resulting from external interactions of pairs of units have disappeared completely. The situation when both kinds of simplifications have been introduced simultaneously, i.e., when we use eq. (6) with $1=0$, corresponds to a large number of structural schemes, including the classical calculation procedures for parachor, refractivity, and polarizability, various thermochemical models, and also various so-called group contribution theories.

As demonstrated in [10], eq. (1) contains completely single contributions of the type displayed in eqs. (5) and (6). Thus, using eq. (4) we take into account single contributions together with the binary ones; this is immaterial for the quantity we study in this paper, but it is important for many other functions $F$.

A useful discussion of the significance of the pressure second virial coefficient $B$ has been written by Kilpatrick [13]. We know that $B$ results from binary interactions only, so we can put $F=B$ in eq. (4). While, in general, some spin distribution effects might appear, Larsen and his colleagues have demonstrated [14] that such effects are negligible, except at very low temperatures. Thus, for temperatures studied here eq. (4) with $F=B$ can be used as it stands. To perform summations indicated by (4), we need the numbers for each kind of graph, i.e., for each kind of molecule belonging to the system. For a given graph, define

$$
a_{i}^{\ell}=\sum_{j=1}^{r} a_{i j}^{l}
$$

and

$$
a^{l}=\sum_{i=1}^{r} a_{i}^{l} .
$$

Then [9]

$$
\begin{aligned}
& \Gamma_{1}=\frac{1}{2} a^{1}=\frac{1}{2} \operatorname{tr} M^{2} \\
& \Gamma_{2}=\frac{1}{2}\left(a^{2}-\operatorname{tr} M^{2}\right) \\
& \Gamma_{3}=\frac{1}{2}\left(a^{3}-a^{1}-\operatorname{tr} M^{3}\right) .
\end{aligned}
$$


In the present work we will not need $\Gamma_{1} s$ for $1>3$, but such $\Gamma_{1} s$ are considered in [9].

\section{Calculations and Results}

Numerical results discussed in this Section come from the thesis of Pérez-Gutiérrez [7] and also from some subsequent calculations [8]. We begin with normal alkanes.

\section{1 Alkanes}

Reliable experimental results for n-alkanes are those of McGlashan and Potter [15]. We assume that walks containing the $\mathrm{CH}_{3}$ group (denoted by subscript e for end) are different from the walks containing only $\mathrm{CH}_{2}$ groups (subscript $\mathrm{m}$ for middle). Thus, eq. (4) takes the form

$$
B=\frac{1}{2} x_{e}^{2} B_{e e}+x_{e} x_{m} B_{e m}+\frac{1}{2} x_{m}^{2} B_{m m}
$$

Parameters $B_{e e}, B_{e m}$, and $B_{m m}$ represent the respective pair interactions for a given length 1 ; for any 1 we have $x_{e}=\Gamma_{e} /\left(\Gamma_{e}+\Gamma_{m}\right)$. Each of the $B_{y y^{\prime}}$ parameters can be represented in terms of $u_{y y^{\prime}}\left(R_{y y^{\prime}}\right)$ by an equation of the form of (2) or else by (3).

Consider first $1=0$, when the end unit is the methyl group and the middle unit is the methylene group. We have in any molecule $r$ carbon atoms and

$$
\Gamma_{0}=r
$$

moreover, for any length $\ell \leqslant r-1$

$$
\Gamma_{\mathrm{e}}=r_{\mathrm{e}}=2
$$

Thus, from eq. (11)

$$
B=\frac{r_{e}^{2}}{2 r} B_{e e}+\frac{\left(r-r_{e}\right) r_{e}}{r} B_{e m}+\frac{\left(r-r_{e}\right)^{2}}{2 r} B_{m m} .
$$

For $\ell=1$, with the end unit $\mathrm{CH}_{3} \cdot \mathrm{CH}_{2}$ and the middle unit $\left(\mathrm{CH}_{2}\right)_{2}$, according to eq. (8), we have $\Gamma_{1}=r-1$, so that

$$
B=\frac{r_{e}^{2}}{2(r-1)} B_{e e}+\frac{\left(r-r_{e}^{-1) r} e\right.}{\left(r^{-1}\right)} B_{e m}+\frac{\left(r-r_{e}-1\right)^{2}}{2(r-1)} B_{m m}
$$


We do not go beyond $\ell=2$. In this last case

$$
B=\frac{r_{e}^{2}}{2(r-2)} B_{e e}+\frac{\left(r-r e^{-2) r} e\right.}{(r-2)} B_{e m}+\frac{\left(r-r_{e}-2\right)^{2}}{2(r-2)} B_{m m}
$$

The experimental data in [15] are for values of $r$ between 3 and 8 . From these data by multiple regression analysis, we have evaluated the three characteristic $B_{y y}$, parameters for each of the lengths $1=0,1$ and 2 and for several temperatures. The resulting parameter values are listed in table 1.

Table 1. Byy' parameters for n-alkanes.

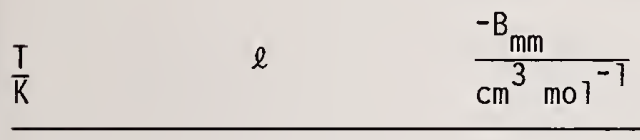

298

$\begin{array}{ll}0 & 1983.4 \\ 1 & 1779.1 \\ 2 & 1989.2\end{array}$

348

$\begin{array}{ll}0 & 1527.5 \\ 1 & 1375.5 \\ 2 & 1529.4\end{array}$

373

$\begin{array}{ll}0 & 1208.7 \\ 1 & 1097.4 \\ 2 & 1206.7\end{array}$

400

$\begin{array}{ll}0 & 965.8 \\ 1 & 876.6 \\ 2 & 953.3\end{array}$

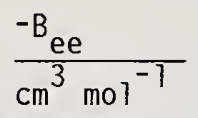

1559.2

454.1

745.8

1138.9

374.1

615.2

858.4

313.9

517.2

664.0

267.1

441.6

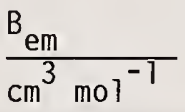

1543.2

237.8

- 224.6

1074.1

116.2

- 236.9

769.9

44.1

- 234.1

565.9

0.3

- 225.7

512.9

417.6

224.9

$-\quad 26.2$

371.1

- 220.0

Parameters of table 7 have been substituted back into eqs. (14). The calculated values of $B$ are compared with the experimental ones in table 2 . The latter data have been obtained using an analytical eq. (9.4) in [15], namely 


$$
\frac{B}{V_{c r}}=0.430-0.886 \frac{T_{c r}}{T}-0.694\left(\frac{T_{c r}}{T}\right)^{2}-0.0375(n-1)\left(\frac{T_{c r}}{T}\right)^{4.5}
$$

The index or refers to the liquid-vapor critical point and $n$ characterizes the size of a molecule (for $n$-alkanes $n=r$ ). McGlashan and Potter [15] claim that the precision of their new experimental results is between $\pm 5 \mathrm{~cm}^{3} \mathrm{~mol}^{-1}$ and $\pm 20 \mathrm{~cm}^{3} \mathrm{~mol}^{-1}$ (worse for longer chains) They also say that the agreement between their eq. (9.4) (our eq. (15)) and the measured values is "almost, if not quite, within the experimental error." Table 2 contains also percentage differences between the calculated values and the experimental ones. Given the assessment of McGlashan and Potter, the differences of the order of 3 percent (slightly less for longer chains) are within limits of precision of the smoothed experimental data.

Table 2. Second virial coefficients of n-alkanes.

\begin{tabular}{|c|c|c|c|c|c|}
\hline n-alkane & $\frac{T}{K}$ & $\frac{-\mathrm{B} \text { exper. }}{\mathrm{cm}^{3} \mathrm{~mol}^{-1}}$ & $\ell$ & $\frac{{ }^{-B} \mathrm{calc} .}{\mathrm{cm}^{3} \mathrm{~mol}^{-1}}$ & $\begin{array}{l}\text { difference } \\
\text { - percent }\end{array}$ \\
\hline \multirow{10}{*}{$\mathrm{C}_{3} \mathrm{H}_{8}$} & 298 & 387.2 & 0 & 453.9 & -17.2 \\
\hline & & & 1 & 453.1 & -17.2 \\
\hline & 323 & 326.3 & 0 & 373.8 & -14.6 \\
\hline & & & 1 & 374.1 & -14.6 \\
\hline & 348 & 278.7 & 0 & 313.6 & -12.6 \\
\hline & & & 1 & 373.9 & -12.6 \\
\hline & 373 & 240.5 & 0 & 266.9 & -11.0 \\
\hline & & & 1 & 267.1 & -11.1 \\
\hline & 400 & 206.0 & 0 & 224.5 & -9.0 \\
\hline & & & 1 & 224.9 & -9.2 \\
\hline \multirow{15}{*}{$\mathrm{C}_{4} \mathrm{H}_{10}$} & 298 & 713.9 & 0 & 566.0 & 20.7 \\
\hline & & & 1 & 540.2 & 24.3 \\
\hline & & & 2 & 745.8 & -4.2 \\
\hline & 323 & 592.5 & 0 & 487.7 & 17.8 \\
\hline & & & 1 & 486.5 & 20.9 \\
\hline & & & 2 & 615.2 & -3.8 \\
\hline & 348 & 500.6 & 0 & 423.1 & 16.2 \\
\hline & & & 1 & 409.1 & 18.3 \\
\hline & & & 2 & 517.2 & -3.3 \\
\hline & 373 & 429.0 & 0 & 370.5 & 13.6 \\
\hline & & & 1 & 359.9 & 16.7 \\
\hline & & & 2 & 441.6 & -2.9 \\
\hline & 400 & 358.9 & 0 & 321.8 & 10.3 \\
\hline & & & 1 & 313.5 & 12.6 \\
\hline & & & 2 & 371.1 & -3.4 \\
\hline
\end{tabular}


Table 2. (Continued)

\begin{tabular}{|c|c|c|c|c|c|}
\hline n-alkane & $\frac{T}{K}$ & $\frac{-\mathrm{B} \text { exper. }}{\mathrm{cm}^{3} \mathrm{~mol}^{-1}}$ & $\ell$ & $\frac{\mathrm{cm}^{-B}}{\frac{\mathrm{calc}}{30 l^{-1}}}$ & $\begin{array}{l}\text { difference } \\
\text { - percent }\end{array}$ \\
\hline \multirow{15}{*}{$\mathrm{C}_{5} \mathrm{H}_{12}$} & \multirow[t]{3}{*}{298} & \multirow[t]{3}{*}{1196.8} & 0 & 1165.1 & 2.6 \\
\hline & & & 1 & 1176.2 & 1.8 \\
\hline & & & 2 & 1092.2 & 8.6 \\
\hline & \multirow[t]{3}{*}{323} & \multirow[t]{3}{*}{974.2} & 0 & 951.8 & 2.3 \\
\hline & & & 1 & 960.4 & 1.4 \\
\hline & & & 2 & 899.6 & 7.7 \\
\hline & \multirow[t]{3}{*}{348} & \multirow[t]{3}{*}{810.5} & 0 & 794.3 & 2.0 \\
\hline & & & 1 & 806.6 & 1.2 \\
\hline & & & 2 & 755.8 & 6.7 \\
\hline & \multirow[t]{3}{*}{373} & \multirow[t]{3}{*}{686.6} & 0 & 674.4 & 1.8 \\
\hline & & & 1 & 679.2 & 1.1 \\
\hline & & & 2 & 645.9 & 5.9 \\
\hline & \multirow[t]{3}{*}{400} & \multirow[t]{3}{*}{594.9} & 0 & 573.3 & 3.6 \\
\hline & & & 1 & 576.9 & 3.0 \\
\hline & & & 2 & 552.9 & 7.1 \\
\hline
\end{tabular}

\begin{tabular}{|c|c|c|c|c|c|}
\hline & 298 & 1895.8 & 0 & 2007.7 & -5.9 \\
\hline & & & 1 & 2034.5 & -7.1 \\
\hline & & & 2 & 1933.3 & -1.2 \\
\hline & 323 & 1512.4 & 0 & 1591.9 & -5.3 \\
\hline & & & 1 & 1611.4 & -6.5 \\
\hline & & & 2 & 1539.1 & -1.8 \\
\hline \multirow{9}{*}{$\mathrm{C}_{6} \mathrm{H}_{14}$} & 348 & 1237.9 & 0 & 1296.3 & -4.8 \\
\hline & & & 1 & 1310.6 & -5.9 \\
\hline & & & 2 & 1257.4 & -1.6 \\
\hline & 337 & 1034.6 & 0 & 1078.4 & -4.2 \\
\hline & & & 1 & 1089.3 & -5.3 \\
\hline & & & 2 & 1049.9 & -1.5 \\
\hline & 400 & 860.3 & 0 & 901.9 & -4.8 \\
\hline & & & 1 & 910.3 & -5.8 \\
\hline & & & 2 & 882.2 & -2.5 \\
\hline
\end{tabular}


Table 2. (Concluded)

\begin{tabular}{|c|c|c|c|c|c|}
\hline n-alkane & $\frac{T}{K}$ & $\frac{-\mathrm{B}_{\text {exper. }}}{\mathrm{cm}^{3} \mathrm{~mol}^{-1}}$ & $\ell$ & $\frac{-\mathrm{B}_{\mathrm{calc} .}}{\mathrm{cm}^{3} \mathrm{~mol}^{-1}}$ & $\begin{array}{l}\text { difference } \\
\text { - percent }\end{array}$ \\
\hline \multirow{15}{*}{$\mathrm{C}_{7} \mathrm{H}_{16}$} & 298 & 2860.1 & 0 & 2989.5 & -4.5 \\
\hline & & & 1 & 3002.0 & -4.8 \\
\hline & & & 2 & 2972.3 & -3.9 \\
\hline & 323 & 2240.7 & 0 & 2332.8 & -4.1 \\
\hline & & & 1 & 2341.8 & -4.5 \\
\hline & & & 2 & 2320.6 & -3.6 \\
\hline & 348 & 1805.5 & 0 & 1873.1 & -3.9 \\
\hline & & & 1 & 1879.8 & -4.1 \\
\hline & & & 2 & 1864.2 & -3.3 \\
\hline & 373 & 1488.2 & 0 & 1539.7 & -3.5 \\
\hline & & & 1 & 1544.6 & -3.8 \\
\hline & & & 2 & 1533.6 & -3.1 \\
\hline & 400 & 1237.1 & 0 & 1274.6 & -3.0 \\
\hline & & & 1 & 1278.6 & -3.4 \\
\hline & & & 2 & 1270.5 & -2.7 \\
\hline \multirow{15}{*}{$\mathrm{C}_{8} \mathrm{H}_{18}$} & 298 & 4186.7 & 0 & 4058.3 & 3.1 \\
\hline & & & 1 & 4035.6 & 4.7 \\
\hline & & & 2 & 4110.2 & 2.8 \\
\hline & 323 & 3227.8 & 0 & 3136.3 & 2.8 \\
\hline & & & 1 & 3117.9 & 3.4 \\
\hline & & & 2 & 3173.2 & 1.7 \\
\hline & 348 & 2563.8 & 0 & 2496.7 & 2.9 \\
\hline & & & 1 & 2483.0 & 3.2 \\
\hline & & & 2 & 2523.7 & 1.6 \\
\hline & 373 & 2087.6 & 0 & 2036.8 & 2.4 \\
\hline & & & 1 & 2025.8 & 2.9 \\
\hline & & & 2 & 2057.1 & 1.4 \\
\hline & 400 & 1713.8 & 0 & 1674.9 & $2: 3$ \\
\hline & & & 1 & 1666.9 & 2.7 \\
\hline & & & 2 & 1688.3 & 1.5 \\
\hline
\end{tabular}

Inspection of table 2 shows that the best results are obtained for $\ell=2$. This supports our basic hypothesis about the influence of internal molecular structure upon external interactions and upon macroscopic properties. The same problem is considered in the following paper [16] from the point of view of a property of liquid n-alkanes which is measured experimentally with high precision, namely molar volume, and the same 
conclusion is reached (the results in [16] are even more convincing, since lengths from $\ell=0$ to $\ell=3$ are considered and a steady improvement observed along with increasing $\ell$; given limited accuracy of the experimental determination of the second virial coefficient, there was little point in going beyond $\ell=2$ in the present work).

The length $\ell=2$ for $n$-propane was not included, since the methylene group here has different surroundings, and the only 2-walk here is different from 2-walks in larger molecules. It appears at first sight that better agreement between calculations and experiment is obtained at higher temperatures. This, however, almost certainly is an artifact, since experiments in general produce more accurate (and also more precise) values of B at higher temperatures. As noted by McGlashan and Potter [15], at lower temperatures vapor condensation in their apparatus is possible. Thus, we are inclined to believe that smaller differences between experimental and calculated values at high temperatures reflect a better quality of experiments, and do not imply that the theory works better at higher temperatures.

We also find in table 2 that the percentage differences between theory and experiment decrease along with the increase of the chain length $r$. This is understandable. Since the methylene group in a propane molecule is in different surroundings than a methylene group in, say, heptane, a $B_{m m}$ parameter which describes heptane molecules ought to serve well for octane too, but it ought not work well for propane. In other words, the discrepancies observed for shorter molecules prove precisely the importance of the effect we are studying.

\subsection{Mixtures of alkanes}

We now pass to mixtures of n-alkanes. Experimental data for binary equimolar mixtures of propane with heptane and of propane with octane have been obtained also by McGlashan and Potter [15]. Values interpolated from their data for temperatures given in table 1 are listed in column 4 of table 3.

Typically, one predicts properties of mixtures in terms of properties of pure components using a number of approximate combining rules. Quantities obtained from such combining rules represent some phenomena which supposely occur in mixtures but which were absent from pure components. Following this route, McGlashan and Potter assumed a principle of corresponding states, and used three combining rules to calculate properties of their mixtures. Namely, for a binary mixture $i+j$,

$$
\begin{gathered}
v_{c r_{j+j}}=\left(v_{c r_{i}}^{1 / 3}+v_{c r_{j}}^{1 / 3}\right) / 8 \\
T_{c r_{i+j}}=T_{c r_{j}}^{1 / 2} \cdot T_{c r_{j}}^{1 / 2}
\end{gathered}
$$

and

$$
n_{i+j}=\left(n_{i}+n_{j}\right) / 2
$$


or

$$
n_{i+j}=n_{i}^{1 / 2} \cdot n_{j}^{1 / 2}
$$

We have performed calculations using the rules recommended by McGlashan and Potter, namely (16), (17), and (18b). The results are listed in column 5 of table 3.

Table 3. Second virial coefficients $B$ of equimolar n-alkane mixtures.

\begin{tabular}{|c|c|c|c|c|c|c|c|}
\hline System & $\frac{T}{K}$ & $\ell$ & Experimental & $\begin{array}{l}-8 /\left(\mathrm{cm}^{3} \mathrm{~mol}^{-1}\right) \\
\text { combining } \\
\text { rules }\end{array}$ & $\begin{array}{l}\text { Graph } \\
\text { theory }\end{array}$ & Graph & $\begin{array}{l}\text { theory diff } \\
\text { percent }\end{array}$ \\
\hline \multirow{6}{*}{$\mathrm{C}_{3} \mathrm{H}_{8}+\mathrm{C}_{7} \mathrm{H}_{16}$} & 348 & 0 & 830 & 834 & 794.2 & & 4.3 \\
\hline & & 1 & & & 800.8 & & 3.5 \\
\hline & 373 & 0 & 696 & 696 & 674.4 & & 3.1 \\
\hline & & 1 & & & 679.0 & & 2.4 \\
\hline & 400 & 0 & 579 & 583 & 573.3 & & 0.98 \\
\hline & & 1 & & & 577.0 & & 0.35 \\
\hline \multirow{4}{*}{$\mathrm{C}_{3} \mathrm{H}_{8}+\mathrm{C}_{8} \mathrm{H}_{18}$} & 373 & 0 & 894 & 894 & 867.3 & & 3.0 \\
\hline & & 1 & & & 876.3 & & 2.0 \\
\hline & 400 & 0 & 715 & 687 & 730.7 & & 2.2 \\
\hline & & 1 & & & 737.8 & & 3.2 \\
\hline
\end{tabular}

Return now to our graph theory formalism. Applying it to mixtures of $n$-alkanes, we find immediately that any combining rules are here completely superfluous: we have clearly the same kinds of interactions between walks in mixtures as in pure components. We can, therefore, use again the parameters of table 1 to predict $B$ values; that is, we calculate values for mixtures basing on data for pure components only. We apply again eqs. (14), now with the parameter $r$ featuring in these equations given by

$$
r=x_{y} r_{y}+x_{y^{\prime}} r_{y^{\prime}}
$$

The results are listed in column 6 of table 3 . Column 7 gives percentage differences between the experimental values and those predicted with the graph theory formalism.

Inspection of table 3 shows that the corresponding states formalism and the walk interaction formalism both predict values for mixtures within limits of experimental precision. The basic problem with the former, and with all schemes involving combining rules, is that one never knows in advance what set of combining rules will produce acceptable values. For instance, talking about eq. (18a) vs (18b), McGlashan and Potter 
say that: "The use of the geometric mean instead of the arithmetic mean for $n$ gives a slightly better fit"; of course, for a different class of systems, the reverse can be true, or perhaps a still different combining rule, such as the harmonic mean $[17,18]$, would produce better values than either of the rules given in eqs. (18).

\subsection{Perfluorocarbons}

We now pass to normal perfluorocarbons $C_{n} F_{2 n+2}$. Dantzler and Knobler [19] have critically analyzed and listed recommended rules for pure components, as well as obtained experimental values for some binary mixtures.

Given the analogical structure of $n$-alkane and n-perfluoroalkane molecules, calculations now have been performed in the same way as for alkanes. The characteristic parameters are listed in table 4 . Walks for perfluorocompounds have been marked by asterisks; thus, for instance $e^{*}$ for $\ell=0$ denotes the $\mathrm{CF}_{3}$ group, so as to distinguish it from e which, for the same length, still denotes the $\mathrm{CH}_{3}$ group.

Table 4. B yy' parameters for n-perfluorocarbons.

\begin{tabular}{|c|c|c|c|c|}
\hline$\frac{\mathrm{T}}{\mathrm{K}}$ & $\ell$ & $\frac{-\mathrm{B}_{\mathrm{m}^{*} \mathrm{~m}^{*}}}{\mathrm{~cm}^{3} \mathrm{~mol}^{-1}}$ & $\frac{-\mathrm{B} \mathrm{e}^{*} \mathrm{e}^{*}}{\mathrm{~cm}^{3} \mathrm{~mol}^{-1}}$ & $\frac{-\mathrm{B} \mathrm{e}^{*} \mathrm{~m}^{*}}{\mathrm{~cm}^{3} \mathrm{~mol}^{-1}}$ \\
\hline \multirow[t]{3}{*}{323} & 0 & 1325.6 & 485.8 & -163.9 \\
\hline & 1 & 1205.7 & 434.3 & 374.7 \\
\hline & 2 & 1208.0 & 744.0 & 736.0 \\
\hline \multirow[t]{3}{*}{373} & 0 & 857.4 & 307.9 & -88.5 \\
\hline & 1 & 784.0 & 288.7 & 255.7 \\
\hline & 2 & 738.0 & 492.0 & 513.0 \\
\hline
\end{tabular}

Experimental and calculated values for pure compounds are listed in table 5. For $\ell=2$, we have only 3 compounds to evaluate 3 parameters; thus, no check of the theory is possible in this case, but a check will be provided by predictions for mixtures discussed below. For $\ell=0$ and $\ell=1$, the calculated values are certainly within limits of the experimental error. We would welcome very much experimental B values for $r>6$, which would enable one to test the present formalism further. 
Table 5. Second virial coefficients B of n-perfluorocarbons.

\begin{tabular}{|c|c|c|c|c|c|}
\hline Compound & $\frac{T}{K}$ & $\ell$ & $\frac{-\mathrm{B} \text { exper. }}{\mathrm{cm}^{3} \mathrm{~mol}^{-1}}$ & $\frac{{ }^{-B} \mathrm{calc}}{\mathrm{cm}^{3} \mathrm{~mol}^{-1}}$ & $\frac{\text { difference }}{\text { percent }}$ \\
\hline \multirow{4}{*}{$\mathrm{C}_{3} \mathrm{~F}_{8}$} & 323 & 0 & 435 & 435.5 & -0.12 \\
\hline & & 1 & & 434.0 & 0.23 \\
\hline & 373 & 0 & 290 & 289.3 & 0.23 \\
\hline & & 1 & & 288.7 & 0.005 \\
\hline \multirow{6}{*}{$C_{3} F_{10}$} & 323 & 0 & 744 & 741.8 & 0.29 \\
\hline & & 1 & & 740.3 & 0.50 \\
\hline & & 2 & & 744.0 & - \\
\hline & 373 & 0 & 492 & 494.7 & 0.55 \\
\hline & & 1 & & 493.6 & 0.03 \\
\hline & & 2 & & 492.0 & - \\
\hline \multirow[t]{6}{*}{$C_{5} F_{12}$} & 323 & 0 & 1188 & 1190.7 & -0.23 \\
\hline & & 1 & & 1194.7 & -0.56 \\
\hline & & 2 & & 1188.0 & - \\
\hline & 373 & 0 & 793 & 789.6 & 0.42 \\
\hline & & 1 & & 792.0 & 0.001 \\
\hline & & 2 & & 793.0 & - \\
\hline \multirow{6}{*}{$\mathrm{C}_{6} \mathrm{~F}_{14}$} & 323 & 0 & 1712 & 1710.9 & 0.06 \\
\hline & & 1 & & 1708.5 & 0.20 \\
\hline & & 2 & & 1712.0 & - \\
\hline & 373 & 0 & 1128 & 1129.4 & -0.12 \\
\hline & & 1 & & 1127.9 & 0.000 \\
\hline & & 2 & & 1128.0 & - \\
\hline
\end{tabular}

\subsection{Mixtures of perfluorocarbons}

We have calculated $B$ values for equimolar mixtures of n-perfluorocarbons from the data given by Dantzler and Knobler [19] (data for pure components from their table 4, the "interaction virial coefficient $\mathrm{B}_{12}$ " from their table 2; they have not listed their experimental values of $B$, only some related parameters). These values are listed in column 4 of table 6 . This time we do not include any results obtained with combining rules. 
Dantzler and Knobler [19] have made such calculations, using a set of combining rules and a set of Kihara interaction potential parameters; good agreement was obtained. They have concluded, however, that "the uncertainty in the potential parameters for the pure components makes it impossible to demonstrate the uniqueness of the chosen parameters and combination rules."

Table 6. Second virial coefficients B of equimolar n-perfluorocarbon mixtures.

\begin{tabular}{llllll} 
System & $\frac{T}{\mathrm{~K}}$ & $\ell$ & $\frac{-\mathrm{B} \text { exper. }}{\mathrm{cm}^{3} \mathrm{~mol}^{-1}}$ & $\frac{{ }^{-B} \mathrm{calc} .}{\mathrm{cm}^{3} \mathrm{~mol}^{-1}}$ & $\frac{\text { difference }}{\text { percent }}$ \\
\hline $\mathrm{C}_{4} \mathrm{~F}_{10}+\mathrm{C}_{5} \mathrm{~F}_{12}$ & 323 & 0 & 953 & 959.4 & -0.67 \\
& 1 & & 956.9 & -0.41 \\
& 2 & 950.0 & 0.31 \\
$\mathrm{C}_{4} \mathrm{~F}_{10}+\mathrm{C}_{6} \mathrm{~F}_{14} 373$ & 0 & 772 & & -2.3 \\
& 1 & & 789.7 & -2.6 \\
\hline
\end{tabular}

We have predicted $B$ values for mixtures from the parameters 1 isted in table 6 . The formalism used was analogous to that for mixtures of n-alkanes. Thus, again the data for pure components were sufficient, and no combining rules necessary. The results obtained are listed in column 5 of table 6 . We find that also for perfluorocarbons the graph theory predictions are within limits of the experimental accuracy.

\subsection{Alkanes + perfluorocarbons}

Some experimental B values for mixtures of $n$-alkanes with $n$-perfluorocarbons are available [20]. Referring to the temperatures for which walk interaction parameters are listed in tables 1 and 4 , we find that there are only five experimental data points at $323 \mathrm{~K}$ and also five points of $373 \mathrm{~K}$. To describe mixtures of $\mathrm{n}$-alkanes with $\mathrm{n}$-perfluoroalkanes we need for any length $l$ of walks a total of 10 parameters: $B_{m m}, B_{e e}, B_{e m}, B_{m^{*} m^{*}}$,

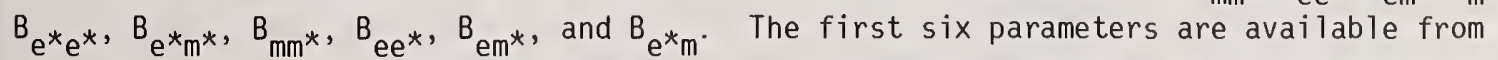
tables 1 and 4 . The last four parameters could be calculated from the first six and from experimental $B$ values for pure components of the type $C_{n} H_{x} F_{2 n+2-x}$, but such reliable values have not been found. Further, it might seem that this time we could imitate others and use some combining rules, e.g., $B_{m^{*}}=B_{m m^{*}}\left(B_{m m}, B_{m^{*} m^{*}}\right)$. Dantzler Siebert and Knobler [20] have found, however, that the combining rules for molecules as a whole which were successful for hydrocarbons and fluorocarbons fail badly in the case of hydrocarbon + fluorocarbon mixtures. We have calculated the $4 \mathrm{~B}_{\mathrm{yy}}$ parameters in question from the experimental data 
for mixtures, and found that typical combining rules do not work for $B_{y y}$ parameters either. The culprit is clearly the strong specific $\mathrm{C}+\mathrm{H}$ interaction. Our four new parameters (+6 "old" parameters) reproduce at each temperature and for each length $\ell$ the experimental $B$ values for mixtures within limits of the experimental accuracy. This, however, is hardly a test of the theory: at each temperature we represent 5 data points with 4 new parameters. For this reason, we do not report the respective numerical values in this paper. Again, we conclude that more reliable experimental values are necessary to test the theory properly: either for pure $\mathrm{C}_{n} \mathrm{H}_{x} \mathrm{~F}_{2 n+2 n-x}$ compounds, or for hydrocarbon + fluorocarbon mixtures, and preferably for both pure components and mixtures.

\section{$5.6 \quad$ Alk-1-enes}

Experimental B values for pure alklenes as well as some of their binary mixtures have been reported by McGlashan and Wormald [21]. A n-alkene molecule contains 4 kinds of segments: terminal $\mathrm{CH}_{3}$, the same as in alkanes, symbol e; middle $\mathrm{CH}_{2}$, also the same as in alkanes, symbol $\mathrm{m}$; terminal $\mathrm{CH}_{2}$, call them $\mathrm{c}$; and $\mathrm{CH}$, call them $\mathrm{t}$. This leads to the total of 10 interaction parameters. Fortunately, $x y^{x} y^{\prime}$ products in eq. (4) for some of the interaction parameters $B_{y y^{\prime}}$ are the same and only 3 parameters are needed for each value of $\ell$ if propene is excluded. Specifically, eq. (4) now takes the form

$$
B=\frac{(r-3)^{2}}{2 r} \quad x_{1}+\frac{1}{2 r} x_{2}+\frac{r-3}{r} \quad x_{3}
$$

with the three characteristic parameters defined in terms of $\mathrm{B}_{\mathrm{yy}} \mathrm{s}^{\mathrm{s}}$ by

$$
\begin{aligned}
& x_{1}=B_{m m} \\
& x_{2}=B_{c c}+B_{t t}+B_{e e}+\frac{1}{2}\left(B_{c t}+B_{c e}+B_{t e}\right) \\
& x_{3}=B_{m e}+B_{m c}+B_{m t}
\end{aligned}
$$

Values of the $X$ parameters are given in table 7 . B values for alk-1-enes calculated from the parameters in table 7 are compared with the experimental data in table 8 . Except for $\ell=0$ for but-1-ene, the agreement is within limits of the experimental error. Increasing the value of length $\ell$ to unity improves dramatically the agreement between calculation and experiment for but-1-ene, and brings this compound into line with higher n-alkenes.

\subsection{Mixtures of alk-1-enes}

The only experimental data for mixtures reported by McGlashan and Wormald [21] are those for propene + hept-1-ene. Once again, to predict values for mixtures from the walk interaction formalism, we do not need any parameters other than those for pure components, 
and no combining rules. A certain extrapolation is involved, since propene has not been included in the evaluation of parameters for pure components listed in table 7 . Experimental as well as predicted values of the pressure second virial coefficients are listed in table 9. The agreement is within limits of the experimental error, except for the temperature of $348 \mathrm{~K}$. We, therefore, suspect that there is a mistake in the reported experimental value for $348 \mathrm{~K}$.

Table 7. Characteristic parameters for alk-1-enes.

\begin{tabular}{|c|c|c|c|c|}
\hline \multirow[t]{2}{*}{$\mathrm{T}$} & \multirow[t]{2}{*}{$\ell$} & $-x_{1}$ & & $-x_{3}$ \\
\hline & & $\mathrm{cm}^{3} \mathrm{~mol}^{-1}$ & $\mathrm{~cm}^{3} \mathrm{~mol}^{-1}$ & $\mathrm{~cm}^{3} \mathrm{~mol}^{-1}$ \\
\hline \multirow[t]{2}{*}{323} & 0 & 2062.5 & 1838.1 & 152.19 \\
\hline & 1 & 3290.4 & 2084.1 & 600.15 \\
\hline \multirow[t]{2}{*}{348} & 0 & 1592.1 & 1390.0 & 130.10 \\
\hline & 1 & 2700.6 & 1359.2 & 847.25 \\
\hline \multirow[t]{2}{*}{373} & 0 & 1399.7 & 1152.7 & 42.30 \\
\hline & 1 & 2339.8 & 1251.0 & 595.78 \\
\hline \multirow[t]{2}{*}{400} & 0 & 1200.4 & 910.2 & 95.47 \\
\hline & 1 & 2024.9 & 994.7 & 514.00 \\
\hline
\end{tabular}

Table 8. Second virial coefficients B of alk-1-enes (n-alkenes).

\begin{tabular}{|c|c|c|c|c|c|}
\hline Compound & $\frac{\mathrm{T}}{\mathrm{K}}$ & $\ell$ & $\frac{-\mathrm{B} \text { exper. }}{\mathrm{cm}^{3} \mathrm{~mol}^{-1}}$ & $\frac{-{ }^{-B} \text { calc. }}{\mathrm{cm}^{3} \mathrm{~mol}^{-1}}$ & $\frac{\text { difference }}{\text { percent }}$ \\
\hline \multirow{8}{*}{$\mathrm{C}_{4} \mathrm{H}_{8}$} & \multirow[t]{2}{*}{323} & 0 & 528 & 449.5 & 23.36 \\
\hline & & 1 & & 548.4 & -3.85 \\
\hline & \multirow[t]{2}{*}{348} & 0 & 447 & 395.2 & 11.50 \\
\hline & & 1 & & 450.1 & -0.80 \\
\hline & \multirow[t]{2}{*}{373} & 0 & 383 & 329.6 & 14.00 \\
\hline & & 1 & & 390.0 & -1.73 \\
\hline & \multirow[t]{2}{*}{400} & 0 & 329 & 287.7 & 12.46 \\
\hline & & 1 & & 337.5 & -2.64 \\
\hline
\end{tabular}


Table 8. (Concluded)

\begin{tabular}{|c|c|c|c|c|c|}
\hline Compound & $\frac{T}{K}$ & $\ell$ & $\frac{-\mathrm{B} \text { exper. }}{\mathrm{cm}^{3} \mathrm{~mol}^{-1}}$ & $\frac{{ }^{-B} \text { calc. }}{\mathrm{cm}^{3} \mathrm{~mol}^{-1}}$ & $\frac{\text { difference }}{\text { percent }}$ \\
\hline \multirow{8}{*}{$\mathrm{C}_{5} \mathrm{H}_{10}$} & \multirow{2}{*}{323} & 0 & 880 & 880.6 & -0.03 \\
\hline & & 1 & & 821.9 & 6.64 \\
\hline & \multirow[t]{2}{*}{348} & 0 & 733 & 734.9 & -0.22 \\
\hline & & 1 & & 719.3 & 1.91 \\
\hline & \multirow[t]{2}{*}{373} & 0 & 622 & 618.0 & 0.57 \\
\hline & & 1 & & 597.8 & 3.82 \\
\hline & \multirow[t]{2}{*}{400} & 0 & 532 & 522.3 & 1.75 \\
\hline & & 1 & & 505.9 & 4.83 \\
\hline
\end{tabular}

$\begin{array}{cccc} & 323 & 0 & 1387 \\ & & 1 & \\ & 348 & 0 & 1136 \\ \mathrm{C}_{6} \mathrm{H}_{12} & & 1 & \\ & 373 & 0 & 950 \\ & & 1 & 798 \\ & 400 & 0 & \end{array}$

$\begin{array}{rr}1474.4 & -6.32 \\ 1402.7 & -1.16 \\ 1179.5 & -3.83 \\ 1152.6 & -1.47 \\ 1002.3 & -5.51 \\ 972.7 & -2.38 \\ 830.4 & -4.05 \\ 806.0 & -0.98\end{array}$

$\begin{array}{cccc} & 323 & 0 & 2075 \\ & & 1 & \\ \mathrm{C}_{7} \mathrm{H}_{14} & 348 & 0 & 1674 \\ & & 1 & 1432 \\ & 373 & 0 & 1146 \\ & & 1 & \end{array}$

$\begin{array}{rr}2161.1 & -4.13 \\ 2137.4 & -2.98 \\ 1684.1 & -0.62 \\ 1668.1 & 0.34 \\ 1441.6 & -0.69 \\ 1431.1 & 0.04 \\ 1180.5 & -3.02 \\ 1171.7 & -2.26\end{array}$

$\begin{array}{cccc} & 323 & 0 & 3000 \\ & & 1 & \\ \mathrm{C}_{8} \mathrm{H}_{16} & 348 & 0 & 2231 \\ & & 1 & 1942 \\ & 373 & 0 & 1592 \\ & & 1 & \end{array}$

$\begin{array}{ll}2905.8 & 3.13 \\ 2959.8 & 1.33 \\ 2226.2 & 0.21 \\ 2230.4 & 0.02 \\ 1915.1 & 1.40 \\ 1937.2 & 0.25 \\ 1556.8 & 2.21 \\ 1575.1 & 1.06\end{array}$


Table 9. Second virial coefficients $B$ for mixtures of propene with hept-1-ene; mole fraction of propene is 0.5005 .

\begin{tabular}{ccccc}
$\frac{T}{\mathrm{~K}}$ & $\ell$ & $\frac{- \text { Bexper. }}{\mathrm{cm}^{3} \mathrm{~mol}^{-1}}$ & $\frac{-\mathrm{B} \text { calc. }}{\mathrm{cm}^{3} \mathrm{~mol}^{-1}}$ & $\frac{\text { difference }}{\text { percent }}$ \\
\hline 323 & 0 & 983 & 947.9 & 3.6 \\
348 & 0 & 783 & 715.7 & 8.6 \\
373 & 0 & 659 & 658.2 & 0.1 \\
400 & 0 & 547 & 533.0 & 2.6 \\
\hline
\end{tabular}

\section{Some Concluding Remarks}

It has been found that the walk interaction formalism, conceived originally for equilibrium properties of liquid phases [10], produces also good results for at least one property of gases, namely the pressure second virial coefficient. We would like very much to test this approach further, but for this we need more reliable experimental data.

The results obtained strengthen the feasibility and the desirability of a proposal made in Section 2: instead of considering interaction potentials for entire polyatomic molecules such as hydrocarbons or fluorocarbons, one should construct $u(R)$ functions for interactions between molecular segments. This would correspond to interactions between walks of length $\ell=0$. Further, extension of the treatment to longer walks is also possible. For any walk length, one can then relate the appropriate $u(R)$ potentials so obtained to the $B_{y y^{\prime}}$ values studied in the present work.

This work forms a part of a research program supported financially by the National Research Council of Canada, Ottawa, and by the National Council of Science and Technology, Mexico City.

\section{References}

[1] Barrett, C. R., Nix, D. M., and Tetelman, A. S., The Principles of Engineering Materials (Prentice-Ha11, Englewood Cliffs, N.J., 1973).

[2] Brostow, W., Science of Materials (John Wiley and Sons, N.Y., 1978).

[3] Bose, T. K., Sochanski, J. S., and Cole, R. H., J. Chem. Phys. 57, 3592 (1972).

[4] Rätzsch, M. T. and Wohlfarth, C., Z. Phys. Chem. 256, 401, 412 (1975). 
[5] Singh, S. and Singh, Y., Physica A 87, 207 (1977); Singh, S., Ibid, 89, 219 (1977).

[6] Kau1, B. K. and Prausnitz, J. M., Ind. Eng. Chem. Fundam. 16, 335 (1977).

[7] Pérez-Gutiérrez, S., Dr.Sc. Thesis, Center for Advanced Studies, Mexico City (1977).

[8] Brostow, W., McEachern, D. M., and Pérez-Gutiérrez, S., in preparation.

[9] Brostow, W. and Schinze1, A., J. Stat. Phys. 4, 103 (1972).

[10] Brostow, W., Phys. Chem. Liquids, $\underline{3}$, 91 (1972).

[11] Essam, J. W. and Fisher, M., Rev. Mod. Phys. 42, 272 (1970).

[12] Harary, F., Graph Theory (Addison-Wesley, Reading, MA, 1969).

[13] Kilpatrick, J. E., Adv. Chem. Phys. 20, 39 (1971).

[14] Larsen, S. Y., Kilpatrick, J. E., Lieb, E. H., and Jordan, H. F., Phys. Rev. 140, A129 (1965).

[15] McGlashan, M. L. and Potter, D. J. B., Proc. Royal Soc. A $\underline{267}, 478$ (1962).

[16] Brostow, W. , Maynadier, P., and Sangster, J., in preparation.

[17] Lin, H. M. and Robinson, Jr., R. L., J. Chem. Phys. 54, 52 (1971).

[18] Kreglewski, A. and Wilhoit, R. C., J. Phys. Chem. 78, 1961 (1974).

[19] Dantzler, E. M. and Knobler, C. M., Ibid, 73, 1335 (1969).

[20] Dantzler Siebert, E. M. and Knobler, C. M., Ibid, 75, 3863 (1971).

[21] McGlashan, M. L. and Wormald, C. J., Trans. Faraday Soc. 60, 646 (1964). 
National Bureau of Standards Special Publication 561, Proceedings of the 10th Materials Research Symposium on Characterization of High Temperature Vapors and Gases held at NBS, Gaithersburg, Maryland, September 18-22, 1978. Issued 0ctober 1979.

\title{
MECHANISMS OF OXIDATION AND HOT CORROSION OF METALS AND ALLOYS AT TEMPERATURES OF 1150 TO $1450 \mathrm{~K}$ UNDER FLOW
}

\author{
Earl A. Gulbransen and Gerald H. Meier \\ Department of Metallurgical and Materials Engineering \\ University of Pittsburgh \\ Pittsburgh, PA 15261
}

Knowledge of vapor species in metal-oxygen-sulfur systems is essential to interpret observations of high temperature oxidation and corrosion in oxygen and sulfur containing gases. A brief description is first given of the mechanisms of high temperature corrosion reactions involving volatile metal, oxide, and sulfide species. The reaction conditions for the several mechanisms are determined by temperature, pressure, flow and the oxygen and sulfur potentials of the environment.

A description is next given of thermochemical analyses and thermochemical diagrams followed by the thermochemistry of gaseous species in the $\mathrm{H}-\mathrm{O}-\mathrm{S}-\mathrm{C}$ system. A thermochemical analysis is then presented of the vapor phase equilibria for the $A 1-0-S, \mathrm{CO}_{-}-\mathrm{O}-\mathrm{S}$, $\mathrm{Cr}-\mathrm{O}-\mathrm{S}$, Fe-0-S, Mo-0-S, Ni-0-S, Si-0-S, Ti-O-S, V-O-S, and $\mathrm{W}-0-\mathrm{S}$ systems. Vapor equilibria diagrams $\left(\log P_{M_{x} O_{y}}\right.$ vs $\log P_{0_{2}}$ and $\log P_{M_{x} S_{y}}$ vs $\log P_{S_{2}}$ ) are given for $1250 \mathrm{~K}$ and extended in the description to temperatures of 1150,1342 , and $1450 \mathrm{~K}$. The influence of vapor species on the mechanisms of high temperature corrosion for the ten metal-oxygen-sulfur systems is described.

\section{Introduction}

The kinetics of reaction of metals and alloys with oxygen and other reactive gases depends on many factors: (1) the nature of the reaction product, (2) the chemical potentials of the one or more reactive gases involved, (3) the equilibrium pressures of the volatile species, (4) the flow rate of the reactive gases, and (5) the geometry and dimensions of the specimen and surrounding container. If melting of oxide or sulfide phases occurs or volatile species form, the rates of reaction are generally accelerated and gas flow becomes important. 
Gulbransen and Jansson [1] ${ }^{1}$ have shown that metal and volatile oxide species are important in the kinetics of high temperature oxidation of carbon, silicon, molybdenum and chromium. Six types of oxidation phenomena were identified: (1) at low temperature, diffusion of oxygen and metal species through a compact oxide film, (2) at moderate and high temperatures, a combination of oxide film formation and oxide volatility, (3) at moderate and high temperature; the formation of volatile metal and oxide species at the metal-oxide interface and transport through the oxide lattice and mechanically formed cracks in the oxide layer, (4) at moderate and high temperature, the direct formation of volatile oxide gases, (5) at high temperature, the gaseous diffusion of oxygen through a barrier layer of volatilized oxides, and (6) at high temperature, spalling of metal and particles. In addition, if the oxide melts, the oxide may drip off and rapid transport of oxygen to the metal may occur by convection in the liquid phase.

Sulfidation reactions follow a similar series of kinetic phenomena as has been observed for oxidation. Unfortunately, few studies have been made of the basic kinetic phenomena involved in sulfidation reactions at high temperature. In this study we will try to predict from thermochemical analyses where the volatile species play an important role for both oxidation and sulfidation. Four temperatures are of interest 1150, 1250, 1342, and $1450 \mathrm{~K}$. These temperatures are related to the melting temperatures of $\mathrm{Na}_{2} \mathrm{SO}_{4}, 1157 \mathrm{~K}$, and $\mathrm{K}_{2} \mathrm{SO}_{4}, 1342 \mathrm{~K}$. $1250 \mathrm{~K}$ is above the melting temperature of $\mathrm{Na}_{2} \mathrm{SO}_{4}$ and near the operating temperature range for gas turbines. $1450 \mathrm{~K}$ is a useful temperature for accelerated corrosion testing.

\section{Thermochemical Analyses}

Following the methods used in earlier papers, we will use standard Gibbs free energy data, $\Delta G_{f}^{o}$ and equilibrium constants, $\log K_{p}$ to evaluate the equilibrium pressures of the volatile species over the several condensed phases as a function of temperature and of the oxygen and sulfur potentials of the gas mixture. Diagrams have been developed [1] for presentation of the complex equilibrium. $\log P_{M_{x} O_{y}}$ vs $\log P_{O_{2}}$ and $\log P_{H_{2}} / P_{H_{2} O}$ and $\log P_{M_{x} S_{y}}$ vs $\log P_{S_{2}}$ and $\log P_{H_{2}} / P_{H_{2} S}$ are of interest to show the influence of the oxygen and sulfur potentials on the equilibrium pressures of the volatile species $M_{x} 0_{y}$ and $M_{x} S_{y}$. To summarize the data $\log P_{M_{x}{ }^{0}}$ and $\log P_{M_{x} S_{y}}$ vs $\frac{1}{T}$ diagrams are used.

Table 3 in the Appendix shows a list of the condensed oxide and sulfide phases and volatile species for the oxides and sulfides of $\mathrm{Al}, \mathrm{Cr}, \mathrm{Co}, \mathrm{Fe}, \mathrm{Mo}, \mathrm{Ni}, \mathrm{Si}, \mathrm{Ti}, \mathrm{V}$, and $\mathrm{W}$ together with the available melting points. One feature of this summary is the lower melting points of the condensed sulfide phases. This is of major importance in comparing the oxidation and sulfidation reactions of these metals.

Thermochemical analyses of the volatile species over the condensed phases allow the prediction of four phenomena related to the six types of oxidation processes. The first is

${ }^{1}$ Figures in brackets indicate the literature references at the end of this paper. 
the temperature where the equilibrium pressure of metal or oxide species reaches $10^{-9}$ atm. Volatility of oxide species and rapid diffusion of metal species through the oxide begins to affect the kinetics of oxidation as determined from vacuum microbalance studies on molybdenum [2].

Second, the temperature at which the pressure of volatile oxide species generated at the metal-oxide interface equals the external gas pressure. The oxide film may fracture leaving the metal surface open for rapid reaction. Also, volatile oxide species may diffuse through fissures in the oxide film leading to very rapid oxidation. This phenomenon was found in the high temperature oxidation of silicon $[1,2]$.

Third, the temperature where complete volatilization of the oxide layer occurs. For this condition, the geometry of the reaction system and the flow rate of the reactive gas over the specimen become important. For fast flow rates very fast oxidation rates have been observed for carbon [1,4] and molybdenum [1,2]. The absence of any condensed reaction product eliminates solid state diffusion as an important reaction step. Adsorption of oxygen, surface reaction with the metal and desorption of the reaction product are the basic mechanisms of reaction [5]. For molybdenum volatilization is important when

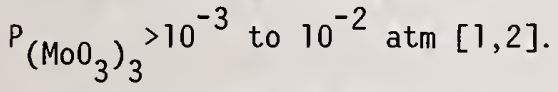

Fourth, the physical state of the volatilized oxide species in the barrier layer at the temperature of the reaction is important. For condensed phases in the barrier layer diffusion of oxygen to the metal interface is easy and very rapid reactions may occur $[1,3]$.

\section{Thermochemistry of Reactive Gas Mixtures}

Table 1 gives oxidizing, sulfiding, and sulphate forming gas mixtures associated with oxygen and sulfur containing gas mixtures. Table 1 shows the equations, the expressions for oxygen, sulfur, and sulfur trioxide chemical potentials expressed as pressures, and the values of $\log K_{R}$ at 1150 and $1342 \mathrm{~K}$.

Table 2 shows the $0_{2}$ and $S_{2}$ pressures for the gas mixtures of table 1 for a $1: 1$ pressure ratio at $1150,1250,1342$ and $1450 \mathrm{~K}$. Low $0_{2}$ pressures are noted for the $\mathrm{H}_{2} / \mathrm{H}_{2} \mathrm{O}$ and $\mathrm{CO} / \mathrm{CO}_{2}$ gas mixtures and high $\mathrm{O}_{2}$ pressures for the $\mathrm{SO}_{2} / \mathrm{SO}_{3}$ gas mixture. High $\mathrm{S}_{2}$ pressures are found for the $\mathrm{H}_{2} / \mathrm{H}_{2} \mathrm{~S}$ gas mixture and low $\mathrm{S}_{2}$ pressures for the $\mathrm{SO}_{2} / \mathrm{SO}_{3}$ gas mixture.

Part $C$ of table 2 shows $\log \mathrm{P}_{\mathrm{SO}_{3}}$ for a $\mathrm{SO}_{2} / \mathrm{SO}_{3}$ gas mixture with $\log \mathrm{P}_{0_{2}}=0$ and and $\log \mathrm{P}_{\mathrm{SO}_{2}}=-4 . \quad \log \mathrm{P}_{\mathrm{SO}_{2}}$ decreases with increase in temperature.

Table 4 in the Appendix shows equilibrium constants, $\log \mathrm{Kp}$, for the condensed oxide and sulfide phases, where known, and for the volatile oxide and sulfide species. Unfortunately, inadequate data is available for many of the condensed phases and vapor species. For liquid phase sulfides, e.g., NiS $y$ of variable composition $\log \mathrm{P}_{\mathrm{S}_{2}}$ are given. 
4. Volatile Species and Mechanisms of Oxidation and Sulfidation

Only one condensed oxide, $\mathrm{Al}_{2} \mathrm{O}_{3}$, having a melting point of $2317 \pm 4 \mathrm{~K}$ is stable [24]. Thermochemical data are available on $\mathrm{Al}_{2} \mathrm{O}_{3}(\mathrm{~s}), \mathrm{Al}(\mathrm{g})$, and the four oxide vapors, $\mathrm{AlO}(\mathrm{g})$, $\mathrm{AlO}_{2}(\mathrm{~g}), \mathrm{Al}_{2} \mathrm{O}(\mathrm{g})$, and $\mathrm{Al}_{2} \mathrm{O}_{2}(\mathrm{~g})$ (see table 4 ).

At room temperature only one sulfide is stable, $\mathrm{Al}_{2} \mathrm{~S}_{3}$, having a melting point of $1370 \mathrm{~K}$ [25]. At elevated temperature the composition A1S with a maximum in the melting temperature of $1470 \mathrm{~K}$ is reported. Thermochemical data are available on the condensed phase $A_{2} S_{3}(s, 1)$ and the three sulfide vapors $A 1 S(g), A l_{2} S(g)$, and $A_{2} S_{2}(g)$ (see table 4).

Figure $1^{2}$ shows a $\log \mathrm{P}_{\mathrm{Al}} \mathrm{x}_{\mathrm{y}}$ vs $\log \mathrm{P}_{\mathrm{O}_{2}}$ and $\log \mathrm{P}_{\mathrm{H}_{2}} / \mathrm{P}_{\mathrm{H}_{2} \mathrm{O}}$ diagram at $1250 \mathrm{~K}$. The

vertical line at the left shows the phase boundary for $A 1(1)$ and $\mathrm{Al}_{2} \mathrm{O}_{3}$ (s). Equilibrium pressures for the vapor species $A 1(\mathrm{~g}), \mathrm{Al}_{2} \mathrm{O}(\mathrm{g}), A 10(\mathrm{~g})$, and $\mathrm{Al}_{2} \mathrm{O}_{2}(\mathrm{~g})$ are shown as horizontal and diagonal lines. $\mathrm{Al}(\mathrm{g})$ and $\mathrm{Al}_{2} \mathrm{O}(\mathrm{g})$ are the important vapor species with the highest pressures occurring at the $\mathrm{Al}(1) / \mathrm{Al}_{2} \mathrm{O}_{3}(\mathrm{~s})$ interface. Figure 2 shows a $\log \mathrm{P}_{\mathrm{Al}} \mathrm{x}^{0} \mathrm{y}$ vs $\frac{1}{\bar{T}}$ chart for the temperature range of 1150 to $1450 \mathrm{~K}$. The lower dashed line is for $\log P_{A 1}{ }_{x y}=-9$. Al(g) may affect the kinetics of oxidation at $1250 \mathrm{~K}$ for $\mathrm{Al}$ activities in alloys of $\log a_{A 1} \geq-2$. At $1342 \mathrm{~K}$ and $1450 \mathrm{~K}$ both $\mathrm{Al}(\mathrm{g})$ and $\mathrm{Al}_{2} \mathrm{O}(\mathrm{g})$ may affect the kinetics of oxidation. No experimental studies have been made to test this prediction.

Figure 3 shows a $\log P_{A 1} S_{x}$ vs $\log P_{S_{2}}$ and $\log P_{H_{2}} / P_{H_{2} S}$ diagram. $A_{2} S_{3}(s)$ is a stable sulfide having a $S_{2}$ equilibrium pressure of $\log P_{S_{2}}=-15.9$ at $1250 \mathrm{~K}$. The vertical line at the right shows the $S_{2}$ gas pressure over $S(1)$. Al(g) is the major volatile species. Figure 4 shows a $\log P_{A 1} S_{x}$ vs $\frac{1}{T}$ diagram. $A 1(g)$ may affect the kinetics of sulfidation for $\log \mathrm{a}_{\mathrm{Al}} \geq-2$ at $1250 \mathrm{~K}$. The sulfide vapors, $\mathrm{A} 7 \mathrm{~S}(\mathrm{~g})$ and $\mathrm{Al}_{2} \mathrm{~S}(\mathrm{~g})$, are important at temperatures above $1450 \mathrm{~K}$ and for high aluminum activities in the alloy.

Figure 5 shows $\log \mathrm{P}_{\mathrm{S}_{2}}$ vs $\frac{1}{\mathrm{~T}}$ and $\log \mathrm{P}_{0_{2}}$ vs $\frac{1}{\mathrm{~T}}$ diagrams for the stabilities of $\mathrm{Al}_{2} \mathrm{~S}_{3}(\mathrm{~s}, 1)$ and $\mathrm{Al}_{2} \mathrm{O}_{3}(\mathrm{~s})$. Both compounds are stable.

We conclude that aluminum in alloys forms stable oxide and sulfide condensed phases. Al (g) may effect the kinetics of oxidation and sulfidation for high aluminum activities in alloys. Above $1370 \mathrm{~K}$ molten $\mathrm{Al}_{2} \mathrm{~S}_{3}$ is formed which may lead to rapid sulfidation reactions.

\subsection{Cobalt-oxygen-sulfur system}

Two condensed oxides $\mathrm{CoO}$ and $\mathrm{Co}_{3} \mathrm{O}_{4}$ exist for the conditions of interest $[25,28]$. CoO and $\mathrm{Co}_{3} \mathrm{O}_{4}$ have the following homogeneity ranges $50.0-51.7 \mathrm{a} / 0$ for $\mathrm{CoO}$ and $56.7-58.5 \mathrm{a} / \mathrm{o}$

${ }^{2} \mathrm{~A} 11$ figures appear in Appendix 2 at the end of this paper. 
for $\mathrm{Co}_{3} \mathrm{O}_{4}$. At $1173 \mathrm{~K} \mathrm{Co}_{3} \mathrm{O}_{4}$ decomposes to $\mathrm{CoO}_{1.07}(51.7 \mathrm{a} / \mathrm{o})$ [28]. Above $1273 \mathrm{~K}$ only stoichiometric $\mathrm{COO}$ exists. $\mathrm{CO} 0$ has a melting temperature of $2083 \mathrm{~K}$ [25]. Thermochemical data is available for $\mathrm{CoO}(\mathrm{s}), \mathrm{CO}_{3} \mathrm{O}_{4}(\mathrm{~s})$, and $\mathrm{Co}(\mathrm{g})$ (see table 4).

Five condensed sulfides exist, $\mathrm{Co}_{4} \mathrm{~S}_{3}, \mathrm{Co}_{9} \mathrm{~S}_{8}, \mathrm{CoS}, \mathrm{Co}_{3} \mathrm{~S}_{4}$ and $\mathrm{CoS}_{2}[25]$. CoS has a melting temperature of $1451 \mathrm{~K}$. A constitution diagram has been developed for the temperature range of interest here based on $\mathrm{Co}_{4} \mathrm{~S}_{3}+x, \mathrm{CoS}_{1}+x$ and the liquid $\mathrm{CoS}_{y}$ [12]. Thermochemical data is available on these condensed phases and on $\mathrm{Co}(\mathrm{g})$ (see table 4).

Figure 6 shows a $\log \mathrm{P}_{\mathrm{Co}_{x}{ }_{y}}$ vs $\log \mathrm{P}_{0_{2}}$ and $\log \mathrm{P}_{\mathrm{H}_{2}} / \mathrm{P}_{\mathrm{H}_{2} \mathrm{O}}$ diagram at $1250 \mathrm{~K}$. The vertical line in the center of the diagram shows $\log P_{0_{2}}=-12.2$ atm for the $\operatorname{Co}(\mathrm{s}) / \mathrm{CoO}(\mathrm{s})$ interface. $\mathrm{Co}(\mathrm{g})$ is not an important vapor species even for high activities of cobalt in alloys at $1250 \mathrm{~K}$. Figure 7 shows a $\log \mathrm{P}_{\mathrm{Co}_{x}{ }_{\mathrm{y}}}$ vs $\frac{1}{\mathrm{~T}}$ diagram. Above $1342 \mathrm{~K}$, Co(g) may affect the kinetics of oxidation for high cobalt activity alloys.

Figure 8 shows a $\log \mathrm{P}_{\mathrm{Co}_{x} \mathrm{~S}_{y}}$ vs $\log \mathrm{P}_{\mathrm{S}_{2}}$ and $\log \mathrm{P}_{\mathrm{H}_{2}} / \mathrm{P}_{\mathrm{H}_{2} \mathrm{~S}}$ diagram at $1250 \mathrm{~K}$. $\operatorname{CoS}_{y}(1)$ is the condensed sulfide phase in contact with $\operatorname{Co}(s) . \quad \log P_{S_{2}}=-5.7$ atm for the $\operatorname{Co}(s) / \operatorname{CoS}{ }_{y}(1)$ interface. $\operatorname{Co}(\mathrm{g})$ is not an important vapor species at $1250 \mathrm{~K}$ even at high activities of cobalt in alloys.

The important feature of the Co-S system is the relatively low stability of the sulfide phases and the formation of a liquid $\operatorname{CoS}_{y}(1)$ phase above $1153 \mathrm{~K}$.

\subsection{Chromium-oxygen-sulfur system}

Only one oxide, $\mathrm{Cr}_{2} \mathrm{O}_{3}(\mathrm{~s})$ is formed under the conditions of interest here. The highest melting point for $\mathrm{Cr}_{2} \mathrm{O}_{3}(\mathrm{~s})$ is given as $2697 \mathrm{~K}$ [24]. Thermochemical data is available on $\mathrm{Cr}_{2} \mathrm{O}_{3}(\mathrm{~s}), \mathrm{Cr}(\mathrm{g})$ and the three oxide species $\mathrm{CrO}(\mathrm{g}), \mathrm{CrO}_{2}(\mathrm{~g})$, and $\mathrm{CrO}_{3}(\mathrm{~g})$ (see table 4).

Five condensed sulfides exist [26], $\mathrm{CrS}, \mathrm{Cr}_{7} \mathrm{~S}_{8}, \mathrm{Cr}_{5} \mathrm{~S}_{6}, \mathrm{Cr}_{3} \mathrm{~S}_{4}$, and $\mathrm{Cr}_{2} \mathrm{~S}_{3} . \mathrm{CrS}$ has a melting temperature of $1840 \mathrm{~K}$ [7]. Thermochemical data are available on these five sulfides and on $\operatorname{Cr}(\mathrm{g})$ and the sulfide vapor $\operatorname{CrS}(\mathrm{g})$ and are shown in table 4.

Figure 9 shows a $\log \mathrm{P}_{\mathrm{Cr}_{x}{ }_{y}}$ vs $\log \mathrm{P}_{\mathrm{O}_{2}}$ and $\log \mathrm{P}_{\mathrm{H}_{2}} / \mathrm{P}_{\mathrm{H}_{2}}$ diagram at $1250 \mathrm{~K}$. The vertical line in the center shows the phase boundary for $\operatorname{Cr}(\mathrm{s}) / \mathrm{Cr}_{2} \mathrm{O}_{3}\left(\mathrm{~s}, \log \mathrm{P}_{0_{2}}=-22.5\right.$ atm. Equilibrium pressures for the vapor species $\mathrm{Cr}(\mathrm{g}), \mathrm{CrO}(\mathrm{g}), \mathrm{CrO}_{2}(\mathrm{~g})$, and $\mathrm{CrO}_{3}(\mathrm{~g})$ are shown in the diagram. $\mathrm{Cr}(\mathrm{g})$ is important at the $\mathrm{Cr}(\mathrm{s})$ and $\mathrm{Cr}_{2} \mathrm{O}_{3}(\mathrm{~s})$ interface and $\mathrm{CrO}_{3}(\mathrm{~g})$ at the $\mathrm{Cr}_{2} \mathrm{O}_{3} / \mathrm{O}_{2}$ interface. Volatility of $\mathrm{CrO}_{3}(\mathrm{~g})$ is expected at the oxide-gas interface at $1250 \mathrm{~K}$ and higher. This has been observed experimentally on superalloys [27]. Figure 10 shows a $\log \mathrm{P}_{\mathrm{Cr}_{x} \mathrm{O} y}$ vs $\frac{1}{\mathrm{~T}}$ diagram for the volatile species. 
Figure 11 shows a $\log \mathrm{P}_{\mathrm{Cr}_{x} S_{y}}$ vs $\log \mathrm{P}_{\mathrm{S}}$ and $\log \mathrm{P}_{\mathrm{H}_{2}} / \mathrm{P}_{\mathrm{H}_{2} S}$ diagram. CrS(s) is the most stable sulfide with $\log \mathrm{P}_{\mathrm{S}_{2}}=-11.05$ at the $\operatorname{Cr}(\mathrm{s}) / \mathrm{CrS}(\mathrm{s})$ interface. The vertical lines in figure 11 show the $S_{2}$ equilibrium pressures for the several equilibria in the $\mathrm{Cr}-\mathrm{S}$ system. $\mathrm{Cr}(\mathrm{g})$ is the important volatile species with the highest pressure at the $\mathrm{Cr}(\mathrm{s}) / \mathrm{CrS}(\mathrm{s})$ interface. $\operatorname{Cr}(g)$ may affect the kinetics of sulfidation for high chromium activities in alloys. This has not been tested experimentally.

Figure 12 shows a $\log \mathrm{P}_{\mathrm{Cr}} \mathrm{S}_{\mathrm{y}}$ vs $\frac{1}{\mathrm{~T}}$ diagram of the vapor species in the $\mathrm{Cr}-\mathrm{S}$ system. Figure 13 shows $\log P_{S_{2}}$ vs $\frac{1}{T}$ and $\log P_{0_{2}}$ vs $\frac{1}{T}$ diagrams for the stabilities of the several sulfides and $\mathrm{Cr}_{2} \mathrm{O}_{3}(\mathrm{~s})$.

We conclude that chromium in alloys form a stable $\mathrm{Cr}_{2} \mathrm{O}_{3}(\mathrm{~s})$ phase in oxygen atmospheres and five sulfide phases of widely varying stabilities. $\mathrm{Cr}(\mathrm{g})$ and $\mathrm{CrO}_{3}(\mathrm{~g})$ may affect the kinetics of oxidation at temperatures of $1250 \mathrm{~K}$ and higher. $\mathrm{Cr}(\mathrm{g})$ may affect the kinetics of sulfidation at $1250 \mathrm{~K}$ and higher.

\subsection{Iron-oxygen-sulfur system}

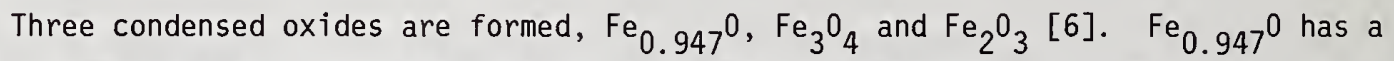
melting temperature of $1650 \mathrm{~K}$ and $\mathrm{Fe}_{3} \mathrm{O}_{4}$ has a melting temperature of $1870 \mathrm{~K}$ [6]. $\mathrm{Fe}_{2} \mathrm{O}_{3}$ decomposes above $1735 \mathrm{~K}[6]$. Thermochemical data are available on the three condensed oxides and on $\mathrm{Fe}(\mathrm{g})$ and the oxide vapor $\mathrm{Fe} 0(\mathrm{~g})$ (see table 4 ).

A constitution diagram has been developed for the $\mathrm{Fe}-\mathrm{S}$ system based on $\mathrm{FeS}_{1}+\mathrm{x}$ and the liquid $\mathrm{FeS}_{y}$ [12] which has a melting temperature of $1261 \mathrm{~K}$. Thermochemical data are available on $\mathrm{FeS}_{1}+x$ and $\mathrm{FeS}_{y}$ and $\mathrm{Fe}(\mathrm{g})$ (see table 4). $\mathrm{FeS}(\mathrm{g})$ also exists but thermochemical data are not available.

Figure 14 shows a $\log \mathrm{P}_{\mathrm{FeO}_{\mathrm{X}}}$ vs $\log \mathrm{P}_{\mathrm{O}_{2}}$ and $\log \mathrm{P}_{\mathrm{H}_{2}} / \mathrm{P}_{\mathrm{H}_{2}}$ at $1250 \mathrm{~K}$. The vertical 1 ines show the oxygen pressures associated with the several oxide equilibria. For the $\mathrm{Fe} / \mathrm{Fe}_{0.947} \mathrm{O}$ equilibria at $1250 \mathrm{~K} \log \mathrm{P}_{0_{2}}=-15.4 \mathrm{~atm}$. Equilibrium pressures of $\mathrm{Fe}(\mathrm{g})$ and $\mathrm{Fe} 0(\mathrm{~g})$ are shown as horizontal and diagonal lines. $\mathrm{Fe}(\mathrm{g})$ and $\mathrm{Fe} 0(\mathrm{~g})$ are not important vapor species at $1250 \mathrm{~K}$. Figure 15 shows a $\log \mathrm{P}_{\mathrm{Fe}_{\mathrm{x}} \mathrm{O}_{\mathrm{y}}}$ vs $\frac{1}{\mathrm{~T}}$ diagram. $\mathrm{Fe}(\mathrm{g})$ is important at temperatures above $1300 \mathrm{~K}$ for alloys of high iron activities.

Figure 16 shows a $\log \mathrm{P}_{\mathrm{Fe}_{x} \mathrm{~S}_{\mathrm{y}}}$ vs $\log \mathrm{P}_{\mathrm{S}_{2}}$ and $\log \mathrm{P}_{\mathrm{H}_{2}} / \mathrm{P}_{\mathrm{H}_{2} \mathrm{~S}}$ diagram at $1250 \mathrm{~K}$. The vertical line in the diagram shows $\log \mathrm{P}_{\mathrm{S}_{2}}=-6.7 \mathrm{~atm}$ for the $\mathrm{Fe}(\mathrm{s}) / \mathrm{FeS}_{1}+\mathrm{x}$ interface. $\mathrm{Fe}(\mathrm{g})$ is not an important vapor species in the $\mathrm{Fe}-\mathrm{S}$ system at $1250 \mathrm{~K}$. Figure 17 shows a $\log \mathrm{P}_{\mathrm{Fe}_{\mathrm{x}} \mathrm{S}}$ vs $\frac{1}{\mathrm{~T}}$ diagram. Enhanced sulfidation may occur for temperature above $1300 \mathrm{~K}$ and for high activity of iron in alloys. 
The major features of the $\mathrm{Fe}-\mathrm{S}$ system are the relatively high melting point of the iron-sulfides, the low thermochemical stabilities of the sulfides and the low equilibrium pressures of the vapor species.

\subsection{Molybdenum-oxygen-sulfur system}

Two condensed oxides form, $\mathrm{MoO}_{2}$ and $\mathrm{MoO}_{3}$ [24]. In addition $\mathrm{Mo}_{3} \mathrm{O}$ and a series of oxides in the range of $\mathrm{Mo}_{4}{ }^{0} 11$ and $\mathrm{Mo}_{26}{ }^{0} 75$ are formed [24]. The melting temperature of $\mathrm{MoO}_{3}$ is $1068 \mathrm{~K}$. Thermochemical data are available on $\mathrm{MoO}_{2}(\mathrm{~s}), \mathrm{MoO}_{3}(1)$ and the vapor species $\mathrm{Mo}(\mathrm{g}), \mathrm{MoO}(\mathrm{g}), \mathrm{MoO}_{2}(\mathrm{~g}), \mathrm{MoO}_{3}(\mathrm{~g})$, and $\left(\mathrm{MoO}_{3}\right){ }_{3}(\mathrm{~g})$ (see table 4 ).

Three condensed sulfides exist $\mathrm{Mo}_{2} \mathrm{~S}_{3}, \mathrm{MoS}_{2}$, and $\mathrm{MoS}_{3}$ [24]. The melting temperature of $\mathrm{MoS}_{2}$ is subject to several opinions ranging from $1458 \mathrm{~K}$ to greater than $2073 \mathrm{~K}$ [28]. Thermochemical data are available on $\mathrm{Mo}_{2} \mathrm{~S}_{3}(s)$ and $\mathrm{MoS}_{2}(s)$. No data are available for volatile sulfide gases.

Figure 18 shows $\log \mathrm{P}_{\mathrm{Mo}_{x} \mathrm{O}_{y}}$ vs $\log \mathrm{P}_{0_{2}}$ and $\log \mathrm{P}_{\mathrm{H}_{2}} / \mathrm{P}_{\mathrm{H}_{2} 0}$ at $1250 \mathrm{~K}$. The vertical lines show the $\mathrm{O}_{2}$ equilibrium pressures for $\mathrm{Mo}(\mathrm{s}) / \mathrm{MoO}_{2}(\mathrm{~s})$ and $\mathrm{MoO}_{2}(\mathrm{~s}) / \mathrm{MoO}_{3}(1)$. At the $\mathrm{Mo}(\mathrm{s}) / \mathrm{MoO}_{2}(\mathrm{~s})$ interface $\log \mathrm{P}_{0_{2}}=-15.4$ atm at $1250 \mathrm{~K}$. Equilibrium pressures for the vapor species $\mathrm{Mo}(\mathrm{g}), \mathrm{MoO}(\mathrm{g}), \mathrm{MoO}_{2}(\mathrm{~g}), \mathrm{MoO}_{3}(\mathrm{~g})$, and $\left(\mathrm{MoO}_{3}\right){ }_{3}(\mathrm{~g})$ are shown on the

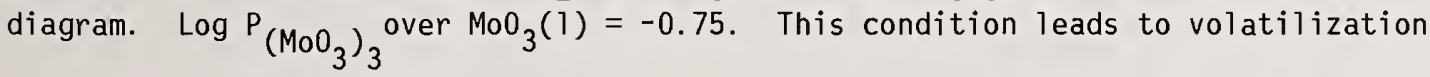
of the $\mathrm{MoO}_{3}(1)$ layer. Figure 19 shows a $\log \mathrm{P}_{\left(\mathrm{MoO}_{3}\right)_{x}}$ vs $\frac{1}{\mathrm{~T}}$ diagram. Complete volatilization of $\mathrm{MoO}_{3}(1)$ is expected. This has been observed experimentally by Gulbransen, Andrew, and Brassart [1,2]. Figure 20 shows a $\log P_{0_{2}}$ vs $\frac{1}{T}$ diagram for the oxygen pressures at the $\mathrm{Mo}(\mathrm{s}) / \mathrm{MoO}_{2}(\mathrm{~s})$ and $\mathrm{MoO}_{2}(\mathrm{~s}) / \mathrm{MoO}_{3}(1)$ interfaces. On a relative basis molybdenum oxides are only moderately stable. The high volatility of $\mathrm{MoO}_{3}$, and its low melting point of $1068 \mathrm{~K}$ suggest poor oxidation behavior in oxygen atmospheres.

Figure 21 shows a $\log \mathrm{P}_{\mathrm{Mo}_{x} \mathrm{~S}_{y}}$ vs $\log \mathrm{P}_{\mathrm{S}_{2}}$ and $\log \mathrm{P}_{\mathrm{H}_{2}} / \mathrm{P}_{\mathrm{H}_{2} \mathrm{~S}}$ diagram. The vertical lines show the $\mathrm{S}_{2}$ equilibrium pressures for the $\mathrm{Mo}(\mathrm{s}) / \mathrm{Mo}_{2} \mathrm{~S}_{3}(\mathrm{~s})$ and $\mathrm{Mo}_{2} \mathrm{~S}_{3}(\mathrm{~s}) / \mathrm{MoS}_{2}(\mathrm{~s})$ interfaces. Mo(g) is the only volatile species in the Mo-s system reported at this date. Mo(g) has a very low equilibrium pressure at $1250 \mathrm{~K}$ over $\mathrm{Mo}(\mathrm{s})$ with $\log \mathrm{P}_{\mathrm{Mo}_{0}}=-19.7 \mathrm{~atm}$. At $1450 \mathrm{~K}$ $\log P_{M_{0}}=-15.9 \mathrm{~atm}$.

Figure 22 shows $\mathrm{S}_{2}$ dissociation pressures at the $\mathrm{Mo}(\mathrm{s}) / \mathrm{Mo}_{2} \mathrm{~S}_{3}(\mathrm{~s})$ and $\mathrm{Mo}_{2} \mathrm{~S}_{3}(\mathrm{~s}) / \mathrm{MoS}_{2}(\mathrm{~s})$ interfaces, $\log \mathrm{P}_{\mathrm{S}_{2}}$ vs $\frac{1}{\mathrm{~T}}$, and the conditions for sulfidation of molybdenum.

We conclude that molybdenum in alloys can lead to rapid oxidation attack due to the formation of $\mathrm{MoO}_{3}(1)$ and the formation of $\left(\mathrm{MoO}_{3}\right)_{3}(\mathrm{~g})$. Rapid attack may occur even for low activities of molybdenum in the alloy. 


\subsection{Nickel-oxygen-sulfur system}

Nickel oxide is the principal condensed oxide [28]. It has a melting temperature of $2233 \mathrm{~K}$. No volatile oxide vapor species have been reported. Thermochemical data on $\mathrm{NiO}(\mathrm{s})$ and $\mathrm{Ni}(\mathrm{g})$ are given in table 4.

For the temperatures of interest here $\mathrm{NiS}_{\mathrm{y}}(1)$ is the condensed sulfide [12]. It has a melting temperature of $918 \mathrm{~K}$. Thermochemical data for $\mathrm{NiS}(1)$ and $\mathrm{Ni}(\mathrm{g})$ are given in table 4.

Figure 23 shows a $\log \mathrm{P}_{\mathrm{Ni}_{x} \mathrm{O}_{y}}$ vs $\log \mathrm{P}_{\mathrm{O}_{2}}$ and $\log \mathrm{P}_{\mathrm{H}_{2}} / \mathrm{P}_{\mathrm{H}_{2} \mathrm{O}}$ diagram for $1250 \mathrm{~K}$. The vertical line shows $\log \mathrm{P}_{0}=-10.7$ atm for the $\mathrm{Ni}(\mathrm{s}) / \mathrm{NiO}(\mathrm{s})$ interface. $\mathrm{NiO}(\mathrm{s})$ is readily reduced in $\mathrm{H}_{2} / \mathrm{H}_{2} \mathrm{O}$ mixtures to $\mathrm{Ni}(\mathrm{s})$ at $1250 \mathrm{~K}$. $\mathrm{Ni}(\mathrm{g})$ is the only vapor species reported. At $1250 \mathrm{~K} \log \mathrm{P}_{\mathrm{Ni}}=-10 \mathrm{~atm}$ at the $\mathrm{Ni}(\mathrm{s}) / \mathrm{NiO}(\mathrm{s})$ interface. $\mathrm{Ni}(\mathrm{g})$ should have no influence on the kinetics of oxidation at $1250 \mathrm{~K}$ even for high activity nickel alloys. No experimental study has shown an influence of nickel volatility on the oxidation reaction at $1250 \mathrm{~K}$.

Figure 24 shows a $\log P_{N i_{x}{ }_{y}}$ vs $\frac{1}{T}$ and $\log P_{0_{2}}$ vs $\frac{1}{\mathrm{~T}}$ diagram for the $\mathrm{Ni}(\mathrm{s}) / \mathrm{NiO}(\mathrm{s})$ interface. Enhanced oxidation due to $\mathrm{Ni}(\mathrm{g})$ may occur at $\mathrm{T}>1325 \mathrm{~K}$ for high activity nickel alloys.

Figure 25 shows a $\log \mathrm{P}_{\mathrm{Ni}} \mathrm{S}_{y}$ vs $\log \mathrm{P}_{\mathrm{S}_{2}}$ and $\log \mathrm{P}_{\mathrm{H}_{2}} / \mathrm{P}_{\mathrm{H}_{2}} \mathrm{~S}$. The vertical line shows $\log \mathrm{P}_{\mathrm{S}_{2}}=-7$ for the $\mathrm{Ni}(\mathrm{s}) / \mathrm{NiS}_{\mathrm{y}}(1)$ interface. NiS $\mathrm{y}_{\mathrm{y}}(1)$ is only formed for $\log \mathrm{P}_{\mathrm{H}_{2}} / \mathrm{P}_{\mathrm{H}_{2}} \mathrm{~S}^{<2.3}$ at $1250 \mathrm{~K}$. $\mathrm{Ni}(\mathrm{g})$ is the only vapor species reported in the $\mathrm{Ni}-\mathrm{S}$ system. At $1250 \mathrm{~K}$ $\log P_{\mathrm{Ni}}=-10 \mathrm{~atm}$ at the $\mathrm{Ni}(\mathrm{s}) / \mathrm{NiS}_{\mathrm{y}}(1)$ interface. $\mathrm{Ni}(\mathrm{g})$ should have no influence on the kinetics of sulfidation at $1250 \mathrm{~K}$ even for high activity nickel alloys. No experimental study has been made on the influence of nickel volatility on the sulfidation reaction.

Figure 26 shows a $\log \mathrm{P}_{\mathrm{Ni}}$ vs $\frac{1}{\mathrm{~T}}$ and $\log \mathrm{P}_{\mathrm{S}_{2}}$ vs $\frac{1}{\mathrm{~T}}$ diagram for the $\mathrm{Ni}(\mathrm{s}) / \mathrm{NiS} \mathrm{S}_{\mathrm{y}}$ (1) interface. Ni(g) has no effect on the sulfidation reaction at $T<1330 \mathrm{~K}$ for high activity nickel alloys.

The important feature of the $\mathrm{Ni}-\mathrm{O}-\mathrm{S}$ system is the relatively low stability of the sulfide phase and the formation of $\mathrm{NiS}_{y}(1)$ at $918 \mathrm{~K}$. The formation of $\mathrm{NiS}_{y}(1)$ can lead to rapid sulfidation reactions.

\subsection{Silicon-oxygen-sulfur system}

$\mathrm{SiO}_{2}$ is the only condensed oxide [28]. $\alpha$-tridymite is the stable polymorph for temperatures of $1140-1743 \mathrm{~K} . \alpha$-cristobalite is the stable polymorph for temperatures of 1743 - $1996 \mathrm{~K}$ (melting point). Thermochemical data are available for $\mathrm{SiO}_{2}(\mathrm{~s})$ and for the vapor species $\mathrm{Si}(\mathrm{g}), \mathrm{Si}_{2}(\mathrm{~g}), \mathrm{Si}_{3}(\mathrm{~g}), \operatorname{SiO}(\mathrm{g})$, and $\mathrm{SiO}_{2}(\mathrm{~g})$ (see table 4 ). 
Two condensed sulfides exist $\operatorname{SiS}(s, 1)$ and $\operatorname{SiS}_{2}(s, 1)[7]$. Both have a melting temperature of $1363 \mathrm{~K}[25]$. Thermochemical data are available for $\mathrm{SiS}_{2}(\mathrm{~s})$ and the vapor species $\mathrm{Si}(\mathrm{g}), \mathrm{Si}_{2}(\mathrm{~g}), \mathrm{Si}_{3}(\mathrm{~g})$, and $\mathrm{SiS}(\mathrm{g})$ (see table 4 ).

Figure 27 shows a $\log \mathrm{P}_{\mathrm{Si}_{\mathrm{x}} \mathrm{y}}$ vs $\log \mathrm{P}_{0_{2}}$ and $\log \mathrm{P}_{\mathrm{H}_{2}} / \mathrm{P}_{\mathrm{H}_{2}}$ at $1250 \mathrm{~K}$. The vertical line shows the $\mathrm{O}_{2}$ equilibrium pressure for the $\mathrm{Si}(\mathrm{s}) / \mathrm{SiO} \mathrm{O}_{2}(\mathrm{~s})$ interface; $\log \mathrm{P}_{0_{2}}=-28.7 \mathrm{~atm}$ and $\log \mathrm{P}_{\mathrm{H}_{2}} / \mathrm{P}_{\mathrm{H}_{2} \mathrm{O}}=6.85$. Si(s) forms a stable oxide which cannot be reduced at $1250 \mathrm{~K}$ in $\mathrm{H}_{2}(\mathrm{~g})$. Equilibrium pressures for the vapor species are shown as horizontal and diagonal lines. High $\mathrm{SiO}(\mathrm{g})$ pressures are noted at the $\mathrm{Si}(\mathrm{s}) / \mathrm{SiO}_{2}(\mathrm{~s})$ interface. This high $\mathrm{SiO}(\mathrm{g})$ pressure can lead to active oxidation as predicted by $C$. Wagner [29] and confirmed experimentally by Gulbransen, Brassart, and Andrew [3] in low pressure oxidation studies where $\log \mathrm{P}_{\mathrm{Si} 0}>\log \mathrm{P}_{0_{2}}$. The transition between passive and active oxidation as a function of temperature is directly related to the thermochemical condition, $\log \mathrm{P}_{\mathrm{SiO}}>\log \mathrm{P}_{\mathrm{O}_{2}}$. Figure 28 shows a $\log P_{S i^{0} y}$ vs $\frac{1}{T}$ plot for the two species $\operatorname{SiO}(g)$ and $\operatorname{Si}(g)$.

Figure 29 shows a $\log \mathrm{P}_{\mathrm{Si}_{x} \mathrm{~S}_{y}}$ vs $\log \mathrm{P}_{\mathrm{S}_{2}}$ and $\log \mathrm{P}_{\mathrm{H}_{2}} / \mathrm{P}_{\mathrm{H}_{2} \mathrm{~S}}$ diagram for $1250 \mathrm{~K}$. The vertical line shows the $\mathrm{S}_{2}$ equilibrium pressure for the $\mathrm{Si}(\mathrm{s}) / \mathrm{SiS}_{2}(\mathrm{~s})$ interface, $\log \mathrm{P}_{\mathrm{S}_{2}}=-3.9$ and $\log \mathrm{P}_{\mathrm{H}_{2}} / \mathrm{P}_{\mathrm{H}_{2} \mathrm{~S}}=0.75 . \quad \mathrm{SiS}_{2}(\mathrm{~s})$ is only formed in relatively high $\mathrm{S}_{2}$ potential environments. Very high $\operatorname{SiS}(g)$ pressures are observed at the $\mathrm{Si}(\mathrm{s}) / \mathrm{SiS}_{2}(\mathrm{~s})$ interface, $\log P_{\text {SiS }}=0.4$ at $1250 \mathrm{~K}$. This high SiS(g) pressure suggests volatility of any $\mathrm{SiS}_{2}(\mathrm{~s})$ phase that forms. Figure 30 shows a $\log \mathrm{P}_{\mathrm{Si}_{x} \mathrm{~S}_{\mathrm{y}}}$ vs $\frac{1}{\mathrm{~T}}$ diagram for $\operatorname{SiS}(\mathrm{g})$ and $\operatorname{Si}(\mathrm{g})$ at the important interfaces. Conditions on the $S_{2}$ potential for forming $\operatorname{SiS}_{2}(s)$ as a function of temperature are shown in figure 31.

Elemental silicon in alloys can lead to rapid oxidation and sulfidation due to the formation of the volatile species $\operatorname{SiO}(\mathrm{g})$ and $\mathrm{SiS}(\mathrm{g})$. Active oxidation can occur for $\log P_{\text {SiO }}>\log P_{0_{2}}$. This has been observed for oxidation of pure silicon where $\log a_{S i}=0$ [3]. For low activity silicon alloys active oxidation could only be observed at pressures below $10^{-6} \mathrm{~atm}$.

Silicon can form $\mathrm{SiS}_{2}$ only in high $\mathrm{S}_{2}$ potential atmospheres. Due to the high volatility of $\operatorname{SiS}(\mathrm{g})$ the $\mathrm{SiS}_{2}(\mathrm{~s}) \mathrm{film}$ would volatilize leading to a rapid depletion of silicon. This phenomenon should occur also in low activity silicon alloys.

\subsection{Titanium-oxygen-sulfur system}

The condensed oxide phases are complex $[17,24,28]$. Four oxides are reported for the $\mathrm{Ti}(\mathrm{s})$ - $\mathrm{TiO}(\mathrm{s})$ region: $\mathrm{Ti}_{6} \mathrm{O}, \mathrm{Ti}_{3} \mathrm{O}, \mathrm{Ti}_{2} \mathrm{O}$, and $\mathrm{Ti}_{3} \mathrm{O}_{2}$. Five oxides exist in the $\mathrm{Ti} 0(\mathrm{~s})$ $\mathrm{TiO}_{2}(\mathrm{~s})$ region: $\mathrm{Ti} 0, \mathrm{TiO}_{2}, \mathrm{Ti}_{2} \mathrm{O}_{3}, \mathrm{Ti}_{3} \mathrm{O}_{5}$, and $\mathrm{Ti}_{4} \mathrm{O}_{7}(\mathrm{~s})$. $\mathrm{TiO}(\mathrm{s})$ has a melting temperature 
of $2023 \mathrm{~K}, \mathrm{Ti}_{2} \mathrm{O}_{3}-2115 \mathrm{~K}, \mathrm{Ti}_{3} \mathrm{O}_{5}-2050 \mathrm{~K}, \mathrm{Ti}_{4} \mathrm{O}_{7}-1950 \mathrm{~K}$, and $\mathrm{Ti}_{2}$ (rutile) - $1950 \mathrm{~K}$. Thermochemical data exist on all of the oxides except $\mathrm{Ti}_{3} \mathrm{O}_{2}(\mathrm{~s})$ and the volatile species

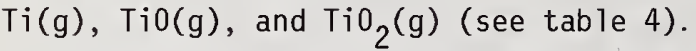

Eleven condensed sulfide phases are reported $[24,28]$. These exist in four regions of the phase diagram $\mathrm{TiS}_{3}, \mathrm{TiS}_{2}-\mathrm{Ti}_{2} \mathrm{~S}_{3}, \mathrm{Ti}_{3} \mathrm{~S}_{4}-\mathrm{TiS}$ and subsulfides. TiS has a melting temperature of $2200 \mathrm{~K}$. $\mathrm{Ti}_{2} \mathrm{~S}(\mathrm{~s})$ has a melting temperature of $1760 \mathrm{~K}$. Thermochemical data are available on $\operatorname{TiS}(\mathrm{s}), \operatorname{TiS}_{2}(\mathrm{~s})$, and $\operatorname{TiS}(\mathrm{g})$ (see table 4).

Figure 32 shows a $\log \mathrm{P}_{\mathrm{Ti}_{x} \mathrm{O}}$ vs $\mathrm{P}_{\mathrm{O}_{2}}$ and $\log \mathrm{P}_{\mathrm{H}_{2}} / \mathrm{P}_{\mathrm{H}_{2} \mathrm{O}}$ at $1250 \mathrm{~K}$. The vertical lines show the $\mathrm{O}_{2}$ equilibrium pressures for the several oxide-metal and oxide-oxide equilibria. For the $\mathrm{Ti}(\mathrm{s}) / \mathrm{TiO}(\mathrm{s})$ interface $\log \mathrm{P}_{\mathrm{O}_{2}}=-35.4 \mathrm{~atm}$ and $\log \mathrm{P}_{\mathrm{H}_{2}} / \mathrm{P}_{\mathrm{H}_{2} \mathrm{O}}=10.25$. At the $\mathrm{Ti}_{4} 0_{7}(\mathrm{~s}) / \mathrm{TiO}_{2}$ (s) interface $\log \mathrm{P}_{0_{2}}=-20.4$ atm and $\log \mathrm{P}_{\mathrm{H}_{2}} / \mathrm{P}_{\mathrm{H}_{2}} \mathrm{O}=2.7$. Figure 33 shows the $0_{2}$ pressures for the several oxide equilibria as a function of temperature between 1150 and $1450 \mathrm{~K}$. The higher oxides of titanium, $\mathrm{Ti}_{4} \mathrm{O}_{7}(\mathrm{~s})$ and $\mathrm{Ti}_{3} \mathrm{O}_{5}(\mathrm{~s})$ can be reduced at $1250 \mathrm{~K}$ in a $\mathrm{H}_{2} / \mathrm{H}_{2} \mathrm{O}$ gas mixture with $\log \mathrm{P}_{\mathrm{H}_{2}} / \mathrm{P}_{\mathrm{H}_{2} \mathrm{O}}=5$ to $\mathrm{Ti}_{2} \mathrm{O}_{3}(\mathrm{~s})$ at $1250 \mathrm{~K}$. However, $\mathrm{Ti}_{2} \mathrm{O}_{3}(\mathrm{~s})$ and $\mathrm{TiO}(\mathrm{s})$ are not reducible in such a gas mixture.

$\mathrm{Ti}(\mathrm{g})$ is the major volatile species in the Ti-0 system at $1250 \mathrm{~K}$. The highest $\mathrm{Ti}(\mathrm{g})$ pressure occurs over the metal phase. Even at $1450 \mathrm{~K} \log \mathrm{P}_{\mathrm{Ti}}=-9.49$. Volatile metal and oxide species do not play a role in the oxidation of titanium and titanium containing alloys for our temperature range.

The important features of the high temperature oxidation of titanium are the high solubility of oxygen in the metal and the complex system of oxides. The limiting 0 content of $\sigma-\mathrm{Ti}$ is $\mathrm{Ti}_{0.48}$, (32 percent).

Figure 34 shows a $\log \mathrm{P}_{\mathrm{Ti}_{x} \mathrm{~S}_{y}}$ vs $\log \mathrm{P}_{\mathrm{S}_{2}}$ and $\log \mathrm{P}_{\mathrm{H}_{2}} / \mathrm{P}_{\mathrm{H}_{2} \mathrm{~S}}$ diagram for $1250 \mathrm{~K}$. The vertical lines show the $S_{2}$ equilibrium pressures for the $\operatorname{Ti}(s) / T i S(s)$ and the

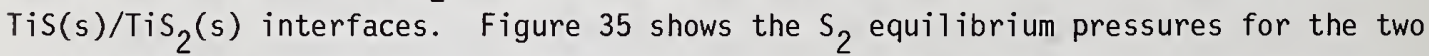
interfaces as a function of temperature. $\mathrm{Ti}(\mathrm{g})$ is the important volatile species in the $\mathrm{Ti}-\mathrm{S}$ system. The highest pressures occur over the metal with $\log \mathrm{P}_{\mathrm{Ti}}=-12.15$ at $1250 \mathrm{~K}$. Volatile metal and sulfide species do not play a role in the sulfidation of titanium and titanium alloys.

\subsection{Vanadium-oxygen-sulfur system}

Twelve condensed oxide phases are reported $[24,28]$. These exist in two regions of the phase diagram: $V-V 0$ and $V 0-V_{2} 0_{5}$. $V 0$ has a wide range of homogeneity and a melting temperature of $2063 \pm 10 \mathrm{~K}$. Ten oxides exist in the $v_{0}-V_{2} 0_{5}$ composition range: $V_{2} 0_{3}$,

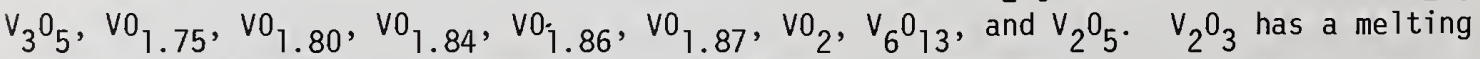
temperature of $2340 \pm 20 \mathrm{~K} . \mathrm{VO}_{2}\left(\mathrm{~V}_{2} \mathrm{O}_{4}\right)$ has a melting temperature of $1818 \pm 15 \mathrm{~K}$ and 
$\mathrm{V}_{2} \mathrm{O}_{5}$ has a melting temperature of $943 \pm 10 \mathrm{~K}$. Thermochemical data are available on $\mathrm{V} 0(\mathrm{~s})$, $\mathrm{V}_{2} \mathrm{O}_{3}(\mathrm{~s}), \mathrm{V}_{2} \mathrm{O}_{4}(\mathrm{~s})$, and $\mathrm{V}_{2} \mathrm{O}_{5}(\mathrm{l})$ and on the gaseous species $\mathrm{V}(\mathrm{g})$, $\mathrm{VO}(\mathrm{g})$, and $\mathrm{VO}_{2}(\mathrm{~g})$ (see table 4).

Four condensed solid sulfides exist $[24,25,28], V_{3} S, V S, V_{2} S_{3}$, and $V_{4}$. Little information has been reported on their melting points. The high temperature polymorph $\alpha-V_{3} S$ is molten at $1673 \mathrm{~K}$. Thermochemical data are available on $V_{2} S_{3}(s)$ and the vapor species $V(g)$ and $V S(g)$ (see table 4 ).

Figure 36 shows a $\log P_{V_{X} O_{y}}$ vs $\log P_{0_{2}}$ and $\log P_{H_{2}} / P_{H_{2}}$ at $1250 \mathrm{~K}$. Log $P_{0_{2}}=-27.2$ atm and $\log \mathrm{P}_{\mathrm{H}_{2}} / \mathrm{P}_{\mathrm{H}_{2} \mathrm{O}}=6.1$ are noted for the $\mathrm{V}(\mathrm{s}) / \mathrm{VO}(\mathrm{s})$ interface. $V 0(\mathrm{~s})$ is the stable oxide phase in a hydrogen gas atmosphere with $\log \mathrm{P}_{\mathrm{H}_{2}} / \mathrm{P}_{\mathrm{H}_{2} \mathrm{O}}=5$. Figure 37 shows the $0_{2}$ equilibrium pressures for the several oxide-metal and oxide/oxide interfaces. $V_{2} 0_{5}(1)$ can form in high oxygen pressure environments at $1250 \mathrm{~K}$ with $\log P_{0_{2}}=-2.4$ atm at the $\mathrm{V}_{2} \mathrm{O}_{4}(\mathrm{~s}) / \mathrm{V}_{2} \mathrm{O}_{5}(1)$ interface.

$\mathrm{VO}_{2}(\mathrm{~g})$ is the most volatile species at $1250 \mathrm{~K}$ with $\log \mathrm{P}_{\mathrm{VO}_{2}}=-10.1 \mathrm{~atm}$. Figure 38 shows the equilibrium pressures of the volatile metal and oxide species as a function of temperature. Volatilization of $\mathrm{VO}_{2}(\mathrm{~g})$ becomes important over $\mathrm{V}_{2} \mathrm{O}_{4}(\mathrm{~s})$ at temperatures above $1342 \mathrm{~K}$. For alloys containing vanadium at low metal activities volatilization of oxide species is not important for temperatures up to $1450 \mathrm{~K}$. No experimental studies on the influence of volatile species on the oxidation of vanadium have been reported.

The important features of the $V-0$ system is the formation of $V_{2} 0_{5}(1)$ in high oxygen gas environments above $943 \mathrm{~K}\left(670^{\circ} \mathrm{C}\right)$ and the formation of a complex series of oxides including the suboxide $\mathrm{V}_{2} \mathrm{O}(\mathrm{s})$.

Figure 39 shows a $\log P_{V_{x} S_{y}}$ vs $\log P_{S_{2}}$ and $\log P_{H_{2}} / P_{H_{2} S}$ diagram at $1250 \mathrm{~K}$. Since thermochemical information is not available on $V_{3} S$, VS, and $V S_{4}$ the diagram is of 1 imited value in predicting $S_{2}$ equilibrium pressures for the $V-S$ system. Figure 39 shows that the volatile species $V(g)$ are not important over $V(s)$ and $V_{2} S_{3}(s)$. We predict that $V(g)$ and VS(g) will not be important volatile species over the $V_{2} S$, VS, and $V_{4}$ condensed phases.

We conclude that the major features of interest in the V-0-S system are the formation of $\mathrm{V}_{2} \mathrm{O}_{5}(1)$ and a complex series of oxides including the suboxide $\mathrm{V}_{2} \mathrm{O}$. The vapor species $\mathrm{VO}_{2}(\mathrm{~g})$ is only important above $1342 \mathrm{~K}$.

\subsection{Tungsten-oxygen-sulfur system}

Five condensed oxides are reported $w_{2}, w_{2.72}, w_{2} .90, w_{2} .96$, and $w_{3}[6] . w_{3}$ has a melting temperature of $1744 \mathrm{~K}$. Thermochemical data are available on the five condensed oxides and on the volatile species $\mathrm{W}$, wo, $\mathrm{WO}_{2}, \mathrm{~W}_{3} \mathrm{O}_{8}, \mathrm{WO}_{3},\left(\mathrm{WO}_{3}\right)_{2},\left(\mathrm{WO}_{3}\right)_{3}$, and $\left(\mathrm{WO}_{3}\right)_{4}$ (see table 4). 
Two condensed sulfides exist $[24,25], W_{2}$ and $W S_{3}$. WS 2 has a melting temperature above $2073 \mathrm{~K}$. WS 3 is prepared chemically and decomposes to $\mathrm{WS}_{2}$ and $S$ on heating at 543 - $773 \mathrm{~K}$ depending on the atmosphere. Thermochemical data are available on $\mathrm{WS}_{2}(\mathrm{~s})$ and $W(g)$ (see table 4).

Figure 40 shows a $\log P_{W 0}$ vs $\log P_{0_{2}}$ and $\log P_{H_{2}} / P_{H_{2} 0}$ diagram at $1250 \mathrm{~K}$. To simplify the complex oxide equilibria between $\mathrm{WO}_{2}(\mathrm{~s})$ and $\mathrm{WO}_{3}(\mathrm{~s})$, the oxide phases $\mathrm{WO}_{2} .90$ and $\mathrm{WO}_{2} .96$ are not considered. The vertical lines show that $\mathrm{O}_{2}$ equilibrium pressures for the several interfaces for $W(s) / W O_{2}(s) \log P_{0_{2}}=-15.24$ atm and $\log P_{H_{2}} / P_{H_{2} 0}=-0.15$ at $1250 \mathrm{~K}$. A11 of the tungsten oxides are easily reduced in hydrogen at $1250 \mathrm{~K}$.

$\left(\mathrm{WO}_{3}\right)_{3}$ is the major volatile species over $\mathrm{WO}_{3}(\mathrm{~s})$ at $1250 \mathrm{~K}$ with $\log \mathrm{P}_{\left(\mathrm{WO}_{3}\right)_{3}}=-7.1 \mathrm{~atm}$. Volatilization of $\left(\mathrm{WO}_{3}\right)_{3}$ can affect the oxidation kinetics at $1250 \mathrm{~K}$ even for $\log \mathrm{a}_{\mathrm{W}}=-1.9$. Figure 41 shows a $\log \mathrm{P}_{\left(\mathrm{WO}_{3}\right)_{\mathrm{x}}}$ vs $\frac{1}{\mathrm{~T}}$ diagram for $\left(\mathrm{WO}_{3}\right)_{\mathrm{x}}(\mathrm{g})$ over $\mathrm{WO}_{3}(\mathrm{~s})$. Mixed oxidation and volatilization should occur in the oxidation of tungsten above $1150 \mathrm{~K}$.

Tungsten behaves like molybdenum in oxidation with volatilization occurring at higher temperatures than for molybdenum. Also $\mathrm{WO}_{3}(\mathrm{~s})$ has a higher melting temperature of $1745 \mathrm{~K}$ than $\mathrm{MoO}_{3}(\mathrm{~s})$. No melting of $\mathrm{WO}_{3}(\mathrm{~s})$ should occur in the temperature range of interest in hot corrosion.

No volatile sulfides of tungsten have been reported. Therefore, $W(g)$ is the only volatile species in the $W-S$ system. Since the volatility of $W(g)$ is $\log P_{W}=-23.14 \mathrm{~atm}$ at $1450 \mathrm{~K}$ volatile species have no effect on the sulfidation of tungsten. Figure 42 shows a $S_{2}$ equilibrium pressure vs $\frac{l}{T}$ diagram for the $W(s) / W S_{2}(s)$ interface. Sulfidation occurs for $S_{2}$ pressures above the equilibrium pressures.

We conclude that mixed oxidation and volatilization processes occur in the oxidation of tungsten at $1150 \mathrm{~K}$ and higher temperatures.

\section{Summary}

Thermochemical analyses has given chemists, engineers and metallurgists a powerful tool for the study of high temperature corrosion processes in oxygen and sulfur containing gases. The following factors are important: (1) melting temperatures of the metal, oxide, sulfide, and sulfate phases, (2) thermochemical stability of the oxide, sulfide, and sulfate condensed phases, (3) solubility of oxygen and sulfur in the metal, (4) vapor species at the several metal-oxide, metal-sulfide, oxide-gas, sulfide-gas, and sulfategas interfaces, (5) vapor and condensed volatilized species in the barrier layer surrounding the reacting metal, and (6) enthalpies of reaction of the oxide, sulfide and sulfate compounds.

Good thermochemical data exist for most of the metal-oxygen systems discussed here. Rapid oxidation reaction can be predicted for molybdenum and silicon due to volatile 
species and for vanadium due to melting of the oxide $V_{2} 0_{5}$. Volatile oxide species begin to play an important role in the oxidation of chromium, tungsten, and aluminum above $1250 \mathrm{~K}$. Titanium is unique in this group of metals since volatile species are not important at $1250 \mathrm{~K}$. However, due to the high solubility of oxygen in the metal and a high enthalpy of reaction, rapid oxidation can occur above $1250 \mathrm{~K}$ since volatile species cannot limit access of oxygen to the reacting metal. High temperatures with melting of the metal can occur.

Less useful thermochemical data exist for the metal-sulfur systems. Useful studies should be made in this area including phase diagram studies and melting points. Many of the metal-sulfide systems have compounds with melting points below $1250 \mathrm{~K}$. Little work has been done on the volatile species on the metal-sulfide systems discussed here. Silicon forms a very volatile sulfide $\operatorname{SiS}(\mathrm{g})$ with high pressures at the $\mathrm{Si}(\mathrm{s}) / \mathrm{SiS}_{2}(\mathrm{~s})$ interface. Rapid sulfidation of silicon can occur at $1250 \mathrm{~K}$.

Work is needed to study experimentally the role of volatile species in high temperature oxidation and sulfidation reactions. These studies should include both weight and total oxygen and sulfur consumed to distinguish condensed oxide and sulfide formation from volatilized oxide and sulfide species.

The authors have found thermochemical analyses a useful discipline for planning and interpreting experimental studies. This is possible today with the availability of the JANAF Thermochemical Tables and other tabulations given in the references.

The authors wish to acknowledge the support in part of the United States Department of Energy. 
Appendix 1. Tables

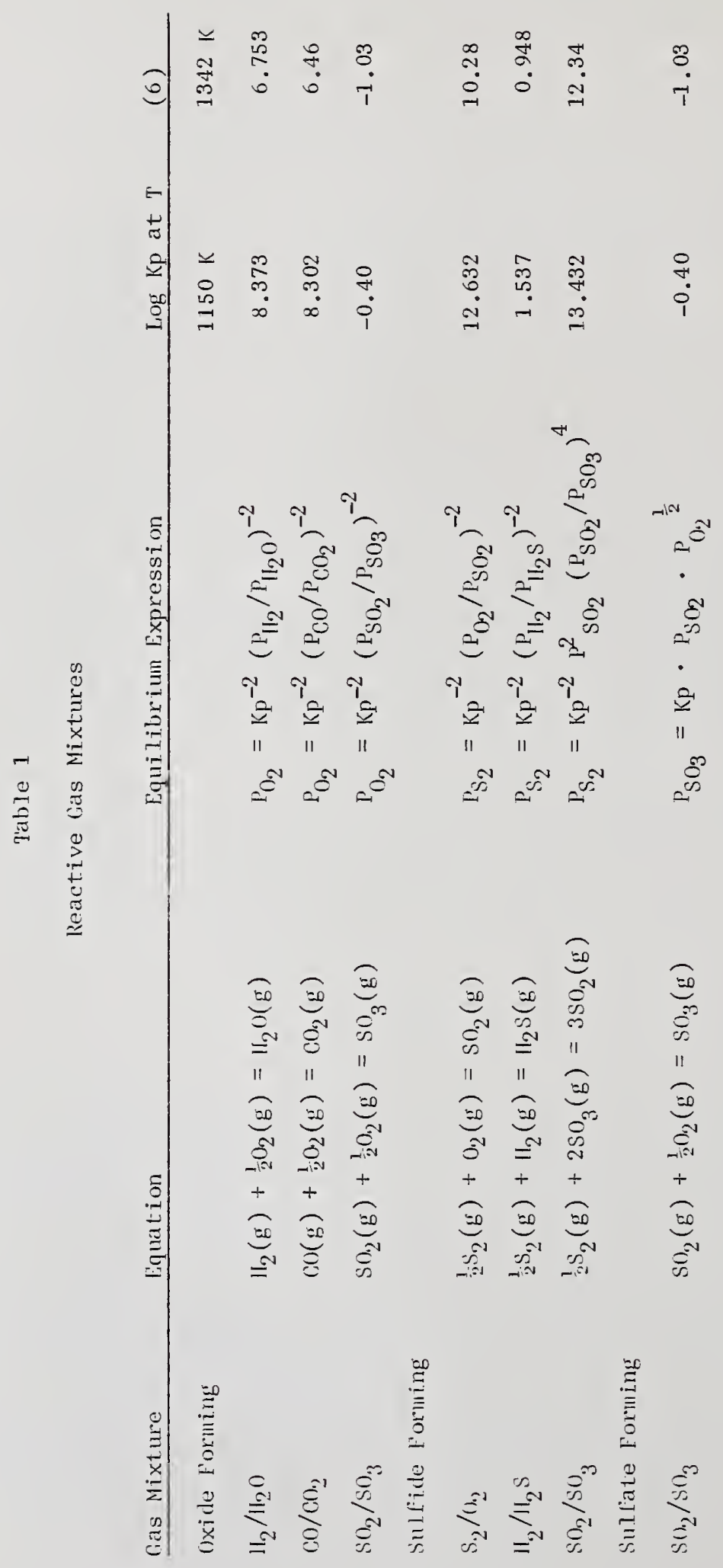


Table 2

A. Oxygen Potential of Three Gas Mixtures for $1: 1$ Concentration

$\log \mathrm{P}_{\mathrm{O}_{2}}$ at $\mathrm{T} \mathrm{K}$

Gas Mixture

$\mathrm{H}_{2} / \mathrm{H}_{2} \mathrm{O}$

$\mathrm{CO} / \mathrm{CO}_{2}$

$\mathrm{SO}_{2} / \mathrm{SO}_{3}$

Gas Mixture

$\mathrm{S}_{2} / \mathrm{O}_{2}$

$\mathrm{H}_{2} / \mathrm{H}_{2} \mathrm{~S}$

$\mathrm{SO}_{2} / \mathrm{SO}_{3}$

Gas Mixture

$\mathrm{SO}_{2} / \mathrm{SO}_{3}$
1150

$-16.75$

$-16.604$

0.80
1342

$-13.51$

$-12.936$

2.06
$-14.93$

$-14.552$

1.50

1450

$-12.05$

$-11.308$

2.62

B. Sulfur Potential of Three Gas Mixtures for $1: 1$ Concentration

$\log \mathrm{P}_{\mathrm{S}_{2}}$ at $\mathrm{T} \mathrm{K}$

$\begin{array}{llll}1150 & 1250 & 1342 & 1450 \\ -25.26 & -22.63 & -20.56 & -18.46 \\ -3.07 & -2.41 & -1.90 & -1.37 \\ -34.86 & -33.64 & -32.68 & -31.7\end{array}$

C. Sulfate Forming Potential for Log $\mathrm{P}_{\mathrm{SO}_{2}}=-4$
and $\log \mathrm{P}_{\mathrm{O}_{2}}=0$ $\log \mathrm{PSO}_{3}$ at $\mathrm{T} \mathrm{K}$

$\begin{array}{llll}1150 & 1250 & 1342 & 1450 \\ -3.90 & -4.25 & -4.53 & -4.81\end{array}$




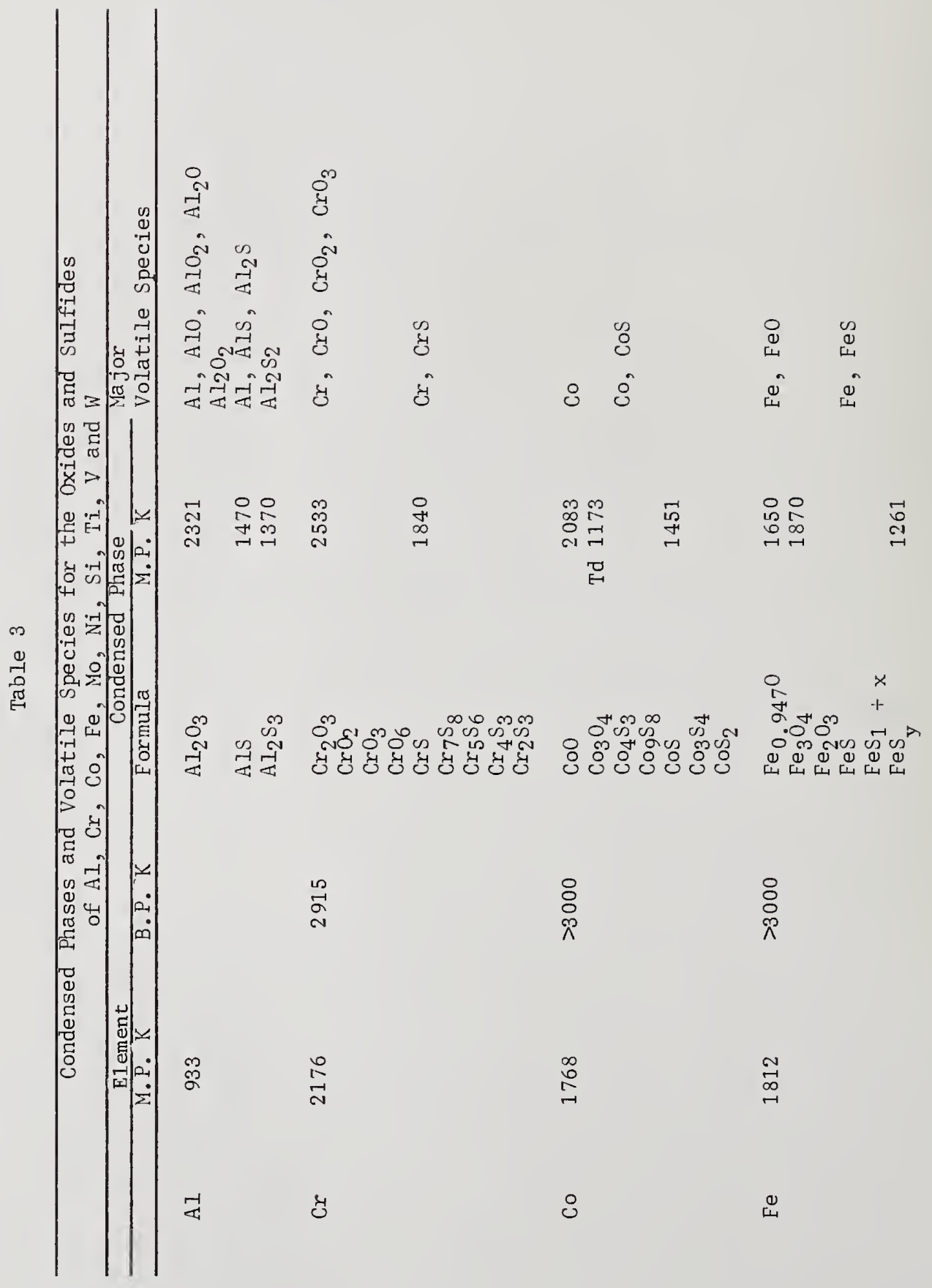




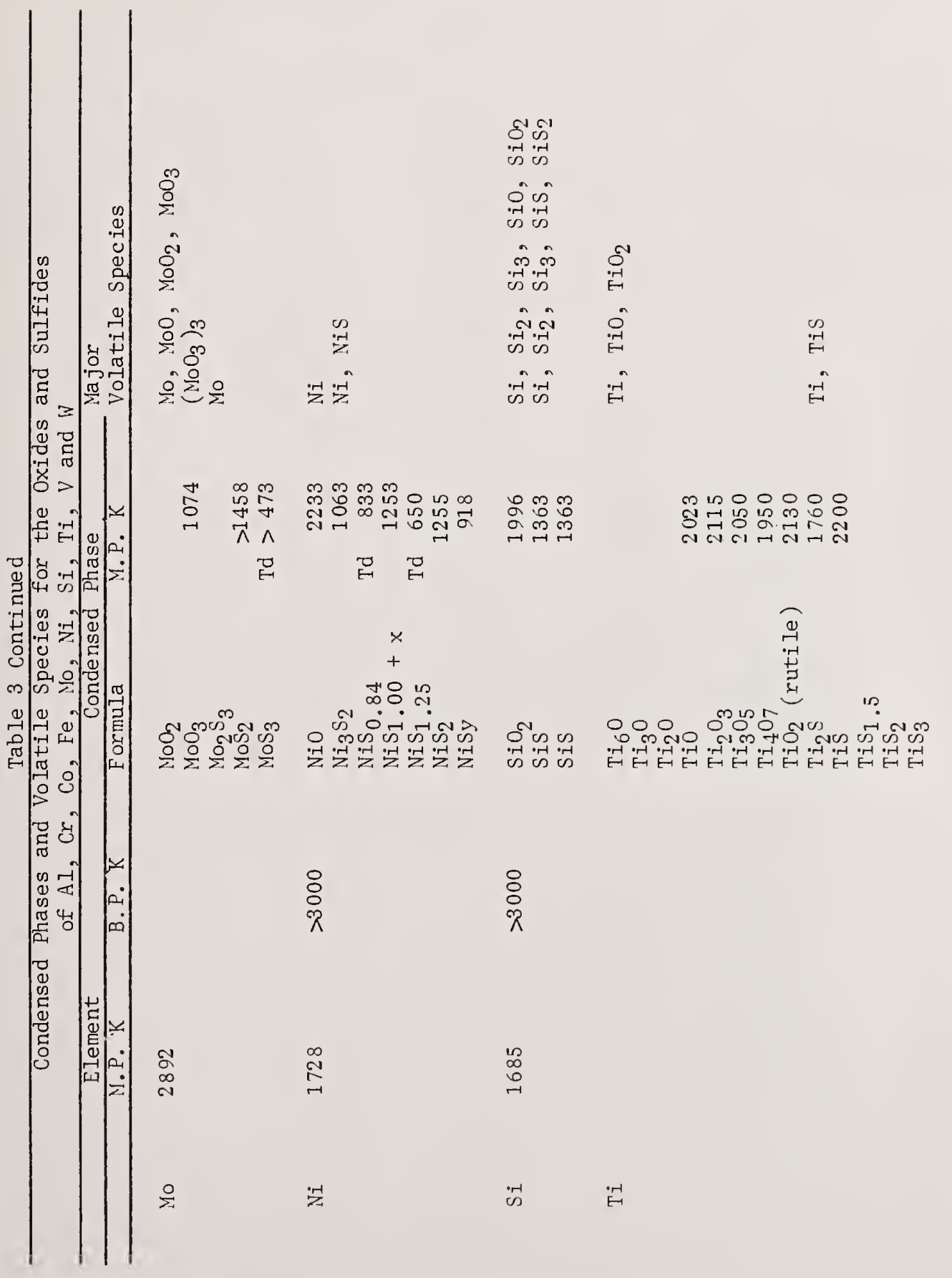




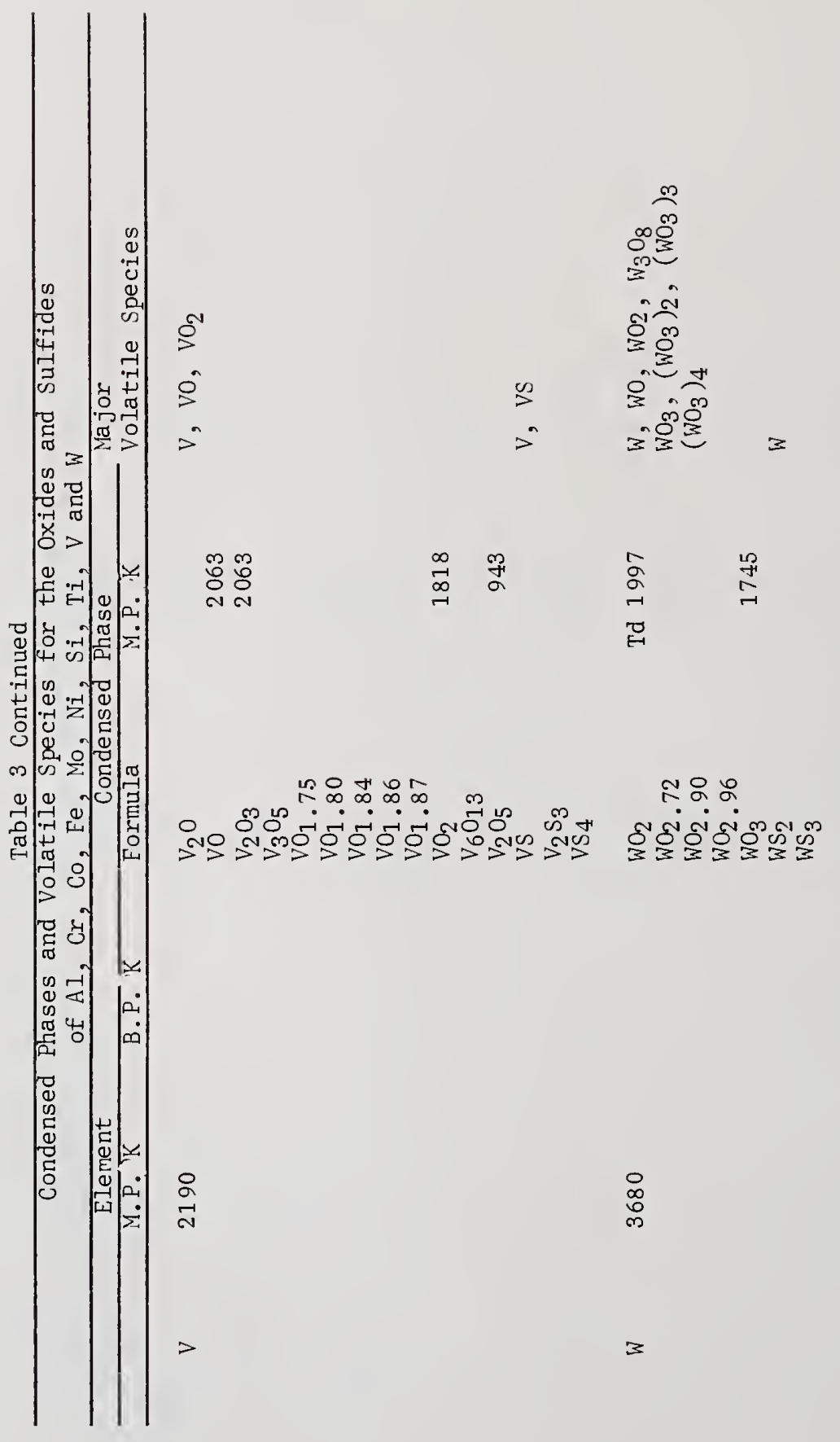


Table 4

Summary Thermochemical Data 1150 - $1450 \mathrm{~K}$

Aluminum $\quad \log \mathrm{Kp}$ at $\mathrm{T} \mathrm{K}$

\begin{tabular}{|c|c|c|c|c|c|c|}
\hline Compound & M.P. K & 1150 & 1250 & 1342 & 1450 & Reference \\
\hline $\mathrm{Al}_{2} \mathrm{O}_{3}(\mathrm{~s})$ & 2315 & 59.57 & 53.43 & 48.59 & 43.71 & 6 \\
\hline $\mathrm{Al}_{2} \mathrm{~S}_{3}(\mathrm{~s}, \mathrm{I})$ & 1370 & 27.18 & 23.84 & 21.24 & 18.65 & 7 \\
\hline $\mathrm{Al}_{2}\left(\mathrm{SO}_{4}\right)_{3}(\mathrm{~s})$ & $\mathrm{Td} 1100$ & 93.52 & 80.62 & 70.28 & 59.84 & 8 \\
\hline $\mathrm{AI}(\mathrm{g})$ & & -8.025 & -6.905 & -6.023 & -5.136 & 6 \\
\hline $\mathrm{A} 10(\mathrm{~g})$ & & 0.358 & 0.589 & 0.767 & 0.946 & 6 \\
\hline $\mathrm{AIO}_{2}(\mathrm{~g})$ & & 8.766 & 8.041 & 7.468 & 6.884 & 6 \\
\hline $\mathrm{Al}_{2} \mathrm{O}(\mathrm{g})$ & & 10.378 & 9.757 & 9.263 & 8.755 & 6 \\
\hline $\mathrm{Al}_{2} \mathrm{O}_{2}(\mathrm{~g})$ & & 17.810 & 16.195 & 14.920 & 13.62 & 6 \\
\hline $\operatorname{AIS}(\mathrm{g})$ & & -3.72 & -3.16 & -2.72 & -2.28 & 6 \\
\hline $\mathrm{A}_{2} \mathrm{~S}(\mathrm{~g})$ & & -3.41 & -2.92 & -2.52 & -2.13 & 6 \\
\hline $\mathrm{AI}_{2} \mathrm{~S}_{2}(\mathrm{~g})$ & & 1.64 & 1.33 & 1.10 & 0.855 & 6 \\
\hline
\end{tabular}

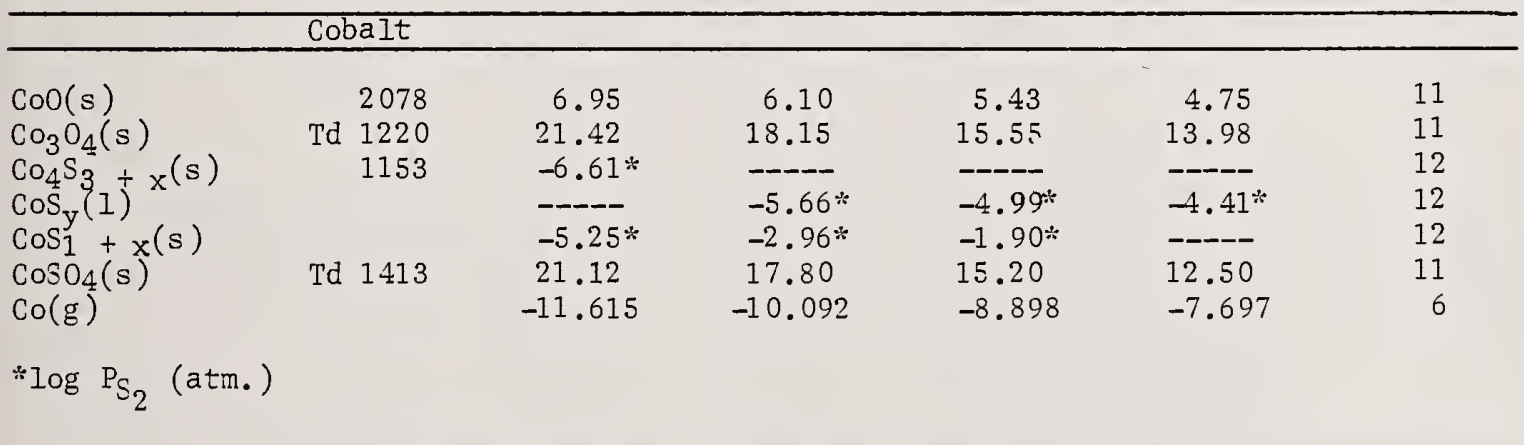

\begin{tabular}{|c|c|c|c|c|c|c|}
\hline \multicolumn{7}{|c|}{ Chromium } \\
\hline $\mathrm{Cr}_{2} \mathrm{O}_{3}(\mathrm{~s})$ & 2533 & 38.07 & 33.95 & 30.69 & 27.40 & 13 \\
\hline $\mathrm{CrO}_{6}(\mathrm{I})$ & 470 & 15.76 & 13.75 & 12.22 & 10.53 & 14 \\
\hline $\operatorname{CrS}(\mathrm{s})$ & 1840 & 6.27 & 5.53 & 4.95 & 4.36 & 15 \\
\hline $\mathrm{Cr}_{7} \mathrm{~S}_{8}(\mathrm{~s})$ & & 50.14 & 44.16 & 39.50 & 34.82 & 16 \\
\hline $\mathrm{Cr}_{5} \mathrm{~S}_{6}^{0}(\mathrm{~s})$ & & 37.33 & 32.71 & 29.16 & 25.57 & 16 \\
\hline $\mathrm{Cr}_{3} \mathrm{~S}_{4}(\mathrm{~s})$ & & 24.32 & 21.15 & 18.76 & 16.32 & 16 \\
\hline $\mathrm{Cr}_{2} \mathrm{~s}_{3}(\mathrm{tr})$ & & 16.75 & 14.52 & 12.84 & 11.13 & 16 \\
\hline $\mathrm{Cr}_{2} \mathrm{~S}_{3}(\mathrm{rh})$ & & $-0.7 \%$ & $-0.6 *$ & $-0 . f^{k}$ & $-0.3 *$ & 16 \\
\hline $\operatorname{cr}(g)$ & & -10.38 & -8.96 & -7.84 & -6.72 & 17 \\
\hline $\mathrm{CrO}(\mathrm{g})$ & & -2.91 & -2.26 & -1.75 & -1.24 & 17 \\
\hline $\mathrm{CrO}_{2}(\mathrm{~g})$ & & 5.26 & 4.96 & +.71 & 4.46 & 17 \\
\hline $\mathrm{CrO}_{3}(\mathrm{~g})$ & & 9.72 & 8.64 & 7.78 & 6.91 & 17 \\
\hline $\operatorname{crs}(\mathrm{g})$ & & -7.16 & -6.16 & -5.30 & -4.50 & 7 \\
\hline
\end{tabular}

* Iog $\mathrm{P}_{\mathrm{S}_{2}}$ (atm.) 
Table 4 Continued

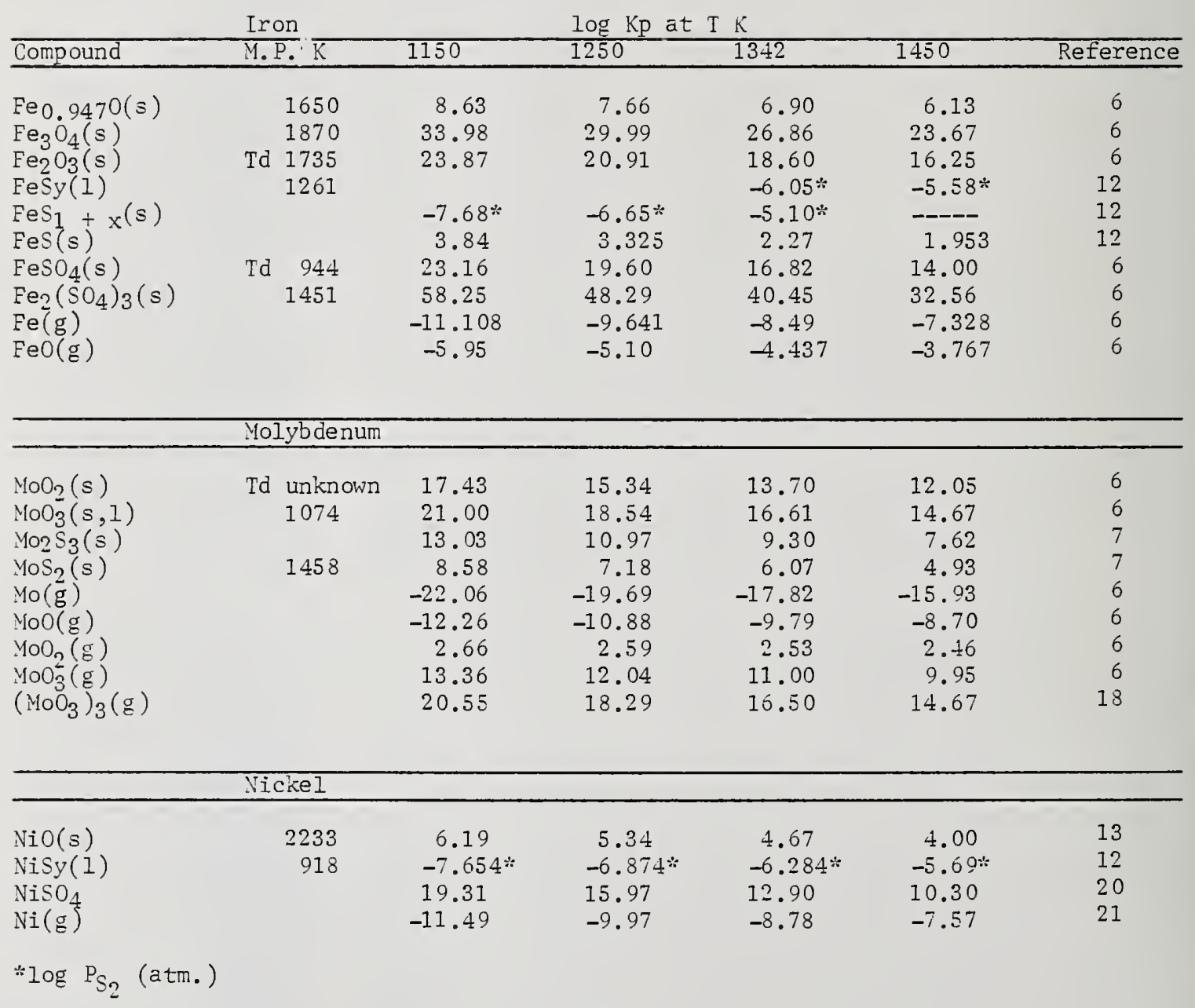


Table 4 Continued

\begin{tabular}{|c|c|c|c|c|c|c|}
\hline \multirow[b]{2}{*}{ Compound } & \multicolumn{2}{|l|}{ Silicon } & \multicolumn{3}{|c|}{$\log \mathrm{Kp}$ at $\mathrm{T}^{\prime} \mathrm{K}$} & \multirow[b]{2}{*}{ Reference } \\
\hline & M.P. K & 1150 & 1250 & 1342 & 1450 & \\
\hline $\mathrm{SiO}_{2}(\mathrm{~s})$ & 1996 & 31.99 & 28.72 & 26.13 & 23.53 & 6 \\
\hline $\mathrm{SiS}_{2}(s, 1)$ & 1363 & 5.01 & 3.93 & 3.09 & 2.30 & 6 \\
\hline $\mathrm{Si}(\mathrm{g})$ & & -12.74 & -11.11 & -9.83 & -8.54 & 6 \\
\hline$S i_{2}(g)$ & & -17.03 & -14.91 & -13.24 & -11.56 & 6 \\
\hline $\mathrm{Si}_{3}(\mathrm{~g})$ & & -18.19 & $-15.9 ?$ & -14.13 & -12.34 & 6 \\
\hline SiO(g) & & 9.07 & 8.6 & 8.37 & 8.05 & 6 \\
\hline $\mathrm{SiO}_{2}(\mathrm{~g})$ & & 14.02 & 12.9 & 12.01 & 11.16 & 6 \\
\hline Sis(g) & & 2.18 & 2.3 & 2.48 & 2.61 & 7 \\
\hline
\end{tabular}

Titanium

\begin{tabular}{|c|c|c|c|c|c|c|}
\hline $\mathrm{TiO}(\mathrm{s})$ & 2023 & 19.66 & 17.70 & 16.16 & 14.62 & 17 \\
\hline $\mathrm{Ti}_{2} \mathrm{O}_{2}(\mathrm{~s})$ & 2115 & 54.66 & 49.20 & 44.91 & 40.57 & 17 \\
\hline $\mathrm{Ti}_{3} \mathrm{O}_{5}(\mathrm{~s})$ & 2050 & 88.53 & 79.69 & 72.70 & 65.66 & 17 \\
\hline $\mathrm{Ti}_{4} \mathrm{O}_{7}(\mathrm{~s})$ & 1950 & 122.18 & 109.90 & 100.22 & 90.44 & 17 \\
\hline $\mathrm{TiO}_{2}$ (rutile) & 2130 & 33.44 & 30.02 & 27.33 & 24.60 & 17 \\
\hline $\operatorname{TiS}(\mathrm{s})$ & 2200 & 12.47 & 10.86 & 9.81 & 8.75 & 7 \\
\hline $\mathrm{TiS}_{2}(\mathrm{~s})$ & & 15.75 & 13.59 & 12.13 & 10.65 & 7 \\
\hline $\operatorname{Ti}(\tilde{\mathrm{g}})$ & & -13.83 & -12.15 & -10.82 & -9.49 & 6 \\
\hline $\mathrm{TiO}(\mathrm{g})$ & & 2.498 & 2.654 & 2.771 & 2.886 & 17 \\
\hline $\mathrm{TiO}_{2}(\mathrm{~g})$ & & 14.86 & 13.71 & 12.79 & 11.86 & 17 \\
\hline Tis(g) & & -7.08 & -6.20 & -5.48 & -4.71 & 7 \\
\hline
\end{tabular}

\begin{tabular}{|c|c|c|c|c|c|c|}
\hline & Vanadiun & & & & & \\
\hline $\mathrm{VO}(\mathrm{s})$ & 2063 & 15.08 & 13.54 & 12.34 & 11.12 & 17 \\
\hline $\mathrm{V}_{2} \mathrm{O}_{3}(\mathrm{~s})$ & 2063 & 42.06 & 37.71 & 34.29 & 30.88 & 17 \\
\hline $\mathrm{V}_{2} \mathrm{O}_{4}(\mathrm{~s})$ & 1818 & 47.52 & 42.45 & 38.48 & 34.45 & 17 \\
\hline $\mathrm{V}_{2} \mathrm{O}_{5}(1)$ & 943 & 48.93 & 43.64 & 39.48 & 35.28 & 17 \\
\hline$V_{0} s_{3}(s)$ & & 42.00 & 37.38 & 33.75 & 30.07 & 23 \\
\hline $\mathrm{VOSO}_{4}(\mathrm{~s})$ & Td 900 & ----- & ----- & -- & ----- & 22 \\
\hline$V(g)$ & & -15.48 & -13.62 & -12.15 & -10.67 & 17 \\
\hline yo(g) & & -0.903 & $-0 . \pm 72$ & -0.135 & 0.201 & 17 \\
\hline $\mathrm{VO}_{n}(\mathrm{~g})$ & & 11.93 & 11.06 & 10.36 & 9.66 & 17 \\
\hline VST(g) & & -7.52 & -6.53 & -5.75 & -4.97 & 7 \\
\hline
\end{tabular}

Tungsten $\quad \log \mathrm{Kp}$ at $\mathrm{T} K$

\begin{tabular}{|c|c|c|c|c|c|c|}
\hline Compound & M.P. K & 1150 & 1250 & 1342 & 1450 & Reference \\
\hline $\mathrm{WO}_{2}(\mathrm{~s})$ & Td 1997 & 17.32 & 15.24 & 13.58 & 11.92 & 6 \\
\hline $\mathrm{WO}_{2}, 72(\mathrm{~s})$ & & 23.22 & 20.44 & 18.24 & 16.03 & 6 \\
\hline $\mathrm{WO}_{2} \mathrm{go}(\mathrm{s})$ & & 24.31 & 21.39 & 19.08 & 16.77 & 6 \\
\hline $\mathrm{WO}_{2} \cdot 96(\mathrm{~s})$ & & 24.76 & 21.78 & 19.44 & 17.08 & 6 \\
\hline $\mathrm{WO}_{3}(\mathrm{~s})$ & 1745 & 24.94 & 21.96 & 19.60 & 17.22 & 6 \\
\hline$W S_{2}(s)$ & & 7.75 & 6.39 & 5.20 & 4.25 & 7 \\
\hline$W(\mathrm{~g})$ & & -31.23 & -28.11 & -25.65 & -23.14 & 6 \\
\hline WO $(\mathrm{g})$ & & -13.80 & -12.28 & -11.08 & -9.87 & 6 \\
\hline $\mathrm{WO}_{2}(\mathrm{~g})$ & & -1.213 & -0.957 & -0.758 & -0.557 & 6 \\
\hline $\mathrm{W}_{3} \mathrm{O}_{8}(\mathrm{~g})$ & & 56.46 & 50.34 & 45.53 & 40.64 & 6 \\
\hline $\mathrm{wO}_{3}(\mathrm{~g})$ & & 10.43 & 9.36 & 8.52 & 7.66 & 6 \\
\hline$\left(\mathrm{WO}_{3}\right)_{2}(\mathrm{~g})$ & & 39.81 & 35.65 & 32.38 & 29.06 & 6 \\
\hline$\left(\mathrm{WO}_{3}\right)_{3}(\mathrm{~g})$ & & 66.08 & 58.83 & 53.14 & 47.36 & 6 \\
\hline$\left(\mathrm{WO}_{3}\right)_{4}(\mathrm{~g})$ & & 89.68 & 79.66 & 71.79 & 63.80 & 6 \\
\hline
\end{tabular}


Appendix 2. Figures

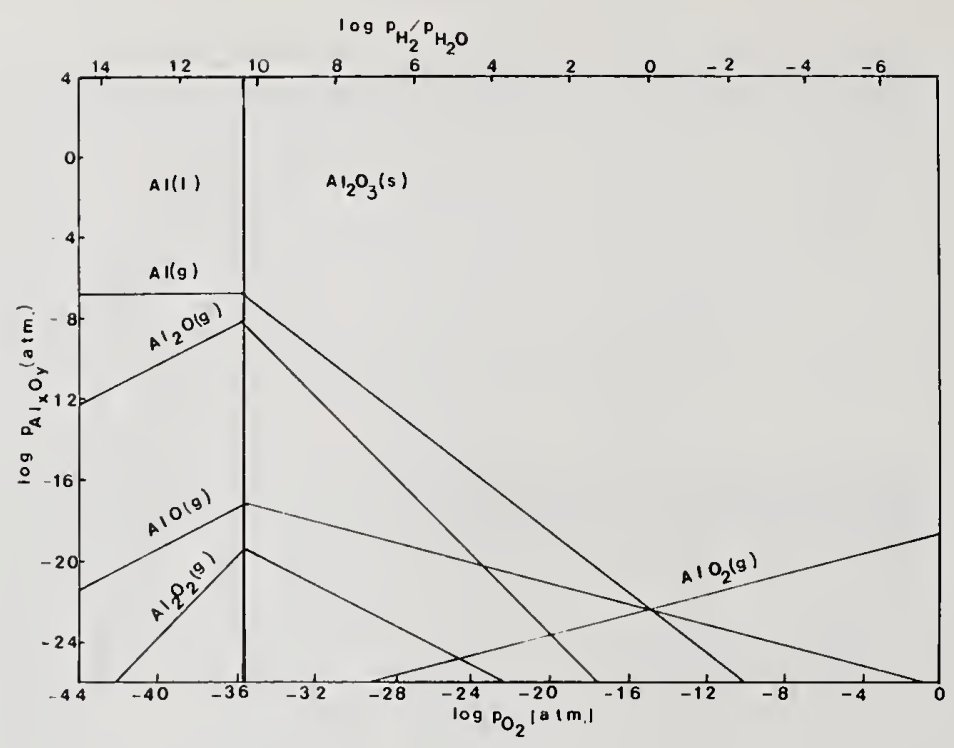

Figure 1. Al-0 system, volatile species, $1250 \mathrm{~K}$.

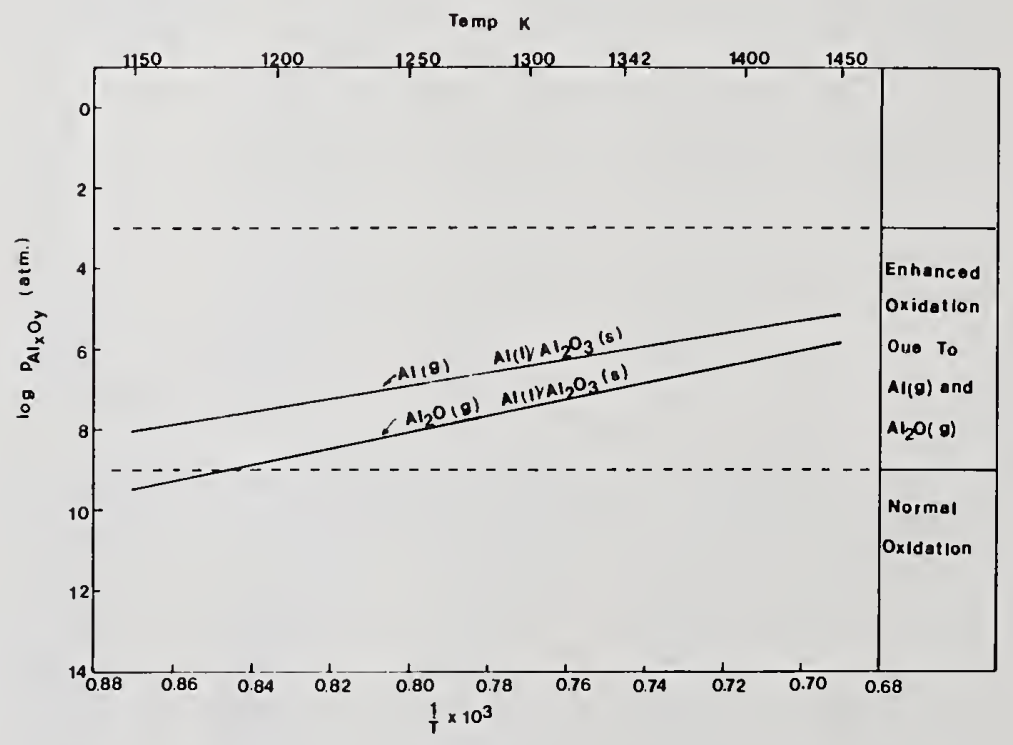

Figure 2. Al-0 system, volatile species vs temperature. 


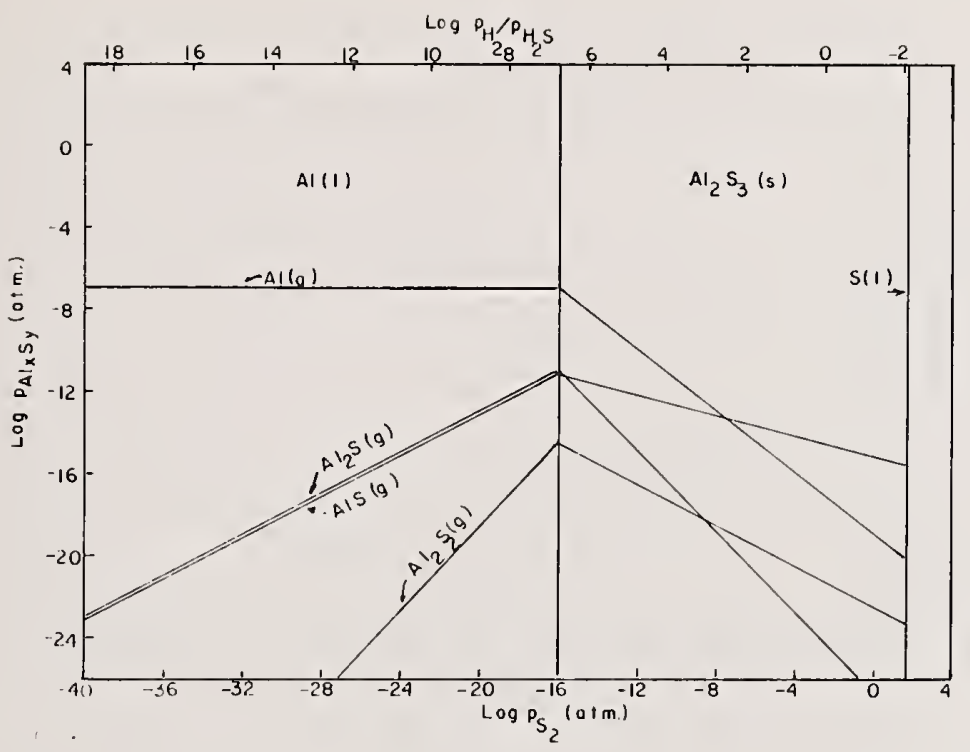

Figure 3. Al-S system, volatile species, $1250 \mathrm{~K}$.

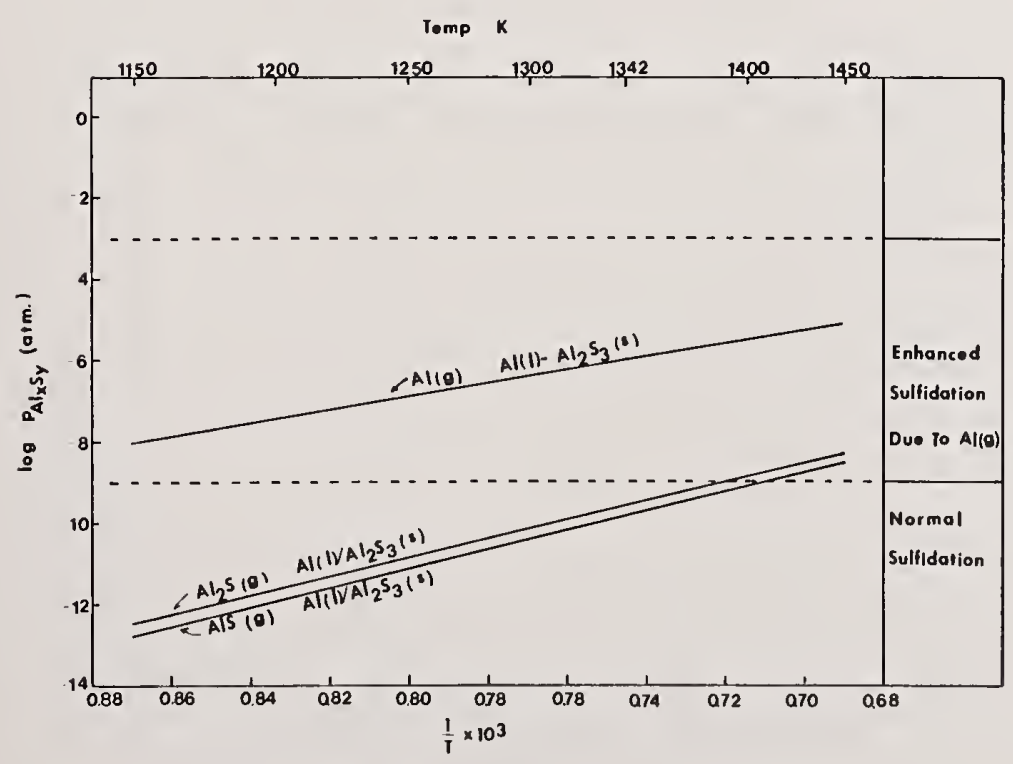

Figure 4. Al-S system, volatile species vs temperature. 


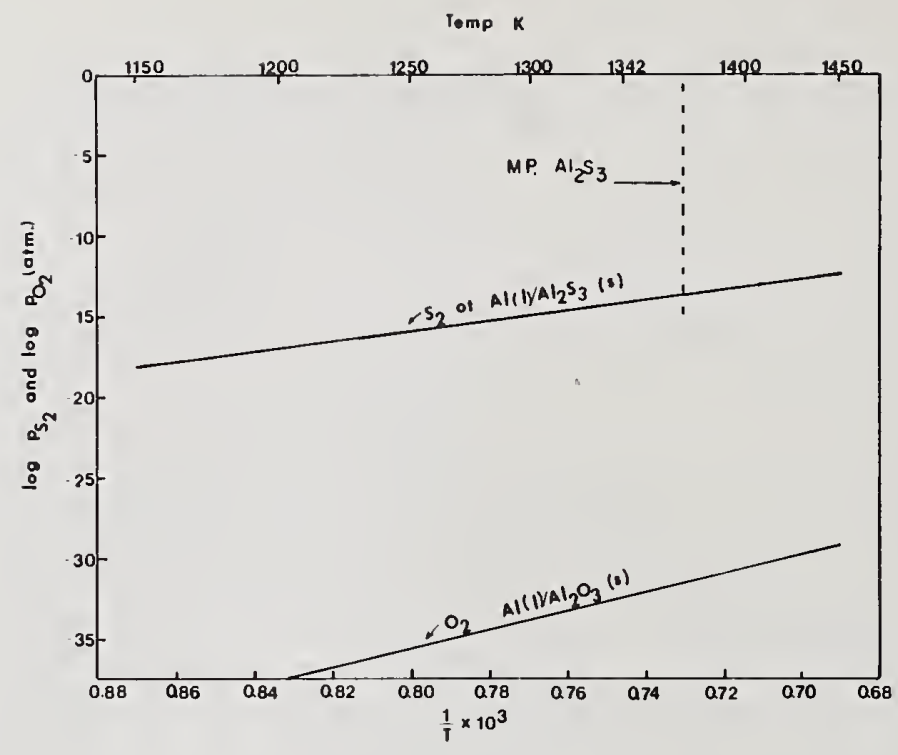

Figure 5. Al-0 and $A 1-S$ systems, $O_{2}$ and $S_{2}$ dissociation pressures.

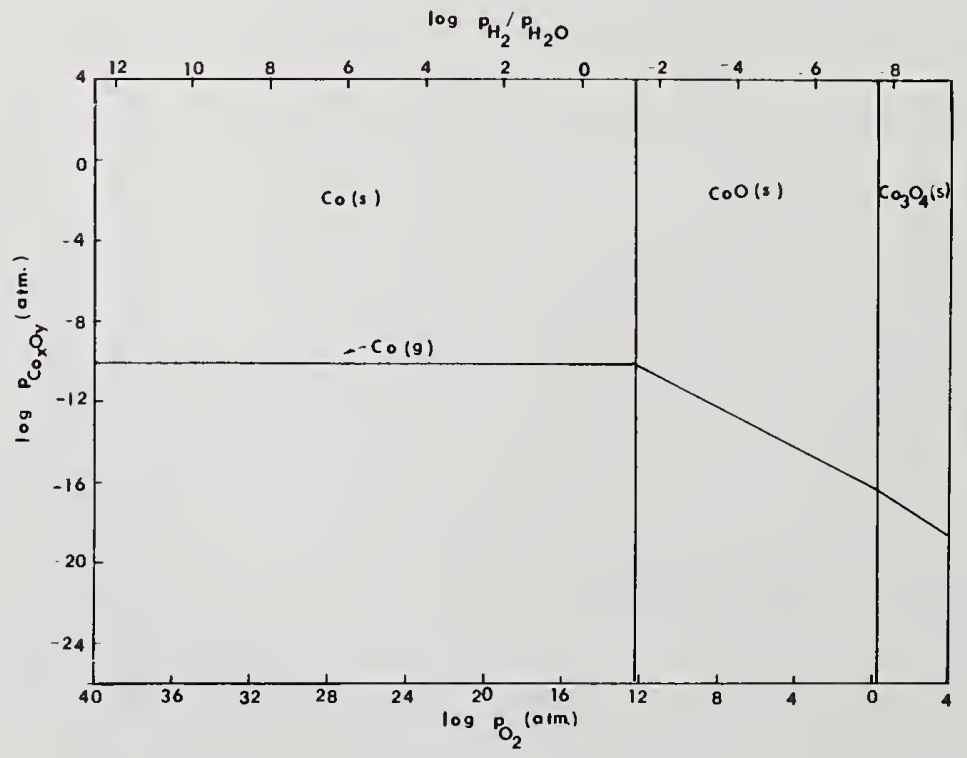

Figure 6. Co-0 system, volatile species, $1250 \mathrm{~K}$. 


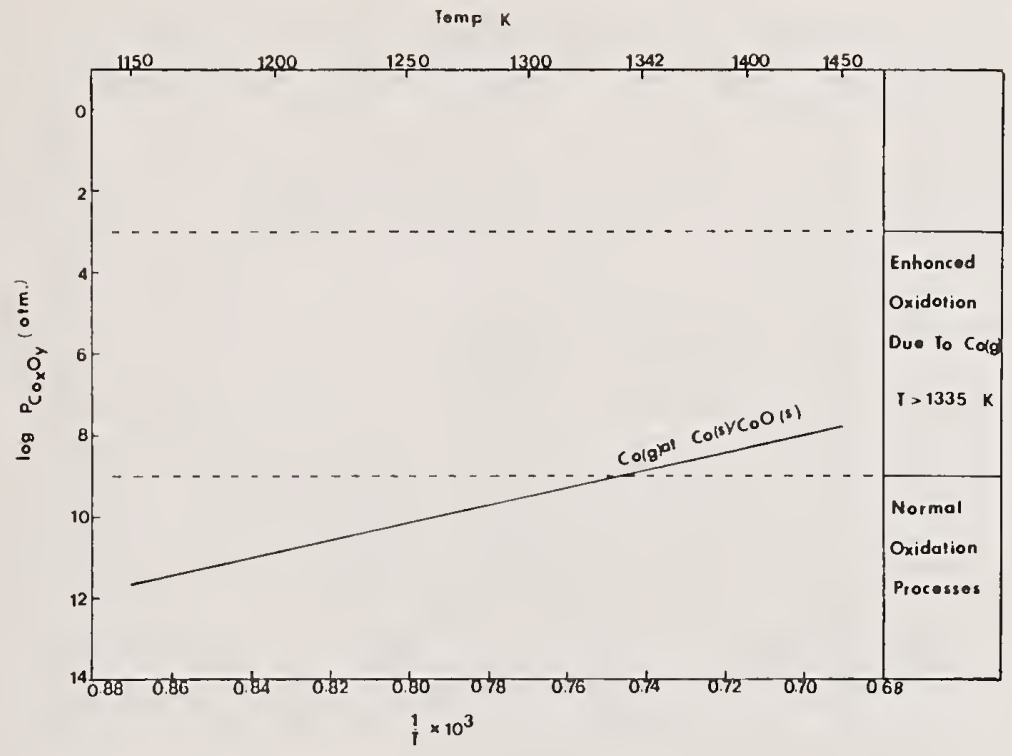

Figure 7. Co-0 system, volatile species vs temperature.

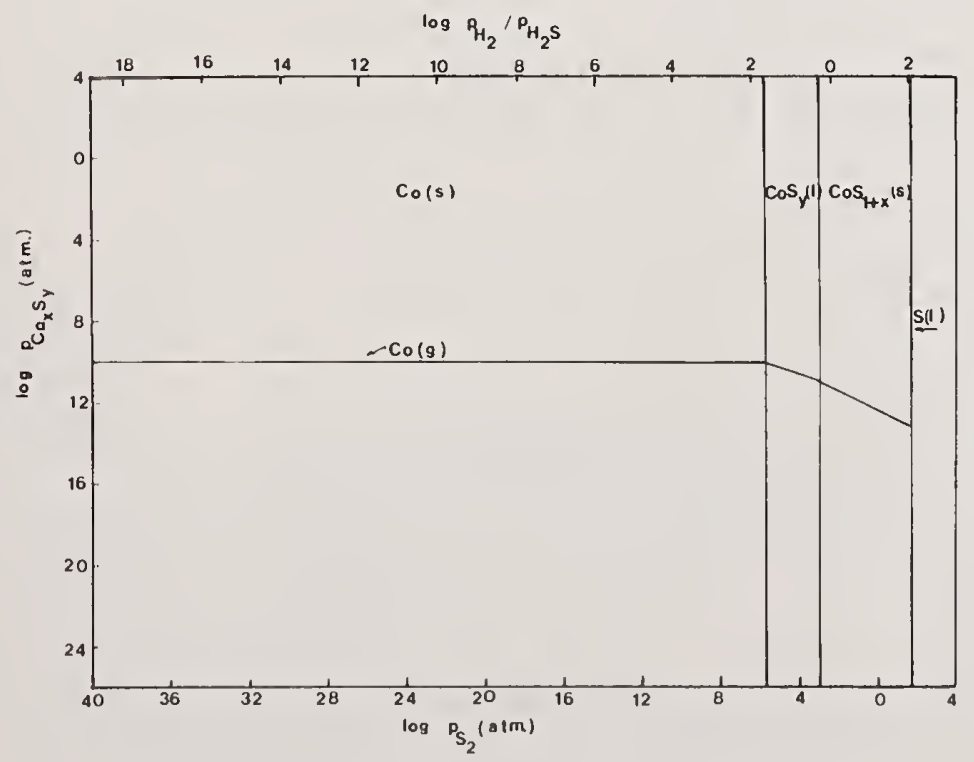

Figure 8. ColS system, volatile species, $1250 \mathrm{~K}$.

1663 


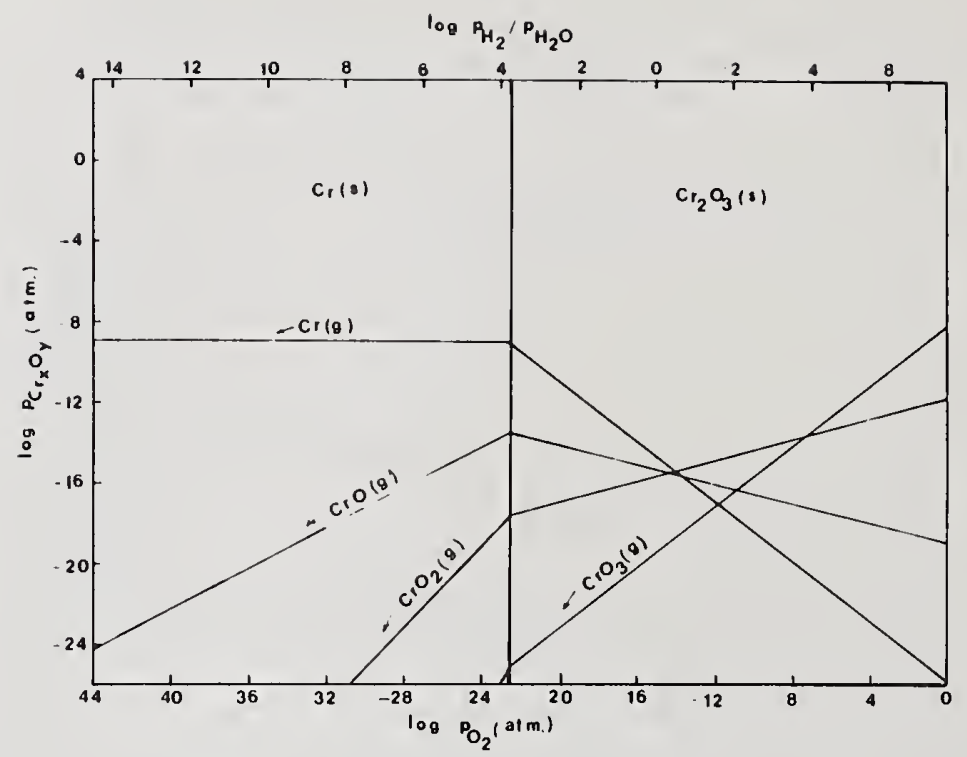

Figure 9. Cr-0 system, volatile species, $1250 \mathrm{~K}$.

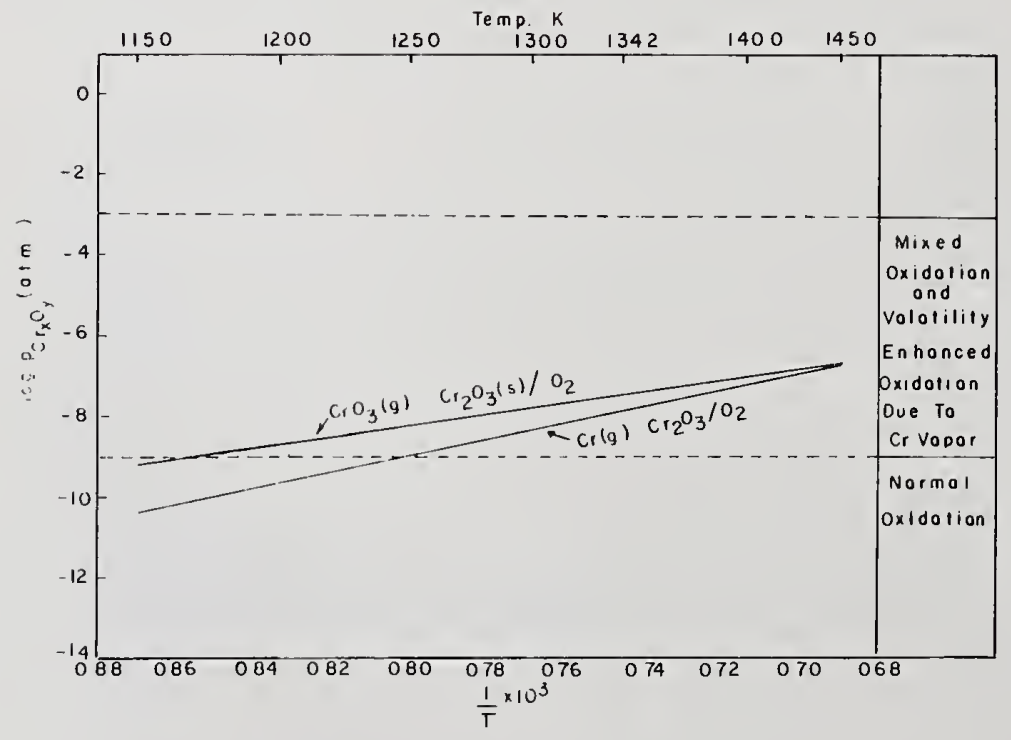

Figure 10. Cr-0 system, volatile species vs temperature. 


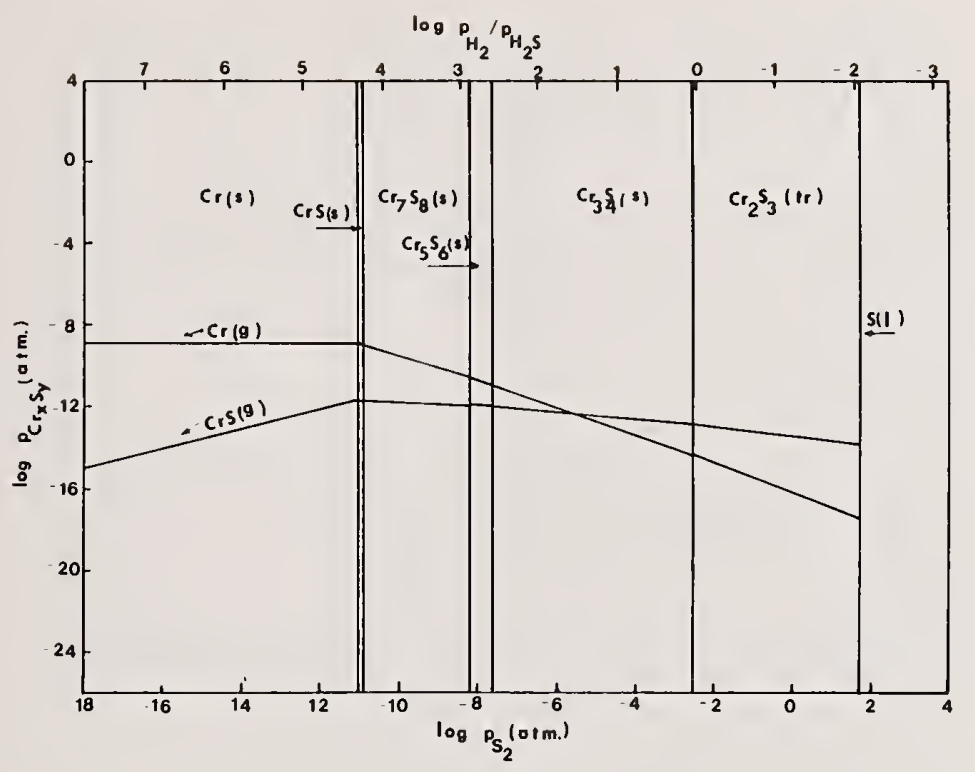

Figure 11. Cr-S system, volatile species, $1250 \mathrm{~K}$.

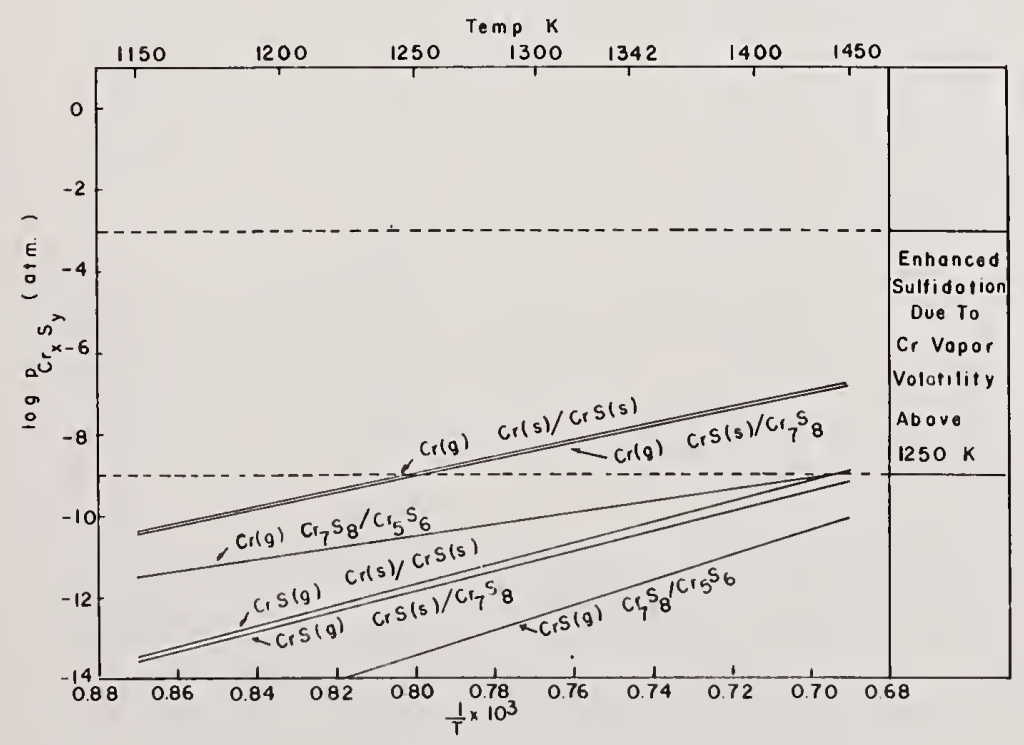

Figure 12. $\mathrm{Cr}_{\mathrm{S}} \mathrm{S}$ system, volatile species vs temperature. 


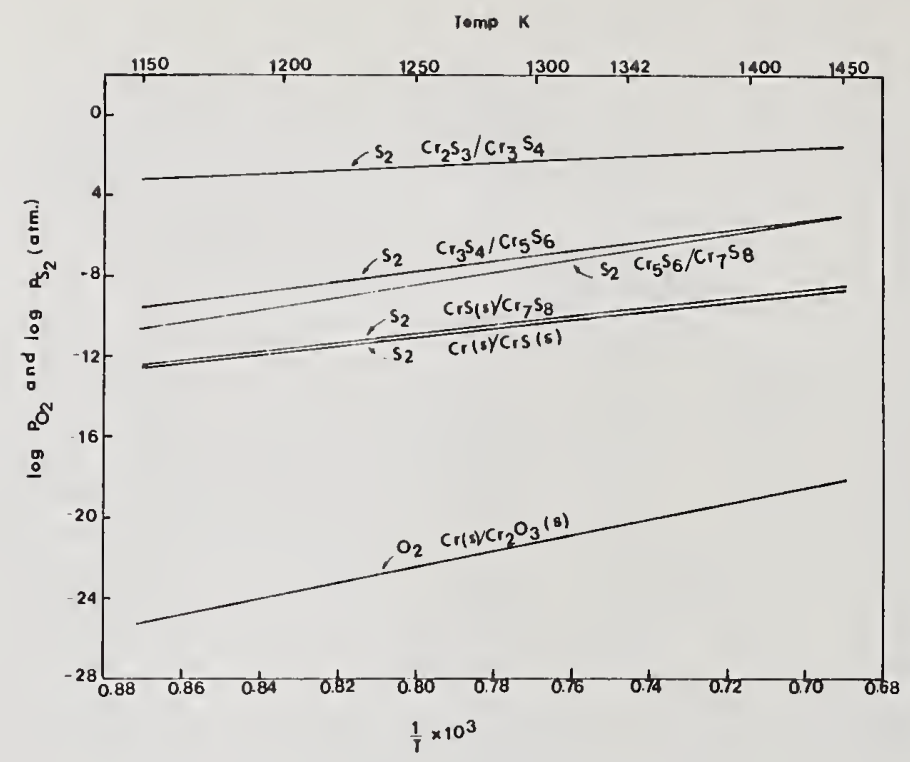

Figure 13. $\mathrm{Cr}-0$ and $\mathrm{Cr}-\mathrm{S}$ systems, $\mathrm{O}_{2}$ and $\mathrm{S}_{2}$ dissociation pressures.

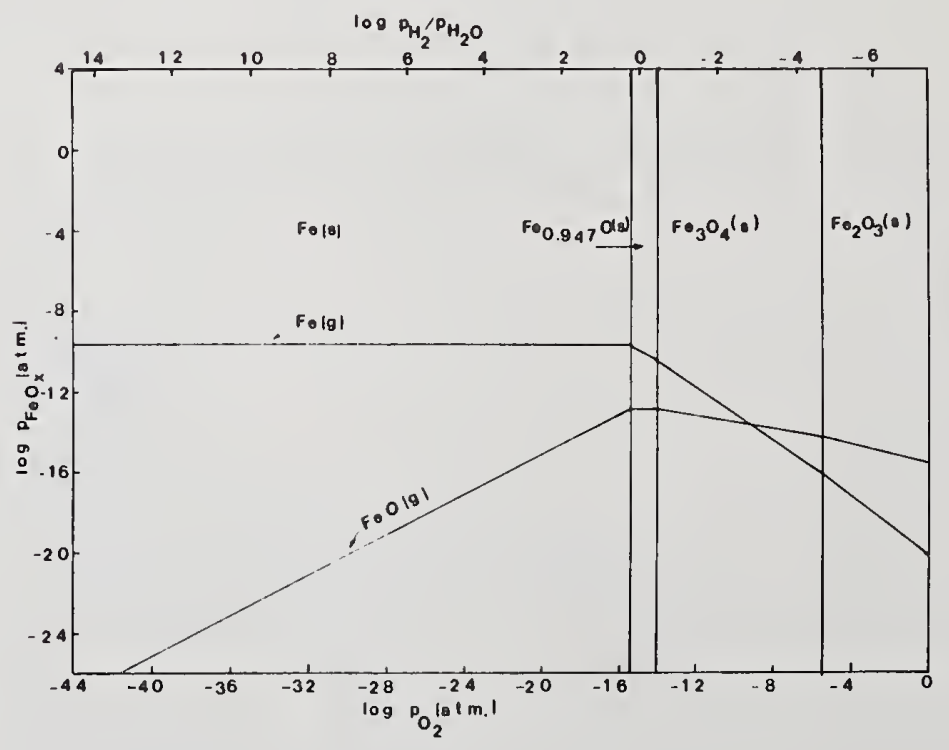

Figure 14. Fe-0 system, volatile species, $1250 \mathrm{~K}$. 


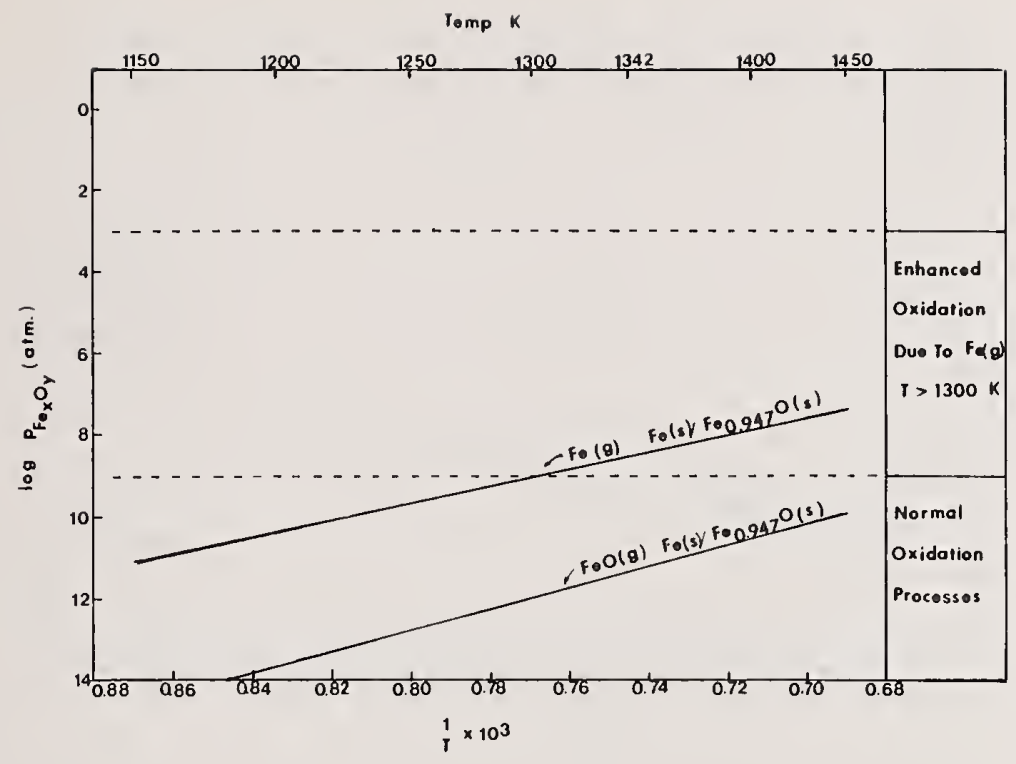

Figure 15. Fe-0 system, volatile species vs temperature.

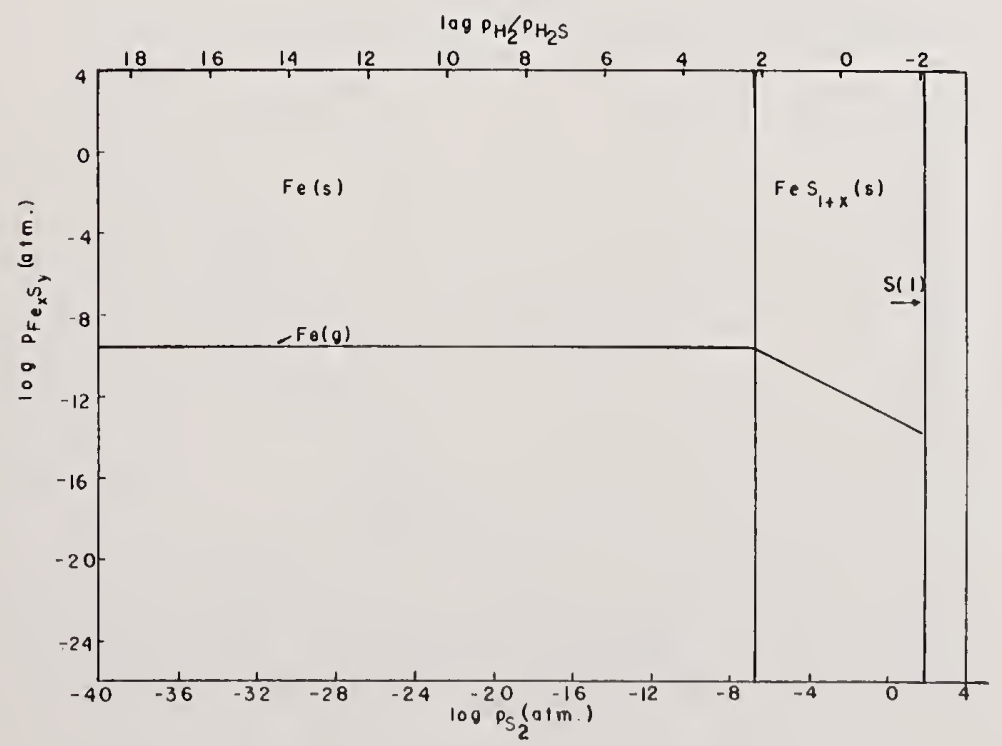

Figure 16. Fe-S system, volatile species, $1250 \mathrm{~K}$. 


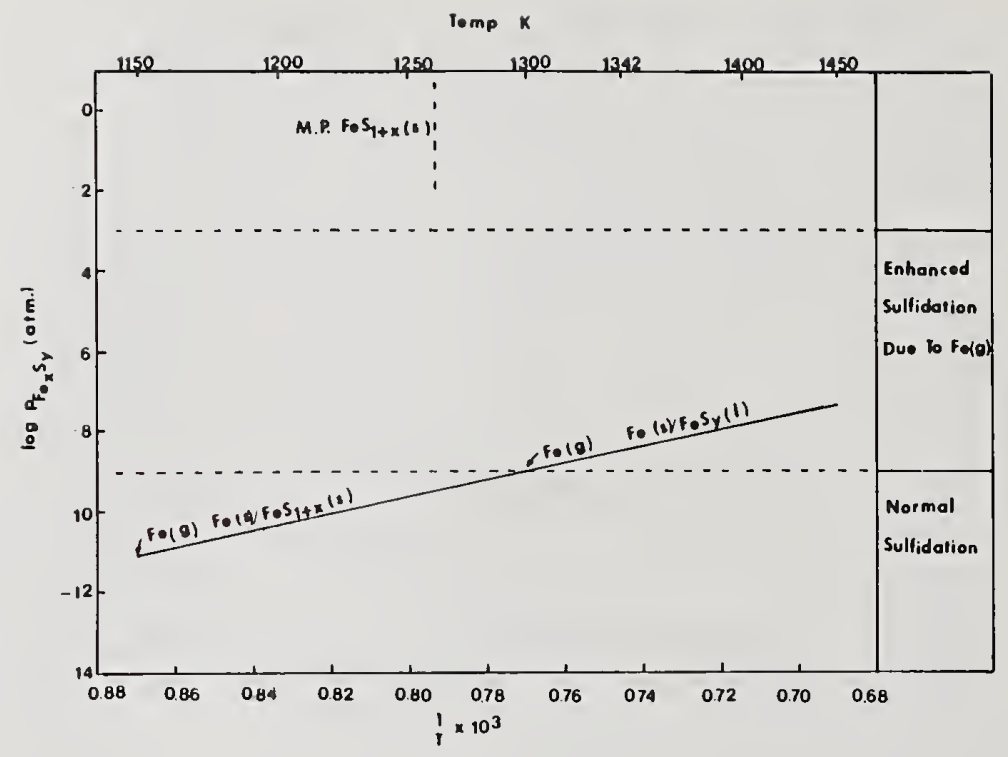

Figure 17. Fe-S system, volatile species vs temperature.

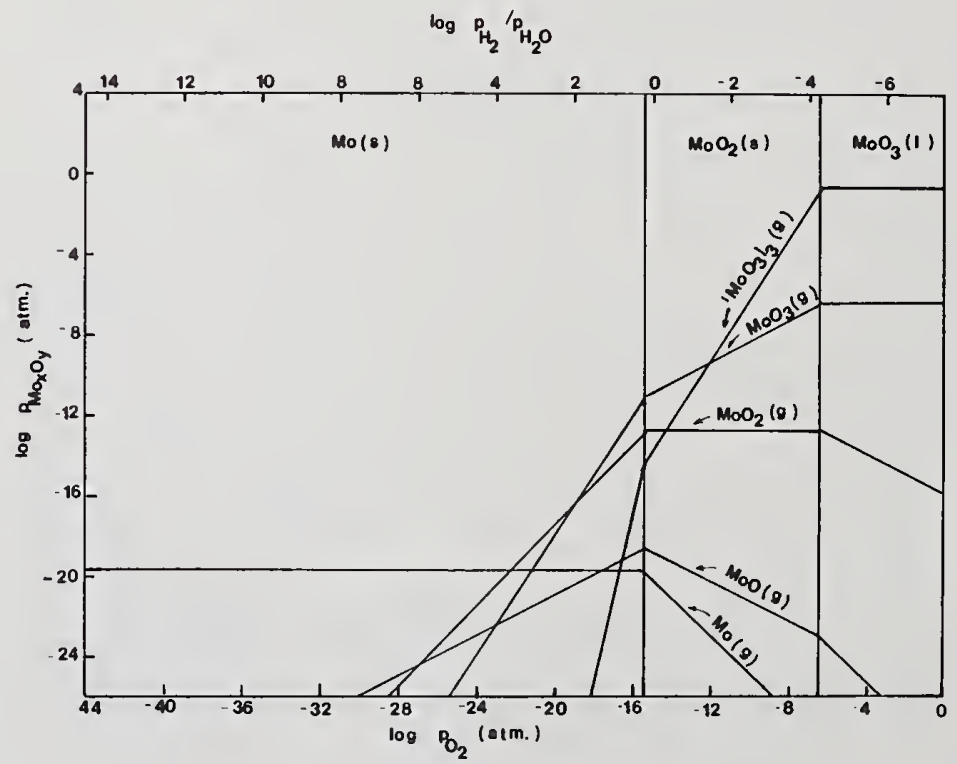

Figure 18. Mo-0 system, volatile species, $1250 \mathrm{~K}$. 


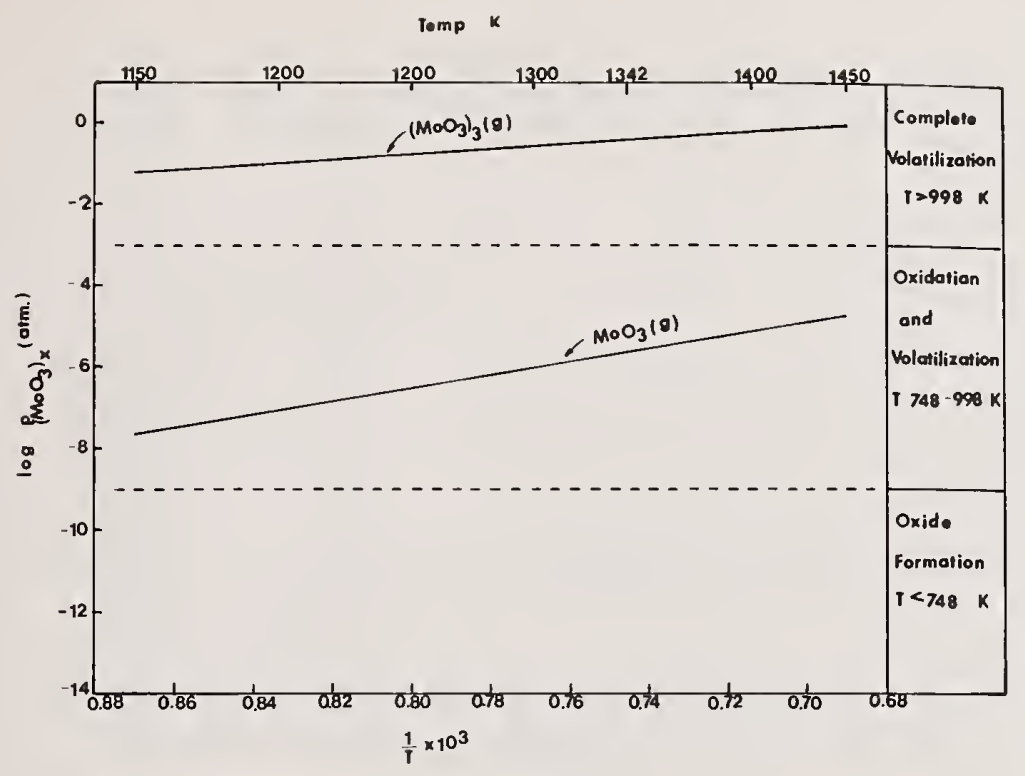

Figure 19. Mo-0 system, volatile species vs temperature.

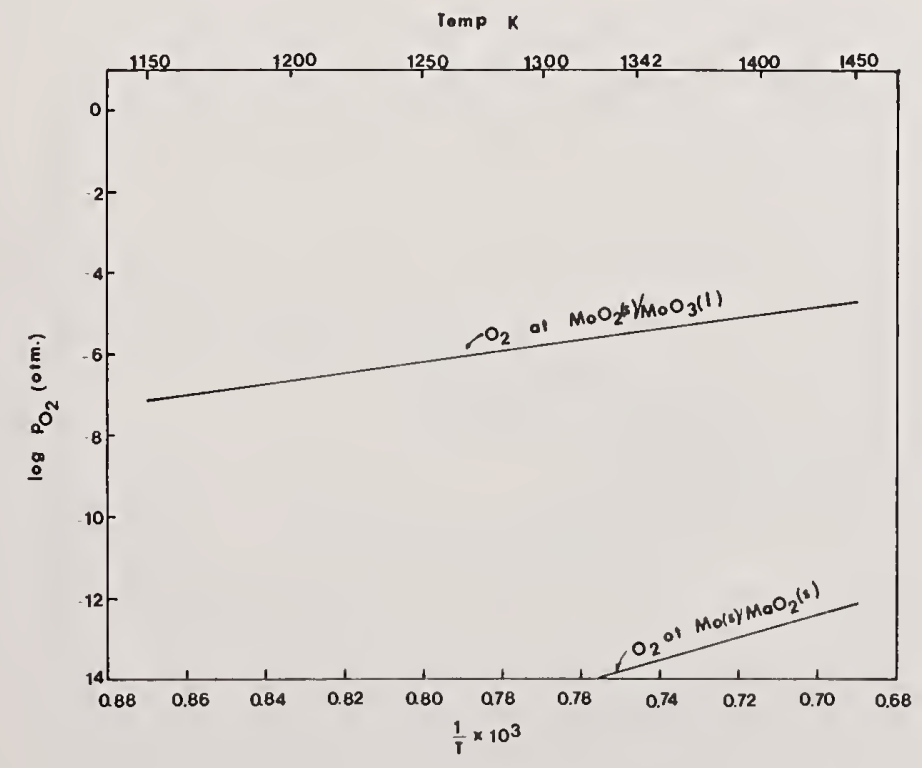

Figure 20. Mo-0 system, $0_{2}$ dissociation pressures. 


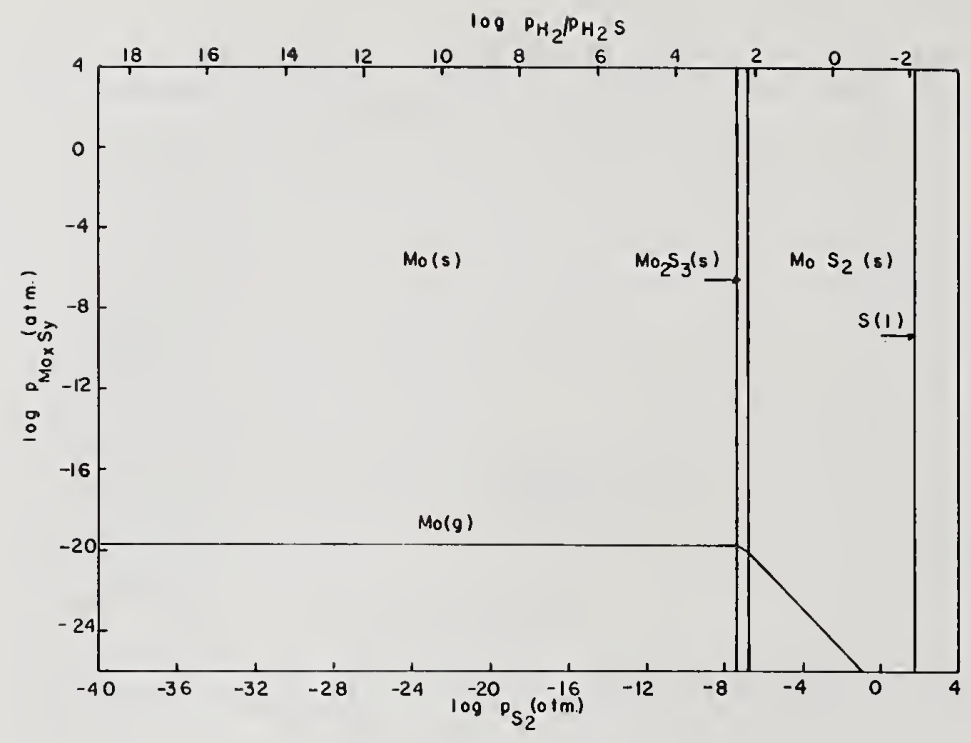

Figure 21. Mo-S system, volatile species, $1250 \mathrm{~K}$.

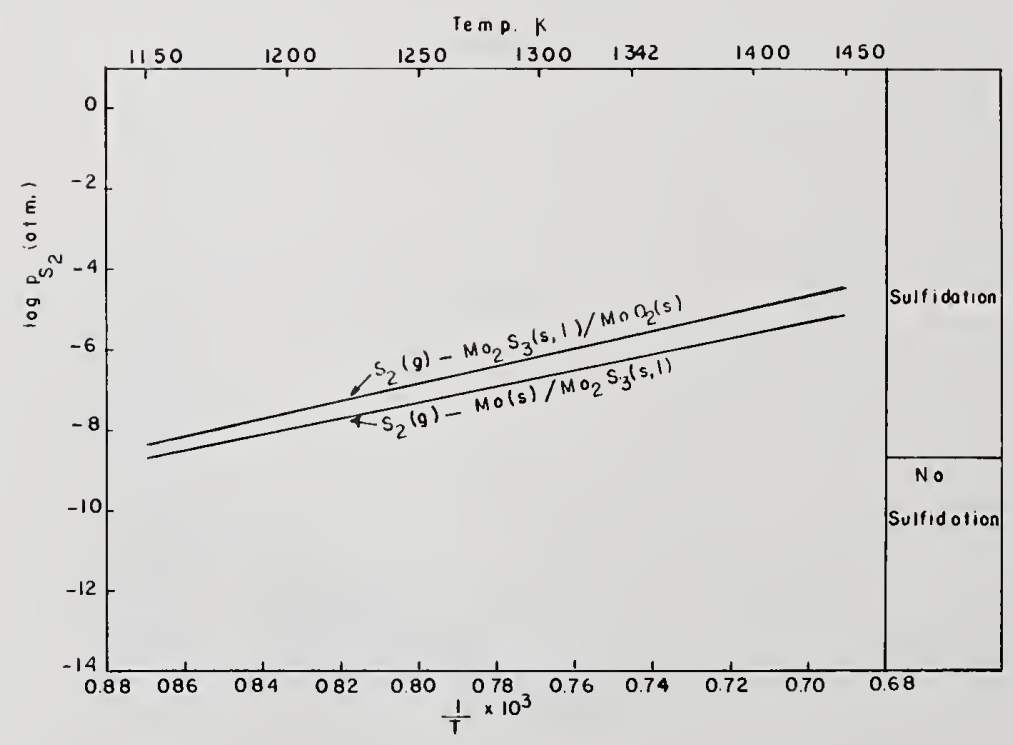

Figure 22. Mo-S system, $S_{2}$ dissociation pressures. 


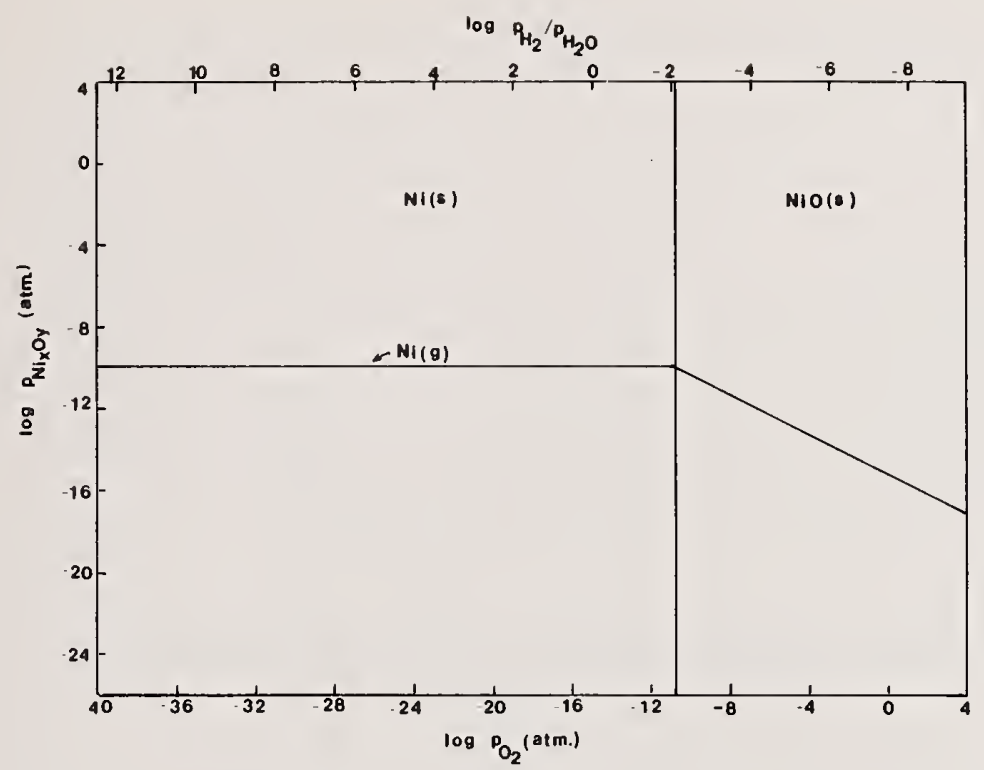

Figure 23. Ni-0 system, volatile species, $1250 \mathrm{~K}$.

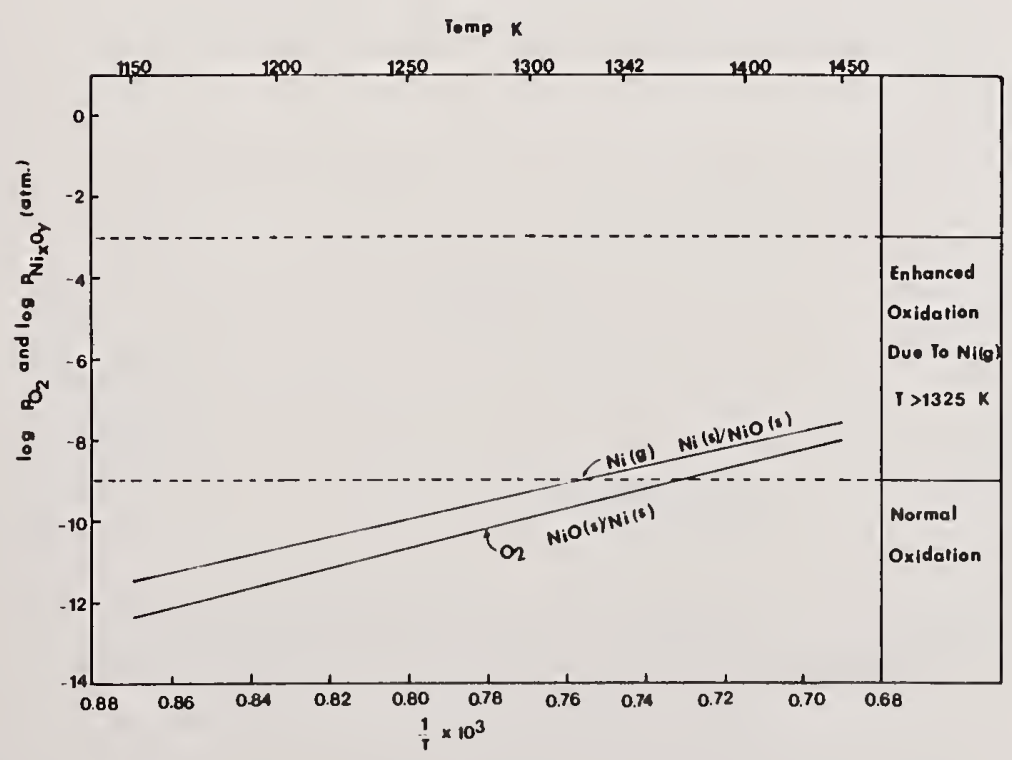

Figure 24. Ni-0 system, volatile species and $0_{2}$ dissociation pressures. 


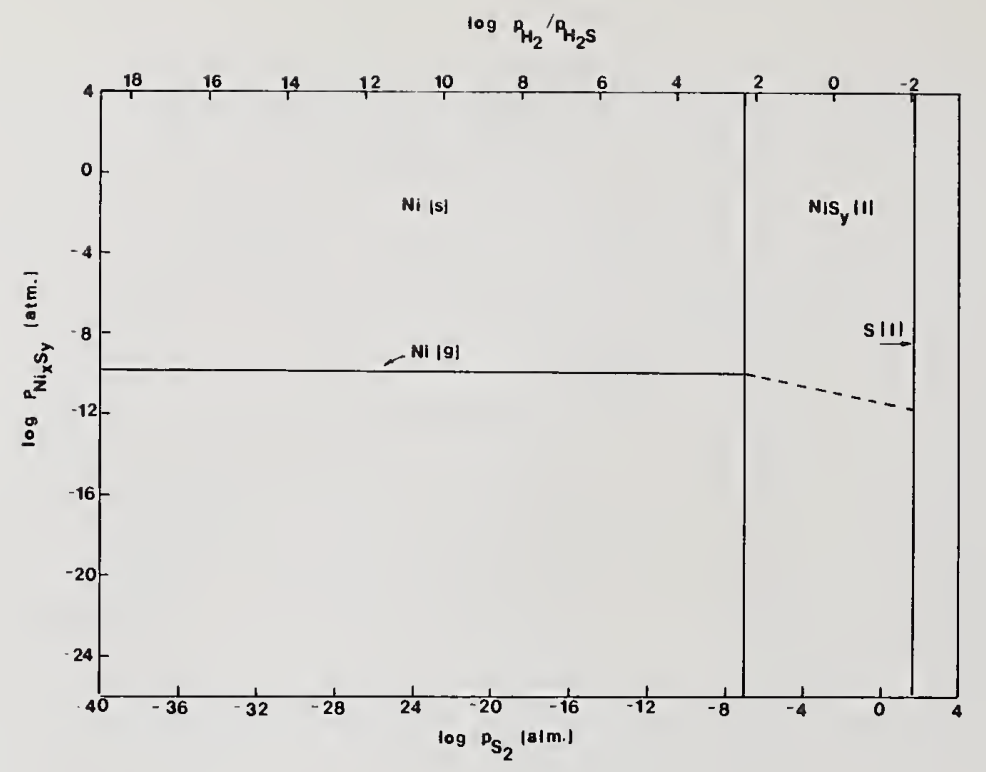

Figure 25. Ni-S system, volatile species, $1250 \mathrm{~K}$.

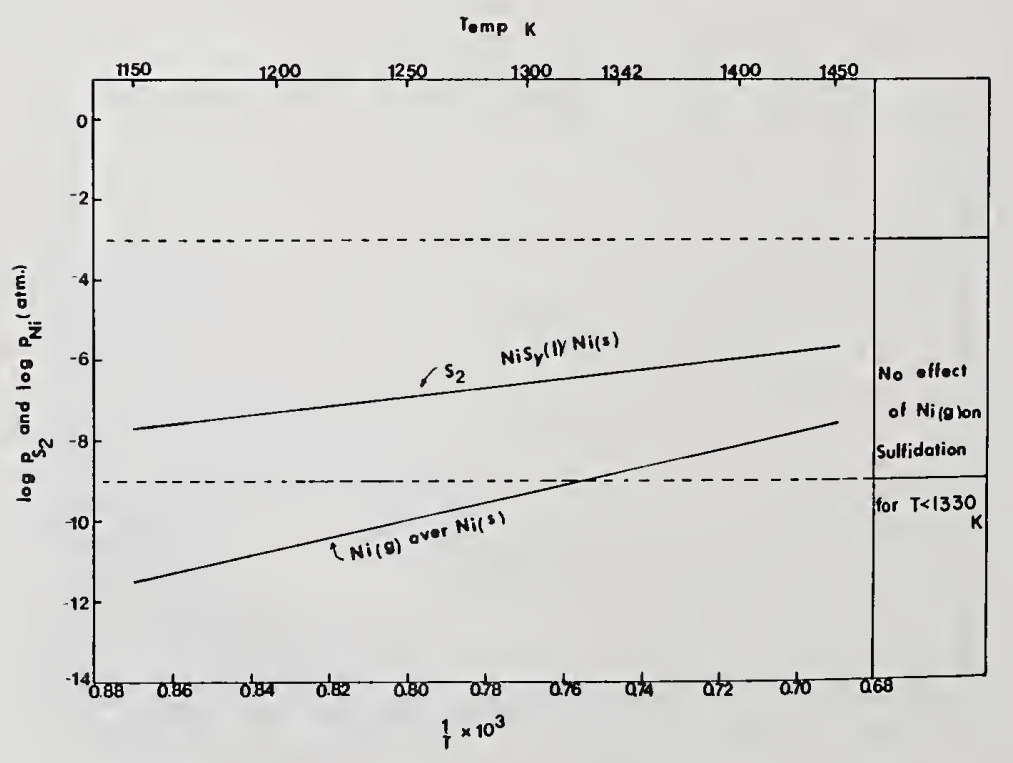

Figure 26. $\mathrm{Ni}-\mathrm{S}$ system, $\mathrm{S}_{2}$ dissociation pressures and volatile species. 


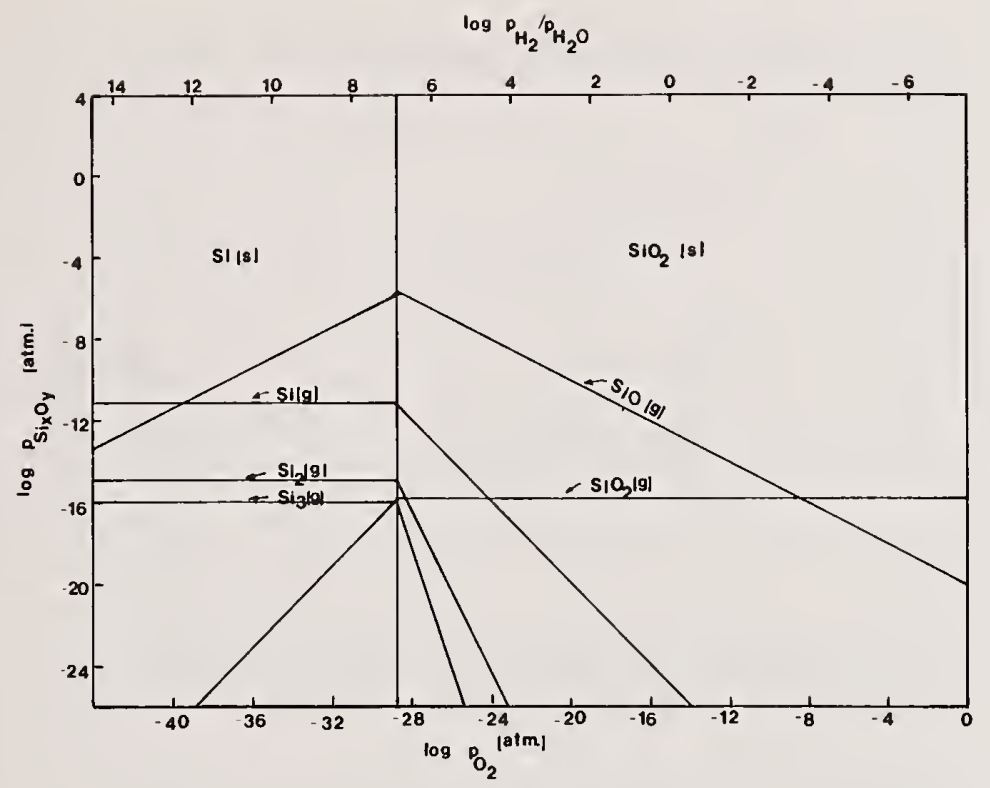

Figure 27. Si-0 system, volatile species, $1250 \mathrm{~K}$.

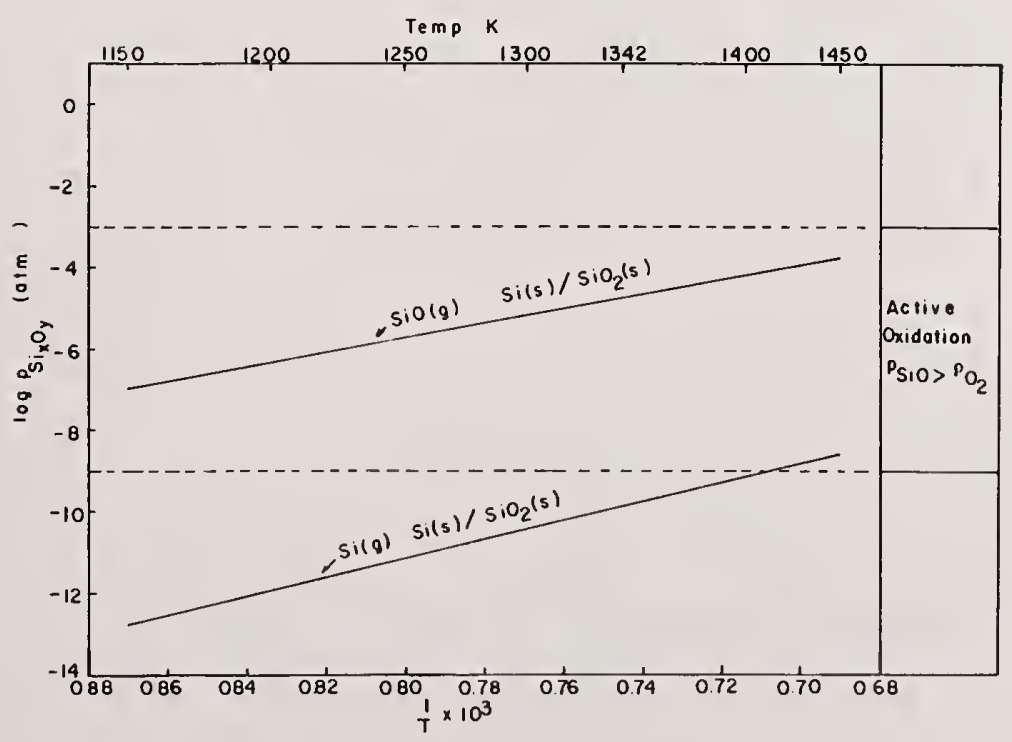

Figure 28. Si-0 system, volatile species vs temperature. 


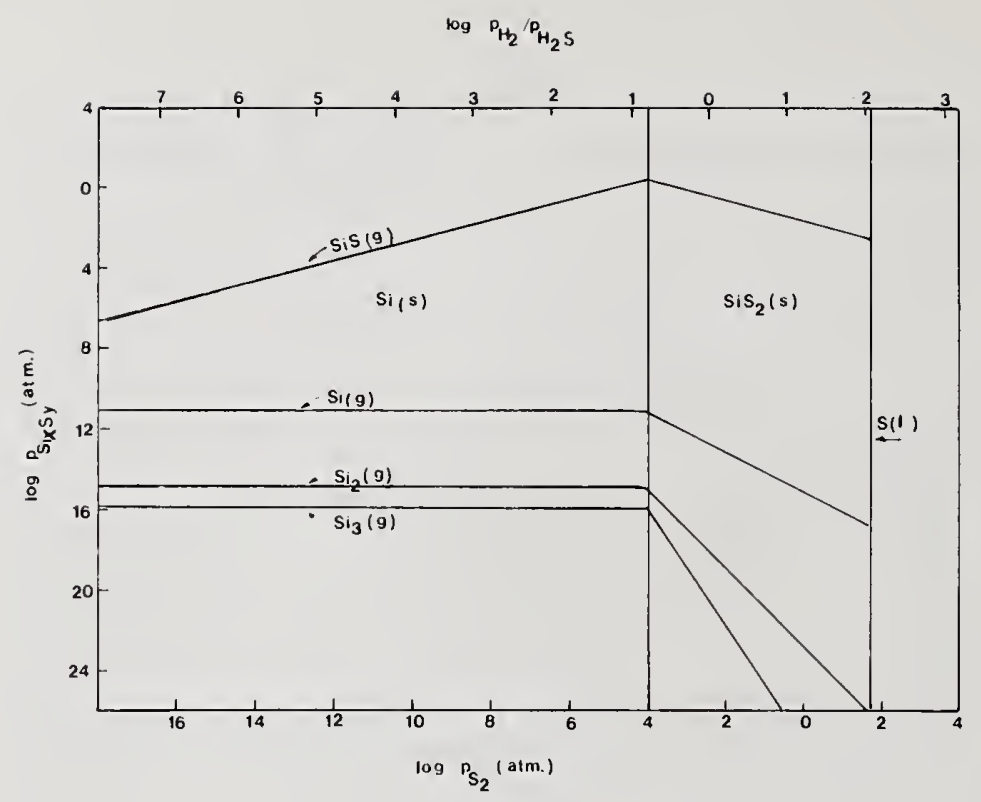

Figure 29. Si-S system, volatile species, $1250 \mathrm{~K}$.

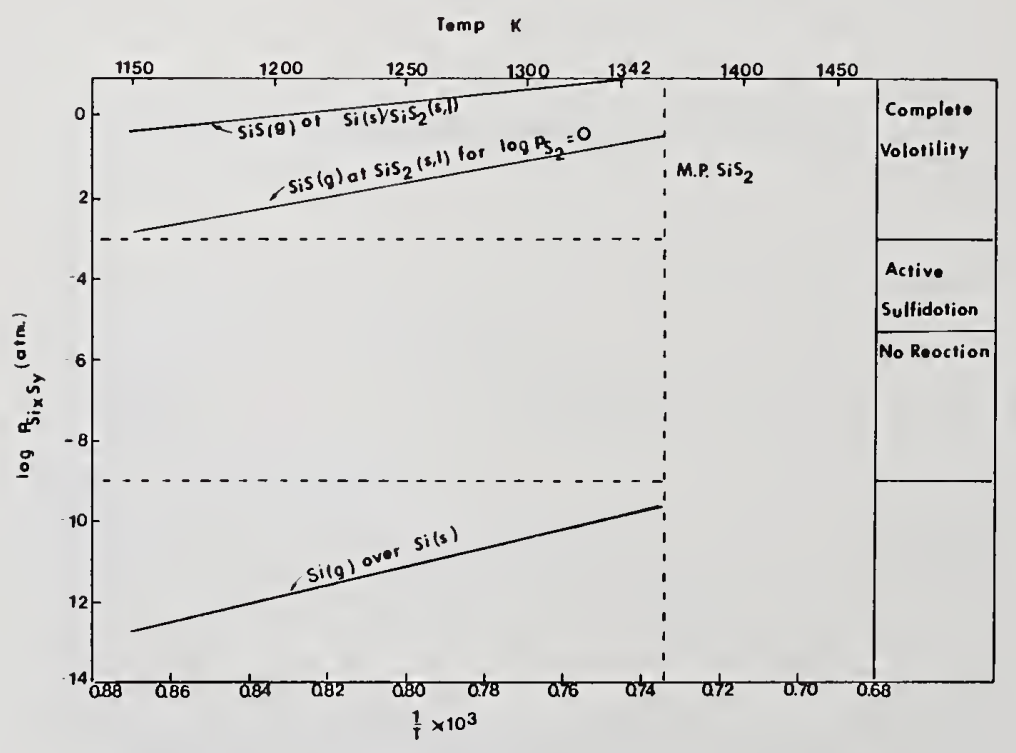

Figure $30 . \quad$ Si-S system, volatile species vs temperature. 


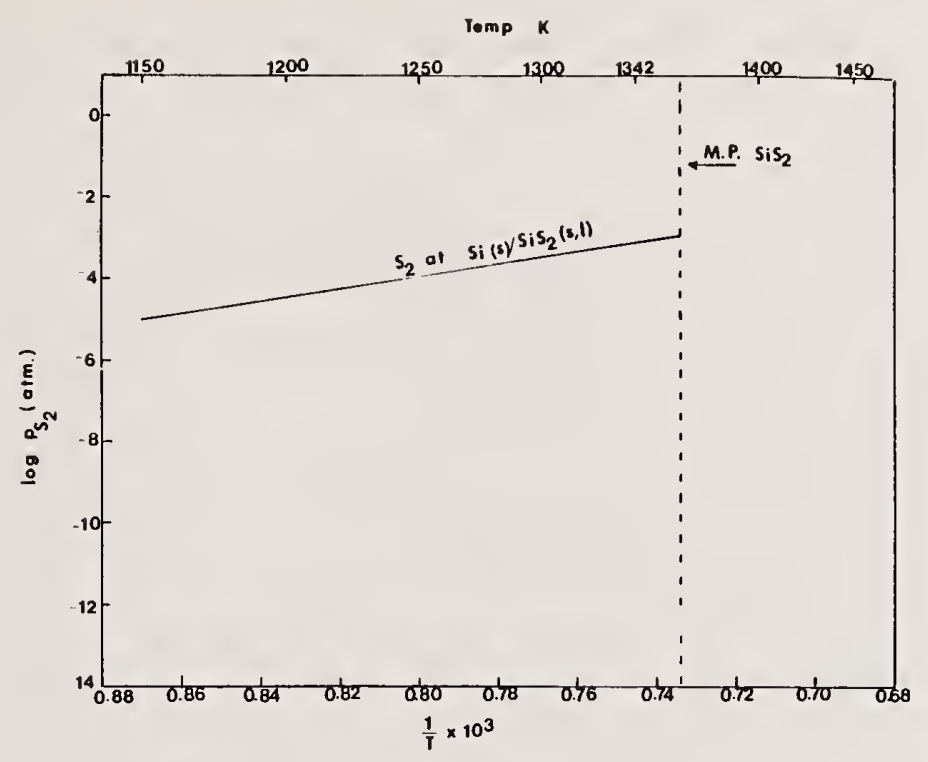

Figure 31 . Si-S system, $S_{2}$ dissociation pressures.

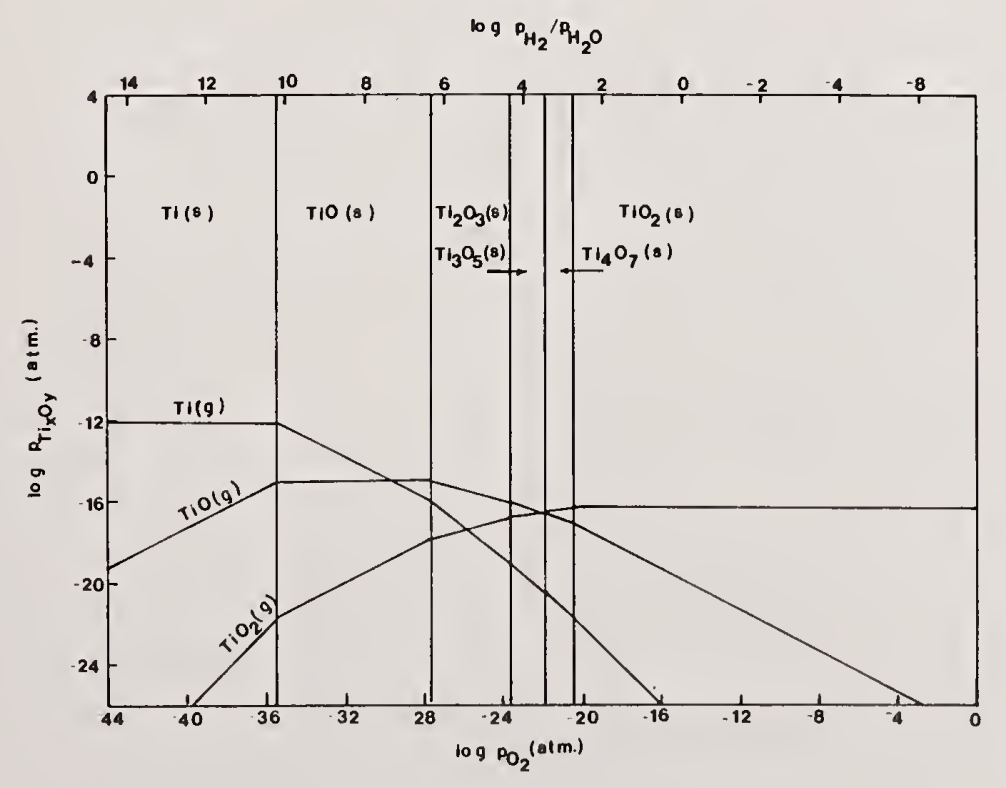

Figure 32 . Ti-0 system, volatile species, $1250 \mathrm{~K}$. 


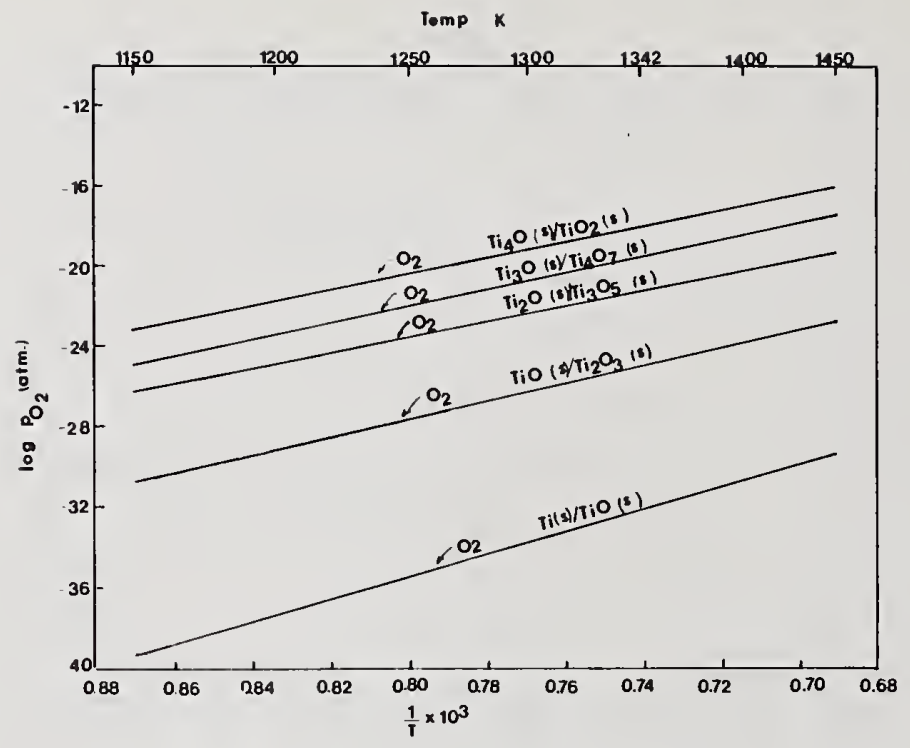

Figure 33. Ti-0 system, $0_{2}$ dissociation pressures.

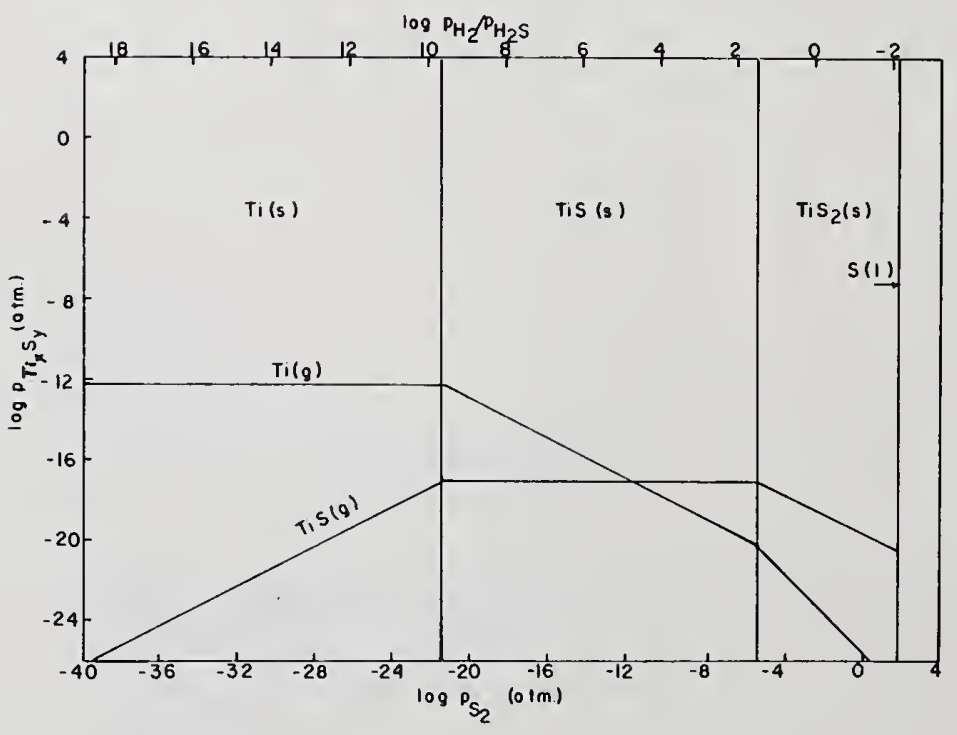

Figure 34 . Ti-S system, volatile species, $1250 \mathrm{~K}$. 


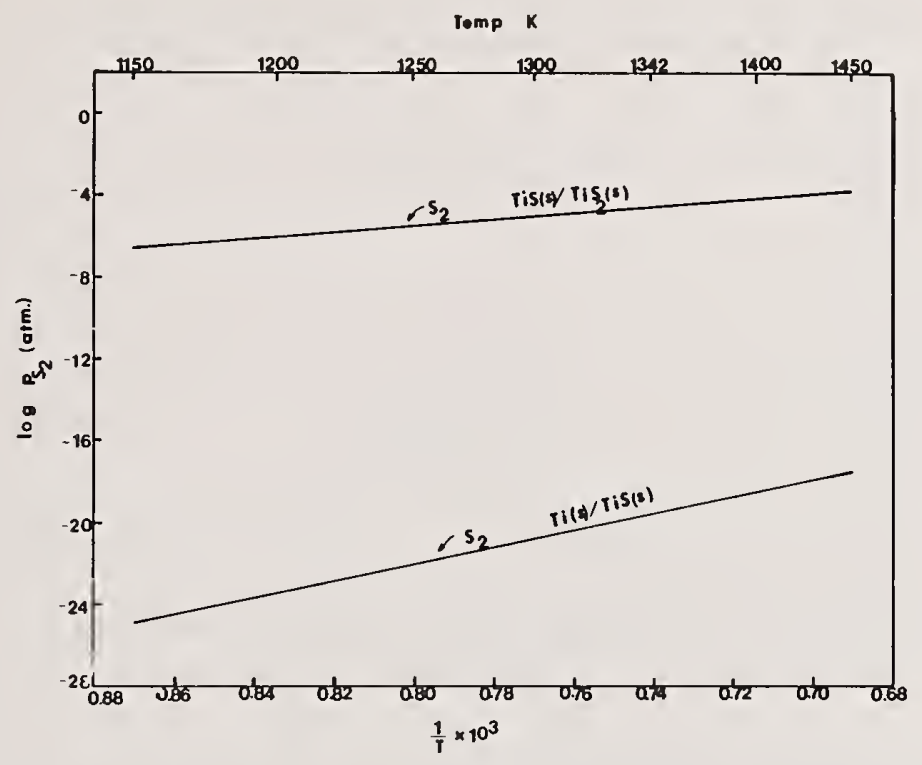

Figure 35 . Ti-S system, $S_{2}$ dissociation pressures.

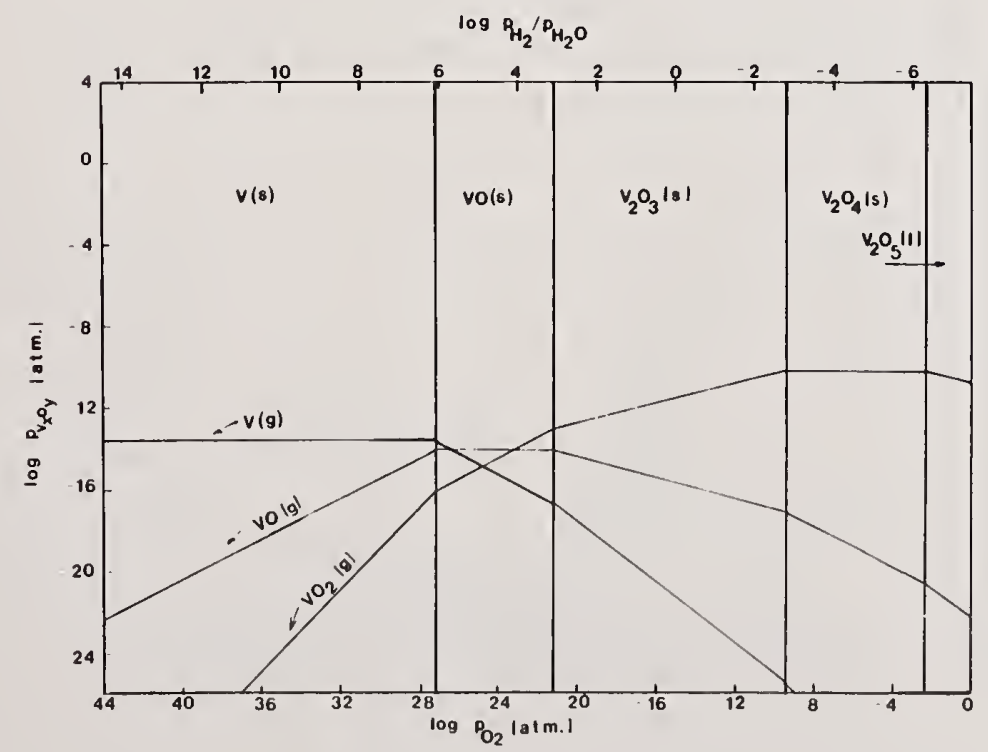

Figure $36 . \quad$ V-0 system, volatile species, $1250 \mathrm{~K}$. 


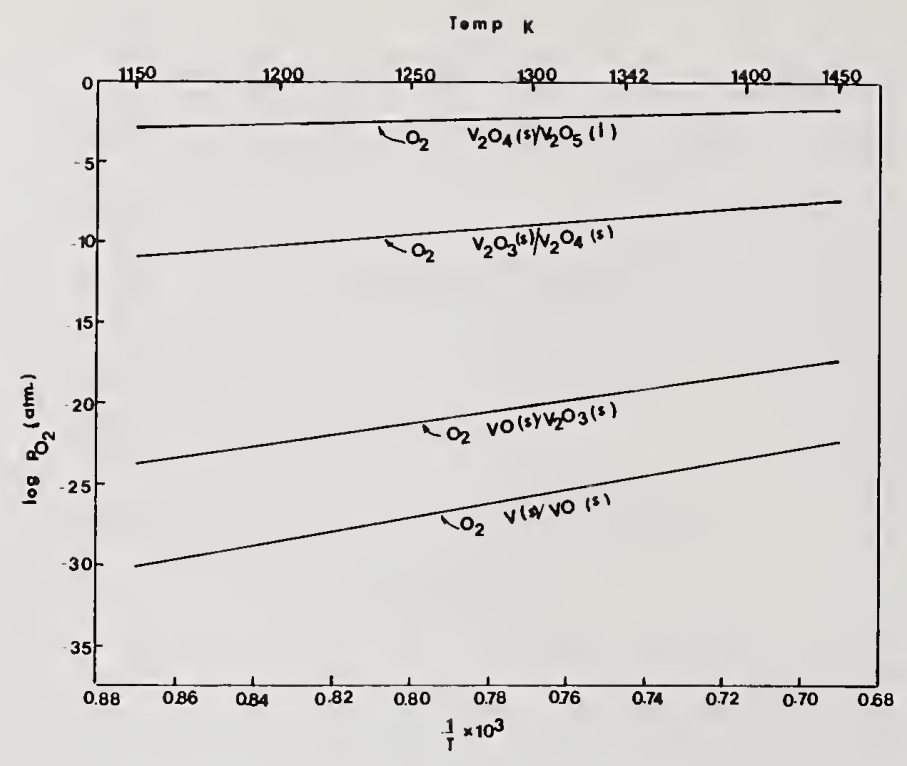

Figure 37 V-0 system, $0_{2}$ dissociation pressures.

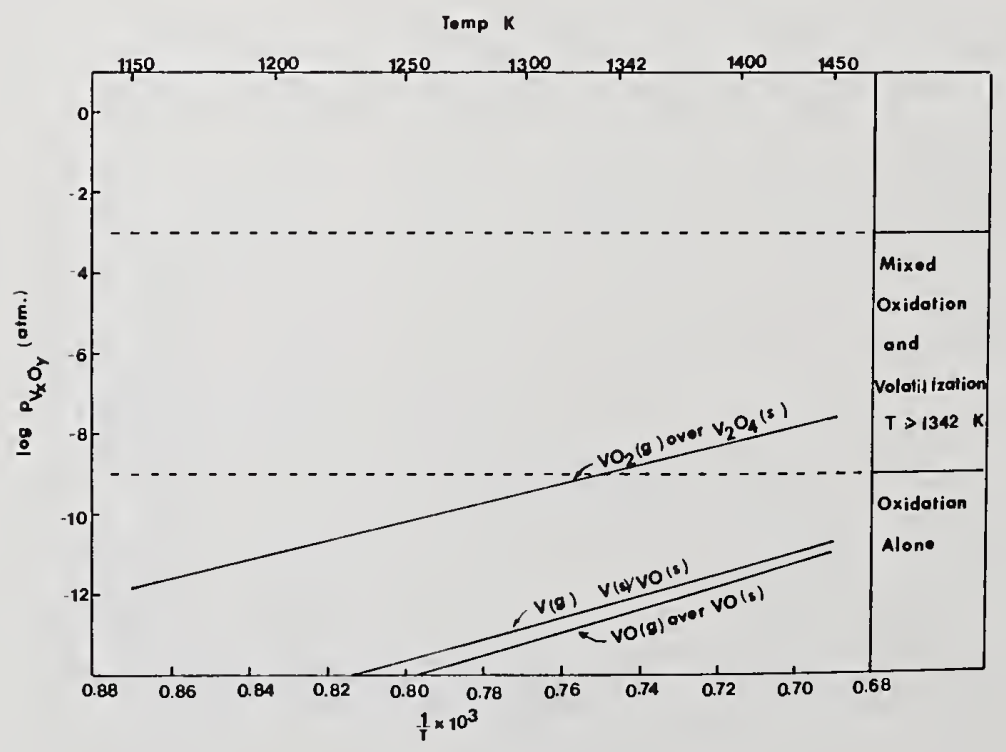

Figure 38. V-0 system, volatile species vs temperature. 


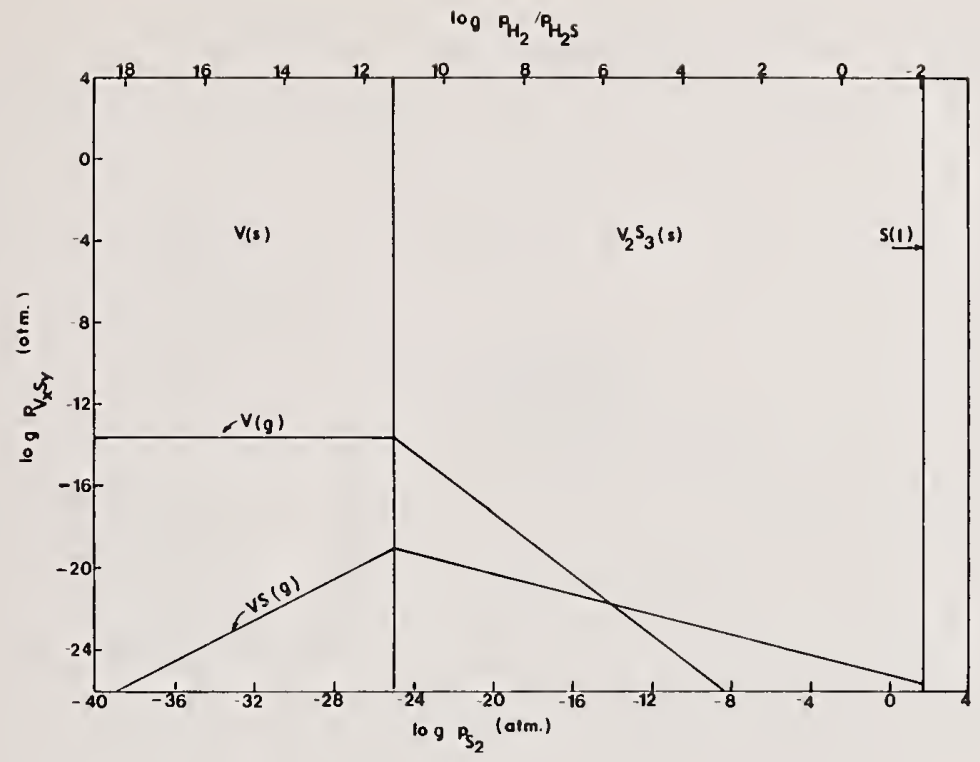

Figure 39. $V$-S system, volatile species, $1250 \mathrm{~K}$.

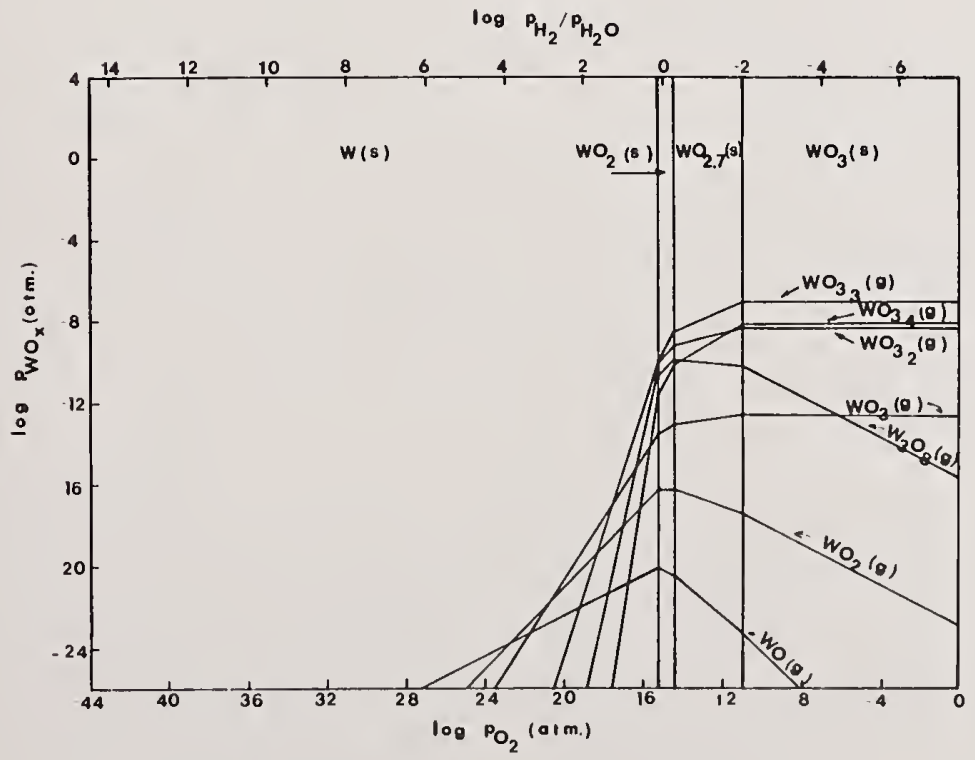

Figure 40. W-0 system, volatile species, $1250 \mathrm{~K}$. 


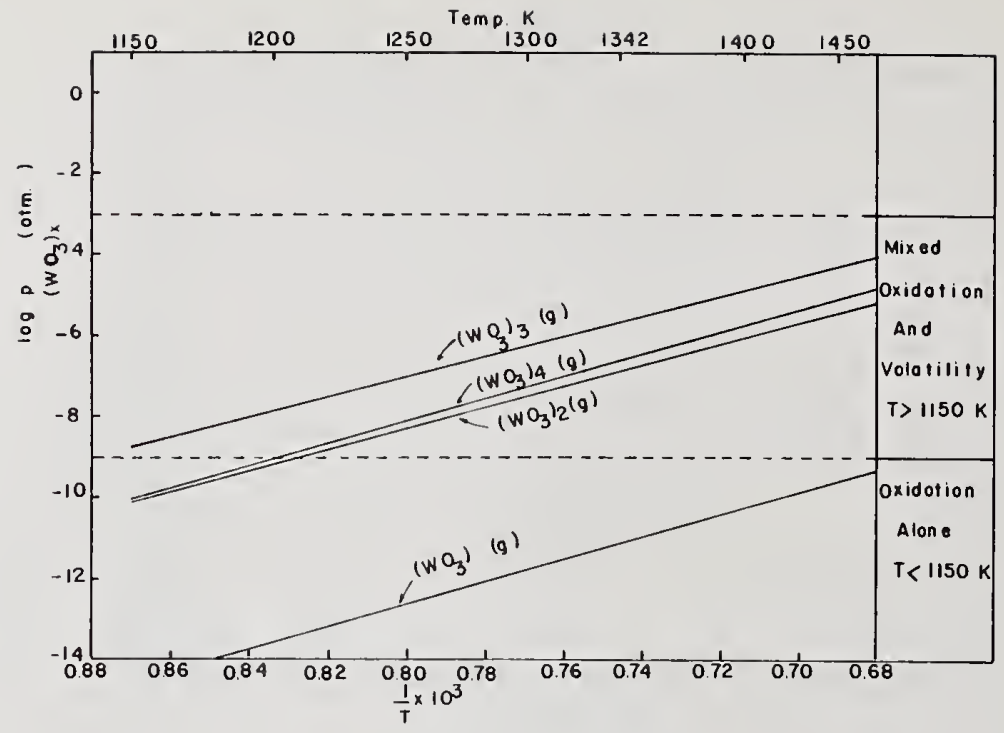

Figure 41 . W-0 system, volatile species vs temperature.

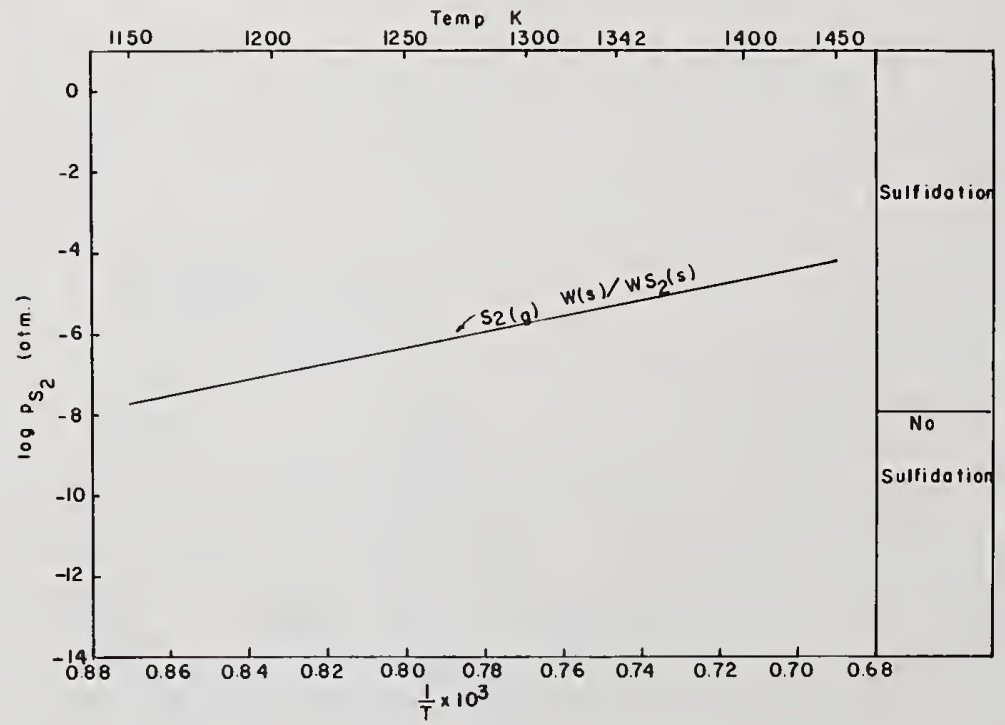

Figure 42. W-S system, $S_{2}$ dissociation pressures. 


\section{References}

[1] Gulbransen, E. A. and Jansson, S. A., Heterogeneous Kinetics at Elevated Temperatures, G. R. Belton and W. L. Worrel1, eds. (Plenum Press, New York, 1970).

[2] Gulbransen, E. A., Andrew, K. F., and Brassart, F. A., J. Electrochem. Soc. 110, 952 (1963).

[3] Gulbransen, E. A., Andrew, K. F., and Brassart, F. A., J. Electrochem. Soc. 113, 834 (1966).

[4] Gulbransen, E. A., Andrew, K. F., and Brassart, F. A., J. Electrochem. Soc. 110, 476 (1963).

[5] Gulbransen, E. A., Ind. and Eng. Chem. 44, 1045 (1952).

[6] JANAF Thermochemical Tables, NSRDS-NBS 37, U.S. Government Printing Office, Washington, DC (1971).

[7] Mills, K. C., Thermodynamic Data for Inorganic Sulfides, Selenides and Tellurides (Butterworths, London, 1974).

[8] Stearn, K. H. and Weise, E. L., NSDRS-NBS 7, U.S. Government Printing Office, Washington, DC (October 1, 1966).

[9] Shick, H., Thermodyanmics of Certain Refractory Compounds, Vol. 2 (Academic Press, New York and London, 1966).

[10] Barin, I. and Knacke, 0., Thermochemical Properties of Inorganic Substances, (Springer-Verlag, Berlin, Heidelberg, New York, 1973).

[11] JANAF Thermochemical Tables, 1974 Supplement, Reprint No. 50 from J. of Phys. and Chem. Ref. Data, 3 , 311-480 (1974).

[12] Rosenquist, J., J. Iron and Steel Inst. 176, 37 (1954).

[13] Wicks, C. E. and Block, F. E., Bulletin 605, Bureau of Mines, U.S. Government Printing Office, Washington, DC (1963).

[14] Fryburg, G. C., Koh1, F. J., and Stearns, C. A., J. Electrochem. Soc. 121, 952 (1974).

[15] Hager, J. P. and E11iott, J. F., Trans. AIME, 239, 513 (1967).

[16] Nishida, K., Nakayama, K., and Narita, T., Corr. Sci. 13, 759 (1973).

[17] JANAF Thermochemical Tables, 1975 Supplement, Reprint No. 60 from J. of Phys. and Chem. Ref. Data, 4, 1-175, (1975).

[18] Gulbransen, E. A., Andrew, K. F., and Brassart, F. A., J. Electrochem. Soc. 110, 242 (1963).

[19] Ingraham, J. R. and Holtz, M. C. B., Canadian Met. Quarterly, 7, 139 (1968).

[20] Warner, J. S., Dr. Eng. Sc. Dissertation, Columbia University, New York, NY.

[21] Stu11, D. R. and Sinke, G., Advances in Chemistry Series, No. 18, American Chemical Society, Washington, DC (1956).

[22] Kellogg, H. H., AIME, 230, 1622 (1964).

[23] Shatynski, S. R., Oxidation of Metals, 11, 307-319 (1977).

[24] Shunk, F. A., Constitution of Binary Alloys, Second Supplement (McGraw-Hill, New York, 1969). 
[25] Hansen, M. and Anderko, K., Constitution of Binary Alloys, Second Edition (McGrawHi11, New York, 1958).

[26] Popma, J. A. and van Bruggen, C. E., J. Inorg. and Nucl. Chem. 31, 73 (1969).

[27] Zaplatynsky, I. , Oxidation of Metals, 11, 289-305 (1977).

[28] Elliott, R. P., Constitution of Binary Alloys, First Supplement (McGraw-Hill, New York, 1965).

[29] Wagner, C., J. Appl. Phys. 29, 1295 (1962).

\section{Discussion}

Moderator (Blander): What are the sources of your data for these calculations?

Response (Gulbransen): There were four or five different sources (see reference citations). But that is the big problem, to find good data for the sulfide systems. We need a lot more work on not only condensed phases, but the volatile species. I think it would be a pay-off of great dividends in making predictions of what we think we can do, and not only avoid making useless experiments, but we can also predict which are the better experiments to make and help interpret the mechanisms of reaction. So, that is why we go through this analysis. So, we spent a lot of time bringing all this together. 
National Bureau of Standards Special Publication 561, Proceedings of the 10th Materials Research Symposium on Characterization of High Temperature Vapors and Gases held at NBS, Gaithersburg, Maryland, September 18-22, 1978. Issued October 1979.

\title{
POTASSIUM SEED ACTIVITIES IN OPEN CYCLE MHD SYSTEMS: PREDICTIONS USING A MULTIPHASE MULTICOMPONENT SOLUTION MODEL ${ }^{1}$
}

\author{
Chen C. Hsu and Carl E. Johnson \\ Chemical Engineering Division \\ Argonne National Laboratory \\ 9700 South Cass Avenue \\ Argonne, IL 60439
}

Although the importance of seed recovery in the open cycle coal fired MHD system has been established, complete understanding of the complex chemistry existing at high temperature between potassium seed and coal-ash constituents is still lacking. A multiphase, multicomponent solution model was developed by modifying the widely used NASA computer program for calculation of complex chemical equilibrium compositions to include a subroutine that calculates solution formation from the condensed phase species. This model has been used to predict the potassium activity in the $\mathrm{K}_{2} \mathrm{O}+\mathrm{SiO}_{2}, \mathrm{~K}_{2} \mathrm{O}+\mathrm{SiO}_{2}+$ $\mathrm{Al}_{2} \mathrm{O}_{3}, \mathrm{~K}_{2} \mathrm{O}+\mathrm{SiO}_{2}+\mathrm{MgO}$, and $\mathrm{K}_{2} \mathrm{O}+\mathrm{SiO}_{2}+\mathrm{CaO}$ systems over the temperature range of 2300 to $1200 \mathrm{~K}$, in which condensed phase components (i.e., $\mathrm{K}_{2} \mathrm{SiO}_{3}, \mathrm{~K}_{2} \mathrm{Si}_{2} \mathrm{O}_{5}$....) were assumed to form an ideal solution. Calculations showed that the addition of $\mathrm{Al}_{2} \mathrm{O}_{3}, \mathrm{CaO}$, or $\mathrm{MgO}$ to the $\mathrm{K}_{2} \mathrm{O}+\mathrm{SiO}_{2}$ binary system resulted in an increase in potassium activity in the gas phase, especially for CaO. Such information improves our predictive capability and may be used in the selection of materials and in establishing optimum conditions for operation of the combustorgenerator high temperature air heater components of an open cycle MHD system. Further, the predictive capability of the program can be used to assess other, more suitable additives and in determining operating conditions for improved potassium seed recovery.

\section{Introduction}

Plant cost analyses $[1]^{2}$ indicate that at least 95 percent of the potassium seed added to an open cycle MHD system must be recovered for MHD systems to be economically

\footnotetext{
${ }^{1}$ Work performed under the auspices of the U.S. Department of Energy.

${ }^{2}$ Figures in brackets indicate the literature references at the end of this paper.
} 
feasible. There are four possible processes by which the seed may be lost in the open cycle MHD system: ( 1 ) the potassium-containing vapor species ( $\mathrm{K}, \mathrm{KCl}, \mathrm{KOH}, \mathrm{KO}, \mathrm{K}_{2} \mathrm{SO}_{4}$ ) may react with slag (in vapor or particulate form) to form seed-slag particles or a deposited film; (2) the seed may be absorbed by the slag film, especially at temperatures below $2000 \mathrm{~K}$, and become irrecoverable because of chemical reaction between the potassium and the slag; (3) the seed absorbed by the slag film may diffuse through the slag layer and react with the ceramic lining and metallic structural materials; and (4) the seed may react or be entrained with particles carried out with the effluent gases. Thus, it becomes apparent that the potassium loss is due to extremely complex seed-slag interactions with the potential for forming a multicomponent solution of seed and slag constituents. Because of difficulties encountered in laboratory experiments to measure the physical and chemical properties of the solution formed from seed and slag [2], computer simulation to predict seed/slag properties becomes a likely alternative.

Various estimates, based on meager data, have been made of the equilibrium concentration of potassium in slag. Although the results of several calculations are not in general agreement, the calculations illustrate the importance of eliminating or separating slag from the high temperature exhaust gas to minimize interaction of seed and slag. Raask [3] estimated that for a coal containing 20 percent ash and a gas containing 0.7 wt percent $\mathrm{K}$, the seed loss would reach 22 percent at $1900 \mathrm{~K}$. Spencer, Hendrie, and Bienstock [4] developed a Margules-type calculational model to predict the properties of the ternary potassia-silica-alumina system. Although activity coefficients were incorporated and the improvement over their ideal solution model [5] was demonstrated, their prediction of the seed loss to the slag in the MHD environment is still greater than that of experiment [6] (50 percent seed loss at $1700 \mathrm{~K}$ ). If 80 percent of the slag is removed by a cyclone combustor, their calculations show a maximum of 10 percent of the potassium trapped. Similar calculations by Blackburn [7] show that the loss of potassium to the silicate phase can be limited to about 5 percent if the condensed slag is removed at $2300 \mathrm{~K}$ at the diffuser exit. The potassium loss can be reduced to about 3 percent if 90 percent of the ash is removed at the combustor. These calculations show a maximum of 64 percent of the potassium lost if all slag remains in the system down to $1300 \mathrm{~K}$.

There have been very few thermodynamic measurements pertinent to the interaction of seed and slag in the MHD system. Results reported on by Dicks, et a1. [8] indicated that the rates of reactions between seed and slag may be sufficiently low that seed losses will be small. In these experiments, all of the mineral slag flows through the channel and the exhaust gases are cooled below $1500 \mathrm{~K}$ in less than one second [8]. More than 80 percent of the seed was recovered simply by water washing of the recovered solids. The balance of the potassium was either lost with the effluent or remained with the slag. This loss is too large, and larger losses would be expected in an MHD power plant, where longer seedslag contact times are necessary to provide for adequate $\mathrm{NO}_{\mathrm{x}}$ decomposition.

Using the Knudsen effusion method, Plante, 01son, and Negas [9] have studied the vaporization of $\mathrm{K}_{2} \mathrm{O}$ in the binary systems $\mathrm{K}_{2} \mathrm{O}+\mathrm{SiO}_{2}$ and $\mathrm{K}_{2} \mathrm{O}+\mathrm{Al}_{2} \mathrm{O}_{3}$. The measurements were 
later extended to other synthetic slag systems [10]. In the $\mathrm{K}_{2} \mathrm{O}+\mathrm{SiO}_{2}$ experiments, the results varied significantly with the orifice size of the Knudsen cell suggesting that equilibrium was not attained with the larger orifice area. A sample of fly ash obtained from an MHD combustor was analyzed [11] using infrared and laser-Raman spectroscopic techniques. These results indicate that the mineral kalsilite, KAlSi0, probably arising from the reaction of $\mathrm{K}_{2} \mathrm{O}$ with aluminum silicates, is the primary species responsible for reducing seed recovery. This material is water insoluble and may explain why aqueous extraction of the fly ash gave a maximum recovery of only about 75 percent potassium [12]. The condensed species $\mathrm{K}_{2} \mathrm{SiO}_{3}, \mathrm{KAlO}_{2}, \mathrm{~K}_{2} \mathrm{SiO}_{2} \mathrm{O}_{5}$, and KAlSi${ }_{n} \mathrm{O}_{2 n+2}(\mathrm{n}=1,2,3)$ are the important compounds that contain potassium at temperatures above $1500 \mathrm{~K}$. The simple silicate begins to form at temperatures below $2600 \mathrm{~K}$ and the potassium aluminosilicates form at temperatures below $2500 \mathrm{~K}$. The discrepancy existing between the predicted and the observed seed recovery values suggests that further study of slag-seed interactions is needed.

Because MHD power generation is a newly developing technology, complete understanding of the complex chemistry between seed and ash constituents at high temperature is still lacking. We have developed and used a computer program of the multiphase, multicomponent solution model (MMSM) [13] which was extended from the NASA CEC code [14], to calculate potassium activities in the $\mathrm{K}_{2} \mathrm{O}+\mathrm{SiO}_{2}, \mathrm{~K}_{2} \mathrm{O}+\mathrm{SiO}_{2}+\mathrm{Al}_{2} \mathrm{O}_{3}, \mathrm{~K}_{2} \mathrm{O}+\mathrm{SiO}_{2}+\mathrm{MgO}$, and $\mathrm{K}_{2} \mathrm{O}+$ $\mathrm{SiO}_{2}+\mathrm{CaO}$ systems. Comparisons of predicted values with the available experimental results are made. The means to increase the potassium activities in the gaseous phase and thereby increase seed recovery is discussed.

\section{Multiphase Multicomponent Solution Model}

After the successful test on the binary solution model [15], which was modified from NASA CEC code [14], the computer program was extended to a multiphase multicomponent solution model (MMSM). We assume that it is possible to form a solution of many components by condensation of a mixture of ideal gaseous species. The system Gibbs free energy, $g$, is expressed as:

$$
g=\sum_{k=1}^{m} n_{k} \mu_{k}+g_{s 01}
$$

where $\mu_{k}$ and $n_{k}$ are the chemical potential and number of moles of $k^{\text {th }}$ gas or pure condensed species, $m$ is the total number of gas and pure condensed species, and $g_{\text {sol }}$ is the Gibbs free energy of the solution. The chemical potentials can be expressed as follows:

for gas species,

$$
\mu_{k}=\mu_{k}^{o}+R T \ln \left(\frac{n_{k}}{n}\right)+R T \ln p
$$


for pure condensed species,

$$
\mu_{k}=\mu_{k}^{\circ}
$$

where $\mu_{k}^{\circ}$ denotes the chemical potential in the standard state, $n$ and $p$ are respectively the total number of moles of gaseous species and its total pressure, and $R$ is the gas constant. The Gibbs free energy of an ideal solution can be expressed explicitly as,

$$
g_{S 01}=n_{S 1} \mu_{S 1}+n_{S 2} \mu_{S 2}+\ldots+n_{S n} \mu_{S n}
$$

where $\mathrm{n}_{\mathrm{S} 1}, \mathrm{n}_{\mathrm{S} 2}, \ldots ., \mathrm{n}_{\mathrm{Sn}}$, are the number of moles of components, S1, S2, . ., Sn, respectively, $\mu_{S 1}, \mu_{S 2}, \ldots ., \mu_{S n}$, are the chemical potential of each respective component which can be expressed as,

$$
\begin{gathered}
\mu_{S 1}=\mu_{S 1}^{\circ}+R T \ln \left[\begin{array}{l}
\sum_{j=S 1}^{n_{S 1}} n_{j} \\
\mu_{S 2}= \\
\mu_{S 2}^{o}+R T \ln \left[\frac{n_{S 2}}{\sum_{j=S 1}^{S n} n_{j}}\right.
\end{array}\right] \\
\cdot \\
\mu_{S n}=\mu_{S n}^{\circ}+R T \ln \left[\begin{array}{c}
n_{S n} \\
\sum_{j n}^{S n} n_{j} \\
j=S 1
\end{array}\right]
\end{gathered}
$$

where $\mu_{S 1}^{o}, \mu_{S 2}, . . ., \mu_{S n}^{\circ}$ are the chemical potentials at standard state of each respective component, and $n_{j}$ is the number of moles of the $j^{\text {th }}$ component. Defining $G$ as

$$
G=g+\sum_{i=1}^{\ell} \lambda_{i}\left(b_{i}-b_{i}^{o}\right)
$$


where $\ell$ is the total number of elements in the system, $\lambda_{i}$ the Lagrangian multiplier, and $b_{i}^{\circ}$ the assigned number of kilogram-atoms of element $i$ per kilogram of total reactants. $b_{i}$ is the number of kilogram-atoms of element $i$ per kilogram of mixture which can be written,

$$
b_{i}=\sum_{j=1}^{t} a_{i j} N_{j}, i=1, \ldots, \ell
$$

where $a_{i j}$ is the stoichiometric ratio of the $i^{\text {th }}$ element in the $j^{\text {th }}$ species, and $t$ is the total number of gas, pure condensed species and solution components. The condition for chemical equilibrium is the minimization of the system free energy. In the process of minimization two constraints are imposed; the first is the mass balance constraint:

$$
\sum_{j=1}^{t} a_{i j} N_{j}-b_{i}^{o}=0, i=1, \ldots, \ell
$$

and the second is $\delta G=0$. The $\delta G$ function can be written as,

$$
\begin{aligned}
\delta G & =\sum_{k=1}^{m}\left(\mu_{k}+\sum_{i=1}^{\ell} \lambda_{i} a_{i k}\right) \delta n_{k}+\left[\left(\frac{\partial g_{s 01}}{\partial n_{S 1}}\right)+\sum_{i=1}^{\ell} \lambda_{i} a_{i S 1}\right] \delta n_{S 1} \\
& +\left[\left(\frac{\partial g_{S 01}}{\partial n_{S 2}}\right)+\sum_{i=1}^{\ell} \lambda_{i} a_{i S 2}\right] \delta n_{S 2}+\ldots \\
& +\left[\left(\frac{\partial g_{S O 1}}{\partial n_{S n}}\right)+\sum_{i=1}^{\ell} \lambda_{i} a_{i S n}\right] \delta n_{S n}+\sum_{i=1}^{\ell}\left(b_{i}-b_{i}^{0}\right) \delta \lambda_{i}=0
\end{aligned}
$$

By the Gibbs-Duhem relation,

$$
\begin{gathered}
\left(\frac{\partial g_{S 01}}{\partial n_{S 1}}\right)_{P, T}=\mu_{S 1} \\
\left(\frac{\partial g_{S 01}}{\partial n_{S 2}}\right)_{P, T}=\mu_{S 2} \\
\vdots \\
\cdot \\
\left(\frac{\partial g_{S 01}}{\partial n_{S n}}\right)_{P, T}=\mu_{S n}
\end{gathered}
$$


so that eq. (9) can be written,

$$
\begin{aligned}
\delta G & =\sum_{k=1}^{m}\left(\mu_{k}+\sum_{i=1}^{\ell} \lambda_{i} a_{i k}\right) \delta n_{k}+\left[\mu_{S 1}+\sum_{i=1}^{\ell} \lambda_{i} a_{i S 1}\right] \delta n_{S 1} \\
& +\left[\mu_{S 2}+\sum_{i=1}^{\ell} \lambda_{i} a_{i S 2}\right] \delta n_{S 2}+\ldots+\left[\mu_{S n}+\sum_{i=1}^{\ell} \lambda_{i} a_{i S n}\right] \delta n_{S n} \\
& +\sum_{i=1}^{\ell}\left(b_{i}-b_{i}^{\circ}\right) \delta \lambda_{i}=0
\end{aligned}
$$

Treating $\delta n_{k}, \delta n_{S 1}, \delta n_{S 2}, \ldots, \delta n_{S n}$, and $\delta \lambda_{i}$ as independent variables, the following equations are obtained,

$$
\begin{aligned}
& \mu_{k}+\sum_{i=1}^{\ell} \lambda_{i} a_{i k}=0 \\
& \mu_{S 1}+\sum_{i=1}^{\ell} \lambda_{i} a_{i S 1}=0 \\
& \mu_{S 2}+\sum_{i=1}^{\ell} \lambda_{i} a_{i S 2}=0 \\
& \cdot \quad \cdot \\
& \mu_{S n}+\sum_{i=1}^{\ell} \lambda_{i} a_{i S n}=0 \\
& b_{i}-b_{i}^{o}=0
\end{aligned}
$$

To solve the corrections to the initial estimates of $n_{k}, \lambda_{i}, n_{S 1}, n_{S 2}, \ldots, n_{S n}$, the descent Newton-Raphson method [16] was used. Gibbs iteration equations are derived by using Taylor series expansion to the first derivative. For Sn-component solutions, they are: 


$$
\begin{aligned}
& -\sum_{i=1}^{\ell} \pi_{i} a_{i S 1}+\left(\frac{\sum_{j \neq S 1_{j}}^{\ell} n_{j}}{n_{S 1} \sum_{j=1}^{\ell} n_{j}}\right) \Delta n_{S 1}-\frac{1}{\sum_{j=1}^{\ell} n_{j}}\left(\sum_{k \neq S 1}^{S n} \Delta n_{k}\right)=-\frac{\mu_{S 1}}{R T} \\
& -\sum_{i=1}^{\ell} \pi_{i} a_{i S 2}+\left(\frac{\sum_{j \neq S 2}^{\ell} n_{j}}{n_{S 2} \sum_{j=1}^{\ell} n_{j}}\right) \Delta n_{S 2}-\frac{1}{\sum_{j=1}^{\ell} n_{j}}\left(\sum_{k \neq S 2}^{S n} \Delta n_{k}\right)=-\frac{\mu_{S 2}}{R T} \\
& \vdots \\
& -\sum_{i=1}^{\ell} \pi_{i} a_{i S n}+\left(\frac{\sum_{j \neq S n^{j}}^{\ell} n_{n_{S n}}^{\ell}}{\sum_{j=1}}\right) \Delta n_{S n}-\frac{1}{\sum_{j=1}^{\ell} n_{j}}\left(\sum_{k \neq S n}^{S n} \Delta n_{k}\right)=-\frac{\mu_{S n}}{R T}
\end{aligned}
$$

where $\pi_{i}=-\frac{\lambda_{i}}{R T}$.

The coefficients of vectors of $\Delta n_{S n}$ and $\Delta n_{k}$ in eqs. (19-21) become the elements of the matrix. The final solutions of the matrix are obtained when the calculated values deviate within a designated limit $\left(5 \times 10^{-6}\right)$. This addition of the solution formation has been incorporated into the NASA-CEC computer code [13]. The execution of the computer program is coupled with a chemical data base consisting of 752 chemical species.

\section{Calculations and Results}

By using the MMSM, the potassium and silica activities were calculated in the $\mathrm{K}_{2} \mathrm{O}+\mathrm{SiO}_{2}, \mathrm{~K}_{2} \mathrm{O}+\mathrm{SiO}_{2}+\mathrm{Al}_{2} \mathrm{O}_{3}, \mathrm{~K}_{2} \mathrm{O}+\mathrm{SiO}_{2}+\mathrm{MgO}$ and $\mathrm{K}_{2} \mathrm{O}+\mathrm{SiO}_{2}+\mathrm{CaO}$ systems. The results are discussed as follows.

\subsection{The $\mathrm{K}_{2} \mathrm{O}+\mathrm{SiO}_{2}$ binary system}

In this system, the three condensed phase species, $\mathrm{K}_{2} \mathrm{SiO}_{3}(\ell), \mathrm{K}_{2} \mathrm{Si}_{2} \mathrm{O}_{5}(\ell)$, and $\mathrm{SiO}_{2}(\ell)$ were assumed to form an ideal solution. For this mixture over the temperature range of 2300 to $1200 \mathrm{~K}$, the concentration of $\mathrm{K}_{2} 0$ varies from 10 to 40 mol percent, while 
the concentration of $\mathrm{SiO}_{2}$ varies from 60 to $90 \mathrm{~mol}$ percent. Thermochemical data for $\mathrm{K}_{2} \mathrm{SiO}_{3}(l)$ and $\mathrm{SiO}_{2}(l)$ were taken from the JANAF Tables [17], and that for $\mathrm{K}_{2} \mathrm{Si}_{2} \mathrm{O}_{5}(l)$ was estimated by Blackburn [18]. The results are given in figure 1 and table 1 . As expected the potassium partial pressures increase while the silica activity, $\mathrm{A}_{\mathrm{SiO}_{2}(\ell)}$, in the solution decreases with temperature and potassium concentration. Because of the greater stability of potassium disilicate, $\mathrm{K}_{2} \mathrm{Si}_{2} \mathrm{O}_{5}$, over that of potassium metasilicate, $\mathrm{K}_{2} \mathrm{SiO}_{3}$ (free energy of formation of $\mathrm{K}_{2} \mathrm{Si}_{2} \mathrm{O}_{5}$ is $180 \mathrm{kcal} \mathrm{mol}{ }^{-1}$, at $1000 \mathrm{~K}$, more negative than for $\mathrm{K}_{2} \mathrm{SiO}_{3}$ ), the activity of $\mathrm{K}_{2} \mathrm{Si}_{2} \mathrm{O}_{5}$ in solution is higher ${ }^{3}$ than that of $\mathrm{K}_{2} \mathrm{SiO}_{3}$ (except in the case of $\mathrm{K}_{2} \mathrm{O}: \mathrm{SiO}_{2}=40: 60$, fig. 2). In table 1 the equilibrium constants, $K_{e q}$, for the reaction

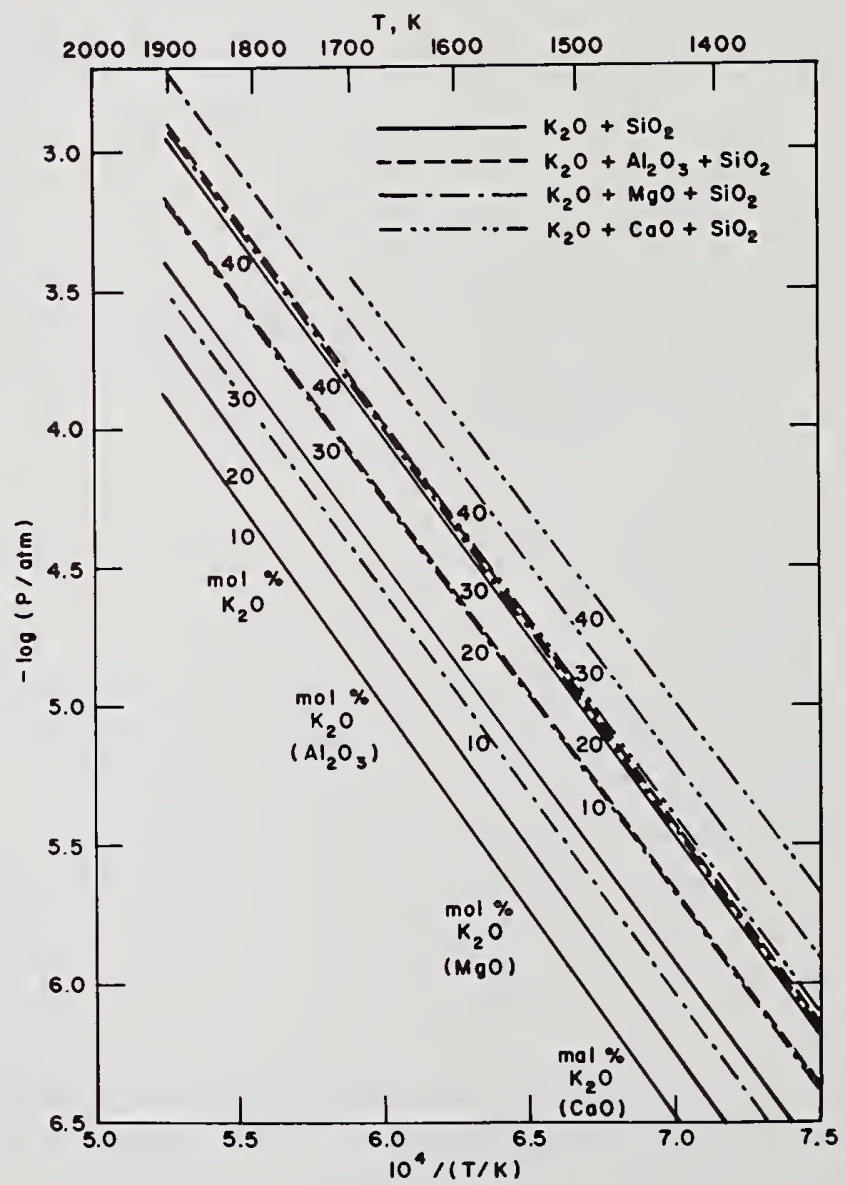

Figure 1. Equilibrium potassium pressures as a function of temperature in the $\mathrm{K}_{2} 0$ $+\mathrm{SiO}_{2}, \mathrm{~K}_{2} \mathrm{O}+\mathrm{Al}_{2} \mathrm{O}_{3}+\mathrm{SiO}_{2}, \mathrm{~K}_{2} \mathrm{O}+\mathrm{MgO}+\mathrm{SiO}_{2}$, and $\mathrm{K}_{2} \mathrm{O}+\mathrm{CaO}+\mathrm{SiO}_{2}$ systems.

'Editor's note: This is mainly due to the higher $\mathrm{SiO}_{2}$ concentration in the disilicate. 


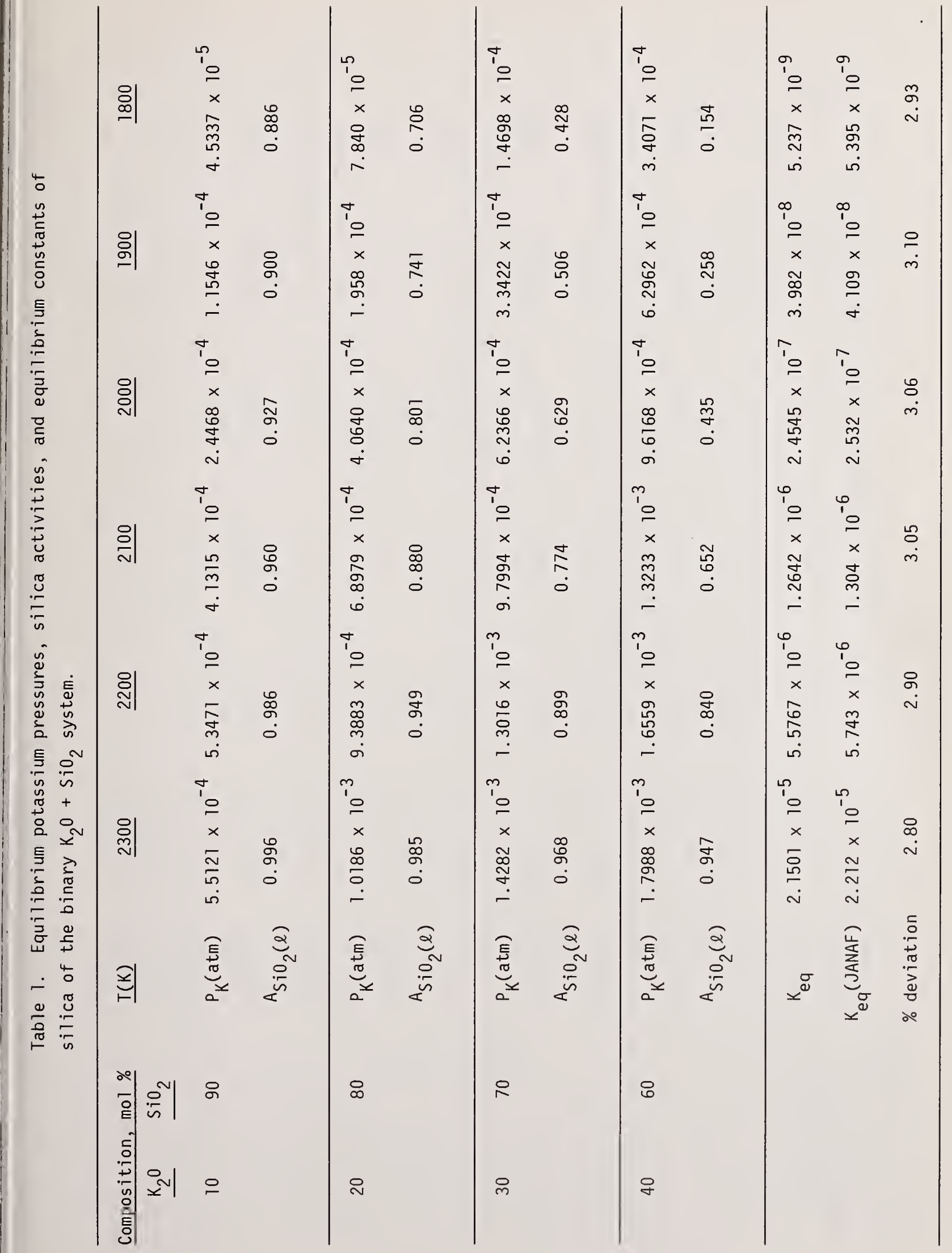




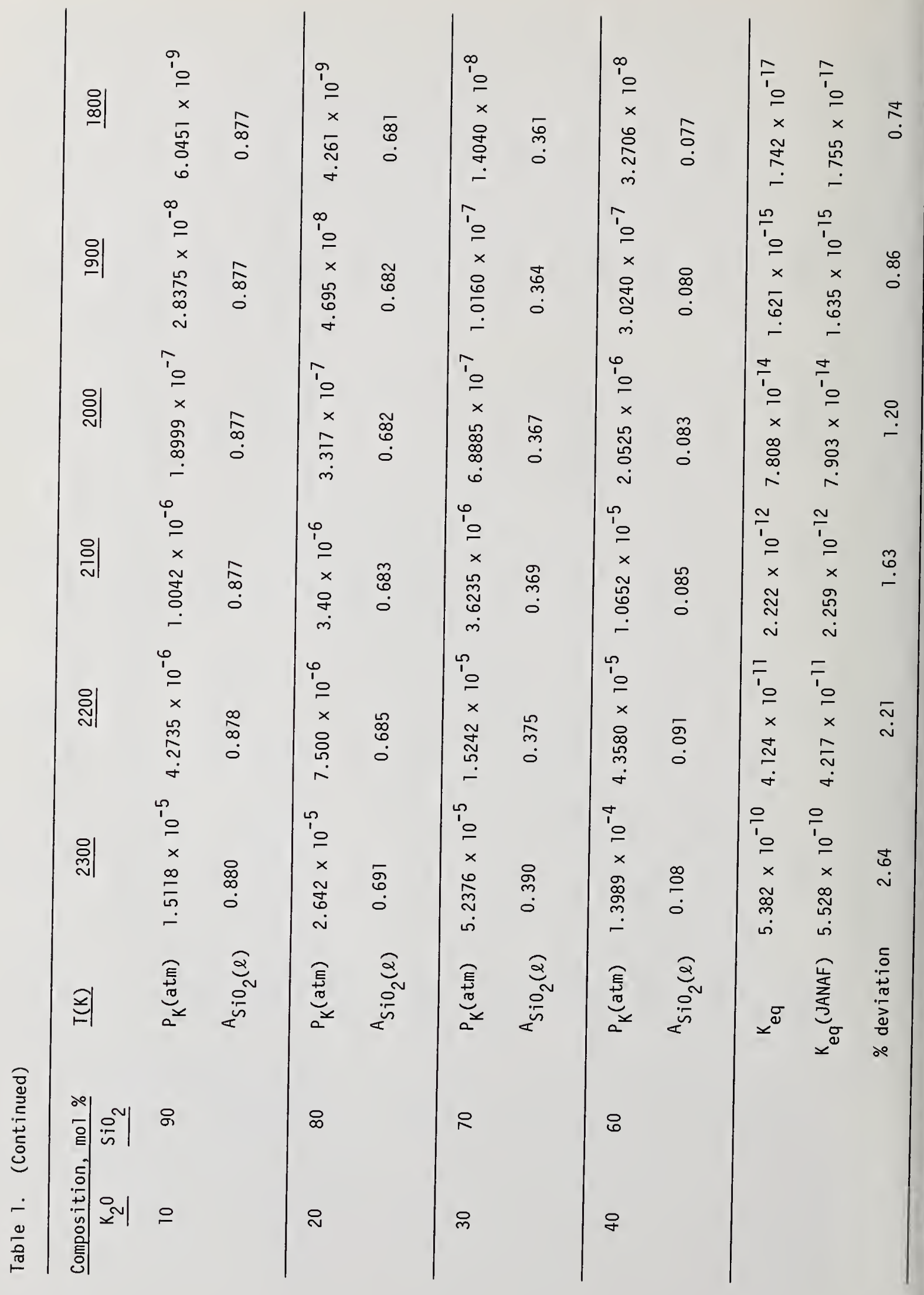




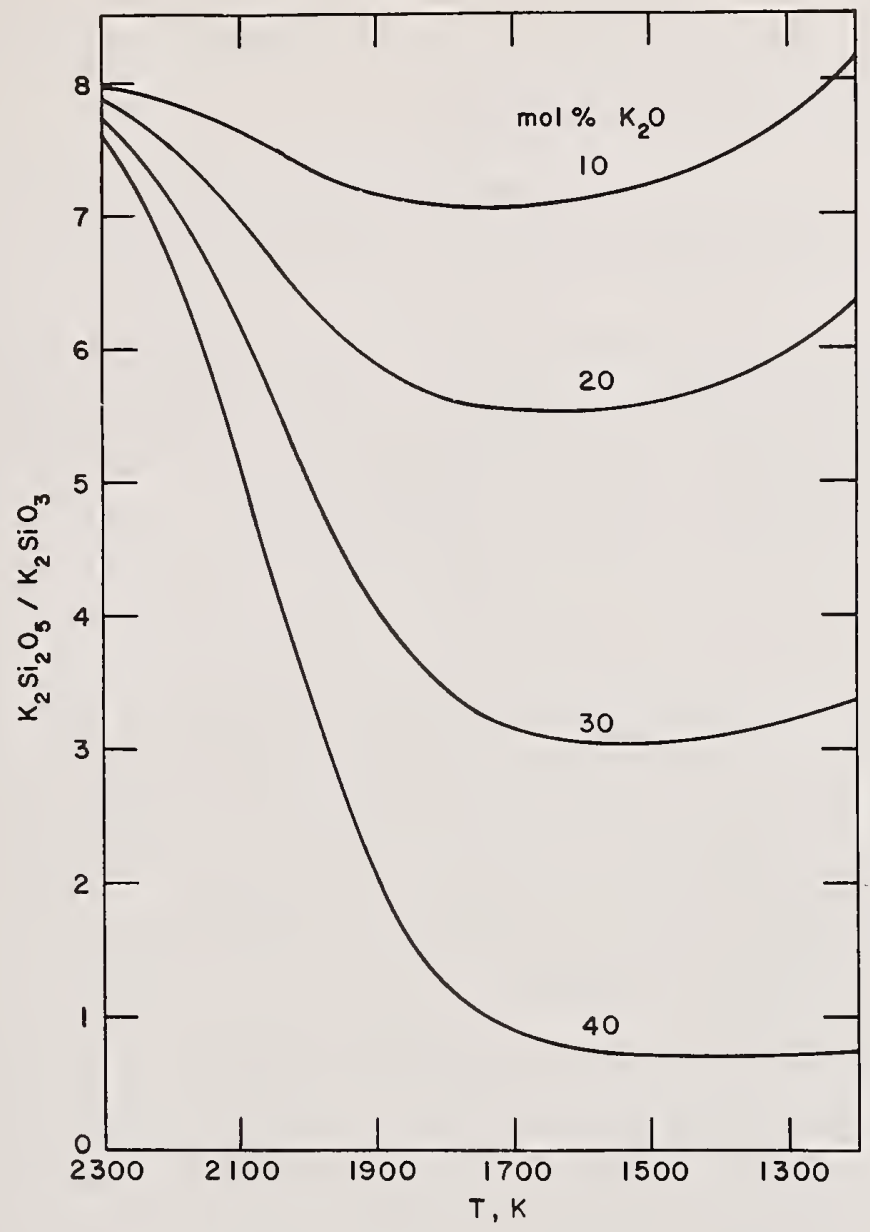

Figure 2. The ratios of $\mathrm{K}_{2} \mathrm{Si}_{2} \mathrm{O}_{5} / \mathrm{K}_{2} \mathrm{SiO}_{3}$ as a function of temperature and the concentration of $\mathrm{K}_{2} \mathrm{O}$ in the $\mathrm{K}_{2} \mathrm{O}+\mathrm{SiO}_{2}$ system.

$$
\mathrm{SiO}_{2}(\ell) \underset{\leftarrow}{\rightarrow} \mathrm{SiO}_{2}(\mathrm{~g})
$$

were computed at various temperatures from the calculated equilibrium concentrations of $\mathrm{SiO}_{2}(\ell)$ and $\mathrm{SiO}_{2}(\mathrm{~g})$ according to

$$
K_{e q}=\frac{\mathrm{P}_{\mathrm{SiO}_{2}}(\mathrm{~g})}{\mathrm{A}_{\mathrm{SiO}_{2}}(\mathrm{l})}
$$


where $\mathrm{A}_{\mathrm{SiO}_{2}}(\ell)$ is evaluated as the mole fraction of the total number of moles of the condensed species. The equilibrium constants from the JANAF Thermochemical Tables [17] were also calculated using the following equation

$$
K_{\text {eq }}(J A N A F)=\exp \left\{\frac{\Delta G_{f}^{\circ}\left[\mathrm{SiO}_{2}(\ell)\right]-\Delta G_{f}^{\circ}\left[\operatorname{SiO}_{2}(g)\right]}{R T}\right\}
$$

where $\Delta G_{f}^{o}$ is the Gibbs free energy of formation of the pure species. The equilibrium constants from our results are slightly lower than that of JANAF Tables at all the composition and the temperature range of interest (maximum deviation is 3.10 percent at $1900 \mathrm{~K}$ ) This agreement indicates self-consistency in the MMSM method.

\subsection{The $\mathrm{K}_{2} \mathrm{O}+\mathrm{SiO}_{2}+\mathrm{Al}_{2} \mathrm{O}_{3}$ ternary system}

In this system, seven condensed phase species were assumed to form a solution: $\mathrm{SiO}_{2}(l), \mathrm{KATO}_{2}(l), \mathrm{K}_{2} \mathrm{SiO}_{3}(l), \mathrm{K}_{2} \mathrm{Si}_{2} \mathrm{O}_{5}(l), \mathrm{KATSiO}_{4}(\ell), \mathrm{KATSi}_{2}{ }_{6}(l)$, and KATSi${ }_{3} \mathrm{O}_{8}(\ell)$. Thermochemical data for $\mathrm{KAlO}_{2}(\ell), \mathrm{KATSiO}_{4}(\ell), \mathrm{KAISi}_{2} 0_{6}(\ell)$, and $\mathrm{KATSi}_{3} 0_{8}(\ell)$ were taken from the estimated values of Spencer, et al. [4]. According to the phase diagram [19], the potassium aluminosilicates form in the potassia-rich region.

The calculations covered only two compositions: $\mathrm{K}_{2} \mathrm{O}=30, \mathrm{SiO}_{2}=50, \mathrm{Al}_{2} \mathrm{O}_{3}=20 \mathrm{~mol}$ percent and $\mathrm{K}_{2} \mathrm{O}=40, \mathrm{SiO}_{2}=50, \mathrm{Al}_{2} \mathrm{O}_{3}=10 \mathrm{~mol}$ percent. The results are shown in figure 1 and table 2. It should be noted that the potassium activities in the gas phase for this system are higher than for the comparable $\mathrm{K}_{2} \mathrm{O}+\mathrm{SiO}_{2}$ system. For instance, the equilibrium potassium pressures at $1900 \mathrm{~K}$ are $5.7097 \times 10^{-4}$ and $3.3422 \times 10^{-4}$ atm for the $\mathrm{K}_{2} \mathrm{O}: \mathrm{SiO}_{2}: \mathrm{Al}_{2} \mathrm{O}_{3}$ - 30:50:20 and $\mathrm{K}_{2} 0: \mathrm{SiO}_{2}-30: 70$ mol percent systems, respectively. This increase in activity is reflected from the fact that the activity of $\mathrm{SiO}_{2}$ in the binary $\mathrm{K}_{2} \mathrm{O}+\mathrm{SiO}_{2}$ system is five times greater than that for the ternary $\mathrm{K}_{2} \mathrm{O}+\mathrm{SiO}_{2}+\mathrm{Al}_{2} \mathrm{O}_{3}$ system (tables $\mathrm{l}$ and 2 ). As a result of higher silica activity in the solution phase, more seed would be tied up in the slag. Also from the ternary $\mathrm{K}_{2} \mathrm{O}+\mathrm{SiO}_{2}+\mathrm{Al}_{2} \mathrm{O}_{3}$ system [19] at the composition of $\mathrm{K}_{2} \mathrm{O}: \mathrm{SiO}_{2}: \mathrm{Al}_{2} \mathrm{O}_{3}$ of $30: 50: 20 \mathrm{~mol}$ percent, the melting temperature is around $1800 \mathrm{~K}$. The equilibrium below $1800 \mathrm{~K}$ indicates a reduction in the total system free energy upon the formation of the ideal solution from these components. (The calculations cover 1900 1200 K.)

In $\mathrm{K}_{2} \mathrm{O}+\mathrm{SiO}_{2}+\mathrm{Al}_{2} \mathrm{O}_{3}$ systems of varying composition there is a shift in importance among the condensed phase species. For a $\mathrm{K}_{2} \mathrm{O}: \mathrm{SiO}_{2}: \mathrm{Al}_{2} \mathrm{O}_{3}$ solution of 40:50:10 mol percent at $1900 \mathrm{~K}$ the decreasing order of importance is $\mathrm{KAlO}_{2}(l), \mathrm{K}_{2} \mathrm{SiO}_{3}(l), \mathrm{K}_{2} \mathrm{Si}_{2} \mathrm{O}_{5}(l), \mathrm{KAlSi}_{3} \mathrm{O}_{8}(l)$, $\mathrm{SiO}_{2}(\ell), \mathrm{KATSiO}_{4}(\ell), \mathrm{KAISi}_{2} \mathrm{O}_{6}(\ell)$. At lower temperatures, the order remains the same except that $\mathrm{K}_{2} \mathrm{SiO}_{3}(l)$ becomes more predominant than $\mathrm{KAlO}_{2}(l)$. Similarly, for the case of $\mathrm{K}_{2} \mathrm{O}: \mathrm{SiO}_{2}: \mathrm{Al}_{2} \mathrm{O}_{3}-30: 50: 20 \mathrm{~mol}$ percent, the order of importance at $1900 \mathrm{~K}$ is: $\mathrm{KAlO}_{2}(\ell)$, $\mathrm{KATSi}_{3} \mathrm{O}_{8}(l), \mathrm{KAlSiO}_{4}(l), \mathrm{SiO}_{2}(l), \mathrm{K}_{2} \mathrm{SiO}_{3}(l), \mathrm{K}_{2} \mathrm{Si}_{2} \mathrm{O}_{5}(l), \mathrm{KAlSi}_{2} \mathrm{O}_{6}(l)$. At $1300 \mathrm{~K}$ for this same system that order of importance is $\mathrm{KAlO}_{2}(l), \mathrm{KAISi}_{3} \mathrm{O}_{8}(\ell), \mathrm{K}_{2} \mathrm{SiO}_{3}(\ell), \mathrm{K}_{2} \mathrm{Si}_{2} \mathrm{O}_{5}(l)$, $\mathrm{SiO}_{2}(\ell), \mathrm{KATSiO}_{4}(\ell)$, and $\mathrm{KATSi}_{2} \mathrm{O}_{6}(\ell)$. 


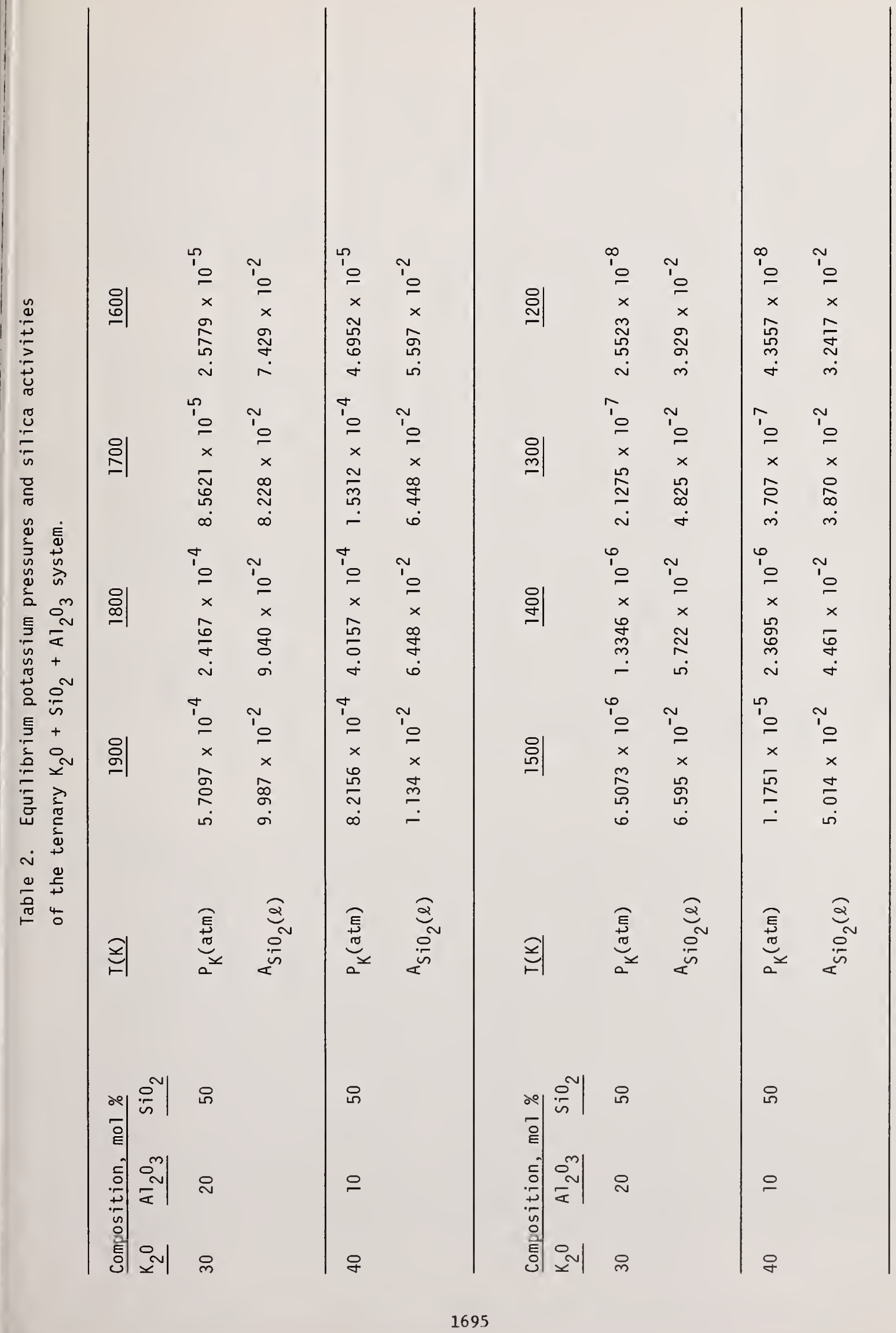




\subsection{The $\mathrm{K}_{2} \mathrm{O}+\mathrm{SiO}_{2}+\mathrm{MgO}$ ternary system}

For the $\mathrm{K}_{2} \mathrm{O}+\mathrm{SiO}_{2}+\mathrm{MgO}$ ternary system in the temperature range of $2200-1200 \mathrm{~K}$ it was assumed that the following six components $\mathrm{K}_{2} \mathrm{SiO}_{3}(\ell), \mathrm{K}_{2} \mathrm{Si}_{2} \mathrm{O}_{5}(\ell), \operatorname{MgO}(\ell), \mathrm{MgSiO}_{3}(\ell)$, $\mathrm{Mg}_{2} \mathrm{SiO}_{4}(\mathrm{~s})$, and $\mathrm{SiO}_{2}(\ell)$ formed an ideal solution. The thermocouple data of $\mathrm{MgO}(\mathrm{s})$, $\mathrm{MgSiO}_{3}(l)$, and $\mathrm{Mg}_{2} \mathrm{SiO}_{4}(\mathrm{~s})$ were taken from the JANAF Tables [17]. Because of the high melting temperature of $\mathrm{MgO}$ and $\mathrm{Mg}_{2} \mathrm{SiO}_{4}$, the solid phase data were chosen to describe the solution phase in the temperature range of interest. The calculations of equilibrium potassium pressure cover the concentration ranges of $\mathrm{K}_{2} \mathrm{O}$ and $\mathrm{MgO}$ from 10 to $40 \mathrm{mo} 1$ percent, while keeping the $\mathrm{SiO}_{2}$ concentration constant at $50 \mathrm{~mol}$ percent. The results of these calculations are shown in figure 1 and table 3.

For the above-mentioned systems and as shown in figure 2 , the calculations indicated that the potassium partial pressures were greater than that of comparable $\mathrm{K}_{2} \mathrm{O}+\mathrm{SiO}_{2}$ and $\mathrm{K}_{2} \mathrm{O}+\mathrm{SiO}_{2}+\mathrm{Al}_{2} \mathrm{O}_{3}$ systems. The formation of magnesium metasilicate and magnesium orthosilicate decrease the activity of silica in the solution. As a result, the activity of potassium is increased. Throughout the whole range of compositions considered, magnesium metasilicate and orthosilicate in the solution phase are more important for the silica-rich melt, whereas potassium metasilicate and disilicate are predominate in the potassia-rich phase. It appears that magnesia would be a good candidate for the ceramic lining material as well as an additive for the fuel in the MHD system to protect the metallic components and increase the potassium activity, thereby improving seed recovery.

It is to be noted that the ternary compounds of the $\mathrm{K}_{2} \mathrm{O}+\mathrm{SiO}_{2}+\mathrm{MgO}$ system, such as $\mathrm{K}_{2} \mathrm{MgSi}_{3} \mathrm{O}_{8}\left(\mathrm{~K}_{2} \mathrm{O} \cdot \mathrm{MgO} \cdot 3 \mathrm{SiO}_{2}\right), \mathrm{K}_{2} \mathrm{MgSi}_{5} \mathrm{O}_{12}\left(\mathrm{~K}_{2} \mathrm{O} \cdot \mathrm{MgO} \cdot 5 \mathrm{SiO}_{2}\right), \mathrm{K}_{2} \mathrm{Mg}_{5} \mathrm{Si}_{12} \mathrm{O}_{30}\left(\mathrm{~K}_{2} \mathrm{O} \cdot 5 \mathrm{MgO} \cdot 12 \mathrm{SiO}_{2}\right)$, and $\mathrm{K}_{2} \mathrm{MgSiO}_{4}\left(\mathrm{~K}_{2} \mathrm{O} \cdot \mathrm{MgO} \cdot \mathrm{SiO}_{2}\right)$ and the compound $\mathrm{K}_{2} \mathrm{Si}_{4}{ }_{9}$, are not included in the calculations because of unavailability of thermochemical data.

\subsection{The $\mathrm{K}_{2} \mathrm{O}+\mathrm{SiO}_{2}+\mathrm{CaO}$ ternary system}

For the $\mathrm{K}_{2} \mathrm{O}+\mathrm{SiO}_{2}+\mathrm{CaO}$ ternary system six condensed phase species were assumed to form an ideal solution: $\mathrm{CaO}(\mathrm{s}), \mathrm{CaSiO}_{3}(\mathrm{~s}), \mathrm{Ca}_{2} \mathrm{SiO}_{4}(\mathrm{~s}), \mathrm{K}_{2} \mathrm{SiO}_{3}(\ell), \mathrm{K}_{2} \mathrm{Si}_{2} \mathrm{O}_{5}(\ell)$, and $\mathrm{SiO}_{2}(\ell)$. Thermochemical data for $\mathrm{CaO}(\mathrm{s}), \mathrm{CaSiO}_{3}(\mathrm{~s})$, and $\mathrm{Ca}_{2} \mathrm{SiO}_{4}(\mathrm{~s})$ were taken from the JANAF Tables [17] and the Geological Survey Bulletin [20]. The available data for solid $\mathrm{CaSiO}_{3}$ were limited to $1700 \mathrm{~K}$ which was then set as the upper temperature limit for this species in the calculation. The calculated results are shown in figure 1 and table 4 . As observed previously, we find the equilibrium potassium pressure is higher than that for the $\mathrm{K}_{2} \mathrm{O}+\mathrm{SiO}_{2}$ binary system. In fact, calculations show the potassium activity to be the highest of the three ternary systems studied. As there are no thermochemical data available of possible ternary compounds for the $\mathrm{K}_{2} \mathrm{O}+\mathrm{SiO}_{2}+\mathrm{CaO}$ system, the added $\mathrm{CaO}$ would tie up $\mathrm{SiO}_{2}$ in the solution as calcium metasilicate and di-orthosilicate which in turn increases the potassium slag activity and content in the gas phase. The potassium activity may change if one includes ternary compounds [19] such as $\mathrm{K}_{2} \mathrm{CaSiO}_{4}\left(\mathrm{~K}_{2} \mathrm{O} \cdot \mathrm{CaO} \cdot \mathrm{SiO}_{2}\right), \mathrm{K}_{2} \mathrm{Ca}_{3} \mathrm{Si}_{6}{ }^{0}{ }_{16}$ 


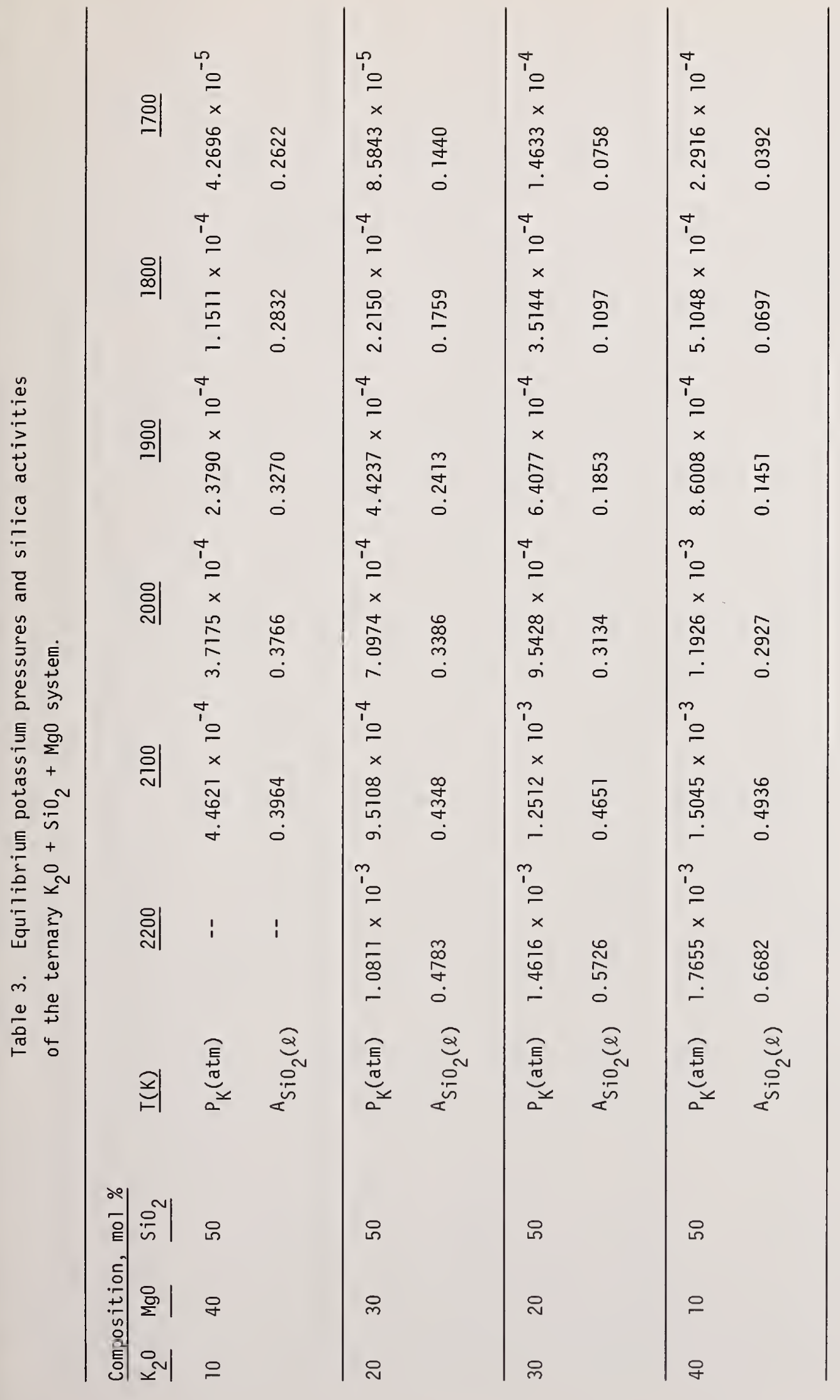




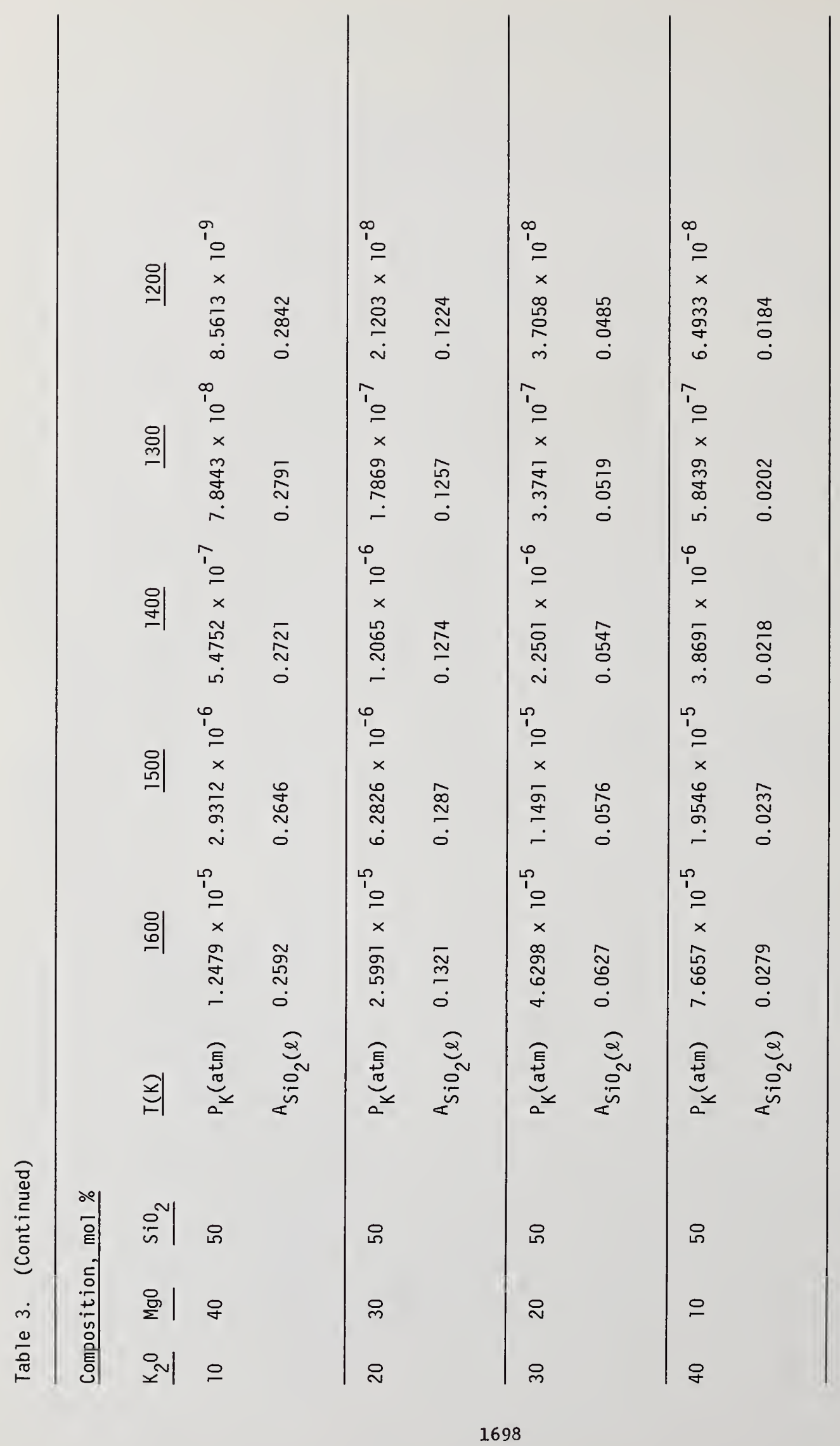




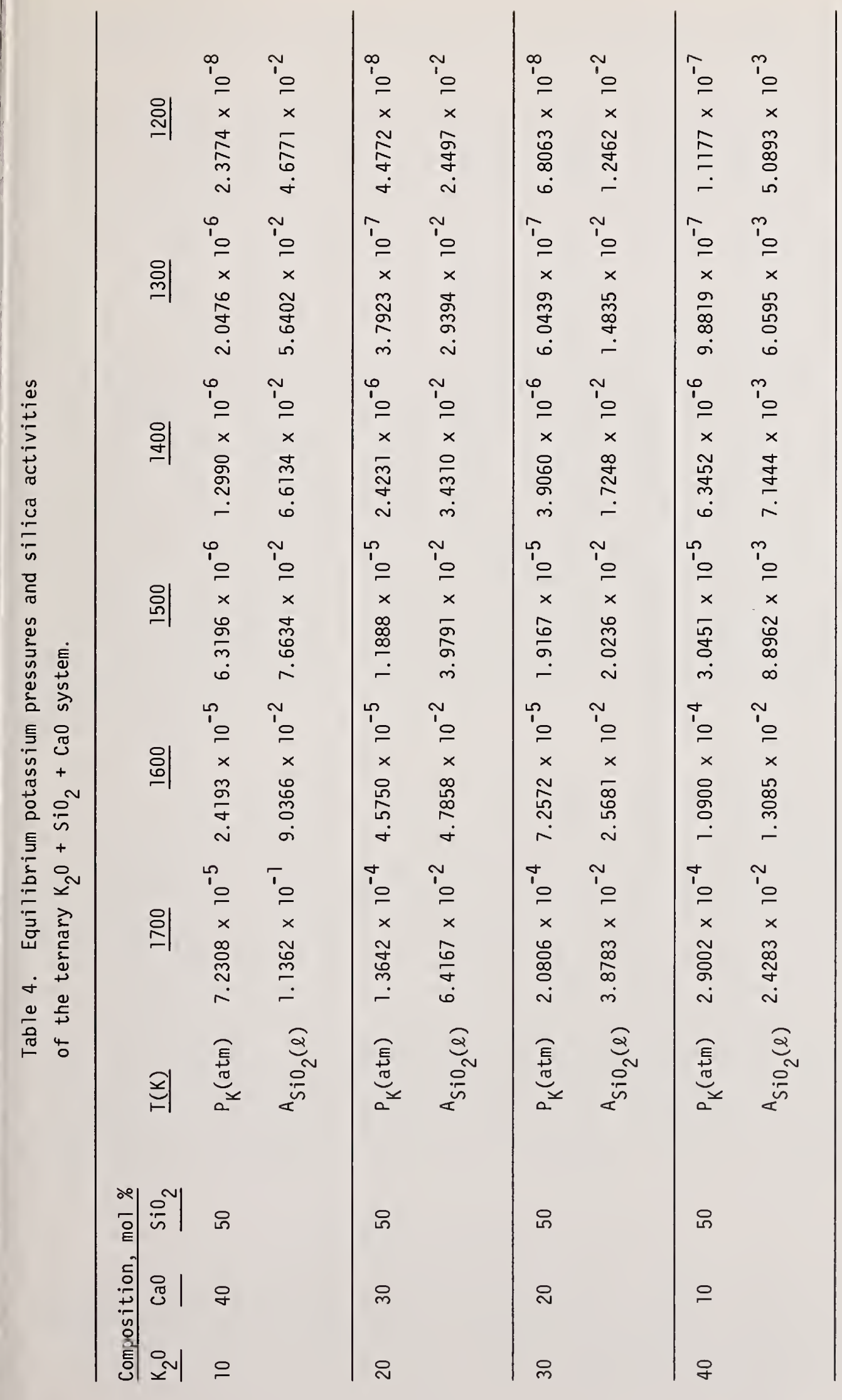


$\left(\mathrm{K}_{2} \mathrm{O} \cdot 3 \mathrm{CaO} \cdot 6 \mathrm{SiO}_{2}\right), \mathrm{K}_{4} \mathrm{CaSi}_{6}{ }^{0}{ }_{15}\left(2 \mathrm{~K}_{2} \mathrm{O} \cdot \mathrm{CaO} \cdot 6 \mathrm{SiO}_{2}\right), \mathrm{K}_{4} \mathrm{CaSi}_{3} \mathrm{O}_{9}\left(2 \mathrm{~K}_{2} \mathrm{O} \cdot \mathrm{CaO} \cdot 3 \mathrm{SiO}_{2}\right)$, and $\mathrm{K}_{8} \mathrm{CaSi}_{10}{ }_{25}$ $\left(4 \mathrm{~K}_{2} \mathrm{O} \cdot \mathrm{CaO} \cdot 10 \mathrm{SiO}_{2}\right)$ in the calculation.

In the silica-rich compositions, calcium metasilicate and di-orthosilicate activities are higher than for potassium silicates, the reverse is true for the potassia-rich compositions. Within the composition range of $\mathrm{K}_{2} \mathrm{O}$ and $\mathrm{Ca} 0$ from 10 to $40 \mathrm{~mol}$ percent with constant 50 mol percent of $\mathrm{SiO}_{2}$, the activity of $\mathrm{CaO}(\mathrm{s})$ is of the least importance in the solution at the temperature of interest.

\section{Discussion}

The MMSM calculations indicate that the vapor phase potassium activity increases with the addition of the metal oxides alumina, magnesia, or calcia to the binary $\mathrm{K}_{2} \mathrm{O}+\mathrm{SiO}_{2}$ system. The most significant increase in potassium activity was found for the addition of calcia; the addition of either magnesia or alumina showing a somewhat smaller impact on potassium activity. For the $\mathrm{K}_{2} \mathrm{O}+\mathrm{CaO}+\mathrm{SiO}_{2}, \mathrm{~K}_{2} \mathrm{O}+\mathrm{MgO}+\mathrm{SiO}_{2}, \mathrm{~K}_{2} \mathrm{O}+\mathrm{Al}_{2} \mathrm{O}_{3}+\mathrm{SiO}_{2}$ ternary systems containing $30 \mathrm{~mol}$ percent $\mathrm{K}_{2} 0$, the estimated potassium pressures are 3.97 , 2.79 , and 1.63 times higher, respectively, than for a $\mathrm{K}_{2} \mathrm{O}+\mathrm{Si}_{2}$ binary solution containing the same amount of $\mathrm{K}_{2} \mathrm{O}$. These results agree very well with the measurements of Cook et al. [21] in which they found that a synthetic slag containing 15 wt percent $\mathrm{Ca} 0$ and 10 wt percent $\mathrm{K}_{2} \mathrm{O}$ gave higher potassium pressures than the binary $\mathrm{K}_{2} 0+\mathrm{SiO}_{2}$ system with 30 wt percent $\mathrm{K}_{2} \mathrm{O}$. Further measurements on a slag containing 13 wt percent $\mathrm{K}_{2} \mathrm{O}$ and 20 wt percent $\mathrm{Al}_{2} \mathrm{O}_{3}$ yielded potassium pressures above that of the binary $\mathrm{K}_{2} \mathrm{O}+\mathrm{SiO}_{2}$ solution with 20 wt percent $\mathrm{K}_{2} \mathrm{O}$.

As has been noted, MMSM calculations showed that the potassium activity was greater for the magnesia and calcia containing systems as compared with the alumina containing system. This greater activity may be partially accounted for by the fact that no ternary compounds were included in the calculations for these systems. The inclusion of the ternary compounds in the calculation for the $\mathrm{K}_{2} \mathrm{O}+\mathrm{SiO}_{2}+\mathrm{MgO}$, and $\mathrm{K}_{2} \mathrm{O}+\mathrm{SiO}_{2}+\mathrm{CaO}$ systems is therefore very likely to reduce the gas phase potassium activity. However, it is still expected that the potassium activity for these systems will be greater than for the $\mathrm{K}_{2} \mathrm{O}+\mathrm{SiO}_{2}$ binary system.

of the four systems considered, the gas phase potassium activity for the binary $\mathrm{K}_{2} 0$ $+\mathrm{SiO}_{2}$ system was the only system measured experimentally [22-23]. Using the mass spectrometric-Knudsen effusion technique, Plante $[22,24]$ measured the equilibrium potassium pressure at various compositions of $\mathrm{K}_{2} \mathrm{O}+\mathrm{SiO}_{2}$ over a range of temperature assuming the vaporization process

$$
\mathrm{K}_{2} \mathrm{O}(\mathrm{soln}) \underset{\mathrm{t}}{\mathrm{K}} \mathrm{K}(\mathrm{g})+1 / 2 \mathrm{O}_{2}(\mathrm{~g}) \text {. }
$$

In the process of his measurements, he pointed out that the potassium pressure was a function of orifice size. Thus, the lower potassia concentration studies must be viewed cautiously as to whether equilibrium was attained in the effusion cell. (But see the report by Plante elsewhere in this volume.) 
Using atomic absorption and a graphite furnace, Eliezer, et al. [23], measured the distribution of potassium atoms in a so-called steady state diffusion system, from which the vapor pressures of potassium were evaluated. Although the atomic absorption technique has good sensitivity, the uncertainty in their reported data is large. Along with Plante's measurements their reported potassium pressures at $\left(\mathrm{K}_{\mathrm{K}_{2} \mathrm{O}}=0.0778\right)$ were compared with our

calculated results and these are shown in figure 3 . Their data are about four and two times smaller than our calculated potassium pressures at 1300 and $1900 \mathrm{~K}$, respectively. Moreover, with the higher potassium pressures of the Plante data (as compared with our results), an order of magnitude discrepancy between Plante's measurements and those of Eliezer, et al., was found. Our predicted results for potassium pressure in the $\mathrm{K}_{2} 0+$ $\mathrm{SiO}_{2}$ system fall between the data of Plante and Eliezer, et al. Within the uncertainty of the thermochemical data we used, the agreement is quite good.

For the ternary systems, much more detailed experimental data are needed to check the predicted values. Thermochemical data of the ternary compounds mentioned before are indispensible to improve the precision of the calculation. Unless more experimental data become available, constrained equilibrium calculations [25] cannot be performed.

It is to be noted that the results presented here represent the chemical properties at equilibrium. For the open cycle MHD system, kinetic factors may need to be considered. The longer the residence time in the MHD channel the better the chance that chemical equilibrium will be attained. In such cases the predicted equilibrium properties will enable one to elucidate the complex chemical reactions that occur in operating an open cycle MHD system.

\section{Conclusions}

A multiphase multicomponent solution model was developed for the computation of complex chemical equilibrium compositions. The usefulness of the model was demonstrated by predicting the potassium activities in the $\mathrm{K}_{2} \mathrm{O}+\mathrm{SiO}_{2}, \mathrm{~K}_{2} \mathrm{O}+\mathrm{SiO}_{2}+\mathrm{Al}_{2} \mathrm{O}_{3}, \mathrm{~K}_{2} \mathrm{O}+\mathrm{SiO}_{2}+$ $\mathrm{MgO}$, and $\mathrm{K}_{2} \mathrm{O}+\mathrm{SiO}_{2}+\mathrm{CaO}$ systems. For the $\mathrm{K}_{2} \mathrm{O}+\mathrm{SiO}_{2}$ binary system reasonable agreement between predicted potassium activities and the experimental results was obtained. The model also correctly predicts an increase of potassium activity on the addition of $\mathrm{Al}_{2}{ }_{3} 3$, $\mathrm{MgO}$, or $\mathrm{CaO}$ to the $\mathrm{K}_{2} \mathrm{O}+\mathrm{SiO}_{2}$ system. This predictive capability may be used in the selection of materials and in establishing optimum conditions for operation of an open cycle MHD system. Further, the predictive capability of the program can be used to assess other more suitable additives and in determining operating conditions for improved potassium seed recovery. 


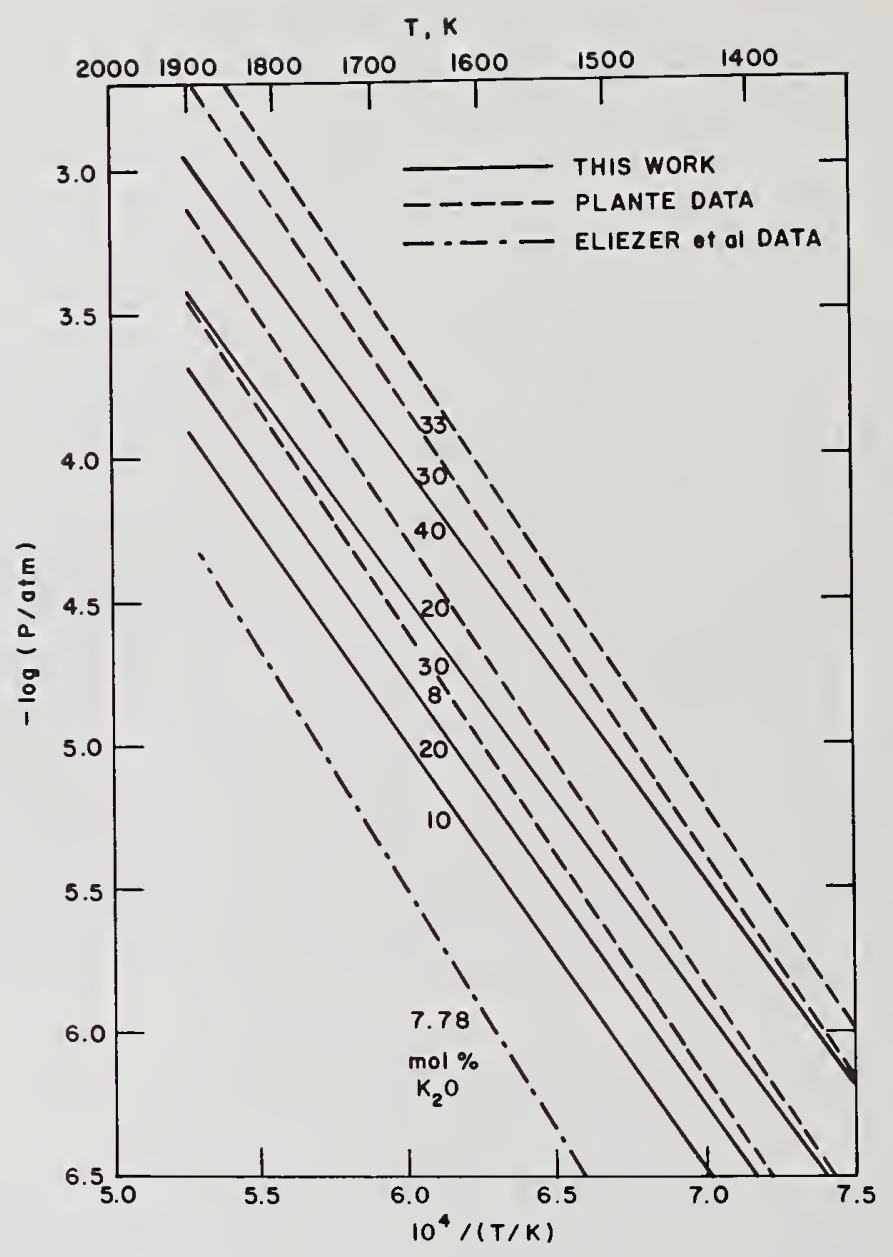

Figure 3. Comparison of predicted equilibrium potassium pressures with experimental results in the $\mathrm{K}_{2} \mathrm{O}+\mathrm{SiO}_{2}$ system.

\section{References}

[1] Hoover, D. Q., et a1., Energy Conversion Alternatives Study (ECAS) Westinghouse Phase I Final Report, Vol. VIII--Open Cycle MHD, NASA-CR-134941 (1976).

[2] Negas, T., Plante, E., 01son, C., and Cook, L., NBS Quarterly Report, p. 24-28 $(1975)^{4}$.

"Editor's note: This is not an archival publication and should be considered as a private communication. However, Plante has described his $\mathrm{K}_{2} \mathrm{O}+\mathrm{SiO}_{2}$ system data in detail elsewhere in this volume. 
[3] J. B. Heywood and G. J. Womack, eds., Open Cycle MHD Generation, p. 680 (Pergamon Press, 1970).

[4] Spencer, F. E., Jr., Hendrie, J. C., Jr. and Bienstock, D., A ternary margules-type model for plasma-slag equilibrium in the potassium-seeded coal combustion for MHD power generation: calculation and comparison with experiment, 6th International Conference on MHD Electrical Power Generation, Vol. II, p. 18, (1975).

[5] Spencer, F. E., Jr. and Orning, A. A., An Algorithm for Calculating Multiphase Chemical Equilibrium, U.S. Bureau of Mines, RI-7786 (1973).

[6] Bienstock, D., Bergman, P. D., Henry, J. M., Demski, R. J., Demeter, J. J., and Plants, K. D., Air pollution aspects of MHD power generation, 13th Symposium Eng. Asp. of MHD, Stanford Univ., paper VII. 1., March 26-28, 1973.

[7] Blackburn, P. E., GASCON and MHDGAS: FORTRAN IV Computer Codes for Calculating Gas and Condensed Phase Compositions in the Coal-Fired Open-Cycle MHD System, Argonne National Laboratory Report, ANL/MHD-77-4 (1977).

[8] Dicks, J. B., et al., Development Program for MHD Direct Coal-Fired Power Generation Test Facility, FE-1760-14, Technical Progress Report for 1975, p. 37-60 (1976).

[9] Plante, E. R., 01 son, C. D., and Negas, T., Interaction of $K_{2} 0$ with slag in opencycle, coal-fired MHD, 6th International Conference on MHD Electrical Power Generation, Vol. II, p. 211 (1975).

[10] Cook, L. P., Plante, E. R., Negas, T., Roth, R. S., and 01son, C. D., Crystallization and vaporization studies on synthetic coal slag compositions, 15th Symposium on Engineering Aspects of MHD (1976).

[11] Karr, C., Jr., Waldstein, P., and Kovach, J. J., Composition of fly ash from a coalfired MHD generator with potash seed, J. of Inst. of Fuel, 177 (1974).

[12] Bienstock, D., Demski, R. J., and Kurtzrock, R. C., High-Temperature Combustion of Coal Seeded with Potassium Carbonate, MHD Generation of Electric Power, U.S. Clearinghouse Fed. Sci. Tech. Inform., P. B. Rep. (1970), No. 190960.

[13] Hsu, C. C., Johnson, C. E., and Land, R. H., Multiphase, Multicomponent Solution Model for Computation of Equilibrium Compositions, to be published as report of Argonne National Laboratory.

[14] Gordon, S. and McBride, B., Computer Program for Calculation of Complex Chemical Equilibrium Composition, Rocket Performance, Incident and Reflected Shocks, and Chapman-Jouguet Detonations, NASA-SP-273 (1971).

[15] Hsu, Chen C., Land, Robert H., and Blander, M., Binary Solution Model for Computation of Equilibrium Compositions, Argonne National Laboratory Report, ANL-77-83 (1978).

[16] Kandiner, H. J. and Brinkley, S. R., Jr., Calculation of complex equilibrium relations, Ind. Eng. Chem. 42, 850 (1950).

[17] Stu11, D. R. and Prophet, H., JANAF Thermochemical Tables, 2nd ed., NSRDS-NBS 37 (1971).

[18] Blackburn, P. E., private communication (1977).

[19] Levin, E. M., McMurdie, H. F., and Ha11, F. P., Phase diagrams for ceramists, The Am. Ceramic Soc. (1964). 
[20] Robies, R. A. and Waldbaum, D. R., Thermodynamic Properties of Minerals and Related Substances at $298.15 \mathrm{~K}\left(25.0^{\circ} \mathrm{C}\right)$ and One Atmosphere (1.013 Bars) Pressure and at Higher Temperatures, Geological Survey Bulletin 1259, U. S. Government Printing Office, Washington (1968).

[21] Cook, L. P., Plante, E. R., Negas, T., Roth, R. S., and 01son, C. D., Crystallization and vaporization studies on synthetic coal slag compositions, 15th MHD Symposium, Philadelphia, Pa., May 1976.

[22] Plante, E. R., Some Vapor Pressure Data on $\mathrm{K}_{2} \mathrm{O}$-Containing Substances, Conference on High Temp. Sci. Related to Open-Cycle, Coal-Fired MHD System, Argonne National Laboratory, Apri1 4-6, 1977.

[23] Eliezer, N., Howald, R. H., Marinkovic, M., and Eliezer, I., Vapor pressure measurements, thermodynamic parameters, and phase diagram for the system potassium oxidesilicon oxide at high temperatures, J. Phys. Chem. 82 (9), 1021 (1978).

[24] Plante, E. R., Quarterly Report of Development, Testing and Evaluation of MHD-Materials, Apri1-June $1976^{5}$.

[25] Blander, M. and Hsu, C. C. , Calculation of Equilibria Between Gases and Condensed Phases, Conference on High Temp. Sci. Related to Open-Cycle, Coal-Fired MHD Systems, Argonne National Lab., April 4-6, 1977.

\section{Discussion}

Comment (Lee): The solution properties of the silicate system (e.g., $\mathrm{K}_{2} \mathrm{O}-\mathrm{SiO}_{2}$ ) you discussed were obtained by assuming ideal mixing of various compounds (e.g., $\mathrm{K}_{2} \mathrm{SiO}_{3}$ ) in solution which were formed from constituent oxides (e.g., $\mathrm{K}_{2} \mathrm{O}, \mathrm{SiO}_{2}$ ). This implies that the compounds are unstable to exist as their own separate phase. If they are stable, then the solution cannot be considered as a single homogeneous phase as you treated, and, depending on the number of the stable compounds, the phase rule should be taken into consideration.

The stability of the compounds can be tested by using activity data for constituent oxides you obtained by the ideal mixing model to calculate the activity of the compounds with the following example of chemical equilibria (in solution):

$$
\mathrm{K}_{2} \mathrm{O}+\mathrm{SiO}_{2}=\mathrm{K}_{2} \mathrm{SiO}_{3}, \mathrm{~K}=\frac{{ }_{K_{2}} \mathrm{SiO}_{3}}{{ }_{\mathrm{K}_{2} \mathrm{O} \cdot{ }^{\mathrm{a}} \mathrm{SiO}_{2}}}
$$

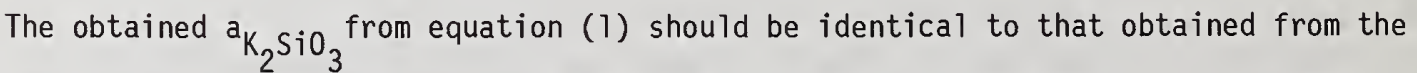
ideal mixing model, if the free energy minimization method is consistent with chemical equilibria in solution. Otherwise, the solution cannot be treated as single phase.

\footnotetext{
${ }^{5}$ See footnote 4 on previous page.
} 
Response (Hsu): Actually, the compounds will not be very stable if the mixture is only the metal oxides, because at high temperature they will react to form a stable compound or compounds, and those are the solution components to form.

Moderator (Blander): If I could just interject to help answer the phase rule question, it (the Hsu method) doesn't violate the phase rule because there is a minimization procedure, and there is an internal equilibrium between all of these different species, and that puts the right number of constraints into it. So, it doesn't violate the phase rule, because each of these species have some relative free energy of formation which goes into an internal equilibrium in the minimization procedure.

This is a very common procedure in geology, where geologists don't know the structure or how to handle the thermodynamics of complex solutions, and every few years a new model pops up in geology, but they never really work over a wide range of compositions, let us say from acidic to basic melts, they don't work. But in a narrow range, they seem to work. And you don't violate the phase rule, if you have these internal equilibria also set up in computer programs. 
National Bureau of Standards Special Publication 561, Proceedings of the 10th Materials Research Symposium on Characterization of High Temperature Vapors and Gases held at NBS, Gaithersburg, Maryland, September 18-22, 1978. Issued October 1979.

\title{
COMPUTER MODELING OF GAS-CONDENSED PHASE EQUILIBRIA: PRESENT STATUS AND SOME FUTURE NEEDS IN SCIENCE AND TECHNOLOGY
}

\author{
Milton Blander \\ Chemical Engineering Division \\ Argonne National Laboratory \\ Argonne, IL 60439
}

\section{Introduction}

The purpose of this discussion is to bring to a focus the possible uses and needs for a central capability for computer calculations of equilibria between high temperature multicomponent vapors and condensed phases. Such calculations have proven to be invaluable in science and in a number of technologies (e.g. metallurgical processing, fluidized bed coal combustion, hot corrosion of turbines, coal-fired MHD generators, chemical processing, cosmochemistry, rocket design, coal gasification, etc.) $[1-13]^{1}$ and are used in at least three chapters in this volume. The methods of calculation involve a computer program which is usually based on the minimization of the free energy of a system and which is coupled with a thermodynamic data base to provide a means for calculating equilibrium phases and their compositions. The use of such programs has greatly expanded the usefulness of thermodynamic data on vapor species. There have been a number of independent computer programs which have been developed, each tailored to specific needs. A great deal of redundancy and wasted effort is inherent in these separate efforts and their success has been limited to laboratories able to devote adequate manpower to setting up and maintaining such programs. A considerable fraction of the effort has been expended on accumulating and updating the different data bases. One can broaden the number of users of such programs and the use of thermodynamic compilations by the development of a central calculational capability which would be easily accessible and which would be coupled to a data base which is being actively updated. Such a capability would decrease the present redundancy in setting up such programs and should provide a large central data base in a useful format. In this discussion, I will examine some typical uses for such programs in order to define the needed capabilities. The range of complexity of the problems to be solved is very broad ranging from simple systems in which a single equilibrium predominates to complex multicomponent systems in which several condensed phases form, with some of these being solution phases. The computer programs should handle all of these possibilities as well as the possibility of departures from equilibrium. One fallout of such a central capability would be an increase in the utility of thermodynamic data banks and a further justification for their existence and growth.

${ }^{1}$ Figures in brackets indicate the literature references at the end of this paper. 


\section{Examples of Problems}

Perhaps the simplest type of problem is one in which the formation of a single solid product from a complex multicomponent gas mixture is the predominant condensation reaction. For example, the formation of sodium sulfate condensates from coal combustion effluents goes according to the reaction $[5,6,7]$

$$
2 \mathrm{NaCl}+\mathrm{SO}_{2}+\frac{1}{2} \mathrm{O}_{2}+\mathrm{H}_{2} \mathrm{O} \underset{\leftarrow}{\leftarrow} \mathrm{Na}_{2} \mathrm{SO}_{4}(\mathrm{~s} \text { or } \ell)+2 \mathrm{HCl}
$$

where the reaction could have been written with $\mathrm{CO}_{2}$ rather than $\mathrm{O}_{2}$ as the oxidizing agent. Such differences are, of course, not significant in an equilibrium calculation but would be important when one is also concerned with kinetic factors. These alkali sulfates are directly related to the severity of corrosion of turbines or other metal parts in the path of the effluent stream. Such simple reactions can be.handled by available computer programs [1] which can also handle the formation of a number of other pure condensed phases. The importance of such calculations is to define the compositions and temperatures at which a condensate forms and the state of the condensate (solid or liquid). Corrosion can be minimized by fixing conditions or introducing additives so that liquids do not form.

The next level of complication occurs with the possibility of the formation of solutions. For example, if both potassium and sodium are present in coal combustion effluents (as would be the case for a coal-fired MHD generator) [3] reactions such as eq. (1) would lead to the formation of a solution of $\mathrm{K}_{2} \mathrm{SO}_{4}$ and $\mathrm{Na}_{2} \mathrm{SO}_{4}$. Therefore, one must incorporate information on the solution chemistry into the calculation. For such a simple binary solution the free energy of the solution is

$$
\underline{G}=n_{1} G_{1}^{0}+n_{2} G_{2}^{0}+2 R T\left(n_{1} l n X_{1}+n_{2} \ln X_{2}\right)+n \Delta G_{m}^{E}
$$

where $n_{i}$ is the number of moles of component $i ; x_{i}$ is the ion fraction of the cation of component $i ; n=\Sigma n_{j}$; the 2 in front of the logarithmic terms comes from the fact that the alkali sulfates have two cations; and $\Delta G_{m}^{E}$ is the excess free energy of mixing per mole which, for a binary system, is usually expressed as

$$
\Delta G_{m}^{E}=\sum_{m=1} \sum_{n=1} a_{m n} x_{1}^{m} x_{2}^{n}
$$

For an ideal solution $\Delta G_{m}^{E}$ is zero. It is usually advantageous to express $\Delta G_{m}^{E}$ in a simple and short expression and, if possible the form used should be chosen to accomplish this. The results of such calculations must be coupled with phase diagram information to determine whether liquids or solids will be present. An independent thermodynamic analysis of the condensed phase diagram would be necessary for a complete analysis. A simple comparison of calculations with the measured phase diagram can often lead to a useful understanding of the chemistry. 
Similar problems occur in cosmochemistry where one is concerned with condensation from a hot solar nebula [9-12]. One has the possibility of forming binary silicate solid solutions by reactions such as

$$
\begin{aligned}
& 2 \mathrm{Mg}+\mathrm{SiO}+3 \mathrm{H}_{2} \mathrm{O} \underset{\leftarrow}{\mathrm{Mg}} \mathrm{SiO}_{4} \text { (s.s.) }+3 \mathrm{H}_{2} \\
& 2 \mathrm{Fe}+\mathrm{SiO}+3 \mathrm{H}_{2} \mathrm{O} \stackrel{\rightarrow}{\leftarrow} \mathrm{Fe}_{2} \mathrm{SiO}_{4} \text { (s.s.) }+3 \mathrm{H}_{2}
\end{aligned}
$$

The chemistry of such condensates is of primary importance in deducing the origins of our solar system. An even more complex possibility lies in deducing the mode of formation of refractory metal particles in certain meteorites [13]. Ten different refractory metals, 0s, $\mathrm{Re}, \mathrm{W}, \mathrm{Mo}, \mathrm{Ir}, \mathrm{Ru}, \mathrm{Pt}, \mathrm{Fe}, \mathrm{Ni}$, and $\mathrm{Rh}$, are found in various metal particles and thermodynamic calculations for a ten component solution are necessary to explain their modes of occurrence. For example, figure 1 gives an example of such calculations if all these metals formed an ideal solution. For a complete picture, one would have to incorporate non-idealities and take into account the possibility of the formation of more than one metallic phase (fcc, bcc and hcp). Thus, in the final analysis, one would have to take into account not only gas-condensed phase equilibria but also equilibria between different condensed phases. Such a complex calculation has never been attempted.

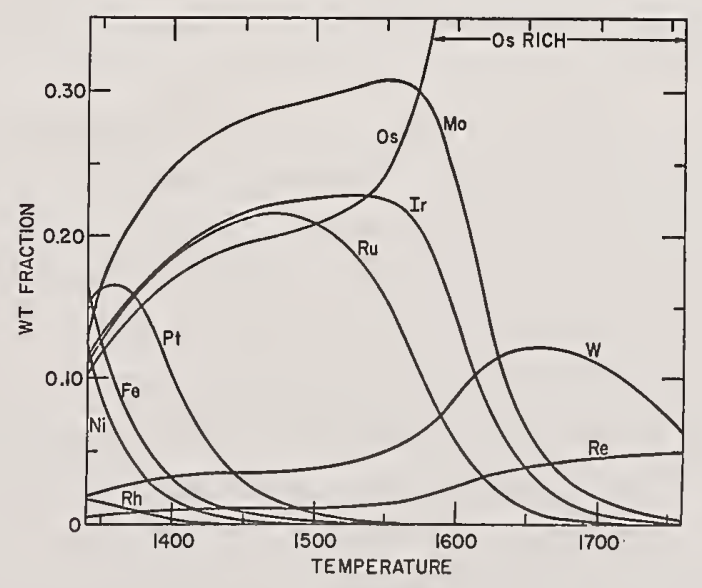

Figure 1. Predicted ideal solution properties of a ten component condensed system.

Complex types of solution chemistry would require more sophistication. For example, in the development of advanced fuel cells with molten alkali carbonates as the electrolyte 
one initally has a melt of carbonates (e.g., $\mathrm{Li}_{2} \mathrm{CO}_{3}+\mathrm{K}_{2} \mathrm{CO}_{3}$ or $\mathrm{Li}_{2} \mathrm{CO}_{3}+\mathrm{Na}_{2} \mathrm{CO}_{3}+\mathrm{K}_{2} \mathrm{CO}_{3}$ ) and a gas containing among other things, $\mathrm{CO}_{2}, \mathrm{H}_{2} \mathrm{O}, \mathrm{O}_{2}$, and $\mathrm{SO}_{2}$. Equilibria between the gas phase and the melt can produce other anionic species such as $\mathrm{OH}^{-}$and $\mathrm{SO}_{4}=$ by equilibria such as

or

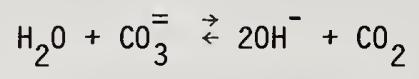

$$
\mathrm{SO}_{2}+\frac{1}{2} \mathrm{O}_{2}+\mathrm{CO}_{3}=\overrightarrow{\mathrm{SO}}=\mathbf{4}+\mathrm{CO}_{2}
$$

The solution chemistry of such systems requires additional terms to take into account the fact that there are at least two cations and two anions

$$
\Delta G_{m}=\sum_{a} n_{a} R T \ln X_{a}+\sum_{c} n_{c} R T \ln X_{c}+n \Delta G_{m}^{E}
$$

where $\Delta G_{m}$ is the free energy of mixing, $X_{a}$ and $X_{c}$ are anion and cation fractions and $\Delta G_{m}^{E}$ is the molar excess free energy of mixing which is known theoretically and can be expressed in terms of the ion fractions and of parameters deduced from lower order systems. Perhaps an even more complex type of solution is that formed by the silicates. For example, the system $\mathrm{CaO}-\mathrm{SiO}_{2}-\mathrm{Al}_{2} \mathrm{O}_{3}-\mathrm{MgO}-\mathrm{FeO}$ is important as a slag phase in coal combustion, in an MHD generator, in steel-making and in possible new processes for making aluminum. It is also important in cosmochemistry as a condensate from a nebula. The thermodynamic properties of such liquids are quite complex and require the development of methods for their accurate representation.

One must also be concerned about multiphase equilibria between very different types of phases. For example, in order to deduce the probable disposition of the trace pollutants, mercury, lead and cadmium, in the effluents from coal combustion, one must consider all the possible phases (e.g., oxide, sulphide) into which these toxic heavy metals can condense. Apparently these metals condense onto the surfaces of particulates. To deduce the details of their chemistry would require extensive equilibrium calculations. In cosmochemistry, different trace elements tend to distribute themselves between metallic (siderophile), silicate (lithophile) and sulphide (chalcophile) phases and some appear to have condensed onto available surfaces. The relative chemical stabilities, the types of other materials in the system, and the surface areas of particulates determine the phases with which such trace elements are associated and fixes their chemistries and their temperatures of condensation.

In addition, one must also consider kinetic factors in discussing gas condensed phase reactions. To perform this, one must place constraints on the equilibrium calculations. For example, metallurgical stack gases can contain sio which, if no other oxides tend to form, would condense as crystalline silica. Observations indicate that only a glass forms probably because of a nucleation constrair.t on the formation of a crystalline phase. Consequently one must place a constraint on the crystalline form and permit the formation of the glass. Similarly, in cosmochemistry one considers constraints on the formation of metal which is prevented by nucleation barriers. Because of this, the chemical potential 
of metal is higher than equilibrium and the chemistry changes to such an extent that sulfides can be formed instead of metal. If such constraints are essentially time independent they can be readily incorporated into an equilibrium calculation by the omission of the blocked equilibrium phases. Time dependent constraints are more difficult to incorporate but can be handled if products which are formed without any kinetic constraints are treated as if they are at equilibrium whereas the formation of the products of kinetically constrained reactions are considered separately. However, such computations can be expensive to run, especially if more than one reaction has such constraints. A sophisticated effort would be needed to minimize the cost of such calculations.

\section{Projected Needs}

From the discussion in the last section we can deduce some of the capabilities which would be useful in a centralized computer program.

1. A broad complete thermodynamic data base is required on both vapor phase and condensed species. A large fraction of the work expended in compiling and updating the data base and entering it into the computer program could be saved for an individual researcher.

2. The capability for calculations of equilibria between a multi-component gas phase and several condensed phases some being solution phases.

3. The capability of an accurate representation of complex solution chemistry in nonideal solution phases. This requires a flexibility which permits the incorporation of a variety of ways of expressing non-ideal solution behavior.

4. Simple formats for easy access and use. In addition, simple, self-explanatory and interactive means of using the program are desirable so as to attract even occasional users. Adaptability to different computers would be desirable to make the program transferable to heavy users.

My own experience with the extension of programs has pointed up the amount of time needed by an individual researcher to modify a known program tailored for other uses and to add to its data base. The development of computer programs that can be used "off the shelf" will greatly increase the uses of such programs and will broaden the usefulness of thermodynamic data on vapor species. The creation of a single complete data base in a unified readily accessible format will save considerable time even for those researchers who use a different computer program. In addition, the use of the program in a centralized capability can be, at least in part, documented and help to provide a justification for thermodynamic research. The recent focus of research support on mission oriented programs has fragmented work involving thermodynamics and, for the case of calculations such as I have discussed, has led to a fair degree of wasteful redundance. 
[1] Gordon, S. and McBride, B. J., Computer program for calculation of complex equilibrium compositions, rocket performance, incident and reflected shocks and Chapman-Jouguet detonations, NASA SP 273 (1971).

[2] Spencer, Jr., F. E., Orning, A. A., and Bienstock, D., Applications of a high temperature chemical equilibrium program to problems in coal combustion, AICHE Symp. Ser. 70 , (137) 522-8 (1974).

[3] Spencer, Jr., F. E., Hendrie, Jr., J. C., and Bienstock, D., A ternary margules-type model for plasma/slag equilibrium in potassium seeded coal combustion for magnetohydrodynamic power generation: calculations and comparison with experiment, Proc. 6th Int'1. Conf. on MHD Electric Power Generation, Washington, D.C., Vol. 2, p. 181 (1975).

[4] Harvey, F. J., The application of complex equilibria calculations to the design of high temperature processes. Presented at a Symposium on Materials Stability in Energy Technology, TMS-AIME fall meeting, Chicago (1977).

[5] Spacil, H. S. and Luthra, K. L., Corrosive environment resulting from alkali metals and trace contaminants in the coal fired combined cycle gas turbine, General Electric Research and Development Center to be issued as a report to DOE (1978).

[6] Spacil, H. S. and Luthra, K. L., Thermochemical model for a coal fired pressurized fluidized bed/gas turbine combination, presented at a Symposium on Materials Stability in Energy Technology TMS-AIME fall meeting, Chicago (1977).

[7] Elliott, J. F. and Yurek, G. J., Formation of inorganic aerosols in coal combustion systems, Ibid.

[8] Goto, S., Equilibrium calculation of multicomponent systems between non-homogeneous phases-equilibrium between matte, slag and gaseous phases, J. Fac. Eng. Univ. Tokyo Ser A., 14, 58-9 (1976).

[9] Lewis, J. S., Metal/silicate fractionation in the solar system, Earth Planetary Sci. Lett. 15, 286-290 (1972).

[10] Lewis, J. S., Low temperature condensation from the solar nebula, Icarus, 16, 241 (1972).

[11] Grossman, L., Condensation in the primitive solar nebula, Geochim. Cosmochim. Acta, $\underline{36}$, 577-619 (1972).

[12] Blander, M., Hsu, C. C., and Fuchs, L. H., Quantitative computer calculations of constrained equilibria in the formation of chondritic meteorites, 11, 251 (1976).

[13] Blander, M., Horowitz, C., and Land, R., Refractory metal condensation from a nebula, 41 st Annual Meeting of the Meteoritical Society, Sudbury, Ontario, August 1978.

\section{Discussion}

(Editor's note): The purpose of Dr. Blander's paper was to introduce the topic of current needs for generally available computer programs capable of handling multicomponent equilibria problems, and to serve as a stimulus for general discussion on this question. 
Comment (Dewing): Since we are invited by the Moderator to promote discussion, I will be happy to cooperate. And the first question I would like to raise is the one that Milt Blander mentioned, the question about up-dating the data base. Now, from a scientific point of view, this is undoubtedly desirable. One always wants the base figures. But from a calculational point of view, I can see it being an absolute menace. If, in the middle of doing some calculations on a certain system, the data base is updated, and the calculations as of next Monday are working on a different base from the ones I do today, you can see all the problems that can arise. And this may even be spread over quite long periods of time. I can readily envisage that I do some calculations on a certain system today, and in a year's time, someone says we11, suppose we don't operate at one atmosphere, suppose we go up to one and a quarter. What difference does it make? And you might find there would be some quite dramatic differences in changing your pressure a little bit. But really, what has happened is that someone has changed the data base during that year, or it might be two years.

So, changes in the data base, although, as I say scientifically desirable, are very much a loaded gun. But secondly, there is the whole question of the general philosophy of approach, because obviously a computer program that is capable of answering any question I might care to ask is going to be so unwieldy, that in fact, it is going to be virtually unusable, unless one has access to the very largest computers. And even then, one is going to be using them very inefficiently. One can see immediately that a program which is capable of putting a man on the moon, whether it can get me to National Airport by 1:00, I don't know, but even if it does, obviously I am not using the major part of that program which I have stored away in the computer and am paying for everything else.

Up to now, at any rate, my personal experience has been that it is easier to write special purpose programs which are fairly compact, fairly efficient and also deal strictly with the limited system which one is considering, than it is to get some all-purpose monster that may or may not deliver when it is required to do so.

However, what might be very desirable to have, if not standardized, at least readily available, would be some logic and algorithms for tackling specific questions, such as the one, do I have a liquid, or do I have a solid and if it is a liquid, what is its composition? This is the kind of question that often comes up, and I kind of solve it on a hand-to-mouth basis each time. But if someone could develop some standard algorithms which could conceivably be available in the form of subroutines, which could be plugged into any other main program, this I think might be useful.

Response (Blander): I think on your first point about the data base, that that is just an organizational thing. Just have data bases which are updated at a particular time of year or whatever, just a seasonal data base, where you know that on January ]st, there will be new data, so if you want to keep the old data, just somehow keep it in, perhaps, and keep the old data in a data base which you can tap into, if you want to. Or, if you have a program, on your own computer. 
A central capability does not necessarily just have to be a very complex program. It can be a selection of programs, some of which are simple, and some of which are more difficult. For an occasional user, I think it is cheaper to say call a central place in Washington or Berkeley, or whereever, a program like this might be sought, it is easier to get them on the telephone line and tap into it, if you are using it once a year, and I think it is a lot cheaper than to write a program and run it your self. It is much easier to call it on the telephone for an occasional user. So, I don't think it is a problem. Even if you want a simple computer program, it is much easier to tap into a collection of computer programs of different degrees of complexity.

Now, the last question you raised a complex form, such a program would automatically give it to you, because you can put into it all the phase diagram information in principle. You can analyze the phase diagram first, get all the parameters you need, and put it in, and it will tell you if it is a liquid or a solid. It can do that, and it is a fairly standard thing. It is being done in another context. People who look at liquid/solid phase equilibrium do that all the time.

Question (Haas): Doesn't that require you to know the answer before you start, if you know how to draw the phase diagram, you know the equation of state.

Response (Blander): No, you don't -- not at the gas phase involved. And if you know the liquid/solid equilibrium, you can do an independent analysis of that and that is getting a lot of programs available, then you are stuck, and you use what you have. But very often, in the silicate system, for example, you have all these solid compounds and for many of those, you know the standard free energies of formation. There is data at some temperatures in some regions of that system that have been measured. There are other binary systems where data is available, and there are independent computer programs now.

Comment (Horton): I do agree with you that it would be nice to have a program which does these various things and that everybody could get a hold of. I would be for a program which was strictly the equilibrium composition part. I think one of the difficulties that I have found with the NASA program is that there is an awful lot of other stuff in there. I surely don't want to know anything about shock waves and anything like that.

Now, the problem can certainly be handled, I think, very nicely by having a central program of the kind described and anybody that wants subroutines in the central place, the subroutines could be made available for these other things. Remark number one. Number two, perhaps you are aware that there is a program by Erickson (Swedish program) which handles condensed phase solutions with non-ideal parameters, if you have the parameters.

About data bases, I thought I would mention that there is an even further one in its birth pangs at the moment, the IUPAC Commission on High Temperature Chemistry is putting together a high temperature thermochemical data base, under the leadership of Ben Alcock of the University of Toronto, and what is going to be included are the various heats of formation, entropies, standard entropies at $298 \mathrm{~K}$, and the heat capacities in the form somewhat like they are in the Gordon and McBride (NASA) program. That thing is going to be updated as fast as it can be updated. 
Comment (Hastie): While we are talking about the data base, the problem that Dr. Dewing mentioned about the change in the basic data every so often is a problem we have right now with the JANAF tables. Sometimes you will see some calculated data in the literature based on the JANAF tables, but the authors won't specify which date for the table they used, and you have to really work hard to figure out which particular set of several updatings of the JANAF tables were being used. So, people are abusing, or are not properly recognizing the changing nature of basic thermodynamic data.

Comment (Hsu): With regard to the question raised about the bulky NASA program. It is, according to my experience, very easy to get rid of those other applications in the program.

According to the proposal for centralization of utility programs which are accessible for general use, I think this is very attractive and desirable. My question is who is going to do this?

I have another question. My experience on the data base is that it is not difficult to add species to the data base, besides the NASA program also has a different program to evaluate those coefficients of thermo-chemical data. So, if one wanted to add a species into the data base, one has to just get the thermo-chemical data and then run the program through. You will get all the coefficients instead of forms, and then you input what is added to the data base.

Comment (Kohl): Bonnie McBride and Sanford Gordon of NASA program fame are, at this very time, seriously considering starting a major effort into developing like the Stage 2 NASA Program, which could handle simple ideal solutions. What they need to get this going is support (moral). If they had anywhere near the support that we have heard at a conference like this, people referring to the NASA Lewis program, if they could get letters asking them to do this and discussing this and kind of complimenting them on the past efforts, they would have enough ammunition to be able to carry out what they would really like to do, and that is to develop sort of the second plateau here, that is very desirable.

Comment (written) (Kohl and Stearns): A description of the multicomponent gas phase equilibrium and condensation phenomena has been carried out to describe the formation of gaseous and condensed phase $\mathrm{Na}_{2} \mathrm{SO}_{4}$ in gas turbine engine hot section environments

[C. A. Stearns, F. J. Kohl and G. C. Fryburg in "Properties of High Temperature Alloys," Z. A. Foroulis and F. S. Pettit, eds., p. 655, The Electrochemical Society Softbound Symposium Series, Vol. 77-1, Princeton, N.J. (1976); also NASA TM X-73476 (1976)]. Measured thermodynamic data for $\mathrm{Na}_{2} \mathrm{SO}_{4}(\mathrm{~g})$ was included in the NASA chemical equilibrium computer program that was used in this study. The CEC program has also been used to examine the formation of $\mathrm{Na}_{2} \mathrm{SO}_{4}(\mathrm{~g})$ and $\mathrm{K}_{2} \mathrm{SO}_{4}(\mathrm{~g})$ in flames [G. C. Fryburg, R. A. Miller, C. A. Stearns, and F. J. Kohl in "High Temperature Metal Halide Chemistry," D. L. Hildenbrand and D. D. Cubicciotti, eds., The Electrochemical Society Softbound Symposium Series, Vo1. 78-1, Princeton, N.J. (1978), pp. 468-483; also NASA TM 73794 (1977)] and to elucidate condensation phenomena for $\mathrm{Na}^{-}$and $\mathrm{S}$-seeded flame systems (see chapter by Rosner et al. elsewhere in this volume). 



\section{SYMPOSIUM PARTICIPANTS}

V. 0. Altemose

Corning Glass Works

Sullivan Park

Corning, NY 14830

Robert M. Atkins

Yale University

Mason Lab. 9 Hillhouse Ave.

New Haven, CT 06520

Giovanni Balducci

University of Rome

Istituto di Chimica Fisica

Rome, Italy 00100

Eugene John Barber

Union Carbide Corp. - Nuclear Div.

P.0. Box P

0ak Ridge, TN 37763

Ezra Bar-Ziv

Israel Atomic Energy Commission

Nuclear Research Center-Negev

P.0. Box 9001

Beer-Sheva, Israel

Leslie E. Bauman

Mississippi State Univ.

P.0. Box 5167

Mississippi State, MS 39762

I. R. Beattie

Univ. of Southampton

Dept. of Chemistry

Southampton S09 5NH

United Kingdom

James $H$. Bechtel

General Motors Research Lab.

Physics Dept.

Warren, MI 48090

Robert G. Behrens

Los Alamos Scientific Laboratory

P.0. Box 1663

Los Alamos, NM 87545

Joseph Berkowitz

Argonne National Laboratory

Physics Division

9700 S. Cass Ave.

Argonne, IL 60439

Richard Beyer

Aberdeen Proving Ground

Aberdeen, MD 21005
Paul Blackburn

Chemical Engineering Division

Argonne National Laboratory

Argonne, IL 60439

Milton $\mathrm{Bl}$ ander

Argonne National Laboratory

9700 S. Cass Ave.

Argonne, IL 60439

John Blanks

Alcoa Aluminum Company of America

Alcoa Technical Center

Alcoa, PA 15069

Mary Blech

General Electric

Nela Park

$\mathrm{Cleveland}, \mathrm{OH} \quad 44112$

David Bonne 11

National Bureau of Standards

Center for Materials Science

Washington, DC 20234

Jean Bouix

University of Lyons

43 boulevard du 11 novembre 1918

69621 Villeurbanne, France

James N. Bower

University of Florida

5 Leigh Hall

Gainesville, FL 32601

Allen Bowling

Univ. of Tennessee

Dept. of Chemistry

Knoxville, TN 37916

John Bradshaw

University of Florida

109 Leigh $\mathrm{Ha} 1 \mathrm{l}$

Gainesville, FL 32611

Leo Brewer

Univ. of California

Dept. of Chemistry

Berkeley, CA 94720

E. David Cater

University of Iowa

Dept. of Chemistry

Iowa City, IA 52242 
I. Chabay

National Bureau of Standards

B1dg. 222, Rm. A121

Washington, DC 20234

Calvin Chan

Univ. of California - Berkeley

Dept. of Mechanical Engineering

Berkeley, CA 94720

Malcolm W. Chase

The Dow Chemical Company

Thermal Research, 1707 B1dg.

Midland, MI 48640

Christian Chatillon

LTPCM/ENSEEG

A324, Physics

Washington, DC 20234

Jack H. Colwell

National Bureau of Standards

A324, Physics

Washington, DC 20234

Alan Covington

NASA - Ames Research Center

Mail Stop 234-1

Moffett Field, CA 94035

Tom Coyle

National Bureau of Standards

Center for Materials Science

Washington, DC 20234

John Creighton

Lawrence Livermore Laboratory

P. O. Box $808(L-35)$

Livermore, CA 94550

David R. Crosley

Ballistic Research Laboratory

Aberdeen Proving Ground

Aberdeen, MD 21005

Larry A. Curtiss

Chemical Engineering Div.

Argonne National Laboratory

Argonne, IL 60439
John W. Daily

University of California

Mechanical Engineering

6163 Etcheverry Hall

Berkeley, CA 94720

Mark A. Dewilde

U.S. Army Ballistic Research Labs

Aberdeen Proving Grounds

Aberdeen Proving Grounds, MD 21030

Ernest W. Dewing

Aluminum Co. of Canada

Research Centre

Kingston, Ontario

Canada K7L $4 Z 4$

Ray Dils

National Bureau of Standards

Center for Mechanical Engineering

and Process Technology

Washington, DC 20234

J. R. Downey

Dow Chemical USA

1707 Building

Midland, MI 48640

Michael C. Drake

National Bureau of Standards

B1dg. 223, Rm. A329

Washington, DC 20234

Alan C. Eckbreth

United Technologies Res. Ctr.

Silver Land

East Hartford, CT 06108

Thomas L. Eddy

West Virginia Univ.

Dept. Mech. Engr. \& Mechanics

Morgantown, WV 26506

Jimmie G. Edwards

Univ. of Toledo

Dept. of Chemistry

Toledo, $\mathrm{OH} 43606$

John R. Ferron

Dept. of Chem. Eng.

Univ. of Rochester

Rochester, NY 14627

A. Fontijn

Aerochem Research Lab. Inc.

P.0. Box 12

Princeton, NJ 14627 
R. M. Fristrom

Applied Physics Laboratory

Johns Hopkins University

8621 Georgia Ave.

Silver Spring, MD 20910

F. W. Froben

Freie Universitat Berlin

FB20, WE2, Boltzmannstrasse 20

1000 Berl in 33, Germany

David J. Frurip

Argonne National Laboratory

B 1dg. 205

Argonne, IL 60439

Richard G. Gann

National Bureau of Standards

Center for Fire Research

Washington, DC 20234

W. C. Gardiner

Univ. of Texas

WEL103W

Austin, TX 78712

Michael Gersh

Aerodyne Research, Inc.

Crosby Dr.

Bedford, MA 01730

Paul W. Gilles

Univ. of Kansas

Dept. of Chemistry

Lawrence, KS 66045

Karl Gingerich

Texas A \& M Univ.

Dept. of Chem.

College Station, TX 77843

James L. Gole

Georgia Inst. of Technology

Chemistry Dept.

Atlanta, GA 30332

William R. M. Graham

Dept. of Physics

Texas Christian Univ.

P. 0. Box 29160A

Ft. Worth, TX 76129

Frank T. Greene

Midwest Research Inst.

425 Volken Blvd.

Kansas City, MD 64410

Robert T. Grimley

Purdue University

Dept. of Chemistry

W. Lafayette, IN 47907
Earl A. Gulbransen

Dept. of Metallurgical and

Materials Engineering

Univ. of Pittsburgh

Pittsburgh, PA 15261

L. Haas

National Bureau of Standards

Washington, DC 20234

Jeffrey C. Halle

Naval Research Lab.

Code 6130 - Chemistry Div.

Washington, DC 20375

D. R. Hardesty

Combustion Research Div.

Sandia Laboratories

Livermore, CA 94550

John Hastie

National Bureau of Standards

Center for Materials Science

Washington, DC 20234

R. H. Hauge

Rice University

Dept. of Chemistry

Houston, TX 77001

D. L. Hildenbrand

SRI International

Menlo Park, CA 94025

James C. Hindman

Argonne National Laboratory

8700 So. Cass Ave.

Argonne, IL 60439

C. Hirayama

Westinghouse Research \&

Development Center

1310 Beulah Road

Pittsburgh, PA 15235

John A. Holy

Monsanto Research Corp.

P. 0. Box 32, A- 158

Miamisburg, $\mathrm{OH} 45542$

William Horton

National Bureau of Standards

Center for Materials Science

Washington, DC 20234

Chen-Chao Hsu

Argonne National Laboratory D-205, 9700 S. Cass Ave.

Argonne, IL 60439 
L. W. Hunter

Applied Physics Laboratory

Johns Hopkins Rd.

Laurel, MD 20810

Marilyn E. Jacox

National Bureau of Standards

Molecular Spectroscopy Div.

Washington, DC 20234

Larry Jeffers

Babcock Wilcox Co.

1562 Beeson St.

Alliance, $\mathrm{OH} 44601$

Jack G. Kay

Drexel Univ.

32nd \& Chestnut Streets

Philadelphia, PA 19104

Werner Klotzbucher

Univ. of Toronto

Erindale College

3359 Mississauya Rd.

Mississauya, Ont. L5L 1C6

Lon B. Knight, Jr.

Furman Univ.

Chemistry Dept.

Greenville, SC 29613

F. Kohl

NASA Lewis Research Ctr.

21000 Brookpark Road

Cleveland, $\mathrm{OH} 44135$

Charles Kolb

Aerodyne Research, Inc.

Crosby Drive

Bedford, MA 01730

Mitsuo Koshi

University of Tokyo

Hongo, Bunkyo-ku, Tokyo

Japan 113

Ralph Krause

National Bureau of Standards Center for Materials Science Washington, DC 20234

Steven C. Lamey

U.S. Dept. of Energy

Morgantown Energy Tech. Ctr.

P.0. Box 880

Morgantown, WV 26505

Kenneth C. Lapworth

National Physical Laboratory (U.K.)

Teddington, England TW11 9LJ
Thomas W. Lassiter

Dept. of Chemistry

Univ. of Tennessee

Knoxville, TN 37916

L. Leibowitz

Argonne National Laboratory

9700 S. Cass Avenue

Argonne, IL 60439

Young F. Lee

Union Carbide Corp.

Metals Division

4625 Royal Ave.

Niagara Falls, NY 14302

D. R. Lide, Jr.

National Bureau of Standards Office of Standard Reference Data Washington, DC 20234

M. C. Lin

Naval Research Laboratory

Code 6110

Washington, DC 20375

Sing-Shong Lin

Army Mat. \& Mech. Research Ctr. Arsenal St.

Watertown, MA 02172

Kenneth A. Lincoln

NASA - Ames Research Ctr.

Mail Stop 234-1

Moffett Field, CA 94035

Frank Lovas

National Bureau of Standards

Center for Thermodynamics and

Molecular Science

Washington, DC 20234

Kenneth McCulloh

National Bureau of Standards

Center for Thermodynamics \&

Molecular Science

Washington, DC 20234

J. R. McDonald

Naval Research Laboratory

Chemistry Division

Washington, DC 20375

Michael E. McIlwain

Argonne National Laboratory

9700 So. Cass Ave.

Argonne, IL 60439 
Julius L. Mack

Dept. of Chemistry

Univ. of D.C.

1331 H. St. NW

Washington, DC 20005

G. Mallard

National Bureau of Standards

Washington, DC 20234

Thomas B. Malloy, Jr.

Mississippi State Univ.

P.0. Box 5167

Mississippi State, MS 39762

Joseph F. Masi

AFOSR/NA

Bldg. 410, Bolling AFB

Washington, DC 20332

Lynn A. Melton

The Univ. of Texas at Dallas

Chemistry Department, Box 688

Richardson, TX 75080

Federico Miller

Laredo Junior College

West End Washington St.

Laredo, TX 78040

Glenn H. Miller

Sandia Laboratories

Division 5216

P. 0. Box 5800

Albuquerque, NM 87185

William Miller

Aero Chem Research Labs

P.0. Box 12

Princeton, NJ 08540

Thomas A. Milne

Solar Energy Research Inst.

1536 Cole Blvd.

Golden, C0 80401

J. W. Mitchell

Bel1 Laboratories

MH 10239 Bell Labs

Murray Hill, NJ 07974

Barbara A. Mueller

Los Alamos Scientific

Laboratory MS-348

P.0. Box 1663

Los Alamos, NM 87645

Clifford E. Myers

Dept. of Chemistry

State Univ. of New York

at Binghamton

Binghamton, NY 13901
Stephen Nicolosi

Brookhaven National Laboratory

Upton, NJ 11973

J. S. Ogden

Univ. of Southampton

Dept. of Chemistry

Southampton S09 5NH

United Kingdom

Roland W. Ohse

Commission of the European

Communities

7500 Karlsruhe 1, postfach 2266

Federal Republic of Germany

George N. Papatheodorou

Argonne National Laboratory

CEN-205

Argonne, IL 60439

Ernest Plante

National Bureau of Standards

Center for Materials Science

Washington, DC 20234

John W. Reed

Monsanto Research Corporation

P.0. Box 32, A-158

Miamisburg, $\mathrm{OH} 45342$

James J. Reuther

Fuel Science Section

The Pennsylvania State Univ.

316 Steidle Bldg.

University Park, PA 16802

Gary H. Rinehart

Los Alamos Scientific Lab.

P.0. Box 1663

Los Alamos, NM 87545

Bruce Rising

Penn State University

316 Steidle Bldg.

University Park, PA 16802

W. M. Roquemore

Air Force Aero Propulsion Lab.

Wright-Patterson AFB, $\mathrm{OH} 45433$

Gerd M. Rosenblatt

The Pennsylvania State Univ.

Dept. of Chemistry, 152 Davey Lab.

University Park, PA 16802

Daniel E. Rosner

Yale University 319 ML

New Haven, CT 06520 
Paul Roth

Univ. of Duisburg

Kommandautenstr.60, 41 Duisburg 1

Duisburg, Germany

D. Sanders

National Bureau of Standards

B1dg. 223, Rm. B328

Washington, DC 20234

T. Sasamoto

Mass. Inst. Tech.

MIT, Ceramic Div.

Rm. 13-4057

Cambridge, MA 02139

Peter K. Schenck

National Bureau of Standards

Rm. A329, Bldg. 223

Washington, DC 20234

Tom Sedgwick

IBM Research

P. 0. Box 218

Yorktown Hts., NY 10598

Sidney A. Self

Stanford University

Dept. of Mechanical Engineering

Stanford, CA 94305

Walter M. Shaub

2027 Winged Foot court

Reston, VA 22091

Richard R. Smardzewski

Naval Research Laboratory

Code 6130

Washington, DC 20375

Richard D. Smith

Battelle-Northwest

Bldg. 329 - 300 Area

Richland, WA 99352

John E. Spencer

General Electric Co.

Nela Park

Cleveland, $\mathrm{OH} 44112$

David R. Squire

U.S. Army Research office

P.0. Box 12211

Research Triangle Park, NC 27709

Carl A. Stearns

NASA - Lewis Res. Ctr.

21000 Brookpark Road

Cleveland, $\mathrm{OH} \quad 44135$
Martin Steinberg

Quantum Institute

Univ. of California

Santa Barbara, CA 93106

Kurt H. Stern

Naval Research Laboratory

Code 6130

455 Overlook Ave.

Washington, DC 20375

David Stevenson

Stanford Univ.

Dept. of Materials Science

Stanfard, CA 94305

Gerald W. Stewart

U.S. Dept. of Energy, Morgantown

Energy Technology Center

P. 0. Box 880

Morgantown, WV 26505

Charter D. Stinespring

U.S. Dept. of Energy

Morgantown Energy Technology Ctr.

P.0. Box 880

Morgantown, WV 26505

E. K. Storms

Los Alamos Scientific Lab.

Los Alamos, NM

David Tevault

Naval Research Lab.

Code 6130

Washington, DC 20375

Stephen S. C. Tong

Corning Glass Works

Sullivan Park

Corning, NY 14830

W. Tsang

National Bureau of Standards

Washington, DC 20234

John Vitko, Jr.

Sandia Laboratories

Div 8342

Livermore, CA 94550

Harue Wada

University of Michigan

4227 East Engineering, Dept.

Mats. and Met. Engineering

Ann Arbor, MI 48109 
Donald Wagman

National Bureau of Standards

Center for Thermodynamics \&

Molecular Science

Washington, DC 20234

\section{B. Webb}

Propellants, Explosives and Rocket

Motor Establishment

Westcott, Aylesbury

Buckinghamshire, England

William Weltner, Jr.

Univ. of Florida

Dept. of Chemistry

Gainesville, FL 32611

Jack Wenze 1

National Bureau of Standards

B1dg. 223

Washington, DC 20234

Dale E. Work

GTE Sylvania

100 Endicott St.

Danvers, MA 01923

Joda Wormhoudt

Aerodyne Research Inc.

Crosby Drive

Bedford, MA 01730

Perry P. Yaney

Dept. of Physics

Univ. of Dayton, 300 College Park

Dayton, $\mathrm{OH} 45469$

L. N. Yannopoulos

Westinghouse Res. \& Dev. Center

Churchi11 Boro

Pittsburgh, PA 15235

Robert J. Zollweg

Westinghouse R \& D Center

1310 Beulah Road

Pittsburgh, PA 15235 



\section{AUTHOR INDEX}

Allen, Jr., J. E. ...... 1153

Allibert, M. . . . . . . 181

Anderson, W. R. . . . . 1153

Arbach, G. U. . . . . . 885

Babel ot, J. F. ...... 83

Baronavski, A. P. . . . . . 817

Beachey, Jocob E. . . . . . 431

Beattie, I. R. . . . . . 597

Behrens, Robert G. . . . . 125

Berkowitz, J. . . . . . . 757

Blander, M. . . . . 1597,1707

Bonne11, David W. . . . . 357

Bouix, J. . . . . . . . 899

Bower, J. . . . . . . . 1079

Bowling, R. A. . . . . . 771

Bradshaw, J. . . . . . 1079

Brostow, W. . . . . . . 1619

Cater, E. David . . . . . 3237

Cercignani, L. . . . . . . 83

Chan, C. . . . . . . . . 847

Chase, M. W. . . . . . . . 1581

Chatilion, C. ...... 181

Chatwani, A. U. . . . . . 1373

Chidsey, I. L. . . . . . . 1181

Creighton, J. R. . . . . . 1223

Crosley, D. R. …1153,1171,1181

Curtiss, L. A. . . . . . 791

Daily, J. W. ........ 847

Davidson, Ernest R. . . . . 545

De La Mora, J. . . . . . . . . 1451

DeWilde, M. A. . . . . . . 1171

Dils, R. R. . . . . . . 1027

Downey, J. R. . . . . . . 1581

Drake, Michael C. . . . 609,1105

Dun, Haiping . . . . . . 411

Eckbreth, A. C. . . . . . 943

Edwards, Jimmie G. . . . . . 67

Fansler, T. B. . . . . 1153

Ferron, J. R. . . . . . 1373

Feugier, A. . . . . . . . 1315

Follansbee, P. S. . . . . . 1027

Fontijn, A. . . . . . . . . 1359

Forsman, James. A. . . . . 211

Fries, James E. . . . . . 237

Fristrom, R. M. . . . . 1071

Froben, F. W. . . . . . 523

Frurip, D. J. . . . . . 1597

Fryburg, George C. . . . 303,1451

Fujiwara, K. . . . . . . 1079

Gardiner, Jr., W. C. . . . . 835

Gersh, M. E. . . . . . 679

Gevantman, L. N. . . . . . 157
Gilles, Paul W. . . . . . . 231

Gingerich, Karl A. . . . . . 289

Grabner, L. H. . . . . . . . 1105

Gransden, S. E. . . . . . . . 557

Greene, Frank T. . . . . . . 431

Greehalch, D. A. . . . . . . 597

Grimley, Robert T. . . . . . 211

Gruen, D. M. . . . . . . . . . 1517

Grunfelder, C. . . . . . . 1071

Gulbransen, E. A. . . . . . . 1639

Hall, R. J. ......... 943

Haller, W. K. . . . . . . 111

Haraguchi, H. . . . . . . . 1079

Hardesty, D. R. . . . . . . . 1407

Hargis, Jr., P. J. . . . . . . . 1135

Harvey, A. B. . . . . . . . . 817

Hastie, John W. . . . . . 357,1105

Hauge, R. H. . . . . . . . 495,557

Hildenbrand, D. L. . . . . . . . 171

Hillel, R. ......... 899

Hirayama, C. . . . . . . . . 1539

Hosha11, C. H. ........ 1071

Hsu, C. C. . . . . . . . 1683

Hunter, L. W. . . . . . . . 1071

Inomata, T. . . . . . . . . 1327

Ip, P. . . . . . . . . 1161

Jacox, Marilyn E. . . . . . . 529

Jensen, D. E. . . . . . . 1195

Johnson, C. E. . . . . . . . 1683

Just, T. H. . . . . . . . . 1339

Kauffman, J. W. . . . . . . 557

Kerr, D. L. . . . . . . . 1373

Kinsman, P. R. . . . . . . . 83

Klotzbucher, Werner, E. .... 567

Knight, L. B. . . . . . . . 545

Kobayashi, Michio . . . . . 283

Kolb, C. E. . . . . . . 4 457,679

Koh1, Fred J. ...... 303, 1451

Korobeinichev, 0. P. . . . . . 479

Lamont, S. . . . . . . . . 817

Larzilliere, Michael ..... 529

Lassiter, T. W. . . . . . . 771

Lide, Jr., D. R. . . . . . . 1571

Lin, C. Y. . . . . . . . . . 1539

Lin, M. C. . . . . . . . . 1249,1551

Long, K. A. . . . . . . . . . 83

Lund, C. M. . . . . . . . . . . 1223

Machaelides, A. . . . . . . . 899

Magi11, J. .......... 83

Mallard, W. G. ...... 865

Margrave, J. L. . . . . . 4 495,557

Martin, R. L. ...... . 545 
Mattes, Brenton L. . . . . . . 411

McDonald, J. R. . . . . . . . 817

Meier, G. H. ......... 1639

Melton, L. A. . . . . . . . 153

Miller, Federico ....... . 39

Miller, G. H. .......... . 1135

Miller, Robert A. ....... 303

Miller, W. J. . . . . . . . 443

Milne, Thomas A. . . . . . . 431

Moriwaki, T. . . . . . . . . 1327

Mulac, A. J. ......... . 1135

Mueller, B. A. . . . . . 143,237

Mueller, Barbara H. . . . : . 237

Muller, III, C. H. ....... 855

Ogden, J. S. .......... 511

Ohse, R. W. . . . . . . . . 83

Okazaki, S. . . . . . . . . 1327

Omenetto, N. ......... 1079

Orlovskii, V. P. . . . . . . . 1497

Øye, H. A. . . . . . . . . 1517

Ozin, Geoffrey A. ....... 567

Papatheodorou, G. N. . . . . . 647

Pasternack, L. . . . . . . . 817

Pattoret, A. . . . . . . . . 181

Plante, E. R. ........ 265

Poh 1, J.W. ......... 1407

Pratt, D. T. ......... 1265

Reuther, J. J. ......... 1281

Rinehart, Gary H. . . . . . . 125

Roquemore, W. M. . . . . . . 973

Rosenblatt, Gerd M. . . . . . 609

Rosner, D. E. ........ 1451

Roth, P. .......... 1339

Sanders, D. M. . . . . . . . 111

Santoro, G. . . . . . . . 1451

Sasamoto, Tadashi ...... 283

Sata, Toshiyuki .. . . . . . 283

Schafer, H. . . . . . . . . 1507

Schenck, P. K. . . . . . . . 865

Schofield, K. . . . . . . . 855

Schweitzer, G. K. . . . . . . 771

Scotti, A. ......... . 83

Sedgwich, T. 0. . . . . . . . 885

Self, S. A. . . . . . . . 915

Seshadri, K. . . . . . . . 1451

Shaub, W. M. . . . . . . . . 1249

Shelton, Robert I. . . . . . . 231

Smyth, K. C. . . . . . . . 865

Spiridonov, V. P. . . . . . . 711

Stearns, Carl A. ..... 303, 1451

Steinberg, M. . . . . . . . 855

Stevenson, David A. . . . . . 411

Storms, E. K. . . . . . . . 143

Strauss, H. L. . . . . . . . 1611

Suzuki, M. ........... 1327

Syverud, A. N. . . . . . . . . 1581
Taylor, J. E. .......... 1353

Tereshenko, A. G. . . . . . . 479

Tevault, D. ........... . 1551

Tiemann, E. . . . . . . . . . 695

Torring, T. . . . . . . . . 695

Travis, J. C. .......... 865

Vick, D. 0. . . . . . . . . 771

Weeks, S. . . . . . . . . . 1079

Weltner, Jr., William . . . . . 587

White, Jr., H. J. . . . . . . 1571

Winefordner, J. D. . . . . . . . 1079

Wormhoudt, J. C. . . . . . . . 457

Yaney, P. O. . . . . . . . . 973

Zasorin, E. Z. .......... 711

Zollweg, R. J. ......... . 1477 
NBS-114A (REV. $9-78)$

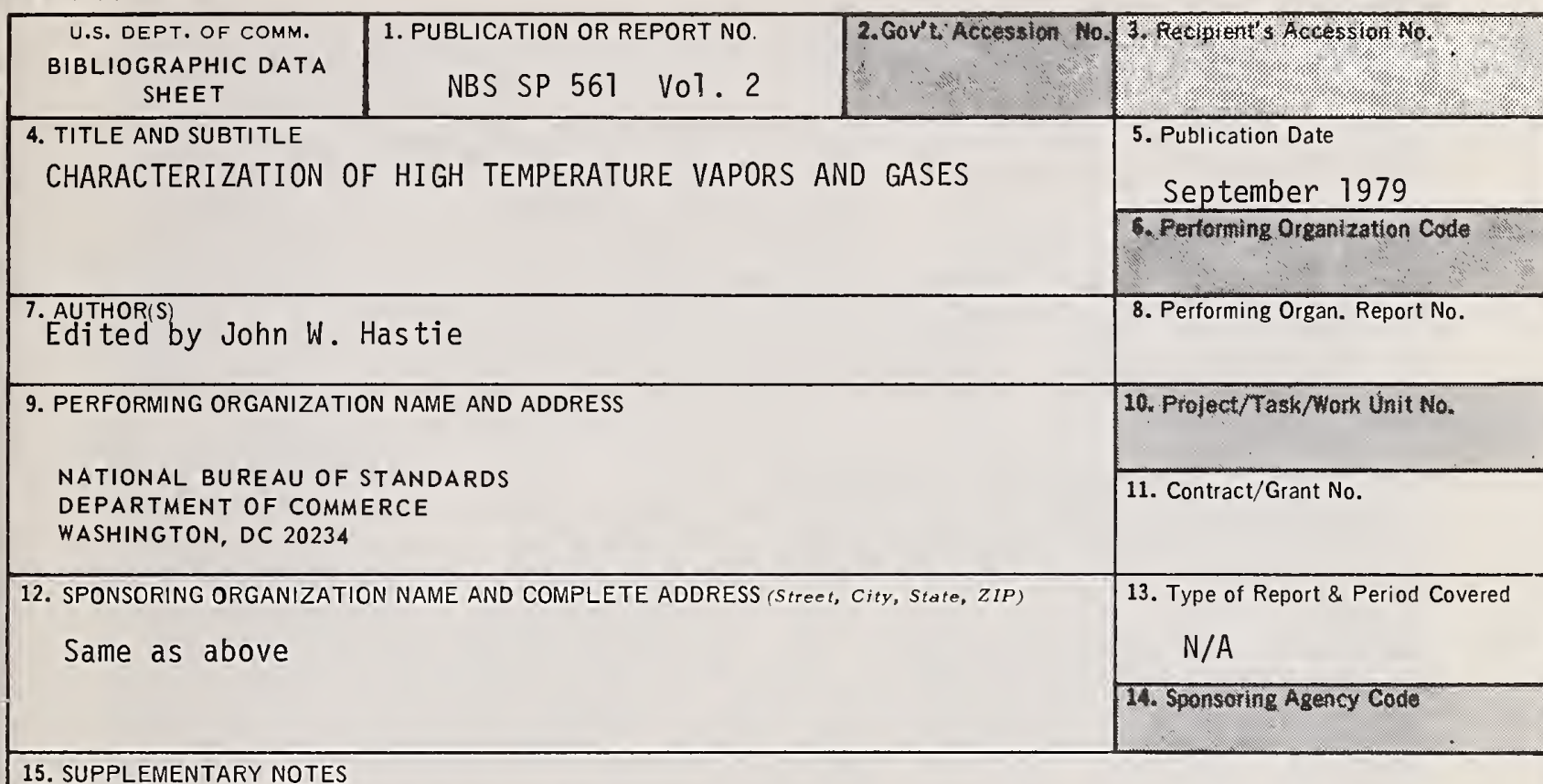

Library of Congress Number 79-600152

$\square$ Document describes a computer program; SF-185, FIPS Software Summary, is attached.

16. ABSTRACT (A 200-word or less tactual summary of most significant information. If documeat includes a significant bibliography or literature survey, mention it here.)

This book is based on the proceedings of the 10th Materials Research Symposium, held at the National Bureau of Standards, Gaithersburg, Maryland, September 18-22, 1978. The symposium was sponsored by the National Measurement Laboratory (formerly Institute for Materials Research). Modern technology increasingly requires materials and processes to function at high temperatures -- a condition where the vapor, gaseous or plasma phase becomes significant. Characterization of such atmospheres, or components thereof, is a challenging problem requiring adaptation of existing and development of new experimental and theoretical techniques. The aim of the symposium was to assess the state-of-the-art ard future directions, and characterization methods for high temperature vapors including, where appropriate, gases and, to a lesser extent, plasmas. In order to prevent, as far as possible, serious omissions of important new or improved techniques, a number of papers not delivered at the Symposium were solicited for inclusion in this publication. A total of eighty-six papers (invited and contributed) or chapters representing nine countries are contained in these volumes. The discussion, which followed most of the delivered papers, is also included.

17. KEY WORDS (six to twelve entries; alphabetical order; capitalize only the first letter of the first key word unleas a proper namo; separated by semicolons)

Combustion; equilibrium; high temperature; isolation; lasers; mass spectrometry; matrix; modeling; vapors
18. AVAILABILITY
x] Unlimited

For Official Distribution. Do llot Release to NTIS

[X] Order From Sup. of Doc., U.S. Government Printing Office, Washington, DC 20402, SD Stock No. SNO03-003-02124-5

$\square$ Order From National Technical Information Service (NTIS), Springfield, VA. 22161

\begin{tabular}{|l|c|}
\hline $\begin{array}{l}\text { 19. SECURITY CLASS } \\
\text { (THIS REPORT) } \\
\text { UNCLASSIFIED }\end{array}$ & $\begin{array}{c}\text { 21. NO. OF } \\
\text { PRINTED PAGES }\end{array}$ \\
\hline $\begin{array}{l}\text { 20. SECURITY CLASS } \\
\text { (THIS PAGE) }\end{array}$ & 22. PrICe \\
UNCLASSIFIED & $\$ 20.00 /$ SET \\
\hline
\end{tabular}




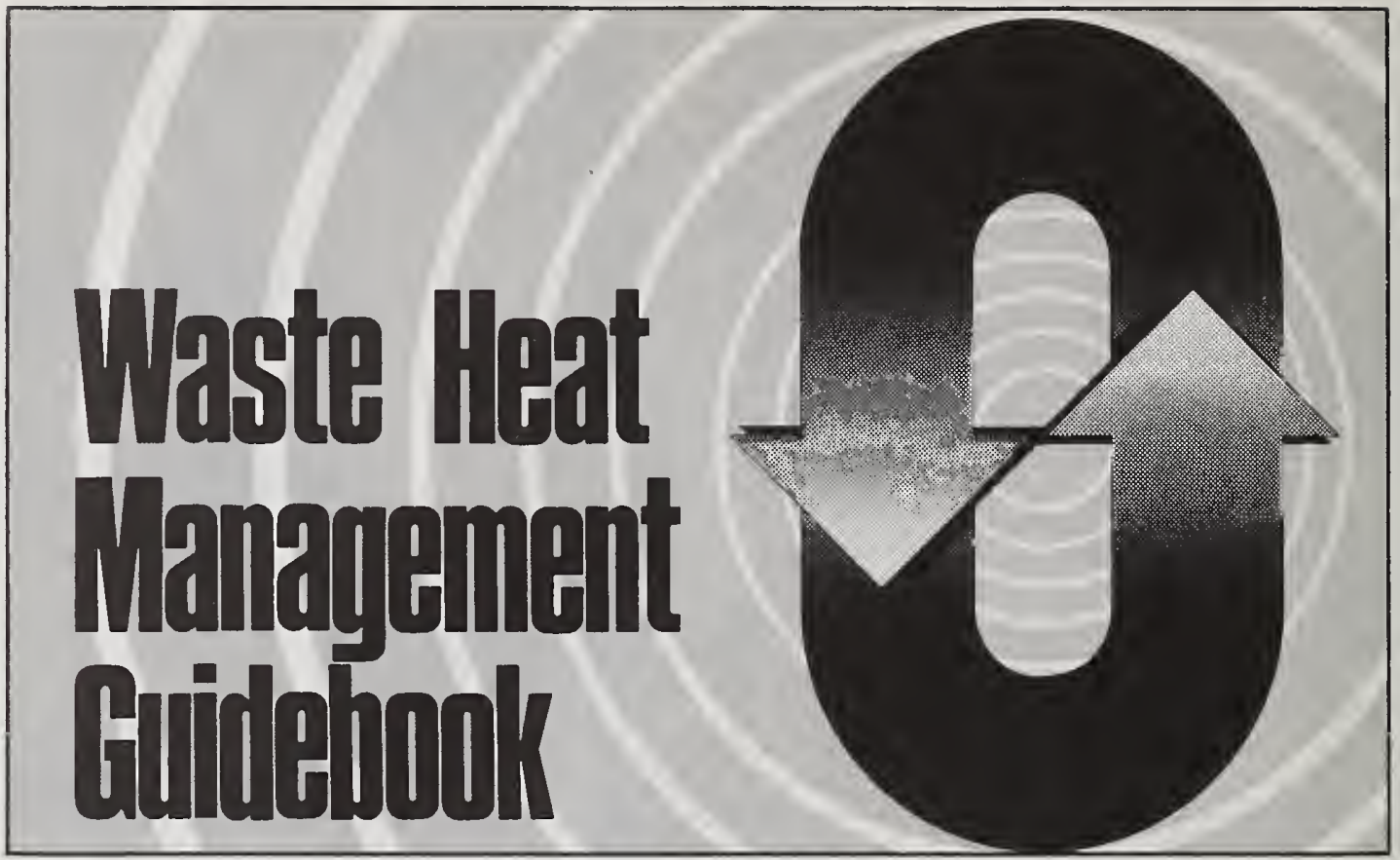

A typical plant can save about 20 percent of its fuel-just by installing waste heat recovery equipment. But with so much equipment on the market, how do you decide what's right for you?

Find the answers to your problems in the Waste Heat Management Guidebook, a new handbook from the Commerce Department's National Bureau of Standards and the Federal Energy Administration.

The Waste Heat Management Guidebook is designed to help you, the cost-conscious engineer or manager, learn how to capture and recycle heat that is normally lost to the environment during industrial and commercial processes.

The heart of the guidebook is 14 case studies of companies that have recently installed waste heat recovery systems and profited. One of these applications may be right for you, but even if it doesn't fit exactly, you'll find helpful approaches to solving many waste heat recovery problems.

In addition to case studies, the guidebook contains information on:

- sources and uses of waste heat

- determining waste heat requirements

- economics of waste heat recovery

- commercial options in waste heat recovery equipment

- instrumentation

- engineering data for waste heat recovery

- assistance for designing and installing waste heat systems

To order your copy of the Waste Heat Management Guidebook, send \$2.75 per copy (check or money order) to Superintendent of Documents, U.S. Government Printing Office, Washington, D.C. 20402 A discount of 25 percent is given on orders of 100 copies or more mailed to one address.

The Waste Heat Management Guidebook is part of the EPIC industrial energy management program aimed at helping industry and commerce adjust to the increased cost and shortage of energy.

U.S. DEPARTMENT OF COMMERCE/National Bureau of Standards FEDERAL ENERGY ADMINISTRATION/Energy Conservation and Environment 


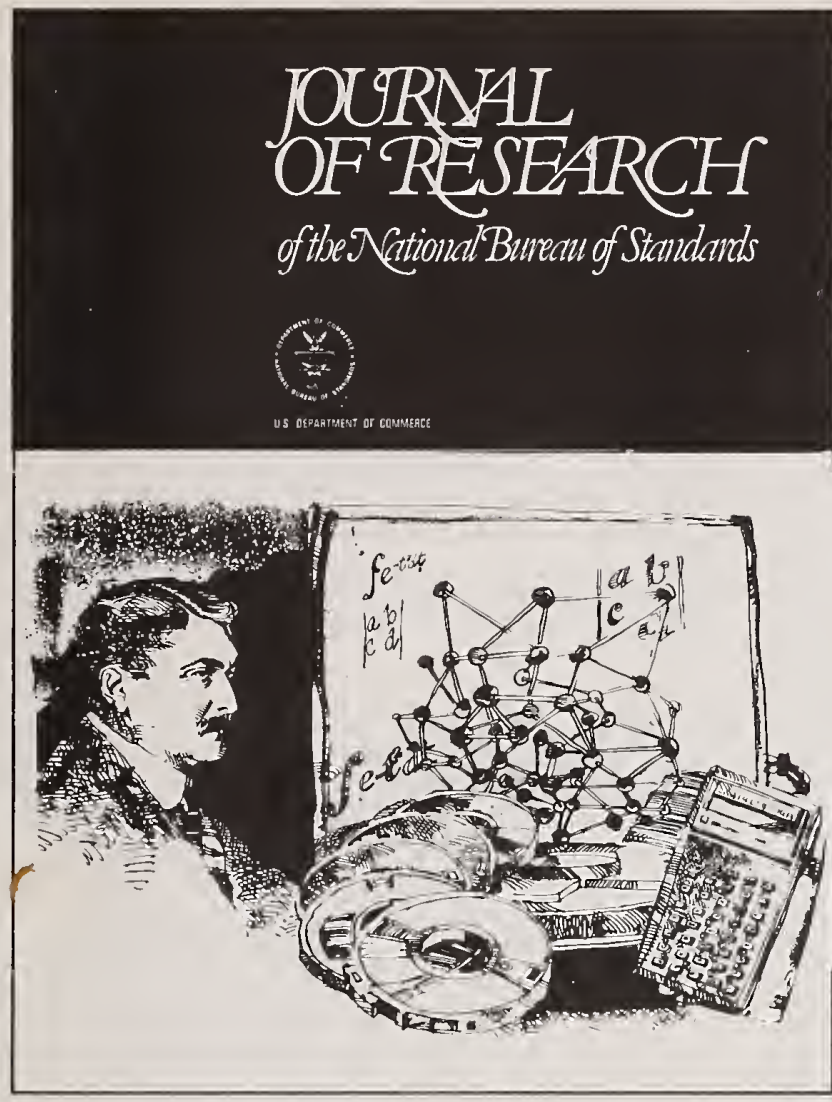

Subseribe nowThe new Tational Bureau of Standards Journal

The expanded Journal of Research of the National Bureau of Standards reports NBS research and development in those disciplines of the physical and engineering sciences in which the Bureau is active. These include physics, chemistry, engineering, mathematics, and computer sciences. Papers cover a broad range of subjects, with major emphasis on measurement methodology, and the basic technology underlying standardization. Also included from time to time are survey articles on topics closely related to the Bureau's technical and scientific programs. As a special service to subscribers each issue contains complete citations to all recent NBS publications in NBS and nonNBS media. Issued six times a year. Annual subscriptions: domestic $\$ 17.00$; foreign $\$ 21.25$. Single copy, $\$ 3.00$ domestic; $\$ 3.75$ foreign.

- Note: The Journal was formerly published in two sections: Section A "Physics and Chemistry" and Section B "Mathematical Sciences."

NBS Board of Editors

Churchill Eisenhart,

Executive Editor (Mathematics)

John W. Cooper (Physics)

Donald D. Wagman (Chemistry)

Andrew J. Fowell (Engineering)

Joseph O. Harrison (Computer Science)

Howard J. M. Hanley (Boulder Labs.)

Subscription Order Form

Enter my subscription to NBS Journal of Research

at $\$ 17.00$. Add $\$ 4.25$ for foreign mailing. No additional postage is

required for mailing within the United States or its possessions.

(SJRA-File Code 2N)

Send Subscription 10

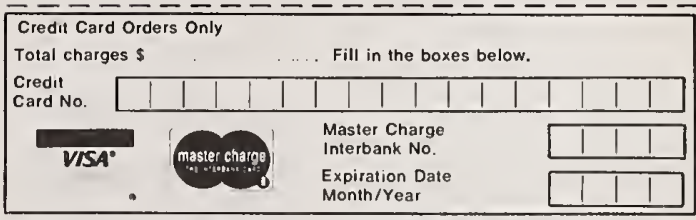

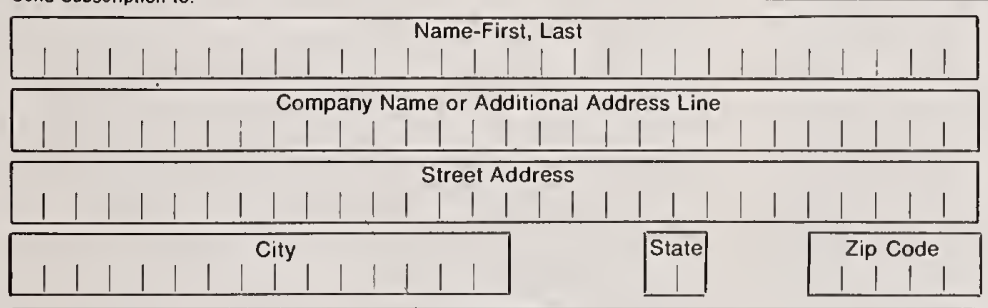

(or) COUNTRY

\begin{tabular}{llllllllllllllllllllll}
\hline & 1 & 1 & 1 & 1 & 1 & 1 & 1 & 1 & 1 & 1 & 1 & 1 & 1 & 1 & 1 & 1 & 1 & 1 & 1 & 1 \\
\hline
\end{tabular}

PLEASE PRINT

$\square$ Remittance Enclosed. Domestic: Check or money order. Foreign: International money order. draft on an American or Canadien Bank, or by UNESCO coupons. Made payable to the Superintendent of Documenls.

$\square$ Charge to my Deposit Accouni No.

MAIL ORDER FORM TO: Superintendent of Documents Government Printing Office Washington, D.C. 20402 



\section{PERIODICALS}

JOURNAL OF RESEARCH-The Journal of Research of the National Bureau of Standards reports NBS research and development in those disciplines of the physical and enginecring scicnces in which the Bureau is active. These include physics, chemistry, engineering, mathematics, and computer sciences. Papers cover a broad range of subjects, with major emphasis on measurement methodology, and the basic technology underlying standardization. Also included from time to tine are survey articles on topics closely related to the Bureau's technical and scientific programs. As a special service to subucribers each issue contains complete citations to all recent NBS publications in NBS and nonNBS media. Issued six times a year. Annual subeription: donrestic $\$ 17.00$; foreign $\$ 21.25$. Single copy, $\$ 3.00$ domestic; $\$ 3.75$ foreign.

Note: The Journal was formcrly published in two sections: Section A "Physics and Chemistry" and Section B "Mathematical Sciences."

\section{DIMENSIONS/NBS}

This monthly magazine is published to inform scientists, engineers, businessmen, industry, teachers, students, and consumers of the latest advances in science and technology, with primary emphasis on the work at NBS. The magazine highlights and reviews such issues as cnergy research, fire protection, huilding technology, metric conversion, pollution abatement, health and safety, and consumer product performance. In addition, it reports the results of Bureau prograns in measurement standards and techniques, properties of matter and materials, engineering standards and services, instrumentation, and automatic data processing.

Annual subscription: Domestic, \$11.00; Foreign $\$ 13.75$

\section{NONPERIODICALS}

Monographs-Major contributions to the technical literature on various subjects related to the Bureau's scientific and technical activities.

Handbooks-Recommended codes of engineering and industrial practice (including safety codes) developed in cooperation with interested industries, professional organizations, and regulatory bodies.

Special Publications-Include proccedings of conferences sponsored hy NBS, NBS annual reports, and other special publications appropriate to this grouping such as wall charts, pocket cards, and bibliographies.

Applicd Mathematics Series-Mathematical tables, manuals, and studies of special intercst to physicists, engineers, chcmists, biologists, niathematicians, computer progranmers, and others engaged in scientific and technical work.

National Standard Reference Data Series-Provides quantitative data on the physical and chemical properties of materials, compiled from the world's literature and critically cvaluated. Developed under a world-wide program coordinated by NBS. Program under authority of National Standard Data Act (Public Law 90-396).
NOTE: At present the principal publication outlet for these data is the Journal of Physical and Chemical Reference Data (JPCRD) published quarterly for NBS by the American Chemical Society (ACS) and the American Institute of Phy'sics (AIP). Subscriptions, reprints, and supplements available from ACS, 1155 Sixtecnth St. N.W., Wash., D.C. 20056.

Building Science Stries-Disseminates technical information developed at the Bureau on building materials, components, systems, and wholc structures. The series presents research results, test methods, and performance criteria related to the structural and environmental functions and the durability and safety characteristics of building elements and systems. Technical Notes-Studies or reports which are complete in themselves but restrictive in their trcatment of a subject. Analogous to monographs but not so comprehensive in scope or definitive in treatment of the subject area. Often scrve as a vehicle for final reports of work performed at NBS under the sponsorship of other government agencies. Voluntary Product Standards-Developed under procedures published by the Department of Commerce in Part 10, Title 15, of the Code of Federal Regulations. The purpose of the standards is to establish nationally recognized requirements for products, and to provide all concerned interests with a basis for conmon understanding of the characteristics of the products. NBS administers this program as a supplement to the activities of the private sector standardizing organizations.

Consumer Information Series-Practical information, based on NBS research and experience, covering areas of interest to the consumer. Easily understandable language and illustrations provide useful background knowledge for shopping in today's technological marketplace.

Order above NBS publications from: Superintendent of Documents, Government Printing Office, Washington, D.C. 20402

Order following NBS publications-NBSIR's and FIPS from the National Technical Information Services, Springficld, Va. 22161.

Federal Information Processing Standards Publications (FIPS PUB) - Publications in this scries collectively constitute the Federal Information Processing Standards Register. Register serves as the official source of information in the Federal Government regarding standards issued by NBS pursuant to the Federal Property and Administrative Services Act of 1949 as amended, Public Law 89-306 (79 Stat. 1127), and as implcmented by Fxecutive Order 11717 (38 FR 12315, lated May 11, 1973) and Part 6 of Title 15 CFR (Code of Federill Regulations).

NBS Interagency Reports (NBSIR)-A special scries of interim or final reports on work performed by NBS for outside sponsors (both govermment and non-government). In general, initial distribution is handled by the sponsor; public distrihution is by the National Tcchnical Information Services (Springfield, Va. 22161) in papcr copy or microfiche form.

\section{BIBLIOGRAPHIC SUBSCRIPTION SERVICES}

The following current-akareness and literature-survey bibliographies are issued periodically by the Bureau:

Cryogenic Data Center Current Awareness Sen ice. A literature survey issued biweekly. Annual subscription: Domestic, $\$ 25.00$; Foreign, $\$ 30.00$.

Liquefied Natural Gas. A literature survey issucd quarterly. Annual suhscription: $\$ 20.00$.
Superconducting Devices and Materials. A literature survey issued quartcrly. Annual subscription: $\$ 30.00$. Send subscription ordcrs and remittances for the preceding bithliographic scrvices to National Bureau of Standards, Cryogenic Data Center (736.00) Boulder, Colorado 80303. 


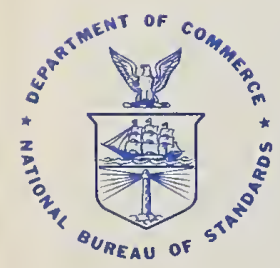


